\title{
PHYSICIANS POSTER SESSIONS
}

P375

Prophylactic DLI vs no DLI for refractory acute leukemia undergoing allo-HSCT with sequential intensified conditioning

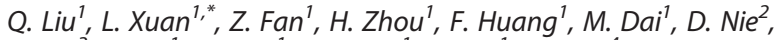

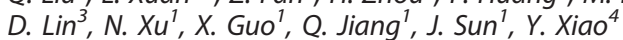

${ }^{1}$ Department of Hematology, Nanfang Hospital, Southern Medical University, ${ }^{2}$ Department of Hematology, Sun Yat-Sen Memorial Hospital, Sun Yat-Sen University, ${ }^{3}$ Department of Hematology, the Third Affiliated Hospital, Sun Yat-Sen University, ${ }^{4}$ Department of Hematology, Guangzhou General Hospital of Guangzhou Military Command, Guangzhou, China

Introduction: The major obstacle is leukemia relapse for refractory leukemia undergoing allogeneic hematopoietic stem cell transplantation (allo-HSCT). We previously introduced a strategy of sequential intensified conditioning and early rapid immunosupressant withdrawal for refractory leukemia undergoing allo-HSCT, with 5-year overall survival (OS) and 3-year relapse rate of $44.6 \%$ and $33.3 \%$. To reduce leukemia relapse, prophylactic donor lymphocyte infusion (DLI) was administered based on our historical strategy.

Material (or patients) and methods: We conducted a prospective multi-center study to assess the efficacy of the new strategy for refractory advanced acute leukemia. Sequential intensified conditioning regimen was: fludarabine $(30 \mathrm{mg} /$ $\mathrm{m}^{2} /$ day, -10 to -6 days $)+$ cytarabine $\left(2.0 \mathrm{~g} / \mathrm{m}^{2} /\right.$ day, -10 to -6 days) plus total body irradiation (4.5 Gy/day, $-5,-4$ days) +cyclophosphamide $(60 \mathrm{mg} / \mathrm{kg} /$ day, $-3,-2$ days)+etoposide (15 mg/kg/day, $-3,-2$ days). Immunosupressant was withdrawn by $10 \% /$ week in patients without acute graft-versus-host disease (aGVHD) by day +30 post-transplantation. For patients without grade II/ $>$ II aGVHD by day +60 post-transplantation, DLI was administered at a median dose of $1.0 \times 10^{8}$ mononuclear cells $/ \mathrm{kg}$ if donor lymphocytes were available. DLI was given once to all patients regardless of minimal residual disease (MRD), and was then administered based on GVHD and MRD status. If patients were MRD negative, DLI was not given again; if patients were MRD positive and without GVHD, DLI was given monthly until GVHD occurred or MRD became negative or for a total of four times.

Results: A total of 153 patients with refractory advanced acute leukemia undergoing allo-HSCT from January 2009 to June 2014 were enrolled. All patients achieved hematopoietic reconstitution except for two who died of infection and two who died of RRT within 2 weeks post-transplantation. At the time of neutrophil reconstitution, all 149 evaluable patients achieved complete remission. The incidence and mortality of regimen-related toxicities were $100.0 \%$ and $1.3 \%$. The 5 -year overall and disease-free survival post-transplantation were $51.1 \% \pm 5.7 \%$ and $49.2 \% \pm 5.3 \%$. The 5 -year relapse rate and non-relapse mortality (NRM) were $27.3 \% \pm 4.4 \%$ and $29.7 \% \pm 5.3 \%$. According to the availability of donor lymphocytes and the criteria for DLI, 144 patients surviving day +60 were divided into two groups ( $80 \mathrm{DLI}$ versus 64 non-DLI). The relapse rate was less and OS was better in patients receiving $\mathrm{DLI}$ than in those not receiving DLI $(22.7 \%$ vs $33.9 \%, P=0.048$; $58.1 \%$ vs $54.9 \%, P=0.043)$. NRM was similar between DLI and non-DLI groups $(P=0.104)$. Multivariate analysis revealed that lower bone marrow blasts on day 0, DLI and chronic GVHD were associated with less relapse and better OS.
Conclusion: The strategy of sequential intensified conditioning followed by early immunosupressant withdrawal and DLI could reduce relapse of refractory acute leukemia after alloHSCT and improve survival.

Disclosure of Interest: None declared.

\section{P376}

Successful treatment of EBV-triggered malignant B-cell lymphoma after allogeneic SCT with EBV-peptide primed Cytokine-induced killer (CIK) cells - new treatment options?

L.-M. Pfeffermann ${ }^{1, *}$, E. Rettinger ${ }^{1}$, A. Willasch ${ }^{1}$, S. Huenecke ${ }^{1}$, M. Bremm ${ }^{1}$, V. Pfirrmann ${ }^{1}$, A. Jarisch ${ }^{1}$, J. Soerensen ${ }^{1}$, E. Ullrich ${ }^{\prime}$, H. Boenig ${ }^{2}$, T. Klingebiel ${ }^{7}$, P. Bader ${ }^{7}$

${ }^{1}$ University Hospital Frankfurt, Goethe University, Department for Children and Adolescents, Division for Stem Cell Transplantation and Immunology, ${ }^{2}$ University Hospital Frankfurt, Goethe University, Institute for Transfusion Medicine and Immunohematology and German Red Cross Blood Donor Service BadenWuerttemberg-Hessen, Frankfurt/Main, Germany

Introduction: Epstein-Barr virus (EBV) reactivation after allogeneic SCT can lead to life-threatening lymphoproliferative disease (PTLD). Development of PTLD is often favored by prolonged immunosuppression post-transplant or by delayed T-cell recovery. Treatment options are limited by reduction of immune suppression, Rituximab transfusion or the application of donor lymphocytes (DLI) or, if possible by the application of EBV-specific T-cells. In patients who did not respond to such treatment, mortality is high and the progression of PTLD might only be stopped by high dose chemotherapy.

A potential novel treatment approach are CIK cells with dual specific cytotoxic capacity which might transfer anti-cancer potential and donor T-cell memory to confer protection against virus infections and treatment of malignancies. Here we report our experience of EBV-specific CIK cell treatment in a patient with an EBV-associated PTLD who had received an allogeneic HSCT for secondary myelodysplastic syndrome following acute myeloid leukemia.

Material (or patients) and methods: EBV-related PTLD occurred during delayed T-cell recovery two months after HSCT. Rituximab was given 4 times, but treatment failed. Immunomagnetic isolation of specific EBV T-cells was hampered by minuscule frequencies of circulating target cells. Only $0.1 \times 10^{3}$ specific T-cells/kg could be isolated from $10^{9}$ WBCs. The patient did not respond and lymphoma progressed. Histopathology showed a monoclonal, highly proliferative large-cell B-NHL. The patient received conventional CIK-cell treatment $\left(1 \times 10^{6}\right.$ T-cells/ $\mathrm{kg}$ ), but EBV driven lymphoma still progressed.

Results: EBV-specific CIK cells were generated from unmobilized leukapheresis $\left(1,7 \times 10^{8}\right.$ WBC total) via stimulation with IFN $\gamma$, IL-2, anti-CD3 and IL-15 under GMP-conditions over a period of 10 days. EBV peptide pool (EBV-select, Miltenyi Biotec) was introduced on day 0 and 2 during culture period. EBV-specific CIK cells $\left(\mathrm{CD}^{+}\right.$and $\mathrm{CD} 8^{+}$) were detected by flow cytometry using specific MHC-dextramers prior and post infusion. Cytotoxic capacity of generated cells was analyzed by europium release assay.

At culture start EBV-specific donor T-cells were barely detectable (5 CD8 ${ }^{+}$EBV-specific T-cells total, $0.02 \%$ of $C D 8^{+}$). However, due to stimulation, we observed a proliferation rate 
of 130 within 10 days resulting in $0.1 \% \mathrm{CD}^{+}$and $0.3 \% \mathrm{CD}^{+}$ EBV-specific cells. In vitro cytotoxicity analysis showed specific lysis of $97.1 \%, 77.0 \%, 60.1 \%$ and $10.5 \%$ against EBV loaded target cells at effector to target ratios of 40:1, 20:1, 10:1 and $5: 1$, respectively, while the anti-tumor potential was retained (K562: 79.5\%, 55.9\%, 41.7\% and 19.3\% and THP-1: $41.7 \%$, $41.2 \%, 39.6 \%$ and $35.1 \%)$.

In total, the patient received a CIK-cell dose of $10 \times 10^{6} / \mathrm{kg}$ including $1 \times 10^{4} / \mathrm{kg} \mathrm{CD} 8^{+}$EBV-specific CIK cells. Treatment with EBV-specific CIK cells resulted in rapid clearance of plasma EBV DNA and large (vol. $27 \mathrm{~cm}^{3}$ ) PTLD-malignant lymphoma disappeared within 7 days after infusion. EBV-specific cells were detectable within the $\mathrm{CD}^{+}$and $\mathrm{CD}^{+}{ }^{+} \mathrm{T}$-cell compartment after infusion. Interestingly, the ratio of $\mathrm{CD}^{+}$and $\mathrm{CD} 8^{+}$ EBV-specific T-cells changed after 2 weeks $\left(1.2 \% \mathrm{CD}^{+}\right.$and $\left.4.1 \% \mathrm{CD}^{+}\right)$. On day 26 , the ratio switched again and $\mathrm{CD}^{+}$ EBV-specific cells were re-dominantly present $\left(2.1 \% \mathrm{CD}^{+}\right.$and $\left.0 \% \mathrm{CD}^{+}\right)$

Conclusion: EBV-specific CIK cells successfully cleared aggressive, highly proliferative $\mathrm{NHL}$.

Disclosure of Interest: None declared.

P377

Human lymphoid tissues harbor a distinct CD69+CXCR6+ natural killer cell population

G. Lugthart ${ }^{1}$, J. Melsen ${ }^{1}$, C. Vervat ${ }^{1}$, M. van Ostaijen-ten Dam ${ }^{1}$, W. Corver ${ }^{2}$, D. Roelen ${ }^{3}$, J. van Bergen ${ }^{3}$, M. van Tol ${ }^{\prime}$, A. Lankester ${ }^{\prime}$, M. W. Schilham ${ }^{1, *}$

${ }^{1}$ Pediatrics, ${ }^{2}$ Pathology, ${ }^{3}$ Immunohematology and Blood transfusion, Leiden University Medical Center, Leiden, Netherlands

Introduction: In recent years, evidence has been provided that natural killer (NK) cells function in the regulation of immune responses. In humans, two NK cell populations are usually distinguished: CD56dimCD16+ NK cells form the predominant population in blood while the CD56brightCD16- NK cell population is more prominent in tissues. Little data regarding the phenotype of human NK cells in lymphoid tissues are available, especially in bone marrow as an important localization of leukemic cells. Therefore, we evaluated the expression of chemokine receptors and adhesion molecules on NK cells in healthy donor blood, bone marrow, spleen and lymph node.

Material (or patients) and methods: Mononuclear cells were isolated from blood, bone marrow, spleen and lymph node by Ficoll gradient centrifugation. Cells were stained with combinations of monoclonal antibodies and analyzed by flow cytometry. For stimulation experiments, cells were incubated with medium or stimulating cytokines and analyzed by intracellular staining for interferon-gamma production or CD107a staining for degranulation. For cytotoxicity experiments, cells were FACS-sorted to purity and used as effector cells in a $51 \mathrm{Cr}$ release assay. NK cell reconstitution was evaluated in marrow of pediatric acute leukemia patients who received an unmanipulated bone marrow $(n=4)$ or peripheral blood stem cell $(n=2)$ transplantation from an HLA-matched unrelated donor after myeloablative conditioning.

Results: Based on the combined expression of CD69 and CXCR6, we identified a major third NK cell subset in lymphoid tissues with a distinct immunophenotype and function. CD69 +CXCR6+ NK cells are absent from blood and represent 30-60\% of NK cells in marrow, spleen and lymph node. Phenotypically, CD69+CXCR6+ lymphoid tissue NK cells are distinct from conventional NK cells and have an intermediate expression of CD56 and high expression of NKp46 and ICAM-1. They lack the early markers c-kit and IL7Ra, as well as killer-cell immunoglobulin-like receptors or other markers acquired late in differentiation. Furthermore, they show a bi-modal expression of the activating receptor DNAM-1. After cytokine stimulation, CD69 + CXCR6+ lymphoid tissue NK cells produce interferon- $\gamma$ at levels comparable to CD56dim NK cells. They constitutively express perforin but require pre-activation to express granzyme $\mathrm{B}$ and exert cytotoxicity. After hematopoietic stem cell transplantation, CD69+CXCR6+NK cells do not exhibit the hyper-expansion as observed for both conventional NK cell populations.

Conclusion: Additional studies in patients with immunohematological diseases are needed to investigate a potential therapeutic function of CD69+CXCR6+ NK cells. The identification of this distinct CD69+CXCR6+ NK cell population provides tools to further evaluate the role of NK cells in human immunity.

Disclosure of Interest: None declared.

\section{P378}

Evaluation of the Donor-Derived Hepatocyte Cell Repopulation in Co-transplantation of HSCT with Mesenchymal Stem Cells in Major Thalassemia Class III

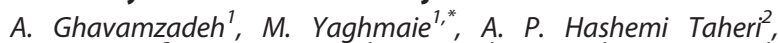
M. Sotoodeh ${ }^{3}$, A. A. Hamidieh ${ }^{7}$, F. Shahi ${ }^{4}$, M. Jalili ${ }^{7}$, A. Kasaeian ${ }^{1}$, M. Jahani ${ }^{1}$, S. A. Mousavi ${ }^{1}$, B. Bahar ${ }^{1}$, K. Alimoghaddam ${ }^{1}$

${ }^{1}$ hematology,oncology and stem cell transplantation research center, ${ }^{2}$ Department of Radiology, shariati hospital, ${ }^{3}$ Pathology Departement, Shariati Hospital, ${ }^{4}$ Cancer Institute - Imam Khomeini, Tehran university of medical sciences, tehran, Iran, Islamic Republic Of

Introduction: Mesenchymal stem cells with a capacity for self-renewal and the potential to differentiate into bone, cartilage, marrow stroma and the other tissues have been identified in human bone marrow. In this study we attempted to determine whether circulating stem cells and mesenchymal cells have a similar potential in major thalassemia class III, detect donor-derived hepatocytes in sex-mismatched recipients and assess the effect of tissue injury on the extent of the repopulation.

Material (or patients) and methods: From 126 sex mismatched major Thalassemia class III with the mean age of $14.28 \pm 5.03$ who underwent transplantation since 1991 to 2014 in Hematology, Oncology and Stem Cell Transplantation Research center, 57 patients received co-transplantation of HSCT with mesenchymal stem cells. 8 patients of this group included in our study for liver biopsy after obtaining informed consent form to evaluate the presence of donor-derived epithelial cells and hepatocytes. Five female patients had received transplants from a male donor and three male patients had received transplantion from female donor. Engraftment of hematopoietic stem cell transplantation was verified by cytogenetic analysis. The biopsies were studied for the presence of donor-derived epithelial cells or hepatocytes using fluorescence in situ hybridization of interphase nuclei by $X$ and $Y$ centromeric probes and immunohistochemical staining for cytokeratin, CD45 (leukocyte common antigen), and a hepatocyte-specific antigen.

Results: In the present study, all sex-mismatched tissue samples demonstrated donor-derived hepatocyte in dependent of donor gender. XY-positive epithelial cells or hepatocytes in female recipient accounted for 11 to 25 percent of the cells in histologic sections of the biopsy specimens in first follow-up (around 2 years after HSCT) and $47-95 \%$ in second follow-up (around 5 years after HSCT) and in male recipient accounted for 4 to 11 percent of the cells in first follow-up and 18 to 70 percent in second follow-up.These cells were detected in liver tissue as early as day 743 and as late as day 2370 after co-transplantation of peripheral blood with mesenchymal stem cells. The presence of donor cells in the biopsy specimens did not seem to depend on the intensity of tissue damage induced by graft-versus-host disease.

Conclusion: The study has shown that some recipient hepatocytes are replaced by donor-derived cells. Circulating stem cells can differentiate into mature hepatocytes and epithelial cells of the liver. The origin of these stem cells and the way in which they generate hepatocytes and epithelial cells have not yet been identified. The fate of the donorderived hepatocyte cell repopulation can only be evaluated using tissue biopsies systematically performed in longitudinal studies. 
References: 1-M Mirzania, A Ghavamzadeh, M Yaghmaie, N Sedighi, N Kamalian, K Alimoghaddam, S H Ghaffari, P Azimi and $\mathrm{B}$ Chahardouli, Hepatocytes of donor origin in recipient liver after hematopoietic SCT in $\beta$-thalassemia major patients, Bone Marrow Transplantation (2010) 45, 694-698.

2-KÖRBLING M; HEPATOCYTES AND EPITHELIAL CELLS OF DONOR ORIGIN IN RECIPIENTS OF PERIPHERAL-BLOOD STEM CELLS N Engl J Med, Vol. 346, No. 10 March 7, 2002, 738-746. Disclosure of Interest: None declared.

\section{P379}

Donor lymphocyte infusions as early interventions after allogeneic hematopoietic stem cell transplantation in 20 patients with various hematological malignancies: single centre experience

M. Krejci ${ }^{1, *}$, Z. Racil ${ }^{1}$, L. Semerad ${ }^{1}$, B. Weinbergerova ${ }^{1}$, B. Robesova', J. Prochazkova', K. Ksenakova', A. Zmijakova' Z. Adam ${ }^{7}$, J. Mayer ${ }^{1}$

${ }^{7}$ Dpt. of Internal Medicine, Hematology and Oncology, UNIVERSITY HOSPITAL BRNO, Brno, Czech Republic

Introduction: Donor lymphocyte infusions (DLI) often are used after allogeneic stem cell transplantation (allo-SCT) to augment the graft-versus-tumor effect. The main indications for DLI are the treatment of post-transplant relapse and correction of incomplete donor chimerism. In some cases, DLI applications were done preemptive to minimize tumor reccurence (Tomblyn $M$ et al., BMT 2008; 42: 569-579). The optimal strategy of timing and cell dose for DLI administration remains unclear, this topic is very heterogenous. Here we present our experience with early DLI administrations in 20 patients (pts) with various hematological malignancies after allo-SCT.

Material (or patients) and methods: We analyzed 20 pts with lymphoid $(n=9)$ or myeloid $(n=11)$ hematological malignancies after allo-SCT. The indications for DLI administrations were as follows: unstable mixed donor chimerism (12 cases, $60 \%$ ), molecular relapse of disease after SCT (6 cases, 30\%), persistence of minimal residual disease (PCR-MRD positivity) after allo-SCT (2 cases, 10\%). Median time interval from alloSCT to DLI administration was 7 months (range 4-47 months). All pts in time of the first DLI dose were in hematological remission of disease, they were free of immunosupressive medication and were free of graft-versus-host disease, too. Patients received DLIs in escalating doses in 6-8 week intervals. Fifteen pts $(75 \%)$ received one DLI dose, three pts $(15 \%)$ received two $\mathrm{DLI}$ doses and two pts $(10 \%)$ received three $\mathrm{DLI}$ doses. The first DLI dose was $5 \times 10 \mathrm{E} 6 \mathrm{CD} 3+$ cells $/ \mathrm{kg}$, the second dose was $1 \times 10 \mathrm{E} 7 \mathrm{CD} 3+$ cells $/ \mathrm{kg}$, and the third dose was $5 \times 10 \mathrm{E} 7 \mathrm{CD} 3+$ cells $/ \mathrm{kg}$.

Results: Overall response after DLI administrations was achieved in 16 of 20 patients (80\%). Complete donor chimerism was achieved in 11 of 12 pts (92\%), molecular remission in 3 of 6 pts (50\%) and PCR-MRD negativity in 2 from 2 pts (100\%). Incidence of acute GvHD was evaluated in all 20 pts, $65 \%(13 / 20)$ of pts had GvHD (grade I+II in 9 pts, grade III +IV in 4 pts). No other significant toxicity according to common toxicity criteria was observed. With median follow-up from the first DLI dose administration 24 months (range 2-41 months), $70 \%$ of all pts (14/20) were alive (all 14 pts in remission of hematological malignancy), 6 pts died (4 deaths from progression of hematological malignancy, 1 dead to pulmonary embolism, 1 dead to infection).

Conclusion: Donor lymphocyte infusions as early interventions after allogeneic hematopoietic stem cell transplantation seems to be promising approach to achievement of complete donor chimerism, overall response rate was $92 \%$. In some cases, DLI administration coud be usefull in molecular relapse or persistance of PCR-MRD positivity after allo-SCT. Early administration of DLI can prevent the relapse of hematological malignancy after allo-SCT.
References: Tomblyn M, Lazarus HM. Donor lymphocyte infusions: the long and winding road: how should it be traveled? Bone Marrow Transplantation, 2008, 42: 569-579.

Disclosure of Interest: None declared.

\section{P380}

Combined isolation of virus specific $T$ cells from seronegative donors, TAA and MiHA specific T cells for the prevention of viral infections and tumor relapses early after alloSCT

M. Roex ${ }^{1, *}$, J. Matamoros Luna ${ }^{1}$, L. Hageman ${ }^{1}$, E. van Liempt ${ }^{1}$, E. van Egmond ${ }^{1}$, S. Veld ${ }^{1}$, C. Hoogstraten ${ }^{1}$, L. Germeroth ${ }^{2}$, P. van

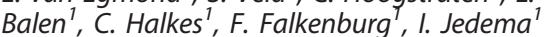

${ }^{1}$ Hematology, Leiden University Medical Center, Leiden, Netherlands, ${ }^{2} J u n o$ Therapeutics, Goettingen, Germany

Introduction: T cell depleted allogeneic stem cell transplantation (alloSCT) followed by a donor lymphocyte infusion (DLI) is a potential curative treatment for patients with hematologic malignancies. However, between the alloSCT and the DLI patients are vulnerable to viral infections and disease relapses. In the EU FP7 program T-control we aim to generate multiantigen specific T-cell products to prevent these complications. Therefore, we strive to isolate donor T-cells directed against HLA-class-I-restricted peptides of CMV, EBV and Adenovirus, HLA-A2-restricted peptides from the tumorassociated antigens (TAA) NY-eso, WT-1, RHAMM, PRAME and proteinase 3 , and the minor histocompatibility antigen (MiHA) HA-1h. Due to the relatively high precursor frequency of virus-specific T cells in seropositive donors, these T cells can be easily isolated. Here, we focus on the isolation of specificities with a very low precursor frequency: TAA and MiHA specific $T$ cells, and virus specific $T$ cells from seronegative donors.

Material (or patients) and methods: Five CMV seronegative donors and two EBV seronegative HLA-A2, B7, and/or B8positive donors were selected for isolation of viral specificities. Four HLA-A2 positive donors were used for the isolation of TAA and MiHA specific T cells. The reversible HLA/peptide streptamer and magnetic bead technique was used for the isolation out of $1-2^{*} 10^{9}$ donor PBMC, using streptamers depending on the HLA-type of the donor. The positive fractions were non-specifically expanded using PHA, IL-2 and allogeneic feeders. The presence of the different specificities was assessed 10-14 days after the isolation by CD8 and tetramer staining. To confirm the presence of the invisible T cell specificities, subsequent enrichment and expansion round were performed until populations were visible. Specificities that were present in a frequency $>1 \%$ were clonally expanded and tested for their functional potential.

Results: After 2 or 3 enrichment and expansion rounds, we have isolated 6 CMV specificities from 4 seronegative donors (pp65 A2 NLV (3x), pp65 B7 TPR, IE-1 A2 VLE, IE-1 B8 OIK) and 4 EBV specificities from 2 seronegative donors (BMLF-1 A2 GLC, EBNA-3a B7 RPP (2x), BZLF-1 B8 RAK). So far, a limited repertoire of CD8/tetramer positive clones from two CMV specificities (pp65 A2 NLV and pp65 B7 TPR) and one EBV specificity (EBNA-3a B7 RPP) were tested for antigen specific reactivity measured by cytokine release (IFN-gamma and GM-CSF) after 24 hours of stimulation with TAP deficient T2 cells and/or allogeneic EBV-LCL loaded with the relevant peptide. This analysis revealed the presence of clones of high avidity recognizing up to $10^{-9} \mathrm{M}$ peptide on T2 cells and $10^{-8} \mathrm{M}$ peptide on allogeneic EBV-LCL. Besides this, all mentioned TAA and MiHA specificities could be isolated from all four HLA-A2 positive donors after 2 to 4 enrichment and expansion rounds, and comprised clones with a range of different functional avidities.

Conclusion: In conclusion, we were able to isolate EBV and CMV specific $T$ cell clones from seronegative donors and TAA and MiHA specificities from HLA-A2 positive donors. These results indicate that the HLA/peptide-streptamer technology allows the simultaneous isolation of both naïve and memory 
virus specific T cells, as well as TAA and MiHA specificities in a multi antigen specific $T$ cell product.

Disclosure of Interest: M. Roex: None declared, J. Matamoros Luna: None declared, L. Hageman: None declared, E. van Liempt: None declared, E. van Egmond: None declared, S. Veld: None declared, C. Hoogstraten: None declared, L. Germeroth Employee of: Juno Therapeutics, P. van Balen: None declared, C. Halkes: None declared, F. Falkenburg: None declared, I. Jedema: None declared.

\section{P381}

ErbB2-chimeric antigen receptor engineering of cytokineinduced killer cells against soft tissue sarcomas: highly increased cytotoxicity in vitro and effective prevention of tumor engraftment in in vivo mouse models

M. Merker ${ }^{1, *}$, E. Rettinger ${ }^{1}$, S. Oelsner ${ }^{1,2}$, V. Pfirrmann ${ }^{1}$, J. Wagner ${ }^{1}$, E. Ullrich ${ }^{1}$, T. Klingebiel ${ }^{1}$, W. Wels ${ }^{2}$, P. Bader ${ }^{1}$

${ }^{1}$ University Hospital Frankfurt, Goethe University, Department for Children and Adolescents, Division for Stem Cell Transplantation and Immunology, ${ }^{2}$ Georg-Speyer Haus, Institute for Tumor Biology and Experimental Therapy, Frankfurt/Main, Germany

Introduction: Pediatric patients with high-risk solid tumors often acquire refractory disease or relapse during treatment. Specifically, patients with stage IV alveolar rhabdomyosarcoma (aRMS) above the age of 10 years cannot be cured by conventional therapies. Cytokine-induced killer (CIK) cells represent a heterogeneous predominantly polyclonal $\mathrm{T}$ cell population with phenotypic and functional properties of natural killer (NK) cells acquired during in vitro expansion. It was shown that CIK cell treatment is safe in the autologous setting, but the cytotoxic potential is limited. Soft tissue sarcoma cells, unlike hematopoietic cells, express ErbB2. ErbB2 might therefore represent an attractive tumor antigen for targeted CIK cell therapy using chimeric antigen receptors (CARs).

Material (or patients) and methods: CIK cells were generated from peripheral blood mononuclear cells (PBMC) in the presence of interferon $\gamma$, anti-CD3 antibody, interleukin (IL)-2 and IL-15. ErbB2-CAR modified and control-vector CIK cells were generated from conventional CIK cells by lentiviral gene transduction on day 4 of culture. Phenotypic analysis and cytotoxic activity were assessed on days $0,7,10$ and between days 12-21 of culture, respectively. To illustrate efficacy of these cells in preclinical in vivo mouse models by in vivo imaging, luciferase gene-transduced $\mathrm{RH} 30$ cells were sorted and administered in immunodeficient NOD/SCID/ $\mathrm{YC}^{-}$(NSG) mice.

Results: With our protocol CIK cells expanded by 8.5 -fold showing gene transduction rates of $8.5 \%-51.30 \%$ at day 12 of culture $(n=19)$. Up to $90 \%$ of gene transduced cells showed surface expression of the ErbB2 CAR $(n=8)$. Flowcytometric analysis of CIK cells showed stem cell memory and effector memory phenotypes. ErbB2 CAR CIK cells efficiently lysed ErbB2-overexpressing breast carcinoma cells (MDA-MB 453) in a 3-hour short-term (europium release) cytotoxicity assay while there was no difference against ErbB2-negative target cells (e.g. AML cell line THP1) when using WT or ErbB2 CAR CIK cells. The surface expression of ErbB2 on different established and primary RMS cell lines differed, but was detectable in all cases. Accordingly, specific lysis of tumor cells was target celldependent and correlated with the percentage and degree of ErbB2 expression on tumors. As one representative example, specific lysis of alveolar RMS cell line RH30 was increased by $150 \%$ during short-term cytotoxicity assay using ErbB2 CAR CIK cells compared with WT CIK cells (20:1 effector to target ratio, $44 \%$ vs. $17 \%$ specific lysis, $n=5 ; P<0.03$ ). Long-term cytotoxicity analysis (brightfield imaging cytometry) demonstrated comparable results even at low effector to target ratios of $1: 1 \quad(36 \%$ vs. $12 \%$ specific lysis, $n=3 ; P<0.03)$. In vivo imaging and $\mathrm{QPCR}$ data demonstrates effective prevention of tumor engraftment compared to untreated and WT CIK cell treated controls if administered $24 \mathrm{~h}$ after application of the tumor cells. Transduced CIK cells could be found even 100 days after administration in the spleens of treated mice.

Conclusion: ErbB2-CAR engineered CIK cells are highly specific and efficient against ErbB2-antigen expressing tumor cell lines in vitro and in vivo. ErbB2-CAR CIK cells may be an approach for improved treatment of patients with high-risk soft tissue sarcoma.

Disclosure of Interest: None declared.

P382

Intravitreal injection of autologous bone marrow stem cells in retinitis pigmentosa patients. Preliminary results of a phase I clinical trial

N. García Inesta ${ }^{1}$, F. Iniesta ${ }^{1}$, A. V. García ${ }^{2}$, J. M. Marín ${ }^{2}$ C. García ${ }^{2}$, M. Ródríguez ${ }^{1}$ M. López ${ }^{1}$, D. Sánchez ${ }^{1}$, C. Asín ${ }^{1}$ M. Molina ${ }^{1}$, S. López ${ }^{1}$, C. Algueró ${ }^{1}$, J. Sánchez ${ }^{1}$, S. Valiente ${ }^{\prime}$ V. Hurtado ${ }^{1}$, J. Gómez ${ }^{1}$, C. Maeztu ${ }^{3}$, A. M. García ${ }^{1}$, M. Blanquer ${ }^{1, *}$, J. M. Moraleda ${ }^{1}$, R. Reigadas ${ }^{2}$, M. Rodríguez $^{2}$

${ }^{1}$ Hematología, ${ }^{2}$ Oftalmología, Hospital Clínico Universitario Vírgen de la Arrixaca, ${ }^{3}$ Oftalmología, Hospital General Universitario Reina Sofía, Murcia, Spain

Introduction: Retinitis pigmentosa (RP) is a group of hereditary progressive retinal diseases affecting 1/4000 people. RP is an external retina dystrophy caused by the progressive death of photoreceptors (PR) by apoptosis, affecting the rods and later the cones. Initially the function of the PR, the retinal pigment epithelium and the inner retina cells is altered, causing the loss of ganglion cells, which ultimately leads to legal blindness. Most RP patients start their visual symptoms in the early adolescence with impaired night vision. Legal blindness is reached at 40-50 years old. Studies in animal models of RP have shown that intravitreal injection of mononuclear cells derived from bone marrow (BM-MNCs) may slow PR degeneration, being a feasible and safe procedure. It has been postulated that the beneficial effect is related to the local production of neurotrophic factors such as the ciliary (CNTF) and glial (GDNF) ones. Our group has designed a prospective, single-center, randomized, parallel, double-blind, placebo controlled Phase I clinical trial, to treat 10 patients with RP performing an intravitreal injection of autologous BMMNCs.

Material (or patients) and methods: The main objective of the study is to evaluate the safety of the intravitreal injection of autologous BM-MNCs in patients with advanced RP. The secondary objective is to determine the ability of BM-MNCs to slow or improve the development of retinal degeneration. After obtaining informed consent, the corresponding tests were performed to verify that all inclusion criteria were met and none of exclusion. The patients included in the clinical trial received a pars plana intravitreal injection of autologous BM-MNCs (0.1 mL) in one eye (experimental eye) and a subconjunctival injection of saline (placebo) $(0.1 \mathrm{ml})$ in the contralateral eye (control eye). The bone marrow was collected in the operating room $(120 \mathrm{~mL})$ and processed by Ficoll to separate the mononuclear cells in an automated system (SEPAX S-100), under GMP, in our Cell Production Unit. Evaluations were schedulled $24 \mathrm{~h}, 8$ days, and 1, 2, 3, 4, 6, 9 and 12 months after the infusion to assess the safety of the procedure, the change in the rate of progression of the disease and some efficacy data.

Results: Seven patients have been included in the clinical trial. A mean of $29,5 \times 106[18,4 \times 106-44,8 \times 106]$ BM-MNCs were injected without incident. There have been no serious or non-serious procedure-related adverse events. The ophthalmologic evaluation performed in 6 patients who have reached 4 months of follow-up showed a gradual improvement in the distant and near visual acuity measured by the ETDRS test (Early Treatment Diabetic Retinopathy Study) and macular sensitivity microperimetry. This improvement was observed both in the experimental and the control eye, being more significant in the experimental one. Viable cells have been observed months after infusion but no ocular complications. 
Conclusion: The procedure is safe and feasible. No major complications or procedure-related morbidity were observed. In 3 patients, the disease appears to have stabilized and some improvement was seen in 4 of the 7 patients infused.

References: Ministerio de Salud, Política Social e Igualdad (EC11-004) and Red Española de Terapia Celular (TerCel) del Intituto de Salud Carlos III (RD12/0019/0001).

Disclosure of Interest: None declared.

\section{P383}

Amyotrophic lateral sclerosis treatment with intramuscular injection of autologous bone marrow stem cells. Preliminary results of a phase I clinical trial

V. Hurtado ${ }^{1}$, N. García ${ }^{1}$, F. Iniesta ${ }^{1}$, S. Valiente ${ }^{1}$, M. Rodríguez ${ }^{1}$, M. D. López ', D. Sánchez ', C. Asin ${ }^{1}$, M. D. M. Molina ${ }^{1}$, S. López ${ }^{\prime}$ C. Algueró ${ }^{1}$, M. Punzano ${ }^{1}$, J. Sanchez García ${ }^{7}$ A. M. García ${ }^{\prime}$ M. Blanquer ${ }^{2, *}$, P. de Mingo ${ }^{3}$, M. V. Saez ${ }^{3}$, C. Pastore ${ }^{4}$, E. Geijó $^{4}$, J. Hernández ${ }^{5}$, S. Martínez ${ }^{6,7}$, J. Gómez ${ }^{1}$ J. M. Moraleda ${ }^{i, 8}$

${ }^{1}$ Hematology Service and Cell Therapy Unit, Clinical University Hospital Virgen de la Arrixaca. IMIB-FFIS, ${ }^{2}$ Hematology Service and Cell Therapy Unit, IMIB-FFIS-HCUVA, ${ }^{3}$ Neurophysiology Departmen, Clinical University Hospital Virgen de la Arrixaca. IMIB-FFIS, El Palmar (Murcia), ${ }^{4}$ Neurobiology Department, University Hospital San Carlos, Alicante, ${ }^{5}$ Anesthesia and resuscitation Service, Clinical University Hospital Virgen de la Arrixaca. IMIB-FFIS, El Palmar (Murcia), ${ }^{6}$ Neuroscience Institute, Alicante, ${ }^{7}$ Miguel Hernández University, Elche (Alicante), ${ }^{8}$ Internal Medicine, University of Murcia, Murcia, Spain

Introduction: Amyotrophic lateral sclerosis (ALS) is histologically defined by the degeneration of the upper and lower motoneurons. The etiopathogenesis of this neurodegeneration is not well understood. Its incidence in Spain is estimated at 1 case per 100.000 inhabitants per year. The average age at diagnosis is 60 years, although some genetic conditions can produce an earlier debut. Its relentlessly progressive course leads the patients to their death in an average of 3-5 years from its diagnosis. There is no curative treatment for ALS, the current treatment being symptomatic.

The results obtained by our team in animal models show that the stem cells from the bone marrow infused intramuscularly produce a trophic effect in the spinal motoneurons, promoting their survival, preventing muscle degeneration and improving the motor function of the animal. These actions are mediated by the release of neutrophic factors such as GDNF, which are captured by the axon terminals and carried to the spinal cord by retrograde axonal transport. Based on these data, our team has launched the TCIM/ELA clinical trial. It is a prospective, unicentric, randomized, parallel, double-blind, placebo controlled phase I clinical trial. The intended recruitment is 20 ALS patients (EudraCT number: 2011004801-25, PEl: 06-074).

Material (or patients) and methods: The main objective was to determine the safety of the intramuscular infusion of autologous mononucleated cells from bone marrow (BM-MNC) in the tibialis anterior muscle (TA) of ALS patients. The secondary objective was to study the ability of such cells to stop or slow the progressive loss of functional TA motor units that characterizes ALS.

After signing the informed consent and checking that all the inclusion and none of the exclusion criteria were fulfilled, the patients were intramuscularly infused with BM-MNC in the TA muscle of one of the lower limbs (experimental lower limb: ELL) and with normal saline solution (placebo) in the contralateral lower limb (control lower limb: CLL). It was randomized which of the lower limbs was the ELL.

The follow up consisted of 5 on-site visits (days 7, 30, 90, 180 and 360 post-injection) and 7 telephone interviews (months 2 , $4,9,15,18,21$ and 24 post-injection).

Results: 18 patients have been included. A mean of $504 \times 10^{6}$ $\left(206 \times 10^{6}-1.058 \times 10^{6}\right)$ BM-MNC were infused. With a mean follow up of 156 days (30-335), 5 procedure-related non-severe adverse events have been registered. All of them were CTCAE grade 1-2 and resolved before day +5 . There also have been 5 non procedure-related serious adverse events. In the 14 patients that have reached the day+90 follow-up, there were no differences in the evolution of the dynamometer-measured TA strength between both legs.

Conclusion: The procedure is safe and feasible. Neither major complications nor morbidities related to the procedure have been observed.

Disclosure of Interest: Ministerio de Salud, Política Social e Igualdad (EC11-325) and Red Española de Terapia Celular (TerCel) del Intituto de Salud Carlos III (RD12/0019/0001 y RD12/0019/0024). None declared.

P384

The mesenchymal stem cell therapy for steroid refractory acute graft versus host disease in Children: a Multicenter Survey by the Turkish Pediatric Bone Marrow Transplantation Study Group (TPBMT-SG)

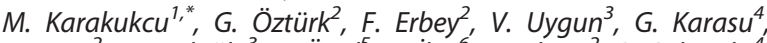

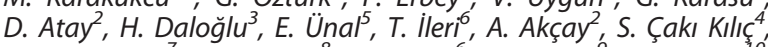
B. S. Karagün ${ }^{7}$, S. Gözmen ${ }^{8}$, M. Ertem ${ }^{6}$, E. Kürekçí, A. Küpesiz ${ }^{30}$, T. Fıskın ${ }^{11}, M$. A. Yeșilipek ${ }^{3}$

${ }^{1}$ Pediatric Hematology/Oncology and BMT Unit, Erciyes University Faculty of Medicine, KAYSERi, ${ }^{2}$ Pediatric Hematology/Oncology and BMT Unit, Acıbadem University Atakent Hospital, Istanbul, ${ }^{3}$ Pediatric Hematology/Oncology and BMT Unit, Bahcesehir University School of Medicine Medical Park Antalya Hospital, Antalya, ${ }^{4}$ Pediatric Hematology/Oncology and BMT Unit, Bahcesehir University School of Medicine Medical Park Goztepe Hospital, İstanbul, ${ }^{5}$ Pediatric Hematology/Oncology and BMT Unit, Erciyes University Faculty of Medicine, Kayseri, KAYSERI, ${ }^{6}$ Pediatric Hematology/Oncology and BMT Unit, Ankara University School of Medicine, Ankara, ${ }^{7}$ Pediatric Hematology/ Oncology and BMT Unit, Acıbadem University Adana Hospital, Adana, ${ }^{8}$ Pediatric Hematology/Oncology and BMT Unit, Ege University Faculty of Medicine, Izmir, 9 Pediatric Hematology/ Oncology and BMT Unit, Gulhane Military Medical Academy, Ankara, ${ }^{10}$ Pediatric Hematology/Oncology and BMT Unit, Akdeniz University Faculty of Medicine, Antalya, ${ }^{11}$ Pediatric Hematology/Oncology and BMT Unit, Medical Park Samsun Hospital, Samsun, Turkey

Introduction: Severe acute graft versus host disease (GvHD) is a life-threatening complication after allogeneic hematopoietic stem cell transplantation. Mesenchymal stem cells (MSCs) play an important role in endogenous tissue repair. And MSCs possess strong immune-modulatory properties making them a promising tool for the treatment of steroid-refractory GvHD. The objective of this study is to evaluate the treatment results of children with refractory GvHD treated with MSCs.

Material (or patients) and methods: The outcome of 93 children with GvHD that were transplanted in 10 pediatric centers in Turkey between 2011 and 2015 was retrospectively analyzed. Forty three patients (46.2\%) underwent HSCT for a hematologic malignancy, and the remaining patients underwent HSCT for non-malignant disease, primarily of genetic origin. Totally 232 MSCs infusions were used when the patient did not respond to the first line treatment modalities with corticosteroids. Ninety three children ( 24 female, 69 male; median age of 7.4 years, (range 8 months-18 years) all had refractory acute GvHD (grade II-IV) after hematopoietic stem cell transplantation (HSCT) were treated with MSCs in combination with immunosuppressive agents. The MSCs from bone marrow or adipose tissue of HLA-unrelated third-party donors were used at the $29.9 \pm 26.8$ th day after the onset of acute GvHD, at a dose of mean $1.74 \pm 0.78 \times 10(6)$ cells $/ \mathrm{kg}$ on first infusion. Sixteen patients received one infusion, 41 patients received two infusions, and 36 patients received three or more infusions with the interval of $11.4 \pm 6.6$ days between first and second infusion.

Results: Two patient (2.2\%) with grade II, 33 patients (35.5\%) with grade III and 58 patients (62.4\%) with grade IV acute 
GvHD received a total of 232 infusions of MSCs. Organ involvement at baseline was $89.2 \%$ gastrointestinal, $87.1 \%$ skin, and $39.7 \%$ liver. Totally, 41 patients $(44.1 \%)$ had a complete response (CR) and 31 patients $(33.3 \%)$ had a partial response (PR, which is determined as one grade reduction) to MSCs treatment, while 21 patients $(22.6 \%)$ gave no response and had a progressive disease. The observed CR for individual organs was $42.7 \%$ for the gastrointestinal, $65.0 \%$ for skin, and $24.3 \%$ for liver. None of the patients had side-effects during or immediately after infusions. The probabilities of overall survival in patients with CR and PR to MSCs treatment were $85.4 \%$ and $51.6 \%$, respectively. All 36 patients without response died through the follow up period because of progressive GvHD.

Conclusion: The clinical trials of therapy with MSCs showed promising results in pediatric patients with severe acute GVHD those are resistant to multiple immunosuppressive agents. This strategy may provide a high rate of overall responses of acute GVHD with a low toxicity profile.

Disclosure of Interest: None declared.

P385

Long-Term Survival of Donors Bone Marrow Multipotent Mesenchymal Stromal Cells Intraosseous Introducted to Patients with Allogeneic Graft Failure

N. Petinati ${ }^{1, *}$, L. Kuzmina ${ }^{2}$, N. Drize $^{1}$, N. Risinskaya ${ }^{3}$, A. Sudarikov $^{3}$, V. Vasillieva ${ }^{2}$, M. Drokov', E. Michaylzova ${ }^{2}$, E. Parovichnikova ${ }^{2}$, V. Savchenko ${ }^{2}$

${ }^{1}$ Lab. Physiology of Hematopoiesis, ${ }^{2}$ Bone Marrow Transplantation, ${ }^{3}$ Molecular Hematology, National Research Center for Hematology, Moscow, Russian Federation

Introduction: Multipotent mesenchymal stromal cells (MSC) are part of bone marrow microenvironment maintaining hematopoiesis. Leukemic cells damage the bone marrow niche for normal hematopoietic stem cells (HSC), changing the stromal microenvironment(SM). After allogeneic hematopoietic stem cell transplantation (allo-HSCT) SM also suffering from the effects of chemotherapy drugs. We can assume that in patients with graft failure and long-term aplasia hematopoietic microenvironment is fatally damaged, and not able to support hematopoiesis. Perhaps in such cases the donor MSCs may be a source of recovery for niches. In the department of Bone Marrow Transplantation 3 patients with graft failure after alloHSCT were observed.

Material (or patients) and methods: A randomized clinical trial (ClinicalTrials.gov NCT01941394) for acute graft-versus host disease prevention with MSCs has been on-going at the National Research Center for Hematology. Bone marrow MSCs have been developed individually for each patient from its own donor of HSC. Three patients with graft failure after allo-HSCT were observed. In all 3 cases second transplantation was done. In an attempt to restore the SM donor MSCs were injected under local anesthesia in the iliac crest after obtaining informed consent from the patient. The cells were injected into the bone tissue in small portions $100-200 \mathrm{mkl}$ through 2 skin punctures, and multiple periosteal punctures. In 2 patients 5 and 3 months after the donor MSCs implantation bone marrow puncture were performed. It was withdrawn by 2-3 $\mathrm{ml}$ of bone marrow from 4 independent puncture from iliac crest of each patient. MSCs were isolated and cultured. At the 2 nd passage DNA were recovered from patients MSCs by standard methods. Determination of chimerism was performed by the method of STR-PCR (polymerase chain reaction with a panel of primers to short tandem repeat loci humans) using the protocol and reagent kit for multiplex analysis of 19-STR markers.

Results: In all patients aplasia was observed for a long time. The aim of MSCs implantation into periosteal was to support the SM in patients bone marrow. After MSCs introduction into the iliac crest no other complications, except standard for bone marrow puncture were observed. On average, 2 weeks after MSCs administration to patients their own hematopoiesis recovered. In another 3 and 5 months after MSCs implantation in the bone marrow of patients donor MSCs were found. Patient 1 died one month after donor MSCs implantation, so his MSCs were not examined. On 2nd passage in all MSCs samples the presence of donor cells was investigated. In MSCs from patient 2 a small percentage of the donor MSCs were detected in 3 of 4 samples (1.6; 0.7; 1.0 and $0 \%$ ), in MSCs from patient 3 only in one sample $18.8 \%$ of donor cells were revealed.

Conclusion: Thus for the first time it is shown directly that functionally adequate donor MSCs can exist in the bone marrow of the patient for a long time. The data indicate that in the case of graft failure after allo- HSCT the implantation of donor MSCs add to restore the hematopoiesis. The data suggest the importance of implantation as much as possible donor MSCs into the greatest number of ileum crest places. Disclosure of Interest: None declared.

\section{P386}

Placenta-Derived Decidual Stromal Cells as Treatment for Haemorrhagic Cystitis in Patients Who Have Undergone Allogeneic Haematopoietic Stem Cell Transplantation (NCT02172963)

W. Aronsson-Kurttila ${ }^{1}$, A. Baygan ${ }^{1}$, M. Remberger ${ }^{2}$, M. Solders ${ }^{1}$, T. Erkers ${ }^{7}$, S. Nava ${ }^{7}$, B. Khoein ${ }^{7}$, B. Sadeghi ${ }^{1}$, H. Kaipe', O. Ringdén ${ }^{1, *}$

${ }^{1}$ Laboratory Medicine, Therapeutic Immunology, KAROLINSKA INSTITUTET, ${ }^{2}$ Center for Allogeneic Stem Cell Transplantation, Karolinska University Hospital, STOCKHOLM, Sweden

Introduction: Haemorrhagic cystitis (HC) is a complication after haematopoietic stem cell transplantation (HSCT) with no effective treatment. Mortality in patients with moderate-tosevere $\mathrm{HC}$ is above $55 \%$. Decidual stromal cells (DSCs) from the placenta protect the foetus from the mother's immune system. Stromal cells have been successfully tested as therapy for HC. Material (or patients) and methods: Eleven patients with grades 3-4 (moderate-to-severe) HC were treated with DSCs after HSCT. Median age was 33 (range 8-50) years. All had pain requiring opiates. We isolated maternal DSCs from the foetal membranes of term placentas, obtained following Caesarian section. DSC passages used were $2^{\text {nd }}(n=1), 3^{\text {rd }}(n=7)$ and $4^{\text {th }}$ $(n=3)$. The DSC viability was median $92 \%$ (range $75-99 \%)$. The DSC dose given i.v. was median $1.5(0.7-2.5) \times 10^{6} \mathrm{cells} / \mathrm{kg}$. Nine patients got one dose, one got two doses, and one patient got four doses.

Results: Five of the patients had no more HC five days after DSC infusion. Patients who received DSCs within three days after HC debut had a median duration of HC of 6 days (5-7) compared to 31 (8-361) and shorter pain duration, as opposed to patients given DSCs later $(P=0.02)$. Three patients received DSCs prepared in albumin instead of $A B$ plasma and had a trend for shorter pain duration $(P=0.07)$. There was no infusion toxicity. Adverse events were those commonly seen after HSCT. One-year transplant-related mortality was $0 \%$. Nine of eleven patients were alive one year after HSCT with a oneyear survival of $81 \%$.

Conclusion: DSCs seems as a promising therapy for $\mathrm{HC}$ after HSCT. Based on this pilot study, we started a randomised placebo-controlled double-blind study using two doses of $1 \mathrm{x}$ $10^{6} \mathrm{DSCs} / \mathrm{kg}$ dissolved in albumin for early HC.

Registered trial at Clinical Trials, NCT02172963.

Disclosure of Interest: None declared. 
P387

Enhanced Engineering of Chimeric Antigen Receptor (CAR)-Modified T Cells Using Non-Viral Sleeping BeautyMediated Transposition From Minicircle Vectors

R. Monjezi ${ }^{1, *}$, C. Miskey ${ }^{2}$, T. Gogishvili ${ }^{1}$, H. Einsele ${ }^{1}$, Z. Ivics ${ }^{2}$, M. Hudecek

${ }^{7}$ Department of Medicine II - Hematology and Medical Oncology, University of Würzburg, Würzburg, ${ }^{2}$ Division of Medical Biotechnology, Paul-Ehrlich-Institute, Langen, Germany

Introduction: Gene-modified T cells expressing a tumorreactive chimeric antigen receptor (CAR) have demonstrated remarkable efficacy in pilot clinical trials. In order to become a standardized and widely available therapy, strategies have to be provided for rapid and transferrable CAR T cell manufacturing, including a gene transfer system that combines efficacy and safety, and is compatible with affordable vector production, handling and storage. Here, we demonstrate that nonviral gene transfer using Sleeping Beauty (SB) mediated transposition of CAR transgenes from minicircle (MC) DNA vectors enables dramatically enhanced gene transfer rates compared to conventional plasmid DNA and rapid production of CAR T cell products with a highly favorable gene integration profile.

Material (or patients) and methods: We prepared a MC transposon donor vector encoding a CD19-specific CAR from a corresponding parental pT2 plasmid through site specific recombination, and a MC encoding SB100X transposase. Each MC contained only the promotor and gene of interest, and was devoid of antibiotic resistance and bacterial origin of replication.

Results: We performed gene transfer into CD8+ and CD4+ T cells of healthy donors $(n=7)$ by nucleofection and analyzed the transposition rate and stability that could be accomplished when CD19-CAR transposon and SB100X transposase were encoded by MCs vs. equimolar amounts of their corresponding plasmid DNA vectors. In each donor, we found a significantly higer percentage of CAR+ T cells when MCs rather than plasmid DNA were utilized for gene transfer (up to 88.3\% CAR+, mean 4.4-fold higher, $P<0,001$ ). Expression of the CD19-CAR was stable over multiple rounds of expansion in both CD8+ and CD4+ T cells. Importantly, the use of MCs was also associated with substantially lower toxicity to $T$ cells. Within 14 days of culture, the yield of CAR+ T cells was on average 5.7-fold higher with the use of MCs compared to plasmids.

In functional studies, CD19-CAR T cells modified with our enhanced SB system exerted high levels of specific cytolytic activity, produced cytokines including IFNg and IL-2, and underwent productive proliferation after stimulation with CD19+ lymphoma cell lines. Moreover, a single administration of SB-modified CD19-CAR T cells lead to complete eradication of systemic lymphoma in a murine xenograft model (NSG/RajiffLuc), and was at least equivalently potent as the use of CD19CAR T cells prepared from the same donor and transduced by lentiviral gene transfer.

To address issues related to biosafety, we determined the gene copy number $(n=5$, range $3-8$ in CD8+ T cells) and performed a comprehensive integration site analysis by Illumina sequencing. The data show a close-to-random integration profile of the CD19-CAR transposon, with neither a preference for highly expressed nor cancer related genes. Importantly, a significantly higher proportion of SB integrations was detected in genomic safe harbor loci compared to lentiviral integrations $(P<0,001)$.

Conclusion: Our data demonstrate the potential to manufacture CAR T cells by non-viral SB-mediated transposition from MC DNA vectors. MC-derived CAR transposons display a highly favorable integration profile and confer stable gene expression that permits potent anti-tumor functions and are intended to facilitate and accelerate the clinical translation of CAR T-cell therapy in Europe.
Disclosure of Interest: R. Monjezi: None declared, C. Miskey: None declared, T. Gogishvili: None declared, H. Einsele: None declared, Z. Ivics Conflict with: pending patent application, M. Hudecek Conflict with: pending patent application.

\section{P388}

Isolation and function of clinical grade cord blood regulatory $T$ cells using reversible streptamer technology R. C. Duggleby 1,", D. Shah ${ }^{1}$, R. Laza ${ }^{2}$, C. Stemberger ${ }^{3}$, S. Gomez ${ }^{4}$, L. Germeroth $^{5}$, A. Jochheim-Richter, S. Mielke, H. Bönig, K. Latham ${ }^{8}$, H. Einsele', S. Querol', A. Madrigal ${ }^{1,10}$, A. Saudemont ${ }^{1,10}$ ${ }^{1}$ Anthony Nolan Research Institute, London, United Kingdom, ${ }^{2}$ Research, Anthony Nolan Research Institute, London, United Kingdom, 'Juno Therapeutics, Munich, Germany, ${ }^{4}$ Anthony Nolan Cell Therapy Centre, Nottingham, United Kingdom, ${ }^{5}$ Juno Therapeutics, Göttingen, ${ }^{6}$ German Red Cross Blood Service Baden-Württemberg-Hesse, Frankfurt, ' 'Universitäts-Klinikum Würzburg, Würzburg, Germany, ${ }^{8}$ Anthony Nolan, London, United Kingdom, ${ }^{9}$ Banc de Sang i Teixits, Barcelona, Spain, ${ }^{10}$ UCL, London, United Kingdom

Introduction: Haematopoietic stem cell transplantation is used to treat haematological malignancies and disorders, with delayed immune reconstitution, with infection and graft versus host disease (GvHD) as the main complications. A number of studies have investigated using regulatory $T$ cells (Tregs) as a cellular therapy for GvHD. Of particular interest is third party cryopreserved umbilical cord blood (CB) as a possible "off-the-shelf" cell source. Streptamer technology offers the possibility of a minimally manipulated cell population, without the presence of the selection antibody. The aim of this project was to test the feasibility of developing, to clinical grade level a CB Treg cell product from cryopreserved $C B$ units. Also being assessed is the practicality of using the product with and without, subsequent clinical grade compatible expansion.

Material (or patients) and methods: CD25+ and CD4+CD25+ cells were isolated using anti-CD25 and anti-CD4 streptamers using a single or double step procedure. Positively labelled cells were magnetically separated and the streptamers dissociated using D-biotin. Selected cells were cultured using conditions based on published clinical grade compatible protocols ${ }^{1}$ with either anti-CD3/28 Dynabeads (Life technologies) or anti-CD3/28 expansion beads (Miltenyi Biotec). Cells were expanded for up to 3 weeks with either a single, double or triple stimulation (one each week). Cells were maintained with a range of IL-2 doses and feeding regimes. Treg function was measured as a function of suppression of CFSE labelled CD4+ cells, from an adult donor, to soluble anti-CD3.

Results: It was observed that the loading of the anti-CD25-fab fragment onto the streptamer complex could alter the purity and CD25 surface levels of the final product. An association was observed between the CD25 surface levels and the suppressive function of the cells. The same product isolated from cryopreserved CB was not suppressive immediate post isolation, regardless of the surface CD25 levels. However, these cells were highly proliferative to anti-CD3/28 Dynabeads, and anti-CD3/28 expansion beads. Using a clinical grade compatible methodology $1 \times 10^{6}$ Tregs could be isolated using a single anti-CD25 streptamer or $\sim 1 \times 10^{6}$ cells using a CD4, CD25 double positive streptamer selection. By expanding up to three weeks a 50-100 fold expansion could be achieved with a double stimulation. Also, from the first stimulation onwards, the cells were highly suppressive. Cell viability and function could be maintained throughout the culture period.

Conclusion: The streptamer technology results in an "untouched" cell product, even if a subsequent expansion is performed prior to clinical use. The technology also, uniquely, allows for multiple positive selections, without flow sorting, with increasing purity. Finally, despite a low suppressive function immediately post isolation, the product could be expanded 100 fold in a 2-3 week culture using clinical grade compatible conditions, to result in a highly suppressive population. 
References: 1. Brunstein, C. G. et al. Infusion of ex vivo expanded $\mathrm{T}$ regulatory cells in adults transplanted with umbilical cord blood: safety profile and detection kinetics. Blood 117, 1061-70 (2011).

Disclosure of Interest: R. Duggleby Funding from: The research leading to these results is funded by the European Union's Seventh Framework Programme (FP7/2007-2013) under grant agreement $n^{\circ}$ 601722., D. Shah: None declared, R. Laza: None declared, C. Stemberger: None declared, S. Gomez: None declared, L. Germeroth: None declared, A. Jochheim-Richter: None declared, S. Mielke: None declared, H. Bönig: None declared, K. Latham: None declared, H. Einsele: None declared, S. Querol: None declared, A. Madrigal: None declared, A. Saudemont: None declared.

\section{P389}

A standardized and characterized GMP-compliant human platelet lysate for efficient expansion of human bone marrow mesenchymal stem cells

S. Viau ${ }^{1}$, L. Chabrand ${ }^{1}$, J. Bouilliez', S. Eap ${ }^{1}$, P. Bertholet ${ }^{1}$, T. Bouckenooghe', F. Goudaliez, B. Delorme

${ }^{1}$ Biotherapy Division, Macopharma, Mouvaux, ${ }^{2}$ Transfusion Division, Macopharma, Tourcoing, France

Introduction: Human platelet lysate (hPL) is rich in growth factors (GF) and nutritive elements and represents an advantageous xeno-free alternative to fetal bovine serum for mesenchymal stem cell (hMSC) proliferation. However, there is a great variability in hPL sources and production protocols, resulting in discrepancies in product quality, low management of product safety and poor batch-to-batch standardization.

Material (or patients) and methods: We describe here the development and the characterization of a standardized hPL prepared from outdated screened normal human donor platelet concentrates (PCs), manufactured on an industrial scale (batch sizes of $10 \mathrm{~L} ; 240$ donors) and under GMP standards (clean room, trained operators and qualified industrial process). PCs were frozen at $-80{ }^{\circ} \mathrm{C}$ and thawed at $+4{ }^{\circ} \mathrm{C}$ to lyse platelets. The number of freeze-thaw cycles was optimized. Cell debris were removed by centrifugation and the supernatant (hPL) was recovered. In order to ensure final aseptic filtration of hPL, we tested 5 different filter sequences and selected a $0.22 \mu \mathrm{m}$ size pore membrane allowing optimal GF recovery (EGF, VEGF, bFGF, TGF-beta1, PDGF-AB and IGF-1). GMP-compliant $10 \mathrm{~L}$ batches of aseptic filtered $\mathrm{hPL}$ were characterized.

Results: We showed a robust standardization between batches in terms of reproducible GF contents, biochemical analyses (such as contents of total proteins, fibrinogen, glucose and iron) and efficacy on BM-hMSC proliferation. Finally, we compared expansion and functional characteristics of BM-hMSCs grown in clinical grade hPL versus MSC-screened FBS batch. We showed 1) a reproducible increase in cell growth kinetics using hPL, 2) a maintenance of BM-hMSC membrane marker expression and clonogenic potential, 3) a similar adipogenic and osteogenic differentiation potential and finally 4) that immunosuppressive properties of BMhMSCs (on PHA-activated allogenic mononuclear cells) cultivated in parallel in both conditions remained also identical.

Conclusion: In conclusion, we demonstrated the feasibility to use a standardized, efficient and GMP-compliant hPL for research and cell therapy applications.

Disclosure of Interest: S. Viau Employee of: Macopharma, L. Chabrand Employee of: Macopharma, J. Bouilliez Employee of: Macopharma, S. Eap Employee of: Macopharma, P. Bertholet Employee of: Macopharma, T. Bouckenooghe Employee of: Macopharma, F. Goudaliez Employee of: Macopharma, B. Delorme Employee of: Macopharma.
P390

Durable remissions, with control and prediction of cytokine release syndrome (CRS), using T cells expressing a CD19-targeted chimeric antigen receptor (CTLO19) to treat relapsed/refractory $(r / r)$ pediatric ALL

S. Grupp ${ }^{1,2, *}$, S. Maude ${ }^{1,2}$, P. A. Shaw ${ }^{3}$, R. Aplenc ${ }^{1,2}$, C. Barker ${ }^{1}$ D. M. Barrett, ${ }^{1,2}$, C. Callahan ${ }^{1}$, F. Chen ${ }^{4}$, J. Finklestein ${ }^{4}$, N. Frey ${ }^{5}$, S. F. Lacey ${ }^{4}$, B. L. Levine ${ }^{6}$, J. J. Melenhorst ${ }^{4}$, L. Motley ${ }^{\prime}$, F. Nazimuddin ${ }^{4}$, D. Porter, S. R. Rheingold ${ }^{1,2}$, C. White ${ }^{\prime}$, P. A. Wood', Z. Zheng ${ }^{6}$, D. T. Teachey ${ }^{1,2}$, C. June ${ }^{6}$

${ }^{1}$ Pediatric Oncology, Children's Hospital of Philadelphia, ${ }^{2}$ Pediatrics, Perelman School of Medicine, University of Pennsylvania, ${ }^{3}$ Biostatistics and Epidemiology, ${ }^{4}$ Translational and Correlational Studies Laboratory, University of Pennsylvania, ${ }^{5}$ Medicine,

${ }^{6}$ Center for Cellular Immunotherapies, Perelman School of Medicine, University of Pennsylvania, Philadelphia, ${ }^{7}$ Cell \& Gene Therapies Unit, Novartis Pharmaceuticals Corporation, East Hanover, United States

Introduction: CARs combine a targeting domain with intracellular signaling domains. We have previously reported on CTL019 cells which show prolonged persistence and durable antitumor activity in pts with ALL (NEJM 2013\&2014). We now report on outcomes and long-term f/u of 59 pts with $r / r$ ALL.

Material (or patients) and methods: $T$ cells collected from the patient were lentivirally transduced with a CD19 $2^{\text {nd }}$ generation CAR and infused into pts with r/r CD19+ ALL. 54/59 pts received lymphodepleting (LD) chemotherapy the week prior to CTL019 infusion. T cell dose range was $10^{7}$ to $10^{8}$ cells/ $\mathrm{kg}$ with a transduction efficiency of $2.3-45 \%$.

Results: We treated 59 pts with CD19+ ALL, ages 20 m-24y. Disease burden after LD chemo was measured 1d prior to CTL019 infusion: 44/59 pts had detectable ALL while 15 were MRD(-). There were no infusional toxicities $>$ gr2. 55 pts (93\%) achieved a CR, including a patient with CD19+ T ALL, 4 did not respond. MRD measured by clinical flow cytometry was $<0.01 \%$ at $28 \mathrm{~d}$ in 50 responding pts and positive at $0.024-1.1 \%$ in 5 pts, with 2 pts becoming negative by $3 \mathrm{~m}$ with no further therapy. With median follow up $12 \mathrm{~m}$ (2-43 m), 34 pts have ongoing CR, with only 6 receiving subsequent SCT. RFS is $76 \%$ at $6 \mathrm{~m}(95 \% \mathrm{Cl} 65-89 \%)$ and $55 \%$ at $1 \mathrm{y}(95 \% \mathrm{Cl} 42-$ $73 \%)$, and OS is $79 \%$ at $1 \mathrm{y}(95 \% \mathrm{Cl} 69-91 \%)$. CTL019 was detected by qPCR in the CSF of $46 / 47$ pts, 4 pts with CNS2a ALL experienced a CR in CSF, and there have been no CNS relapses including pts with multiple pre-CTL019 CNS recurrences.

All but 7 (88\%) pts developed gr1-4 CRS at peak T cell expansion. In addition to pre-infusion disease burden, the peak levels of 24 cytokines, including IFNy, IL-6, sgp130, sIL6R, and GM-CSF measured within 28d after CTL019 infusion were highly associated with severe CRS. Analyzing cytokines from the first $3 \mathrm{~d}$ after infusion prior to severe CRS, only 2 cytokines, sgp130 and IFNY, were significantly associated with later risk of severe CRS after multiple comparisons adjustment. Using regression modeling, we could accurately predict which patients would develop severe CRS using 3 analytes measured in the first $72 \mathrm{~h}$ : IFN $\gamma$, sgp130, and IL1RA (PPV 75\%, NPV 94\%, sensitivity $86 \%$, specificity $89 \%$, AUC 0.93 ). Treatment for CRS was required for hemodynamic or respiratory instability in $27 \%$ of patients and was reversed in all cases with IL6 blockade (tocilizumab), together with short courses of corticosteroids in 10 pts. Persistence of CTL019 cells can be detected by flow cytometry and/or qPCR, and results in the PD marker of CTL019 function, which is B cell aplasia requiring IVlg, which has continued for 2-39 $\mathrm{m}$ after infusion in pts with ongoing responses.

Conclusion: CTL019 cells can undergo robust in vivo expansion and can persist for $>3 y$ in pts with $r / r$ ALL, allowing for the possibility of long term disease control without subsequent therapy such as SCT. This approach also has promise as salvage therapy for patients who relapse after allo SCT with a low risk of GVHD. CTL019 therapy can be associated with 
significant CRS that responds rapidly to IL6-targeted cytokine blockade, and can be predicted in the first $3 \mathrm{~d}$ after infusion using the model described here. CTL019 therapy has received Breakthrough Therapy designation from the FDA in pediatric and adult ALL, and phase 2 multicenter and global registration trials are well underway.

Disclosure of Interest: S. Grupp Funding from: Novartis, Conflict with: Patent interest managed under the UPenn COI policy, S. Maude Funding from: Novartis, P. A. Shaw: None declared, R. Aplenc: None declared, C. Barker: None declared D. M. Barrett: None declared, C. Callahan: None declared, F. Chen: None declared, J. Finklestein: None declared, N. Frey: None declared, S. F. Lacey: None declared, B. L. Levine Funding from: Novartis, Conflict with: Patent interest managed under the UPenn COI policy, J. J. Melenhorst: None declared, L. Motley: None declared, F. Nazimuddin: None declared, D. Porter Funding from: Novartis, Conflict with: Patent interest managed under the UPenn COI policy, S. R. Rheingold: None declared, C. White: None declared, P. A. Wood Employee of: Novartis, Z. Zheng: None declared, D. T. Teachey: None declared, C. June Funding from: Novartis, Conflict with: Patent interests managed under the UPenn COI policy.

\section{P391}

Donor lymphocyte infusion (DLI) in patients with hematological malignancies induce multiepitope-specific cytotoxic T-cell (CTL) responses against several leukemiaassociated-antigens (LAA)

S. Hofmann ${ }^{1, *}$, M. Götz ${ }^{1}$, M. Wiesneth ${ }^{2}$, H. Döhner ${ }^{1}$, D. Bunjes ${ }^{1}$, J. Greiner ${ }^{3}$

${ }^{1}$ Internal Medicine III, University Hospital, ${ }^{2}$ Institute of Transfusion Medicine, Ulm, ${ }^{3}$ Internal Medicine, Diakonieklinikum Stuttgart, Stuttgart, Germany

Introduction: T-cell responses play a central role in maintaining remission and prolonging overall-survival in patients with hematologic malignancies after allo-HSCT and delayed DLI. The role of LAA and $\mathrm{mHag}$ has to be elucidated although there is evidence that the graft-versus-leukemia-effect observed after DLI is based on CTL-mediated immunity which is reactive against $\mathrm{mHag}$ and LAA.

Material (or patients) and methods: In this study, we analyzed peripheral blood and serum samples of 12 patients (pts) with AML (5 pts), CML (2 pts), Multiple Myeloma (3 pts) and CLL (2 pts) in the course of allo-HSCT and before and after DLI for specific CTL responses against several LAA and the corresponding cytokine profile. Of the $5 \mathrm{AML}$ patients three have a NPM1mutation, one a FLT3-ITD mutation and one a t $(6 ; 9)$ translocation. The indications for DLI were in 4 pts preemptive DLI in mixed chimerism, in 4 pts preemptive DLI in molecular relapse and in 4 pts therapeutic DLI. 5 pts underwent a single DLI, 4 pts received a second and $1 \mathrm{pt}$ a third DLI. Epitopes derived from PRAME, NPM1 mut, RHAMM, WT-1 and other LAA were tested. Immune reactions of CTL were measured in ELISpot assays (INF-gamma and Granzyme B) and tetramer staining. Immune inhibitory as well as immune stimulatory cytokines were analyzed for further interpretation of the clinical data.

Results: 5 pts showed no clinical response (NR) and 7 pts reached a clinical response (R). 6 pts $(5 R, 1 N R)$ experienced GvHD whereas 6 pts (2R, 4 NR) did not have any signs of GvHD. 10/12 pts showed immunological responses by means of an increase in the number of the recognized epitopes by CTLs after DLI. Higher cytokine levels for IL-7, IL-15, IL-2 and IFN-gamma were measured after DLI indicating T-cell activation and thus suggesting reversal of T-cell exhaustion.

Conclusion: Taken together, an increase in the frequency of specific CTLs was detected in the majority of patients independent of their clinical response. Therefore our data support the hypothesis of exhausted T-cells that can be restored by DLI in some patients. Our results support the application of checkpoint inhibitors in combination with DLI to regain the functionality of the T-cells.

Disclosure of Interest: None declared.
P392

DLI application to revert mixed chimerism and treat relapse after Fludarabine-Melphalan \pm Alemtuzumab reduced-intensity conditioning regimen for adults with haematological malignancies

T. D. Pereira ${ }^{1, *}$, R. Danby ${ }^{1}$, P. Andy ${ }^{1}$, T. Littlewood ${ }^{1}$, V. Rocha ${ }^{1}$

${ }^{1}$ CHURCHILL HOSPITAL, OXFORD, Oxford, United Kingdom

Introduction: One of the strategies adopted by enhancing the graft-versus-tumour effect to prevent and treat relapse after allogeneic HSCT is via donor lymphocyte infusion (DLI).

The purpose of this study is evaluating DLI efficacy in treating mixed chimerism and relapse, in hematological malignancies. Material (or patients) and methods: Retrospective analysis of 58 transplanted adult patients, in Churchil Hospital, who received DLI, either for mixed chimerism or relapse, from 2001 to 2014.

Results: We splited the population into two subgroups (mixed chimerism and relapse). All non-related transplants received alemtuzumab.

A total of 20 patients were submitted to DLI for low chimerism. The majority of cases (18) received alemtuzumab (90\%) and only 3 cases had a related donor. Fifty percent of patients in this group were transplanted for acute leukaemia (all AML). The median days from transplant to first DLI in this group was 396 and one single DLI was sufficient to revert from mixed to full donor chimerism $50 \%$ of cases. The main reason for stopping DLI was recovery of full donor chimerism (73\%) being only $5.3 \%$ due to relapse. Acute GVHD grade III-IV was experienced in $40 \%$ of patients and limited chronic GVHD in $15 \%$ (none presented extensive GVHD). Most of the patients (90\%) were still alive at the end of the period considered (only two deaths with a median follow up of 61 months). The two causes of death were not related to DLI and the patient who relapsed after DLI was still alive at the end of follow-up.

For relapse, of a total of 38 patients, half were transplanted from a related donor and $60.52 \%$ were T-cell depleted. The median days from transplant to first DLI was 405. Acute leukaemias $(57.89 \%)$ were the main reason of transplant (18 $\mathrm{AML}$ and $4 \mathrm{ALL}$ ); in this group, 21 out of 23 received chemotherapy before DLI but only 10 were in CR pre DLI. On the other hand, the remaining 16 patients (non-acute leukaemia patients) received chemotherapy pre DLI in 10 cases, but only 2 were in CR before DLI. Just one DLI was used in $17.94 \%$ (7) of cases.

Considering response after DLI, in the leukaemia group, 5 out of 10 patients who achieved CR after chemotherapy before DLI remained in CR at the end of follow-up and only 2 out of 12 patients who didn't achieve remission before DLI, did it so after the infusion. Both patients who received $D L I$ in $C R$, in non-leukaemia group remained in CR after DLI but only 3 out of 13 who received $\mathrm{DLI}$ in non-CR, achieved CR afterwards. Twelve patients treated by leukaemia relapse were still alive at the end of period analyzed, as well as 11 patients in the nonleukaemia group. GVHD was the reason for stopping DLI in $7.9 \%$ of relapsed patients, being $31.25 \%$ grade III-IV. Among those who experienced chronic GHVD (15.79\%), half presented extensive manifestation of the disease. With a median follow up of 42 months, 23 patients in relapse group were alive, being 12 of them from acute leukaemia subgroup.

Conclusion: DLI has been successful in reverting low chimerism and prevent relapse after transplantation, with encouraging results- in this cohort being as high as $90 \%$ of cases. In less extent, it is still capable of re-inducing few remissions after post-transplant relapse, even though these results still needs improvements.

Disclosure of Interest: None declared. 
P393

High TRM even in patients responding to MSC therapy for steroid-resistant aGVHD

U. Salmenniemi ${ }^{1, *}$, J. Nystedt ${ }^{2}$, M. Putkonen ${ }^{1}$, M. Kauppila ${ }^{1}$, T. Salmi ${ }^{1}$, S. salomäki ${ }^{1}$, M. Valta ${ }^{1}$, K. remes ${ }^{1}$, M. Itälä-Remes ${ }^{1}$, M. korhonen ${ }^{2}$

${ }^{1}$ Dept of Hematology, Turku University Hospital, Turku, ${ }^{2}$ Finnish Red Cross Blood service, Helsinki, Finland

Introduction: Steroid resistant aGVHD is a treatment challenge but promising results have been achieved with mesenchymal stem cells (MSC). We report here the outcome in 17 adult patients treated with MSC.

Material (or patients) and methods: Patients had undergone allogeneic SCT at Turku University Hospital between 3/2012 and $5 / 2015$. All patients received methylprednisolone $2 \mathrm{mg} / \mathrm{kg}$ as first-line treatment for aGVHD. Steroid refractoriness was defined as a progression or lack of response after 5 days' treatment. Glucksberg criteria were used for aGVHD grading, and responses were defined as CR, VGPR (decrease in overall grade by $\geq 2$ grades), $\mathrm{PR}$, or NR after 28 days' treatment. Survival was calculated from the onset of aGVHD to death. MSCs were produced in compliance with national ATMP hospital exemption and GMP requirements at the Advanced Cell Therapy Centre of the Finnish Red Cross Blood Service. MSCs were derived from bone marrow of third party donors, expanded in platelet lysate and quality controlled before batch release.

Results: The median age of patients was 50 years (21-66), alloSCT indications were AML/MDS $(n=8)$, MPN (4), lymphoma/MM (4), and ALL (1), and disease status at alloSCT was CR1 (8), PR/VGPR (1), refractory (6) and stable/untreated (2). Conditioning was MAC for 10 patients, RIC/RIT (3) or sequential FLAMSA-RIC (4).

aGVHD occurred after a median of 55 (18-229) days posttransplant with overall grade II in one patient (6\%), III in 8 $(47 \%)$, and IV in $8(47 \%)$ patients. All patients had Gl tract involvement together with skin (71\%) and liver (24\%) gvhd. Stage III-IV GI tract, skin or liver involvement occurred in 10 $(59 \%), 5(29 \%)$, and $2(12 \%)$ patients, respectively. Patients received a median of 5 (1-6) biweekly MSC infusions at a median cell dose of $2.0 \times 10 \mathrm{E} 6 / \mathrm{kg}$ (1.4-2.7). Median time from the aGVHD onset to the first MSC infusion was 11 days (5-278). Overall response rate to MSCs was 53\%: CR in 4 (24\%), VGPR in $3(18 \%)$ and PR in $2(12 \%)$ patients. Eight patients (47\%) did not respond. The median survival from the onset of aGVHD was 101 days (16-1156) and survival was significantly longer for responders than nonresponders (estimated medians 424 vs 47 days, $P=0.03$ ). However, only $4(24 \%)$ patients were alive at the end of follow-up, $33 \%$ of responders vs $13 \%$ of nonresponders (1/4 CR, 2/3 VGPR, 0/2 PR, 1/8 NR). TRM was $56 \%$ and $75 \%$ among responders and nonresponders, and the respective relapse rates $11 \%$ vs $13 \%$. The main cause of death was infection in responders and aGVHD in nonresponders.

Conclusion: MSCs are an effective therapy for ca. half of patients with steroid resistant aGVHD. However, further improvements are needed since the other half do not respond, and mortality is high even among the responders.

Disclosure of Interest: None declared.

\section{P394}

Towards a more economical large-scale production of clinical grade MSCs

V. Valceckiene ${ }^{1, *}$, M. Stoskus ${ }^{1}$, A. Bukauskas ${ }^{1}$, L. Griskevicius ${ }^{1,2}$

${ }^{1}$ Hematology, Oncology and Transfusion Medicine Center, Vilnius University Hospital Santariskiu Klinikos, ${ }^{2}$ Clinics of Internal, Family Medicine and Oncology, Faculty of Medicine, Vilnius University, Vilnius, Lithuania

Introduction: Production of large scale mesenchymal stromal cells (MSCs) using standard cell culture conditions requires extensive resources, space, human labor and is time consuming. Recent introduction of single-use bioreactors offers an attractive alternative that addresses these issues, however, at a significantly increased production cost for disposables and cell media. Here we describe an innovative approach that allows a more economical large-scale production of MSCs by using CellEffic BM device and T-flasks for enrichment of MSCs from bone marrow (BM) with a subsequent large scale-expansion in the Quantum Cell Expansion System.

Material (or patients) and methods: $17-30 \mathrm{ml}$ of BM were obtained from the iliac crest of 6 healthy adult volunteers. BM cells were processed with CellEffic BM device (Kaneka Corporation, Osaka, Japan) and seeded in four $150 \mathrm{~cm}^{2}$ cell culture flasks. Cell medium was exchanged every 3 days. After 14-22 days of culture cells were harvested and reloaded on Quantum ${ }^{\circledR}$ Cell Expansion System (Terumo BCT, Inc, Lakewood, CO, USA). After 7-10 days of culture cells were harvested and cryopreserved for therapeutic use. All cells were grown in high glucose DMEM medium supplemented with $5 \%$ human platelet lysate and $5 \mathrm{U} / \mathrm{ml}$ heparin. The harvested batches of MSCs were tested for sterility, mycoplasma DNA, endotoxin levels as well as CD105, CD90, CD73, CD45 and CD34 expression by flow cytometry. Trilineage differentiation capacity was checked by in vitro differentiation assays (ScienCell Research Laboratories, Carslbad, CA, USA) on selected batches of MSCs.

Results: First passage of CellEffic BM device concentrated $\mathrm{BM}$ cells in cell culture flasks yielded $18-80 \times 10^{6}$ (mean 41 $\times 10^{6}$ ) of MSCs. The yield of MSCs after expansion on Quantum ${ }^{\circledR}$ system was $280-690 \times 10^{6}$ (mean $515 \times 10^{6}$ ). Tests for infections were negative. All MSCs were positive for CD105, CD90, CD73 and negative for CD45 and CD34. Selected batches of MSCs showed trilineage differentiation capacity.

Conclusion: CellEffic device concentrates MCSs from BM allowing passage 1 growth on a much smaller surface area (up to $600 \mathrm{~cm}^{2}$ ) and making it easier to handle standard cell culture in T-flasks. The growth of first passage MSCs in cell culture flask format considerably decreases the cost of MSCs production compared to the growth on Quantum ${ }^{\circledR}$ system. Second passage on Quantum ${ }^{\mathbb{R}}$ Cell Expansion System simplifies the open, labor-intensive manual tasks associated with large-scale MSCs culture in the T-flasks. Produced cells are consistent with the International Society of Cell Therapy (ISCT) standards for human MSCs.

Disclosure of Interest: None declared.

\section{P395}

CLEC12A-mediated antigen uptake and cross-presentation by human dendritic cell subsets efficiently boosts tumorreactive $\mathrm{T}$ cells

W. Hobo ${ }^{1, *}$, T. Hutten ${ }^{1}$, S. Thordardottir ${ }^{1}$, H. Fredrix ${ }^{1}$, L. Janssen ${ }^{1}$ R. Woestenenk ${ }^{1}, J$. Tel ${ }^{2}$, B. Joosten ${ }^{2}, A$. Cambi $^{2}$, M. Heemskerk ${ }^{3}$ G. Franssen ${ }^{4}$, O. Boerman ${ }^{4}$, A. Bakker ${ }^{5}$, J. Jansen', N. Schaap ${ }^{6}$, H. Dolstra

${ }^{7}$ Laboratory Medicine - Laboratory of Hematology, ${ }^{2}$ Tumor Immunology, Radboud university medical center, Nijmegen, ${ }^{3}$ Hematology, Leiden university medical center, Leiden, ${ }^{4}$ Nuclear Medicine, Radboud university medical center, Nijmegen, ${ }^{5}$ Merus pharmaceuticals, Utrecht, "Wematology, Radboud university medical center, Nijmegen, Netherlands

Introduction: Potent immunotherapies are urgently needed to boost anti-tumor immunity in hematological malignancies to enable disease control and cure. Because of their efficient antigen processing and presentation machinery, powerful $T$ cell stimulation and cross-talk capacity, natural dendritic cell (DC) subsets hold great potential for in vivo targeting with hematological tumor antigens. In this context, the endocytic C-type lectin receptors (CLRs) are attractive DC targeting molecules. Here, we investigated whether CLEC12A is an efficient and versatile target for in vivo tumor antigen delivery into human DC subsets, enabling effective induction of $\mathrm{CD4}{ }^{+}$ and $\mathrm{CD} 8^{+} \mathrm{T}$ cell responses. 
Material (or patients) and methods: CLEC12A expression and internalization capacity on myeloid and plasmacytoid DCs ( $\mathrm{mDCs}$ and $\mathrm{pDCs}$ respectively) in peripheral blood of healthy donors was studied using flow cytometry. In addition, CLEC12A and DEC205 internalization and intracellular routing to early endosomes $\left(\mathrm{EEA} 1^{+}\right)$and lysosomes $\left(\mathrm{LAMP} 1^{+}\right)$was investigated in monocyte-derived DCs (moDCs) using confocal microscopy. The effect of CLEC12A antibody binding on moDC phenotype and functionality was studied in vitro in an allogeneic mixed lymphocyte reaction. Furthermore, MoDCs, $\mathrm{mDCs}$ and $\mathrm{pDCs}$ were labeled with anti-CLEC12A/keyhole limpet hemocyanin (KLH) of anti-CLEC12A/HA1 synthetic I ong peptide conjugates. Upon maturation, DCs were co-cultured with $\mathrm{KLH}$ - or HA1-reactive T cells obtained from patients following allogeneic stem cell transplantation and/or KLH-pulsed dendritic cell vaccination. Subsequently, T cell proliferation, cytokine secretion and degranulation was analyzed.

Results: We confirmed that CLEC12A is selectively expressed by all cells of the myeloid lineage, including myeloid and plasmacytoid DC subsets ( $\mathrm{mDCs}$ and $\mathrm{pDCs}$, respectively). Moreover, we demonstrated that these DC subsets efficiently internalize CLEC12A, whereupon it quickly translocates to the early endosomes and subsequently routes to the lysosomes. Notably, CLEC12A antibody targeting did not negatively affect DC maturation nor function. Furthermore, CLEC12A-mediated delivery of KLH resulted in profound proliferation and cytokine secretion by $\mathrm{KLH}$-experienced $\mathrm{CD} 4^{+} \mathrm{T}$ cells. Most importantly CLEC12A-targeted delivery of HA-1 long peptide resulted in efficient antigen cross-presentation by $\mathrm{mDCs}$ and $\mathrm{pDCs}$, enabling strong activation of $\mathrm{HA}-1$-specific $\mathrm{CD}^{+} \mathrm{T}$ cells of patients after allogeneic stem cell transplantation.

Conclusion: Collectively, these data indicate that CLEC12A is an effective new candidate with great potential for in vivo antigen delivery into $\mathrm{mDCs}$ and $\mathrm{pDCs}$, thereby employing the specialized functions and cross-talk capacity of these DC subsets to boost tumor-reactive T cell immunity in hematological malignancies.

Disclosure of Interest: W. Hobo: None declared, T. Hutten None declared, S. Thordardottir: None declared, H. Fredrix: None declared, L. Janssen: None declared, R. Woestenenk: None declared, J. Tel: None declared, B. Joosten: None declared, A. Cambi: None declared, M. Heemskerk: None declared, G. Franssen: None declared, O. Boerman: None declared, A. Bakker Employee of: Merus pharmaceuticals, J. Jansen: None declared, N. Schaap: None declared, H. Dolstra: None declared.
P396

Cerebral cytokine release syndrome and complete remission of acute leukemia after CD19 chimeric antigen receptor modified $\mathbf{T}$ cell therapy

Y. $\mathrm{Hu}^{1, *}$, Z. $\mathrm{Wu}^{2}$, J. Sun ${ }^{1}$, J. $\mathrm{Yu}^{1}$, C. $\mathrm{Pu}^{2}$, L. Xiao ${ }^{2}$, H. Huang ${ }^{1}$

${ }^{1}$ The First Affiliated Hospital, School of medicine, Zhejiang University, Hangzhou, ${ }^{2}$ Shanghai Sidansai Biotechnology Limited Company, Shanghai, China

Introduction: Anti-CD19 chimeric antigen receptor modified $T$ cells (CART) have shown amazing clinical efficacy on refractory or relapsed acute $\mathrm{B}$ cell leukemia. Cytokine release syndrome (CRS) is the most common and severe complication after CART therapy and become the limiting factor for the successful clinical adaptation. So understanding the biology of the CRS and developing effective clinical management schemes will help to increase the safety, potency, and broad applicability of CART-cell technology.

Material (or patients) and methods: The patient received a diagnosis of ALL with BCR/ABL p210(+) in May 2013. She had a first relapse 14 months after the original diagnosis and had a second remission after reinduction chemotherapy and dasatinib treatment but complicated with central nervous system leukemia(CNSL) for 3 times. Afterwards she underwent 2 times of relapse in bone marrow. She did not have a response to further chemotherapy after relapse for the third time. Peripheral-blood mononuclear cells (PBMCs) were collected by means of apheresis before administration of lymphocytedepleting chemotherapy regimen FC (Fludarabine $30 \mathrm{mg} / \mathrm{m}^{2}$ day 1 to 3, Cyclophosphamide $750 \mathrm{mg} / \mathrm{m}^{2}$ day 3). CD19 CART cells were generated 11 days after PBMCs collection. The patient received an infusion of CD19 CART cells with the total dose of $6.7 \times 10^{7}$ cells per kilogram), given over a period of 3 consecutive days.

Results: 10 days after infusion, CR were achieved in bone marrow. The patient got fever at day 2 after infusion peaking at day 5 . She suffered from headache and vomit since day 3 . Serum cytokine levels are shown in Fig. 1. She also had recurrent right-sided facial and limb paresis that lasted approximately 20 minutes since day 3 . Papilloedema were observed by ophthalmoscope. Intracranial hypertension were considered. A bolus infusion of $125 \mathrm{~mL}$ of $20 \%$ mannitol was administered intravenously every 6 hours. But the symptoms continue to progress. Lumbar puncture was performed on day 5 and showed that cerebrospinal pressure was higher than $400 \mathrm{mmH} 2 \mathrm{O}$. The CSF contained $20 \mathrm{WBC} / \mu \mathrm{L}$ and $4.0 \mathrm{~g} / \mu \mathrm{L}$ protein; FACS showed $99 \%$ cells were CD3+

[P396]

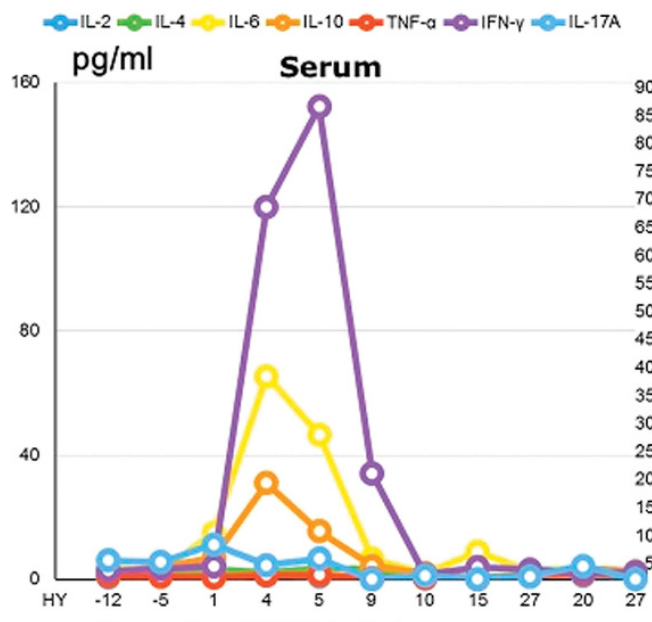

Days after CART infusion

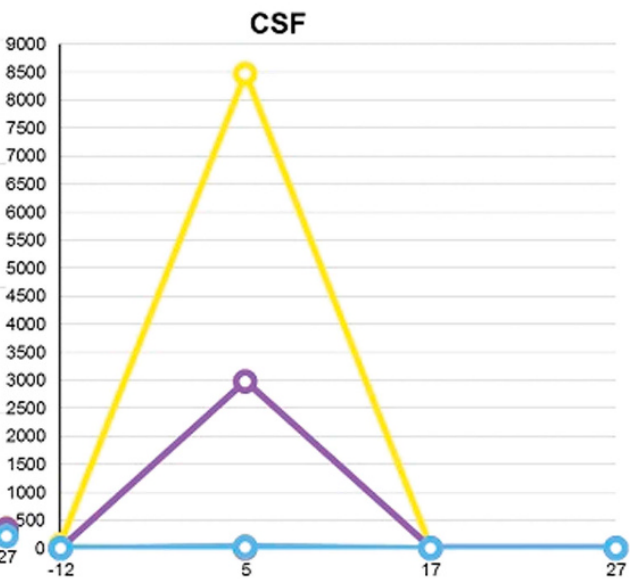

Days after CART infusion 
T cells. qPCR analysis showed 3032265 CAR copies/mg DNA. CSF cytokine showed IL- 6 was $8475 \mathrm{pg} / \mathrm{ml}$ and IFN- $\gamma$ were $2977 \mathrm{pg} / \mathrm{ml}$ while serum cytokine showed IL-6 was $46 \mathrm{pg} / \mathrm{ml}$ and IFN- $\gamma$ were $152 \mathrm{pg} / \mathrm{ml}$. Severe cerebral CRS was considered. Methyprednisone were applied to the patient on day 5 . Soon the symptoms of headache and vomit dissappeared. Cytokine levels in serum and cerebral decreased to normal level. She followed up in out-patient department and dasatinib was tapered.

Conclusion: For the first time we reported the cerebral CRS with high cytokine levels in CSF than in serum. We also applied methyprednisone and mannitol for the succeful treatment strategy. Our results perfect the mechnism, diagnosis and treatment of CRS

Disclosure of Interest: None declared.

P397

G-CSF mobilized versus conventional donor lymphocytes for relapse or mixed chimerism after HSCT: a single center retrospective study

Y. Tirefort ${ }^{1, *}$, S. Federico ${ }^{1}$, S. Masouridi-Levrat ${ }^{1}$, A. Pradier $^{1}$, C. Dantin ${ }^{1}$, Y. Beauverd ${ }^{1}$, C. Stephan ${ }^{1}$, K. Polchlopek', Y. Chalandon

${ }^{1}$ Hematology, University Hospital of Geneva, Geneva, Switzerland

Introduction: Donor lymphocyte infusions (DLI) are commonly administered after allogeneic hematopoietic stem cell transplantation (HSCT) to prevent and treat the relapse,to establish full donor chimerism, and to treat and prevent infections (1). G-CSF-mobilised lymphocytes from the original graft (G-DLI) are widely used in parallel with conventionally collected DLI (C-DLI). There are very few studies comparing the efficiency and toxicity of G-DLI and C-DLI after HSCT (2). We compared the outcome of patients who received C-DLI to those who had G- DLI.

Material (or patients) and methods: We retrospectively analysed the data of 191 patients transplanted in our centre from 2000 to 2015 for haematological malignancies and who received either G-DLI $(n=104)$ or C-DLI $(n=87)$.Indications for DLI included relapse (50.8\%),mixed chimerism (29.3\%),MRD (9.9\%),protocol driven (5.2\%),infection $(2.6 \%)$ or graft failure (2\%).Differences between survival curves of G-DLI and C-DLI recipients were determined using Log-rank test. Cox regression was used to examine the independent effect on OS of the following factors:age, conditioning (RIC or MAC), donor type (related or unrelated donor),Disease Risk Index (DRI: low, intermediate, high), disease status (CR or no $C R$ ),number of $\mathrm{T}$ cells infused (less or more than $10 \times 10^{6} \mathrm{CD} 3$ cells/ $\mathrm{Kg}$ ),year of DLI (before or after 2009) and type DLI (G-DLI or C-DLI). Cumulative incidence estimates of non-relapse mortality (NRM) were calculated with death from relapse or other causes as competing event.DLI efficiency for donor chimerism was scored as obtaining full donor chimerism after the last DLI, improvement in chimerism ( $>20 \%$ increase after the last DLI), or no response.For DLI given for relapse the efficiency was defined as CR, PR or no response after the last DLI.

Results: Accordingly to previous reports, we found no differences in OS between patients receiving G-DLI $(n=104)$ or C-DLI $(n=87)$ when all indications for DLI were taken into account $(P=0.121)$. Similarly, the cumulative incidence of postDLI acute and chronic GVHD were similar $(P=0.852$ and $P=0.343$ resp)in the G-DLI and C-DLI groups. Patients receiving $\mathrm{DLI}$ for relapse had similar OS in the G-DLI (40 patients) and C-DLI (57 patients) groups $(P=0.292)$.In contrast, when analysis was restricted to patients receiving DLI for mixed chimerism,we found significantly improved OS in patients receiving C-DLI $(n=18)$ as compared to patients receiving G-DLI $(n=38) \quad(P=0.0288)$. The positive effect of C-DLI on OS remained significant (HR $0.18395 \% \mathrm{Cl} 0.04128$ $0.814 ; P=0.02572$ ) in the multivariate analysis taking into account the aforementioned clinical factors. While there was no statistically significant difference in obtaining a full donor chimerism after G-DLI infusion as compared to C-DLI
$(P=0.077)$, the NRM was significantly reduced in the group of patients treated with C-DLI for mixed chimerism as compared with patient receiving $\mathrm{G}-\mathrm{DLI}(P=0.0466)$.

Conclusion: Our results suggest that G-DLI and C-DLI have similar impact on survival when given for relapse and mixed chimerism. There was no difference on GVHD incidence. However when administered for mixed chimerism only, there was a significant advantage in OS for C-DLI as compared to G-DLI. Further investigations are needed to assess safety and efficacy of G-DLI when compared to C-DLI.

References: (1) Frey NV and Porter DL. Best Pract Res Clin Haematol 2008 21: 205.

(2) Abbi KK et al.BMT 2013; 48:357.

Disclosure of Interest: None declared.

P398

Results of Sepax 2 validation for washing of PBSC transplants after thawing and process optimization

L. Adamusová ${ }^{1,2,3,{ }^{*}}$, Z. Kořistek ${ }^{1,3}$, J. Smejkalová ${ }^{1}$, P. Vrublová ${ }^{1,3}$, L. Grebeníček ${ }^{1}$, R. Hájek ${ }^{1,3}$

${ }^{1}$ Department of Haematooncology, University Hospital Ostrava, ${ }^{2}$ Department of Biology and Ecology, ${ }^{3}$ Faculty of Medicine, University of Ostrava, Ostrava, Czech Republic

Introduction: Cryopreservation of hematopoietic stem cells (HSC) for autologous transplantation requires cryoprotective agent (CPA), typically dimethyl sulfoxide (DMSO). However, infusion of cryopreserved HCSs with DMSO could be associated with adverse reactions during and after the infusion. Therefore, removal of DMSO from grafts is generally recommended, but it can lead to a significant loss of HSCs. The most advanced approach is to use automatic systems, which enable standard conditions during the washing and the process could be further optimized. Washing of grafts not only reduces DMSO toxicity, but have more advantages: volume of HSCs infusion is reduced and does not demand central venous catheter, transplantation could be easily performed in outpatient setting.

Material (or patients) and methods: A fully-automated, mobile, closed capability system SEPAX 2 (Biosafe, Switzerland) with SmartWash software was used for the HSC graft washing. Validation procedures were performed in triplicates. For ethical reasons, all tested cryopreserved transplants came from deceased patients. Process included adjustment of washing and washing solution, verification of washing effectiveness and safety and determination of shelf life of washed HSC transplants under different conditions. Following tests were used after thawing, immediately after washing and in regular time intervals during storage of washed transplants in room temperature and in $4-8^{\circ} \mathrm{C}$ : sterility, WBC, CD34+ cells and CFU-GM counts, vitality and concentration of DMSO.

Results: Two cryopreserved HSC transplants were used for optimization of washing and washing solution. Formation of precipitates in grafts occurred immediately after using of washing solution consisted from $0.9 \%$ saline solution with $2.5 \%$ of human albumin. Precipitates contained fibrin fibers and cellular elements including CD34+ cells. If the washing solution was enriched with ACD-A citrate solution in ratio of $1: 12$, the precipitates formations were not observed. Four HSC grafts were used for further studies. Ratios of WBC, CD34+ cells, CFU-GM counts and vitality after and before washing exceeded 0.9 (recovery $>90 \%$ ). Residual concentrations of DMSO were $<1 \%$. Sterility was confirmed during all processes. Determination of shelf life of washed transplants was based on CFU-GM recovery ( $>80 \%$ was acceptable). The room temperature shelf life was 2 hours and shelf life in $4-8{ }^{\circ} \mathrm{C}$ was 4 hours. Vitality, WBC and CD34+ cell counts were $>80 \%$ compared to numbers obtained immediately after washing even after 8 hours of storage.

Conclusion: The washing procedure using SEPAX 2 with SmartWash software was reliable, cell recoveries and vitalities were satisfactory. The main obstacle, formation of clots or precipitates in the HSC transplants after washing, was solved 
by the adding of ACD-A citrate solution to the washing solution. The grafts should be stored at $4-8{ }^{\circ} \mathrm{C}$ and infused within four hours after washing. Based on these data, the system and method has been successfully used for washing of HSC transplants in a patient with end-stage renal disease and anuria resulting in hematopoietic recovery without any delay. Disclosure of Interest: None declared.

\section{P399}

Low miRNA-15a and miRNA-126 level prior to autologous hematopoietic stem cell transplantation are associated with shorter time to engraftment

M. Nowicki $i^{1, *}$, J. Szemraj ${ }^{2}$, A. Wierzbowska ${ }^{1}$, R. Małachowski ${ }^{1}$ O. Grzybowska-Izydorczyk ${ }^{1}$, A. Pluta ${ }^{1}$, M. Czemerska ${ }^{1}$, K. Stępka ${ }^{1}$ T. Robak', A. Szmigielska-Kapłon ${ }^{7}$

${ }^{1}$ Medical University of Lodz, Copernicus Hospital, Department of Hematology, ${ }^{2}$ Department of Medical Biochemistry, Medical University of Lodz, Łódź, Poland

Introduction: MicroRNAs are a class of small (19-25 nucleotides), endogenous RNA which play a significant role in regulation of gene expressions, cell proliferation, differentiation, apoptosis. It also can modulate angiogenesis, mobilization of hematopoietic stem cells and homing after transplant targeting bone marrow niche cytokines.

The aim of our study was to evaluate the miRNA's involved in hematopoiesis, homing and mobilization of hematopoietic stem cells in autologous transplantation setting. Levels of miRNA-15a, miRNA-16 and miRNA-126 were assessed before autologous hematopoietic stem cells transplantation and their influence on engraftment was evaluated.

Material (or patients) and methods: Thirty four patients were enrolled to the study $(17 \mathrm{~F}, 17 \mathrm{M})$. The median (Me) age was 59 years. The investigated group consisted of 29 Multiple Myeloma, 4 Non-Hodgkin Lymphoma, and 1 Hodgkin Lymphoma. The blood (plasma samples) were collected on 2 time points: before chemotherapy "BC" and on the day of HSCT "0" (after chemotherapy). The miRNA level evaluated by Real Time PCR method.

Results: Our study revealed decreased level of miRNA-15a on day " 0 " as compared to baseline $(\mathrm{BC})$ : ( $\mathrm{Me}=1$ vs $2,61, P=0,01$ ). The level of miRNA-16 and miRNA-126 has not changed significantly on day " 0 " as compared to baseline.

To evaluate influence of miRNA level on engraftment time we divided patients according to median miRNA levels into two groups: "high" and "low expressors" (above and below median).

We observed, that in the group of miRNA-15a "low expressors" and miRNA-126 "low expressors" on "BC" day, the time to engraftment was shorter than in "high expressors" ( $\mathrm{Me}=12$ vs 15 days, $P=0,04)$ and $(M e=12$ vs 14 days, $P=0,02)$ respectively. The miRNA-16 level measured in "BC" time point did not differ and no correlation with engraftment was found. We found no correlation between miRNA-15a, miRNA-16 and miRNA-126 level on day" 0 " and time to engraftment.

Conclusion: In conclusion, miRNA-15a level change significantly after conditioning treatment. Low level of miRNA-15a and miRNA-126 before chemotherapy correlates with faster engraftment after HSCT.

Disclosure of Interest: None declared.

\section{P400}

International validation of the prognostic value of the Lymphocyte/Monocyte ratio: one size does not fit all M. S. Santos ${ }^{1, *}$, R. Tenreiro ${ }^{1}$, M. Pereira ${ }^{1}$, R. Guilherme ${ }^{1}$, M. Gomes ${ }^{7}$, P. César ${ }^{i}$, J. P. Carda ${ }^{7}$, E. Cortesão ${ }^{7}$, A. I. Espadana ${ }^{\prime}$, C. Geraldes', L. Ribeiro ${ }^{\prime}$

${ }^{1}$ Hematology, CHUC-HUC, Coimbra, Portugal

Introduction: Autologous stem cell transplantation (ASCT) is largely employed in primary refractory or relapsed Hodgkin Lymphoma (HL). In allogenic stem cell transplantation several prognostic factors at day 100 have been studied to predict clinical outcomes including absolute lymphocyte count (ALC), absolute monocyte count (AMC) and ALC/AMC ratio, with higher associating with improved survival. Some studies have also demonstrated that these are prognostic markers in patients with $\mathrm{HL}$ after ASCT for overall survival (OS) and progression freesurvival (PFS); an ALC/AMC ratio of 1.3 has been proposed as the optimal cut-off in a 131 patient single-center cohort.

Material (or patients) and methods: We aim to evaluate the applicability of the Day 100 ALC/AMC ratio as a prognosticator of OS and PFS in patients with HL after ASCT, in a different patient cohort. We did a retrospective analysis on patients with HL submitted to ASCT between 01-01-1996 e 31-12-2014 $(n=66)$. Patients with ALC and AMC data at approximately day100 (median day101, 80-130) who had achieved complete remission (CR) at day100 were included.

Results: We studied 35 patients (69\%male) with a median age of 28-years-old $(\mathrm{y})(15-61)$. In $86 \%(n=30)$, the histologic subtype was nodular sclerosis. At the time of diagnosis, $63 \%$ $(n=22)$ presented advanced stage disease and $37 \%(n=13)$ presented early stage disease. Most patients received $1^{\text {st }}$ line chemotherapy based on the ABVD protocol( $n=27)$. Fourteen patients $(40 \%)$ had radiation therapy before ASCT. Nine patients (26\%) presented CR>12 months, 8 (23\%) CR $<12$ months and 18 (51\%) didn't achieve CR. Twenty eight patients were in CR at the time of ASCT(46\% RC1; $43 \%$ RC2; $11 \%$ $\mathrm{RC}>2$ ). All patients were conditioned with a BEAM regimen. $A$ mean of $4,79 \times 10^{6}$ cells $\mathrm{CD} 34+/ \mathrm{Kg}$ were transplanted. At day 100 , the median ALC was $1.65 \times 10^{9} / L(0,3-3,6)$ and median the AMC was $0,5 \times 10^{9} / \mathrm{L}(0,1-1,4)$. OS and PFS, at $10 \mathrm{y}$, for this group was $90 \%$ and $84.2 \%$, respectively. Contrary to the published series, the ALC/AMC ratio of 1.3 did not adequately predict survival. In our series, lower ALC predicted longer survival, with an optimal cut-off of $2.15 \times 10^{9} / \mathrm{L}$, with a median PFS of $3.9 \mathrm{y}$ in the $>2.15$ cohort, and not reached in the $<2.15$ cohort $(P=0.015)$; considering the ALC/AMC ratio, higher ratios associated with poor outcomes, with an optimal cut-off of 6.075; patients with ratios $<6.075$ had a median PFS of $0.86 y$, and those with ratios $>6.075$ had not reached the median, with $96.3 \%$ alive without progression at $10 \mathrm{y}(P<0.0001)$. A comparison of our series with the published cohort identified a higher proportion of patients transplanted in CR (in our series), compared to PR (in the published data). Subgroup analyses performed on our data in the CR and PR subsets were concordant with our results for our full cohort.

Conclusion: Day 100 ALC/AMC ratio is a simple and low cost prognosticator of OS and PFS. However, published cut-offs appear to be series-specific and influenced by local patient-, disease- and treatment-specific characteristics; further studies should help to validate the proposed cut-offs and to identify their specific applicability to different populations.

Disclosure of Interest: None declared.

\section{P401}

Bone Marrow Transplantation in Children: a Single-Centre Experience in Saudi Arabia

M. M. Alam ${ }^{1, *}$, I. Abosoudah ${ }^{1}$, S. AL Harbi ${ }^{1}$, M. Elhadidy ${ }^{1}$, A. Ali ${ }^{1}$, M. Bayoumy ${ }^{1}$

${ }^{1}$ Department of Oncology, Section of Pediatric Hematology/ Oncology/BMT, King Faisal Specialist Hospital and Research Centre, Jeddah, Saudi Arabia

Introduction: Bone Marrow Transplantation (BMT) has frequently considered as a curative treatment for children with serious hematological and malignant disorders. The aim of this study was to determine the indication, frequencies of transplant-related morbidity and outcome at our tertiary health care facility.

Material (or patients) and methods: We retrospectively analyzed the clinical, laboratory and outcome data of all 131 pediatric patients consecutively underwent BMT between 2005 and 2014. 
Results: The mean age of the study population at the time of transplant was $6.5 \pm 4$ years. Out of them males were 85 $(64.9 \%)$ and females were $46(35.1 \%)$. Majority of patients have hematological nonmalignant disorder $(n=51,38.2 \%)$ followed by hematological malignancy $(n=50 ; 38.2 \%)$; then solid tumors $(n=30 ; 22.8 \%)$.

Most of the patients received allogeneic transplant $(n=92$; $70.2 \%)$ and remaining $(n=39 ; 29.8 \%)$ were autologous transplant. Source of donor in all allogeneic cases were full matched related donor. Source of stem cell in $(n=51$; $38.9 \%)$ patients was bone marrow and $(n=43 ; 32.9 \%)$ was peripheral stem cell. Mean stem cell dose CD 34 was 7.2 $(+/-0.25) \times 10^{6} / \mathrm{kg}$.

Acute GVHD was observed in $26(19.8 \%$ in all and $28.3 \%$ in allogeneic transplant patients). Out of them grade I, II, III and IV GVHD were observed in 9, 10, 5 and 2 patients respectively. Chronic GVHD was observed in $9(6.9 \%)$ cases. Most of patients were engrafted $(n=109 ; 83.2 \%)$ with median duration of ANC engraftment is 20.9 (Range: 9-48) days and median platelet engraftment days of 28 (Range: 7-200) days.

CMV reactivation was observed in 38 patients (29\%) cases within 100 day of post BMT. Out of them majority were asymptomatic $(n=35 ; 26.7 \%)$ and remaining $(n=3 ; 2.3 \%)$ had clinical manifestation/organ involvement (retinitis/pneumo$\mathrm{nia} / \mathrm{colitis} /$ hepatitis and skin manifestation). Most of CMV cases were resolved $(n=35 ; 26.7 \%)$. In most case $(35 / 38 ; 92.1 \%)$, the level of antigenemia declined within 2 weeks as a result of the continuation of GCV therapy, but only three out of 38 patients subsequently developed CMV disease. There is no difference in engraftment failure in patients who had CMV reactivation and no CMV reactivation $18.5 \%$ vs $16 \%$, however patients who had CMV reactivation had significantly higher rate of GVHD as compared to patients without CMV reactivation (12$/ 38=31.6 \%)$ vs $14 / 93=15.1 \%)[P=0.031]$.

Overall survival (OS) and Event free survival (EFS) rate were $69.5 \%$ and $75.6 \%$ respectively. Overall survival time was 35.5 $(+/-2.8)$ months and EFS time is $31.6(+/-2.6)$ months with mean duration of follow up $72(+/-4.4)$ months. Cause of death in majority of patients (31/40) were either progressive disease and/or relapse, however in other $9(9 / 40=6.9 \%)$ patients who died in remission the possible causes of death were as follow (5 sepsis, 3 GVHD and/or VOD, 1 secondary pulmonary fibrosis).

Conclusion: In conclusion, recent development in better understanding of GVHD, process of engraftment and advances in the field of infection control after BMT have had a great impact on the improvement in transplant outcomes. The results of the pediatric BMT program at our institution have been comparable to those reported in the literature as far as transplant-related morbidity and mortality is concerned. The duration of follow-up is short and the long-term outcome is yet to be determined.

Disclosure of Interest: None declared.

\section{P402}

The utility of autologous haematopoietic stem cell backup harvests for children receiving living unrelated or cord progenitor stem cells allogeneic haematopoietic transplantation? A large single paediatric centre study

P. Sivaprakasam ${ }^{1, *}$, M. Cummins ${ }^{1}$, J. Moppett ${ }^{1}$, J. Collins ${ }^{1}$,

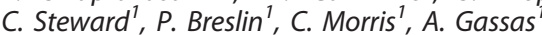

${ }^{7}$ Paediatric Stem Cell Transplantation, BRISTOL ROYAL HOSPITAL FOR CHILDREN, Bristol, United Kingdom

Introduction: Autologous haematopoietic stem cells (HSCs) harvested as back-up prior to allogeneic hematopoietic stem cell transplantation (HSCT) may potentially be useful in the treatment of graft failure.

Material (or patients) and methods: A retrospective review was done on all children who received a HSCT in Bristol Royal Hospital for Children (BRCH) and had an autologous HSC backup harvested between January 1990 to December 2015 to evaluate the value of this procedure by establishing how many of these harvested back-up HSCs were reinfused.

At BRCH, between 1990-2012, autologous back-up harvest was performed on all children receiving an unrelated donor, cord or haploidentical HSCT. However from 2012, patients receiving fully matched unrelated donor recipients were excluded.

Results: 594 living unrelated/cord stem cells and 78 haploidentical HSCTs (total $n=672$ ) were performed. 5 patients had their autologous HSCs reinfused (1- X-ALD; 1-ALL; 3-AML). Indications were as follows: 4- Primary graft failure; 1- graft dysfunction with disseminated viral infection. Three patients are still alive, one lost to follow-up and one died of relapse. Median time to follow up is 48 months (7-154 months).

Conclusion: Our data demonstrates that the utility of autologous back-up HSCs is $<1 \%$ and the storage of these cells in terms of resources and cost involved may not be justified. Furthermore, particularly in children where at least one parent is available, the current success of haploidentical HSCT makes it an attractive choice for engraftment failure salvage rather than patients' own HSCs.

References: The harvest and use of autologous back-up grafts for graft failure or severe GVHD after allogeneic hematopoietic stem cell transplantation: a survey of the European Group for Blood and Marrow Transplantation. M Stelljes et al. Bone Marrow Transplantation (2008) 42, 739-742; doi:10.1038/ bmt.2008.254; published online 25 August 2008.

Are backup BM harvests worthwhile in unrelated donor allogeneic transplants? C Stotler et al. Bone marrow transplantation June 2009; 45(1):49-52.

Disclosure of Interest: None declared.

P403

Influence of donor age in allogeneic hematopoietic cell transplantation outcomes

R. Saldaña Moreno ${ }^{1, *}$, M. D. Madrigal Toscano ${ }^{1}$, A. Salamanca Cuenca', C. Fraidias Hidalgo ${ }^{2}$, V. Verdugo Cabeza de Vaca', M. Á. Correa Alonso' ${ }^{1}$ S. Garzón López ${ }^{1}$

${ }^{1}$ Hematology, Hospital de Jerez de la Frontera, Jerez de la Frontera, 'Facultad de Medicina, Universidad de Cádiz, Cádiz, Spain

Introduction: Allogeneic stem cell transplantation (HSCT) is, nowadays, the only potential curative approach for most hematologic malignancies. Development of some strategies as reduced-intensity conditioning and improvement of the surrounded supportive care, has allowed the HSCT to be also an option for elderly patients. As the age of patients increases, sibling donors do it as well. Nevertheless, the way in which advanced age affects on the results of HSCT remains uncertain. The aim of this study is to describe the influence of donor age on the transplant outcome, in terms of overall survival, and secondary, to compare those results depending on kinship with the donor (related or non related).

Material (or patients) and methods: Analytical, observational and retrospective study. We included 172 patients diagnosed with different hematological diseases who were treated with allogeneic stem cell transplantation at Hospital de Jerez de la Frontera between January 2000 and March 2015. We analysed overall survival (\% patients alive) at 100-days, 1-year and 5 -years post-HSCT in different groups of patients, depending on donor's age and type of transplant (related/non-related).

Results: In the survival analysis, the Kaplan-Meier estimator detected a 5-year overall survival (OS) of $44.3 \%$ for the whole sample. According to donor age, patients who received transplant from older donors (50 years or above) showed a poorer OS $(31.7 \%$ vs $49.3 \% ; P=0.145)$. In the stratified subanalysis, 5-year OS was higher among younger patients with younger donors than among younger patients with older donors (51.8\% Vs $40.3 \%)$, as well as in older patients groups with younger rather than older donors $(36.5 \%$ Vs $28.2 \%$; $P$ 0.0427). Based on kinship, OS was higher in the related and unrelated younger donor groups than in related or unrelated older donor groups ( $P$ 0.116). 


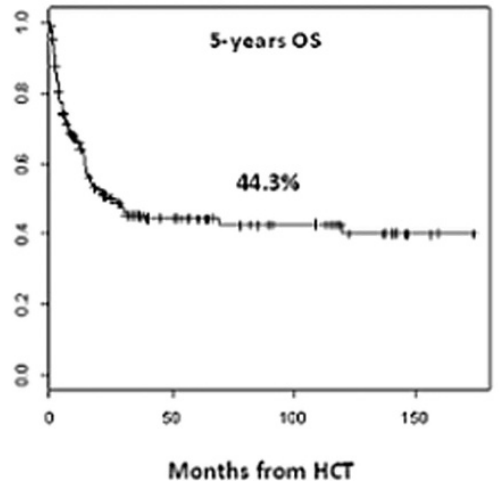

Months from HCT
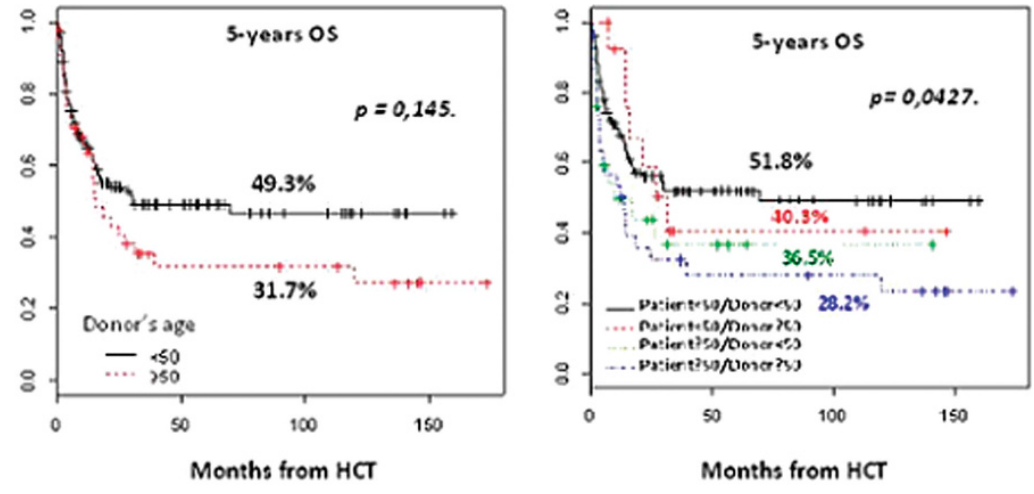

Conclusion: In our cohort, patients with younger donors had better outcomes in terms of overall survival when compared to those with older donors. It seems also to be a trend that patients with unrelated younger donor have better outcomes than those with related older donor, but more studies may be performed to clarify this issue.

References:

Disclosure of Interest: None declared.

\section{P404}

Cytomegalovirus and Effect on Early Chimerism in Patients with Myeloid Disorders Undergoing Stem Cell Transplantation Using Reduced Toxicity Ablative Conditioning Regimen

S. Farhan ${ }^{1, *}$, E. Peres $^{1}$, N. Janakiraman ${ }^{1}$

${ }^{1}$ Henry Ford Hospital, Detroit, United States

Introduction: The influence of cytomegalovirus (CMV) on the chimerism in reduced toxicity ablative conditioning SCT in myeloid disorders is ill defined. A recent report published in Blood by Sellar et al showed that in patients who received alemtuzumab-based regimen, the group of patients who were recipient positive $(\mathrm{R}+)$ / Donor negative (D-) had CMV-specific $T$ cells that are exclusively of recipient origin and significantly influenced the chimerism status toward recipients. To explore the impact of CMV in recipients and donors on early chimerism, we undertook a retrospective analysis of patients with myeloid disorders who received four days of fludarabine and busulfan with or without anti-thymocyte globulin (ATG) at our center in the last 10 years.

Material (or patients) and methods: Chimerism assay was performed using a quantitative fluorescence-based short tandem repeat-polymerase chain reaction (STR-PCR) with capillary electrophoresis for PCR product resolution.

Results: 42 patients with myeloid disorders received fludarabine and busulfan $x 4$ doses. Of the 42 patients, 25 had ATG. There were 28 males and 14 females with a median age of 62 years. Median time to follow up was 8 months (0.8-54 months). Disease risk was considered advanced in 21 , intermediate in 4 and early in 17. Total Recipient cell chimerism showed increase recipient chimerism in $5 / 11$ (45\%) of $R+D$ - vs $2 / 6(33.3 \%)$ of R-D- in the group of patients who received ATG, $P=1.0$, with a mean of recipient chimerism at day 100 of $20.4 \%$ in the $R+D$ group compared to $17 \%$ in the R-D- group. In the group who did not get ATG, recipient chimerism increase was $3 / 4(75 \%)$ in the $R+D$ - patients compared to $4 / 4(100 \%)$ in patients who were $\mathrm{R}-, P=1.0$. The mean of recipient chimerism at day 100 in the R+D- no ATG group was $23.25 \%$ with a median of $12 \%$ while the mean and median at day 100 in the R- no ATG group were $35.25 \%$ and $19.5 \%$ respectively $(P=0.573)$.When looking at the increase in recipient chimerism in the group of patients who were R+D-, in those who got ATG it was $45 \%$ increase vs $75 \%$ increase in those who did not get ATG $(P=0.569)$ with a median of $12 \%$ vs $0 \%$ respectively $(P=0.49)$. Also increase of recipient chimerism was $33.3 \%$ in patients who were $\mathrm{R}$ - and got ATG vs $100 \%$ in R- no ATG patients $(P=0.076)$ with median at day 100 of 0 vs $19.5 \%(P=0.098)$.

Conclusion: In this small cohort from a single center, we found that in patients with myeloid disorders who received reduced toxicity ablative conditioning regimen and ATG, there was no statistically significant increase in recipient chimersim in the R+D- group compared to R-D- group. This is different from what Sellar et al found in a small group of patients who received alemtuzumab. These results may indicate a difference between ATG and alemtuzumab in the effect of CMV on the recipient chimerism, which need to be studied further in a larger retrospective or prospective study. This is especially important in myeloid disorders since persistent or increase in recipient chimerism may identify high-risk patient cohorts who may benefit from additional therapeutic interventions.

References: Rob S. Sellar et al. CMV promotes recipient T-cell immunity following reduced-intensity T-cell-depleted HSCT, significantly modulating chimerism status. Blood. 2015 Jan 22;125(4):731-9.

Disclosure of Interest: None declared.

\section{P405}

Differences in side effects, sick leave and the will to donate again: the Nordic Register of Haematopoietic Stem Cell Donors

S. Pahnke ${ }^{1,2, *}$, A. Fischer-Nielsen ${ }^{3}$, E. Haastrup ${ }^{3}$, D. Heldal ${ }^{4}$, M. Itälä-Remes ${ }^{5}$, M. Kauppila ${ }^{6}$, G. Larfors', R. 'Niittyvuopio', H. Hägglund ${ }^{1}$

${ }^{1}$ Haematology, Uppsala University Hospital, Uppsala, ${ }^{2}$ Haematology, Falun County Hospital, Falun, Sweden, ${ }^{3}$ Clinical Immunology, Copenhagen University Hospital, Copenhagen, Denmark, ${ }^{4}$ Haematology, Oslo University Hospital, Oslo, Norway, ${ }^{5}$ Stem Cell Transplantation Unit, ${ }^{6}$ Haematology, Turku University Hospital, Turku, ${ }^{7}$ Stem Cell Transplantation Unit, Helsinki University Hospital, Helsinki, Finland

Introduction: Given that the number of allogeneic transplantations of haematopoietic stem cells are increasing, approaching 25000 per year worldwide ${ }^{1}$, and more than 27 million potential voluntary donors are registered in donor registries world wide ${ }^{2}$, the safety and follow-up of both related and unrelated donors are of great importance.

Material (or patients) and methods: The Nordic Register of Haematopoietic Stem Cell Donors, NRHSD, is a voluntary, Ethics Committee reviewed, register of Bone Marrow (BM) and Peripheral Blood Stem Cell (PBSC) donors from 10 transplant centres in Sweden, Norway, Finland and Denmark between 1998 and 2014. The register includes information about donor characteristics and the donation process as well as reports on short term side effects, sick leave and the willingness to donate again. As by 31 December 20141980 evaluable donors had, after written consent, been registered. 
Results: Mean age at donation was 42.8 years. BM and unrelated donors were significantly younger than PBSC and related donors; mean ages 37.3 vs 44.7 and 36.7 vs 46.3 years, $(P<0.0001)$. No significant age difference was seen between male and female donors.

$25 \%$ donated $\mathrm{BM}$ and $75 \%$ PBSC. Unrelated donors were significantly more likely to donate bone marrow than related donors, $35 \%$ vs $20 \%(P<0.0001)$.

Side effects were reported by $80 \%$ of BM donors, the most common being skeletal/muscle pain (65\%), fatigue (21\%), hematoma at aspiration site (10\%) and nausea (8\%). 45\% had fully recovered from symptoms within 1 week while $5 \%$ had symptoms lasting $>4$ weeks.

PBSC donors reported side effects in $83 \%$, the most common being skeletal/muscle pain (78\%), headache $(25 \%)$ and fatigue (11\%). $89 \%$ had fully recovered within 1 week and less than $1 \%$ had symptoms lasting more than 4 weeks.

When asked in a follow-up questionnaire, $79 \%$ of donors would be willing to donate again, 19\% were uncertain and $2 \%$ would not consider donating again. Significant differences in the willingness to donate again were seen between unrelated $(92 \%)$ and related $(72 \%), \mathrm{BM}(85 \%)$ and PBSC $(77 \%)$, and between younger $(<50$ years, $84 \%)$ and older donors ( $>50$ years, $73 \%)$ in univariate analyses $(P<0.001)$. No differences were seen between male and female donors. In a multivariate Poisson regression analysis, only being an unrelated donor remained an independent factor.

Donors aged 18-65 years were analysed for days of sick leave, with $71 \%$ needing less than 1 week and $29 \%$ more. There were significant differences $(P<0.0001)$ in sick leave $>1$ week between unrelated (40\%) and related donors (22\%), BM (55\%) and PBSC (19\%), and between younger (18-50 years, 35\%) and older donors (50-65 years, 23\%). No differences in sick leave $>1$ week were seen between male and female donors. Conclusion: This is a first report from the NRHSD register including 1980 haematopoietic stem cell donors. An equal proportion of about $80 \%$ of BM and PBSC donors experienced any side effect but BM donors needed a longer time to full recovery after donation. Unrelated, BM and younger donors were more likely to be willing to donate again when compared respectively with related, PBSC and older donors but also more likely to need longer sick leave, something that to our knowledge has not been previously reported.

References: 1. European Society for Blood and Marrow Transplantation Press Kit 2014, www.EBMT.org

2. Bone Marrow Donors Worldwide, 16 November 2015, www.bmdw.org

Disclosure of Interest: None declared.

\section{P406}

The impact of autologous stem cell transplantation in multiple myeloma on treatment outcomes: a single center experience

V. Pop ${ }^{1, *}$, V. Pop ${ }^{2}$, O. Rukavitsin ${ }^{2}$, P. Simashova ${ }^{2}$

${ }^{1} 1$ Federal State Budgetary Institution Main Military Clinical Hospital named after ac. N.N. Burdenko of the Ministry of Defense of the Russian Federation, Moscow, Russian Federation, ${ }^{2}$ Hematology, 1 Federal State Budgetary Institution Main Military Clinical Hospital named after ac. N.N. Burdenko of the Ministry of Defense of the Russian Federation, Moscow, Russian Federation

Introduction: A main approach to treatment of symptomatic multiple myeloma (MM) in eligible patients (pts) is ASCT that can lead to improving of response, as well as increased survival rates.

The primary aim was to assess progression free survival (PFS) and overall survival (OS).

Material (or patients) and methods: The study included a cohort of pts with MM who underwent of ASCT for the period from 2001 to 2015. Induction therapy prior to ASCT included VAD-regimen and bortezomib-containing regimens with subsequent high-dose cyclophosphamide therapy. OS and progression free survival (PFS) estimated by the KaplanMeier method.

Results: The analyzed group consisted of 82 pts with primary diagnosed MM (57 males/25 females; mean age 54 yrs (range 34-67). Ig $\mathrm{MM}$ was diagnosed in 48pts (58,5\%), IgA MM in 15 $(18,3 \%)$, Bence Jones myeloma in $16(19,5 \%)$, primary plasma cell leukemia in 3pts (3,7\%). In accordance with clinical stage, (Durie B.G.M. and Salmon S.E.) pts were distributed as follows: IA - 4 (4.9\%), IIA - 24 (29.3\%), IIB - 2 (2.4\%), IIIA - 26 (31.7\%), IIIB $26(31.7 \%)$. Induction therapy was VAD in 28pts (34.1\%), bortezomib (VDD, VD and VAD-like) in 54 pts (65.9\%). The median of follow-up was $24.7 \mathrm{~m}$ (0.6-138.7 m). The cohort studied performed 94 ASCT. 12pts received 2 ASCT including planned tandem ASCT $(n=6)$ and 2nd ASCT due to progression $(n=6)$. The impact of induction therapy (with or without bortezomib) did not affect the results of survival $(P=0.62)$. Renal impairment and advanced stage of $\mathrm{MM}$ as a poor prognostic factors also had no effect on the survival rate after ASCT $(P=0.3)$. PFS and OS after ASCT $(n=82)$ was $23.2 \mathrm{~m}$ and $43.4 \mathrm{~m}$, respectively. OS from the diagnosis of MM was $57.6 \mathrm{~m}$. OS after 1 st-line therapy $(n=69)$ was $68,5 \mathrm{~m}$ compared to $27,8 \mathrm{~m}$ after 2 nd-line $(n=13), P=0.03$. Median PFS after the $1 \mathrm{st}$ and 2nd line therapy did not differ $(P=0.22)$. OS was significantly greater in the group of pts with $C R$ after induction therapy $(n=26)$ than with PR $(n=56): 93,6 \mathrm{~m}$ vs $42,8 \mathrm{~m}$, $P=0.03$.

Conclusion: PFS and OS after ASCT in our study was 23.2 months and 43.4 months, respectively. ASCT followed by high-dose chemotherapy demonstrated improving responses in patients with poor prognostic factors (renal impairment). No survival benefit was seen for patients treated with ASCT in different regimens of induction chemotherapy and if they have renal impairment.

Disclosure of Interest: None declared.

\section{P407}

The efficacy of bortezomib-based induction regimen followed by autologus stem cell transplantation for multiple myeloma in Chinese patients

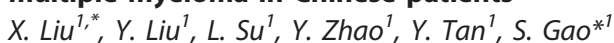

${ }^{1}$ Cancer Center, the first hospital of Jilin University, changchun, China

Introduction: To evaluate long-term effect of novel agents followed by autologous hematopoietic stem cell (ASCT) in Chinese patients with multiple myeloma (MM) in order to find out the optimal therapeutic regimen for transplant-eligible patients.

Material (or patients) and methods: A retrospective analysis was performed upon clinical data of $18 \mathrm{MM}$ patients who received bortezomib-based induction regimen followed by ASCT from Janurary 2011 to December 2015. Stem-cell mobilization was undertaken with cyclophosphamide plus granulocyte colony-stimulating factor after the patients got partial response (PR). The conditioning regimen was high-dose melphalan+/-bortezomib. Thirteen patients received maintenance therapy with thalidomide plus dexamethasone (TD), and 4 patiants got maintenance therapy with lenalidomide. All patients were followed up to December 2015.

Results: Symptomatic MM patients [12 males, 6 females; median age, 56 (range 36 63 years)] received bortezomibbased induction regimen. The analysis of cytogenetics was performed in 13 patients by fluorescence in situ Hybridization (FISH) (10 patients belong to high-risk group, 3 patients belong to standard-risk group).The median cycle was 4 cycles(3-6 cycles). Overall response rate (ORR) [complete remission (CR) +near complete remission $(\mathrm{nCR})+$ partial remission $(\mathrm{PR})], \geq$ very good partial remission (VGPR) and CR rate of post-induction were $94.4 \%, 88.9 \%$, and $55.6 \%$, respectively. The median mononuclear cells (MNCs) and $\mathrm{CD}^{+} 4^{+}$cells were 5.07 $(8.64 \sim 18.1) \times 10^{8} / \mathrm{kg}$ and $5.07(2.71 \sim 14.6) \times 10^{6} / \mathrm{kg}$ after mobilization. The median number of MNCs and CD34 $4^{+}$cells for infusion were $4.5(2.84 \sim 10.15) \times 10^{8} / \mathrm{kg}$ and $2.358(1.65 \sim 5.3)$ 


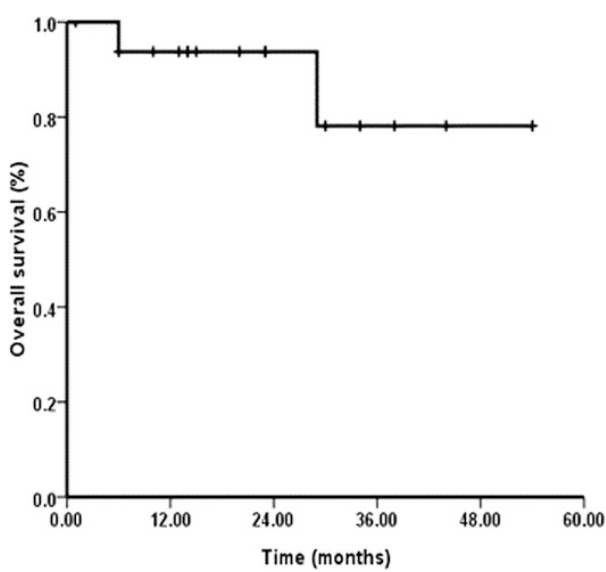

Figure 1

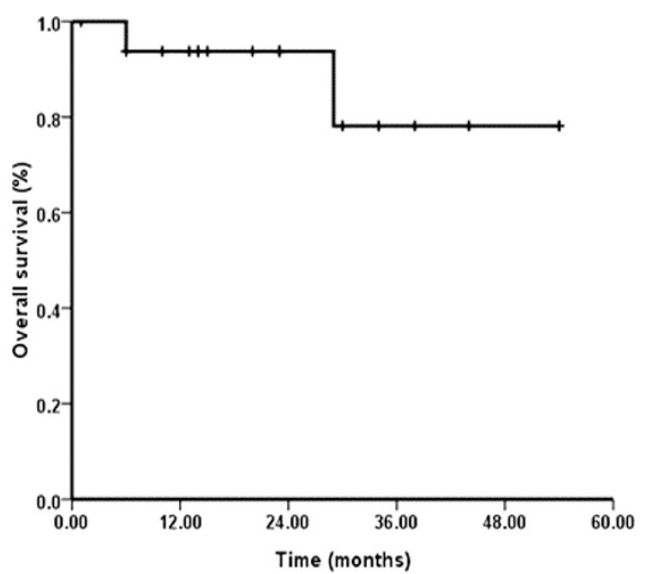

Figure 2 $\times 10^{6} / \mathrm{kg}$ after conditioning regimen. The engraftment rate was $100 \%$, and the median time of neutrophil and platelet engraftment was $11(9 \sim 13)$ days and $11(8 \sim 14)$ days. A highrisk patient died because of severe infection. After ASCT, ORR, $\geq$ VGPR and CR rate were $94.4 \%, 94.4 \%$, and $72.2 \%$, respectively. $C R$ and $\geq$ VGPR rate were improved that compared with post-induction $(P>0.05)$. The median time of follow-up was 21.5 months (range 6 54 months). A 4-year rate of progression-free survival (PFS) (Figure 1) and overall survival (OS) (Figure 2) was $54.9 \%$ and $78.1 \%$. A 4-years rate of cumulative incidence of relapse (CIR) was $24.7 \%$. Nine patients in high-risk group were still alive, 4-years rate of PFS was $63.4 \%$.

Conclusion: Bortezomib-based induction regimen followed by ASCT was safety and efficacy for multiple myeloma patients in Chinese population, especially for those in high-risk group, and PFS and OS could be improved.

References: 1. Jimenez-Zepeda VH, Duggan P, Neri P, et al. Bortezomib and melphalan conditioning increases the rate of complete response and MRD negativity for patients with multiple myeloma undergoing sigle antologous stem cell transplant. Leuk Lymphoma. 2015; 8:1-4.

2. Shah N, Callander N, Ganguly S, et al. Hematopoietic Stem Cell Transplantation for Multiple Myeloma:Guidelines from American Society for Blood and Marrow Transplantation.

Biol Blood Marrow Transplant. 2015;21(7):1155-66.

Disclosure of Interest: None declared.

\section{P408}

New microscopic cell counter using autofocusing system does not require CD45 staining to enumerate CD34+ stem cells: comparison with flow cytometric analysis

Y.-H. Lee ${ }^{1,2, *}$, J. H. Oh ${ }^{3}$, Y. Jung ${ }^{3}$, H. Koh ${ }^{2}$, S. R. Bong ${ }^{3}$, M. S. Kim ${ }^{3}$, S. H. Hong ${ }^{3}$, J. Y. Kim ${ }^{3}$ Y. Y.R. Kim ${ }^{3}$, J. K. Hwang ${ }^{3}$, S. $\mathrm{Han}^{3}$, K. H. Kim ${ }^{3}$, W. $-Y . \mathrm{Sim}^{3}$, C. Chung

${ }^{1}$ Department of Pediatrics, Hanyang University Medical Center, ${ }^{2}$ Department of Translational Medicine, Hanyang University Graduate School of Biomedical Engineering, ${ }^{3}$ NanoEntek, Seoul, Korea, Republic Of

Introduction: Flow cytometric analysis has been a standard method to enumerate CD34+ stem cells in the field of hematopoietic stem cell transplantation. However, there are still some limitations such as expensive instrumentation, high reagent costs, and less reproducibility among technicians and laboratories in most cases. We compared the CD34+ cell data between flow cytometric analysis and the microscopic cell counter using a single stain with CD34 and autofocusing system which was recently obtained patent.

Material (or patients) and methods: We have developed a new CD34+ cell counting device (ADAM II) with a microchip and autofocusing systems. For the ADAM II analysis, samples were just reacted into the ADAM II-CD34 tube containing lyophilized fluorescence mixture. Without staining CD45, these reacted samples were loaded into the chips and read by ADAM II device. We used 18 samples of G-CSF mobilized peripheral bloods (PBs) and harvested peripheral blood stem cells (PBSCs) after G-CSF mobilization from adult volunteer donors. Each informed consent was written from the volunteers prior to obtain samples and this study was approved by the Institutional Review Board of the Hanyang University Hospital. We compared the enumeration of total/ viable CD34+ cells and assessed the linearity and reproducibility between 2 different assays of ADAM II and flow cytometry. We performed these assays using same ADAM II and 2 different instruments of flow cytometry (FACS Calibur, FACS Canto II) at 2 different institutes.

Results: Total 36 samples were used to compare ADAM II and flow cytometry (FACS Calibur and FACS Canto II). The CD34+ counts obtained by ADAM II showed high correlation with those of FACS Calibur (absolute number of CD34+ cells: $r^{2}=0.98$, percentage of CD34+ cells: $\left.r^{2}=0.99\right)$ and FACS Canto II (absolute number of CD34+ cells: $r^{2}=0.98$, percentage of CD34+ cells: $r^{2}=0.99$ ). Reproducibility was assessed by 20 times of replicate measurements with known CD34+ cell concentrations. Four PBSC samples at CD34+ cells concentrations of $27.24,52.56,390.98$ and 1373.23 cells/ $\mu$ l had CVs of $19.8 \%, 16.0 \%, 6.47 \%$, and $7.2 \%$ respectively. A dilution test of the ADAM II method demonstrated a linearity of $r^{2}=0.99$. The ADAM II method was sufficiently precise over the expected ranges in artificially diluted samples. The enumeration of CD45 + cells using flow cytometry were also comparable to the total cell counts in bright images of ADAM II.

Conclusion: We obtained a high correlation of data between flow cytometry and ADAM I| for enumeration of total/viable CD34+ cells, even without staining CD45 which is usually recommended for enumeration of CD34+ cells by flow cytometric analysis. These results suggest that ADAM II CD34+ cell counting device can be potentially be applied in the field of stem cell assay with the advantages of reproducibility, accuracy, convenience, and inexpensiveness.

Disclosure of Interest: None declared. 
P409

Subcutaneous fat derived SVF efficiently improve the behavior of rat model after stroke: A preclinical study A. A. Hamidieh ${ }^{1, *}$, R. Mohseni ${ }^{2}$, A. Shoae-Hassani ${ }^{2}$ ${ }^{1}$ Hematology, Oncology and Stem Cell Transplantation Research Center, ${ }^{2}$ Applied Cell Sciences Department, School of Advanced Technologies in medicine, Tehran University of Medical Sciences, Teheran, Iran, Islamic Republic Of

Introduction: Brain stroke may lead to delayed fatality when death does not occur shortly after the onset. No success was achieved from decades of research for therapeutic alternatives for stroke. Despite the numerous promising preclinical assays including neuroprotective, anti-hypertensive, and antiinflammatory drugs, only symptomatic treatments are currently available. So we conducted a cell therapy panel based on stromal vascular fraction (SVF) via different anatomical sites for this kind of injury. The SVF include adipose stromal cells (ASCs), hematopoietic stem cells (HSCs), progenitor cells, circulating blood cells, fibroblasts, pericytes, endothelial cells, lymphocytes, monocytes and macrophages. Isolated ASCs have the self-renewal capacity and could be differentiate into pre-neural and neural like cells. From among all the factors which may influence the proliferation and the differentiation capacity of ASCs isolated from SVF, the harvest site of adipose tissue has gained great importance.

Material (or patients) and methods: The procedure conducted in 2 phases: in vitro and in vivo. First the adipose tissue was harvested randomly from the subcutaneous inguinal, abdominal inguinal, flank, neck and omentum. Following, grown cells were characterized, following specific induction, regarding neurogenic differentiation capability through biochemical, histochemical, gene expression and microscopy techniques, even in three-dimensional (spheroids) cultures. In the next step SVF was administered to the intracerebral hemorrhage rat model to assess the in vivo outcomes via adhesive removal test.

Results: Upregulation of nestin and MAP-2 was observed in differentiated ASCs most from subcutaneous fat tissue, while the expression of GFAP and p75NGFR was elevated in differentiated ASCs from other sites. At 4 weeks after infusion, there was a significant recovery in behavioral performance when SVF from subcutaneous site were delivered. Rat with tail intra-arterial transplant of SVF exhibited improvement on the adhesive-removal test $(P<0.05)$ and the modified neurologic severity scores $(P<0.05)$ at 14 days compared with received SVF from the other anatomical sites and controls.

Conclusion: The SVF source is an important factor of differentiation, protein expression, and paracrine ability to stimulate neuronal growth. The therapeutic efficiency of SVF is related to its anatomical site due to the number of ASCs, HSCs, their survival potential and the differentiation potency into the pre-neural cells. Subcutaneous derived SVF efficiently improves the behavior of stroke model in our preclinical studies.

Disclosure of Interest: None declared.

P410

Effects of platelet-rich plasma injections in elite athletes with patellofemoral pain syndrome on isokinetic knee profile: a case-series

M. Ansari ${ }^{1, *}$, S. mohammadi ${ }^{1}$, M. Nikbakht ${ }^{1}$, A. Ghavamzadeh ${ }^{1}$ Hematology- oncology and SCT research center, Tehran University of Medical sciences, tehran, Iran, Islamic Republic Of

Introduction: Patellofemoral pain syndrome (PFPS) is among the most common injuries encountered in sports and exercise medicine. PFPS can negatively affect the efficacy of the knee extensor mechanism, which in turn can deteriorate the athletic performance. The usual underlying pathology is the osteochondral degenerative process of the patellofemoral (PF) compartment. Despite the increasing level of evidence in favor of platelet-rich plasma (PRP) use in osteochondral lesions, high quality reports of its applications in treatment of PF lesions are scarce, and are usually advising against. Isokinetic testing has become a mainstay of laboratory-based athletic performance assessment.

Material (or patients) and methods: Thirteen elite male basketball and volleyball players with grade III PF osteochondral injuries in MRI (13 knees, mean age $=26.1$, SD: 5.3 years) with failure of prior conservative PFPS treatments (including physical therapy, insoles, NSAIDs, protective taping and exercise therapy) were enrolled in the study during the offseason period. No severe boney deformity of patellofemoral compartment was detected in radiologic assessments.

The involved knees received 4 biweekly ultrasound-guided injections of 5 milliliters of unactivated autologous PRP. Based on the patients' blood platelet counts, our laboratory-based platelet harvest method was set to yield a PRP platelet count of 1 million per microliter +/- 10\%. Simultaneously, the athletes continued with their individualized off-season preparation program.

Isokinetic testing was carried out before the first injection, and 4 weeks after the final PRP injection, and assessed the concentric knee extension forces at 60 degrees per second.

Results: All athletes finished the study without any considerable adverse effect. Adverse effects included 2 cases of mild knee effusion following the first injection, which were selflimited.

Mean Isokinetic Peak Torque Per Body Weight and Average Peak Torque significantly increased by $19.6 \%$ and $19.8 \%$, respectively (table 1 ) after the injections.

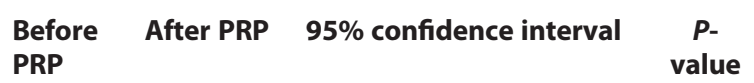

Conclusion: Unactivated autologous PRP injections in athletes with grade III PFPS in absence of remarkable boney deformities may improve the isokinetic profile of knee extensor mechanism. This improvement is possibly due to regenerative effects of PRP on pre-existing PF osteochondral lesions.

The authors recommend studies with proper control groups, and more rigorously controlled variables including the MRI or arthroscopic findings.

Disclosure of Interest: None declared.

\section{P411}

A potential of countering neurologic impairment after infusion of G-CSF with or without autologous mobilized peripheral blood mononuclear cells: Serial cytokine changes in children with cerebral palsy

Y.-H. Lee Le, $^{1, *}$, H. Koh ${ }^{2}$, W.-J. Rah ${ }^{1}$

${ }^{1}$ Department of Pediatrics, Hanyang University Medical Center, ${ }^{2}$ Department of Translational Medicine, Graduate School of Biomedical Science \& Engineering, Hanyang University, Seoul, Korea, Republic Of

Introduction: This study was performed to assess serial cytokine changes and their clinical impact in children with cerebral palsy (CP) who participated in a clinical trial of intravenous infusion of granulocyte-colony stimulating factor (G-CSF) followed by autologous mobilized peripheral blood mononuclear cells (mPBMCs).

Material (or patients) and methods: Peripheral blood (PB) samples were collected from $16 \mathrm{CP}$ children at enrollment (M0), and 1 month (M1) and 7 months (M7) after G-CSF infusion followed by collection of $\mathrm{MPBMC}$, as well as at the end of the study (M13). At M1 and M7, PB samples were obtained before infusion of MPBMC or placebo. Plasma samples were stored at $-80^{\circ} \mathrm{C}$ until the end of the experiment. Levels of cytokines G-CSF, brain derived neurotrophic factor (BDNF), vascular endothelial growth factor (VEGF), insulin like growth factor (IGF)-1, interleukin (IL)-6, IL-8, and IL-10 were measured by enzyme-linked immunosorbent assays. 
Results: G-CSF levels were significantly elevated at M1 and decreased to baseline by M7. VEGF, IL-6, and IL-10 behaved in the same way whereas BDNF and IGF-1 followed the reverse pattern, falling initially and then returning to baseline. There were no significant differences in cytokine levels between the mPBMC and placebo groups over 6 months. However, when clinical responders and non-responders were compared, IL-6 $(P=0.05)$ as well as G-CSF $(P=0.001)$ were higher in the responders than the non-responders at $M 1$, while BDNF $(P=0.03)$ and IGF-1 $(P=0.001)$ were lower. In addition, BDNF was higher at baseline in the responders than the nonresponders $(P=0.03)$.

Conclusion: G-CSF itself, as well as G-CSF-induced cytokines such as IL-6, may help to improve neurologic function in children with CP. Further work is needed to clarify the effect of G-CSF on its own (without harvesting mPBMCs).

Disclosure of Interest: None declared.

\section{P412}

Biological and functional characterization of bone marrow-derived mesenchymal stromal cells isolated from pediatric patients affected by acute myeloid leukemia

L. Tomao ${ }^{1, *}$, M. Algeri ${ }^{1}$, N. Starc ${ }^{2,3}$, S. Biagini ${ }^{2}$, A. Pitisci ${ }^{2}$, A. Conforti ${ }^{2}$, M. E. Bernardo ${ }^{2}$, F. Locatelli ${ }^{2}$

${ }^{1}$ Pediatric Hematology/Oncology, Bambino Gesù CHildren's Hospital, ${ }^{2}$ Pediatric Hematology/Oncology, IRCCS Bambino Gesù Children's Hospital, 'University of Rome "Tor Vergata", Rome, Italy

Introduction: Mesenchymal stromal cells (MSCs) play a fundamental role in the bone marrow microenvironment, where they promote the formation of the hematopoietic niche (1). Whether abnormalities of these cells may contribute to acute myeloid leukemia (AML) pathogenesis in pediatric patients is not entirely known (2).

In this study, we characterized MSCs isolated from pediatric patients affected by AML (AML-MSCs) at diagnosis and/or after remission or relapse.

Material (or patients) and methods: MSCs were expanded ex vivo from bone marrow of $12 \mathrm{AML}$ pediatric patients (mean age: 9 years; range: 1-15) at diagnosis, at remission and/or relapse (if it occurred). Morphology, proliferative capacity (in terms of population doublings), immunophenotype (by flowcytometry), differentiation potential (by histochemical staining and quantification by spectrophotometry) and immunomodulatory properties of AML-MSCs, at any disease stage, were analysed and compared with those of MSCs isolated from 10 healthy donors (HD-MSCs, mean age: 21 years; range: 5-34).

Results: Morphology, proliferative capacity, immunophenotype and in vitro life-span did not differ between AML-MSCs and HD-MSCs, while AML-MSCs derived from patients at remission showed an increased osteogenic potential as compared with HD-MSCs $(P=0.006)$. The immunomodulatory properties of MSCs (activated or not with INF- $\gamma$ and TNF-a) were evaluated in an allogeneic setting (AML-MSCS/HDperipheral blood mononuclear cells; HD-PBMCs) by measuring PHA-induced $\mathrm{T}$ cell proliferation and $\mathrm{CpG}$-induced $\mathrm{B}$ cell proliferation. Non-activated AML-MSCs were able to reduce T cell proliferation up to $85 \%$ (MSCs/PBMCs ratio 1:2) and $69 \%$ (MSCs/PBMCs ratio 1:10), whereas HD-MSCs reduced up to 90\% (ratio 1:2) and 81\% (ratio 1:10). After MSCs activation the inhibition rates were even more robust, being $88 \%$ and $77 \%$ for AML-MSCs (ratio 1:2 and 1:10, respectively), $89 \%$ and $84 \%$ for HD-MSCs (ratio 1:2 and 1:10, respectively). The inhibition of B cell proliferation was obtained exclusively after MSCs activation, with a maximal inhibition rate of $87 \%$ for AMLMSCs and $90 \%$ for HD-MSCs.

Conclusion: Our data demonstrate that AML-MSCs maintain morphology, proliferative capacity, immunophenotype, differentiation potential and immunomodulatory properties typical of HD-MSCs. The solely osteogenic potential resulted significantly increased in AML patients at remission, a result that could be explained taking in exam the described inhibitory effect of blast cells towards MSCs osteogenic potential (3), since the increment of this capability at remission could be the consequence of a bursting effect linked to leukaemia cells elimination.

References: (1) Lichtman MA. Interrupting the inhibition of normal hematopoiesis in myelogenous leukemia: a hypothetical approach to therapy. Stem Cells. 2000; 18(5):304-306.

(2) Low JH, Ramdas P, Radhakrishnan AK. Modulatory effects of mesenchymal stem cells on leucocytes and leukemic cells: A double-edged sword? Blood Cells Mol Dis. 2015 Dec;55(4):351-7. (3) Geyh S, Rodríguez-Paredes M, Jäger P, Khandanpour C et al. Functional inhibition of mesenchymal stromal cells in acute myeloid leukemia. Leukemia. 2015 Nov 25.

Disclosure of Interest: None declared.

\section{P413}

Allogeneic stem cell transplantation results in reduced isolation and altered characteristics of multipotent bone marrow stromal cells

L. M. Siol ${ }^{1}$, J. Schneider ${ }^{1}$, M. Wass ${ }^{1}$, S. Paul ${ }^{1}$, K. Nerger ${ }^{1}$, T. Weber' ${ }^{1}$, C. Müller-Tidow ${ }^{1}$, J. Lützkendorf ${ }^{1}$, L. P. Müller ${ }^{1, *}$

${ }^{1}$ Klinik für Innere Medizin IV, Universitätsklinikum Halle, MartinLuther-Universität Halle-Wittenberg, Halle, Germany

Introduction: Bone marrow multipotent mesenchymal stromal cells (MSC) contribute to the hematopoietic stem cell niche and affect hematopoietic neoplasia. Due to their immunomodulatory potential, the use of in vitro expanded, autologous or allogeneic MSC is under investigation for various clinical conditions. No data exist on the effect of allogeneic stem cell transplantation (SCT) on the ability to isolate MSC and their functional characteristics.

Material (or patients) and methods: MSC were isolated by standard methods from bone marrow aspirates of 368 donors of which 48 had previously undergone allogeneic SCT. Presence of colony forming units-fibroblast (CFU-F) as the typical in vitro growth pattern of MSC was evaluated; CFU-F cells were characterized as MSC by standard criteria at later passages. Clinical data were evaluated and univariate and multivariate analysis for the end point failure of CFU-F growth (i. e. failure of MSC-isolation) as well as number of MSC at $1^{\text {st }}$ passage per $\mathrm{ml}$ bone marrow aspirate was performed. The study was approved by the internal review board.

Results: Median donor age was 61y (15-86y) with 222 male $(60 \%)$ and $146(40 \%)$ female as well as 198 donors (54\%) with previous chemotherapy and 76 donors (21\%) without malignant disease. In 28\% (103/368) of all donors no MSC could be isolated. Multivariate analysis revealed that female gender (Hazard ratio 0.50, $\mathrm{Cl} 0.31-0.81)$, age $>40 \mathrm{y}(0.98,0.96-$ $0.99)$, AML $(0.49,0.26-0.94)$ and allogeneic SCT $(0.38,0.19-0.76)$ were significantly $(P<0.05)$ and independently associated with failure to isolate MSC. In donors with previous allogeneic SCT univariate analysis revealed that the number of failed MSC-isolations was significantly increased for patients with TBI-based conditioning ( $n=23)$ compared to the respective non-TBI group ( $n=25 ; 65 \%$ vs. $32 \%, P=0.02)$. When compared to the respective control group no significant difference was seen for patients with diagnosis of AML or MDS, unrelated donor, HLA-mismatch donor, busulfan- or myeloablative conditioning, female gender, discrepant CMV- and ABOstatus. Repeated MSC-isolations in 25 patients showed that allogeneic SCT reduced the chance to isolate MSC: while before SCT in $4 \%$ ( 1 of 25 isolations) no MSC could be isolated, isolations failed in $48.3 \%$ (14 of 29 isolations) at day $30-120$ after SCT and in $61.5 \%$ (8 of 13 isolations) at day $>120$ after SCT $(P<0.0001)$. Functional analysis revealed that the number of MSC at $1^{\text {st }}$ passage was not affected by a previous allogeneic SCT when compared to the respective controls $(8502 \mathrm{MSC} / \mathrm{ml}$ vs. $20286 \mathrm{MSC} / \mathrm{ml} ; \quad P=0.5)$. However, proliferation beyond $1^{\text {st }}$ passage was reduced in MSC from patients after allogeneic SCT compared to MSC from donors without SCT. 
Conclusion: This analysis shows that the ability to isolate MSC from the bone marrow is reduced not only by general factors like older age and female gender as well as hematological malignancies but also by allogeneic SCT. First, these data thereby directly help to select donors for clinical use of in vitro expanded MSC. Second, our study supports the notion that allogeneic SCT functionally damages MSC as part of the bone marrow niche. This gives a rationale to define MSC-specific molecular targets to improve engraftment and reduce relapse of hematological malignancies.

Disclosure of Interest: None declared.

\section{P414}

Comprehensive characterization of bone-marrow mesenchymal stromal cells isolated from patients affected by primary immunodeficiency

N. Starc ${ }^{1, *}$, D. Ingo ${ }^{2}$, M. Algeri ${ }^{1}$, L. Tomao ${ }^{1}$, A. Conforti ${ }^{1}$, A. Pitisci ${ }^{1}$, S. Biagini ${ }^{1}$, M. Migliavacca ${ }^{2}$, I. Brigida ${ }^{2}$, A. Aiuti ${ }^{2}$, G. Palumbo ${ }^{\prime}$, P. Rossi ${ }^{3}$, F. Locatelli, ${ }^{4,5}$, M. E. Bernardo ${ }^{2}$

${ }^{1}$ Pediatric Hematology/Oncology, IRCCS Bambino Gesù Children's Hospital, Rome, '2San Raffaele Telethon Institute for Gene Therapy (TIGET), Pediatric Immunohematology and Bone Marrow Transplantation Unit, San Raffaele Scientific Institute, Milan, ${ }^{3}$ University Department of Pediatrics, DPUO, Bambino Gesù Children's Hospital, University of Rome Tor Vergata, ${ }^{4}$ Pediatric Hematology/Oncology, IRCCS Bambino Gesù Children's Hospital, Rome, ${ }^{5}$ University of Pavia, Pavia, Italy

Introduction: Mesenchymal stromal cells (MSCs) represent a key component of bone marrow (BM) microenvironment, where they promote the formation of the hematopoietic niche and exert a fundamental role in the development and differentiation of the hematopoietic compartment. MSCs display unique immunoregulatory properties, interacting with all cells of the immune system. In this study, we investigated the biological and functional properties of MSCs derived from patients affected by primary immunodeficiency diseases (PID). Material (or patients) and methods: MSCs were isolated and ex-vivo expanded from the BM of 21 patients (median age 5) affected by PID (PID-MSCs: 9 Wiskott-Aldrich Syndrome, WAS; 7 Chronic Granulomatous Disease, CGD; 5 Adenosine Deaminase-Severe Combined Immunodeficiency, ADA-SCID) and from 15 healthy-donors (HD-MSCs, median age 21). The following characteristics of MSCs were analyzed: morphology, clonogenic (CFU-F) and proliferative capacity (population doublings), osteogenic/adipogenic differentiation, immunophenotype, immunomodulatory properties and analysis of cytokine production following co-colture in an allogeneic setting with peripheral blood mononuclear cells (PBMCs) stimulated with either PHA or CpG. We also evaluate the inhibitory effect of MSCs on the differentiation and maturation of monocytes into dendritic cells (DCs). Expression of WAS and gp91phox proteins by flow-cytometry were analyzed in WASand CGD-MSCs, respectively.

Results: WAS-, CGD- and ADA-MSCs displayed a reduced clonogenic efficiency, as compared with pediatric HD-MSCs. While WAS- and ADA-MSCs showed similar proliferative capacity as compared with HD-MSCs, CGD-MSCs exhibited a reduced proliferation in culture and early senescence (between passage 6 and 8). No differences were found in terms of morphology and immunophenotype. With regard to differentiation capacity, WAS- and CGD-MSCs showed a reduced ability to differentiate into both osteoblasts and adipocytes, while ADA-MSCs exhibited a reduced ability to form osteoblasts only, as compared with HD-MSCs. PID-MSC inhibitory effect on T-cell proliferation was similar to that of the HD counterpart, independently from the type of disease, with a median percentage of inhibition of $87 \%(S D \pm 12)$ and $73 \%(S D \pm 29)$ at MSCs:PBMCs ratios 1:2 and 1:10, respectively. PID-MSCs ability to suppress $B$ cell and plasmacell proliferation (MSCs:PBMCs ratio 1:10) was comparable to HD-MSCs. An increase in anti-inflammatory cytokines and soluble factors and a decrease in pro-inflammatory cytokines was found in supernatants collected from co-cultures of PHA- and CpGstimulated PBMCs with HD- and patient-MSCs, as compared with stimulated PBMC cultures. PID-MSCs maintain the ability to inhibit the differentiation and the maturation of monocytes to dendritic cells in a dose-dependent manner, as well as their HD counterpart. WAS- and HD-MSCs did not express WAS protein; CGD-MSCs showed no expression of gp91phox, while HD-MSCs exhibited $3.5-7 \%$ of the protein.

Conclusion: Our results indicate that while PID-MSCs maintain some of the biological and functional properties of HD-MSCs, they are defective in others. Whether these defects have an impact on the pathophysiology of the diseases will be further investigated.

References: Di Nicola et al. Blood 2002.

Bernardo and Fibbe. Cell Stem Cell 2013.

Disclosure of Interest: None declared.

\section{P415}

Mesensphere cultures but not adherent culture conditions conserve the stem cell properties of human primary bone marrow lin-/CD45-/CD271+/CD140a- stroma cells

R. Ghazanfari ${ }^{1}$, H. Li ${ }^{1}$, D. Zacharaki ${ }^{1}$, H. C. Lim ${ }^{7}$, S. MéndezFerrer $^{2}$, S. Scheding ${ }^{1,3, *}$

${ }^{7}$ Stem Cell Center, University of Lund, Lund, Sweden, ${ }^{2}$ Stem Cell Institute, University of Cambridge, Cambridge, United Kingdom, ${ }^{3}$ Hematology, University Hospital Lund, Lund, Sweden

Introduction: Self-renewal and multilineage differentiation are two key properties of stem cells. However, despite countless studies on the use of so-called "mesenchymal stem cells", it has not yet been demonstrated that human adult bone marrow mesenchymal stromal cells (BM-MSCs) fulfill stringent stem cell criteria. We have recently shown that low/ negative expression of CD140a (PDGFRa) on human lin $^{-} /$ $\mathrm{CD} 45^{\circ} / \mathrm{CD} 271^{+}$bone marrow cells allows to isolate a close to pure population of BM-MSCs ${ }^{1}$. Based on these findings, the current study aimed to investigate the in vitro and in vivo stem cell properties of this putative stem/progenitor cell population. Material (or patients) and methods: BM aspirates were obtained from healthy donors. Following lineage depletion, mononuclear cells were antibody-labelled, FACS sorted (single-cell and bulk), analyzed for single cell gene expression (Fluidigm), differentiation potential, and propagated as either CFU-F in standard adherent MSC cultures or as non-adherent mesenspheres, which retain some primitive MSC features ${ }^{2}$. These cells were then compared side-by-side for their self-renewal in vitro, and self-renewal and differentiation potential in vivo by serial xenotransplantation into NSG mice.

Results: Analysis of the expression of selected relevant genes in single-cell sorted lin $^{-} / \mathrm{CD} 45^{-} / \mathrm{CD} 271^{+} / \mathrm{CD} 140 \mathrm{a}^{-}$cells demonstrated homogeneous mRNA expression levels of genes that are highly expressed in MSCs, such as VCAM1, BMP5, LEPR, ANGPT1, CXCL12, and others. When cultured as CFU-F or mesenspheres, freshly isolated human $\mathrm{lin}^{-} / \mathrm{CD} 45^{-} / \mathrm{CD} 271^{+} / \mathrm{CD} 140^{-}$cells initially exhibited similar progenitor cell frequencies and in vitro selfrenewal capacities, measured as clonal efficiency ("clonogenicity") and replating ability, respectively. Furthermore, CFU-Fs and mesenspheres displayed similar surface marker profiles and comparable in vitro tri-lineage differentiation (osteoblasts, adipocytes, chondrocytes). Next, to evaluate in vivo selfrenewal and differentiation potential of these putative stromal stem cells, $\operatorname{lin}^{-} / \mathrm{CD}_{4} 5^{-} / \mathrm{CD} 271^{+} / \mathrm{CD} 140 \mathrm{a}^{-}$-derived CFU-F and spheres were transplanted s.c. into NSG mice. Adherent CFU-Fs did not show in vivo self-renewal, whereas mesensphere selfrenewal was clearly demonstrated by increasing numbers of human spheres recovered after primary and secondary transplantation ( $1.16 \pm 0.06$ and $2.34 \pm 0.13$-fold, respectively, $n=3)$. Moreover, $\operatorname{lin}^{-} / \mathrm{CD}_{45} / \mathrm{CD} 271^{+} / \mathrm{CD} 140 \mathrm{a}^{-}$-derived spheres differentiated into bone, fat and stromal tissues in vivo in primary as well as secondary transplantations.

Conclusion: Taken together, our data demonstrate that primary human $\operatorname{lin}^{-} / \mathrm{CD} 45^{\circ} / \mathrm{CD} 271^{+} / \mathrm{CD} 140 \mathrm{a}^{-}$cells present a rare, homogenous population of bone marrow stroma cells 
that fulfill strict stem cell criteria, i.e. in vitro and - importantly - in vivo self-renewal and multilineage differentiation potential. These findings address the long-open question of the potential stem cell properties of adult human MSCs.

References: 1) Li, H., et al., Low/Negative Expression of PDGFRalpha Identifies the Candidate Primary Mesenchymal Stromal Cells in Adult Human Bone Marrow. Stem Cell Reports, 2014. 3 (6): p. 965-974.

2) Isern, J., et al., Self-Renewing Human Bone Marrow Mesenspheres Promote Hematopoietic Stem Cell Expansion. Cell Reports, 2013. 3(5): p. 1714-1724.

Disclosure of Interest: None declared.

\section{P416}

Basal CD34+ cell count predicts peripheral blood hematopoietic progenitor stem cell mobilization in healthy donors after administration of granulocyte colonystimulating factor

M. Martino ${ }^{1, *}$, R. Fedele ${ }^{1}$, A. Dattola ${ }^{1}$, A. Pontari ${ }^{1}$, S. Barilla", G. Irrera ${ }^{1}$, G. Messina ${ }^{1}$, T. Moscato ${ }^{1}$, G. Console ${ }^{1}$, G. Tripepi ${ }^{2}$

${ }^{1}$ Oncology and Hematology, Hematology, Stem Cell Collection and Transplantation Unit, Azienda Ospedaliera BMM, ${ }^{2}$ National Research Council (CRN), CRN Institute of Clinical Physiology, Reggio Calabria, Italy

Introduction: Despite the extensive use of granulocyte colonystimulating factor (G-CSF) in healthy donors for peripheral blood hematopoietic progenitor stem cell (HPC) mobilization, there is still a lack of consistent pre-mobilization variables that accurately predict a donor's response to this cytokine.

Material (or patients) and methods: The purpose of this study was to identify clinically significant factors that could influence the effectiveness of CD34+ cell mobilization with special focus on the value of the basal CD34+ cell count in 119 healthy donors undergoing HPC mobilization. Donors received G-CSF subcutaneously at $10 \mathrm{mg} / \mathrm{kg}$ per day in a single dose and HPC collection was started on day five after four days of G-CSF. The total number of CD34+ cells $/ \mathrm{mL}$ in peripheral blood on the first day of apheresis (after G-CSF administration) was used to evaluate the effectiveness of mobilization. This variable was examined separately by linear regression analysis against independent variables (sex, age, weight, height, blood volume, baseline and on day five white cell count, baseline CD34+ cell count, baseline hemoglobin and platelets). In a second step, logistic regression analysis was done to determine the variables influencing the number of CD34+ cells collected $/ \mathrm{kg}$ of donor body weight $(<4$ vs. $\geq 4 \times 106$ CD34+ cells $/ \mathrm{kg}$ ). The independent variables analyzed in this step were the same as those in the previous one with the addition of the volume of blood processed.

Results: By multivariate analysis, baseline CD34+ cell count $(P=0.001)$ and white cell count on day five $(P<0.001)$ were the only two variables that correlated with the number of CD34+ cells in blood the day of apheresis ( $R$ of the model $=0.59$ ). The donor's age was the only variable that significantly correlated with the number of CD34+ cells $/ \mathrm{kg}$ collected $(P=0.030)$.

Conclusion: Our study shows that the number of CD34+ cells circulating in peripheral blood in steady state can be used as a useful indicator of CD34+ cell mobilization after G-CSF administration.

Disclosure of Interest: None declared.

\section{P417}

First Results of a Prospective Study on Erythropoietin Biosimilar (Epoetin zeta) Use in Patients Undergoing Allogeneic Hematopoietic Stem Cell Transplantation for Hematological Malignancies

M. Michallet ${ }^{1, *}$, M. Sobh ${ }^{1}$, J. Monfray ${ }^{1}$, H. Labussière ${ }^{1}$, M. Balsat ${ }^{1}$ C. Lejeune ${ }^{1}$, S. Ducastelle ${ }^{1}$, F. Barraco ${ }^{1}$, X. Thomas ${ }^{1}$, F. -E. Nicolini ${ }^{1}$ ${ }^{1}$ Centre Hospitalier Lyon Sud, Pierre Benite, France

Introduction: We performed a prospective observational study in patients undergoing allogeneic hematopoietic stem cell transplantation (allo-HSCT) for hematological malignancies, to evaluate the effect of a new Erythropoiesis-stimulating agent (ESA) biosimilar, epoetin zeta (Hospira) on hemoglobin $(\mathrm{Hb})$ and platelet $(\mathrm{Pt})$ recovery, safety, quality of life $(\mathrm{QOL})$, overall survival (OS) and relapse incidence. Results of this study were compared to two reference populations, one receiving epoetin beta (Roche) and one control group not receiving ESA.

Material (or patients) and methods: The study included adult patients with $\mathrm{Hb}$ level $\leq 11 \mathrm{~g} / \mathrm{dl}$ occurring after all types of allo-HSCT for any hematological disease. Epoetin zeta (30,000 IU) was administered s.c. once per week for up to 6 months, and $\mathrm{Hb}$ levels were monitored weekly. Injections were stopped once the $\mathrm{Hb}$ level reached $12 \mathrm{~g} / \mathrm{dl}$ without transfusion. If after 4 injections, no improvement was observed, doses were doubled, and if after 8 injections, no improvement was observed, the patient was withdrawn from the study. The QOL was measured at baseline and at 1, 2, 3 and 6 months by the Functional Assessment of Cancer Therapy-Anemia (FACT-An) scale. Epoetin zeta responders were defined as having $\mathrm{Hb}$ level $\geq 12 \mathrm{~g} / \mathrm{dl}$ (complete response, $\mathrm{CR}$ ) or $\mathrm{a} \geq 2 \mathrm{~g} / \mathrm{dl}$ increase (partial response, $\mathrm{PR}$ ) compared with baseline value, in the absence of transfusion. Patients receiving epoetin zeta (group 1) were compared to a similar population receiving epoetin beta with the same procedures (group 2) and to a matched population not treated with ESA (group 3), taking into account the following variables: sex, age, diagnosis, disease status at allo-HSCT, conditioning regimen and HSC source.

Results: Between December 2011 and September 2014, 58 patients (from 168 screened) were included in group 1, and compared to 59 patients in group 2 and 65 patients in group 3 . The main exclusion criteria were ESA contra-indication and patient refusal. Patients in group 1 had lower $\mathrm{Hb}$ baseline levels compared to group 2; patient characteristics for each group are summarized in Table 1. The median number of injections/patient was 10 (range: 6-14) in group 1 and 8 (range: $2-28$ ) in group 2. The cumulative incidence of CR was $80 \%$ in group 1 and $71 \%$ in group 2 . The median time to achieve CR was 48 days (range: $35-70$ ) in group 1 , and 39 days (range: 14-180) in group 2. Eight patients withdrew due to ESA inefficacy in group 1 and 8 in group 2. Adverse events were all thromboembolic: 2 events in group 1 and 5 events in group 2, compared to 2 events in group $3(P=0.34)$. The multivariate analysis studying different confounding factors on the cumulative incidence of $C R$ showed a significant positive impact of younger age $(P=0.001)$, and a negative impact of being female or having major $A B O$ incompatibility. We did not find any significant difference in terms of OS and relapse rate between the 3 groups.

Conclusion: We describe here, for the first time, preliminary data for ESA biosimilar epoetin zeta (Hospira) in allo-HSCT patients showing comparable efficacy and safety to an existing ESA, epoetin beta (Roche) with no impact on OS and relapse incidence, compared to a control group. A study on quality of life and transfusion evaluations as well as a cost-effectiveness are ongoing and results will be presented.

Disclosure of Interest: None declared.

P418

Preemptive use of plerixafor on day 5 of G-CSF treatment. Experience of University Hospital of Salamanca

M. López-Parra ${ }^{1}$, O. López Villar ${ }^{1, *}$, J. M. Bastida Bermejo ${ }^{1}$, O. López-Godino', M. Cabrero', M. I. Ramos Sevillano", B. Oreja Martín ${ }^{7}$, F. López Cadenas ${ }^{1}$, J. Dávila Vals' ${ }^{1}$, M. J. Nieto ${ }^{1}$, C. del Cañizo

${ }^{1}$ Hematology, Hospital Universitario de Salamanca, Salamanca, Spain

Introduction: The use of high dose of chemotherapy with autologous stem cell transplantation (ASCT) is the standard treatment of multiple myeloma (MM) and lymphomas. Any patients didn't undergo the transplant because they suffer a 
failed collection for CD34+ cells after the treatment with G-CSF.

Plerixafor reversibly inhibits binding of stromal cell-derived factor 1 alpha to the chemokine receptor CXCR4 resulting in mobilization of bone marrow hematopoietic stem cells. Plerixafor given on day 4 of G-CSF treatment is more effective than G-CSF alone, but this strategy involves the visit to the hospital on day 4 of treatment (to monitor circulating CD34+ cells) and on 5 day (to apheresis).

Material (or patients) and methods: Retrospective analysis from 01.01.2009 to 01.09 .2015 of patients aged 18-70 years with MM or lymphoma who on day 5 of G-CSF treatment $(10 \mu \mathrm{g} / \mathrm{kg} /$ day) the circulating CD34+ cells level was $<10 / \mu \mathrm{l}$; or if the product of first day of apheresis contained CD34+ cells $<1 \times 10^{6} / \mathrm{kg}$; or if the patients who have had a previous mobilization failure.

The patients received plerixafor $(0,24 \mathrm{mg} / \mathrm{kg}$ or $0,16 \mathrm{mg} / \mathrm{kg}$ if the patient had renal failure) on day 5 of G-CSF treatment. The aim was to collect at least $2 \times 10^{6}$ CD $34+$ cells $/ \mathrm{kg}$ in the apheresis product.

The patients could receive 2 consecutive doses of plerixafor. Results: 392 patients underwent stem cell mobilization from January 2009 to September 2015. Of these, 54 (14\%) patients required rescue treatment with plerixafor. The median age was 58 years (28-70). The diagnoses were MM in 24 (44\%) patients, non-Hodgkin lymphoma in 27 (50\%) patients and Hodgkin lymphoma in 3 patients (6\%). The reason to need plerixafor was: circulating CD34+ cells level $<10 / \mu \mathrm{l}, \mathrm{CD} 34+$ cells $<1 \times 10^{6} /$ $\mathrm{kg}$ in the first day of apheresis and previous mobilization failure in $78 \%, 15 \%$ and $7 \%$ of the patients, respectively.

The median circulating CD34+ cell level on day 6 (after G-CSF following one dose of plerixafor) was 4 times higher than the day 5 (6,5 and 28, respectively). Only 6 patients (11\%) had mobilization failure after plerixafor treatment.

$56 \%$ of patients needed one day of apheresis to collect CD34+ cell $>2 \times 10^{6} / \mathrm{kg}$ and $38 \% 2$ days.

Any patients had serious side effects after plerixafor treatment. $70 \%$ patients underwent autologous transplantation. The median CD34+ cells infused was 2,7x106/kg (2-7). The median time to neutrophil engraftment $(>500 / \mu \mathrm{l})$ and platelet engraftment $(>20000 / \mu \mathrm{l})$ was +11 (9-14) y +12 (8-21), respectively, following transplantation. There was one primary graft failure.

Conclusion: Treatment with plerixafor in the day +5 of G-CSF in patients with lymphoma and MM who either have suffered a failure of previous mobilization, or don't achieve the desired threshold circulating CD34+ cells after treatment with G- CSF, or that after the first apheresis don't get a count of CD34+ cells in the product $>1 \times 10^{6} / \mathrm{kg}$, has proven effective in achieving an apheresis product with optimum counting of CD34+cells to perform autologous transplantation, obtaining a graft suitable. It is a safe treatment. This strategy saves one visit to the hospital (the monitoring CD34+cells visit) to the $80 \%$ of patients who requiring autologous transplantation.

References: DiPersio JF et al. J Clin Oncol 2009:27; 4767-4773 ME Horwitz et al. Preemptive dosing of plerixafor given to poor stem cell mobilizers on day 5 of G-CSF administration. Bone Marrow Tranasplantation 2012, 47; 1051-1055

Disclosure of Interest: None declared.

\section{P419}

Clinical Outcome and Immune Reconstitution in a $\beta$ T-cell Depleted Allogeneic Stem Cell Transplantation from Matched Related and Unrelated Donors

M. D. de Witte ${ }^{1,2, *}$, G. Fleurke ${ }^{2}$, I. Slaper ${ }^{3}$, E. Spierings ${ }^{1}$, J. Kuball ${ }^{1,2}$ ${ }^{1}$ Laboratory of Translational Immunology, ${ }^{2}$ Hematology, ${ }^{3}$ Cell Therapy Facility, University Medical Center Utrecht, Utrecht, Netherlands

Introduction: Specific depletion of a $\beta$ T-cells is proposed to result in a decreased incidence of aGVHD, whereas the remaining innate cells such as NK cells and $\gamma \delta T$ cells may provide control of infected and transformed cells the first months post SCT. This strategy has been pioneered in haploindentical transplantation with encouraging results (1). Here we extend aßT- cell depleted allo-SCT to patients with a matched related and unrelated donor.

Material (or patients) and methods: 55 patients with hematological malignancies (including AML, ALL, MM, NHL, MPN) received an aßT-cell depleted allo-SCT of a HLA matched sibling (MRD) or HLA matched (9 or 10/10) unrelated donor (MUD). aßT-cell reduction was performed by negative selection with anti-aßTCR antibodies in combination with magnetic microbeads, using the automated CliniMACS device (Miltenyi Biotec, Bergisch Gladbach, Germany). The maximal contamination with aßT-cells was $5 \times 10^{5} / \mathrm{kg}$. The conditioning regimen consisted of: ATG (Genzyme ${ }^{\circledR}$ ) $6 \mathrm{mg} / \mathrm{m}^{2}+$ fludarabine $120 \mathrm{mg} /$ $\mathrm{m}^{2}+$ busilvex $A \cup C=90$. Part of the patients received mycophenolic acid as GVHD prophylaxis for 28 days. Patients were retrospectively analyzed for clinical parameters including immune reconstitution, engraftment, infections, GVHD, relapse, NRM and OS and compared to an historical control cohort of recipients of T cell replete allo-SCT. In addition in a
Grade I-IV

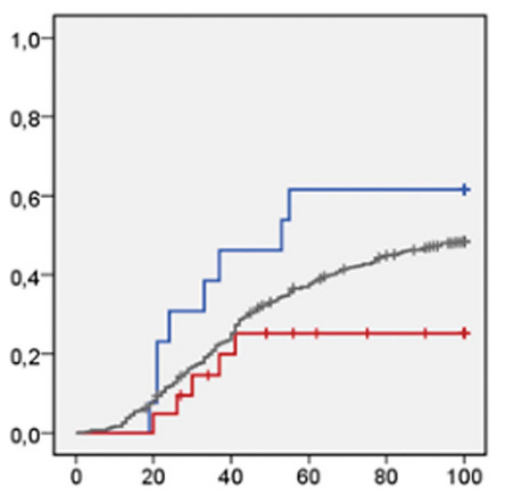

Grade II-IV

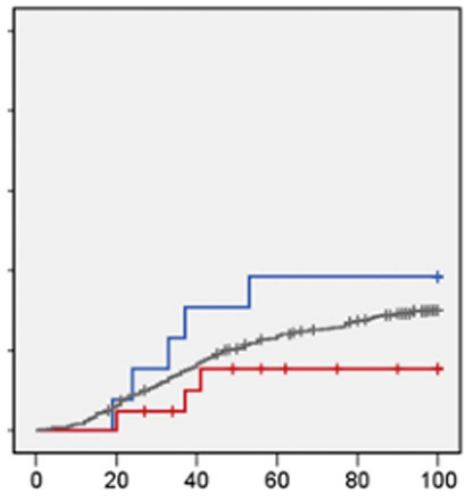

Grade III-IV

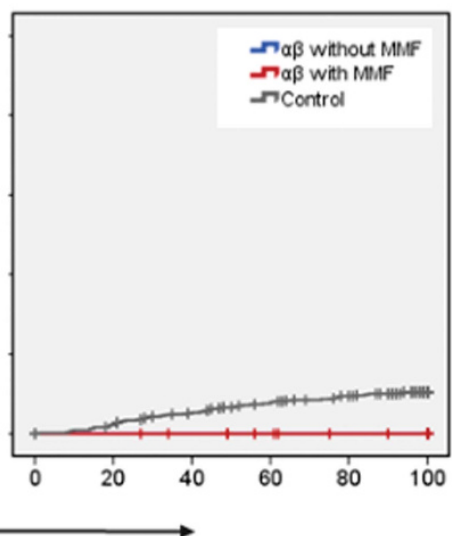

Time post SCT (days) 
subset of patients NGS of the TCR $\beta$ chain was performed using the Illumina/MiSeq sequencing platform after isolation of diverse immune subsets within the a $\beta T$-cell repertoire.

Results: aßT-cell depletion with anti-aßTCR antibodies resulted in a 4.1 (range 1.7-5.2) log depletion of a $\beta T$ cells, resulting in a median contamination of $16 \times 10^{3} \mathrm{a} \beta \mathrm{T}-\mathrm{cells} / \mathrm{kg}$ $\left(0.8 \times 10^{3} / \mathrm{kg}-200 \times 10^{3} / \mathrm{kg}\right.$ ). Primary engraftment (chimerism $>95 \%)$ was observed in all patients $(n=55)$. Immune reconstitution primarily consisted of NK cells. In addition, $\gamma \delta T$ cells were detectable at normal numbers the first half year post SCT, whereas the adaptive immune repertoire showed a delayed reconstitution. The incidence of CMV infections was $54 \%$ in patients after aßT-cell depleted allo-SCT without MMF, $23 \%$ in patients after a $\mathrm{\beta T}$ cell depleted allo-SCT with MMF and $38 \%$ in T cell replete allo-SCT control cohort. The incidence of EBV infections was 30,8\%; 9,5\% and $8,7 \%$ respectively. The incidence of aGVHD > grade II within 100 days in patients of a aßT-cell depleted allo-SCT was 0\% (figure 1). During this short time of follow-up (1-20 months) we observed no significant differences in EFS, NRM and OS as compared to historical control cohorts. With NGS of the TCR $\beta$ repertoire, a surprising diversity was observed in defined immune subsets ranging from clonal expansion of regulatory $T$ cells to broad repertoires in effector memory cells.

Conclusion: aßT-cell depletion in allografts of MRD/MUD results in a swift reconstitution of innate cells (NK cells and $\gamma \delta T$-cells), followed by a subsequent reconstitution of the adaptive immune repertoire. The incidence of severe aGVHD is $0 \%$, without a significant increase in infections or relapse shortly post allo-SCT. These results will be confirmed during extended follow-up and in a planned prospective multicenter study.

References: 1. Bertaina A, Merli P, Rutella $S$, et al. HLAhaploidentical stem cell transplantation after removal of $a \beta 1 \mathrm{~T}$ and $B$ cells in children with nonmalignant disorders. Blood 2014;124(5):822-826.

Disclosure of Interest: $M$. de Witte Funding from: Dutch Cancer Society, G. Fleurke: None declared, I. Slaper: None declared, E. Spierings: None declared, J. Kuball Funding from: Dutch Cancer Society.
P420

FACTORS PREDICTING PERIPHERAL BLOOD PROGENITOR CELL MOBILIZATION IN HEALTHY DONORS RECEIVING GRANULOCYTE COLONY STIMULATING FACTOR: EXPERIENCE FROM A SINGLE CENTRE (2008-2015)

R. Bailen ${ }^{1}$, A. Pérez-Corral ${ }^{1, *}$, C. Pascual ${ }^{1}$, V. Pradillo ${ }^{1}$, M. Bastos ${ }^{1,2}$ J. Gayoso 1,2, M. Kwon ${ }^{1,2}$, C. Muñoz ${ }^{1}$ P. Balsalobre ${ }^{1,2}$, D. Serrano ${ }^{1,2}$, J. Anguita ${ }^{1,2}$, J. L. Díez-Martín ${ }^{1,2}$

${ }^{1}$ Hemathology, ${ }^{2}$ Instituto de Investigación Sanitaria Gregorio Marañón, Hospital General Universitario Gregorio Marañón, Madrid, Spain

Introduction: Poor collection results are unexpected after granulocyte colony stimulation factor (G-CSF)-induced peripheral blood stem cell (PBSC) collection in healthy donors. Factors predicting CD34+-cell yield after G-CSF mobilization in these donors are still poorly known. In this study, we analyzed the differences between poor and normal mobilizers in allogenic transplantation in our centre from 2008 to 2015.

Material (or patients) and methods: We retrospectively analyzed the baseline characteristics and the efficacy results of G-CSF induced PBSC mobilizationof 138 adult healthy donors in our institution, from 2008 to 2015. All donors received $10 \mathrm{mg} / \mathrm{kg}$ of G-CSF once a day subcutaneously for four days. Leukapheresis using a continuous blood cell separator (Cobe Spectra and Optia ${ }^{\mathbb{R}}$ Terumo BCT) was started in the $5^{\text {th }}$ day of G-CSF treatment. Donors were classified as poor mobilizers if they had less than $20.000 \mathrm{CD} 34+\mathrm{cell} / \mathrm{mL}$ peripheral blood count in the $5^{\text {th }}$ day of G-CSF treatment or if they needed 3 or/ and more leukapheresis for graft collection. We compared baseline characteristics between the two groups to identify factors related to poor mobilization. Statistical analysis was performed with the software Stata ${ }^{\mathbb{R}}$ 13.1.

Results: Donor characteristics and variables analyzed are shown in Table 1. Age, weight, total G-CSF dose and platelet count before and after mobilization were significantly different between poor and normal mobilizers, as previously reported by other groups[1],[2]. Normal mobilizers $(n=124)$ were younger (median age 42.3 years versus $50.7, P=0.006$ ), had a higher platelet count before $\left(221.0 \times 10^{9} / \mathrm{L}\right.$ vs. $197.0 \times 10^{9} / \mathrm{L}$,

\begin{tabular}{|c|c|c|c|}
\hline & $\begin{array}{l}\text { Poor mobilizers } \\
\qquad(n=14)\end{array}$ & $\begin{array}{l}\text { Normal mobilizers } \\
\qquad(n=124)\end{array}$ & $\bar{p}$ \\
\hline Age (years) & $50.7(44.5-62.2)$ & $42.3(33.6-54.4)$ & 0.006 \\
\hline Sex (male) & $5(37 \%)$ & $72(58 \%)$ & 0.16 \\
\hline Comorbidity (if any) & $4(29 \%)$ & $31(25 \%)$ & 0.75 \\
\hline Chronic treatment (if any) & $5(36 \%)$ & $34(27 \%)$ & 0.5 \\
\hline $\begin{array}{l}\text { Priorto mobilization: } \\
\text { - Haemoglobin }(\mathrm{g} / \mathrm{dL}) \\
\text { - Platelet count }\left(\mathrm{x} 10^{9 / \mathrm{L}}\right) \\
\text { - WBC cell count }\left(/ \mathrm{x} 10^{9 / L}\right)\end{array}$ & $\begin{array}{c}13.9(13.5-15.1) \\
197.0(173.0-214.0) \\
6.1(5.1-7.0)\end{array}$ & $\begin{array}{c}14.4(13.5-15.6) \\
221.0(181.0-263.0) \\
6.5(5.5-7.8)\end{array}$ & $\begin{array}{l}0.33 \\
0.049 \\
0.33\end{array}$ \\
\hline Weight (kg) & $70(58-73)$ & $74.6(64-88)$ & 0.039 \\
\hline G-CSF received (million UI) & $78(60-78)$ & $78(78-96)$ & 0.036 \\
\hline $\begin{array}{l}\text { At day } 1 \text { of collection: } \\
\text { - Haemoglobin }(\mathrm{g} / \mathrm{dL}) \\
\text { - Platelet count }\left(\times 10^{9} / \mathrm{L}\right) \\
\text { - WBC count }\left(\times 10^{9} / \mathrm{L}\right) \\
\text { - CD34+ cells }\left(\times 10^{9} / \mathrm{L}\right) \\
\text { - CD34+ cells collected (absolute) }\end{array}$ & $\begin{array}{c}13.7(12.8-14.7) \\
195.5(147.0-209.0) \\
42.6(32.8-52.8) \\
18.3(16.1-21.4) \\
102.5(93-142)\end{array}$ & $\begin{array}{c}14(13-14.8) \\
206.5(171.0-249.0) \\
49.1(40.7-56.9) \\
62.9(39.6-93.6) \\
330(215-467)\end{array}$ & $\begin{array}{c}0.52 \\
0.047 \\
0.12 \\
<0.001 \\
<0.001\end{array}$ \\
\hline Days of apheresis (days) & $2.5(2-3)$ & $1(1-2)$ & $<0.001$ \\
\hline Total CD34 collected (cells $/ \mathrm{kg})$ & $3.6(2.9-4.1)$ & $5.2(4.3-6.2)$ & $<0.001$ \\
\hline
\end{tabular}

Table 1. Baseline and collection characteristics of poor and normal mobilizers. WBC, white blood cell. Qualitative variables expressed in number (\%). Quantitative variables expressed in median (interquartil range). 
$P=0.049)$ and after $\left(206.5 \times 10^{9} / \mathrm{L}\right.$ vs. $\left.195.5 \times 10^{9} / \mathrm{L}, P=0.047\right)$ mobilization, weight higher $(74.6 \mathrm{~kg}$ vs. $70 \mathrm{~kg}, P=0.04)$, and had received a higher total mean daily G-CSF dose $(74.6 \mathrm{mUI}$ vs. $70 \mathrm{mUI}, P=0.036)$. Platelets at baseline were also significantly correlated $(P<0.001)$ with $\mathrm{CD} 34+$ cell count $/ \mathrm{mL}$ on day 5th in univariate analysis but in the multivariate analysis we did not identify reliable parameters predicting the CD34+-cell yield. Despite $\mathrm{CD} 34+\times 10^{6}$ cells per $\mathrm{kg}$ of receptor weight collected were lower in the poor mobilization group (5.2 vs. 3.6, $P<0.001)$, no differences on engraftment with neutrophils $>5.0 \times 10^{9} / \mathrm{L}$ ( 16 days (15-18) vs. 16 days (14-18), $\left.P=0.96\right)$ and platelets $>20.0 \times 10^{9} / \mathrm{L}$ (15 days (12-30) vs. $\left.12(11-15), P=0.96\right)$ were found.

Conclusion: In this cohort of healthy donors in a single institution, older age, less weight, lower total G-CSF dose and lower platelet counts seems to be related with poorer mobilization. Further studies are needed to validate or not these findings in multivariate analysis and define useful baseline characteristics that predict mobilization in healthy donors.

References: [1] Anderlini P et al. Factors affecting mobilization of CD34+ cells in normal donors treated with filgrastim. Transfusion 1997;35(5):507-12.

[2] Suzuya $\mathrm{H}$ et al. Factors associated with granulocyte colonystimulating factor-induced peripheral blood stem cell yield in healthy donors. Vox Sanguinis 2005:89(4):229-35.

Disclosure of Interest: None declared.

\section{P421}

One more step further to optimize stem cell collection

S. M. Tan ${ }^{1, *}$, T. C. Ong ${ }^{1}$, M. Z. Zakaria ${ }^{1}$, N. Othman ${ }^{1}$, S. Q. Chui ${ }^{1}$, S. Bai Renganathan', K. M. Chang'

${ }^{1}$ Haematology, Hospital Ampang, Ampang, Malaysia

Introduction: Peripheral blood stem cell (PBSC) harvesting and storage incur significant cost and resources. Our implementation of initiating PBSC mobilization based on peripheral blood CD34 count (PB-CD34) cutoff points at least 10/uL since June 2011 has proven to be cost effective. To further optimize PBSC collection, we move in with large volume apheresis in general for all machines (COMTEC, AMICUS and COBE Spectra) provided patient can tolerate as well as using predictive algorithms for OPTIA cell seperator in particular since 2015 .

Material (or patients) and methods: We compared the outcome of PBSC collection in 2015 ever since the large volume apheresis and predictive algorithms kicked off for individual who can tolerate as compared to previous year.

Results: A total of 343 patients/donors with 451 apheresis were recorded in 2015 as compared to 297 patients/donors with 406 apheresis in 2014. We routinely use OPTIA machine for all allogeneic PBSC collection as less red cell contamination as well as $42 \%$ of our autologous setting. The collection efficiency (CE2) of all machines almost comparable whereas the predictive algorithms in OPTIA for allogeneic is more reliable as compared to the autologous setting. We were able to increase the total number of autologous PBSC collection from 283 to 345 as a result of increase number of successful mobilization achieving target cell dose of $>2 \times 10^{6} / \mathrm{kg}$ in only one apheresis session (from a total of 186 to 238 apheresis) by using large volume apheresis. Furthermore, the predictive algorithms enable us to just processing the necessary blood volume and shorter procedure time for those with high prePB-CD34 count. Such achievement brought huge impact to a resource limited center like us.

Conclusion: We managed to further improve our stem cell mobilization and storage program using large volume apheresis and predictive algorithms after implementation of one step measure by deciding PBSC mobilization based on PBCD34 cutoff points at least 10/uL.

References: 1. Cousins AF, Sinclair JE, Alcom MJ, et al. HPC-A dose prediction on the optia cell separator based on a benchmark CE2 collection efficiency: Promoting clinical efficiency, minimizing toxicity, and allowing quality control. J Clin Apher. 2015 Jan 24. doi: 10:1002/jca.21380 [Epub ahead of print].

2. Fontana S, Groebli R, Leibundgut K, et al. Progenitor cell recruitment during individualized high-flow, very-largevolume apheresis for autologous transplantation improves collection efficiency. Transfusion. 2006;46(8):1408-16.

3. Gasova Z, Marinoy I, Vodvarkova S, et al. PBPC collection techniques standard versus large volume leukapheresis (LVL) in donors and in patients. Transfus Apher Sci 2005;32(2)167-76. Disclosure of Interest: None declared.

\section{P422}

Cost analysis of a randomized stem cell mobilization study in multiple myeloma

V. Varmavuo ${ }^{1,2,{ }^{*}}$, R. Silvennoinen ${ }^{2,3}$, P. Anttila ${ }^{3}$, M. Säily ${ }^{4}$ M. Sankelo ${ }^{5}$, J. Lievonen ${ }^{3}$, M. Putkonen ${ }^{6}$, J. Ahonen ${ }^{7}$, E. Mahlamäki ${ }^{8}$, P. Mäntymaa ${ }^{8}$, E.-R. Savolainen ${ }^{9}$, K. Remes $^{6,10^{\prime}}$, E. Jantunen ${ }^{2}$

${ }^{1}$ Department of Medicine, Kymenlaakso Central Hospital, Kotka, ${ }^{2}$ Department of Medicine, Kuopio University Hospital, Kuopio, ${ }^{3}$ Comprehensive Cancer Center, Helsinki University Hospital, Helsinki, ${ }^{4}$ Department of Medicine, Oulu University Hospital, Oulu, ${ }^{5}$ Department of Internal Medicine, Tampere University Hospital, Tampere, ${ }^{6}$ Department of Medicine, Turku University Hospital, Turku, ${ }^{7}$ Hospital Pharmacy of Kuopio University Hospital, Kuopio University Hospital, ${ }^{8}$ Laboratory of Eastern Finland, Kuopio, ${ }^{9}$ Nordlab Oulu, Medical Research Center, Oulu University Hospital, Oulu, ${ }^{10}$ University of Turku, Turku, Finland

Introduction: Autologous stem cell transplantation (ASCT) upfront is the standard therapy for younger myeloma (MM) patients. The patients are usually mobilized with cyclophosphamide $(\mathrm{CY})$ followed by G-CSF or with G-CSF alone. A limited number of prospective studies are available comparing costs of different mobilization strategies.

Material (or patients) and methods: Eighty transplant-eligible patients up to 70 years of age with untreated MM were included in this prospective study ${ }^{1}$. Patients were randomized at inclusion into one of the two mobilization arms. The patients were treated with RVD (lenalidomide, bortezomib, dexamethasone) induction for three 21- day cycles.

The mobilization in arm A was $C Y 2 \mathrm{~g} / \mathrm{m}^{2}$ on day (d) +1 plus filgrastim $5 \mu \mathrm{g} / \mathrm{kg} / \mathrm{d}$ starting on $\mathrm{d}+4$ and in arm B filgrastim $10 \mu \mathrm{g} /$ $\mathrm{kg} / \mathrm{d}$ alone starting on $\mathrm{d}+1$. Plerixafor was given subcutaneously with a dose of $240 \mu \mathrm{g} / \mathrm{kg}$, if blood (B) CD34 ${ }^{+}$level was $<10 \times 10^{6}$ \% $\mathrm{L}$ in both arms provided that WBC count was at least $10 \times 10^{9} / \mathrm{L}$ in arm $A$ or $d+5$ had been achieved in arm $B$. The aphereses were initiated if the B-CD34 ${ }^{+}$cell level was $>10 \times 10^{6} / \mathrm{L}$ on $\mathrm{d}+10$ or $\mathrm{d}+5$ in arms $A$ and $B$, respectively. High-dose melphalan $\left(200 \mathrm{mg} / \mathrm{m}^{2}\right)$ was used as high-dose therapy and G-CSF was given after the graft infusion if the number of collected $\mathrm{CD} 34^{+}$cells was $<3 \times 10^{6}$ \% kg. Fifty-eight patients (31 patients in arm A and 28 patients in arm B) who were successfully collected and transplanted were included in this cost analysis.

The costs of different procedures were collected from transplantation units and the average values of costs were used in cost analysis calculations. The estimated average costs are presented in Table 1.

Results: The median cost of the mobilization phase was $3855 €(2615 €-16400 €)$ in arm A and $785 €(560 €-13126 €)$ in $\operatorname{arm} B(P=<0.001)$. The median costs of the collection phase were $7752 €(3376 €-13376 €(\operatorname{arm} A), 3376 €-14504 €$ (arm B) in the both arms $(P=0.915)$. The combined median cost of the mobilization and the collection phases was $11622 €$ $(5991 €-28678 €)$ in arm $A$ and $8631 €(3936 €-27630 €)$ in arm $B(P=0.062)$. There was no significant difference in the use of plerixafor between the arms ( 2 patients vs. 5 patients, respectively) $(P=0.170)$

The median costs of high-dose therapy and the early post-transplant phase was $21454 €(14965 €-76210 €)$ in arm $A$ and $20808 €(14454 €-29000 €)$ in arm $B(P=0.785)$. The total median costs of stem cell mobilization, collection and 
Table 1. The estimated average costs

\begin{tabular}{|c|c|c|}
\hline Phase & Cost (per process/dose/item) & Price $(€)$ \\
\hline \multirow[t]{3}{*}{ General costs } & Inpatient/hospital day (incl. routine treatments) & 1000 \\
\hline & Platelets ( 4 units / ' 1 bag') & 454 \\
\hline & Red blood cells ( 1 unit / '1 bag') & 142 \\
\hline \multirow[t]{5}{*}{ Mobilization phase } & Cyclophosphamide $\left(2 \mathrm{~g} / \mathrm{m}^{2}\right)+$ mesna & 195 \\
\hline & G-CSF & outpatient/inpatient \\
\hline & $-30 \mathrm{MU}$ & $70 / 11$ \\
\hline & $-48 \mathrm{MU}$ & $110 / 15$ \\
\hline & Plerixafor $\left(\right.$ Mozobil $\left.{ }^{\mathbb{R}}\right) 24 \mathrm{mg}$ & 6250 \\
\hline \multirow[t]{2}{*}{ Collection phase } & Measurement of circulating $\mathrm{CD} 34^{+}$cells & 150 \\
\hline & Apheresis (incl. all material and laboratory costs) & 2026 \\
\hline
\end{tabular}

transplantation were $34997 €(23366 €-82366 €)$ and $31981 €$ $(22871 €-53067 €)$, respectively $(P=0.118)$.

Conclusion: Total cost of ASCT seems to be comparable in myeloma patients after RVD induction whether they have been mobilized with $\mathrm{CY}+\mathrm{G}-\mathrm{CSF}$ or G-CSF alone.

References: 1 . Silvennoinen $\mathrm{R}$ et al. A randomized phase II study of stem cell mobilization with cyclophosphamide+G-CSF or G-CSF alone after lenalidomide-based induction in multiple myeloma. Bone Marrow Transplant. 2015 Oct 5 e-Pub.

Disclosure of Interest: V. Varmavuo Conflict with: Consultancy fees from Celgene, Roche, Amgen, Genzyme/Sanofi., R. Silvennoinen Conflict with: Honoraria from Celgene, Genzyme and Sanofi., P. Anttila: None declared, M. Säily: None declared, M. Sankelo: None declared, J. Lievonen: None declared,

M. Putkonen: None declared, J. Ahonen: None declared, E. Mahlamäki: None declared, P. Mäntymaa: None declared, E.-R. Savolainen: None declared, K. Remes Conflict with: Advisory Board Membership of Amgen, Celgene and Takeda, and lecture fees and congress travel costs from Amgen, Celgene, Roche and Takeda., E. Jantunen Conflict with: Horonaria from Sanofi, Celgene and Amgen.
P423

The "HELP" Scoring System to Predict Chemo-mobilization Outcome in Non-Hodgkin Lymphoma Patient Undergoing Autologous Stem Cell Transplant

B. Yap ${ }^{1}$, L. See ${ }^{1}$, Z. Lao ${ }^{2,}{ }^{*}$, C. Phipps Diong ${ }^{2}$, J. Q. S. Koh ${ }^{3}$ C. H. Yeap 2 , A. Y. Leung ', S. F. S. A. Wahid', I. Surapol', C. H. Tzeng, Y. T. Goh ${ }^{2}$

${ }^{1}$ Duke-NUS Graduate Medical School, ${ }^{2}$ Haematology, Singapore General Hospital, Singapore, Singapore, ${ }^{3}$ Monash University, Australia, Australia, ${ }^{4}$ Queen Mary Hospital, Hong Kong, Hong Kong, ${ }^{5}$ UKM Medical Centre, Malaysia, Malaysia, ${ }^{6}$ Mandihol University, Thailand, Thailand, "7Taipei Veterans General Hospital, Taiwan, Taiwan, Province of China

Introduction: Autologous Stem Cell Transplantation (ASCT) is the preferred treatment for relapsed/refractory Non-Hodgkin Lymphoma (NHL). Minimum threshold for successful mobilization is defined as $\geq 2 \times 10^{6}$ CD34+ cells/kg body weight following chemo-mobilization with various agents. However, a proportion of patients fail mobilization regardless of the agents utilised. Studies have identified clinical parameters in univariate models to predict mobilization outcome. Recently,

[P423]

Table 1: Points allocation for each pre-mobilization parameter (Point-based Scoring System)

\begin{tabular}{cccc}
\hline Factors & Regression Coefficient & Categories & Points \\
\hline Total Cycles of Chemotherapy & $1.21(0.24-2.17)$ & $>6$ Cycles & 0 \\
Platelet & $1.22(0.42-2.02)$ & $<6$ Cycles & 1 \\
& & $\geq 200 \times 10^{9} / \mathrm{L}$ Platelet & 1 \\
WBC & $2.75(0.68-4.82)$ & $<7 \times 10^{9} / \mathrm{L}$ WBC & 0 \\
Hemoglobin & $1.32(0.39-2.26)$ & $\geq 12 \times 10^{9} / \mathrm{L}$ WBC & 3 \\
& $1.03(0.20-1.85)$ & Yes Hemoglobin & 0 \\
Prior Ifosphamide Exposure & & No & 1 \\
& & & 0 \\
\hline
\end{tabular}


Xiaohong Han et al. created a model based on Chinese patients in a single-centre study [1]. However, its utility may be restricted due to it being derived from Chinese patients alone. In this multi-national retrospective study, we attempt to identify pre-mobilization parameters and develop a scoring system to predict mobilization outcome in NHL patients in the Asian population.

Material (or patients) and methods: A total of $238 \mathrm{NHL}$ patients were recruited consecutively from Singapore, Thailand, Malaysia, Taiwan and Hong Kong. Parameters collected included: age, gender, disease stage, previous bone marrow infiltration, bone marrow radiotherapy, prior lines/cycles/types of chemotherapy, hematocrit, hemoglobin, red blood cell (RBC) count, platelets, white blood cell (WBC) count (including neutrophils, lymphocytes and monocytes) and CD34+ cells just prior to chemo-mobilization. Univariate and multivariable logistic regression were performed. The Hosmer-Lemeshow test and Area under the Receiver Operating Characteristic Curve (AUROC) were performed to test the model's goodness of fit and discriminatory ability, respectively. Internal validation was performed via bootstrapping. Two scoring systems (formula-based and point-based) were developed based on the odds ratios and regression coefficients, respectively. BlandAltman plot was used to compare both scoring systems.

Results: Univariate and multivariable analyses showed that $\leq 6$ cycles of chemotherapy, platelets $\geq 200 \times 10^{9} / L, W B C \geq 7 \times 10^{9} /$ $\mathrm{L}$, hemoglobin $\geq 12 \times 10^{9} / \mathrm{L}$ and no prior ifosphamide exposure were predictive of successful mobilization with odds ratio $(P$ value) of $3.34(0.014), 3.40(0.003), 15.63(0.009), 3.75(0.006)$ and $2.79(0.015)$ respectively. This model was well-calibrated and discriminated with Hosmer-Lemeshow P-value of 0.702 and Area under the Receiver Operating Characteristic Curve (AUROC) of 0.812 . Internal validation yielded a mean AUROC of 0.807 (0.805-0.809). Formula-based and point-based scoring system with predictive scores of 15-60 and 0-7 respectively had probabilities of successful mobilization between 0.34-1.00. Bland-Altman plot showed excellent agreement $\left(R^{2}=0.987\right)$ between the two scoring system. Patients with 2-7 points on the point-based scoring system have a high probability of successful mobilization $(0.85-1.00)$. Patients with $0-1$ point have a low probability of successful mobilization ( 0.34 and 0.63 respectively).

Conclusion: The novel "HELP" point-based scoring system (Table 1) (Hemoglobin: $\geq 12 \times 10^{9} / \mathrm{L}$, Exposure to $\leq 6$ cycles of chemotherapy, no prior exposure to Ifosphamide, Leucocytes: $\geq 7 \times 10^{9} / \mathrm{L}$, Platelets: $\geq 200 \times 10^{9} / \mathrm{L}$ ) provides an objective tool to estimate mobilization outcome in $\mathrm{NHL}$ patients undergoing chemo-mobilization for ASCT.

References: 1. Han, X., et al., Predictive factors for inadequate stem cell mobilization in Chinese patients with $\mathrm{NHL}$ and $\mathrm{HL}$ : 14-year experience of a single-center study. J Clin Apher, 2012. 27(2): p. 64-74.

Disclosure of Interest: None declared.

P424

Graft composition and engraftment: is it possible to create a predictive risk score model that could help on planning future transplant procedures?

M. Vacca 1, ${ }^{1,}$ A. Pandolfi ${ }^{2}$, F. Ipsevich ${ }^{2}$, A. Mengarelli ${ }^{3}$, F. Marchesi $^{3}$, A. Ostuni, V. Pavone, M. R. Morciano, L. Abbruzzese, M. Caputo ${ }^{6}$, G. Bonsignore, , G. Di Peri, A. Crescimanno ${ }^{8}$, J. Montoro Gomez ${ }^{9}$ D. Laszlò ${ }^{10}$, A. Gardellini ${ }^{10}$, P. Accorsi ${ }^{11^{\prime}}$, C. Passeri $i^{11}$ O. Iuliani ${ }^{11}, C$.' Savignano ${ }^{12}$, C. Rinaldi ${ }^{12}$, M. Forgiarini' ${ }^{12}$, A. Lanti $^{13}$, Á Ferraro $^{13}$, E. Fiorelli ${ }^{13}$, F. Lanza ${ }^{14}$, P. Pezzotti ${ }^{15}$, L. Pierelli ${ }^{16}$ on behalf of SIdEM PLT engraftment study group

${ }^{7}$ Medicina Trasfusionale, ${ }^{2}$ Immunoematologia e Medicina Trasfusionale, Azienda ospedaliera San Camillo Forlanini Rome, Rome, ${ }^{3}$ Exp Clin Oncology Hematology, Regina Elena Cancer Institute, Roma, ${ }^{4}$ Medicina Trasfusionale, Policlinico Bari, Bari, ${ }^{5}$ ematologia, ${ }^{6}$ Medicina Trasfusionale, AO Card Panico, Tricase, ${ }^{7}$ Medicina Trasfusionale La Maddalena, ${ }^{8}$ Ematologia, OO Riuniti Villa Sofia Cervello, Palermo, Italy, ${ }^{9}$ Hematology, University Hospital La Fe, Valencia, Spain, ${ }^{10}$ Hematoncology, European Institute of Oncology, Milano, ${ }^{11}$ Medicina Trasfusionale, AUSL Pescara, Pescara, ${ }^{12}$ Medicina Trasfusionale, A.O. Universitaria, Udine, ${ }^{13}$ Medicina Trasfusionale, Policlinico Tor Vergata, Roma, ${ }^{14}$ Hematology, Istituti Ospitalieri, Cremona, ${ }^{15}$ Epidemiology Infectious desease, Istituto Superiore Sanità, Rome, ${ }^{16}$ Medicina Sperimentale, Università La Sapienza, Roma, Italy

Introduction: Delayed haematological recovery after autologous stem cell transplantation (ASCT) is associated with a significant increase in both relapse and non-relapse mortality. Modern cell separator devices, with high collection efficiency, ensure a safe dose of Human Progenitor Cells (HPC)-Apheresis (CD34 ${ }^{+} \geq 4 \times 10^{6}$ \% $\mathrm{kg}$ ) that led to rapid granulocytes (10-12 days) and Platelets (11-14 days) recovery. Nevertheless delayed granulocyte (rarely) or PLT (more frequently) engraftment can occur even in absence of any known risk factor. In case of high contamination of HPC-A collections by mature cells, a detrimental effect on engraftment has been suggested due to their interference with viability and

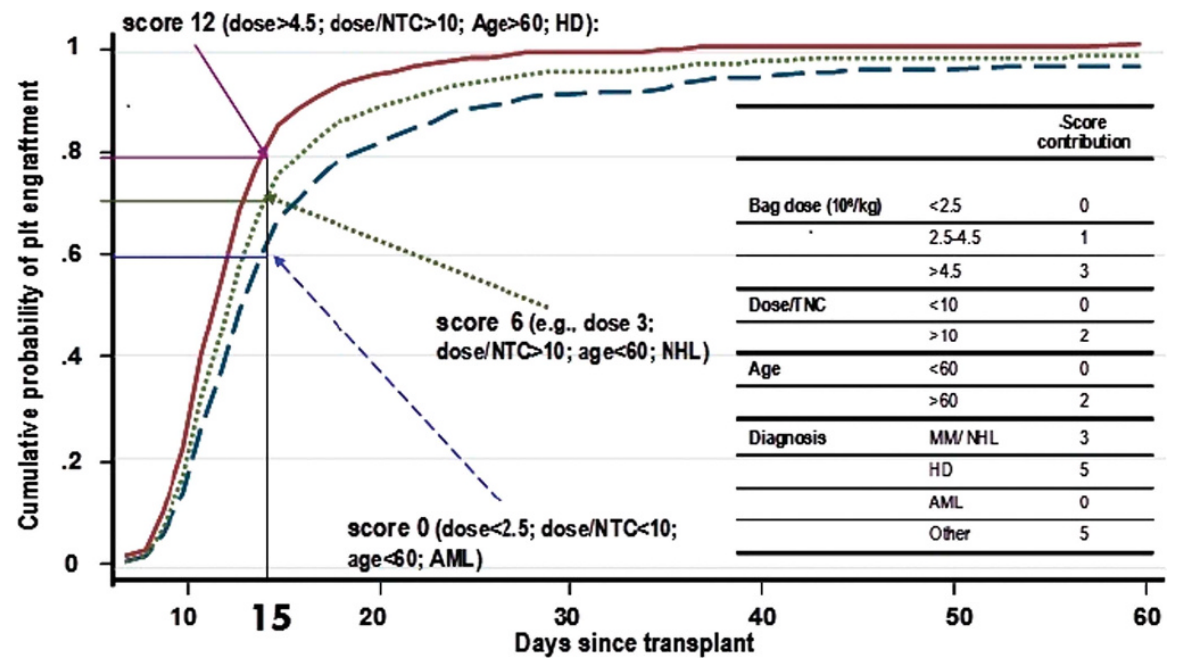


homing of Progenitor Cells during thawing and reinfusion. Our goals were to evaluate the influence of the graft composition on engraftment kinetics and, considering others patients features, to create a predictive risk score model of time to engraftment useful to plan future transplant procedures.

Material (or patients) and methods: We collected retrospective data from eight transplant centres in Italy on 762 transplanted patients with autologous HPC-A in 2009-2012. To evaluate time to PLT and granulocyte engraftment (i.e., 20,000 mmc and 500 $\mathrm{mmc}$, respectively) Kaplan-Meier curves and proportional hazards Cox regression model were used. The following characteristics were evaluated: transplantation centre, age at transplantation, gender, diagnosis, and graft composition $\left(\mathrm{CD} 34^{+}\right.$per $10^{6} / \mathrm{Kg}$, TNC per $\left.10^{9}\right)$ ]. In particular, instead of TNC we evaluated a variable obtained as the ratio between $\mathrm{CD} 34^{+}$and TNC. The scores was calculated using the coefficients of each statistically significant factor included in the final multiple Cox models.

Results:: Our 762 patients (MM 49.5\%, NHL 32.5\%, HD, 11.2\%, AML $3.9 \%$, other $2.9 \%$ ) received a median of $4.9 \times 10^{6}$ CD34 cells $/ \mathrm{kg}$ (range 0.2.-16.0), median days to achieve engraftment were 11 days (2-100) and 13 days (7-108) for granulocyte and for PLT, respectively. Total nuclear cells infused per $10^{9} / \mathrm{Kg}$ were 0.35 (0.03-4.14). Factors who resulted independently associated with time to PLT engraftment were: $\mathrm{CD} 34^{+}$dose (stratified as $<2.5,2.5-4.5,>4.5$ ), ratio between CD34 ${ }^{+} / \mathrm{TNC}$ $(£ 10,>10)$, age at transplantation $(£ 60,>60)$, and diagnosis The figure shows the estimated cumulative probability of PLT engraftment, as well as the score contribution of each of the factors associated, for different groups of patients with different characteristics and different total score. Patients scoring 0,6 and 12 had an estimated probability of around $60 \%, 70 \%, 80 \%$ of engraftment at 15 days since transplantation, respectively. When evaluating time to granulocyte engraftment, we found that only $\mathrm{CD}_{3} 4^{+}$and ratio between CD34+/TNC resulted independently associated.

Conclusion: The study confirms the role of $\mathrm{CD}_{3} 4^{+}$cells on engraftment; TNC have a detrimental effect as expressed by the ratio with the $\mathrm{CD}_{3} 4^{+}$suggesting that selectivity during mobilization and collection must be considered as an additional requisite to reduce time to engraftment. Other factors such as diagnosis and age play a role on platelet but not in granulocyte engraftment. A predictive risk score model of time to engraftment can be useful to plan future transplant procedures.

Disclosure of Interest: None declared.

\section{P425}

Neurological events during the infusión of hematopoietic stem cells cryoprserved with dimethyl sulfoxide for autologous transplantation

M. López-Parra', O. López Villar ${ }^{1, *}$, F. López-Cadenas ${ }^{1}$, B. Oreja Martín ${ }^{1}$, M. I. Ramos-Sevillano ${ }^{1}$, M. J. Nieto ${ }^{1}$, C. del Cañizo

${ }^{1}$ Hematology, Hospital Universitario de Salamanca, Salamanca, Spain

Introduction: Dimethyl sulfoxide (DMSO) is a solvent that is routinely used as a cryoprotectant for storage of hematopoietic progenitor cells from the marrow or blood. The DMSO is generally considered to be relatively innocuous, but any adverse effects have been associated with its infusion during the autologous stem cell transplantation (ASCT). Any centres have described cases of neurological toxicity.

Material (or patients) and methods: Retrospective analysis of the incidence of neurological events during the infusion of hematopoietic stem cells of peripheral blood for ASCT that have been cyopreserved with DMSO.

Each unit of the apheresis product freeze keeps a cell concentration $<2 \times 10^{5}$ cells $/ \mathrm{ml}$ and a final concentration of $10 \%$ DMSO.

Cryopreservation was performed in the biological freezer CM-2010 and then, the units were stored in a liquid nitrogen container. Thawing was performed in a water bath at $37^{\circ} \mathrm{C}$. All patients received intravenous premedication before the infusion with hydrocortisone $100 \mathrm{mg}$, acetominophen $1 \mathrm{~g}$ and dexchlorpheniramine $10 \mathrm{mg}$.
Prior to the infusion the blood pressure, oxygen saturation and heart rate were taken and monitored every 5 minutes. Infusions were performed through a subclavian route.

Results: From 01.01.2009 the 01.03.2015 have been done in our center 324 infusions of ASCT. 9 (3\%) patients had neurological symptoms during the infusion. The median age was 59 years (15-67). The diagnoses were multiple myeloma in 6 patients (chemotherapy conditioning with melphalan-200), Hodgkin lymphoma in 2 patients and non-Hodgkin lymphoma in 1 patient (BEAM condicioning type). Median CD34+ and mononuclear cells infused was $2,3 \times 10^{6} / \mathrm{kg}$ (2 to 12$)$ and $9,9 \times 10^{8} / \mathrm{kg}(4$ to 22) respectively. The volume of product infused was $320 \mathrm{ml}$ (160-920). The relationship between DMSO infused and the weight of the recipient was $0,4 \mathrm{ml} / \mathrm{kg}(0,2-0,9)$. 9 patients developed a depression of the central nervous system and hypotension during the procedure. 7 (78\%) presented complex partial seizure. One of these patients had a history of epilepsy. 2 patients had symptoms compatible with acute cerebrovascular accident (hemiplegia and oral commissure deviation). Analytical studies following episode (blood count, renal and liver function and ions) were normal. In $100 \%$ of cases the symptoms were reversible and didn't repeat. 7 patients have brain magnetic resonance with normal results in 6 of them, and ischemic lesions in one patient. One patient needed support in the Intensive Care for 48 hours. In the absence of other causes, we attribute the reaction to DMSO toxicity.

All patients achieved an adequate graft (median time to neutrophil engraftment $(>500 / \mu \mathrm{l})$, and platelet engraftment $(>20000 / \mu \mathrm{l})$ was 11 days and 10 days respectively, following transplantation. Median days of infusion until discharge was 15 (13-20).

Conclusion: The presence of neurologic complications during the infusion of autologous hematopoietic stem cell cryopreserved with DMSO is rare and reversible. However, it is a serious situation that may compromise the patient's life, so it should be known by the medical team.

References: L. J Fry et al. Assessing the toxic effects of DMSO on cord blood to determine exposure time limits and the optimum concentration for cryopreservation. Vox Sanguinis, 2015, 109, 181-190.

Stroncek DF et al: Adverse reactions in patients transfused with cryopreserved marrow. Transfusion 1991; 31.521-526.

Disclosure of Interest: None declared.

\section{P426}

Haematopoietic Progenitor Cell Collections Using the Spectra Optia ${ }^{\circledR}$ MNC Protocol (v.11) on patients $<12.5$ kgs.

N. Comey ${ }^{1, *}$, P. Lynam ${ }^{2}$, O. Smith ${ }^{2}$, C. Owens ${ }^{3}$, M. Capra ${ }^{3}$, J. Pears ${ }^{3}$, A. O'Marcaigh ${ }^{2}$

${ }^{1}$ Haematology / Oncology Department, ${ }^{2}$ Haematology, ${ }^{3}$ Oncology, Our Lady's Children's Hospital Crumlin, Dublin, Ireland

Introduction: Challenges of apheresis in small children $(<12.5 \mathrm{~kg})$ include small total blood volume (TBV), access issues, tolerable anticoagulant doses, and safe collection volume limits. Paediatric patients, and in particular, those with TBV $(<12.5 \mathrm{~kg})$ present a unique set of challenges when performing MNC collections on Spectra Optia ${ }^{\circledR}$.

Limitations include:

1.Total Blood Volume: For paediatric patients blood volume is calculated at $70-80 \mathrm{ml} / \mathrm{kg}$.

2. Inlet Flow Rate/ AC: Inlet:AC ratio was increased above the 15:1 maximum recommendation to facilitate a minimum IFR of $10 \mathrm{ml} / \mathrm{min}$ and the risk of clotting in the circuit compensated by the addition of Heparin (10iu/ml) to the AC.

3. Collection parameters: Processing of 2 TBVs with collection of product volume not exceeding $13 \%$ of TBV was achieved by using the collection phase control on the advance control options, selecting inlet volume as a trigger for collection. On 4 of the collections, Optia defaulted to collect 0 chambers when patient data was inputted.

Material (or patients) and methods: Procedural data was collected from the Optia Apheresis reporting system for 11 


\begin{tabular}{|c|c|c|c|c|c|c|c|c|}
\hline Patient & $\begin{array}{l}\text { Day of } \\
\text { collection }\end{array}$ & $\begin{array}{l}\text { Weight } \\
\text { (Kg) }\end{array}$ & Diagnosis & $\begin{array}{c}\text { CD34 pre-PBSC } \\
\left(10^{6} / L\right)\end{array}$ & $\begin{array}{c}\text { CD34 Yield } \\
\left(10^{6} / \mathrm{Kg}\right)\end{array}$ & $\begin{array}{c}\text { Target CD34 dose } \\
\left(10^{6} / \mathrm{Kg}\right)\end{array}$ & $\begin{array}{l}\text { CD34 } \\
\text { CE\% }\end{array}$ & $\begin{array}{l}\text { No TBV } \\
\text { processed }\end{array}$ \\
\hline 1 & D1 & 6.9 & Neuroblastoma & 200 & 9.1 & 3 & 36 & 2 \\
\hline 2 & D1 & 10.7 & Neuroblastoma & 83 & 6.2 & 6 & 44 & 2 \\
\hline 3 & D1 & 11 & $\begin{array}{c}\text { Atypical TeratoidRhab- } \\
\text { doid Tumour }\end{array}$ & 330 & 18.9 & 3 & 53 & 2 \\
\hline \multirow[t]{3}{*}{4} & D1 & 11.5 & Medulloblastoma & 41 & 1.8 & 9 & 40 & 2 \\
\hline & D2 & & & 51 & 3.1 & & 50 & 2 \\
\hline & D3 & & & 87 & 4.1 & & 41 & 1.9 \\
\hline \multirow[t]{2}{*}{5} & D1 & 10.6 & $\begin{array}{c}\text { Atypical TeratoidRhab- } \\
\text { doid Tumour }\end{array}$ & 27 & 1.7 & 3 & 43 & 2 \\
\hline & D2 & & & 24 & 1.4 & & 65 & 2 \\
\hline \multirow[t]{2}{*}{6} & D1 & 10.6 & Neuroblastoma & 43 & 2.1 & 3 & 60 & 1.9 \\
\hline & D2 & & & 81 & 3.4 & & 59 & 2 \\
\hline 7 & D1 & 10.0 & Neuroblastoma & 8 & 0.54 & 3 & 39 & 2 \\
\hline
\end{tabular}

collections from patients $<12.5 \mathrm{Kg}$. Laboratory analyses of pre-apheresis peripheral blood and harvested apheresis products were also examined. Spectra Optia ${ }^{\mathbb{R}}$ MNC performance was measured by CD34+ collection efficiency, expressing the total number of CD34+ cells collected as a proportion of the total number of CD34+ processed during apheresis.

Results: A total of 11 collections (7 patients) were completed during the period examined. Collection efficiency (CE) $>40 \%$ was achieved in 9/11 collections. $7 / 11$ products collected have been successfully infused with engraftment times observed within our target ranges in all cases.

Conclusion: From these data we conclude that with manipulation and operator intervention, Spectra Optia ${ }^{\mathbb{R}}$ MNC program can be used effectively for HPC collection in this patient cohort. References: Glicher's "modified rule of five", pg 228 of Mc Leod B, Apheresis:Principles and practice.

Disclosure of Interest: None declared.

\section{P427}

Feasibility and safety of bone marrow harvest in subarachnoid anaesthesia: a single-center experience of 140 healthy donors

S. Marotta ${ }^{1, *}$, S. Pagliuca ${ }^{2}$, F. Grimaldi ${ }^{2}$, C. Maglione ${ }^{3}$, M. Di Fraia $^{4}$, A. Raiola ${ }^{5}$, L. Pezzullo ${ }^{6}$, B. Serio ${ }^{6}$, P. Ricci ${ }^{7}$, F. Pane ${ }^{8}$, C. Selleri', G. De Rosa ${ }^{10}$, A. Risitano ${ }^{11}$

${ }^{1}$ Department of Hematology and Transplantation Unit, ${ }^{2} \mathrm{Hema-}$ tology and Transplantation Unit, Federico II University of Naples, ${ }^{3}$ Department of Anesthesiology and Reanimation, AORN A. Cardarelli, ${ }^{4}$ Department of Anesthesiology and Reanimation, Federico II University of Naples, Naples, ${ }^{5}$ Departmento of Bone Marrow Transplantation, Istituto San Martino, Genoa, ${ }^{6}$ OO.RR. San Giovanni di Dio e Ruggi d'Aragona, Salerno, "University of Naples Ferderico II, ${ }^{8}$ Hematology and Transplantation Unit, University of Naples Federico II, Naples, ' $U$ niversity of Salerno, OO.RR. San Giovanni di Dio e Ruggi d'Aragona, Salerno, Italy, Salerno, ${ }^{10}$ Hematology and Transplantation Unit, University of Naples Federicoll, ${ }^{11}$ Hematology and Bone Marrow Transplantation Unit, University of Naples Federico II, Naples, Italy

Introduction: Donation of hematopoietic stem cells (HSC) for an allogeneic transplantation is generally considered a procedure with negligible risks. Although in the past 2 decades, bone marrow (BM) as HSC source, had been largely replaced by peripheral blood (PBSC), allogeneic BM HSC remains the preferred source in non-malignant disorders or in case of HLA-disparity (e.g., haploidentical donors). The main arguments dissuading from BM donation are the risks of general anaesthesia, which includes arrhythmias, myocardial infarction, stroke, pulmonary embolism or edema, malignant hyperthermia and anaphylaxis.

Material (or patients) and methods: Here we report our experience on 140 consecutive healthy donors who underwent a BM harvest in subarachnoid anaesthesia for an allogeneic HSC donation. All procedures were performed between 1995 and 2015, in subjects who have fulfilled our institutional criteria of eligibility to HSCT donation. The protocol of subarachnoid anaesthesia consisted of a fine (25$27 \mathrm{G}$ ) needle injection of $12 \mathrm{mg}$ of hyperbaric bupivacaine $0.75 \%$ or ropivacaine $0.2 \%$ in L2-L3 or L4-L5 space, with donor in setting position. BM harvest was performed according to standard guidelines, with multiple punctures from both iliac crests; the total BM blood volume harvested ranged between $15-25 \mathrm{mg} / \mathrm{Kg}$, depending also on recipient weight. During the procedure, the donors received fluid infusion through a peripheral line; after about half of the procedure, the subjects also received autologous red cell transfusions (2-3 units), which have been collected in the 30 days before BM harvest. Results: All the subjects completed the procedure, which lasted on average 90 minutes (range 75-150); no donor required[U1] the need of a subsequent general anaesthesia (in 1 case a double dose of subarachnoid anaesthesia and in 2 cases a deeper analgesia with morphino-mimetics agents were required). Adverse events ( $A E)$ were grouped in early (during the procedure) and late. Among these 140 cases, only 2 late severe $A E(S A E)$ were recorded, both independent from the anaesthesia. Indeed one subject experienced an extrapiramidal syndrome about 4 hours after the procedure, independent from the anaesthesia, secondary to a metoclopramide injection given for nausea (early raising up). Another subject experienced a reaction to intravenous iron therapy which required steroids and anti-histaminic medications. Both subjects recovered in a few hours without any sequaelae. Early AE included hypotension ( $60 \%$ of cases), followed by nausea (about $40 \%$ ) and vomiting (15\%); all these events were mild or moderate ( $\leq 3$ according to ICD scale) and easily managed by liquid infusions, anti-emetic and inotropic agents. Late AE were negligible, with headache due to iron-deficient anaemia and bone pain reported by a minority of donors. After the procedure, all the patients received antimicrobials intravenously for 24 hours and then orally, together with iron/vitamin supplementation. The hospitalization lasted about 48 hours, with admission the evening before the procedure and discharge the following morning.

Conclusion: In conclusion, BM harvest in subarachnoidal anaesthesia is a safe and very well tolerated procedure for 
HSCT donation. The avoidance of a general anaesthesia eventually improves donor compliance, as well as the access to HSCT donation for subjects with possible comorbidities.

Disclosure of Interest: None declared.

\section{P428}

Cost Effectiveness of G-CSF biosimilars in peripheral blood stem cell collection for Myeloma patients

S. Elmoamly ${ }^{1, *}$, J. Sudhir ${ }^{1}$, M. Saif ${ }^{1}$, E. Tholouli ${ }^{1}$, F. Dignan ${ }^{1}$, A. Rocci ${ }^{1}$, R. Krishna ${ }^{2}$

${ }^{1}$ Haematology, Central Manchester University NHS trust, Manchester, ${ }^{2}$ Haematology, Leicester Royal Infirmary, Leicester, United Kingdom

Introduction: The use of biosimilar Granulocyte ColonyStimulating Factors (G-CSF) such as Ratiograstim ${ }^{\circledR}$ Tevagrastim ${ }^{\circledR}$, Biograstim ${ }^{\circledR}$, Zarzio ${ }^{\circledR}$, Nivestim ${ }^{\circledR}$ ) were approved by the European Medicines Agency (EMA) for all the registered indications of the originator (Neupogen ${ }^{\circledR}$ )

Material (or patients) and methods: We aimed to retrospectively analyse the cost effectiveness of biosimilar G-CSF (i.e. Zarzio ${ }^{\mathbb{R}}$ Nivestim ${ }^{\mathbb{R}}$ ) compared to the originator (Neupogen ${ }^{\mathbb{R}}$ ) in mobilizing Stem Cells in peripheral blood (PB) of patients with multiple myeloma (MM). All patients with MM undergoing stem cell mobilization from 1 January 2014 to 1 August 2015 at the Manchester Royal Infirmary (Manchester UK) have been enrolled. Patients have been divided according to the G-CSF brand they received for mobilization purposes and the following data has been collected: use of Plerixafor, dose of G-CSF received, pre-apheresis CD34 counts and the count of stem cells harvested.

Results: 53/86 patients received Neupogen 11/53 (21\%) required additional Plerixafor for mobilization. The average dose of Neupogen used was $843.5 \mathrm{mcg} /$ day, increased to $910.9 \mathrm{mcg} /$ day in patients requiring Plerixafor. An average pre CD34 count of 68.4 cells/ul with Neupogen alone and 15.6 cells/ul with Neupogen plus Plerixafor with an average of 5.88 million stem cells collected with Neupogen alone and 1.44 million stem cells collected with Neupogen plus Plerixafor. Zarzio was used in 23 patients and 4 (17\%) required additional Plerixafor. The average dose of Zarzio used was $840 \mathrm{mcg} /$ day with average of $780 \mathrm{mcg} /$ day when Plerixafor was added. Average pre CD34 count of 46.4 cells/ul with Zarzio alone and 19.5 cells/ul with Zarzio plus Plerixafor with an average of 4.2 million stem cells collected with Zarzio alone and 2.1 million stem cells collected with Zarzio plus Plerixafor. Ten patients used Nivestim with 1 patient (10\%) requiring additional Plerixafor. The average dose of Nivestim used was $723.3 \mathrm{mcg} /$ day with average of $1560 \mathrm{mcg} /$ day when Plerixafor was added. Average pre CD34 count was 83.25 cells/ul with Nivestim alone and 56 cells/ul with Nivestim plus Plerixafor with an average of 4.9 million stem cells collected with Nivestim alone and 2.6 million stem cells collected with Nivestim plus Plerixafor.

A cost analysis showed that, $£ 506.14$ ( $€$ 700.91) were spent per day for each patient using Neupogen for mobilization compared to $£ 99.5$ ( $€ 137.79)$ for Zarzio and $£ 80.7(€ 111.75)$ for Nivestim, suggesting a five-fold increase in cost when the originator has been used.

Conclusion: Our findings showed a non inferiority of biosimilar G-CSF (i.e. Zarzio and Nivestim) when compared to the originator Neupogen when used for stem cell mobilization in MM patients. The drastic reduction in cost with a similar efficacy is sustaining the current change of practice in MM patients. Further investigations are required in different populations such as the healthy donors in the allotransplant setting.

Disclosure of Interest: None declared.
P429

Evaluation of the Spectra Optia Apheresis System Mononuclear Cell (MNC) Collection: A Single Center Experience

T. Jisr, ${ }^{1, *}$, G. Rawas ${ }^{1}$, W. Halabi ${ }^{1}$, A. Fanous ${ }^{1}$, A. Mekdad ${ }^{1}$, A. Mugharbil' ${ }^{2}$ A. Ibrahim ${ }^{2}$

${ }^{1}$ Labaratory Mecdicine and Blood Bank, ${ }^{2}$ Hematology Oncology, MAKASSED UNIVERSITY HOSPITAL, Beirut, Lebanon

Introduction: The Spectra Optia (SO) apheresis system has been recently introduced in our center. SO device is designed to perform a wide range of therapeutic procedures, including peripheral blood stem cell (PBSC) collection in mobilized donors and patients (pts). The device was studied to evaluate the cellular composition of PBSCs harvested in pts with multiple myeloma (MM), non Hodgkin's lymphoma (NHL) and Hodgkin's lymphoma(HL) planed for autologous peripheral stem cell transplantation (APBSCT), and to optimize the collection of PBSCs using the CD34+ precount and collection efficiency(CE2) of apheresis device which is calculated as follows:

CE2 $=$ total CD34+cells collected $\times 10^{6} /$ CD34+ precount $/ \mu \mathrm{l} x$ blood processed (Liters).

The blood volume processed is calculated as follows: Desired CD34+x $10^{6} / \mathrm{kg} \times$ recipient weight $(\mathrm{kg}) /$ CE2 $\times$ CD34+ precount/ $\mu \mathrm{l}$

Material (or patients) and methods: In this single center study which enrolled pts undergoing PBSC mobilization and planed for APBSCT, we evaluated SO system's mononuclear cell collection performance, with respect to CD34+ cells and MNC collection efficiency, platelet reduction pre to post apheresis, and product purity in view of using prediction algorithms to optimize the procedure and predict the CD34+ yield, blood volume processed and platelets loss. We also evaluated neutrophil recovery in pts who underwent APBSCT. Results: Between 30/3/2015 and 30/11/2015, 16 pts underwent PBSC harvesting by SO device. Median age was 56 y (2369). There were 4 females and 12 males. Diagnosis was MM in 10 pts, $\mathrm{HL}$ in 4 pts and $\mathrm{NHL}$ in 2 pts. The number of aheresis procedures was 22. Mobilization consisted in G-CSF alone in $11 \mathrm{pts}$, chemotherapy and G-CSF in $4 \mathrm{pts}$ and G-CSF+CXCR4 inhibitor in one patient. Median count of CD34+ cells precollection was $48.6 / \mu \mathrm{l}$ (16.5-212). Median total blood volume processed was $13 \mathrm{~L}$ (6.3-19.9). Median count of CD34+ cells collected was $3.610^{6} / \mathrm{kg}(1-15)$. Median MNC collection efficacy was $45 \%$ (13-95). Median CD34+ cell collection efficacy was $44.5 \%$ (15-86\%). Median platelet reduction pre to post apheresis was 30\% (7-50\%). Median Product hematocrit and granulocytes product was $5 \%(3-9)$ and $55 \%$ (5-87), respectively. There were no device related severe adverse events.

Ten of the 16 pts underwent myeloablative high dose chemotherapy followed by APBSCT which was performed for $\mathrm{MM}$ in 8 pts and $\mathrm{HL}$ in 2 pts. The median count of CD34+ cells re-infused was $3.6310^{6} / \mathrm{kg}(2-8)$. All the pts received G-CSF post-APSCT until neutrophil recovery. The median day for neutrophil recovery was 11 (9-14). Median duration of severe neutropenia (ANC $<0.510^{9} / \mathrm{L}$ ) was 7 days (5-10).

Conclusion: The study results confirm that the SO apheresis system's MNC collection protocol is safe and effective. The neutophil recovery in pts auto-transplanted was not inferior compared to historical controls. In addition, this system will help us to use prediction algorithms for whole blood processing to achieve a desirable and optimal yield based on CD34+ precounts and CE2 of the apheresis device.

Disclosure of Interest: None declared.

\section{P430}

Improving collection efficiency during stem cell apheresis using a quick cell count

T. Netelenbos ${ }^{1, *}$, A. van Oostrum ${ }^{1}$, Y. Rozier ${ }^{1}$, J. Muijsenberg ${ }^{1}$, J. Overdevest ${ }^{1}$, A. Noort ${ }^{1}$, P. Spruitenburg ${ }^{1}$, J. J. Zwaginga ${ }^{1}$

${ }^{1}$ Immunohematology and bloodtransfusion, LUMC, Leiden, Netherlands 
Introduction: Stem cell collection by apheresis requires knowledge of CD34 levels in the donor to calculate the processed volume needed to obtain the acquired amount of CD34 cells $/ \mathrm{kg}$ recipient. The formula to calculate this volume however, considers the collection efficiency (CE) a fixed value. In real practice the $\mathrm{CE}$ can vary considerably among procedures. Therefore, feedback on CE during a collection could enable adjustments to the procedure and better prediction of its required duration.

Material (or patients) and methods: We prospectively collected data of stem cell apheresis procedures (autologous and allogeneic) performed in our center between 1-1-2015 and 1-12-2015 using the Spectra Optia ${ }^{\mathbb{R}}$. Using a Hematology Analyzer, KX-21 N Sysmex, white blood cell (WBC) and mononuclear cell (MNC) counts were measured in the peripheral blood of the donor before the collection and in samples taken from the collection bag during the apheresis. This quick cell count takes approximately 15 minutes. Furthermore, in each donor CD34 counts and percentage CD34 within the MNC counts were measured by flow cytometry. On 75 samples collected during apheresis both MNC counts as well as CD34 counts were performed. The MNC counts from the samples taken during the procedure were used to predict the CD34 yield in the bag, considering the percentage CD34 within the MNC fraction to remain constant before and during the harvesting. A correlation between the predicted and measured CD34 was made. In a subsequent cohort of 53 stem cell apheresis procedures, the CD34 prediction by the MNC count early in the apheresis procedure (after emptying of four collection chambers) was used to estimate the CE. In some cases a $2^{\text {nd }}$ sample was drawn. The CE during apheresis was estimated using the formula: CE predicted=percentage CD34 within MNC pre apheresis * MNC in sample / (CD34 pre apheresis * processed volume). When the predicted CE was lower than $30 \%$, attempts were made by the apheresis operator to improve it by adjusting the collection preference, the thrombocyte count or hematocrit. The CE from the completed procedure was assessed using the formula: $\mathrm{CE}$ total procedure $=$ CD34 yield in product / CD34 pre apheresis * total processed volume.

Results: The correlation between the predicted CD34 count by using MNC count and the measured CD34 by flow cytometry was excellent (correlation coefficient 0.96 ). From 45 donors we predicted the CE in 88 samples taken during apheresis. In $60.4 \%$ of cases the CE of the total procedure was higher than that of the predicted CE calculated from the MNC counts from the first sample (median increase of CE from 45.2 to $48.2 \%$ ). Focusing on the procedures with a predicted CE below $30 \%$, in 7 out of 8 cases the CE could be improved to $>30 \%$ (median $42.9 \%)$.

Conclusion: During stem cell apheresis a quick and easy MNC count of the stem cell product enables the CE to be predicted so that adjustments of the procedure can be made in order to obtain the stem cells more efficiently. Without time consuming and costly CD34 counts by flow cytometry this enables higher CD34 yields and better time management.

Disclosure of Interest: None declared.

P431

The snapshot CD34+cells extraction efficiency: a new method to validate an apheresis device

V. Lapierre ${ }^{1, *}$, N. Chaput ${ }^{1}$, D. Tramalloni ${ }^{1}$, K. Alexandrova ${ }^{7}$

${ }^{1}$ Cell Therapy Center, Gustave Roussy Cancer Campus, Villejuif, France

Introduction: The validation of a new apheresis device is not obvious because $1 /$ the comparison of different devices on consecutive days even in the same patient does not take into account the variation of biologic and clinical parameters of the patient, 2/the comparison of the collection efficiency does not take into account the variation of the CD34+ cells in the blood during the procedure. We propose a new approach to validate the performance of an apheresis device based on a snapshot CD34+cells extraction efficiency.

Material (or patients) and methods: This study compared the Spectra device which is characterized by continuous cell collection with high centrifugation speed and the cMNC-Optia device which is characterized by continuous cell collection with low packing factor which is supposed to decrease the collection of platelets in the bag. Twenty consecutive patients with a programme of a minimum of two CD34+cells collections participated in this validation study. Ten patients were collected day1 with Spectra and day2 with cMNC-Optia then 10 consecutive patients were collected day 1 with cMNCOptia and day2 with Spectra. The snapshot CD34+ cells extraction efficiency was calculated with the CD34+cells count contained in the blood collected directly $1 /$ from inlet line and 2 / from return line. These samples were collected 2 times, after the first and after the second blood mass treated.

Results: For the snapshot CD34+cells extraction efficiency, there was no difference between CMNC-Optia and Spectra at one blood mass (89.63\% [74.43-98.94] vs $88.86 \%$ [63.72-100], $P=0.33)$ and 2 blood mass treated $(90.18 \%$ [56.17-98.00] vs 92.56\% [44.54-100], $P=0.91$ ). Moreover, the platelet count in the collection bag, was not different between CMNC-Optia and Spectra at one blood mass $\left(1.9 \times 10^{11}[0.4-2.3]\right.$ vs $2.4 \times 10^{11}[0.2-$ 4.0], $P=0.35)$ and 2 blood mass $\left(3.5 \times 10^{11}[0.8-5.3]\right.$ vs $\left.4.6 \times 10^{11}[0.6-8.8], P=0.48\right)$. By contrast, the polynuclear neutrophil cell contamination in the collection bag was lower with CMNC-Optia compared with Spectra at one blood mass $(2.2 \%$ [0.1-11.0] vs $17.4 \%$ [2.0-56.4], $P=0.01)$ and 2 blood mass treated (2.8\% [0.1-12.1] vs $20.9 \%$ [1.4-35.6], $P=0.02$ ).

Conclusion: The snapshot CD34+cells extraction efficiency is an easy and reproducible method to compare the real efficiency of CD34+cell extraction by an apheresis device. Using this method, the Spectra and the CMNC-Optia are comparable and the CMNC-Optia has been validated in our apheresis unit. In our study, the platelet contamination in the collection bag does not differ between CMNC-Optia and Spectra. However, considering the PNN contamination, the bags collected with cMNC-Optia have the best purity.

Disclosure of Interest: None declared.

\section{P432}

Poor stem cell harvest may not always be related to poor mobilization: lessons gained from a mobilization study in $\boldsymbol{\beta}$-thalassemia major patients

V. Constantinou ${ }^{1,2, *}$, A. Bouinta ${ }^{1}$, G. Karponi ${ }^{2}$, F. Zervou' ${ }^{2}$, M. Iskas ${ }^{1}$, G. Stamatoyannopoulos ${ }^{3}$, A. Anagnostopoulos ${ }^{1,2}$, E. Yannaki ${ }^{1,2,3}{ }^{\prime}$ ${ }^{1}$ Hematology-BMT Unit, ${ }^{2}$ Gene and Cell Therapy Center, George Papanikolaou General Hospital, Thessaloniki, Greece, ${ }^{3}$ University of Washington, Seattle, United States

Introduction: Hematopoietic stem cell (HSC) mobilization and leukapheresis have been recently shown to be safe and effective in $\beta$-thalassemia patients, in the context of clinical trials for obtaining HSCs for thalassemia gene therapy (GT). In one of these trials for optimizing mobilization strategies for GT of $\beta$-thalassemia (Yannaki et al, Hum Gene Ther 2013), we observed that in some patients, HSC harvest was poor under standard leukapheresis settings, despite successful mobilization. The factors associated with the observed discrepancy between CD34+cell yield and mobilized CD34+cells were analyzed and are presented here.

Material (or patients) and methods: Twenty adult $\beta$ thalassemia patients were mobilized with Plerixafor, or G-CSF +Plerixafor after primary mobilization failure, with a goal to collect $6 \times 10^{6}$ CD34 ${ }^{+}$cells/kg (EudraCT\#2009-014136-37). Leukapheresis was performed with a Cobe Spectra device at $3 \mathrm{X}$ total blood volume. In 4 patients who all mobilized adequately, modification of the standard apheresis variables was needed to rescue the CD34+ harvest. Hematological and clinical parameters were analyzed in order to define predictive factors for the disproportional to mobilization $\mathrm{CD}_{3} 4^{+}$cell harvests. 
Results: On the basis of circulating CD34+ cells, 18/20 patients mobilized successfully with Plerixafor ( $\geq 20$ CD34+ cells/ $\mu \mathrm{l}$, range $25-220)$. In 3 of these adequately mobilized patients $(\mathrm{P} 10,14,20)$, there was an impressive mismatch between the numbers of circulating CD34+cells and the CD34+cell dose harvested by $1^{\text {st }}$ leukapheresis, resulting in poor yields in spite of successful mobilization (mean blood CD34+ 60.7 $\pm 22.5 / \mu \mathrm{l}$, mean CD34+ harvest $\left.1.0 \pm 0.38 \times 10^{6} / \mathrm{Kg}\right)$. In contrast, the rest of the patients who mobilized similarly (mean blood CD34+ 79.8/ $\mu$ l) yielded, as expected for their circulating CD34+cells, significantly higher CD34+cell doses/apheresis $\left(4.72 \pm 3.2 \times 10^{6} / \mathrm{Kg}, P=0.0001\right)$. In P14 and P20 the total CD34 +cell yield was rescued after adjusting the collection layer deeper, towards the RBC layer, in subsequent leukaphereses sessions, whereas in another patient (P19), in whom the apheresis settings modifications were made upfront because of suspected harvest failure, harvest was also successful (mean blood CD34+ 56.5/ $\mu$ l, mean CD34+ harvest $3.25 \times 10^{6} / \mathrm{Kg} /$ apheresis). All 4 patients were splenectomised and presented both at baseline and before $1^{\text {st }}$ apheresis, (relevant and absolute) lymphocytosis as well as reticulocytosis $(P=0.003$ and 0.005 , respectively). Splenectomy was correlated with absolute lymphocytosis and reticulocytosis at baseline $(P=0.01$ and 0.03 , respectively) and before 1 st apheresis, $(P=0.0003$ and 0.09 , respectively), as compared to patients with intact spleen. Not unexpectedly, by collecting the CD34 cells deeper, within the RBC layer, the hemoglobin $(\mathrm{Hb})$ content of the leukaphereses products (LPs) in these patients was significantly higher than the average $\mathrm{Hb}$ concentration in the LPs obtained from donors in whom leukaphereses were performed under standard operating settings (4.56 vs $1.09 \mathrm{~g} /$ $\mathrm{dl}, P<0.0000001)$.

Conclusion: Irrespective of the mobilization efficiency, lymphocytosis and reticulocytosis move the mononuclear cell layer towards the red blood cell layer during apheresis and thus could identify subjects who are at risk of poor CD34+cell harvests. In these subjects adjustment of the operating settings is needed in order to rescue the $\mathrm{CD}_{3}{ }^{+}$cell harvests. Disclosure of Interest: None declared.

\section{P433}

G-CSF activates coagulation and complement cascade without increasing the risk of a thrombotic event in healthy donors

L. Baumann 1,*, G. Ehninger ${ }^{2}$, A. Quade ${ }^{3}$, M. Punzel ${ }^{1}$, H. Schmidt ${ }^{1}$ ${ }^{1}$ Cellex GmbH, Cologne, '2University Hospital Carl Gustav Carus, Medizinische Klinik 1, Dresden, ${ }^{3}$ Labor Dr. Quade und Kollegen, Cologne, Germany

Introduction: Allogeneic stem cell transplantation has become a standard therapy in malignant but also nonmalignant diseases. To donate, the donor must first undergo granulocyte-colony stimulating growth factor (G-CSF) treatment. In recent years, some incidents of thrombosis have been reported following subcutaneous injections of G-CSF. In vitro experiments have also shown that initiating the mobilization of stem cells from the bone marrow involves three evolutionarily ancient proteolytic cascades (coagulation, fibrinolysis and complement). We examined whether some evidence of changes in the coagulation cascade could be detected in healthy unrelated donors.

Material (or patients) and methods: Unrelated donors of peripheral stem cells underwent physical examination 30 days prior to stem cell apheresis. Coagulation parameters including partial prothrombin time (PT), partial thromboplastin time (aPTT), fibrinogen, D-dimer and C3-complement (C3), as well as soluble interleukin 2 - receptor (IL2-R) level were assessed at this time and immediately before commencing apheresis. 518 donors were examined. If there was no medical problem and informed consent was obtained, all donors received lenograstim at a dose of $7.5 \mu \mathrm{g}$ per $\mathrm{kg}$ body weight for 5 days prior to stem cell collection. The donors were aged between 18-59 and had no risk factors for thrombosis. CD34+ cell counts, as well as leucocyte count, hemoglobin and platelet were also analyzed before apheresis. The data were compared using standard paired t-tests. The donors were split into groups according to their mobilization of CD 34+ cells (group 1: $<45$ CD34+ cells/ $\mu l$, group $246-100$ CD34+ cells/ $\mu l$, group $3>100 \mathrm{CD} 34+$ cells $/ \mu \mathrm{l})$.

Results: The PT decreased from $95.63( \pm 9.09) \%$ to $88.43 \%$ $( \pm 8.14)(P<0.001)$. The aPTT decreased from $26.09( \pm 1.94) \mathrm{sec}$ to $24.70( \pm 1.88) \mathrm{sec}(P<0.001)$. D-dimers increased from 0.26 $( \pm 0.13) \mathrm{mg} / \mathrm{L}$ to $0.48( \pm 0.44) \mathrm{mg} / \mathrm{L}(P<0.001)$. Fibrinogen levels increased from $267.59( \pm 60.59) \mathrm{mg} / \mathrm{dL}$ to $304.97( \pm 59,33) \mathrm{mg} /$ $\mathrm{dL}$. When we compared the different groups there were no significant difference in poorly mobilizing donors compared to very highly mobilizing donors. All the assessed parameters of coagulation showed statistically significant changes when compared to the status before apheresis, but all values stayed within normal ranges. In addition, we analyzed further parameters to assess the activation of other cascades as well. C3 levels increased from $118.77( \pm 24.04) \mathrm{mg} / \mathrm{dL}$ to 121.53 $( \pm 19.96) \mathrm{mg} / \mathrm{dL}(P<0.001)$ and soluble IL2-R increased from $381.89( \pm 152.61) \mathrm{U} / \mathrm{L}$ to $929.39( \pm 436.402) \mathrm{U} / \mathrm{L} \quad(P<0.001)$. Regular questionnaires were obtained from $41 \%$ of the donors examined; none reported any sign of thrombosis.

Conclusion: Our data confirm that G-CSF activates the coagulation and complement cascades in donors treated with lenograstim. By inducing a hypercoagulable state it could be hypothized that the risk of thrombosis may be increased. All values remained within normal ranges and none of the 518 donors had a thrombotic event, thus the clinical risk of thrombosis seems to remain minimal. Our data show that other parameters like complement C3 and soluble IL2-R also change during lenograstim treatment. These changes might correlate with side effects of G-CSF, but do not further harm the donor.

Disclosure of Interest: None declared.

\section{P434}

Survey concerning bone marrow harvest

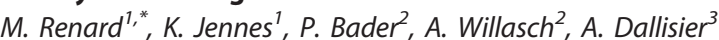
${ }^{1}$ Paediatric Oncology, UNIVERSITY HOSPITAL LEUVEN, Leuven, Belgium, '2Paediatric Oncology, University Hospital Goethe Frankfurt, Frankfurt, Germany, ${ }^{3}$ Paediatric Disease Working Party, EBMT, Paris, France

Introduction: Bone marrow is the oldest stem cell source for stem cell transplantation. During the nineties peripheral stem cells became the main source but a place remains for bone marrow for particular indications. Guidelines concerning the procedure to harvest bone marrow are lacking. Every centre uses its own procedure based on their own experience.

Material (or patients) and methods: A survey was conducted by the eBMT to obtain an overview of the different procedures for bone marrow harvest.

Results: Of the 261 centres, responses were collected from 48 centres (8 adult only, 23 paediatric and 17 combined centres). Most centres $(n=42)$ have more than 10 years of experience in harvesting bone marrow and physicians with more than 2 years of experience.

Over a period of 5 years, a mean of 55 collections per centre were performed (median: 35), half of them in paediatric patients. There is a large range, with some centres performing only 5 harvests and others 346 in 5 years.

In most centres (83\%), G-SCF is not given prior to the procedure and the procedure is usually done under general anaesthesia $(87.5 \%)$, with the donor in prone position $(79.2 \%)$ and the posterior iliac spine as preferred location (87.5\%). An 11 gauge $(25 \%)$ or 15 gauge needle $(14.6 \%)$ is used most frequently in adults. In paediatric patients, the size of the needle used is not as consistent.

For collection, a bag is preferably used $(89.6 \%)$, containing heparin alone $(33 \%)$, or ACD alone $(21 \%)$ or both $(21 \%)$.

The procedure itself varied widely. A single aspirate mostly consists of $5 \mathrm{ml}(62.5 \%)$ or $10 \mathrm{ml}(29 \%)$ and the way in which 
the puncture is performed is very different in terms of the number of skin holes, distance between different skin holes, how many times marrow is aspirated from one bone puncture, etc.

Graft manipulation is not done in $31.2 \%$, while it is done in $64.6 \%$ of centres.

The minimum number of TNC aimed at is also very different between centres: $2 \times 10^{*} 8 / \mathrm{kg}$ of the recipient is the minimum goal in $56.3 \%$ of centres, $3 \times 10^{*} 8 / \mathrm{kg}$ in $20.8 \%$ and $4 \times 10^{*} 8 / \mathrm{kg}$ in $12.5 \%$ of centres. $64 \%$ of centres do not perform a CD34 count during the procedure and those who do (26\%) have very different targets.

An upper limit volume to collect from the donor is fixed in $90 \%$, usually $20 \mathrm{ml} / \mathrm{kg}$ of the donor, ranging from 15 to $30 \mathrm{ml} / \mathrm{kg}$.

In almost half of the centres (46\%), a blood transfusion is given after the procedure, being autologous in $26 \%$.

A blood test is performed in $76 \%$ of the centres on the day after the procedure. Most patients/donors stay one night in the hospital (50\%) while $32 \%$ stay 2 nights. $68 \%$ of the centres prescribes pain therapy (mainly paracetamol). Iron therapy is routinely prescribed in $58 \%$, only occasionally in $26 \%$, and EPO is not often prescribed (18\%). The donor is re-evaluated after one week in $44 \%$, after 4 weeks in $66 \%$ and after one year in $22 \%$ of centres. Only $8 \%$ of the centres continues to follow-up the donor for more than one year. Usually there are no long-term problems after donation (86\%), but $6 \%$ mentions long-term problems after donation.

Conclusion: Bone marrow is the oldest stem-cell source. This survey reveals quite some heterogeneity in the procedure, the follow-up of the donor and method of target acquisition. Further research is required to identify elements to optimize the harvest procedure and to optimize donor safety.

Disclosure of Interest: None declared.

\section{P435}

Bone marrow source and chronic graft versus host disease have significant impact on survival in acute myeloid leukemia patients who underwent unrelated allogeneic hematopoietic cell transplantation

M. Sánchez-Escamilla ${ }^{1,2, *}$, A. Bermúdez ${ }^{1,2}$, L. Yáñez ${ }^{1,2}$, M. D. L. M. Colorado ${ }^{1,2}$, M. López-Duarte ${ }^{1,2}$, A. Casado-Díez ${ }^{1,2}$, B. LópezPereira $^{1,2}$, C. Amunarriz ${ }^{1,3}$, C. Richard ${ }^{1,2}$, E. Conde ${ }^{1,2}$

"Hematology, University Hospital "Marqués de Valdecilla", ${ }^{2}$ Hematology, IDIVAL, ${ }^{3}$ Hematology, Blood and tissue bank of Cantabria, Santander, Spain

Introduction: Allogeneic hematopoietic cell transplant (alloHCT) is potentially curative for acute myeloid leukemia (AML) patients. We analysed overall survival (OS), disease-free survival (DFS), total mortality (TM) and treatment-related mortality (TRM) in AML patients who underwent unrelated alloHCT.

We also analysed the impact on OS of patient's age, cytogenetic, disease status at transplantation, EBMT score, stem cell source, graft-vesus-host disease (GvHD), conditioning regimen and degree of HLA mismatching.

Material (or patients) and methods: We analyzed retrospectively 68 patients with AML who underwent unrelated AlloHCT between 2001-2014. Male/female relation was 29/39 $(42 / 57 \%)$, median age at transplant was 43 years (IQR 29-54). Intermediate and high-risk cytogenetic were observed in 42 $(61 \%)$ and $11(16 \%)$ patients. EBMT risk-score was $\leq 3,4-6$ and $\geq 7$ points in $31(45.5 \%), 33(48.5 \%)$ and $2(2.9 \%)$ patients. HCTcomorbidity index (HCT-Cl) was $0,1-2$ and $\geq 3$ in 10(14.7\%), 25 $(36.7 \%)$ and $25(36.7 \%)$ patients.

Transplants were HLA matched in 46(67.6\%) patients. Status at transplant was first complete remission (CR1) in 36(52.9\%). Stem cell source was bone marrow in $54(79.4 \%)$ patients. The median CD34+ dose was $2.84(1.50-4.87) \times 10^{6} / \mathrm{kg}$. Conditioning remigen was myeloablative in $58(85.2 \%)$. GvHD prophylaxis was made with CsA/Tacrolimus+MTX in 45(66.1\%). ATG was used in 17 patients (25\%).
Results: Median days to recover 500 granulocytes/uL and 20000 platelets/uL were 16 (IQR 13-17) and 16 (IQR 13-20). Two patients had secondary graft failure. Full donor chimerism $(>95 \%)$ by day +30 was observed in $48 / 60$ patients.

Median length of immunosuppression was 181 (IQR 109-243) days. Acute GvHD (aGvHD) was developed by 43/68 (63.2\%) patients and 12/45 were severe forms. The most frequent presentation was cutaneous (21/43). Seven patients died before day +100 so $61 / 68$ patients were evaluable for chronic GvHD (cGvHD). cGvHD was developed in 31/61 (50.8\%) patients: mild 22, moderate 4 and severe 6 . Median time to develop aGvHD and cGvHD was 32 (IQR 23-42) and 137 (IQR 113-177) days.

With a median follow up of 27 months, OS at day $+100,1$ and 2 years was $89.7 \%( \pm 3.0), 73.5 \%( \pm 5.4)$ and $61.9 \%( \pm 6.0)$. DFS at day $+100,1$ and 2 years was $86.6 \%( \pm 4.2), 68.7 \%( \pm 5.7)$ and $61.9 \%( \pm 6.0)$. In CR1 patients, the median follow up was 40 months and OS at day $+100,1$ and 2 years was $100 \%, 88.9 \%$ $( \pm 5.2)$ and $82.1 \%( \pm 6.7)$. TM was $30 / 68(44 \%)$ and in $24 / 30$ patients knew the cause of death. Most frequent causes were GvHD and infection in 9 patients, relapse in 7 patient and infection in 3 patients. TRM at day +100 and +365 was $8.8 \%$ $( \pm 3.4)$ and $16.5 \%( \pm 4.5)$. In CR1, TRM at day +100 was $8.3 \%$ $( \pm 4.6)$.

In multivariate analysis, bone marrow as source (HR: 0.23 (IQ 95\% 0.06-0.82)), cGvHD (HR: 0.21 (IQ 95\% 0.05-0.81)), CR1 (HR: 0.127 (IQ 95\% 0.01-0.96)) and time to transplant $\leq 12$ months (HR: 0.155 (IQ 95\% 0.02-0.94)) were significant statistically associated with better survival. On the other hand, aGvHD (HR: 5.04 (IQ 95\% 1.20-21.05)) and age higher than 45 years (HR: 3.78 (IQ 95\% 1.02-14.00)) were associated with worse survival rates.

Conclusion: CR1 status, bone marrow as source, development of CGvHD and short time to transplant were significantly associated with an improved survival in AML patients who underwent unrelated Allo-SCT.

Disclosure of Interest: None declared.

\section{P436}

Salvage use of allogeneic HSCT after reduced intensity conditioning from unrelated donors in multiple myeloma: outcomes from 10/10 HLA-matched unrelated donors, 9/10 HLA-mismatched unrelated donors and cord blood. A study by the EBMT and Eurocord

M. Michallet ${ }^{1}$, M. Sobh ${ }^{1, *}$, V. Dubois ${ }^{2}$, S. lacobelli ${ }^{3}$, L. Koster ${ }^{4}$, A. Van Biezen ${ }^{4}$, P. Ceballos ${ }^{5}$, R. Tabrizi ${ }^{6}$, J. Finke ${ }^{7}$, J. El-Cheikh ${ }^{8}$, M. Schipperus ${ }^{9}$, E. Meijer ${ }^{10}$, J. H. Veelken ${ }^{11}$, E. Petersen ${ }^{12}$, N. Russell ${ }^{13}$, E. Tholouli ${ }^{14}$, J. Passweg ${ }^{15}$, F. Garban ${ }^{16}$, H. Schoemans ${ }^{17}$, P. Chevalier ${ }^{18}$, N. Maillard ${ }^{19}$, L. Volin $20^{\prime}$ S. Francois ${ }^{21}$, B. Lioure ${ }^{22}$, Y. Beguin ${ }^{23}$, E. Gluckman ${ }^{24}$, A. Ruggeri' ${ }^{25}$ ', L. Garderetet, N. Kröger ${ }^{26}$ on behalf of Plasma Cell Disorder subcommittee of the EBMT chronic malignancies working party ${ }^{1}$ Hematology, Centre Hospitalier Lyon Sud, Pierre Bénite, ${ }^{2}$ Histocompatibility, Etablissement Français du Sang, Lyon, France, ${ }^{3}$ Centro Interdipartimentale di Biostatistica e Bioinformatica, Università Tor Vergata, Rome, Italy, ${ }^{4} E B M T$ Data Office, Leiden, Netherlands, ${ }^{5}$ Hematology, CHU Lapeyronie, Montpellier, ${ }^{6}$ Hematology, CHU Bordeaux, Bordeaux, France, 'University of Freiburg, Freiburg, Germany, ${ }^{8}$ Institut Paoli Calmettes, Marseille, France, ${ }^{9}$ Haga Hospital (Leyenburg), The Hague, ${ }^{10} \mathrm{VU}$ University Medical Center, Amsterdam, ${ }^{11}$ Leiden University Medical Center, Leiden, ${ }^{12}$ University Medical Centre Utrecht, Utrecht, Netherlands, ${ }^{13}$ Nottingham University, Nottingham, ${ }^{14}$ Manchester Royal Infirmary, Manchester, United Kingdom, ${ }^{15}$ University Hospital Basel, Basel, Switzerland, ${ }^{16}$ Hopital A. Michallon, Grenoble, France, ${ }^{17}$ University Hospital Gasthuisberg, Leuven, Belgium, ${ }^{18} \mathrm{CHU}$ Nantes, Nantes, ${ }^{9}$ Hopital La Miletrie, Poitiers, France, ${ }^{20}$ HUCH Comprehensive Cancer Center, Helsinki, Finland, ${ }^{21} \mathrm{CHRU}$ Angers, Angers, ${ }^{22}$ Nouvel Hopital Civil, Strasbourg, France, ${ }^{23}$ University of Liege, Liege, Belgium, ${ }^{24}$ Eurocord - Hopital St Louis, ${ }^{25}$ Hospital Saint Antoine, Paris, France, ${ }^{26}$ University Hospital Eppendorf, Hamburg, Germany 
Introduction: Allogeneic HSCT for multiple myeloma (MM) is performed in about $70 \%$ of cases in Europe at a late time in the treatment course after one or two autologous HSCTs. As MM occurs in a more aged population, the chance to find a suitable related donor tends to decrease. In the absence of HLA-identical sibling donor, a 10/10 HLA-matched unrelated donor (MUD) remains the first option and if absent, 9/10 HLA-mismatched UD (MMUD) or cord blood (CB) can be used. However, the outcome of MM patients transplanted from different unrelated sources has not been very well documented. The study objective was to evaluate the outcome of MM patients receiving reduced intensity conditioning (RIC) followed by allo-HSCT from UD after one or two auto-HSCT, with a comparison of different HSC sources.

Material (or patients) and methods: We included $M M$ patients who received RIC-allo-HSCT after one or two autoHSCT between January 2001 and December 2013 from UD as reported in the EBMT registry. Only patients with complete HLA typing were included. HLA results were reviewed and classified by an independent HLA expert on HLA A,B,C,DR,DQ for PBSC and on HLA A,B (generic level) DR (allelic level) for CB. There were 570 patients, $346(61 \%)$ were males, median age at allo-HSCT was 55 years (19-74), the Ig subtype was lgG in $48 \%$ of patients, light chain in $26 \%$ and $\operatorname{lgA}$ in $20 \%$. Overall, $62 \%$ were in stage III ISS; 119 (21\%) patients had karyotype abnormalities, majority with del(13q), 12 patients presented with $\mathrm{t}(4 ; 14)$ and/or del(17p). Allo-HSCT was performed after a median time of 15 months following one $(n=336,59 \%)$ or two ( $n=234,41 \%)$ auto-HSCT. Donors were 10/10 HLA-MUD for 419 (74\%) patients, 9/10 HLA-MMUD for 93 (16\%) and at least $4 / 6$ HLA CB in 58 (10\%) (19 single, 39 double). At transplantation, $466(82 \%)$ were in > = PR (122 CR). In the MUD and MMUD groups, RIC included fludarabine+busulfan+ATG in $45 \%$ of patients, fludarabine+TBI in $30 \%$, fludarabine+melphalan+ either ATG, Campath or bortezomib in $26 \%$ of patients. In the CB group, RIC mainly included cyclophosphamide+fludarabine+TBI ( $81 \%$ of cases)

Results: Engraftment rate was 94\%, $96 \%$ and $89 \%$ in the MUD, MMUD and CB groups respectively; acute GVHD grade II-IV occurred in $33 \%$ of patients, $41 \%$ and $42 \%$ respectively while chronic GVHD was present in 152/373 patients (56 limited, 96 extensive), 39/83 (15 limited, 24 extensive) and 15/49 (11 limited, 4 extensive) respectively. After a median follow-up of 26 months, the probability of overall survival at 3 years was $47 \%(41-52), 45 \%(34-56)$ and $38 \%(25-51)$ in the MUD, MMUD and $\mathrm{CB}$ groups respectively $(P=0.35)$. The cumulative incidence of relapse at 3 years in the respective groups was $50 \%$ (44-55), 35\% (25-46) and 54\% (40-67), $P=0.013$. Non-relapse mortality rates at 2 years were $22 \%(18-26), 33 \%(23-43)$ and $27 \%$ (16-39) in the MUD, MMUD and CB groups respectively, $P=0.23$. Results of ongoing multivariable analysis will be presented.

Conclusion: Preliminary results of this large study with fully documented HLA, showed that salvage RIC-Allo-HSCT from UD after one or two auto-HSCT could lead to promising outcomes. Overall, 10/10 HLA-MUD remains the first choice but results from either 9/10 HLA-MMUD or at least 4/6 HLA-CB are comparable and both sources should be equally considered. Disclosure of Interest: None declared.

P437

Patient/CBU Four Loci High Resolution Matching May Significantly Change CBU Selection

O. Nikolajeva ${ }^{1, *}$, K. Aitchinson ${ }^{2}$, E. Francis ${ }^{2}$, I. Evseeva ${ }^{2}$, J. A. Madrigal $^{1}$

${ }^{1}$ Anthony Nolan Research Institute, ${ }^{2}$ Anthony Nolan, Iondon, United Kingdom

Introduction: Currently the minimum requirement of patientCBU match for cord transplant is at the antigenic level for HLA-

[P436]
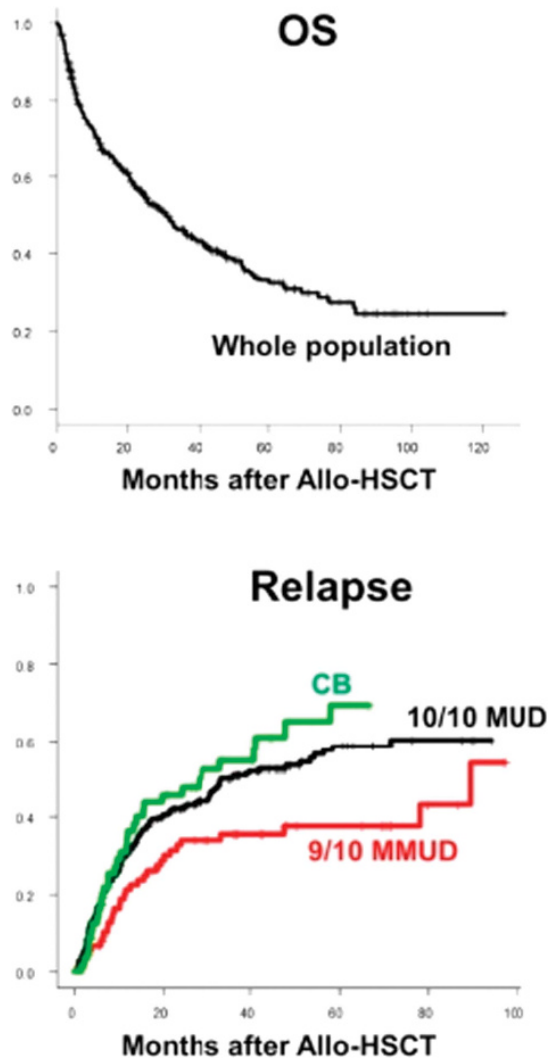

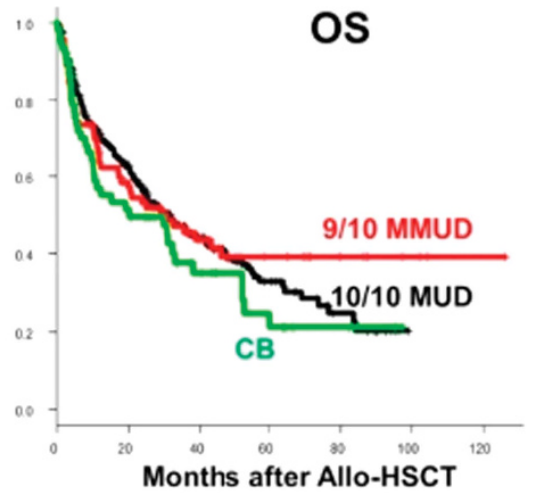

NRM

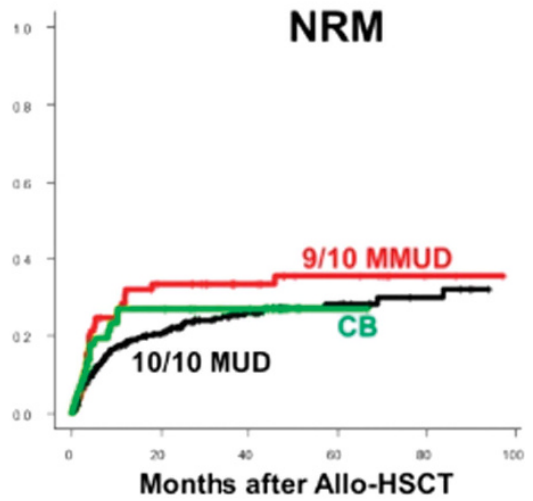


$A$ and $-B$ and allelic level for HLA-DRB1. There are emerging data that four locus allele level matching (including HLA-C) plays a role in transplant outcome ${ }^{1}$. A retrospective analysis of HLA matching of CBUs used for UK patients in 2013 -2014 was performed.

Material (or patients) and methods: 144 patients (65 adults and 79 children) underwent double unit ( $n=172$ units) or single unit $(n=58)$ transplant using 230 CBUs. CBU and recipient phenotypes were obtained from the Anthony Nolan database. The level of matching was determined at low (LR) and high resolution (HR). Where unavailable, HR matching was predicted based on published HLA haplotype frequencies ${ }^{2}$

Results: The majority of CBUs obtained a match grade of $4 / 6$ or above $(6 / 6(12 \%), 5 / 6(39 \%)$ and $4 / 6(49 \%))$. Evaluation of four loci matching revealed that all $6 / 6$ matched units had a HR match of between $7 / 8$ and $8 / 8$ except one case (4/8).

Within the $5 / 6$ match group, $63 \%$ were HR matched at $6-7 / 8$ (23\% at $5 / 8$ and $14 \%$ at $4 / 8)$.

In the $4 / 6$ match group only $60 \%$ of units were matched at the $5-6 / 8$ level and $36 \%$ showed HR matching at $\leq 4 / 8$.

Conclusion: Our study suggests that four loci HR matching may significantly change CBU selection. A few $\mathrm{CBUs}$ were selected for single CBT with level of HLA matching and/or TNC dose suitable for double CBT, but beyond UK recommendations for single $\mathrm{CBT}^{3}$.

CBU HR typing is therefore necessary at the search stage for use in the selection process to ensure the best unit is chosen to improve transplant outcomes in accordance to the previously published data. Third Generation Sequencing will deliver allelic level typing on all future CBUs stored by Anthony Nolan.

References: 1 Effect of donor-recipient HLA matching at HLA A, B, C, and DRB1 on outcomes after umbilical-cord blood transplantation for leukaemia and myelodysplastic syndrome: a retrospective analysis. M. Eapen, JP. Klein, GF. Sanz et al. Lancet Oncology; 2011 Dec;12(13):1214-21.

$2 \mathrm{https}: / /$ bioinformatics.bethematchclinical.org/hla-resources/ ${ }^{3}$ Recomebdation for a standart UK approach to incomporating umbilicl cord blood into clinical transplantation practice:an update on cord blood unit selection donor selection algorithmsand conditioning protocols. R.Haugh, R.Danby, N.Russell et al. BJH:18 NOV 2015, DOI: 10.1111/bjh.13802.

Disclosure of Interest: None declared.

P438

CBBs DNA collections can be successfully used for population studies of mitochondrial DNA and Y-chromosome variation. The Pavia CBB experience

P. Bergamaschi, ${ }^{1,{ }^{*}}$, I. Sbarsi ${ }^{1}$, A. L. Cremaschi ${ }^{1}$, S. Brandini ${ }^{2}$, A. Raveane ${ }^{2}$, A. Olivieri ${ }^{2}$, V. Grugni ${ }^{2}$, V. Genovese ${ }^{7}$, B. Romano ${ }^{1}$, M. Guarene ${ }^{1}$, A. Pasi ${ }^{1}$, C. Perotti ${ }^{1}$, O. Semino ${ }^{2}$, A. Torroni ${ }^{2}$ ${ }^{1}$ Service of Immunohematology and Transfusion Medicine, Fondazione IRCCS Policlinico San Matteo, ${ }^{2}$ Dept. of Biology and Biotechnology (DBB), University of Pavia, Pavia, Italy

Introduction: Non-Caucasian individuals are poorly represented in donor registries with unequal access to SCT as respect to Caucasians. Less stringent HLA-matching requirements make $C B$ an alternative for patients from non-Caucasian ethnic groups, also because the proportion of non-Caucasians is higher in $C B$ than in BM donors. So CBBs and registries are highly committed to increasing the genetic diversity of their donors pool. Initiatives include brochures and promotional materials inseveral languages and involvement of bilingual translators and cultural mediators. As our CBB stores a DNA archive of all its $C B$ units and also the demographics are recorded (geographical and ethnic origin of the family can be easily reconstructed, including grandparents), we investigated the potential application in population genetics. Studies based on uniparental markers -mitochondrial DNA (mtDNA) and $Y$ chromosome (MSY)- provide information concerning the relationship among human populations that are useful to trace back ancient migratory events. In particular, the ancestors of Native Americans are thought to have reached the Americas from Beringia at the time of the Last Glacial Maximum and then spread across the double continent. Very recently, the resolution of Native American-specific haplogroups has improved thanks to the sequencing of entire mitogenomes, the counterpart being represented by MSY, an independent source of genetic information.

Material (or patients) and methods: In this setting, we review the data on the ethnic composition of our CBB inventory and found 48 CB DNA samples stored from 1997 to 2014 suitable to be enrolled in a study on the origin of South American populations. For all CB donors included in this study the maternal or paternal geographical origin was documented to be from Central and South America (namely: Argentina, Brazil, Ecuador, Cuba, Chile, El Salvador, Peru, Dominican Republic, Venezuela). According to the geographical origin reported for maternal and paternal lineages respectively, 43 DNAs were employed for mtDNA and 12 for MSY analyses. Results: By surveying the mtDNA control-region, 27 subjects were found to harbor Native American mtDNAs; 14 of these, nine from Ecuador and five from Peru, were then completely sequenced (Illumina, San Diego, CA) and used for phylogeny construction in a dataset of 497 samples (185 from Ecuador and 312 from Peru). For MSY, by RFLP analysis, only 1 CB DNA from El Salvador was found to belong to the Native American haplogroup Q.

Conclusion: Purity and concentration of stored CB DNAs were always acceptable for our purposes. CB DNAs obtained from blood (namely cord blood, as collected from the cord vessels) provide optimal concentrations for sequencing by NGS platforms, less time consuming than traditional Sanger methods (used for DNAs isolated from saliva and mouthwash), impacting on the overall working load. In a multidisciplinary approach aiming to study the origins of human populations and based on the potential interactions between archeology, linguistics, climatology and genetics, we imagined a role for stem cell donors repositories. In this scenario, in parallel to the mission of improving its genetic diversity, a CBB can thus contribute to population genetics studies by making available its satellite sample collections and donors information. Networking of CBBs could be of valuable contribution too.

Disclosure of Interest: None declared.

\section{P439}

MOBILIZED HEMATOPOIETIC STEM CELLS FROM

PERIFERAL BLOOD AS PREFERED SOURCE FOR AUTOLOGOUS TRANSPLANTATION IN HEMATOLOGICAL PATIENTS - 15 YEARS OF EXPERIENCE

R. M. Grubovic ${ }^{1, *}$, L. Cevreska ${ }^{2}$, B. Georgievski ${ }^{2}$, S. GenadievaStavric $^{2}$, S. Useini ${ }^{1}$, A. Pivkova ${ }^{2}$, L. Cadievski ${ }^{2}$, E. Petkovic ${ }^{7}$, M. Grubovic ${ }^{1}$

'Institute for Transfusion Medicine of RM, Medical Faculty Skopje, ${ }^{2}$ University Hematology Hospital, Skopje, Macedonia, The Former Yugoslav Republic of

Introduction: Mobilized peripheral blood stem cells (PBSC) are the preferred source for $99 \%$ autologous stem cell transplantation (SCT) and they are widely used to treat various hematologic malignances. The aim of this study is to present our experience in apheresis collection of mobilized autologous PBSC in hematological patients for the period of 15 years.

Material (or patients) and methods: This is a retrospective study performed in the Institute for Transfusion Medicine of Republic of Macedonia and University Clinic for Hematology from January 2001 till December 2015. Patients with hematological diseases who underwent autologous PBSC collection and transplantation were included in the study. All subjects in the study were fully informed on the donation procedure and signed an informed consent for donation. Minimum dose required to ensure successful and sustained engraftment was $2 \times 106 / \mathrm{kg} \mathrm{CD34+} \mathrm{cells} \mathrm{and} 2 \times 108 / \mathrm{kg}$ mononucleated cells (MNC). PBSC harvesting was performed with 
continuous flow cell separator Baxter C53000 and COBE Spectra using conventional-volume apheresis processing 2-2.5 total blood volumes per apheresis. Mobilization regimens included granulocyte colony-stimulating factor (G-CSF) alone, or combination of G-CSF and disease-specific chemotherapy.

Results: There were 527 apheresis procedures performed in 255 hematological patients, median 2.1 collections/patient (range 1-5) for the mentioned period. They were 156 males and 99 females, aged $18-65$. The single procedure usually took 3-4 hours and the volume of collected stem cells was $50-220 \mathrm{ml}$. Sufficient number of cells was collected by 2 apheresis procedures mostly (78\%). The only adverse effects of the apheresis procedure were bone pain as reaction of G-CSF and numbness of the extremities as reaction of ACD-A (hypocalcemia), which occur rarely and were very mild. The main indications for transplantation were: multiple myeloma (52\%), acute myeloid leukemia (21.2\%), Hodgkin disease (15\%), non-Hodgkin lymphoma (9.5\%), acute lymphoblastic leukemia $(1.5 \%)$ and chronic lymphoblastic leukemia $(0.8 \%)$.

Conclusion: The tolerance of apheresis collection of PBSC in our patients was good. Collection of mobilized hematopoietic peripheral blood stem cells is an effective and safe procedure. Disclosure of Interest: None declared.

\section{P440}

Mobilization of autologous and allogeneic peripheral blood stem cells for transplantation in hematological malignancies using biosimilar G-CSF

S. Michaell,", J.-M. Hoffmann ${ }^{1}$, K. Lorenz', A. Publicover ${ }^{2}$, A. Schmitt ${ }^{1}$, A. Nagler ${ }^{3}$

${ }^{7}$ Department of Internal Medicine V, University Clinic Heidelberg, Heidelberg, Germany, ${ }^{2}$ Human Dendritic Cell Laboratory, Institute of Cellular Medicine, Newcastle University, Newcastle, United Kingdom, ${ }^{3}$ Hematology Division, BMT and Cord Blood Bank, Tel-Aviv University, Chaim Sheba Medical Center, Tel-Aviv, Israel

Introduction: Biosimilars of the granulocyte colony stimulating factor (G-CSF) filgrastim were approved by the European Medicines Agency (EMA) for registered indications of the originator G-CSF including prevention and treatment of neutropenia, as well as mobilization of peripheral blood stem cells in 2008. Nevertheless, there is still ongoing debate regarding the quality, efficacy and safety of biosimilar G-CSF.

Material (or patients) and methods: We performed a metaanalysis of clinical studies on the use of biosimilar G-CSF for mobilization and transplantation of hematopoietic stem cells as available in public data-bases.

Results: More than 1,892 individuals, mostly with hematological malignancies but also including 351 healthy donors have been successfully mobilized for autologous or allogeneic stem cell transplantation using biosimilar G-CSF (Zarzio ${ }^{\mathrm{TM}}$ : 1,239 individuals; Ratiograstim ${ }^{\mathrm{TM}} /$ Tevagrastim $^{\mathrm{TM}}$ : 653 individuals). 740 patients with multiple myeloma, 491 with Non-Hodgkin's lymphoma (NHL), 150 with Hodgkin's lymphoma (HL), and 160 with other diseases are included in this meta-analysis, as well as 161 siblings and 190 volunteer unrelated donors.

All data sets were weighted for the number of patients and parameters and then subjected to statistical meta-analysis employing the U-Mann-Whitney test followed by the HodgesLehmann estimator to assess differences between biosimilar and originator G-SCF.

No significant differences $(P>0.05)$ were observed for the following parameters in autologous and allogeneic stem cell mobilization: number of apharesis, $\mathrm{CD} 34^{+}$progenitor cells $/ \mathrm{kg}$ body weight, engraftment, $>0.5 \mathrm{G} / \mathrm{L}$ neutrophil count and platelets $>20 \mathrm{G} / \mathrm{L}$ - Using the Hodges-Lehmann estimator bioequivalence was demonstrated within the $20 \%$ range.

Conclusion: In summary, for biosimilar and originator G-CSF bioequivalence was observed for the yield of $\mathrm{CD} 34^{+}$stem cells as well as for the engraftment of the transplants.

Disclosure of Interest: None declared.
P441

Allogeneic Peripheral Blood Stem Cell Transplant for Severe Aplastic Anemia

S. Apte ${ }^{1, *}$

${ }^{1}$ Haematology \& BMT, Sahyadri speciality hospital, Pune, India

Introduction: Aplastic anemia encompasses a heterogeneous group of diseases with distinct pathophysiologies and a common clinical endpoint of marrow failure. Patients with severe aplastic anemia (SAA) can be treated with immunosuppressive therapy or hematopoietic stem cell transplantation (HSCT). Allogeneic stem cell transplantation is the preferred treatment modality in young patient with HLAmatched family stem cell donor.

Material (or patients) and methods: In this retrospective study data from patients with SAA, who underwent allogeneic HSCT at our center from 1999 to 2013, was reviewed under following headings.

1. Patient (Age, sex, transfusions, infections, $\mathrm{PNH}$ )

2. Donor (Age, sex, relation, HLA match)

3. Transplant (conditioning, graft source, GvHD prophylaxis, MNC/CD34-dose)

4. Engraftment $(\mathrm{y} / \mathrm{n}$, day, chimerism)

5. GvHD (acute/chronic, grade, treatment, outcome)

6. Complications (infections, VOD, CMV)

7. follow up and overall outcome

Results: Results: Data of 53 patients was analyzed.

Overall survival at two years was $75.47 \%$. Significant improvement in OS after $2009(86 \%)$ as compared to before 2009 (69\%)

13 patients died. 7 due to aGvHD+sepsis, 4 due to sepsis, and 1 due to accident.

51/53 patients engrafted. 2 died before engraftment.

10 patients had grade 3-4 aGvHD. 2 improved. 7 died due to sepsis, 1 had graft failure following intensive immunosuppression. Conclusion: Allogeneic HSCT is a curative and cost effective treatment modality for patients with SAA who qualify for it. Analysis of our center's data shows a definite improvement in OS of patients who undergo HSCT, parallel to the global trends. Thus, it should be offered to all eligible patients with SAA, as primary treatment.

Conclusion: PBSCT is preferred source of stem cells in third world countries as it causes rapid engraftment.If done early then the EFS is more than $90 \%$.The cost of Allo PBSCT is much cheaper than Triple Immunosuppression for SAA.

Disclosure of Interest: None declared.

\section{P442}

Effect of graft source on safety and efficacy in patients undergoing hematopoietic stem cell transplantation

S. Mielke ${ }^{1, *}$, J. Maertens ${ }^{2}$, D. Selleslag ${ }^{3}$, P. Lewalle ${ }^{4}$, I. Walker ${ }^{5}$ D.-C. Roy ${ }^{6}$, G. Bos', S. Devine ${ }^{8}$, D. Milojkovic ${ }^{9}$, L. Gerez ${ }^{10}$, K. Meewisse ${ }^{10}, K_{\text {. Reitsma }}{ }^{10}$, M. Rüdiger ${ }^{10}$, J. Rovers ${ }^{10}$

${ }^{1}$ Division of Hematology and Oncology, Department of Medicine II, Julius-Maximilian-University, Würzburg, Germany, ${ }^{2}$ Department of Haematology, University Hospital Gasthuisberg, Leuven, ${ }^{3}$ Department of Hematology, AZ Sint-Jan Brugge-Oostende AV, Brugge, ${ }^{4}$ Laboratory of Experimental Hematology, Institut Jules Bordet, ULB, Brussels, Belgium, ${ }^{5}$ Department of Medicine, Juravinski Hospital and Cancer Centre, Hamilton, ${ }^{6}$ Blood and Marrow Transplantation Program, Div. of Hematology-Oncology, Hôpital Maisonneuve-Rosemont, University of Montreal, Quebec, Canada, ${ }^{7}$ Department of Hematology, University Hospital Maastricht, Maastricht, Netherlands, ${ }^{8}$ Department of Hematology, The Ohio State University James Cancer Hospital, Columbus, United States, ${ }^{9}$ Department of Hematology, Hammersmith Hospital, Imperial College Healthcare NHS Trust, London, United Kingdom, ${ }^{10}$ Kiadis Pharma, Amsterdam-Duivendrecht, Netherlands

Introduction: Donor availability remains a major challenge in allogeneic stem cell transplantations (HSCT). For patients who cannot find an HLA-matched sibling donor, current standard of care is a fully matched unrelated donor (MUD). However, 
not for all a MUD can be found and additional alternatives such as single loci mismatched unrelated donors, umbilical cord blood or haploidentical donors are used.

Material (or patients) and methods: In this retrospective, multicenter study (CR-AIR-006; NCT02188290) data was collected on outcome of HSCT in patients with AML or ALL (both in remission) or MDS, using either a fully matched (8/8 or 10/10) unrelated donor (MUD), a single-locus mismatched $(9 / 10)$ unrelated donor (MMUD), umbilical cord blood (UCB) or a haploidentical $(3 / 6,4 / 6,5 / 10,6 / 10)$ donor (HAPLO). Data from transplantations performed between January 2010 and January 2013 (MUD, MMUD, UCB) or between January 2006 and July 2013 (HAPLO) was collected from centers participating in ATIR101 studies (CR-AIR-007; NCT01794299) and will serve as historic control group in the development of postHSCT donor lymphocyte infusion (ATIR101). Haploidentical donor transplantations were conducted using myeloablative conditioning and a CD34+ cell selected graft. Transplantrelated mortality (TRM), nonrelapse mortality (NRM), and overall survival (OS) up to 12 months post HSCT were compared between the four groups. In addition, incidence and severity of acute and chronic graft-versus-host disease (GvHD) up to 12 months was compared between groups. To determine clinical benefit the composite end-point of GVHDfree, Relapse-Free Survival (GRFS) was used.

Results: Data on 158 subjects was collected: HAPLO $=35$; $M U D=64 ; \quad M M U D=37 ; \quad U C B$ (double cord) =22. Relapse occurred in $15 \%$ of the 158 patients with 12 months cumulative incidences of $20 \%$ in the HAPLO group, $14 \%$ in the MUD group, $16 \%$ in the MMUD group and $9 \%$ in the UCB group. NRM was lower for the MUD group (9\%) compared to the other groups (HAPLO: 66\%; MMUD: 25\%; UCB: 36\%). Acute GVDH grade III-IV was less frequent in the HAPLO group (6\%) compared with other groups (MUD: 11\%; MMUD: 16\%; UCB: $27 \%$ ), and there was also less chronic GvHD in the HAPLO groups (11\%) compared to the other groups (MUD: $39 \%$; MMUD: 30\%; UCB: 32\%). Twelve month OS was lower for the HAPLO treatment group (20\%) compared to all other treatment groups (MUD: 86\%; MMUD: 64\%; UCB: 55\%). However, when looking at the GRFS it is clear that MUD
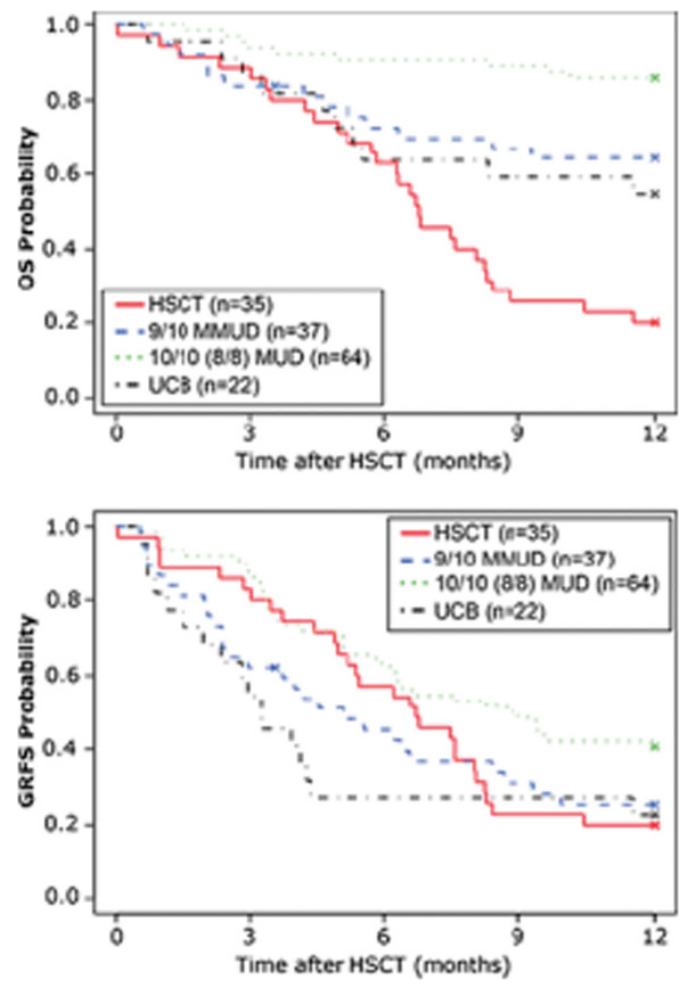

transplantations have the best outcome, and that all other alternative donor sources have a low GRFS at 1-year postHSCT.

Conclusion: Our data show that the current alternatives (HAPLO, MMUD or UCB) have a worse outcome compared to standard of care (MUD). Use of MMUD or UCB donors shows high rates of GVHD and TRM. Use of T-cell depleted haploidentical donors has substantially less GVHD, but more infections and thus higher rates of TRM. Adding additional donor lymphocytes (ATIR101) post-HSCT could overcome limitation of this CD34+ selected HAPLO regimen.

Disclosure of Interest: S. Mielke: None declared, J. Maertens: None declared, D. Selleslag: None declared, P. Lewalle: None declared, I. Walker: None declared, D.-C. Roy: None declared, G. Bos: None declared, S. Devine: None declared, D. Milojkovic: None declared, L. Gerez Employee of: Kiadis Pharma, K. Meewisse Employee of: Kiadis Pharma, K. Reitsma Employee of: Kiadis Pharma, M. Rüdiger Employee of: Kiadis Pharma, J. Rovers Employee of: Kiadis Pharma.

\section{P443}

CD34+ subsets in leukapheresis products collected after chemomobilization with or without Plerixafor in Lymphoma and Myeloma patients: a retrospective study M. R. Morciano ${ }^{1}$, A. Mele ${ }^{1}$, G. Greco ${ }^{1}$, R. De Francesco ${ }^{1}$, C. De Risi ${ }^{1}$, S. Sibilla ${ }^{1}$, D. Carlino', M. Fina ${ }^{1}$, B. Vannata ${ }^{1}$, B. Rossini $^{\prime}$, V. Federico ${ }^{1}$, A. Greco ${ }^{1}$, E. Prete ${ }^{1}$, S. Citiso ${ }^{1}$, V. Pavone ${ }^{1, *}$

${ }^{1} \mathrm{OU}$ Hematology and TMO, Hospital Card. G. Panico, Tricase, Italy

Introduction: CD34+ cell dose is the most reliable marker to evaluate the graft quality and to predict engraftment in autologous transplant. Nevertheless,there is an increasing interest in evaluating the role of CD34+ subsets in hematopoietic recovery above all, since Plerixafor $(P)$ is used to enhance CD34+ cells mobilization in Lymphoma and Myeloma Multiple (MM) patients (pts). In our studies we compared cellular composition of leukapheresis products (LPs) Lymphoma and MM pts mobilized with $P$ associated to granulocyte-colony-stimulating factor $(G)$ and chemotherapy $(C H T+G+P$, group B) or from pts mobilized with $C H T+G$ (group A).

Material (or patients) and methods: Samples of first fresh LPs were obtained from 152 pts with Lymphoma and MM mobilized with $\mathrm{CHT}+\mathrm{G}(n=116)$ and $\mathrm{CHT}+\mathrm{G}+\mathrm{P}(n=36)$ from July 2010 to October 2015. The relative and absolute number of CD34+ cells and the CD34+ phenotypic profile were performed by flow cytometry.

Results: After the first apheresis, the median CD34+ yield was $7.0 \times 10^{6} / \mathrm{kg}$ in group $\mathrm{A}$ and $3.13 \times 10^{6} / \mathrm{kg}$ in group $\mathrm{B}(P<0.001)$. The total median yield of the CD34+ cells was significantly higher in group $\mathrm{A}$ as compared to group $\mathrm{B}(P<0.001)$, achieved with a median of one apheresis procedure than group $B$ (two procedure, $P<0.001$ ) (Tab. 1). We characterized CD38, CD90, HLA-DR, CD117 and CD33 expression on CD34+ cells via flow cytometry. In 20 pts, 10 in group $A$ and 10 in group $B$,we also evaluated the surface expression of CD133 and adhesion molecules, such as CD184, CD49d, CD49e and CD62L. As shown in Fig. 1 LPs from group B had a significantly higher CD34+HLA-DR- and CD34+CD117- cells median percentage when compared to group $A(P=0.003 ; P=0.001)$. The median percentage of CD34+CD33- cells was lower in group $B$ than in group $A(P=0.009)$. Also CD34+CD38- median percentage in group $B$ was higher than group $A$,but this difference wasn't statistically significant $(P=0.827)$. We didn't note any significant difference between the two groups for CD34+CD90+ $(P=0.857)$ and CD34+CD133+ cells $(P=0.078)$. The surface expression of CD184, CD49d and CD62L on CD34+ cells was respectively higher in pts from group $B$ than in pts from group $A$, without statistically significant difference $(P=0.781 ; P=0.052 ; P=0.166) . C D 34+C D 49 e+$ median percentage was significantly higher in group $A$ than group $B$ $(P=0.021)$. 
Table 1 Patients' characteristics at mobilization and CD34+ cells dose in LPs

\begin{tabular}{|c|c|c|c|}
\hline & $\begin{array}{c}\text { Mobilization with } \\
\text { CHT+G (group A) } \\
\mathrm{n}=111\end{array}$ & $\begin{array}{c}\text { Mobilization with } \\
\begin{array}{c}C H T+G+P \text { (group B) } \\
\mathrm{n}=36\end{array}\end{array}$ & $\mathrm{P}$ \\
\hline Age & $53(16-71)$ & $54(22-73)$ & \\
\hline Gender (M/F) & $59 / 52$ & $29 / 7$ & \\
\hline Diagnosis (Lymphoma/MM) & $71 / 40$ & $31 / 5$ & \\
\hline Time to collection (days) & $13(10-21)$ & $16(9-22)$ & 0,001 \\
\hline CD34/ $\mu \mathrm{L}$ before apheresis & $78,4(10,98-1039,95)$ & $33,24(10,03-160,98)$ & 0,001 \\
\hline N.of apheresis & $1(1-3)$ & $2(1-3)$ & 0,001 \\
\hline CD34+ $\times 10^{6} / \mathrm{kg}$ in first LP & $7,01(1,14-27,60)$ & $3,13(0,85-9,69)$ & $<0,0001$ \\
\hline CD34+ $\times 10^{6} / \mathrm{kg}$ total yield & $7,09(3,38-35,70)$ & $4,79(1,74-11,06)$ & $<0,0001$ \\
\hline
\end{tabular}

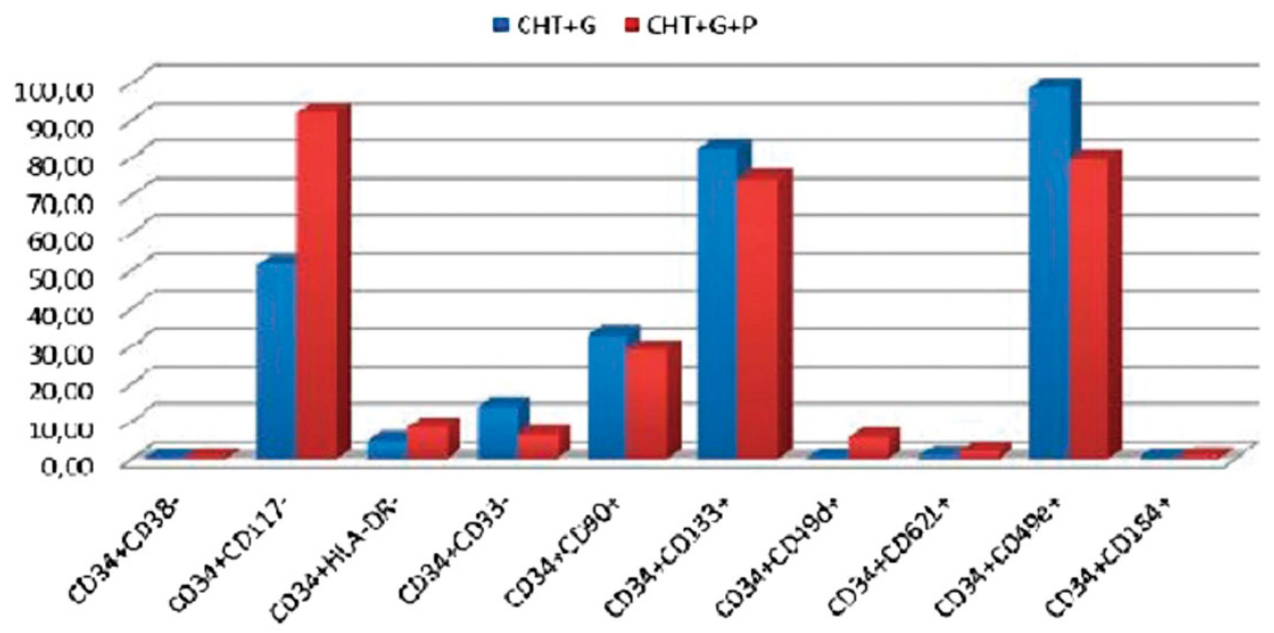

Fig. L. Distribution of CD34+ subsets in LPs of patients mobilized with $\mathrm{CI} I T+G$ vs $\mathrm{CITT}+\mathrm{G}+P$

Conclusion: Our results show that LPs collected from Lymphoma and $\mathrm{MM}$ pts mobilized with $\mathrm{P}$ contain more primitive CD34+ cells than LPs collected without $P$. In addition $P$ mobilizes CD34+ cells with a favorable expression profile in terms of homing and bone marrow retention.

Disclosure of Interest: None declared.

\section{P444}

CD34+ cells cryopreserved with 5\% dimethyl sulfoxide compared to $10 \%$ : better viability of CD34+ and no neurological side effects

A. Mele ${ }^{1}$, A. Musarò ${ }^{7}$, M. R. Morciano ${ }^{1}$, C. De Risi ${ }^{1}$, G. Greco ${ }^{1}$, S. Citiso ${ }^{1}$, E. Prete ${ }^{1}$, V. Federico ${ }^{1}$, M. Caputo $^{2}$, S. Sibilla ${ }^{1}$, D. Carlino ${ }^{1}$, B. Rossini ${ }^{1}$, L. Abbruzzese ${ }^{2}$, V. Pavone ${ }^{1, * *}$

${ }^{1} \mathrm{OU}$ Hematology and TMO, ${ }^{2}$ Transfusion Medicine, Hospital Card. G. Panico, Tricase, Italy

Introduction: Dimethyl sulfoxide(DMSO)is used as a cryoprotectant in stem cell preservation,but is associated with toxicity and adverse reaction in the transplant recipient. Cryopreservation using $5 \%$ DMSO instead of $10 \%$ DMSO is a strategy to reduce the adverse effects. We compare the use of 5\%DMSO versus $10 \%$ DMSO in terms of median and viability of CD34+ cells infused,haematological engraftment, number of febrile neutropenia and treatment related mortality (TRM).

Material (or patients) and methods: 144 consecutive patients with haematological malignancies submitted to transplant (125 autologous(87\%) and 19 allogeneic(13\%)) were included in the study. $82(57 \%)$ were stored in $10 \%$ DMSO (from January 2012 to September 2013) and 62(43\%) inDMSO5\%(from October 2013 to December 2015).

Results: Patients were similar in terms of median age, diagnosis, number of previous treatments, disease status at transplant. Median time to transplant from diagnosis was similar in the two groups but higher number of patients in DMSO10\% were transplanted after one year from diagnosis (86\%vs37\%, $P<0,001)$. Median of CD34+ cells infused was similar in the two groups,table 1. Reduction in DMSO concentration to $5 \%$ was associated with higher CD34+ cells viability ( $99 \%$ vs $81,5 \%$; $P<0,0084)$. No significant difference in median time to neutrophil (ANC) and platelets (PLT) engraftment was demonstrated in the two groups of patients, table 1.DMSO concentration did not influence median day of hospitalization and febrile neutropenia. No differences in terms of mucositis, diarrhea, renal or hepatic toxicity were observed. No neurological side effects were observed in the group of patients with DMSO5\%, instead 3 events (4\%) were registered in the DMSO10\% group, $(P=0,23)$. After 20 months of median follow-up (range, 1-39), the estimated 1yTRM was $6 \%$ in the DMSO5\% vs $13 \%$ in DMSO10\%, $P=0,45$.

Conclusion: Our results suggest that reduction in DMSO concentration to $5 \%$ was associated to higher viability of CD34 + cells infused without affecting median CD34+ cells infused and haematological reconstitution. At the same time, an equivalent 1YTRM was experienced between the two groups of patients studied. Interesting, neurological side effects were 


\begin{tabular}{|c|c|c|c|}
\hline $\begin{array}{l}\text { Table 1: Results of cryopreserved HPC and } \\
\text { haematoogical recovery }\end{array}$ & $\begin{array}{l}\text { Group } \\
5 \% \text { DMSO }\end{array}$ & $\begin{array}{l}\text { Group } \\
10 \% \text { DMSO }\end{array}$ & $\mathrm{p}$ \\
\hline $\begin{array}{l}\text { Median CD } 34+\text { infused cells }\left(\times 10^{6} / \mathrm{Kg}\right) \text { (range) } \\
>4 \times 10^{6} / \mathrm{Kg}(\mathrm{N}, \%)\end{array}$ & $\begin{array}{c}4,47(1,91-8,35) \\
25(40 \%)\end{array}$ & $\begin{array}{c}3,85(1,17-12) \\
34(48 \%)\end{array}$ & 0,317 \\
\hline $\begin{array}{l}\text { Median viabilityCD } 34+(\mathrm{r} \text { ange }) \\
>70 \%(\mathrm{~N}, \%)\end{array}$ & $\begin{array}{c}99 \%(94-100) \\
62(100 \%)\end{array}$ & $\begin{array}{c}81,5(31-99,6) \\
63(82 \%)\end{array}$ & 0,004 \\
\hline $\begin{array}{l}\text { Median dasy to }>0,5 \text { ANC } \times 10^{\circ} 9 / L \text { (range) } \\
\quad>12 \text { days }(\mathrm{N}, \%) \\
\text { Median days to }>20 \text { platelets } \times 1 \% 9 / L \text { (range) } \\
\quad>14 \text { days }(\mathrm{N}, \%)\end{array}$ & $\begin{array}{l}10(8-16) \\
3(5 \%) \\
12(9-26) \\
15(29 \%)\end{array}$ & $\begin{array}{l}10(6-27) \\
6(12 \%) \\
13(5-42) \\
15(28 \%)\end{array}$ & $\begin{array}{c}0,34 \\
0,8\end{array}$ \\
\hline $\begin{array}{l}\text { Median days of hospitalization from day } 0 \\
\quad>24 \text { days }(\mathrm{N}, \%)\end{array}$ & $\begin{array}{c}21(14-30) \\
17(28 \%)\end{array}$ & $\begin{array}{l}21(12-69) \\
10(20 \%)\end{array}$ & 0,89 \\
\hline Febrile neutroperia & $43(69 \%)$ & $53(66 \%)$ & 0,853 \\
\hline Neurological toxicity & 0 & $3(4 \%)$ & 0,23 \\
\hline Mucositis & $48(77 \%)$ & $58(76 \%)$ & 0,97 \\
\hline Diarrhea & $38(61 \%)$ & $48(63 \%)$ & 0,74 \\
\hline Renal toxicity & $2(3 \%)$ & $2(3 \%)$ & 0,23 \\
\hline $\mathrm{H}$ epatic toxicity & $2(3 \%)$ & $2(3 \%)$ & 0,44 \\
\hline
\end{tabular}

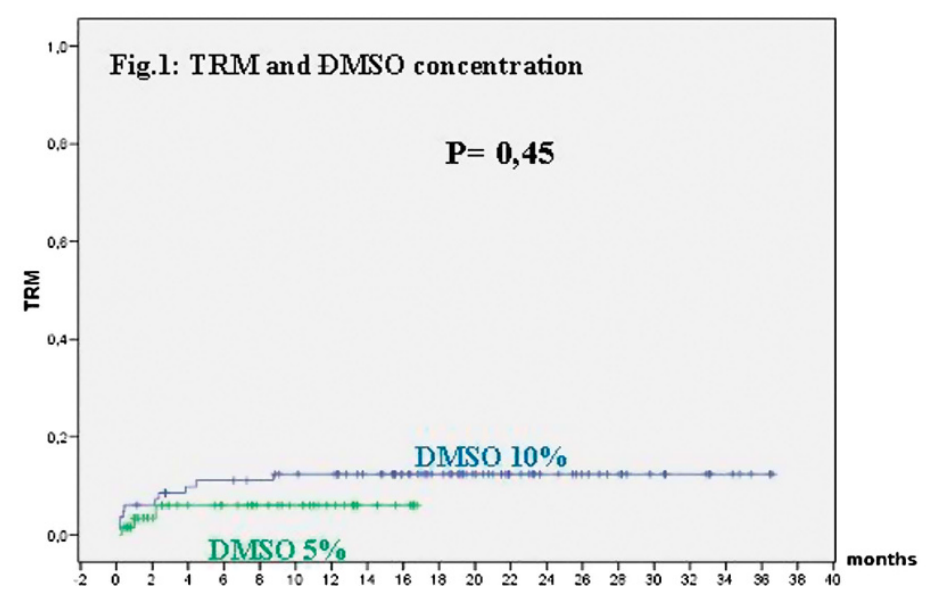

not observed in DMSO5\%. Further studies and longer followup is needed to evaluate fully efficacy and long term safety of cryopreservation of cells with 5\%DMSO.

Disclosure of Interest: None declared.

P445

Early recovery of CMV-specific T cells after HLA-

haploidentical HSCT with post-transplant cyclophosphamide is associated with a reduced risk of severe infections: a validation study

M. Noviello ${ }^{1, *}$, R. Greco ${ }^{2}$, V. Valtolina ${ }^{3}$, A. Forcina ${ }^{2}$, M. Pozzato ${ }^{2}$, M. T. Lupo Staghellini ${ }^{2}$, F. Giglio ${ }^{2}$, M. Morelli ${ }^{2}$, A. Assanelli ${ }^{2}$, S. C. Dalto ${ }^{2}$, S. Piemontese ${ }^{2}, M$. Bernardi ${ }^{2}$, C. Corti ${ }^{2}$, L. Vago ${ }^{4}$, N. Cieri ${ }^{5}$, J. Peccatori ${ }^{2}$, C. Bonini ${ }^{1}$, A. Bondanza ${ }^{6}$, F. Ciceri ${ }^{2}$

${ }^{1}$ Experimental Hematology Unit, Division of Immunology, Transplantation and Infectious Diseases, ${ }^{2}$ Hematology and Bone Marrow Transplantation Unit, Division of Regenerative Medicine, Stem Cell and Gene Therapy, San Raffaele Scientific Institute, ${ }^{3}$ MolMed, ${ }^{4}$ Unit of Molecular and Functional Immunogenetics, Division of Regenerative Medicine, Stem Cell and Gene Therapy, San Raffaele Scientific Institute, ${ }^{5}$ Università degli studi di Milano, ${ }^{6}$ Innovative Immunotherapies Unit, Division of Immunology, Transplantation and Infectious Diseases, San Raffaele Scientific Institute, Milan, Italy
Introduction: After HLA-haploidentical hematopoietic stem cell transplantation (haplo-HSCT), the profound state of immune incompetence secondary to aggressive GvHD prophylaxis results in high incidence of opportunistic infections. We have recently described that the frequency of functional CMV-specific T cells measured by day 90 with IFN- $\gamma$ ELISpot besides clearly associating with lower CMV-reactivation rates, appeared to protect from severe infections overall. Moreover, we proposed a cutoff of $1 \mathrm{CMV}$ spot forming cells (sfc) per microL for the identification of patients at lower risk for CMV reactivations and severe infections.

Material (or patients) and methods: To validate such threshold in an independent cohort, we analysed 30 adult patients undergoing haplo-HSCT and receiving a T-cell replete graft followed by immunomodulation with sirolimus and pulse cyclophosphamide as in vivo T-cell allo-depleting agent (sirPTCy). Patients were evaluated for CMV specific immune reconstitution by IFN- $\gamma$ ELISpot and for infectious serious adverse events (SAEs) up to one year after transplant.

Results: Early after transplant, a high rate of bacterial infections was documented, since all patients experienced at least one episode of febrile neutropenia, which in one case evolved in septic shock. Also viral reactivations were common with more than $50 \%$ of patients (17/30) developing CMV reactivations requiring pre-emptive antiviral systemic treatment. Invasive 
fungal infections were reported in 5 out of 30 patients. Severe infections occurring in the long-term follow up were caused by viruses (6/12 cases), bacteria (5/12) or fungi (1/12). Polyclonal T-cell reconstitution was fast: at day 30 , median CD3 cells were 366.5 per microL (range 35-2564), CD4 112 per microL (22-2137), CD8 197 per microL (5-1885). Higher T-cell counts, however, did not associate with a lower risk of CMV disease or infectious severe events. The frequency of CMV-specific T cells was evaluated by IFN- $\gamma$ ELISpot. After the normalization of ELISpot results for the CD3 content of samples analysed, median CMV sfc were 0.9 per microL (range 0.0-18.4) at day 30. When comparing clinical outcomes according to CMV immune recovery, patients who did not reached the previously described threshold of 1 CMV sfc per microL had higher rate of CMV disease compared to patients who reached the threshold $(27 \%$ vs $0 \%$; $P$ value $<0.05$ by the Log Rank test). Most importantly, the early recovery of protective CMV immunity strongly associated with a lower risk of subsequent severe infections for any cause $(P$ value $>0.05)$.

Conclusion: After haplo-HSCT with sir-PTCy GvHD prohpylaxis, the threshold for protective CMV-specific immunity of $1 \mathrm{CMV}$ sfc per microl allows to discriminate patients at higher risk for severe infectious events and can be potentially exploited to minimize overtreatment in the sir-PTCy setting. These results confirm the clinical utility of CMV-specific T-cell evaluation after haplo-HSCT and warrant the investigation of the threshold of 1 CMV sfc per microL as a surrogate biomarker for general immune competence in larger prospective trials.

Disclosure of Interest: None declared.

\section{P446}

ALFA/Beta T AND CD19+ lymphocite depleted hematopoietic stem cell transplantation from haploidentical donors in pediatric patients: a single center experience

M. Tumino 1,*, A. Marzollo ${ }^{1}$, M. V. Gazzola ${ }^{2}$, E. Calore ${ }^{1}$ C. Mainardi ${ }^{1}$, M. Pillon ${ }^{1}$, R. Destro ${ }^{3}$, A. Strano ${ }^{3}$, M. Barioni ${ }^{3}$, E. Carraro ${ }^{1}$, G. Basso ${ }^{7}$, C. Messina ${ }^{1}$

${ }^{1}$ Dept. SDB, PEDIATRIC HEMATO-ONCOLOGY UNIT, UNIVERSITY OF PADOVA, ${ }^{2}$ Dept. SDB, Stem Cell Transplantation Laboratory University of Padova, ${ }^{3}$ Dept. SDB, Stem Cell Transplantation Laboratory - University of Padova, Padova, Italy

Introduction: Transplantation of haploidentical hematopoietic stem cells (haploHSCT) has become an accepted option for pediatric patients with malignant and non malignant diseases (MD, NMD) who lack a matched related or unrelated donor. In order to prevent severe GvHD, many techniques of cells manipulation have been used. In this setting, immune recovery delay and high rate of rejection represent major concerns.

Material (or patients) and methods: We retrospective analyzed a cohort of 24 pediatric patients with MD and NMD, who received $\alpha \beta T$ - and B-cell depleted allografts from haploidentical family donors (haploHSCT a $\mathrm{\beta}$ T- and B-cell depleted). Myeloablative conditioning regimen and serotherapy with ATG were used for the most. Graft manipulation was carried out with anti-TCRa $\beta$ and anti-CD19 Abs and immunomagnetic microbeads. Gamma/delta T cells, NK, and other cells remained in the grafts. This procedure was compared with standard transplant procedures for each groups: matched sibling donor stem cell transplantation (MSD-SCT: 27 patients) and matched unrelated donor SCT (MUD-SCT: 58 patients).

Results: In haploHSCT a $\mathrm{\beta}$ T- and B-cell depleted SCT patients, primary engraftment was achieved in all patients, acute GvHD (aGvHD) grade III-IV and chronic GvHD (cGvHD) occurred in $25 \%$ and $12 \%$ respectively (MSD-SCT primary engraftment, aGvHD III-IV and CGvHD, respectively $100 \%, 15 \%$ and $5 \%$. MUD-SCT primary engraftment, aGvHD III-IV, and CGvHD respectively $100 \%, 48 \%$ and $16 \%$ ). No differences occurred between three groups in terms of OS, TRM and PFS as both as MD and NMD (MD P-value: OS 0.52, TRM 0.96, PFS 0.29. NMD $P$-value: OS 0.28 , TRM 0.29 , PFS 0.13 ). In the setting of MD, relapses represent the major cause of death, especially in the state of non complete remmission before transplant.
Conclusion: Compared with standard transplant procedures (MUD and MFD) we may consider haploHSCT a $\mathrm{B}$ - and B-cell depleted a valid alternative in pediatric MD and NMD. In MD newer cellular approaches are mandatory to controll relapses after transplantation. In this context, prompt availability of the donor such as in the haploidentical procedures represents an advantage.

Disclosure of Interest: None declared.

\section{P447}

Unmanipulated haploidentical (uHaplo) bone marrow (BM) and peripheral blood stem cells (PBSC) transplantation following myeloablative conditioning (MAC) and posttransplantation Cy (PT-CY) in high-risk hematologic malignancies. A single Centre experience

M. D. Simone ${ }^{1, *}$, A. Proia ${ }^{1}$, M. B. Pinazzi ${ }^{1}$, F. Spirito ${ }^{1}$, N. Natale ${ }^{2}$, C. Armentano ${ }^{1}$, A. Matteocci ${ }^{3}$, L. Pierelli ${ }^{3}$, L. G. Pacilli ${ }^{1}$, A. Severino ${ }^{1}$ Hematology and Stem Cell Transplantation, ${ }^{2}$ Pediatrics, ${ }^{3}$ Transfusion Medicine and Stem Cell Therapy, SAN CAMILLO HOSPITAL, ROME, Italy

Introduction: uHaplo HSCT, with PT-CY as GvHD prophylaxis, provided an opportunity of cure for nearly all patients. The Genoa group has shown, that a MAC with PT-CY (on day +3 and +5$)$, cyclosporine $A(C S A)$ from day 0 and mycophenolate mofetil (MMF) from day +1 , is higly effective on engraftment and remission, with acceptable non relapse mortality (NRM) and GvHD in havily pre-treated hematologic Malignancies (HMs).

Material (or patients) and methods: In our institution, 25 high-risk HMs received the same TBF MAC protocol: Thiotepa $(10 \mathrm{mg} / \mathrm{kg})$, iv Busulfan $(9,6 \mathrm{mg} / \mathrm{kg})$ and Fludarabine $(150 \mathrm{mg} /$ $\mathrm{mq})$. GvHD prophylaxis consisted of PT-Cy $(50 \mathrm{mg} / \mathrm{kg})$ on day +3 and +4 , CSA $2,5 \mathrm{mg} / \mathrm{kg} /$ day and MMF $45 \mathrm{mg} / \mathrm{kg} /$ day from day +5 , in order to respect $C y$-induced tolerance. In 17 patients stem cells source was BM, in 7 patients were G-CSF mobilized PBSC and in 1 case BM plus PBSC. 22 adults (13 male) with median age 47 years, (range 22-65) and 3 children ( 2 male and age 4, 7 e 7 years old); with high risk ALL ( 2 children; 5 adults), AML (1 child; 9 adults), 2 MDS, 5 Lymphomas and one Richter evolution of CLL; underwent uHaplo HSCT. At time of transplant 17 patients were in CR (8 above first CR), 1 in a good PR and 7 in refractory disease.

Results: Sustained donor engraftment occurred in 20 patients $(80 \%)$, with a median time to neutrophil recovery $\left(>0,5 \times 10^{9} /\right.$ $\mathrm{L})$ and platelet recovery $(>20 \times 1 \%$ L) of 19,5 and 25 days from HSC infusion. 5 patient was not evaluable for engraftment: 2 early deaths, 1 early relapse and 2 graft-failure (either with MDS). For patients receiving BM or PBSC grafts, the median time to neutrophils recovery was 21 (range 13-34) and 16,5 days (range 15-21), respectively, and platelets recovery of 25,5 (range 15-40) and 25 days (range 20-28), respectively. The incidence of grade II-IV aGVHD and CGvHD was $16 \%$ and $20 \%$, respectively. With a median follow-up of 24 months (range, 3-37), 13 patients are alive and disease free. The cumulative incidence of transplant-related mortality (TRM) and relapse was $12 \%$ and $28 \%$, respectively. The incidence of relapse was $11 \%$ for patients in remission and $71 \%$ for patients with active disease. Causes of death were: sepsis $(n=2)$, fatal aGvHD $(n=1)$, pneumonia $(n=2)$ and relapse $(n=7)$.

Conclusion: u-Hplo HSCT with both BM and PBSC with our current protocol is a feasible approach in pediatric and adult patients which promised high engraftment rates and acceptable NRM and rate of GvHD, associated with a durable remission in a proportion of patients with high-risk HMs.

Disclosure of Interest: None declared. 
P448

Haploidentical hematopoietic stem cell transplantation (hHSCT) in patients affected by Immunodeficiencies (ID): a single-centre experience

M. Comini ${ }^{1}$, F. Bolda ${ }^{1}$, A. Beghin ${ }^{1}$, S. Guarisco ${ }^{2}$, E. Soncini', F. Porta ${ }^{2, *}$, A. Lanfranchi ${ }^{1}$

${ }^{1}$ Stem Cell Laboratory, Section of Hematology and Blood Coagulation, ${ }^{2}$ Pediatric Onco-Haematology and BMT Unit, Children's Hospital, Spedali Civili of Brescia, Brescia, Italy

Introduction: Haploidentical hematopoietic stem cell transplantation (hHSCT) is a therapeutic approach for severe combined immunodeficiency (SCID), primary immunodeficiency diseases, inherited metabolic disorders and malignant and non-malignant hematological diseases in the absence of an HLA-matched donor.

Material (or patients) and methods: We retrospectively analyzed 74 patients (pts) with primary immunodeficiency diseases that underwent T cell-depleted hHSCT between 1991 and 2015 in our center: Children's Hospital of Brescia.

Median patient age at transplant was 15 months (range 2 months-16 years).

We treated 51 SCID, 8 Omenn's Syndrome, 3 Osteopetrosis, 2 WAS, 2 CID, 1 Hyper-lgE Syndrome, 2 LAD, 2 Lymphohistiocytosis, 2 MHC class II deficiency, 1 Osteogenesis Imperfecta. Results: Pts received conditioning, according to the European Group for Bone Marrow Transplantation-European Society for Immunodeficiencies Guidelines, excluding those pts with severe infections at HSCT.

Stem cell source for hHSCT were Bone Marrow (HPC-M) and Mobilized Peripheral Blood (HPC-A).

19 out of the 74 pts received a contemporary infusion of HPC$M$ and HPC-A, 9 pts received both HPC-M and HPC-A, but in two different steps; 39 pts infused only HPC-M and 7 pts only HPC-A.

7 pts underwent prenatal HSCT, without conditioning: 6 pts received positive selection of CD34+ cells from HPC-M and 1 from maternal HPC-A, with intraperitoneal injection at $22^{\text {Th }}$ of pregnancy.

43 pts underwent one HSCT, a second HSCT was performed in 27 pts and 4 pts underwent a third HSCT.

Different graft manipulation methods were performed: $\mathrm{T}$ cell depleted with Campath $1 \mathrm{M}(n=33), \mathrm{CD} 34+$ positive selection $(n=95), C D 3+/ C D 19+$ depletion $(n=2)$ and TCR $a / \beta+/ C D 19+$ depletion $(n=1)$.

The mean number of infused CD34+ cells was $15.4 \times 10^{6} / \mathrm{Kg}$, of infused $\mathrm{CD} 3+$ was $0.69 \times 10^{5} / \mathrm{Kg}$.

Median times to absolute neutrophil count $>0.5 \times 10^{9} / \mathrm{I}$ and to platelets $>50 \times 10^{9} /$ were 16.6 and 20 days respectively.

Chimerism analysis by STR-PCR on PMN and PBL, was achieved by day 22 and in prenatal HSCT the evaluation of donorchimerism was performed at birth both by flow cytometer analysis and STR-PCR assay in T-cell specific lineages.

5-years overall survival (OS) was: $48 \%$ in SCID (51 pts) and 57\% in the other ID (23 pts).

21 -years OS remained $48 \%$ in SCID and became $52 \%$ in the other ID.

In the 19 pts that received a co-infusion of CD34+ cells selected from HPC-M and HPC-A (mean number of infused cells $\left.=20,82 \times 10^{6} / \mathrm{Kg}\right)$, 5-years OS was $38 \%$ in SCID (13 pts) and $100 \%$ in the other ID (6 pts), with no differences at 18 years. Conclusion: Haplo-HSCT is a great alternative stem cell source thanks to the advances in transplantation techniques.

A 4 log T-depletion and an infusion of a high dose of hematopoietic progenitors are important to minimize GvHD and to ensure engraftment. TCR $\alpha / \beta+/ C D 19+$ depletion seems to give excellent results. This strategy permits the infusion of $a$ good number of CD34+, but also other lineages cells that are important for the outcome and engraftment of transplantation. References: 1- Kanakry CG et al. Nat Rev Clin Oncol. 2015 Aug 25.

2- Apperley J et al. Biol Blood Marrow Transplant. 2015 Nov 10. Disclosure of Interest: None declared.

\section{P449}

Haploidentical hematopoietic stem cell transplantation for acquired severe aplastic anemia

M. Miao ${ }^{1, *}$, X. Zhang ${ }^{1}$, T. Xu ${ }^{1}$, S. Jin ${ }^{1}$, H. Wang ${ }^{1}$, X. Yang ${ }^{1}$, Z. Pan ${ }^{1}$, J. He ${ }^{1}$, A. Sun ${ }^{1}$, D. Wu ${ }^{1}$

${ }^{1}$ The First Affiliated Hospital of Soochow University,Jiangsu Institute of Hematology, Suzhou, China

Introduction: To evaluate the efficiacy and safety of haploidentical hematopoietic stem cell transplantation (haplo-HSCT) in patients with acquired severe aplastic anemia (SAA), who lacked suitable related or unrelated HLAmatched donors

Material (or patients) and methods: 39 SAA patients underwent haplo-HSCT from Jul 2012 to Jun 2015 at our center. There were 23 males and 16 females at a median follow-up of 11 (range, from 0 to 36) months. The median time from diagnosis to transplantation was 1 (range, from 0.5 to 52 ) months. The median ages of SAA patients and related haploidentical donor were 23 years (range, 9 to 51years) and 45 (range, from 21 to 61) years, respectively. All patients were given BuCy plus ATG conditioning regimen. GVHD prophylaxis regimen consisted of cyclosporine $A(C s A)$, mycophenolate motetil (MMF), and short-term methotrexate.

Results: Stem cells were collected from bone marrow in $23.08 \%(n=9)$ of patients, peripheral blood in $2.56 \%(n=1)$, bone marrow plus peripheral blood in $74.36 \%(n=29)$ patients. 36 patients received haplo-HSCT combined with the third part of cord blood transfusion $92.31 \%$. The median stem cell dose transplanted was 9.76 (range, from 4.02 to 20.10 ) $\times 10^{8} / \mathrm{kg}$ for mononuclear cells, while 3.4 (range, from 1.05 to 8.60 ) $\times 10^{8} / \mathrm{kg}$ for CD34 cells. 36 patients achieved neutrophil engraftment at a median of 12 (range, from 9 to 28), and 29 patients achieved platelet engraftment at a median of 29 (range, from 10 to 26) days. Cumulative incidence of $\mathrm{II}^{\circ} \sim \mathrm{IV}^{\circ}$ acute graft versus host disease (aGVHD) was $8.9 \pm 4.9 \%$. 6 patients died of transplantrelated mortality (TRM), including 4 from severe infection, 1 from TMA and 1 from encephalorrhagia. The 2-year overal survival rate of all patients was $83.2 \% \pm 6.4 \%$.

Conclusion: Haplo-HSCT is likely to be an option for SAA patients without suitable related or unrelated HLA-matched donors, in consideration of the acceptable TRM and severe GVHD incidences.

Disclosure of Interest: None declared.

\section{P450}

Th9 cells reconstitution and its clinical significance after allogeneic hematopoietic stem cells transplantation N. Pang ${ }^{1}$, M. Jiang ${ }^{1, *}$ on behalf of 25, J. Qu ${ }^{1}$ on behalf of 25, X. Guo ${ }^{1}$, X. Duan ${ }^{1}$, H. Yuan ${ }^{1}$, J. Xu ${ }^{1}$

${ }^{1}$ Hematologic Disease Center, HEMATOLO GIC DISEASE CENTER THE FIRST AFFILIATED HOSPITAL OF XINJIANG MEDICAL UNIVERSITY, Urumqi, China

Introduction: Allogeneic hematopoietic stem cells transplantation (allo-HSCT) is the main treatment for high risk malignant hematological diseases. Th9 cell has recently been described as a new effector T cell subset, and play an important role in immune response. However, the role of Th9 cell in allo-HSCT has rarely been reported. The purpose of this study is to analyze the reconstitution of Th9 cells after HLA-matched and HLA-haploidentical peripheral blood hematopoietic stem cells transplantation (PBSCT) and explore the relationship between Th9 cells and acute graft-versus-host disease (aGVHD).

Material (or patients) and methods: 50 patients were enrolled in this study, 25 patients received related HLAhaploidentical PBSCT, the other 25 patients received related HLA-matched PBSCT, and 19 out of 50 evaluable patients developed aGVHD after transplantation. Flow cytometry and ELISA assay were used to analyze percentages of Th9 cells, levels of Interleukin-9 (IL-9), IL-4, interferon- $\gamma$ (IFN- $\gamma$ ) and transforming growth factor beta (TGF- $\beta$ ). 
Results: In the present study, we found that (1) Th9 cells (and IL-9) in HLA-matched group showed increasing from 60 days and reaching normal levels at 90 days, whereas in HLAhaploidentical group, Th9 cells increase from 90 days gradually and recover to healthy levels at 150 days after transplantation; (2) Compared to HLA-matched group, HLA-haploidentical group showed faster recovery of IFN- $\gamma$ and IL-4, and slower recovery of IL-9 and TGF- $\beta$; (3) IL-9 levels of patients without aGVHD are sigfnicantly higher than that of patients with aGVHD, and serum IL-9 level was negatively correlated with the severity of aGVHD in both groups.

Conclusion: In summary, our results indicate that peripherial blood Th9 cells and IL-9 levels recover slower in HLAhaploidentical PBSCT than in HLA-matched PBSCT. IL-9 is strongly correlated with aGVHD severity. Th9 cells maybe help induce immune tolerance after allo-HSCT.

Disclosure of Interest: None declared.

\section{P451}

TcR a $\beta$ (+) Depleted Haploidentical Hematopoietic Stem

Cell Transplantation in Children: The Single Center Experiences

M. Karakukcu, ${ }^{1, *}$ E. Ünal ${ }^{1}$, E. Yılmaz ${ }^{1}$, A. Özcan ${ }^{1}$, G. Uçan ${ }^{1}$, T. Patıroğlu', M. A. Özdemir ${ }^{1}$

${ }^{1}$ Pediatric Hematology/Oncology and BMT Unit, Erciyes University Faculty of Medicine, KAYSERI, Turkey

Introduction: Haploidentical hematopoietic stem cell transplantation (HSCT) provides an opportunity for nearly all patients without matched donor. Erciyes University is one of the reference pediatric centers for $T c R$ a $\beta(+)$ depleted haploidentical HSCT in Turkey. We would like to share our experience with a follow up period of three years.

Material (or patients) and methods: Between December 2012 to December 201535 haploidentical HSCT in 30 children (13 relapsed/refractory AML, 6 relapsed/refractory ALL, 4 severe aplastic anemia (SAA), 2 relapsed Familial $\mathrm{HLH}, 2$ Gricelli syndromes, $2 \mathrm{SCID}, 1 \mathrm{JMML}$ ) were included in the study. The regimen included ATG, Fludarabine, Thiotepa, Melphalan. Mycophenolate mofetil (MMF) was given as GvHD prophylaxis if the graft contained $>2.5 \times 104 / \mathrm{kg}$ TCR aß (+) lymphocytes counts.

Results: The median of transfused CD34 cells were 19,73 (range $3,99-43,66) \times 10^{6} / \mathrm{kg}$. The graft had a purity of $99.9 \%$. TCR a $\beta$ depletion with a median of 12.6 (range 0.1 to 137,2 ) $x$ $10^{3} / \mathrm{kg}$ TCRaß cells. The median engraftment days for myeloid and platelet were both 12th day of HSCT (range 9 to 28, 9 to 33 day) respectively. Grade II skin GvHD was detected in 5 patients, and treated with methyl prednisolon without complications. Moreover grade II-III gastrointestinal, liver GvHD were observed in 4, 2 children, respectively. Although the patients with gastrointestinal GvHD were treated with steroid, budenosid, cyclosporine, MSC; one patient did not respond and died. The mean day of discharge was 30th day of HSCT. MMF was given as GVHD prophylaxis in 16 patients and 14 patients did not receive any immune suppressive drug. Twelve of 20 patients with hematological malignancies were not in remission. Two patients were died within the first 6 months due to sepsis. Six of the patients died because of relapses; one of the each other three patient died due to acute gastrointestinal GvHD, graft failure and PTLD. Transplant related mortality in these patients was $16,6 \%$. Nineteen patients are currently alive, with a median follow up of 18 months (range 1 to 34 months). Overall survival was $63,3 \%$ in these group.

Conclusion: Our primary results underline that haploidentical HSCT with depletion of $\mathrm{TCR} \alpha \beta(+)$ can be an option in experienced center in countries which unrelated donor programs are not satisfactory, as in Turkey. The availability of a haploidentical donor in most families is a potential advantage. Moreover probably more potent graft-versustumor effect can be induced with haploidentical HSCT.

Disclosure of Interest: None declared.
P452

CMV REACTIVATION IN HAPLOIDENTICAL HEMATOPOIETIC STEM CELL TRANSPLANT: RETROSPECTIVE STUDY AND COMPARISON WITH A NON HAPLOIDENTICAL DONOR TRANSPLANT GROUP

N. Arratibel ${ }^{1, *}$, E. Perez-Lopez ${ }^{1}$, O. Lopez-Godino ${ }^{1}$, M. Cabrero ${ }^{1}$ M. Rojas ${ }^{2}$, S. Franco ${ }^{3}$, A. A. Martín ${ }^{1}$, L. Lopez-Corral ${ }^{1}$, S. Alonso ${ }^{\prime}$, A. Veiga ${ }^{1}$, M. Baile ${ }^{1}$, O. Ferre ${ }^{1}$, F. Lopez-Cadenas ${ }^{1}$, D. Caballero ${ }^{\prime}$, L. Vazquez ${ }^{7}$

${ }^{1}$ Hematology Department, Salamanca University Hospital, Salamanca, Spain, ${ }^{2}$ Hematology Department, Hospital General de Occidente, Guadalajara, Mexico, ${ }^{3}$ Hematology Department, Navarra University Clinic, Pamplona, Spain

Introduction: HLA-Haploidentical hematopoietic stem cell transplant (haploHSCT) is a potentially curative strategy for patients lacking a HLA matched donor or a suitable unrelated donor. However, infectious complications are still not explored enough, particularly in terms of CMV reactivations, with a high incidence reported in some studies, mainly in patients receiving marrow grafts; moreover no direct comparison with Non Haploidentical (NH) donors have been reported.

Material (or patients) and methods: Between August-2012 and August-2015, 213 patients received an HSCT in our centre. In this study we retrosvectively analyse CMV reactivation determined by PCR and response to pre-emptive therapy in the early post-HSCT period ( 6 months) in patients receiving an haplo ( $n=34,16 \%)$ comparing them with a control group of Non haplo HSCT (88 MRD and 91 MUD) $(n=179)$.

Results: Median age was 52 years (range 16-70), 59 for haploHSCT and 51 for control group. Reduced intensity conditioning (RIC) regimen had been used in $85 \%$ of haploHSCT and $65 \%$ of control group.

HaploHSCT characteristics: Conditioning regimen consisted of Fludarabine $(30 \mathrm{mg} / \mathrm{m} 2 \mathrm{x}$ or $50 \mathrm{mg} / \mathrm{kg} \times$ 4days in RIC or MA regimen) and Busulfan ( $3.2 \mathrm{mg} / \mathrm{kg} \times 2$ in RIC or 3 days in MA). Cyclophosphamide was used for GVHD prophylaxis in $94 \%$. Median of days to reach more than $500 \times 10^{9}$ granulocytes and more than $20 \times 10^{9}$ platelets were $18(13-30)$ and $23(0-103)$ respectively. Incidence of acute GVHD was $63,6 \%$ (grade I-II, $60,6 \%$, and III-IV 3\%), with no steroid-refractory cases.

CMV reactivation: $70 \%$ of haploHSCT patients presented CMV reactivation, versus $29,1 \%$ in control group $(P=0.000)$. Median number of CMV reactivation episodes was 1 (1-3) in haploHSCT vs $1(1-10)$ in the control group $(P=0.109)$. Median time to CMV PCR detection was 33 days (4-62) and 40 (0-186) in haploHSCT and control group respectively $(P=0.026)$. Average maximum CMV IU by PCR was 14250 in haplo vs 9525 in the control group $(P=0.619)$. First antiviral pre-emptive therapy (Valganciclovir in 63\%) was effective in $88 \%$ in haploHSCT vs $65 \%$ in control group $(P=0.034)$. Main reason for antiviral treatment switch was failure in CMV IU reduction, and Foscarnet was the most used therapy in refractory cases. Fifteen patients developed CMV disease ( 2 in haplo and 13 in control group). HaploHSCT, serological CMV status (positive patient/negative donor), MA conditioning regimen and development of acute GVHD were variables associated with a higher CMV reactivation in the univariate analysis. All of them kept it significant impact in a multivariate model (Table. 1) 
Table 1 Risk factors related to CMV reactivation. Uni and Multivariant analyse.

\begin{tabular}{|c|c|c|c|}
\hline & $\begin{array}{c}\text { CMV } \\
\text { reactiva- } \\
\text { ción }\end{array}$ & $P\left(x^{2}\right)$ & OR (IC 95\%); $\mathrm{P}$ \\
\hline $\begin{array}{l}\text { Haplo } \\
\text { Control } \\
\text { group }\end{array}$ & $\begin{array}{l}70,6 \% \\
29,1 \%\end{array}$ & 0.000 & $19.4(6.66-56.91) 0.000$ \\
\hline \multicolumn{4}{|c|}{ GVHD acute } \\
\hline $\begin{array}{l}\text { No } \\
\text { GVHD }\end{array}$ & $18,9 \%$ & 0,00 & $3.69(1.35-10.11) 0.011$ \\
\hline $\begin{array}{l}\text { I-II } \\
\text { III-IV }\end{array}$ & $\begin{array}{l}34,7 \% \\
65,8 \%\end{array}$ & 0 & $18.29(5.47-61.10) 0.000$ \\
\hline \multicolumn{4}{|c|}{ CorticoR GVHDa } \\
\hline $\begin{array}{l}\text { Yes } \\
\text { No }\end{array}$ & $\begin{array}{l}69 \% \\
37 \%\end{array}$ & 0.002 & \\
\hline \multicolumn{4}{|l|}{$C M V R / D$} \\
\hline $\begin{array}{l}-/- \\
-/+ \\
+/- \\
+/+\end{array}$ & $\begin{array}{c}5,6 \% \\
23,8 \% \\
51,5 \% \\
33,3 \%\end{array}$ & 0,001 & $\begin{array}{l}0.082(0.009-0.73) 0.025 \\
2.27(1.05-4.88) 0.036\end{array}$ \\
\hline \multicolumn{4}{|c|}{ Conditioning } \\
\hline $\begin{array}{l}\text { AMA } \\
\text { AIR }\end{array}$ & $\begin{array}{l}44,1 \% \\
31,7 \%\end{array}$ & 0,055 & $2.24(1.05-4.77) 0.037$ \\
\hline
\end{tabular}

Conclusion: Based on these results haploHSCT is associated with a higher CMV reactivation compared to non-haplo HSCT, despite a lower incidence of all other risk factors as aGVHD or MA conditioning in the haplo group. However, response to pre-emptive therapy is comparable and no higher incidence of CMV disease was observed.

Disclosure of Interest: None declared.

\section{P453}

Unmanipulated haploidentical hematopoietic stem cell transplantation in children with post-transplant cyclophosphamide in advanced acute leukemia. Single center experience

O. V. Paina ${ }^{1, *}$, A. Borovkova ${ }^{1}$, N. Stancheva ${ }^{1}$, P. Kozhokar ${ }^{1}$, S. Razumova , K. Ekushov', I. Moiseev ${ }^{1}$, O. Pirogova', E. Babenko ${ }^{1}$, O. Slesarchuk ${ }^{1}$, L. Zubarovskaya ${ }^{1}$, B. Afanasyev ${ }^{7}$

${ }^{1}$ R.M.Memorial Institute of children Oncology, Hematology and Transplantation, I.P.Pavlov 1st St.Petersburg State Medical University, St.Petersburg, Russian Federation

Introduction: For patient with advanced hematological malignancies or high risk of relapse acute leukemia (AL) a suitable alternative donor source is the HLA-haploidentical family donor who is available for most patients. Haploidentical hematopoietic stem cell transplantation (Haplo-HSCT) has been associated with excessive graft failure, graft-versus-host disease (GVHD) and relapse rate. Post-transplant cyclophosphamide (PTCY) have been successfully performed to eliminate T-cells in vivo. The strategy has been used in many transplant centers in adult patients but its feasibility is unknown in children. We retrospectively compared the outcome of unmanipulated Haplo-HSCT with PTCY and ATGbased T-cell depletion in children with advanced acute leukemia. Purpose: to compare the feasibility and outcome of Haplo-HSCT with PTCY vs conventional prophylaxis of GVHD in children with advanced acute leukemia. Primary end points: acute GVHD (aGVHD), chronic GVHD (cGVHD).

Secondary end points: engraftment rate, relapse rate, nonrelapse mortality (NRM), overall survival (OS).
Material (or patients) and methods: 56 children (range from 0-19 y.o. median 7 y.o.) with AL (ALL-34pts, AML-22pts) in high high risk of relapse (HR) or advanced disease (AD) (1-2 hematological remission 10pts, relapse or resistant disease 46 and $14 \mathrm{pts}$ had cytoreduction chemotherapy prior conditioning regimen) were eligible to undergo haplo-HSCT with PTCY. The control group (conventional prophylaxis of GVHD) included 39 pts with AL (ALL-25pts, AML-14, 1-2 hematological remission $8 \mathrm{pts}$, relapse or resistant disease $31 \mathrm{pts}$ and $11 \mathrm{pts}$ had cytoreduction chemotherapy prior conditioning regimen). The MAC+ ATG based on GIAC protocol was performed in $21 \mathrm{pts}$, MAC+PTCy $50 \mathrm{mg} / \mathrm{kg}$ on D+3,+4 in $20 \mathrm{pts}, \mathrm{RIC}+$ ATG regimen based on Flu-Mel in $18 \mathrm{pts}, \mathrm{RIC}+\mathrm{PTCy} 50 \mathrm{mg} / \mathrm{kg}$ on $\mathrm{D}+3,+4$ in 36pts. All pts PTCy-group received prophylaxis of aGVHD based on calcineurin inhibitors (CNI) with mycophenolate mofetil (MMF)-18 or sirolimus-38. G-CSF-primed T-cell replete BM was used as a graft source in 53pts (median CD34 cells $11 \times 10^{6} / \mathrm{kg}$ ), G-CSF mobilized peripheral blood (positively immunoselected $\mathrm{CD}_{3} 4^{+}$by CliniMACS, Miltenyi Biotec) and G-CSF-primed BM-3pts (median $\mathrm{CD}_{3} 4^{+}$cells $7 \times 10^{6} / \mathrm{kg}$ ). The control group received G-CSF-primed T-cell replete BM- 20pts (median CD34 ${ }^{+}$cells $7 \times 10^{6} / \mathrm{kg}$ ), combined transplant-19pts (median $\mathrm{CD}_{3} 4^{+}$cells $6 \times 10^{6} / \mathrm{kg}$ ). All pts in control group received $\mathrm{CNI}$ with $\mathrm{MMF}$ and methotrexate. The median follow-up of surviving patients was 10 months. Statistical analyses were performed using SPSS V19.

Results: $67,9 \%$ of patients achieved engraftment in PTCYgroup vs $87,2 \%$ control-group $(P=0,08)$. Cumulative incidences of aGVHD grate I-II $(31,6 \%$ vs $53 \%, P<0,000)$, grade III-IV $(15,8 \%$ vs $32,4 \%, P=0,009)$ and CGVHD $(21 \%$ vs $35,3 \%$ $P=0,009)$ were significantly lower in PTCY-group compared to control-group. Cumulative incidence of relapse $(42,1 \%$ vs $52,9 \% P=0,5)$, NRM $(18,4 \%$ vs $20,6 \%)$ was not statistical significant. 1y-OS was the same in both group (PTCy-group$50 \%$ vs $51,3 \%$ in control-group $P=0,4$ ).

Conclusion: Unmanipulated Haplo-HSCT in children with PTCY in advanced acute leukemia is a safe and feasibility option. PTCY in combination with $\mathrm{CNI}$ and MMF or sirolimus had acceptable incidence of aGVHD and CGVHD. Future studies are needed to increase OS and reduce relapse rate in post-transplant period. Disclosure of Interest: None declared.

\section{P454}

T-Cell Replete Haploidentical PBSC Transplantation Using Intensified Myeloablative Conditioning, Post-Transplant Cyclophosphamide and Sirolimus

R. Greco ${ }^{1, *}$, S. Piemontese ${ }^{1}$, N. Cieri ${ }^{2}$, F. Giglio ${ }^{1}$, M. Morelli ${ }^{1}$ C. Messina ${ }^{1}$, M. Marcatti ${ }^{1}$, S. Marktel ${ }^{1}$, M. Carrabba ${ }^{\prime}$, M. Bernardi ${ }^{1}$, C. Corti ${ }^{1}$, A. Assanelli ${ }^{1}$, M. T. Lupo Stanghellini ${ }^{1}$ C. Bonini ${ }^{2}$, L. Vago ${ }^{1,3}$, F. Ciceri ${ }^{1}$, J. Peccatori ${ }^{1}$

${ }^{1}$ Unit of Hematology and Bone Marrow Transplantation, ${ }^{2}$ Experimental Hematology Unit, ${ }^{3}$ Unit of Immunogenetics, Leukemia Genomics and Immunobiology, IRCCS San Raffaele Scientific Institute, Milano, Italy

Introduction: The use of haploidentical hematopoietic stem cell transplantation (HSCT) with post- transplant cyclophosphamide (PT-Cy) and calcineurin inhibitors (CNI) as graft-versus-host disease (GVHD) prophylaxis is rapidly increasing.

Herein we describe long-term outcomes of haploidentical HSCT from PBSC grafts, using intensified myeloablative conditioning, and GVHD prophylaxis with PT-Cy and sirolimus. Material (or patients) and methods: In our center we collected 77 adult patients (pts) receiving haploidentical HSCT for high-risk hematological malignancies, mainly acute myeloid leukemia $(n=44)$. Median age was 52 years (range 21-77). At HSCT, $66 \%$ of patients were not in complete remission. According to the Disease Risk Index (DRI) the pts were stratified in very high $(n=18)$, high $(n=32)$, intermediate $(n=24)$, or low $(n=1)$ risk. Graft source was mainly PBSCs $(97 \%)$.

All pts received a myeloablative conditioning regimen. The majority of pts $(77 \%)$ received treosulfan $(14 \mathrm{~g} / \mathrm{m} 2 /$ day $)$ on days -6 to -4 , fludarabine $(30 \mathrm{mg} / \mathrm{m} 2 /$ day) on days -6 to -2 , and 
melphalan $(70 \mathrm{mg} / \mathrm{m} 2 /$ day $)$ on days -2 and -1 . All pts received PT-Cy $(50 \mathrm{mg} / \mathrm{kg} /$ day) on days 3 and 4 . Sirolimus was given from day 5, and withdrawn 3 months after HSCT. Mycophenolate mofetil (MMF) was initiated on day 5, and withdrawn on day 30.

Results: Median follow up was over 13 months (range 2-32) All the recipients of haploidentical HSCT, except one who failed to engraft, experienced a sustained donor cell engraftment. The median time to a neutrophil count of $\geq 500 / \mu \mathrm{L}$ was 18 days. Post-HSCT recovery of lymphocyte subsets was broad and fast, with a median time to CD3 $>100 / \mathrm{ml}$ of 35 days; at 1 month after HSCT we observed a median of $170 \mathrm{CD} 3+/ \mathrm{ml}, 72$ CD4+/ml and $100 \mathrm{CD} 8+/ \mathrm{ml} \mathrm{T}$ cells. CMV reactivation occurred in $62 \%$ pts who were at high risk (recipient seropositive).

The cumulative incidence of grades II-IV and III-IV acute GVHD at 100 days was $36 \%$ and $21 \%$, respectively. The cumulative incidence of chronic GVHD was $21 \%$ at 1 year and $35 \%$ at 2 year; we observed severe chronic GVHD only in 8 pts.

The cumulative incidences of relapse and non-relapse mortality (NRM) were $39 \%$ and $26 \%$ at 2 years, respectively; NRM was higher in pts affected by acute leukemia $(P=0.02)$. Two-year overall survival (OS) was $37 \%$ and disease free survival (DFS) $35 \%$. There was a significant trend towards a longer OS and DFS in patients with complete remission status at HSCT: 2-year OS was $67 \%(P=0.008)$ and 2-year DFS was $57 \%(P=0.026)$. We did not found an effect of Sorror-comorbidity index on survival. Thirty nine pts have died. The causes of death were relapse $(n=23)$, infection $(n=12)$, GVHD $(n=4)$.

The composite end point of GVHD-free/relapse-free survival (GRFS) was $40 \%$ at 1 year and $18 \%$ at 2 year, in which events include grade 3-4 acute GVHD, systemic therapy-requiring chronic GVHD, relapse, or death.

Conclusion: These outcomes suggest that haploidentical HSCT from PBSCs grafts using double alkylating chemotherapy, PT-Cy and sirolimus, is associated with acceptable NRM and severe GVHD, providing relevant survival and effective clinical immune reconstitution in high-risk diseases.

Disclosure of Interest: None declared.

\section{P455}

Sequential chemotherapy combining Thiotepa, Etoposide and Cyclophosphamide followed by reduced-intensity conditioning in T-cell replete HLA-haploidentical hematopoietic stem cell transplantation for the treatment of refractory hematological malignancies

R. Dulery ${ }^{1,2,3, *}$, A.-L. Ménard ${ }^{4}$, F. Giannotti ${ }^{1}$, A. Ruggeri ${ }^{1}$ E. Brissot ${ }^{1,2,3}$, G. Battipaglia ${ }^{1}$, R. Belhocine ${ }^{1}$, S. Eder ${ }^{1}$, F. Isnard ${ }^{1}$, A. Le Bourgeois', A. M. Rosu', N. Santoro', A.-C. Mamez ${ }^{1}$ S. Lapusan ${ }^{1}$, A. Vekhoff', O. Legrand ${ }^{1,2}$, M.-T. Rubio ${ }^{1}$, M. Mohty ${ }^{1,2,3}$ ${ }^{1}$ Department of Hematology and Cellular Therapy, Saint Antoine Hospital, AP-HP, ${ }^{2}$ Université Pierre \& Marie Curie, ${ }^{3}$ UMRs 938, INSERM, Paris, ${ }^{4}$ Department of Hematology, Henri Becquerel Center, Rouen, France

Introduction: The results of conventional allo-SCT in refractory hematologic malignancies are poor. The sequential FLAMSA strategy has shown promising results in refractory AML. However, sequential strategies have not been validated in a haploidentical (haplo) setting. We developed a new sequentia approach combining chemotherapy with broad anti-tumor activity, followed by reduced-intensity conditioning (RIC) regimen for the treatment of wide spectrum refractory hematologic malignancies.

Material (or patients) and methods: Fifteen patients with refractory hematologic malignancies received an haplo-SCT and were compared to 17 patients with unrelated donor (MUD) who received the same regimen. TEC-RIC regimen consisted in total dose Thiotepa of 5 to $10 \mathrm{mg} / \mathrm{Kg}$, Etoposide $400 \mathrm{mg} / \mathrm{m}^{2}$, Cyclophosphamide $1600 \mathrm{mg} / \mathrm{m}^{2}$ (D-15 to -10), and after a 3 days rest, Fludarabine $150 \mathrm{mg} / \mathrm{m}^{2}$, iv Busulfan $6.4 \mathrm{mg} /$ $\mathrm{kg}$ and Thymoglobuline $5 \mathrm{mg} / \mathrm{Kg}$ (D-6 to -2). GVHD prophylaxis consisted in Cyclosporine and Mycophenolate Mofetil. High dose post-transplant Cyclophosphamide was added in case of haplo. Prophylactic infusion of donor lymphocytes ( $p D L I)$ was scheduled by D+120 after withdrawal of immunosuppression, in the absence of GVHD.

Results: Median age was 44 years (range 17-72). All patients had refractory hematologic diseases: $17 \mathrm{AML}, 5 \mathrm{ALL}, 2 \mathrm{MDS}, 3$ CMML, 2 MPN and 3 lymphomas. Fifteen leukemic patients had persistent excess marrow blasts at transplant, 3 refractory MRD/ cytogenetic and 2 persistent pancytopenia. They had a median of 2 prior treatment lines (range 1-4), including 3 autologous and 4 allo-SCT. MUD were HLA $10 / 10(n=12)$ or $9 / 10(n=5)$. Graft source was peripheral blood stem cells in $91 \%$ of patients. Median follow-up was 13 months (2-32). Median time for neutrophil engraftment was 18 days (13-25) in haplo patients and 14 days $(11-24)$ in MUD patients $(P=0.016)$. Toxicities of conditioning in the haplo setting were all reversible and included $60 \%$ of mucositis (median grade $=1$ ) and 1 case of VOD. No significant differences in terms of toxicities or viral reactivations were observed between haplo and MUD patients. At $D+30$, all patients reached complete remission. Cumulative incidence of acute GVHD at D+100 was significantly lower in haplo (grade II-IV: $27 \%$ and grade III-IV: 7\%) than in MUD (grade IIIV: $53 \% ; P=0.046$ and grade III-IV: $35 \% ; P=0.03$ ). Chronic GVHD was observed in $6 / 13$ evaluable patients in haplo compared to $6 / 12$ in MUD. Non-relapse mortality (NRM) was $13 \%$ at $\mathrm{D}+100$ and $20 \%$ at 1 year in haplo (24\% and $29 \%$ in MUD, respectively; $P=\mathrm{NS}$ ). Cumulative incidence of relapse was $20 \%$ at 1 year in haplo and $24 \%$ in MUD. Five patients received pDLI after haplo and 4 of them were alive in complete remission at last follow-up. At 1 year, estimated event-free survival was $60 \%$ and $47 \%$ and overall survival was $73 \%$ and $47 \%$ in haplo and MUD patients, respectively $(P=N S)$.

Conclusion: TEC-RIC sequential conditioning regimen prior to haplo-SCT is relatively safe and even compares favorably to MUD. It is highly effective and therefore could be proposed to any type of refractory and high-risk hematologic malignancy. A prospective multicenter study based on this new sequential approach including post-transplant immuno-intervention is currently being scheduled.

Disclosure of Interest: None declared.

\section{P456}

HLA-haploidentical transplantation using T-cell-replete grafts and high-dose cyclophosphamide posttransplantation in the treatment of multiple myeloma: a single center experience

S. Häbe ${ }^{1, *}$, G. Totok', D. Prevalsek ${ }^{1}$, S. Fritsch ${ }^{1}$, M. Hubmann ${ }^{1}$, A.-K. Zöllner ${ }^{1}$, T. Köhnke ${ }^{1}$, C. Schulz ${ }^{1}$, G. Ledderose', A. Hausmann ${ }^{2}$, W. Hiddemann ${ }^{7}$, J. Tischer ${ }^{7}$

${ }^{1}$ Department of Internal Medicine III, Ludwig-Maximilians-University Hospital of Munich-Grosshadern, ${ }^{2}$ Department of Internal Medicine I, Klinikum München-Schwabing, Munich, Germany

Introduction: Hematopoietic stem cell transplantation might be a valuable treatment option for patients suffering from high-risk and relapsed multiple myeloma (MMY) while providing long term control in selected patients. However, not in all of our patients a suitable HLA-matched donor could be identified in time. Successful treatment of HLA-haploidentical transplantation (haplo-HSCT) using T-cell-replete grafts (TCR) and high-dose cyclophosphamide post-transplantation (PTCY) was observed in the treatment of lymphoma (Burroughs LM, Biol BMT 2008), but there is little data on the outcome of this setting in the treatment of MMY.

Material (or patients) and methods: We retrospectively evaluated the outcome of haplo-HSCT using T-cell-replete grafts and PTCY in four patients with MMY treated between 2013 and 2015 at our center (male $n=3$; median age: 54 years). At first diagnosis poor cytogenetic features were documented in three patients $(t(4 ; 14)$, del $(13 q)$, del $(17 p))$. All Patients had relapsed after autologous transplantation (tandem $n=3$ ) within 21 (range 3-41) months, and had received multiple prior therapies including IMIDs, bortezomib and cyclophosphamide. At time of haplo-HSCT three patients presented in very good partial response while one patient showed PR. All related donors (male 
$n=2)$ were fully HLA-haplotype mismatched (5/10 HLA-loci). Reduced-intensity conditioning consisted of fludarabine, cyclophosphamide and melphalan $(140 \mathrm{mg} / \mathrm{m} 2)$. Post-grafting immunosuppression was tacrolimus and mycophenolate mofetil (both started on day +5$)$, and stimulated peripheral blood stem cells were the graft source.

Results: No graft rejection occurred; neutrophil/platelet engraftment was achieved in all patients at a median of 18 (range: 16-20) and 20 (range: 13-26) days, respectively. Acute GvHD grade III (skin $n=4$; skin and gut $n=2$ ) was observed in all patients, and moderate chronic GvHD in one. Treatmentrelated grade III-IV toxicities occurred in three patients, while there was a low rate of infections (CMV reactivation: 1/2 patients at risk; no PTLD; no invasive fungal infection). One patient developed VOD resolving completely under treatment with defibrotide; no patient died transplant related.

At day +30 full donor chimerism was achieved in all patients, and two patients showed sCR while another one achieved sCR at day +100 . However, one patient remained immune-fixation positive. No patient received maintenance therapy. Up to date (follow up: 29 and 8 months) two patients are still in SCR, and one patient had relapsed (immune-fixation positive) at seven months after transplantation. Despite of promptly initiated immunotherapy (lenalidomide and DLI), this patient presented with extramedullary disease at 15 months after transplantation.

Conclusion: Sequential therapy in the setting of TCR haploHSCT using PTCY is feasible and well tolerated and might provide anti-myeloma activity in high-risk patients with multiple prior treatments.

Disclosure of Interest: None declared.

P457

Haploidentical Stem Cell Transplantation with TCRaß and CD19 Depletion in Children and Young adults with NonMalignant Disorders

S. P. Yadav ${ }^{1, *}$, Y. R. Chopra ${ }^{1}$, S. Nivargi ${ }^{1}$, N. Rastogi ${ }^{1}$, S. Kohli ${ }^{1}$, S. Katewa'

${ }^{1}$ Pediatric Hematology Oncology \& Bone Marrow Transplant Unit, Fortis Memorial Research Institute, Gurgaon, India

Introduction: T-cell depleted haploidentical related donor stem cell transplant (SCT) is widely used to treat many disorders. T-cell receptor alpha beta (TCRaß) and CD19 depletion has been used to reduce the risk of posttransplant complications and enhance immune reconstitution. We report here our experience with TCRaß/CD19 depletion in children and young adults with non-malignant disorders.

Material (or patients) and methods: Three children with Fanconi Anemia-1, Wiskott-Aldrich Syndrome (WAS)-1 and Thalassemia Major-1 and one young adult with Thalasemia Major were transplanted from haploidentical related donors after TCRaß+/CD19+ graft depletion. First child, a 5-yr-oldmale, with Fanconi anemia received conditioning Rabbit AntiThymoglobulin (ATG), Fludarabine, Cyclophophaamide (FluCy) and $T B \mathrm{BI}$ and had primary rejection. He underwent second transplant from same donor (mother) with Campath, FluCy and engrafted. Second child, a 5-month-old-male, with WAS received Campath, Fludarabine, Treosulfan and Thiotepa (FluTreoThio) and engrafted. Third patient, a 22-year-old-male, with thalassemia underwent SCT from his mother with conditioning ATG, FluCy, thiotepa and TBI and had rejection. He underwent three months later a second SCT from his father with conditioning Campath FluTreoThio and engrafted. Fourth patient, a 10-yr-old-male with thalassemia had initially undergone a T-cell replete haploidentical SCT from his mother with ATG FluCy, Thiotepa and TBI followed by post transplant cyclophosphamide but had primary rejection. He underwent a second SCT with TCRaß/CD19 depletion from his father as donor with conditioning Campath FluTreoThio and engrafted. Results: Four patients underwent 6 haploidentical TCRa $\beta$ +/CD19+ depleted SCT. The median numbers of CD34+ and TCRa + cells in the graft were $13 \times 106 / \mathrm{kg}$ and $12.5 \times 103 / \mathrm{kg}$, respectively. Acute graft-versus-host disease (GVHD) was observed in 3 out of 6 SCT all had grade II acute GVHD and responded to first-line therapy and no chronic GVHD was seen. Primary non-engraftment was observed in 2 patients. Both these patients were successfully re-transplanted with different conditioning. Preliminary data on immune reconstitution were not encouraging. All had non-significant numbers of T-lymphocytes detected on day +30 and on day +100 . Cytokine storm was seen in 4 out of 6 SCT. The child with WAS developed transplant-associated thrombotic microangiopathy needing mechanical ventilation and dialysis. He recovered fully with Rituximab, defibrotide and plasmapheresis. First three cases had CMV reactivation which was treated. Third case developed JC virus encephalitis 10-months post his second SCT which was managed successfully with tapering immuno-suppression and combination of intravenous cidofovir, weekly mefloquine and resperidone. First three cases are alive and disease free. Fourth case developed BK virus induced hemorrhagic cystitis and encephalitis and died on day+98 post his second SCT. With a median follow-up period of 13 months (range 3-18 months), the cumulative probabilities of overall patient survival and transplant-related mortality were $75 \%$ and $25 \%$, respectively.

Conclusion: TCRaß+-depleted HSCT is a feasible option for children with non-malignant disorders without a matched related donor. This procedure is associated with high rejection rates and high infection incidence needing greater supportive care.

Disclosure of Interest: None declared.

\section{P458}

Pediatric Haploidentical Stem Cell Transplantation with Post-Transplantation Cyclophosphamide for NonMalignant Disorders: A Feasible Option

S. P. Yadav ${ }^{1, *}$, D. Thakkar ${ }^{1}$, N. Rastogi ${ }^{1}$, S. Kohli ${ }^{1}$, S. Nivargi ${ }^{1}$, Y. R. Chopra', S. Katewa ${ }^{1}$

${ }^{1}$ Pediatric Hematology Oncology \& Bone Marrow Transplant Unit, Fortis Memorial Research Institute, Gurgaon, India

Introduction: Due to unavailability of matched related donor many children needing transplant are unable to avail this treatment as unrelated donor are either not available or too expensive for most children in the developing countries like India. T-cell replete haploidentical stem cell transplantation (SCT) with post-transplantation cyclophosphamide (PTCy) has shown encouraging results for the treatment of hematologic malignancies. However this technique has rarely been applied to treat non-malignant disorders. Here we present our experience using same in 19 children with various nonmalignant disorders.

Material (or patients) and methods: Donors were mobilized with Granulocyte colony stimulating factor 10 microgram $/ \mathrm{kg}$ daily for four days, the PBSC were collected with one large volume apheresis procedure. The conditioning was with Fludarabine, Cyclophosphamide, Thioptepa and total body irradiation in all 16 children, Fludarabine and Treosulfan in 2 (age less than 1) and Fludarabine and Busulfan in 1.Serotherapy was part of conditioning, Rabbit Anti-thymoglobulin $4.5 \mathrm{mg} / \mathrm{kg}$ in 16 and Campath $1 \mathrm{mg} / \mathrm{kg}$ in 3 . All received post transplant cyclophosphamide (PT-Cy) $50 \mathrm{mg} / \mathrm{kg}$ on day 3 and 4 as graft vs. host disease (GVHD) prophylaxis along with tacrolimus and mycophenolate mofetil. After a signed informed consent, 19 patients who needed a transplant, were allografted; median age was 3 years (range 1-18), 18 were boys and $1 \mathrm{girl}$, the diagnosis were: Primary immunodeficiency- 6 , thalassemia major-7, sickle cell disease-3, pure red aplasia-2, acquired aplastic anemia-1.

Results: All the donors shared 5 out of 10 alleles with the recipient; in $42 \%$ of the cases the donor was the Mother, in $32 \%$ the Father and in other $26 \%$ one sibling. A median of 10 million of CD34cells $/ \mathrm{kg}$ was infused (range 5-24 million/ $/ \mathrm{kg}$ ). Three children died before engraftment ( 2 due to multi-drug resistant bacterial sepsis and one due to stroke).The engraftment rate was $84 \%$, median time to achieve 500 neutrophil or more was 15 days (range 13-21) and a self- sustained platelet count of 20.000 or more was 12 days (range $9-20$ ). Chimerism 
at day+100 was available in 16 cases; 14 of them had full donor hematopoiesis. One had mixed chimerism and 1 fully recipient and both had thalassemia. One with mixed chimerism had rejection despite giving one dose of donor lymphocyte infusion $(2 \times 104 / \mathrm{kg})$. Both of them underwent second PBSCT from a different haploidentical donor (one TCR alpha-beta depleted and other $\mathrm{T}$ cell replete) and both engrafted but one died of Veno-occlusive-disease (VOD) and other with BK virus encephalitis.

The median follow-up of remaining patients is 13 months (range 3-30), the cumulative incidence of graft versus host disease (GVHD) acute and chronic extensive was $26 \%$ and $16 \%$ respectively. Grade-III acute GVHD was seen in 2 patients. Three patients have died, the causes were; Post-transplant lympho-proliferative disoder-1,Veno-occlusive disease- 1 and Myxedema -1 . Overall 58\% children are alive and diseases free at last follow up.

Conclusion: The use of T-cell replete haploidentical SCT with PTCy and a reduced intensity conditioning for treating pediatric non-maligant disorder is feasible and a good alternative for children with non-malignant disorders and without suitable matched donors.

Disclosure of Interest: None declared.

\section{P459}

CD3+/19+ depleted haploidentical stem cell transplantation for advanced stage sickle cell disease

J. Foell ${ }^{1}$, B. Pfirstinger ${ }^{1}$, D. Wolff', E. Holler ${ }^{2}$, S. Corbacioglu ${ }^{1, *}$ ${ }^{1}$ Pediatric Hematology, Oncology and Stem Cell Transplantation, University Childrens Hospital REGENSBURG, ${ }^{2}$ Internal Medicine III, University Hospital REGENSBURG, Regensurg, Germany

Introduction: Sickle cell disease (SCD) is an inherited disorder with an estimated incidence of over 350.000 affected newborns per year worldwide. Despite significant improvements in the supportive management of SCD-related complications, the disease cause substantial morbidity and mortality with reduced life expectancy. Allogeneic haematopoetic stem cell transplantation (SCT) is currently the only curative therapy for SCD and only offered if a matched sibling donor (MSD) or an unrelated donor (MUD) is available. Since transplant related mortality is an inacceptable outcome in hemoglobinopathies graft-versus-host-disease (GvHD) is the worst transplant related morbidity (TRM). With these priorities CD3/CD19 depleted SCT (T-haplo-SCT) is the prioritized therapeutic option due to the lowest incidence of GVHD including MSD SCT.

Material (or patients) and methods: We report of 9 patients with severe SCD, median age $17 \mathrm{yrs}, 5$ females and 4 males. 7 with acc. TCD $>200 \mathrm{~cm} / \mathrm{s}, 3$ with cerebral infarcts, 6 with acute chest crisis $>2,7$ with multifocal bone infarcts and 1 with complete alloimmunization. All were transplanted with CD3/CD19 depleted SCT. The conditioning regimen consisted of thiotepa (TT)/ fludarabin (FLU)/melphalan (Mel) and campath in one and treosulfan (Treo)/TT/Flu and ATG in the following eight patients. Immunosuppression was carried out with CSA and MMF.

Results: Final engraftment was achieved in all patients with stable chimerisms beyond $90 \%$, established with prolonged immunosuppression. Decreasing mixed chimerisms could be rescued via increasing immunosuppression in 2 patients. The first child transplanted with Flu/TT/Mel/ Campath experienced a fulminant graft rejection of stem cells from his mother, and underwent a successful second T-haplo-SCT within weeks with stem cells from his father and a conditioning regimen using Flu/cyclophosphamide and TBI (2 Gy). In all 9 patients the conditioning regimens were well tolerated. No high grade transplant related toxicity and in particular no VOD or clinical viral complications were observed. SCD-related adverse events like bone pain secondary to engraftment were the primary adverse event, neurological complications like headache and paresthesias and in one patient a neurological deficit occurred after haematological engraftment in a few patients. All were transient. In five patients Glucksberg Grade I aGvHD (skin only) was observed and the oldest patient (31 yrs) presented a moderate steroid responsive chronic GvHD (dry mouth, mild fasciitis and eyes).

Conclusion: In our experience T-SCT with ex vivo CD3+/CD19 + depleted peripheral stem cells is a safe procedure even for patients with severe SCD. T-haplo-SCT is therefore an option for cure for a wide range of SCD-patients with no MSD and advantages concerning the incidence of aGvHD. Increasing evidence of the safety of T-SCT in SCD warrants earlier curative intervention reducing even further TRM.

Disclosure of Interest: None declared.

\section{P460}

Improved Outcome of Refractory or Relapsed Acute Leukemia in Children with T-cell-Rich Haploidentical Stem Cell Transplantation

S. Kobayashi ${ }^{1, *}$, H. Sano ${ }^{1}$, K. Mochizuki ${ }^{1}$, M. Akaihata ${ }^{1}$, Y. Ohara ${ }^{1}$, N. Takahashi ${ }^{1}$, H. Ohto', A. Kikuta ${ }^{1}$

${ }^{1}$ Department of Pediatric Oncology, ${ }^{2}$ Department of Blood Transfusion and Transplantation Immunology, Fukushima Medical University, Fukushima, Japan

Introduction: T-cell-rich (TCR) HLA-haploidentical SCT (haplo$\mathrm{SCT}$ ) is a form of T-cell therapy that has a high degree of efficacy in hematologic malignancies. We previously reported the safety profile assessing graft-versus-host disease (GVHD) prophylaxis that was conducted with anti-human thymocyte immunoglobulin (ATG), tacrolimus, methotrexate (MTX) and prednisolone (PSL) in unmanipulated haplo-SCT (Clin Transplant 2010, Transfus Med 2014). We evaluated efficacy and toxicity of TCR haplo-SCT in children with very high risk refractory/relapsed acute leukemia (VHR-R/R AL).

Material (or patients) and methods: VHR-R/R AL were defined as: relapse after SCT, very early or early relapse, induction failure (two or more) and relapse of risk factor with MLL rearrangement, $\mathrm{Ph}+, \mathrm{Mo} 7$ and 5q-. From August 2000 to April 2014, consecutive 38 patients with VHR-R/R AL who underwent TCR-haplo-SCT were included in the study. The median age of the patients was $8.2(0.3-19.1)$ years old. The diagnosis included ALL $(n=27), \operatorname{AML}(n=8)$, and M/NKL $(n=3)$. The disease status at TCR-haplo-SCT was 18 in CR (positive MRD: 8 patients), and 20 in non-CR. HLA disparities were $2 / 8$ in one patient, $3 / 8$ in nine patients, and $4 / 8$ in 28 patients. Donors included fathers $(n=21)$, mothers $(n=14)$, and siblings $(n=3)$. Thirty-one patients received myeloablative conditioning (TBI based: 20 patients, Bu based: 11 patients) and 34 patients of them received ATG (rabbit, thymoglobulin $2.5 \mathrm{mg} /$ $\mathrm{kg}$ ) containing regimen. The GVHD prophylaxis was conducted with tacrolimus, MTX and PSL. Thirty-four patients received peripheral blood stem cells and four patients received BM.

Results: Neutrophil engraftment (defined as $>0.5 \times 10^{9} / \mathrm{L}$ ) was $95 \%$ with a median day of 13 (range, 10-15). At a median 1,640 days follow-up (range, 320-5,510 days), in patients without events, the actuarial three-year overall survival (OS) and disease-free survival (DFS) were $57 \%$ and $39 \%$, respectively. On competing-risk analysis, one-year cumulative incidences of grade II-IV acute GVHD and chronic GVHD were $67 \%$ and $63 \%$, respectively; three-year cumulative incidences of relapse and non-relapse mortality (NRM) were $40 \%$ and $21 \%$, respectively. On univariate analysis, three-year OS in patients with acute GVHD vs. without acute GVHD was $70 \%$ vs. $22 \%(P=.0006)$, respectively; in patients with $C R$ vs. non-CR at TCR-SCT was $83 \%$ vs. $32 \%(P=.007)$, respectively; in patients infused with CD3 cell doses $\geq 5 \times 10^{8} / \mathrm{kg}$ vs. $<5 \times 10^{8} / \mathrm{kg}$ was $83 \%$ vs. $25 \%(P=.005)$, respectively; and in patients with age at TCR-SCT $<9$ vs. $\geq 9$ years was $79 \%$ vs. $34 \%(P=.008)$, respectively. In contrast, the occurrence of acute GVHD had no significant difference in infused CD3 cell doses.

Conclusion: These data suggest that TCR haplo-SCT following low-dose ATG containing conditioning combined with our GVHD prophylaxis is well tolerated, facilitate engraftment, and has significant anti-leukemic activity, particularly in pediatric patients with refractory/ relapsed population.

Disclosure of Interest: None declared. 
P461

Outcome of haploidentical $\mathbf{T}$ replete hematopoietic transplantation (haplo-HSCT) with post infusion Cyclophosphamide (PT-Cy) in peripheral T cell Lymphomas (T-NHLs)

S. Bramanti, ${ }^{1,2}$, , R. Devillier ${ }^{1}$, A. Granata ${ }^{1}$, S. Furst ${ }^{1}$, S. Harbi ${ }^{1}$, S. Barbara', C. Faucher ${ }^{1}$, L. Morabito ${ }^{2}$, R. Crocchiolo ${ }^{2}$, J. El Cheikh ${ }^{\prime}$, J. P. Weiller ${ }^{1}$. C. Chabannon ${ }^{3}$, C. Carlo stella ${ }^{4}$, F. Legrand', A. Santoro ${ }^{4}$, D. Blaise ${ }^{1}$

${ }^{7}$ Bone Marrow Unit, Institute Paoli Calmettes, Marseille, France,

${ }^{2}$ Bone Marrow Unit, Instituto Clinico Humanitas, Milano, Italy,

${ }^{3}$ Bone Marrow, Institute Paoli Calmettes, Marseille, France, ${ }^{4}$ Bone

Marrow, Instituto Clinico Humanitas, Milano, Italy

Introduction: T-NHLs are a rare and heterogeneous group of aggressive lymphoma with a poor outcome after conventional therapy. Many studies confirm the existence of the graft versus lymphoma effect of allogeneic transplantation. We report on haplo-HSCT performed in patients with poor prognosis T-NHLs Material (or patients) and methods: Patients and Methods. Between April 2009 and April 2015, 16 consecutive patients affected by T-NHL received T-cell replete haplo-HSCT with PT$\mathrm{Cy}$ in 2 centers. All patients received a nonmyeloablative conditioning regimen (NMAC). GVHD prophylaxis consisted of $50 \mathrm{mg} / \mathrm{kg}$ of PT-Cy (day +3 and +4$)$, followed by tacrolimus or cyclosporine and mycophenolate mofetil (MMF) from day +5 . Unmanipulated marrow or peripheral blood stem cells were infused on day 0 . All the donor/recipient pairs exhibited a median of 5 mismatches (range 5-7) on the unshared haplotype. Results: Patient characteristics were reported in the Table 1. The median day to ANC $>0.5 \times 10^{9} / \mathrm{L}$ and platelet count more than $20 \times 10^{9} / \mathrm{L}$ was 26 and 38 respectively. No graft failure occurred.The cumulative incidence aGVHD grade 2-3 was observed in 4 patients (25\%). No grade 4 aGVHD was observed and no patients died from aGVHD. 1 patient developed mild cGVHD. Four patients had CMV reactivation, 2 no documented pneumonia, 1 hemorrhagic cystitis, and 1 EBV reactivation. After a median follow-up of 25 months (range 4-38), 1 patients died from liver failure, and 2 patients for progressive disease. 11 patients are still alive, without immunosuppressive drugs, and all but one in complete remission. 3-year OS and PFS was depicted in Figure 1.

Table 1. Patient characteristics.

\begin{tabular}{lc}
\hline Sex M/F & $12 / 4$ \\
\hline Median age & $57(20-68)$ \\
Indication for Haplo & \\
Relapse after HDC & $4(25 \%)$ \\
Tandem HDC-ALLO & $1(6 \%)$ \\
Several CT lines (w/out HDC) & $2(12 \%)$ \\
First remission & $9(56 \%)$ \\
Disease status at Haplo & \\
CR & $12(75 \%)$ \\
PR & $3(18 \%)$ \\
PD & $1(13 \%)$ \\
Histological subtypes & \\
Angioimmunoblastic & 3 \\
Mycosis Fungoides & 1 \\
T peripheral NOS & 12 \\
Conditioning regimens & \\
Baltimore & \\
Thiotepa-based & $14(88 \%)$ \\
Stem cell source & $2(12 \%)$ \\
BM & \\
PBSC & $5(31 \%)$ \\
\hline
\end{tabular}

Conclusion: This study suggests a strong anti-lymphoma effect of haplo-HSCT with PT-Cy. We can confirm that the procedure is well tolerated without prohibitive toxicities. However, more patients should be included to better define the role of haplo-HSCT in patients with NHL-T.

References: $x x x$

Disclosure of Interest: None declared.

\section{P462}

An exploratory, open-label, multicenter study to evaluate safety and efficacy of a two-dose regimen of ATIR101 in patients with a hematologic malignancy, who received a CD34-selected hematopoietic stem cell transplantation from a haploidentical donor

S. Mielke ${ }^{1, *}$, D.-C. Roy ${ }^{2}$, R. Freudenthal ${ }^{3}$, L. Gerez ${ }^{3}$, K. Reitsma ${ }^{3}$, M. Rüdiger ${ }^{3}$, J. Rovers ${ }^{3}$

${ }^{1}$ Division of Hematology and Oncology, Department of Medicine II, Julius-Maximilian-University, Würzburg, Germany, ${ }^{2}$ Blood and Marrow Transplantation Program, Div. of Hematology-Oncology, Hôpital Maisonneuve-Rosemont, University of Montreal, Quebec, Canada, ${ }^{3}$ Kiadis Pharma, Amsterdam-Duivendrecht, Netherlands

Introduction: Previous studies demonstrated that donor lymphocytes, selectively depleted of alloreactive T-cells (ATIR101), could be given safely in the haploidentical HSCT setting at doses up to $2 \times 10^{6}$ viable T-cells $/ \mathrm{kg}$. In 42 patients a single dose of ATIR101 was given and no grade III/IV acute GVHD has been reported. This confirms the efficacy of the elimination method of allo-reactive T-cells and attributes to its beneficial safety profile. In the ongoing phase 2 study, CRAIR-007 (NCT01794299), preliminary data shows that addition of ATIR101 at 28 days post-HSCT results in a reduction of transplant-related mortality (TRM) and improvement of overall survival and event-free survival. Incidence of life-threatening infections might be further reduced with the additional of an additional dose of donor lymphocytes.

Material (or patients) and methods: In an open-label, multicenter phase 2 study (CR-AIR-008; NCT02500550), 15 patients with acute myeloid leukemia (AML), acute lymphoblastic leukemia (ALL) or myelodysplastic syndrome (MDS) will undergo a haploidentical HSCT with adjuvant administration of ATIR101. Conditioning regimen consists of either TBI (1200 cGy in 6 fractions) or melphalan $\left(60 \mathrm{mg} / \mathrm{m}^{2}\right.$ once daily for 2 days), in combination with thiotepa $(10 \mathrm{mg} / \mathrm{kg})$, fludarabine ( $30 \mathrm{mg} / \mathrm{m}^{2}$ once daily for 5 days) and ATG ( $2.5 \mathrm{mg} / \mathrm{kg}$ once daily for 4 days). Patients will receive a T-cell depleted graft (CD34+ selection) from a related, haploidentical donor, targeted to contain at least $5 \times 10^{6} \mathrm{CD} 34+$ cells/kg but if possible $8-11 \times 10^{6} \mathrm{CD} 34+$ cells $/ \mathrm{kg}$ with a maximum of $3 \times 10^{4} \mathrm{CD} 3+$ cells $/ \mathrm{kg}$ as assessed by flow cytometry. First ATIR101 infusion at a dose of $2 \times 10^{6}$ viable T-cells $/ \mathrm{kg}$ is given between 28 and 32 days after the HSCT. Patients will receive a second ATIR101 infusion at a dose of $2 \times 10^{6}$ viable T-cells $/ \mathrm{kg}$ between 70 and 74 days after the HSCT. To assess safety of the second dose administration, the first 6 patients treated will be evaluated for the occurrence of dose limiting toxicity (DLT), defined as acute GVHD grade III/IV within 120 days post HSCT (or within 42 days after the second ATIR101 infusion in case of prior dose delays).

Results: Regulatory authorities in Canada, Belgium, United Kingdom and Germany have approved the clinical protocol. The study has been initiated and enrolment of the study is expected to continue until May 2016, with first report of safety of the additional dose administration of ATIR101 to be expected before July 2016.

Conclusion: Results of this study will be used to optimize the treatment regimen for a randomized, phase III study, comparing T-cell depleted HSCT+ATIR101 versus T-cell replete HSCT using post transplantation cyclophosphamide (PTCy).

Disclosure of Interest: S. Mielke: None declared, D.-C. Roy: None declared, R. Freudenthal Employee of: Kiadis Pharma, L. Gerez Employee of: Kiadis Pharma, K. Reitsma Employee of: 


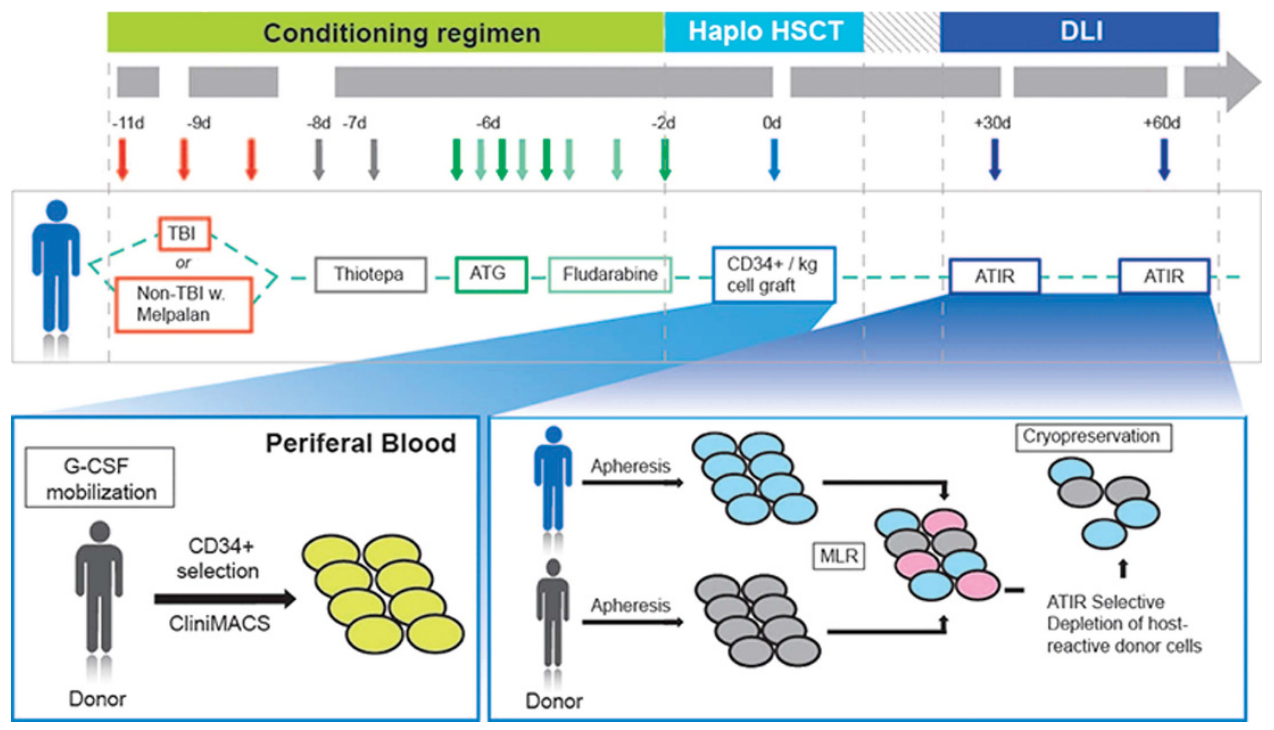

Kiadis Pharma, M. Rüdiger Employee of: Kiadis Pharma, J. Rovers Employee of: Kiadis Pharma.

\section{P463}

Outcome of haploidentical hematopoietic stem cell transplantation for non-Hodgkin lymphoma

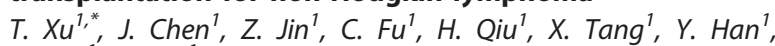
A. $\operatorname{Sun}^{1}$, D. $W u^{1}$

${ }^{1}$ Jiangsu Institute of Hematology, The First Affiliated Hospital of Soochow University, Suzhou, China

Introduction: To explore the efficacy and safety of haploidentical hematopoietic stem cell transplantation(HSCT) for refractory, relapsed or highly aggressive non-hodgkin lymphoma(NHL) patients.

Material (or patients) and methods: 26 refractory\#x03001; relapsed or highly aggressive $\mathrm{NHL}$ patients who received haploidentical HSCT from Jan 2004 to Mar 2015 were analyzed retrospectively. 17 patients were treated with the conditioning regimen consisting of modified busulfan/cyclophosphamide $(\mathrm{Bu} / \mathrm{Cy})$ plus anti-human thymocyte globulin(ATG). 9 patients were given the regimen comprised of total body irradiation(TBI)/Cy plus ATG. All patients were pretreated with cyclosporin $A(C s A)$, methotrexate(MTX) 、 mycophenolate mofetil(MMF) and ATG to prevent graft-versus-host disease (GVHD).

Results: The patients included 4 cases of diffuse large B-cell lymphoma, 1 case of follicular lymphoma, 5 cases of B-lymphoblastic lymphoma/leukemia, 9 cases of T- lymphoblastic lymphoma/leukemia, 1 case of anaplastic large cell lymphoma(ALK-), 5 cases of peripheral T-cell lymphoma(NOS), and 1 case of NK/T-cell lymphoma. 19 patients were refractory or relapsed. All patients achieved full donor chimerism. With a median follow-up of 13.5(4-136) months, 20 cases(76.92\%) survived, 15(57.69\%) survived without lymphoma, and 7 (26.92\%) relapsed. 6 patients died, 4 because of recurrence/ progress, 2 because of the complication of HSCT. 8 patients developed acute GVHD grades II-IV, which risk at 100 days was $30.80 \%$. The risk of chronic GVHD at 2 years was $25.10 \%$. The estimated 2-year recurrence rate was $42.20 \%$. The estimated 1 year overall survival(OS) and disease-free survival(DFS) rate was $84.60 \%$ and $61.10 \%$, respectively. The 2 -year OS and DFS rate was $71.60 \%$ and $48.90 \%$, respectively. Univariate analysis showed that disease status (complete remission/ not complete remission) before haploidentical HSCT may be a factor affecting OS and DFS.
Conclusion: Haploidentical HSCT is effective for relapsed, refractory or highly aggressive NHL. It may prolong DFS in part of those patients and even cure them.

Disclosure of Interest: None declared.

\section{P464}

Haploidentical Hematopoietic Stem Cell Transplant for Thalassaemia Major Using pre transplant immunosupression and Post Transplant Cyclophosphamide

V. Khandelwal ${ }^{1, *}$, D. choudhary ${ }^{1}$, S. sharma ${ }^{1}$, G. kharya ${ }^{1}$, E. kaul' ${ }^{1}$, S. kothari ${ }^{1}, D$. doval ${ }^{1}$, R. setia ${ }^{1}, A$. handoo ${ }^{\prime}$

${ }^{1}$ haematooncology and BMT, B L K Superspeciality hospital, delhi, India

Introduction: Hematopoietic stem cell transplant (HSCT) can cure thalassaemia major (TM). This option is however sometimes limited by availability of matched sibling or related unaffected donors. Over the years results of haploidentical transplants have improved significantly. We report a child with TM being successfully treated using Fludarabine-BusulphanThymoglobulin based conditioning along with Post Transplant Cyclophosphamide(PT cy).

Material (or patients) and methods: 9 year old boy with TM on regular transfusions was taken up for haploidentical HSCT from his mother in the absence of suitably matched donor. He was started on hypertransfusion (target $\mathrm{Hb} 10-11 \mathrm{gm} / \mathrm{dl}$ ) and hydroxyurea $(20 \mathrm{mg} / \mathrm{kg})$ from day -60. He also received Kelfer and Asunra as chelation therapy. He was given 2 cycles of pre transplant immunosupression which included Fludarabine $35 \mathrm{mg} / \mathrm{m} 2$ along with Dexamethasone for 5 days on D-68 to D-64 and second cycle on D-40 to D-36. He was conditioned using Thymoglobulin $1.5 \mathrm{mg} / \mathrm{kg} /$ day (D-11 to D-9),Fludarabine $35 \mathrm{mg} / \mathrm{m} 2$ (D-7 to D-2),Busulphan $3.2 \mathrm{mg} / \mathrm{kg} /$ day from D-7 to D -4. GVHD prophylaxis included PTCy $50 \mathrm{mg} / \mathrm{kg} /$ day on D3 and 4, Tacrolimus to maintain a level of $5-15 \mathrm{ng} / \mathrm{ml}$ (till 6 months post HSCT) \& MMF (till D35) starting from D5. Patient received unmanipulated peripherally harvested haploidentical cells from his mother with a target MNC $>8 \times 10^{8} / \mathrm{kg}$. He received $13.9 \times 10 \wedge 8 / \mathrm{kg}$ mononuclear cells, $10.64 \times 10 \wedge 6 / \mathrm{kg}$ CD34 cells.

Results: Polymorphonuclear cell and platelet engraftment were seen on $D+13$ and $D+18$ respectively. Whole blood chimerism on day +21 showed $99.63 \%$ donor cells. There is no evidence of acute or chronic GVHD. He was discharged on D+29 in good clinical condition. He is currently D+ 100 posts HSCT clinically well, on Tacrolimus. Post HSCT he has no transfusion requirement and chimerism D+ 60 posts HSCT is $99 \%$. 
Conclusion: Haploidentical HSCT for TM using pre transplant immunosupression and PTCy appears to be a promising technique with early and sustained engraftment.

Disclosure of Interest: None declared.

P465

Autologous hemapoietic stem cell transplantation combined with the infusion of haploidentical granulocytecolony stimulating factor mobilized peripheral blood stem cells improves the outcome of patients with hematologic malignancies

C. Zhang ${ }^{1}$ on behalf of Yes, X.-H. Chen ${ }^{1}$, Y. Liu' , L. Gao ${ }^{1}$, L. Gao ${ }^{1}$, P.-Y. Kong ${ }^{1}$, Y.-M. Feng ${ }^{1}$, H. Liu', X. Zhang,

'Department of hematology, XINQIAO HOSPITAL,THIRD MILITARY MEDICAL UNIVERSITY, Chongqing, China

Introduction: Relapse following autologous hematopoietic stem cell transplantation (auto-HSCT) is an important issue because of the failure to eradicate minimal residual malignant disease. Non-engraftment haploidentical cellular therapy represents a potential new direction in the therapy of hematologic malignancies with graft-versus leukemia effects and the enhancement of long-time survival. Role of auto-HSCT combined with the infusion of haploidentical granulocytecolony stimulating factor mobilized peripheral blood stem cells (G-PBSCs) in the treatment of hematologic malignancies is still unknown.

Material (or patients) and methods: Fifty patients with hematologic malignancies with medium-high risk were randomly divided into two groups following the induction remission; the patients were treated with auto-HSCT combined with $(n=24)$ or without $(n=26)$ the infusion of G-PBSCs.

Results: The median recovery times for neutrophils and platelets with similar numbers of autologous stem cell infusion were 13 days and 17 days, respectively, in the G-PBSC group and 12 days and 14 days, respectively, in the control group $(P>0.05)$. The 2-year overall survival was $76.2 \%$ and $50.0 \%$ for the G-PBSC and control groups, respectively $(P<0.05)$. The $2-$ year disease-free survival was $66.7 \%$ and $45.0 \%$ for the G-PBSC and control groups, respectively $(P<0.05)$. Two patients with graft-versus-host disease with $100 \%$ donor chimerism were identified and died, and mixed donor chimerism (range, 1-7\%) was successfully and persistently detected in an additional four patients.

Conclusion: Auto-HSCT combined with the infusion of G-PBSCs may represent a promising treatment with a longer survival time and low rate of relapse and acceptable complications.

Disclosure of Interest: None declared.

\section{P466}

Impact of cyclosporine-A concentration in T cell-replete haploidentical allogeneic stem cell transplantation X. Yang ${ }^{1, *}$, A. Sun ${ }^{1}$, S. Yang ${ }^{1}$, X. Hu ${ }^{1}$, D. $\mathrm{Wu}^{7}$

${ }^{1}$ The First Affiliated Hospital of Soochow University,Jiangsu Institute of Hematology, Suzhou, China

Introduction: This study was to investigate whether CSA (cyclosporine-A) levels impact on clinical outcomes of patients in the T cell-replete haploidentical allo-sct (allogeneic stem cell transplantation) setting.

Material (or patients) and methods: we retrospectively analyzed 140 consecutive patients who conducted T cell-replete haploidentical allo-sct in our institution to assess effect of CSA concentration in early stage on clinical outcomes including hematopoietic recovery, aGVHD (acute graft versus host disease), infection, DFS (disease free survival), and OS (overall survival).

Results: The median concentrations of CSA in the blood in the 1st, 2nd, 3rd and 4th week after allo-sct were $218 \mathrm{ng} / \mathrm{ml}$ (rang:54-1377ng/ml), 235ng/ml (rang:27-1500ng/ml), 263ng/ $\mathrm{ml}$ (rang:20-1500 ng $/ \mathrm{ml}$ ), and $270 \mathrm{ng} / \mathrm{ml}$ (rang:4-1500 $\mathrm{ng} / \mathrm{ml}$ ); $46 \%, 40 \%, 27 \%$ and $18 \%$ of the patients had CsA blood levels below $200 \mathrm{ng} / \mathrm{mL}$ during these weeks. 39 patients developed grade 2-4 aGVHD for a cumulative incidence of $27.8 \%$ at a median of 32 days.CSA levels during $1 \mathrm{st}, 2 \mathrm{nd}$, and 4th week didn't affect patients' hematopoietic recovery, aGVHD, infection, DFS, and OS significantly $(P>0.05)$. However, patients having CSA concentration below $200 \mathrm{ng} / \mathrm{ml}$ in the 3 rd week had a higher cumulative incidence of grade 2-4 aGVHD $(P=0.02)$. In a multivariate logistic regression analysis, low CSA concentration (below $200 \mathrm{ng} / \mathrm{ml}$ ) in the 3 rd week remained the independent risk factor of grade $2-4$ aGVHD $(P=0.02)$. CSA level in the 3rd week was not associated with patients' engraftment, infection, DFS, and OS $(P>0.05)$.

Conclusion: The analysis presented here emphasize that adequate management of CSA levels in the early stage, especially during the periengrafment period, can improve clinical outcomes in the T cell-replete haploidentical allo-sct setting.

Disclosure of Interest: None declared.

P467

Donor-specific anti-human leukocyte antigen antibodies make less advantage of paternal donor on primary graft failure in haplo-identical hematopoietic stem cell transplantation

Y. JI, ${ }^{1,3, *}$, Y.-J. Chang ${ }^{1,2,3}$, Y. Wang ${ }^{1,2,3}$, X.-J. Huang ${ }^{1,2,3}$

${ }^{1}$ Peking University People's Hospital, ${ }^{2}$ Peking University Institute of Hematology, ${ }^{3}$ Beijing Key Laboratory of Hematopoietic Stem Cell Transplantation, Beijing, China

Introduction: Who should be the best donor for related human leukocyte antigen (HLA) haplotype-mismatched hematopoietic stem cell transplant (HSCT) is always a hot topic. From 1201 recipients undergoing HLA-mismatched HSCT in our institute, it is found out that father donors were associated with less nonrelapse mortality, acute graft-versus-host disease (GVHD), and better survival compared with mother donors [1]. Recently, based on our published data [2], high level of donor specific anti-donor HLA antibodies (DSAs) were associated with an increased incidence of primary graft failure. Thus, whether paternal donors should be selected if HSCT recipient has positive DSAs?

Material (or patients) and methods: A total of 143 patients were chosen from prospectively recruited 345 subjects underwent haploidentical HSCT between May 2012 and March 2014, who received stem cells from paternal donors with positive DSAs (group A) or negative DSAs (group B) or maternal donor with negative DSAs (group C). The levels of DSAs in recipients' plasma/serum were detected by flow cytometry, and the median fluorescent intensity (MFI) $>2000$ was defined as positive outcome. In this study, primary graft failure included graft rejection (GR) and poor graft function (PGF). The former was the failure to engraft neutrophils by day +28 for three consecutive days and absence of donor hematopoiesis and the latter was defined as the presence of three cytopenic counts beyond day +28 with the presence of complete donor chimerism.

Results: All of 143 patients achieved myeloid engraftment within +28 days, and the median times to neutrophil and platelet engraftment were 12 and 17 days in group $A(n=10)$, 12 and 18 days in group $B(n=100), 13$ and 19 days in group C $(n=33)$ respectively. The cumulative incidences of poor graft function at 1 year after HSCT were $30.0 \%, 8.0 \%$ and $9.1 \%$ respectively $(P=0.086)$, which indicated the negative effect of DSAs on primary graft failure for father donors. However, the grade 2-4 acute graft versus host disease (aGVHD) was similar among these three groups $(43.0 \%, 50.0 \%$ and $45.5 \%$ respective, $P=0.914)$. Although the incidence of chronic GVHD $(20.0 \%$ vs. $33.0 \%$ vs. $33.3 \%)$, disease free survival $(90.0 \%$ vs. $89.0 \%$ vs. $78.0 \%$ ), transplant related mortality ( $0 \%$ vs. $8.0 \%$ vs. $18.2 \%$ ), and overall survival ( $100.0 \%$ vs. $89.0 \%$ vs. $78.8 \%$ ) in group A, B and $C$ were not significantly different, there was the tendency that the outcomes of HSCT with paternal donors with or without positive DSAs were superior to maternal donors. 
Conclusion: Although the DSAs lead to higher incidence of primary graft failure in haploidentical HSCT with paternal donor, these donor still should be selected rather than maternal donor with negative DSAs.

References: 1. Yu Wang, et al. Blood, 2014, 124: 843-50. 2. Ying-Jun Chang, et al. Journal of Hematology \& Oncology, 2015, 8:84.

Disclosure of Interest: None declared.

\section{P468}

Unmanipulated haploidentical versus matched unrelated donor allogeneic stem cell transplantation in adult patients with acute myelogenous leukemia in first remission: A retrospective pair-matched comparative study

Y. Sun 1, ${ }^{1,}$ E. Beohou' ${ }^{2}$, M. Labopin ${ }^{3}$, L. Volin ${ }^{4}$, N. Milpied ${ }^{5}$, I. YakoubAgha $^{6}$, S. Piemontese, E. Polge ${ }^{8}$, M. Houhou', X.-J. Huang ${ }^{9}$ M. Mohty ${ }^{10}$, A. Nagler ${ }^{11}$, N.-C. Gorin ${ }^{10}$ on behalf of ALWP

${ }^{1}$ Peking University People's Hospital, Peking University Insitute of Hematology, Beijing, China, ${ }^{2}$ Acute Leukemia Working Party of the $E B M T,{ }^{3}$ Acute Leukemia Working Party of the EBMT, Hospital SaintAntoine, Paris University UPMC, INSERM U938, Paris, France, ${ }^{4}$ Helsinki University Central Hospital, helsinki, Finland, ${ }^{5}$ Service d'Hématologie et de Thérapie Cellulaire, CHU Haut-Lévêque, Bordeaux, France; Université Bordeaux Segalen, Bordeaux, ${ }^{6} \mathrm{Hema}$ tology, CHRU de Lille, lille, France, "7 Hematology and Bone Marrow Transplantation Unit, San Raffaele Scientific Institute, Milan, Italy, ${ }^{8}$ Acute Leukemia Working Party of the EBMT, Paris, France, ${ }^{9}$ Peking University People's Hospital, Peking University Institute of Hematology, Beijing, China, ${ }^{10}$ Acute Leukemia Working Party of the EBMT, Department of Hematology and Cell therapy, Hospital SaintAntoine APHP, Paris University UPMC, INSERM U938, Paris, France, ${ }^{11}$ EBMT Acute Leukemia Working Party and Registry, Hematology Division, BMT and Cord Blood Bank, Tel-Aviv University, Chaim Sheba Medical Center, Tel-Hashomer, Ramat-Gan, Israel

Introduction: For adult patients with acute myelogenous leukemia (AML) in first remission (CR1) without HLA genoidentical sibling, a HLA 10/10 matched unrelated donor (URD) is the best alternative choice. An haploidentical donor (HID) is a recently developed alternative option. The "Beijing group" has pioneered non T depleted HID, using the combination of G-CSF primed bone marrow plus Peripheral blood. This team has reported a 2 years leukemia free survival (LFS) as high as $70 \%$. There has been some concern that the population may have been selected for good prognostic factors such as younger age and longer diagnosis to transplant interval. We used the EBMT registry to compare HID and 10/10 matched URD transplants in a pair matched analysis.
Material (or patients) and methods: Study inclusion criteria included adult ( $>=18$ ) patients, with de novo AML only with intermediate risk cytogenetics transplanted in CR1, following exclusively a non-TBI myeloablative conditioning regimen in the period from January 2008 to December 2012.Because the patient population was small, a 1:1 ratio matched pair analysis with the following matching factors was used: (1) Age \pm 5 years,(2) Interval from CR1 to transplant less than or greater than 6 months, and (3) Numbers of induction courses to reach CR1 of one or more than one. We were able to match 87 HID with 87 URD patients. The primary endpoint was LFS, and the secondary endpoints were overall survival (OS), relapse incidence (RI), non-relapse mortality (NRM).

Results: The two groups were comparable except for older donors (40.5 versus 34.1 years, $P=0.02$ ) and more patient and donor CMV positive by serology in the HID group (table). URD and HID patients had similar LFS, OS RI and NRM: the 5 year adjusted probabilities were $60.3 \%$ in the URD group versus $73.5 \%$ in the HID group for LFS $(P=0.15), 63.6 \%$ versus $78.2 \%$ for OS $(P=0.15), 24.0 \%$ versus $12.7 \%$ for $\mathrm{RI}(P=0.08), 15.7 \%$ versus $13.8 \%$ for NRM $(P=0.96)$, respectively. The incidence of severe grade III-IV acute GVHD $(9.4 \%$ versus $9.2 \%, P=1)$ and chronic GVHD (34.9\% versus $42.5 \%, P=0.39$ ) were also comparable in the two groups.

Conclusion: This retrospective study suggests that HID transplantation can achieve results similar to URD.This preliminary study albeit with a low statistical power, supports planning randomized studies to compare HID to URD.

Disclosure of Interest: None declared.

\section{P469}

CD4 T-cells directed against mismatched HLA-DP isolated following donor CD4 T-cell infusion after allogeneic stem cell transplantation show differential tissue recognition

P. van Balen ${ }^{1, *}$, M. van de Meent ${ }^{1}$, S. A. van Luxemburg-Heijs ${ }^{1}$, C. J. Halkes ${ }^{1}$, I. Jedema ${ }^{1}$, J. Falkenburg ${ }^{1}$

'Hematology, LEIDEN UNIVERSITY MEDICAL CENTER, Leiden, Netherlands

Introduction: Expression of HLA-Class II molecules is under non-inflammatory conditions predominantly restricted to hematopoietic cells. However, donor CD4 T-cells directed against mismatched HLA-DP can cause Graft-versus-Host Disease (GVHD) after allogeneic stem cell transplantation (alloSCT) or donor lymphocyte infusions from HLA 10/10 matched but HLA-DP mismatched donors due to upregulation of HLA-Class II expression under inflammatory conditions. It is often assumed that allo-HLA-DP directed CD4 T-cells recognize peptides from household genes presented in HLA-DP and that

\begin{tabular}{|c|c|c|c|c|}
\hline Variable & All patients & $H I D$ & URD & $\mathrm{P}$ value \\
\hline Follow up for survivors ${ }^{a}$ & $41[1.8-87.9]$ & $49.7[23.5-70.4]$ & $34.8[1.8-87.9]$ & $<0.0001$ \\
\hline Patient age (in yrs) ${ }^{a, c}$ & $33.3[18-55.6]$ & $33[18-55]$ & $33.6[19.3-55.6]$ & 0,75 \\
\hline $\begin{array}{l}\text { Slow remitters ( }>1 \text { induc- } \\
\text { tion courses to reach CR1) } \text { (ion }^{b}\end{array}$ & $48(27.6 \%)$ & $24(27.6 \%)$ & $24(27.6 \%)$ & 1 \\
\hline CR1 to Tx(in months) ${ }^{a, c}$ & $4[1-9]$ & $4[1-9]$ & $4.1[1.4-8.4]$ & 0,91 \\
\hline Donor age (in yrs) ${ }^{a}$ & $37[13-63]$ & $40.5[13-63]$ & $34.1[20.6-57.1]$ & 0,0235 \\
\hline Female Donor to Male & $34(19.5 \%)$ & $23(26.4 \%)$ & $11(12.6 \%)$ & 0,0835 \\
\hline Recipient $^{\text {b }}$ & & & & \\
\hline patient CMV Positive ${ }^{b}$ & $128(74.9 \%)$ & $83(95.4 \%)$ & $45(53.6 \%)$ & $<0.0001$ \\
\hline Donor CMV Positive & $119(68.4 \%)$ & $83(95.4 \%)$ & $36(41.4 \%)$ & $<0.0001$ \\
\hline
\end{tabular}

${ }^{\mathrm{a}}$ median $[$ Range $]{ }_{i}^{\mathrm{b}} \mathrm{N}(\%){ }_{i}^{\mathrm{c}}$ Matching Factors. 
LFS

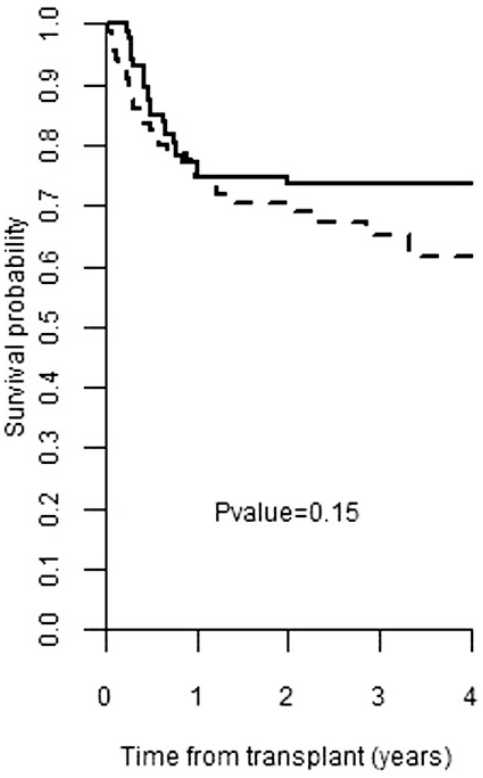

number of at-risk patients

\begin{tabular}{|c|c|c|c|c|}
\hline- & HID 87 & 65 & 63 & 48 \\
\hline & JRD 87 & 57 & 44 & 27 \\
\hline
\end{tabular}

every HLA-DP expressing cell is a target cell for these T-cells. However, in vitro experiments illustrated that allo-HLA-DP directed CD4 T-cells were not always recognizing patient derived fibroblasts induced to express HLA-DP. We hypothesized that HLA-DP directed CD4 T-cells can have tissue specificity if the presented peptides in HLA-DP are encoded by genes with tissue specific expression.

Material (or patients) and methods: In a randomized clinical trial we treat patients three months after T-cell depleted alloSCT from HLA 10/10 matched, HLA-DP mismatched, donors with $0.25 \times 10^{6} / \mathrm{kg}$ donor CD4 T-cells to promote immune reconstitution. In 3 patients, severe GVHD occurred after the infusion. To characterize the immune responses in these patients, in vivo activated T-cells were clonally isolated at the time of occurrence of GVHD and tested for reactivity against a panel of target cells, including patient and donor EBV-LCL, third party B-cells, antigen presenting cells (APC) and different GVHD target cells (skin fibroblast, third party colon carcinoma cells, biliary epithelial cells and lung fibroblasts) expressing the mismatched, patient variant, HLA-DP molecule. Results: Allo-HLA-DP directed CD4 T-cells showing differential recognition of target cells were found in all 3 patients. A total of 43 allo-HLA-DPB1*04:01 reactive CD4 T-cell clones were isolated from patient 1 of which $12(28 \%)$ recognized only patient EBV-LCL and B-cells and $31(72 \%)$ recognized also one or more GVHD target cells. From patient 2 a total of 295 alloHLA-DPB 1*03:01 reactive CD4 T-cell clones were isolated from which $22(7 \%)$ recognized only patient EBV-LCL and B-cells and 273 (93\%) recognized also one or more nonhematopoietic target cells. Further testing of T-cells that only recognized the hematopoietic cells reveals that these CD4 T-cells recognized also third party EBV-LCL, B-cells and malignant APCs. In patient 3 allo-reactive CD4 T-cell clones were isolated directed against mismatched HLA-DPB1*19:01 (3 different clones) and mismatched HLA-DRB3*02:02 (12 clones), an HLA molecule considered to be irrelevant in donor selection. In both groups CD4 T-cells were present with

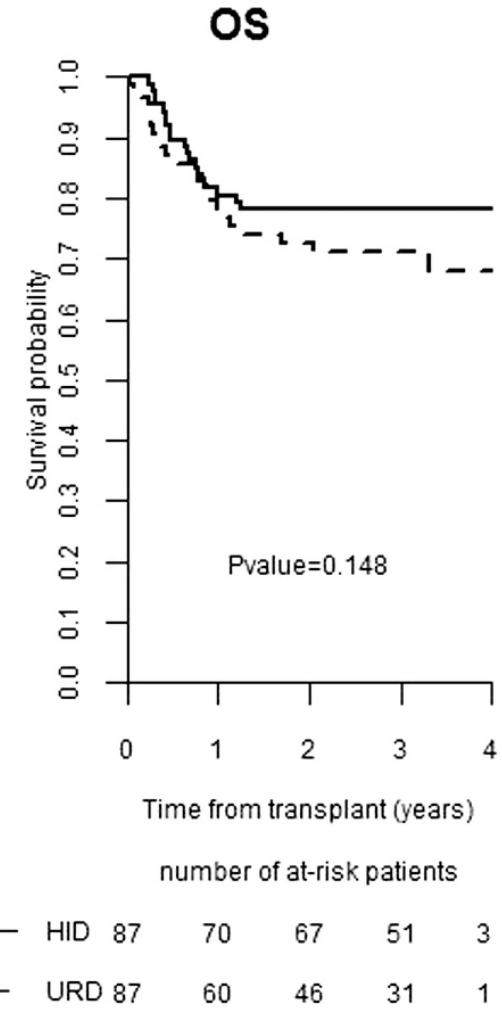

hematopoiesis restricted recognition (1 HLA-DPB1*19:01 and 4 HLA-DRB3*02:02 specific clones).

Conclusion: These results illustrate that donor CD4 T-cells directed against mismatched HLA-DP or HLA-DRB3 can show differential recognition of target cells including specificity for only cells of hematopoietic origin. These findings have potential clinical relevance if we succeed to detect the peptides presented in HLA-Class II molecules that are targeted by hematopoiesis specific allo-HLA-Class II directed CD4 T-cells.

Disclosure of Interest: None declared.

\section{P470}

Down-regulated Nrf2 antioxidant signaling in chronic graft-versus-host disease following allogeneic hematopoietic stem cell transplantation

P. Chen ${ }^{1, *}$, M. Hong ${ }^{1}$, J. Fang ${ }^{1}$

${ }^{1}$ Institute of Hematology, Union Hospital, Tongji Medical College, Huazhong University of Science and Technology, Wuhan, China, Wuhan, China

Introduction: Chronic graft-versus-host disease (cGVHD) is an important long-term complication after allo-HSCT. NF-E2related nuclear factor 2 (Nrf2) is a vital transcriptional factor which induces expression of antioxidant genes including NAD (P) $\mathrm{H}$ : quinone oxidoreductase 1 (NQO1) and heme oxygenase 1(HO-1). According to recent studies, host Nrf2-glutathione redox dysregulation predicts severity of GVHD and Nrf2 may be a critical regulator of acute GVHD in experimental transplantation. It remains unclear whether Nrf2 antioxidant signaling affects the development of cGVHD. This study aimed to investigate the activity of Nrf2 antioxidant pathway in both CGVHD patients and murine CGVHD model.

Material (or patients) and methods: In clinical study, 48 patients undergoing allo-HSCT were evaluated and divided into cGVHD $(n=26)$ or non-cGVHD $(n=22)$ group with signed informed consents. Peripheral blood samples were collected 
with a median time 8 months post allo-HSCT. Nrf2, NQO1 and HO-1 mRNA levels in peripheral blood mononuclear cells (PBMCs) were detected by real-time RT-PCR. Oxidative activity in PBMCs was assessed by intracellular reactive oxygen species (ROS) production. For murine model, all mice were randomly allocated into control (wild type), total body irradiation (TBI), synBMT (non-cGVHD) and alloBMT (cGVHD) group. In alloBMT group, irradiated female $\mathrm{CB} 6 \mathrm{~F} 1$ recipients were transplanted with spleen cells $\left(6-7 \times 10^{7}\right)$ plus bone marrow cells $\left(8 \times 10^{6}\right)$ from male $B A L B / c$ donors. In synBMT group, donors were changed to be male CB6F1 mice. All mice were sacrificed on day 27-33 post BMT. Clinical CGVHD score and histologic analysis (H\&E and Masson's Trichrome) were used to evaluate cGVHD. BM and liver samples were collected to explore the expression of Nrf2, NQO1 and HO-1 by western blot and realtime RT-PCR.

Results: In clinical study, Nrf2, NQO1 and HO-1 mRNA levels in cGVHD group were significantly lower than those in noncGVHD group $(0.29 \pm 0.03$ vs. $0.49 \pm 0.06,0.34 \pm 0.05$ vs $0.54 \pm 0.08,0.89 \pm 0.11$ vs. $1.41 \pm 0.23, P<0.05)$. ROS level in cGVHD group was significantly higher than that in non-cGVHD group $(1.02 \pm 0.05$ vs. $0.7 \pm 0.06, P<0.05)$. These results suggested down-regulated Nrf2 antioxidant signaling and increased oxidative activity in CGVHD patients.In experimental transplantation study, clinical cGVHD score and histologic analysis consistently identified the establishment of CGVHD murine model. Nrf2, NQO1 and HO-1 mRNA levels as well as protein levels in BM and liver of alloBMT group all significantly decreased than those in synBMT group (Table 1 \& Figure 1). These results demonstrated down-regulation of Nrf2 antioxidant pathway in mice with cGVHD.

Conclusion: This study firstly provided the evidence of downregulation of Nrf2 antioxidant signaling in both CGVHD patients and mice cGVHD model. It suggests that defects of Nrf2 antioxidant pathway may contribute to development of cGVHD. It deserves further study that whether Nrf2 can be a novel target against CGVHD.

Disclosure of Interest: None declared.
P471

Endothelial Microparticles delivering microRNA-155 into T lymphocytes is involved in the initiation of a mouse aGVHD model

\section{R. Zhang ${ }^{1, *}$, Q. Wu ${ }^{1}$, M. Hong ${ }^{1}$, L. Xia ${ }^{1}$}

${ }^{1}$ Institute of Hematology, Union Hospital, Tongji Medical College, Huazhong University of Science and Technology, Wuhan, China

Introduction: Endothelial microparticles (EMPs) have been regarded as an important transcellular delivery system in cellto-cell transfer and communication, having a crucial role in pathological process of acute graft-versus-host disease (aGVHD). And microRNA-155 (miR-155) was reported to be upregulated in the effector T lymphocytes of aGVHD mice. However, how miR-155 encapsulated within EMPs ultimately influences and manipulates T lymphocytes in the initiation of aGVHD has not been previously explored.

Material (or patients) and methods: We firstly established the $\mathrm{C} 57 \mathrm{BL} / 6\left(\mathrm{H}-2^{\mathrm{b}+}\right)$ donor to BALB/C $\left(\mathrm{H}-2^{\mathrm{d}+}\right)$ recipient $\mathrm{MHC}-$ mismatched aGVHD model, and recipient mice were monitored for survival rate, clinical symptoms and pathological evidences of aGVHD. We then compared dynamic change of EMPs in plasma of aGVHD and non-aGVHD mice $(+4 d,+8 d$, $+12 \mathrm{~d},+16 \mathrm{~d}$ and aGVHD point) using flow cytometry. RT-PCR was used to detect the levels of miR-155 in MPs and T lymphocyte from peripheral blood. We also compared the expressions of miR-155 in aGVHD-involved organs: bone marrow, spleen and liver. Nextly, we divided the experiments into eight groups: BM group, aGVHD group, aGVHD+EMPs group, aGVHD+simvastatin group, aGVHD+EMPs+simvastatin group, aGVHD+miR-155 antagomir group, aGVHD+NC antagomir group, aGVHD+miR-155 antagomir+EMPs group [EMPs was derived from TNF-a-stimulated mouse aortic endothelial cells (MAECs)]. We observed the clinical manifestations and histopathological severities of aGVHD in each group. RT-PCR was used to detect the levels of miR-155 in T lymphocyte and flow cytometry was used to observe the change of T lymphocytes subsets. Simultaneously, ELISA was used to detect the concentration of TNF- $a$, IFN- $\gamma$, IL-2, IL-4, IL-6, IL-10 and IL-17 in the serum of each group.

Results: In this study, we firstly found that EMPs from peripheral blood of aGVHD mice was significantly elevated from $+8 \mathrm{~d}$ after allo-HSCT compared with non-aGVHD mice and

[P470]

Table 1 Relative mRNA level by real time RT-PCR

\begin{tabular}{|c|c|c|c|c|c|c|}
\hline & \multicolumn{3}{|c|}{ Mice liver (mean \pm SEM) } & \multicolumn{3}{|c|}{ Mice bone marrow (mean \pm SEM) } \\
\hline & $c G V H D(\mathrm{n}=10)$ & Non-cGVHD $(\mathrm{n}=7)$ & $\mathrm{P}$ value & $c G V H D(\mathrm{n}=10)$ & Non-cGVHD $(\mathrm{n}=7)$ & $\mathrm{P}$ value \\
\hline Nrf2 & $0.12 \pm 0.03$ & $0.38 \pm 0.11$ & 0.018 & $0.57 \pm 0.05$ & $1.00 \pm 0.12$ & 0.003 \\
\hline NQO1 & $0.85 \pm 0.07$ & $1.53 \pm 0.20$ & 0.002 & $0.50 \pm 0.07$ & $0.79 \pm 0.09$ & 0.029 \\
\hline HO-1 & $1.77 \pm 0.47$ & $4.51 \pm 0.87$ & 0.009 & $0.52 \pm 0.06$ & $1.14 \pm 0.21$ & 0.005 \\
\hline
\end{tabular}

Figure 1.
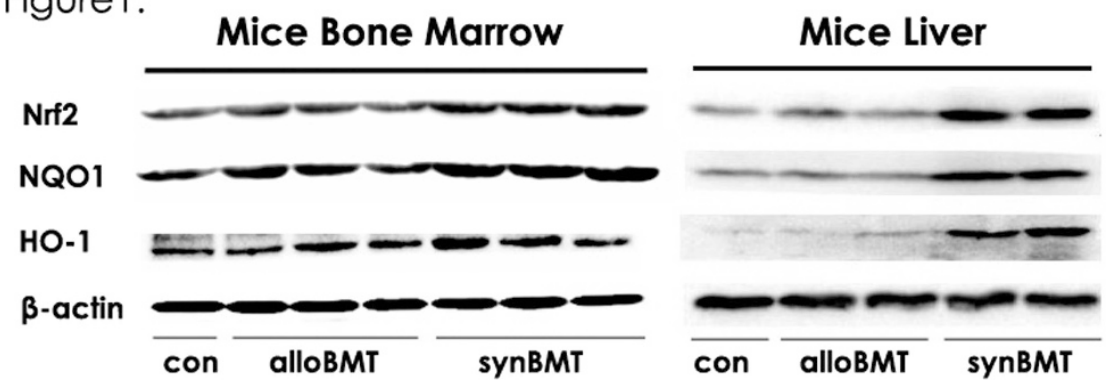
slightly decreased at aGVHD point. Similarly, in aGVHD mice, miR-155 in plasma MPs was significantly higher on $+8 d,+12 d$, $+16 \mathrm{~d}$ after allo-HSCT while slightly lower at aGVHD point. While the increment of miR-155 in T lymphocytes of aGVHD mice were postponed than in plasma MPs and didn't occur only until aGVHD. In addition, the expression of miR-155 elevated from $+4 d$ in bone marrow cells, $+12 d$ in liver and $+16 \mathrm{~d}$ in spleen cells, respectively. EMPs-treated aGVHD mice exhibited exacerbated clinical manifestations and histopathological severities of aGVHD. On the other hand, administration of simvastatin, which was described as an endothelial cell protector, significantly attenuated the clinical severity of aGVHD, the histopathology of aGVHD-involved organs and the overall mortality from aGVHD. In addition, specific inhibition of miR-155 in EMPs by miR-155 antagomir have made $T$ lymphocytes defective differentiation toward Th1 and Th17 and skewed differentiation toward Th2 and Tregs, and resulted in ameliorated clinical and pathological manifestations of aGVHD.

Conclusion: Taken together, our study indicates that miR-155 delivered by EMPs plays a critical role in the initiation of mouse aGVHD through promoting the Th1 and Th17 responses while repressing the expansions of Th2 and Tregs, and might be used as a potential diagnostic and therapeutic target of aGVHD after allo-HSCT.

Disclosure of Interest: None declared.

\section{P472}

Assessment of the TCR-repertoire of Treg cells after bone marrow transplantation to identify protective Treg cell clones for cellular GvHD-prevention and therapy

S. Raha ${ }^{1, *}$, S. Ravens ${ }^{1}$, R. Geffers ${ }^{2}$, A. Ganser ${ }^{1}$, I. Prinz ${ }^{1}$, C. Koenecke

${ }^{1}$ Hannover Medical School, Hannover, ${ }^{2}$ Helmholtz Center for Infection Research, Braunschweig, Germany

Introduction: Treatment of Graft-versus-Host Disease (GvHD) relies on unspecific immunosuppressive drugs that leave the recipient prone to opportunistic pathogens. Potential strategies for a more specific prevention and treatment of GvHD are based on $\mathrm{CD}^{+} \mathrm{CD} 25^{+} \mathrm{Foxp}^{+}$regulatory T cells (Treg cells) that have been shown to be effective when given in large amounts as an addition to the stem-cell graft. This research project aims at establishing a method to analyse the Treg cell repertoire during experimental acute GvHD using high-throughput Illumina sequencing. This will allow us to identify GvHDspecific $T$ cell receptor (TCR) rearrangements, which might designate alloantigen-specific protective Treg cell clones. Such protective Treg cell clones will be tested for its survival promoting impact after bone marrow transplantation (BMT).

Material (or patients) and methods: Experiments were performed in a C57BL/6 into BALB/c murine model of acute GvHD. We used a C57BL/6 Foxp3-IRESeGFP reporter that allowed isolation of Treg cells using FACS. For BMT, $5 \times 10^{6}$ Bone marrow (BM) and $0.5 \times 10^{6}$ conventional T cells with or without Treg cells (ratio 1:1) were intravenously injected into lethally irradiated BALB/c recipients. GFP ${ }^{+}$Treg cells were reisolated on different time points after BMT for further analysis. Total RNA of sorted ex vivo Treg cells was isolated and, using TCR specific primers, transcribed into full length CDNA using rapid amplification of CDNA-ends (RACE)-PCR. Sequencing of the repertoire was done on the high-throughput platform Illumina. Subsequent bioinformatics analysis of TCR clones was done using the online annotation platform IMGT.org, R-packages and in-house Unix and Python scripts.

Results: We were able to establish a RACE-PCR protocol to reverse transcribe RNA from as little as $3 \times 10^{4} \mathrm{~T}$ cells, allowing us to analyse recovered Treg cells on early time points after GvHD induction. Introduction of specific primer sites for Illumina sequencing was done with custom primers, optimized for the RACE protocol. Recovery of Treg cells on days 4, 8 and 14 after BMT yielded an average of $108 \%$ increase in GFP ${ }^{+}$Treg cell numbers between days 8 and 14. In one GvHD experiment, we detected 8265 clonotypes, of which the most frequent clonotypes comprised $\sim 0.2 \%$ of the Treg cell input sample. At day 14 of the same experiment the sequence read was 39995, in which the most frequent clonotypes made up $\sim 39 \%$. Our sequencing results indicate a lower Treg cell diversity as compared to the input Treg cell repertoire (four independent experiments). Interestingly, three weeks after BMT, we observed a rebound of the Treg cell diversity.

Conclusion: In this study, we were able to analyse the Treg cell TCR-repertoire early after BMT. Our data shows that the diversity of the TCR repertoire of Treg cells narrows early after BMT in mice, which are protected from acute GvHD. Therefore, we hypothesize that a fraction of dominant donor Treg cell clones are responsible for protection from acute GvHD. Such clones are currently tested for their protective capacity in GvHD. Our results will be of value for optimized Treg celltherapy to protect patients from GvHD.

IP and CK contributed equally to this work.

Disclosure of Interest: None declared.

\section{P473}

Pre-transplant treatment of hematopoietic grafts with Fas Ligand for acute graft-versus-host disease prevention: Preliminary Results for Launching a Clinical Study

T. Zuckerman ${ }^{1,2, *}$, M. Meiron ${ }^{3}$, L. Pinkas ${ }^{3}$, H. Levi ${ }^{3}$, G. Rodinov ${ }^{3}$, S. Yarkoni ${ }^{3}$, T. Katz ${ }^{1,2}$, J. M. Rowe ${ }^{4}$, E. Ganon ${ }^{1}$

${ }^{1}$ Hematology and BMT, RAMBAM HEALTH CARE CAMPUS, ${ }^{2}$ Bruce Rappaport Faculty of Medicine, Technion, Haifa, ${ }^{3}$ Cellect Bio, Kfar-Saba, ${ }^{4}$ Hematology, Shaare Zedek Medical Center, Jerusalem, Israel

Introduction: Graft-versus-host disease (GVHD) mediated by donor alloreactive $\mathrm{T}$ lymphocytes is the major obstacle of allogeneic hematopoietic stem cell transplantation (HSCT). Fas Ligand (FasL) has been shown to induce apoptosis of donor $\mathrm{T}$ cells while sparing early hematopoietic progenitors and hence not jeopardizing hematopoietic engraftment. A negative selection process that is based on differential sensitivity of hematopoietic cells to FasL-induces apoptosis which eliminated mature $T$ cells and may significantly improve transplant outcome as it generates a potent graft containing early T cells important for engraftment, diminishes aGVHD and supports post-transplantation immune recovery. The aim of this study is to evaluate the efficacy of aGVHD prevention and safety of transplantation of human mobilized peripheral blood xenograft pre-exposed to FasL.

Material (or patients) and methods: Apheresis-derived mobilized peripheral blood cells (MPBCs) were collected from a healthy donor upon obtaining inform consent (IRB Number 0506-14-RMB). Cells of the treatment group were subjected to 4- hour incubation in the presence of $50-100 \mathrm{ng} / \mathrm{ml}$ FasL. The control groups received cells that were not incubated (control 1) or incubated for 4 hours without FasL (control 2). For efficacy study, $5 \times 10^{6}$ MPBCs cells were transplanted to NOD. SCID IL2-Rgamma-null (NSG) mice irradiated with 2 cGy. This is used as a severe GVHD model for xenograft. The mice were monitored for engraftment, and GVHD occurrence up to 40 days post transplantation. For safety study, $10 \times 10^{6}$ MPBCs cells were intravenously transplanted to NOD.SCID mice irradiated with $3 \mathrm{cGy}$. The mice were monitored for 9 weeks for clinical signs of toxicity followed by evaluation of blood tests and histopathology of major organs.

Results: Engraftment of hCD $45^{+}$cells was evident in 100\% of mice in the two models. Upon incubation of the hematopoietic graft with FasL, improvement of mice survival was demonstrated in GVHD models (NSG) and in the toxicological model (NOD.SCID). Kaplan Meier analysis demonstrated extension of the survival curve in the severe GVHD model, with median survival time (MST) of 21 days for both control groups and 24 days for the treatment group $(P<0.01)$. In addition, $20 \%$ of the treated group survived until the study end. In the safety model $100 \%$ of the FasL treated group survived compared to $50 \%$ death in the control group. The 
GVHD clinical score was significantly lower in the treatment group compared to control groups; average clinical score was $6.05 \pm 0.75,5.55 \pm 0.50$, and $2.84 \pm 0.71$ for control 1 , control 2 and treatment group, respectively, on day 22 ( $p<0.05$ and $<0.001$ ). Reduction of 1.25 fold in RBCs and 2.4 fold in platelet count and increase of 1.6 fold in lymphocytes were found in the control group compared to the FasL treated group.

Conclusion: Overall, these results demonstrate that apoptoticbased functional selection of the hematopoietic graft significantly reduced aGVHD without impairing human hematopoietic cell engraftment. Results of safety studies showed improved survival in the treated compared to the control group. Based on these data a phase I/lla study is planned.

Disclosure of Interest: None declared.

\section{P474}

Calcineurin inhibitor increases chronic GVHDby enhancing IL-5 production in mice

W. S. Lee ${ }^{1, *}$, Y.-D. Joo ${ }^{2}$, J.-S. Chung ${ }^{3}$, S.-K. Seo ${ }^{4}$

${ }^{1}$ Int. Medicine, Hemato-Oncology, Inje University Busan Paik Hospital, ${ }^{2}$ Int. Medicine, Hemato-Oncology, Inje University Haeundae Paik Hospital, ${ }^{3}$ Int. Medicine, Hemato-Oncology, Pusan National University Hospital, ${ }^{4}$ Microbiology and Immunology, Inje University, College of Medicine, Busan, Korea, Republic Of

Introduction: Although calcineurin inhibitors (CNIs) arethe most widely used for pharmacologic GVHD prophylaxis in clinic,it isconsidered that CNIs contribute tothe development of chronic GVHD. However, the underlying mechanism remains unclear. Here, weinvestigated the effects of tacrolimus (FK506) on developing chronic GVHD andidentified critical mediator and its downstream effector mechanism.

Material (or patients) and methods: Lethally irradiated $\mathrm{B} 6 \mathrm{D} 2 \mathrm{~F} 1\left(\mathrm{H}-2^{\mathrm{b} / \mathrm{d}}\right)$ recipients were injected with $5 \times 10^{6} \mathrm{TCD}$ BM plus $2 \times 10^{6} \mathrm{~T}$ cells from $\mathrm{B} 6\left(\mathrm{H}-2^{\mathrm{b}}\right)$ donorand were given $15 \mathrm{mg} / \mathrm{kg}$ i.p. once daily from 0 to day +10 .Mice were monitored every other day for survival and clinical score. Serum cytokines were measured using the mouse Flex-Set cytokine bead array.Cell phenotypes and intracellular cytokine profileswere analyzed by flow cytometry. Skin tissues were collected from each group of recipients on day 21 and stained with $\mathrm{H} \& \mathrm{E}$.

Results: Although survival was statistically improved byFK506 treatment, we observed worse skin GVHD scores in recipient of FK506 than control recipient. In contrast of control, massive infiltration of eoshinophils was observed in the skin tissues from recipients of FK506. Profibrotic cytokine IL-13 and IL-33 in the skin tissues were significantly elevated in FK506 treatment compared with vehicle. Apparently, IL-5 was increased in serum and lymphoid organs from recipient of FK506on day 5-7before skin GVHD. Blockade of IL-5 signaling with administration of anti-IL-5mAb significantly reduced skin GVHD severityin the recipient of FK506.

Conclusion: Our results demonstrate that 1) $\mathrm{CNI}$ treatment increases fibrotic skin GVHD, 2) IL-5-mediated eosinophil activation is critical molecular mechanism, and 3) blockade of IL-5 signaling reduces skin GVHD induced by CNI treatment. Our study suggests that neutralizing IL-5 during early time after HSCTis a possible strategy to reduceCNls-mediated chronicGVHD.

Disclosure of Interest: None declared.

\section{P475}

TLR2 signaling in donor $\mathrm{T}$ cells against calcineurin inhibitor responsible for persistence of GVHD in mice model

Y.-D. Joo ${ }^{1, *}$, W.-S. Lee ${ }^{2}$, J.-S. Chung ${ }^{3}$, S.-K. Seo ${ }^{4}$

${ }^{7}$ Hematology, Haeundae Paik Hospital, College of Medicine, Inje University, ${ }^{2}$ Hematology, Pusan Paik Hospital, College of Medicine, Inje University, ${ }^{3}$ Hematology, Pusan National University Hospital, School of Medicine, ${ }^{4}$ Microbiology and Immunology, College of Medicine, Inje University, Busan, Korea, Republic Of
Introduction: Toll-like receptors (TLRs) play a fundamental role in innate immunity through their capacity to recognize pathogen-associated molecular patterns. Also, TLRs have been shown to play an important role in T cells as costimulatory receptors. However, the significance of TLR signaling in alloreactive donor $T$ cells affecting GVHD remains unclear. Here, we investigated TLR2 signaling in donor T cells on GVHD and its function to resist against calcinurine inhibitors (CNIs). Material (or patients) and methods: Mixed lymphocyte reaction (MLR) cultures were set up with CFSE-labelled naïve $\mathrm{BALB} / \mathrm{C}$ T-cell responder and $\mathrm{B} 6 \mathrm{TLR}^{-1-}$ splenic stimulator per well in the presence or absence of TLR2 agonist $\mathrm{Pam}_{3} \mathrm{CSK}_{4}$ (PAM). The MLR cultures were treated with various concentrations of CNIs (CsA and FK506) and determined the resistance through proliferation and cytokine levels. Lethally irradiated B6.WT or B6. TLR2 ${ }^{-/}\left(\mathrm{H}-2^{\mathrm{b}}\right)$ recipients were injected with $5 \times$ $10^{6} \mathrm{TCD}-\mathrm{BM}$ plus $5 \times 10^{6} \mathrm{~T}$ cells from BALB/C $\left(\mathrm{H}-2^{\mathrm{d}}\right)$ donor and were given $15 \mathrm{mg} / \mathrm{kg}$ i.p. once daily from 0 to day +10 . Additionally, recipients were treated with PAM on day +3 and +5 after transplantation. The survival and clinical GVHD score were monitored.

Results: Stimulation with TLR2 agonist promoted alloreactive T cell proliferation and survival. IL-2 and IFN-g production were also significantly increased by TLR2 ligation. In the condition of high dose $\mathrm{CNI}$ treatment, the activation of allogeneic $\mathrm{T}$ cells was prevented in either TLR2 stimulation or not. However, as the lower dose of CNI treatment, TLR2 stimulation led proliferation of allogeneic $T$ cells but not without TLR2 stimulation. The severity of GVHD in recipients treated with FK506 was higher in PAM treatment than control treatment. Conclusion: Our results demonstrate that TLR2 stimulation directly enhances allogeneic T cell responses and provides the resistance against CNIs, which might be responsible for meek efficacy of CNIs on GVHD prophylaxis.

Disclosure of Interest: None declared.

\section{P476}

Screening of biological marker gene associated with B cell in CGVHD patients

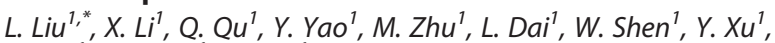
X. Wu ${ }^{1}$, G. Chen ${ }^{1}, D . W u^{7}$

${ }^{1}$ Jiangsu Institute of Hematology, The First Affiliated Hospital of Soochow University, Suzhou, China

Introduction: Allogeneic hematopoietic stem cell transplantation (allo-HSCT) is a widely used therapy for a lot of malignant and nonmalignant hematologic diseases. Graft-versus-host disease (GVHD) remains one of the major barriers to a more widespread and successful application of allo-HSCT. Despite immune-suppressive prophylaxis, most survivors develop chronic GVHD (cGVHD). Identification of biologic markers of the syndrome could facilitate significant advances in understanding to help guide prevention and treatment approaches. Data in humans support a role of both T- and B-cells in a highly complex network leading to cGVHD. However, there was no report about biological marker gene associated with $B$ cell in cGVHD patients. So we screened biological marker gene associated with B cell in cGVHD patients.

Material (or patients) and methods: Microarrays were used to detect expressed gene profiles of peripheral blood mononuclear cells total RNA from subjects in the mild-moderate CGVHD, severe CGVHD, non-cGVHD, immune tolerance and healthy donors groups. Found out the genes associated with $B$ cell in CGVHD patients reached statistical significance after a false discovery rate (FDR) correction was applied to the data. Real-time quantitative RT-PCR was used to confirm the B cell genes.

Results: 284 genes were found by microarrays between CGVHD patients and patients without CGVHD, 5 genes (CDKN2A, SOX4, ZBTB32, CD70, IL21R) associated with B cell. 800 genes were found by microarrays between immune tolerance patients and patients without CGVHD, 3 genes (NFAM1, CD27, BST1) associated with B cell. Real-time 
quantitative RT-PCR confirmed significantly lower expression of CDKN2A in CGVHD relative to without CGVHD patients $(P=0.001)$. Significantly higher expression of CD27 and lower expression of BST1 in immune tolerance relative to without cGVHD patients $(P=0.038, P=0.022)$.

Conclusion: Significantly lower expression of CDKN2A in cGVHD relative to without CGVHD patients. Significantly higher expression of CD27 and lower expression of BST1 in immune tolerance relative to without cGVHD patients. The changes of these genes may be potential biomarkers for cGVHD and immune tolerance.

Disclosure of Interest: None declared.

P477

Efficacy of bone marrow derived MSC for steroid refractory acute GVHD is related to the age of the MSC donor

L. Van Der Wagen ${ }^{1, *}$, K. Westinga ${ }^{2}$, M. van Gelder ${ }^{2}$, J. J. Boelens ${ }^{3}$, I. Slaper-Cortenbach ${ }^{2}$, J. Kuball

${ }^{i}$ Hematology, ${ }^{2}$ Cell Therapy Facility, ${ }^{3} B M T$ Unit Pediatrics, University Medical Center Utrecht, Utrecht, Netherlands

Introduction: Acute graft versus host disease (aGVHD) remains a life-threatening complication and substantially reduces efficacy of allo-HSCT. In particular, the outcome of patients with severe steroid-refractory aGVHD continues to be poor. Administration of Mesenchymal Stromal Cells (MSC) has been reported by others and us[1-4] as an interesting treatment option. However, variability in products and the inability to link surrogate markers of the product to clinical efficacy could be a major threat in daily clinical practice.

Material (or patients) and methods: We evaluated the impact of individual donors as well as donor properties such as age on clinical response in a cohort of 102 patients with grade II-IV steroid refractory aGVHD treated with bone marrow derived MSC Primary outcome measures were one year overall survival (OS) and response. Cox proportial hazards models, competing risk analysis and Kaplan Meier estimates were used for analyzing response and OS.

Results: 102 patients received MSC from ten different donors. Median number of infusions was 3 (range 1-4). 75,5\% of patients received all MSC infusions from the same donor, $20,6 \%$ with MSC from 2 donors and 3,9\% with MSC from 3 different donors. Two donors (donor 1 and donor 2) were used to treat $28,4 \%$ and $43,1 \%$ of patients respectively. When testing impact on one year OS of an individual product no differences between patients treated with either the 2 main contributing donors or the patients treated with the 'other' MSC donors could be observed. Also no significant differences in achieving complete resolution of GVHD symptoms were observed between these three groups in a Cox proportional hazards model. However, when donor age was used to cluster

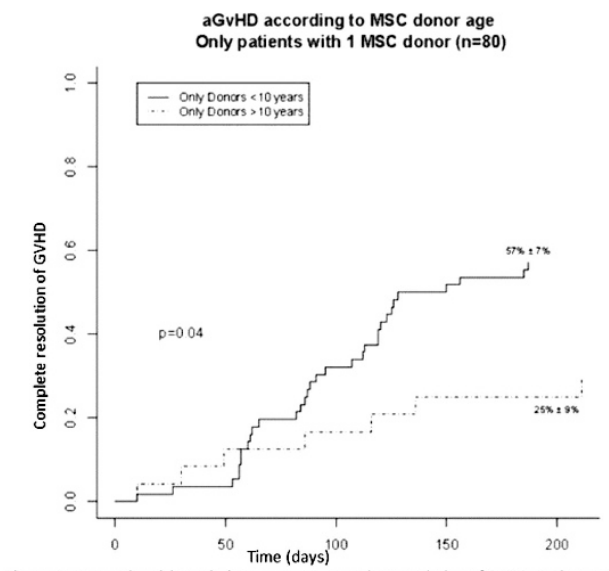

Figure 1: Competing risk analysis on outcome complete resolution of GVHD. Patients onfy treated with MSC derived from donors < 10 years of age had a significantly higher chance to reach CR GVHD compared to patients only treated with MSC derived from donors $>10$ years of age (Gray's test, $p=0.04$ ). Competing events were death or relapse. cohorts (donor age $<$ and $>10$ years) significant differences were observed with both improved CR GVHD rates (Figure 1) as well as a survival benefit for patients only treated with MSC derived from young donors.

Conclusion: In steroid refractory acute GVHD patients treated with MSC, we did not observe differences in efficacy from a specific donor. However patients treated only with MSC derived from young donors had significantly improved CR GVHD and OS.

References: 1. Te Boome, L.C., et al., Biomarker profiling of steroid-resistant acute GVHD in patients after infusion of mesenchymal stromal cells. Leukemia, 2015. 29(9): p. 1839-46. 2. Le, B.K., et al., Mesenchymal stem cells for treatment of steroid-resistant, severe, acute graft-versus-host disease: a phase II study. Lancet, 2008. 371(9624): p. 1579-1586.

3. von, B.M., et al., Treatment of refractory acute GVHD with third-party MSC expanded in platelet lysate-containing medium. Bone Marrow Transplant., 2009. 43(3): p. 245-251.

4. Lucchini, G., et al., Platelet-lysate-expanded mesenchymal stromal cells as a salvage therapy for severe resistant graftversus-host disease in a pediatric population. Biol. Blood Marrow Transplant., 2010. 16(9): p. 1293-1301.

Disclosure of Interest: None declared.

P478

Treatment of Chronic Graft versus Host Disease with a Combination of B-cell Depletion and Tyrosine Kinase Inhibition

L. Van Der Wagen ${ }^{1, *}$, L. te Boome ${ }^{1}$, I. Nijhof ${ }^{1}$, M. Schoordijk2, E. Meijer ${ }^{2}$, J. Kuball ${ }^{7}$

${ }^{1}$ Hematology, University Medical Center Utrecht, Utrecht, ${ }^{2} \mathrm{Hema-}$ tology, VU Medical Center, Amsterdam, Netherlands

Introduction: Chronic Graft Versus Host Disease (cGVHD) still has a large impact on morbidity and mortality after allogeneic hematopoietic stem cell transplantation (HSCT). Affected patients require long term use of immunosuppressive drugs, mainly corticosteroids, which lead to development of severe side effects. Therapeutic approaches for CGVHD are limited. Generally recommended first line therapy consists of glucocorticoid therapy combined with a calcineurin inhibitor. Options for second line therapy are numerous but no consensus on the most favourable choice of agents has been reached.

Material (or patients) and methods: As both monotherapy with rituximab[1-3] and monotherapy with tyrosine kinase inhibition $[4,5]$ have shown to be effective in reducing cGVHD symptoms. We aimed to test whether the sequential therapy of the anti CD20 antibody rituximab followed by a 6 month treatment period with the tyrosine kinase inhibitor nilotinib is a good treatment strategy for patients with sclerotic cGVHD (EudraCT nr 2008-004125-42).

Results: We included 29 patients, 4 patients went offstudy, 13 patients have currently completed the follow up. Of these 13 patients, 1 patient reached a complete response, 8 patients showed a partial response and 4 patients remained in stable disease. Moreover, 2 out of 5 patients who suffered from severe ulcerations at the start of the study had a complete resolution of ulcers at the end of the treatment period. There is also a significant decrease in cGVHD affected body surface area (Figure 1). Patients with a (partial) response also show a decrease in self attributed severity of CGVHD and their immunosuppressive drugs could be tapered.

Conclusion: The sequential therapy of B-cell depletion and tyrosine kinase inhibition provides a new and interesting alternative treatment option for this difficult and heavily pretreated patient category. Approximately $70 \%$ of patients achieve a (partial) response.

References: van, D.S., et al., The immunological phenotype of rituximab-sensitive chronic graft-versus-host disease: a phase II study. Haematologica, 2011. 96(9): p. 1380-1384.

2. Cutler, C., et al., Rituximab for steroid-refractory chronic graft-versus-host disease. Blood, 2006. 108(2): p. 756-762. 


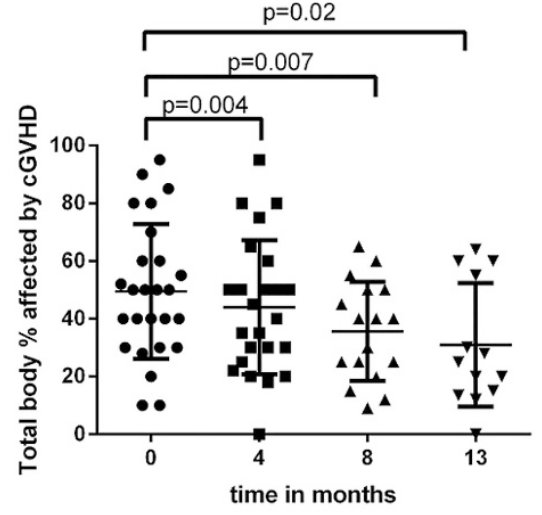

Figure 1. Percentage of total body surface area affected by chronic Graft versus Host Disease is plotted against time in months after start of the study. Each dot represents 1 patient. Puvalues are calculated by means or Wicoxon.

matched-pairs signed rank test.

3. Kharfan-Dabaja, M.A., et al., Efficacy of rituximab in the setting of steroid-refractory chronic graft-versus-host disease: a systematic review and meta-analysis. Biol Blood Marrow Transplant, 2009. 15(9): p. 1005-13.

4. Magro, L., et al., Imatinib mesylate as salvage therapy for refractory sclerotic chronic graft-versus-host disease. Blood, 2009. 114(3): p. 719-722.

5. Olivieri, A., et al., Imatinib for refractory chronic graft-versushost disease with fibrotic features. Blood, 2009. 114(3): p. 709-718.

Disclosure of Interest: None declared.

\section{P479}

Extracorporeal Photopheresis for Treatment of Children with Acute Graft-Versus-Host Disease (GVHD): A Multicentre Retrospective Analysis

L. Strocchio ${ }^{1,},{ }^{*}$, P. Merli ${ }^{1}$, D. Pagliara ${ }^{1}$, A. Bertaina ${ }^{1}$, B. Lucarelli ${ }^{7}$ S. Ceccarelli ${ }^{1}$, M. Montanari ${ }^{1}$, C. Messina ${ }^{2}$, M. Zecca ${ }^{3}$, F. Fagioli ${ }^{4}$, F. Locatelli ${ }^{1}$

${ }^{7}$ Pediatric Hemato-Oncology, IRCCS Bambino Gesù Children's Hospital, Rome, ${ }^{2}$ Pediatric Hemato-Oncology, University Hospital of Padova, Padova, ${ }^{3}$ Pediatric Hemato-Oncology, Fondazione IRCCS Policlinico San Matteo, Pavia, ${ }^{4}$ Pediatric Hemato-Oncology, Ospedale Infantile Regina Margherita, Turin, Italy

Introduction: Extracorporeal photopheresis (ECP) is a promising immunomodulatory therapy for both acute and chronic GVHD. We present our multicenter experience on the use of ECP for children developing acute GVHD (aGVHD) after allogeneic HSCT. The aim of this analysis was to evaluate the clinical effect of ECP, its impact on intensity of immunosuppressive therapy, safety and tolerance of the procedure in a pediatric population.

Material (or patients) and methods: From January 2009 to June 2013, 93 pediatric patients (pts) underwent ECP procedures at four Italian Centres (Roma, Pavia, Padova and Torino). Median age at transplantation was 8 years (range $0.8-$ 22). Indications for HSCT were both malignant (79 pts) and non-malignant (14 pts) conditions. The conditioning regimen was myeloablative in almost all patients (89 pts). The donor was an HLA-identical sibling for 13 pts (14\%), a MatchedUnrelated Donor (MUD) for 59 pts (63.4\%) and a PartiallyMatched Family Donor for 21 pts (22.6\%). The source of stem cells was bone marrow in 47 cases $(50.5 \%)$, cord blood in 10 (10.8\%) and peripheral blood after mobilization in 36 (38.7\%). Overall, grade aGVHD at ECP start was Grade I in 7 pts (7.5\%), II in 59 pts (63.4\%), III in 23 pts (24.7\%) and IV in 4 pts (4.3\%). ECP schedule was 3 procedures per week for 46 pts and 2 per week for 47 pts. Systemic corticosteroids were administered to 85 pts (before starting ECP in 58; during ECP in the remaining 27).
Results: No serious adverse event was recorded. A complete response (CR) was obtained in $73 \%$ of patients, a partial $/$ mixed response or stable disease was observed in $15 \%$, and $12 \%$ of patients developed progressive disease. Median time to steroid tapering was 10 days (95\% Confidence Interval, Cl, 914), while median time to best overall response was 25 days (95\% Cl, 15-30). The time elapsing from aGVHD onset to ECP start, the number of procedures per week and the number of mononuclear cells per microliter at ECP start did not show significant influence on ECP response rate (RR). Similarly, ECP resulted in comparable response rate if employed as first, second, third, or subsequent line of therapy. Not surprisingly, patients with grade I-II aGVHD showed better responses than those with grade III-IV aGVHD (RR $85 \%$ versus $48 \%, P=0.0015$ ). For the whole cohort, the 5-year cumulative incidence of chronic (cGVHD) was $40.4 \%$ (95\% Cl, 28.5-52), with a $71 \%$ incidence for ECP non-responders $(95 \% \mathrm{Cl}, 46.6-85.7)$ compared to $28.8 \%$ for patients who obtained CR after the procedures $(95 \% \mathrm{Cl}, 16.1-42.9)(P<0.0001)$. Most importantly, ECP-response resulted in a significantly superior overall survival for patients with CR after ECP [3-year OS 77.9\% (95\% Cl, 58.8-88.9) versus $21.2 \%(95 \% \mathrm{Cl}, 6.4-41.6)$ for patients with partial, mixed or no response, $P<0.0001$ ] (Figure I).

Conclusion: ECP is a safe and effective treatment in children experiencing aGVHD after allogeneic HSCT, allowing rapid steroid tapering. ECP can be effective also after several lines of immunosuppressive therapy. Most importantly, CR to ECP results in a lower incidence of CGVHD and a better overall survival.

Disclosure of Interest: None declared.

\section{P480}

Gastrointestinal fibrosis and obstruction following extensive steroid refractory severe acute graft versus host disease in children successfully treated with mesenchymal stromal cells

F. Calkoen ${ }^{1}$, J. Schweizer ${ }^{2}$, L. Mearin ${ }^{2}$, L. M. Ball ${ }^{3, *}$

${ }^{1}$ Pediatrics, ${ }^{2}$ Pediatrics gastroenterology and hepatology, ${ }^{3}$ Pediatrics Hematopietic stem cell transplantation, Willem Alexander Childrens Hospital, LUMC, Leiden, Netherlands

Introduction: Acute gastrointestinal graft-versus-host disease (Gl aGvHD) complicating hematopoietic stem cell transplantation (HSCT) is a cause of severe morbidity and mortality. Steroid therapy resistant cases have a poor prognosis. Mesenchymal stromal cells (MSC) have been reported as salvage treatment.

Material (or patients) and methods: We report the occurrence of gastrointestinal fibrosis and obstruction developing in a 4 children treated with MSC who survived extensive GI aGVHD. Results: From Dec 2004 to Nov 2014, children with severe steroid refractory GI aGvHD (> Grade III), who received MSC as salvage treatment were evaluated. 23 patients. 11 male 12 female aged 10 months- 18 years were included. GI aGVHD was histologically confirmed in 20/22 patients. One child died within 7 days of MSC infusion and was excluded from further analyses.

Eleven children responded completely (CR: no evident GVHD clinical/biopsy proven) and 6 partially (PR: at least one grade) at 30 days following the $1^{\text {st }}$ MSC infusion, (1-3 infusions total), with 5 no response or progression. Four out of seventeen patients surviving $>100$ days post initial MSC infusion developed Gl fibrosis and obstruction. One girl, aged 15 months with total bowel involvement at 3 months post MSC infusions, required total parenteral nutrition (TPN), but died 3 years later from pulmonary cGHVD. In the 2 nd patient a boy aged 15 yrs, fibrosis developed 2 months post MSC and was localized to the terminal ileum and caecum. He underwent surgical resection, but later developed chronic diarrhea due to cGVHD of the pancreas. Another boy, aged 33/4 yrs required local surgical resection of a pre-terminal ileum stricture causing complete bowel obstruction, occurring 
4 months after MSC infusion and remains well with no GI or cGVHD problems. Finally a boy, aged 17 yr developed multiple stenotic lesion throughout the ileum and jejunum with intermittent bowel obstruction at 4 months post MSC infusion, whereby surgical intervention and possible small bowel transplant may be required.

Conclusion: Fibrosis and obstruction have been rarely reported as a complication of severe CGVHD of the gut (1). MSC may have a fibrogenic potential in a mouse model of hepatitis but has never been described in GVHD setting (2). The role in the development of complications following survival from extensive mucosal damage as seen in these patients is unclear, but stenosis and fibrosis is more likely a reflection of the natural progression of disease following improved overall survival with MSC from such devastating bowel disease. However these cases highlight the need for extended follow-up in children enrolled in novel treatment studies as response rates alone do not reflect the burden of complications ensuing after resolution of severe aGVHD.

References: 1. Strasser, S Mc Donald G in Thomas' Hematopoetic Cell Transplantation 2004

2. Baertschiger et al., PLoS ONE 4, 2009

Disclosure of Interest: None declared.

\section{P481}

Low Dose Alemtuzumab without Methotrexate for Graft versus Host Disease Prevention

M. Liga ${ }^{1, *}$, P. Tsirigotis ${ }^{2}$, E. Triantafyllou ${ }^{1}$, A. Sarantopoulos ${ }^{1}$, A. Siorenta ${ }^{3}$, K. Gkirkas ${ }^{2}$, M. Stamouli ${ }^{2}$, K. Petevi ${ }^{2}$, A. Spyridonidis ${ }^{1}$ ${ }^{1}$ Bone Marrow Transplantation Unit, University Hospital of Patras, Patras, ${ }^{2}$ Department of Internal Medicine, University of Athens, ${ }^{3}$ Immunology Lab \& National Tissue Typing Centre, General Hospital of Athens "G.Gennimatas", Athens, Greece

Introduction: In a previous dose evaluation study, by using progressively decreasing doses of Alemtuzumab (AL), we showed that a low dose of $10 \mathrm{mg}$ and $20 \mathrm{mg} \mathrm{AL}$ was effective in preventing graft versus host disease (GVHD) after allogeneic stem cell transplantation (allo-SCT) from matched related (MRD) or unrelated (MUD), and mismatched unrelated (MMUD) donor, respectively. In the present trial we prospectively evaluated the efficacy of low dose AL in a large group of 148 consecutive patients.

Material (or patients) and methods: One hundred and forty eight patients with various hematological malignancies were included in the study. Patients underwent allo-SCT in two affiliated units in Greece. AL was administered intravenously as part of the conditioning regimen on days -2 and -1 before graft infusion. Patients with MSD or MUD ( $8 / 8$ allele matched) donor received $10 \mathrm{mg}$ total dose $A L$, while a total dose of $20 \mathrm{mg} \mathrm{AL}$ was administered in cases with a MMUD (7/8 allele matched). Single agent cyclosporine (CyA) was administered as post-transplant GVHD prophylaxis. The graft source was mobilized peripheral blood stem cells (PBSC) in all cases. Patient characteristics are shown in Table 1.

Results: Twenty-five out of 148 developed acute GVHD grade 2-4 in a median of 43 days after allo-SCT (range, 10-150 days), while 10 patients developed aGVHD-grade 3-4. The cumulative incidence of aGVHD-grade 2-4 and grade 3-4 was 20\% and $7 \%$ respectively. Chronic GVHD developed in 52 patients in a median of 150 days (range, 110-700 days) after allo-SCT. The cumulative incidence of chronic GVHD was $41 \%$. Relapse occurred in 24 patients in a median of 7.5 months (range, 1-24 months), for a cumulative incidence of $20.5 \%$. Thirty-one patients died from causes not directly related to relapse. Causes of non-relapse mortality (NRM) were sepsis in 10, GVHD in 7 , viral infection $(B K, J C)$ in 5 , hemorrhage in 2, VOD in 2, pulmonary toxicity in 4 , and solid tumor in 1 patient. The cumulative incidence of NRM was $25 \%$. Overall (OS) and event free survival (EFS) at 2 years were $62 \%$ and $55 \%$, respectively. In multivariate analysis, transplantation from a MMUD was the most significant factor associated with increased NRM and decreased OS.

Conclusion: Low dose AL is effective in preventing GVHD after allo-SCT from MRD and MUD. However transplantation from a MMUD was associated with a worse outcome due to increased NRM.

Disclosure of Interest: None declared.

\section{P482}

Imbalances in the reconstitution of naïve and memory $T$ cell subsets in chronic graft versus host disease

M. Soares ${ }^{1, *}$, R. Azevedo ${ }^{1}$, I. Ferreira ${ }^{7}$, S. Bucar ${ }^{1}$, A. Alho ${ }^{1}$, E. Espada ', C. Juncal', N. Camacho ${ }^{3}$, C. Martins $^{3}$, J. Carmo ${ }^{3}$ F. Lourenço ${ }^{3}$, R. Moreno ${ }^{3}$, C. Vaz ${ }^{4}$, A. Campos ${ }^{4}$, F. Campilho ${ }^{4}$, R. Ferreira ${ }^{4}$, J. Ritz ${ }^{5}$, J. Lacerda ${ }^{7}$

${ }^{1}$ Jlacerda lab, Hematology and Transplantation Immunology, Instituto de Medicina de Molecular, Faculdade de Medicina da Universidade de Lisboa, ${ }^{2}$ Serviço de Imunohemoterapia, ${ }^{3}$ Serviço de Hematologia e Transplantação de Medula, Hospital de Santa Maria, Lisboa, ${ }^{4}$ Serviço de Transplantação de Medula Ossea, Instituto Português de Oncologia, Porto, Portugal, ${ }^{5}$ Division of Hematologic Malignancies and Cancer Vaccine Center, Dana-Farber Cancer Institute, Boston, United States

Introduction: Absence of tolerance after hematopoietic stem cell transplantation (HSCT) results in the emergence of chronic Graft versus Host Disease (cGVHD), a potentially

Table l.

\begin{tabular}{|c|c|c|c|}
\hline \multicolumn{2}{|c|}{ Patient character istics } & \multicolumn{2}{|c|}{ Transplant characteristics } \\
\hline No. of pts & 148 & Disease Status at HSCT & \\
\hline Age, median (range) & $45 y(17-70)$ & CR-1 & 87 \\
\hline Male / Female & $81 / 67$ & CR-2 & 21 \\
\hline Disease & & REL/REFR & 27 \\
\hline AMI & 69 & Untreated & 13 \\
\hline ALL & 41 & Donor & \\
\hline Mixed AL & 4 & Sibling & 89 \\
\hline MDS & 13 & $\operatorname{VUD}(\mathrm{S} / \mathrm{S}-7 / \mathrm{S}$ match $)$ & $59(35-24)$ \\
\hline AAPNH & 3 & Campath Dose & \\
\hline Lymphoma (NHL, HD) & 10 & $10 \mathrm{mg}$ & 110 \\
\hline MPN (CML,OMF) & 8 & $20 \mathrm{mg}$ & 35 \\
\hline Disease Risk & & $30 \mathrm{mg}$ & 3 \\
\hline Standard & 46 & Sex mismatch & 49 \\
\hline High & 102 & ABO incompatibility & 61 \\
\hline
\end{tabular}


life-threatening condition associated with impaired regulatory $T$ cell (Treg) reconstitution ${ }^{(1)}$. To better understand this relationship, we evaluated the kinetics of the different $T$ lymphocyte subsets emerging in patients who engraft with unrelated donor hematopoietic stem cells.

Material (or patients) and methods: 39 consecutive patients were monitored. 11 were excluded from the analysis due to disease relapse and/or death due to infection or acute GVHD in the first 9 months post-HSCT. 28 patients (16M, $12 \mathrm{~F}$; median age 49) were eligible for the analysis, of whom 25 are alive with a median follow-up of 2 years. The diagnosis were: AML $(n=16)$, ALL $(n=1)$, Blastic CML $(n=2), \mathrm{NHL}(n=5), \mathrm{HL}(n=1)$, myeloma $(n=2)$, myelofibrosis $(n=1)$. All unrelated donors $(17 \mathrm{M}, 11 \mathrm{~F}$; median age 33$)$ were $10 / 10(n=14)$ or $9 / 10(n=14)$ allele HLA matched. Stem cells source was equally distributed between PBPC and BM ( $n=14$ for both). All patients received the same reduced intensity conditioning (RIC) regimen with Fludarabine/Melphalan/ATG and GVHD prophylaxis with MMF plus cyclosporine. 19 developed acute GVHD (5 Gl, 9 GII, 5 GIII) and 12 patients developed CGVHD (5 mild, 3 moderate, 4 severe). Peripheral blood was collected at months 1, 2, 3, 6, 9 , 12,18 and 24 after HSCT. Flow cytometry was performed in whole blood using CD31FITC, Ki-67PE, CD3PerCPCY5.5, CD45RA and CD25 in PE-CY7, CD4APC, CD62L and CD127 in APCeFlour780 and Foxp3eFluor450. Data was acquired on a LSR Fortessa(BD Biosciences) and analyzed using Flow Jo ${ }^{\circledR}$ Naïve $T$ cells were identified as CD45RA+CD62L+, Central Memory (CM) as CD45RA-CD62L+, Effector Memory (EM) as CD45RA-CD62L- and Terminal Effectors expressing CD45RA (TEMRA) as CD45RA+CD62L-. CD31 in CD4+CD45RA+CD62L+ was used to identify Recent Thymic Emigrants (RTE). CD4, CD25, CD127and Foxp3 were used to identify Tregs.

Results: Patients with cGVHD had statistically significantly less CD4+ Naïve, CD4+ RTE, total Treg, Treg Naïve and Treg RTE cells than those without CGVHD. This was associated with lower Ki67 in CM and EM subtypes of both total CD4+ and Treg cells. In contrast, patients with cGVHD had significantly higher numbers of Naïve and TEMRA CD8+ cells. This was evident from the initial time points after HSCT, persisted throughout the follow-up period, and was not due to significant increases in proliferation, as assessed by Ki67 expression.

Conclusion: In a homogenous population of patients submitted to unrelated donor HSCT after an ATG-containing RIC regimen, we report a clear association between cGVHD development and impaired CD4 Naïve and RTE reconstitution after 6 months post HSCT. This impairment is prominent in the Treg compartment, establishing a clear link between the emergence of cGVHD and the lack of Treg-mediated immune tolerance. We further highlight an early and persistent increase in CD8 Naïve and TEMRA cells in patients developing CGHVD. We speculate that low Treg cell numbers coupled to the appearance CD8 Naïve cells early after HSCT may result from defective negative selection processes generating an excess of host-reactive CD8 Naïve that subsequently differentiate into an TEMRA phenotype mediating CGVHD.

References: 1. Matsuoka K, et al. JCl, 2010.

Disclosure of Interest: This work was supported by a Harvard Medical School Portugal Collaborative Research Grant. None declared.

\section{P483}

Effect of Infliximab in Steroid-Refractory Acute GVHD

M. Nygaard, ${ }^{1, *}$ N. Smedegaard Andersen ${ }^{1}$, D. Hovgaard ${ }^{1}$, C. Moser', G. Olesen ', I. Schjødt ', C. Heilmann ${ }^{3}$, H. Sengeløv' ${ }^{1}$ Department of Hematology, ${ }^{2}$ Department of Clinical Microbiology, ${ }^{3}$ Department of Pediatrics, University Hospital Rigshospitalet, Copenhagen, Denmark

Introduction: Steroid refractory acute Graft versus Host Disease (aGVHD) is a life threatening condition, but there is no consensus regarding second line treatment. We present the results of Infliximab therapy evaluated by recently proposed reponse criteria for studies on treatment of aGVHD (1)(2)(3).
Material (or patients) and methods: We retrospectively analyzed the outcomes of 54 patients treated with infliximab for steroid refractory aGVHD between 2000 and 2010. Both classical aGVHD occurring within 100 days after HCT and lateonset aGVHD were included. Complete response (CR) was defined as resolution of all indicators of aGVHD, very good partial response (VGPR) was defined as described by Martin et al.(1) Partial response (PR) was improvement of one or more grades in an affected organ without progression or de novo aGVHD in other organs. Response was evaluated at day 28 and endpoint was 6 months freedom from treatment failure (6MFTF), defined as absence of death, relapse of malignancy or need for additional systemic immunosuppression(3).

Results: There were 35 male and 19 female patients with a mean age of 49.6 years (range 25-73). Conditioning was myeloablative in 16 and non-myeloablative in 38 patients. There were 29 related and 25 unrelated donors. Overall aGVHD grade was II $(n=17)$, III $(n=25)$ and IV $(n=12)$. Involvement of the gut was observed in 42 (78\%) of the patients. Fifty (92\%) of the patients received MP $2 \mathrm{mg} / \mathrm{kg}$ and the remaining $1 \mathrm{mg} / \mathrm{kg}$ or less.

Mean number of Infliximab doses given by day 28 were 3 (range 1-5). Mean total number of Infliximab doses given were 4 (range 1-12). Median days from start of steroid treatment till start of Infliximab were 6 (range 1-69). At day 28, CR/VGPR were obtained in $31(56 \%)$ of the patients. 21 patients (39\%) achieved 6 months freedom from treatment failure, and the majority of these patients had CR/VGPR at day $28(n=17$, $81 \%)$. Reasons for treatment failure within 6 months were death $(n=21)$, additional immunosuppression $(n=11)$ and relapse $(n=1)$. Response was seen in both gut, skin and liver manifestations. Low grade of aGVHD was the only baseline characteristic significantly associated with achievement of day 28 CR/VGPR and 6MFTF.

Day 28 responders had 6 months OS at 69\% versus 17\% in non-responders, $P=0.001$, and a 24 months OS at $57 \%$ vs $8 \%$ in non-responders, $P<0.0001$, respectively. All patients alive were observed for more than 24 months. Causes of death were primarily infections (48\%) and GVHD (30\%).

Conclusion: We show that CR/VGPR at day 28 is an important predictor of survival after second line therapy with infliximab for steroid-resistant acute GVHD. Treatment with infliximab resulted in a 2-year survival rate among responders at $57 \%$, despite a large proportion (69\%) of patients with grade 34 aGVHD.

References: 1. Martin PJ et al. Endpoints for clinical trials testing treatment of acute graft-versus-host disease: a joint statement. Biol Blood Marrow Transplant 2009 Jul;15(7):777-84.

2. MacMillan ML et al. The best endpoint for acute GVHD treatment trials. Blood 2010 Jul 1;115(26):5412-7.

3. Sengsayadeth $\mathrm{S}$ et al. Six-month freedom from treatment failure is an important end point for acute GVHD clinical trials. Bone Marrow Transplant. 2014 Feb;49(2):236-40.

Disclosure of Interest: None declared.

\section{P484}

Graft-versus-Host Disease after matched sibling or unrelated donor allografts; the role of ant-thymocyte globulin

M. Brune ${ }^{1, *}$, M. Lisak ${ }^{1}$, M. Remberger ${ }^{2}$, M. Nicklasson ${ }^{1}$, K. Boroskina ${ }^{3}, H$. Anderson ${ }^{4}$, J. Mattsson ${ }^{3}$

${ }^{1}$ Section of Hematology \& Coagulation, Sahlgrenska Academy, Gothenburg, ${ }^{2}$ OnkPat, Karolinska Institutet, Karolinska University Hospital, Huddinge, ${ }^{3}$ Center for Allogeneic Stem Cell Transplantation, Karolinska University Hospital, Stockholm, ${ }^{4}$ Dpt of Cancer Epidemiology, Clinical Sciences, Lund, Lund, Sweden

Introduction: Chronic GvHD (cGvHD) is the main sequel after alloSC affecting more than $50 \%$ of patients, and is associated with reduced quality of life, late infections and mortality. Pretransplant anti-T-cell globulin (ATG) is widely used in matched unrelated transplants (MUD) and do reduce incidence and severity of cGvHD. 
For this report, we retrospectively analyzed clinical outcome after 4 years alloSCT in two Swedish centers (Huddinge, Stockholm and Sahlgrenska, Gothenburg). The primary objective was to assess and compare clinical outcome, and in particular the prevalence of cGvHD in pts transplanted either from an HLA-identical sibling donor (MSD), or a MUD. In the latter group all pts received ATG (Thymoglobuline ${ }^{\circledR}$ or ATGFresenis ${ }^{\mathbb{R}}$ ). As surrogate marker for severity of cGvHD and for patients' strain, we chose standard therapy i.e. glucocorticosteroid (GC), and/or any other immunosuppressant (IS).

Material (or patients) and methods: Thirty-six pts with missing data, or individualized GvHD prophylaxis, were excluded. Remaining 269 consecutive pts, allografted 20112014 included 155 males and 114 females, age 52 (18-72) yrs). Pts were allografted from an MSD $(n=69)$ or a matched $(\geq 9 / 10)$ MUD $(n=200)$. Median CD34 ${ }^{+}$dose was $6(1-14) \times 10^{6} /$ $\mathrm{kg}$. Diagnoses were 148 acute leukemia, $87 \mathrm{MDS} / \mathrm{MPN}, 21$ lymphoma and 14 chronic leukemia. Disease status were CR1 $(n=142)$, advanced disease $(n=72)$, not defined $(n=55)$. Pts received reduced (RIC; $n=179$ ), or myeloablative (MAC; $n=190$ ) conditioning, and in the MUD group also thymoglobuline (median $4.1(2.5-8) \mathrm{mg} / \mathrm{kg}$, or ATG-Fresenius $(30-40 \mathrm{mg} /$ kg; $n=13)$. Post-Tx IS was CyA/Mtx $(n=236)$, or tacrolimus/ sirolimus $(n=13)$. Chi-square tests were used to compare discrete variables between the MSD and MUD groups. For survival type variables Kaplan-Meier curves and cumulative incidence functions, log-rank tests and Cox regression were used.

Results: As per 2015-12-01, median follow-up for living pts was 24 (1-55) months (mo). Comparisons between MSD and MUD groups revealed an even distribution of age and diagnoses, but significantly more males and RICs in the MUD group (61 vs $47 \%$ and 60 vs $45 \%$, respectively). Leukemia-free survival (LFS) was superior in the MSD group $(P=0.008)$; at 24 months post-Tx LFS was $75 \%$ in the MSD and $51 \%$ in the MUD group. Overall survival (OS) showed a trend towards better $O S$ in the MSD group $(P=0.09)$. The cumulative incidence of relapse was higher in the MUD group $(P=0.018)$; at 3 yrs $39 \%$ vs MSD $24 \%$. The better results in the MSD group remained after adjusting for differences in prognostic factors.

In the MSD and MUD groups the incidence of acute GvHD was similar (71 vs $63 \%$; n.s.). In contrast, the incidence of cGvHD the first year post-Tx was higher in the MSD group (66 vs 36\%; $P<0.001)$. At 12 mo the prevalence of ongoing GC therapy was $56 \%$ (27/48 pts) in the MSD group and 25\% (30/119) in the MUD group $(P<0.001)$. MSD patients had higher GC dose $(P<0.001)$, and more often additional IS therapy $(P<0.01)$. A similar pattern was observed at 24 mo with a significantly higher prevalence of ongoing GC therapy in the MSD group; $48 \%$ vs $17 \%$ in MUD pts.

Conclusion: Due to lower NRM and relapse rate, MSD pts had superior LFS compared to MUD/ATG pts. However, the prevalence of CGvHD and ongoing GC therapy was significantly higher in the MSD group. More studies are needed to define an expedient dose of ATG in MUD transplants - and possibly also in MSDs.

Disclosure of Interest: None declared.

P485

High incidence of severe chronic GVHD after HSCT with sibling donors.

An single center analysis

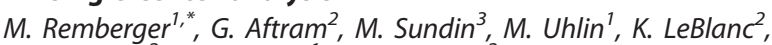
A. Björklund ${ }^{2}$, J. Mattsson ${ }^{1}$, P. Ljungman ${ }^{2}$

${ }^{7}$ Center for Allogeneic Stem Cell Transplantation, ${ }^{2}$ Hematology,

${ }^{3}$ Pediatrics, KAROLINSKA INSTITUTET, Stockholm, Sweden

Introduction: Major obstacles to success after HSCT are acute and chronic GVHD, infections, and relapse of malignancy.

Material (or patients) and methods: Consecutive patients who underwent HSCT between the years 2000 and 2014 either (1) with a HLA-identical sibling donor and not receiving ATG $(n=230)$ or (2) with an HLA-A, $-\mathrm{B}$, and -DR matched unrelated donor (URD) and receiving ATG $(n=445)$ were eligible for the study. Only patients with hematological malignancies were included. The median age was 45 ( $<1$ to 72 ) years and there were 116 children ( $<18$ years of age) (17\%). Most patients $(n=380,56 \%)$ had an acute leukemia: $153 \mathrm{AML}$ and $127 \mathrm{ALL}$. Two hundred and eighty-four (42\%) were in first complete remission (CR) or chronic phase (CP) and were considered to be low risk, while 391 (58\%) were in later stages and were considered to be high risk.

Results: Patients with a sibling donor had significantly better overall survival (OS; $67 \%$ vs. $56 \%, P=0.02)$, better relapse-free survival (RFS; $60 \%$ vs. $50 \%, P<0.01$ ), and lower transplantrelated mortality (TRM; $14 \%$ vs. $21 \%, P=0.025$ ) than patients with URDs. Both the incidence of overall cGVHD (51\% vs. $28 \%$, $P<0.001)$ and severe cGHVD $(12 \%$ vs. $3 \%, P<0.001)$ were higher in the sibling donor cohort than in patients transplanted with URDs. A sibling donor also correlated to more overall and severe CGVHD in multivariate anaysis.

In the sibling donor cohort, age (RH 0.61, $P=0.002)$, PBSCs (RH 26.3, $P=0.006$ ), and an Immunized Female donor to Male recipient (HR 2.83, $P=0.03$ ) were associated with an increased frequency of severe cGVHD. Patients with all three risk-factors had an incidence of severe cGVHD of $31 \%$, compared to $0 \%$, $10 \%$ and $15 \%$ in patients with 0,1 and 2 risk-factors.

Conclusion: In this study, we have isolated a population of patients having a high risk for severe chronic GVHD. As severe chronic GVHD is associated with considerable morbidity and mortality, actions to reduce the incidence among patients at high risk must be considered. However, since according to the present and previous findings the overall survival is better for patients with sibling donors, any change should be done carefully in larger prospective studies.

Disclosure of Interest: None declared.

\section{P486}

Improvement of GvHD-control among pediatric leukemia patients after TCRaß+/CD19+-depletion of transplant from matched unrelated and haploidentical family donors: a single-center retrospective analysis of two GVHDprophylaxis regimens

Z. Shekhovtsova', L. Shelikhova', D. Balashov', E. Kurnikova', I. Muzalevsky ${ }^{2}$, A. Kazachenok ${ }^{3}$, J. Skvortsova ${ }^{1}$, M. Ilushina ${ }^{\prime}$, I. Shipitsina', 'D. Shasheleva', R. Khismatullina', A. Livshits $^{\prime}$, S. Blagov ${ }^{1}$, A. Abrosimov ${ }^{1}$, E. Gutovskaya ${ }^{1}$, J. Starichkova ${ }^{4}$, D. Litvinov ${ }^{5}$, G. Novichkova ${ }^{3}$, A. Maschan ${ }^{3}$, M. Maschan ${ }^{1, *}$

${ }^{1} \mathrm{HSCT},{ }^{2}$ Laboratory of Transplant Processing and Cell Preparations, "Dmitry Rogachev Federal Research Centre of Pediatric Hematology, Oncology and Immunology", Moscow, Russian Federation, ${ }^{4}$ Informatic technologies, ${ }^{5}$ Oncohematology, "Dmitry Rogachev Federal Research Centre of Pediatric Hematology, Oncology and Immunology", Moscow, Russian Federation

Introduction: Graft-versus-host disease (GvHD) remains to be a factor, which induces significant morbidity and mortality after allogeneic hematopoietic stem cell transplantation (HSCT). We did a retrospective analysis of two GvHDprophylaxis regimens in a cohort of pediatric patients with acute leukemia who underwent HSCT from HLA-matched unrelated and haploidentical family donors after TCRa $\beta$ +/CD19+-depletion in our center.

Material (or patients) and methods: Between May 2012 and June 2015108 transplantations from 63 HLA-matched unrelated and 45 haploidentical donors were performed for children ( 73 boys and 35 girls with median age $=9$ years, range 0,6-23 years) with acute lymphoblastic $(n=46)$ and acute myeloblastic $(n=62)$ leukemia. TCRa $\beta+/ C D 19+-d e p l e t i o n$ of HSCT with CliniMACS technology was implemented in all cases. For all patients it was a first allogeneic HSCT. The majority (88\%) of the patients received Treosulfan-based condition regimen. Remaining $12 \%$ of patients received TBIbased conditioning regimen. Two regimens of GVHD prophylaxis were used. Regimen $1(n=59)$ : ATGAM $50 \mathrm{mg} / \mathrm{kg}$ and 
post-transplant Tacro/MTX $(n=47)$ or no post-transplant prophylaxis $(n=12)$; regimen $2(n=49)$ : thymoglobulin $5 \mathrm{mg} /$ $\mathrm{kg}$, rituximab $200 \mathrm{mg} / \mathrm{m}^{2}$ and post-transplant bortezomib $(n=$ 36) or Tacro/MTX $(n=8)$, no post-transplant prophylaxis $(n=5)$ or other agents as post-transplant GvHD-prophylaxis. The median dose of CD34+ cells in the transplant was $8 \times 10^{6} / \mathrm{kg}$ (range $1-21$ ), TCRa $/ \beta-17 \times 10^{3} / \mathrm{kg}$ (range $0,6-190$ ).

Results: Cumulative incidence of neutrophil engraftment at 30 days was 96\%; cumulative incidence of platelets engraftment at 60 days was 98\%; two patients did not achieve engraftment and required second transplantation. Median time to neutrophil and platelet engraftment was 15 and 14 days, respectively. Cumulative incidence of acute GvHD II-IV grade was $23 \%$ (95\% Cl: $16-32)$; grade III-IV - 7\% (95\% Cl: 4-14) and chronic GvHD - 25\% (95\% Cl: 18-35). Cumulative incidence of acute GvHD was significantly lower within the group with regimen 2: $9 \%$ (95\% Cl: 3-26) vs. 30\% (95\% Cl: 22 46), $P=0,02$. Regimen 2 was also effective in prevention of chronic GvHD: $\mathrm{Cl}$ at 1 year after HSCT was $14 \%$ vs. $29 \%$, $P=0,12$. Median time of follow-up for survivors was 22 months (range 91 days -40 months). Cumulative incidence of relapse at 2 years after $\mathrm{HSCT}$ was $30 \%(95 \% \mathrm{Cl}: 23-42)$ and TRM - $17 \%$ (95\% Cl: 10-26), without significant statistical difference between leukemia or donor type, and GvHD-prophylaxis regimens. EFS (event $=$ death or relapse) at 2 years after HSCT was $55 \%$ (95\%Cl: $45-66)$, OS - 58\% (95\%Cl: 48-69). Univariate analysis of event-free or overall survival probabilities did not reveal any statistical difference.

Conclusion: In our retrospective single-center study we revealed, that Thymoglobulin/Rituximab/Bortezomib improves GVHD control in recipients of $T C R a / \beta$ - depleted grafts in comparison to ATGAM/Tacro/MTX.

Disclosure of Interest: None declared.

\section{P487}

Extracorporeal Photopheresis for the Treatement of Acute and Chronic Graft Versus Host Disease: A Retrospective Multicentric Analysis on 94 Patients

M. Malagola ${ }^{1, *}$, V. Cancelli ${ }^{1}$, C. Skert ${ }^{1}$, P. Ferremi Leali ${ }^{2}$, E. Ferrari ${ }^{2}$ A. Tiburzi ${ }^{2}$, M. Sala ${ }^{2}$, I. Donnini ${ }^{3}$, P. Chiusolo ${ }^{4}$, A. Muffetti ${ }^{5}$, M. Battista ${ }^{6}$, A. Turra ${ }^{7}$, F. Cattina ${ }^{7}$, B. Rambaldi ${ }^{1}$, A. Bosi ${ }^{3}$, S. Sica ${ }^{4}$, P. Corradini ${ }^{5}$, F. Patriarca ${ }^{6}$, M. Marini $^{2}$, D. Russo $^{7}$

${ }^{1}$ Experimental and Clinical Sciences, CHAIR OF HEMATOLOGY, BONE MARROW TRANSPLANT UNIT, UNIVERSITY OF BRESCIA, AO SPEDALI CIVILI BRESCIA, ${ }^{2}$ Immunohematology and Transfusione Medicine Unit, AO Spedali Civili di Brescia, Brescia, ${ }^{3}$ Bone Marrow Transplant Unit SODC Ematologia, AOU Careggi, Firenze, ${ }^{4}$ UO Ematologia, Policlinico Universitario A Gemelli, Università Cattolica del Sacro Cuore, Roma, ${ }^{5}$ SC Ematologia, Istituto Nazionale Tumori, Milano, ${ }^{6}$ Clinica Ematologica, AOU Santa Maria della Misericordia, Udine, Italy

Introduction: Extracorporeal photopheresis (ECP) is considered a valid second-line treatment of both acute and chronic GVHD, which represent the most frequent causes of morbidity and mortality after allo-SCT in hematological malignancies. According to an observational multicentric study, we report here a retrospective analysis on the use and results of ECP in 94 patients with acute and chronic GVHD, recruited in 6 italian Centres.

Material (or patients) and methods: 94 patients with aGVHD grado grater than $2(n=45)$ and CGVHD $(n=49)$ were submitted to ECP after first-line steroid-based therapy. ECP treatment consisted on conventional bi-weekly procedure for 4 weeks. Then, most of the patients were treated with biweekly procedures every 2 weeks for 4 other weeks. Response to steroid was assessed after 7 days for aGVHD and 15 days for CGVHD.

Results: aGHVD: 45 patients with aGVHD were treated with ECP. In 22 (49\%) ECP was used as salvage treatment, being these patients non responsive to steroid (NR). 19/22 (86\%) achieved a CR after ECP, whose median duration was 77 days (range 20-1112). The median duration of steroid therapy was
101 days (range 24-772), with a median of 22 days (range 7-80) on full steroid dose. The median duration of steroid on tapering was 82 days (range 0-756). 8/22 (36\%) patients developed a cGVHD (extensive in 6 cases and limited in 2 cases). After a median follow up of 419 days (range 61-2149), $11 / 22(50 \%)$ patients are alive. In $23 / 45$ cases (51\%) ECP was used earlier, being these patients responsive to steroid, with the aim to reach faster or consolidate the $C R$ and to reduce the duration of steroid treatment. Among these patients, 22 (96\%) achieved the CR after ECP. In this setting of patients, the median duration of steroid was 88 days (range 32-719), with a median of 18 days (range 4-69) on full dose steroid therapy. The median duration of steroid on tapering was 68 days (range 0-700). The median duration of ECP treatment was 194 days (range 22-933). 7/23 (30\%) patients developed a cGHVD (extensive in 6 cases and limited in 1 case). With a median follow up of 741 days (range 81-1819), 17/22 (77\%) patients are alive.

CGVHD: 49 patients with CGVHD were treated wiht ECP. 12/49 (24\%) and 37/49 (76\%) had limited and extensive cGVHD, respectively. The median time of CGVHD onset was 193 days (range 43-3756), the median time from steroid to ECP was 26 days (range $0-1347$ ) and the median duration of ECP was 276 days (range 19-2861). The median duration of steroid therapy in this group was 276 days (range 19-2861). 22/49 patients (45\%) and $17 / 49$ patients (35\%) achieved a CR and PR, respectively. After a median follow up of 811 days (range 152$4676), 44 / 49$ patients (90\%) are alive, 28 of whom (64\%) on immunosuppressive therapy.

Conclusion: ECP confirmed to be an effective treatment in aGVHD steroid-resistant patients as it can induce a CR in more than $80 \%$ of the cases, with $50 \%$ of these patients becoming long-term survivors. In aGVHD steroid-responsive patients it can probably allow a faster steroid tapering, with more than $70 \%$ of patients becoming long term survivors. In cGVHD steroid-resistant patients, ECP are associated with an overall response rate of $80 \%$, with $90 \%$ of patients who become longterm survivors.

Disclosure of Interest: None declared.

\section{P488}

Gut ultrasound as a "decision support" method for GVHD diagnosis

M. Drokov 1,*, E. Parovichnikova ${ }^{1}$, D. Dubnyak ${ }^{1}$, G. Yatsyk $^{2}$, A. Kireeva ${ }^{2}$, O. Pirikova ${ }^{2}$, L. Kuzmina ${ }^{1}$, V. Vasilyeva ${ }^{1}$, O. Koroleva ${ }^{1}$, E. Mikhalcova ${ }^{1}$, N. Popova ${ }^{1}$, V. Savchenko ${ }^{1}$ on behalf of CIC 930 ${ }^{1}$ Bone Marrow Transplant Department, ${ }^{2} \mathrm{MRI}$ and ultrasound department, NATIONAL RESEARCH CENTER FOR HEMATOLOGY, Moscow, Russian Federation

Introduction: As has been shown before (Malard et al., 2014) gut ultrasound is a suitable method for evaluating the thickness of the intestinal wall. However, this cheap and available method still couldn't take place in the diagnosis of graft-versus-host disease (GVHD) in patients after allogeneic stem cell transplantation. So we try to provide comparative study with a gut ultrasound as "decision support" method.

Material (or patients) and methods: Since June in 2014 till November 2015 - 64 patients with hematological malignancies were transplanted from allogeneic donors(matched related $n=28$, MUD $n=19$, mismatched unrelated $n=17$ )in CIC930. The ONLY inclusion criteria for the study was the stool volume more than $500 \mathrm{ml} /$ day. Gut ultrasound was performed at Aloka Alfa 10 ultrasound system (Japan) within 72 hours after the first signs of volume diarrhea (>500 ml/day). 5 patients constituted "negative" control group (no diarrhea, before HSCT). 14 patients after allogeneic HSCT with a median age 37 years (27-53 years; 7 males, 7 females, AML $n=5$, ALL $n=4$, MDS $n=1$, LPD $n=2, \mathrm{CML} n=1, \mathrm{CMML} n=1)$ and 3 patients with MM and median age 51 years (35-64 years)after autologous HCST were included. Acute or chronic GVHD with gut involvement ("alloimmune gut damage")was diagnosed and treated in 9 patients after allo-HSCT (acute GVHD II-IV 


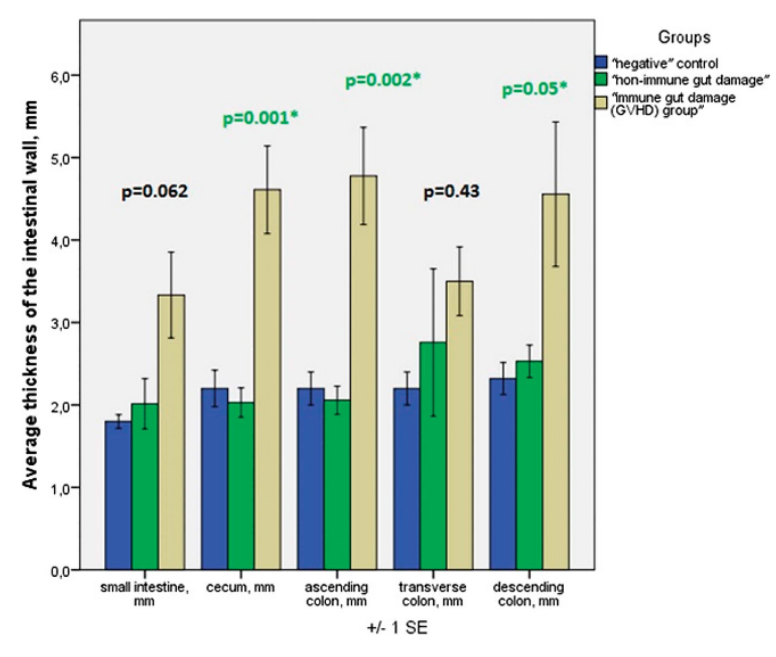

$(n=7)$, and chronic GVHD $(n=2))$.Median stool volume in this group was $600 \mathrm{ml}(500-3500 \mathrm{ml}), \mathrm{WBC}$ median at time of diarrhea onset-3.1*1 $10^{9} / \mathrm{I}\left(0.4-8.4^{*} 10^{9} / \mathrm{I}\right)$, median time to diarrhea after allogeneic HSCT was 48.5 day (20-113 days).5 patients after allogeneic HSCT with stool $>500 \mathrm{ml}$ but without GVHD (diarrhea resolved without the use of steroids) and 3 patients with MM after autologous HCST became "positive" control group ("non-alloimmune" gut damage) median stool volume was $1000 \mathrm{ml} \quad(500-1500 \mathrm{ml})$, median WBC-0.3*10 ${ }^{9} / \mathrm{I}$ (0.03$\left.5.86^{*} 10^{9} / \mathrm{l}\right)$, median time of diarrhea onset after HSCT was 6.5 days (5-17).

Results: Average thickness of the intestinal wall shown at chart 1. This chart show us that thickness of the cecum $(P=0.001)$, ascending $(P=0.002)$ and descending colon $(P=0.05)$ wall is significantly greater in "alloimmune gut damage (GVHD) group" 4.6 (2.5-7 mm), 4.8(2.5-8 mm),4.6(2.5$10 \mathrm{~mm})$ than in "negative" control: $2.2(1.7-3 \mathrm{~mm}) ; 2.2(2-3 \mathrm{~mm})$; $2.3(2-3 \mathrm{~mm})$ and in group with "non-alloimmune gut damage": 2 (1.5-2.5 mm); 2.1 (1.5-2.5 mm); 2.5 (1.7-3 mm).

Conclusion: Despite the fact that the study is small, we revealed significant differences in the thickness of intestinal (cecum, ascending and descending colon) wall in "alloimmune gut damage" group and may conclude that the use of gut ultrasound as a "decision support" method as non-invasive and inexpensive approach for GVHD diagnosis looks very attractive for BMT centers.

References: Malard F, Mohty M. New insight for the diagnosis of gastrointestinal acute graft-versus-host disease. Mediators Inflamm. 2014.

Disclosure of Interest: None declared.

\section{P489}

Identification of potential risk factors of acute graft versus host disease and infectious complications after allogeneic hematopoietic stem cells transplantation

M. Rybicka ${ }^{1}$, M. Markiewicz ${ }^{1, *}$, E. Pietruszka ${ }^{1}$, K. Goraus ${ }^{1}$, A. Suszka-Switek ${ }^{2}$, R. Wiaderkiewicz, M. Dzierzak-Mietla ${ }^{\prime}$, K. Bialas ${ }^{7}$, S. Kyrcz-Krzemień ${ }^{1}$

${ }^{1}$ School of Medicine in Katowice, Medical University of Silesia, Department of Hematology and Bone Marrow Transplantation, ${ }^{2}$ School of Medicine in Katowice, Medical University of Silesia, Department of Histology and Embryology, Katowice, Poland

Introduction: The allogeneic Hematopoietic Stem Cells Transplantation (alloHSCT) is associated with the risk of Graft versus Host Disease (GvHD) and infections. The patogenesis of acute GvHD is related to T-lymphocytes, which identify alloantigens on host's Antigen Presenting Cells, induce production of interferon (IFN) gamma and interleukin (IL) -2 , recruit the immunological effectory cells and destroy tissues and organs.
Material (or patients) and methods: The study involved 62 patients, $30(48 \%)$ male and 32 (52\%) female, aged at median 49.5 (19-68) years, after myeloablative (MAC) $n=26$ (42\%) or reduced intensity (RIC) $n=36(58 \%)$ conditioning therapy before alloHSCT from sibling $(n=12)$ or unrelated $(n=50)$ donor, performed for acute myeloblastic leukemia (AML) in Katowice, Poland, in years 2012-14. 54 (87\%) patients achieved complete remission (CR) before alloHSCT. All patients received standard immunosuppressive therapy with cyclosporin-A and methotrexate plus pre-transplant anti-thymocyte globulin in unrelated transplant setting. Blood samples were collected pre-transplant before start and on day -1 after the conditioning therapy, and on days: $+2+4,+6,+10,+20,+30$ following alloHSCT. The IL-2 and IFN-gamma serum concentrations were determined with use of ELISA assay. Analysis of potential risk factors was performed with Logistic Regression and Cox Proportional Hazards Model for aGvHD and with Logistic Regression for infections. Variables used in statistical analysis were: IL-2 and IFN-gamma concentrations, patients' age, use of $\mathrm{MAC} / \mathrm{RIC}, \mathrm{CR} /$ non-CR status before transplantation.

Results: The Logistic Regression revealed that independent risk factors of aGvHD were: non-CR status before alloHSCT $(\mathrm{OR}=10.52, P=0.040)$ and use of $\mathrm{MAC}(\mathrm{HR}=4.80, P=0.007)$. The Cox Proportional Hazards Model revealed following independent risk factors for aGvHD: non-CR status before alloHSCT (HR=3.38, $P=0.006)$, use of $M A C \quad(H R=2.30$, $P=0.032)$ and high level of IFN-gamma at day $+6(H R=1.03$, $P=0.032)$. MAC was also the independent risk factor for infectious complications in Logistic Regression analysis $(\mathrm{OR}=$ 4.04, $P=0.024$ ).

Conclusion: High level of IFN-gamma at day +6 , non-CR status before alloHSCT and use of MAC are independent risk factors of aGvHD. MAC is also the independent risk factor of infectious complications.

Disclosure of Interest: None declared.

\section{P490}

Glycoprotein YKL40: a Novel Biomarker of Chronic Graft Versus Host Disease Activity?

N. Durakovic ${ }^{1, *}$, Z. Peric ${ }^{1}$, V. Kusec ${ }^{2}$, L. Desnica ${ }^{3}$, D. Pulanic ${ }^{1}$, R. Vrhovac ${ }^{1}$, S. Z. Pavletic ${ }^{4}$, D. Nemet ${ }^{7}$

${ }^{1}$ Hematology, University of Zagreb School of Medicine, UHC Zagreb, ${ }^{2}$ Clinical Division of Laboratory Diagnostic, ${ }^{3}$ Hematology, UHC Zagreb, Zagreb, Croatia, ${ }^{4}$ Graft-versus-Host and Autoimmunity Unit, National Cancer Institute, Bethesda, United States

Introduction: Even though considerable effort has been invested into identifying a reliable biomarker for chronic Graft vs Host Disease (cGVHD), thus far the search has not been successful. Glycoprotein YKL40 has been investigated in various conditions and has been found to be a marker of an ongoing inflammation as well as oncogenic transformation. In a cross-sectional study we investigated the level of YKL-40 in patients suffering from CGVHD in reference to transplanted patients without CGVHD (controls).

Material (or patients) and methods: The study was conducted in UHC Zagreb from July 2013 to October 2015. A total of 56 transplanted patients were included, 35 cGVHD patients and 21 controls. Median age was 45 years (range 9-60) in patient group and 40 years (range 16-59) in control group, there were 18 and 10 females and 17 and 11 males, respectively. Median time from transplant to study enrollment was 463 days (range 61-7853) in patient group, and 428 days (range 190-1770) for controls. Median time from CGVHD diagnosis to enrollment was 154 days (range 1-7125). There were 17 and 8 patients that received myeloablative, 18 and 13 received reduced conditioning in patient and control group, respectively. There was no difference between the source of transplanted cells between groups, 15 and 8 patients that received bone marrow, 20 and 13 received PBSC in patient and control group, respectively. Using global $\mathrm{NIH}$ score to estimate disease severity 4 patients were considered to have mild, 15 moderate and 16 severe cGVHD. YKL-40 levels were measured by ELISA and compared between cGVHD 
patients and controls. We also tested for correlation in reference to disease severity and activity in cGVHD patients (estimated using Global NIH score, Clinicians impression of activity and Intensity of immunosuppression).

Results: YKL-40 levels were found to be significantly higher in cGVHD patients in reference to controls (median 707 (71-4000) and $314 \mathrm{ng} / \mathrm{ml}(105-2790)$ respectively, $(P=0.003)$. Even if patients with myelofibrosis (condition shown to increase the level of YKL-40) were excluded from analysis (total 6 patients, 3 in CGVHD group and 3 controls), the difference between groups remained statistically significant $(P=0.017)$. Furthermore, YKL-40 levels were found to correlate significantly with disease severity estimated using global $\mathrm{NIH}$ score. YKL-40 levels also positively correlated with Clinician's impression of activity $(P=0.016)$ but not with Intensity of immunosuppression $(P=0.085)$. When tested for correlation with CRP as a measure of active inflammation, no statistically significant correlation was found.

Conclusion: Although the number of patients included in this cross-sectional study is limited, our results show that YKL-40 glycoprotein might be a simple and inexpensive biomarker of cGVHD and its activity. However, further investigation is warranted with validation in a larger group of patients, as well as longitudinal testing of YKL-40 glycoprotein levels in patients at risk of developing CGVHD.

Disclosure of Interest: None declared.

\section{P491}

Arginine and Tryptophan Metabolism Predict Risk

of Acute and Chronic GVHD in Patients Receiving Allogeneic Hematopoietic Stem Cell Transplantation

N. R. Guedes ${ }^{1, *}$, P. Yamakawa ${ }^{1}$, T. Carneiro ${ }^{1}$, M. Vescovi ${ }^{1}$, A. Pereira' ${ }^{2}, Y$. Novis ${ }^{2}, V$. Rocha ${ }^{2}$, I. Silva ${ }^{3}$, C. Arrais-Rodrigues ${ }^{4}$

${ }^{1}$ Department of clinical and experimental oncology, Unifesp, ${ }^{2}$ Hospital Sirio Libanes, ${ }^{3}$ Unifesp, ${ }^{4}$ Department of clinical and experimental oncology, Unifesp/Hospital Sirio Libanes, São Paulo, Brazil

Introduction: There are no validated diagnostic or predictive biomarkers for acute or chronic graft versus host disease (GVHD) routinely used in clinical practice of hematopoietic stem cell transplantation (HSCT). The characterization of metabolomic profiles consists of a collection of assays that characterize panels of metabolites related to specific pathways. Arginine and their metabolites are involved in immune regulation by modulating $T$ cell activation and function, while tryptophan catabolites are potent inhibitors of $\mathrm{T}$ cell activation and can induce T-cell apoptosis and immune tolerance. Little is known of how these metabolite might influence the HSCT endpoints.

Material (or patients) and methods: We performed metabolomics screening using mass spectral analysis of plasma samples collected at engraftment in patients receiving an allogeneic HSCT. The Absolute IDQ ${ }^{\mathbb{R}}$ p180 kit assay was used for the quantification 188 analytes, including amino acids, acylcarnitines, sphingomyelines, phosphatidylcholines, hexoses and biogenic amines. Twenty seven patients $(59 \%$ male, median age 57 years, range 18-73), receiving stem cells from mobilized peripheral blood $(n=16)$, bone marrow $(n=6)$, or umbilical cord $(n=5)$, from haploidentical $(n=11)$, matched unrelated $(n=8)$, or matched related donors $(n=8)$ were studied. The most common diagnoses were acute leukemias $(n=9)$ and lymphoma $(n=11)$. All patients received reduced intensity conditioning regimens. Antithymocyte globulin was used in 4 patients (15\%) and total body irradiation in 18 (67\%). Median follow up time was 24 months (range 4-35).

Results: Median levels of ornithine were significantly higher at the time of engraftment in patients who later developed acute GVHD as compared to those who did not $(P=0.009)$. Besides, the ornithine/arginine ratio was also significantly higher in those presenting acute GVHD $(P=0.01)$, expressing a higher arginase 1 activity. Cumulative incidence $(\mathrm{Cl})$ of acute GVHD was significantly higher in those with ornithine levels above the median level $(64 \%$ vs $8 \%, P=0.002)$. For chronic GVHD, median levels of kynurenine, a tryptophan metabolite, were significantly lower in those who developed chronic GVHD $(P=0.01)$, as well as the kynurenine/ tryptophan ratio $(P=0.03)$, expressing decreased indoleamine 2,3-dioxygenase activity. $\mathrm{Cl}$ of chronic GVHD was significantly higher in those with lower levels of kynurenine ( $83 \%$ vs $35 \%, P=0.03$ ) and lower kynurenine/ tryptophan ratio (78\% vs $0 \%$ ).

Conclusion: Higher concentration of arginine metabolites was observed in patients with acute GVHD, while lower kynurenine/tryptophan ratio was associated with a higher risk of chronic GVHD. These data suggest increased activity of arginase 1 in acute GVHD, and decreased indoleamine 2,3dioxygenase activity in chronic GVHD. These markers might help to predict the development of GVHD early after HSCT. The mechanisms by which the metabolism of amino acids interferes with the risk of GVHD after transplant deserve further investigation.

Disclosure of Interest: None declared.

\section{P492}

Efficacy of Extracorporeal Photopheresis in chronic cutaneous, oral, ocular, gut and liver GvHD: findings from GvHD Registry

N. Alband ${ }^{1}$, S. Saglam ${ }^{1}$, T. Basu ${ }^{2}$, F. Child ${ }^{1, *}$

${ }^{1}$ Dermatology, St John's Institute of Dermatology, ${ }^{2}$ Dermatology, Kings College Hospital, London, United Kingdom

Introduction: Extracorporeal photopheresis (ECP) is a novel cell-based immunomodulatory therapy used second line in the management of steroid resistant, dependent or intolerant chronic mucocutaneous graft versus host disease (GvHD) and in patients with involvement of other organs who have failed first and second line treatment options ${ }^{1}$. Previous studies have demonstrated efficacy of ECP but it remains unclear as to the optimum length of treatment and whether specific skin subtypes respond differently.

Material (or patients) and methods: We reviewed 144 consecutive patients on an intention-to-treat basis with chronic GvHD (cGvHD) who received ECP between 2011 to 2014. Of these, 41 were excluded from the study due to lack of sufficient data or non-attendance for treatment. Patients underwent fortnightly ECP for 6 months and were then tapered to monthly or six weekly treatment depending on clinical response. Response assessments were carried out every three months using National Institute of Health (NIH) scoring criteria until completion of ECP. Primary end point was response to ECP at the end of treatment and secondary end point was reduction in immunosuppression.

Results: A total of $103(72 \%)$ had completed at least 6 months of ECP. Of these, $90(87 \%)$ had cutaneous GvHD (43\% lichenoid disease, 39\% sclerodermoid, 18\% eczematoid), 21 patients (20\%) had oral GvHD, 11 (11\%) liver GvHD, 10 (10\%) lung GvHD, and $10(10 \%)$ gut GvHD. Thirty eight patients had multiorgan involvement.

The median duration of ECP treatment was 14 months. However median length of treatment for patients with cutaneous sclerodermoid, lichenoid and eczematoid were 15, 15 and 12 months respectively.

In total, $63 \%$ of patients with cutaneous GvHD had $\geq 50 \%$ improvement in symptoms and signs of GvHD. Based on analysis of sub-types of skin GvHD, ECP was effective in sclerodermoid $(P<0.0001)$, lichenoid GvHD $(P$ 0.0008) and eczematoid GvHD ( $p$ 0.0353) Sixty eight percent were able to reduce immunosuppression by $>50 \%$ ( $14 \%$ stopped completely). $80 \%$ had reduction in steroid dose. In those with liver GvHD a trend in improvement of liver function tests was seen but did not reach statistical significance. No significant improvement was observed in lung, oral $(P$ 0.7) and ocular $\left(\begin{array}{l}P \\ 0.1)\end{array}\right)$ GvHD. A statistically significant improvement was seen in patients with gut GvHD ( $P$ 0.03). 
Fifty six patients had consistently completed Dermatology Life Quality Index (DLQI) and chronic GvHD symptom scale (cGvHD) questionnaires during their clinic visits. Of these, $45 \%$ had $\geq 25 \%$ improvement in both their DLQI and cGvHD symptoms scale. Thirty eight percent of patients had $\geq 50 \%$ improvement in their DLQI and $20 \%$ had $\geq 50 \%$ improvement in their cGvHD symptoms scale.

Conclusion: We provide further evidence of the efficacy of ECP in chronic cutaneous GvHD. The average length of treatment is longer than in previous studies, and data suggest that patients with sclerodermoid and lichenoid disease have a better response than those with eczematoid disease. Our results also demonstrate a significant improvement in patients with chronic gut GvHD. Further prospective studies are needed to evaluate the efficacy of ECP in liver, lung, oral and ocular GvHD.

References: 1. Klassen J, The role of photopherisis in the treatment of graft-versus host disease, Curr Oncol. 2010 Apr; 17(2)55-58.

Disclosure of Interest: None declared.

\section{P493}

Effect of extracorporeal photopheresis on the mobilisation of granulocytic myeloid-derived suppressor cells in chronic graft-versus host disease

N. Matthews ${ }^{1, *}$, C. Barker ${ }^{1}$, A. Alfred ${ }^{1}$, P. Taylor ${ }^{7}$

${ }^{7}$ Dept of Photopheresis, Rotherham NHS Foundation Trust, Rotherham, United Kingdom

Introduction: Extracorporeal photopheresis (ECP) is a therapy for treating steroid-refractory chronic graft versus host disease (cGVHD) and facilitating immunotolerance. Recent data suggests that therapeutic response to ECP is associated with a sustained induction of circulating granulocytic myeloid-derived suppressor cells (G-MDSCs) in acute GVHD patients, but not in those with CGVHD (1). However, data about G-MDSCs in longterm ECP patients ( $>6$ months) have not been reported. Here, we have used immunophenotyping to monitor the effect of ECP on G-MDSC mobilisation in CGVHD patients starting ECP and in a cohort of patients that are established on ECP.

Material (or patients) and methods: A total of 29 patients: 11 with steroid refractory or steroid-dependent CGVHD before starting ECP (Pre-ECP), 18 established (>6 months; mean treatment duration of 19 months) CGVHD patients receiving ECP (Est-ECP) and 9 healthy controls were recruited. Pre-ECP patients had GVHD affecting skin (10/11), mouth (3/11) and gut (2/11).Est-ECP patients initially had GVHD affecting skin (16/18), mouth (4/18), gut (6/18) and liver (4/18). PBMC were isolated and immunophenotyped for markers of G-MDSCs (CD14 ${ }^{\text {-ve }}$, CD16, CD66b, HLA-DR ${ }^{\text {-ve }}$, CD33 $^{\text {int }}$ ) by flow cytometry. Statistical analysis was by two-tailed Mann Whitney test using GraphPad 6.

Results: Est-ECP patients had substantially greater frequencies of circulating G-MDSCs than healthy controls $(P<0.0001$; median: $4 \%$ and IQR $1.6 \%-26 \%$ vs $0.3 \%$ and IQR $0.1 \%-0.35 \%$, respectively), but a significantly lower frequency than in Pre-ECP cGVHD patients $(P<0.02$; median: $13 \%$ and IQR: $1.5 \%-26 \%$ ). Monitoring of G-MDSCs in both ECP cohorts (in 6/11 of the PreECP patients and 12/18 of the Est-ECP patients) over 12 weeks of ECP showed marked flux in some patients and relative stability in most others. The frequency of G-MDSCs in Est-ECP patients showed an inverse relationship with time where patients with a complete response to long-term ECP therapy had low G-MDSC frequencies far closer to those of healthy controls (median:0.6\% and IQR $0.5 \%-2.3 \%$ ). Analysis over 3 months showed that most Est-ECP patients had stable G-MDSC frequencies. However, further immunophenotyping showed that patients with very low levels of, or undetectable circulating CD19 B-cells $(<1 \%$ of total lymphocytes), had significantly higher frequencies of G-MDSCs than patients with higher frequencies of B-cells $(P=0.0013$; median: $31 \%$ and IQR $26 \%-42 \% ; n=4$ vs median $3.7 \%$ and IQR $1.9 \%-8.1 \% ; n=14$, respectively).

Conclusion: Consistent with Rieber et al (1) there was no pattern of sustained G-MDSC induction in ECP-treated CGVHD patients. In contrast, the data suggest that a high frequency of circulating G-MDSCs is associated with a state of dysregulated immunity and that the immunomodulatory therapeutic response to ECP in CGVHD patients is indicated by a decrease in circulating G-MDSC frequency.

References: Rieber, N. et al. 2014. Bone Marrow Transplantation 49:545-552.

Disclosure of Interest: None declared.

\section{P494}

Extracorporeal photopheresis in patients with steroidrefractory acute graft-versus-host disease: does the number of UVA-treated leukocytes impact response to therapy?

N. Worel ${ }^{1, *}$, E. Lehner ${ }^{1}$, W. Rabitsch ${ }^{2}$, M. Mitterbauer ${ }^{2}$, W. Bauer ${ }^{3}$, A. Schulenburg', G. Hopfinger', U. Just', G. Stary', P. Kalhs' ${ }^{2}$, H. Greinix ${ }^{4}$

${ }^{7}$ Transfusion Medicine, ${ }^{2} B M T$ Unit, Internal Medicine I, ${ }^{3}$ Dermatology, Medical University of Vienna, Vienna, ${ }^{4}$ Dept. of Haematology, Medical University of Graz, Graz, Austria

Introduction: Acute Graft-versus-Host disease (aGvHD) is a serious complication of allogeneic hematopoietic cell transplantation (HCT) causing significant morbidity and mortality. Steroids are the established $1^{\text {st }}$-line therapy with $40 \%$ of patients (pts) responding. The optimal therapy for steroidrefractory (SR) aGVHD is undefined. Extracorporeal photopheresis (ECP) has an objective efficacy in the treatment of SR aGvHD and an excellent safety profile. As several ECP devices are available, yielding in different numbers of ultraviolet $A$ (UVA) treated cells we aimed to analyze the impact of UVA treated leukocytes and cellular subtypes (lymphocytes, monocytes, neutrophils) on response rates to ECP at 1 and 3 months. Material (or patients) and methods: Between 2000 and 2013, 99 pts with aGVHD underwent ECP including 58 with SR disease and 15 given ECP as $1^{\text {st }}$-line treatment. For HCT, 76 pts received myeloablative conditioning with Cyclosporine A (CsA) and methotrexate, 23 received reduced intensity protocols with $\mathrm{CsA}$ and mycophenolate mofetil. The preferred graft source was mobilized peripheral blood (82\%). The majority of donors was HLA identical (70\%) and unrelated (84\%). Response to ECP was classified as complete response (CR) if organ manifestations resolved, partial response (PR) if $>50 \%$ response was reached or no response (NR) if pts did not improve or required additional immunosuppression. CR and $P R$ were used to define overall response (OR) to ECP.

Results: AGvHD occurred 16 days (median, range 7-115) post $\mathrm{HCT}$ and was treated with $2 \mathrm{mg} / \mathrm{kg} /$ day steroids (median, range 1.5-6.5). ECP was initiated 21 days post HCT (median, range 8-121) and given 2-3 times per week/weekly. Treatments within 1 week were counted as 1 cycle. At start of ECP, 62 pts had aGvHD grade I+II, and 37 grade III+IV. Median steroid dose at onset of ECP was $2 \mathrm{mg} / \mathrm{kg} /$ day (range, 0.5-3.6). Pts received 4 (median, range 1-16) ECP cycles. Four weeks after start of ECP the median steroids dose was $0.6 \mathrm{mg} / \mathrm{kg} / \mathrm{day}$ (range, 0-3.6). OR at 1 and 3 months was $72 \%(49 \% \mathrm{CR})$ and $81 \%(74 \% C R)$, respectively. Maximal response was observed after a median of 4 (range, 1-14) ECP cycles. The mean number of infused UVA-irradiated leukocytes and MNCs in a single ECP treatment was $2.5 \times 10^{9}(\mathrm{SD}, \pm 1.0)$ and $1.2 \times 10^{9}(\mathrm{SD}, \pm 0.6)$. In univariate analyses severity of aGVHD (grade $>\mathrm{II}$ ), and number of involved organs at start of ECP influenced OR at 1 month $(P<0.01)$. OR at 3 months was influenced by the number of involved organs at onset of aGvHD $(P=0.023)$. In multivariate analyses only the number of involved organs at start of ECP was an independent predictor for OR at 1 month $(P=0.002)$. The number of UVA-treated cells did not correlate with response to ECP at 1 or 3 months. Kaplan-Meier estimates for overall survival at 1 year for pts achieving CR or PR at 1 month were $79 \%$ vs. $46 \%$ for those with NR $(P=0.025)$.

Conclusion: In summary, our results in patients with SR aGvHD demonstrate high overall response rates to ECP with improved overall survival in patients responding at 1 month. 
We could not identify a threshold of leukocytes or leukocyte subtypes associated with success of ECP. Prognostic biomarkers are highly warranted to predict response to ECP.

Disclosure of Interest: None declared.

\section{P495}

Organsiderosis and Hemophagocytosis during acute GVHD

A. Nogai ${ }^{1}$, Y. Shi ${ }^{2}$, S. Cordes $^{2}$, K. Riesner ${ }^{2}$, S. Mertlitz ${ }^{2}$ J. Mengwasser' ${ }^{2}$, D. Pérez-Hernandez ${ }^{3}$, J.-H. Erdmann ${ }^{2}$, R. Ziebig ${ }^{4}$, G. Dittmar ${ }^{3}$, O. Penack,

${ }^{1}$ Division of Hematology and Oncology, ${ }^{2}$ Division of Hematology, Oncology and Tumor Immunology, Charité, Berlin, ${ }^{3}$ Mass Spectrometry, Max-Delbrück-Center for Molecular Medicine, ${ }^{4}$ Department for Laboratory Medicine and Pathobiochemistry, Charité, Berlin, Berlin, Germany

Introduction: Iron overload prior to allo-HSCT is associated to increased transplant related mortality - possibly mediated by toxic effects of labile plasma iron. Despite its significant impact on allo-HSCT outcome and despite the close connection between iron metabolism and inflammation, few data is available on the role of iron metabolism during GVHD.

Material (or patients) and methods: We retrospectively reviewed clinical data and ferritin levels of 104 successive alloHSCT recipients at Charité, CBF. In patients with high ferritin levels vs. low levels, we performed iron staining and looked for the presence of hemophagocytes in bone marrow (BM) biopsies. In material from preclinical murine models, we measured hepatic iron contend by photometry, as well as by iron staining. We performed gene array as well as proteomic analyses.

Results: Clinical data: We found no correlation of ferritin levels prior to allo-HSCT and the occurrence of any grade aGVHD. In patients with GVHD, ferritin levels were significantly higher as compared to allo-HSCT recipients without GVHD (Fig 1a). Very high ferritin levels $(>10.000 \mu \mathrm{g} / \mathrm{l})$ exclusively occurred in patients during GVHD (Fig 1a) and these patients had significantly reduced overall survival (Fig 1b). Since serum ferritin is primarily produced by macrophages, we hypothesized that macrophage activation may have occurred.
We therefore investigated BM biopsies from patients with high vs. low ferritin levels after allo-HSCT for hemophagocytes, which is a hallmark of macrophage activation. In 9 from 11 patients with ferritin levels $>10.000$ BM biopsies were available. In 8 of these 9 aspirates, hemophagocytes were seen. In the remaining patient, siderophages without clear evidence of hemophagocytosis were detectable. Furthermore, other parameters of hemophagocytic syndrome were present. Preclinical data: We found significantly elevated liver iron contend in allo-BMT recipients with GVHD vs syn-BMT recipients at different time points after allo-BMT. Gene array and proteomic analyses of liver tissue during GVHD vs. no GVHD identified several iron-related and macrophage-related pathways to be significantly activated during GVHD (Fig 1c+d). Conclusion: Our clinical data demonstrates that GVHD is associated with highly elevated ferritin levels and to the presence of hemophagocytes in the BM. Our experimental results show that the iron metabolism is profoundly affected, leading to GVHD-associated iron overload. Based on our finding that very high ferritin levels and hemophagocytes in the BM were exclusively present in patients with GVHD, we speculate that alloreactivity leads to macrophage activation, hemophagocytosis and consequently release of high iron amounts in a subset of patients with GVHD.

Disclosure of Interest: None declared.

\section{P496}

Plasma levels of IL-17, IL-6, INF- $\gamma$, TNF- $a$ after hematopoietic stem cell transplantation with posttransplant cyclophosphamide

O. Pirogova ${ }^{1, *}$, E. Surkova ${ }^{1}$, S. Lapin ${ }^{1}$, I. Moiseev ${ }^{1}$, E. Babenko ${ }^{1}$, A. Alyanskiy ${ }^{1}$, B. Afanasyev

${ }^{1}$ R.M. Gorbacheva Memorial Institute of Children Hematology, Oncology and Transplantation, First Pavlov State Medical University, St. Peterburg, Russian Federation

Introduction: There are a number of studies investigating predictive value of cytokines for graft versus host disease (GVHD), but this research was performed with classical GVHD prophylaxis. It is unclear whether it could be translated to prophylaxis with PTCy. We investigated such common proinflammatory cytokines as IL-17, IL-6, IFN- $\gamma$, TNFa in patients

[P495]
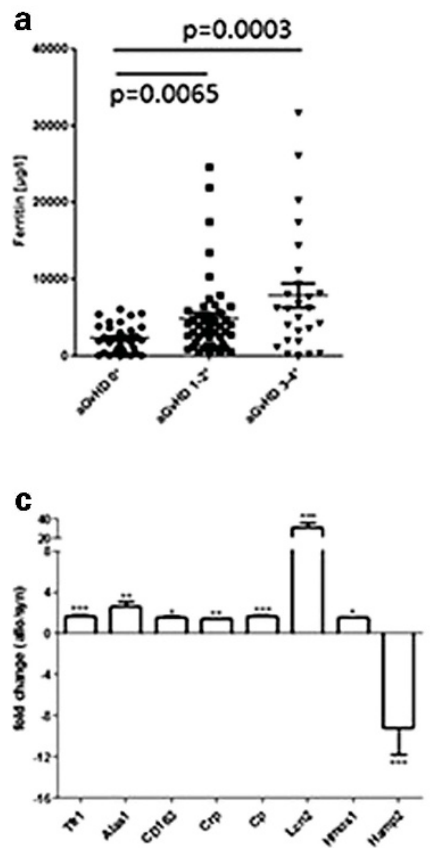

b

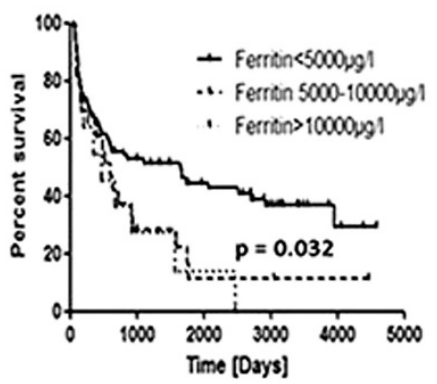

d

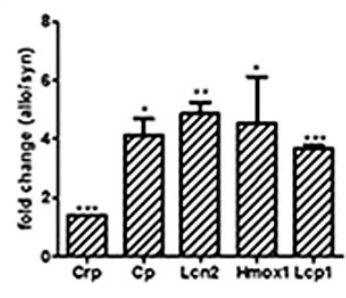

Fig. 1: A+8) Clinical data of 104 successive patients undergoing allo-HSCT at Charité CBF.

A) Elevated Ferritin levels in patients during aGvHD. B) Patients with increased ferritin levels after allo-HSCT have a worse overall survival. $C+D)$ Analyses of liver tissue at day +14 syn vs allo in the LP.B6 murine GVHD model.

C) Gene array data on iron related genes as well as macrophage activation related

Drocen 0.14 genes. D) Proteomic data on iron related proteins as well as macrophage activation related proteins. 
with allogeneic peripheral blood stem cell transplantation (PBSCT).

Material (or patients) and methods: 26 unrelated PBSCT recipients were included in this study. All patients had hematological malignancies and received Flu/Bu2 conditioning; cyclophosphamide on D+3, +4, tacrolimus, MMF as prophylaxis GVHD. 12 recipients (46\%) had aGVHD (I-IV). 14 patients constituted the control group without GVHD. The plasma levels of IL-17, IL-6, IFN- $\gamma$, TNFa were evaluated by ELISA on day $0,+7,+21.15 \%$;font-family:"Arial","sans-serif"' $>\gamma$, TNFa in patients with allogeneic peripheral blood stem cell transplantation (PBSCT).

Results: In the whole group the dynamics of IL-17, IL-6, IFN- $\gamma$ were similar with highest value on day 0 and declining afterwards. The differences between time points were statistically significant $(P<0,02)$. For TNFa there were no statistical differences between the measurements $(P>0.05)$. For 3 out of 4 cytokines concentration was lower in GVHD group. However, a statistically significant difference was obtained only for IL-17, probably due to the small sample of patients. IL-17 plasma levels on day 0 was $198,8 \pm 378,2$ vs $457,8 \pm 634,4 \mathrm{pg} / \mathrm{ml}(P=0.04)$, on day +7 was $172,2 \pm 265,3$ vs $265,7 \pm 484,4 \quad(P>0.05)$, on day +21 was $121,5 \pm 139,4$ vs $225 \pm 442,1 \quad(P>0.05)$ for GVHD and nonGVHD, respectively. IL-6 plasma levels on day 0 was $49,3 \pm 121,3$ vs $5,2 \pm 3,2$ $(P>0.05)$, on day +7 was $17,5 \pm 33,4$ vs $11,9 \pm 17,8(P>0.05)$, on day +21 was $26,7 \pm 66,3$ vs $15,4 \pm 12,9(P>0.05)$ for GVHD and nonGVHD, respectively. IFN- $\gamma$ plasma levels on day 0 was $69,4 \pm 107,5$ vs $57 \pm 54,8 \quad(P>0.05)$, on day +7 was $70 \pm 79,3$ vs $79,6 \pm 75,1(P>0.05)$, on day +21 was $67,4 \pm 67,4$ vs $80,1 \pm 47,5(P>0.05)$ for GVHD and nonGVHD, respectively. TNFa plasma levels on day 0 was $5,5 \pm 11,6$ vs $7,8 \pm 10,5$ $(P>0.05)$, on day +7 was $1,7 \pm 1,6$ vs $2,3 \pm 4,1(P>0.05)$, on day +21 was $0,8 \pm 0,7$ vs $0,85 \pm 0,5(P>0.05)$ for GVHD and nonGVHD, respectively.

Conclusion: We have observed usual dynamics of cytokines in alloHSCT, but unlike in patients with classical GVHD prophylaxis, patients with higher cytokine levels were less likely develop GVHD. This may be explained by more prominent PTCy alloreactive T-cell depletion in patients with higher levels of T-cell activation. Further studies on larger number of patients are required to confirm this phenomenon.

Disclosure of Interest: None declared.

\section{P497}

Placenta-derived decidual stromal cells for treatment of severe acute graft-versus-host disease

M. Solders ${ }^{1,2}$, A. Baygan ${ }^{1}$, M. Remberger, ${ }^{2,3}$, T. Erkers', S. Nava ${ }^{1}$, B. Gustafsson ${ }^{2}$, J. Winiarski ${ }^{4}$, B. Sadeghi ${ }^{1}$, H. Kaipe ${ }^{1,5}$, O. Ringdén ${ }^{1, *}$ ${ }^{1}$ Division of Therapeutic Immunology, Karolinska Institutet, ${ }^{2}$ Center for Allogeneic Stem Cell Transplantation, Karolinska University Hospital, ${ }^{3}$ Department of Pathology-oncology, Karolinska Institutet, ${ }^{4}$ Department of Pediatrics, ${ }^{5}$ Department of Clinical Immunology and Transfusion Medicine, Karolinska University Hospital, Stockholm, Sweden

Introduction: Severe acute graft-versus-host disease (GVHD) is a life-threatening complication after allogeneic haematopoietic cell transplantation (HSCT). The immune modulation exerted by decidual stromal cells (DSCs) plays a part in fetomaternal tolerance during pregnancy. This provides a rationale for using DSCs to treat allogeneic inflammatory disorders. In the present study, we wanted to evaluate if placenta-derived decidual stromal cells were safe and effective in treating severe acute GVHD.

Material (or patients) and methods: DSCs were obtained from term placentas from healthy mothers during elective Caesarian-section birth. The DSCs were given to 38 patients with severe acute GVHD grade II-IV. The patients were subsequently divided into two groups. The first group (Previous) comprises of 17 patients treated between 20112013. This group were treated a median of day 13 (range 1-37) after initiation of high-dose steroids. They received a median of 1 (1-5) infusion and the DSCs were thawed with human $A B$ serum supplementation. Cell dose and passage of the DSCs were $2,0(0.9-2.8) \times 10^{6}$ cells/kg and $2(2-4)$, respectively. The second group (Current), comprises the subsequent 21 patients treated between 2013-2015. This group was treated earlier after steroids, median day 7 (0-35), received more DSC infusions $2(1-6)$ and the cells were thawed using human serum albumin as supplementation. The cell dose was lower $1,2(0.9-2.9) \times 10^{6}$ cells $/ \mathrm{kg}$ and the passage was higher $4(2-4)$. The DSCs given to the Current group had a higher viability 95 $(69-100) \%$ compared to the Previous group 89.5 (70-97)\%. Looking closer at steroid refractory patients, refractivity to steroids was defined as no response after 7 days or progressive disease after 2 days. 32 retrospective controls with steroid-refractory acute GVHD were also included in the analysis.

Results: Apart from the time between that steroids were started and the intervention, the Current group could be significantly distinguished by earlier and more repeated interventions, reduced cell dose, higher cell passage and different thawing protocols. The GVHD response rate, no response/partial response/complete response was 7/5/5 in the Previous group and 0/10/11 in the Current group. One-year survival was $47 \%$ and $75 \%$ in the two groups, respectively $(P<0.01)$. Looking at the steroid-refractory patients, one-year survival was $3 \%$ in the controls, $38 \%$ in the Previous group and $72 \%$ in the Current group $(P<0.05)$. The median steroid dose at four weeks after initiation of steroids was $1.67 \mathrm{mg} / \mathrm{kg}$ in the controls, $0.56 \mathrm{mg} / \mathrm{kg}$ in the Previous group and $0.54 \mathrm{mg} / \mathrm{kg}$ in the Current group $(P<0.01)$.

Conclusion: DSCs is a promising novel treatment for severe acute GVHD. Prospective randomized trials are needed to evaluate to which extent this is true.

Disclosure of Interest: None declared.

\section{P498}

Hyperacute graft versus host disease (haGVHD): risk factors, outcomes and impact of Tacrolimus/Sirolimus prophylaxis

O. Lopez Godino ${ }^{1, *}$, E. Pérez-López ${ }^{1}$, L. López Corral ${ }^{1}$, S. Alonso ${ }^{7}$ R. Pérez Lópezz, M. Cabrero', J. Labrador ${ }^{7}$, F. Sánchez-Guijo ${ }^{\prime}$, D. caballero ${ }^{\top}$, L. Vázquez $^{\top}$

${ }^{1}$ Hematology department, ${ }^{2}$ Pharmacy department, Hospital Universitario de Salamanca, Salamanca, Spain

Introduction: HaGVHD is associated with poor prognosis in the context of aGVHD due to its severity and to its worse response to steroids. Most reports include mayority patients receiving calcineurin inhibitors/MTX prophylaxis.

Material (or patients) and methods: We prospectively analysed 627 patients consecutively transplantated in our Unit between 1995-2014 excluding paediatrics and 2nd alloHSCT. HaGVDH was defined as that occurring within 14 days after HSCT.

Results: 70 patients (11\%) developed II-IV haGVHD, 264 (42\%) other acute II-IV GVHD (oaGVHD). Median of days to haGVHD and oaGVHD were +10 (4-14) and +35 (12-362) respectively. Patient's characteristics are shown in table 1. Differences between haGVHD and oaGVHD include a higher skin involvement and a higher proportion skin III-IV grades in haGVHD: 75 Vs $45 \%, P=0,00$ and $44 \%$ Vs $18 \%, P=0,03$ respectively. In multivariate analysis, risk factors associated with haGVHD include sex mismatch ( $P=0,004$; OR 2,1; IC95\% $1,02-4,4)$, conditioning regimen other than FludarabinaBusulfan $(P=0,00)$, Tacrolimus-Sirolimus prophylaxis $(P=0,001$; OR 2,1; IC95\% $1,4-3,4)$ and unrelated donor +/mismatch $(P=0,004$, OR 3,2, IC95\% 1,4-7,1), whereas only mismatch unrelated donor was associated with a higher risk of oaGVHD ( $P=0,003$, OR 3,2, IC95\% 1,4-7,1).

Steroids resistance was similar in both groups (haGVHD 23\% Vs $17 \%$ in oaGVHD). Factors influencing higher risk of steroid resistance in the multivariate analysis were HLA mismatch $(P=0,04$; OR 5,9; IC95\% $1,05-33,7)$ and grade III-IV $(P=0,004$, 
OR 11,7, IC95\% 2,2-62) in haGVHD and grades III-IV $(P=0,00$, OR 21, IC95\% 9,2-48) in the group of oaGVHD.

With a median follow-up of 38 months, OS and TRM for the whole series is $57 \%$ and $19 \%$. Comparing haGVHD Vs oaGVHD, haGVHD hadn't impact neither in OS $(56 \%$ Vs $51 \% ; P=0,5)$ and TRM (33\% Vs 25\%; $P=0,17$ ) (Figure 1).

Although Tacrolimus/sirolimus prophylaxis was associated with a higher risk of haGVHD, this scheme was also associated with a better OS in the global series ( $P=0,002, \mathrm{HR} 0,38, \mathrm{IC} 95 \%$ $0,2-0,7)$.

\begin{tabular}{|c|c|c|c|}
\hline Value $n$ (range) & $\begin{array}{c}\text { All } \\
\text { patients }\end{array}$ & $\begin{array}{c}\text { II-IV } \\
h a G V H D\end{array}$ & $\begin{array}{c}I I-I V \\
\text { oaGVHD }\end{array}$ \\
\hline Male sex & 365 & 50 & 143 \\
\hline Sex mismatch & 137 & 27 & 51 \\
\hline Median age patient & $49(16-69)$ & $\begin{array}{c}49(17- \\
65)\end{array}$ & $50(16-69)$ \\
\hline \multicolumn{4}{|l|}{ Donor } \\
\hline Sibling & 412 & 26 & 161 \\
\hline Matched Unrelated & 109 & 25 & 44 \\
\hline $\begin{array}{l}\text { Unrelated with } 1 \text { or } 2 \\
\text { mismatch }\end{array}$ & 77 & 18 & 39 \\
\hline Haploidentical & 23 & 0 & 10 \\
\hline $\begin{array}{l}\text { Related or unrelated } \\
\text { with mismatch }\end{array}$ & 92 & 20 & 47 \\
\hline \multicolumn{4}{|l|}{ Diagnosis } \\
\hline AML/MDS/MPS Ph- & 293 & 18 & 127 \\
\hline ALL & 73 & 15 & 24 \\
\hline $\mathrm{HL} / \mathrm{NHL} / \mathrm{CLL}$ & 151 & 29 & 66 \\
\hline CML & 40 & 3 & 15 \\
\hline$A A$ & 16 & 0 & 4 \\
\hline MM & 53 & 5 & 26 \\
\hline \multicolumn{4}{|l|}{ EBMT status disease at HSCT } \\
\hline Early & 249 & 27 & 94 \\
\hline Intermediate & 246 & 32 & 107 \\
\hline Late & 95 & 11 & 44 \\
\hline Myeloablative & 237 & 28 & 85 \\
\hline \multicolumn{4}{|l|}{ Conditioning regimen } \\
\hline FluBu +/- Thiotepa & 234 & 8 & 102 \\
\hline FluMel & 179 & 34 & 80 \\
\hline FluMel Thiotepa & 6 & 3 & 3 \\
\hline BuCy+/- Thiotepa & 78 & 6 & 32 \\
\hline СуТВI & 68 & 16 & 28 \\
\hline Others & 53 & 3 & 18 \\
\hline $\begin{array}{l}\text { PB cell source/ Median } \\
\text { CD34×106/kg }\end{array}$ & $\begin{array}{l}526 / 5,3 \\
(0,9-22)\end{array}$ & $\begin{array}{c}58 / 5,1 \\
(1,2-9,6)\end{array}$ & $\begin{array}{c}217 / 5,5 \\
(1,3-16,3)\end{array}$ \\
\hline \multicolumn{4}{|l|}{ GVHD prophylaxis } \\
\hline CSA+MTX & 329 & 18 & 130 \\
\hline Tacrolimus+MTX & 76 & 10 & 35 \\
\hline $\begin{array}{l}\text { Calcineurin inhibitor } \\
+\mathrm{MMF}\end{array}$ & 64 & 8 & 28 \\
\hline Tacrolimus+Sirolimus & 141 & 32 & 64 \\
\hline Others & 17 & 2 & 5 \\
\hline In vivo $T C D$ & 69 & 4 & 21 \\
\hline
\end{tabular}

Conclusion: According to our centre experience, haGVHD is characterized for a higher skin preference; it was not associated with a poorer outcome neither with a higher steroid resistance as compared with oaGVHD; althought tacrolimus/sirolimus is one of the risk factors to

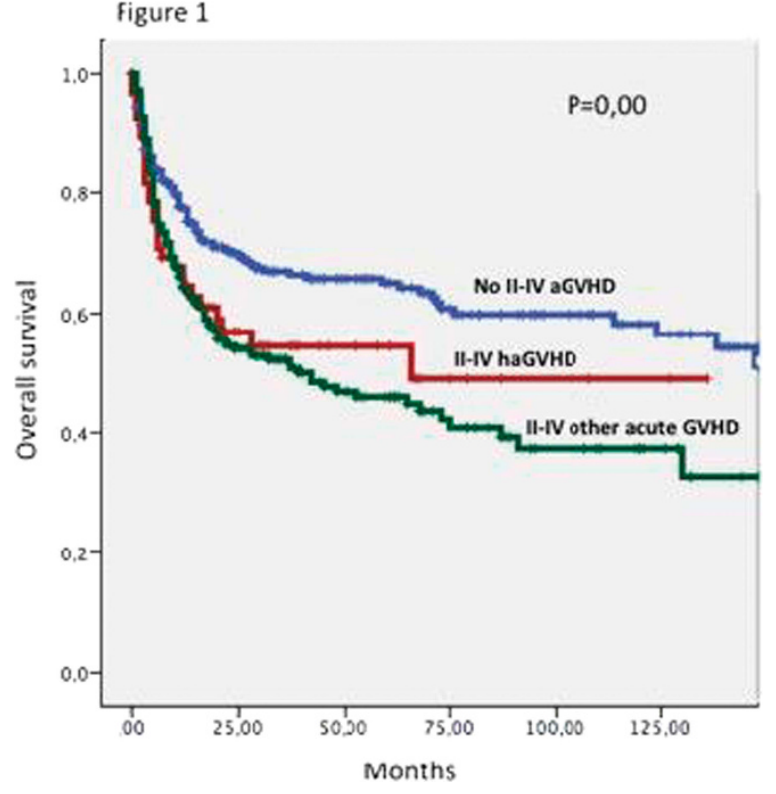

develop haGVHD, this regimen is also associated with a better OS.

Disclosure of Interest: None declared.

P499

Microbiome Changes After Conditioning Regimen for allogeneic hematopoietic stem cell transplantation P. Chiusolo ${ }^{1, *}$, E. Metafuni ${ }^{1}$, F. Paroni Sterbini ${ }^{2}$, S. Giammarco ${ }^{1}$, L. Masucci', S. Sica

${ }^{1}$ HEMATOLOGY, ${ }^{2}$ MICROBIOLOGY, FONDAZIONE POLICLINICO UNIVERSITARIO AGOSTINO GEMELLI, ROME, Italy

Introduction: The intestinal microbiome is a modulator of gastrointestinal immune homeostasis as well as hematopoietic cells too ${ }^{1}$. The main bacterial phylum dominating in human adult intestine are Bacteroidetes, Firmicutes, Proteobacteria and Actinobacteria ${ }^{2}$. However, conditioning regimen appears to be the main responsible for microbiome composition changes after stem cell transplantation (SCT) ${ }^{3}$.

Material (or patients) and methods: The aim of our study was to analyze the human gut microbiome composition before and after SCT and to identified the relationship, if any, between microbiome imbalance and post-transplant outcome. For this purpose, we prospectively studied 17 patient (pts) undergoing an allogeneic SCT (matched sibling $n=9$, unrelated $n=8)$. The diagnosis was $\operatorname{AML}(n=10), \operatorname{MDS}(n=2), \operatorname{ALL}$ $(n=2), \mathrm{NHL}(n=1), \mathrm{HL}(n=1)$ and $\mathrm{AA}(n=1)$; the age ranged between 21 and 62 ys (median 47). Pts were prepared with a myeloablative regimen $(n=11)$ reduced intensity regimen $(n=6)$. Antibiotic prophylaxis was given to the allogeneic SCT recipient. Stool samples were collected at baseline before starting SCT conditioning and on day $+15,+30,+60,+100$. Another sample was provided at the time of GvHD onset. Samples were stored at $-80^{\circ} \mathrm{C}$ until they were processed. DNA was isolated with QIAamp DNA Stool Mini kit. The hypervariable V1-V3 region of the $16 \mathrm{~s}$ RNA gene was amplified by PCR and sequenced on 454 GS Junior Titanium. Taxonomic composition was defined according to Greengenes database. IBM SPSS Statistics 22 was used for statistical analysis.

Results: We identified a change in microbiome composition since baseline to day $+15,+30$ and +60 . In particular, a reduction of Firmicutes was documented at day $+15(19.05 \%$ vs $46.82 \% ; P=0.019)$ and $+30(27.06 \%$ vs $46.82 \% ; P=0.017)$, while an increase was founded between day +30 to day +60 (50.09\% vs $27.06 \% ; P=0.005)$. Contrarily, a Proteobacteria increase was found since baseline to day $+30(27.38 \%$ vs 
$0.04 \% ; P=0.014)$ and a new reduction was recorded between day +30 and $+60(6.25 \%$ vs $27.38 \%$; $P=0.05)$. At the time of GvHD onset, patients showed a reduction of Bacteroidetes with an increase of Proteobacteria $(46.89 \%$ vs $30.93 \%$ and $5.24 \%$ vs $21.22 \%$, respectively; $P=0.04$ )

Conclusion: We concluded that, starting from baseline microbiome composition, the conditioning regimen promotes changes in the microbiome after allo-SCT. In particular, after an opening reduction of Firmicutes Phylum (Bacilli, Lactobacilli, Clostridium, Enterococci, Streptococci) since baseline to day +30 , it arise at day +60 . On the contrary, Proteobacteria phylum increases since baseline to day +30 and than decreases at day +60 . Moreover, pts who developed GvHD harboured more Proteobacteria and less Bacteroidetes than pts without this complication.

References: 1) Shono Y, Docampo MD, Peled JU et al "Intestinal microbiota-related effects on graft-versus-hostdisease." Int J Hematol 2015; 101: 428.

2) Nuding S, Antony L, Stange EF "The hst and the flora" J Clin Investig 2014; 124: 4162.

3) Taur Y, Xavier JB, Lipuma L et al "Intestinal domination and the risk of bacteriemia in patients undergoing allogeneic hematopoietic stem cell transplantation" Clin Infec Dis 2012; 55: 905.

Disclosure of Interest: None declared.

\section{P500}

CD34+ selected ex-vivo T-cell depleted grafts for allogeneic hematopoietic cell transplantation is associated with low incidence of acute and chronic graft-versus-host disease (GVHD) and high chronic-GVHD/relapse-free survival

P. Barba ${ }^{1,2, *}$, P. Hilden ${ }^{2}$, S. Devlin ${ }^{2}$, M. Maloy ${ }^{2}$, J. Barker ${ }^{2}$, H. Castro-Malaspina ${ }^{2}$ A. Jakubowski', G. Koehne ${ }^{2}$, E. Papadopoulos', C. Sauter', M. van den Brink', J. Young' ${ }^{2}$, S. Avecilla ${ }^{2}$, R. Meagher', S. Giralt ${ }^{2}$, M. A. Perales ${ }^{2}$, D. Ponce ${ }^{2}$

${ }^{1}$ Hospital Universitari Vall d'Hebron, Barcelona, Spain, ${ }^{2}$ Memorial Sloan Kettering Cancer Center, New York, United States

Introduction: GVHD contributes significantly to transplantrelated morbidity and mortality after allo-HSCT. While clinical characteristics, therapeutic approaches and outcomes of patients developing acute (a-) and chronic (c-) GVHD after unmodified grafts for allo-HSCT have been widely analyzed, there is paucity of studies having specifically addressed these issues in the CD34+ selected ex-vivo TCD setting.

Material (or patients) and methods: We evaluated all consecutive patients with acute leukemia in complete remission or myelodysplastic syndrome (MDS) with $\leq 5 \%$ blasts undergoing CD34+ selected TCD allo-HSCT from peripheral blood mobilized progenitors in a single center from 01/2008 to

FIGURE. Probability of CRFS

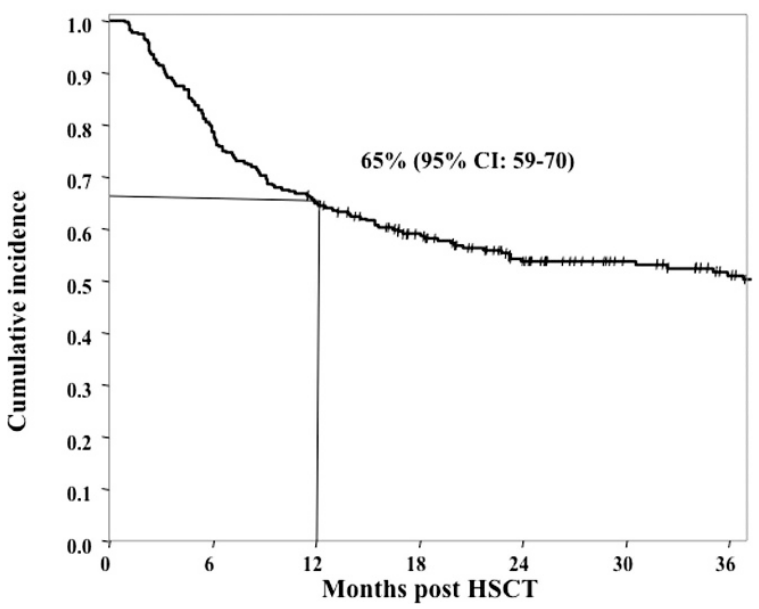

06/2014. IBMTR and NIH consensus criteria were used for diagnosis and grading of aGVHD and CGVHD, respectively. Several outcomes were evaluated including the recently defined $C G V H D$-free/ relapse-free survival (CRFS).

Results: A total of 255 patients with a median age of 56 years (range 20-73) were included (67\% AML, 13\% ALL, 20\% MDS). Seventy-seven patients presented with grade I-IV aGVHD at a median onset of 70 days (range 15-452). The majority developed aGVHD prior to day $100(49 / 77,64 \%)$ whereas $28 / 77$ (36\%) had aGVHD after day 100 . With a median followup of 22 months (range 12-85), the cumulative incidences (cum.inc.) of grade II-IV and III-IV aGVHD at day 180 were $18 \%$ (95\% Cl: 14-23) and 6\% (95\% Cl: 3-9), respectively. Among the 63 patients with aGVHD on day 180, the skin was the most commonly affected organ $(n=41,65 \%)$, followed by gastrointestinal tract $(n=33,52 \%)$, and liver $(n=5,8 \%)$. Treatment response (complete or partial) by day 28 in these patients was $81 \%$. Multivariate analysis was performed to determine risk factors for day 180 grade III-IV aGVHD. It demonstrated that recipient CMV seronegativity was the only variable associated with higher grade III-IV aGVHD [HR 5 (95\% Cl: 1.4-18.2), $P=0.01]$, whereas the use of HLA-mismatched donors [HR 2.7 (95\% Cl: 0.9-7.9), $P=0.08$ ] showed a trend. CGVHD developed in 13 patients at a median of 162 days (95-1645): 9 had classical cGVHD (6 interrupted/progressive, 3 de novo) and 4 had overlap syndrome. The cum.inc. of any grade of cGVHD at 3 years was $5 \%$ ( $95 \% \mathrm{Cl}: 3-8)$. Of the 229 patients who were alive and in remission at day 100, 201(88\%) had no GVHD after day 100 . The 1 -year probability of CRFS was $65 \%$ (95\% Cl: $59-$ 70) (Figure).

Conclusion: CD34+ selected TCD allo-HSCT is a calcineurin inhibitor (CNI)-free intervention associated with low rates of aGVHD and CGVHD. Recipient CMV seronegativity was associated with higher grade III-IV aGVHD at day 180 . The 1-year CRFS demonstrated to be high in this population. The development of a CNI-free allo-HSCT intervention is a promising and feasible approach in patients with acute leukemia and MDS in remission.

Disclosure of Interest: None declared.

\section{P501}

Major histocompatibility complex (MHC) gamma-block (GB) mismatch is associated with graft-versus-host disease (GVHD) in allogeneic hematopoietic stem cell transplantation (HSCT)

A. Jimenez ${ }^{1, *}$, R. L. Mitchell' ${ }^{1}$, P. Kongtim ${ }^{2}$, G. Patel ${ }^{3}$, I. Kurbegovic ${ }^{3}$, S. Piggott ${ }^{3}$, S. Kanangat ${ }^{3}$, D. Katz', S. Nathan ${ }^{1}$, J. Maciejewski ${ }^{1}$, G. Akpek

${ }^{1}$ Department of Hematology, Oncology and Cell Therapy, Rush University Medical Center, Chicago, United States, ${ }^{2}$ Department of Internal Medicine, Thammasat University, Bangkok, Thailand, ${ }^{3}$ Department of Pathology, Rush University Medical Center, Chicago, United States

Introduction: HLA mismatches at the class I and/or II loci are the major risk factor for GVHD. The MHC GB contains many inflammatory and immune regulatory genes including the complement C4 genes and is not typically considered in HSCT donor selection. We evaluated the association between MHCGB mismatch and cumulative incidence of GVHD and transplant related mortality (TRM) at day 100 after HSCT.

Material (or patients) and methods: We retrospectively analyzed $53 \mathrm{HSCT}$ donor/recipient pairs that matched (at least 10/10) HLA antigen and allele levels and underwent allogeneic HSCT at our institution between 2013 and 2015. Donor and recipient DNA from each sample were amplified by PCR using sequence specific primers for 25 single nucleotide gene polymorphism (SNPs); pairs with identical electrophoretic mobility patterns were considered GB-matched. Of 53 pairs, 23 were found to be GB mismatches. Standard transplant outcomes between GB-matched and mismatched pairs were compared. Primary study endpoint was rate of acute and chronic GVHD. Secondary endpoints were overall survival (OS), 
relapsed free survival (RFS), relapse rate and TRM. OS and PFS were calculated and plotted using the Kaplan-Meier method. Univariate comparisons were made by the log-rank test. A Cox proportional hazards model and the Fine and Gray method were used for multivariate regression analyses (MVA). Comparative results were provided after adjusting for other significant factors for a particular endpoint.

Results: We analyzed 53 recipient/donor pairs undergoing matched related donor $(n=22)$, matched $(10 / 10)$ unrelated donor $(n=26)$, haploidentical $(n=4)$ and syngeneic donor $(n=1)$ HSCT. Median follow up for surviving patients was 14 months (1-32 months). Source of stem cells was peripheral blood in all cases. Conditioning regimen intensity was reduced in 38 patients (66\%). Transplant indications included: acute myeloid leukemia $(n=20)$, acute lymphoblastic leukemia $(n=7)$, non-Hodgkin lymphoma $(n=9)$, and others $(n=17)$. Disease status at transplant was: complete response $(n=35)$, partial response $(n=7)$, refractory disease $(n=10)$ and early relapse $(n=1)$. Fifty five percent of the patients were male. Median age at the time of HSCT was 47.3 years (range 19-67). Based on univariate analysis, recipients with GB-mismatches had a higher incidence of grade $2-4$ acute GVHD $(20.5 \%$ v $13 \%$; $P=0.044)$, chronic GVHD (28.4\% v $16.3 \% ; P=0.048)$ and 1 -year TRM as compared to those with no mismatches $(24 \%$ v $8 \%$ respectively; $P=0.038$ ) (Table 1 , Figure 1 ). MVA showed that GB SNP mismatch was associated with higher TRM (HR: 1.5, $95 \% \mathrm{Cl} 1.17-2.44 ; P=0.020)$ and a trend for severe acute GVHD ( $\mathrm{HR}$ 2.450, 95\% Cl 0.96-6.22; $\mathrm{p}=0.060$ ) after controlling for donor type.

Table 1: Outcomes

\begin{tabular}{lccc}
\hline & $\begin{array}{c}\text { GB } \\
\text { Matched }\end{array}$ & $\begin{array}{c}\text { GB Mis- } \\
\text { matched }\end{array}$ & P \\
\hline 1-yr PFS & 76.5 & 62.5 & 0.846 \\
Median PFS & $\mathrm{NR}$ & $\mathrm{NR}$ & \\
1-yr OS & 86 & 67.4 & 0.211 \\
Median OS & $\mathrm{NR}$ & $\mathrm{NR}$ & \\
Cl of relapse at $1 \mathrm{yr}$ & 15 & 4 & 0.273 \\
1 yr TRM & 8 & 24.3 & 0.038 \\
Cl of aGVHD grade 2-4 at & 13 & 20.5 & 0.044 \\
100 days & & & \\
Cl of cGVHD at 1 yr & 16.3 & 28.4 & 0.048 \\
Cl of cGVHD extensive at & 8.6 & 16.4 & 0.071 \\
1 yr & & & \\
\hline
\end{tabular}

Conclusion: GB SNP mismatches appear to be associated with a higher risk of severe GVHD and TRM. Higher TRM at day 100 may be related to increased incidence of acute GVHD. While GB typing is not routinely performed in recipient/donor matching, it might be considered to help guide donor selection and minimize the risk of GVHD and transplantassociated mortality.

Disclosure of Interest: None declared.

\section{P502}

Antithymocyte globulin for steroid-refractory acute graftversus-host-disease: retropective analysis of unrelateddonor umbilical cord blood and sibling transplants

R. Hernani Morales ${ }^{1,2, *}$, J. Sanz ${ }^{1,2}$, G. Iacoboni $^{1}$, M. J. Arilla ${ }^{1}$, A. Regadera ${ }^{7}$, P. Montesinos ${ }^{1}$, I. Lorenzo ${ }^{7}$, F. Lopez ${ }^{1}$, I. Jarque ${ }^{1}$ S. Romero', A. Balaguer', G. Sanz ${ }^{1,2}$, M. A. Sanz ${ }^{1,2}$

${ }^{1}$ DEPARTMENT OF HEMATOLOGY, LA FE HOSPITAL, ${ }^{2}$ MEDICINE DEPARTMENT, UNIVERSITY OF VALENCIA, VALENCIA, Spain

Introduction: To our knowledge, there is no specific information on therapy and factors affecting response to antithymocyte globulin (ATG) in umbilical cord blood transplants (UCBT). The aim of this study was to analyze the efficacy of ATG for the treatment of steroid refractory acute graftversus-host disease (SR-aGVHD) in adults with hematological malignancies undergoing unrelated-donor UCBT or allogeneic stem cell transplantation from HLA-matched sibling donors (MST) and to identify prognostic factors for outcome.

Material (or patients) and methods: From January 1997 to January 2014, 89 patients (UCBT 44; MST 45) developed grade $\geq 2$ SR-aGVHD that required salvage therapy with ATG and were included in the study.

Results: Median age was 44 years (range, 16-66 years). Fiftyone patients (57\%) had been diagnosed of acute leukemia and 61 patients (69\%) received a mieloablative conditioning. Staging of aGVHD at time of initiation of ATG was as follows: grade II in 23 patients (26\%); III in 37 patients (42\%); IV in 29 patients (33\%). ATG-response was achieved in $14(61 \%), 16$ $(43 \%)$ and $8(28 \%)$ patients with initial grade of 2,3 and 4 , respectively $(P=0.05)$. There were no differences in outcome after UCBT or MST. Regarding the impact on the type of organ involvement, 13 of the 47 patients (28\%) with liver involvement (with or without other organ involvement), 17 of 32 patients (53\%) with gastrointestinal involvement (without liver and with or without skin involvement) and 8 of 10 patients $(80 \%)$ with isolated skin involvement were ATG-responders $(P=0.003)$ (Figure 1). Type of organ involvement prior to therapy (RR 3.5, 95\% Cl, 1.67-7.33, $P<0.001$ ) and diagnosis of acute leukemia (RR $0.3,95 \% \mathrm{Cl}, 0.11-0.84, P=0.019$ ), were associated with better response to ATG. Cumulative incidence of non-relapse mortality (NRM) at 5 years was $85 \%$. Type of organ involvement prior to therapy ( $\mathrm{HR} 0.68,95 \% \mathrm{Cl}, 0.49-0.95$, $P=0.02)$ and lower platelet count (HR $0.95,95 \% \mathrm{Cl}, 0.9-0.99$, $P=0.039)$ were associated with increased NRM. 5-y overall survival was $8 \%$. Type of organ involvement prior to therapy (HR 0.62, 95\% Cl, 0.44-0.88; $P=0.008$ ), higher LDH (HR 1.04; $95 \% \mathrm{Cl}, 1.01-1.07 ; P=0.04)$ and lower platelet count (HR 0.93; $95 \% \mathrm{Cl}, 0.87-0.98 ; P=0.01$ ) were associated with lower survival. Early response at day +6 discriminated for survival probability. 5 -y OS was $14 \%, 5 \%$ and $0 \%$ for patients with complete/partial response, stable disease and progression to ATG, respectively $(P<0.001)$.

Conclusion: This study showed similar outcome after ATG for SR-aGVHD in patients receiving single UCBT or MST. Response rate for patients with liver involvement was disappointing. ATG was associated with elevated morbidity and mortality that hampered long-term clinical benefit for most patients, even those with initial organ response to salvage therapy.

Disclosure of Interest: None declared.

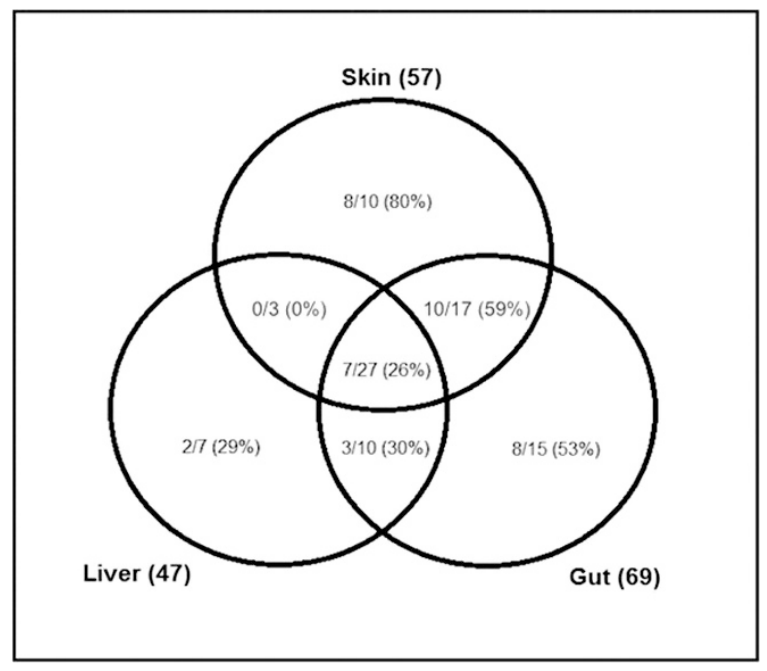


P503

JAK-inhibition to treat steroid-refractory acute and chronic graft versus host disease (SR-GvHD) - A single center experience

S. Syed Abd Kadir ${ }^{1, *}$, C. Wolschke ${ }^{1}$, F. Ayuk ${ }^{1}$, S. Janjetovic ${ }^{1}$, U. von Pein ${ }^{1}$, A. Van Randenborgh ${ }^{1}$, M. Christopeit ${ }^{1}$, N. Kröger ${ }^{1}$

${ }^{1}$ Department of Stem Cell Transplantation, University Medical Center Hamburg-Eppendorf, Hamburg, Germany

Introduction: Graft-versus-host disease (GvHD), which is a serious complication following allogenic stem cell transplantation (allo-SCT) may develop in $20-80 \%$ recipients. Steroid remains the first line therapy and those who are steroid refractory have dismal outcome. Ruxolitinib (Jakavi ${ }^{\mathbb{R}}$ ) is an JAK1/2 inhibitor approved for treatment of Myelofibrosis. It significantly reduced inflammatory cytokine and previous work from us and others, have shown dramatic effect of T-cells.

Material (or patients) and methods: We report 9 consecutive patients receiving Ruxolitinib for SR-GvHD in our center, between May and November 2015. The patients were 46 to 74 years old, with median age of 63. Type of transplantation included matched related donor $(n=2)$, matched unrelated donor $(n=3)$ and mismatched unrelated donor $(n=4)$. All patients received Mycophenolate mofetil (MMF) and Cyclosporin A (CsA) as GvHD prophylaxis. In addition, 8 patients received anti-thymoglobulin (ATG) and 1 patient received post-transplantation Cyclophosphamide. GvHD was evaluated according to NIH criteria. Four patients had severe acute SRGvHD (skin, $n=4$; liver, $n=2$; gut, $n=2$ ). Six patients with chronic SR-GvHD had 2-3 organ involvements; skin (6), liver (5), gut (4), mouth (2) and eye (1). The onset of cGvHD was between 106 to 154 days (median 130.3 days) after allo-SCT in 4 patients and 45 days after donor lymphocyte infusion in 1 patient. One patient had GvHD relapse after 1 year of stable CGvHD, in which Ruxolitinib was prescribed for. Ruxolitinib was prescribed with dosage 10-20 mg twice daily for SR-aGvHD and 5-10 mg twice daily for SR-cGvHD.

Results: Corticosteroid therapy was tapered down and eventually stopped when Ruxolitinib was started. CsA and MMF were continued for all aGvHD. We observed complete response (CR) in 3 patients with SR-aGvHD. One patient had partial response (PR) and progressed to $C G v H D$. One patient is still receiving Ruxolitinib after achieving $C R$ until this review. No relapse GvHD observed in the 3 patients after stopping Ruxolitib with median duration of follow up 45 days after stopping. All patients developed cytopenia, with 1 patient warranted Ruxolitinib withdrawal. In SR-cGvHD, Ruxolitinib was added after MMF alone $(n=2)$ or MMF and Calcineurin inhibitor (CsA or Tacrolimus, $n=4)$, and all are undergoing extracorporeal photopheresis (ECP). One patient died of sepsis. The remaining 5 patients had early near CR but signs of GvHD progressed after 1-2 weeks. Ruxolitinib were withdrawn in 3 patients after 15 to 82 days (median 52.3 days) due to severe cytopenia. One patient stopped after 85 days as she has stable disease. One patient is still on Ruxolitinib at the time of review with stable disease.

Conclusion: This case series demonstrated a more effective role of Ruxolitinib in aGvHD, but limited due to cytopenia. In the setting of cGvHD, perhaps Ruxolitinib can be used as an adjunct therapy to ECP. Further prospective clinical trial on the use of Ruxolitinib in aGvHD and CGvHD is required to determine it's optimal dosage, timing, efficacy and safety.

References: Spoerl, Silvia, et al. "Activity of therapeutic JAK 1/2 blockade in graft-versus-host disease." Blood 123.24 (2014): 3832-3842. Zeiser, R., et al. "Ruxolitinib in corticosteroidrefractory graft-versus-host disease after allogeneic stem cell transplantation: a multicenter survey." Leukemia 29.10 (2015): 2062-2068.

Disclosure of Interest: None declared.

\section{P504}

Single-needle off-line extracorporal photopheresis: feasibility and side-effects

S. Flommersfeld ${ }^{1, *}$, E. Wollmer ${ }^{2}$, R. Marschall', G. Bein ${ }^{3}$ U. J. Sachs $s^{3}$

${ }^{1}$ Transfusionsmedizin, ${ }^{2}$ Haematology, Oncology, PHILIPS-UNIVERSITÄT MARBURG, Marburg, ${ }^{3}$ Transfusionsmedizin, Justus-Liebig University, Giessen, Germany

Introduction: Extracorporal photopheresis (ECP) is an established second-line treatment for both graft-versus-hostdisease (GVHD) after stem cell transplantation (SCT) and the bronchiolitis obliterans syndrome (BOS) after lung transplantation. A single-needle, off-line protocol was established in our center since we considered many patients unsuitable for a dual-needle system approach. Buffy coat is collected on a MCS + device (Haemonetics, Munich, Germany), exposed to UVA light in the presence of 8-methoxypsoralen in the Macogenic system (Macopharma, Langen, Germany), and re-transfused. Here, we analyse the patients' feasibility and report on the number of side effects observed in ECP.

Material (or patients) and methods: A total of 793 ECP procedures in 44 patients were analysed retrospectively to collect data on ECP feasibility related to the venous access site, and side effects.

Results: The mean age of patients was 49 years, 35\% were female, and ECP was initiated 492 days (range 21-2,117) after transplantation. The mean duration of ECP was 20 weeks (range 1-93) with 18 procedures per patient (range 2-54). In the initial patient assessment, 14/44 patients were scheduled for central venous access (CVA), whereas $4 / 44$ had one, and 26/44 patients had two sufficient peripheral veins. In total, 534 procedures were performed via single-needle peripheral vein access, of which 85 procedures $(15.9 \%)$ required change of the punctured vein during the procedure. 31/534 procedures $(5.8 \%)$ were terminated early because an acceptable apheresis flow rate could not be maintained. The same termination rate was observed in the CVA cohort $(16 / 259=6.2 \%)$. The decision to cease ECP because of limited venous access was made in 5/44 patients (median week 9, range 1-26); 3/5 with CVA. Clinical side effects were observed in $18 / 793$ procedures $(2.3 \%)$ including, citrate reactions (10), vasovagal reactions (2), dyspnoea (2) hypopotassemia (2), and Gl bleeds associated with low platelet counts (2), equally distributed between the two cohorts. Major incidents observed in the CVA cohort included catheter-associated infections (5), fibrin obstruction requiring lysis (4), and catheter-associated thrombosis (2). Transfusion support with RBCUs or platelets was required in $10 / 44$ patients for $28 / 793$ procedures $(3.5 \%)$, again with no difference between the two groups.

Conclusion: A major challenge in ECP is venous access. Singleneedle, off-line protocols using peripheral veins are feasible in the majority of patients $(63.6 \%)$, and most procedures are uneventful (63.4\%). Occasionally, peri-procedural re-puncturing may be required. Based on our data, we conclude that $24 / 44$ patients $(54.5 \%)$ would also qualify for a peripheral dualneedle approach, but only in 308/793 (38.8\%) procedures. Early termination of single-needle ECP procedures is not more frequent than in procedures with central lines. Of note, central lines put significant additional risk on patients. Central lines should only be considered for ECP if no reliable peripheral access can be established. Side effects of ECP not related to the venous access are rare and easily manageable.

Disclosure of Interest: None declared. 


\section{P505}

Extracorporeal photopheresis for acute graft-versus-host disease after allogeneic hematopoietic stem cell transplantation - a multicentre experience

S. Eder ${ }^{1, *}$, R. Belhocine ${ }^{1}$, L. Margo ${ }^{2}$, M.-T. Rubio ${ }^{1}$, A. Ruggeri ${ }^{1}$ R. Duléry ${ }^{1}$, J. Gauthier, A. Le Bourgeois ${ }^{1}$, G. Battipaglia', F. Giannotti', E. Brissot ${ }^{1}$, I. Yakoub-Agha ${ }^{2}$, M. Mohty ${ }^{1}$

${ }^{1}$ Service d'Hématologie Clinique et de Thérapie Cellulaire, Hôpital Saint-Antoine, Paris, ${ }^{2}$ Service des Maladies du Sang, Hôpital Claude Huriez, CHRU Lille, Lille, France

Introduction: Acute graft-versus-host disease (aGvHD) is a major limitation after allogeneic hematopoietic stem cell transplantation. The standard-treatment consists of methylprednisolone (MP) and a calcineurin-inhibitor. No standardized second-line treatment for aGvHD exists. Here, we report a multi-centre experience with extracorporeal photopheresis (ECP) for aGvHD: the objective was to investigate the efficacy of ECP for patients with steroid-refractory/-dependent aGvHD as well as an early intervention in patients with low-grade aGvHD to avoid steroids. Furthermore, we evaluated the reduction of immunosuppressive therapy.

Material (or patients) and methods: Until December 2015 29 patients with aGvHD were treated. Five patients had grade I GvHD (whereas three were not treated with MP and two patients had ongoing MP-therapy due to engraftment syndrome), nine patients had a maximum grade II GvHD, 12 patients were graded as III and three patients had grade IV. Organ involvement was as follows: skin only in 20, gastrointestinal tract (GIT) only in one, skin and GIT in three and skin liver, GIT in five patients. Treatment before ECP consisted of MP in 26 patients, and four patients had a second-line therapy (two with basiliximab, one with mycophenolic acid and one with methotrexate). One patient received a third-line therapy (sirolimus). Before start of ECP, 18 patients were steroidrefractory/-dependent.

Results: The median number of ECP sessions per patient was 12 (range, 4-36), five patients received ECP three times, nine patients twice and 15 patients once a week. Best response to ECP was complete remission (CR) in $46 \%$, partial response (PR) in $21 \%$ and no response in $32 \%$, after a median number of 4 treatments (range, 2-9). Response was better for grade I/II: all patients received CR/PR compared to $53 \%$ with grade III/IV. We evaluated the tapering of immunosuppressive therapy between first and last session of ECP: mean reduction of MP was $97 \%$ (range, 60-100) and mean reduction of calcineurininhibitor was 69\% (range, 40-100). Ten patients could terminate ECP due to resolution of aGvHD, eight patients are still under continuously ECP. Reasons for interrupting ECP were no response, relapse/death or venous access.

Thirteen of 23 eligible patients (57\%) developed chronic GvHD, whereas seven patients were treated with ECP for that as well as three patients for overlap syndrome. Four patients relapsed from their underlying disease and ten patients died (three during ECP: two after relapse and one after septic shock).

Conclusion: In this multi-centre experience, ECP was efficient and safe in patients with steroid-refractory/-dependent aGvHD and also when used upfront in patients with low-grade GvHD. We were able to taper adjuvant immunosuppressive therapy with a mean reduction of steroids of $97 \%$ and a mean reduction of calcineurin-inhibitor of $69 \%$. Best responses were seen in patients with grade I/II GvHD, suggesting that ECP should be started as early as possible.

Further studies should aim at determining the optimum schedule for ECP in order to avoid recurrence of aGvHD upon discontinuation and to prevent the development of chronic GvHD (57\% in our cohort after aGvHD).

Disclosure of Interest: None declared.
P506

Prospective clinical study identifies cytokeratin (CK)-18 and CK18 fragments as sensitive and specific biomarkers of acute hepato-intestinal GVHD (NCT00935324)

S. Sauer ${ }^{1, *}$, A. Radujkovic ${ }^{1}$, U. Hegenbart ${ }^{1}$, A. Ho $^{1}$, P. Dreger ${ }^{1}$, T. Luft

${ }^{1}$ University Hospital Heidelberg, Heidelberg, Germany

Introduction: Cytokeratin-18 (CK18) is an abundant protein in liver and gut epithelial cells. Retrospective analyses have suggested that both, CK18 and its apoptotic degradation product CK18 fragments (CK18F) could indicate apoptotic end organ damage induced by acute hepato-intestinal GVHD and, thus, may be used as an acute GVHD biomarker. Here, we present the first results of a prospective clinical study aiming to assess the capacity of CK18 and CK18F as biomarkers to predict acute hepato-intestinal GVHD activity and its response to treatment.

Material (or patients) and methods: Main endpoints of the study were (1) prediction of imminent acute hepato-intestinal GVHD by comparing CK18/CK18F serum levels measured 7 to 14 days prior to symptom onset with baseline levels (preconditioning, at transplant and one week after alloSCT) of the same subject; and (2) prediction of response to GVHD treatment by measuring CK18/CK18F kinetics 3, 7 and 14 days after start of immunosuppressive therapy for hepato-intestinal GVHD. Blood samples were collected in the study population prior to the initiation of the conditioning regimen and from the day of transplantation on a weekly basis for one year. The M30-Apoptosense ELISA Kit was used for measuring serum levels of CK18F, CK18 was analyzed with the M65 (EpiDeath) ELISA Kit (both provided by PEVIVA, Sweden). Serum levels measured at the pre-specified landmarks were compared by Wilcoxon's signed rank test. The study was registered at ClinicalTrials.gov (NCT00935324).

Results: 109 patients were enrolled, median age was 53 years (range 19 - 69), 72 were male. The underlying disease was AML in 39 patients, MPN/ MDS in 18 patients, ALL in 10 patients, and lymphoproliferative malignancies in 42 patients. Donors were MRD (36), MUD (52), or MMUD (21). Stem cell source was PB in 100 patients and BM in 9 patients. RIC protocols $(n=89)$ prevailed over myeloablative $(n=7)$ and aplasia conditioning protocols $(n=13)$. Immunosuppression was based on a calcineurin inhibitor (CNI) backbone with mycophenolate or short-course methotrexate, plus ATG in mismatched or unrelated transplants. Acute hepato-intestinal GVHD grade II-IV developed in 38 patients $(34,9 \%)$ at a median of 53 days post-transplant and was steroid-refractory in 12 patients. In patients with biopsy-proven acute hepato-intestinal GVHD, CK18 significantly raised 7 to 14 days before clinical GVHD onset and can therefore serve as an early predictor of impending acute hepato-intestinal GVHD (median at baseline vs preGVHD, $149 \mathrm{ng} / \mathrm{ml}$ vs $527 \mathrm{ng} / \mathrm{ml} ; P=0.012$ ), and peaked at symptom onset (median $1281 \mathrm{ng} / \mathrm{ml} ; P=0.003$, compared to baseline). CK18F kinetics paralleled that of CK18 but was less pronounced. In contrast, patients with isolated acute skin GVHD, patients without acute GVHD (cut-off day+53), and patients with GVHD-typical symptoms but a negative biopsy did not show any increase CK18 or CK18F in serum (Figure 1). However, CK18/CK18F levels were not correlated with aGVHD severity. Neither marker predicted refractory GVHD or nonrelapse mortality after aGVHD at any of the time points measured.

Conclusion: This prospective study identifies CK18 and CK18F as specific biomarkers to support the diagnosis and prediction of suspected imminent and clinically manifest acute hepatointestinal GVHD. Neither CK18 nor CK18F can be used to predict the prognosis of an ongoing GVHD episode.

Disclosure of Interest: None declared. 


\section{P507}

Extracorporeal photophoresis for the treatment of acute or chronic GvHD supports immune suppressive cells

S. Anita ${ }^{1, *}$, L. Wang ${ }^{1}$, A. Hückelhoven ${ }^{1}, \mathrm{M} . \mathrm{Ni}^{1}$, J. Hong ${ }^{1}$, P. Wuchter ${ }^{1}$, J. Puthenparambil ${ }^{1}$, A. Ho ${ }^{1}$, P. Dreger ${ }^{1}$, W. Krüger ${ }^{2}$, M. Schmitt'

${ }^{1}$ Department of Internal Medicine V, University Clinic Heidelberg, Heidelberg, ${ }^{2}$ University of Greifswald, Greifswald, Germany

Introduction: Acute and chronic graft-versus-host disease (GvHD) constitute a serious problem for patients after allogeneic stem cell transplantation. A promising therapeutic approach represents the extracorporeal photopheresis (ECP). ECP modulates the immune system however the mechanism has still to be elucidated. Therefore we analyzed the clinical and immunological effects in 20 patients during ECP therapy. Material (or patients) and methods: Nine patients with aGvHD of the gut overall 'II-IV suffering from extensive diarrhea were treated with ECP next to extensive immunosuppressive therapy including steroids, calcineurin inhibitors and/or mycophenolate mofetil (MMF) and pentostatin and/or ruxolitinib treatment in advance. Furthermore 11 patients with cGvHD of the skin or lung despite triple-drug therapy comprising steroids, cyclosporine A, mTOR inhibitors and/or MMF received ECP treatment. Clinical and immunological parameters were analyzed.

Results: Within the intensive ECP treatment for patients with aGvHD of the gut, the average stool volume and frequency declined and consistency changed from loose to formed bowels and steroids could be tapered down to a mean of $22 \%$ of the initial dose. CGvHD patients stabilized under ECP treatment. Steroid dose in CGvHD patients could be reduced by a mean of $38 \%$.

Clinically responding patients showed immunologically an increase of CD14 ${ }^{+} \mathrm{HLA}-\mathrm{DR}{ }^{-/ l o w}$ myeloid derived suppressor cells (MDSCs) defined by CD33 and CD11b. Activated CD $33^{\text {hi }}$ CD $11 b^{\text {hi }}$ MDSCs increased over time paralleled by a decrease of inactivated $C D 33^{-/ l o w} C D 11 b^{\text {hi }}$ MDSCs. V $\delta 2^{+} \gamma \delta$ $T$ cells increased along with the up-regulation of CD4/CD8 double-negative $\mathrm{CD}^{+}{ }^{+} \mathrm{T}$ cells. Furthermore, Tc17 and Th17 cells were up-regulated in patients with complete clinical response. Moreover, not only $\mathrm{CD} 4^{+} \mathrm{CD} 25^{\text {hi }}$ Foxp $3^{+}$regulatory $\mathrm{T}$ cells but also $\mathrm{CD} 8^{+} \mathrm{Foxp}^{+}$regulator $\mathrm{T}$ cells increased under insensitive ECP treatment.

Conclusion: ECP treatment might stabilize and even improve the clinical situation in patients suffering from acute or chronic GvHD. In responding patients we observed immunologically a modulation of the immune system. According to our data ECP enhances the immunosuppressive cell subsets like MDSCs, Tc17, Th17 and Treg cells.

Disclosure of Interest: None declared.

\section{P508}

The Impact of CD3 Cell Dose on the outcome of HLAmatched Allogeneic Hematopoietic Stem Cell Transplantation

M. O. Jamil' ', S. Hashmi 2, ${ }^{2}$, M. Rizwan ${ }^{7}$, M. Hathcock ${ }^{3}$, E. Jacob ${ }^{4}$, A. Saad

${ }^{1}$ Blood and Marrow Translantation, University of Alabama at Birmingham, Birmingham, 2Internal Medicine, ${ }^{3}$ Mayo Clinic, Rochester, ${ }^{4}$ Division of Transfusion, Mayo Clinic, Rocester, United States

Introduction: Allogeneic hematopoietic stem cell transplantation (allo HCT) is prescribed using a target CD34+ cell dose of $2-5 \times 10^{6} / \mathrm{kg}$ body weight. The CD3+ (T cell) content of the graft is a key mediator of acute graft versus host disease (aGVHD). $\mathrm{CD} 3+\mathrm{T}$ cells are also essential for engraftment, immune reconstitution, and disease control (graft versus tumor effect). The graft CD3+ cell dose is not accounted for except in T cell depleted transplants. We hypothesize that there is graft CD3+ $T$ cell dose range that is associated with better survival outcome.
Material (or patients) and methods: This is a retrospective study of a total of 604 patients treated at Mayo Clinic and University of Alabama at Birmingham, USA) who underwent HLA-matched (related and unrelated) peripheral Blood stem cell (PBSC) transplant between 2007 and 2014. We excluded patients who received $>$ one allo transplant, with syngeneic donor, or received donor lymphocyte infusion, antithymocyte globulin, alemtuzumab, or post-transplant cyclophosphamide. Patients were divided into 3 groups based on the CD3+ cell dose (measured as $\times 10^{7} / \mathrm{kg}$ of recipient body weight); group $A$ $(<30)$, group B $(30-60)$, group $C(>60)$. We estimated the overall survival (OS), disease free survival (DFS), incidence of aGVHD and chronic GVHD (cGVHD), relapse rate, and NRM in all gorups.

Results: Patient characteristics are summarized in Table 1. The median CD3+ T cell dose was $27.7 \times 10^{7} / \mathrm{kg}$ (range: $2-5-246$ ). GVHD prophylaxis was calcineurin inhibitor (cyclosporin or tacrolimus) plus mini methotrexate in 598/604 patients (99\%). Median duration of follow up of surviving patients was 17.1 months (0.6-90 months). The following outcome results are described in groups $A, B$ and $C$ respectively. The incidence of grade II-IV aGVHD was 29,34 , and $43 \%(P=\mathrm{NS})$, whereas grade III-IV aGVHD was $10 \%, 12 \%$ and $10 \%(P=N S)$. The incidence of cGVHD was $10 \%, 5 \%$, and $15 \%$. The incidence of relapse was $17 \%, 12 \%$, and $8 \%(P=N S)$, whereas NRM was $31 \%, 27 \%$, and $40 \%(P=N S)$. The median OS was 34, 62, and 16 months (logrank $P=0.05$ ) (figure $1 \mathrm{a}$ ), whereas median DFS was 32,55 months, and 18 months (logrank $P=0.08$ ) (figure $1 \mathrm{~b}$ ).

Conclusion: $\mathrm{CD} 3+\mathrm{T}$ cell content of PBSC grafts is vastly variable. In our series, a CD3+ T cell dose $>60 \times 10^{7} / \mathrm{kg}$ tended to have worse NRM and shorter DSF and OS, while CD3+ T cell dose $30-60 \times 10^{7} / \mathrm{kg}$ tended to have better DFS and OS. These finding require further testing on larger cohort or observational prospective clinical trial.

Disclosure of Interest: None declared.

\section{P509}

Incidence, clinical features, and outcomes of patients with renal graft versus host disease following allogeneic stem cell transplant

S. Hashmi 1,*, J. Reneau ${ }^{2}$, M. Alexander ${ }^{3}$, N. Leung ${ }^{4}$, M. Patnaik ${ }^{1}$ ${ }^{1}$ Hematology, ${ }^{2}$ Internal Medicine, ${ }^{3}$ Pathology, ${ }^{4}$ Nephrology, Mayo Clinic, Rochester, United States

Introduction: Renal graft versus host disease (rGHVD) is a rare and poorly defined complication of allogeneic hematopoietic stem cell transplantation (HCT). Previous publications regarding rGVHD have reported an incidence ranging from $1-3.5 \%$. The presentations include nephrotic range proteinuria and renal pathology consistent with minimal change disease (MCD) or membranous nephropathy (MN).

Material (or patients) and methods: This was a single institution, retrospective observational study to evaluate the incidence of rGVHD, its clinical presentation, and response to therapy.

Results: Eleven (1\%) cases of renal GVHD were identified among 1,063 successive allogeneic HCT performed at the Mayo Clinic between 1990 and July, 2014. The median age at transplant was 52 years (range 32 to 67) and $72 \%$ were male. Five had matched related donors, 4 had matched unrelated donors, and 2 had HLA mismatched unrelated donors. Six patients had peripheral blood stem cell grafts and 4 had bone marrow grafts. One patient had a failed bone marrow graft followed by a second transplant with a peripheral blood stem cell graft from the same donor. Five patients underwent myeloablative conditioning and six received reduced intensity conditioning. Two patients experienced only acute GVHD (aGVHD) following transplant, 5 experienced both aGVHD and chronic GHVD (cGVHD), and 2 experienced only cGVHD. Of these patients, 1 had aGHVD and 5 had CGVHD at the time of rGVHD diagnosis. The median time to proteinuria was 619 days (131 to 4847 days), with the median daily proteinuria being

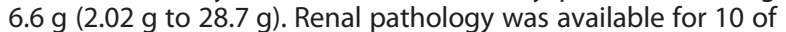


Table 1: Patient characteristics

\begin{tabular}{|c|c|c|c|}
\hline & $\begin{array}{c}\text { Group } A(\mathrm{n}=334) C D 3+\text { cell } \\
\text { dose }\left(<30 \times 10^{7} / \mathrm{kg}\right)\end{array}$ & $\begin{array}{c}\text { Group } B(\mathrm{n}=230) C D 3+\text { cell } \\
\text { dose }\left(30-60 \times 10^{7} / \mathrm{kg}\right)\end{array}$ & $\begin{array}{c}\text { Group } C(\mathrm{n}=40) C D 3+\text { cell } \\
\text { dose }\left(>60 \times 10^{7} / \mathrm{kg}\right)\end{array}$ \\
\hline Age: median (range) & 53 (11-72yrs) & 55 (20-72yrs) & 57 (10-70yrs) \\
\hline \multicolumn{4}{|l|}{ Sex } \\
\hline Males & 207 (62\%) & $136(59 \%)$ & $22(55 \%)$ \\
\hline Females & $126(38 \%)$ & $94(41 \%)$ & $18(45 \%)$ \\
\hline \multicolumn{4}{|l|}{ Race } \\
\hline Caucasian & $299(89 \%)$ & $212(92 \%)$ & $33(83 \%)$ \\
\hline Blacks & $9(3 \%)$ & $7(3 \%)$ & 0 \\
\hline Others & $25(8 \%)$ & $11(5 \%)$ & $7(17 \%)$ \\
\hline \multicolumn{4}{|l|}{ Donor source } \\
\hline Related & $178(53 \%)$ & $165(72 \%)$ & 37 (84\%) \\
\hline Unrelated & $155(47 \%)$ & $65(28 \%)$ & $2(16 \%)$ \\
\hline $\begin{array}{l}\text { Median CD34+ cell dose } \\
/ \mathrm{kg}\left(\mathrm{X} 10^{6}\right)(\text { range })\end{array}$ & $5.8(2.5-13.6)$ & $5.8(1.6-5036)$ & $5.2(1.2-10.6)$ \\
\hline $\begin{array}{l}\text { Median CD3+ cell dose } / \mathrm{kg} \\
\left(\mathrm{X} 10^{7}\right) \text { (range) }\end{array}$ & $19.4(2.5-30)$ & $40(30.3-59.8)$ & $68(60.1-246.6)$ \\
\hline \multicolumn{4}{|l|}{ Regimen type } \\
\hline Myeloablative & $144(44 \%)$ & $88(38 \%)$ & $12(30 \%)$ \\
\hline $\begin{array}{l}\text { Reduced intensity/ } \\
\text { non- myeloablative }\end{array}$ & $186(56 \%)$ & $141(62 \%)$ & $28(70 \%)$ \\
\hline Missing & $3(<1 \%)$ & $1(<1 \%)$ & \\
\hline
\end{tabular}
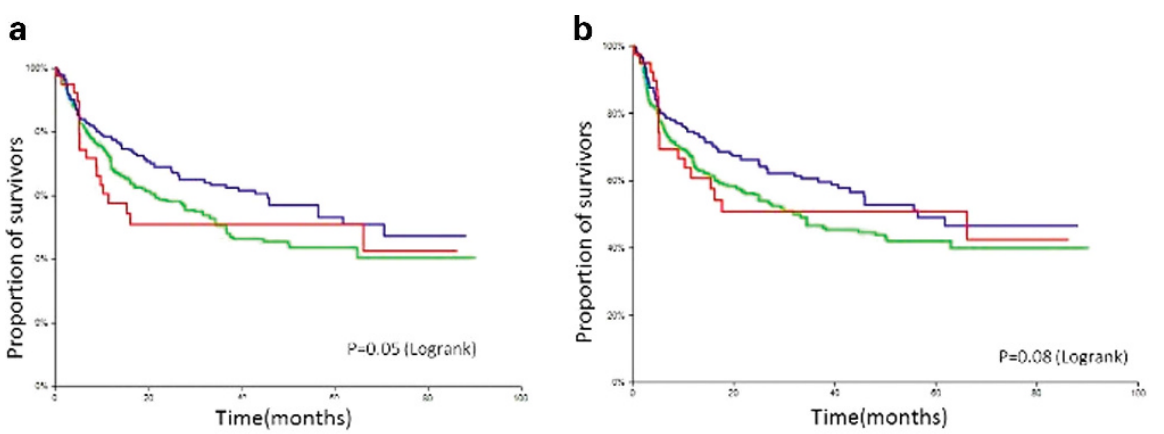

Figure 1: $A)$ overall survival $(O S)$ and $B)$ disease-free survival for the three groups
based on $C D 3+T$ cell dose $\left(\times 10^{7}\right.$ cells $\left./ K_{B}\right)$.
Group $A(<30)$
Group B $(30-60)$
Group C $(>60)$

11 patients. MCD was noted in 4 patients with concomitant segmental $M N$ and thrombotic microangiopathy (TMA) noted in one and concomitant IgA nephropathy in another. MN was noted in 4 patients with concomitant TMA in one. There was one case of immune complex mediated glomerulonephritis. Only minor glomerular abnormalities were noted in the final patient. Nine patients were treated with prednisone, some in combination with cyclosporin (2) thalidomide (1), and mycophenolate mofetil (2). One patient was treated with mycophenolate and one patient was treated with cyclosporine and hydroxychloroquine. Those that failed initial therapy were treated with second line agents including ATG (1) or rituximab (3). One patient died 58 days following onset of rGVHD due to renal failure. A second patient died 56 days following transplant due to complications of acute GHVD. Of the remaining 9 patients, all had resolution of proteinuria to $<1 \mathrm{gram} /$ day after a median of 136 days ( 22 to 509 days).
Conclusion: Cases of suspected rGVHD are rare following allogeneic stem cell transplant $(\sim 1 \%)$. The presentation is heterogeneous. In our study, there were a wide variety of pathologic findings with MCD and MN being common. Most patients responded well to immunosuppressive therapy.

Disclosure of Interest: None declared.

\section{P510}

Optimal blood concentration of tacrolimus (FK506) for prevention of moderate to severe chronic graft versus host disease in allogeneic haematopoietic stem cell transplantation from unrelated donors

S. Park ${ }^{1,}$, K. Kim ${ }^{1}$, J. H. Jang ${ }^{1}$, S. J. Kim ${ }^{1}$, W. S. Kim ${ }^{1}$, C. W. Jung ${ }^{1}$ ${ }^{1}$ Samsung Medical Center, Seoul, Korea, Republic Of

Introduction: Tacrolimus (FK506) is widely used for prevention of graft versus host disease (GVHD) after allogeneic hematopoietic stem cell transplantation (allo-HSCT). However, 
target blood levels varies among institutions and it has rarely been studied that how the blood level of tacrolimus affect the incidence of chronic GVHD (cGVHD).

Material (or patients) and methods: A total of 223 patients who underwent allo-HSCT for malignant and non-malignant hematologic disease from a HLA-matched or mismatched unrelated donors between Mar 2007 and Nov 2014 were retrospectively reviewed. Peripheral blood was used as a stem cell source in almost cases $(n=219,98.3 \%)$, and the patients received methotrexate and tacrolimus as a prophylaxis regimen for GVHD. According to institutional strategy, administration of tacrolimus were adjusted with target level of 10-15, 8-12, and 5-10 (ng/ml) during the first, second, and third month after transplantation. Primary objective was the association of blood tacrolimus level and the occurrence of moderate to severe CGVHD. Overall survival (OS) and cumulative incidence of relapse (CIR) according to tacrolimus levels were also assessed as secondary end points.

Results: The median age at the time of allo-HSCT was 43 years and acute leukemia was the most common underlying disease $(n=166,74.4 \%)$. The average monthly tacrolimus blood concentration $\left(\mathrm{TAC}^{\mathrm{avr}}, \mathrm{ng} / \mathrm{ml}\right)$ was calculated in each patient: $0-1,1-2$, and 2-3 months after allo-HSCT. The median value of TAC $^{\text {avr }}$ was $12.2,8.6$, and $6.4 \mathrm{ng} / \mathrm{ml}$ during the first, second, and third month, respectively. In Cox-proportional hazard model, TAC $^{\text {avr }}$ at the second month showed significant association with the occurrence of moderate to severe cGVHD in multivariate analysis adjusted for gender, age, total body irradiation, anti-thymocyte globulin, acute GVHD $\geq$ grade 2, HLA-matching status and TAC ${ }^{\text {avr }}$ levels of other periods. The risk of CGVHD development was lowest in patients with $2^{\text {nd }}$ month TAC ${ }^{\text {avr }}$ of $<8 \mathrm{ng} / \mathrm{ml}(P=0.036)$. Other than tacrolimus level, ATG administration and HLA matching status $(\geq 7 / 8$, high resolution) were significantly advantageous for less occurrence of cGVHD ( $p<0.001$ for ATG, $p=0.010$ for HLA matching). The multivariate analysis for OS revealed that the TAC $^{\text {avr }}$ level $<8 \mathrm{ng} / \mathrm{ml}$ during 1-2 month after HSCT showed a significant association with better OS $(P=0.006)$, and $\mathrm{CIR}$ did not differ by tacrolimus levels.

Conclusion: Blood concentration of tacrolimus below $8 \mathrm{mg} / \mathrm{ml}$ during the second month after transplantation was significantly associated with the decreased risk of moderate to severe CGVHD.

Disclosure of Interest: None declared.

P511

The impact of graft-versus-host disease on dendritic cell homeostasis and their potential use as biomarker to predict the severity of chronic graft-versus-host disease S. Thiant ${ }^{1,2, *}$, J. Roy ${ }^{1,2}$, J. Trottier ${ }^{1}$, M.-P. Giard ${ }^{1}$, R. Parat ${ }^{1}$, D.-C. Roy ${ }^{1,2}$, M. Guimond $d^{2,3}$

${ }^{1}$ Division of Hematology-Oncology, HOPITAL MAISONNEUVEROSEMONT, ${ }^{2}$ Université de Montréal, ${ }^{3}$ Department of Microbiology-Immunology, HOPITAL MAISONNEUVE-ROSEMONT, Montreal, Canada

Introduction: Graft-versus-host disease (GVHD) is the principal complication of allogeneic stem cell transplantation (allo-SCT) and occurs when GVHD T cells recognize differences in major and/or minor histocompatibility antigens expressed by recipient cells. Following cytotoxic regimens, the inflammatory milieu represents a fertile ground for alloreactivity and thymic insults resulting from GVHD are largely responsible for thymic dysfunction and immune-incompetence. Studies have also demonstrated that GVHD insult to the peripheral niche is perhaps the most important factor for limiting thymic independent $\mathrm{T}$ cell regeneration. Given that patients with chronic GVHD (cGVHD) are profoundly lymphopenic and that dendritic cells (DCs) play a critical role in T cell homeostasis, we postulated that changes in DC counts could precede the development of clinical signs of acute and/or chronic GVHD. Material (or patients) and methods: 44 patients that received HLA-matched allogeneic SCT were included in this study. Controls consist of 15 patients that received autologous $\mathrm{SCT}$ and 30 healthy donors. Flow cytometric analysis was used to measure plasmacytoid DCs (pDCs), myeloid DC type 1 $(\mathrm{mDC} 1), \mathrm{mDC} 2$ and $\mathrm{mDC} 3$. Blood samples were obtained at day 0,1 month post-SCT and then every other month until 1 year. The percentage and absolute counts of naïve $\mathrm{CD}^{+}$or $\mathrm{CD}^{+}$lymphocytes $\left(\mathrm{T}_{\mathrm{NA}}\right)$; $\left(\mathrm{CD}^{+}{ }^{+} \mathrm{CD} 45 \mathrm{RA}^{+} \mathrm{CCR7}{ }^{+}\right)$, central memory $\left(T_{C M}\right) ;\left(C D 3^{+} C D 45 R A^{+} C C R 7^{\text {neg }}\right)$, effector memory $\left(T_{E M}\right)$; ( $\mathrm{CD}^{+} \mathrm{CD} 4 \mathrm{RA}^{\text {neg }} C \mathrm{CR} 7^{\text {neg }}$ ) and terminal differentiation $\left(\mathrm{T}_{\mathrm{TD}}\right)$; $\left(C D 45 R^{+} C C R 7^{\text {neg }}\right.$ ) was determined by flow cytometry. B lymphocytes and regulatory $\mathrm{CD} 4+\mathrm{T}$ cells were also evaluated. Results: One month after allo-SCT, we found a significant increase in blood DCs followed by a gradual decrease and stabilization by 3 months post-allo-SCT. During the first year, all $\mathrm{DC}$ subsets including $\mathrm{pDCs}, \mathrm{mDC} 1, \mathrm{mDC} 2, \mathrm{mDC} 3$ as well as $\mathrm{CD}^{+}$and $\mathrm{CD}^{+}{ }^{+}$cells were significantly diminished compared to autologous and healthy controls. Plasmacytoid DCs regeneration was in general the most affected and we found a positive correlation between pDC cells count and the number of naïve and central memory $\mathrm{CD}^{+}$lymphocytes $(r=X)$. Similarly, pDCs counts also correlated with the number of naïve and memory $\mathrm{CD}^{+} \mathrm{T}$ cells. In contrast, effector $\mathrm{CD} 4^{+}$ and $\mathrm{CD}^{+} \mathrm{T}$ cell showed a stronger correlation with $\mathrm{mDC} 1$. Patients with grade II-IV acute GVHD had lower DC counts compared with mild grade 0-1 aGVHD and a similar trend was also observed during the development of CGVHD, Importantly, loss of pDCs and $\mathrm{mDC} 1$ that preceded the development of cGVHD by 1 month was associated with the development of grade 3 cGVHD whereas loss of DCs during cGVHD was associated with milder 0-2 cGVHD.

Conclusion: Thus far, our data support a model wherein loss of $\mathrm{pDCs}$ and $\mathrm{mDC1}$ could represent potential biomarkers to predict the severity of cGVHD.

Disclosure of Interest: None declared.

\section{P512}

GVHD prophylaxis with abatacept for patients with nonmalignant diseases - a single-center experience

S. Radygina ${ }^{1, *}$, A. Vasilieva ${ }^{1}$, D. Balashov ${ }^{7}$, L. Shelikhova ${ }^{1}$, E. Gutovskaia ${ }^{1}$, A. Livshits', I. Shipitsina ${ }^{1}$, A. Laberko A. Abrosimov ${ }^{1}$, R. Khismatullina ${ }^{1}$, M. Maschan ${ }^{1}$, G. Novichkova ${ }^{3}$, A. Maschan ${ }^{1}$

${ }^{7}$ bone marrow transplantation, ${ }^{2}$ immunology, ${ }^{3}$ pediatric hematology and oncology, Dmitry Rogachev Federal Research and Clinical Centre of Paediatric Haematology, Oncology and Immunology, Moscow, Russian Federation

Introduction: GVHD and associated with it morbidity and mortality remain significant and unresolved problem after alloHSCT. Interactions between T-cell receptor (TCR) and antigen presenting cells (APCs) play the major role in T-cell activation but without co-stimulatory signaling from APCs to T-cell can't potentiate aGVHD. Abatacept is a recombinant fusion protein that interrupts the T-cell co-stimulatory signal mediated through the CD28-CD80/CD86 pathway. We presented results of the single-center, open-label, controlled, randomized study to evaluate the efficacy of GVHD prophylaxis with abatacept for patients with non-malignant diseases.

Material (or patients) and methods: Sixty patients, included in the study, (median age 6,6 years, range 0,2-23,0 years) with different non-malignant diseases (SAA, $n=25$; PIDs, $n=32$; FA, $n=3)$ were transplanted from MRD $(n=18), \operatorname{MUD}(n=39)$ and MMRD $(n=3)$. Forty patients received TCRab/CD19 depleted grafts (MUD, $n=37$; MMRD, $n=3$ ); 20 patients (MUD, $n=4$; MRD, $n=16$ ) were transplanted with non-manipulated SCT. Patients received tacrolimus (after TCRab/CD19 depleted SCT) or tacrolimus/methotrexate (after non-manipulated graft) as a standard protocol of aGVHD prophylaxis.

All patients were randomized into two groups: control group received standard protocol of GVHD prophylaxis; patients from study group received abatacept $(10 \mathrm{mg} / \mathrm{kg} ;-1,+5,+14,+28$ days) as the additional agent to the standard GVHD prophylaxis. 


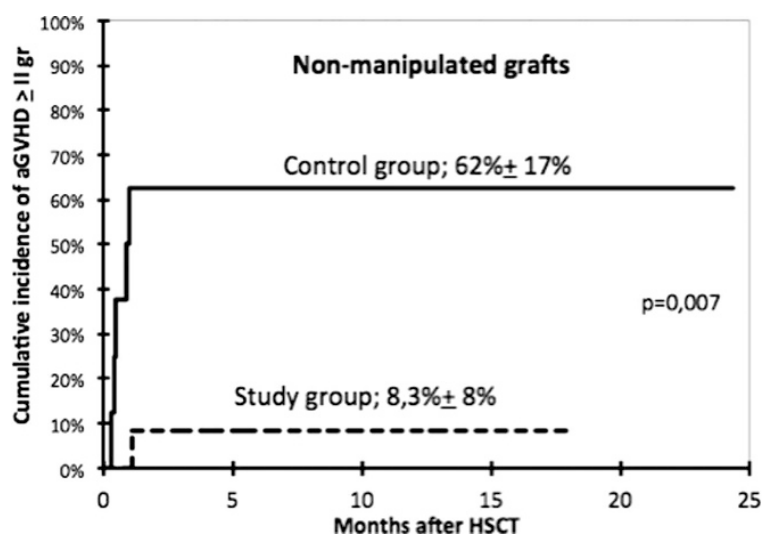

Results: We compared kinetics of immune recovery and graft failure rates in both groups and did't find any difference. We did not observed statistically significant difference in the incidence of aGVHD ( $\geq$ Grade II) between randomized groups following TCRab/CD19-depleted SCT (control group, $n=20, \mathrm{Cl}$ $33,8 \% \pm 11,4 \%$; study group, $n=20, \mathrm{Cl} 31,2 \% \pm 10,8 \% ; P=0,79$ ). However, the probability of aGVHD ( $\geq$ Grade II) after HSCT with non-manipulated graft was significantly higher in control group $(n=8 ; \mathrm{Cl} 62 \% \pm 17 \%)$ to compare with study group $(n=12 ; \mathrm{Cl} 8,3 \% \pm 8 \%) ; P=0,007$ (see Figure).

Conclusion: The efficacy of co-stimulatory signal blockage after TCRab/CD19 graft depletion has not been seen due to high efficacy of GVHD prevention with graft engineering. Importantly, that all cases of GVHD in that groups were mild and treated successfully with first-line therapy.

Nevertheless, significantly decreased incidence of aGVHD in non-manipulated SCT group demonstrates the efficacy of abatacept in GVHD prevention. Importantly, addition of abatacept to standart GVHD prophylaxis did not influence on the kinetics of the immune recovery and rejection rates.

Disclosure of Interest: None declared.

\section{P513}

Gut impairment and host macrophages reactivity before conditioning regimen predict the development of acute graft versus host disease

T. Hueso ${ }^{1, *}$, L. Magro ${ }^{2}$, V. Coiteux ${ }^{2}$, J. Gauthier ${ }^{2}$, T. Jouault ${ }^{1}$ J.-L. Desseyn ${ }^{1}$, I. Yakoub-Agha ${ }^{2}$, D. Seguy ${ }^{1}$

${ }^{1}$ UMR-995, ${ }^{2}$ Hematology, Lille, France

Introduction: Acute graft versus host disease (aGvHD) remains the first cause of early death after allogeneic stem cell transplantation (allo-SCT). In mouse model, gut injury induced by conditioning regimen plays a critical role in aGvHD development, by increasing microbial translocation and macrophages reactivity responsible for alloreactivity. In human, few data are available about gut health before conditioning regimen and the role of circulating monocytes in aGvHD pathogenesis. In such patients, plasmatic concentration of citrulline, a non proteic amino acid, is a marker of small bowel functional enterocyte mass. The aim of this work was to assess the gut health and the reactivity of circulating monocytes derived macrophages before the conditioning regimen, and to study their relationship with the occurrence of aGvHD.

Material (or patients) and methods: One month before undergoing allo-SCT, citrulline plasma level ( $\mu \mathrm{mol} / \mathrm{l}$ ) was measured by HPLC; circulating monocytes were separated using Ficoll gradient and counted using the trypan blue dye exclusion in 40 patients. After overnight platting, circulating monocytes derived macrophages were stimulated with pathogens associated molecular patterns (PAMP): LPS, PAMcsk4, Flagellin, MDP and curdlan, which recognize pattern recognition receptors (PRR) as TLR, NLR or CLR. TNF- $\alpha$, IL-1 $\beta$, IL-6, IL-10 and INF- $\gamma$ (pg/ml) were measured in stored culture supernatants for these different conditions using multiplex fluorescent immunoassay. BMI, albuminemia and CRP were also evaluated. Analysis compared patients who declared a grade I to IV aGVHD (aGvHD group) within the 100-day postransplant period and those who did not (no aGvHD group). Twelve healthy donors (HD group) constituted a control group. Results are expressed in median and interquartile (med [Q1-Q3]).

Results: In aGvHD group ( $n=16)$, citrulline was lower than in no aGvHD group $(n=24)$, (22 [18-25] vs. 26 [21-30], $P=0.04)$. Conversely, IL-6 was higher in aGvHD group after LPS (154 [71373 ] vs 79 [49-107], $P=0.05), \operatorname{MDP}(P=0.035)$ and curdlan $(P=0.07)$ stimulation compare to no aGvHD group and to HD group after LPS, PAM, FLA and curdlan stimulation $(P<0,04)$. IL-10 was also higher in aGvHD group after LPS (66 [21-105] vs 21 [10-46], $P=0.003)$, PAM $(P=0.007)$, FLA $(P=0.023)$ MDP $(P=0.035)$ or curdlan $(P=0.004)$ stimulation compare to no aGvHD group and to HD group after each PAMP stimulation $(P<0.05)$. IL-6 and IL-10 correlated positively whatever the PAMP used. The secretion of others cytokines, BMl, albuminemia and CRP were similar between aGvHD and no aGvHD groups while INF- $\gamma$ remained undetectable. Results in no aGvHD group were not different than those observed in HD group.

Conclusion: These results suggest that infra-clinical preconditioning gastro-intestinal damages allow microbial translocation and modulate the macrophages reactivity predisposing to aGvHD development.

\section{References:}

1. Crenn, P. et al. Gastroenterology 119, 1496-1505 (2000).

2. Hempel, L. et al. Bone marrow transplantation 20, 365-368

(1997).

3. Hill, G. R. \& Ferrara, J. L. Blood 95, 2754-2759 (2000).

4. Kennedy, G. A. et al. The Lancet Oncology 15, 1451-1459 (2014).

5. Seguy, D. et al. Transplantation 94, 287-294 (2012).

Disclosure of Interest: None declared.

P514

ATG and statins reduce incidence of severe chronic GVHD by distinct mechanisms - influence of Vitamin B6

K. Schmidt ${ }^{1}$, K.-H. Kellner ${ }^{2}$, A. Radujkovic ${ }^{1}$, N. Lehners? ${ }^{1}$,

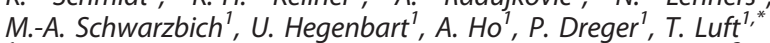
${ }^{7}$ Medicine V, UNIVERSITÄTSKLINIKUM HEIDELBERG, Heidelberg, ${ }^{2}$ Dr. Kellner Technische Unternehmensberatung, Karlsruhe, Germany

Introduction: Severe chronic graft versus host disease (cGVHD) is a serious complication after allogeneic stem cell transplantation (alloSCT). It affects survival by causing debilitating morbidity due to fibrotic and inflammatory changes in a variety of connective tissues. In contrast, mild CGVHD is strongly associated with better overall survival (OS) due to lower relapse rates, so that permissive immunosuppressive drug management is actively pursued by many clinicians. Although alloreactive T lymphocytes are clearly involved in the induction of both grades of CGVHD, it is unpredictable which patients are prone to develop severe rather than mild chronic disease. Monokine induced by interferon gamma (MIG, CXCL9) has recently been correlated with CGVHD. Anthranilic acid (AA) reduced the severity of acute GVHD in mice. AA is the product of a pathway involving indoleamine 2,3-dioxygenase (IDO), tryptophane (Trp), kynurenine (Kyn), and Vitamin B6. Based on our observation that anti-thymocyte globulin (ATG) and statins independently reduce the incidence of severe CGVHD, we investigated if MIG, IDO, Trp, Kyn and Vitamin B6 serum levels are correlated with cGVHD.

Material (or patients) and methods: The incidence of cGVHD was evaluated in 554 patients who consented to participate in this observational study and who survived the first 6 months after alloSCT. 325 patients received ATG (largely unrelated donor transplants), and 244 patients received pravastatin at a dose of $20 \mathrm{mg} / \mathrm{d}$ starting from day-1 of alloSCT as per institutional policy. 176 patients received both, ATG and pravastatin, whereas 159 
patients received neither. Chronic GVHD was diagnosed and graded as severe or non-severe applying the National Institutes of Health's 2005 consensus criteria.

Day +100 serum samples for measuring CXCL9/MIG, IDO, Trp and Kyn by ELISA were available for 350 patients and at onset of cGVHD for 185 patients. Furthermore, VitB6 was measured by HPLC on day +100 in 268 patients.

Results: Chronic GVHD occurred in 295 patients (54\%), 58 (11\%) of whom developed severe cGVHD of skin, lungs or eyes, $40(7 \%)$ isolated severe CGVHD of the gastrointestinal tract, and 197 (36\%) developed non-severe cGVHD. ATG and statin use were associated with reduced incidence of any type of severe cGVHD. In contrast, only ATG reduced the incidence of mild chronic GVHD. Increased levels of CXCL9 and Kyn were observed for both mild and severe CGVHD at disease onset. Patients who received ATG prior to transplantation showed significantly lower serum levels of CXCL9 at CGVHD onset, but not on $d+100$. In contrast, statins did not influence CXCL9 levels, but were associated with lower serum levels of Trp and Kyn and increased IDO levels on day+100. Kyn serum levels on day+100 despite statin usage predicted higher incidence of severe cGVHD. This suggested that Kyn catabolism requiring Vitamin B6 plays an important role in the development of severe CGVHD. Indeed, d100 Vitamin B6 serum levels were the strongest predictor of an impending severe GVHD in uni- and multivariable analyses.

Conclusion: Our data suggest that ATG and statins minimize severe CGVHD by distinct mechanisms involving inhibition of CXCL9 producing $T$ cells and activation of IDO and Kyn catabolism.This is supported by the observation that $\mathrm{d} 100$ Vitamin B6 levels strongly predicted cGVHD. The synergism of statin and Vitamin B6 is clinically relevant and warrants further studies.

Disclosure of Interest: K. Schmidt: None declared, K.-H. Kellner Personal Interest: ELISAs for Trp, Kyn and IDO, A. Radujkovic: None declared, N. Lehners: None declared, M.-A. Schwarzbich: None declared, U. Hegenbart: None declared, A. Ho: None declared, P. Dreger: None declared, T. Luft: None declared.

P515

GVHD-related mortality is associated with high levels of CCR7+ CD4 T lymphocytes in the graft

V. Gomez Garcia De Soria ", , I. Portero ${ }^{2}$, C. Fernandez Arandojo ${ }^{7}$, C. Muñoz

${ }^{1}$ HEMATOLOGY, ${ }^{2}$ IMMUNOLOGY, HOSPITAL DE LA PRINCESA, MADRID, Spain

Introduction: Graft versus host disease (GVHD) remains the major complication associated with hematopoietic stem cell transplantation (HSCT). Its pathogenesis involves the migration of donor T cells towards the secondary lymphoid organs, which is mainly steered by the chemokine receptor CCR7. We have recently demonstrated that the percentage of donor CCR7+ T cells in the infunded graft is significantly associated with the development and severity of GVHD. However, there are no studies analyzing the impact of the migratory capacity of donor T cells on the outcome of the patients.

Material (or patients) and methods: Apheresis samples from 108 donors were collected in this single center study. The minimum and maximun follow up was from 1 to 4 years. The expression of the chemokine receptor CCR7 was analyzed on CD4+ and CD8+ T cells from the donor graft by flow cytometry. Its value was expressed as percentage from the total cells infunded and used as a surrogate marker of migratory capacity. Treatment-related mortality and relapse- related mortality were analyzed by Kaplan-Meier method and log-rank test after dichotomizing the values of CCR7+ T cells above the 75 percentil and below the 25 percentil.

Results: To study whether the percentage of CCR7+ T cells in the apheresis correlates with survival after the transplantation, the patients were divided into "high CCR7 expression" (\% of CCR7+ cells higher than 75 percentil) and "low CCR7 expression" (\% of CCR7+ cells less than 25 percentil) both for CD8+ and CD4+ T lymphocytes. No significant association was found between CCR7+ CD8+ T cells and mortality. Regarding CD4+ T cells, the mortality related to GVHD was significantly higher $(P=0,02)$ in the "high CCR7 CD4" group. However, mortality related to relapse was not associated with the percentage of CCR7+ CD4+ T cells $(P=0.7)$ in both groups. Conclusion: Donor grafts with high percentage of CCR7+ T cells content are associated with increased treatment-related mortality. These results suggest that an immunotherapy aimed at reducing high levels of CCR7+ T cells could prevent GVHD-related mortality without affecting the incidence of relapse leading to mortality.

Disclosure of Interest: None declared.

\section{P516}

Clinical Efficacy of Umbilical Cord-Derived Mesenchymal

Stem Cells in the Prophylaxis of Chronic Graft-versus-Host Disease after HLA-haploidentical Stem Cell

Transplantation

L. Gao ', J. Liu', Y. Zhang ${ }^{2}$, Y. Liu' ${ }^{1}$, C. Zhang ${ }^{1}$, L. Gao ${ }^{1}$ L. Zhu ${ }^{1}$, Q. Wen ', P. Wang ${ }^{1}$, X.-H. Chen ${ }^{1}$, P.-Y. Kong ', J. Zhong ${ }^{1,3}$, Y. Su $^{4}$, H. M. $L i^{5}$, X. Zhang ${ }^{1, *}$

${ }^{7}$ Department of hematology, Xinqiao Hospital, Third Military Medical University, ${ }^{2}$ Department of Health Statistics, College of Military Preventive Medicine, Third Military Medical University, Chongqing, China, "3Pathology of Keck School of Medicine, University of Southern California, Los Angeles, California, United States, ${ }^{4}$ Department of Hematology, General Hospital of Chengdu Military Region of PLA, Chengdu, ${ }^{5}$ Department of Hematology, Affiliated Hospital of Kunming Medical College, Kunming, China

Introduction: Although mesenchymal stem cells (MSCs) possess immunomodulatory properties and exhibit promising efficacy against chronic graft-versus-host disease (cGVHD), little is known regarding MSCs in the prophylaxis of cGVHD after HLA-haploidentical hematopoietic stem cell transplantation (HLA-haplo HSCT).

Material (or patients) and methods: In this multicenter clinical trial, we investigated the effects of MSCs derived from the umbilical cord as a prophylactic therapy for 42 recipients after HLA-haplo HSCT. The findings led us to compare the outcome of a cohort of MSC transfusion recipients after HLAhaplo HSCT to that of a similar historical cohort of recipients who never received MSCs.

Results: We found that the 2-year cumulative incidence of CGVHD in the MSCs group compared with the non-MSCs control group was $29.2 \%$ (15.3-43.1\%) vs. $51.9 \%$ (36.5-63.5\%). Nine patients in the non-MSCs control group had severe lung CGVHD, but none in the MSCs group developed typical lung cGVHD. After the MSC transfusion, the number of NK cells decreased, but the numbers of memory $B$ lymphocytes and regulatory $T$ (Treg) cells and the ratio of Th1:Th2 increased.

Conclusion: Our findings suggest that the continuous transfusion of MSCs might prevent cGVHD symptoms in patients after HLA-haplo HSCT, accompanied by an alteration of the numbers and subtypes of T/B/NK cells leading to the acquisition of immune tolerance.

Disclosure of Interest: None declared. 
P517

The Safety and Tolerability of RGI-2001 in the In Vivo Modulation of Regulatory T-cells (Tregs) in Patients Undergoing Allogeneic Hematopoietic Stem Cell Transplantation (AHSCT) in a First-in-Human (FIH) Phase I/Ila Dose-Finding Study

Y.-B. Chen ${ }^{1, *}$, Y. A. Efebera ${ }^{2}$, L. Johnston ${ }^{3}$, E. Ball ${ }^{4}$, D. Avigan ${ }^{5}$, L. J. Lekakis ${ }^{6}$, C. R. Bachier ${ }^{7}$ J. Bui ${ }^{8}$, P. Martin ${ }^{9}$, O. Duramad ${ }^{10}$

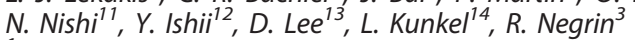

${ }^{1}$ Bone Marrow Transplant Program, Massachusetts General Hospital Cancer Center, Boston, ${ }^{2}$ Division of Hematology, The Ohio State University James Cancer Hospital, Columbus, ${ }^{3}$ Blood and Marrow Transplantation, Stanford University, Stanford, ${ }^{4}$ Division of Bone Marrow Transplantation, University of California San Diego, Moores UCSD Cancer Center, La Jolla, ${ }^{5}$ Hematology-Oncology, Beth Israel Deacons Medical Center, Boston, ${ }^{6}$ Division of Hematology/Oncology, University of Miami Hospital and Clinics, Sylvester Comprehensive Cancer Center, Miami, ${ }^{7}$ Texas Transplant Institute, a member of the Sarah Cannon Blood Cancer Network, San Antonio, ${ }^{8}$ Department of Pathology, University of California San Diego, La Jolla, ${ }^{9}$ Division of Clinical Research, Fred Hutchinson Cancer Research Center, Seattle, ${ }^{10}$ Department of Immunology, REGiMMUNE Corp., Berkeley, United States, ${ }^{11}$ Department of Development, REGiMMUNE Corp., ${ }^{12}$ REGiMMUNE Corporation, Tokyo, Japan, ${ }^{13}$ Medical Affairs 360, San Diego, ${ }^{14}$ Department of Development, REGiMMUNE, Berkeley, United States

Introduction: RGI-2001 is a liposomal formulation of KRN7000 (a-galactosylceramide). CD1d mediated activation by RGI-2001 stimulates an immunologic cascade resulting in regulatory T-cell (Treg) expansion (ref. 1). A Phase I/II open-label, multicenter, dose-escalation study of RGI-2001 administered as a single intravenous infusion in patients (pts) with hematologic malignancies undergoing allogeneic hematopoietic stem cell transplantation (AHSCT) was conducted to determine the safety, tolerability, pharmacokinetics, pharmacodynamics (PD), and acute graft-versus-host disease (GvHD) outcome at day 100.

Material (or patients) and methods: Bone marrow (BM) or peripheral blood stem cells (PBSC), related or unrelated, with $\leq 1 \mathrm{HLA}$ allele or antigen mismatch was allowed. GvHD prophylaxis included a calcineurin inhibitor with methotrexate and/or mycophenolate mofetil or sirolimus (SIR). RGI-2001 was administered within 30 minutes after AHSCT. In phase lla, pts were randomized to $1.0 \mu \mathrm{g} / \mathrm{kg}$ vs. $100 \mu \mathrm{g} / \mathrm{kg}$ and a contemporaneous control was enrolled to evaluate Treg kinetics.

Results: A total of 57 pts ( 20 acute myeloid leukemia, 9 acute lymphoblastic leukemia, 7 myeloproliferative, and 21 other malignancies) received RGI-2001. Median age was 51 years old (19 to 72 years); $24.6 \%$ received unmodified BM and $75.4 \%$ received PBSC grafts. Clinically significant elevated acute inflammatory cytokines and infusion-related toxicities were not observed. There was no apparent effect on engraftment, and no DLTs. The Day 100 mortality was 5.3\% (3/57), deaths were unrelated to RGI-2001. The control group mean Treg frequency (\%) in the CD4 population remained below $6 \%$ through day 29. Expansion cohorts at $1 \mu \mathrm{g} / \mathrm{kg}$ and $100 \mu \mathrm{g} / \mathrm{kg}$ demonstrated a mean increase in Treg \% with largest \% increases in RGI-2001+SIR (Figure 1). The $\mathrm{t} 1 / 2$ at $100 \mu \mathrm{g} / \mathrm{kg}$ was 23.4 hours. The PD effect as measured by onset of Treg \% expansion occurred on day 8 and persisted through day 29 . There were no reports of Grade (G) IV GVHD in any cohorts. In the $1 \mu \mathrm{g} / \mathrm{kg}$ group $37 \%$ (7/19) had GII-IV, 5\% (1/19) had GIII; GII-IV GvHD in pts with the SIR use was $25 \%(2 / 8)$. In the $100 \mu \mathrm{g} / \mathrm{kg}$ group the GII-IV GVHD was $43 \%$ (9/21) with $9.5 \%(2 / 21) \mathrm{GIII}$, there was no GvHD in the 3 pts treated with $100 \mu \mathrm{g} / \mathrm{kg} \mathrm{RGI}-2001$ and SIR.

Table 1. Dose Escalation Schedule and Patient Numbers

\begin{tabular}{lccc}
\hline Part & $\begin{array}{c}\text { Dose } \\
\text { Group }\end{array}$ & $\begin{array}{c}\text { RGI-2001 Dose } \\
(\mathrm{mg} / \mathrm{kg})\end{array}$ & $\begin{array}{c}\text { Number of } \\
\text { Patients }\end{array}$ \\
\hline Phase I $(n=28)$ & 1 & 0.001 & 2 \\
& 2 & 0.01 & 6 \\
& 3 & 0.1 & 4 \\
& 4 & 1 & 5 \\
Phase Ila $(n=40)$ & 5 & 10 & 5 \\
& 6 & 100 & 6 \\
& 1 & 1 & 14 \\
& 2 & 100 & 15 \\
& Control & n.a. & 11 \\
\hline
\end{tabular}

[P517]

Figure 1. Treg Frequency and Counts in Peripheral Blood after Administration of RGI-2001
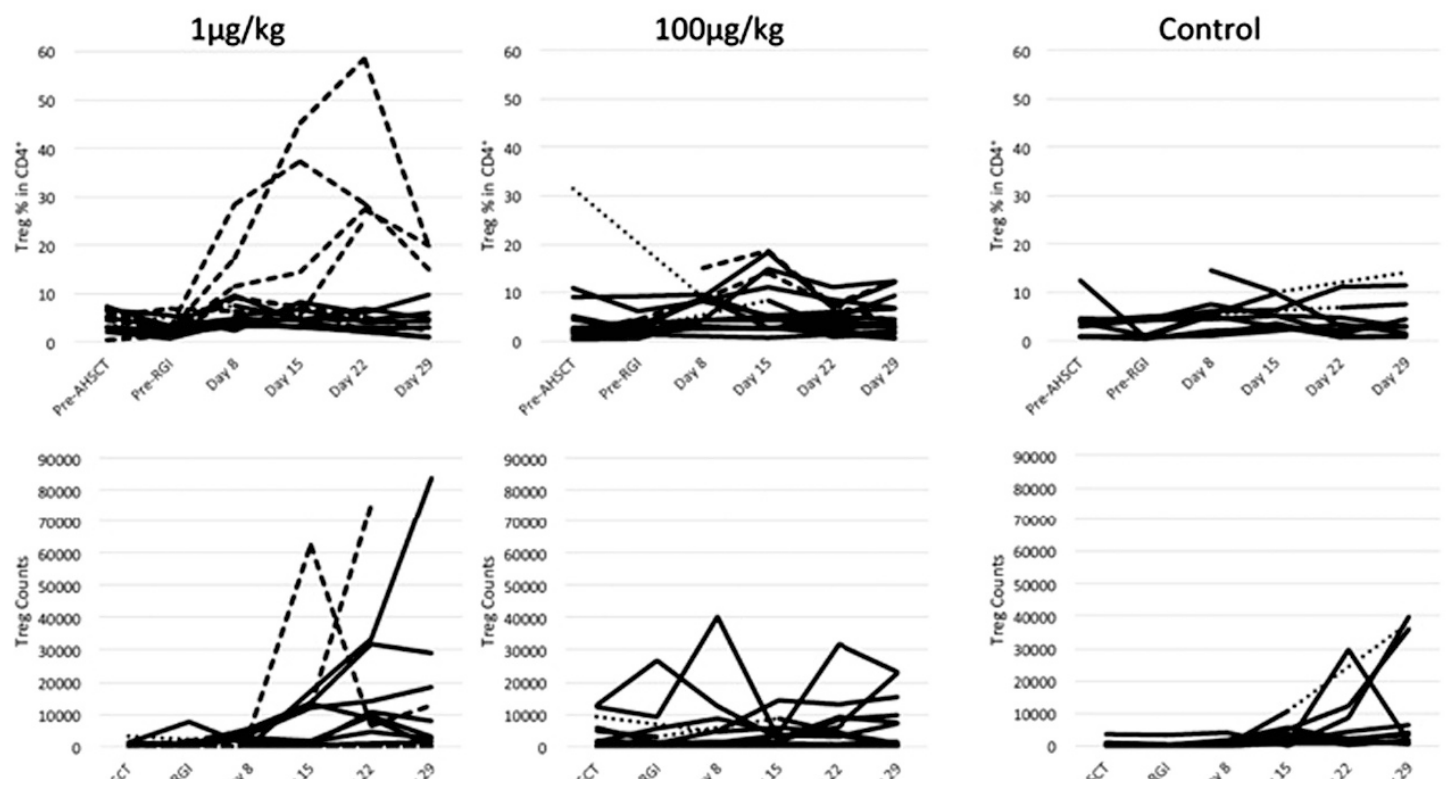
Conclusion: RGI-2001 was safe and well-tolerated up to $100 \mu \mathrm{g} / \mathrm{kg}$ in pts with hematological malignancies undergoing AHSCT. In vivo expansion of Treg was observed in pts treated with RGI-2001. SIR based regimens appeared most permissive to Treg expansion, and further studies with SIR/RGI-2001 is warranted.

References: 1. Duramad et al., Biol Blood Marrow Transplant. 2011; 17: 1154-1168

Disclosure of Interest: Y.-B. Chen: None declared, Y. A. Efebera: None declared, L. Johnston: None declared, E. Ball: None declared, D. Avigan: None declared, L. J. Lekakis: None declared, C. R. Bachier: None declared, J. Bui: None declared, P. Martin: None declared, O. Duramad Employee of: REGiMMUNE Corp., N. Nishi Employee of: REGiMMUNE Corp., Y. Ishii Employee of: REGiMMUNE Corp., D. Lee Funding from: REGiMMUNE Corp., L. Kunkel Employee of: REGiMMUNE Corp., R. Negrin: None declared.

\section{P518}

Transplantation-associated thrombotic microangiopathy in allogeneic hematopoietic stem cell transplantation: risk and prognostic factors

Y. Ye ${ }^{1, *}$, W. Zheng ${ }^{1}$, J. Wang ${ }^{1}$, Y. Hu ${ }^{1}$, Y. Luo ${ }^{1}$, Y. Tan ${ }^{1}$, M. Zhang ${ }^{1}$, H. Huang

${ }^{1}$ Bone Marrow Transplantation Center, The First Affiliated Hospital, Zhejiang University, Hangzhou, China

Introduction: Transplantation-associated thrombotic microangiopathy (TA-TMA) is a significant complication of hematopoietic stem cell transplantation. However, it remains controversial which clinical or laboratory markers are of evident risk and prognostic value.

Material (or patients) and methods: From 2006 to 2013, a nested case control study was carried out in our center to study the risk and prognostic factors of TA-TMA. 654 consecutive patients who underwent allogeneic hematopoietic stem cell transplantation were studied. Risk factors were examined using a nested cohort and non-relapse mortality was compared.

Results: Prognostic factors were studied in the TA-TMA cohort. 21(3.2\%) patients matched the established diagnostic criteria. 49 patients without TA-TMA were randomly selected as the control. Serum LDH concentration $>500 \mathrm{U} / \mathrm{L}$, secondary hypertension, and Grades 2 to 4 aGVHD were independent risk factors for TA-TMA occurrence. 2 year none-relapse-mortality cumulative incidence of the two groups were significantly different $(61.9 \%$ vs $8.2 \%, P<0.01)$. Significant gastrointestinal bleeding, liver dysfunction and $\mathrm{Hb}$ count $<90 \mathrm{~g} / \mathrm{L}$ at TA-TMA diagnosis were related with higher risk of TMA related death. Conclusion: We concluded that allo-HSCT recipients with grades 2 to 4 acute GVHD patients with serum LDH concentration $>500 \mathrm{U} / \mathrm{L}$ or unexplained secondary hypertension

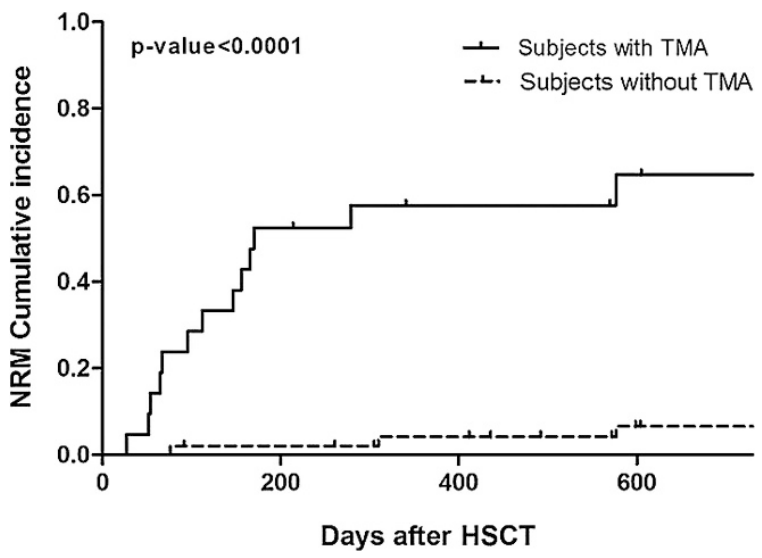

should be monitored closely for TA-TMA. Liver dysfunction was not rare among TA-TMA patients and had prognostic value. Disclosure of Interest: None declared.

P519

increase in eosinophil counts in bone marrow is closely related with acute graft versus host disease

Y. Shimomura ${ }^{1, *}$, Y. Ochi ${ }^{1}$, Y. Koba ${ }^{1}$, Y. Ono ${ }^{1}$, N. Hiramoto ${ }^{2}$ S. Yoshioka ${ }^{1}$, S. Tabata ${ }^{1}$, N. Yonetani ${ }^{1}$, A. Matsusita H. Hashimoto ${ }^{2}$, T. Ishikawa ${ }^{7}$

${ }^{1}$ Kobe City Hospital Organization Kobe City Medical Center General Hospital, Department of hematology, ${ }^{2}$ Foundation for Biomedical Research and Innovation, Department of cell therapy, Kobe, Japan

Introduction: Hematopoietic stem cell transplantation (HSCT) can be used to treat hematological malignancies and aplastic anemia. Approximately half of HSCT recipients develop graft versus host disease (GVHD), which can range from mild to severe in severity and which may significantly impair patient quality of life. Eosinophil infiltration has been observed in the tissues of patients with acute GVHD (aGVHD), and it may be harmful to organs. Eosinophilia in peripheral blood after HSCT has been reported to be associated with chronic GVHD, but the relationship between eosinophilia and aGVHD is controversial. In some diseases, eosinophils pass from bone marrow to infiltrated tissue without causing eosinophilia. It is unclear whether eosinophils in bone marrow after HSCT are involved in the development of aGVHD. We investigated the relationship between increases in eosinophil counts in bone marrow and aGVHD after HSCT.

Material (or patients) and methods: A retrospective case analysis was performed of 147 patients who underwent allogeneic HSCT in our hospitals from October 2010 to January 2015. We excluded 11 patients without evidence of engraftment [A1] and 6 patients who did not undergo bone marrow examination. Microscopic examinations of bone marrow smears were performed approximately 28 days after HSCT. Patients were grouped using the upper $25 \%$ percentile (eosinophils $=3 \%$ ) as a cutoff; specifically, those in whom at least $3 \%$ of the total nuclear cells were eosinophils comprised the "eosinophil-positive group," and the remaining patients comprised the "eosinophil-negative group." The primary endpoint was the cumulative incidence function (CIF) of severe aGVHD (grades 2-4), which was assessed using the Gray test. The Fine and Gray proportional hazards model was used to assess the prognostic value of each clinical variable. Results: The median duration of follow-up of survivors in our cohort was $653(81-1746[A 1])$ days. The primary diagnoses were acute myeloid leukemia $(n=50)$, acute lymphoblastic leukemia $(n=30)$, myelodysplastic syndrome $(n=14)$, malignant lymphoma $(n=31)$, aplastic anemia $(n=4)$, and others $(n=1)$. The patients were treated with bone marrow transplantation $(n=77)$, peripheral blood stem cell transplantation $(n=17)$, and cord blood transplantation $(n=36)$. The eosinophil-negative and eosinophil-positive groups consisted of 96 and 34 patients, respectively. The CIF values of aGVHD were 55.8 and $82.3 \%(P=0.001)$ in the eosinophil-negative group and eosinophil-positive group, respectively, and the CIFs of severe aGVHD (grades 2-4) in these two groups were 33.9 and $50.7 \% \quad(P=0.038)$, respectively. No significant difference in overall survival, event-free survival, non-relapse mortality, and relapse rates was observed between eosinophil-negative group and eosinophil-positive group. Univariate analysis revealed that eosinophil positivity and the donor source affected the CIF of aGVHD, and age $>50$ years, sex, myeloablative conditioning regimen[A2], and GVHD prophylaxis had no effect on aGVHD. After adjusting for the aforementioned variables using multivariate analysis, eosinophil positivity remained an independent prognostic factor for aGVHD (hazard ratio $=2.04 ; 95 \%$ confidence interval $=1.10-3.82 ; P=0.024)$. 
Conclusion: Our study revealed that an increase in eosinophil counts in bone marrow 28 days after HSCT is closely associated with aGVHD.

Disclosure of Interest: None declared.

\section{P520}

Brincidofovir is an oral antiviral therapy which is effective and well tolerated in patients with refractory viral reactivation in the post stem cell transplant setting

K. Foley ${ }^{1}$, R. Thompson ${ }^{2}$, E. Davies ${ }^{7}$, M. Dennis ${ }^{2}$, F. Dignan ${ }^{1, *}$, M. Saif', D. Kaye ${ }^{2}$, E. Tholouli ${ }^{1}$

${ }^{1}$ Haematology, Central Manchester Teaching Hospitals, ${ }^{2}$ Haematology, The Christie NHS Foundation Trust, Manchester, United Kingdom

Introduction: Brincidofovir is a lipid conjugate of the nucleotide analogue antiviral drug, Cidofovir (CDV). The antiviral activity of Brincidofovir has been demonstrated in vitro to be significantly greater than CDV against doublestranded DNA (dsDNA) viruses. Because of its potent, broadspectrum antiviral activity, Brincidofovir is being developed for the prevention and treatment of clinically significant infection caused by dsDNA viruses, including cytomegalovirus (CMV) and adenovirus (AdV) infections.

Brincidofovir is therefore potentially more efficacious and better tolerated than other antiviral agents currently used to treat viral reactivation in the post stem cell transplant setting. Material (or patients) and methods: Through a compassionate access program we collected data on Brincidofovir use in 11 patients across 2 transplant centres in Manchester, UK. All patients had undergone reduced intensity conditioning allogeneic stem cell transplant for a range of primary haematological malignancies.

Results: Brincidofovir was primarily used to treat asymptomatic CMV viraemia, with 1 patient treated for CMV colitis and 1 for AdV hepatitis. All patients were polymerase chain reaction (PCR) positive in blood and/or urine for at least 1 secondary virus.

Reasons for using Brincidofovir were multifactorial, including contraindications to alternative antivirals due to renal impairment in $6 / 11(55 \%)$ or cytopenias $3 / 11(27 \%)$. Several patients had experienced treatment failure on prior lines of antiviral therapy 3/11 (27\%). 4/11 patients (36\%) were started on Brincidofovir to enable outpatient treatment, after either failing Valganciclovir or at high risk for developing cytopenias. All patients had previously received at least 1 alternative antiviral treatment.

$8 / 11$ patients $(73 \%)$ had a reduction in viral load to $500 \mathrm{IU} / \mathrm{ml}$ or undetectable levels, measured by viral DNA PCR. Only 1 patient (9\%) experienced treatment failure.

$8 / 11$ patients $(73 \%)$ had renal impairment $(\mathrm{CrCl}<50 \mathrm{ml} / \mathrm{min})$ prior to treatment, with improvement in 3/11 (37\%). No patient experienced renal deterioration. Although transaminitis is a known side effect, no patient had a rise in transaminase levels attributable to Brincidofovir. There was no change in cytopenias during treatment.

Diarrhoea is a commonly reported side effect, as evident in our patient cohort. $3 / 11$ patients $(27 \%)$ had diarrhoea at start of treatment, with 6/11 (55\%) developing diarrhoea while on treatment. However, an alternative diagnosis was thought more likely in all patients, including graft versus host disease, sapovirus infection and CMV colitis. While 1 patient had Brincidofovir stopped solely due to diarrhoea, there were 5/11 cases $(45 \%)$ where diarrhoeal symptoms contributed to the decision to stop treatment.

Conclusion: Although a small sample size, our data confirms Brincidofovir is effective in patients who have failed previous antiviral treatment, and who are unable to tolerate alternative antiviral therapy due to renal or marrow compromise.

The drug was generally well tolerated, with diarrhoea as the only reported adverse effect, which led to discontinuation of Brincidofovir in a number of cases; although notably there was no significant improvement in diarrhoea after cessation of the drug.
Disclosure of Interest: None declared.

P521

Micafungin as anti-fungal prophylaxis in allogeneic hematopoietic stem cell transplant (HSCT) recipients K. Perruccio ${ }^{1, *}$, F. Arcioni ${ }^{1}$, I. Capolsini ${ }^{1}$, A. Carotti ${ }^{2}$, C. Cerri ${ }^{1}$ G. Gurdo ${ }^{1}$, E. Mastrodicasa ${ }^{1}$, O. Minelli ${ }^{3}$, A. Velardi ${ }^{2}$, M. Caniglia $^{1}$ ${ }^{7}$ Pediatric Oncology Hematology, ${ }^{2}$ Hematology and Clinical Immunology, ${ }^{3}$ Transfusion medicine and Cell collection, Perugia, Italy

Introduction: After allogeneic HSCT, invasive aspergillosis (IA) is one of the most lethal infections. Risk factors are: transplant from a matched unrelated donor (MUD) or cord blood, grade II-IV acute Graft-versus-Host Disease (GvHD), and chronic GvHD ${ }^{(1,2)}$. In this study, we focused on the efficacy of the echinocandin Micafungin as primary (during the conditionig regimen, until engraftment) and secondary (until immune reconstitution or immune suppression suspension) antifungal prophylaxis. It has been demonstrated to exert in vitro and in vivo activity against Aspergillus $s p p^{(3)}$

Material (or patients) and methods: From January 2012 to November 2015, at our Pediatric Transplant Unit, 27 allogeneic HSCT (median age: 17 years; range: 6 months- 23 years) were performed, mainly for acute leukemia (median follow-up: 16 months; range: 2-42). Donors were: 5 HLA-identical siblings, 6 MUDs, 1 unrelated cord blood unit, 8 haploidentical family members. Haploidentical transplants were T-depleted, except 1 case which was T-replete. Micafungin was administered as primary prophylaxis to 8 patients (3 HLA-matched, 4 MUDs and 1 haplo T-depleted); Fluconazole to the cord blood recipient; all others received liposomal Amphotericine B. As secondary prophylaxis, micafungine was administers to 5 patients with chronic GvHD (4 MUDs and 1 cord blood recipients) who could not assume azoles for interference with immune suppressive treatments, while the others received Posaconazole or Voriconazole.

Results: Analyses showed 3 cases of proven/probable IA, with $11 \%$ incidence and $4 \%$ mortality. Two cases were adults (20 and 21 years old). See Table 1 for IA incidence and outcome.

Table 1. Incidence of IA and outcomes

\begin{tabular}{|c|c|c|c|c|}
\hline Prophylaxis & $\begin{array}{c}\text { Proven/ } \\
\text { probable } \\
\text { IA }\end{array}$ & $a G v H D \geq \| I^{*}$ & Outcome & IA Treatment \\
\hline Micafungin & $\begin{array}{c}1 / 8 \\
(12.5 \%)\end{array}$ & YES & Resolution & $\begin{array}{l}\text { L-Amph-B } \\
+ \text { Caspo** }^{* *}\end{array}$ \\
\hline Ambisome & $\begin{array}{c}2 / 18 \\
(11 \%)\end{array}$ & $\begin{array}{l}1 \mathrm{YES} \\
1 \mathrm{NO}\end{array}$ & $\begin{array}{l}1 \text { Death } \\
1 \text { Resolu- } \\
\text { tion }\end{array}$ & Voriconazole \\
\hline
\end{tabular}

${ }^{*} \mathrm{GGvHD}=$ acute GvHD; ${ }^{*} \mathrm{~L}$-Amph-B = liposomal Amphotericine

$\mathrm{B} ;$ Caspo $=$ Caspofungin.

None of the 5 patients with chronic GvHD who received Micafungin as secondary prophylaxis developed IA. Micafungin was cost effective, as the treatment cost $>50 \%$ less than other antifungal agents.

Conclusion: Compared with our historical control group of 28 allogeneic transplants (4 matched-sibling, 5 MUD, 5 unrelated cord blood unit, and $14 \mathrm{~T}$-cell-depleted haploidentical transplants), the incidence of IA was not significantly higher (11\% vs $7 \%, P=1, \mathrm{NS})$ and the mortality rate was the same (4\% vs $3.5 \%$, $P=0.98$, NS). No differences in incidence of breakthrough IA emerged between Micafungin-treated and non treated patients ( $12.5 \%$ vs $11 \%, P=0.59$, NS). Consequently, Micafungin was safe, feasible and cost effective in HSCT recipients.

References:

1. Girmenia C, Raiola AM, Piciocchi A, et al. Incidence and outcome of invasive fungal diseases after allogeneic stem cell transplantation: a prospective study of the Gruppo Italiano 
Trapianto Midollo osseo (GITMO). Biol Blood Marrow Transplant.2014;epub:S1083-8791(14)000153-0.

2. Girmenia C, Barosi G, Piciocchi A et al. Primary prophylaxis of invasive fungal diseases in allogeneic stem cell transplantation: recise recommendations from a consensus process by Gruppo Italiano Trapianto Midollo Osseo. Biol Blood Marrow Transplat.2014;epub:S1083-8791(14)00133-5.

3. Enoch DA, Idris SF, Aliyu SH et al. Micafungin for the treatment of invasive aspergillosis. J Infect. 2014;68(6):507-26. Disclosure of Interest: None declared.

P522

Staphylococcus epidermidis septicemia is important predictor of lower survival after hematopoietic stem cell transplantation

K. Bogusz ${ }^{1, *}$, E. Snarski ${ }^{1}$, P. Rusicka ${ }^{2}$, K. Hałaburda ${ }^{1}$, T. Torosian ${ }^{1}$, M. Rokicka ${ }^{7}$, G. Basak , P. Boguradzki ${ }^{1}$, M. Tormanowska ${ }^{1}$, G. Charliński ${ }^{1}$, M. Paluszewska ${ }^{1}$, W. Wiktor Jędrzejczak ${ }^{1}$

${ }^{1}$ Department of Hematology, Oncology and Internal Diseases, Medical University of Warsaw, Warsaw, ${ }^{2}$ Department of Hematology, Oncology and Internal Diseases, Medical University of Warsaw, Warszawa, Poland

Introduction: One positive blood culture with Staphylococcus epidermidis in a hematopoietic stem cell transplantation (HSCT) recipient is often regarded as contamination. Such case does not fill classical criteria for laboratory confirmed bloodstream infection (LBSI), however we hypothesized that in HSCT setting even one positive blood culture with this bacteria is a sign of clinically significant bloodstream infection (BSI).

Material (or patients) and methods: To answer this question we established advanced system for patient data management in 2007. The 272 patients transplanted between 2007 and 2013 were prospectively followed until January 2015. Two hundred twelve patients had complete medical records and were included in this study.

Results: The median 5-year overall survival (5yOS) for the whole group of patients was $58.1 \%: 66.4 \%$ for patients without BSI, $51.2 \%$ for patients with antibiotic-susceptible BSI, and $0 \%$ for patients with antibiotic-resistant BSI. Among patients with Staphylococcus epidermidis positive blood culture, 10\% had LBSI and $90 \%$ had only one positive blood culture and did not fulfil LBSI criteria. The patients with at least one positive blood culture for Staphylococcus epidermidis had 5yOS of $34.9 \%$ (Image1). They had similar survival to patients with other types of sepsis $(P=0.5501)$ but differed significantly from patients without sepsis $(P=0.0229)$. The risk of developing Staphylococcus epidermidis BSI was almost identical in autologous and allogenic transplantation (per day of hospitalization: $0.35 \%$ in allo HSCT recipients and $0.32 \%$ in auto HSCT recipients,

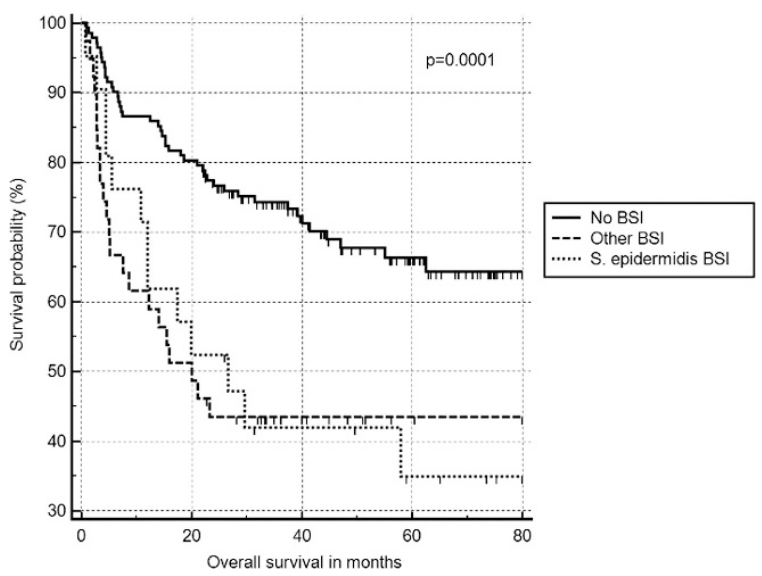

without significant difference $P=0,923$; per day of neutropenia: $0.55 \%$ in allo HSCT group and $0.52 \%$ in auto HSCT group, without significant difference $P=0.912$ ). In univariate and multivariate analysis Staphylococcus epidermidis remained significant factor for reduced 5yOS after HSCT (HR 2.46 (1.065.7), $P=0.003$ in univariate analysis and HR $2.02(1.08-3.8)$, $P=0.027$ in multivariate analysis).

Image 1. Survival in patients with $\mathrm{S}$. epidermidis sepsis compared to patients with sepsis with other types of bacteria and to the patients without sepsis. No BSI - no bloodstream infection; S. epidermidis BSI - patients with at least one Staphylococcus epidermidis positive blood culture; Other BSI - patients with other blood stream infections.

Conclusion: The single positive blood culture with Staphylococcus epidermidis is regarded often as simple "contamination". To our knowledge this is the first report that shows that long term survival after HSCT is significantly lower in patients who experience specifically an episode of single positive blood culture with Staphylococcus epidermidis during HSCT hospitalization.

Disclosure of Interest: None declared.

P523

Genotypical characterisitcs of BK virus infections and predictive value of blood quantitative BK virus PCR in hemorragic cystitis after hematopoietic stem cell transplantation: retrospective study in 102 patients

L. Souchet ${ }^{1, *}$, S. Burrel ${ }^{2}$, N. Beauhaire, M. Uzunov ${ }^{2}$, M. Ouzegdouh ${ }^{1}$, N. Jacque ${ }^{1}$, D. Roos-Weil', D. Boutolleau', S. Nguyen ${ }^{1}$

${ }^{1}$ hematology, ${ }^{2}$ virology departement, Pitié Salpêtrière hospital, Paris, France

Introduction: BK virus (BKV) hemorragic cystitis $(\mathrm{HC})$ after allogeneic hematopoietic stem cell transplantation (HSCT) is defined by the association of micro or macroscopical hemorragic cystitis and BKV viremia or viruria. It occurs generally after hematopoietic reconstitution. BKV viruria is more studied than BKV viremia after HSCT, however positive predictive value of BKV viruria in $\mathrm{HC}$ is weak after HSCT. Actually $50 \%$ of patients have BKV viruria though only 10 to $20 \%$ of patients develop HC. The aim of this retrospective study is to evaluate the BKV blood quantitative PCR value in BKV infections, and to describe genotypical characteristics of BKV infections after HSCT.

Material (or patients) and methods: One hundred and two patients who received a HSCT between the 1st january 2007 and the 31th december 2008 in Pitié Salpêtrière Hospital were included in this retrospective study. The median follow-up was 1 year after HSCT. The BKV load was measured in total blood the day of the HSCT, every 2 weeks during the first 3 months, and 6,9 and 12 months after HSCT. The threshold of BKV positivity was 25 copies $/ \mathrm{mL}$ of total blood.

Results: Cumulative incidence of BKV viremia during the first year post HSCT was 39\%. The median viral load was 475 copies $/ \mathrm{mL}$ of total blood. Factors associated with BKV viremia were female gender $(P=0,013)$, cord blood tranplantation $(P=0,003)$ and ancestral haplotype 44.1 in donors $(P=0,03)$. We did not find viral genotype predominance, nor virulence factors after sequencing of the regulating area NCCR. $21,6 \%$ of patients developed BKV HC in a median of 30 days post HSCT. BKV viremia was associated with a higher risk of $\mathrm{HC}$ $(P<0,0001)$, especially when viremia persisted at least 2 weeks $(P<0,001)$. Furthermore the viral load of patients was higher in patients with BKV HC than in patients without BKV HC (1801 vs 0 copies $/ \mathrm{mL}$ of total blood, $P=0,0004)$. Viral load threshold superior to 550 copies $/ \mathrm{ml}$ of total blood predicted the occurrence of BKV HC (sensibility $85 \%$, specificity $95 \%$ ). In this retrospective study only $4,9 \%$ of patients developed symptomatic BKV HC, and less of $1 \%$ had more than a grade 2 HC. No patient needed a specific antiviral treatment.

Conclusion: BKV HC is a frequent complication after HSCT, however rarely symptomatic in this retrospective study. 
Systematic screening of viruria need to be discussed because of its low specificity. We observed that viremia seems to be more predictive of BKV $\mathrm{HC}$ occurence than viruria. A prospective follow-up of BKV viremia in all transplanted patients in Pitié Salpêtrière Hospital is ongoing, to determine the correlation between BKV viruria and viremia and to define the risk factors for severe $\mathrm{HC}$.

Disclosure of Interest: None declared.

\section{P524}

Epidemiology of bloodstream infections after myeloablative and non-myeloablative allogeneic hematopoietic stem cell transplantation: a single center cohort study

L. I. Gjærde ${ }^{1, *}$, C. Moser ${ }^{2}, H$. Sengeløv ${ }^{3}$

${ }^{1}$ Faculty of Health and Medical Sciences, University of Copenhagen, ${ }^{2}$ Department of Clinical Microbiology, ${ }^{3}$ Department of Hematology, Rigshospitalet, Copenhagen, Denmark

Introduction: Bloodstream infection (BSI) is a potentially lifethreatening complication after allogeneic hematopoietic stem cell transplantation (allo-HSCT). We analyzed the incidence, antibiotic resistance patterns, and risk factors for BSIs in the year following allo-HSCT with a myeloablative (MA) or nonmyeloablative (NMA) conditioning regimen.

Material (or patients) and methods: All patients who underwent their first allo-HSCT at Rigshospitalet, Copenhagen, from 2008 to 2013 were included. In this period, all patients received prophylactic antibiotics (ceftazidime for MA conditioned patients or oral ciprofloxacin for NMA conditioned patients) when absolute neutrophil count was $<0.5 \times 10^{9} / \mathrm{L}$. We retrospectively collected data on BSIs from initiation of conditioning to day 360 after transplant. BSI was defined as isolation of a bacterial or fungal pathogen from a blood culture; for common contaminants, two positive blood cultures within 72 hours with identical species were required. Multidrug resistance was defined as non-susceptibility to $\geq 1$ agent in $\geq 3$ therapeutically relevant antimicrobial classes. We used a multivariate Cox proportional hazards model, stratifying by conditioning regimen and censoring subjects at death, to estimate hazard ratios (HR) for the development of first BSI. A priori defined covariates were age, sex, disease stage (intermediate vs. early and late vs. early), stem cell donor (unrelated vs. sibling and other vs. sibling), stem cell source (umbilical cord vs. bone marrow and peripheral blood vs. bone marrow), time to engraftment, and acute graft-versus-host disease grade II-IV (aGVHD, time-dependent).

Results: Our study comprised $207 \mathrm{MA}$ and 253 NMA conditioned patients with a median age of 39 and 61 years, respectively. At least one BSI occurred in 34\% (95\% confidence interval $(\mathrm{Cl}): 28 \%, 41 \%)$ of MA conditioned patients and in $17 \%$ (95\% Cl: $12 \%, 22 \%)$ of NMA conditioned patients (Figure). Median time to first BSI was 12 days (inter-quartile range: 7, 54 days) in MA conditioned patients and 117 days

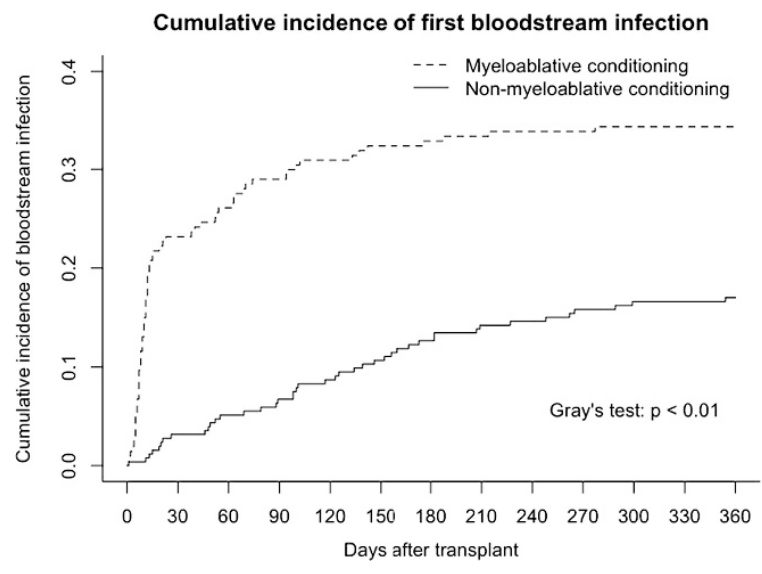

(inter-quartile range: 51, 178 days) in NMA conditioned patients. 30- day all-cause mortality after first BSI was $11 \%$ for MA conditioned patients versus $26 \%$ for non-MA conditioned patients (log-rank test: $P=0.04$ ). Of all isolates, 68\% were Gram-positive bacteria (GPB), 23\% Gram-negative bacteria (GNB), and 9\% fungi. The total GPB/GNB-ratio was 3.0, but it declined from 2008 to 2013 ( $p$ for trend $=0.04$ ). MA conditioned patients had a higher GPB/GNB-ratio than NMA conditioned patients (4.5 vs. 1.9, $P=0.03$ ). Of all $G N B, 44 \%$ were multidrug resistant, a proportion which remained stable from 2008 to 2013. Factors significantly associated with a higher hazard of first BSI were aGVHD (HR 2.42, 95\% Cl: 1.43, 4.10) and late vs. early disease stage (HR 2.73, 95\% Cl: 1.15, 6.46).

Conclusion: After allo-HSCT, MA conditioned patients experienced BSIs earlier and more often than NMA conditioned patients. However, 30- day all-cause mortality after first BSI was significantly higher in NMA conditioned patients. BSIs were mostly caused by GPB, but the proportion of GNB increased from 2008 to 2013, and a substantial number of GNB were multidrug resistant. Independent risk factors for first BSI were aGVHD and late disease stage.

Disclosure of Interest: None declared.

\section{P525}

Usefulness of an Immunodeficiency Scoring Index for predicting outcomes of respiratory virus infections in allogeneic stem cell transplant recipients

L. WANG ${ }^{1, *}$, J. ALLEN ${ }^{2}$, C. DIONG ${ }^{1}$, Y.-T. GOH ${ }^{1}$, S. GOPALAKRISHNAN ${ }^{1}$,

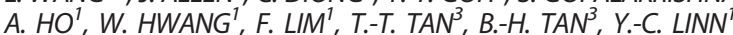

${ }^{1}$ Haematology, Singapore General Hospital, ${ }^{2}$ Academic Medicine Research Institute, Infectious Disease, Singapore General Hospital, Singapore, Singapore

Introduction: Respiratory virus infection (RVI) is a cause of morbidity and mortality in allogeneic hematopoietic stem cell transplant (HSCT) recipients. Here we review the types, incidence, risk factors and outcomes of RVI, and explore the applicability of a published Immunodeficiency-Scoring Index for respiratory syncytial virus (ISI-RSV) in our cohort of patients. Material (or patients) and methods: All patients who underwent HSCT in the Singapore General Hospital from Dec 2010 to Mar 2015 were included and results of all nasal/throat swabs done for RV by PCR were analysed. Each episode of PCR-proven RVI was scored using the ISI-RSV and correlations with outcomes assessed. Pneumonia was defined as the presence of symptoms with a new radiographic infiltrate.

Results: RVI occurred sporadically throughout the year in our tropical climate, with peaks in June to July and December to January. A total of 195 patients underwent HSCT during this period; 95 had a total of 191 episodes of RVI (Table), including 46 patients whith 1 and 49 with 2 or more episodes. Of note, RVI resulted in pneumonia in $23 \%$ to $63 \%$ of patients and was the direct cause of death in a small proportion of patients. Cumulative incidence for first episode of RVI was $30 \%$ by $6 \mathrm{mth}$, $52 \%$ by 12 mth and $61 \%$ by 2 years post-HSCT.

By the ISI-RSV (based on neutrophil <500/uL, lymphocyte $<200 / u L$, age $>40$ years, ablative conditioning, GVHD, corticosteroid use in prior 30 days, pre-engraftment or within 30 days of HSCT, score $0-11$ ), $38 \%, 46 \%$ and $16 \%$ were low-, intermediate- and high-risk episodes. Risk stratification significantly correlated with pneumonia and need for mechanical ventilation $(P<0.05)$ for RSV, influenza, adenovirus and coronavirus. Receiver operating characteristic (ROC) analysis showed a PPV of $>80 \%$ for ISI-RSV of $>=8$ for pneumonia due to RSV, influenza, adenovirus and para-influenza, and NPV of $>80 \%$ for ISI-RSV of $=<2$ (AUC 0.7391, 0.7699, 0.825 and 0.77 respectively). ROC analysis showed poor predictive value of ISI-RSV for pneumonia for coronavirus and rhinovirus.

There is no difference in survival at follow up to $50 \mathrm{mth}$ amongst the 95 patients with PCR-proven RVI, the 69 patients who were PCR-negative and the 30 patients who never had any swabs done $(P=0.285)$. 


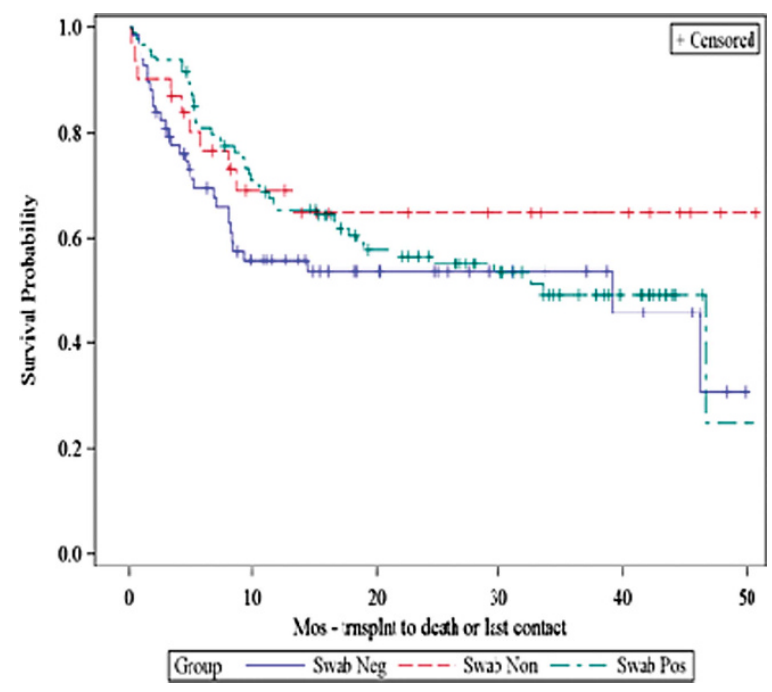

\begin{tabular}{|c|c|c|c|c|}
\hline Virus (episodes) & $\begin{array}{l}\% \text { of } 191 \\
\text { episodes }\end{array}$ & $\begin{array}{l}\% \text { com- } \\
\text { munity } \\
\text { acquired }\end{array}$ & $\begin{array}{c}\% \text { with } \\
\text { pneu- } \\
\text { monia }\end{array}$ & $\begin{array}{c}\% \\
\text { death }\end{array}$ \\
\hline Rhinovirus (39) & 20.42 & 64.10 & 23.08 & 5.13 \\
\hline RSV (37) & 19.37 & 75.68 & 32.43 & 10.81 \\
\hline $\begin{array}{l}\text { Para-influenza } \\
\text { (28) }\end{array}$ & 14.66 & 67.86 & 25.00 & 10.71 \\
\hline Influenza (26) & 13.61 & 96.15 & 42.31 & 0.00 \\
\hline $\begin{array}{l}\text { Coronavirus } \\
\text { (19) }\end{array}$ & 9.95 & 78.95 & 31.58 & 0.00 \\
\hline Adenovirus (11) & 5.76 & 36.36 & 63.64 & 9.09 \\
\hline $\begin{array}{l}\text { Metapneumo- } \\
\text { virus (6) }\end{array}$ & 3.14 & 83.33 & 33.33 & 0.00 \\
\hline $\begin{array}{l}\text { Concurrent } \\
>=2 \text { virus (21) }\end{array}$ & 10.99 & 80.95 & 38.10 & 14.29 \\
\hline
\end{tabular}

Note: bocavirus (4) and enterovirus (1) not included.

Conclusion: RVI is a non-negligible cause of pneumonia in allo-HSCT patients, even for the seemingly benign viruses such as rhinovirus and coronavirus. ISI-RSV of extreme high or low value may predict poor or benign outcomes and may be useful for guiding the management of HSCT recipients with RSV as well as non-RSV RVI.

References: Shah DP, Ghantoji SS, Ariza-Heredia EJ et al. Immunodeficiency scoring index to predict poor outcomes in hematopoietic cell transplant recipients with RSV infections. Blood 2014,123:3263-8

Disclosure of Interest: None declared.
P526

Reducing transplant and infection- related mortality after allogeneic stem cell transplantation. The impact of non pharmacological procedures

L. Yañez San Segundo ${ }^{1, *}$, A. Bermudez ${ }^{1}$, A. Insunza ${ }^{1}$, J. Calvo ${ }^{2}$, L. Martinez Martinez ${ }^{2}$, E. Conde

${ }^{1}$ Hematology, ${ }^{2}$ Microbiology, Hospital Universitario Marques de Valdecilla, Santander, Spain

Introduction: Transplant-related mortality (TRM) and infection- related mortality (IRM) following allogeneic hematopoietic stem cell transplantation (HSCT) is related to several factors especially disease stage, type of donor and age at transplant. Furthermore, the outcome of transplants performed since 2000 appears to be better than that of transplants done in the previous decade. The aims of this study were to determine the main changes associated with the procedure in the last decade and how they have affected the outcome of transplants.

Material (or patients) and methods: We analyzed 518 consecutive HSCT performed in our center. They were divided in 4 time periods: 2000-2003 $(n=104), 2004-2006(n=94)$, 2007-2009 ( $n=142)$ and 2010-2013 $(n=178)$. Patients' and transplant characteristics and infectious risk factors, including hypogammaglobuinemia, ferritin levels, antifungal prophylaxis, CMV serology, graft-versus-host disease (GVHD) development, protective isolation (HEPA filter and water filter), a central venous catheter protocol to prevent infections and galactomannan screening were retrospectively evaluated. Except antibacterial prophylaxis during the neutropenic phase which was not administered, anti infectious prophylaxis were done following recommended guidelines.

Results: Changes during these eras include underlying disease $(P<0.02)$, increasing patient age $(P<0.001)$, the presence of hipogammaglobulinemia $(P<0.01)$, more unrelated and mismatched transplants $(p<0.005)$, different GvHD prophylaxis ( $p<0.001$ ) and different management of prevention and the screening of infections. Transplant-related mortality (TRM) and infection-related mortality (IRM) differed significantly between the transplant eras $(P<0.001$ in both). Disease status at transplant, gastrointestinal toxicity related with the conditioning regimen, severe acute or extensive chronic GVHD, the protective isolation and early diagnosis of invasive fungal infection showed a significant impact $(P<0.001)$ in TRM. IRM was significantly related to hospital length stay, parenteral nutrition, severe acute GVHD, the protective isolation, a centralvenous catheter protocol and galactomannan screening $(P<0.0001)$. Multivariate analysis showed hospitalization length stay (HR 2.2, IC95\% 1.4-3.4, $P=0.001$ ) and parenteral nutrition (HR 2.1, IC95\% 1.2-3.6, $P=0.008$ ) as the main risk factors and water filter (HR 0.4, IC95\% 0.3-0.7, $P<0.0001$ ) and galactomannan assay (HR 0.5, IC95\% 0.3-0.9, $P=0.024$ ) as the principal protective factors for infection-related mortality.

Conclusion: In conclusion, TRM and IRM have been significantly reduced among the last decade. Complications related with the conditioning regimen are the major factors for IRM. The use of protective isolation, especially water filters that reduce waterborne gram-negative hospital-acquired infections and the early diagnosis of fungal infection based on microbiological assays have shown a benefit impact in TRM and IRM.

Disclosure of Interest: None declared.

P527

Is there an interest of new techniques for dosing posaconazole in invasive fungal infection prophylaxis?

L. Gabellier ${ }^{1, *}$, M. Vincent ${ }^{2}$, O. Mathieu ${ }^{2}$, Y. Hicheri $^{1}$

${ }^{1}$ Department of Clinical Hematology, ${ }^{2}$ Department of Clinical Pharmacology \& Toxicology, CHRU de Montpellier, Montpellier, France

Introduction: Invasive fungal infections (IFI) are a major cause of morbidity and mortality in patients treated for acute 
myeloid leukemia (AML) or those receiving allogeneic stem cell transplant (SCT). The interest of IFI prophylaxis by posaconazole (PCZ), an anti-fungal molecule with intramembrane mechanism of action, in these settings is well proved. Despite the threshold of $0.7 \mathrm{mg} / \mathrm{l}$ considered to the goal in prophylaxis indication, usefulness of therapeutic drug monitoring (TDM) of PCZ is still questionable because both poor plasma levels reached in practice and the absence of demonstrated correlation between blood and membrane levels. We evaluate the interest of the dosage of different sub-fractions of posaconazole and correlate them with confusion parameters known to influence results of $P C Z$ monitoring.

Material (or patients) and methods: Samples from the TDM of PCZ (oral suspension) performed in hematological malignancies between January and April 2015 in our laboratory were prospectively collected. In more, we measured the total fraction (TF) after hydrolysis of the glucuroconjugate, and the fraction in erythrocyte membrane (EF) of PCZ. We calculated the conjugated fraction (GF = TF - UF) and the ratio of conjugation. Results were correlated with 13 confusion parameters: indication of prophylaxis, body-mass index and surface area, ethnicity, fever, nutrition mode, hemoglobin concentration, white blood cell count, glomerular filtration rate (GFR), albumin, intake of proton pump inhibitors, immunosuppressive drugs (IS), and corticosteroids.

Results: 95 blood samples from 28 patients were collected (median: 3/patient). The prophylaxis indication was AML for 32 samples (33.7\%) and SCT for 63 (66.3\%). The median concentrations [min;max values] were $0.51 \mathrm{mg} / \mathrm{l}[<0.10 ; 2.49$ ] $2.46 \mathrm{mg} / \mathrm{l}[<0.10 ; 11.07], 2.35 \mathrm{mg} / \mathrm{l}[<0.10 ; 9.44]$ and $0.12 \mathrm{mg} / \mathrm{l}$ $[<0.10 ; 0.45]$ for UF, TF, GF and EF respectively. Median conjugation ratio was $82.2 \%$ [35.3;100]. All dosage fractions were statistically lower in the AML group compared to the SCT group, and in patients with GFR $\geq 90 \mathrm{ml} / \mathrm{min} / 1,73 \mathrm{~m}^{2}$ compared to those with a lowered GFR. Moreover, intake of an IS was correlated with a significant increase in UF, TF and GF, but not in the EF. Parenteral nutrition significantly lowered the unconjugated, the total and the erythrocytic fraction of PCZ Hemoglobin concentration $\geq 110 \mathrm{~g} / \mathrm{L}$ allowed an increase of total fraction and GF, but not UF or EF. A normal albumin rate ( $\geq 35 \mathrm{~g} / \mathrm{L}$ ) only increased significantly the UF and the GF. Fever within the 24 hours before blood sample $\left(\geq 38^{\circ} \mathrm{C}\right.$ ) decreased only the UF. No statistical difference was found for the other parameters.

Conclusion: Our study highlights the feasibility of new techniques of dosage of PCZ. We report a high proportion of glucuronides, so that the total fraction of PCZ is largely above the threshold of $0.7 \mathrm{mg} / \mathrm{l}$. It seems that the success of prophylaxis in patients with low blood level of PCZ (UF) may be explained by those fractions, at least as a better marker of exposure. In contrast, PCZ distributes poorly in erythrocytes. A saturation point could be assumed for EF because it did not increase with IS, high albumin and hemoglobin levels. As only 3 confusion parameters (prophylaxis indication, GFR, nutrition) influenced this rate, the EF might be more correlated with effective deep exposure and/or clinical effects of PCZ. This study has to be continued in the tablet era of posaconazole. Disclosure of Interest: None declared.

\section{P528}

Strategies for monitoring, prophylaxis and treatment of fungal infection in children undergoing hematopoietic stem cell transplantation, in light of azole resistance: Leiden experience

L. M. Ball, ${ }^{1, *}$, A. M. S. van Walraven ${ }^{1}$, M. T. van der Beek ${ }^{2}$, E. J. Kuijper ${ }^{2}$ ${ }^{1}$ Pediatrics, Willem Alexander Childrens Hospital, LUMC, ${ }^{2}$ Medical Microbiology, Leiden University Medical Centre, Leiden, Netherlands

Introduction: In the Netherlands, up to $20 \%$ of aspergillus isolates are azole resistant. ${ }^{1}$ In pediatric patients diagnosed with acute leukemias, bone marrow failure or neutropenic
MDS standard azole prophylaxis plus Ambisome as 1st line therapy for proven/probable infection with subsequent voriconazole for susceptible isolates is recommended. SCT unit protocol dictates screening pre-HSCT by high resolution $(\mathrm{HR}) \mathrm{CT}$ thorax / sinuses, plus therapeutic drug monitoring (TDM) of azoles with dose adjustment.

Material (or patients) and methods: An audit of practice of protocol standards adherence, monitoring effectiveness and treatment outcome.

Results: From Jan 2010 to Dec 2014, 75 patients, 46 boys: 31 girls were included, median age 8 y $4 \mathrm{~m}(5 \mathrm{~m}-17 \mathrm{y} 8 \mathrm{~m})$. Diagnosis: ALL $n=30$ AML $n=19$, MDS/JMML $n=15 \mathrm{BM}$ failure $n=11$

$5 / 60$ sinus $C T$ s undertaken were abnormal, sinus exploration in one revealed no fungus. 71/75 HRCT scans showed abnormalities in 14 children. Nine were documented before referral with positive galactomannan (GM) in $4 / 9,1 / 9$ culture positive (zygomyces) 2/9 viral positive from bronchio-alveolar lavage (BAL) and treated pre-SCT resulting in varying improvement at admission.

5/14 were new abnormalities: BAL GM positive 2/5; no positive cultures: $2 / 5$ viral positive. Nine patients were transplanted under antifungal therapy.

Of 64 eligible patients, 3 had no azole prophylaxis at admission. 45/61 received itraconazole and TDM was assessed in 32 , of whom only 12 had therapeutic levels at admission. Switch to iv voriconazole due to oral intolerance $(n=7)$ or inadequate levels $(n=11)$ was required but levels remained sub-therapeutic in $5 / 18$.

At maximum of 15 months post SCT, a total of 23 probable or proven fungal infections (21 pulmonary, 1 sinus, 1 ophthalmic, 1 saddle embolus-zygomyces) were documented. The majority $16 / 23$ were documented before admission for SCT $(3 / 16>1$ year pre SCT).

Most $(n=13)$ were diagnosed $<4$ months prior to admission. Of these 4 received voriconazole prophylaxis during SCT, later 2 switched to- Ambisome due to respiratory symptoms, atypical CT/ GM positive BAL. (2 died; 1 CMV/aspergillus infection, 1 relapse).

2/13 received SCT under voriconazole; 1 died relapse.

7/13 received SCT under Ambisome because of progressive CT findings or clinically active fungal disease, two of whom had progressed under voriconazole, one with documented azole resistance. Renal toxicity in 2 patients resulted in switch to voriconazole and 4/13 received granulocyte transfusions 2/7 died; 1 GVHD and 1 progressive fungus (zygomyces).

7 infections occurred at a median of 7 months post SCT ( 2-15 months). 4 patients treated with additional immune suppression with GVHD, 2 chemotherapy after relapse post SCT, 1 delayed engraftment. Two children died with azole resistance disease and two from relapse.

Conclusion: Azoles were generally sub-therapeutic at admission and may be associated with high numbers of HR patients referred for SCT with active fungal disease. ${ }^{2}$

Diagnostically chest HR-CT proved useful. Diagnostic yield of BAL culture was poor, no antifungal selection based on azole sensitivity testing was possible and new diagnostic testing is needed to comply with protocol aims. Ambisome use however resulted in excellent survival with minimal toxicities.

References: 1. Verweij P et al NEJM 2007346:1481.

2. Pacsual et al Clin inf Dis 2008 46:201.

Disclosure of Interest: None declared.

\section{P529}

Clinical Impact of Rhinovirus Infection Early after Hematopoietic Stem Cell Transplantation (HCT)

M. Taremi ${ }^{1, *}$, D. P. Shah ${ }^{1}$, D. El Haddad ${ }^{1}$, F. El Chaer ${ }^{1}$, L. El Haddad $^{1}$, R. F. Chemaly ${ }^{1}$

${ }^{1}$ Infectious Diseases, The University of Texas M.D. Anderson Cancer Center, Houston, United States

Introduction: Little is known on the clinical impact of rhinovirus infections (RhVl) among HCT recipients. Recent 
data suggest that rhinovirus might be associated with severe pneumonia in this patient population.

Material (or patients) and methods: We reviewed the clinical course and outcome of $\mathrm{RhVl}$ in $44 \mathrm{HCT}$ recipients during the first 100 days after transplant at M.D. Anderson Cancer Center between July 2014- September 2015. Rhinovirus was detected in 44 respiratory samples (40 nasal washes, and 4 bronchoalveolar lavages) by RT-PCR. Demographics, clinical characteristics and outcomes data were gathered from medical records.

Results: Majority of patients were Caucasians (59\%), male (75\%), with a median age of 47 years (range: $21-72$ ) and $64 \%$ were allograft recipients (12 unrelated, 8 haploidentical, 7 match-related and 1 cord blood). All 44 patients presented with signs and symptoms of upper respiratory tract infection (URTI), 12 (27\%) of which developed pneumonia documented by imaging. The frequency and outcome of pneumonia were similar among recipients of allogeneic and autologous transplant. Nosocomial infection occurred in 18 patients $(41 \%)$, and the majority were infected before engraftment $(14 / 18,78 \%)$. Amongst the 44 patients, more patients with RhVI before engraftment developed pneumonia (6/14, 43\%) when compared to patients with RhVl after engraftment $(6 / 30$, 20\%; $P=0.113)$. There was no significant difference in neutropenia and lymphopenia between patients with URTI and pneumonia, although there was a trend towards severe lymphopenia (absolute lymphocyte count $<200$ ) in the latter $(P=0.064)$. No death occurred within day 30 from diagnosis; however at day 90, 4 patients died and rhinovirus pneumonia may have contributed to the demise of 1 patient. Among the 28 patients with repeated nasal washes, 17 (61\%) had persistent shedding with a median duration of 115 (range: 22-406 days) and occurring in 12/17 (70\%) patients with UTRI and only in 5/17 (29\%) patients with pneumonia.

Conclusion: Our retrospective single center study showed that despite a high rate of nosocomial acquisition of RhVI particularly during the pre-engraftment period, the clinical impact was not significant. However, the long duration of shedding and the risk for nosocomial acquisition underscore the importance of strict infection control measures.

References: 1. Ghosh S, Champlin R, Couch R, Englund J, Raad I, Malik S, Luna M, Whimbey E. Rhinovirus infections in myelosuppressed adult blood and marrow transplant recipients. Clin Infect Dis. 1999; 29(3):528-32.

2. Ison MG, Hayden FG, Kaiser L, Corey L, Boeckh M. Rhinovirus infections in hematopoietic stem cell transplant recipients with pneumonia. Clin Infect Dis. 2003;36(9):1139-43.

Disclosure of Interest: None declared.

\section{P530}

Incidence of EBV-PTLD and Adenovirus Infections in Pediatric Allogeneic Transplants with Campath ${ }^{\mathbb{R}}$ as Part of Pre-Transplant Conditioning: A Retrospective Study

M. Cupit ${ }^{1, *}$, A. Nageswara Rao ${ }^{2}$, D. Warad ${ }^{2}$, V. Rodriguez, S. Khan ${ }^{2}$

${ }^{1}$ Mayo Medical School, ${ }^{2}$ Division of Pediatric Hematology/ Oncology, Mayo Clinic, Rochester, United States

Introduction: Campath ${ }^{\circledR}$ (alemtuzumab) is used as part of the conditioning regimen for pediatric allogeneic hematopoietic stem cell transplants (HSCT) in our institution. Campath ${ }^{\circledR}$ binds to CD52 antigens and decreases the incidence of severe acute and chronic graft versus host disease (GVHD) through T cell depletion. However, the risk of viral infections is a concern, with reported rates of Epstein-Barr virus associated posttransplant lymphoproliferative disorder (EBV-PTLD) and adenovirus infections among pediatric HSCT recipients receiving Campath ${ }^{\circledR}$ of $14 \%$ and up to $70 \%$, respectively. We have previously reported on the decreased incidence of serious GVHD and the low incidence of cytomegalovirus in patients receiving Campath ${ }^{\mathbb{R}}$. The purpose of this study was to review the incidences of adenovirus infections and EBV-PTLD in our institution.
Material (or patients) and methods: A retrospective review of related and unrelated allogeneic HSCT recipients, ages 0-21 years, who received Campath ${ }^{\mathbb{R}}$ as part of conditioning, treated between January 2003 and October 2014. In patients who are seropositive for EBV and receiving matched unrelated donor (MUD) transplants, our institutional practice is to obtain EBV PCR (polymerase chain reaction) at two week intervals beginning at the time of engraftment and continuing until day +100 . Adenovirus and EBV PCRs are also obtained when clinically indicated.

Results: Our review included 45 patients (19 female; 26 male) patients. Median age at time of transplant was 10 years (range: 0.7-19.7 years) and median follow up 2.5 years (range: $0.11-$ 12.2 years).

Forty-eight transplants were performed, with 3 patients receiving 2 transplants. Transplant indications included malignant disorders $(n=24)$ in complete remission (CR1 and/ or CR2): acute lymphoblastic leukemia $(n=7)$, acute myelogenous leukemia $(n=5)$, chronic myelogenous leukemia $(n=5)$, myelodysplastic/ myeloproliferative disorders $(n=6)$, non-Hodgkin lymphoma $(n=1)$; and benign disorders $(n=21)$ : bone marrow failure syndromes $(n=14)$, immune deficiencies $(n=2)$, metabolic $(n=4)$, sideroblastic anemia $(n=1)$.

Forty-two transplants were MUD transplants; and six transplants were matched related donor transplants. Graft sources were bone marrow $(n=32)$, peripheral blood stem cells $(n=14)$, and cord blood $(n=2)$.

Thirty-seven patients had EBV positive recipient and/or donor serology. Four patients (all MUD transplants) were noted to be EBV positive on PCR post- transplant. One patient (2.2\%) developed EBV-PTLD 128 days after transplant with involvement of the cecum and transverse colon, which resolved with resection and rituximab. Four patients (8.9\%) were PCR positive for adenovirus. One of these patients with Omen syndrome developed pneumonitis 67 days prior to transplant and was treated with cidofovir. The remaining 3 patients developed diarrhea 41, 98, and 68 days after transplant. Treatment included (cidofovir $=1$, cidofovir followed by ganciclovir $=1$, and supportive care $=1$ ), and all patients had clinical resolution of symptoms.

Conclusion: Our review suggests that the incidence of adenovirus infections may be lower than reported in literature. The risk of developing EBV-PTLD is also low.

Disclosure of Interest: None declared.

\section{P531}

Nanosphere's Verigene ${ }^{\circledR}$ System to detect MultidrugResistant Gram-negative Bacterial Outbreak: a prospective study in $\mathbf{6 0}$ patients

M. C. Barbanti ${ }^{1}$, N. Mancini ${ }^{2}$, L. Infurnari ${ }^{2}$, R. Pasciuta ${ }^{2}$, A. Forcina ${ }^{1}$, L. Vago ${ }^{1}$, F. Giglio ${ }^{1}$, C. Messina ${ }^{1}$, M. Morelli ${ }^{1}$ T. Perini ${ }^{1}$, S. Girlanda ${ }^{1}$, M. Carrabba ${ }^{1}$, M. T. Lupo Stanghellini ${ }^{\prime}$, S. Marktel ${ }^{1}$, A. Assanelli ${ }^{1}$, M. Bernardi ${ }^{1}$, C. Corti ${ }^{1}$, J. Peccatori ${ }^{1}$ M. Clementi ${ }^{2}$, F. Ciceri ${ }^{1}$, R. Greco ${ }^{1, *}$

${ }^{1}$ Unit of Hematology and Bone Marrow Transplantation, ${ }^{2}$ Laboratory of Microbiology and Virology, Vita-Salute San Raffaele University, IRCCS San Raffaele Scientific Institute, Milano, Italy

Introduction: Infections are the major cause of morbidity and mortality in hematological patients, mostly after allogeneic hematopoietic stem cell transplantation (HSCT). Although the proportion of infection-related deaths has decreased in the past two decades, the alarming emergence of multidrug resistant (MDR) gram-negative pathogens represents a significant challenge. Timely microbiological diagnosis, active surveillance and early therapeutic strategies are critical for the management of MDR infections after allogeneic HSCT.

Material (or patients) and methods: In the last 18 months, all blood cultures positive for gram-negative bacteria at San Raffaele Hematology and BMT Unit were prospectively collected and in parallel tested with Verigene ${ }^{(R)}$ Gramnegative blood culture (BC-GN) test (Nanosphere, Northbrook, 
IL, USA). Verigene ${ }^{\circledR}$ is a microarray-based system allowing the identification of genus, species, and genetic resistance determinants for a broad panel of gram-negative bacteria directly from positive blood cultures. Clinical data were collected for each patient.

Results: Initially, we evaluated the reliability of the BC-GN test on 60 consecutive patients undergoing chemotherapy $(n=17)$, autologous $(n=6)$ or allogeneic $(n=37)$ HSCT. Considering the bacteria detectable by the system, the concordance with standard blood cultures was $100 \%$. Resistance genes (CTX-M or carbapenemases, as KPC and VIM) were detected in $21.67 \%$ of the isolates and $100 \%$ were concordant with the phenotypic antibiotic resistance. We observed only $6.67 \%$ of phenotypic resistances not detected by the test, belonging to other kinds of resistance mechanisms not related to the genes included in the panel. Overall, Verigene BC-GN assay correctly identified $49 / 60$ of all the gram-negative pathogens $(6$ Klebsiella pneumonia, 30 Escherichia coli, 6 Pseudomonas aeruginosa, 4 Acinetobacter spp, 2 Enterobacter spp, 2 Citrobacter spp), yielding a general sensitivity of $83 \%$ with a positive predictive value of $98 \%$

Then, we examined the potential clinical impact of this molecular approach in allogeneic HSCT recipients $(n=37)$, either in an inpatient $(n=29)$ or outpatient $(n=8)$ management. In the majority of cases we were able to early start targeted antibiotic therapy (82.35\%), sparing or interrupting non-specific antimicrobial therapy (respectively, $80.56 \%$ and $61.54 \%$ ), thus reducing useless and/or potentially more toxic antibiotics and their potential impact in favoring antimicrobial resistance. In $96.77 \%$ of patients, immunosuppressive prophylaxis was not reduced, thanks to a rapid control of sepsis, therefore avoiding the risk of GVHD. Early contact isolation was possible in $35.13 \%$ of patients, preventing the spread of infections among patients. Infection-related mortality was reported in only two patients. An outpatient management was continued in $4 / 8$ hemodynamically stable patients, avoiding hospitalization. While conventional microbiological methods required 2-4 days, BC-GN test provided results within 2 hours from blood culture positivity. The mean gain in time comparing $\mathrm{BC}-\mathrm{GN}$ test to fast blood cultures was reported to be about 20 hours.

Conclusion: Verigene BC-GN test provids highly accurate identification and earlier information on antibiotic susceptibility, with a relevant clinical impact in appropriate early targeted antimicrobial treatments.

Disclosure of Interest: None declared.

\section{P532}

ROLE OF ORAL RIBAVIRIN FOR TREATMENT OF RESPIRATORY SYNCYTIAL VIRUS INFECTION IN PATIENTS UNDERGOING IN ALLOGENEIC TRANSPLANTATION

M. D. Madrigal Toscano ${ }^{1,1, *}$, J. A. Raposo Puglia ${ }^{1}$, R. Saldaña Moreno ${ }^{1}$, V. Verdugo Cabeza de Vaca ${ }^{1}$, V. Rubio Sánchez ${ }^{1}$ S. Garzón López ${ }^{1}$

${ }^{1}$ Hematology, Hospital de Jerez de la Frontera, Jerez de la Frontera, Spain

Introduction: Respiratory Syncytial Virus (RSV) infections have been recognized as a significant cause of morbidity and mortality in patients undergoing hematopoietic stem cell transplantation (HSCT). The majority of publications are retrospective studies that suggest use nebulised ribavirin (RBV) with more experience but more precautions and side effects.

Material (or patients) and methods: Retrospective descriptive study about our experience of all HSCT patients treated in our institution with oral RBV in April 2015. The nasopharyngeal aspirate samples (ANF) were processed with the technical immunochromatographic Clearview ${ }^{\mathbb{R}}$.

Results: Overall, 6 patients had received oral RBV. Patient demographic and clinical characteristics are shown in Table 1. We suffered an outbreak of RSV infection on April 2015 and we had difficulty to treat all patients in the unit for the use of the

[P532]

\begin{tabular}{|c|c|c|c|c|c|c|}
\hline & CASE 1 & CASE 2 & CASE 3 & CASE 4 & CASE 5 & CASE 6 \\
\hline AGE & 45 & 41 & 32 & 48 & 18 & 46 \\
\hline GENDER & Male & Male & Female & Female & Male & Female \\
\hline UNDERLYING DISEASE & LMA & LLA-L1 & $\begin{array}{l}\text { LMA }^{\circ} \\
\text { Fanconi } \\
\text { Anemia }\end{array}$ & $\begin{array}{c}\text { LNH } \\
\text { Mantle }\end{array}$ & $\begin{array}{l}\text { SMD } \\
\text { AREB II }\end{array}$ & $\begin{array}{l}\text { SMD } \\
\text { AREB I }\end{array}$ \\
\hline CONDITIONING & AIR & $M-A$ & AIR & $M-A$ & $M-A$ & AIR \\
\hline TYPE OF TPH & Alo DNE & $\begin{array}{l}\text { Alo } \\
\text { DE }\end{array}$ & $\begin{array}{c}2^{\circ} \text { Alo } \\
\text { DE }\end{array}$ & $\begin{array}{l}\text { Alo } \\
\text { DE }\end{array}$ & $\begin{array}{l}\text { Alo } \\
\text { DNE }\end{array}$ & $\begin{array}{l}\text { Alo } \\
\text { DNE }\end{array}$ \\
\hline $\begin{array}{l}\text { RISK FACTORS } \\
\text { - } \quad \text { Lymphopenia }<200 / \mu \mathrm{l} \\
\text { - Age }>65 \text { years } \\
\text { - } \text { Alo-TPH }<1 \text { month } \\
\text { - Severe ID } \\
\text { - }\end{array}$ & $\begin{array}{l}\text { Yes } \\
\text { No } \\
\text { Yes } \\
\text { Yes } \\
\text { No }\end{array}$ & $\begin{array}{l}\text { No } \\
\text { No } \\
\text { Yes } \\
\text { Yes } \\
\text { No }\end{array}$ & $\begin{array}{l}\text { Yes } \\
\text { No } \\
\text { No } \\
\text { No } \\
\text { No }\end{array}$ & $\begin{array}{l}\text { Yes } \\
\text { No } \\
\text { No } \\
\text { Yes } \\
\text { No }\end{array}$ & $\begin{array}{l}\text { No } \\
\text { No } \\
\text { No } \\
\text { Yes } \\
\text { No }\end{array}$ & $\begin{array}{l}\text { No } \\
\text { No } \\
\text { No } \\
\text { Yes } \\
\text { No }\end{array}$ \\
\hline ONSET OF INFECTION & +21 & +35 & +47 & +330 & +1009 & +1492 \\
\hline $\begin{array}{l}\text { INVOLVEMENT OF } \\
\text { RESPIRATORY TRACT }\end{array}$ & UPR & UPR & UPR & LRT & UPR & $\begin{array}{l}\text { UPR and } \\
\text { LRT }\end{array}$ \\
\hline $\begin{array}{c}\text { DURATION OF } \\
\text { TREATMENT (days) }\end{array}$ & 17 & 18 & 14 & 35 & 15 & 18 \\
\hline CLINICAL RESPONSE & Yes & Yes & Yes & No & Yes & No \\
\hline VIRAL CLEARANCE & Yes & ND & ND & No & Yes & Yes \\
\hline THERAPEUTIC RESCUE & No & No & No & $\begin{array}{c}\text { Not } \\
\text { possible }\end{array}$ & Yes & Yes \\
\hline SECONDARY EFFECTS & No & No & Sickness & No & No & No \\
\hline ACTUAL CONDITION & Live & Live & Live & Exitus & Live & Live \\
\hline
\end{tabular}

LMA: acute myeloblastic leukemia, SMD: Myelodysplastic syndrome, LNH: lymphoma non-Hodgkin, LLA: acute lymphoblastic leukemia, AIR: reduced-intensity conditioning, M-A: myeloablative, Alo: allogenic, DNE: unrelated donor, DE: related donor. ND: not available. ID: immunosuppression. LRT: lower respiratory tract. UPR: upper respiratory tract. 
same inhaled. Oral RBV is less well studied in HSCT patients. Following the recommendations of Casey and col (1) we commenced on oral RBV at $20 \mathrm{mg} / \mathrm{kg} /$ day given in 3 divided doses. After 2 weeks of treatment an objective clinical response was observed in 4 of the 6 patients $(66 \%)$, although one of them was necessary oral RBV retreatment with by recurrence of symptoms (other 14 days after which it became asymptomatic and without detection of RSV in the ANF ). In the 2 patients who did not respond to oral RBV, infection progress to lower respiratory tract and required hospitalization. One of them was treated with RBV inhaled during 10 days along with immunoglobulins and steroids, with good response. However, in the other patient treatment was not possible due to side effects including cough, wheezing and progressive respiratory failure that was finally died.

Conclusion: - Our experience is that Oral RBV controlled the infection (or prevented the progression to lower) in $66 \%$ of the patients with excellent tolerance and with the benefit of antiviral therapy in an ambulatory care in this patient group. Further prospective randomized studies are needed to support the use of oral RBV in patients transplanted.

Disclosure of Interest: None declared.

\section{P533}

Serum galactomannan-based preemptive management of invasive mold infections in autologous hematopoietic cell transplantation

M. E. Martínez Muñoz, ${ }^{1, *}$, F. Portero ${ }^{1}$, E. Ojeda ${ }^{7}$, G. Bautista ${ }^{1}$, R. Forés ${ }^{1}$, I. Krsnik ${ }^{7}$, A. de Laiglesia' ${ }^{7}$, B. Navarro ${ }^{7}$, A. Ramos ${ }^{1}$, J. R. Cabrera ${ }^{1}$, R. F. Duarte

${ }^{1}$ Hospital Universitario Puerta de Hierro, Madrid, Spain

Introduction: Serum galactomannan (sGM) based preemptive strategies are used for the management of invasive mold infections (IMI) in hematology patients. We have previously shown that effective anti-mold prophylaxis in high-risk patients makes sGM surveillance unreliable [RF Duarte, et al; Clin Infect Dis 2014]. Thus, these strategies would be particularly suited for patients not receiving anti-mold prophylaxis. However, it remains to be tested whether they would be useful to manage patients for whom anti-mold prophylaxis is usually not recommended for an intermediate or low risk of IMI, such as autologous HCT recipients.

Material (or patients) and methods: We present a singlecenter study on the use of sGM as part of a preemptive strategy to diagnose IMI in 87 consecutive episodes of autologous HCT in 85 patients: 44 men; median age 52,range 16-69; 38 multiple myelomas, 29 non-Hodgkin lymphomas, 13 Hodgkin lymphomas, 2 systemic amyloidosis, 2 acute promyelocytic leukemias, 1 Crohn's disease. Patients received itraconazole prophylaxis up until 2011 (26; 30\%) and fluconazole thereafter $(61 ; 70 \%)$, and underwent twice weekly sGM surveillance throughout the risk episode.

Results: A total of 686 sGM tests were performed in this series, median 8 tests per episode (2-12). A vast majority of $658 \mathrm{sGM}$ tests $(95.8 \%)$ in 72 episodes (82.8\%) were all negative. Fifteen episodes (17.2\%) had a median of 1 positive sGM test result (1-5) with a median optical index (OI) of 0.9 (0.5-5.3). There were no episodes of IMI according to EORTC/MSG criteria. One patient in the series $(1.1 \%)$ died from septicemia with a single sGM positive test result (OI: 0.9 ), no other features of IMI, and no necropsy (EORTC/MSG unclassified episode). In 4 episodes ( $4.6 \%$ of total, $26.7 \%$ of sGM positive episodes), despite sGM positive test results (median 1.5, 1-4; median OI 0.65, 0.5-1.1) patients were afebrile (surveillance GM tests), did not receive antifungal treatment and recovered from neutropenia with no other features of IMI (false positive GM episodes). Finally, 11 sGM positive episodes $(12.6 \%$ of total, $73.3 \%$ of positive episodes), in patients who survived the risk episode and did not develop other features of IMI, were not evaluable for sGM test performance as patients received antifungal treatment (non-evaluable GM episodes). In 6 of them, the positive sGM tests results occurred in the absence of persistent fever (surveillance GM tests). Of note, there were no differences between patients on itraconazole or fluconazole prophylaxis in the occurrence of false positive GM episodes ( 1 and 3 out of 4 , respectively) or non-evaluable GM episodes ( 3 and 8 out of eleven, respectively).

Conclusion: This study reproduces the very low incidence of IMI in recipients of autologous HCT. Regardless of the use of mold-active prophylaxis, our data show that sGM-based preemptive strategies are not reliable to manage IMI in this setting of low prevalence of infection. The vast majority of sGM tests are negative, and beyond confirmed false positive GM episodes, the remaining positive sGM test results can mislead diagnostic and therapeutic efforts, in particular when used as surveillance in patients without persistent fever.

Disclosure of Interest: None declared.

\section{P534}

Screening of paediatric allogeneic stem cell transplant recipients for invasive aspergillosis - increased diagnostic accuracy by using a dual parameter system combining galactomannan and fungal DNA detection assays

J. Springer ${ }^{1}$, J. Löffler ${ }^{1}$, J. Hafner ${ }^{2}$, C. Wirth ${ }^{3}$, C. P. Heußel ${ }^{4}$, A. Ullmann ${ }^{1}$, C. Löffler ${ }^{1}$, H. Einsele ${ }^{i}$, V. Wiegering ${ }^{2}$, M. Woelfl ${ }^{2}$, P. G. Schlegel ${ }^{2}$, M. Eyrich ${ }^{2, *}$

${ }^{1}$ University Hospital Würzburg, Department of Internal Medicine II, ${ }^{2}$ University Hospital Würzburg, Children's Hospital, ${ }^{3}$ University Hospital Würzburg, Department of Radiology, Würzburg, ${ }^{4}$ University Hospital Heidelberg, Department of Radiology, Heidelberg, Germany

Introduction: Invasive fungal infections with Aspergillus species, mostly Aspergillus fumigatus, are a frequently occurring event after allogeneic stem cell transplantation (alloSCT) also in the paediatric setting. As in adults, invasive aspergillosis (IA) in children is associated with a poor prognosis and requires intensive and prolonged treatment. Diagnostic workup of febrile pulmonary infections is usually difficult, as fungi poorly grow in microbial cultures. In adult patients, prospective screening of sera for the fungal cell wall component galactomannan (GM) and for DNA in parallel has resulted in increased diagnostic accuracy for $I A$, however, this approach has not been extensively evaluated in children so far.

Material (or patients) and methods: In this study, we included a biweekly screening for the presence of GM (by Platelia ${ }^{\text {B }}$ ELISA) and a sensitive real time PCR assay into the routine microbial workup of 41 children shortly before or after stem cell transplantation. We prospectively collected a mean of $n=13$ serum specimens per patient (range: 3 to 33 serum specimens).

Results: In this cohort, we classified 4 probable, and 2 possible cases of IA, according to the current definitions of the EORTC, resulting in an overall incidence of suspected IA of $15 \%$. Together with a clinical course highly suggestive for IA, all four probable cases exhibited simultaneous positive results in both test systems, whereas none of the possible or unclassified cases showed this pattern at any time. Interestingly, optical density values of GM assays were higher and positive intervals of these tests were substantially longer in children than in a comparable previous control group (adults after alloSCT). This indicates that combining both fungal test systems is of high diagnostic value also in the paediatric setting. A multivariate analysis comprising clinical as well as laboratory data is currently performed and will provide a better understanding of the interactions between parameters.

Conclusion: In summary, our data indicate that simultaneous prospective detection of GM and DNA in serum is of high relevance also in the paediatric setting and can help to increase the diagnostic accuracy in febrile pulmonary infections after alloSCT.

Disclosure of Interest: None declared. 


\section{P535}

Secondary Antifungal Prophylaxis in Allogeneic Hematopoietic Stem Cell Transplantation

M. S. Pepeler ${ }^{1, *}$, S. Yıldız ${ }^{2}$, Z. A. Yegin ${ }^{1}$, Z. N. Özkurt ${ }^{1}$, Ö. Güzel Tunçcan ${ }^{3}$, G. Erbas ${ }^{4}$, N. Köktürk ${ }^{5}$, Z. Yıldırım ${ }^{5}$

${ }^{1}$ Hematology, ${ }^{3}$ Internal Medicine, ${ }^{3}$ Infectious Diseases, ${ }^{4}$ Radiology, ${ }^{5}$ Pulmonary Diseases, Gazi University Faculty of Medicine, Ankara, Turkey

Introduction: Invasive fungal infections(IFI) are considered to be significant causes of morbidity and mortality in patients with hematological malignancies.The incidence of IFI has been increased due to high dose chemotherapy regimens and hematopoietic stem cell transplantation(HSCT).Secondary antifungal prophylaxis is reported to be a feasible approach in patients with prior IFI(1-5).

Material (or patients) and methods: A total of 57 patients [M/F: 41/16; median age: 30(16-62)] who had undergone allogeneic HSCT with secondary antifungal prophylaxis were included in this retrospective study.

Results: Pretransplant EBMT score and Sorror comorbidity index were $4(0-6)$ and 2(0-7). Pretransplant performance status was found to be $1(0-4)$ in the study population. Primary diagnosis was AML in $31(54,4 \%), A L L$ in $14(24,6 \%), N H L$ in 2 $(3,5 \%)$, SAA in $6(10,5 \%)$, MDS in $2(3,5 \%)$ and CLL in $2(3,5 \%)$ patients. A total of 49 HSCTs (86\%) were performed from related, while 7 HSCTs (12,3\%) from unrelated and 1(1,8\%) from haploidentical donor. CSA and methotrexate were used in $49(86 \%)$ patients and CSA and MMF in $8(14 \%)$ patients for GVHD prophylaxis. Conditioning regimens were myeloablative in $42(73,7 \%)$ and RIC in $15(26,3 \%)$ patients. Neutrophil and platelet engraftment periods were 18(10-43) and 19(11-46) days. Granulocyte colony stimulating factor was used in 31 patients $(54,4 \%)$ until neutrophil engraftment. Granulocyte infusions were performed in 2 patients with active fungal infection. Liposomal amphoterisin B was used in 35 patients $(61,4 \%)$, voriconazole in $17(29,8 \%)$, posaconazole in $1(1,8 \%)$ and caspofungin in $2(3,5 \%)$ patients for secondary antifungal prophylaxis. At pretransplant evaluation, 32 patients $(56,1 \%)$ were found to have possible, 12 patients $(21,1 \%)$ probable and 13 patients $(22,8 \%)$ proven fungal infections. In 46 patients $(81 \%)$ lung was the primary infective focus, while skin in 1 $(1,8 \%)$, cranium in $1(1,8 \%)$, gastrointestinal tract in $1(1,8 \%)$ and sinus in $3(5,3 \%)$ patients. Fungemia was demonstrated in 4 (7\%) patients, whereas hepatosplenic candidiasis was observed in $1(1,8 \%)$ patient. Fungal agent was documented in 10 patients,which was aspergillus in 7, candida in 2 and rhizopus in 1 patient. Pretransplant galactomannan was found to be positive in 6 patients $(10,5 \%)$. A total of 12 patients $(21,1 \%)$ were transplanted under active fungal infection. In 19 patients $(33,3 \%)$ recurrent fungal infection was seen. The median duration of the antifungal therapy was 25(6-96) days. Clinical response was achieved in $21(36,8 \%)$ patients. A total of 9 patients $(15,8 \%)$ developed concomittant GVHD with a median grade of 2(1-3). Median follow-up period was 154(73285) days in the study cohort. Pretransplant performance status and EBMT score were found to have a significant impact on overall survival. There was no survival difference between the patients who received either amphoterisin $B$ or voriconazole.

Conclusion: As the optimal agent, timing and duration of the secondary antifungal prophylaxis in allogeneic HSCT recipients remain to be uncertain, further efforts are warranted to improve the transplant outcomes particularly in patients with a history of prior fungal infection.

References: 1. Plos 2014;9(12):e115461.

2. Biol Blood Marrow Transplant 2014;20:1198-1203.

3. Trans Infect Dis 2013;15:243-50.

4. Bone Marrow Transplant 2013;48:141-3.

5. Trans Proc 2008;40:1575-85.

Disclosure of Interest: None declared.
P536

Hemocoagulase for the treatment of severe hemorrhagic cystitis following hematopoietic stem cell transplantation M. Xue ${ }^{1, *}$, F. Chen ${ }^{1}$, D. $\mathrm{Wu}^{1}$, S. Xue ${ }^{1}$

${ }^{7}$ The First Affiliated Hospital of Soochow University, Jiangsu Institute of Hematology, Suzhou, China

Introduction: The purpose of this study is to investigate the clinical efficacy of hemocoagulase for severe hemorrhagic cystitis (HC) following allogeneic hemotopoietic stem cell transplantation (HSCT).

Material (or patients) and methods: We treated Twenty patients with severe HC (macroscopic hematuria with clots and macroscopic hematuria with renal or bladder dysfunction, with symptoms of cystitis) following HSCT with hemocoagulase. All the patients were initially treated with hyperhydration, forced diuresis and red cell and platelet transfusion support. For the patients with cytomegalovirus (CMV) replication in plasma determined by polymerase chain reaction, ganciclovir or foscarnet sodium was given. When patients developed macroscopic hematuria with clots, hemocoagulase was given, $1 \mathrm{U}$ iv twice per day for 5 days as a course. If the macroscopic hematuria disappeared at the sixth day after stopping the drug, hemocoagulase was no longer given. If not, the next course was given. During the treatment, the blood clotting factors was monitored for 2 to 3 times weekly. If the fibrinogen was decreased, the plasma or fibrinogen was transfused to correct the blood coagulation abnormality. The urine speciments reserved before and after hemocoagulase respectively were examined by naked eye and microscope to evaluate the efficacy.

Results: Patients included 12 males and 8 females with a median age of 27 years (range, 13-57). The median onset time of severe $\mathrm{HC}$ was 28 days after HSCT(range, 14 to 70 days). The $\mathrm{HC}$ was cured in 18 patients, Patients included 12 males and 8 females with a median age of 27 years (range, 13-57years). The median time to onset of severe HC was 28 days after HSCT (range, 14 to 70 days). The HC was cured (disappearance of macroscopic hematuria without relapse) in 18 patients ( 2 cases were cured after 1 courses, 9 cases were cured after 2 courses, 2 cases were cured after 3 courses, 2 cases were cured after 4 courses, 2 cases were cured after 5 courses, 1 cases were cured after 9 courses), improved (amelioration of macroscopic hematuria) in 1 patients after 3 courses and uncontrolled (persistence of macroscopic hematuria with red cell transfusion requirements) in 1 patients who died of pulmonary infection and pneumorrhagia. For the patients with response, macroscopic hematuria disappeared at a median time of 28 days after the treatments (range, 4-127 days). Among 20 patients, 7 cases had no decrease of fibrinogen ( 2 cases were cured after 1 courses, 5 cases were cured after 2 courses), the other 13 cases had obvious decrease of fibrinogen, while after the infusion of plasma or fibrinogen, the fibrinogen recoveried. There was no patient required interruption of treatment.

Conclusion: Hemocoagulase seems to be a safe and effective drug for severe HC following HSCT.

Disclosure of Interest: None declared.

\section{P537}

Origin region has an impact on the spectrum of Gram Negative Bacilli infections in allogeneic stem cell transplantation patients

M. Sánchez-Escamilla ${ }^{1,2, *}$, L. Yáñez ${ }^{1,2}$, A. Casado-Díez ${ }^{1,2}$ S. García-Ávila ${ }^{1,2}$, M. Celis $^{1,2}$, I. Ormazabal ${ }^{1,2}$, A. Bermúdez ${ }^{1,2}$ G. Pérez-Vázquez ${ }^{1,2}$, A. Insunza ${ }^{1,2}$, C. Richard ${ }^{1,2}$, E. Conde ${ }^{1,2}$

${ }^{1}$ Hematology, University Hospital "Marqués de Valdecilla",

${ }^{2}$ Hematology, IDIVAL, Santander, Spain

Introduction: Gram-negative bacilli (GNB) infections are a well-known cause of mortality in allogeneic stem cell transplantation (Allo-SCT). Our hospital provides hematopoietic transplantation service to several Spanish regions. The 
objective of the study is to evaluate the influence of the patient's region of origin on GNB infections after Allo-SCT.

Material (or patients) and methods: We retrospectively analyzed the incidence of GNB infections, in relation to the patien's region of origin in 218 Allo-SCT performed between 2010 and 2014. GNB infection was defined as infection proven by culture in blood or urinary samples guided by clinical symptoms. The presence of different GNB in the same culture, or the reappearance of the same GNB after a negative culture were considered as different episodes.

Results: Patient data are summarized in table 1.

The patients' origin was Cantabria (51\%), Basque Country (21\%), Aragon (9\%), La Rioja (9\%), Canary Islands (6\%) and Other (4\%).

118/218 patients (54.1\%) developed GNB infections. The highest incidence was observed in patients from Canary Islands (77\%) and Aragon (70\%), followed by Cantabria (56\%), La Rioja (52\%), Basque Country (40\%) and Other (38\%). GNB bacteriemia was more frequent in patients from Cantabria (43\%) than from other regions (21-38\%), but not reaching significance $(P=0.259)$. We did not observe differences $(P=0.106)$ in the day of first GBN detection: day+58 in Cantabria, day+66 in Basque Country, day +76 in Aragon, day +53 in La Rioja, day+71 in Canary Islands and day+5 in Other. We found 304 GNB positive cultures. The most frequent pathogen was E. Coli $(n=134 ; 44.1 \%)$ followed by $E$. cloacae $(n=39 ; 12.8 \%)$, K. pneumoniae $(n=35 ; 11.5 \%)$ and $P$. aeruginosa $(n=26 ; 8.6 \%)$.

$P$. aeruginosa infections were significatively more frequent in patients from La Rioja (25\%) than from other regions: Cantabria (7.6\%), B. Country (5.4\%), Aragon (8.1\%) and C. Islands $(3.4 \%),(P=0.041)$. On the other hand, K. pneumoniae infections are more frequent in patients from Other regions $(66.7 \%)$ and C. Islands (34.5\%) compared to the rest of locations $(<10 \%)$.

We did not observe any significant differences between $E$. Coli and E. cloacae infections.

Conclusion: In our study we have shown that the incidence and spectrum of GNB infections after Allo-SCT varies depending on the patient's region of origin. In our opinion this factor should be taken into account when managing prophylaxis and treatment of microbial infections in Allo-SCT. Further studies, like the hypothetically different antibiotic resistance patterns of the GNB detected could be of vital importance.

Disclosure of Interest: None declared.
P538

Clinical associations between pre-transplant serum ferritin, red blood cell indexes and early post-HSCT complications

A. Chukhlovin ${ }^{1}$, M. Shaheen ${ }^{2, *}$, M. Ivanova ${ }^{1}$, I. Moiseev ${ }^{1}$, S. Bondarchuk ${ }^{3}, Y$. Eismont ${ }^{4}$

${ }^{1}$ R.Gorbacheva Memorial Institute of Children Oncology, Hematology and Transplantology, St. Petersburg State I. Pavlov Medical University, St. Petersburg, Russian Federation, ${ }^{2}$ Hematology and Bone Marrow Transplantation Department, Tishreen Hospital, Damaskus, Syrian Arab Republic, ${ }^{3}$ Department of Hematology, Faculty Therapy Clinics, S.M.Kirov Military Medical Academy, ${ }^{4}$ Department of Clinical Microbiology, St. Petersburg State I.Pavlov Medical University, St. Petersburg, Russian Federation

Introduction: Red blood cell (RBC) quality and turnover, multiple $\mathrm{RBC}$ transfusions cause a risk for iron overload (IO). Blood ferritin content is a well-known surrogate marker of IO. These factors, as well as donor/recipient $\mathrm{ABO}$ group mismatch, are considered important reasons of complications following allogeneic hematopoietic stem cell transplantation (allo-HSCT). Controversial results exist about the role of $1 \mathrm{O}$ in GVHD, infections, and other HSCT complications. The aim of our single-center study was to assess RBC parameters and initial ferritin levels as potential risk factors for the common HSCT outcomes.

Material (or patients) and methods: In a single-center study, we have reviewed medical records of ninety-one consecutive patients (42 males and 49 females), including cases of AML $(n=68)$, MDS $(n=10)$, aplastic anemia $(n=6)$, beta-thalassemia $(n=2)$ with a median age at HCT of 31.6 years (range, 5 to 60), who underwent allo-HCT with unmanipulated grafts between Jan 2013 and Dec 2014. Standard assays of RBC indexes, ABO groups, and serum ferritin (SF) levels were performed.

Results: The median pre-HSCT SF concentration was 765 (range, 12 to 4247 ) $\mathrm{ng} / \mathrm{mL}$. The patients with SF of $\geq 500 \mathrm{ng} /$ $\mathrm{mL}$ were considered a high-ferritin (HF) group. Increased pretransplant ferritin levels were significantly associated with toxic and infectious complications of HSCT, i.e., number of febrile neutropenic episodes $(P=0.005)$, number of bacterial infection episodes $(P=0.009)$, pneumonias $(P=0.04)$, and demand for multiple RBC transfusions $(P=0.04)$ within 100 days post-HCT. A significant association was found between pre-HSCT ferritin $(>773 \mathrm{ng} / \mathrm{mL})$ and overall survival $(P=0.04)$, disease free survival $(P=0.019)$, and mortality $(P$ $=0.02$ ) among the group. No significant relationships were observed between the initial ferritin levels and incidence of mucositis, or graft-versus-host disease $(P>0.05)$. Moreover, we had tested correlation between RBC parameters, other clinical features, including cytomegalovirus (CMV) reactivation. There

\begin{tabular}{|c|c|c|c|c|c|c|c|}
\hline & Cantabria & Pais & Aragón & La Rioja & Canarias & Others & P-value \\
\hline & $\mathrm{n}=111$ & $\mathrm{n}=45$ & $\mathrm{n}=20$ & $\mathrm{n}=21$ & $\mathrm{n}=13$ & $\mathrm{n}=8$ & \\
\hline Male $(\%)$ & $63(56,8 \%)$ & $\begin{array}{l}25 \\
(55,6 \%)\end{array}$ & $\begin{array}{l}12 \\
(60 \%)\end{array}$ & $\begin{array}{l}13 \\
(61,9 \%)\end{array}$ & $7(53,8 \%)$ & $\begin{array}{l}3 \\
(37,5 \%)\end{array}$ & $p=0.904$ \\
\hline Age, value ( $\pm \mathrm{SD})$ & $51( \pm 16.1)$ & $\begin{array}{l}46 \\
( \pm 12,3)\end{array}$ & $\begin{array}{l}42 \\
( \pm 15,7)\end{array}$ & $\begin{array}{l}47 \\
( \pm 12,5)\end{array}$ & $44( \pm 15,6)$ & $\begin{array}{l}48 \\
( \pm 15,0)\end{array}$ & $\mathrm{p}=0.179$ \\
\hline $\begin{array}{l}\text { HLA matched } \\
\text { related }\end{array}$ & $41(37,8 \%)$ & $\begin{array}{l}20 \\
(44.4 \%)\end{array}$ & $0(0)$ & $\begin{array}{l}7 \\
(33.3 \%)\end{array}$ & $0(0)$ & $4(50 \%)$ & \\
\hline $\begin{array}{l}\text { HLA matc hed } \\
\text { unrelated }(\%)\end{array}$ & $34(30.6 \%)$ & $\begin{array}{l}10 \\
(22.2 \%)\end{array}$ & $9(45 \%)$ & $\begin{array}{l}9 \\
(42.8 \%)\end{array}$ & $8(61.5 \%)$ & $\begin{array}{l}3 \\
(37.5 \%)\end{array}$ & $\mathrm{p}=0.001$ \\
\hline HLA mismatch & $35(31.5 \%)$ & $\begin{array}{l}15 \\
(33.3 \%)\end{array}$ & $\begin{array}{l}11 \\
(55 \%)\end{array}$ & $\begin{array}{l}5 \\
(23.8 \%)\end{array}$ & $5(38,4 \%)$ & $\begin{array}{l}1 \\
(12.5 \%)\end{array}$ & \\
\hline $\mathrm{BM}$ origin ( $\%$ ) & $81(73 \%)$ & $\begin{array}{l}31 \\
(68,9 \%)\end{array}$ & $\begin{array}{l}16 \\
(80 \%)\end{array}$ & $\begin{array}{l}10 \\
(47,6 \%)\end{array}$ & $\begin{array}{l}11 \\
(47,6 \%)\end{array}$ & $\begin{array}{l}7 \\
(87,5 \%)\end{array}$ & $\mathrm{p}=0.244$ \\
\hline CR to SCT $(\%)$ & $60(54 \%)$ & $18(40 \%)$ & $\begin{array}{l}14 \\
(7096)\end{array}$ & $\begin{array}{l}10 \\
(47,6 \% 6)\end{array}$ & $9(69,2 \%)$ & $\begin{array}{l}3 \\
(37,5 \%)\end{array}$ & $\mathrm{p}=0.017$ \\
\hline $\begin{array}{l}\text { AMI and MDS } \\
(\%)\end{array}$ & $66(59,5 \%)$ & $\begin{array}{l}26 \\
(57,896)\end{array}$ & $\begin{array}{l}16 \\
(80 \%)\end{array}$ & $\begin{array}{l}13 \\
(61,9 \%)\end{array}$ & $\begin{array}{l}10 \\
(76,9 \%)\end{array}$ & $\begin{array}{l}5 \\
(62,5 \%)\end{array}$ & $\mathrm{p}=0.820$ \\
\hline
\end{tabular}


were no significant connections between donor RBC blood antigens, or donor/recipient $\mathrm{ABO}$ mismatch, and posttransplant infectious complications. Meanwhile, presence of A antigen (blood group II or IV) in the patients, but not in donors, showed a highly significant correlation with CMV reactivation $(P<0.01)$.

Conclusion: Measurement of serum ferritin, as a surrogate laboratory marker for iron overload, is quite feasible in the most hematological clinics. A baseline increase of serum ferritin levels is associated with higher risk of febrile episodes, infectious conditions, and slower recovery of myeloid cells, thus being of distinct predictive value. Of interest is an association between the pre-transplant ferritin and increasing demand for RBC transfusions post-HSCT.

Disclosure of Interest: None declared.

\section{P539}

Invasive fungal disease in pediatric recipients of allogenic hemopoietic stem cell transplantation: incidence, risk factors and outcome. A single center experience

N. Decembrino ${ }^{1, *}$, A. Comelli ${ }^{1}$, G. Giorgiani ${ }^{1}$, L. Rubert ${ }^{1}$, T. Mina ${ }^{7}$ A. Tolva ${ }^{7}$, F. Introzzi ${ }^{1}$, E. Bergami ${ }^{1}$, S. Recupero ${ }^{1}$, F. Bonetti ${ }^{\prime}$, P. Comoli ${ }^{1}$, C. Cavanna ${ }^{2}$, P. Marone ${ }^{2}$, M. Zecca $^{1}$

${ }^{1}$ Pediatric Hematology and Oncology, ${ }^{2}$ Microbiology and Virology Unit, IRCCS San Matteo Pavia, Pavia, Italy

Introduction: Invasive fungal disease (IFD) are a significant cause of morbidity and mortality among allogeneic HSCT recipients. Large studies that focus on paediatric population are lacking. Single centre data analysis could provide the basis for risk stratification, prevention, and management of IFDs. Material (or patients) and methods: We retrospectively analyzed all children who received allogeneic HSCT at Fondazione IRCCS Policlinico San Matteo of Pavia between 2011 and 2015. Only patients lost to follow up before day-100 were excluded. IFD were defined according to EORTC criteria ${ }^{1}$ Only proven and probable cases (PP-IFD) were analyzed. PPIFDs were classified as early (days 0 to 40 ), late (days 41 to $100)$, very late (>101 days) $)^{2}$. Since 2014, antifungal prophylaxis with fluconazole or mould active was employed, according to GITMO guidelines ${ }^{3}$.

Results: A total of 150 allo-HSCT were analysed, mean age 9.6 years ( 3 months to 22 years): 24 (16\%) MFD, 73 (49\%) MUD, 53 (35\%) MMFD; 13 were second allogeneic HSCT. Underlying disease included malignant (69\%) and non malignant (31\%) disorders. Eleven patients (7\%) had a previous PP-IFD. 55\% of patients received fluconazole and $45 \%$ a mould active prophylaxis. Cumulative incidence of PP-IFD was $15 \%$ with a total of 22 IFD (9 proven and 13 probable): 9 occurred in HSCT from MMFD and 13 in MUD HSCT. 16 IFD were early (73\%), 4 (18\%) late, 2 (9\%) very late. Aspergillus spp was the most frequent causative organism (64\%), followed by Candida spp (18\%) and Mucorales (18\%). Among risk factors, age $\geq 14$ years, alternative donor source, myeloablative conditioning regimen, mucositis, grade III-IV aGVHD and CMV infection were associated to IFD in univariate analysis; in multivariate analysis only mucositis and CMV infection retained significance. The overall survival (OS) and transplant related mortality (TRM) of the entire population were $60 \%$ (46-73) and 29\% (20-44). In patients with PP-IFD the OS was $19 \%$ vs $65 \%(P=0.0001)$ and TRM was $71 \%$ vs $24 \%(P<0.00005)$. $13 / 22$ patients $(59 \%)$ with PP-IFD died; fungal infection was considered the primary cause of death in 10 patients (45.5\%).

Conclusion: In our population, PP-IFD increased HSCT related mortality and length of hospitalization. MMFD recipients developed less PP-IFD than MUD, possibly due to earlier engraftment and reduced aGVHD incidence, confirming it as a promising and safe transplant modality for patients without a related donor. Type of antifungal prophylaxis and previous IFD did not influence outcome, confirming that a risk stratificationbased prevention strategy is successful.

References: 1. De Pauw, B. et al. Clin. Infect. Dis 46, 1813-1821 (2008).
2. Girmenia C et al, Biol Blood Marrow Transplant 20 (2014) $872 \mathrm{e} 880$

3. Girmenia C. et al. Biol. Blood Marrow Transplant. 20, 1080-1088 (2014).

Disclosure of Interest: None declared.

\section{P540}

Routine Vaccination Programme (RVP) Practice after Adult and Paediatric Allogeneic Haematopoietic Stem Cell Transplant (HSCT): A British Society of Blood and Marrow Transplantation (BSBMT) Survey of UK NHS-Based Programmes

P. D. E. Miller ${ }^{1, *}$, T. de Silva ${ }^{2}$, R. Skinner ${ }^{3}$, M. Gilleece ${ }^{4}$, A. Peniket ${ }^{5}$, A. Hamblin ${ }^{5}$, D. Greenfield ${ }^{6}$, C. Anthias ${ }^{1}$, K. Peggs ${ }^{7}$, A. Madrigal ${ }^{1}$, J. A. Snowden ${ }^{6}$ on behalf of the BSBMT Clinical Trials Committee ${ }^{7}$ Anthony Nolan Research Institute, London, ${ }^{2}$ Department of Infection and Tropical Medicine, Sheffield Teaching Hospitals NHS Foundation Trust, Sheffield, ${ }^{3}$ Department of Paediatric and Adolescent Haematology and Oncology, Newcastle upon Tyne Hospitals NHS Foundation Trust, Newcastle upon Tyne, ${ }^{4}$ Department of Clinical Haematology, Leeds Teaching Hospitals NHS Trust, Leeds, ${ }^{5}$ Department of Clinical Haematology, Oxford University Hospitals NHS Foundation Trust, Oxford, ${ }^{6}$ Department of Clinical Haematology, Sheffield Teaching Hospitals NHS Foundation Trust, Sheffield, ${ }^{7}$ Department of Clinical Haematology, University College London Hospitals NHS Foundation Trust, London, United Kingdom

Introduction: Antibodies to vaccine preventable diseases decline in the first year following allogeneic HSCT. Guidelines recommend that recipients are vaccinated as part of routine care $^{1-4}$.

Material (or patients) and methods: To present a comprehensive overview of post HSCT vaccination practice in the UK, we conducted a survey of all 27 adult and 12 paediatric NHS allogeneic HSCT programmes. We defined RVP as a series of scheduled vaccinations administered following allogeneic HSCT as part of standard post-transplant care. We invited programmes to participate in an online survey comprising 26 questions examining service organisation, initiation and delay of vaccination, and vaccine selection.

Results: The response rate from programme directors or delegated specialist staff was $100 \%$ for adult programmes, and $83 \%$ for paediatric. All adult and paediatric programmes recommend RVP. Programmes use a range of guidelines as their main source of information to direct RVP. Most adult programmes draw from international HSCT specific guidelines $^{1-2}(26 \%)$ or versions modified locally (44\%), whereas most paediatric programmes draw from national HSCT specific guidelines $^{3-4}$ (60\%). $48 \%$ of adult programmes commence RVP at 12 months, with $15 \%$ commencing at 3 months, and $4 \%$ at 18 months post HSCT. Most paediatric programmes commence RVP at 12 months for recipients of sibling donors (80\%) and matched unrelated donors (60\%), with some $(20 \%)$ commencing the latter group at 18 months. Most adult programmes $(74 \%)$ do not use a marker of immune reconstitution to guide initiation of RVP, while $70 \%$ of paediatric programmes use lymphocyte subsets alone $(40 \%)$ or with immunoglobulin levels (30\%). $70 \%$ of adult and $80 \%$ of paediatric programmes delay inactive vaccines for mild to severe CGVHD by NIH criteria. $23 \%$ of adult programmes and $60 \%$ of paediatric programmes report that either moderate or severe CGVHD is the lowest grade that would prompt a delay in live vaccines. While most adult (96\%) and paediatric (90\%) programmes delay live vaccines in patients receiving single agent low dose corticosteroid, the approach to inactive vaccines is less consistent with programmes delaying across a range of doses and combinations of IST. There is variation in the vaccines recommended as part of RVP. $67 \%$ of programmes recommend MMR, 38\% HPV, 29\% Hepatitis B, and $5 \%$ varicella vaccine. Recommended formulations of pneumococcal, meningococcal, and diphtheria-tetanus- 
pertussis vaccines vary between programmes, and in up to $14 \%$ selection is at the discretion of primary care.

Conclusion: This survey has demonstrated variation in RVP practice between UK allogeneic HSCT programmes. Some variation, particularly between adult and paediatric programmes, reflects the various sources of guidance. RVP practice around CGVHD and IST varies greatly and in some cases does not accord with best practice recommendations. This survey highlights the need for an up-to-date paediatric and adult UK HSCT specific RVP guideline with the aim of harmonising care.

References: 1. Rubin, L et al. (2014) Clin Infect Dis, 58, 1-57.

2. Tomblyn, M et al. (2009) Biol Blood and Marrow Transplant, $15,1143-1238$

3. RCPCH (2002) Immunisation of the Immunocompromised Child.

4. CCLG (2014) Vaccinations for Paediatric Patients Treated with Standard-Dose Chemotherapy and HSCT Recipients.

Disclosure of Interest: None declared.

\section{P541}

HHV-6 chromosomal integration in allogeneic haematopoietic stem cell transplantation in adult and paediatric transplant centres

P. Hubacek', I. Zelezna ${ }^{2}$, J. Sumova ${ }^{2}$, B. Krausova ${ }^{2}$, P. Keslova ${ }^{3}$, D. Janeckova ${ }^{3}$, R. Formankova ${ }^{4}, A$. Briksi $^{2}, H$. Cechova ${ }^{5}$, P. Riha ${ }^{3}$ V. Valkova ${ }^{5}$, M. Markova-Stastna ${ }^{5}$. M. Kouba ${ }^{5}$. M. Zajac ${ }^{2}$, P. Cetkovsky ${ }^{5}$, P. Sedlacek ${ }^{4, *}$

${ }^{1}$ Dept. of Medical Microbiology, 2nd Medical Faculty of Charles University and Motol University Hospital, ${ }^{2}$ Dept. of Medical Microbiology, ${ }^{3}$ Dept. of Paediatric Haematology and Oncology, Motol University Hospital, ${ }^{4}$ Dept. of Paediatric Haematology and Oncology, 2nd Medical Faculty of Charles University and Motol University Hospital, ${ }^{5}$ Institute of Haematology and Blood Transfusion, Prague, Czech Republic

Introduction: Chromosomal integration of HHV-6 is an interesting biological phenomenon. According to published knowledge, Ci-HHV-6 genome is at least partly transcribed with a pro-inflammatory impact documented recently by increased risk of cardiac angina pectoris. Therefore we wanted to find out the Ci-HHV-6 frequency and related complications in our allogeneic haematopoietic stem cell transplant (alloHSCT) recipients.

Material (or patients) and methods: Between January 2003 and November 2015, we tested for presence of HHV-6 7,970 samples from 330 children and 652 adults after alloHSCT. At least one sample was tested pre- and one after recovery of haematopoiesis was obtained in 839 donor/recipient combinations. DNA from whole blood and and other biological samples (e.g. BALs, tissues, nails etc.) was extracted by appropriate Qiagen kits according to the manucture's instructions. Testing for HHV-6, other viruses and albumin gene was performed using RQ-PCR. In the recipients, $\mathrm{Ci}-\mathrm{HHV}-6$ was confirmed by HHV- 6 detection in the nails. Viral quantity in the sample was normalised to 100,000 human genome equivalents assessed by quantification of human albumin gene and expressed in normalised viral copies (NVCs). In the available tissue samples, the DNA chimaerism studies based on STR polymorphism were performed.

Results: HHV-6 DNA was detected in 984 (12.3\%) samples from 100 children (30.3\%) and 104 adult (15.9\%) patients after the HSCT in 46 children and 25 adults before HSCT. In none of the patients, virostatic treatment only against HHV-6 was started. From these, Ci-HHV- 6 was confirmed in 4 patients (in recipient) and 7 donors as in engrafted post-transplant blood cells by long lasting high HHV-6 positivity and viral/human DNA ratio about $1: 1$. In 7 patients with $\mathrm{Ci}-\mathrm{HHV}-6$, we detected HHV-6 A, while in the rest HHV-6B was detected. Two patients carrying $\mathrm{Ci}-\mathrm{HHV}-6$ before alloHSCT and 4 transplanted from $\mathrm{Ci}$ HHV-6 positive donor died from post-transplant complications. If possible, tissue samples were tested too. In Ci-HHV-6 positive recipients, median of the HHV-6 DNA detected quantity in the tissues was 21,731 (log 4.34), while in the situation of HSCT with $\mathrm{Ci}-\mathrm{HHV}-6$ donor, median of the detected quantity was $12,945$ ( $\log 4.11)$ with different levels in the tissues according to the presence of donor cells, which was also shown by chimaeric studies. In two patients, ganciclovir resistence (A594V and L595S) developed quickly after 39 and 53 days of treatment (126 and 186 days after HSCT). In both patients, donor was immunologically naive to CMV. In one patient, multiple viral infections were observed including EBV-LPD from donor Ci-HHV-6 positive cells. Patient subsequently deceased without any GvHD due to relapse of the primary leukaemia. Comparing to HHV-6, CMV was detected in 663 patients $(67.5 \%)$ from the cohort and virostatic treatment was started in 360 of them.

Conclusion: As in our previous studies, we confirmed higher frequency of Ci-HHV-6 A in our cohort. Comparing to related CMV was HHV-6 detected less frequently and despite the described pro-inflammatory impact of Ci-HHV-6 on the carriers, it seems that such impact of $\mathrm{Ci}-\mathrm{HHV}-6$ can be observed only in $\mathrm{Ci}-\mathrm{HHV}-6$ positive recipient.

Disclosure of Interest: Supported by grant of Grant Agency of $\mathrm{MH}$ of Czech Republic NT/13691-4 and project for conceptual development of research organization 00064203. None declared.

\section{P542}

HHV6 Specific T-cells are Predictor of Clinically Relevant HHV6 Infection after Allogeneic HSCT

R. Greco ${ }^{1, *}$, M. Noviello ${ }^{2}$, L. Crucitti ${ }^{3}$, S. Racca ${ }^{4}$, V. Valtolina ${ }^{2}$ M. Pozzato ${ }^{1}$, M. Morelli ${ }^{1}$, F. Giglio ${ }^{1}$, D. Mannina ${ }^{1}$, M. C. Barbanti ${ }^{1}$ A. Forcina ${ }^{1}$, S. Rolla ${ }^{4}$, A. Assanelli ${ }^{1}$, M. Carrabba ${ }^{1}$, S. Marktel ${ }^{1}$, M. Bernardi ${ }^{1}$, C. Corti ${ }^{7}$, M. T. Lupo Stanghellini ${ }^{1}$, L. Vago ${ }^{1,5}$ J. Peccatori ${ }^{7}$, M. Clementi ${ }^{4}$, C. Bonini ${ }^{2}$, F. Ciceri ${ }^{1}$

${ }^{1}$ Unit of Hematology and Bone Marrow Transplantation, ${ }^{2}$ Experimental Hematology Unit, Division of Immunology, Transplantation and Infectious Disease, IRCCS San Raffaele Scientific Institute, ${ }^{3}$ Università degli Studi di Milano, ${ }^{4}$ Laboratory of Microbiology and Virology, Vita-Salute San Raffaele University, ${ }^{5}$ Unit of Immunogenetics, Leukemia Genomics and Immunobiology, IRCCS San Raffaele Scientific Institute, Milano, Italy

Introduction: Human herpesvirus-6 (HHV6) reactivation is associated with severe clinical manifestations and increased mortality in recipients of allogeneic HSCT (allo-HSCT). Only limited experiences are reported on HHV6-specific immune responses after HSCT, and their correlation with clinical outcome is unexplored.

Material (or patients) and methods: From February 2013 to October 2015, we conducted a prospective observational study to investigate HHV6 reactivation in 213 consecutive adult patients who received allo-HSCT for high-risk hematological malignancies. Donors were haploidentical (104), sibling (39), unrelated (63), cord blood (7). Viral load was weekly monitored by quantitative PCR in plasma within the first month after HSCT. Numbers of IFNץproducing HHV6-T-cells were determined by enzyme-linked immunospot assay (ELISPOT). We challenged pts PBMC against a library of overlapping peptides covering the entire sequence of the immunodominant virus protein U54. Pts were evaluated at a median of 34d after HSCT (HHV6-; 57 pts) in the absence of HHV6 reactivation, or by the 4th day after the first HHV6 DNAemia (median 32d) for reactivating patients (HHV6+; 54 pts).

Results: HHV6-reactivation occurred in $56 \%$ of pts at 100 days, with a median time of 28 days after HSCT. HHV6 was detected in plasma for $86 \%$ of pts, while $33 \%$ resulted positive in other materials: 9 BM aspirates, 39 gut biopsies, 3 BAL, 5 CSF. All pts received acyclovir as prophylaxis. Only $41 \%$ of reactivating pts presented a clinically relevant HHV6 infection (HHV6 positivity in presence of HHV6-related clinical symptoms and/or HHV6disease). Clinical manifestations were: fever (25), skin rash (37), hepatitis (19), diarrhoea (28), encephalitis (5), BM suppression (30). According to center guidelines, antiviral treatment was given in $23 \%$ of reactivating pts, for uncontrolled clinically relevant HHV6 infection. 
Overall survival (OS) was not different in HHV6 reactivating pts compared to controls $(P=0,2)$. Relapse incidence and TRM were not affected by HHV6. All HSCT recipients showed a better OS with CD3+ cells $\geq 200 / \mathrm{mcl}$ at 30 days $(P<0.001)$, independently of HHV6. In univariate analysis, we identified the following risk factors for HHV6-reactivation: active disease status before HSCT $(P=0,052)$, haploidentical HSCT $(P=0,003)$, PT-Cy use $(P<0.001)$, CMV reactivation $(P=0,001)$, GvHD $(P=0,003), \mathrm{CD} 3+$ cells $<200 /$ $\mathrm{mcl}$ at 30 days $(P=0,013)$.

The number of IFNY-producing HHV6-specific T-cells was significantly higher in HHV6 reactivating patients $(P=0.0149$; mean number of specific T-cells 43,48 per $10 \wedge 5$ PBMC) than in HHV6- pts, especially in the presence of clinically relevant HHV6 infection $(P<0,0001$; mean number of specific T-cells 81,46 per $10 \wedge 5$ PBMC). No influence of IFNy-producing CMV specific T-cells, absolute counts of CD3+ T cells or GvHD was observed.

Conclusion: In this study, we observed that active disease status before HSCT, haploidentical donors, especially using PT-Cy, CMV reactivation, GvHD and lower $\mathrm{CD} 3$ + counts at 30 days, are strong predictors of HHV6 reactivation. These associations may have implications for HHV6 monitoring and pre-emptive protocols. Interestingly, HHV6-specific T-cells, detectable by ELISPOT assay despite extremely low T-cell numbers and immunosuppressive therapy, are significantly associated with HHV6 clinically relevant infections, representing a new and promising tool to unravel the role of HHV6 positivity in allo-HSCT recipients.

Disclosure of Interest: None declared.

\section{P543}

Persistent recipient-derived human adenovirus (hAdV) -specific $T$ cells promote $h A d V$ control after allogeneic hematopoietic stem cell transplantation

R. Schultze-Florey ${ }^{1,2, *}$, S. Tischer ${ }^{2,3}$,W. Kuehnau ${ }^{4}$, A. Heim ${ }^{5}$, B. Eiz-Vesper ${ }^{2,3}$, B. Maecker-Kolhoff ${ }^{1,2}$

${ }^{1}$ Pediatric Hematology and Oncology, ${ }^{2}$ Integrated Research and Treatment Center Transplantation (IFB-TX), Institute for Transfusion Medicine, ${ }^{4}$ Institute of Human Genetics, ${ }^{5}$ Institute of Virology, Hannover Medical School, Hannover, Germany

Introduction: Patients after hematopoietic stem cell transplantation (HSCT) are at high risk for viral infections or reactivation. In pediatric patients, human adenovirus (hAdV) reactivation is frequent and often associated with high morbidity and mortality. Viral clearance is usually achieved by a functional anti-hAdV T-cell response. Depending on conditioning regimen, the use of T-cell depleting antibodies, in vitro T-cell depletion and donor hAdV status recovery of antiviral T-cell responses may be delayed for several weeks. In patients with CMV reactivation persistence of recipient CMV-specific $T$ cells mediating viral control has been reported mainly after nonmyeloablative conditioning regimens. Here, we establish the platform to evaluate donor-/recipient provenience in virusspecific $\mathrm{T}$ cells and demonstrate persistence of host hAdVspecific T cells in two pediatric HSCT recipients.

Material (or patients) and methods: Patient 1 was an 18month-old boy with alpha-mannosidosis. After conditioning with fludarabine, busulfan, melphalan, and ATG he underwent CD34-selected HSCT from a 10/10 HLA-matched unrelated donor without pharmacological immunosuppression. On day 5 after transplantation hAdV was detected in stool and later in blood by PCR. He received 2 courses of cidofovir. Patient 2 was a 5-year-old boy who underwent HSCT for hyper IgM syndrome from a 10/10 HLA-matched unrelated donor after conditioning with fludarabine, treosulfan, and alemtuzumab. He received unmanipulated bone marrow. GvHD prophylaxis was performed with cyclosporine and MMF. 18 days after transplantation he reactivated $\mathrm{hAdV}$ in stool and low copy number in blood. After 9 courses of cidofovir he presented a decrease in his donor chimerism to $85 \%$.

$\mathrm{T}$ cells reactive to $\mathrm{hAdV}$ hexon and penton peptide pools were quantified using interferon-gamma (IFN- $\gamma$ ) Elispot assay. hAdVspecific T-cells were enriched by IFN- $\gamma$ secretion assay using peptides or peptide pools (hexon and penton) as stimulators followed by magnetic separation. The eluate was subjected to molecular chimerism by short tandem repeat analysis.

Results: In both patients hAdV-specific T cells against hexon and penton peptide pools could be detected during hAdV reactivation followed by decrease in $\mathrm{hAdV}$ viral load. At this time both patients showed a mixed chimerism in peripheral blood total leukocytes prompting us to question whether the drop in donor chimerism may be caused by expansion of autologous hAdV-specific T cells. Patient 1 demonstrated an autologous fraction in hexon- $(61 \%)$ and penton- $(46 \%)$ specific T cells. In patient 2 recipient chimerism was $53 \%$ in hAdVspecific $T$ cells at the beginning of T-cell response. Sequential analysis revealed an increasing donor chimerism in hAdVspecific T cells over the next two months to $83 \%$. This suggests that recipient immunity to hAdV may be transient until robust donor-derived immune responses are generated. In both patients total peripheral blood donor chimerism improved to $92 \%$ and $97 \%$, respectively, after clearance of hAdV.

Conclusion: hAdV-specific T cells may survive even myeloablative conditioning and contribute to control of hAdV infection early after stem cell transplantation. Drops in donor chimerism after HSCT may be caused by persisting virusspecific T cells fighting viral infections / reactivations.

Disclosure of Interest: None declared.

P544

Incidence of Cytomegalovirus Reactivation after

Autologous Peripheral Blood Stem Cell Transplantation

(APSCT) in 227 Myeloma and Lymphoma Patients: A

Single-Center Study at the American University of Beirut Medical Center (AUBMC)

R. Assi ${ }^{1,}{ }^{*}$, R. Massoud ${ }^{1}$, N. Kreidieh ${ }^{1}$, R. Mahfouz ${ }^{1}$, S. KanjSharara?', A. El-Zakhem ${ }^{1}$, M. Kharfan-Dabaja ${ }^{2}$, A. Bazarbachi ${ }^{7}$ J. El Cheikh ${ }^{i}$

${ }^{1}$ Bone Marrow Transplantation Program, American University of Beirut Medical Center, Beirut, Lebanon, '2Department of Blood and Marrow Transplantation, Moffitt Cancer Center, Tampa, Fl, Tampa, United States

Introduction: Cytomegalovirus (CMV) reactivation is not uncommon and could determine a CMV-related disease in immunocompromised patients. CMV disease may involve almost any organ, particularly lung and gastrointestinal tract. CMV reactivation and end-organ disease after allogeneic hematopoietic stem cell transplantation have been well studied. On the contrary, hematologic patients treated with high-dose chemotherapy and who underwent autologous stem cell transplantation (ASCT) was historically considered to have a low risk of CMV reactivation or end-organ disease. Previous studies on lymphoma and myeloma patients suggested an incidence of CMV reactivations of about $30 \%$ $>40 \%$.

The aim of this study is to evaluate the incidence, risk factors, and outcome of CMV reactivation in adult patients with hematologic malignancies who underwent ASCT at AUBMC.

Material (or patients) and methods: A total of 227 consecutive autografts were performed at the AUBMC in Lebanon between January 2005 and December 2013. Among patients, 123 were male and 104 were female. 87 ASCT were performed for patients with multiple myeloma (MM), 74 for those affected by non-Hodgkin's lymphoma (NHL), whereas the remaining 66 patients had Hodgkin's lymphoma (HL). CMV DNA load in the blood has been determined by polymerase chain reaction in cases of clinical suspicion of reactivation; therefore, no routine monitoring strategy was adopted. In the presence of signs and symptoms of CMV reactivation an antiviral treatment was instituted. The median follow up for patients who underwent ASCT was 5 years (range: 2 to 10 years).

Results: Overall, 30 patients (13\%) had a CMV reactivation and 20 of them (9\%) required a specific antiviral treatment for a symptomatic CMV reactivation. Among these 20 patients, 12, 5 and 3 had MM (13,7\%), NHL (6,7\%) and HL (4,5\%), respectively. 
All cases of CMV reactivation were observed in seropositive patients, with no documented cases of primary CMV infection. All patients were treated with a specific antiviral therapy, with a global rate of hospitalization of $85 \%$. Transplant related mortality has doubled in patients who experienced a CMV reactivation $(6,6 \%$ vs $3 \% ; P=0.298)$. However, this difference did not reach statistical significance likely because of sample size. Finally, we did not observe any impact for age, sex, type of disease, pretransplant types (i.e chemotherapy, monoclonal antibodies, bortezomib and lenalidomide) or lines of therapy (first vs $2^{\text {nd }}$ line or beyond) on the incidence CMV reactivation following ASCT.

Conclusion: Our data suggest that CMV reactivation is a relatively frequent complication in ASCT recipients particularly in $\mathrm{MM}$ patients and may contribute to increased cost, morbidity and mortality.

Disclosure of Interest: None declared.

\section{P545}

Audit of the adherence to and effectiveness of the antifungal prophylaxis strategy in the Glasgow adult allogeneic HSCT population

R. McNeil ${ }^{1, *}$, I. G. McQuaker ${ }^{2}$, A. Clark ${ }^{2}$, B. Jones ${ }^{3}$, A. Parker

${ }^{1}$ Haematology, St John's Hospital, Livingston, ${ }^{2}$ Haematology,

${ }^{3}$ Microbiology, Queen Elizabeth University Hospital, Glasgow, United Kingdom

Introduction: Invasive fungal infection (IFI) carries a significant morbidity and mortality in the allogeneic transplant (HSCT) population. We carried out an audit of the incidence of IFI and prophylaxis used in our unit over 2 years.

Material (or patients) and methods: $A$ retrospective audit was carried out for all patients receiving an allogeneic HSCT in the Glasgow Adult Unit 1/5/2010 - 30/04/2012. Patients were identified from the transplant database. Case notes were reviewed for all patients until 6 months post transplant. Itraconazole levels were obtained from the laboratory database. Data was audited against the Unit IFI prophylaxis policy and for the development of features consistent with a diagnosis of proven or possible IFI based on EORTC criteria.

Results: There were 92 patients transplanted during this period, and case notes were available for 89 . The median age for the audit population (89) was $46(18-64)$ with 39 female and 50 male. Stem cell source was BM 6, cord 3, PBSC 80. The non-cord donors were 30 matched siblings and 56 VUD. There were 24 myeloablative transplants ( $16 \mathrm{~T}$ depleted) and $68 \mathrm{RIC}$ transplants (66 T depleted). The incidence of grade 3-4 GVHD was $14 \%$.

Itraconazole was started in $87 \%$ (77) with 7 on posaconazole (3 cord transplants, 4 previous $\mathrm{IFI}$ ) and 4 voriconazole (previous IFI). All patients were started on prophylaxis as per unit protocol. An itraconazole level was carried out at least once in $86 / 89$ patients despite 11 not receiving itraconazole. One patient died within 2 days of transplant and has not been analysed further. The median number of itraconazole levels/ patient was 6 (1-15). There were 4 or more levels recorded in $78 \%$ of patients median $0.87 \mathrm{mg} / \mathrm{ml}(0.11-4.05) .58 \%$ had 1 or no sub-therapeutic levels whilst $<10 \%$ had more than 4 results $<0.5 \mathrm{mg} / \mathrm{ml}$. There were no cases of proven or probable IFI identified in the 89 patients in the 6 months post transplant.

Conclusion: Antimould prophylactic agents are expensive with itraconazole less than quarter of the cost of posaconazole. The unit IFI prophylaxis policy was developed to maximise efficacy balanced against cost effectiveness. We used posaconazole in patients receiving cords or proven/ probable IFI pre transplant unless the referring team had started voriconazole, the remainder had itraconazole prophylaxis from admission with liquid changing to iv if the liquid was not tolerated or severe mucositis developed until engraftment (neutrophil $>0.5$ ). Levels were to be monitored weekly aiming for a trough of $0.5 \mathrm{mg} / \mathrm{ml}$, the dose was increased if this was not achieved. Post engraftment patients could be switched to capsules if they were unable to tolerate liquid. In patients requiring steroid for GVHD therapy posaconazole or ambisome prophylaxis was used. This audit demonstrates that the antifungal prophylaxis was adhered to in the majority of patients with consistent therapeutic trough levels in most patients adjusting dose according to levels. Itraconazole was generally well tolerated using drug levels to confirm compliance, only a minority of patients $(<10 \%)$ switched to an alternative azole as a result of Gl toxicity. The policy was extremely effective in this high risk T depleted population with no patients developing features consistent with proven or probable IFI.

Disclosure of Interest: R. McNeil Funding from: Travel grant from Gilead, I. G. McQuaker: None declared, A. Clark: None declared, B. Jones Funding from: MSD, Gilead, Pfizer research grant, Personal Interest: MSD, Gilead, Astellas for consultancy and fees for lectures, A. Parker Funding from: audit carried out with funding from an educational grant from MSD, Personal Interest: Advisory work for MSD, Basilea.

\section{P546}

Bacteremia during Neutropenic Episodes in Children Underwent Hematopoietic Stem Cell Transplantation with Ciprofloxacin and Penicillin Prophylaxis

S. Pakakasama ${ }^{1, *}$, W. Choeyprasert ${ }^{2}$, U. Anurathapan ${ }^{1}$, S. Hongeng ${ }^{1}$ ${ }^{1}$ Pediatrics, Faculty of Medicine Ramathibodi Hospital, Mahidol University, Bangkok, ${ }^{2}$ Pediatrics, Faculty of Medicine, Chiang Mai University, Chiang Mai, Thailand

Introduction: Bacteremia during neutropenic episodes is a cause of morbidity and mortality in patients undergoing hematopoietic stem cell transplantation (HSCT). The incidence of bacteremia during pre-engrafted neutropenic period was $20-44 \%$ with mortality rate of $10-50 \%$. Fluoroquinolones has been recommended as an antibiotic prophylaxis in patients with high risk of chemotherapy-induced neutropenia. Addition of penicillin $\mathrm{V}$ to fluoroquinolone prophylaxis reduced the incidence of streptococcal bacteremia in patients with cancer. Therefore, our center has been used the combination of ciprofloxacin and penicillin $\mathrm{V}$ as antibiotic prophylaxis in children underwent HSCT. The objective of this study was to analyze the incidence, characteristics, and risk factors of bacteremia of these patients during neutropenic episodes. Material (or patients) and methods: We retrospectively enrolled children and adolescents underwent HSCT between January 2002 and September 2014 at our institute. The patients received oral ciprofloxacin $20-30 \mathrm{mg} / \mathrm{kg} /$ day and penicillin $\vee 25-50 \mathrm{mg} / \mathrm{kg} /$ day started at the beginning of preparative regimen. Discontinuation of antibiotic prophylaxis was indicated when patients developed fever, clinically documented infection, clinically suspected infection, intolerance to the medications, or engraftment. We collected data including patients' characteristics, HSCT procedure and complications, febrile illness and bacteremia during neutropenic periods, bacterial infections, morbidity, and mortality.

Results: There were 215 patients with a median age of 8.3 years (range, 0.1-21.6 years). We performed HSCT in both malignant $(49 \%)$ and non-malignant $(51 \%)$ diseases. Most of the patients (83.7\%) develop fever during neutropenic periods in which 33 patients $(15.3 \%)$ had bacteremia. The most common gram-negative bacteria was Escherichia coli (28.2\%) and Streptococcal viridans (12.8\%) was the most common of gram-positive bacteria. Antimicrobial sensitivity test revealed that $32 \%$ of gram-negative bacteria and $14.3 \%$ of grampositive bacteria were multi-drug resistant. The risk factors for developing bacteremia were patients who received ATGcontaining preparative regimen (OR 2.67; $95 \% \mathrm{Cl}, 1.24-5.77)$ and using umbilical cord blood (OR $8.27 ; 95 \% \mathrm{Cl}, 1.38-49.44$ ). These 2 factors were still statistically significant using multivariate analysis. Four patients died from severe sepsis during the pre-engrafted period.

Conclusion: This study demonstrated the feasibility of ciprofloxacin and penicillin $\mathrm{V}$ prophylaxis in children 
underwent HSCT. ATG-containing regimen and umbilical cord blood were the significant risk factors for bacteremia during neutropenic episodes.

Disclosure of Interest: None declared.

\section{P547}

Definition and characterization of novel CD8+ T cell epitopes derived from JCV/BKV polyomaviruses with clinical relevance

S. Michael ${ }^{1, *}$, J. Mani ${ }^{1}$, L. Wang ${ }^{1}$, A. Hückelhoven ${ }^{1}$, A. Schmitt ${ }^{1}$, A. Gedvilaite, ${ }^{2}$ J. $\mathrm{Nan}^{3}$, C. Kleist ${ }^{4}, \mathrm{~A}^{\mathrm{H}} \mathrm{Ho}^{1}$

${ }^{1}$ Department of Internal Medicine V, University Clinic Heidelberg, Heidelberg, Germany, ${ }^{2}$ Department of Eukaryote Genetic Engineering, Institute of Biotechnology, Vilnius University, Vilnius, Lithuania, ${ }^{3}$ Department of Hematology, ZongDa Hospital, Southeast University, Nanjing, China, ${ }^{4}$ Department of Nuclear Medicine, University Clinic Heidelberg, Heidelberg, Germany

Introduction: Human JC and BK polyomaviruses (JCV/BKV) can establish a latent infection without any clinical symptoms in healthy individuals. In immunocompromised hosts infection or reactivation of JCV and BKV can cause lethal progressive multifocal leukoencephalopathy (PML) and hemorrhagic cystitis, respectively. Vaccination with JCV/BKV derived antigen epitope peptides or adoptive transfer of virus-specific T cells would constitute an elegant approach to clear virus-infected cells. Although only few immunodominant $T$ cell epitopes of JCV and BKV have been described, there is a fervent need for new epitopes.

Material (or patients) and methods: In this study, we identified novel $T$ cell epitopes by screening libraries of overlapping peptides derived from the major capsid protein VP1 of JCV. All identified T cell specificities were confirmed to be naturally processed using virus like particles (VLPS). Some epitopes were characterized by fine mapping with overlapping peptides and nonamer peptide sequences were identified. The cytokine release profile of the epitope-specific $T$ cells was analyzed by enzyme-linked immunospot (ELISPOT) assays and by flow cytometry.

Results: We detected several possible novel HLA-A*02 restricted epitopes in 31 tested healthy donors which were tested in with a pool of 86 overlapping pentadecamer peptides arranged in a matrix format. Responses against two of the immunogenic pentadecamer peptides (OP-29 and OP-72) were observed in more than $30 \%$ of the tested HLA-A*02-positive donors. With overlapping nonamer peptides the epitope sequence was defined. Natural processing was demonstrating by the use of VLPs. For further characterization of the epitope the cytokine profile of $\mathrm{CD}^{+} \mathrm{T}$ cells recognizing the newly defined epitope was analyzed. The virus-specific $T$ cell responses were mostly effector memory $T$ cells with a significant release of pro-inflammatory effector cytokines (IFN- $\gamma$, TNF- $a$, and IL-2).

Conclusion: We demonstrated that $\mathrm{T}$ cell responses were of polyfunctional nature with the potential of epitope-specific killing and cross-reactivity between JCV and BKV. These novel epitopes might constitute a new potential tool to design effective diagnostic and therapeutic approaches against both polyomaviruses.

Disclosure of Interest: None declared.

P548

Protective effect on chronic graft-versus-host disease occurrence in allogeneic hematopoietic stem cell transplantation patients with Epstein Barr virus viremia treated by Rituximab

S. Xue ${ }^{1, *}$, S. $\mathrm{Ji}^{1}$, X. $\mathrm{Bao}^{1}, \mathrm{~T} . \mathrm{Tao}^{1}, \mathrm{X} . \mathrm{Ma}^{1}, \mathrm{D} . \mathrm{Wu}{ }^{1}$

${ }^{1}$ The First Affiliated Hospital of Soochow University,Jiangsu Institute of Hematology, Suzhou, China

Introduction: Chronic graft-versus-host disease (cGVHD) is an important cause of late morbidity, mortality and impaired quality of life in patients after allogeneic hematopoietic stem cell transplantation (allo-HSCT). It has been demonstrated that B cells are involved in CGVHD pathogenesis, which is evidenced by the presence of antibodies reactive to the host tissue in allo-HSCT patients with active CGVHD. It has also been proven that Rituximab could have a distinctly therapeutic effect on CGVHD in allo-HSCT patients. And now the prophylactic effect of Rituximab on cGVHD is being focused on. Herein, the prophylactic effect of Rituximab on cGVHD in a group of Chinese allo-HSCT patients is retrospectively evaluated.

Material (or patients) and methods: Between January 2012 and August 2014, a total of 102 allo-HSCT patients from the First Affiliated Hospital of Soochow University, who underwent a myeloablative conditioning regimen and suffered EBV viremia within post-transplantation 100 days with a median time of 54 days (range 14-99), were included in this study. Among them, 50 (49\%) patients (Rituximab group) received Rituximab treatment by $375 \mathrm{mg} / \mathrm{m} 2$ weekly to deal with EBV viremia, while other 52 cases (control group) were treated by other anti-EBV agents. A competing risk model was adopted to compare the cumulative incidence of CGVHD, relapse rate and transplantation related mortality (TRM) between Rituximab group and control group. Death and relapse were treated as competing events in the analysis of cGVHD. Overall survival (OS) and progression-free survival (PFS) were estimated by the Kaplan-Meier method.

Results: All patients either in Rituximab group or in control group achieved viral load negativity except 3 patients who died before achieving viral negativity. In the Rituximab group, the median start time of Rituximab administration is posttransplantation 64 days (range 23-101) and the median count number of Rituximab administrations was 1 (range 1-4). The overall cumulative incidence of CGVHD in the cohort at 6-, 12-, 24 -month were $23.5 \%, 42.2 \%$ and $46.1 \%$, respectively. Retrospectively, the cumulative incidence of cGVHD in Rituximab group was lower at each time point $(14.0 \%$ vs. $32.7 \%, 34.0 \%$ vs. $50 \%, 36.1 \%$ vs. $54.1 \%$ at $6-, 12-, 24-$ month, respectively, $P=0.059)$ when compared with controls. Moreover, there was no significant difference regarding cumulative relapse rate $(P=0.48)$ and TRM $(P=0.39)$ between two groups. Multivariable analyses corrected by other factors showed that Rituximab was one of the independent factors for the reduction of cumulative incidence of CGVHD (Hazard ratio $(\mathrm{HR})=0.37,95 \% \mathrm{Cl}=0.178-0.767, P=0.0075)$. Additionally, survival analyses showed that there was no significant difference between two groups regarding overall survival (OS) $(P=0.667)$ or progression free survival (PFS) $(P=0.571)$.

Conclusion: Rituximab administrated in post-transplantation early phase could reduce the cumulative incidence of cGVHD and play a protective role in CGVHD occurrence among alloHSCT patients but without increasing relapse rate and TRM.

Disclosure of Interest: None declared.

\section{P549}

Efficacy and safety of micafungin for antifungal prophylaxis in patients receiving allogeneic stem cell transplantation

S. Santarone $e^{1, *}$, P. Olioso ${ }^{1}$, A. Natale ${ }^{1}$, G. Papalinetti ${ }^{1}$, T. Bonfini ${ }^{1}$, P. Accorsi ${ }^{1}$, S. Angelini ${ }^{1}$, G. Parruti ${ }^{1}$, P. Di Bartolomeo ${ }^{1}$

${ }^{1}$ Hematology, BMT Center, Ospedale Civile, Pescara, Italy

Introduction: Invasive fungal infections (IFIs) are major causes of morbidity and mortality in patients receiving allogeneic SCT. The aim of this prospective, single-arm and single-center, surveillance study was to establish the safety and efficacy of the echinocandin micafungin as primary or secondary antifungal prophylaxis in patients undergoing allo-SCT for high risk hematological disorders.

Material (or patients) and methods: Between February 2011 and July 2015, 54 consecutive patients (males 28) were enrolled. The median age at time of transplant was 51 (17-67) years. The diagnosis as AML in 27, ALL 10, MDS 8, CML 3, MF 2, 
Lymphoma 3, Plasmacell leukemia 1. Fourteen patients have received a previous transplant (autologous in 10, allogeneic 4). Seven patients were diagnosed with probable IFI before transplant. Sixteen patients were transplanted from HLA identical sibling donor, 20 from haploidentical family donor, and 18 from unrelated donor. Stem cell source was bone marrow in 28 and PBSC in 15. Conditioning regimen was myeloablative in 39 patients and reduced intensity in 15 . GvHD prophylaxis consisted of CSA and short course MTX for patients transplanted from HLA identical sibling donor, CSA+MTX+ATG+Mycophenolate mofetil+Basiliximab in haploidentical SCT, and CSA+MTX+ATG in unrelated SCT. For both primary (47 patients) and secondary ( 7 patients) prophylaxis, micafungin $(50 \mathrm{mg})$ was administered once daily in $1 \mathrm{~h}$ i.v. infusion from the beginning of conditioning therapy until recovery from neutropenia (first day of an absolute neutrophil count $\geq 0.5 \times 10^{9} / \mathrm{L}$ for 3 consecutive days). Routine microbiological monitoring included both galactomannan assays and blood, urine, stool, oropharynx and sputum cultures, either performed two times per week. Only probable and proven IFI according to the EORTC/MSG criteria were recorded.

Results: After a median follow-up of 18 (4-55) months, 38 patients (70\%) are living and disease-free. Eight patients (15\%) died for relapse of the original disease (median time to death 394 days) and 8 (15\%) for transplant-related causes (nonfungal infections in 4, GvHD in 1, VOD in 1, small bowel volvulus in 1), with a median time to death of 66 days. No death was related to any fungal infection or to the study drug. Overall, the median duration of micafungin administration was 24 days (5-45). No infusion-related adverse event was reported. Discontinuation of micafungin occurred in 3 patients due to transiently elevated bilirubin levels between day 9 and 15 post-transplant. There was no episode of fungal growth from blood cultures. Only one patient showed positive stool cultures for Candida glabrata during administration of micafungin. No patient developed proven IFI either during micafungin prophylaxis or after drug discontinuation. Three patients $(5.5 \%)$ were diagnosed with probable IFI due to at least 3 consecutive galactomannan positive tests associated with positive chest computed tomography scans. One of them received secondary prophylaxis for previous probable pulmonary aspergillosis.

Conclusion: This study confirms that micafungin prophylaxis may be efficacious in preventing IFIs in patients receiving alloSCT. The safety and tolerability profile was remarkably good. Disclosure of Interest: None declared.
P550

Nurse-led 'prescription' significantly reduces the time between onset of suspected neutropenic sepsis and the administration of intravenous antibiotics

S. Clare ${ }^{1}$, S. Rowley ${ }^{1, *}$, J. Lambert ${ }^{1}$, D. Comerford ${ }^{1}$, K. White ${ }^{1}$, G. Cooper ${ }^{1}$, S. Thomas ${ }^{1}$, J. Derbyshire ${ }^{1}$, S. Jordan ${ }^{1}$

${ }^{1}$ Haematology, UCLH NHS Foundation Trust, London, United Kingdom

Introduction: For every hours delay in septic patients receiving intravenous antibiotics the chances of survival reduce by $7.6 \%$ (Kumar et al 2006). In multivariate analysis, time to initiation of effective antimicrobial therapy was the single strongest predictor of outcome (Rosa 2014). A recent prospective study demonstrated mortality rates of $35 \%$ in severe sepsis, $47 \%$ in septic shock and $85 \%$ in multi-organ failure in patients with haematological malignancies (Azoulay et al 2013). At UCLH, delays in the administration of antibiotics for suspected neutropenic sepsis were identified from a pre interventional audit sample $(n=36)$; delays mainly resulted from the wait for busy doctors to attend the patient; to reduce this critical period, the haematology service developed and introduced a Patient Group Direction (PGD) in order for haematology nurses to independently 'prescribe' and administer the first line antibiotic of Tazobactum or Ceftazadine.

Material (or patients) and methods: Ward nursing staff were trained, assessed and mandated to undertake the PGD. A clinical audit was then initiated to investigate possible reductions in the time of first temperature to the administration of intravenous antibiotics following the introduction of PGD.

Results: The pre-intervention audit delays occurred both during the day and at night; significant delays (38,61\%) over and above the recommended 60 minute maximum were identified: $61-120 \mathrm{~min} 16(26 \%)$ and $>120 \mathrm{~min} 22$ (35\%). The initial post implementation audit $(n=200)$ indicated the Mean time to first line antibiotic delivery had reduced to 26 minutes, prompting a revised target time of 30 minutes to be used for the continuing audit of practice. The prospective audit $(n=380)$ continues to report improvements (Table 1) with a Mean time to first line antibiotic administration currently running at $22 \mathrm{~min}$ (range $5-270 \mathrm{~min}$ ).

Conclusion: Nurse-led 'prescription' through Patient Group Directive significantly reduces the time between onset of temperature and the administration of intravenous antibiotics for neutropenic patients. Success factors included the mandating of nursing responsibility for PGD even when a ward doctor was available. These data highlight the importance of maximising the nurses ever presence in the ward environment in improving patient outcome.

Continued and contrasting delays in the delivery of second line antibiotics (Gentimycin or Ciprofloxacin) have been highlighted and a second PGD has subsequently been developed for second line antibiotics, including an electronic

\begin{tabular}{lllllc}
\hline & \multicolumn{1}{|c|}{$\begin{array}{l}>60 \\
\text { minutes }\end{array}$} & $\begin{array}{l}30-60 \\
\text { minutes }\end{array}$ & $\begin{array}{l}\text { Total } \\
\text { minutes } \\
(\mathrm{N}=380)\end{array}$ & $\begin{array}{l}\text { Incomplete } \\
\text { Data }\end{array}$ \\
\hline $\begin{array}{l}\text { Spike-to-needle } \\
1^{\text {st }} \text { Abx. }\end{array}$ & $307(81 \%)$ & $65(17 \%)$ & $6(2 \%)$ & 378 & $2(1 \%)$ \\
$\begin{array}{l}\text { Spike-to-needle } \\
2^{\text {nd Abx. }}\end{array}$ & $39(12 \%)$ & $205(64 \%)$ & $78(24 \%)$ & 322 & $58(15 \%)$ \\
$\begin{array}{l}\text { Spike-to-Dr. } \\
\text { Review }\end{array}$ & $160(45 \%)$ & $151(43 \%)$ & $44(12 \%)$ & 355 & $25(7 \%)$ \\
\hline & & & & & Table 1
\end{tabular}


algorithm that facilitates safe dosage calculations for nephrotoxic antibiotics.

References: Azoulay E, Mokart D, Pene F, et al (2013) Outcomes of critically ill patients with hematologic malignancies: prospective multi-centre data from France and Belgium - a groupe de recherché respiratoire en reanimation onco-hematologique study. Journal of Clinical Oncology 31:2810-2818.

Rosa RG, Goldani LZ (2014) Cohort study of the impact of time to antibiotic administration on mortality in patients with febrile neutropenia. Antimicrobial Agents and Chemotherapy 58(7):3799-803.

Kuderer NM, Dale DC, Crawford J, et al (2006) Mortality, morbidity, and cost associated with febrile neutropenia in adult cancer patients. Cancer 106:2258-66.

Disclosure of Interest: None declared.

\section{P551}

Risk factors for cytomegalovirus infection of 79 allogeneic hematopoietic stem cell transplantation patients

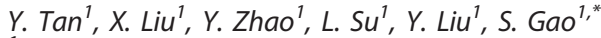

${ }^{1}$ Cancer center, the First Hospital of Jilin University, Changchun, China

Introduction: Cytomegalovirus (CMV) infection is a common complication in allogeneic hematopoietic stem cell transplantation (allo-HSCT). The aim of the study is to investigate the incidence, risk factors and clinical significance of CMV infection of allo-HSCT patients.

Material (or patients) and methods: The clinical data of the patients received allo-HSCT from May 8, 2015 to June 19, 2011 in our hospital were retrospectively analyzed for the incidence and risk factors of CMV infection. The correlation between cytomegalovirus infection and other complications was also analyzed.

Results: A total of 79 patients were included, in which 33 cases were with sibling donors, 42 cases were with haploidentica related donors and 4 cases were with unrelated matched donors. The conditioning regimens were modified BU/CY, ATG was applied in haploidentical related donor and unrelated matched donor transplantations. The incidence of CMV infection was $48.1 \%(38 / 79)$, the median time of was 39 day after transplantation (19 67 days). CMV pneumonia was occurred in 1 patient and the patient died 1 week later. Univariate analysis showed that conditioning regimen with ATG $(P<0.001)$, the lower number of stem cells infused $(P=0.029)$, older age $(P<0.001)$ and female donors $(P=0.012)$ were risk factors for $\mathrm{CMV}$ infection. Multivariate analysis showed that conditioning regimen with ATG $(P<0.001)$, the lower number of stem cells infused $(P=0.014)$ and older age $(P=0.002)$ were associated with CMV infection. The incidence of acute GVHD in patients with CMV activated was significantly higher than that in CMV negative patients $(65.79 \%$ vs $14.63 \%$, $P<0.001)$. Although the incidence of hemorrhagic cystitis in patients with CMV was higher than that in the negative patients $(26.32 \%$ vs $9.75 \%)$, but there was no significant difference $(P=0.054)$. CMV activation had no effect on the long-term survival of the patients $(P=0.747)$.

Conclusion: The CMV activation rate was high in allo-HSCT Conditioning regimen with ATG, lower number of stem cells infused, and older age were associated with CMV infection. CMV infection was associated with the occurrence of aGVHD, but had no effect on long-term survival.

References: 1. Takenaka K, Nishida T, Asano-Mori Y, Oshima K, Ohashi K, Mori T, Kanamori H, Miyamura K, Kato C, Kobayashi $\mathrm{N}$, Uchida N, Nakamae H, Ichinohe T, Morishima Y, Suzuki R, Yamaguchi T, Fukuda T. Cytomegalovirus reactivation after allogeneic hematopoietic stem cell transplantation is associated with a reduced risk of relapse in patients with acute myeloid leukemia who survived to day 100 after transplantation: the Japan society for hematopoietic cell transplantation transplantation-related complication working group. Biol Blood Marrow Transplant. 2015 Nov; 21(11):2008-16.
2. Xiao Y, Song J, Jiang Z, Li Y, Gao Y, Xu W, Lu Z, Wang Y, Xiao $H$. Risk-factor analysis of poor graft function after allogeneic hematopoietic stem cell transplantation. Int J Med Sci. 2014 Apr 30; 11(6):652-7.

Disclosure of Interest: None declared.

\section{P552}

Long term follow-up-of HCV-infected Hematopoietic Cell Transplantation patients and effects of classic and new antiviral therapies

V. Gomez Garcia De Soria ${ }^{1, *}$, S. Veramendi ${ }^{1}$, M. L. Garcia Buey ${ }^{2}$, A. Marinero ${ }^{2}, R$. De la Cámára

${ }^{7}$ HEMATOLOGY, ${ }^{2}$ GASTROENTEROLOGY, HOSPITAL DE LA PRINCESA, MADRID, Spain

Introduction: Hepatitis $\mathrm{C}$ virus (HCV) infection is associated with morbidity and mortality in long-term survivors after HCT. Chronic HVC is the leading cause of cirrhosis after HCT. Although the number of new infections has highly declined since $90 \mathrm{~s}$, there are many HCT survivors chronically infected through blood transfusions. The management of hepatitis C (HCV) infection has changed radically because of the approval of several directacting antiviral agents. We aimed to identify in our database all long-term HCT survivors, HCV positive in order treat them with the new antiviral therapies if they were PCR positive.

Material (or patients) and methods: The medical records of HCT patients seen at Princesa Hospital from May 1985 to April 2015 were reviewed retrospectively. Diagnosis of HCV was made by serological testing, PCR or both. Genotyping of $\mathrm{HCV}$ was performed too. We analysed the therapies received, the response and side effects.

Results: During the last 30 years, 1859 HCT were performed (990 allogeneic HCT). Hepatitis diagnosis of HCV was made in $36 \mathrm{HCT}$ patients (28 allogeneic and 8 autologous). 11 patients were diagnosed by serological testing and 25 by PCR and serology. Genotype information exists for 22 patients $(1 \mathrm{~b}=20 ; 1 \mathrm{a}=1 ; 2=1)$. Underlying disease were: $7 \mathrm{AML} ; 11 \mathrm{ALL}$, $7 \mathrm{AAS}, 6 \mathrm{CML}, 3 \mathrm{MDS}, 1 \mathrm{CLL}$ and $1 \mathrm{NHL}$. The median follow up from HCT is 13,97 years and the maximum follow-up is 30 years.

Of the analysed cohort 8 of 36 patients have died. The main causes of death were relapse and secondary tumours. Liver disease was directly implicated as the cause of death in only one patient (an autologous HCT recipient without response to interferon therapy).

Overall $15 / 36$ were treated with interferon- based therapy with (9 patients) or without the addition of ribavirin ( 6 patients). In addition 3 patients were treated more than once, with interferon (INF) alone as first treatment and with combination of INF or pegylated-INF plus ribavirin as second course. Only $2 / 6$ have sustained response to monotherapy with interferon and $6 / 9$ to combination therapy. The most common side effects were asthenia, loss of weight, muscular pain, anaemia and/or leucopenia.

Overall $6 / 36$ have been recently treated with new antiviral therapies. The choice of treatment combination was decided by the hepatologist specialist. 3 patients received ledipasvirsofosbuvir; 2 ombitasvir-paritaprevir-ritonavir (OPR) plus dasabuvir and 1 OPR plus dasabuvir and ribavirin too. One patient had previously received pegylated-INF and ribavirin without response. $5 / 6$ completed the treatment and $1 / 6$ is ongoing. All these 6 cases became PCR negative and continue negative at last follow up. The side effects are minimal, only one patient had cephalea and mild itching. None of the patients reactivated graft versus host disease. Three more HVC infected HCT patients will start treatment in the near future.

Conclusion: The availability of newer potent antiviral therapies for HCV infected patients with minimal toxic effects compared with the toxic effects of classical therapies, requires an early and preferential treatment of this patient population to avoid long-term liver damage.

Disclosure of Interest: None declared. 
P553

Incidence of Cryptosporidium after autologous and allogeneic hematopoietic stem cell transplantation V. Bordon ${ }^{1, *}$, C. Dhooge ${ }^{1}$, E. Claerebout ${ }^{2}$, S. Casaert ${ }^{2}$, G. Laureys ${ }^{1}$ ${ }^{1}$ Pediatric Hematology, Oncology and Stem Cell Transplantation, Ghent University Hospital, '2 Laboratory of Parasitology, Department of Virology, Parasitology and Immunology Faculty of Veterinary Medicine, Ghent, Belgium

Introduction: Cryptosporidium spp. are protozoan parasites with a worldwide distribution. The characteristics that make of the Cryptosporidium spp. a frequent waterborne infectious pathogen are the facts that Cryptosporidium spp. have a broad range of hosts, including humans, following by a low infectious dose and a relative resistance to water chlorination. In the immune-competent host, cryptosporidiosis is a major cause of childhood diarrhea. In the immunocompromised host, this pathogen can cause prolonged diarrhea and malnutrition, but also liver and biliary tract disease, as well as extra-intestinal problems. The aim of this study is to evaluate the incidence and the severity of Cryptosporidium infections in children after autologous or allogeneic HSCT in a prospective manner.

Material (or patients) and methods: PCR was used to amplify the $18 \mathrm{~S}$ rDNA gene of Cryptosporidium spp. was used to detect Cryptosporidium spp. in faeces, every 2 weeks starting from the conditioning period and in case of diarrhea.

Results: Thirty consecutive patients after allo- HSCT and 11 after auto-HSCT were analyzed. Allo-HSCT was performed for acute or chronic hematological malignancies in 15 patients $(50 \%)$, congenital disorders as primary immune deficiencies or metabolic diseases in 13 patients (43\%), 2 pts were transplanted for other reasons. The donor was a MUD in 19 procedures (64\%), a MSD in $7(23 \%)$ and 4 transplants were performed with a haploidentical donor. The graft was bone marrow in 16 transplants (54\%), PBSC in $7(23 \%)$ and CBU in 7 (23\%). Conditioning was myeloablative in 24 allo-HSCT, RIC in 4 and no conditioning was given in 2 patients.

Auto-HSCT was performed in all cases with PBSC. In auto-HSCT the diagnosis was neuroblastoma in 5 pts, Ewing tumor in 3, brain tumor in 2 and in 1 case the diagnosis was a resistant autoimmune disease.

In the allo-HSCT group a Cryptosporidium infection was detected in 4 of 30 pts. (13\%). Seven out of these 30 patients received a transplant for a severe primary immune deficiency (PID), 3 out these 7 patients showed a Cryptosporidium infection (42\%). The diagnosis in these 3 patients was: SCID, $\mathrm{X}$-LP and hyper IgM Syndrome. There was only 1 patient with Cryptosporidium infection in the group of hematological malignancies. The infection was treated with specific antibiotics in 2 cases. In all cases the infection was limited in time, extra-gastrointestinal symptoms did not appear.

There was no Cryptosporidium infection detected in the autologous group.

Conclusion: Cryptosporidium

is a potentially dangerous protozoan infection. This infection is not always easy to detect. Overall, the incidence of this infection in our HSCT population was low, except for PID pts. In PID patients 3 out of 7 (42\%) patients had Cryptosporidium infection post SCT with need for specific treatment in 2 cases. We recommend a Cryptosporidium serial surveillance in this group, since the chronic and severe T-cell deficiency of these patients made them more susceptible for Cruyptosporidium infection.

Disclosure of Interest: None declared.
P554

Late-onset severe pneumonia after allogeneic hematopoietic stem cell transplantation: prognostic factors and treatments

X.-D. Mo ${ }^{1, *}$, X.-H. Zhang ${ }^{1}$, L.-P. Xu', Y. Wang ${ }^{1}$, C.-H. Yan ${ }^{1}$, H. Chen ${ }^{1}$, Y.-H. Chen', W. Han ${ }^{1}$, F.-R. Wang ', J.-Z. Wang ${ }^{\prime}$, K.-Y. Liu', X.-J. Huang ${ }^{7}$

${ }^{1}$ Institute of Hematology, Beijing, China

Introduction: Late-onset severe pneumonia (LOSP) is a severe complication affecting the clinical outcomes of allogeneic hematopoietic stem cell transplantation (allo-HSCT) recipients. Thus, we aimed to evaluate the prognostic factors associated with and treatments for LOSP in patients who underwent human leukocyte antigen-related allo-HSCT.

Material (or patients) and methods: Fifty consecutive patients who underwent non-T cell-depleted allo-HSCT at the Peking University Institute of Hematology and they met the criterion of LOSP after allo-HSCT from June 1, 2012 to June 30, 2015 were enrolled.

Results: The median time from allo-HSCT to the incidence of LOSP was 231 (90-1487) days. Twenty-eight patients harbored one or more pathogens (infectious LOSP; I-LOSP), whereas 22 did not harbor any pathogens (non-infectious LOSP, NI-LOSP). Smoking before allo-HSCT ( $0 \%$ vs. $35.4 \%, P=0.002$ ) and male sex $(20.0 \%$ vs. $61.9 \%, P=0.026)$ were associated with poorer outcomes. Patients with a lower bronchoalveolar lavage fluid (BALF) neutrophil percentage had better outcomes relative to those with higher BALF neutrophil percentage $(45.5 \%$ vs. $16.7 \%, P=0.012$ ). Pathogens were identified in blood and/or BALF samples from 28 patients; 16 had positive blood samples, 26 had positive BALF samples, and 14 had simultaneous positive blood and BALF samples. The 100- day survival rate of patients with I-LOSP was poorer than that of patients with NILOSP. All patients received methylprednisolone (MP) along with broad-spectrum antimicrobial therapies, and patients given late ( $\geq 1$ week after LOSP diagnosis) and low-dose MP therapy $(\leq 2 \mathrm{mg} / \mathrm{kg} / \mathrm{d})$ had the best outcomes. In the multivariate analysis, non-smoking before allo-HSCT and late and low-dose MP therapy were significantly associated with a better OS after LOSP.

Conclusion: LOSP is a severe complication after allo-HSCT. Patients who smoked before allo-HSCT had poorer survival outcomes. The correct timing and corticosteroid dosage in the context of broad-spectrum antimicrobial therapy might further improve the outcomes of patients with LOSP.

Disclosure of Interest: None declared.

P555

Elevated expression of Tim-3 on CD8 T cells correlates with Cytomegalovirus Reactivation After Allogenic Stem Cell Transplantation

X. $\mathrm{Li}^{1}$, B. Yang ${ }^{1}$, L. X $\mathrm{U}^{1}$, B. $\mathrm{Liu}^{1}, \mathrm{X} . W \mathrm{Wu}^{1, *}, \mathrm{D} . W \mathrm{U}^{1}$

${ }^{1}$ The First Affiliated Hospital of Soochow University,Jiangsu Institute of Hematology, Suzhou, China

Introduction: T-cell immunoglobulin and mucin domaincontaining molecule 3 (Tim-3) represents a novel mechanism of T-cell dysfunction and exhaustion in virus infections. However, the role of Tim-3 in the pathogenesis of early cytomegalovirus (CMV) reactivation after allogeneic stem cell transplantation (allo-HSCT) is not well understood. In this study, we aimed to investigate the role of Tim-3 during CMV infection after allo-HSCT.

Material (or patients) and methods: We detected 33 patients and 10 healthy controls in this study. Collected peripheral blood (PB) of patients 30 days, 60 days, 90 days after allo-HSCT who were neither aGVHD nor suffered from other virus infection, such as HBV, HIV, EBV, et al. Plasma samples were assessed for CMV-DNA by PCR, (Peripheral blood mononuclear cells) PBMCs were isolated for flow cytometry to detect Tim-3 expression on CD8+ T cells, apoptosis of Tim-3- and Tim-3+ 
T cells (Annexin V, AV) and IFN- $\gamma$ secreted by Tim-3- and Tim-3 + T cells.

Results: The rate of CMV reactivation was 51.5\% (17/33) of all the patients. Hence we divided them into three groups: CMV positive group, CMV negative group, healthy control group. An elevated frequency of Tim-3+CD8+ cells was observed in transplanted patients compared to healthy controls, and in transplanted patients, the Tim-3 expression on CD8+ T cells was significantly higher in CMV positive patients than CMV negative cases $(7.048 \pm 3.060 \%$ for healthy controls, $15.55 \pm 6.314 \%$ for CMV negative patients, and $38.98 \pm 17.50 \%$ for CMV positive patients, $P<0.05)$. The frequency of Tim-3-expression $T$ cells correlates with viral load in CMV reactivation. After effective antivirus treatment and CMV became negative, the frequency of Tim-3+CD8+ $\mathrm{T}$ cells was decreased. IFN- $\gamma$ secreted by Tim-3-CD8+ T cell was significantly higher than that produced by Tim-3+CD8+ T cells in each group $(P<0.05)$. In healthy controls, the apoptosis of Tim-3+CD8+ T lymphocytes were significantly increased than that of Tim-3-CD8+ counterparts $(2.926 \pm 0.654 \%$ vs. $1.546 \pm 0.411 \%, P=0.001$ ), while in allo-HSCT patients, there were no differences in apoptosis between Tim-3+CD8+ and Tim-3-CD8+ T lymphocyte $(2.069 \pm 1.720 \%$ vs. $1.632 \pm 0.789 \%$ in CMV negative group, $2.274 \pm 1.185 \%$ vs. $1.871 \pm 0.265 \%$ in CMV positive group, $P>0.05$ )

Conclusion: Tim-3 expression on T cells results in the changes of $\mathrm{T}$ cell function and level of apoptosis, and closely correlated with CMV infection after allo-HSCT.

Disclosure of Interest: None declared.

\section{P556}

Expansion of T or B Lymphocytes after Unrelated Cord Blood (UCB) Allogeneic Stem Cell Transplantation in Adults Correlates with CMV Reactivation and Is Associated with a Better Outcome

Y. Le Bris, ${ }^{1,}$, T. Guillaume ${ }^{2}$, M. Iliaquer ${ }^{3}$, J. Martin ${ }^{4}$, P. Peterlin ${ }^{2}$ A. Menard ', M. Eveillard' ', S. Wuilleme ${ }^{1}$, N. Robillard ', J. Delaunay', M. Mohty ${ }^{5}$, A. Garnier', P. Moreau' ${ }^{2}$, M. C. Bene ${ }^{1}$, P. Chevallier ${ }^{2}$

${ }^{1}$ Hematology Biology, ${ }^{2}$ Department of Hematology, ${ }^{3}$ Virology, ${ }^{4}$ Immunology, CHU Nantes, Nantes, ${ }^{5}$ INSERM U938, Paris University UPMC, Paris, France

Introduction: Peripheral lymphocytosis encountered after myeloablative or reduced-intensity conditioning allogeneic stem cell transplantation (allo-SCT) is an ill-defined feature. Most reports in the literature deal with large granular lymphocytes ( $L G L)$ expansions and only seldom of B-cell increases. Most available data were mainly described in the setting of allo-SCT using bone marrow or peripheral blood as stem cell source. Here, we report data regarding the incidence and features of lymphocyte expansions after unrelated cord blood (UCB) transplantation.

Material (or patients) and methods: Ninety-nine UCB alloSCT performed in adults between October 2005 and October 2014 were considered for the purpose of this study. Most patients received double CB units $(n=94)$ and a RIC regimen $(n=89)$, for various hematological diseases. Whenever detected, we collected the date of onset and termination of peripheral blood lymphocyte expansions $\left(\geq 4 \times 10^{9} / \mathrm{L}\right)$ among the 86 UCB-SCT patients alive at 3 months post-transplant. LGL expansion was defined as sustained LGL above $0.5 \times 10^{9} / \mathrm{L}$. Concomitant immunophenotypic results allowed to discriminate expansions of cytotoxic T-cells, NK-cells and B-cells.

Results: Lymphocytosis was observed in 21 cases (24\%; 10 females and 11 males; median age: 58 y., range: 32-69). Most patients had a myeloid-lineage disease $(67 \%)$ and were in complete remission at time of UCB-SCT (76\%). The median onset of lymphocyte expansion after UCB-SCT was 12.6 months (range, 1.4-49). The median initial lymphocyte count was $4.76 \times 10^{9} / \mathrm{L}$ at time of expansion diagnosis. The median duration of expansion was 12 months (range: 1-52). Twenty patients could be further analyzed phenotypically, showing 8 CD8+ T, $1 \mathrm{NK}$ and $1 \mathrm{~T}-\mathrm{NK}$ LGL expansions. T-LGL expansions were monoclonal $(n=3)$, oligoclonal $(n=2)$ or polyclonal $(n=2)$. Interestingly, 7 cases of polyclonal B-lymphocytes expansions were also documented while 3 patients presented both T CD8+ and B expansions. Of note, B-cell expansions were $\mathrm{CD} 5+$

Lymphocyte expansions were from donor origin for 12/14 tested patients. Acute and chronic GVHD developed respectively in $31 \%$ and $68 \%$ of lymphocytosis patients, and in $57 \%$ and $45 \%$ of the 65 patients without lymphocyte expansion $(P=\mathrm{NS})$. CMV reactivation was significantly more frequent in the group of lymphocytosis patients ( $76 \%$ vs. $29 \%, P=0.0001)$. At time of analysis, 1 patient had relapsed and 4 had died, the causes of death being disease in 1 case and transplant-related mortality in 3 . These events were significantly lower than in the group of patients without lymphocytosis $(P=0.003$ for relapses and $P=0.04$ for death). Two-year disease-free survival and overall survival were significantly different at respectively $85 \%$ vs. $55 \%(P=0.01)$ and $85 \%$ vs. $63 \% .(P=0.03)$.

Conclusion: Lymphocyte expansion, at $24 \%$, is not a rare event in adults receiving UCB allo-SCT. These expansions involve equally the $\mathrm{T}$ or B-lineages. The latter are often $\mathrm{CD} 5+$ suggesting a proliferation of innate B1 cells from the UCB. Lymphocyte expansions are significantly associated with previous reactivation of CMV. Because these cells were of donor origin, it can be postulated that they represent primoactivation upon encounter with CMV. Finally, both types of lymphocyte expansions are associated with a significant favorable outcome, suggesting a possible bystander GVL effect.

Disclosure of Interest: None declared.

P557

Biochemical markers as the early predictors of infections in lymphoma patients undergo autologous hematopoietic stem cell transplantation

Y. Dubinina ${ }^{1, *}$, V. Sarzhevskiy ${ }^{1}, N$. Mochkin ${ }^{1}$ on behalf of Melnichenko V., Smirnova E., Kolesnikova D., Bannikova A., Zamyatin M., Gusarov V., Prokhorova E.

${ }^{1}$ National Medical Surgical Center n.a.N.Pirogov, National Medical Surgical Center n.a.N.Pirogov, Moscow, Russian Federation

Introduction: Preclinical identification the infectious complications can reduce the mortality rate and access the efficacy of antimicrobial therapy during autologous hematopoietic stem cell transplantation (autoHSCT) [1-2].

Material (or patients) and methods: The prognostic significance of three inflammatory markers was studied: presepsin (PSP), procalcitonin (PCT) and C-reactive protein (CRP). PSP, PCT and CRP were evaluated on the day of admission (DA), D+ 1, D+3, D+7 and on the day of discharge (DD). 28 patients were included in the study (12 - Hodgkin lymphoma, 6 - non-Hodgkin's lymphoma, 10 - multiple myeloma; 20 women and 8 men). The median age was 40 (23-66) years. The conditioning regimens were CBV, BEAM or melphalan. Depending on the presence of infectious complications, the patients were divided into 2 groups: group 1 - patients without complications $(n=12)$, group 2 - patients with complications ( $n=16 ; 15$ with febrile neutropenia (FN) and 1 - with sepsis). Results: The median of FN development was 5,5 days. On the DA the median PSP in the group 1 was $166,5 \mathrm{pg} / \mathrm{ml}(77,2-476)$, on the DD - $199 \mathrm{pg} / \mathrm{ml}(90-298)(\mathrm{p}=0.78)$. Median PSP in the group 2 on the DA and also on the DD were not significantly different $(P=0.08)$. In the comparative analysis of PSP in groups 1 and 2 there were no significant difference on the DA and on the D+1 (table 1). Significant difference in PSP between the analyzed groups was found from the D+3 till DD (graph 1) 
Table 1. Comparative dynamics of the PSP in groups 1 and 2 .

\begin{tabular}{lccccc}
\hline & $D A$ & $D+1$ & $D+3$ & $D+7$ & $D D$ \\
\hline Median PSP, group 1 & 166,5 & 144 & 130 & 199 & 199 \\
Median PSP, group 2 & 129 & 153,5 & 231 & 354 & 288,5 \\
$\mathbf{P}$ & 0,56 & 0,72 & 0,01 & 0,011 & 0,022 \\
\hline
\end{tabular}

It was found a significant increasing of the $\mathrm{PCT}$ in both groups. Median PCT on the DA and the DD in group 1 was $0.023 \mathrm{ng} / \mathrm{ml}$ $(0,02-0,112)$ and $0.07 \mathrm{ng} / \mathrm{mL}(0,02-0,356)$, respectively $(P=0.04)$, and in the group $2-0.039 \mathrm{ng} / \mathrm{ml}(0,02-0,158)$ and $0.106 \mathrm{ng} / \mathrm{mL}$ $(0,045-3,67)$, respectively $(P=0.001)$. Unlike the PSP, the PCT didn't significantly differ between groups on D+3, D+7 and DD. Also it was found a significant increasing of the CRP during autoHSCT in both groups. Median CRP on the DA and the DD in the group 1 was $2,25 \mathrm{mg} / \mathrm{l}(0,6-20,4)$ and $14,85 \mathrm{mg} / \mathrm{l}(3,7-50)$, respectively $(P=0.001)$, while in the group $2-3,2 \mathrm{mg} / \mathrm{l}(0,2-53)$ and $19,7 \mathrm{mg} / \mathrm{l}(5,1-152,2)$, respectively $(P=0.025)$. But CRP didn't significantly differ between groups during the study.

Conclusion: The preliminary data showed that PSP is the most appropriate early predictor of infections in lymphoma patients undergo autoHSCT.

References: 1. Reinhart K., Bauer M., Riedemann N. C. et al. New approaches to sepsis: molecular diagnostics and biomarkers. Clinical Microbiology Reviews, 2012; vol. 25, no. 4, 609-634. 2. Naurois de J., Novitzky-Basso I., Gill J.M. et al. Management of febrile neutropenia: ESMO clinical practice guidelines. An. Of Oncol.2010; 21 (Suppl. 5), 252-253. Disclosure of Interest: None declared.

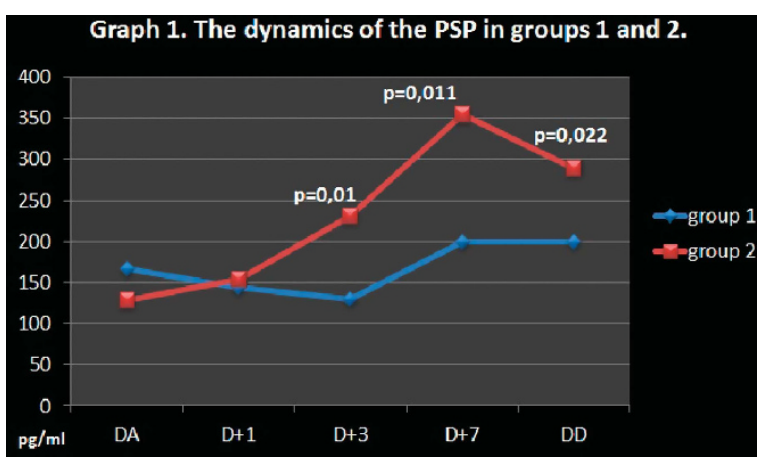

P558

Morbidity 15-30 years after allogeneic haematopoietic stem cell transplantation

J. N. Stuart ${ }^{1, *}$, R. Szydlo ${ }^{2}$, J. Todd ${ }^{3}$, J. F. Apperley ${ }^{2}$, N. Salooja ${ }^{2}$ ${ }^{1}$ Imperial College London, ${ }^{2}$ Haematology, ${ }^{3}$ Endocrinology, Hammersmith Hospital, Imperial College Healthcare Trust, London, United Kingdom

Introduction: Long term survivors of allogeneic haematopoietic stem cell transplantation (HSCT) have an increased prevalence of cardiovascular and endocrine complications. The changing burden of morbidity in patients surviving 15-30 years after HSCT was evaluated using patients' medication lists at measured time points.

Material (or patients) and methods: In this single center study 178 patients surviving longer than 15 years post allogeneic HSCT were identified from our transplant database for whom follow up data beyond 15 years was available for 141. The remaining 37 were abroad or lost to follow up. Details of medication use were collected for each patient at 15, 20, 25 and 30 years post-HSCT from hospital records or from the general practitioner and trends compared using linear chi squared analysis.

Results: Of 141 patients (80 male) the median follow up was 22.3 years (range 15-35) and median age at follow up was 55 years (range 32-79). The majority of patients had CML (120/141) and 16 patients had acute leukaemia. 138/141 had TBI conditioning with a median of $12 \mathrm{~Gy}$ (range 10-14.4). 35 patients had some form of cGVHD documented either presently or in the past. Use of drugs associated with cardiovascular risk factors namely lipid-lowering, anti-diabetic, anti-hypertensive and antiplatelet drugs increased significantly at each successive five year time point after HSCT (Figure 1). At 15, 20, 25 and 30 years after transplant, the percentage of patients taking lipid-lowering medications increased significantly from $18.4 \%$ at 15 years to $29.4 \%, 47.5 \%$ and $64.3 \%$ respectively $(P<0.001)$. Use of antiplatelet medication increased from $5.0 \%$ at 15 years to $15.0 \%$ at 25 years $(P<0.001)$ and anti-diabetic drugs from $4.3 \%$ at 15 years to $17.5 \%$ at 25 years $(P<0.001)$ Use of these drugs was higher at all time points than age and sex matched UK population statistics from the Health and Social Care Information Centre (1). Use of bone protectant medication between 15y-30y, and thyroxine between $15 y-25 y$ also increased significantly (both $P<0.05)$ following HSCT. Use of prophylactic antibiotics remained constant at approximately $65 \%$ of patients and an

Medication use $15-30$ years after HSCT

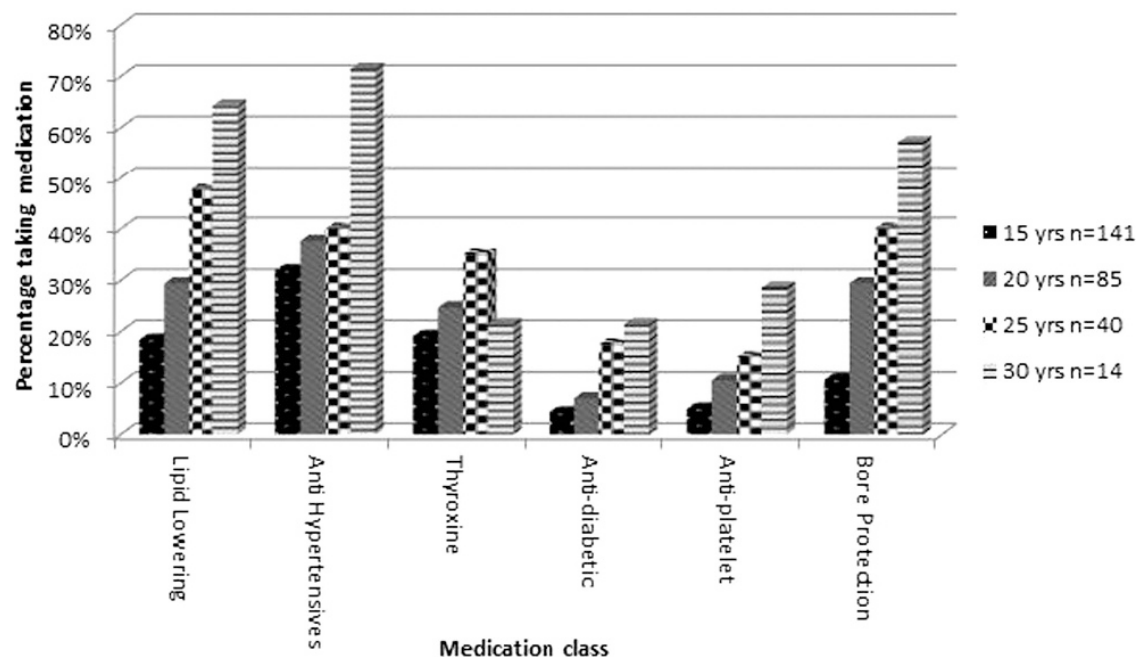


increase in male patients using testosterone from $6.3 \%$ at $15 y$ to $10.5 \%$ at $25 y$ did not reach statistical significance $(P=0.424)$.

Conclusion: Existing data indicates that allogeneic HSCT is associated with increased rates of cardiovascular risk factors and hypothyroidism 15 years from HSCT. Our data show that the burden of morbidity continues to increase substantially 15 30 years post HSCT and highlight that these patients require ongoing medical follow up.

References: 1. Scholes, S., Faulding S., Mindell J., 2014, Use of Prescribed Medicines. http://www.hscic.gov.uk/catalogue/ PUB16076/HSE2013-Ch5-pres-meds.pdf [Accessed 11/12/2015]

Disclosure of Interest: None declared.

\section{P559}

Assessment of iron overload (IO) in the post-allogeneic hematopoietic stem cell transplant (HSCT) setting

J. Zubicaray Salegui ${ }^{1, *}$, I. Lopez Oreja ${ }^{1}$, A. Ugarte $^{2}$, J. M. Alustiza ${ }^{2}$ J. I. Emparanza ${ }^{3}$, N. Argoitia ${ }^{1}$, N. Uresandi ${ }^{1}$, M. Araiz ${ }^{1}$, J. C. Vallejo

${ }^{1}$ Hematology, ${ }^{2}$ Radiology, ${ }^{3}$ Epidemiology, Osakidetza, Donostia, Spain

Introduction: Post-transplant $\mathrm{IO}$ has been found to have a detrimental effect on long-term survival of HSCT recipients (Meyer et al, BBMT 2013), so therapeutic intervention is warranted with pharmacological chelation or phlebotomies (Vallejo et al, Haematologica 2014). Assessment of IO by using serum ferritin (SF) is controversial, since it is an imperfect surrogate marker. Estimation of liver iron concentration (LIC) by MRI is a well-recognized method to quantify IO. However, several problems of standardization and universal accessibility to MRI are not solved yet. There are 3 different methods to estimate LIC by MRI: Signal Intensity Ratio (SIR), T2 Relaxometry and T2* Relaxometry. All of them have been validated and have advantages and disadvantages. SIR methods are cheap, simple, reproducible, easily standardized and accurate enough for the vast majority of cases in clinical practice. As a consequence, the estimating of IO by using a SIR approach is a strategy applicable world-wide. Aim: to estimate the degree of $\mathrm{IO}$ in the post-HSCT setting in order to intervene therapeutically.

Material (or patients) and methods: We prospectively studied all pts who underwent allo-HSCT during a six-month period (16/10/14-24/04/15) in our Center. Fourteen pts were male, and 10 female. Median age was 52 years (20-69). The baseline disease was: $11 \mathrm{AML}, 3 \mathrm{MDS}, 3 \mathrm{ALL}, 3 \mathrm{NHL}, 2 \mathrm{MM}$, and $1 \mathrm{CLL}$. Thirteen transplants were from family donor (4 of them haplo-identical), and 11 from URD (9 or 10/10). Stem cell source were $\mathrm{PB}$ in 22 , and $\mathrm{BM}$ in 2 cases. Conditioning therapy was busulphan-based in 17 (Cy-Bu, Flu-Bu), melphalan-based in 3 (Flu-Mel), TBI-based in 2 (TBI-Cy), and other in 2 (Cy-Flu). Pts and donor were $A B O$ matched in 17 cases, minor mismatched in 4 , and major mismatched in 2. Pts who suffered early death $(n=3)$, relapse $(n=1)$, or intolerance to MRI $(n=1)$ were considered not evaluable. Pts who had received $\geq 25$ PRBC transfusions and had SF levels $\geq 900 \mathrm{ng} /$ $\mathrm{mL}$ underwent a liver MRI employing a SIR method (group A) $(n=12)$. The rest of the pts did not undergo an MRI (group B) $(n=7)$.

Results: Pts of group A had received a median of 36 PBRC transfusions (range: 25-103) and had SF levels at pre-HSCT, day +90 , and day +180 of $1957 \mathrm{ng} / \mathrm{mL}(1223-4954), 1900 \mathrm{ng} / \mathrm{mL}$ (1260-5420) and $1825 \mathrm{ng} / \mathrm{mL}$ (975-5780), respectively. Pts of group B had received a median of 11 PBRC transfusions (2-18), and had SF levels at pre-HSCT, day +90 , and day +180 of 623 $\mathrm{ng} / \mathrm{mL}(102-1670), 669 \mathrm{ng} / \mathrm{mL}$ (313-1155), and $530 \mathrm{ng} / \mathrm{mL}$ (453-802), respectively. All pts in groups A and B were transfusion-independent by day +180 . Liver MRI in $100 \%$ pts of group A showed IO, with a median LIC of 131 micromoles Fe/g dw (range: 60-204) (normal LIC: $\leq 35$ ). IO was moderate (LIC: $36-80$ ) in 2 cases, and severe in 10 cases (LIC $>80$ ). All pts of group A started chelation therapy with deferasirox $(n=9)$ or a program of phlebotomies $(n=3)$.
Conclusion: Assessment of post-HSCT iron overload is warranted in order to implement a therapeutic strategy to reduce it and prevent the patients from the associated morbidity and mortality. In this study, it was showed that combination of history of PRBC transfusions, serum ferritin levels, and liver MRI employing a SIR method for selected patients, was an efficient method to assess post-transplant iron overload.

Disclosure of Interest: None declared.

\section{P560}

Effect of episil ${ }^{\mathbb{R}}$ oral liquid on oral mucositis severity and duration in HSCT patients

K. G. Legert, ${ }^{1, *}$, A. Svanberg ${ }^{2}$, T. Zuckerman ${ }^{3}$, R. Ram ${ }^{4}$, A. Kolomansky ${ }^{5}$, J. Romejko-Jarosinska ${ }^{6}$, B. Nasilowska-Adamska ${ }^{1}$ Dept of Orofacial Diagnosis and Surgery, Dentmed. Karolinska Institutet, Huddinge, ${ }^{2}$ Dept of Haematology, Uppsala University hospital, Uppsala, Sweden, ${ }^{3}$ Rambam Health Care Campus, Haifa, ${ }^{4}$ Tel Aviv Medical Center and Tel Aviv University, ${ }^{5}$ Tel-Aviv Sourasky Medical Center, Tel Aviv, Israel, ${ }^{6}$ Maria Sklodowska Curie Memorial Cancer Center and Institute of Oncology, ${ }^{7}$ Institute of Haematology and Transfusion Medicine, Warsaw, Poland

Introduction: episil ${ }^{\mathbb{R}}$ oral liquid is a medical product indicated for the management and relief of pain caused by oral mucositis. It is a lipid-based liquid that spreads on the mucosal surface, where it transforms into a highly bioadhesive film which mechanically protects the damaged mucosa. We studied the effects of episil ${ }^{\mathbb{R}}$ plus standard of care (SOC) versus SOC alone on oral mucositis in patients receiving conditioning treatment for hematopoietic stem cell transplantation (HSCT). The aim was to investigate the severity and duration of oral mucositis symptoms, as well as the safety and tolerability of episil ${ }^{\mathbb{R}}$.

Material (or patients) and methods: This was an open-label parallel-group study at 12 sites across 4 countries, and was conducted from February to October 2013. Patients scheduled for myeloablative or intense reduced-intensity conditioning treatment (radiation-based or non-radiation-based) followed by HSCT $(n=116)$ were randomized 1:1 to SOC (which included basic oral hygiene) or SOC plus episil ${ }^{\mathbb{R}}$ treatment. episil $^{\mathbb{R}}$ treatment comprised 3 pump strokes and was administered 3 times a day. Treatment with SOC or SOC plus episil ${ }^{\circledR}$ started on the first day of conditioning and lasted for 28 days; HSCT was done after 1-7 days of conditioning.

Results: Of the 109 patients (median age 50 years) who received study treatment (intention-to-treat [ITT] population), 23 were excluded from the per-protocol (PP) population because of inadequate compliance $(<50 \%$ of study days with $100 \%$ compliance). Oral mucositis incidence was $76-77 \%$ in both treatment groups. Overall, the incidence and severity of oral mucositis were considerably lower than anticipated. Nevertheless, $\mathrm{AUC}_{(0-28 \text { days })}$ for WHO Oral Toxicity Score, which reflects oral mucositis severity across the 28 days of treatment, was significantly lower for SOC plus episil ${ }^{\mathbb{B}}$ than for SOC alone in the PP population (adjusted mean 0.5 versus $0.7, P=0.028$ ), but not in the ITT population (adjusted mean 0.7 versus 0.7 , $P=0.889$ ). Median duration of oral mucositis was also significantly reduced in the $\mathrm{SOC}$ plus episil ${ }^{\mathbb{R}}$ group compared with SOC alone in the PP population (5.5 versus 10 days, $P=0.015$ ), but not in the ITT population (8 versus 10 days, $P=0.202)$. In general, SOC plus episil ${ }^{\mathbb{R}}$ was safe and well tolerated, with an adverse event (AE) profile comparable to that of SOC alone. Across the 2 treatment groups there were 5 serious AEs, none of which was judged to be related to study treatment. Local tolerability of episil ${ }^{\mathbb{R}}$, assessed as AEs associated with the oral cavity, was good, with $1 \mathrm{AE}$ in the SOC plus episil ${ }^{(\mathbb{R}}$ group (versus $2 \mathrm{AEs}$ in the SOC alone group). There were 5 adverse device effects (ADEs), the most common of which was vomiting ( 3 events). There were no serious ADEs. Conclusion: episil ${ }^{\mathbb{R}}$ had positive effects on oral mucositis severity and duration in patients receiving conditioning 
treatment for HSCT (PP population). In addition, episil ${ }^{\mathbb{R}}$ was well tolerated in this patient group.

Disclosure of Interest: K. Legert Funding from: Camurus $A B$, sponsor of the study, A. Svanberg Funding from: Camurus $A B$ (sponsor of the study), T. Zuckerman Funding from: Camurus $A B$ (sponsor of the study), R. Ram Funding from: Camurus $A B$ (sponsor of the study), A. Kolomansky Funding from: Camurus $A B$ (sponsor of the study), J. Romejko-Jarosinska Funding from: Camurus $A B$ (sponsor of the study), B. Nasilowska-Adamska Funding from: Camurus $A B$ (sponsor of the study).

P561

Dynamics of immunosuppressive biomarkers in allogeneic hematopoietic stem cell transplantation received recombinant thrombomodulin

K. Ishii ${ }^{1,{ }^{*}}$, S. Fujita ${ }^{1}$, Y. Azuma $^{1}$, Y. Tsubokura ${ }^{1}$, H. Yoshimura ${ }^{1}$, M. Hotta , T. Nakanishi ${ }^{1}$, A. Nakaya ${ }^{1}$, A. Satake ${ }^{7}$, T. Ito ${ }^{1}$, S. Nomura ${ }^{1}$ Department of Internal Medicine, Kansai Medical University, Hirakata, Japan

Introduction: Regulatory T-cells (Treg) are important players to ameliorate acute GVHD (aGVHD). It was reported that HLA-G was associated with Treg in alloHSCT. Recombinant thrombomodulin (rTM) is a new drug for treating disseminated intravascular coagulation (DIC) approved by the Japanese Ministry of Health, Labour and Welfare in 2008. We reported that rTM had the possibility to play a therapeutic role for aGVHD and SOS induced by suppression on adhesion molecules and inflammatory cytokines as well as coagulating factor in the previous EBMT annual meeting. It is to be elucidated how these immunosuppressive factors act when attributable biomarkers to aGVHD are suppressed with rTM. We analyzed dynamics of immunosuppressive biomarkers in alloHSCT patients who received rTM.

Material (or patients) and methods: Patients eligible for alloHSCT for hematopoietic disorders who secured attending physicians clearance were permitted in this study at Kansai Medical University Hospital (KMUH). Blood samples were collected from the patients before and after transplantation through day 35. Levels of cytokines (IL-6, TNF-a, HMGB1, MCP-1, RANTES, TGF- $\beta$ ) and soluble molecules (VCAM-1, E-selectin, PAl-1, PDMP, HLA-G, CTLA-4) were measured by ELISA. CTLA-4 is found in Treg and considered to be important to their function. rTM was administered as a prophylactic therapy for coagulopathy in day 4-14 after alloHSCT. The historical control group was employed, who did not receive rTM at the KMUH $(n=57)$.

Results: 36 patients were analyzed between June 2010 and February 2015. We observed lower and later cases of aGVHD who received $\mathrm{rTM}$ compared to the control group (control vs rTM. incidence rate: $58.1 \%, 36.1 \%, P=0.023$; median days when appeared aGVHD after alloHSCT: 18days, 28days, $P=0.005$, respectively). TNF-a was an attributable factor to later aGVHD among all the biomarkers $(P=0.003$, odds ratio $=0.634$ ). Immunosuppressive biomarkers, which were IL-10, TGF- $\beta$, HLA-G, and CTLA-4, exhibited increment since day 14 after alloHSCT. With higher proportion of CTLA-4, cord blood exhibited the greatest increase compared to the other sources. However, it did not translate into later aGVHD or lower incidence of aGVHD. Mild correlation between HLA-G and CTLA-4 was exhibited $\left(P=0.002, R^{2}=0.039\right)$. Step-wise multi linear regression analysis revealed that HLA-G was the only independent factor for increasing levels of CTLA-4 among all the biomarkers $(P=0.002$, standardized partial regression coefficient $=0.196$ ). Meanwhile, II-10 was significantly associated with HLA-G dynamics $(P=0.000$, standardized partial regression coefficient $=0.675$ ). Unexpectedly, neither HLA-G nor CTLA-4 was associated with risk factor for aGVHD. Also, well we did not identify any other attributable biomarkers for aGVHD.

Conclusion: HLA-G was released in dependent manner with IL-10 and then activated CTLA-4 in alloHSCT received rTM. Treg expressing CTLA-4 seemed to exert its function mainly in a contact-dependent manner ${ }^{1}$. We had a limitation to follow the dynamics of biomarkers until day35 after alloHSCT in which half cases of aGVHD developed beyond this period. However, we could not confirm that HLA-G or CTLA-4 contribute to the inhibition of aGVHD as rTM suppressed inflammatory cytokines such as TNF-a involved in aGVHD.

References: ${ }^{1}$ Pankratz $\mathrm{S}$. et al. Human $\mathrm{CD} 4{ }^{+} \mathrm{HLA}-\mathrm{G}^{+}$regulatory $T$ cells are potent suppressors of graft-versus-host disease in vivo. FASEB J. 2014;28:3435.

Disclosure of Interest: None declared.

P562

Absolute Lymphocyte Count Recovery Predicts Clinical Outcome after Allogeneic Hematopoietic Stem Cell Transplantation in a Japanese Population

K. Kurata ${ }^{1,}$, K. Yakushijin ${ }^{1}$, I. Mizuno ${ }^{2}$, Y. Inui ${ }^{1}$, H. Gomyo ${ }^{2}$ A. Okamura ${ }^{1}$, H. Ichikawa $^{1}$, Y. Mizutani ${ }^{1}$, S. Kakiuchi ${ }^{1}$, Y. Miyata $^{1}$ R. Tominaga ${ }^{2}$, A. Kitao ${ }^{1}$, Y. Sanada ${ }^{1}$, Y. Saito ${ }^{1,3}$, Y. Minami ${ }^{1,4}$ S. Kawamoto ${ }^{1}$ A. Maeda ${ }^{2}$, K. Yamamoto', T. Murayama ${ }^{1,2}$ M. Ito ${ }^{1,5}$, H. Matsuoka ${ }^{1}, H$. Minami ${ }^{1}$

${ }^{1}$ Department of Medical Oncology/Hematology, Kobe University Hospital, Kobe, ${ }^{2}$ Department of Hematology, Hyogo Cancer Center, Akashi, ${ }^{3}$ Division of Molecular and Cellular Signaling, Kobe University Graduate School of Medicine, ${ }^{4}$ Department of Blood Transfusion, Kobe University Hospital, ${ }^{5}$ Division of Medical Biophysics, Kobe University Graduate School of Health Sciences, Kobe, Japan

Introduction: Rapid lymphocyte recovery is generally associated with better clinical outcomes in hematopoietic stem cell transplantation (HSCT). Although several studies have shown the impact of absolute lymphocyte count (ALC) recovery after allogeneic HSCT in recent years, there are only a few reports on Asian populations including patients administered mycophenolate mofetil (MMF) as graft-versus-host disease (GVHD) prophylaxis.

Material (or patients) and methods: We retrospectively reviewed the medical records of 153 adult patients (median age, 52 years [range, 18-66]) who underwent allogeneic HSCT at Hyogo Cancer Center and Kobe University Hospital (Hyogo, Japan) from January 2010 to September 2015. The patients were divided into 2 groups according to ALC on day 28: "low ALC group" $\left(\leq 0.16 \times 10^{9}\right.$ cells $\left./ L\right)$ and "high ALC group" $\left(>0.16 \times 10^{9}\right.$ cells $/$ L)

Results: The median follow-up time of the surviving patients was 768 days (2-1983 days). The diagnoses were acute myeloid leukemia $(n=69)$, acute lymphoblastic leukemia $(n=24)$, myelodysplastic syndrome $(n=20)$, chronic myeloid leukemia $(n=5)$, and malignant lymphoma $(n=31)$. The patients were conditioned either with myeloablative conditioning regimens $(n=71)$ or reduced-intensity conditioning regimens $(n=82)$. The GVHD prophylaxis was either cyclosporine-based $(n=30)$ or tacrolimus-based $(n=123)$. Methotrexate was used in 77 patients and MMF in 71 patients. The median neutrophil engraftment time was 14 days (range, 8-58). At 3-year post-HSCT, the overall survival (OS), relapse rate (RR), non-relapse mortality rate (NRM), and cumulative incidence of acute GVHD were $54 \%, 37 \%, 16 \%$, and $28 \%$, respectively. For patients in the high ALC group, the 3-year OS was significantly higher $(60 \%$ vs. $27 \% ; P<0.01)$ and the 3 -year NRM significantly lower $(10 \%$ vs. $38 \% ; P<0.01)$ than those of patients in the low ALC group. The 3 -year RR (38\% vs. $35 \%$; $P=0.81)$ and cumulative incidence of acute GVHD (30\% vs. $25 \% ; P=0.5$ ) were the same in the both groups. A multivariate analysis of OS showed that a high ALC was significantly associated with better OS (HR 0.47, 95\% confidence interval $0.27-0.81, P<0.01$ ).

Conclusion: Early ALC recovery on day 28 after HSCT may be an important prognostic indicator for HSCT in the Japanese population, including patients administered MMF as GVHD prophylaxis. However, larger prospective studies are warranted to confirm our findings.

Disclosure of Interest: None declared. 


\section{P563}

Symptoms and distress in patients after blood stem cell transplantation - comprehensive assessment and need for further treatment

K. Schäfer-Eckart ${ }^{1, *}$, M. Horneber ${ }^{1}$, M. Rottorf ${ }^{1}$, S. Cebulla ${ }^{1}$, K. Wendelin ${ }^{1}$, S. Dressler ${ }^{1}$, M. Wilhelm

${ }^{1}$ Med. Klinik 5, Klinikum Nürnberg, PMU, Nürnberg, Germany

Introduction: Despite advances in hematopoietic stem cell transplantation, long-term post-transplant complications are common and related with a considerable burden of symptoms and distress. The objectives of this study were to assess patients' symptom experience and distress after stem cell transplantation, to identify the need for further investigations and to offer treatment options.

Material (or patients) and methods: A longitudinal design was used to collect data in a single outpatient BMT clinic. Between August and November 2015, in the first phase of the study 38 individuals at least six months after autologous (auto) or at least 12 months after allogeneic (allo) transplantation (tx) were asked to participate and to complete a standardized set of questionnaires including BFI (Brief Fatigue Inventory), EORTC QLQ-C30 and QLQ-FA13 (core questionnaire and fatigue module), HADS (Hospital Anxiety and Depression Scale) and RS-13 (Resilience Scale). Cutoff scores for further investigations were fatigue: severity $\geq 4$ and impact $\geq 5$ (BFI score), psychological distress: HADS sum score $\geq 13$, neurocognitive deficits or sleep disorders: scores of $<83$ and insomnia > 33 (QLQ-C30).

Results: Thirty-six patients (pts) participated, two were not interested. Median age was 51 years (range 25-71) and 18 patients were females. Seventeen pts had acute and chronic leukemia, 15 multiple myeloma, and 4 lymphoma. Twenty patients had received allogeneic and 16 autologous transplantation. Thirty-two pts were in complete remission. Nine pts were on active treatment, 4 because of a relapse and 5 for maintenance therapy. The number of individuals that should be further investigated by means of structured clinical interviews either for fatigue or psychologic distress (SCID-I) were $11(30 \%)$ and $6(17 \%)$ respectively. Nineteen pts (53\%) were identified as candidates for sleep diagnostics and 13 (36\%) for neurocognitive testing.

Conclusion: The number of individuals at-risk identified with a symptom-oriented screening is high. These first data demonstrate the importance of a thorough symptom assessment during the first years after auto/allo tx to improve differential diagnosis and subsequent supportive care. The study is ongoing and results of the structured clinical interviews, consecutive treatments and follow-up consultation will be presented.

Disclosure of Interest: None declared.

\section{P564}

Use of Defibrotide to treat Transplant-Associated

Thrombotic Microangiopathy - a Retrospective Study of the Paediatric Diseases and Inborn Errors Working Parties of EBMT

L. Yeates 1,* M. Slatter ${ }^{1}$, S. Bonanomi ${ }^{2}$, W. Lim ${ }^{3}$, S. Ong ${ }^{3}$ A. Dalissier ${ }^{4}$ W. Barberi ${ }^{5}$, A. Schulz ${ }^{6}$, M. Duval, C. Heilmann ${ }^{8}$ A. Willekens ${ }^{9}$, H. Hwang ${ }^{3}$, C. Uderzo ${ }^{2}$, P. Bader ${ }^{10},{ }^{\prime}$ A. Gennery on behalf of Paediatric Diseases and Inborn Errors Working Parties of EBMT

${ }^{1}$ Institute of Cellular Medicine, Newcastle University, Newcastle upon Tyne, United Kingdom, ${ }^{2}$ Pediatric Department - BMT, S. Gerardo Hospital, Monza, Italy, ${ }^{3}$ Department of Haematology, Singapore General Hospital, Singapore, ${ }^{4}$ Faculté de Médecine St-Antoine, Paris, France, ${ }^{5}$ Department of Cellular Biotechnologies and Hematology, Sapienza University, Rome, Italy, ${ }^{6} \mathrm{Ulm}$ University Medical Centre, Ulm, Germany, ${ }^{7}$ Transplantation de cellules souches hématopoïétiques et immunotherapies, Montreal, Canada, ${ }^{8}$ Paediatric Clinic II, The National University Hospital, Copenhagen, Denmark, ${ }^{9}$ Clinical Haematology, Research Group Haematology-Immunology of the Free
University, Brussels, Belgium, ${ }^{10}$ Division for Stem Cell Transplantation and Immunology Department for Children and Adolescents, Theodor-Stern-Kai, Frankfurt, Germany

Introduction: Endothelial cell (EC) activation syndromes include veno-occlusive disease (VOD) and transplantassociated thrombotic microangiopathy (TMA), are common complications following haematopoietic stem cell transplantation (HSCT), and cause significant morbidity and mortality. TMA is caused by widespread endothelial dysfunction, and leads to multi-organ failure for many patients. Associated factors include CMV infection, and GVHD. Prognosis is often poor with upto $100 \%$ mortality. Treatment includes removing precipitating factors, plasma exchange, rituximab, and more recently defibrotide (DFT), a polydisperse oligonucleotide with local-acting antithrombotic, anti-ischemic and anti-inflammatory activity. We surveyed paediatric patients treated with DFT and compared data with adult patients.

Material (or patients) and methods: A PDWP/IEWP retrospective analysis was performed for patients receiving DFT as primary treatment for TMA, and compared with an adult cohort. Standard diagnostic inclusion criteria were used ${ }^{1}$.

Results: 42 patients (24 paediatric $<18$ yrs and 18 adult), identified from 8 centres. Median age at HSCT was 96 months (range 5-208: paediatric) and 497.5 months (range 225-766: adult) for adult patients. Paediatric indications for HSCT: malignancy 17 cases, non-malignancy 7 cases (primary immunodeficiency 4, metabolic disease 1 , bone marrow failure 2), adult indications: malignancy 17 , bone marrow failure 1.23 paediatric and 14 adult patients received myeloblative conditioning, 1 paediatric, 4 adult patients received reduced intensity conditioning. 23 paediatric patients had a potential identifiable trigger (most commonly GvHD) vs 9 adult patients $(P=0.001)$. Median time post-HSCT to recognition of TMA was 52 days (range 10-215, paediatric) and 31.5 days (range 16-152, adult). Median time to treatment with DFT was day $0[-10$ to 32 , paediatric], median dose $30 \mathrm{mg} / \mathrm{kg} /$ day (range 25-60) and day +1 [-23 to 10 , adult], median dose $400 \mathrm{mg}$ tds [range $200 \mathrm{mg}$ bd - $800 \mathrm{mg}$ qds] (some patients were already receiving DFT for VOD before a diagnosis of TMA). There was no significant difference to time to treatment between paediatric and adult groups. Median length of treatment was 53 days (range 2-184, paediatric) and 18.5 days (range 2-63, adult). Resolution of TMA was seen in $18(75 \%)$ paediatric and $14(78 \%)$ adult cases $(P=\mathrm{ns})$. Non-resolution of TMA led to death in $5 / 6$ paediatric and $3 / 4$ adult cases.

Conclusion: TMA usually develops within 100 days post-HSCT, diagnosis can be challenging, and following removal of precipitating factors, effective specific treatment is difficult and often unsuccessful. Unresolved TMA is associated with a poor outcome; therefore the development of an effective treatment strategy is required. As TMA is an EC activation syndrome we reasoned that DFT may be an effective treatment. This retrospective survey uncovered 42 patients, treated with DFT for TMA. For a condition with historically high mortality, the TMA-related survival in this unselected population was surprisingly high, and equally good in children and adults. Whilst there are many flaws in this opportunistic retrospective survey, there is sufficient evidence to propose a controlled clinical trial to investigate further the efficacy of DFT in the treatment of TMA.

References: 1. Cho, B.-SY, et al. Transplantation 2010;90:918. Disclosure of Interest: None declared.

\section{P565}

Immunohematologic complications after hematopoietic stem cell transplantation across major ABO-barriers: comparison of three different transfusion strategies L. Infanti, ${ }^{1,2}$, D. Eissa ${ }^{1}$, J. Passweg ${ }^{2}$, A. Holbro ${ }^{3}$, A. Buser ${ }^{7}$ ${ }^{1}$ Blood Transfusion Service, Swiss Red Cross, ${ }^{2}$ Hematology, University Hospital, ${ }^{3}$ Blood Transfusion Center, Swiss Red Cross, Basel, Switzerland 
Introduction: In stem cell transplantation (HSTC) across major ABO-incompatibility, delayed RBC engraftment, pure red cell aplasia (PRA), and passenger lymphocyte syndrome (PLS) in case of bidirectional ABO-barrier can occur. RBC transfusion policy in the peri-transplantation period may influence the occurrence of immunhematologic complications.

Material (or patients) and methods: Data of HSCTs performed from $01 / 2006$ to $12 / 2014$ were retrospectively evaluated, excluding HSCT with cord blood units. The number of RBC transfusions, the occurrence of PRA, PLS, and survival were evaluated up to day 180 or to last follow-up. RBC transfusion policy included administration of RBC units of either donor ABO-blood, recipient ABO-blood group or of blood group O starting from the day of HSCT. Until December 2010, basically no blood group O RBC units were given; if Anti$A / B$ titer was $>1: 16$, donor-type RBC units were administered from day -3 to reduce the titer to $<1: 2$; if Anti-A/B titer was $>1: 128$ or no sufficient reduction was achieved with $A B O-$ incompatible RBC transfusions, plasmaphereses were performed. From January 2011, ABO-incompatible RBC transfusions were no longer given, and blood group O RBC units were transfused in case of bidirectional ABO-barrier.

Results: Results: Data of 105 HSCT in 102 patients (59 males, 43 females) were analyzed. Median age was 46 years (range: $10-71 ; 7$ cases $<18$ years). Diagnoses were AML $(N=26)$, ALL ( $N=26)$, lymphoid neoplasia $(N=18)$, multiple myeloma $(N=11)$, other myeloid neoplasia $(N=10), \operatorname{MDS}(N=8)$, other $(N=3)$. Donors included 58 males and 30 females (gender unknown in 14 cases) with median age 39.5 years (range: 1365); 34 were related and 71 unrelated. Stem cell source was $\mathrm{BM}$ in 15 and $\mathrm{PBSC}$ in 90 cases. $\mathrm{A}$ major $\mathrm{ABO}$-barrier $(\mathrm{A} / \mathrm{O}, \mathrm{B} / \mathrm{O}$, $A B / O, A B / A, A B / B)$ was present in 78 cases, a bidirectional barrier $(A / B, B / A)$ in 27 cases. WBC and platelet engraftment occurred in median at day 16 and day 17 after HSCT. The results of RBC engraftment and immunhematologic complications by RBC transfusion policy groups are presented in the table.

There was no difference in the occurrence of PRA and PLS, and in $\mathrm{RBC}$ transfusion requirements. For the time of $\mathrm{RBC}$ engraftment and for one-year-survival, a trend in favor of the patients transfused with RBC blood $O$ and of those receiving $\mathrm{RBC}$ of $\mathrm{ABO}$-donor-type was observed, respectively. Conclusion: Different RBC transfusion policies in HSCT across $\mathrm{ABO}$-major barriers seem not to play a role in the occurrence of immunhematologic complications. The impact of different transfusion strategies on clinical outcomes and the observed effect on survival need further evaluation in prospective studies.

Disclosure of Interest: None declared.

\section{P566}

Outcome of stem cell transplant patients admitted to the Intensive Care Unit. A Center Experience

L. Sánchez ${ }^{1}$, J. C. Ballesteros ${ }^{2}$, L. Vazquez ${ }^{3, *}, N$. Arratibel ${ }^{3}$ L. Lopez-Corral', M. Cabrero ${ }^{3}$, M. Perez-Cheng ${ }^{\prime}$, D. Caballero ${ }^{3}$ A. Rodriguez-Encinas ${ }^{2}$

${ }^{7}$ Intensive care unit, ${ }^{2}$ Salamanca University Hospital, salamanca, Spain, ${ }^{3}$ Hematology Department, Salamanca University Hospital, salamanca, Spain

Introduction: The prognosis of malignant hematological diseases has experienced an important advance over the last decades, mainly in patients who undergo hematopoietic transplantation, which has led to many of these patients to be admitted into Intensive Care Units. However, there is still some controversy on the real benefit of treatment in these patients. The aim of this retrospective study was to establish short-term mortality rate and prognosis factors in a group of patients with hematological diseases and hematopoietic transplantation who were admitted to our unit (multipurpose Intensive Care Unit in a tertiary hospital).

Material (or patients) and methods: Retrospective study of all patients with hematopoietic transplantation who required admission to the Intensive Care Unit at any point during their evolution after the transplantation over a period of 5 years (2011-2015). We analyze the demographic characteristics of the patients, severity of the case, type of transplant, reasons for admission to the ICU, number of organ failures and need for ventilatory support.

Results: In the period of our study, 47 patients with hematopoietic transplantation were admitted, with an average age of 48.68 years (70\% men), APACHE II score 22.57. The average stay in the ICU was 9.47 days. $53 \%$ of the patients were neutropenic at admission to the ICU. Diagnosis was acute leukemia in $26 \%$ of the patients, non-Hodgkin and Hodgkin lymphomas in $25 \%$, multiple myeloma in $17 \%$ of them, myelodysplastic syndrome in $11 . \%$ and bone marrow aplasia in $4 \%$ of the patients. In $26 \%$ of the patients, more than 2 transplants were carried out. Out of the 61 transplantation episodes, $47 \%$ were autologous and $53 \%$ were allogeneic. The most common reason for admission to the ICU was respiratory failure (48\%), followed by septic shock (36\%). At admission patients presented with an average of 2 organ failures, being the most common one hemodynamic (72\%), followed by respiratory failure (53\%). 49\% were admitted within the first 30 days after transplantation and $45 \%$ after day $90.64 . \%$ of the patients required invasive mechanical ventilation, and in $15 \%$ non-invasive mechanical ventilation was initially implemented with a failure rate of $86 \%$. A tracheotomy was performed in $11 \%$ of the cases and $15 \%$ required treatment with continuous venovenous hemodiafiltration. The mortality rate in the ICU was $57 \%$. According to the type of transplant, mortality in the ICU for patients with an allogeneic transplant was $70 \%$ and for

\begin{tabular}{|c|c|c|c|c|}
\hline & $\begin{array}{c}\text { Donor-type RBCs } \\
\mathrm{N}=25\end{array}$ & $\begin{array}{l}\text { Recipient-type RBCs } \\
\qquad \mathrm{N}=51\end{array}$ & Blood group $O R B C s \mathrm{~N}=29$ & $\begin{array}{c}\mathrm{P} \\
\text { value }^{a}\end{array}$ \\
\hline ABO-major/bidirectional barrier, $\mathrm{N}$ & $21 / 4$ & $48 / 3$ & $9 / 20$ & 0.000 \\
\hline Anti-A/Anti-B titer median (range) & $24(1-128) / 8(2-64)$ & $8(1-1024) / 8(1-128)$ & $8(1-128) / 6(0-16)$ & $0.8 / 0.1$ \\
\hline Anti-A /B reduction, $\mathrm{N}$ & $13^{b}$ & $3^{c}$ & 0 & \\
\hline Total RBC transfusions, N median (range) & $12(0-69)$ & $13(0-80)$ & $10(0-81)$ & 0.32 \\
\hline RBC engraftment, day median (range) & $69(7-395)$ & $34(12-754)$ & $24(14-136)$ & 0.089 \\
\hline Pure red cell aplasia, $\mathrm{N}$ & 5 & 7 & 3 & 0.200 \\
\hline Passenger lymphocyte syndrome, $\mathrm{N}$ & 0 & 0 & 2 & 0.200 \\
\hline One Year Survival \% & $84 \pm 15$ & $62 \pm 14$ & $69 \pm 17$ & 0.079 \\
\hline
\end{tabular}

${ }^{a}$ chi-square test; ${ }^{\mathrm{b}}$ donor-type RBC transfusions; ${ }^{\mathrm{c}} 2$ donor-type RBC transfusions, 1 plasmapheresis

There was no difference in the occurrence of PRA and PLS, and in RBC transfusion requirements. For the time of RBC engraftment and for one-yearsurvival, a trend in favor of the patients transfused with RBC blood $\mathrm{O}$ and of those receiving RBC of ABO-donor-type was observed, respectively. 
patients with an autologous transplant was $48 \%(P=0.064)$. Regarding the severity in patients who die are higher the APACHE II score (24.74 vs. $19.65 ; P=0.024)$ and the number of organs failure (2.44 vs $1.88 ; P=0.25$ ).

Conclusion: Patients with a hematological transplant show a high mortality rates in the ICU and a high consumption of resources. A significant relation between APACHE II and mortality was observed. That could help to a better selection of patients before admission.

Disclosure of Interest: None declared.

\section{P567}

Validation of Refined Disease Risk Index (DRI) and analysis of host and transplant factors in two Brazilian institutions for Allogeneic Hematopoietic Stem Cell Transplantation (HSCT)

L. J. Arcuri 1,* A. Ribeiro ${ }^{2}$, S. Maradei ${ }^{1}$, V. Mattos ${ }^{2}$, A. Oliveira $^{1}$, C. Silva ${ }^{2}$, S. Lermontov ${ }^{1}$, N. Hamerschlak ${ }^{2}$, R. Castro ${ }^{1}$

${ }^{1}$ Centro de Transplante de Medula Óssea, Instituto Nacional de Cancer, Rio de Janeiro, ${ }^{2}$ Transplante de Medula Ossea, Hospital Israelita Albert Einstein, Sao Paulo, Brazil

Introduction: HSCT is a potentially curative therapy for many hematologic malignances. Risk assessment before transplant is crucial for balancing morbidity and mortality associated with this procedure. Currently some algorithms are used in clinical practice for this assessment. The Disease Risk Index, which was refined in 2014, was developed as a tool for comparing different populations.

Material (or patients) and methods: This is a retrospective study. All patients with hematologic malignances who underwent first allogeneic HSCT between Jan'2010 to Dec'2014 were included. DRI was defined retrospectively. Survival curves were calculated using Kaplan-Meier survival curves. Risk factor for death were estimated by Cox model, stratified for center.

Results: Two hundred seventy seven patients were included. Median follow-up was 34 months. Median age was 36 (range 1-75). The cohort was heterogeneous: 101 AML patients; 77 ALL patients; 35 lymphoproliferative disorders and 24 myelodisplastic syndromes. Myeloablative conditioning regimen was utilized in $217(78 \%)$ and stem cell source was bone marrow in 169 patients $(61 \%)$, peripheral blood in $76(27 \%)$ and cord blood in $32(12 \%)$. There were more matched-sibling donors 145 (52\%) - than unrelated donors, 112 (40\%). Twenty (7\%) were haploidentical transplants. Eighteen patients (7\%) had low DRI, 165 (60\%) patients had intermediate DRI, 72 (26\%) had high and $22(8 \%)$ had very high DRI. Mortality at 100 days was $25 \%$. Kaplan-Meier estimate of overall survival at 1 and 2 years was $54 \%$ and $42 \%$. Overall survival curves according to DRI score are reported in figure 1 . Multivariate analyses identified age ( $<18$ years, $\mathrm{HR}=0,6 ; P=0,01$, compared to 18 50 years), unrelated ( $\mathrm{HR}=2,0 ; P<0,001)$, haploidentical donors

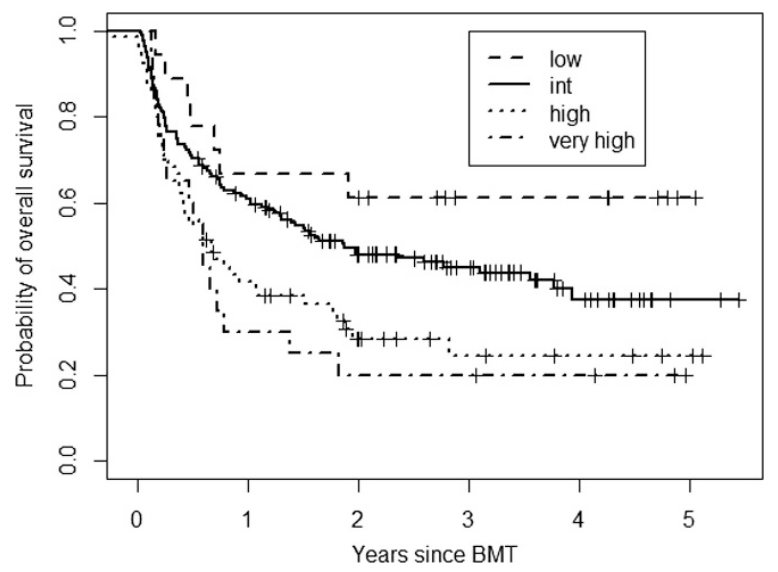

$(\mathrm{HR}=2,7 ; P=0,005)$ and $\mathrm{DRI}(\mathrm{HR}=1,7 ; P=0,002$ for high/very high risk, compared to intermediate risk) as risk factors. Age $>50$ years $(H R=1,3 ; P=N S)$, conditioning intensity and stem cell source were not related to death in multivariate analysis, although cord blood was associated to worse outcome in univariate analysis $(\mathrm{HR}=1,8 ; P=0,02$, and $\mathrm{HR}=$ 1,$1 ; P=$ NS in multivariate analysis). Interaction test was negative for DRI and age, and DRI and donor type.

Conclusion: DRI predicted outcome in our population, which also included alternative donors (as haploidentical and cord blood) and children, and allowed to us to make inferences about other risk factors. Our data suggests that age alone does not negatively influences outcomes, confirming previous reports. Unexpectedly, unrelated donors were associated to poorer outcome. This could be explained by patient referral and delay until transplant.

References: Armand P, Gibson CJ, Cutler C, Ho VT, Koreth J, et al. A disease risk index for patients undergoing allogeneic stem cell transplantation. Blood 2012; 120: 905-913.

Armand P, Kim HT, Logan BR, Wang Z, Alyea EP, et al. Validation and refinement of the Disease Risk Index for allogeneic stem cell transplantation. Blood 2014; 123: 36643671.

Sorror ML. Hematopoietic cell transplantation (HCT)-specific comorbidity index: a new tool for risk assessment before allogeneic HCT. Blood 2005; 106: 2912-2919.

McCurdy SR, Kanakry JA, Showel MM, Tsai H-L, Bolanos-Meade $\mathrm{J}$, et al. Risk-stratified outcomes of nonmyeloablative HLAhaploidentical BMT with high-dose posttransplantation cyclophosphamide. Blood 2015; 125: 3024-3031.

Disclosure of Interest: None declared.

\section{P568}

Defibrotide as a potential treatment for thrombotic microangiopathy (TMA) after haematopoietic stem cell transplantation (HSCT)

L. Poon ${ }^{1, *}$, Y. C. Yuen ${ }^{2}$, X. C. Lim $^{3}$, C. M. Woon ${ }^{2}$, L. K. Tan ${ }^{1}$, W. J. Chng ${ }^{1}$, B. Tan ${ }^{1}$, Y. ${ }^{-1}$, J. Lieow' ${ }^{1}$, L. P. Koh ${ }^{1}$

${ }^{1} 1$ Department of Haematology-Oncology, Stem Cell Transplantation Unit, National University Cancer Institute Singapore, National University Health System, ${ }^{2}$ Department of Pharmacy, ${ }^{3}$ Dept of Internal Medicine, National University Health System, Singapore, Singapore, Singapore

Introduction: Thrombotic microangiopathy (TMA) is a serious complication after haematopoietic stem cell transplantation (HSCT) and effective treatment remains undefined.

Material (or patients) and methods: 7 patients with posttransplant TMA from a single center in Singapore were treated with defibrotide between 2011 and 2015. Diagnosis of TMA was made based on the International Working Group of the European Group for Bone Marrow Transplantation (IWGEBMT) criteria while response was defined as per Blood and Marrow Transplant Clinical Trials Network (BMT CTN) recommendations.

Results: TMA was diagnosed at a median of 68 days (range 36476) after HSCT. All patients received defibrotide at a dose of $20-30 \mathrm{mg} / \mathrm{kg}$. Patient characteristics and risk factors for TMA are as summarized in Table 1. All except one patient had calcineurin inhibitor $(\mathrm{CNI})$ stopped, and none of the patients received plasma exchange (PE). No other TMA treatment was tried prior to defibrotide. 3 out of 7 patients had a response to defibrotide, of whom 2 has remained in remission while 1 patient has died of disease relapse while in remission from her TMA. All 3 responders had defibrotide started within 2 weeks of diagnosis, compared to only 2/4 non responders. Long term outcomes of TMA remained poor with only 2 of 7 survivors in our series of patients.

Conclusion: Conclusion: Our study showed that defibrotide was effective in about $42 \%$ of patients with transplantation associated TMA. Early use of defibrotide at diagnosis might help improve response rates.

Disclosure of Interest: None declared. 
Table 1: Characteristics of 7 patients with TMA treated with defibrotide

\begin{tabular}{|c|c|c|c|c|c|c|c|c|c|}
\hline $\begin{array}{l}\text { Sex/ } \\
\text { Age }\end{array}$ & $\begin{array}{c}\text { Diagnosis/ Transplant } \\
\text { type/ Type of } \\
\text { conditioning }\end{array}$ & $\begin{array}{l}\text { Day of } \\
\text { TMA } \\
\text { diagno- } \\
\text { sis }\end{array}$ & $\begin{array}{l}\text { Clinical } \\
\text { Symptoms }\end{array}$ & $\begin{array}{l}\text { Risk factors } \\
\text { for TMA }\end{array}$ & $\begin{array}{l}\text { Time from } \\
\text { Diagnosis } \\
\text { to DFT use } \\
\text { (Days) }\end{array}$ & $\begin{array}{l}\text { Duration } \\
\text { DFT (days) }\end{array}$ & $\begin{array}{l}\text { Out- } \\
\text { come } \\
\text { (TMA) }\end{array}$ & $\begin{array}{l}\text { Outcome } \\
\text { (Patients) }\end{array}$ & $\begin{array}{l}\text { Cause of } \\
\text { Death }\end{array}$ \\
\hline M/ 29 & ALL (CR2)/UCBT/RIC & $D+68$ & Renal & CMV/GVHD & 2 & 50 & $\mathrm{R}$ & Alive & - \\
\hline $\mathrm{M} / 40$ & $\begin{array}{c}\text { DLBCL (CR3)/Haplo/ } \\
\text { RIC/PT Cy }\end{array}$ & $D+44$ & $\begin{array}{l}\text { CNS, } \\
\text { Renal }\end{array}$ & $\mathrm{CMV} / \mathrm{GVHD}$ & 23 & 24 & NR & Dead & IPS \\
\hline$F / 51$ & $\mathrm{AML}(\mathrm{CR} 2) / \mathrm{UCBT} / \mathrm{RIC}$ & $D+61$ & $\begin{array}{l}\text { CNS, } \\
\text { Renal }\end{array}$ & $\begin{array}{l}\text { CMV/ } \\
\text { GVHD }\end{array}$ & 1 & 14 & NR & Dead & AKI \\
\hline$F / 45$ & $\begin{array}{c}\text { T-ALL (CR1)/ MSD/ } \\
\text { MA }\end{array}$ & $D+476$ & Renal & GVHD & 11 & 36 & $\mathrm{R}$ & Dead & Disease \\
\hline M/ 37 & AML (CR1)/MSD/MA & $D+36$ & - & CMV & 14 & 34 & $\mathrm{R}$ & Alive & - \\
\hline F/ 41 & B-ALL (CR1)/UCBT/MA & D178 & DAH & $\begin{array}{l}\text { CMV/ } \\
\text { GVHD }\end{array}$ & 56 & 17 & NR & Dead & $\mathrm{DAH}$ \\
\hline M/ 55 & AML (CR2)/Haplo/RIC & $D+252$ & - & $\begin{array}{l}\text { PTB/ CMV/ } \\
\text { GVHD }\end{array}$ & 6 & 11 & NR & Dead & IPS \\
\hline
\end{tabular}

Legend: R: Responders, NR non responders, IPS: Idiopathic pneumonia syndrome, AKI: Acute kidney injury, DAH: Diffuse alveolar hemorrhage, DFT: Defibrotide.

P569

Eccrine squamous syringometaplasia in children undergoing stem cell transplantation is associated with treosulfan based regimens

L. M. Balli, ${ }^{1,}$, D. Bresters ${ }^{1}$, M. van Kester ${ }^{2}$, R. van Doorn ${ }^{2}$

${ }^{7}$ Pediatrics, Willem Alexander Childrens Hospital, LUMC,

${ }^{2}$ Dermatology, Leiden University Medical Centre, Leiden, Netherlands

Introduction: Eccrine squamous syringometaplasia (ESS) is a specific clinical skin eruption that has been described in adults undergoing hematopoietic stem cell transplantation (HSCT) and high dose chemotherapy. ${ }^{1}$ This entity together with the incidence or risk factors have not been reported in children.

Material (or patients) and methods: All children undergoing HSCT in our unit form October 2013 until July 2015 were included. ESS was defined as confluent erythematous macular areas/plaques in the flexural skin areas, the axillae and/or groin, occurring shortly after HSCT, with no evidence of skin mycosis, HHV6 infection and in the absence of aGVHD. Possible risk factors including age, gender, underlying diagnosis, conditioning regimen and co-occurrence of mucositis were documented.

Results: Forty-seven patients were included, 27 boys and 20 girls with median age 10 y $4 \mathrm{~m}$ (range $10 \mathrm{~m}-17 \mathrm{y} 10 \mathrm{~m}$ ), with an underlying diagnosis of hematological malignancy $n=22$ : immune deficiencies $n=2$; hemoglobinopathies $n=7$ : aplastic anemia $n=5$ other $n=11$.

The cumulative incidence of ESS was 17\% (8/47) developing within 6-16 days post HSCT. Pain was a common factor often requiring opiate analgesia. No specific skin preparation alleviated symptoms. The skin rash was self-limiting within weeks, typically with widespread desquamation and discolouration of the involved areas. A typical skin lesion in one child was biopsied for histopathological examination. This showed epidermal hyperkeratosis and keratinocyte apoptosis with dermal proliferation of fibroblasts around adnexal structures, characteristic in reports of established ESS.

Children receiving treosulfan regimens had a significant higher incidence versus those receiving non-treosulfan based chemotherapy or total body irradiation $(6 / 18$ versus $2 / 29 P=0.05$ Chi square). The severity and frequency of mucositis did not differ between children with or without ESS and there was no statistical increase in the occurrence of acute skin GVHD ( $p$ $=0.29$ ).
Conclusion: Chemotherapy-induced ESS is associated a rare, distinctive clinical skin eruption primarily located in the skin folds. It is thought to reflect sensitivity of adnexal epithelium to chemotherapeutic agents secreted by sweat glands. We observed that patients undergoing HSCT with treosulfan are more at risk of developing ESS. It should be considered in the differential diagnosis of erythematous eruptions during HSCT. Topical treatment with free radical scavengers for chemotherapy induced symptoms have been successfully used in adults ${ }^{2}$ It remains to be determined if such strategies would help to prevent or alleviate ESS in children undergoing HSCT.

References: ${ }^{1}$ Valks R et al Arch Dermatol 1997; 133:873-78.

${ }^{2}$ Kluschke F et al. Abstract 5064, ASCO 2012.

Disclosure of Interest: None declared.

\section{P570}

Hepatic sinusoidal obstruction syndrome (SOS) following hematopoietic stem cell transplantation (HSCT): incidence, treatment and outcome in a cohort of 1,540 patients

M. Abecasis", , J. Schuh ${ }^{2}$, I. Coelho ${ }^{1}$, S. Leocádio ${ }^{2}$, F. Moita ${ }^{1}$, G. Teixeira ${ }^{1}$, I. Ferreira ${ }^{1}$, N. Miranda ${ }^{1}$

${ }^{1}$ hematology, instituto portugues oncologia, ${ }^{2}$ hematology, Centro Hospitalar de Lisboa Central, lisboa, Portugal

Introduction: HSCT-associated SOS is a well-recognized regimen-related toxicity whose diagnosis and treatment is particularly difficult. It usually develops by 30 days after HSCT, but it may occur later and its reported incidence varies from 5 to $60 \%$.

Material (or patients) and methods: We retrospectively reviewed the occurrence, treatment and outcome of SOS cases occurring in our center between May 1987 and December 2014. Although our review covered the time period that was mentioned it must be said that the first cases were only diagnosed in 1996 . Out of 1,540 patients receiving autologous ( $n=922)$ or allogeneic $(n=618)$ HSCT for malignant and nonmalignant conditions, 34 (2\%) fulfilled the Seattle, Baltimore or Bearman clinical criteria for the diagnosis of severe VOD ${ }^{1}$. All cases underwent ultrasonography of the liver with Doppler studies, by 2 dedicated radiologists, to look specifically for ascites and reversal of flow in the portal vein, hepatic artery resistance index $>0.75$ or an abnormal portal vein waveform and $71 \%$ had findings suggestive of SOS $^{2}$.

Results: Twenty two $(65 \%)$ of these patients had received Busulphan containing conditioning regimens without 
pharmacokinetic monitoring of drug levels. Median age was $30 y(1-57)$ and most cases occurred between days +2 to +21 from HSC infusion (median onset $\mathrm{d}+14$ ). The most frequent underlying diagnoses were acute leukemia (35\%), nonHodgkin's lymphoma (12\%) and breast cancer (12\%). SOS was more frequently observed after allogeneic than autologous transplantation (3.2 vs $1.1 \% P<0.01$ ). We had access to defibrotide in 1998 and since then we have used it to treat 20 patients. Most were given a dose of $25 \mathrm{mg} / \mathrm{kg}$ per day in 4 divided doses and were treated for a median of 15 days (range 3-58). Eleven had a complete resolution of SOS and survived with no significant treatment-associated toxicity. Of the remaining 14 patients, treated with ancillary or supportive measures only, 9 survived. There were a total of 14 deaths directly attributed to SOS, occurring more frequently in older patients (mean $40 y$ vs $24 y ; P<0.01$ ).

Conclusion: This report includes a large number of patients transplanted at a single center over a long period of time, with a lower incidence of SOS than in most other studies. We believe this is mainly the result of misdiagnosed cases in the years 1987 to 1995, and also due to the fact that most of our patients had moderate to severe disease, as it is known that only $25 \%$ of patients who develop SOS have severe disease. Defibrotide itself has proven to be an effective and safe treatment in our hands but challenges still remain regarding the SOS, namely the need to identify its risk factors, new criteria for its diagnosis and the need to search for relevant biomarkers ${ }^{3}$.

References: 1 . Chao N. Blood 2014, 123: 4023

2. Abecasis M et al. Bone Marrow Transplant 1999, 23: 843

3. Mohty M et al. Bone Marrow Transplant 2015, 50: 781

Disclosure of Interest: None declared.

\section{P571}

Influence of chronic graft versus host disease and late effects in patients underwent allogeneic stem cell transplantation with long term follow up

M. Celis ${ }^{1, *}$, S. García-Ávila ${ }^{1,2}$, B. López-Pereira ${ }^{1}$, A. Bermúdez ${ }^{1}$ L. Yañez ${ }^{1}$, R. Lobeira ${ }^{7}$, M. Jimenez-Paiz ${ }^{1}$, A. Insunza ${ }^{1}$, M. LópezDuarte', M. Colorado ${ }^{1}$, M. Sánchez-Escamilla ${ }^{7}$, A. Casado ${ }^{1}$, C. Richard ${ }^{1}$, E. Conde

${ }^{1}$ Hematology, University Hospital Marqués de Valdecilla., ${ }^{2}$ Hematology, IDIVAL, Santander, Spain

Introduction: Allogenic stem cell trasplantation (AlloSCT) is a curative option in patients with hematologic diseases. The quality of life (QoL) in these patients could be influenced by chronic GvHD and late effects. AIMS: To evaluate cGvHD and analize the impact on QoL. The secondary point is to describe late effects and drugs consumption in long term survivors.
Material (or patients) and methods: In our center 307 patients underwent AlloSCT between 2000-10. A crosssectional study was performed.We included 102 patients with $>5$ years long-term follow up, $>18$ years at enrolment and in complete remission.Data were collected by clinical history and personal telephone interviews.To evaluate QoL validated questionnaires EQ-5D-5 L and FACT-BMT were utilized.We added specific questions related with cutaneous-mucous damage, hormone dysfunction, osteoarticular disease, hypoacusia, cataracts, infections, cardiovascular factor risks (CVFR), secondary neoplasm and drugs consumption.

Median age at transplantation was 42 years(IQR 30-51) and 51 $(50 \%)$ were males.Conditioning regimen was myeloablative in $67(65,7 \%)$. Bone marrow was use as stem cell source in 84 (82\%). Donor was related in 65(63,7\%). HLA was matched in 88 (85\%). ATG was used in $15(14.7 \%)$. GvHD prophylaxis was with Csa+ MTX or MMF in $97(94 \%)$.

Results: CGvHD was developed in 67(65.7\%) patients. cGvHD was limited/extensive in $41(61,2 \%) / 26(38.1 \%)$, mild, moderate and severe in 29(43,3\%), 27(40.3\%) and $11(16.4 \%)$ patients. cGvHD was quiescent, progressive and "de novo" in 31(46.3\%), 13(19.4\%) and 23(34.3\%) patients. Median follow up was 103 months(IQR 80-140). At the interview, mucous symptoms were the most frequent 57(55.9\%). Table 1 describes CGvHD symptoms and others that are important for patients life. cGvHD was related with significant lower score in QoL scales (EQ-5D-5 L) versus non-cGvHD (72vs83\%) and in physical well being (FACT-BMT) (21.8 vs 25.1). Progressive CGvHD was statistically significative in the same questionnaires $60 \%$ in QoL scale (EQ-5D-5 L) and 23 in PWB(FACT-BMT) comparing with quiescent, "de novo" and non-cGvHD.

Considering late effects, $44(43 \%)$ patients developed hormonal dysfunction ( $16.7 \%$ thyroid, $24.5 \%$ gonadal, $2 \%$ both), 52 $(51 \%)$ osteoarticular damage $(13.7 \%$ osteoporosis, $31,3 \%$ articular pain, $4.9 \%$ avascular necrosis, $0.9 \%$ osteomalacia), 6 (5.9\%) had hypoacusia and $34(33.3 \%)$ cataracts. Relevant infections were observed in $24(23.5 \%)$ patients $(12.7 \%$ pneumoniae, 2\%pielonefritis, 8.8\%herpes-zoster).

Cardiovascular risk factors (CVRF) were detected in $48(47 \%)$ patients(1 factor:27, >1 factor:18) and 3(2.9\%) developed severe cardiovascular events.

Secondary tumors were observed in 6 (5.9\%) (colon:3, lung:1, skin:1, lymphoma:1).

Nowadays, 15 (14.7\%) patients are receiving inmunosupression therapy (prednisone:14, azathioprine:1). Most frequent drug compsuntions are eyewash (33.3\%), hormonal substitutive therapy $(25.4 \%)$, hypolipidemic agents $(24.5 \%)$, bronchodilators $(20.6 \%)$, analgesics (19.6\%), antihypertensives (16.7\%) and antidepressant (13.7\%).

Conclusion: In our series of long-term survivors $37 \%$ of patients develop moderate-severe cGvHD and $14.7 \%$ need long-term inmunosupressive therapy. cGvHD decreases QoL

Table 1

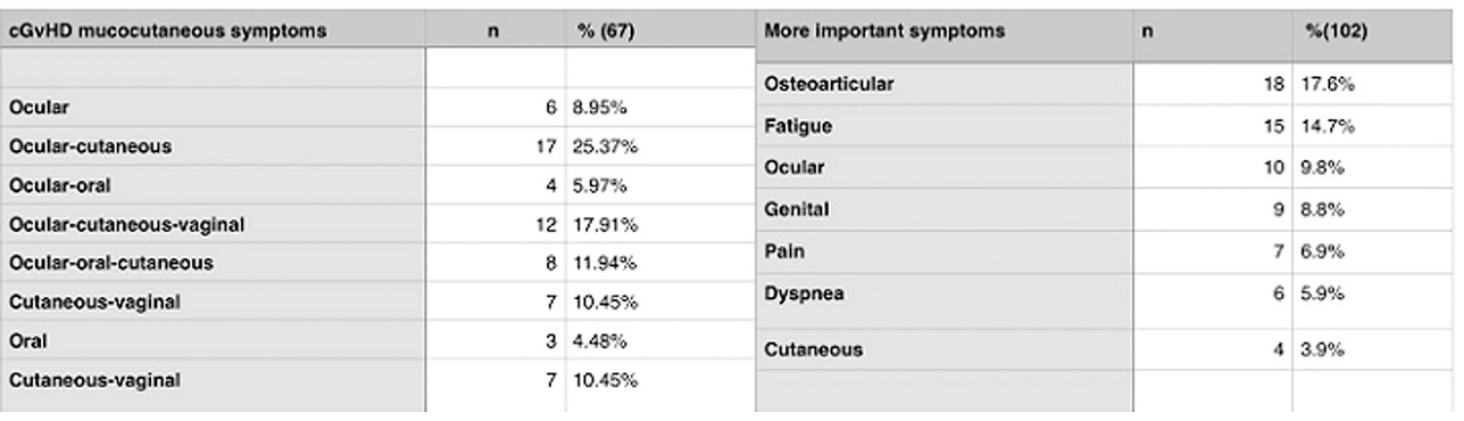


and raises drug compsuntion of eyewash and bronchodilators. Half of patients have some osteoarticular symptoms and $47 \%$ present any cardiovascular risk factors (CVRF). Most used drugs are related with CVRF control.

Disclosure of Interest: None declared.

P572

Risk factors for second solid cancers in allogeneic haematopoietic cell recipients: a Brazilian nested casecontrol study

R. Tavares $^{1}$, L. J. Arcuri ${ }^{1}$, M. Silva ${ }^{1, *}$, V. Kussumi ${ }^{1}$, S. Dechery ${ }^{1}$, M. Moreira', D. Lerner ${ }^{1}$, S. Maradei ${ }^{\prime}$, S. Lermontov', R. Castro ${ }^{\prime}$, L. Bouzas

${ }^{1}$ Centro de Transplante de Medula Óssea, Instituto Nacional de Cancer, Rio de Janeiro, Brazil

Introduction: Advances in allogeneic hematopoietic cell transplantation (HCT) techniques led to better long-term survival. Thus, late complications are being seen more often. Risk factors for secondary malignancies include high doses of total body irradiation (TBI), younger age at HCT, HLAmismatched donor and chronic graft versus host disease (cGVHD). The objective of this study is to determine risk factors for secondary neoplasm.

Material (or patients) and methods: This is a single center nested case-control study conducted at Brazil. Patients who received HCT between 2000 and 2009 and had secondary neoplasms were included. All living patients had at least 5-years follow-up. Controls were paired in a 2:1 ratio. Patients who underwent HCT just before or after a case, and had equal or greater follow up time than the case, were selected as controls, eliminating survival bias. The study was approved by local ethics committee. Risk factor for secondary cancer were estimated by conditional logistic regression.

Results: During the study period, 520 patients were transplanted and 19 developed post-HCT secondary neoplasm. The histological types were: melanoma (3), non-melanoma skin cancer (3), esophagus carcinoma (3), prostate carcinoma (2), cervical in situ neoplasia (2), uterine carcinoma (1), breast cancer (1), non-Hodgkin lymphoma (1), choroidal carcinoma (1), tongue carcinoma (1) and rectosigmoid adenocarcinoma (1). Median age at transplant was 39 years for cases and 26 years for controls $(P<0,01)$. All cases were transplanted due to hematologic malignancy, most with advanced disease at HCT, while 4 controls were transplanted for benign diseases. Distribution of prior radiotherapy, conditioning intensity and TBI-conditioning were not different between cases and controls. Bone marrow we the stem cell source of $63 \%$ of cases and $71 \%$ of controls $(P=0,10)$. Moderate to severe CGVHD was diagnosed in $68 \%$ of cases and in $63 \%$ of controls. In univariate analysis, severe cGVHD (HR=5,6, $P=0,03)$ and age at transplantation $(\mathrm{HR}=2.1$ for each 10-years increase, $P=0,01)$ were risk factors for secondary neoplasm. In multivariate analysis, only age remained as risk factor $(H R=3,2$ for each 10-years increase, $P=0,03)$. Severe cGVHD $(H R=3,7$, $P=0,18)$ and unrelated donor $(H R=7,5, P=0,16)$ did not reach statistical significance in multivariate analysis despite high hazard ratios.

Conclusion: Increasing age at HCT was significantly associated to higher risk for secondary neoplasm. However, earlier reports suggested that younger patients would be at higher risk. This may be related to survival bias, which is almost eliminated by our methodology, or to higher doses of TBI done in the past. Our results also suggest that secondary neplasm might be increased in unrelated transplantation and in patients who develop severe cGVHD.

References: Curtis RE, Rowlings PA, Deeg HJ et al (1997) Solid cancers after bone marrow transplantation. $N$ Engl J Med 336:897-904.

Socie $\mathrm{G}$ et al (2000) New malignant diseases after allogeneic marrow transplantation for childhood acute leukemia. J Clin Oncol 18:348-357.
Omori et al. Eleven secondary cancers after hematopoietic stem cell transplantation using a total body irradiation-based regimen in 370 consecutive pediatric and adult patients; SpringerPlus 2013, 2:424.

Disclosure of Interest: None declared.

\section{P573}

Secondary solid tumors after allogeneic stem cell transplantation: active surveillance in long term

\section{follow-up}

M. T. Lupo-Stanghellini ${ }^{1, *}$, A. Assanelli ${ }^{1}$, R. Greco ${ }^{1}$, F. Giglio ${ }^{1}$ S. Mastaglio ${ }^{7}$, M. Morelli ${ }^{1}$, F. Pavesi ${ }^{1}$, E. Sala ${ }^{7}$, M. Brambilla ${ }^{\prime}$, S. Piemontese', L. Vago ${ }^{1}$, C. Messina ${ }^{7}$, S. C. Dalto ${ }^{1}$, L. Francesca ${ }^{\prime}$, T. Perini ${ }^{1}$, S. Girlanda ${ }^{1}$, A. Malerba ${ }^{1}$, M. Marcatti ${ }^{1}$, E. Guggiari ${ }^{\prime}$, S. Marktel ${ }^{1}$, M. Carrabba ${ }^{1}$, F. Lunghi ${ }^{1}$, M. Bernardi ${ }^{1}$, C. Bonini ${ }^{\prime}$, C. Corti ${ }^{7}$, J. Peccatori ${ }^{1}$, F. Ciceri ${ }^{1}$

${ }^{1}$ Unit of Hematology and Bone Marrow Transplantation, ${ }^{2}$ Experimental Hematology Unit, Division of Immunology, Transplantation and Infectious Diseases, IRCCS San Raffaele Scientific Institute, Milano, Italy

Introduction: Allogeneic hematopoietic cell transplantation (HCT) is an effective therapeutic option for high-risk hematological malignancies; $80 \%$ of those who survive the first 2-years ( $y$ ) are expected to become long-term survivors.

The prevalence of chronic health conditions approaches $75 \%$ among HCT-survivors and that for severe or life-threatening conditions exceeds 20\%. Secondary tumors (ST) are among the most significant late effects.

Material (or patients) and methods: A standardized followup of HCT survivors is applied at our center, according to Jacie Standards. We analyze data collected between 2011 and 2015 in 292 adult patients (pts) who underwent an HCT at our Institution. Data were prospectively collected in Institutional database. A written consent was given by pts allowing the use of medical records for research in accordance with the Declaration of Helsinki.

Eligible pts were $>/=18 \mathrm{y}$, with post HCT follow-up $>/=2 \mathrm{y}$. Each patient was candidate to annual screening for ST according to recommended guideline for HCT survival or where otherwise not specified - general population.

Results: Median age was 48-y (r10-76) at transplant and 54-y (r20-82) at follow-up. Pts features are reported in table. A treosulfan-based conditioning regimen was administered in 242 pts and GvHD prophylaxis mostly relies upon the combination of rapamycin/mycophenolate (180pts).

The median time of follow-up was 4.7-y ( $\mathrm{r} 2-22$; cumulative follow-up 1660 y).

The incidence of new ST diagnosis during the time of observation was $16 \%$ - 47 cases.

The characteristics of the 47 ST are shown in Table. There were 10 invasive solid cancers and 37 carcinomas in situ. Most (96\%) ST were diagnosed 1 to $4(62 \%)$ and 5 to $9(34 \%)$-y post HCT. The median duration of follow-up of the $47 \mathrm{pts}$, from the time of development of ST, was 2-y (r0.5-11 years); $64 \%$ of pts were followed for more than $5-y$, whereas $17 \%$ were followed for more than $10-y$ post $\mathrm{HCT}$.

Of the 47 PTS 6 have died. The overall survival probabilities at 1 and 5-y following development of an invasive or in situ ST was $92 \%$ and $72 \%$ respectively.

The 5-y cumulative incidences of death because of ST was $24 \%$. ST was the most commonly reported cause of death for pts who died subsequent to the development of a ST. Among the 6 deaths in the study population, 3 were because of ST, 1 death from GVHD and 2 deaths from recurrent primary disease.

Conclusion: HCT survivors are at a defined relevant risk of developing ST. Active surveillance, adequate counseling, primary/secondary prevention are crucial within routine HCT long-term follow-up to enhance early diagnosis/treatment and overall outcome.

Disclosure of Interest: None declared. 


\begin{tabular}{|c|c|c|c|c|c|c|}
\hline & All pts & ST pts & Cancer subgroup & Site & Behavior & $N$ \\
\hline \multirow[t]{2}{*}{$N$} & 292 & 47 & Lung & & & \\
\hline & & & Adenocarcinoma & Lung & $\begin{array}{l}\text { Invasive } \\
\text { In situ }\end{array}$ & $\begin{array}{l}2 \\
1\end{array}$ \\
\hline \multirow[t]{3}{*}{$\operatorname{Sex}(M / F)$} & $184 / 10$ & $32 / 15$ & Female reproductive & & & \\
\hline & 8 & & Adenocarcinoma & Uterus & Invasive & 1 \\
\hline & & & Intraepithelial neoplasia & Cervix & In situ & 6 \\
\hline \multirow[t]{2}{*}{ Age (at TX / at ST) } & 48 / 61 & $52 / 61$ & Breast & & & \\
\hline & & & Adenocarcinoma & _ & In situ & 1 \\
\hline Primary disease & & & Male reproductive & & & \\
\hline Acute Leukemia & 157 & 23 & Adenocarcinoma & Prostate & Invasive & 3 \\
\hline Hodgkin Disease & 27 & 1 & Gastrointestinal tract & & & \\
\hline $\mathrm{NHL}$ & 29 & 6 & Adenocarcinoma & Colon & Invasive & 1 \\
\hline Myeloma & 16 & 1 & & Stomach & In situ & 1 \\
\hline Myelodisplasia & 32 & 6 & & Pancreas & Invasive & 1 \\
\hline Others & 31 & 10 & & & & \\
\hline Donor type & & & Genitourinary tract & & & \\
\hline MRD & 91 & 14 & Adenocarcinoma & Kidney & In situ & 1 \\
\hline $\mathrm{MUD}+\mathrm{CB}$ & $85+3$ & $16+1$ & Transitional cell carcinoma & Bladder & In situ & 2 \\
\hline \multirow[t]{2}{*}{ HAPLO } & 113 & 16 & Thyroid & & & \\
\hline & & & Papillary carcinoma & - & In situ & 1 \\
\hline \multirow[t]{2}{*}{ GvHD history } & 164 & 20 & Neck & & & \\
\hline & & & Squamous cell carcinoma & Larynx & In situ & 1 \\
\hline \multirow[t]{3}{*}{$T B I$} & 73 & 13 & Non melanoma skin cancer & & & \\
\hline & & & & - & In situ & 19 \\
\hline & & & & & Invasive & 6 \\
\hline
\end{tabular}

P574

Secondary hemosiderosis management is required in a high proportion of patients after HCT: a prospective evaluation of 252 consecutive cases

M. T. Lupo-Stanghellini ${ }^{1, *}$, L. Malabarba ${ }^{2}$, E. Guggiari ${ }^{1}$ A. Assanelli ${ }^{1}$, R. Greco ${ }^{1}$, L. Barzizza ${ }^{2}$, S. Malato ${ }^{1,2}$, F. Giglio ${ }^{\prime}$, M. Morelli ${ }^{1}$, A. Forcina ', F. Pavesi', M. Carrabba', 'S. Marktel',' M. Brambilla ${ }^{1}$, F. Lunghi ${ }^{1}$, M. Bernardi ${ }^{1}$, B. Laura $^{2}$, C. Corti ${ }^{1}$, J. Peccatori ${ }^{1}$, F. Ciceri ${ }^{1,2}$

${ }^{1}$ Unit of Hematology and Bone Marrow Transplantation, ${ }^{2}$ Immunohematology and transfusional medicine, IRCCS San Raffaele Scientific Institute, Milano, Italy

Introduction: The negative impact of hyperferritinemia on survival after allogeneic stem cell transplantation is well recognized. Even if ferritin is an imperfect surrogate marker of iron stores, pre transplant iron chelation may be associated with improved survival and reduced non-relapse mortality. ${ }^{1-2}$ Similarly, lowering ferritin after transplantation through phlebotomy and/or iron chelation seems rational, although the impact on survival is not yet known and needs to be studied in controlled trials.

Material (or patients) and methods: Here we are reporting our experience in the past 5 years. A standardized follow-up of HCT survivors is prospectively applied at our center, according to Jacie Standards. We analyze data collected between January 2011 and Nov 2015 in 252 adult patients (pts) who underwent an HCT at our Institution. Data were prospectively collected in Institutional database. A written consent was given by pts allowing the use of medical records for research in accordance with the Declaration of Helsinki.

Eligible pts were $>/=18$ years, with transfusional hyperferritinemia, $>6$ months after transplantation with adequate engraftment and in complete stable remission. Iron overload was documented with biochemistry and magnetic resonance imaging $[\mathrm{MRI}]$ was used to confirm hepatic hemosiderosis.
Results: Fifty pts (14 female, median age 58y, range 25-74) were candidate to treatment for secondary hemosiderosis. Median time from transplant to treatment was 559 days (14 pts during the $1^{\text {st }}$ year after transplantation). Nineteen pts were presenting GvHD at time of iron overload treatment and systemic immunosuppression was ongoing in 14/19 pts. Pre-transplant, baseline and posttreatment parameters (serum ferritin, transferrin saturation, liver iron concentration by MRI) are reported in table 1 .

The majority of pts were treated with phlebotomy (39/50) according to standard guideline. No severe adverse events occurred in this cohort of pts.

Eleven pts received deferasirox at a starting dose of $10 \mathrm{mg} / \mathrm{Kg} /$ day (because of poor efficacy after adequate treatment $2 / 11$ - or contraindication - 9/11 - to phlebotomy). No severe adverse events occurred also in this cohort of pts, but one pt developed skin rash that completely resolved at discontinuation of drug and 2 pts experienced acute renal injury that was controlled with drug reduction in one case but that required drug discontinuation in the other.

Eighteen pts obtain the normalization of ferritin value and stopped the treatment.

\begin{tabular}{|c|c|}
\hline $\begin{array}{c}\text { Pre- } \\
\text { transplant }\end{array}$ & Baseline \\
\hline
\end{tabular}

\begin{tabular}{|c|c|c|c|}
\hline Evaluable pts & 50 & 50 & 18 \\
\hline $\begin{array}{l}\text { Median serum ferri- } \\
\text { tin }(\mathrm{ng} / \mathrm{ml})\end{array}$ & 1781 & 1778 & 380 \\
\hline $\begin{array}{l}\text { Median transferrin } \\
\text { saturation }(\%)\end{array}$ & 55 & 46 & 26 \\
\hline LIC by MRI (mmol/gr) & - & 170 & 100 \\
\hline $\begin{array}{l}\text { Median packed red } \\
\text { blood cell (units) }\end{array}$ & - & 40 & - \\
\hline
\end{tabular}


Conclusion: Overall both pts under phlebotomy and pts under treatment with deferasirox are presenting significant reduction in ferritin and transferrin saturation. Practicability and safety of both iron chelation and phlebotomy will provide effective arms in control iron overload, also in the early phase after transplantation.

Being ferritin an imperfect iron surrogate marker next step will be to guide iron overload treatment with the support of liveriron-concentration provided by MRI to better customize the therapy.

References: 1. Jaekel N et al, Bone Marrow Transplant. 2015 Sep 14. doi: 10.1038/bmt.2015.204.

2. Armand $P$ et al, Biol Blood Marrow Transplant. 2014 Aug; 20(8):1248-51.

Disclosure of Interest: None declared.

P575

Sinusoidal Obstruction Syndrome after High-dose Intravenous Busulfan/Melphalan with Autologous Peripheral Blood Stem Cell Transplantation in High Risk Ewing Sarcoma

M. E. Abate ${ }^{1, *}$, A. Paioli ${ }^{1}$, M. Cesari ${ }^{1}$, E. Palmerini ${ }^{1}$, A. Longhi ${ }^{1}$, S. Ferrari ${ }^{1}$

${ }^{1}$ Istituto Ortopedico Rizzoli, Bologna, Italy

Introduction: The consolidation therapy combining busulfan and melphalan followed by autologous peripheral blood stem cell (aPBSC) transplantation is frequently used for high risk Ewing sarcoma (ES) patients, even though its role remains under investigation. Sinusoidal obstruction syndrome (SOS), previously called veno-occlusive disease, is a potential fatal complication that can occur early after hematopoietic stem cell transplantation (HSCT).

Material (or patients) and methods: Medical records of 69 consecutive ES patients entered at our Institution in the last eight years and undergoing aPBSC transplantation after intravenous busulfan ('BU) and melphalan (MEL) were retrospectively analyzed with regard to incidence, risk factors and outcome of SOS.

Patients were defined high risk for localized poor responder (histologically or radiologically) or metastatic disease.

We analyzed if any correlation existed between incidence of SOS and clinical features as gender, age (18 years or more versus $<18$ years), body mass index and prior radiation therapy.

All patients underwent baseline liver ultrasonography and blood tests. Doppler examinations were performed at the time of the clinical diagnosis of SOS and subsequently to confirm complete remission (CR).

Diagnosis of SOS was based on the presence of two of the following clinical findings: 1) bilirubin $>2 \mathrm{mg} / \mathrm{dl}$; 2) ascite and/ or unexplained weight gain $>5 \%$ above baseline weight; 3 ) hepatomegaly. Defibrotide therapy was started as soon as the medication was available after diagnosis of SOS at the dose of $6.25 \mathrm{mg} / \mathrm{kg}$ body weight every 6 hours for a minimum duration of 21 days.

Results: The median age at diagnosis of patients receiving BU/MEL was 18 years (range 3-39 years). 4 (5.8\%) patients developed SOS. Among these, 3 patients had prior radiation therapy (on right pelvis in two patients, on T12 vertebra in one patient). The median time within SOS occurred after HSCT was 24 days (range 8-40 days). There was one death due to severe SOS and multiorgan failure. This was the only case in which a delay in starting defibrotide therapy occurred. 3 patients with mild to moderate SOS started defibrotide treatment within 3 days from clinical diagnosis of SOS and achieved CR at day of treatment 3,5 and 13 respectively. No statistically significant difference was observed regarding the risk factors analyzed. However, the 4 patients who developed SOS were all in the group of 41 adult patients, thus representing $9.8 \%$.

Conclusion: The incidence of SOS is clinically relevant in the adult group of our series treated with intravenous busulfan/ melphalan and autologous HSCT. Prior radiation therapy should be taken into account. Defibrotide is an effective therapy especially if started soon after diagnosis of SOS. However, severe SOS still represents a challenge due to high mortality rate.

Disclosure of Interest: None declared.

\section{P576}

Late Toxicities in Long-Term Survivors of Adult Allogeneic Hematopoietic Cell Transplantation (Allo-HCT) Using ExVivo CD34+ Selection-Based Graft-Versus-Host Disease (GVHD) Prophylaxis

M. Scordo ${ }^{1, *}$, G. L. Shah ${ }^{1}$, S. Devlin ${ }^{2}$, M. Maloy ${ }^{7}$, S. Kosuri ${ }^{1}$ D. Adrianzen Herrera ${ }^{3}$, J. Nieves' ${ }^{1}$, S. T. Avecilla ${ }^{4}$, R. C. Meagher B. Gyurkocza ${ }^{1}$, H. Castro-Malaspina ${ }^{1}$, R. Tamari ${ }^{1}$, M.-A. Perales ${ }^{\prime}$, S. A. Giralt ${ }^{1}$, B. Shaffer ${ }^{1}$

${ }^{1}$ Dept. of Medicine, Adult BMT Service, ${ }^{2}$ Dept. of Biostatistics and Epidemiology, Memorial Sloan Kettering Cancer Center, ${ }^{3}$ Dept. of Medicine, Mount Sinai St. Luke's and Mount Sinai West Hospitals, ${ }^{4}$ Dept. of Laboratory Medicine, Memorial Sloan Kettering Cancer Center, New York, United States

Introduction: Ex-vivo $\mathrm{CD} 34+$ selection results in favorable disease control and lower incidence of GVHD after allo-HCT without the need for pharmacologic immunosuppression, though the late adverse events of this approach in long-term survivors are not well characterized.

Material (or patients) and methods: Toxicities ( $\geq$ grade 3 by CTCAE 4.0) were retrospectively collected on 131 adults who underwent allo-HCT with ex-vivo CD34+ selection
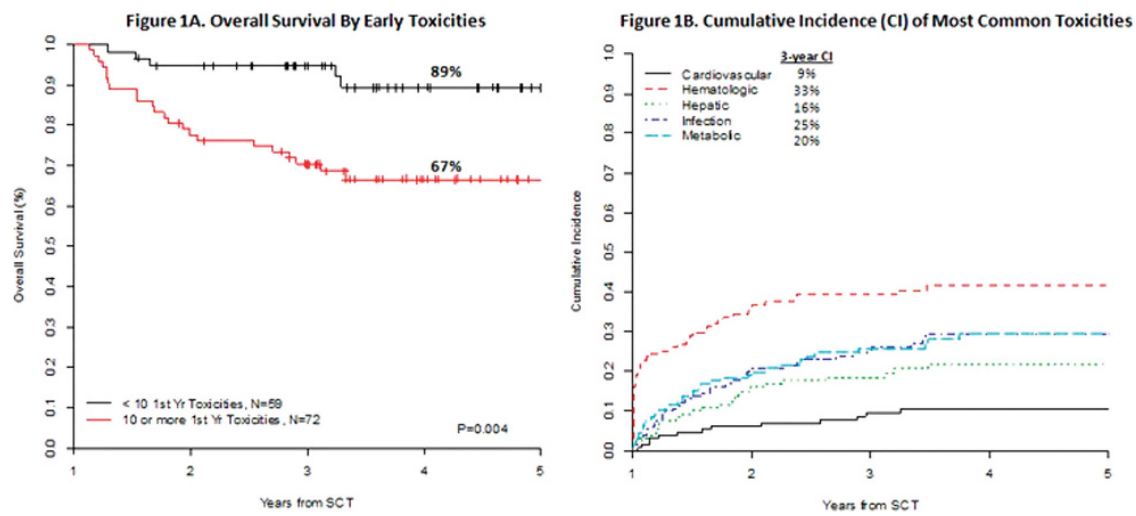
(ClinicMACS ${ }^{\circledR}$ system) between 2006-2012 and who were alive without relapse/progression at 1 year. Cox regression was used to compare the risk of toxicities across patient and treatment characteristics.

Results: Patients (pts) with acute leukemia/myelodysplastic syndrome $(74 \%)$, multiple myeloma $(15 \%)$, and other histologies (11\%) were included. Median age was 54 (19-72), and 46 pts were $\geq 60$ years old. Thirteen $(10 \%)$ pts in our cohort had grade 2-4 acute GVHD. Myeloablative chemotherapy- and total body irradiation-based conditioning was used in $71 \%$ and $29 \%$, respectively. Median HCT-Comorbidity Index (HCT-Cl) was 2 (0-10). Donor allografts were HLA matched or mismatched sibling in 52 pts $(40 \%)$ and matched unrelated in $53(40 \%)$ or mismatched-unrelated in $26(20 \%)$. During the study period, 8 pts (6\%) died of relapsed disease, 20 pts (15\%) died of non-relapse mortality (NRM), and 9 pts (7\%) had relapse without death. At 4 years post-HCT, overall survival (OS) for the cohort was $77 \%$ (median not reached). A HCT$\mathrm{Cl} \geq 3$ was associated with poorer OS, with HR 2.85 (95\% confidence interval $[\mathrm{Cl}]$ : $1.33-6.10), P=0.007$. Toxicities by major group and time period after allo-HCT are shown in Table 1. Pts who experienced greater than the median number of early toxicities had a 4-year OS of $67 \%$ versus $89 \%$ for pts with $<10$ early toxicities, hazard-ratio $(\mathrm{HR})$ of $4.16(95 \% \mathrm{Cl}$ 1.58-10.95), $P=0.004$ (Figure $1 \mathrm{~A}$ ). Having $\geq 10$ early toxicities correlated with late hematologic toxicities, $\mathrm{HR} 2.88(95 \% \mathrm{Cl}$ 1.58-5.24), $P=0.001$, and infections, HR 3.89 (95\% Cl 1.77-8.54), $P=0.001$. Of all late toxicities in $>1$-year survivors (554), $37 \%$ were hematologic ( $92 \%$ were cytopenias), $17 \%$ were infections, $18 \%$ were metabolic, $9 \%$ were hepatic, $4 \%$ were cardiovascular and $15 \%$ were other. There were 7 late viral reactivation events, 1 event of viral organ disease and 2 cases of Epstein-Barr virus post-transplant lymphoproliferative disorder. Cumulative incidences of the most common late toxicities after 1 year are shown in Figure 1B. Allograft type, disease, sex, and acute GVHD in the first year did not correlate with incidence of toxicities in late survivors.

Table 1. Common Toxicities by Group and Time Period

\begin{tabular}{lcccc}
\hline Group & $\begin{array}{c}\leq 1 \text { Year } \\
\text { (Early) } \\
\mathrm{N}=131\end{array}$ & $\begin{array}{c}1 \text { to } \leq 2 \\
\text { Years } \\
\mathrm{N}=131\end{array}$ & $\begin{array}{c}\geq 2+ \\
\text { Years } \\
\mathrm{N}=102\end{array}$ & Total \\
\hline Hematologic & 472 & 136 & 68 & 676 \\
Metabolic & 398 & 60 & 38 & 496 \\
Infection & 299 & 63 & 29 & 391 \\
Hepatic & 115 & 31 & 21 & 167 \\
Cardiovascular & 68 & 15 & 7 & 90 \\
Oral/Gl & 125 & 7 & 0 & 132 \\
Pulmonary & 34 & 11 & 6 & 51 \\
Neurologic & 28 & 6 & 6 & 40 \\
Total & 1549 & 329 & 175 & 2043 \\
\hline
\end{tabular}

Conclusion: One-year survivors of ex-vivo CD34+ selected alloHCT have excellent OS and low incidence of severe toxicities. Pre-transplant HCT-Cl and early toxicity predict for outcome and late adverse events. While late cytopenias occurred, late viral reactivation and viral organ disease are rare suggesting adequate immune reconstitution. These data may serve as a guide for pts in the context of the BMT-CTN 1301 PROGRESS II trial of calcineurin inhibitor-free interventions for GVHD prevention.

Disclosure of Interest: None declared.
P577

Coronary Calcium Scoring is More Accurate than Framingham Risk Scores in Detecting Early Coronary Artery Disease (CAD) in Allogeneic Stem Cell Transplant (Allo-SCT) Survivors

N. A. Jain ${ }^{1}$, M. Y. Chen ${ }^{2}$, A. Remaley ${ }^{2}$, S. Shanbhag ${ }^{2}$, P. Anandi ${ }^{1}$ K. Lu ${ }^{1}$, P. A. Pophali ${ }^{1}$, S. Ito ${ }^{1}$, E. Koklanaris ${ }^{1}$, C. S. Hourigan ${ }^{1}$ A. J. Barrett ${ }^{1}$, M. Battiwalla ${ }^{1, *}$

${ }^{1}$ Hematology Branch, ${ }^{2}$ Cardiovascular and Pulmonary Branch, $\mathrm{NIH}$, Bethesda, United States

Introduction: Premature coronary artery disease (CAD) is a potentially lethal complication in allogeneic stem cell transplantation (allo-SCT) survivors. Early intervention, such as lipid lowering agents, could reduce this risk but needs a validated CAD screening strategy. Clinical screening for CAD traditionally relies upon the Framingham risk scores (FRS) in the general population but the optimum screening strategy in the allo-SCT survivors is not known.

Material (or patients) and methods: This is an interim analysis of an ongoing prospective non-randomized single institution study using cardiac CT (Agatston coronary calcium scoring (CCS) with concomitant coronary $\mathrm{CT}$ angiography) versus FRS for screening asymptomatic allo-SCT survivors. 67 subjects (40 males; 27 females) were studied with a median age of 49 years (range, 24-76) at transplant and a median follow up interval of 10.5 years (range, 3-22). CAD was defined as presence of obstructive or non-obstructive lesions by CT angiography.

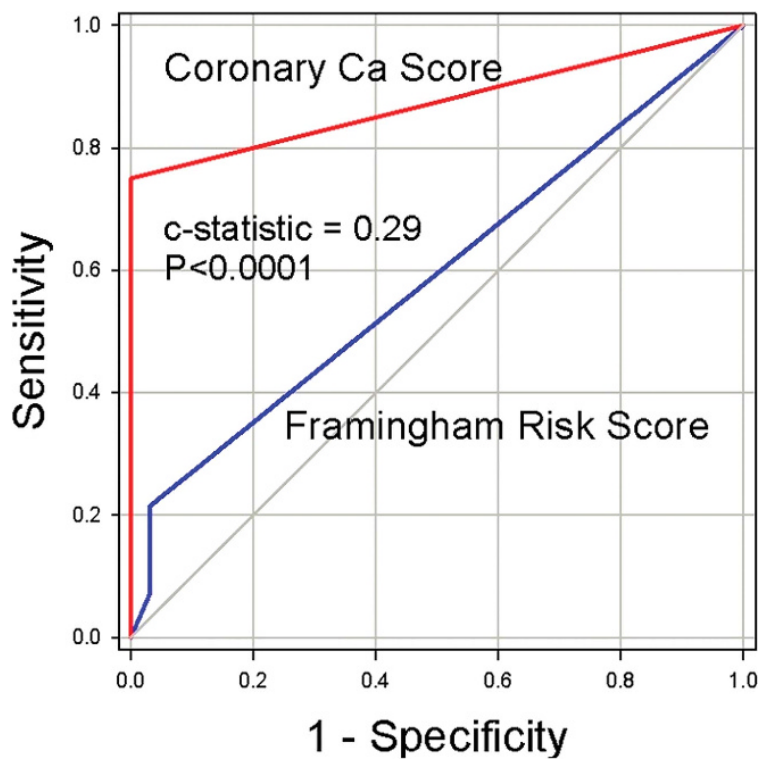

Results: CAD was detected in 30 of $67(44.7 \%)$ allo-SCT survivors. Characteristics of coronary plaques were: $29.3 \%$ calcified, $42.7 \%$ mixed, and $28 \%$ non-calcified. Coronary lesions were mostly non-obstructive (89.3\%), but obstructive lesions were seen in $10.7 \%$ of subjects. Lesion distributions by arterial territory were: left main $11.4 \%$, left anterior descending $35.8 \%$, left circumflex $21.4 \%$, and right coronary artery $31.4 \%$. Additionally, 15 (50\%) patients with CAD had either aortic root or mitral valvular calcification. 10-year FRS classified 3 as high risk, 5 as intermediate risk, and 59 as low risk. Radiation exposure was negligible, at a median of $0.8 \mathrm{mSv}$ for CCS and $1.1 \mathrm{mSv}$ for the coronary CT angiogram. There were no adverse events. CCS had a sensitivity of $75 \%(95 \% \mathrm{Cl}, 55 \%$ $>89 \%)$ with a specificity of $100 \%(95 \% \mathrm{Cl}, 89 \%>100 \%)$ for CAD detection whereas FRS had a sensitivity of only $21 \%(95 \%$ $\mathrm{Cl}, 8 \%>41 \%)$ with a specificity of $97 \%(95 \% \mathrm{Cl}, 84 \%>100 \%)$. 
The overall accuracy of CCS was superior to FRS (c-statistic $0.29, P<0.0001)$. In addition, CCS was able to detect CAD in 2 of 7 subjects who could not have angiograms due to renal dysfunction. CCS alone may be adequate for screening and avoids the use of IV contrast. Current 2010 AHA/ACC guidelines suggest a role for CCS for screening asymptomatic nontransplant individuals with intermediate FRS. However, cardiac CT detected early CAD even in 24 of $59(40.7 \%)$ of low FRS survivors.

Conclusion: Early CAD was detected in $45 \%$ of allo-SCT survivors by cardiac CT screening. CCS with or without CT angiogram is a safe, feasible and sensitive screening technique for CAD. It is informative even in asymptomatic, low-risk survivors and is far more sensitive than the FRS.

Disclosure of Interest: None declared.

P578

A Phase 3 Multicenter Randomized Double-Blinded Trial of Topical Caphosol to Prevent Oral Mucositis in Children Undergoing Hematopoietic Cell Transplantation: a Report of the Children's Oncology Group Study ACCL1031

N. Treister 1,*, M. Nieder ${ }^{2}$, C. Baggott ${ }^{3}$, E. Olson ${ }^{4}$, L. Chen ${ }^{5}$, A. August ${ }^{6}$, L. Sung ${ }^{7}$

${ }^{1}$ Brigham and Women's Hospital, Boston, ${ }^{2}$ Moffitt Cancer Center, Tampa, ${ }^{3}$ Stanford Medical Center, Stanford, ${ }^{4}$ Children's Healthcare of Atlanta, Atlanta, ${ }^{5}$ Children's Oncology Group, Monrovia, ${ }^{6}$ Children's Mercy, Kansas City, United States, ${ }^{7}$ The Hospital for Sick Children, Toronto, Canada

Introduction: The primary objective was to determine if topically administered Caphosol, rinsed orally four times daily at the initiation of conditioning, reduces the duration of severe oral mucositis (OM) compared to placebo among children and adolescents undergoing hematopoietic cell transplantation (HCT). Material (or patients) and methods: This was a Children's Oncology Group multicenter randomized double blinded placebo-controlled clinical trial. Patients between the ages of 4 and 21 who were scheduled to undergo myeloablative HCT for any indication were randomized to Caphosol or placebo saline rinses four times daily from initiation of conditioning through day +20 . Subjects were assessed daily for OM using the World Health Organization (WHO) Oral Toxicity Scale, mouth pain categorical scale (0-10) and the Oral Mucositis Daily Questionnaire (OMDQ). The primary endpoint was duration of severe $\mathrm{OM}(\mathrm{WHO} \geq 3)$. Secondary endpoints included incidence of severe OM, incidence and total dose of opioid analgesic use (morphine equivalents); incidence and duration of total parenteral nutrition administration; and the incidence of fever and neutropenia and invasive bacterial infections.

Results: The study enrolled 220 participants with a median age of 13.7 years (range 4-22); 163 (74\%) received allogeneic $\mathrm{HCT}$. The majority were being treated for malignant conditions and received primarily bone marrow or peripheral blood stem cell grafts. There were 38 subjects (19 in each arm) that were inevaluable for the primary endpoint due to an insufficient number of completed WHO assessments ( $<11$ days). The

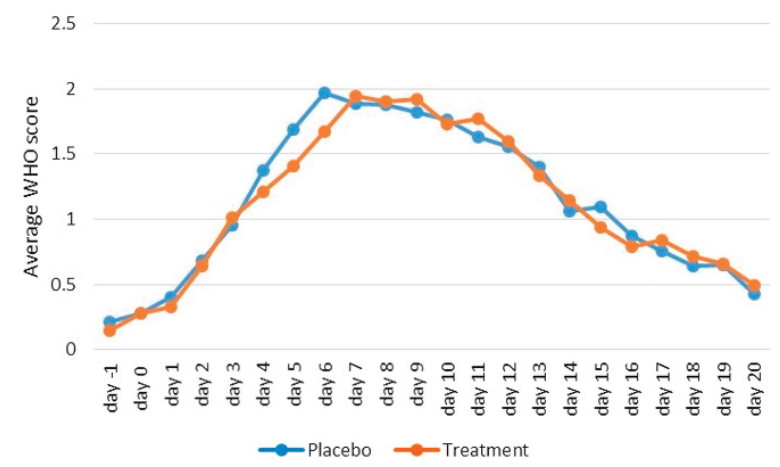

mean $( \pm$ SD) duration of severe OM was not reduced among Caphosol ( $4.5 \pm 4.8$ days) versus placebo $(4.5 \pm 5.0$; $P=0.99)$ recipients (Figure 1). The incidence of severe $\mathrm{OM}$ in the Caphosol and placebo arms was 63\% (57/91) and 68\% (62/91) respectively $(P=0.44)$. There were no significant differences in any of the other secondary endpoints between the groups. There were no toxicities attributed to study rinses.

FIGURE 1. Average daily mucoistis scores among those randomized to Caphasol versus placebo.

Conclusion: Caphosol did not reduce severe OM when compared to placebo among children and adolescents undergoing myeloablative HCT. Studies to identify effective interventions for $\mathrm{OM}$ are needed in this population.

Disclosure of Interest: None declared.

\section{P579}

Five years follow up of Quality of life in pediatric patients after allogeneic SCT:patient self-reports compared to healthy peers, to parent proxy-reports and changes in QOL P. De Vos ${ }^{1, *}$, S. Van Lancker ${ }^{1}$, V. Bordon ${ }^{1}$, K. De Waele ${ }^{2}$, C. Dhooge

${ }^{1}$ Pediatric Hematology, Oncology and Stem Cell Transplantation, ${ }^{2}$ Pediatric endocrinology, Ghent University Hospital, Ghent, Belgium

Introduction: Allogeneic stem cell transplantation proved to increase survival rate in life-threatening diseases as cancer in pediatric patients. In consequence patients and parents face new and major challenges on return to ordinary life. To address the physical and psychosocial rehabilitation a multidisciplinary long term follow up outpatient clinic was founded in 2010. In this Quality of Life study we wanted to find out if there is a difference in QOL in patients at one time point compared to healthy children and if there a difference in QOL self patient -report versus parent proxy-reports at the same time point. And moreover: does the QOL changes over repeated measurements, in patients and parents and is there an evolution?

Material (or patients) and methods: We used the PedsQL to measure the impact of disease burden in patients and parents. At first we used the generic module and, in regard to the finding fatigue was a common complaint, we added the fatigue module. From the age 5-7 both children and parents filled in the questionnaires, for preschool children only parents. The PedsQL has values 0 to 100 , where high values mean high levels of QOL. The average time between repeated measurements was 1 year.

Results:

\begin{tabular}{lll}
\hline GeneralQOL & Mean patients & Mean healthy pears \\
\hline Physical & 79,12 & 87,53 \\
Emotional & 76,20 & 79,33 \\
Social & 82,07 & 85,15 \\
School & 75,78 & 81,12 \\
Total score & 78,47 & 83,84 \\
\hline
\end{tabular}

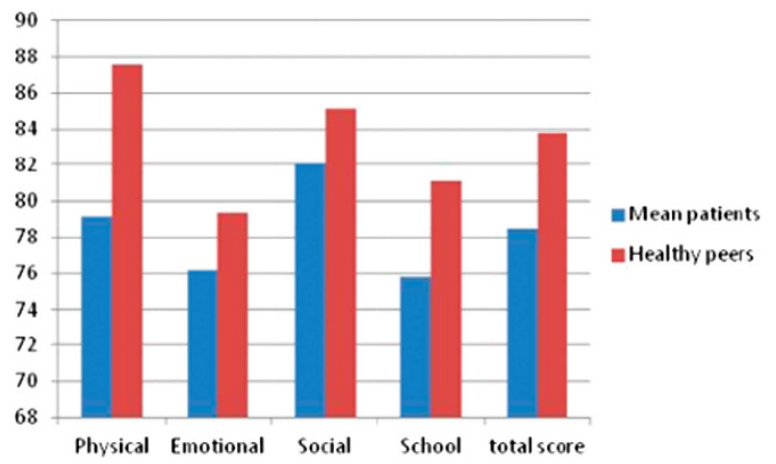


QOL seems to be lower in the group of patients compared to healthy pears (total score 78 versus 84 ) and mostly due to differences in physical wellbeing. Patients report on the emotional and social scale is similar to healthy pears. QOL reported by the parents shows a significant different perception of emotional and social wellbeing of their children. Further we noted that parents underestimate fatigue complaints in their children. Looking at the repeated $\mathrm{QOL}$ measurements, a small improvement on the general QOL scale is seen, in contrast fatigue appears to be increasing.

Conclusion: Special attention to QOL, involving both patients and parents, remains important. Changes over time and the clinical significance of measurements needs further exploration. Our first goal is the development of a physical rehabilitation program as part of the long term follow-up after SCT.

References: Health and Quality of Life Outcomes 2007, 5:43, James W. Varni et al.

Disclosure of Interest: None declared.

\section{P580}

Results of HRQOL-measures (EORTC-QLQ-C30 -3.0) of young adults after pediatric allogeneic SCT and before transition to adult outpatient care

V. Bordon ${ }^{1}$, P. De Vos ${ }^{1, *}$, S. Van Lancker ${ }^{1}$, K. De Waele ${ }^{2}$ C. Dhooge

${ }^{1}$ Pediatric Hematology, Oncology and Stem Cell Transplantation,

${ }^{2}$ Pediatric endocrinology, Ghent University Hospital, Ghent, Belgium

Introduction: As part of the awareness of the possible impact of allogeneic transplant on quality of life (QOL) we started in 2010 with a multidisciplinary outpatient clinic for long-term follow-up of patients(pts) from at least 1 year post SCT. Among them, there are a significant number of adolescents and young adults. Apart from a thorough medical check-up, pts were seen for neurocognitive assessment and their actual QOL, i.c. the psychological or social sequelae of their disease and treatment. For the assessment of HRQL the EORTC-QLQ-C30 was chosen, as this questionnaire has been used in a wide range of international clinical trials.

Material (or patients) and methods: 35 patients filled in the questionnaire at least once during their follow-up. Although this instrument is used mainly from 18 years on, we wanted to use it also in the group 16-17 year olds in addition to assessment with the PedsQL 13-18 years. Our aims were to explore the impact of allogeneic SCT during childhood on QOL in the young adults compared to the general population. The questionnaire consists of three parts: the 'Global Health Status'(GHS), five functional scales (physical, role, emotional, cognitive and social) and nine symptom scales. All have values ranging from 0 to 100 . For the GHS and the functional scales a

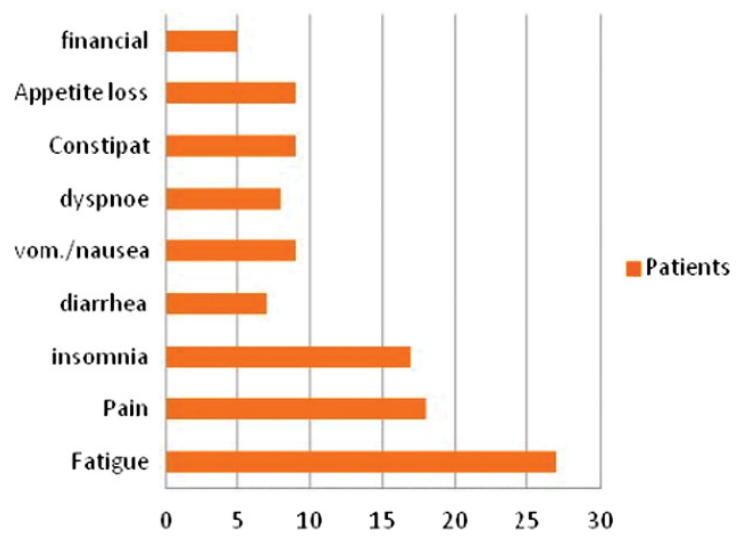

high score means a high QoL and high/healthy level of functioning, in contrast to the symptom scales where a high score means more symptom burden.

Results: The GHS and the level of functioning is comparable to the general population, with exception of physical functioning, which is lower and emotional functioning which is higher. However, within the assessed group a large majority has at least one symptom, about one in three has 2 symptoms and one in five 4 symptoms. The most important symptom burden seems to be caused by three main complaints: fatigue, pain and insomnia. In addition at least $20 \%$ of the patients report other symptoms than these stated as the most common. Compared to the general population- even if the same symptoms appear- the degree of burden and suffering is generally much higher.

Conclusion: Global health status and level of functioning in young adults post SCT is comparable to their healthy peers. However special attention is needed for prevention and treatment of the high symptom burden especially fatigue, pain and insomnia.

Disclosure of Interest: None declared.

\section{P581}

Regrafting For Primary and Secondary Graft Failure

P. Topcuoglu ${ }^{1, *}$, E. Atilla ${ }^{1}$, S. Civriz Bozdag ${ }^{1}$, M. Kurt Yuksel ${ }^{1}$, S. K. Toprak', O. Arslan ', M. Beksac', M. Ozcan 1', G. Gurman ${ }^{1}$ ANKARA UNIVERSITY SCHOOL OF MEDICINE DEPARTMENT OF HEMATOLOGY, Ankara, Turkey

Introduction: Primary Engraftment Failure is defined as no evidence of engraftment or hematological recovery of donor cells whereas secondary graft failure refers to the loss of a previously functioning graft. A second hematopoietic stem cell transplantation (HSCT) from the same donor or an alternative donor is largely employed treatment for graft failure.

Material (or patients) and methods: Between 1992 and 2015, 13 patients who had second HSCT for graft failure (11 primary/2 secondary) diagnosed at Ankara University Department of Hematology were included in our retrospective study. Results: The median age of the group was 29 (range, 16-64), 9 of them were male. 12 patients had received stem cells from the same donors (2 patients: full-matched unrelated, 2 patients: 1 mismatch unrelated, 8 patients:Full-matched related). Stem cell source were same in both transplants in 8 patients (6 peripheral blood and 2 bone marrow). 1 patient had received HLA full-matched graft from unrelated donor at first transplant then haploidentical graft from her daughter at second transplant. Five patients received bone marrow stem cells in first transplantation and switched to peripheral blood as a stem cell source in second transplantation. The median time to second transplant in primary graft failure was 52 days (range, 26-89 days) while in secondary graft failure two patients re-transplanted in 203 and 94 days after the first one. Four patients with primary graft failure survived after second transplant, median neutrophil engraftment was detected at day 14 and platelet engraftment at day 18. Eight of 9 patients died because of severe infections while 1 had massive alveolar hemorrhage following second transplant. Median overall survival in 11 primary graft failure patients was 53 months (range, 9-130) whereas 13 months (range,10-17 months) in secondary graft failure patients. The incidence of engraftment in second transplant has not been effected by time between two transplants, $\leq 30$ days vs $>30$ days or related vs unrelated donors $(P>0.05)$

Conclusion: Graft failure remains an important contributor to morbidity and mortality after allogeneic HSCT. Retransplantation from another donor or the same donor may be the treatment of choice but stil the major challenging problem.

Disclosure of Interest: None declared. 
Table. Patient Characteristics (Anti-thymocyte globulin: ATG, Busulphan:Bu, Cyclosporin: CSA, Cyclophosphamide:Cy, Fludarabine:Flu, GVHD:Graft versus host disease, Melphalan:Mel, Methotrexate:Mtx, Mycophenolate Mofetil:MMF, Metilprednisolon:MP, TBI:Total Body Irridation)

\begin{tabular}{|c|c|c|c|c|c|c|c|c|}
\hline $\begin{array}{l}\text { Patient } \\
\text { No }\end{array}$ & $\begin{array}{l}\text { Graft } \\
\text { Failure }\end{array}$ & Diagnosis & $\begin{array}{c}\text { 1st Transplant } \\
\text { Conditioning } \\
\text { Regimen }\end{array}$ & $\begin{array}{l}\text { GVHD pro- } \\
\text { phylaxis }\end{array}$ & $\begin{array}{c}\text { 2nd Transplant } \\
\text { Conditioning } \\
\text { Regimen }\end{array}$ & $\begin{array}{l}\text { GVHD pro- } \\
\text { phylaxis }\end{array}$ & $\begin{array}{c}\text { Time between } \\
\text { transplants } \\
\text { (days) }\end{array}$ & $\begin{array}{l}\text { Engraftment } \\
\text { after 2nd trans- } \\
\text { plant/Ex }\end{array}$ \\
\hline 1 & Primary & MDS & $C y+A T G$ & CSA+MTX & Flu+TBI & $\mathrm{CSA}+\mathrm{MT}$ & 60 & $\mathrm{No} /+$ \\
\hline 2 & $\begin{array}{c}\text { Secondar } \\
y\end{array}$ & CMML & Flu+Bu+ATG & $\mathrm{CSA}+\mathrm{MMF}$ & Flu+Mel & $\begin{array}{c}\mathrm{X} \\
\text { CSA+MMF }\end{array}$ & 203 & $\mathrm{No} /+$ \\
\hline 3 & Primary & MDS & Flu+Bu+ATG & $\mathrm{CSA}+\mathrm{MMF}$ & Flu+Bu+ATG & N/A & 49 & $\mathrm{No} /+$ \\
\hline 4 & Primary & AML & $\mathrm{TBI}+\mathrm{Cy}+\mathrm{ATG}$ & $\mathrm{CSA}+\mathrm{MMF}$ & Flu & $\mathrm{CSA}+\mathrm{MMF}$ & 65 & $\mathrm{No} /+$ \\
\hline 5 & Primary & $\mathrm{AA}$ & Cy+ATG & CSA & $C y+A T G$ & $\mathrm{CSA}+\mathrm{MT}$ & 51 & Yes/- \\
\hline 6 & Primary & AA & Flu+Cy+ATG & $\begin{array}{c}C y+C S A \\
+M \\
M F\end{array}$ & Flu+TBI & $\begin{array}{c}\mathrm{X} \\
\text { CSA+MTX }\end{array}$ & 70 & $\mathrm{No} /+$ \\
\hline 7 & Primary & CML & $\mathrm{Bu}+\mathrm{Cy}$ & CSA+MTX & $\mathrm{Bu}+\mathrm{Cy}$ & $\mathrm{MP}+\mathrm{MMF}$ & 42 & $\mathrm{No} /+$ \\
\hline $\begin{array}{l}8 \\
\text { CSA } \\
+ \text { MTX }\end{array}$ & $\begin{array}{c}\text { Secondary } \\
94\end{array}$ & $\begin{array}{c}\mathrm{AA} \\
\mathrm{No} /+\end{array}$ & $\mathrm{Cy}+\mathrm{ATG}$ & CSA+MTX & Cy+ATG & & & \\
\hline 9 & Primary & AML & $C y+T B I$ & CSA+MTX & $\mathrm{Bu}+\mathrm{Cy}$ & $\mathrm{CSA}+\mathrm{MT}$ & 89 & Yes/- \\
\hline 10 & Primary & MDS & $\mathrm{Cy}+\mathrm{ATG}$ & $\mathrm{CSA}+\mathrm{MTX}$ & Flu & $\begin{array}{c}\mathrm{X} \\
\text { CSA+MTX }\end{array}$ & 30 & Yes/- \\
\hline 11 & Primary & $A A$ & $C y+A T G$ & $\mathrm{CSA}+\mathrm{MTX}$ & Flu & $\mathrm{CSA}+\mathrm{MT}$ & 26 & Yes/- \\
\hline 12 & Primary & FAA & Cy & CSA & $\mathrm{Cy}+\mathrm{ATG}+\mathrm{Flu}$ & $x$ & 30 & $\mathrm{No} /+$ \\
\hline 13 & Primary & FAA & Flu+Cy+ATG & CSA+MTX & Flu $+C y+A T G$ & $\begin{array}{c}\text { CSA } \\
\text { CSA+MTX }\end{array}$ & 69 & $\mathrm{No} /+$ \\
\hline
\end{tabular}

P582

Secondary malignancies following allogeneic stem cell transplantation in childhood - a single centre experience P. Keslova ${ }^{1, *}$, P. Sedlacek ${ }^{1}$, R. Formankova ${ }^{1}$, P. Riha ${ }^{1}$, J. Stary ${ }^{1}$ ${ }^{1}$ Department of Pediatric Haematology and Oncology, University Hospital Motol, Prague, Czech Republic

Introduction: Allogeneic haematopoietic stem cell transplantation (HSCT) is a potentially curative therapy for a variety of malignant and non malignant disorders. With improved outcomes after HSCT, increasing attention has been drawn to late complications in long-term survivors. The development of secondary malignancies is recognized as one of the most serious complications.

Material (or patients) and methods: The increased incidence of secondary cancers may be result of the chemotherapy and radiotherapy used for conditioning or primary therapy before transplantation, immunosuppresion or immune dysregulation after transplantation. We analysed the data of occurrence of secondary malignancies in our centre, HSCT Unit, University Hospital Prague. 496 patients underwent allogeneic transplantation between $11 / 1989$ to $12 / 2014$.

Results: We documented secondary malignancy in 29 patients (5.8\%). 13 patients developed post - transplant lymphoproliferative disorder (PTLD) at median time 0.3 (0-1.8) years after HSCT, EBV - PTLD was diagnosed in 12 of them (92\%). Secondary solid tumours were diagnosed in 16 pts at median time 11.4 (5.4-17.8) years after HSCT (carcinomas of oral cavity $n=3$, melanoma $n=1$, peritoneal mesothelioma $n=1$, breast cancer $n=1$, malignant schwannomas $n=2$, thyroid carcinomas $n=8)$. 15 out of 16 patients $(93,8 \%)$ had total body irradiation (TBI) $12-14.4$ Gy as a part of conditioning regimen. 9 out of 13 patients in PTLD group died, 6 for PTLD. All patients in solid tumours group underwent surgery and/or chemoradiotherapy and are alive.

Conclusion: The incidence and number of complications following allogeneic HSCT in childhood are increasing in time. The early diagnosis of secondary malignancies is one of the key tasks of long - life multidisciplinary post-transplant care.

Disclosure of Interest: Supported by MHCZ for conceptual development 00064203 University Hospital Motol. None declared.

\section{P583}

Relevance of anti-HLA antibodies in unrelated donor allogeneic haematopoietic stem cell transplantation

R. Cekauskiene ${ }^{1,2, *}$, B. Valatkaite ${ }^{1,2}$, L. Griskevicius ${ }^{1}$, A. Jakubauskas ${ }^{1,2}$ ${ }^{1}$ Hematology, Oncology and Transfusion medicine center, Vilnius University Hospital Santariskiu Klinikos, ${ }^{2}$ State Research Institute Centre for Innovative Medicine, Vilnius, Lithuania

Introduction: Role of anti-HLA antibodies (Abs) in solid organ transplantation is well established. There are accumulating data indicating importance of anti-HLA Abs in haploidentical peripheral blood stem cell or umbilical cord blood transplantations. However, the contribution of anti-HLA Abs to risks of graft failure, delayed engraftment, graft versus host disease (GvHD) and other early complications in unrelated donor (UD) allogeneic haematopoietic stem cell transplantation (alloHSCT) is of uncertain significance. The main objective of this prospective study is to evaluate the clinical relevance of anti-HLA Abs in UD alloHSCT.

Material (or patients) and methods: To date, 58 patients were recruited into the study. Patients' and their donors' DNAs have been typed using sequence based technique (HLA-A, HLA-B, HLA-C, HLA-DRB1, and HLA-DQB1 loci) and Luminex technology (HLA-DPB1 and HLA-DRB3,4,5 loci). The 35 patients' sera samples before conditioning and 1, 2, 3, and 6 months after alloHSCT have been collected and screened for the presence of anti-HLA Abs using Luminex-based solid phase assays.

Results: Of the 35 patient-donor pairs studied, 24 (68.5\%) patients received a 10/10 allele matched UD at HLA-A, HLA-B, HLA-C, HLA-DRB1 and HLA-DQB1 loci. 6 patients had 1 mismatch at HLA class I loci and 3 patients had 1 mismatch 
at HLA class II loci. After HLA-DRB3,4,5 and HLA-DPB1 loci typing only 3 patients (8.6\%) were found to be 14/14 matched. 31 patient had one or two DPB1 mismatches. Pre-transplant anti-HLA Abs were detected in 15 patients (42.8\%) and 13 of them were women. All multiparous female (10 patients) were found to be carriers of anti-HLA Abs. The 7 (20\%) patients were negative for the anti-HLA Abs before alloHSCT, but became positive after transplantation. 11 (31.4\%) patients were antiHLA Ab negative before and after alloHSCT. The only patient with secondary graft failure had 2 DPB1 mismatches; according to the DPB1 T-Cell Epitope algorithm, the predicted immunogenicity of the HLA-DPB1 matching was determined to be non-permissive in the host versus graft direction. In this patient anti-HLA Abs were detected against both HLA classes during all monitoring steps, but there were no donor specific antibodies. Statistical analysis did not find any significant correlation between HLA mismatches, anti-HLA Abs, engraftment time, GvHD development, and other post-transplant complications.

Conclusion: Current data revealed no impact of anti-HLA Abs on the outcome and early complications after alloHSCT possibly due to small patient numbers. All multiparous women were found to be carriers of anti-HLA Abs which may be directed against mismatched HLA alleles. Further recruitment of patients into this study is ongoing.

Disclosure of Interest: None declared.

\section{P584}

Engraftment syndrome- Clinical features and Predictive factors in Autologous stem cell transplant

R. Jain ${ }^{1,2}$, V. Sheth ${ }^{2,3, *}$, A. Gore ${ }^{2}$, A. Ghanekar ${ }^{2}$, M. Shapira ${ }^{4}$ S. Grisariu ${ }^{4}$, A. Batia ${ }^{4}$, R. Or Or T. Saikia $^{2}$

${ }^{1}$ Stem cell transplant, Jaslok hospital, ${ }^{2}$ Stem cell transplant, Prince Aly Khan hospital, ${ }^{3}$ Stem cell transplant, Hadassah, Bombay, India, ${ }^{4}$ Stem cell transplant, Hadassah, Jerusalem, Israel

Introduction: Engraftment Syndrome(ES) maybe observed in patients who undergo autologous stem cell transplant (SCT). To investigate clinical criteria for ES diagnosis and analyse the risk factors for this complication, we reviewed 178 consecutive peripheral blood auto-SCT cases (Lymphoma and Myeloma) performed during the past 9 years at two tertiary care centres. Material (or patients) and methods: We analysed all patients with a non-infectious fever, developed within 7 days of engraftment (first day of ANC of 500 on two consecutive days) in 178 patients undergoing autologous stem cell transplant. Results: A total of $46 / 178$ (25.8\%) patients developed noninfectious fever and one or more clinical signs of ES within 7 days of engraftment.

In all, 29(61\%) fulfilled the Maiolino and $12(26 \%)$ the Spitzer criteria. The incidence of engraftment syndrome using the Maiolino criteria in our study was 29 (15\%), which compares well with Spanish study (13\% using same criteria) and the original Maiolino study (20\%).

All patients with ES satisfactorily recovered and discharged with a median of 20 days from hospital. There was no significant difference in number of days of hospitalisation and days of antibiotics between the ES and non ES arms.

All patients recovered without any morbidity and only $1(2 \%)$ patient required readmission for fungal pneumonitis. $8(17 \%)$ patients required ICU admission due to delay in initiation of steroids.

Non of the factors including number of chemotherapy cycles, conditioning regime, disease status, CD34 collection, growth factors and day of WBC engraftment except female $(P=0.064)$ were statistically significant (in uni or multivariate analysis).
Non-infectious fever

Skin rash

Pulmonary infiltrates

Diarrhea

Clinical criteria of ES

Maiolino (+) Spitzer (+)

Maiolino (+) Spitzer (-)

Maiolino (-) Spitzer (+)

Conclusion: Our study shows that engraftment syndrome is common in Autologous transplant setting. Maiolino criteria to diagnose ES is more sensitive in our setting. If detected and treated early there is not much morbidity or mortality related to ES.

Disclosure of Interest: None declared.

\section{P585}

Large granular lymphocytosis and its impact on chronic graft-versus-host disease

R. Fedele ${ }^{1, *}$, B. Oliva ${ }^{2}$, C. Garreffa ${ }^{2}$, A. Meliadò ${ }^{1}$, S. Barillà ${ }^{1}$ G. Gallo ${ }^{7}$, C. Rigolino ${ }^{1}$, M. Cuzzola ${ }^{1}$, T. Moscato ${ }^{1}$, G. Irrera ${ }^{1}$, G. Console ${ }^{7}$, G. Messina ${ }^{7}$, M. Martino ${ }^{1}$

${ }^{1}$ Oncology and Hematology, Hematology and Bone Marrow Transplant Unit, ${ }^{2}$ Clinical Phatology, Hematology Laboratory, Azienda Ospedaliera BMM, Reggio Calabria, Italy

Introduction: Polyclonal or oligoclonal T-cell large granular lymphocyte (T-LGL) expansion generally occurs in patients with viral infections, autoimmune diseases, malignancies, and solid organ or allogeneic hematopoietic stem cell transplantation (allo-SCT).

Material (or patients) and methods: We have analyzed 97 patients receiving allo-SCT and surviving beyond 100 days to examine the occurrence of large granular lymphocytosis (LGL). LGL was defined as the presence of at least two of the following criteria: (1) lymphocytosis above 3.0x10E9/L in at least three consecutive determinations over a time frame of 23 months; (2) predominance $(>30 \%$ ) of $L G L$ in peripheral blood, (3) confirmation of monoclonality by T-cell receptor analysis using PCR.

Median age of patients at transplantation was 41 years (17-64), 49 males and 48 females. The conditioning regimen was myeloablative in 42 patients and at reduced intensity in 57 patients. Bone marrow in 25 and peripheral blood stem cells in 74 transplantations were used as graft source, respectively. Donors were matched-siblings in 77 cases, and matchedunrelated in 22 cases. Graft-versus-host disease (GVHD) prophylaxis was cyclosporine-based in all cases. Acute GVHD grade 2-3 occurred in 15 patients and was resolved in all cases without any sequelae. Chronic GVHD occurred in 40 patients (mild in 16, moderate in 18, severe in 6 patients). Particularly, of the 40, 13 were affected by scleroderma-like GVHD.

Results: In 16 of the 97 patients LGL was present during their transplantation outcome with a median onset of 320 days from transplant and 13 of the 16 had chronic GVHD (9 of 97 patients had contemporarily scleroderma-like chronic GVHD and LGL). Patients with LGL didn't show a significant improvement on OS but showed an association to chronic graft-versus-host disease $(P=0.014)$ and scleroderma-like chronic GVHD $(P=0.001)$. No significant associations were found among LGL and other factors (CMV serostatus of donor 
and recipients, conditioning regimen, type of donor, stem cell source, donor and recipient's sex).

Conclusion: In conclusion, we demonstrated that LGL is more frequent than we expected and it is associated to chronic GVHD above all in scleroderma-like features. A wider and thorough study is needed to confirm this association and to explain if $L G L$ is involved in the immunological mechanism of chronic GVHD particularly scleroderma-like considering that it is documented in literature the association between LGL and autoimmune diseases.

It would be interesting to know if $L G L$ is predictive of scleroderma-like chronic GVHD.

Disclosure of Interest: None declared.

P586

Multicenter and Retrospective study for Risk factors affecting DLCOc prior Allogeneic Hematopoietic Stem Cell Transplant

R. Parody ${ }^{1, *}$, O. Lopez-Godino ${ }^{2}$, A. Esquirol ${ }^{3}$, J. L. Piñana ${ }^{4}$, G. Orti ${ }^{5}$, F. J. Marquez-Malaver ${ }^{6}$, F. Díaz-Chantar, N. Rodriguez, L. Vazquez ${ }^{2}$, L. Lopez-Corral ${ }^{2}$, P. Barba ${ }^{5}$, E. Perez ${ }^{2}$, C. Solano ${ }^{8}$, R. Martino ${ }^{9}$, J. A. Rodriguez-Portal', J. A. Perez-Simón ${ }^{1}$ ${ }^{1}$ Hematology, H.Universitario Virgen del Rocío, Sevilla, ${ }^{2}$ Hematology, H.Universitario de Salamanca, Salamanca, ${ }^{3}$ Hematology, Santa Creu i Sant Pau, Barcelona, ${ }^{4}$ Hematology, H.Clinico de Valencia, Valencia, ${ }^{5}$ Hematology, H.Vall d'Hebron, Barcelona, ${ }^{6}$ Hematology, H.Virgen del Rocío, ${ }^{7}$ Pneumology, H.Universitario Virgen del Rocío, Sevilla, ${ }^{8}$ Hematology, H.Clínico de Valencia, Valencia, ${ }^{9}$ Hematology, H.Santa Creu i Sant Pau, Barcelona, Spain

Introduction: The basal value of diffusing capacity of the lung for transferring carbon monoxide (DLCOC value, corrected by hemoglobin level) is a well recognized impact factor for nonrelapse mortality in stem cell transplantation (SCT), and a relative contraindication if it is $<50 \%$. Around $40-50 \%$ of patients have a DLCOc $<80 \%$ pre-transplant, and there is scarce literature about risk factors affecting this value.

Material (or patients) and methods: We designed a retrospective multicenter study with 589 patients undergoing to allogeneic transplant (alloHSC) to analyze the impact of pre-transplant variables on DLCOc, such as age, sex, basal disease, status at transplant, time from diagnosis to alloHSC, prior lung history and different previous treatments (for the whole group and separately for myeloid and lymphoid malignancies).

Results: 326 patients (55.3\%) had a myeloid and $43 \%$ a lymphoid malignancy. Disease status was non-early in $63.4 \%$. DLCOc was $<80 \%$ in 324 patients (55\%). In univariate analysis the only significant factors for DLCO $<80 \%$ were age ( $p: 0.002)$ and female sex $(p:<0.003)$, with a trend for smokers (p:0.1), prior radiotherapy (p:0.1), non-early disease ( $p: 0.1)$ and prior chemotherapy with alquilating agents (table 1). For lymphoid malignancies the use of bendamustine also showed a trend for a decreased DLCOc (68.2 vs $31.8 \%$, p:0.1). For myeloid malignancies the use of alquilating agents significantly influenced on DLCOc (68.9 vs $31.1 \%, \mathrm{p}: 0.02)$ and anthracyclines showed a trend (56.3 vs $43.7, \mathrm{p}: 0.1)$. In multivariate analysis significant risk factors were female sex $(\mathrm{OR}=2.3 ; 95 \% \mathrm{Cl}: 1.5$ to 3.6; $P<0.001)$ and smoking $(\mathrm{OR}=1.9 ; 95 \% \mathrm{Cl}: 1.2$ to $3 ; \mathrm{p}$ : $0.0049)$ whereas the age $>50$ was protector $(\mathrm{OR}=0.4 ; 95 \% \mathrm{Cl}$ : 0.2 to $0.6 ; \mathrm{p}:<0.001)$. In addition to these factors, for myeloid malignancies, others risk factors were myelodisplasia (OR: 3.7; $95 \% \mathrm{Cl}, 1.03$ to $17 ; \mathrm{p:}: 0.05)$, and prior exposure to alquilating agents (OR: $4.2 ; 95 \% \mathrm{Cl} 1.1$ to $18 ; \mathrm{p}: 0.03$ ), whereas for lymphoid malignancies a trend was found for methotrexate $(P=0.07)$, prior autoSCT (p:0.08), and bortezomib (p:0.1). Overall survival (figure 1) was significantly lower for patients with pre-SCT-DLCOc $<80 \%(67.6 \%$ at 1 year, $53.6 \%$ at 3 ys) with respect to DLCOc $\geq 80 \%$ (73.7\% at 1 year, $64.6 \%$ at 3 ys, p:0.01).
Table 1. Characteristics of the patients

\begin{tabular}{|c|c|c|c|}
\hline $\mathrm{n}(\%)$ & $\begin{array}{c}D L C O c<80 \% \\
\quad(\mathrm{n}=324)\end{array}$ & $\begin{array}{c}D L C O C \geq 80 \% \\
(\mathrm{n}=265)\end{array}$ & $P$ \\
\hline Median age (range) & 45 & 49 & 0.002 \\
\hline $\begin{array}{l}\text { Time to transplant } \\
\text { (ms) }\end{array}$ & 9.8 & 8.2 & 0.4 \\
\hline \multicolumn{4}{|l|}{ Sex } \\
\hline Female & $152(62.3)$ & $92(37.7)$ & 0.003 \\
\hline Male & $172(49.9)$ & $173(50.1)$ & \\
\hline Basal disease & & & 0.6 \\
\hline Myeloid & $175(53.7)$ & $151(46.3)$ & \\
\hline Lymphoid & $140(55.3)$ & $113(44.7)$ & \\
\hline Status & & & $\begin{array}{c}\mathbf{0 . 1} \\
\text { (early } \\
\text { vs } \\
\text { non- } \\
\text { early) }\end{array}$ \\
\hline Early & $109(50.7)$ & $106(49.3)$ & \\
\hline Intermediate & $146(57.9)$ & $106(42.1)$ & \\
\hline Advanced & $69(56.6)$ & $53(43.4)$ & \\
\hline \multicolumn{4}{|l|}{ Prior chemother. } \\
\hline Cyclophosphamide & $92(55.4)$ & $74(44.6)$ & 0.9 \\
\hline Cytarabine & $205(54.8)$ & $169(55.2)$ & 0.9 \\
\hline Anthracyclinics & $237(55.1)$ & $193(44.9)$ & 0.6 \\
\hline Alquilants & $130(58.6)$ & $92(41.4)$ & 0.1 \\
\hline Cis/Carbo-platin & $46(50)$ & $46(50)$ & 0.2 \\
\hline Purine analogues & $94(58.8)$ & $66(41.2)$ & 0.2 \\
\hline Prior radiotherapy & $46(14.2)$ & $28(10.6)$ & 0.1 \\
\hline Smoking & $91(60.3)$ & $60(39.7)$ & 0.1 \\
\hline Prior lung history & $53(56.4)$ & $41(43.6)$ & 0.7 \\
\hline
\end{tabular}

Conclusion: Intrinsic variables such as age, sex and prior history of smoking are the most important risk factors for a decreased DLCOc pre SCT. Alquilating agents may adversely impact on DLCO but more information about cumulative doses is needed. In addition overall survival is significantly decreased for DLCOc values under $80 \%$.

Disclosure of Interest: None declared.

\section{P587}

Outcome of Allogeneic Hematopoietic stem cell transplantation in Autosomal Recessive Hyper IgE syndrome due to DOCK8 deficiency Single Center Experience

S. A. Elshourbagi ${ }^{1, *}$, R. arnaout ${ }^{1}, H$. aldhekry ${ }^{1}, H$. almousa ${ }^{1}$, A. alghonaim ', B. alsaud ${ }^{1}$, S. almuhsen ${ }^{1}$, M. ayas ${ }^{1}$, A. alseraihy ${ }^{\prime}$, A. alahmari ${ }^{1}, G$. aldawsari ${ }^{1}, N$. ades ${ }^{1}$

${ }^{1}$ pediateics, king faisal specialist hospital\&research center Riyadh, riyadh, Saudi Arabia

Introduction: Autosomal recessive Hyper-IgE syndrome due to DOCK8 mutation is a combined primary immunodeficiency, characterized by severe eczema, recurrent infections, and susceptibility to autoimmunity, malignancy, and multiple allergies, in addition to unusual high serum IgE level.

Material (or patients) and methods: In our center we have a large number of DOCK8 patients. During a period of 10 years (2005-2015), we transplanted 12 patients with documented DOCK-8 mutation confirmed by molecular genetics.

Results: 1 patient did not receive any conditioning because of poor clinical condition and he died from severe cutaneous and 
gut GVHD. The rest of the patients received HSCT from HLA full matched donor with chemoablation with $\mathrm{Bu} / \mathrm{Cy}$ for all with $100 \%$ lymphoid and myeloid engraftment (STR).Among those patients who received chemoablation, GVHD developed in 6 patients mostly grade I and II. In addition 2 patients died:1 died of severe GVHD and the other died of sepsis. 8 patients are alive and well.

Conclusion: In conclusion, we highly recommend early HSCT if fully matched donor is available to prevent the high mortality associated with this disease.

References: Zhang Y1, Yu X2, Ichikawa M3, Lyons JJ2, Datta S2, Lamborn IT1, Jing H1, Kim ES1, Biancalana M4, Wolfe LA5, DiMaggio T1, Matthews HF4, Kranick SM6, Stone KD2, Holland SM7, Reich DS8, Hughes JD9, Mehmet H9, McElwee J9, Freeman AF7, Freeze HH4, Su HC10, Milner JD11.Autosomal recessive phosphoglucomutase 3 (PGM3) mutations link glycosylation defects to atopy, immune deficiency, autoimmunity, and neurocognitive impairment.J Allergy Clin Immunol. 2014 May;133(5):1400-9, 1409.e1-5. doi: 10.1016/j. jaci.2014.02.013.

Alsum $Z^{1}$, Hawwari A, Alsmadi O, Al-Hissi S, Borrero E, AbuStaiteh A, Khalak HG, Wakil S, Eldali AM, Arnaout R, AlGhonaium A, Al-Muhsen S, Al-Dhekri H, Al-Saud B, Al-Mousa H., Clinical, immunological and molecular characterization of DOCK8 and DOCK8-like deficient patients: single center experience of twenty-five patients. J Clin Immunol. 2013 Jan;33(1):55-67. doi: 10.1007/s10875-012-9769-x.

Cuellar-Rodriguez J1, Freeman AF1, Grossman J2, Su H3, Parta M4, Murdock H3, Shah N5, Bollard C6, Kong HH7, Moutsopoulos N8, Stone K9, Gea-Banacloche J10, Holland SM1, Hickstein DD11, Matched related and unrelated donor hematopoietic stem cell transplantation for DOCK8 deficiency, Biol Blood Marrow Transplant. 2015 Jun;21(6):1037-45. doi: 10.1016/j. bbmt.2015.01.022.

Jennifer Cuellar-Rodriguez, Alexandra F Freeman, Juan GeaBanacloche, Helen Su, Jennifer K. Grossman, Terry J Fry, Steven M. Holland, Dennis D. Hickstein, Allogeneic Hematopoietic Stem Cell Transplant Reverses The Phenotype Of DOCK8 Deficiency, November 15, 2013; Blood: 122 (21).

McDonald DR, Massaad MJ, Johnston A, Keles S, Chatila T, Geha RS, Pai SY. Successful engraftment of donor marrow after allogeneic hematopoietic cell transplantation in autosomalrecessive hyper-lgE syndrome caused by dedicator of cytokinesis 8 deficiency, J Allergy Clin Immunol. 2010 Dec;126 (6):1304-5.e3. doi: 10.1016/j.jaci.2010.07.034.

Gatz SA, Benninghoff U, Schütz C, Schulz A, Hönig M, Pannicke U, Holzmann KH, Schwarz K, Friedrich W, curative treatment of autosomal-recessive hyper-lgE syndrome by hematopoietic cell transplantation, Bone Marrow Transplant, 2011 Apr;46 (4):552-6. doi: 10.1038/bmt.2010.169.

Disclosure of Interest: None declared.

\section{P588}

Comparative study of post-transplant lymphoproliferative disorders after solid organ transplantation versus hematopoietic stem cell transplantation

S. Romero ${ }^{1, *}$, J. Montoro ${ }^{1}$, J. Sanz ${ }^{1}$, M. Guinot ${ }^{1}$, A. Balaguer ${ }^{1}$, G. Iacoboni $^{1}$, E. Mayordomo ${ }^{2}$, R. Lopez-Andujar ${ }^{3}$, I. Beneyto ${ }^{4}$ L. Almenar ${ }^{5}$, A. Sole, G. Sanz ${ }^{1}$, M. A. Sanz ${ }^{1}$

${ }^{1}$ Hematology and Hemotherapy, ${ }^{2}$ Anatomic Pathology, ${ }^{3}$ Hepatobilio-pancreatic surgery and transplant unit, ${ }^{4}$ Nephrology,

${ }^{5}$ Cardiology, ${ }^{6}$ Pneumology, Hospital La Fe, Valencia, Spain

Introduction: Post-transplant lymphoproliferative disorder (PTLD) is a well-recognized complication of both solid organ transplantation (SOT) and allogeneic hematopoietic stem cell transplantation (HSCT). However, differences in clinical and biological characteristics between the two procedures are not well established.

Material (or patients) and methods: We retrospectively evaluated 84 consecutive patients diagnosed of PTLD at a single institution between 1993 and 2014. Clinical, biological characteristics and outcomes of both groups were compared.
Results: Sixty-three cases occurred after SOT (24 hepatic, 20 renal, 10 cardiac, 8 pulmonary and 1 double-leg transplant) and 21 after HSCT (18 unrelated donor -17 umbilical cord blood-, 2 haploidentical and 1 HLA-identical sibling). There was a male predominance $(73 \%$ in SOT and $62 \%$ in HSCT). Median age at transplant was 53 years (range, 11-76) in SOT and 43 years (range, 18-59) in HSCT $(P=0.04)$. EBV serostatus before transplant was positive in $78 \%$ and $95 \%$ for SOT and HSCT recipients, respectively. Table 1 summarizes clinical and biological characteristics at PTLD diagnosis. Briefly, HSCT patients had $85 \%$ of B symptoms compared to $40 \%$ in the SOT group $(P=0.002)$. Waldeyer's ring, spleen, central nervous system and liver involvement was $24 \%, 43 \%, 29 \%$ and $52 \%$, respectively, in the HSCT group; and 5\% $(P=0.04), 17 \%(P=0.03), 7 \%(P=0.03)$ and $13 \%(P<0.001)$, respectively, in the SOT group. There was a higher Ann Arbor stage in the HSCT group compare to the SOT group $(P=0.03)$. Time from transplant to PTLD was 4 months (range, 2-27) and 60 months (range, 3-246) for HSCT and SOT group $(P<0.001)$, respectively.

\begin{tabular}{|c|c|c|c|}
\hline & SOT & HSCT & P-value \\
\hline $\begin{array}{l}\text { Time from transplant } \\
\text { to PTLD (months), } \\
\text { median (range) }\end{array}$ & $60(3-246)$ & $4(2-27)$ & $<0.0001$ \\
\hline B symptoms, n (\%) & $22(40)$ & $16(85)$ & 0.002 \\
\hline $\begin{array}{l}\text { Nodal involvement, } \\
\text { n (\%) }\end{array}$ & $33(59)$ & $13(65)$ & 0.8 \\
\hline Waldeyer's ring & $3(5)$ & $5(24)$ & 0.04 \\
\hline Spleen & $10(17)$ & $9(43)$ & 0.03 \\
\hline $\begin{array}{l}\text { Extranodal involve- } \\
\text { ment, } n(\%)\end{array}$ & $35(58)$ & $18(86)$ & 0.045 \\
\hline $\begin{array}{l}\text { Central nervous } \\
\text { system }\end{array}$ & $4(7)$ & $6(29)$ & 0.03 \\
\hline Liver & $8(13)$ & $11(52)$ & $<0.001$ \\
\hline
\end{tabular}

Most patients (95\%) had received calcineurin inhibitors with other immunosuppressive agents. PTLD treatment was different between both groups $(P=0.002)$; in the SOT group, $24 \%$ received rituximab as monotherapy and $68 \%$ chemotherapy, as compared with $71 \%$ and $29 \%$ in the HSCT group, respectively.

The median follow-up after PTLD diagnosis and overall survival of all patients was 27.5 month and 11 months, respectively. At last follow-up, $29 \%$ were alive and $71 \%$ were died. In SOT group, median OS was 19 month and OS at 4 years $35 \%$. In HSCT group, median OS was 1.6 months and OS at 4 years $10 \%(P<0.001)$. Most deaths were directly related to PTLD.

Conclusion: PTLD after HSCT appears to be a different entity because of the earlier appearance after transplant, a more aggressive clinical presentation (B symptoms, nodal and extranodal involvement, higher Ann Arbor staging system) and a poorer survival outcomes compared with PTLD after SOT.

Disclosure of Interest: None declared.

\section{P589}

Metabolic Syndrome After Hematopoietic Stem Cell

Transplantation In Children

S. Aksoylar 1,*, G. Guner ${ }^{1}$, D. Gokcen Simsek ${ }^{2}$, S. Gozmen ${ }^{1}$, S. Darcan ${ }^{3}$, S. Kansoy ${ }^{7}$

${ }^{1}$ Pediatric Hematology\&Oncology, BMT Unit, ${ }^{2}$ Pediatric Endocrinology, ${ }^{3}$ Pediatric Endocrinilogy, Ege University, Izmir, Turkey

Introduction: The early and late complications after hematopoietic stem cell transplantation (HSCT) determine the patient's prognosis and life quality. In recent years, the developmental risk of metabolic syndrome (MS) is shown to have increased among the patients administered HSCT in adults. In limited number of studies made on this subject it is noted that MS can be an important problem in childhood. 
In this study, it was aimed to determine the metabolic syndrome development frequency after HSCT in children administered HSCT and to find out the effective risk factors.

Material (or patients) and methods: The study included 36 children, who were administered HSCT in Ege University, and upon whose application at least two years passed. As the control group 18 healthy children of 11 to 17 ages were used. All of the cases and the healthy children in the control group were assessed with Modified WHO (World Health Organization) Criteria (Obesity, Abnormal glucose balance, dyslipidemia and hypertension) in terms of metabolic syndrome. The found values were compared to the patients previously known values before HCST. The comparison with healthy control group was performed with the patient group at the same age rank.

Results: 25 of the 36 patients (70\%) were male and 11 of them (30\%) were female, the mean age was $10,6 \pm 3,6(1,2-15)$ years and the mean period that passed after HCST was found to be $4,1 \pm 2,1(2-13,5)$ years. The primary disease was heterogeneous (leukemia 8, bone marrow failure 9, thalassemia major 7 , histiocytosis 4, solid tumor 3, and metabolic disease 1 patient). Allogenic HCST was performed to 33 cases. Conditioning regimen was non-myeloablative in 10 cases $(27,8 \%)$ and whole body irradiation (TBI) was administered to 2 cases. Six cases before HCST $(16,6 \%)$ and 16 cases after HCST (66\%) used corticosteroid.

Growth retardation was determined in 9 cases (25\%). Two cases were diagnosed metabolic syndrome $(5,6 \%)$ with WHO criteria. When considered in terms of the sub-components of metabolic syndrome, 2 cases $(5,6 \%)$ were found to have obesity, 17 cases $(47 \%)$ to have abnormal glucose balance, 11 cases $(30,7 \%)$ to have dyslipidemia and 3 cases $(8,6 \%)$ to have hypertension. The MS rate was not different when compared with healthy control group ( $0 \%$ vs $11 \%$ ). The rate of obesity and hypertension also was not different from that of healthy control group.

Dyslipidemia consisted of high triglyceride level by $19,5 \%$, high total cholesterol level by 5,6\% and low HDL level by 5,6\%. Total cholesterol levels of the cases were found to have increased in comparison to pre-HSCT and healthy control group.

The whole of the abnormal glucose balance consisted of insulin resistance. Insulin resistance was found to be different as $33 \%$ in heathy control group and $77,8 \%$ in case group.

Myeloablative conditioning (65\% vs $20 \%$ ) and the older age at which HSCT was performed were considered to be risk factors in terms of insulin resistance.

Conclusion: MS incidence was found 5,6\%. Growth retardation, hyperinsulinemia and high total cholesterol level were found to have significantly increased in an evaluation made 4 years after HSCT. Age and conditioning were found to be the risk factors in terms of insulin resistance. Getting older and the length of the time that passed after HSCT can affect the results. The long-term follow up of the cases administered HSCT in childhood in terms of MS is important in respect of the increase of life quality at late age.

Disclosure of Interest: None declared.

\section{P590}

Multidisciplinary team inclusive of psychological assessment provides benefit on Quality of Life (QoL) in young adult recipients of HSCT

S. Giuliani ${ }^{1, *}$, F. Giglio ${ }^{2}$, L. Sciuto ${ }^{1}$, G. Marini ${ }^{1}$, O. C. Sartorelli ${ }^{1}$ R. Greco ${ }^{2}$, C. Corti ${ }^{2}$, M. Bernardi ${ }^{2}$, J. Peccatori ${ }^{2}$, L. Sarno ${ }^{1}$, F. Ciceri ${ }^{2}$ ${ }^{1}$ Clinical and Health Psychology Unit, ${ }^{2}$ Unit of Hematology and Bone Marrow Transplantation, IRCCS San Raffaele Scientific Institute, Milano, Italy

Introduction: The care of young adults undergoing allogeneic hematopoietic stem cell transplantation (HSCT) poses both clinical and psychological issues beyond those faced by other age groups. HSCT occurs in a period of patients' life at high risk of depression as literature on healthy young adults shows: the perceived gap between personal expectations and their achievements could be very wide in HSCT young adult patients and could represent a risk factor of a poor perceived QoL regardless the treatment efficacy.

Particularly interesting is the association between QoL and patients' psychological variables potentially associated to their perception of QoL, as far as specific intervention can be timely addressed to help them to develop a more adaptive coping style.

In 2011 a specific multidiscliplinary approach including psychological assessment was reserved to young adults treated at our Institution for haematological malignancies from diagnosis, through all treatment phases.

The aim of our study was to evaluate the efficacy of this approach in terms of QoL in this cohort of patients before HSCT and the correlation between QoL and the psychological variables (coping styles, distress, anxiety and depression) measured during the pre-transplant phase.

Material (or patients) and methods: From February 2011 to April 2013, 35 young adult patients (17\% female, $83 \%$ male; mean age: 24,5 ; range $18-30$ y; $40 \%$ Lymphomas, $45,7 \%$ acute leukemia, $14,3 \%$ other hematological disease) candidate to HSTC were studied. Since diagnosis a multidisciplinary team consisting of haematologists, psychologist, nurses, physiotherapist took care of the patients.

Self-administered questionnaires were used during pre-HSCT assessment to evaluate patients' QoL [Medical Outcomes Study SF-36 (Ware e Sherbourne, 1992)], anxious-depressive symptomatology [Hospital Anxiety and Depression Scale HADS (Zigmond \& Snaith, 1983)], level of distress [Psychological Distress Inventory PDI (Morasso et al., 1996)] and coping styles Mental Adjustment to Cancer Scale MAC (Watson et al, 1988)]. The association between QoL and all these variables was studied in univariate analysis (statistical test SPSS-20).

Results: The results show that patients describe a good QoL, both mental and physical: most of them show low levels of distress (77\%) and do not present any anxious (71.5\%) or depressive (88.6\%) symptoms and adopt "fighting spirit" coping strategy (74,28\%). Mental QoL is positively associated $(P<0.05)$ with "fighting spirit" and negatively associated with anxiety, depression, helpless and anxious coping style, while physical QoL is negatively associated with distress.

Conclusion: These data suggest that a multidisciplinary approach for HSCT young adults patients should be developed in order to assess and to act on psychological variables in the pre HSCT phase. Even when the patients are not at risk to experience poor QoL at the beginning of HSCT, anxiety, depression, distress and coping strategies should be assessed during all the HSCT phases since they influence patient doctor communication and compliance to treatment and could be modified by HSCT side effects. Young adults patients would seem to benefit from being treated in a multidisciplinary setting with a structural implementation of psychological assessment.

Disclosure of Interest: None declared.

\section{P591}

Venous thromboembolism following hematopoietic stem cell transplantation - a systematic review and metaanalysis

M. F. Zahid' , M. Patnaik', M. Litzow², S. Hashmi',*

${ }^{1}$ Medical Graduate, Aga Khan University, Karachi, Pakistan, 2 Blood and Marrow Transplant Program, Mayo Clinic College of Medicine, Rochester, United States

Introduction: Venous thromboembolism (VTE) is a common complication of hematopoietic stem cell transplantation (HSCT) with variable incidence. Graft versus host disease (GVHD) is another complication of HSCT that may modify the risk of VTE. Our objective was to explore the incidence of VTE [deep venous thrombosis (DVT) and pulmonary embolism (PE)] following HSCT and to assess the likelihood of VTE following allogeneic and autologous HSCT, and evaluate its association with GVHD. 
Venous thromboembolism (VTE) is a common complication of hematopoietic stem cell transplantation (HSCT) with variable incidence. Graft versus host disease (GVHD) is another complication of HSCT that may modify the risk of VTE.

Objectives: To explore the incidence of VTE [deep venous thrombosis (DVT) and pulmonary embolism (PE)] following HSCT and to assess the likelihood of VTE following allogeneic and autologous HSCT, and evaluate its association with GVHD. Material (or patients) and methods: A Comprehensive search of Medline In-Process \& Other Non-Indexed Citations, MEDLINE, EMBASE, Cochrane Central Register of Controlled Trials, and Scopus was conducted to search for both retrospective and prospective HSCT studies which had reported VTE. Random-effects meta-analysis was used to pool incidence rates.

Results: We included 17 studies reporting on allogeneic and 10 on autologous-HSCT; enrolling 6693 patients; of which 5 were randomized. The overall incidence of VTE after HSCT was $5 \%(4-7 \%)$. Incidence in allogeneic-HSCT was $4 \%(2-6 \%)$ and in autologous-HSCT was 4\%(1-15\%). Eleven and 9 studies reported data on acute and chronic GVHD, respectively. The incidence of VTE in chronic-GVHD was 35\%(20-54\%), whereas in acute-GVHD it was $47 \%(32-62 \%)$.

Conclusion: The overall incidence of VTE after HSCT is $5 \%$. It is evident that patients suffering from both acute and chronic GvHD are at an elevated risk of both PE and DVT. Based on the results of this meta-analysis, VTE is a fairly common complication after HSCT, emphasizing the importance of assimilating guidelines for both treatment and prophylaxis in this patient population.

References: 1. Labrador J, Lopez-Anglada L, Perez-Lopez E, Lozano FS, Lopez-Corral L, Sanchez-Guijo FM, et al. Analysis of incidence, risk factors and clinical outcome of thromboembolic and bleeding events in 431 allogeneic hematopoietic stem cell transplantation recipients. Haematologica. 2013;98(3):437-43.

2. Gerber DE, Segal JB, Levy MY, Kane J, Jones RJ, Streiff MB. The incidence of and risk factors for venous thromboembolism (VTE) and bleeding among 1514 patients undergoing hematopoietic stem cell transplantation: implications for VTE prevention. Blood. 2008;112(3):504-10.

3. Gonsalves A, Carrier M, Wells PS, McDiarmid SA, Huebsch LB, Allan DS. Incidence of symptomatic venous thromboembolism following hematopoietic stem cell transplantation. J Thromb Haemost. 2008;6(9):1468-73.

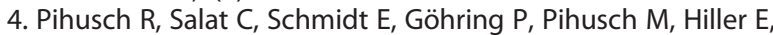
et al. Hemostatic complications in bone marrow transplantation: a retrospective analysis of 447 patients. Transplantation. 2002;74(9):1303-9.

Disclosure of Interest: None declared.

\section{P592}

Title: Assessment of Quality of Life after Haematopoietic stem cell transplantation: South Wales Blood and Marrow Transplant programme

S. Thompson ${ }^{1, *}$, K. Wilson ${ }^{1}$, W. Ingram ${ }^{1}$, D. Balasuriya ${ }^{1}$, G. Scott ${ }^{1}$ T. Banks', J. Bridgeman ${ }^{2}$

${ }^{1}$ Haematology, University Hospital Wales, Cardiff, ${ }^{2}$ Tenovus Cancer Care, Cardiff, United Kingdom

Introduction: Quality of life (QoL) has become increasingly recognised as an important outcome measure after Haematopoietic stem cell transplantation (HSCT). To enable its assessment within our patient population, a questionnaire was designed that evaluated well-being within five domains; Physical, social and family, emotional, functional and additional concerns. The total score available across the five domains was 200, with higher scores indicating better quality of life.

Material (or patients) and methods: The surveys were undertaken at three months, six months, one year and then annually post-transplant when patients attended their key visits in the outpatient department.

Results: Data was analysed up to five years post transplant with 1160 patients being surveyed in total. QoL scores were examined by transplant type, age and gender across the five domains. It is seen that more questionnaires have been received from patients in the early post transplant period with 236 responses from patients three months post transplant falling to 63 at five years. The average overall QoL score at three months was 146 rising to 154 at six months, remaining similar at the remaining time points. Scores from patients from autologous transplants were generally higher than for allogenic transplants at all time points. Patients under 45 had a lower score in the early post transplant period, but this increased faster than in the older age group and eventually superseded it. The trajectory of the QoL scores over time varied significantly across the five domains.

Conclusion: Analysis of the questionnaires show overall QoL is most affected in the early transplant period, and this improves with time. This is consistent with other published data examining the impact of HSCT. However this improvement is not seen consistently across all domains with some domains showing a deterioration over time. The ability to assess QoL is important and allows an insight into this important aspect of the patient journey. Knowing how and when QoL is most affected allows potential targeting of resources and support to maximise patients well being.

Disclosure of Interest: None declared.

\section{P593}

Utility of the HCT-Cl in the Prognosis of HSCT Patients Admitted to the Intensive Care Unit

S. Mehta ${ }^{1, *}$, K. '. Seeger ${ }^{1}$, J. Galvin ${ }^{1}$, J. Mehta ${ }^{1}$

${ }^{1}$ Stem Cell Transplant, Northwestern Medicine, Chicago, United States

Introduction: The use of current intensive care unit (ICU) based prognostic indices (APACHE II, SAPS II, and SOFA) have not shown to be useful for ICU patients who have received an allogeneic stem cell transplant (HSCT). We proposed the use of the Hematopoietic Cell Transplantation-Specific Comorbidity Index (HCT-Cl) as a potentially more useful index for prognosis and prediction of admission to the ICU. The HCT-Cl was validated in HSCT patients and is widely used as a prognostic index for 2-year overall survival and non-relapse mortality in HSCT patients.

Material (or patients) and methods: Data was collected retrospectively from 323 patients undergoing allogeneic stem cell transplant at a single center from 2011-2014. Of the patients analyzed, 47 patients required ICU support during their HSCT admission. Of these patients the median age at time of transplantation was 56 (range: 23-70) with $60 \%$ male and $40 \%$ female. The indications for HSCT were: AML (40\%), NHL (26\%), ALL (13\%), MM (11\%), MDS (6\%), and CLL (4\%). The HSCT donor types were: matched-unrelated (36\%), double cord (36\%), matched-related $(26 \%)$, and syngeneic $(2 \%)$. The HCT-Cl was scored at time of HSCT. The low range for HCT-Cl was defined as

\section{0d Overall Survival}

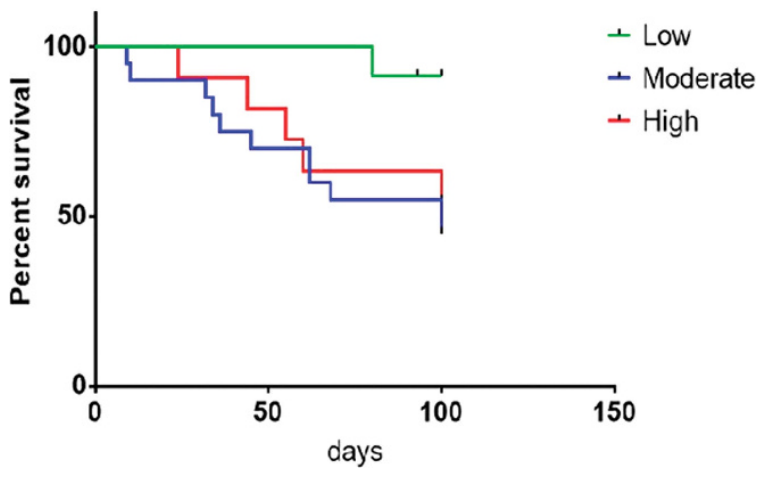


0-2 (28\%), the moderate range 3-4 (42\%) and high range was $>5$ (30\%).

Results: We found that HSCT patients requiring ICU support with a low HCT-Cl score had a significantly better 100 day overall survival in comparison to similar patients with moderate and high HCT-Cl scores $(P=0.04)$. We also found that HSCT patients requiring ICU support with low HCT-Cl scores had a significantly lower number of visits to the ICU during their admission in comparison to HSCT patients with both moderate $(P=0.04)$ and high $(P=0.03) \mathrm{HCT}-\mathrm{Cl}$ scores. However, the HCT-Cl scores did not predict length of stay in the ICU $(P=0.96)$.

Conclusion: There is a current need for a useful prognostic index for patients requiring ICU support and these finding demonstrate the potential utility of the $\mathrm{HCT}-\mathrm{Cl}$ for this purpose. Disclosure of Interest: None declared.

\section{P594}

Granulocyte transfusion against mucositis and infectious complications after allogeneic hematopoietic stem cell transplantation

S. Berglund ${ }^{1,2, *}$, E. Watz $z^{3,4}$, M. Remberger ${ }^{5}$, U. Axdorph-Nygell, ${ }^{3,6}$, M. Sundin ${ }^{7}$, M. Uhlin ${ }^{1,3}$, J. Mattsson ${ }^{1,3}$

${ }^{1}$ Centre for Allogeneic Stem cell Transplantation, Karolinska University Hospital, Stockholm, Sweden, ${ }^{2}$ Dept of Oncology, Johns Hopkins University, Baltimore, United States, ${ }^{3}$ Department of Oncology-Pathology, Karolinska Institute, ${ }^{4}$ Department of Clinical Immunology and Transfusion Medicine, Karolinska University Hospital, ${ }^{5}$ Centre for Allogeneic Stem cell Transplantation, ${ }^{6}$ Department of Clinical Immunology and Transfusion, ${ }^{7}$ Division of Pediatrics, Department of Clinical Science, Intervention and Technology, KAROLINSKA INSTITUTE, Stockholm, Sweden

Introduction: Transient severe neutropenia with a high risk for severe and treatment-refractory infection is common after allogeneic hematopoietic stem cell transplantation (HSCT). Tissue damage caused by HSCT conditioning results in mucositis with ulceration of the mucosa accompanied by severe pain, diarrhea, and damaged barrier function, further increasing the susceptibility to infection. Granulocyte transfusion $(G C X)$ is a valuable treatment alternative in patients with severe infection and insufficient response to conventional treatment, and also in severe mucositis.

Material (or patients) and methods: In this retrospective, single center study we analyzed 85 patients with mucositis or severe infections who received 421 GCX between 1998 and 2014. Clinical outcome, effect of GCX, adverse effects and implications of donor pre-treatment were studied.

Results: Treatment response was classified as partial or complete. In the patients treated due to infection, a complete response was defined as a $\geq 50 \%$ reduction of plasma C-reactive protein (CRP) levels, normalized body temperature, and a marked reduction of clinical symptoms. For mucositis, a complete response was defined as resolution of symptoms or a decrease of $\geq 2$ grades on the WHO oral mucositis scoring scale. Responses that did not fulfill the criteria for complete response were classified as partial.

The overall response rates (including both partial and complete responses) were similar in patients treated with GCSF-GCX and with S-GCX, $83 \%$ and $82 \%$, respectively. However, there were significantly more complete responses in the GCSF-GCX group compared to the S-GCX group (63 vs. $30 \%, p<0.01)$. The rate of complete responses in patients with mucositis was fivefold higher in the GCSF-GCX group (57 vs. $11 \%, P=0.04)$. A similar trend towards more complete responses was also observed when analyzing patients treated for infection ( 68 vs. $38 \%$, respectively, $P=0.07$ ).

Adverse events (AEs) were reported in 36 cases, six were severe or life threatening and three were fatal. In the nine cases of severe to fatal AEs, eihgt pulmonary reactions were reported. All severe pulmonary reactions were seen in patients

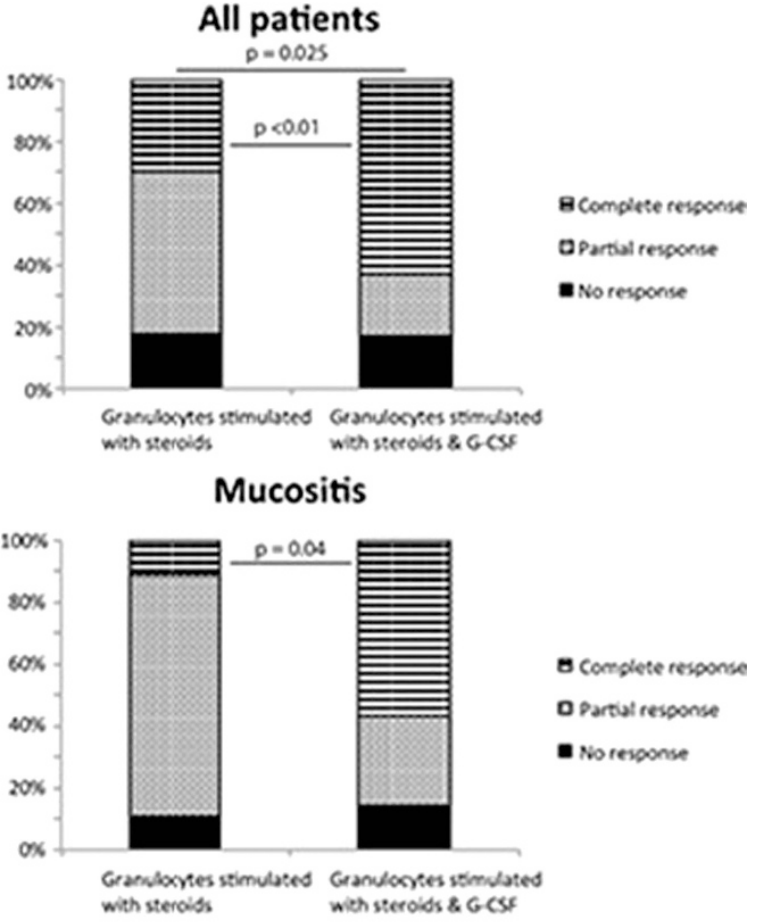

Infection

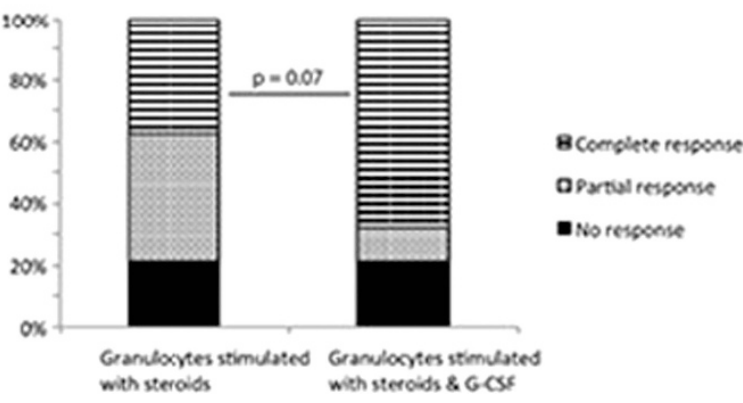

with systemic infection. There was no difference in reported $\mathrm{AE}$ between patients receiving GCSF-GCX or S-GCX.

Conclusion: GCX was observed to be a safe and effective treatment for mucositis after HSCT in this study, with a high rate of complete response and few severe AEs. The benefits of GCX in patients with infections were not as clear, and the use of GCX in these patients is complicated by the risk for severe AEs.

Disclosure of Interest: None declared.

\section{P595}

Impact of different methods of T-cell depletion on poor graft function incidence after allogeneic hematopoietic stem cell transplantation

S. Masouridi Levrat ${ }^{1, *}$, F. Simonetta ${ }^{1}$, Y. Tirefort ${ }^{1}$, O. Tsopra ${ }^{1}$ C. Dantin ${ }^{1}$, Y. Beauverd', A. Simon ${ }^{1}$, K. Polchlopeck ${ }^{\prime}$, C. Stephan ${ }^{\prime}$, Y. Chalandon ${ }^{1}$

'Hematology, Geneva University Hospitals, Geneva, Switzerland

Introduction: Poor graft function (PGF) after allogeneic hematopoietic stem cell transplantation (HSCT) is a serious complication with high mortality, associated with clinical postHSCT conditions such as viral infections, GvHD and myelotoxic treatment. Nevertheless, transplantation-related risk factors for PGF are not established. In the present study we investigated the impact of in vivo and ex vivo T-cell depletion (TCD) 
including use of anti-thymoglobulin (ATG) or alemtuzumab on PGF incidence.

Material (or patients) and methods: We retrospectively evaluated the PGF incidence in 227 patients who underwent allogeneic HSCT for hematologic malignancies at our center from 2004 to 2014 from HLA identical siblings (SIB) or HLA 10/10 matched unrelated donors (MUD) and received either T-cell replete grafts (no TCD group, $n_{1}=48$ ), or TCD grafts obtained by in vivo ATG administration as part of the conditioning regimen (ATG group, $n_{2}=112$ ), or partially TCD grafts obtained through incubation with alemtuzumab in vitro washed before infusion followed on day +1 by an add-back of donor T CD3+ cells (pTCD group, $n_{3}=67$ ). Criteria defining primary PGF were: 1) failure to obtain by day +60 ANC $>0.5 \times 10^{9} / \mathrm{L}$ without G-CSF support and/or Plt $>50 \times 10^{9} /$ $L$, 2) full donor chimerism, 3) no relapse of hematological malignancy and 4) no evidence of peripheral origin cytopenia. Secondary PGF was defined with the same criteria but occurring after initial hematologic recovery and lasting for at least a 4 weeks period. Cox regression was used to examine the independent effect on PGF of factors including age $(\geq$ vs $<50$ years), conditioning regimen (MAC vs RIC), stem cell dose ( $\geq$ vs $<5 \times 10^{6} \mathrm{CD} 34+/ \mathrm{kg}$ ), disease status (CR vs no CR), CMV serology status, year of transplantation (before vs after 2009), donor type (SIB vs MUD), stem cell source (BM vs PBSC) and T-cell depletion. Cumulative incidence estimates of PGF

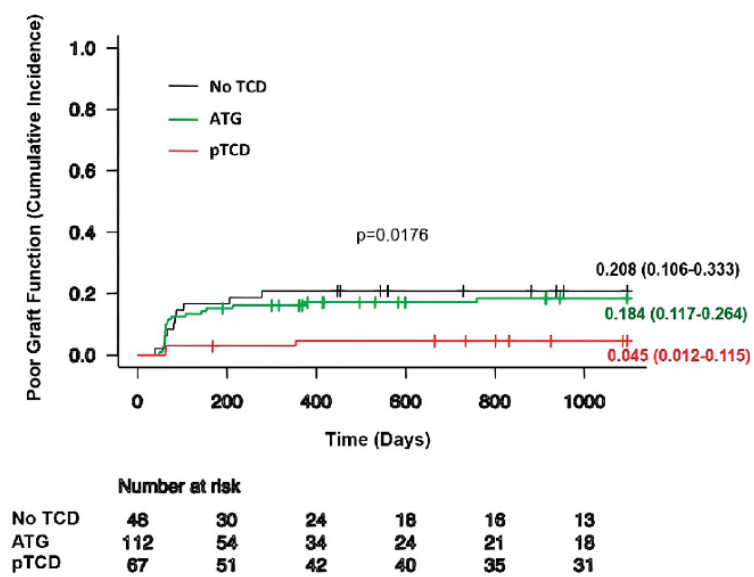

[P596] were calculated with death and relapse defined as competing events.

Results: $33 / 227$ (15\%) patients presented PGF. Ten patients had primary and 23 secondary PGF. 4/33 patients suffered from a viral infection, 9/33 from GvHD, 19/33 from both viral infection and GvHD, while no underlying reason could be identified in one patient. Factors associated with lower risk of PGF were: younger age $(P=0.0061)$, higher stem cell dose $(P=0.01), C M V$ negative donor/recipient pair $(P=0.04)$ and TCD $(P=0.014)$. 3-year PGF cumulative incidence was significantly decreased in the pTCD group $(0.045,95 \% \mathrm{Cl} 0.012-$ $0.115)$ compared to no TCD group $(0.208,95 \% \mathrm{Cl} 0.106-0.333)$ and the ATG group $(0.184,95 \% \mathrm{Cl} 0.117-0.264)(P=0.0176)$. [Figure 1].

Conclusion: The results of this study suggest that partial ex vivo TCD appears to decrease the incidence of PGF in patients undergoing allogeneic HSCT, while in vivo ATG TCD has no beneficial or detrimental impact. This may be in relation with the add-back of lymphocytes on day +1 in the pTCD group.

Disclosure of Interest: None declared.

P596

Long-term Survival and Quality of Life Analysis after Autologous Stem Cell Transplantation for Lymphoma S. Faict ${ }^{1}$, A. De Becker ${ }^{1, *}$, K. Fostier ${ }^{1}$, F. Trullemans ${ }^{1}$, R. Schots ${ }^{1}$ ${ }^{1}$ Hematology, UZ Brussel - Vrije Universiteit Brussel, Brussels, Belgium

Introduction: Autologous stem cell transplantation (ASCT) is frequently applied in the treatment of lymphoma. Survival outcomes are influenced by various prognostic factors. Currently, little is known about the quality of life (QoL) in long-term survivors after ASCT. The objective of this study is to document a single-center's experience on long-term overall survival (OS) and QoL outcomes in lymphoma patients after ASCT.

Material (or patients) and methods: Information was collected on the subtype and staging, the timing of transplant and comorbidities for all consecutive lymphoma patients, who received ASCT in our Centre from 2003 until 2013. Patients with a minimum follow-up of 1 year were inquired to complete the EORTC QLQ-C30 questionnaire and results were compared to a healthy reference population (1).

Results: A total of 85 lymphoma patients were identified with a median follow-up period of 76 months. The estimated OS 
probability was $62,7 \%$ (S.E. $5,5 \%$ ) at 5 years and $60,8 \%$ (S.E. $5,6 \%$ ) at 10,9 years. Indicators of a poor prognosis were: age $\geq 60$ years, high-intermediate to high-risk IPI for NHL and HCT-comorbidity index $\geq 3$. Of long-term ( $>1$ year) survivors 40 completed the EORTC QLQ-C30. They experienced lower cognitive and social functioning and reported more fatigue, dyspnea and financial difficulties, when compared to the reference population. A small but significant proportion of patients reported significant complaints related to pain $(22,5 \%)$ and fatigue (10\%). Very long-term survivors (over 5 years post ASCT) had a better physical and role functioning, with less fatigue, dyspnea, insomnia and loss of appetite as compared to patients who were transplanted more recently. Conclusion: Negative prognostic factors for OS at time of ASCT were age $\geq 60$ years, high-intermediate to high-risk IPI for $\mathrm{NHL}$ and $\mathrm{HCT}-\mathrm{Cl} \geq 3$. Surprisingly, the overall long-term impact of ASCT on QOL was limited but we did note some adverse effects on cognitive and social functions. These negative consequences decreased over time.

References: 1. Hinz A., Singer S., Brähler E., European reference values for the quality of life questionnaire EORTC QLQ-C30: Results of a German investigation and a summarizing analysis of six European general population normative studies, Acta Oncologica, 2014;53:958-965

Disclosure of Interest: None declared.

P597

Long term renal outcome after allogeneic haemopoietic stem cell transplant in an Asian patient population

W. ZHOU ${ }^{1, *}$, C. DIONG ${ }^{1}$, Y.-T. GOH ${ }^{1}$, S. GOPALAKRISHNAN ${ }^{1}$, A. $\mathrm{HO}^{1}$, W. HWANG ${ }^{1}$, L.-P. KOH ${ }^{1}$, M. KOH ${ }^{1}, Y . \mathrm{LOH}^{1,2}$, R. SULTANA ${ }^{\prime}$, P. TAN ${ }^{1}$, Y.-C. LINN

${ }^{7}$ Haematology, Singapore General Hospital, ${ }^{2}$ Raffles Cancer Centre, ${ }^{3}$ Duke-NUS Medical School, Singapore, Singapore

Introduction: Chronic kidney disease (CKD) is a known long term complication of allogeneic haemopoietic stem cell transplant (allo-HSCT) and is well described in Western patients. This study aims to determine the incidence, risk factors, and survival impact of CKD in long term survivors of allo-HSCT in a cohort of Asian patients.

Material (or patients) and methods: Records of patients who survived for at least 2 years post allo-HSCT and had available serum creatinine readings for $>3$ months were analysed. CKD was defined as GFR (glomerular filtration rate, using the MDRD formula) $<60 \mathrm{ml} / \mathrm{min} / 1.73 \mathrm{~m}^{2}$ for $\geq 3$ months and persisted till last seen.

Results: A total of 247 patients (including 12 children) transplanted between 1986 and 2013 in our single center met the above criteria. The median age at transplant was 42.5 years old (3-66 years). Ethnic distribution included Chinese (79.3\%), Malay (8.90\%), Indian (3.6\%) and others (8.1\%).

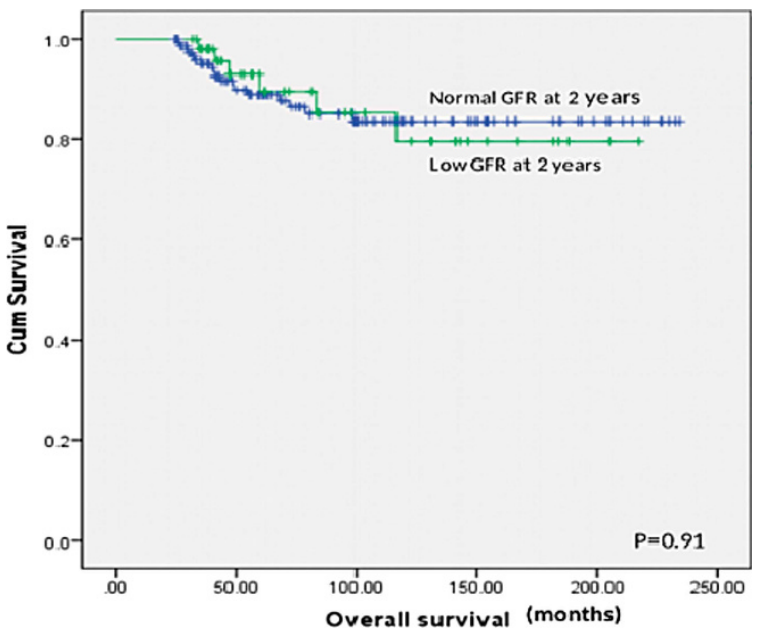

Conditioning was myeloablative in $70.4 \%$ of patients. Donor source was siblings in $78.1 \%$, unrelated donors in $17.8 \%$ and cord blood in $4.0 \%$ of patients. Sixty percent received mobilized stem cell while $36 \%$ received marrow. Median duration of follow-up was 8.25 years (2.0-28.5 years), with 23 patients $(9.3 \%)$ lost to follow up.

Allo-HSCT resulted in significant impairment in renal function. GFR was reduced by a median of $37 \%$ at 1 year and $38 \%$ at 2 years compared to GFR at D0, $P<0.0001$ (Wilcoxon signed rank test). The cumulative incidence of CKD was $10 \%$ at 2 years which continued to increase to $20 \%$ by 25 years.

Univariate logistic regression analysis showed that age at transplant, unrelated donor, lymphoproliferative disease, diabetes mellitus at transplant, duration of foscarnet, and duration of ganciclovir+valganciclovir were significantly associated with development of CKD $(P<0.10)$. The former 3 risk factors remained significant with multivariate analysis $(P<0.05)$. Conditioning regimen, GFR at D0, hypertension, disease risk index at transplant, use of amikacin, amphotericin B, vancomycin, calcineurin inhibitors, development of graft vs host disease, thrombotic microangiopathy, veno-occlusive disease and acute kidney injury post HSCT did not turn out to be significant independent risk factors for development of CKD.

Presence of low GFR $\left(<60 \mathrm{ml} / \mathrm{min} / 1.73 \mathrm{~m}^{2}\right)$ at 2 years post HSCT did not affect long term survival, being $79.5 \%$ at 18 years compared to $83.5 \%$ at 19.5 years for patients with low vs normal GFR at 2 years $(P=0.911)$, see figure. Of the 44 patients who have developed CKD, 3 progressed to end stage renal failure at 11, 19 and 22 years post HSCT and required renal replacement therapy.

Conclusion: Our data represents a single center series of patients with well-captured data and long duration of follow up. CKD is a significant long term complication of allo-HSCT and its incidence in our Asian patient cohort is not different from the Caucasians. Of the multiple possible factors that may insult kidney function, it appears that those that independently contributory to CKD development are inherent (age, unrelated donor and lymphoproliferative disease) rather than modifiable. Nevertheless, development of CKD is logically a multifactorial process involving complex interactions of risk factors such as renal-toxic drugs and vasculo-endothelial complications that may not be detected within this retrospective analysis, and their avoidance as much as possible is still essential.

Disclosure of Interest: None declared.

\section{P598}

Extramedullary relapse following allogeneic

haematopoietic stem cell transplantation for adult acute leukemia and myelodysplastic syndrome: a single center analysis

Y. Katayama ${ }^{1, *}$, K. Iwato ${ }^{2}$, K. Toishigawa ${ }^{1}$, T. Ohchi ${ }^{1}$, T. Okatani ${ }^{1}$ R. Imanaka ${ }^{7}$, K. Kyo ${ }^{1}$, M. Itagaki', S. Katsutani ${ }^{1}$, H. Asaoku', T. Kyo ${ }^{1}$

${ }^{1}$ Division of Haematology, ${ }^{2}$ Division of transfusion, ${ }^{3}$ Division of Laboratory, HIROSHIMA RED CROSS HOSPITAL AND ATOMICBOMB SURVIVORS HOSPITAL, Hiroshima, Japan

Introduction: Allogeneic haematopoietic stem cell transplantation (allo-HSCT) is an effective treatment for acute leukemia and myelodysplastic syndrome (MDS). However, the management of patients relapsing after allo-HSCT is controversial. Extramedullary relapse (EMR) after allo-HSCT, either as an isolated site of relapse or in combination with bone marrow relapse, has been reported with poor prognosis and a lack of efficacious treatment. We performed a retrospective survey of EMR following allo-HSCT for adult patients with acute leukemia and MDS in our institution.

Material (or patients) and methods: We have retrospectively analyzed the records of 354 adult patients with acute leukemia and MDS who underwent allo-HSCT in our hospital between January 2005 and December 2015. The underlying disease was acute myeloid leukemia (AML) in 199 patients, acute lymphoblastic leukemia (ALL) in 71 patients, and MDS in 84 
patients. EMR was confirmed by tissue biopsy or cerebrospina fluid examination.

Results: Sixty-nine patients relapsed after allo-HSCT: 48 patients with $A M L$, 11 with ALL, and 10 with MDS. In addition, 14 of 69 patients developed EMR after allo-HSCT. The median time to EMR following allo-HSCT was day 457.5 (range 26 907). The underlying disease was AML in 8 patients (16.7\% of AML relapse), ALL in 5 patients (45.5\% of ALL relapse), and MDS in no patients. Ages ranged from 18 to 61 years (median 28.5); 6 patients were female and 8 were male. Seven patients were first complete remission (CR), 2 patients were second $C R$, 1 patient was third $C R$, and 4 patients had no remission at alloHSCT. Ten patients were treated with full-intensity conditioning regimens: high dose TBI and high dose cyclophosphamide containing regimens. Four patients were treated with reducedintensity conditioning regimens: fludarabine, melphalan and low dose $\mathrm{TBI}$ containing regimens. The donor source was sibling Bone Marrow (BM) in 1 patient, unrelated BM in 9 patients, and unrelated cord blood in 4 patients. Eight patients had developed chronic graft versus host disease (GvHD) before EMR. Skin and soft tissue was the most common site of EMR in AML patients, but the central nervous system (CNS) was the greatest EMR in ALL patients. EMR in multiple sites was observed in 6 patients. Three patients were noted with another lesion by performing an FDG-PET/CT examination. Four patients received local radiation only, and 5 patients had chemotherapy and/or radiation followed by a second alloHSCT. Currently, 6 of 14 patients $(42.9 \%)$ are alive and 5 patients are disease-free after therapy for EMR. The median time to relapse following therapy for EMR was day 372 (range 139 -918). In total, 8 patients have died. The cause of death are BM relapse (5 patients), treatment related mortality after a second stem cell transplantation ( 2 patients), and lung GvHD after the first allo-HSCT (1 patient).

Conclusion: We suggest that EMR of acute leukemia after the first allo-HSCT is common, but EMR of MDS is rare. Many patients have multiple sites of EMR, and an FDG-PET/CT is useful to find other sites of EMR. EMR of acute leukemia after the first allo-HSCT has a poor prognosis, and BM relapses after EMR have a very poor prognosis because of lack efficacious treatment. There is a need for novel treatment strategies to manage EMR of acute leukemia and MDS after allo-HSCT.

Disclosure of Interest: None declared.

P599

Quality of Life after Hematopoietic Stem Cell Transplantation in Patients with Chronic Graft-Versus-Host Disease

Z. Peric ${ }^{1,2, *}$, L. Desnica ${ }^{1}$, N. Durakovic ${ }^{1,2}$, D. Pulanic ${ }^{1,2}$, A. Ostojic $^{1}$ E. Prenc ${ }^{2}$, R. Serventi-Seiwerth ${ }^{1}$, R. Vrhovac ${ }^{1,2}$, S. 'Z. Pavletic, D. Nemet ${ }^{1,2}$

${ }^{1}$ University Hospital Centre Zagreb, ${ }^{2}$ Faculty of Medicine, University of Zagreb, Zagreb, Croatia, ${ }^{3}$ National Cancer Institute, Bethesda, United States

Introduction: Chronic graft-versus-host disease (cGVHD) is the leading cause of late morbidity and mortality after hematopoietic stem cell transplantation (HSCT) and shows a negative relationship with patient-reported quality of life (QOL). $\mathrm{NIH}$ scoring system for CGVHD was developed in 2005., in order to advance clinical trials for CGVHD prevention and treatment.

Material (or patients) and methods: The aim of this study was to investigate the ability of two standard QOL questionnaires (SF-36 and QLQ C30) to evaluate QOL in cGVHD patients graded according to $\mathrm{NIH}$ global severity scoring system. QOL was assessed in a cross-sectional way in patients who underwent HSCT in University Hospital Centre Zagreb and were alive and in complete remission for more than 1 year after HSCT.

Results: The study included 58 patients (30 female, 28 male) with a median age of 43 years (range 18-71). Patients

[P598]

Table 1. Characteristics of adult patients with acute leukemia who dereloped EMR after allo-HSCT

\begin{tabular}{|c|c|c|c|c|c|c|c|c|c|c|c|c|}
\hline Sex & Age & Disease & $\begin{array}{c}\text { Disease } \\
\text { Status }\end{array}$ & $\begin{array}{l}\text { EM before } \\
\text { allo-HSCT }\end{array}$ & $\begin{array}{l}\text { Donor } \\
\text { source }\end{array}$ & $\begin{array}{l}\text { Day } \\
\text { at EMIR }\end{array}$ & Relapse Site & $\begin{array}{l}\text { Therapy } \\
\text { for EMR }\end{array}$ & $\begin{array}{c}\text { relapse after } \\
\text { therapy } \\
\text { for EMR }\end{array}$ & $\begin{array}{l}\text { OS from } \\
\text { allo-HSCT }\end{array}$ & $\begin{array}{l}\text { OS from } \\
\text { EMR }\end{array}$ & $\begin{array}{l}\text { Cause } \\
\text { of Death }\end{array}$ \\
\hline female & 20 & $\begin{array}{l}\text { AML } \\
\text { MO }\end{array}$ & CR2 & No & $\begin{array}{c}\text { unrelated } \\
\text { BMI }\end{array}$ & 738 & $\begin{array}{l}\text { ovary, } \\
\text { breast }\end{array}$ & $\begin{array}{l}\text { chemotherapy, } \\
\text { radiation, } \\
\text { 2nd SCT }\end{array}$ & No & +2819 & +2081 & No \\
\hline male & 54 & $\begin{array}{l}\text { A.ML } \\
\text { MO }\end{array}$ & $\mathrm{CRl}$ & No & $\begin{array}{c}\text { unrelated } \\
\text { BMI }\end{array}$ & 592 & $\begin{array}{l}\text { soft tissue, } \\
\text { bone marrow }\end{array}$ & $\begin{array}{c}\text { DLI, } \\
\text { radiation }\end{array}$ & $\begin{array}{l}\text { BMI relapse, } \\
\text { dayl } 140\end{array}$ & 878 & 286 & $\begin{array}{l}\text { TRM of } \\
\text { 2nd SCT }\end{array}$ \\
\hline male & 45 & $\begin{array}{l}\text { A.ML } \\
\text { MI4 }\end{array}$ & CRl & CNS & $\begin{array}{l}\text { unrelated } \\
\text { BMI }\end{array}$ & 281 & ocular & radiation & No & 480 & 199 & $\begin{array}{c}\text { lung GrHD of } \\
\text { SCT }\end{array}$ \\
\hline female & 61 & AII & NR & No & cord blood & 267 & $\begin{array}{l}\text { skin, } \\
\text { soft tissue }\end{array}$ & chemotherapy & No & 300 & 33 & relapse \\
\hline female & 28 & $\begin{array}{l}\text { AML } \\
\text { M5 }\end{array}$ & CRl & No & $\begin{array}{l}\text { unrelated } \\
\text { BMI }\end{array}$ & 628 & $\begin{array}{l}\text { soft tissue, } \\
\text { mediastinal } \\
\text { tumor }\end{array}$ & $\begin{array}{l}\text { chemotherapy, } \\
\text { radiation, } \\
\text { 2nd SCT }\end{array}$ & $\begin{array}{c}\text { BM relapse, } \\
\text { day } 918\end{array}$ & 1854 & 1226 & relapse \\
\hline male & 29 & $\begin{array}{l}\text { AIIL } \\
\text { M5 }\end{array}$ & CRl & No & $\begin{array}{c}\text { unrelated } \\
\text { BMI }\end{array}$ & 411 & $\begin{array}{l}\text { testicular, } \\
\text { heart }\end{array}$ & $\begin{array}{l}\text { chemotherapy, } \\
\text { radiation, } \\
\text { 2nd SCT }\end{array}$ & $\begin{array}{l}\text { BMI relapse, } \\
\text { day } 372\end{array}$ & 833 & 422 & relapse \\
\hline female & 22 & $\begin{array}{l}\text { AML } \\
\text { M5 }\end{array}$ & NR & $\begin{array}{c}\text { skin, } \\
\text { lymph node }\end{array}$ & $\begin{array}{l}\text { unrelated } \\
\text { BMI }\end{array}$ & 26 & $\begin{array}{l}\text { skin, } \\
\text { lymph node }\end{array}$ & chemotherapy & No & 347 & 321 & relapse \\
\hline male & 18 & $\begin{array}{l}\text { AMIL } \\
\text { M7 }\end{array}$ & CRl & No & $\begin{array}{c}\text { unrelated } \\
\text { BMI }\end{array}$ & 165 & $\begin{array}{l}\text { thymus, } \\
\text { stomach }\end{array}$ & $\begin{array}{l}\text { chemotherapy, } \\
\text { radiation, } \\
\text { 2nd SCT }\end{array}$ & No & +277 & +112 & No \\
\hline male & 25 & ALL & $\mathrm{CRl}$ & No & cord blood & 652 & CNS & radiation & No & +1407 & +755 & No \\
\hline female & 32 & ALL & CRl & No & $\begin{array}{c}\text { unrelated } \\
\text { BMI }\end{array}$ & 129 & $\mathrm{CNS}$ & radiation & No & +2243 & +2114 & No \\
\hline female & 48 & ALL & CR2 & $\begin{array}{l}\text { Abdominal } \\
\text { tumor }\end{array}$ & cord blood & 504 & ovary & $\begin{array}{l}\text { chemotherapy, } \\
\text { radiation }\end{array}$ & $\begin{array}{c}\text { Kidney relapse, } \\
\text { day537 }\end{array}$ & +1270 & +766 & No \\
\hline male & 42 & ALL & CR3 & No & cord blood & 907 & CNS & radiation & No & +1172 & +265 & No \\
\hline male & 21 & ALL & $\mathrm{NR}$ & CNS & $\begin{array}{l}\text { sibling } \\
\text { BMI }\end{array}$ & 234 & CNS & $\begin{array}{l}\text { radiation, } \\
\text { 2nd SCT }\end{array}$ & No & 290 & 56 & $\begin{array}{l}\text { TRM of } \\
\text { 2nd SCT }\end{array}$ \\
\hline male & 23 & ALL & $\mathrm{NR}$ & No & $\begin{array}{c}\text { unrelated } \\
\text { BMI }\end{array}$ & 650 & kidney & $\begin{array}{c}\text { chemotherapy, } \\
\text { radiation }\end{array}$ & $\begin{array}{l}\text { BM relapse, } \\
\text { dayl39 }\end{array}$ & 819 & 169 & relapse \\
\hline
\end{tabular}




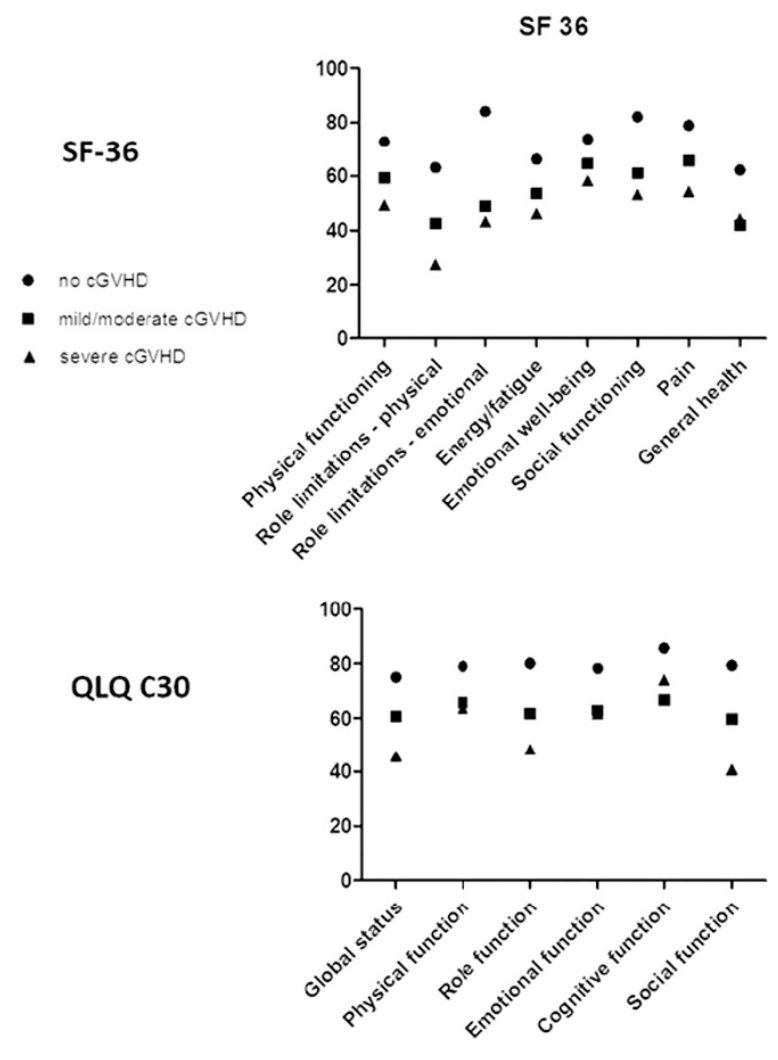

completed QOL forms at a median of 659 days (range 3617853) after HSCT. According to NIH criteria, 21 patients did not meet criteria for CGVHD diagnosis. cGVHD was diagnosed in 37 patients; mild in 1, moderate in 16 and severe in 20 patients, at a median of 298 days after HSCT (range 103-3886). The median of CGVHD organs involved was 2 (range 1-6) and patients received a median of 2 (range 1-4) previous lines of immunosupressive treatment.

When we compared patients with and without CGVHD, those having CGVHD had signifcantly lower scores of global health status as well as significantly lower QOL in terms of all SF-36 subscales and most QLQ C30 functional subscales $(P<0.05$ in all subgroups). In QLQ C30 symptom scales, CGVHD patients reported significantly more pain, dyspnea and sleeping disorders $(P=0.01, P=0.04$ and $P=0.04$, respectively). Furthermore, patients with severe CGVHD had lower QOL scores than patients with moderate CGVHD (Figure 1.). This difference was most evident in physical functioning and role limitations due to physical health in SF-36 $(P=0.01$ and $P=0.04)$ as well as in global status and social functioning scores in QLQ C30 $(P=0.001$ and $P=0.001$, respectively).

Conclusion: In accordance with few previous studies that have adressed this problem, NIH severity scoring system for cGVHD is correlated with patient-reported QOL, particularly in the physical domains as detected by SF-36. In addition, QLQ C30 questionnaire adds more information on social functioning of cGVHD patients and should be used as a valuable instrument of evaluation in the social domains of QOL. In future studies we aim to continue longitudinal QOL assessement by the same means in order to improve care in cGVHD patients.

Disclosure of Interest: None declared.
P600

Optimizing the outcomes of allogeneic hematopoietic stem cell (HSC) transplantation for hematological malignancies: the perfect combination of conditioning regimen, disease stage and type of HSC donor M. Michallet ${ }^{1, *}$, M. Sobh ${ }^{1}$, H. Labussière ${ }^{1}$, M. Balsat ${ }^{1}$, C. Lejeune ${ }^{1}$, S. Ducastelle ${ }^{1}$, F. Barraco ${ }^{1}$, X. Thomas ${ }^{1}$, F. E. E. Nicolini ${ }^{1}$ ${ }^{1}$ Centre Hospitalier Lyon Sud, Pierre Benite, France

Introduction: The objective of this study is to evaluate the impact of the conditioning regimen intensity taking into account the disease stage and the type of HSC donor with its HLA matching on allogeneic transplantation outcomes in a large population of patients with high-risk hematological malignancies.

Material (or patients) and methods: A total of 542 patients who received allo-HSCT between January 2006 and December 2014 in our center were included, 321 (59\%) were males, the median age at allo-HSCT was 49 years (range: 18-70). There was 256 (47\%) acute leukemia (202 AML, 54 ALL), 61 (11\%) MDS, 60 (11\%) multiple myeloma, 46 (8\%) NHL, 25 (5\%) Hodgkin's disease, 23 (4\%) myeloproliferative neoplasms, 21 (4\%) CML, 12 (2\%) CLL and the rest with other hematological diseases. All patients were classified as at high-risk according to either clinical, immunophenotypic, cytogenetic or molecular markers. Conditioning regimen was classified as recently published (Gyurkocza et al. Blood 2014), therefore 282 (52\%) received MAC and 260 (48\%) received RIC/NMA; at allo-HSCT $320(59 \%)$ patients were in CR and $222(41 \%)$ in less than CR. HSC donor was identical siblings (Sib) for 199 (37\%) patients (100 BM, 99 PBSC), 10/10 HLA matched unrelated (MUD) for 159 (29\%) (79 BM, 80 PBSC), 6/6 HLA matched double cord blood (CB) units for 12 (2\%), 9/10 HLA mismatched unrelated (MMUD) for 114 (21\%) (54 BM, 60 PBSC), and the rest of 58 (11\%) were $5 / 6$ or $4 / 6$ mismatched double CB units. For sex mismatching, in $119(22 \%)$, it was female donor to a male patient; 295 (54\%) were ABO compatible, 105 (20\%) had minor incompatibility and $142(26 \%)$ had major incompatibility.

Results: The median follow-up for surviving patients was 29 months (range: 4-96). We conducted a cox multivariate model for OS including patient age, disease status at allo$\mathrm{HSCT}$, conditioning regimen, type of donor and HLA matching, in addition to $A B O$ and sex mismatching, with stratification on the type of disease; this model showed a significant impact of disease status in favor of $\mathrm{CR}(\mathrm{HR}=1.5,95 \% \mathrm{Cl}$ : $1.2-2.0$, $P=0.001)$, conditioning regimen in favor of $M A C(\mathrm{HR}=0.68$, $95 \% \mathrm{Cl}: 0.53-0.88, P=0.003$ ) and type of donor in favor of Sib ( $\mathrm{HR}=0.68,95 \% \mathrm{Cl}: 0.5-0.9, P=0.01)$. Interestingly, we were able to find an optimal association between these 3 factors leading to significantly better results in terms of OS and NRM independently of the disease type. When in $C R$, patients receiving MAC from Sib or from MUD had significant better OS and NRM compared to the rest of patients with 5-years rates of $71 \%$ vs $36 \% \quad(P<0.0001)$ and $15 \%$ vs $37 \% \quad(P=0.001)$ respectively. If not in $\mathrm{CR}$, only patients who received HSC from Sib either after RIC or MAC showed significantly better OS and NRM compared to the rest of patients with 5-years rates of $50 \%$ vs $26 \%(P=0.001)$ and $22 \%$ vs $45 \%(P=0.008)$ respectively (see Figure). Considering only MMUD, patients receiving CB with RIC had better OS and NRM rates compared to $9 / 10$ MMUD (RIC or MAC) and to MAC CB $(P=0.07)$.

Conclusion: We provide in this large study, a practical daily clinical practice outcome preview after allo-HSCT, independently of the type of disease, for the combination of significant impacting factors namely disease status at allo-HSCT, conditioning regimen and type of HSC donor for a with a superiority for MAC when used in CR from Sib or MUD. Disclosure of Interest: None declared. 

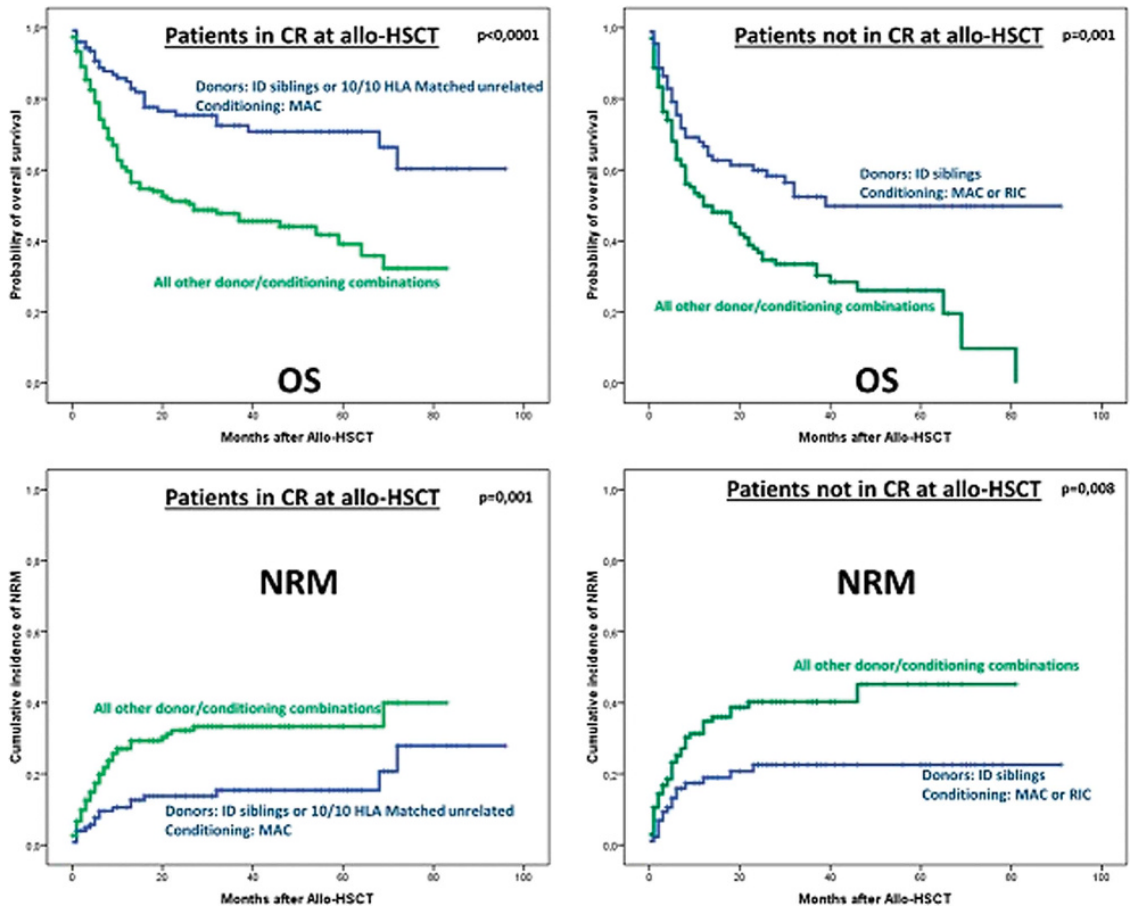

P601

Effect of Cytomegalovirus Reactivation on Prognosis After Allogeneic Transplantation for Acute Leukemia with Active Disease Conditioned with a Sequential Regimen and AntiThymocyte Globulin

M. Gunduz ${ }^{1}$, M. Kurt Yuksel, ${ }^{1,}$, U. Sahin ${ }^{1}$, M. Merter ${ }^{1}$, Z. Narli Ozdemir $^{7}$, S. Civriz Bozdag', S. K. Toprak ${ }^{7}$, P. Topcuoglu', O. Arslan 1, M. Ozcan 1, T. Demirer', O. Ilhan', H. Akan", M. Beksac ${ }^{1}$, G. Gurman ${ }^{1}$

${ }^{1}$ ANKARA UNIVERSITY HEMATOLOGY AND BONE MARROW TRANSPLANTATION UNIT, Ankara, Turkey

Introduction: Acute leukemia with active disease has a dismal prognosis. Allo-HSCT is the only curative option with a limited success. Cytomegalovirus (CMV) reactivation might exert antileukemic effects. However, anti-thymocyte globulin (ATG) use have been reported to overcome this effect [1]. In this paper, we aimed to investigate the frequency of CMV reactivation and its effect on prognosis among an ATG treated patient group.

Material (or patients) and methods: Patients who underwent allo-HSCT in Ankara University Bone Marrow Transplantation Unit between January 2010 and December 2014, using a related or matched unrelated donor (MUD) for acute leukemia with active disease conditioned with a sequential protocol (either with FLAMSA or Mito-FLAG) and received ATG for prophylaxis of graft versus host disease (GvHD) were retrospectively reviewed. GvHD prophylaxis was given with an ATG-Fresenus dose of $30 \mathrm{mg} / \mathrm{kg}$ for match related and unrelated donors, and $60 \mathrm{mg} / \mathrm{kg}$ for mismatch unrelated donors, infused within three consecutive days, as well as CsA and methotrexate. Blood viral load by CMV PCR were screened twice weekly during transplantation and on a regular basis thereafter. Preemptive therapy with gancyclovir was given for at least 14 days until complete disapperance of viremia to

nts having a CMV viral load of $>100$ copies $/ \mathrm{mL}$. Chi-square and Mann-Whitney $U$ tests were used for analyses of variance. Kaplan-Meier method was used for survival estimates. $P<0.05$ was considered statistically significant.

Results: The median age of 10 retrieved patients was 24 (23-58). Males consisted $60 \%$. Acute myleoblastic leukemia (AML) was present in $60 \%$ and acute lymphocytic leukemia (ALL) in $40 \%$. The donor was MUD in $60 \%$, the rest being related donor. Half of the MUD had 9/10 match, the rest having full match. The stem cell source was peripheral blood in $90 \%$ and bone marrow in $10 \%$. Conditioning regimen was myeloablative in $50 \%$. As a sequential therapy $80 \%$ received FLAMSA and the rest MitoFLAG. Median time for follow-up was 6 (1-36) months. Overall survival was $10 \%$. CMV reactivation was observed in $60 \%$. MUD transplanted patients had a higher rate of CMV reactivation compared to related donors ( $83 \%$ vs $25 \% ; P=0.07$ ). Related donor transplanted patients had a beter overall survival compared to MUD ( $P=0.004$, log rank test). Acute GvHD frequency was significantly higher among MUD group $(P=.04)$ and related to a worse overall survival ( $P=.02$, log rank test). CMV reactivated patients had a worse overall survival, however, not statistically significant $(P=0.13)$. 
Table 1. Selected characteristics of patients

\begin{tabular}{llcc}
\hline & Related Donor & MUD & P \\
& & & .43 \\
Diagnosis, \% (n) & $25(1)$ & $50(3)$ & \\
$\quad$ ALL & $75(3)$ & $50(3)$ & \\
$\quad$ AML & & & .05 \\
Sequential therapy, \% (n) & $50(2)$ & $100(6)$ & \\
$\quad$ FLAMSA & $50(2)$ & - & \\
$\quad$ Mito-FLAG & & & 1.0 \\
Conditioning, \% (n) & $50(2)$ & $50(3)$ & \\
$\quad$ Myeloablative & $50(2)$ & $50(3)$ & \\
$\quad$ Reduced intensity & & & .07 \\
CMV reactivation, \% (n) & $75(3)$ & $17(1)$ & \\
$\quad$ Absent & $25(1)$ & $83(5)$ & \\
$\quad$ Present & & & .04 \\
Acute GvHD, \% (n) & $100(4)$ & $33(2)$ & \\
$\quad$ Absent & & $67(4)$ & \\
$\quad$ Present & & & .75 \\
Chronic GvHD, \% (n) & $75(3)$ & $83(5)$ & \\
$\quad$ Absent & $25(1)$ & $17(1)$ & \\
$\quad$ Present & & & \\
\hline
\end{tabular}

Conclusion: Allo-HSCT from MUD in this setting has worse outcomes, probably due to increased risk of acute GvHD and CMV reactivation. The use of ATG may have an additive unfavorable effect.

References: 1. Am J Hematol.2015;90(6):E117-21.

Disclosure of Interest: None declared.

P602

Comparison of reduced intensity conditioning regimens utilizing Campath and ATG as T cell depleting strategy: A single centre retrospective study

M. Saif ${ }^{1, *}$, S. Elmoamly ${ }^{1}$, M. Zine ${ }^{1}$, A. Turner ${ }^{2}$, F. Dignan ${ }^{1}$, E. Tholouli

${ }^{1}$ Haematology, ${ }^{2}$ Microbiology, Central Manchester University NHS trust, Manchester, United Kingdom

Introduction: In vivo T-cell depletion is widely used to reduce graft versus host disease in allogeneic Stem Cell Transplantation (SCT). We retrospectively analysed outcome of patients with myeloid disorders who received either Alemtuzumab or Antithymocyte globulin (ATG) as a part of reduced intensity conditioning regimens in hematopoietic stem cell transplant recipients over a period of 3 years.

Material (or patients) and methods: Patients were identified from department transplant data base. Data was collected using patient medical records, clinical work station and electronic patient records. The conditioning regimens included Fludarabine, Alemtuzumab and Melphalan (FMC, $n=73$ ) and Fludarabine, Busulphan and ATG (FBA, $n=45)$.

Results: Patient demographics for both groups are shown in table 1. Median HCT-Comorbidity index was 2 for FMC (range $0-5$ ) and 1 for FBA (range 0-5). Median time to neutrophil engraftment was 13 days (range 9-23) for FMC and 17 days (range 11-27) for FBA. Median time to platelet engraftment was 13 days (8-33) for FMC and 20 days (range 11-34) for FBA respectively. In FMC group forty three patients (59\%) reactivated cytomegalovirus (CMV), 10 (13\%) had Ebstein barr virus (EBV) in blood and 4 (5\%) were found to have adenovirus on peripheral blood PCR analysis. In FBA cohort, 18 patients (40\%) had CMV reactivation, 16 patients (35\%) had EBV viremia and 2 (4\%) had adenoviremia. On chi square analysis, there was no statistically significant difference in incidence of CMV viremia between the two groups $(p=0.08)$. FBA cohort had a higher incidence of EBV viremia which was statistically significant $(P=0.017)$. Median PBL and CD3 chimerism was $100 \%$ and $95 \%$ at 3 months for both groups. Primary graft rejection was seen in only one patient in FMC cohort. There were no graft failures in FBA group. Pre-emptive DLI was given in $27 \%$ patients in FMC group whilst $24 \%$ received DLI in FBA cohort. This difference was not statistically significant. In both groups there was no statistically significant difference in overall survival (OS) in patients who reactivated CMV and those who did not have CMV reactivation. Non relapse mortality (NRM) at 2 years in FMC cohort was $24 \%$ in FMC and $41 \%$ in FBA cohort. Estimated OS at four years using kaplan meier analysis in FMC group was 58\% and in FBA group it was $37 \%$.

\begin{tabular}{lcc}
\hline & $F M C$ & $F B A$ \\
\hline Median age & $59 \begin{array}{c}\text { (range } 41-71 \\
\text { years) }\end{array}$ & $\begin{array}{c}58 \text { (range } 35-71 \\
\text { years) }\end{array}$ \\
Male/Female & $44(60 \%) / 29(40 \%)$ & $32(71 \%) / 13(29 \%)$ \\
Median follow up & 19 (range & 21 (range 1-58) \\
HCT-Cl & $4-49$ months) & $1(0-5)$ \\
CR at HSCT & $2(1-5)$ & $45(100 \%)$ \\
MUD & $65(89 \%)$ & $35(77.7 \%)$ \\
Sibling & $49(67 \%)$ & $10(22.3 \%)$ \\
Mismatch $(9 / 10$ or & $24(33 \%)$ & $3(6.6 \%)$ \\
less) & $5(7 \%)$ & $31 / 14$ \\
AML/MDS & $64 / 9$ & \\
\hline
\end{tabular}

Conclusion: Our retrospective data show that Alemtuzumab based conditioning regimens in myeloid disorders have acceptable morbidity despite a high incidence of viremia, very low risk of graft rejection and comparable overall survival to the conditioning regimens utilizing ATG as in vivo T-cell depletion strategies. There appears to be favourable NRM for FMC when compared to FBA.

Disclosure of Interest: None declared.

P603

Treosulfan-based conditioning regimen for allogeneic stem cell transplantation in adult patients with hematological malignancies is non toxic, but not myeloablative

N. Popova, , E. Parovichnikova ${ }^{1}$, L. Kuzmina ${ }^{1}$, V. Vasilyeva ${ }^{1}$, T. Sorokina ${ }^{1}$, M. Drokov', E. Mikhaltsova ${ }^{1}$, O. Koroleva ${ }^{1}$, D. Dubnyak', V. Savchenko ${ }^{\prime}$ on behalf of CIC 930

${ }^{1}$ Bone morrow transplant department, National Research center for Hematology, Moscow, Russian Federation

Introduction: For many decades the conditioning regimen consisting of busulfan and cyclophosphamide (allo-HSCT) (Santos GW, Tutschka et al., 1983) is one main of myeloablative conditioning regimens (MAC).

As has been shown before the rate of transplanted-related mortality as a result of early complications such as VOD, toxicity after MAC is still high. However alternative regimens now available and allowed to reduce toxicity and at the same time get a favorable outcome, e.g. the use of treosulfan. (Brigit Greystoke, Sonia Bonanomi at al., 2007).

We studied conditioning regimen with treosulfan as myeloablative, but reduced toxicity regimen for allo-HSCT in adult patients.

Material (or patients) and methods: Between 2013-2015y.y. 18 patients with different hematological malignancies were included in the study. There were $8 \mathrm{M}$ and $10 \mathrm{~F}$ with a median age of 27 years (19-40). 9 patients were diagnosed with AML, 6 patients - with ALL, 2 patients with myeloproliferative neoplasms and myelodysplastic syndrome in 1 patient.

In the AML group 5 patients had normal karyotype and 4 patients cytogenetic abnormalities were detected (intermediate and high risk). Whereas in the ALL group only 1 patient 
had a normal karyotype and 4 patients were detected with $\mathrm{Ph}$ + and 1 patient had an aberrant MLL gene.

All patients underwent conditioning with treosulfan $36 \mathrm{~g} / \mathrm{m}^{2}$ and cyclophosphamide $120 \mathrm{~g} / \mathrm{m}^{2}$ followed allo-HSCT (MRD $n=7$, MUD $n=6$, mismatch MUD $n=5$ ). There was the second transplantation after graft rejection in 2 cases. As stem cell source, there were BM in 13 cases, PB - in 5 cases.

Graft-versus-host disease (GvHD) prophylaxis consisted of the the standard protocol consisting with cyclosporine A+methotrexate / mofetil mycophenolate.

Results: No major toxicities were registered in the study group.

On day +30 full donor chimerism was detected only in 12 patients (66\%). Later the mixed chimerism was observed in 3 cases out of these 12. So graft rejection (GR) was established. We considered for GR 50\% and more of recipient haemopoiesis. We administered $\mathrm{DLI}$ to insure donor chimerism and prevent relapse.

On day +90 GR was diagnosed in 8 cases $(44,4 \%)$. The relapse occurred in $3(16,7 \%)$ cases (hematologic $n=2$, extramedullary $n=1) .3$ patients died due to progression of the disease $(n=1)$ and infections $(n=2)$.

At the time of analysis 15 patients are still alive, and complete donor chimerism is detected only in 6 patients.

Conclusion: Though the study is small, treosulfan-containing regimen is accompanied by high risk of $\mathrm{GR}(47,1 \%)$ in patients with hematological malignancies. The frequency of relapse is not so evident $(17,6 \%)$.

This regimen approach should be further tested. The lowtoxicity effects were proved in many studies, so probably treosulfan-conditioning program as low-toxicity and nonmyeloablative regimen should be tested in elderly patients and patients with significant co-morbidities who are not eligible for allo-HSCT with MAC.

Disclosure of Interest: None declared.

\section{P604}

What is the optimal conditioning regimen for patients with chemosensitive Hodgkin's lymphoma undergoing autologous stem cell transplantation? A retrospective comparison of single-agent high-dose melphalan and BEAM

P. Kaloyannidis ${ }^{1, *}$, S. Kanfar ${ }^{1}$, J. Apostolidis ${ }^{2}$, K. Azar ${ }^{1}$, W. Anzi ${ }^{1}$, M. Al Jamily" ${ }^{1}$ S. Gigantes ${ }^{2}$, I. Baltadakis', D. Karakasis', A. Al Shaghier ${ }^{\prime}$, K. Al Anezi ${ }^{1}$, N. Harhalakis ${ }^{2}$, H. Al Hashmi ${ }^{1}$

${ }^{7}$ Adult Hematology and SCT, King Fahad Specialist Hospital, Dammam, Saudi Arabia, '2Department of Hematology, BMT Unit, Evangelismos Hospital, Athens, Greece

Introduction: High-dose chemotherapy and autologous stem cell transplantation (ASCT) is the treatment of choice for selected patients (pts) with relapsed/refractory Hodgkin lymphoma $(\mathrm{HL})$. Though the most widely used conditioning regimen, it still remains questionable if BEAM represents the optimal standard of care. Single-agent high-dose melphalan (HDM): $200 \mathrm{mg} / \mathrm{m}^{2}$ has been used by some centers, demonstrating sufficient efficacy, acceptable toxicity and costeffectiveness. However, the overall experience is limited and so far there are no data comparing BEAM with HDM.

Material (or patients) and methods: We retrospectively analyzed the medical records from HL-pts with chemosensitive disease pre-ASCT [complete (CR) or very good partial remission (VGPR)], autografted in two different institutions, using either $\operatorname{HDM}(n=17 \mathrm{pts})$ or BEAM ( $n=17 \mathrm{pts})$ and compared the results in terms of efficacy and toxicity. For the statistical analyses the chi-square, the T-test and the Kaplan-Maier method were used. The two groups of pts were selected to be similar regarding age ( 45 vs.36ys $P=\mathrm{ns}$ ), sex ( 8 vs.7 females and 9 vs. 10 males) and disease status before ASCT ( 6 vs. 8 in CR and 11 vs. 9 in VGPR). The pts in the BEAM-group had been salvaged with a median of $3(2-8)$ cycles of either ESHAP $(n=10)$, mini-BEAM $(n=2)$, Gemcitabine-based $(n=2)$, while the pts in the HDM-group had previously received a median of $4(2-6)$ cycles of either $\operatorname{ESHAP}(n=13)$, DICEP $(n=7)$, ICE $(n=2)$, or Brentuximab vedotin $(n=2)$ as salvage regimens. The median number of the infused CD34+ cells were $5,7 \times 10^{6} / \mathrm{kg}$ for the HDM-group and $9,2 \times 10^{6} / \mathrm{kg}$ for the BEAM-group $(P=0,008)$.

Results: All pts engrafted, the median day for neutrophils $>1000 / \mathrm{mm}^{3}$ was 12 vs.16 $(P<0,001)$ and for platelets $>25000 / \mathrm{mm}^{3} 14$ vs.10 $(P=0,05)$ for the HDM- and BEAMgroup, respectively. The 4-ys overall survival and progression free survival were similar, $67 \%$ and $63 \%$ respectively, for both groups. No treatment related mortality (TRM) was noticed in the BEAM-group, while in the HDM-group 1/17 died due to TRM (MERS-CoV pneumonia). No other organ or tissues toxicities (WHO grade $\geq 3$ ) were observed with both regimens. Interestingly, for the HDM-group the median total hospitalization period (including regimen administration) was 17(14-48) days and compared favorable to the required total hospitalization period for the BEAM-regimen 22(16-31) days.

Conclusion: In conclusion, this study, though retrospective and with a small series of pts, demonstrates that for HL-pts with chemosensitive disease before ASCT, HDM as compared to the standard BEAM regimen, showed similar efficacy, no grade $\geq 3$ regimen-related toxicity (organ/tissue) and acceptable TRM. Moreover, the significant shorter periods in terms of neutrophils-recovery and hospitalization, may contribute to a better cost effectiveness for the HDM-regimen compared to multi- agents conditioning regimens.

References: Stewart D. A Guo D, Sutherland JA et al. Singleagent high-dose melphalan salvage therapy for Hodgkin'sdisease: Cost, safety, long term efficacy. Ann Oncol 1997; 8:1277-1279. Magagnoli M, Castagna L, Bramanti S et al. Single-agent highdose melphalan followed by peripheral blood stem cell (PBSC) in lymphoma patients: an effective and well tolerated conditioning regimen. Bone Marrow Transplant 2004;33: 1067-1068.

Disclosure of Interest: None declared.

\section{P605}

Myeloablative conditioning and CD34+ selection results in higher chronic-GVHD free/relapse free-survival compared to reduced-intensity conditioning and unmodified graft in patients with AML and MDS $>\mathbf{5 0}$ years receiving

HLA-matched allogeneic HCT

P. Barba ${ }^{1,2, *}$, R. Martino ${ }^{3}$, Q. Zhou ${ }^{2}$, C. Cho $^{2}, H$. Castro-

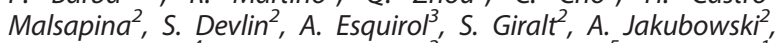
L. Lopez-Corral', E. Papadopoulos' ${ }^{2}$ J. L. Pinana ${ }^{5}$, M. L. Fox ${ }^{\prime}$, D. Valcarcel', C. Solano , D. Caballero ${ }^{4}$, J. Sierra ${ }^{3}$, M.-A. Perales ${ }^{2}$ ${ }^{1}$ Hospital Universitari Vall Hebron, Barcelona, Spain, ${ }^{2}$ Memorial Sloan Kettering Cancer Center, New York, United States, ${ }^{3}$ Hospital de la Santa Creu i Sant Pau, Barcelona, ${ }^{4}$ Hospital Universitario Salamanca, Salamanca, ${ }^{5}$ Hospital Clínico Universitario-INCLIVA, University of Valencia, Valencia, Spain

Introduction: Allogeneic hematopoietic cell transplantation (allo-HCT) with reduced-intensity conditioning (RIC) and t cell depletion (TCD) by CD34+ selection are two strategies used to reduce transplant related mortality (TRM) and morbidity in older patients. However, these strategies have not been compared before.

Material (or patients) and methods: All consecutive patients $>50$ years with acute myeloid leukemia (AML) and myelodysplastic syndrome (MDS) in complete remission receiving 8/8 HLA matched related or unrelated donors TCD at Memorial Sloan Kettering Cancer Center or RIC in a Spanish consortium of 4 centers using the same protocol between 2005 and 2014 were included. TCD patients received a myeloablative conditioning (MAC) with or without $\mathrm{TBI}$ and no post-HCT immunosuppression. RIC patients received fludarabine-based conditioning either with busulfan $(8 \mathrm{mg} /$ $\mathrm{kg}$ ) or melphalan $(140 \mathrm{mg} / \mathrm{m} 2)$ and GVHD prophylaxis consisting of a calcineurin-inhibitor in combination with another drug. Main transplant outcomes including chronic-GVHD-free/ relapse-free survival (CRFS) were evaluated.

Results: A total of 356 patients were included (204 TCD, 152 RIC) with a median age of 61 (range 50-73) and 60 (range 50-71) 
RFS by Transplant Approach and $\mathrm{HCT}-\mathrm{Cl}$

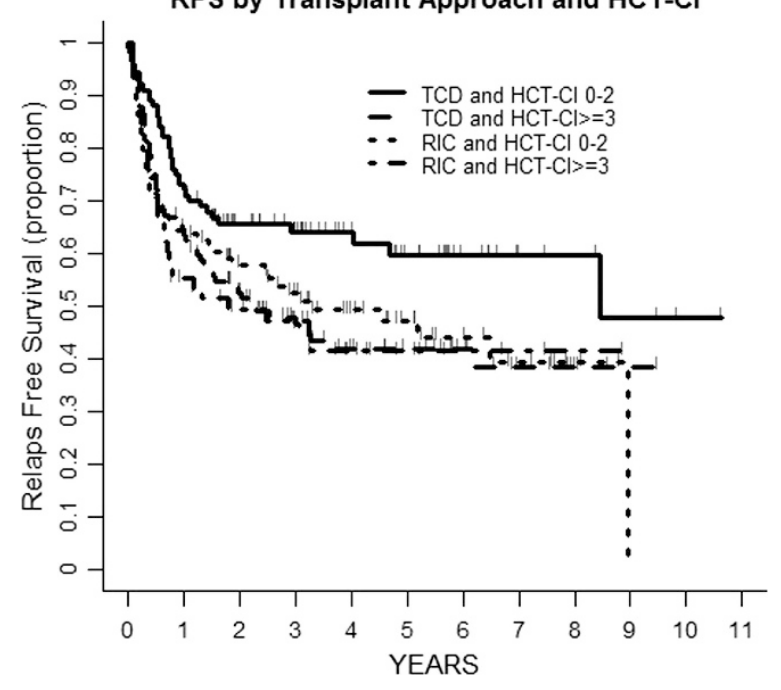

years, respectively. The TCD cohort had more unrelated donors $(60 \%$ vs. $32 \%, P<0.001)$ and higher comorbidity burden (HCT-CI $>=351 \%$ vs. $38 \%, P<0.001)$ than the RIC cohort. There were no additional differences between cohorts including donor/ recipient gender, type of diagnosis, disease status, Disease Risk Index and follow-up time. Main transplant outcomes are summarized (Table). The TCD cohort had less grade 2-4 acute GVHD, less chronic GVHD and higher CRFS than the RIC cohort. In the analysis of risk factors the use of TCD (vs. RIC) was associated with lower relapse risk (HR $0.5[95 \% \mathrm{Cl} 0.3-0.8] P=0.001)$ and higher CRFS (HR $0.3\left[\begin{array}{ll}95 \% & 0.2-0.4]\end{array} P<0.001\right.$ ). There were no statistical differences between both approaches in terms of TRM, relapse-free survival (RFS) and overall survival (OS). When the transplant approach was analyzed according to the comorbidity burden, TCD patients with $\mathrm{HCT}-\mathrm{Cl}=0-2$ showed higher RFS $(P=0.04)$ (Figure), lower relapse $(P=0.009)$ and higher CRFS $(P<0.001)$ than TCD patients with HCT-Cl $>=3$ and RIC patients ( $\mathrm{HCT}-\mathrm{Cl}=0-2$ and $\mathrm{HCT}-\mathrm{Cl}>=3$ ).

Table. Patients' outcomes according to transplant approach

\begin{tabular}{|c|c|c|c|}
\hline Outcome & $\begin{array}{c}\text { CD34+ selec- } \\
\text { tion } \%(95 \% \\
\text { Cl) }\end{array}$ & RIC \% (95\%Cl) & P-value \\
\hline 3-year RFS & $55(48-62)$ & $51(42-58)$ & 0.13 \\
\hline 3-year OS & $58(51-65)$ & $56(48-64)$ & 0.29 \\
\hline $\begin{array}{l}\text { 3-year } \\
\text { relapse }\end{array}$ & $19(14-25)$ & $33(25-41)$ & 0.001 \\
\hline 3-year TRM & $26(20-32)$ & $17(10-23)$ & 0.1 \\
\hline $\begin{array}{l}\text { 180- day } \\
\text { grade } 2-4 \\
\text { aGVHD }\end{array}$ & $18(12-23)$ & $46(38-54)$ & $<0.001$ \\
\hline $\begin{array}{l}\text { 3-year } \\
\text { cGVHD }\end{array}$ & $6(3-9)$ & $55(47-63)$ & $<0.001$ \\
\hline $\begin{array}{l}\text { 3-year } \\
\text { CRFS }\end{array}$ & $51(44-58)$ & $7(4-13)$ & $<0.001$ \\
\hline
\end{tabular}

Conclusion: The use of CD34+ selected graft and a myeloablative conditioning is associated with higher CRFS and at least similar RFS and OS compared to unmodified allo$\mathrm{RIC}$ in patients $>50$ years with AML and MDS. This approach is being explored prospectively in a randomized phase 3 trial (BMT CTN 1301).

Disclosure of Interest: None declared.
P606

Pharmacokinetics of fludarabine in paediatric patients undergoing stem cell transplantation

P. J. Shaw ${ }^{1, *}$, M. Gabriel ${ }^{1}$, S. Keogh ${ }^{1}$, S. Lee ${ }^{2}$, C. Nath ${ }^{2}$

${ }^{1}$ BMT Service, 'Biochemistry, Children's Hospital at Westmead, Sydney, Australia

Introduction: Fludarabine phosphate is commonly used as part of conditioning regimens in paediatric stem cell transplantation. There is little data available on how to best dose paediatric patients, especially in children weighing less than $10 \mathrm{~kg}$.

Material (or patients) and methods: The clinical pharmacokinetics of fludarabine phosphate was evaluated by studying the active metabolite, (9B-D-arabinosyl-2 fludarabine, F-Ara-A). Eighty six transplants were studied in 84 children 0.1 to 19 years. Fludarabine was used with busulphan in 63 transplants. A weight-based dose of 1 to $2 \mathrm{mg} / \mathrm{kg}$ was used in 13 transplants where the patient weighed $<11 \mathrm{~kg}$, whilst 73 children received surface area-based doses of 25 to $50 \mathrm{mg} / \mathrm{m}^{2}$. Fludarabine was infused over 0.4 to $2 \mathrm{hrs}$. F-Ara-A concentrations were measured in 5 to 8 blood samples collected over timed intervals after the infusion. Pharmacokinetic parameters, including area under the concentration curve (AUC) and clearance $(\mathrm{CL})$ were determined using trapezoidal rule implemented by the Kinetica (4.0) software (Innaphase, USA). Dose group (25, 30 or $40 \mathrm{mg} / \mathrm{m}^{2}$ ) was plotted against median AUC values to determine linearity of the pharmacokinetics. Individual clearance values were used to predict AUC for a $25 \mathrm{mg} / \mathrm{m}^{2}$ dose and the median and interquartile range were determined and tested for correlation with age, weight and GFR. Patients with low $\left(<25^{\text {th }}\right.$ percentile), target ( $25^{\text {th }}$ to $75^{\text {th }}$ percentile), and high ( $>75^{\text {th }}$ percentile) exposure were also compared with respect to age, weight and glomerular filtration rate (GFR), using the Kruskal-Wallis Test.

Results: Clearance (CL) of F-Ara-A ranged from $0.3 \mathrm{~L} / \mathrm{h}$ to $17.8 \mathrm{~L} / \mathrm{h}$, with median $5.1 \mathrm{~L}$.h and an interquartile range: 2.8 $-8.3 \mathrm{~L} / \mathrm{h}$. Dose $\left(\mathrm{mg} / \mathrm{m}^{2}\right)$ was linearly related AUC $(\mathrm{mg} / \mathrm{L} . \mathrm{h})$ according to the equation $A U C=0.1306$.dose $\left(r^{2}=0.997\right)$. The predicted AUC for a $25 \mathrm{mg} / \mathrm{m}^{2}$ dose was median $4.1 \mathrm{mg} / \mathrm{L} . \mathrm{h}$, interquartile range:3.6-4.9 mg/L.h, and was not found to correlate significantly with age, weight or body surface area. There was, however, a significant negative correlation with glomerular filtration rate $(r=-0.31 P<0.005)$, so patients with low GFR tend to have high predicted AUC values. Patients predicted to have low or high exposure for the $25 \mathrm{mg} / \mathrm{m}^{2}$ dose had significantly altered GFR $(P<0.05)$, but there was no difference in weight or age.

Conclusion: The linear relationship between dose $/ \mathrm{m}^{2}$ and exposure in the clinically important dose range of 25 to $40 \mathrm{mg} / \mathrm{m}^{2}$ is reassuring. Unlike Busulphan, on our data to date, surface area-based dose provided equivalent exposure of the fludarabine metabolite for children of all weights, including the young babies. There was no obvious cut off where weight rather than surface area should be used to calculate dose to avoid overexposure. Renal function also affects the clearance and is one reason why some children have low or high exposure. Further data is needed, combined with evaluation of pharmacodynamics, to define any role for real-time monitoring in this patient group.

Disclosure of Interest: None declared.

\section{P607}

Treosulfan-based conditioning regimen for autologous and allogeneic hematopoietic stem cell transplantation for Neuroblastoma

P. Merli ${ }^{1, *}$, A. Bertaina ${ }^{1}$, L. Strocchio ${ }^{1}$, B. Lucarelli ${ }^{1}$, A. Castellano ${ }^{1}$, A. Serra ${ }^{1}$, A. Cacchione , G. M. Milano ${ }^{1}$, L. De Sio ${ }^{1}$, M. D. De Pasquale', D. Pagliara', A. Mastronuzzi ${ }^{1}$, F. Locatelli ${ }^{1}$

${ }^{1}$ Hemato-Oncology, Bambino Gesù Children's Hospital, Rome, Italy

Introduction: Autologous hematopoietic stem cell transplantation (auto-HSCT) has a well-defined indication in the 
treatment of neuroblastoma; on the contrary, the role of allogeneic HSCT (allo-HSCT) for this disease is still debated, mainly due to the reported high rates of transplant related mortality (TRM). Moreover, in both settings, data comparing different conditioning regimens are lacking.

The alkylating agent Treosulfan (L-threitol-1,4-bis-methanesulfonate; dihydroxybusulfan) (Treo) has shown promising in vitro results against neuroblastoma cell lines. Furthermore, a favorable toxicity profile makes this drug attractive in the transplant setting.

Material (or patients) and methods: We retrospectively analyzed the data about neuroblastoma patients who underwent a Treo-based conditioning regimen for either auto- or allo-HSCT between July 2010 and February 2015 at IRCCS Ospedale Pediatrico Bambino Gesù in Rome.

Results: Ten high-risk patients received a Treo-based conditioning, 5 as preparative regimen for an auto-HSCT and 5 for an allo-HSCT (4 from a Matched Unrelated Donor and 1 from an HLA-identical sibling). Median age at diagnosis was 3.5 years (range 1.7-7.2). All patients but one had metastatic disease at diagnosis and had previously undergone an auto-HSCT with a busulfan-based conditioning regimen. Four children were in complete remission at time of Treo-based transplantation, while 2 were in very-good partial response and 4 had partial response. Median age at time of HSCT was 5.85 years (range 3.1-14.6). In all cases Treo was administered at the dose of $42 \mathrm{mg} / \mathrm{m}^{2}$ in 3 days, combined with Melphalan $\left(110 \mathrm{mg} / \mathrm{m}^{2}\right)$ for 7 patients, with Cyclophosphamide $(120 \mathrm{mg} / \mathrm{kg}$ in 2 days) for 2 patients, and with both Cyclophosphamide and Melphalan (100 mg/kg in 2 days and $110 \mathrm{mg} / \mathrm{m}^{2}$, respectively) for 1 patient. Rabbit Anti-Thymocyte Globulins (ATG Fresenius) were administered in MUD-HSCT recipients. Treo-based conditioning was well tolerated in most cases. More frequent side effects (detailed in Table I) were cutaneous toxicity, mucositis and infectious complications. No cases of veno-occlusive disease were recorded. Two patients (1 undergoing auto-HSCT and 1 allo-HSCT) died of transplant-related complications: one because of idiopathic pneumonia and the other (the patient who received Treo associated with both Cyclophosphamide and Melphalan) due to multiorgan failure. Interestingly, these two patients were those with the highest tumor burden in the cohort; in fact, both were in partial response at time of transplantation, with 5 and 10 areas of uptake at MIBGscintigraphy, respectively. Of the 4 patients at risk, 1 developed Grade II intestinal GVHD. With a median follow-up of 315 days, 7 out of 10 patients are alive, with a good performance status.

Conclusion: Treosulfan, associated with either Melphalan or Cyclophosphamide, seems a reliable option for both tandem auto- and allo-HSCT. TRM seems related to the tumor burden at the time of transplantation.

References: Lanvers-Kaminsky C, Bremer A, Dirksen U, Jürgens $\mathrm{H}$, Boos J. Cytotoxicity of treosulfan and busulfan on pediatric tumor cell lines. Anticancer Drugs. 2006; 17(6):657-62.

Disclosure of Interest: None declared.

\begin{tabular}{|lcc|}
\hline & Any Grade & $\geq$ Grade 3 \\
\hline Mucositis & $8(80 \%)$ & $2(20 \%)$ \\
Cutaneous & $6(60 \%)$ & $1(10 \%)$ \\
Infective & $5(50 \%)^{*}$ & $5(50 \%)^{*}$ \\
Hepatic & $6(60 \%)$ & 0 \\
Pulmonary & $1(10 \%)$ & $1(10 \%)$ \\
Renal/Bladder & 0 & 0 \\
Cardiovascular & 0 & 0 \\
Neurological & 0 & 0 \\
$*$ 4 febrile neutropenias; 1 Candida krusei sepsis & \\
\hline
\end{tabular}

P608

Low-dose antithymocyte globulin, post-transplant cyclophosphamide and sirolimus as graft-versus-host disease prophylaxis in unrelated donor transplants

R. Greco ${ }^{1, *}$, L. Crucitti ${ }^{2}$, L. Vago ${ }^{1,3}$, N. Cieri ${ }^{2,4}$, F. Giglio ${ }^{1}$, M. T. Lupo Stanghellini ${ }^{1}$, M. Morelli ${ }^{1}$, M. C. Barbanti ${ }^{1}$, S. Piemontese ${ }^{1}$, T. Perini ${ }^{7}$, E. Sala ${ }^{1}$, F. Pavesi ${ }^{1}$, C. Messina ${ }^{1}$, R. Milani ${ }^{\prime}$, M. Marcatti ${ }^{1}$, S. Marktel ${ }^{1}$, M. Carrabba ${ }^{1}$, M. Bernardi ${ }^{1}$, C. Corti ${ }^{7}$ A. Assanellii ${ }^{1}$, C. Bonini ${ }^{4}$, F. Ciceri ${ }^{1}$, J. Peccatori ${ }^{1}$

${ }^{1}$ Unit of Hematology and Bone Marrow Transplantation, IRCCS San Raffaele Scientific Institute, ${ }^{2}$ Università degli Studi di Milano, ${ }^{3}$ Unit of Immunogenetics, Leukemia Genomics and Immunobiology, ${ }^{4}$ Experimental Hematology Unit, ${ }^{5}$ Immunohematology and Transfusion Medicine Unit, IRCCS San Raffaele Scientific Institute, Milano, Italy

Introduction: Graft-versus-host disease (GvHD) still represents a major complication after allogeneic hematopoietic stem cell transplantation (allo-HSCT). We investigated whether the incorporation of low dose anti-thymocyte globulin (ATG) to PTCy and sirolimus GvHD prophylactic approach, may alleviate some of the increased alloreactivity associated with unrelated volunteer donor (URD) transplants.

Material (or patients) and methods: We evaluated a cohort of 21 haematological patients receiving URD allo-HSCT in the San Raffaele BMT Unit, from 2013 to 2015. All subjects had high-risk hematologic malignancies, most commonly myeloid diseases (52\%). A total of 11 patients had active disease at $\mathrm{HSCT}$, while 9 were in hematologic remission. According to disease risk index defined by Armand et al 9 patients were high risk, 10 intermediate and 2 low. Myeloablative conditioning consisted of treosulfan $(14 \mathrm{~g} / \mathrm{m} 2 /$ day) on days -6 to -4 , fludarabine $(30 \mathrm{mg} / \mathrm{m} 2 /$ day) on days -6 to -2 , and melphalan ( $70 \mathrm{mg} / \mathrm{m} 2 /$ day) on days -2 and -1 , followed by T-replete G-CSF-mobilized PBSCs $(n=19)$ or BM grafts $(n=2)$. Whether 12 patients received stem cells from HLA- matched URD (10/10 HLA matched), the remaining 9 had one-locus HLA-mismatched. Patients received ATG-Fresenius $5 \mathrm{mg} / \mathrm{kg}$ per day IV on day -4 to -2 . In vivo B-cell depletion was obtained by rituximab (i.v. $375 \mathrm{mg} / \mathrm{m} 2$ given as a single dose on day -1). Postgrafting immunosuppression consisted of PTCy $(50 \mathrm{mg} /$ $\mathrm{kg} /$ day) on days 3 and 4 . Sirolimus was given orally from day 5 to sixth month after HSCT. Mycophenolate mofetil (MMF) was initiated orally at $10 \mathrm{mg} / \mathrm{kg}$ t.i.d. on day 5 , and withdrawn on day 30 after HSCT.

Results: Median follow up was 247 days (range 29-486) from HSCT. All patients were engrafted in a median time of 17 days (range, 14-51 days), with full donor chimerism achieved by day 30. Poor graft function was observed in 11 patients. Post-HSCT recovery of lymphocyte subsets was lengthy as compared to other unmanipulated $\mathrm{HSCT}$, with a median time to $\mathrm{CD} 3>100 / \mathrm{ml}$ of 41 days (range, 22-389 days) by flow-cytometry, while 5 patients never reached immune reconstitution.

All except one developed febrile neutropenia during aplasia. We observed a high rate of serious infections: severe pneumonia in 7 cases ( 5 required NIV, 4 were admitted to ICU), CMV reactivation in 13 (3 CMV disease), HHV6 reactivation in 10 , BK virus cystitis in 2 . Although the overall mortality associated with these complications was limited, as evidenced by a favourable NRM, it caused significant morbidity in patients and often required aggressive therapies, potentially contributing to poor graft function. The incidences of acute and chronic GVHD were low. Grade II-IV acute GvHD was reported in $10 \%$ patients, while 5 patients developed chronic GvHD (mild in 4 cases, only one moderate).

Non-relapse mortality (NRM) at 100 days and 1 year was $4.8 \%$ and $20.7 \%$, respectively. The estimated 1 -year overall survival (OS) and relapse incidence were $51 \%$ and $22.5 \%$ respectively.

Conclusion: Our study suggests that URD allo-HSCT with ATG, PTCy and sirolimus-based GvHD prophylaxis, can be very effective in preventing GvHD; however a delayed immune 
reconstitution and high rate of infectious complications are observed warranting further clinical-biological studies.

Disclosure of Interest: None declared.

\section{P609}

Reduced-Intensity Busulfan or TBI/Cyclophosphamide/

Fludarabine-Based Conditioning for Allogeneic

Hematopoietic Stem Cell Transplantation in Children with Acquired Severe Aplastic Anemia

R.-J. Sun ${ }^{1, *}$, T. Wu ${ }^{1}$, M. Xiong ${ }^{1}$, Z.-J. Wei ${ }^{1}$, Y.-L. Zhao ${ }^{1}, X^{\prime}-$ Y. Cao ${ }^{1}$, D.-Y. Liu', Y. ${ }^{1} u^{1}$, J.-P. Zhang ${ }^{1}$, S.-Q. Ji', D.-P. Lu ${ }^{1}$

${ }^{1}$ Department of Bone Marrow Transplantation, Hebei Yanda

Ludaopei Hospital, Langfang, China

Introduction: Clinical outcomes of allogeneic hematopoietic stem cell transplantation (HSCT) for severe aplastic anemia (SAA) have been improved with the conditioning including fludarabine, low-dose cyclophosphamide and antithymocyte globulin (ATG). But graft rejection is around 17\% in unrelated transplant (URT). We added either low-dose busulfan or total body irradiation (TBI) into original regimen in certain patients in order to reduce graft rejection and improve survival. In present study, the outcomes with our new conditioning in pediatric SAA were analyzed retrospectively.

Material (or patients) and methods: Between September 2012 and August 2015, total 38 children with acquired SAA underwent HSCT in our hospital were included. The median disease course was 18 (1-91) months. Six cases received one course of ATG before transplant. The donors are identical sibling in 1 , unrelated in 23 and haploidentical in 14 . Conditioning consisted of busulfan (pediatric standard dose) for 2 days (26 cases) or TBI 200 cGy (7 cases); fludarabine $\left(30 \mathrm{mg} / \mathrm{m}^{2}\right)$ for 4 days; cyclophosphamide $\left(500 \mathrm{mg} / \mathrm{m}^{2}\right)$ for 4 days; and either ATG (36 cases, Thymoglobuline, $7.5 \mathrm{mg} / \mathrm{kg}$ or ATG-F, $20 \mathrm{mg} / \mathrm{kg}$ ) or alemtuzumab (2 cases, $1 \mathrm{mg} / \mathrm{kg}$ ). For GVHD prophylaxis, CsA/tacrolimus, MMF and MTX were used in patients received ATG; CsA/tacrolimas alone was used in patients received alemtuzumab.

Results: All patients had durable engraftment. The median time to neutrophil engraftment was 14 (11-19) days; the median time to platelet engraftment was 12 (5-35) days. Two of 38 patients (5.3\%) developed grade III-IV aGVHD. Fifteen cases (39.5\%) had cGVHD (12 in limited, 3 in extensive). Two patients died from intracerebral hemorrhage, fungal pneumonia at 9 months, 22 months post-HSCT, respectively. With a median follow-up 17 (4-36) months, overall survival was 94.7\%. Conclusion: With our new conditioning, a favorable outcome of HSCT for pediatric acquired SAA has been achieved with no graft rejection and much better survival even in this transplant setting mainly with alternative donors.

Disclosure of Interest: None declared.

\section{P610}

Oral Fludarabine shows similar efficacy in transplant outcome as intravenous fludarabine when used as part of FMC RIC conditioning

S. Easdale ${ }^{1}$, S. Wigfall ${ }^{1}$, A. Holyome ${ }^{1}$, V. Soni ${ }^{1}$, J. Brennan ${ }^{1}$, M. Ethell ${ }^{1}$, M. Potter ${ }^{1}$, C. Anthias,"

${ }^{1}$ Haematology, Royal Marsden Hospital, London, United Kingdom

Introduction: Increasing numbers of patients require reduced intensity conditioning regimens for haematopoietic progenitor cell (HPC) transplants for haematological malignancies, of which the most commonly used in the UK, is fludarabine-melphalan campath (FMC). In 2013 our centre introduced oral (PO) fludarabine in place of intravenous (IV) fludarabine. Here we compare the efficacy and cost saving between the two regimens. Material (or patients) and methods: Data were collected from 181 patients who received FMC conditioning with $50 \mathrm{mg} /$ $\mathrm{m}^{2}$ oral fludarabine (93 patients) or $30 \mathrm{mg} / \mathrm{m}^{2}$ IV fludarabine (88 patients) daily from day-7 to day-3 prior to HPC transplantation between January 2011 and May 2015. Patients receiving IV fludarabine and PO fludarabine were admitted on day- 7 and day -2 respectively prior to stem cell infusion. The patients were diagnosed with a range of haematological malignancies, with acute myeloid leukaemia accounting for $47.5 \%$ of the total number of patients.

Patient characteristics between the two groups were comparable with median age of the patients being 56.6 years and 54.9 years in the IV and PO groups respectively. The percentage of sibling allografts compared to MUD allografts was similar in each group, (19 in the IV group and 20 in PO group respectively) $P$-value 0.88 .

Day 100 mortality, graft failure and time to engraftment were compared and a cost analysis was performed on both groups. Results: Median time to engraftment was 19 days in the IV group and 17 days in the PO group, $P$-value 0.594 . Day 100 mortality for the IV fludarabine and PO fludarabine was 11.3 and $9.6 \%$ respectively ( $P$-value 0.0946$)$. The rates of primary graft failure were similar in both arms; $2.27 \%$ (IV) and $3.22 \%$ (PO), $P$ value 0.696 .

The second aim of the study was to determine cost saving benefits. On average each patient in the oral fludarabine group remained at home for 5 days longer than those in the IV group (a total of 465 bed days saved). Each night stay in our centre costs approximately $£ 420$, making a significant cost saving per patient having oral fludarabine.

Conclusion: Our data show, that IV fludarabine can be replaced with oral fludarabine with no significant difference in outcome following HPC with respect to engraftment data and results in a saving of 5 hospital days per patient. Our findings are consistent with previously published data ${ }^{[1,2}$ and ${ }^{3]}$ using oral fludarabine in other conditioning regimens.

References: 1 -Velázquez-Sánchez-de-Cima et al. Oral versus intravenous fludarabine as part of a reduced-intensity conditining for allogenic stem cell transplantation. Acta Haematol 2014; 132:125-128.

2 - Delago J, Marco et al. Reduced Intensity Conditioning allogenic stem cell transplantation using oral fludarabineas part of the conditioning regime. Cytotherapy 2009; 11:356-361. 3 - P A von dem Borne et al. Comparable engraftment and chimerism kinetics using oral and intravenous fludarabine as part of a reduced intensity conditioning regime. Bone Marrow Transplant 2008:42:137-138.

Disclosure of Interest: None declared.

P611

Epigenetic priming with 5-Azacytidine prior to allogeneic stem cell transplantation for myeloid malignancies with in vivo T-cell depletion

S. A. Mayer ${ }^{1, *}$

${ }^{1}$ Medicine, Weill Cornell Medical College, New York, United States

Introduction: Relapse is the main cause of death in allogeneic stem cell transplant for myeloid malignancies. This study aims to increase the sensitivity of the malignancy to the conditioning regimen without increasing toxicity by pretreating with azacytidine, an approach investigated for lymphoma and AML.(1, 2). Material (or patients) and methods: Adults with poor risk AML, MDS or MPN, bone marrow blasts $<=5 \%$, and adequate organ function and performance status were eligible. Priming: Azacytidine $75 \mathrm{mg} / \mathrm{m} 2 / \mathrm{d} \times 5$, days -11 to -7 Conditioning: Fludarabine $40 \mathrm{mg} / \mathrm{m} 2 / \mathrm{d}$, days -6 to -3 Melphalan $140 \mathrm{mg} / \mathrm{m} 2$, day-3.

GVHD - prophylaxis Alemtuzumab $20 \mathrm{mg} / \mathrm{d} \times 3$ to 5 days Tacrolimus for 3-6 months post transplant

Graft: GCSF- mobilized PBSCs from either a sibling (MRD) or unrelated donor (MUD), matched for HLA at loci $A, B, C, D R$ and $\mathrm{DQ}$.

Primary objectives were overall survival (OS) and progression free survival (PFS)

Secondary objectives were incidence of GVHD and transplant related mortality (TRM).

Statistics: Kaplan Meier method for survival and cumulative incidence for competing risks of GVHD- Relapse/death and TRM - relapse. 
Results:

Table: Demographics

\begin{tabular}{lc}
\hline Number of patients & 18 \\
\hline Age (median, range) & $62(39-71)$ \\
Gender & F 11 \\
Diagnosis & M 7 \\
& AML 14 \\
& MDS 3 \\
Remission Status & MPN 1 \\
Graft source & CR 12 \\
& Active disease 6 \\
& MUD 12 \\
\hline
\end{tabular}

Median follow up is 139 days (39-296) and 3 patients have died. The causes of death were relapse, pneumonia and atypical hemolytic uremic syndrome.

All patients engrafted for neutrophils at a median of 12 days (9-16), while $17 / 18$ had platelet engraftment at a median of 16 days (8- not reached).

Estimated OS at 300 days was $66 \%(+/-33 \%)$ and PFS at 270 days was $60 \%(+/-33 \%)$. The relapse rate and TRM at 250 days were $18 \%(+/-24 \%)$ and $22 \%(+/-32 \%)$, respectively. The rate of acute GVHD at day 100 was $17 \%(+/-18 \%)$ and the chronic GVDH incidence was estimated at $28 \%(+/-29 \%)$ at 300 days.

Most common toxicities were renal failure and infections (7 $=>$ grade 3 ). We found $6 / 12$ patients to have $=>$ grade 3 renal toxicity with 2 requiring hemodialysis. After reducing azacytidine to $50 \mathrm{mg} / \mathrm{m} 2 / \mathrm{d}$ we observed $1 / 6$ case of grade 3 renal dysfunction.

Conclusion: Preconditioning treatment with azacytidine is tolerable with a toxicity profile similar to standard conditioning with fludarabine/melphalan (3). Azacytidine at $75 \mathrm{mg} / \mathrm{m} 2 \mathrm{x}$ 5 has significant renal toxicity, which abates with dose reduction. The relapse rate is low for this high-risk population and longer follow up and more patients are needed to evaluate the effect of epigenetic priming on PFS and OS. Biomarker studies for DNA methylation pre- and post azacytidine will be useful in assessing the impact of this intervention.

\section{References:}

1. Mechanism-based epigenetic chemosensitization therapy of diffuse large B-cell lymphoma. Clozel T, Yang S, Elstrom RL, et al. Cancer Discov. 2013 Sep;3(9):1002-19.

2. Phase 1 study of epigenetic priming with decitabine prior to standard induction chemotherapy for patients with AML. Scandura JM, Roboz GJ, Moh M, et al. Blood. 2011 Aug 11;118 (6): $1472-80$

3. Fludarabine, melphalan, and alemtuzumab conditioning in adults with standard-risk advanced AML and MDS. van Besien $\mathrm{K}$, Artz A, Smith S, et al. J Clin Oncol. 2005 Aug 20;23(24):5728-38.

Disclosure of Interest: None declared.

\section{P612}

Efficacy of High Dose Corticosteroid Premedication for the Prevention of Anti-thymocyte Globulin Related Side

\section{Effects}

M. Merter ${ }^{1}$, U. Sahin ${ }^{1}$, E. Atilla ${ }^{1}$, S. Civriz Bozdag ${ }^{1}$, M. Kurt Yüksel ${ }^{1}$ P. Topcuoglu', O. Arslan ${ }^{1}$, M. Ozcan ${ }^{1}$, T. Demirer ${ }^{1}$, O. Ilhan ${ }^{\prime}$, H. Akan ${ }^{1}$, M. Beksac', G. Gurman ${ }^{1}$, S. K. Toprak ${ }^{1, *}$

${ }^{7}$ Hematology Department, Ankara University School of Medicine, Ankara, Turkey

Introduction: Anti-thymocyte globulin (ATG) is commonly used for graft versus host disease prophylaxis in allogeneic hematopoietic stem cell transplantation (allo-HSCT). However, side effects can occur despite premedication. We have been trying a new premedication protocol (cohort-A) that consists of a higher dose of methylprednisolone than our previous protocol (cohort-B) aiming at reducing the frequency and severity of ATG related acute and late complications. In this retrospective study, we compared these two protocols in terms of adverse reactions and changes in clinical and laboratory parameters during/after ATG infusion.

Material (or patients) and methods: Cohort- $A$, containing 17 patients was compared to a historical cohort- $B$, containing 15 patients matched for age, sex, underlying disease, ATG dose, disease status and conditioning regimen. All patients received ATG-Fresenius (ATG-F) infused over 10 hours for 3 days. Patients at cohort-B received $1 \mathrm{mg} / \mathrm{kg}$ methylprednisolone, pheniramine and paracetamol 1 hour before ATG infusion and patients at cohort-A received methylprednisolone $1 \mathrm{mg} / \mathrm{kg}$ 12 hours before, $2 \mathrm{mg} / \mathrm{kg} 2$ hours before and $1 \mathrm{mg} / \mathrm{kg}$ in the middle of ATG-F infusion together with pheniramine and paracetamol. Vital signs, any positive findings in physical examination, platelet transfusion requirement and diuretic use were evaluated, as well as, laboratory parameters including leukocyte and thrombocyte counts, serum glucose, liver and kidney function tests, lactate dehydrogenase (LDH), C-reactive protein (CRP), procalcitonin, fibrinogen, d-dimer, prothrombin and activated partial thromboplastin time. Patients were observed for 4 consecutive days. Lymphocyte counts at the beginning of conditioning regimen and before the first ATG-F dose were also recorded. Mann-Whitney $U$ and Chi-square tests were used for comparisons between two groups. $P<0.05$ was considered statistically significant.

Results: General characteristics of two cohorts are shown in table 2. Laboratory parameters were similar between two groups. For the clinical parameters, only "weight gain" on day 2 was significantly higher in cohort- $A$, remaining parameters were similar. D-dimer and CRP values increased to higher levels towards day 3 and tended to decrease thereafter, regardless of the premedication protocol (Figure 1). D-dimer and LDH levels on day 2, day 3 and day 4 were positively correlated with pre-ATG lymphocyte count on the first day $(P<0.05, P<0.001 ; P<0.05, P<0.001 ; P<0.001, P<0.001$, respectively).

Table 1: General characteristics of cohorts

\begin{tabular}{lcc}
\hline & Cohort-A & Cohort-B \\
\hline $\mathbf{N} \quad 17$ & 15 \\
Age (median) & 39 & 28 \\
$\begin{array}{l}\text { Sex (M/F) } \\
\text { Underlying disease }\end{array}$ & $8 / 9$ & $8 / 7$ \\
(AML/ALL/CML/aplastic & & $6 / 4 / 2 / 2 / 1 / 2 / 1$ \\
anemia/MDS/Hodgkin & & \\
lymphoma/ & & \\
myelofibrosis) & & \\
$\begin{array}{l}\text { Conditioning (MA / RIC) } \\
\text { Total ATG-F dose (30/ }\end{array}$ & $11 / 6$ & $9 / 6$ \\
$\mathbf{6 0 / 9 0}$ mg/kg) & $7 / 8 / 2$ & $6 / 7 / 2$ \\
\hline
\end{tabular}

Conclusion: Advantage of using higher dose of corticosteroids has been previosly reported to have beneficial effects in the prevention of adverse events related to ATG [1]. However, our study did not suggest such advantages. Positive correlation of d-dimer, CRP and LDH levels with pre-ATG lymphocyte counts might be a clue for ATG associated cytokine release due to lysis of lymphocytes and destruction of T regulatory cells. PreATG lymphocyte counts may help to predict adverse reactions due to ATG. Further studies are warranted.

References: 1-Bone Marrow Transplant.2002;30(6):347-54.

Disclosure of Interest: None declared. 
P613

Thiotepa, Etoposide, Cyclophosphamide, ARA-C and Melphalan (TECAM) conditioning protocol for autologous stem cell transplantation - An alternative non BCNU containing regimen

S. Grisariu ${ }^{1}$, M. Y. Shapira ${ }^{1, *}$, R. Or ${ }^{7}$, B. Avni ${ }^{1}$

${ }^{1}$ Bone Marrow Transplantation, Hadassah Medical Center, Jerusalem, Israel

Introduction: High dose chemotherapy and ASCT is the current standard of care for chemosensitive relapsed $\mathrm{NHL}$ and $\mathrm{HL}(1)$. High dose BCNU has been associated with Idiopathic Pneumonitis syndrome after ASCT (2). This high incidence of IPS and relatively short EFS and OS has led our group to create a novel alternative conditioning regimen, consisting of etoposide $200 \mathrm{mg} / \mathrm{m} 2$ from day -6 to -3 , thiotepa $40 \mathrm{mg} / \mathrm{m} 2$ from day -5 to -2 , cytrabine $200 \mathrm{mg} / \mathrm{m} 2$ from day -4 to -1 , cyclophosphamide $60 \mathrm{mg} / \mathrm{kg}$ on day -3 and melphalan $60 \mathrm{mg} /$ $\mathrm{m} 2$ on day -2 and -1 (TECAM). The aim of this report is to assess the safety and efficacy of this regimen.

Material (or patients) and methods: We retrospectively analyzed our cohort of $\mathrm{NHL}$ and $\mathrm{HL}$ patients, who underwent ASCT with the TECAM conditioning, between 2000 and 2013. Two hundred and twelve patients, 114 males and 98 females, with a median age of 46 years old were analyzed.

Results: Three years overall survival among $138 \mathrm{NHL}$ and 74 $\mathrm{HL}$ patients was found to be $66 \%(95 \% \mathrm{Cl} 57 \%$ to $74 \%)$ and $82 \%(95 \% \mathrm{Cl} 70 \%$ to $90 \%)$, respectively (Fig 1). Pretransplantation stage IV disease, higher ECOG performance status and progressive disease at transplantation were found to be statistically significant bad prognostic factors. Age and $\mathrm{BMI}$ adjustments of conditioning protocol had no influence on survival. Seven patients (3.3\%) died during the first 100 days. Three months post ASCT, $61.3 \%$ of patients were in $\mathrm{CR}, 4.7 \%$ in PR and $20.75 \%$ were found to be with progressive disease. No patient was reported to suffer from IPS.

Conclusion: We conclude that our TECAM conditioning regimen shows similar efficacy to the commonly used BEAM protocol with a similar toxicity profile, excluding pulmonary fibrosis. Compared to other groups using TECAM, we had significantly less mortality(3). Our results merit considering TECAM as an alternative conditioning for ASCT in high risk lymphoma patients.

References: 1. Moskowitz AJ, Moskowitz $\mathrm{CH}$. Controversies in the treatment of lymphoma with autologous transplantation. Oncologist 2009;14:921-929.

2. Chen Y.B. et al. Impact of conditioning regimen on outcomes for patients with lymphoma undergoing high-dose therapy with autologous hematopoietic cell transplantation. Biol Blood Marrow Transplant:21:1046-1053.

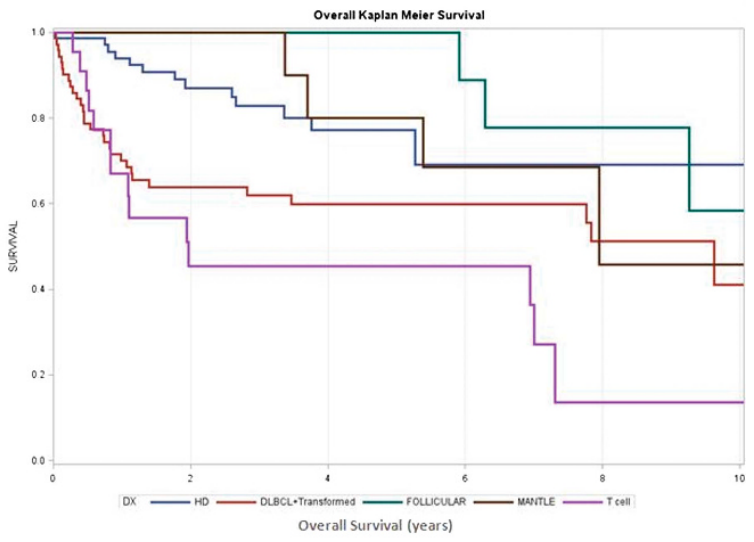

Fig 1. Overall survival of lymphoma patients (by disease type) after high dose ther apy with TECAM protocol followed by ASCT.
3. Rosenberg D. et al. Comparison of the Low Toxicity TECAM Conditioning Regimen to BEAM in Patients with Lymphoma Requiring Autologous Stem Cell Transplantation. Abstract 3188, ASH meeting Dec 2015.

Disclosure of Interest: None declared.

P614

Encouraging short and long term survival after allogeneic stem cell transplantation for AL amyloidosis; a noninterventional study of the Chronic Malignancy Working Party of the EBMT

S. O. Schönland ${ }^{1, *}$, U. Hegenbart ${ }^{1}$, S. lacobelli ${ }^{2}$, J. Hoek ${ }^{3}$ J. Passweg ${ }^{4}$, D. Bordessoule, J.-H. Bourhis ${ }^{6}$, M. Schipperus', T. deWitte, ${ }^{8}$ L. Garderet ${ }^{9}$, N. Kröger ${ }^{10}$ on behalf of Chronic Malignancy Working Party (CMWP)

${ }^{1}$ Amyloidosis Center, Heidelberg, Germany, ${ }^{2} 2$ Centro Interdipartimentale di Biostatistica e Bioinformatica, Roma, Italy, ${ }^{3} E B M T$ Data Office, Leiden, Netherlands, "University Hospital, Basel, Switzerland, ${ }^{5}$ CHRU Limoges, Limoges, ${ }^{6}$ Gustave Roussy, Institute de cancérologie, Villejuif, France, Haga Hospital Leyenburg, Le Hague, ${ }^{8}$ Radboud university medical centre, Nijmegen, Netherlands, ${ }^{9}$ Hospital Saint Antoine, Paris, France, ${ }^{10}$ UKE, Hamburg, Germany

Introduction: Systemic light chain (AL) amyloidosis is a rare protein misfolding and deposition disorder which is caused mostly by a monoclonal plasma cell disorder with a poor prognosis if the heart is severely affected. In a retrospective EBMT analysis allogeneic stem cell transplant (allo-SCT) has emerged as an effective treatment but was associated with a high non-relapse-mortality (NRM) (Schönland et al., Blood 2006).

Material (or patients) and methods: We performed a prospective non-interventional study (NIS) within the EBMT using allo-SCT in patients with AL amyloidosis. Primary and secondary endpoint were best hematologic remission, organ response, acute and chronic Graft-versus-host-disease (GvHD), NRM and overall survival (OS). Fourteen patients have been included and were transplanted between 2006 and 2014. Median age at allo-SCT was 50 years (range, 41-60). Five patients had cardiac and 10 patients kidney involvement. As underlying hematologic disease 13 patients had a clonal plasma cell dyscrasia. All but one patient were in a good performance status (80-100\%) assessed by Karnofsky Index. Previous chemotherapy included high-dose melphalan (HDM) with autologous stem cell transplantation in 11 patients as well as bortezomib, lenalidomide, rituximab and steroids. Disease stages at allo-SCT were: 2 CRs, 1 VGPR, 6 PRs, 3 no responses and 1 progression. Two patients received myeloablative and 12 reduced intensity conditioning (RIC, TBI 2 Gy/fludarabine in 10 patients). Three patients received T cell depletion ( 2 with ATG, one with Campath). Donors were: 8 identical siblings, 1 mismatched relative, 1 syngeneic, 3 matched unrelated donors and 1 mismatched unrelated donor. Source of stem cells was peripheral blood in 13 and cord blood in 1 patient, respectively. Time from diagnosis to allo-SCT was 23 months in median (range, 4-61).

Results: 13 out of 14 patients engrafted after allo-SCT. Acute GvHD grade II-IV occurred in 4 and chronic GvHD in 9 patients ( 4 with limited and 5 with extensive disease, respectively). 3 patients died without relapse or progression (due to acute GvHD, infection and suicide), one patient due to progressive disease. 1- and 2-year-mortality were 14 and $21 \%$, respectively. Best HR after allo-SCT was CR in 7 (50\%). Ten patients were alive after a median follow-up of 53 months with an OS plateau beginning at 28 months post allo-SCT of 70\%. Four patients relapsed or progressed and 7 patients were alive and relapse-free.

In addition to the results of our NIS, we updated the 19 patients of our published cohort (Schönland et al., Blood 2006). 1- and 2-year-mortality were 42 and 53\%, respectively. Five (26\%) patients were still alive after a median follow-up 
of 208 months, four of these living longer than 10 years after allo-SCT.

Conclusion: These preliminary results of our NIS show that allo-SCT is nowadays feasible and effective in selected patients with $\mathrm{AL}$ amyloidosis. In opposite to our retrospective analysis we observed a rather low NRM using mostly RIC Furthermore, the NIS and the updated results of our published cohort revealed a rewarding long term survival. Therefore, allo-SCT might be a reasonable treatment option in young, medically fit and heavily pretreated patients (e.g. early failure after HDM).

Disclosure of Interest: None declared.

\section{P615}

Similar outcome after allogeneic stem cell transplantation with a modified FLAMSA conditioning protocol containing treosulfan in an elderly population with AML

U. Holtick, ${ }^{1, *}$, M. Herling ${ }^{1}$, N. Pflug ${ }^{1}$, G. Chakupurakal ${ }^{1}$, S. Leitzke ${ }^{1}$, D. Wolf ${ }^{2}$, M. Hallek', C. Scheid ${ }^{1}$, J. Chemnitz ${ }^{1}$

${ }^{1}$ Department I of Internal Medicine, University of Cologne, Cologne, ${ }^{2}$ University of Bonn, Bonn, Germany

Introduction: Reduced intensity conditioning (RIC) regimens as compared to myeloablative protocols have demonstrated lower toxicity profiles at similar efficacy in the context of allogeneic haematopoietic stem cell transplantation. The FLAMSA RIC regimen, combining a cytoreductive part and a transplant-conditioning part, has been described to be efficacious in patients with high-risk and refractory AML. The original protocol protocol containing $4 \mathrm{~Gy} \mathrm{TBI}$, cyclophosphamide and anti-thymocyte globulin (ATG) implicates acute toxicity mainly due to TBI preventing its usage in patients with advanced age and/or severe co-morbidities. To increase safety of the conditioning, we previously reported the feasibility to substitute TBI with treosulfan in elderly AML patients. We now present long-term follow-up data of patients treated with FLAMSA treosulfan in comparison to the original FLAMSA TBI protocol.

Material (or patients) and methods: We retrospectively analysed clinical data of 130 consecutive patients with highrisk primary or relapsed primary AML after allogeneic stem cell tranplantation following FLAMSA conditioning at our center. Fifty-eight patients were treated with FLAMSA treosulfan due to age and/ or co-morbidities. Seventy-two patients were treated with FLAMSA $4 \mathrm{~Gy}$ TBI. No prophylactic donor lymphocyte infusions were administered.

Results: Median age of the whole cohort was 53 years (19-73 years). Median age of patients treated with FLAMSA treosulfan was 60 years, whereas median age of FLAMSA TBI patients was 46 years. Donors were matched related, matched unrelated or mismatched unrelated in 33,49 and $18 \%$ of the transplants. Twenty-five percent of the patients were transplanted with blast persistence. Median follow-up was 36 months. The 4-year overall survival of the whole cohort is $45 \%$ with a 4 -year relapse-free survival of $40 \%$. The cumulative incidence of nonrelapse mortality at 4 years was $28 \%$ in the FLAMSA treosulfan group as compared to $13 \%$ in the FLAMSA TBI group. Relapse incidence at 4 years was higher in patients treated with FLAMSA TBI (46\% versus $32 \%)$. The resulting overall and relapse-free survival rates at 4 years were 47 and $41 \%$ for patients treated with FLAMSA TBI as compared to 43 and $40 \%$ in patients treated with FLAMSA treosulfan. Relapse rates were independent of the ELN score, but even more prominent for patients treated in blast persistence with more than $10 \%$ blasts (relapse incidence $70 \%$ for TBI versus $35 \%$ for treosulfan patients) with superior overall and relapse-free survival in patients treated with FLAMSA treosulfan.

Conclusion: FLAMSA treosulfan is an effective treatment option for high-risk AML patients with co-morbidities or advanced age. Our data indicate improved anti-leukemic activity by FLAMSA treosulfan especially in patients with blast persistence prior to transplant. The higher non-relapse mortality is most like due to the older patient population.
Translating FLAMSA treosulfan to younger patients, lower non-relapse mortality and higher anti-leukemic activity might add up to improved overall survival.

Disclosure of Interest: None declared.

\section{P616}

Reduced intensity conditioning with Fludarabine, BCNU and Melphalan (FBM) for allogeneic hematopoietic cell transplantation in elderly AML patients: remission status at transplant predicts outcome

V. Wais ${ }^{1, *}$, L. Kündgen ${ }^{2}$, S. Bohl', S. von Harsdorf ${ }^{1}$, R. F. Schlenk ${ }^{7}$ K. Döhner', V. Teleanu' ${ }^{1}$, L. Bullinger ${ }^{1}$, K. Drognitz', J.-C. Moulin' ${ }^{\prime}$, M. Binnenhei ${ }^{2}$, M. Bentz', H. Döhner ${ }^{1}$, D. Bunjes' ${ }^{1}$, M. Ringhoffer $^{\prime}$, F. Kuchenbauer

${ }^{1}$ University Hospital of Ulm, Ulm, ${ }^{2}$ Municipal Hospital of Karlsruhe, Karlsruhe, Germany

Introduction: With the introduction of reduced-intensity conditioning (RIC), the age limit for allogeneic transplantation of AML patients has risen to 65 and above. In order to balance a low transplant-related mortality (TRM) with high antileukemic efficacy, Marks et al. introduced FBM conditioning consisting of $150 \mathrm{mg} / \mathrm{m} 2$ Fludarabine, $300 \mathrm{mg} / \mathrm{m} 2$ BCNU and $110 \mathrm{mg} / \mathrm{m} 2$ Melphalan as RIC for elderly patients with encouraging results. However, no detailed analysis of factors predicting outcome in this subpopulation has been presented so far.

Material (or patients) and methods: In this two-center retrospective study, 51 patients (pts) with a median age of $64 \mathrm{y}$ (range, 44-75y) diagnosed with AML were uniformly treated according to the FBM protocol. GvHD prophylaxis consisted of Tacrolimus (TAC) or Ciclosporin A (CYA) and mycophenolate mofetil (MMF) in all patients. MMF was tapered after d50 and TAC or CYA after d90 in the absence of GvHD. Pts with matched unrelated donors (MUD) $(n=33)$ or mismatched unrelated donors (MMUD) $(n=9)$ received additional ATG or low dose alemtuzumab as GvHD and rejection prophylaxis. Stem cell source was peripheral blood in $90 \%(n=46)$ and marrow in $10 \%(n=5)$ of the patients. The median follow-up at the time of analysis was 27 months. At the time of transplant 38 pts were in complete remission; the rest of the pts had active disease (partial remission or refractory disease). Molecular (NPM1, FLT3-ITD and CEBPAa) and cytogenetic characteristics (evaluable in 50 patients) were available for all patients.

Results: Acute regimen-related toxicity was low, with a d30 mortality of $0 \%$ in both centers. After 2 years, treatment failure was mainly due to relapse (CIR) with $36 \%$ (SE: $8 \%$ ) in comparison to TRM (20\%, SE: $7 \%)$. The resulting progressionfree survival (PFS) and overall survival (OS) at 2 years was $50 \%$ (SE: $5 \%$ ) and $61 \%$ (SE: 7\%), respectively.

The median EBMT score was 4 (range 2-6). The EBMT score had no impact on different outcome variables (CIR $(P=0.465)$, PFS $(P=0.352)$ and OS $(P=0.171))$. Based on cytogenetic and molecular data patients were categorized according to the European LeukemiaNet (ELN) classification. Neither the ELN score itself nor stratification according to FLT3-ITD or NPM1 mutation status or cytogenetic risk group impacted on outcome in our cohort. By analyzing further risk factors such as remission status, donor type, age, CMV status, CD34+ cell number and acute or chronic GvHD we found the disease status at the time of transplant being the dominant predictor for OS. After 2 years patients transplanted not in remission had only an OS of $20 \%$ (SE: $8 \%$ ) as compared to $75 \%$ (SE: $12 \%$ ) in patients transplanted in CR $(P<0.001)$.

Conclusion: In this cohort of elderly patients with $A M L$ receiving allogeneic transplantation after $\mathrm{FBM}$ conditioning, disease status at the time of transplant was the dominant prognostic factor for outcome. At the present follow-up neither molecular status nor cytogenetics impacted OS, PFS or CIR, significantly. We conclude that alternative strategies have to be applied using FBM conditioning in refractory patients, i.e. the early tapering of immunosuppression and the 
use of donor lymphocytes or cytoreduction immediately before the start of conditioning.

Disclosure of Interest: None declared.

P617

Which conditioning regimen is more effective for high-risk patients with acute myeloid leukaemia/myelodysplastic syndrome: comparing Low-dose decitabine combined with modified BUCY with modified BUCY followed by allogeneic stem cell transplantation

J. $\mathrm{Cao}^{1}$, X. Shi ${ }^{1}$, L. Ge ${ }^{1}$, A. Sun ${ }^{1}$, X. Zhu' ${ }^{1}$, H. Qiu' ${ }^{1}$, Z. Jin ${ }^{1}$, M. Miao',

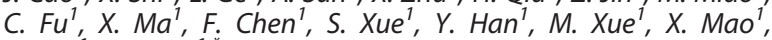
D. Wu ${ }^{1}, X$. Tang ${ }^{1,}$

${ }^{1}$ The First Affiliated Hospital of Soochow University, Jiangsu Institute of Hematology, Suzhou, China

Introduction: Allogeneic hematopoietic stem cell transplantation (allo-HSCT) is the only curative treatment options to hematologic malignancies. However, majority of patients with refractory or resistant hematologic malignancies can not achieve remission before transplantation. This clinical study will investigate the security and efficacy of conditioning regimen containing low-dose decitabine combined with modified BUCY regimen for advanced AML/MDS patients, explore the role of immunomodulatory activity post transplantation and compared this regimen with conventional modified BUCY regimen.

Material (or patients) and methods: Between January 2012 and March 2015, a total of 156 patients were enrolled in this study. In which, there were 46 patients who received a conditioning regimen of low-dose DAC (DAC group) and a modified BUCY regimen(Con group) followed by allo-HSCT, and the second cohort consisted of 110 who only received a modified BUCY regimen. Comparing the baseline of two groups, there were no significant difference except there were more advanced stage patients in DAC group $63 \%$ vs $32.7 \%$, $P=0.007)$ A modified BUCY conditioning regimen include semustine $\left(250 \mathrm{mg} / \mathrm{m}^{2} / \mathrm{d}\right)$ for $1 \mathrm{~d}(-10 \mathrm{~d})$, cytarabine $\left(2 \mathrm{~g} / \mathrm{m}^{2} \mathrm{q} 12\right.$ h) for $2 \mathrm{~d}(-9 \mathrm{~d}$ to $-8 \mathrm{~d})$, busulfan $(0.8 \mathrm{mg} / \mathrm{kg} / 6 \mathrm{~h})$ for $3 \mathrm{~d}(-7 \mathrm{~d}$ to $-5 \mathrm{~d})$, and cyclophosphamide $\left(1.8 \mathrm{~g} / \mathrm{m}^{2} / \mathrm{d}\right)$ for $2 \mathrm{~d}(-4 \mathrm{~d}$ to $-3 \mathrm{~d})$. Meanwhile, patients in the DAC group received the DAC treatment for 3 to $4 \mathrm{~d}$ with a total of $100 \mathrm{mg} / \mathrm{m}^{2}$ before modified BUCY regimen.

Results: In the DAC group, all patients engrafted successfully, including 29/46(63\%) non-remission (NR) patients. However, there were seven patients presented graft failure in Con group. The median time of neutrophil and platelet recovery in two groups were 12 (10-21) vs $12 \mathrm{~d}$ (range: $10-23 \mathrm{~d})(\mathrm{p}$ and 13 (10-35) days vs $14 \mathrm{~d}$ (range: 9-40 d) respectively. There were no significant differences between two groups. The transplantation-related mortality (TRM) rate was $0 \%$ vs $16.4 \%$ $(P=0.004)$ for DAC group and Con group. The median followup were 200(20-985)d and 177.5(3-1093)d. The cumulative rate of aGVHD and CGVHD were $26.7 \%$ vs $46.8 \%(P=0.034)$ and $68.4 \%$ vs $70.7 \%(P=0.598)$ respectively. The cumulative relapse rate was $26.5 \%$ and $37.8 \%(P=0.706)$ respectively. The estimated 2-year overall survival ( $2 \mathrm{yr}$-OS) and 2 year disease-free survival ( $2 \mathrm{yr}$-DFS) rate were $76.6 \%$ vs $51.2 \%$ $(P=0.01)$ and $63.3 \%$ vs $41.4 \%(P=0.046)$ respectively. Furtheremore, for the patients who were in advanced stage before transplant, the The estimated $2 \mathrm{yr}$-OS and $2 \mathrm{yr}$-DFS rate were $77.3 \%$ vs $44.8 \%(P=0.009)$ and $66.5 \%$ vs $46.7 \%(P=0.031)$ respectively.
Conclusion: 1. Low-dose decitabine combined with modified $\mathrm{BUCY}$ is a safe and effective conditioning regimen for high-risk patients with AML/MDS with low toxicity and well tolerance. All patients of DAC group engrafted and transplantationrelated mortality (TRM) rate was 0 .

2. $100 \%$ NR patients of DAC group achieved complete remission with full donor chimerism at d30. 3.Comparing with Con group, DAC group patients had ralative lower incidence of aGVHD and TRM but relative higher estimated 2-yr OS and DFS, especially for advanced stage patients.

Disclosure of Interest: None declared.

\section{P618}

Early NK cell reconstitution predicts overall survival in T-cell replete allogeneic hematopoietic stem cell transplantation

L. Minculescu ${ }^{1, *}$, H. V. Marquart ${ }^{1}$, L. Smidstrup Friis ${ }^{2}$, S. Lykke Petersen $^{2}$, I. Schjødt ${ }^{2}$, L. P. Ryder ${ }^{7}$, N. Smedegaard Andersen ${ }^{2}$, H. Sengeloev ${ }^{2}$

${ }^{1}$ Dept of Clinical Immunology, ${ }^{2}$ Department of Hematology, RIGSHOSPITALET, Copenhagen, Denmark

Introduction: Early immune reconstitution plays a critical role in clinical outcome after allogeneic hematopoietic stem cell transplantation (HSCT). Natural killer (NK) cells are the first lymphocytes to recover after transplantation and is considered a powerful effector cell in HSCT. We aimed to evaluate the clinical impact of early NK cell recovery in T-cell replete transplant recipients.

Material (or patients) and methods: Immune reconstitution was studied in 298 adult patients undergoing HSCT for acute myeloid leukemia (AML), acute lymphoblastic leukemia (ALL) and myelodysplastic syndrome (MDS) from 2005 to 2013. Lymphocyte subpopulations were evaluated by flow cytometry including staining with anti-CD16/56, anti-CD3, anti-CD4 and anti-CD8.

Results: In multivariate analysis NK cell numbers day 30 (NK30) >150cells/ $\mu \mathrm{L}$ were independently associated with superior overall survival (hazard ratio $0.79,95 \%$ confidence interval $0.66-0.95, P=0.01$ ). Cumulative incidence analyses showed that patients with NK30 $>150$ cells/ $\mu \mathrm{L}$ had significantly less transplant related mortality (TRM), $P=0.01$. Patients with NK30 >150cells/ $\mu \mathrm{L}$ experienced significantly lower numbers of life threatening bacterial infections as well as viral infections, including CMV. No association was observed in relation to relapse.

Conclusion: These results suggest an independent protective effect of robust early NK cell reconstitution on TRM which translates into improved overall survival after T-celle replete HSCT.

References: 1) Savani BN, Mielke S, Adams S, Uribe M, Rezvani $\mathrm{K}$, Yong AS et al. Rapid natural killer cell recovery determines outcome after T-cell-depleted HLA-identical stem cell transplantation in patients with myeloid leukemias but not with acute lymphoblastic leukemia. Leukemia 2007; 21: 2145-2152. 2) Dunbar EM, Buzzeo MP, Levine JB, Schold JD, Meier-Kriesche HU, Reddy V. The relationship between circulating natural killer cells after reduced intensity conditioning hematopoietic stem cell transplantation and relapse-free survival and graftversus-host disease. Haematologica 2008; 93: 1852-1858.

3) Norell H, Moretta A, Silva-Santos B, Moretta L. At the Bench: Preclinical rationale for exploiting NK cells and gammadelta $\mathrm{T}$ lymphocytes for the treatment of high-risk leukemias. J. Leukoc. Biol. 2013; 94: 1123-1139.

Disclosure of Interest: None declared. 


\section{P619}

Long-term immune profile and T-cell repertoire analysis after autologous hematopoietic stem cell transplantation in systemic sclerosis patients

L. C. M. Arruda $a^{1,2,3, *}$, F. Brigant ${ }^{3}$, E. Clave ${ }^{3,4}$, C. Douay ${ }^{3,4}$, Z. Marjanovic ${ }^{5}$, C. Deligny ${ }^{5}$, G. Maki $^{6}$, A. Toubert ${ }^{3,4,6}$, H. MoinsTeisserenc ${ }^{3,4,6}$, D. Farge $e^{3,4,}$

${ }^{1}$ Center for Cell-based Therapy, Regional Blood Center of Ribeirão Preto, ${ }^{2}$ Department of Biochemistry and Immunology, Ribeirão Preto Medical School, University of São Paulo, Ribeirão Preto, Brazil, '3 INSERM UMR-1160, Institut Universitaire d'Hématologie, ${ }^{4}$ Université Paris Diderot, Sorbonne Paris Cité, ${ }^{5}$ Unité Clinique de Médecine Interne, Maladies Autoimmunes et Pathologie Vasculaire, AP-HP, Hôpital Saint-Louis, ${ }^{6}$ Laboratoire d'ImmunologieHistocompatibilité, AP-HP, Hôpital Saint Louis, Paris, France

Introduction: Autologous Hematopoietic Stem Cell Transplantation (AHSCT) has become the best treatment option for severe rapidly progressive systemic sclerosis (SSc)[1], inducing de novo tolerance through the reset of immune response [2]. Long-term immune reconstitution analysis is warranted to further understand the mechanisms of response to aHSCT and improve its efficacy.

Material (or patients) and methods: Peripheral blood was collected from 10 SSc patients before and during yearly clinical follow-up until at least 6 years (long-term) post-AHSCT. PBMCs were immunophenotyped by FACS to evaluate $C D 3+(C D 4$ +/CD8+), CD4(+CD45RA+/CD45RO+) T-cells, CD19+ B-cells and CD3-CD16+CD56+ NK-cells reconstitution through linear regression slope (LRS). Anti-Scl-70 antibodies were detected by ELISA. T-cell receptor (TCR) Vb usage and profile was analysed by Immunoscope [2], including 18 controls. Relapse post-AHSCT was defined by $25 \%$ modified Rodnan skin score (mRSS) increase, 10\% Forced Vital Capacity (FVC) decrease or restarting immunosuppression.

Results: At long-term, patients were classified as responders ( $N=5$, group $\mathrm{A})$ and non-responders $(N=5$, group B). All patients had similar clinical evaluation at entry. Reinjected CD34+-cells number was higher in group $B(P=0.02)$. After transplant, only group $B$ patients received mycophenolate (1-2 g/day). All patients had a mRSS fall greater than $25 \%$ up-to long-term as compared to baseline, more pronounced in group A $(P=0.004)$. The relative FVC\% changes compared to pre-transplant differed significantly between the groups $(P=0.040$, ANCOVA). Significant FVC improvement above $10 \%$ compared to pretransplant values was observed only in group A $(P=0.026)$. After aHSCT, there was an overall trend toward increased $\mathrm{CD} 3+(\mathrm{LRS}=16.8), \mathrm{CD} 3+\mathrm{CD} 4+(\mathrm{LRS}=12.5)$, $\mathrm{CD} 3+\mathrm{CD} 8+(\mathrm{LRS}=21.8)$ and $\mathrm{CD} 4+\mathrm{CD} 45 \mathrm{RA}+(\mathrm{LRS}=12.9) \mathrm{T}$-cell changes in group $B$ vs A (LRS $=8.7,9.7,5.0$ and 2.6 , respectively). Memory $\mathrm{CD} 4+\mathrm{CD} 45 \mathrm{RO}+\mathrm{T}$-cells were similar in both groups $A(L R S=7.2)$ and $B(L R S=10.5)$. B-cells had a positive slope in group $A(L R S=11.1)$ and negative in group $B$ $(\mathrm{LRS}=-11.6)$. Four group $A$ patients positive for anti-Scl-70 at entry became negative post-transplant while 3 out 4 group $B$ patients remained positive. Same TCR-BV family usage was observed before aHSCT and at long-term in all 10 patients. TCR repertoire was disturbed before AHSCT, with fewer polyclonal $\mathrm{Vb}$ families than in controls. The percentage of polyclonal families significantly increased at long-term when compared to baseline $(P=0.046)$, with no difference between groups.

Conclusion: Higher numbers of reinfused CD34+-cells may contribute to clinical relapse, which was best assessed by longterm changes in FVC\% rather than in mRSS. Higher B-cells expansion was observed in group $A$, requiring further analysis on B-cells subpopulations and generation. TCR repertoire diversity was not related to clinical response. Despite immunossuppressive drugs reintroduction after AHSCT, higher T-cell increase remained in relapsing patients, illustrating the difficulty of adequate treatment in this setting.

References: [1] van Laar J and Farge D et al. JAMA. 2014;311 (24):2490-2498. [2] Farge D et al. Arthritis Rheum. 2005; (52):1555-1563.
Acknowledgments: Funding was provided by "Groupe Francophone de Recherche sur la Sclerodermie" and "Association des Sclerodermiques de France".

Disclosure of Interest: None declared.

\section{P620}

Bone Marrow Eosinophilia Correlates with Progression Free and Overall Survival After Allogeneic Hematopoietic Stem Cell Transplantation

Y. Georgieva', A. Michova', P. Ganeva ${ }^{2}$, Y. Petrov ${ }^{2}$ V. Madzharova', B. Spassov', G. Balatzenko', G. Arnaudov', G. Mihaylov', M. Guenova ${ }^{1, *}$

${ }^{1}$ Laboratory of Hematopathology and Immunology, ${ }^{2}$ Hematology Clinic, ${ }^{3}$ Laboratory of Cytogenetics and Molecular Biology, National Specialised Hospital for Active Treatment of Hematological Diseases, Sofia, Bulgaria

Introduction: Many studies have shown the beneficial influence of eosinophils (Eo) in diverse neoplasms independent of other standard prognostic factors. Besides, eosinophilia in the blood and tissue is a common feature of graft versus host disease (GvHD) and contributes to the allogeneic response, as revealed in investigations in animal models and clinical observations. Data from a number of cohorts indicate that elevated Eo blood counts could be associated with better outcomes following allogeneic hematopoietic stem cell transplant (HSCT). However, there are still few data on the possible underlying immunologic mechanisms.

The aim of the study was to evaluate how the existence and degree of peripheral blood (PB) and bone marrow (BM) eosinophilia after allo-HSCT correlated with the reconstitution of lymphocytic subpopulations and influenced the transplant outcomes.

Material (or patients) and methods: A total of 64 consecutive pts who received allogeneic HSCT at the National Hematology Hospital, Sofia were included in this study, 45 male and 19 female, including 35 from related and 29 from unrelated donors, at a mean age of 37.4 years (20-59). PB and BM Eo were evaluated at day 100 and at a next time point depending on the follow-up schedule. Eosinophilia was defined as an Eo count $>0.5 \times 10(9) / \mathrm{L}$ in $\mathrm{PB}$ and $>3.5 \%$ in the BM. Information about the following events was recorded: peripheral cell engraftment, acute (a)GvHD and chronic (c) GvHD, viral reactivation, and deaths. Lymphocyte populations and subpopulations were determined in the peripheral blood at multiple points after HSCT by 8-colour flow cytometry (FACSCanto II, BD Diva v.6.1) and monoclonal antibodies combination including CD3; CD4; CD7; CD8; CD19; CD28; CD45; CD45RA; CD56; CD57; CD62L.Progression free survival (PFS) was defined as the time from transplantation until relapse, while overall survival (OS) - until death from any cause. Results: A biphasic pattern of eosinophilia was noted after HSCT. The first peak occurred prior to day 100 then the second one, beyond day 100. Botha at day 100 and beyond PB eosinophilia was detected in $6.7 \%$ of all pts, while increased BM Eo were seen in $25 \%$ and $28 \&$, respectively, regardless of the donor type. No differences in engraftment, viral reactivation, aGVHD or cGVHD depending on Eo counts were established. PB eosinophilia did not correlate with clinical outcomes, however increased BM Eo were associated with better PFS and OS (statistical significance of data beyond day $100-88 \%$ vs $53 \%$ PFS in pts with low Eo, log rank $P=0.016$, and OS $94 \%$ vs $40 \%$, log rank $P=0.001$ ). Interesting correlations of bone marrow eosinophilia were found with the reconstitution of particular lymphocytic subpopulations after HSCT (Th1, central memory CD62L+CD45RA-CD4+, effector memory CD62L-CD45RA-CD4+ T-cells, etc.)

Conclusion: Better outcomes could be expected in patients with BM eosinophilia regardless of the PB Eo counts. Interestingly, immunologic mechanisms might be speculated, however the precise pathophysiology behind eosinophilia remains to be investigated. Further studies are also warranted 
to elucidate the causative mechanisms behind the clinical benefits of increased eosinophils.

Ackowledgements: The study was partially supported by the National Science Fund.

Disclosure of Interest: Y. Georgieva: None declared, A. Michova: None declared, P. Ganeva: None declared, Y. Petrov: None declared, V. Madzharova: None declared, B. Spassov: None declared, G. Balatzenko: None declared, G. Arnaudov: None declared, G. Mihaylov: None declared, M. Guenova Funding from: Novartis, Amgen, Genzyme, Gylead (lectures, advisory boards).

P621

Evaluation of chimerism in the CD34+ sorted cells with the STR-PCR method

M. Comini ${ }^{1}$, A. Beghin ${ }^{1}$, F. Bolda ${ }^{1}$, R. Baffelli ${ }^{1}$, S. Villanova ${ }^{1}$ M. Zucchi , A. Di Palma ${ }^{2}$, S. Perucca ${ }^{2}$, S. Bernardi', F. Cattina ${ }^{2}$, V. Cancelli ${ }^{2}$, B. Rambaldi ${ }^{2}$, M. Malagola ${ }^{2,}$, , A. Lanfranchi ${ }^{1}$

Stem Cell Laboratory, Section of Hematology and Blood Coagulation, Children's Hospital, Spedali Civili of Brescia, ${ }^{2}$ Chair of Hematology, Unit of Blood Diseases and Adult Stem Cell Transplantation, University of Brescia, Brescia, Italy

Introduction: Analysis of donor chimerism is an important diagnostic tool to assess the risk of relapse after Hematopoietic Stem Cell Transplantation (HSCT), especially in patients lacking a specific marker suitable for monitoring of minimal residual disease. We investigated the predictive role of donor chimerism analysis in sorted CD34+ bone marrow (BM) cells patients (pts) with hematological disease.

Material (or patients) and methods: We analyzed 25 pts affected by ALL $(n=8), \operatorname{CML}(n=1), \operatorname{AML}(n=14), \operatorname{MDS}(n=1)$ and Myelofibrosis $(n=1)$.

Fresh mononuclear cells (MNC) were isolated from anticoagulated BM using Ficoll-Paque density gradient centrifugation. CD34+ cells were selected using a CD34 MicroBeads Kit and the AutoMACS automated selection device (MiltenyiBiotec, Bergisch Galdbach, Germany) according to the manufacturer's instructions. STR amplifcation: PCR was performed using the AmpFISTR Profiler Plus Kit Amplification as recommended by the manufacture.

Results: We sorted CD34+ cells from BM and we performed STR-PCR in order to detect host cells in all fractions.

Sorted CD34+ cells STR analysis showed a complete donor chimerism (CC) in 17 pts: 7 ALL, 7 AML, 1 CML, 1 MDS and 1 Myelofibrosis, while the CD34+ fraction from $1 \mathrm{ALL}$ and $7 \mathrm{AML}$ showed a mixed donor chimerism (MC).

In samples with MC $<90 \%$ of donor there is correlation with molecular MRD (Table 1).

In pt 4 the analysis of CD34+ sorted cells showed MC $(98,49 \%$ of donor) corresponding with the presence of a complex karyotype clone, with no molecular evidences and after chemotherapy treatment we observed a conversion from MC to CC. Instead pt 5 showed a spontaneous conversion from MC to CC.

Conclusion: The STR-PCR based on CD34+ cells sorted could be used as an useful diagnostic tool, providing to clinicians additional informations, specially when MRD is not available in patients who lack specific molecular markers.

This method could be an accurate alternative approach in the evaluation of chimerism, suggesting the possible persistence of disease: in fact our preliminary data indicate that decrease of CD34+ chimerism of less than $90 \%$ is a strong evidence of relapse.

References: 1-Monitoring of donor chimerism in sorted CD34+ peripheral blood cells allows the sensitive detection of imminent relapse after allogeneic stem cell transplantation; Bornhäuser M et al.; Haematologica. 2009 Nov;94(11):1613-7. 2-Genomic quantitative real-time PCR proves residual disease positivity in more than $30 \%$ samples with negative mRNAbased qRT-PCR in Chronic Myeloid Leukemia; Pagani IS et al.; Oncoscience. 2014 Jul 23;1(7):510-21.

Disclosure of Interest: None declared.

\begin{tabular}{|c|c|c|c|c|c|c|}
\hline PT & HSCT DATE & $\begin{array}{c}\text { CHIMIERISM } \\
\text { ANALYSIS } \\
\text { DATE }\end{array}$ & DISEASE & $\begin{array}{l}\text { MOLECULAR } \\
\text { MARKER }\end{array}$ & $\%$ OF DONOR & MRD \\
\hline 1 & $20 / 12 / 2013$ & $25 / 06 / 2015$ & L.AM & $\begin{array}{c}\text { WT1; COMPLEX } \\
\text { KARYOTYPE }\end{array}$ & 96 & $\operatorname{Neg}($ L.AIP/WT1) \\
\hline 2 & $06 / 11 / 2013$ & $28 / 05 / 2015$ & L.AM & $\begin{array}{c}\text { NORMAI } \\
\text { KARYOTYPE }\end{array}$ & 96,8 & $\mathrm{Neg}$ (WT1) \\
\hline 3 & $23 / 04 / 2009$ & $\begin{array}{l}02 / 07 / 2015 \\
28 / 09 / 2015 \\
26 / 11 / 20015\end{array}$ & LAM & $\begin{array}{c}\text { WT1; COMPLEX } \\
\text { KARYOTYPE }\end{array}$ & $\begin{array}{c}74,3 \\
0 \\
37,1\end{array}$ & $\begin{array}{c}\text { Pos (WT1) } \\
\text { Pos WT1 } \\
\text { (morphological } \\
\text { relapse) } \\
\text { Pos (WT1) }\end{array}$ \\
\hline 4 & $13 / 12 / 2012$ & $\begin{array}{l}23 / 04 / 2015 \\
27 / 08 / 2015 \\
13 / 11 / 2015\end{array}$ & L.AM & $\begin{array}{c}\text { WT1; COMPLEX } \\
\text { KARYOTYPE }\end{array}$ & $\begin{array}{c}93 \\
98,49 \\
100\end{array}$ & $\begin{array}{l}\text { Neg (WT1) } \\
\text { Neg(WT1) } \\
\text { Neg(WT1) }\end{array}$ \\
\hline 5 & $18 / 06 / 2014$ & $\begin{array}{l}09 / 04 / 2015 \\
16 / 07 / 2015\end{array}$ & LAM & $\mathrm{T}(3 ; 3)$ & $\begin{array}{l}98,3 \\
100\end{array}$ & $\begin{array}{l}\text { Neg (WT1) } \\
\text { Neg WT1 }\end{array}$ \\
\hline 6 & $26 / 11 / 2014$ & $\begin{array}{l}10 / 07 / 2015 \\
12 / 11 / 2015\end{array}$ & LAI & $\begin{array}{c}\text { NORMAI } \\
\text { KARYOTYPE }\end{array}$ & $\begin{array}{c}100 \\
31,20 \%\end{array}$ & $\begin{array}{l}\text { Neg WT1 } \\
\text { Pos WT1 }\end{array}$ \\
\hline 7 & $22 / 12 / 2011$ & $20 / 08 / 2015$ & LAM & WT1 & 97,84 & Neg WT1 \\
\hline 8 & $12 / 06 / 2014$ & $25 / 06 / 2015$ & LAL & del 7 & 97,9 & $\begin{array}{c}\mathrm{Neg} \text { (FISH persex } \\
\text { mm) }\end{array}$ \\
\hline 9 & $07 / 01 / 2015$ & $06 / 08 / 2015$ & L.AM & WT1 & 90 & Neg WT1 \\
\hline
\end{tabular}




\section{P622}

Confirmation of the efficacy of the PowerPlex $16 \mathrm{HS}$ System (Promega USA) panel (15 STR markers plus Amelogenine locus) for the evaluation of Chimerism status after Haematopietic Stem Cell Transplantation in a large series of Donor/Recipent couples

M. Parma ${ }^{1, *}$, F. Colnaghi ${ }^{2}$, A. Colombo ${ }^{2}$, F. Mottadelli ${ }^{2}$ E. Terruzzi ${ }^{1}$, E. Elli ${ }^{1}$, V. Rossi ${ }^{2}$, M. Fedele ${ }^{1}$, R. Renso ${ }^{1}$, G. Cazzaniga ${ }^{2}$, P. Pioltelli ${ }^{1}$

${ }^{1}$ Hematology, ${ }^{2}$ MBBM Foundation, OSPEDALE SAN GERARDO, Monza, Italy

Introduction: The analysis of Short Tandem Repeats (STR) polymorphism is actually the widely employed assay for the evaluation and quantification of Chimerism Status (CS) after hematopoietic stem cell transplantation (HSCT). STR polymorphism analysis is based on a panel of STR markers largely variable in the population, and it has been mutuated from forensic medicine.

In the last years, we tested on one hundred Donor/Recipient (D/R) couples a STR panel derived from the commercial kit for forensic use PowerPlex 16 HS System (Promega USA) which is constituted from 15 highly polymorphic STR, and we showed as 10/15 markers are enough to give at least one informative marker for each D/R couple. Aim of our study is to confirm the previous data on a largest series of $D / R$ couples.

Material (or patients) and methods: We studied STR polymorphism with PCR amplification and semi-quantitative detection of number of repeats of each STR marker. Every STR which presents at least two repeats of difference between donor and recipient individuals, has been considered "informative marker" and suitable for CS monitoring. We analysed 274 consecutive D/R couples of allogeneic HSCT performed in our centre. $95 \%$ of patient was Italian from all the regions, 5\% was from other countries. At last we compared actual with previous results.

Results: Comparing our actual data with previous results we confirm that each couple $D / R$ have at least one informative marker (100\% efficiency). The percentage of D/R couple with 2 markers remain the same $(41 \%)$, instead we observed a decrease in D/R couple with 3 or more markers (20\% and $11 \%$ vs $24 \%$ and $18 \%$ respectively) in advantage of the couples with only one marker ( $25 \%$ vs $16 \%$ ).

Amelogenine, Penta D, Penta E and TH01 are confirmed as the more variable STRs in our population and at least one of them is informative in $71 \%$ of our population (previous data $78 \%$ ). D3S1358, D18S51, TPOX, D21S11 and D8S1179 are confirmed as useful markers, to these we add FGA which is informative in $25 \%$ of our population. These last markers summed to the previous 4 markers constitute an informative panel in 97,5\% of our population. We confirmed D16S539, D5S818, D7S820, Vwa and CSF1PO as the less informative markers, adding to these D13S317. These last ones are present only in $31 \%$ of our population (previous detection $41 \%$ ) and are almost always associated to other more frequent markers.

Conclusion: We substantially confirm our previous data. This panel is useful in CS analysis, even if about $25 \%$ of D/R couple have only one marker. It could be employed a panel restricted to the ten more variable markers (9/10 are the same of previous experience, FGA substitutes D13S317), leaving the extended panel to a limited number of $D / R$ couples $(<5 \%)$ who cannot obtain an useful marker between these 10 ones. Disclosure of Interest: None declared.

\section{P623}

Mixed chimerism at three months after allogeneic hematopoietic stem cell transplantation is a significant predictor of disease relapse in high-risk acute myeloid leukemia patients in first complete remission

M. Michallet ${ }^{1, *}$, M. Sobh ${ }^{1}$, H. Labussière ${ }^{1}$, M. Balsat ${ }^{1}$, C. Lejeune ${ }^{1}$, I. Mollet ${ }^{2}$, X. Thomas ${ }^{1}$, F. Barraco ${ }^{1}$, V. Dubois ${ }^{2}$, F.-E. Nicolini ${ }^{1}$

${ }^{i}$ Centre Hospitalier Lyon Sud, Pierre Benite, ${ }^{2}$ Etablissement

Français du Sang, Lyon, France
Introduction: The aim of our study is to evaluate the impact of chimerism evaluation 3 months after allo-HSCT in AML patients who were in first complete remission (CR1) before transplantation and remained in clinical and morphological CR at day 90 post-transplantation, on overall survival (OS) and relapse incidence.

Material (or patients) and methods: We evaluated 194 AML patients who received allo-HSCT at our center between January 2006 and December 2014 and for whom chimerism follow-up has been performed at 3 months. There were $103(53 \%)$ males and 91 (47\%) females with a median age of 43 years (range: 1867). Patients were classified according to the European LeukemiaNet classification for cytogenetic and molecular biology markers (Dohner et al. Blood 2010), accordingly, 64\% patients were unfavorable and $36 \%$ were in intermediate II risk group. At allo-HSCT, all patients were in CR1; $136(70 \%)$ received a full intensity conditioning (MAC) and 58 (30\%) received a reduced intensity conditioning (RIC). HSC donors were 72 (37\%) HLA identical siblings (44 BM and 36 PBSC), 54 (28\%) 10/10 HLA matched unrelated donors (35 BM and 19 PBSC), 33 (17\%) 9/10 HLA mismatched unrelated donors (16 BM and 17 PBSC) and 35 (18\%) double cord blood units (only 7 were 6/6 HLA matched). Chimerism analysis was performed on marrow and/or blood samples every month following allo-HSCT using polymerase chain reaction (PCR) based on informative polymorphic short tandem repeat, a mixed chimerism was defined by having $5 \%$ or more of recipient cells.

Results: At day 90 after transplantation, all patients remained in clinical and cytological CR at time of chimerism evaluation, $155(80 \%)$ had a full donor chimersim (FDC) and $39(20 \%)$ had a mixed chimerism (MC) ranging from $65 \%$ to $95 \%$ of donor cells. Among patients with MC, 9 (23\%) received increasing doses of DLI (5 of them reached FDC at 6 months), while 20 $(51 \%)$ could not receive DLI (7 because transplanted from cord blood, and 13 because of the presence of GVHD), the rest of patients were left with a transient $M C$ and regained FDC during follow-up.

After a median follow-up of 34 months (range: 4-96) for the surviving patients, the median OS in patients with FDC was not reached with a 3 years probability of $62 \%(95 \% \mathrm{Cl}$ : 58-66), and for patients with MC, it was 18 months (12-24) with a 3 years probability of $32 \%(95 \% \mathrm{Cl}: 23-41),(P=0.01)$. Twenty-two patients in the $\mathrm{MC}$ group have progressed during the followup and 17 among them died from disease progression. The cumulative incidence of relapse at 3 years was $25 \%(95 \% \mathrm{Cl}$ : 21-29) for FDC patients and it was 70\% (95\% Cl: $62-80)$ for MC patients, $(P<0.001)$. The impact of mixed chimerism was still valid in multivariate analysis after including patient age, type of donor, HSC source and risk group, and was independent from the intensity of the conditioning regimen with a Hazard Ratio of 4.7 , and a $95 \% \mathrm{Cl}: 2.6-8.4, P<0.001$.

Conclusion: We demonstrated that chimerism evaluation at day 90 after allo-HSCT is an independent predictor of disease relapse in patients who remain in $C R$ at that time and significantly impacts on long term survival. The standardization of this evaluation may lead to the identification of patients with high-risk of relapse risk suggesting the need of early preemptive intervention.

Disclosure of Interest: None declared.

\section{P624}

Impact of molecular residual disease after allografting in myelofibrosis patients

N. Kröger ${ }^{1, *}$, A. Badbaran 1, T. Zabelina 1, M. Christopeit ${ }^{1}$, C. Wolschke', F. Ayuk', A. R. Zander', H. Alchalby', U. Bacher', I. Triviai ${ }^{1}$, B. Fehse ${ }^{\text {I }}$

${ }^{1}$ Department of Stem Cell Transplantation, University Medical Center Hamburg-Eppendorf, Hamburg, 2'University Hospital Göttingen, Göttingen, Germany

Introduction: Residual molecular disease (MRD) in hematological malignancies has become a valid tool to predict clinical relapse after allogeneic stem cell transplantation (ASCT). 
Material (or patients) and methods: We screened 154 patients with myelofibrosis who underwent ASCT for molecular residual disease by qPCR or next-generation sequencing (NGS) for JAK2V617F, MPLW515L and MPLW515K or Calreticulin (L367f*46, and K385f*47) mutations in peripheral blood (PB) on days +100 and +180 after transplantation. Out of 154 pts, 103 were JAK2V617F $(n=101), 31$ Calreticulin (CALR), and $4 \mathrm{MPL}$ mutated, while 13 pts were triple negative. 136 pts could be followed after ASCT with one molecular marker. The median age of the pts was 58 years (range, 32-75 y). Patients had either primary myelofibrosis $(n=90)$, post ET/PV myelofibrosis $(n=40)$, myelofibrosis in acceleration or were transformed to AML $(n=6)$. Conditioning mainly relied on a busulfan-based reduced-intensity regimen. Donor were HLA-identical sibling $(n=26)$, matched unrelated $(n=71)$ or mismatched unrelated $(n=39)$. JAK2V617F, MPL, and CALR mutations were measured by usage of Taqman PCR or in case of CALR Type 2 mutation by digital PCR on day +100 and day +180 from PB as described elsewhere.

Results: After a median follow up of 78 months (range, 49-101 months) the 5-year estimated overall survival was $60 \%$ (95\% $\mathrm{Cl}: 50-70 \%)$ and the cumulative incidence of relapse at 5 years was $26 \%$ (95\% Cl: $18-34 \%$ ) for the entire study population. On days +100 and +180 after transplantation in $27 \%$ and $12 \%$ of the patients the underlying mutation was still detectable in peripheral blood. The percentage of complete clearance was higher in CALR-mutated patients (96\%) in comparison to MPL (75\%) and JAKV2617F (70\%) mutated pts. Whereas there was a trend for better survival for CALR-mutated patients in comparison to JAK2-mutated patients $(71 \%$ vs $57 \% ; P=0.48)$, once a patient achieved molecular remission post-transplant the risk of relapse remained low independently of the underlying mutation. Patients who were alive and without relapse at days +100 or +180 but with still detectable mutation in PB had a significantly higher risk of relapse than those who were molecular negative (62\% vs $10 \%, P<0.001$; and $70 \%$ vs $10 \%, P<0.001$, respectively). In a multivariate analysis only high-risk disease status (HR 2.5; 95\% Cl: 1.18-5.25, $P=0.016$ ) and detectable MRD at day 180 (HR 8.36, 95\% Cl: 2.76-25.30, $P<0.001)$ were significant factors for a higher risk of relapse.

Conclusion: JAK2V617F, CALR, and MPL genetic lesions allow to monitor MRD in around $90 \%$ of the myelofibrosis patients after ASCT by $\mathrm{qPCR}$ or digital PCR in the PB. Persistence of MRD on days +100 or +180 in peripheral blood can be used to taper immunosuppressive drugs or to apply donor lymphocyte infusion to prevent clinical relapse.

Disclosure of Interest: None declared.

\section{P625}

Long-term persistence but lack of graft-vs-leukemia efficacy of cord blood cells after secondary haploidentical hematopoietic cell transplantation

N. F. Russkamp ${ }^{1, *}$, U. Schanz ${ }^{1}$, A. M. S. Müller ${ }^{1}$

${ }^{1}$ Department for Hematology, University Hospital Zurich, 8091, Switzerland

Introduction: The prognostic relevance of decreasing levels of donor chimerism after hematopoietic cell transplantation (HCT) for hematological malignancies is a matter of debate but always a cause of concern. Particularly cord blood transplants (CBT) are afflicted with a high rate of graft failures (up to $10 \%>20 \%$ ) due to limited numbers of hematopoietic stem cells in the graft. Likewise, overcoming the immune barrier was a major problem following haploidentical HCT prior to the era of post-transplant cyclophosphamide use.

Material (or patients) and methods: Here, we report on the dynamics of donor chimerism in a $28-y / 0$ male patient with BCR-ABL1-positive acute B-lymphoblastic leukemia (B-ALL) who underwent standard induction therapy (including TKI) followed by myeloablative conditioning (cyclophosphamide, ATG, 12 Gy total body irradiation) and allogeneic double-unit CBT due to lack of an HLA-identical donor. Ten months post
HCT the patient relapsed and was given initially TKI, then with morphological relapse re-induction chemotherapy followed by reduced-intensity conditioning (fludarabine, cyclophosphamide, alemtuzumab, NO post-HCT cyclophosphamide) and a second HCT from his haploidentical mother.

Results: 3 months after the first $\mathrm{HCT}$ (CBT) the patient achieved $100 \%$ donor chimerism (FISH: $100 \%$ XX; 0\% XY). With molecular recurrence of the B-ALL donor chimerism decreased to a minimal level of $63 \%$ (XY 37\%). Ever since the second HCT (haplo) the patient has been in complete morphological and molecular remission (100\% XX, 0\% XY); however, for more than 20 months now there is a stable mixed chimerism of cells derived from the cord-blood and the haploidentical graft as assessed by short tandem repeat analysis in the blood and the marrow. The patient was suffering from chronic GVHD of the oral cavity $\left(\mathrm{NIH}^{\circ} \mathrm{l}\right)$, the lungs (bronchiolitis obliterans, $\mathrm{NIH}^{\circ} \mathrm{II}$ ) and the skin and is currently under ongoing pharmacological immunosuppression. Conclusion: Although the CB-graft did not provide Graft-vsleukemia efficacy cells of this source can persist long-term in a host given a secondary conditioning and transplant. In contrast, the haploidentical T-cell replete graft offers stronger anti-leukemic and anti-host reactivity, yet not resulting in complete eradication of the cord-blood derived cells.

Disclosure of Interest: None declared.

\section{P626}

RIC allogeneic stem cell transplantation for high risk CLL followed by preemptive MRD-based immunointervention intermediate results from the phase II ICLL03 RICAC-PMM trial (GCFLLC/MW - GOELAMS - SFGMTC French intergroup) (NCT00935324)

O. Tournilhac ${ }^{1, *}$, M. Le Garff-Tavernier ${ }^{2}$, R. Tabrizi', S. NGuyen Quoc ${ }^{4}$, F. Legrand-Izadifar ${ }^{5}$, P. Chevallier ${ }^{6}$, O. Reman ${ }^{7}$, C. Tomowiak C. Borel ${ }^{9}$, S. Francois ${ }^{10}$, D. Bordessoule ${ }^{17}$, S. Maury ${ }^{12}$, G. Guillerm $^{13}$ L. Vincent ${ }^{14}, K$. Bilger ${ }^{15}, R$. Lemal ${ }^{16}$, C. Quiney , C. Bellanger ${ }^{16}$, L. Calvet $^{16}$, L. Veronese ${ }^{17}$, A. Cabrespine ${ }^{16}$, M. Coudert $^{18}, H$. MerleBeral $^{19}$, N. Dhedin ${ }^{20}$

${ }^{1}$ Service de thérapie cellulaire et d'hématologie clinique adulte, $\mathrm{CHU}$ ESTAING, Clermont-Ferrand, ${ }^{2}$ Service d'Hématologie Biologique, Groupe Hospitalier Pitié-Salpêtrière, Paris, ${ }^{3}$ Service des maladies du sang, Groupe Hospitalier Haut Levêque, Pessac, ${ }^{4}$ Service d'hématologie clinique, Groupe Hospitalier Pitié-Salpêtrière, Paris, ${ }^{5} 5$ Service d'hématologie clinique, département de greffe de moelle, CHU Nice, Nice, "Service d'hématologie clinique, CHU Nantes Hôtel Dieu, Nantes, ${ }^{7}$ Hématologie Clinique, CHU Caen, Caen, ${ }^{8}$ Service Oncologie Hématologique et Thérapie Cellulaire, CHU Poitiers, Poitiers, ${ }^{9}$ Service d'hématologie, Institut Universitaire du Cancer Toulouse - Oncopole, Toulouse, ${ }^{10}$ Service Maladies du sang, CHU Angers, Angers, ${ }^{11}$ Service de thérapie cellulaire et d'hématologie clinique adulte, CHU Dupuytren, Limoges, ${ }^{12}$ Service d'hématologie clinique, Hôpital Henri Mondor, Créteil, ${ }^{13}$ Hématologie clinique, Hôpital Augustin Morvan, Brest, ${ }^{14}$ Département Hématologie Clinique, Hôpital St Eloi, Montpellier, ${ }^{15}$ Service hématologie, CHU Strasbourg, Strasbourg, ${ }^{16}$ Service de thérapie cellulaire et d'hématologie clinique adulte, ${ }^{17}$ Service cytogénétique, CHU ESTAING, Clermont-Ferrand, ${ }^{18}$ Unité de recherche clinique, Groupe Hospitalier Pitié-Salpêtrière, ${ }^{19} 2$ Service d'Hématologie Biologique, Groupe Hospitalier Pitié Salpétrière, ${ }^{20}$ Service de Greffe de Moelle, Unité Adolescents et jeunes adultes, Hôpital St Louis, Paris, France

Introduction: Reduced intensity conditioning (RIC) allogeneic stem cell transplantation (ASCT) remains a valuable and potentially curative strategy in high-risk CLL. Post-ASCT relapse remains an important cause of failure predicted by positive minimal residual disease (MRD) at $12 \mathrm{~m}$. ASCT is also associated with the burden of chronic graft versus host disease (cGVHD). We evaluated the efficacy and safety of a preemptive immunointervention (PrIm) based on serial MRD assessment in high-risk CLL (NCT01849939).

Material (or patients) and methods: Main inclusion criteria were (1) EBMT criteria for ASCT, (2) CLL in PR/CR, (3) mass $\leq 5 \mathrm{~cm}$, (4) age $\leq 70$, (5) SORROR score $\leq 2$ and (6) HLA donor $(10 / 10)$. RIC regimen included fludarabine $120 \mathrm{mg} / \mathrm{m} 2$, IV 
busulfan $6.4 \mathrm{mg} / \mathrm{kg}$, rabbit ATG $5 \mathrm{mg} / \mathrm{kg}$ and CsA prophylaxy. Centralized 10-color flow cytometry blood MRD was assessed before and at M1, M2, M3, M4, M5, M6, M9 and M12 postASCT. MRD[-] was defined by blood detection $<10 \mathrm{e}-4$ at 2 consecutive time-points, confirmed on bone marrow aspiration. PrIm algorithm, applied from D30 to M12, considered IWCCL response criteria, blood MRD levels and GVHD occurrence/severity. PrIm comprised tutored CsA early $(\leq 4$ $5 \mathrm{~m})$, standard $(6 \mathrm{~m})$ or late $(7 \mathrm{~m})$ tapering and discontinuation (T\&D). Early CsA T\&D was followed if failure by 1-3 increasing DLI doses $(1,5$ or $10 \times 10 \mathrm{e} 6 \mathrm{CD} 3 / \mathrm{kg})$. Primary end point was the rate of MRD[-] at M12.

Results: The 43 planned inclusions have been done. Before their last line prior to ASCT, pts had: (a) presence of a del(17p) in $1^{\text {st }}$ line $(n=24)$ or in relapse $(n=3)$, (b) absence of del( $\left.17 \mathrm{p}\right)$ but relapse $\leq 2 y$ post fludarabine-based combination $(n=14)$ or post-autologous transplantation $(n=1)$. At time of transplantation, $11 / 43(26 \%)$ had achieved CR, 28/43 (65\%) had achieved PR, 2/43 (5\%) had a SD with 2 unknown status. Six patients had undetectable MRD (limit of detection: $5 \times 10^{-6}$ ). For the 37 other patients, the median pre-ASCT MRD level was $2.35 \%(0.014$ to $70 \%)$.

This abstract describes the outcome of the 30 first consecutive pts including 26 pts evaluable [alive with $>12 \mathrm{~m}$ post-ASCT follow-up] and 4 pts non evaluable [death at M10 $(n=1$; sepsis) and at M8 ( $n=1 \mathrm{GVHD})$, EBV induced lymphoproliferation $(n=1)$ and immediate graft rejection $(n=1)]$. The PrIm algorithm has been applied in the 26 evaluable pts. At M3, 10 pts were already MRD[-], and 16 pts were still MRD[+]. Then 10 pts became MRD[-] and 6 remained MRD[+]. At 12 months, $67 \%$ blood MRD[-] was achieved (ITT analysis). Gr $\geq 2$ aGVHD rate was (11/30) $37 \%$ (consecutive to early CSA T\&D in 2 cases and to early CSA T\&D followed by DLI in 1 case) and the rate of extensive cGVHD was (5/29) $17 \%$ (consecutive to CsA T\&D in 1 case). We observed the occurrence of $\mathrm{Gr} \geq 2$ aGVHD and extensive CGVHD in $2 / 8$ and $1 / 8$ pts after early CsA T\&D. We also observed one case of $\mathrm{Gr} \geq 2$ aGVHD among the 5 pts who had early CSA T\&D followed by DLI. Eighteen severe adverse events were reported, including the 2 aforementioned deaths. These events were not related to the study procedure (Prlm). Conclusion: These preliminary data highlight the feasibility, safety and efficacy of a standardized Prlm strategy in high-risk CLL. As DLI appears to have limited impact when CSA T\&D fails, the preemptive use of new agents should be considered at this point for persistent MRD[+] after 3-6 $\mathrm{m}$ post-ASCT.

Disclosure of Interest: None declared.
P627

Prognostic value of early donor CD34 positive cells chimerism assessment after allogeneic stem cell transplantation in AML/MDS patients

P. Zielinska ${ }^{1, *}$, A. Wieczorkiewicz-Kabut ${ }^{1}$, M. DzierzakMietla ${ }^{1}$, A. Koclega ${ }^{1}$, K. Bialas ${ }^{7}$, I. Grygoruk-Wisniowska ${ }^{1}$, K. Jagoda $^{\prime}$, S. Kyrcz-Krzemien

${ }^{1}$ Hematology and BMT, MEDICAL UNIVERISTY OF SILESIA, Katowice, Poland

Introduction: The investigation of lineage-specific chimerism has become an important tool for the management of patients undergoing allogeneic hemopoietic stem cell transplantation. The aim of the study was early evaluation of donor CD34 positive cells chimerism after allogeneic hematopoietic stem cell transplantation (alloSCT).

Material (or patients) and methods: A total of 55 patients with acute myeloid leukemia (AML) and myelodysplastic syndrome (MDS) who underwent allo-SCT were included in the study (median age 36 years, range: 19-67 years). The study group consisted of 32 males and 25 females who underwent allo-SCT from unrelated or sibling donor (12 patients). Peripheral blood was source of stem cells in all patients. Flow cytometric analysis and cell sorting was performed in bone marrow collected at day 30 after allo-SCT. For the purpose of this study we analyzed immature progenitor cells (CD34 +CD19-). Lineage specific donor chimerism was assessed using STR method and was calculated following the defined genetic profiles of the donor and the recipient. The cells were sorted using FACS Aria III.

Results: All patients engrafted and were followed up for a median period of 23 months ( 2 to 44 months). Median time to relapse was approx. 100 days. In patients who remained in remission of the underlying disease, chimerism in CD34+ cells never fell below 97\%. Interestingly, all patients who relapsed presented with lower donor chimerism in CD34 positive cells in comparison to the group of patients in remission of the underlying disease.

Conclusion: Currently, fluorescence-based PCR amplification of short tandem repeats is the gold standard for analyzing post-transplant chimerism and has been used in most studies. Assessment of donor chimerism in CD34 positive cells may be worth applying in selected AML/MDS patients with high risk of relapse.

References: 1. Mattsson J, Uzunel M, Tammik L, Aschan J, Ringden O. Leukemia lineage-specific chimerism analysis is a

\begin{tabular}{|c|c|c|c|c|c|c|}
\hline $\begin{array}{l}\mathrm{Pt} \\
\text { no }\end{array}$ & $\begin{array}{c}\text { Time of } \\
\text { survival } \\
\text { since } \\
\text { relapse } \\
\text { (days) }\end{array}$ & $\begin{array}{l}\text { Time to } \\
\text { relapse } \\
\text { (days) }\end{array}$ & $\begin{array}{l}\text { Assessment } \\
\text { of BM blasts } \\
\text { at day } 30 \\
\text { after alloSCT } \\
\quad(\%)\end{array}$ & $\begin{array}{c}\text { MRD } \\
\text { at day+30 } \\
\text { after alloSCT }\end{array}$ & $\begin{array}{c}\text { Donor chimerism } \\
\text { in unsorted BM cells } \\
(\%)\end{array}$ & $\begin{array}{c}\text { Donor lineage specific } \\
\text { chimerism } \\
\text { in } \\
\text { CD } 34+\text { cells }\end{array}$ \\
\hline 1 & 38 & 323 & $<1$ & negative & 87 & 49 \\
\hline 2 & 100 & 28 & 2 & positive & 100 & 36 \\
\hline 3 & 55 & 52 & 1 & positive & 87 & 13 \\
\hline 4 & 220 & 55 & 1 & positive & 100 & 100 \\
\hline 5 & unknown & 57 & 3 & positive & 84 & 51 \\
\hline 6 & 360 & 161 & 1 & positive & 100 & 16 \\
\hline 7 & 312 & 215 & 2 & positive & 99 & 0 \\
\hline 8 & 153 & 293 & 0 & positive & 100 & 3 \\
\hline 9 & 45 & 69 & 1 & positive & 56 & 8 \\
\hline
\end{tabular}


sensitive predictor of relapse in patients with acute myeloid leukemia and myelodysplastic syndrome after allogeneic stem cell transplantation. Leukemia 2001: 15, 1976-1985.

2. Rosenow F, Berkemeier A, Krug U, et alStelljes M. CD34(+) lineage specific donor cell chimerism for the diagnosis and treatment of impending relapse of $\mathrm{AML}$ or myelodysplastic syndrome after allo-SCT. Bone Marrow Transplant. 2013; 4. 3. Lange T, Hubmann M, Burkhardt R, et al. Monitoring of WT1 expression in PB and CD34(+) donor chimerism of BM predicts early relapse in AML and MDS patients after hematopoietic cell transplantation with reduced-intensity conditioning. Leukemia 2011; 25(3):498-505.

Disclosure of Interest: None declared.

\section{P628}

Monitoring of $\mathrm{T}$ cell chimerism and early intervention improves outcome in paediatric patients undergoing haematopoietic stem cell transplantation for malignant diagnosis

P. Sivaprakasam 1,*, M. Cummins ${ }^{1}$, J. Moppett ${ }^{1}$, C. Steward ${ }^{1}$, A. Gassas ${ }^{1}$, B. Pat ${ }^{1}$, L. Keen ${ }^{2}$, E. Wroe ${ }^{2}$, A. Stanton ${ }^{2}$, A. Poles', C. Morris

${ }^{1}$ Paediatric Stem Cell Transplantation, BRISTOL ROYAL HOSPITAL FOR CHILDREN, ${ }^{2}$ Histocompatibility and Immunogenetics, $H$ \& I, NHSBT, Bristol, United Kingdom

Introduction: Chimerism is routinely used for monitoring donor graft function following haemopoietic stem cell transplant (HSCT). The role of monitoring $T$ cell lineagespecific chimerism (TCC) and its impact on outcome is not well established in paediatric HSCT for malignant diagnosis.

Material (or patients) and methods: In this study, we intended to assess the implication of monitoring TCC and evaluating outcome of early intervention with donor lymphocyte infusion (DLI) following a decrease in TCC.

Between January 2009 and December 2015, 134 paediatric patients underwent an allogeneic HSCT at Bristol Royal Hospital for Children for malignant diagnosis. Lineage specific chimerism analyses of patients were collated. Frequency of 'significant drop' in TCC (>5\% drop in donor T cell chimera), need for early intervention with DLI and respective patient outcome were studied.

Results: Of the 134 patients, 34 demonstrated a significant drop in TCC with no significant drop in whole blood and/or myeloid chimerism. Early weaning of immunosuppression was attempted with first episode of dropping TCC, in all patients, when possible. All 13 patients who received at least one DLI are alive (1-relapsed but still alive). A total of 21 DLIs were administered (median dose $5 \times 10^{5} / \mathrm{kg}$ CD3 cells. range: $1 \times 10^{4} /$ $\mathrm{kg} \mathrm{CD3}$ to $1 \times 10^{7} / \mathrm{kg}$ CD3 cells) at a median of 100 days post HSCT (range: 68-253 days) to achieve full donor chimera in all but one, who relapsed. Of the remaining 21 patients who did not receive DLI, seven died of relapse; two of transplant related complications. DLI was not attempted in those patients as four received cord transplant; eight improved TCC with early immunosuppression withdrawal; two had graft rejection; four had early relapse post HSCT; two died of TRM and one lost to follow up. Comparing outcome in patients who received DLI based on reduction in TCC versus patients who did not receive DLI, demonstrated a statistically significant improvement in outcome $(P=0.026)$.

Conclusion: Monitoring of TCC in children undergoing HSCT for malignant diagnosis is very useful. Early intervention with DLI based on reduction of TCC had a significant improvement in outcome.

References: 1. Early Mixed T-Cell Chimerism After Allogeneic Hematopoietic Stem Cells Transplantation Is Highly Predictive For Progression Free Survival. Jérôme Cornillon et al. November 15, 2013; Blood: 122(21) 2079.

2. Donor Chimerism Early after Reduced-Intensity Conditioning Hematopoietic Stem Cell Transplantation Predicts Relapse and Survival. John Koreth et al. BBMT. October 2014. Volume 20, Issue 10, Pages 1516-1521.

Disclosure of Interest: None declared.

\section{P629}

Clinical significance of the expression of WT1 gene before Allogeneic hematopoietic stem cell transplantation in AML patients

S. Rostami, ${ }^{1, *}$, B. Chahardouli ${ }^{1}$, N. Alizad Ghandfroush ${ }^{1}$, M. Jahani ${ }^{1}$, S. A. Mousavi ${ }^{1}$, B. Bahar ${ }^{1}$, H. Kamran Zadeh ${ }^{1}$, M. Vaezi ${ }^{1}$, K. Alimoghaddam ${ }^{1}$, A. Ghavamzadeh ${ }^{1}$

${ }^{1}$ Haematology-Oncology \& Stem cell transplantation Research center, Tehran, Iran, Islamic Republic Of

Introduction: Allogeneic stem cell transplantation represents a curative treatment option for patients with acute myeloid leukemia (AML) with high-risk features at diagnosis. However, the percentage of patients who relapse after transplantation is still high WT1 is an important biomarker for monitoring minimal residual disease in acute myeloid leukemia.

Material (or patients) and methods: To evaluate the prognostic value of the expression of the Wilms' tumor gene (WT1) before allogeneic hematopoietic stem cell transplantation (allo-HSCT) for predicting the relapse of patients with $A M L$, the level of WT1 expression in peripheral blood (PB) samples was quantitated by RQ-PCR in 230 patients with AML who were treated with allo-HSCT.

Results: The WT1 gene expression more than normal peripheral blood ( $>20$ copies $/ 10^{4} \mathrm{ABL}$ ) before allogeneic HSCT was defined as WT1 positivity. Of these 230 patients, 85 were WT1 positive before receiving the conditioning regimen, with a median of 1968 of WT1 (range, 21 to 25466 copies/104 ABL). Twenty seven of the $85(32 \%)$ patients relapsed, compared with $11(8 \%)$ of the remaining 134 patients with normal WT1 expression before allogeneic HSCT. There was significant difference in overall and relapse-free survival rates between WT1 positive and WT1 negative patients $(P=0.009$ and $P=0.016)$.

Conclusion: Pre-HSCT WT1 expression is a useful marker for identifying patients with a high risk of relapse after allo-HSCT who then can be closely monitored and treated much earlier and more effectively by intervention such as early withdrawal of immune suppression or donor lymphocyte infusion as compared to patients in overt relapse.

Disclosure of Interest: None declared.

\section{P630}

Evaluation of chimerism in bone marrow enriched CD34+ cells for monitoring of minimal residual disease after allogeneic stem cell transplantation

S. Vasileiou ${ }^{1, *}$, I. Baltadakis ${ }^{1}$, M. Garofalaki ${ }^{1}$, M. Gonianaki ${ }^{1}$ A. Loidoris", E. Tziotziou', M. Vardaka', C. Giatra?', M. Papageorgiou', M.-E. Karatza ${ }^{1}$, P. Papaioannou ${ }^{1}$, S. Gigantes ${ }^{\prime}$, I. Apostolidis ${ }^{1}$, D. Karakasis ${ }^{1}$, N. Harhalakis ${ }^{1}$

${ }^{1}$ BMT Unit, Evagelismos Hospital, Athens, Greece

Introduction: Monitoring of minimal residual disease (MRD) after allogeneic hematopoietic stem cell transplantation (alloSCT) has become mandatory in the effort to predict the risk of hematologic relapse. The purpose of this study was the evaluation of CD34+ cell donor chimerism as a means of MRD assessment in patients transplanted for myeloid malignancies that lack a reliable molecular marker.

Material (or patients) and methods: The study enrolled 10 patients that underwent allo-SCT for myelodysplastic syndrome (MDS, $n=4$ ), de novo/secondary acute myeloid leukemia (AML, $n=5$ ), or myelofibrosis $(n=1)$. A total of 16 chimerism assays were performed in CD34+ cells, which were enriched from bone marrow (BM) samples either by use of magnetic microbeads coated with anti-CD34 monoclonal antibody $(n=14)$ or by fluorescence-activated cell sorting (FACS, $n=2$ ). Percentage of CD34+ cells in initial BM samples as well as purity of the resulting CD34+ cell populations were analyzed by flow cytometry. Chimerism was measured by PCRbased analysis of Short Tandem Repeats (STRs).

Results: The median percentage of CD34+ cells in unprocessed BM mononuclear cells was $0.73 \%$ (range: $0.06-3.65 \%$ ). Enrichment of CD34+ cells resulted in a median purity of $71 \%$ (range: $40-93 \%$ ). 
Regarding immunomagnetic CD34+ cell separation, purity was superior for BM samples with initial concentrations of CD34+ cells $\geq 1 \%$ compared to $<1 \%$ ( $88-93 \%$ versus $40-73 \%$, respectively). Starting from 1-3 $\mathrm{ml}$ whole BM specimens, the yield of total CD34 + cells for chimerism studies ranged from $10^{3}$ to $2.8 \times 10^{5}$ (median: $\left.3.7 \times 10^{4}\right)$. High-level donor CD34+ cell chimerism (94.7-99.4\%, median: 97\%) was observed in 7 patients, none of whom developed relapse at a median follow-up of 5 (range: 3-17) months. On the other hand, a decline in donor CD34+ cell chimerism was encountered in the 3 remaining patients at a median of 3 (range: 2-5) months post transplant. In one of those 3 patients, low donor CD34+ cell chimerism (5.5\%) coincided with early relapse of AML (BM blasts: 12\%). In the other 2 patients, decreased values of donor CD34+ cell chimerism (49.5 and 46.4\%) were detected while in hematologic complete remission (CR) of the disease (secondary AML and MDS, respectively). All the above 3 patients were treated with salvage or preemptive azacitidine and showed a response in CD34+ cell donor chimerism (64-95.6\%) with achievement or maintenance of hematologic $C R$.

Conclusion: Donor chimerism of BM CD34+ cells is a relevant marker of the activity of myeloid malignancies following alloSCT and may guide to early interventions in the aim of preventing hematologic relapse, particularly with high-risk AML or MDS. Relatively simple and time sparing immunomagnetic selection techniques usually allow for considerable CD34+ cell purity and informative chimerism results, thereby facilitating routine clinical application of the procedure.

Disclosure of Interest: None declared.

P631

Clinical Characteristics and Frequency of Poor Graft Function After Allogeneic Hematopoietic Stem Cell Transplantation

U. Sahin 1,*, M. Kurt Yuksel', M. Merter ${ }^{1}$, M. Gunduz ${ }^{1}$, Z. Narli Ozdemir $^{1}$, S. Civriz Bozdag', S. K. Toprak', P. Topcuoglu ${ }^{1}$ O. Arslan', M. Ozcan 1', T. Demirer', O. Ilhan ', H. Akan', M. Beksac ${ }^{7}$, G. Gurman ${ }^{1}$

${ }^{1}$ ANKARA UNIVERSITY HEMATOLOGY AND BONE MARROW TRANSPLANTATION UNIT, Ankara, Turkey

Introduction: Poor graft function (PGF) is characterized by pancytopenia and a hypoplastic marrow, with complete donor chimerism [1]. This study aims to investigate the frequency, risk factors and prognosis of PGF after allogeneic hematopoietic stem cell transplantation (allo-AHSCT).

Material (or patients) and methods: Clinical data of 341 patients who underwent allo-HSCT in Ankara University Bone Marrow Transplantation Unit between Jan 2009 and Oct 2015 were retrospectively reviewed for the development of PGF, demographics, disease and transplant characteristics. PGF was defined as neutropenia (neutrophil count $<1.0 \times 10^{9} / \mathrm{L}$ ) after day +30 , associated with hypoplastic-aplastic bone marrow, complete donor chimerism and absence of relapse. Chi-square and Mann-Whitney $U$ tests were used for analyses of variance and Kaplan-Meier method for survival estimates. $P<0.05$ was considered statistically significant.

Results: Overall PGF occured in 19 out of 341 transplants (5.6\%). Median age of the patients with PGF was 33 (18-63) and $57.9 \%$ were male. Common diagnoses were aplastic anemia (AA) $(n=5)$, myelofibrosis $(n=4)$, acute lymphoblastic leukemia (ALL) $(n=4)$, non-Hodgkin lymphoma (NHL) $(n=3)$, chronic myleoid leukemia (CML) $(n=2)$ and myelodysplastic syndrome (MDS) $(n=1)$. Median time to neutrophil engraftment (neutrophil count $>0.5 \times 10^{9} / \mathrm{L}$ ) was 20 (14-44) days and median time to PGF diagnosis was 58 (32-163) days. Donor type was matched unrelated in $31.6(n=6)$, related in 63.1 $(n=12)$ and haploidentical in $5.3 \%(n=1)$ Conditioning regimen was myeloablative in $57.9 \%(n=11)$. Stem cell source was bone marrow in $27.3 \% \quad(n=5)$. Cytomegalovirus (CMV) reactivation requiring treatment was observed in $73.7 \%$ $(n=14)$, BK-virus infection in $15.8(n=3)$, invasive fungal infections in $47.4 \%(n=9)$, systemic bacterial infections in $57.9 \%(n=11)$, hemosiderosis in $21.1 \%(n=4)$, acute graft versus host disease (GvHD) in $42.1 \%(n=8)$ and hepatic venooclusive disease in $5.3 \%(n=1)$. PGF ended up with loss of engraftment in $36.8 \%(n=7)$ and progressive severe GvHD in $10.5 \%(n=2)$, resolved in $26.3 \%(n=5)$ and persisted in $26.3 \%$ $(n=5)$. Add-back stem cell infusion was given to 2 patients with AA, however, did not prevent engraftment loss. Two patients underwent retransplantation, including $1 \mathrm{ALL}$ and 1 AA. Median follow-up after allo-HSCT was 6 (2-77) months. Overall survival was $52.6 \%$.

Conclusion: PGF is a serious condition frequently ending up with loss of engraftment, morbidity and mortality. It is common in myelofibrosis patients, which consist nearly quarter of this cohort. Thus, poor bone marrow microenvironment may play a role in PGF development, as well as other factors causing suppression of bone marrow, such as infections, medications and GvHD [2].

References: 1. Bone Marrow Transplant.2014;49(5):720-1.

2. Int J Med Sci.2014;11(6):652-7.

Disclosure of Interest: None declared.

\section{P632}

Reconstitution of CD34+ progenitor cells after allogeneic stem cell transplantation for patients with post-transplantation cyclophosphamide

V. Vasilyeva, ${ }^{1, *}$, E. Parovichnikova ${ }^{1}$, L. Kuzmina ${ }^{1}$, M. Drokov ${ }^{1}$ E. Mikhaltsova', O. Koroleva ${ }^{1}$, D. Dubnyak', I. Galtceva ${ }^{1}$, V. Savchenko on behalf of CIC 930

${ }^{1}$ Bone morrow transplant department, ${ }^{2}$ Laboratory of immunophenotyping of blood cells, National Research center for Hematology, Moscow, Russian Federation

Introduction: Allogenic hematopoietic stem cell transplantation (allo-HSCT) is a potentially curative method for hematological malignancies. Last 10 years high-dose posttransplantation cyclophosphamide (CY) slowly replaces

Table 1. Frequency of PGF and selected characteristics of patients according to disease

\begin{tabular}{lccccccc}
\hline & $A A$ & $M F$ & $A L L$ & NHL & CML & MDS \\
\hline Frequency \% (n/N) & $14.3(5 / 35)$ & $30.8(4 / 13)$ & $6.6(4 / 61)$ & $17.6(3 / 17)$ & $10.5(2 / 19)$ & $3.6(1 / 28)$ & .24 \\
Days to PGF diagnosis Median (min-max) & $58(32-83)$ & $45(33-62)$ & $67(32-153)$ & $85(79-91)$ & $52(52-52)$ & $68(45-163)$ & .77 \\
Bone marrow source \% (n) & $3(60)$ & - & $1(25)$ & - & - & $1(100)$ & .36 \\
MUD \% (n) & $3(60)$ & $1(25)$ & - & $2(67)$ & - & - & .08 \\
Myeloablative conditioning \% (n) & $2(20)$ & $1(25)$ & $3(75)$ & $2(67)$ & $2(100)$ & $1(100)$ & .39 \\
Hemosiderosis \% (n) & $2(50)$ & $1(25)$ & - & $1(33)$ & - & - & .66 \\
CMV \% (n) & $4(80)$ & $2(50)$ & $3(75)$ & $3(100)$ & $1(50)$ & $1(100)$ & .66 \\
Acute GVHD \% (n) & $1(20)$ & $1(25)$ & $3(75)$ & $2(67)$ & - & $1(100)$ & .23 \\
OS \% & 20 & 75 & 50 & 67 & 100 & - & .33 \\
& & & & & & &
\end{tabular}


standard immunosuppression therapy (IST). Despite the fact that selective $\mathrm{T}$ cell depletion by post-transplant Cy is well known, effects on the other cell population such as CD34+ progenitors are still not studied well enough.

Material (or patients) and methods: Recovering of CD34+ cells was analyzed among 29 patients who received allogenic hematopoietic stem cell transplantation (allo-HSCT) for different hematological malignancies. Median of age was 36 years (24-60 years). 16 patients were males, 13 - females. 22 received RIC, 7 - myeloablative regime. Match unrelated donor (MUD) was in 17 cases, "Mismatch" MUD - 2, Match related donor (MRD) - 9, "Mismatch" MRD - 1. We divided patients into two group depending on type of immunosuppression therapy (IST). Standard IST consisted of CSA, MMF or MTX at standard dose were administrated for 23 patients. CY as alternative IST was indicated to 6 patients. 4 of these patients were in relapse or disease progression, and 2 patients had second unsure remission.

Peripheral blood (PB) was the source of stem cells (SC) for 12 patients in standard IST group, bone morrow (BM) - 10, and 1 patient had PBCS and BM. Median for PBCS was $5,1(3,5-12,6)$ ${ }^{*} 10^{6} / \mathrm{kg}$, for BMSC $\left.-3(1,2-3,85)\right)^{*} 10^{8} / \mathrm{kg}$.

In alternative IST group PBSC was the source of SC for 3 patients, median $5(4-5,2)^{*} 10^{6} / \mathrm{kg}$. BM for 3 patients too, median $2,7(2,5-3)^{*} 10^{8} / \mathrm{kg}$

Peripheral blood samples were collected in EDTA-tubes at day of engraftment after allo-HSCT $(\mathrm{Me}=20$ day (14-35)), at days $+30,+60$ and +90 . We used standard ISHAGE (International Society for Hematotherapy and Graft Engineering) protocol for CD34 ${ }^{+}$cell enumeration.

Results: Successful engraftment was observed in all patients except one from alternative IST group. We associate it not with using CY after allo-HSCT, but with the type of hematological malignancy - myeloproliferative neoplasm with myelofibrosis. Recovering of CD34+ cells are shown at figure 1. The percent of CD34+ cell population at day of engraftment after posttransplant $C Y$ alone was statistically lower $(0,019 \% \pm 0,00754 \%$, $\left.P=0,047^{*}\right)$ than in patients with standard IST $(0,119 \% \pm 0.0265 \%)$.

Conclusion: As we have shown earlier: post-transplant CY spared memory T-cells in contrast with standard IST (ASH 2015, abstract 5475 ). Due to this study we may conclude that patients with post-transplant CY had a different CD34+ reconstitution profile. This interesting fact needs further investigation.

Disclosure of Interest: None declared.

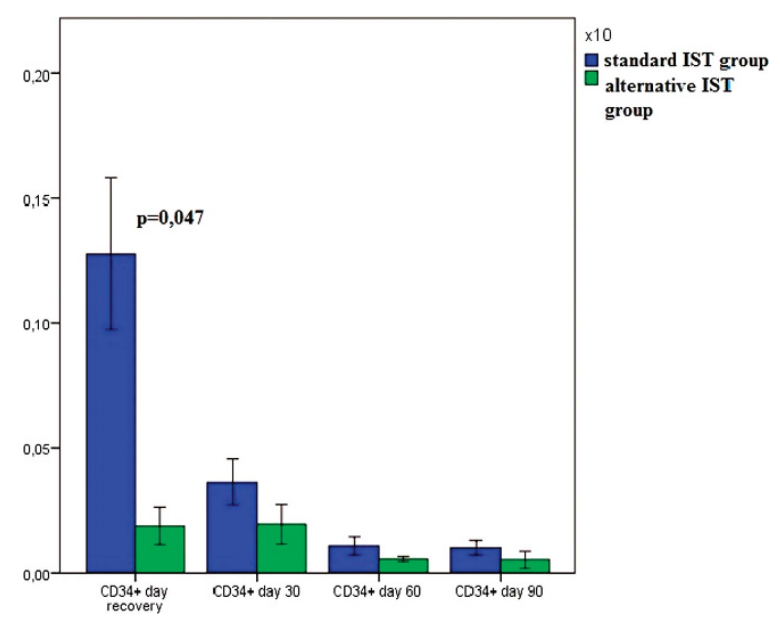

P633

Chimerism on $\mathbf{3 0}$ day after allogeneic hematopoietic stem cell transplantation predicts early relapses

V. Lavrinenko ", , Y. Mareiko ${ }^{1}$, E. Berezovskaya ${ }^{1}$, M. Belevtsev ${ }^{1}$, O. Bydanov" ${ }^{1}$, O. Aleynikova ${ }^{1}$

${ }^{1}$ Belarusian Research Center for Pediatric Oncology, Hematology and Immunology, Minsk, Belarus

Introduction: Hematopoietic stem cell transplantation (HSCT) is a potentially curative treatment for patients with high-risk malignant hematological diseases. However relapse after transplantation is still associated with a poor prognosis and remains the main reason for treatment failure. Increasing mixed chimerism (MC) is often preceding a hematological relapse but there are still absent strict criteria for relapse prediction and only dynamic changes in chimerism level are mostly assayed. The aim of this study was analyzing of the predictive value of chimerism level on day +30 after alloHSCT as universal marker for all patients with malignant hematological diseases.

Material (or patients) and methods: In the assay 57 patients with $\mathrm{AL}, \mathrm{MDS}$ or NHL, 10 (1-29) years old who underwent 61 alloHSCT (4 patients received 2nd HSCT) after MAC (49) or RIC (3) were included. Chimerism analysis was performed by polymerase chain reaction (PCR) of STR markers (AmpFISTR ${ }^{\mathbb{R}}$ SGM Plus ${ }^{\mathbb{R}}$ PCR Amplification Kit, Applied Biosystems) in peripheral blood (PB) and/or bone marrow (BM). If chimerism level was $97-100 \%$, the result was confirmed by Real-time PCR of InDel polymorphisms as more sensitive method.

Results: On day+30 ten (16.4\%) patients after alloHSCT had MC or low level MC (II-MC) with median 98.38\% (92.62-99.1) donor cells and 51 (83.6\%) patients had complete donor chimerism (CC) with median $99.91 \%$ (99.45-100). It was possible to compare chimerism level in bone marrow and peripheral blood on +30 day in 43 patients. Similar results were obtained in 39 pairs of BM and PB samples: CC was in 34 pair of samples, MC was in 5 pair. In 4 cases chimerism in bone marrow and peripheral blood samples differed: 1 patient had MC in PB and CC in BM, 3 had CC in PB and MC in BM. After a median follow-up in surviving patients of 34 months (range, 6.4 to 66 months), OS was $80.4 \%$ in CC group vs $34.3 \%$ in II-MC/MC group (at 6 months, $90.2 \%$ vs $80 \%$ ), $P=0.0022$. LFS was $63.7 \%$ in CC group vs $30 \%$ in II-MC/MC group (at 6 months, $88.2 \%$ vs $40 \%), P=0.0027$. All five (100\%) relapses in patients with II-MC/MC on 30 day were early isolated bone marrow and occurred during the first 6 months after transplantation (median 84, range 54-147 days), the cumulative incidence of relapse was $50 \%$. Patients with CC on 30 day during the first 6 months after transplantation had only 1 extramedullary relapse in patient after $2^{\text {nd }}$ alloHSCT, later they had 7 more relapses

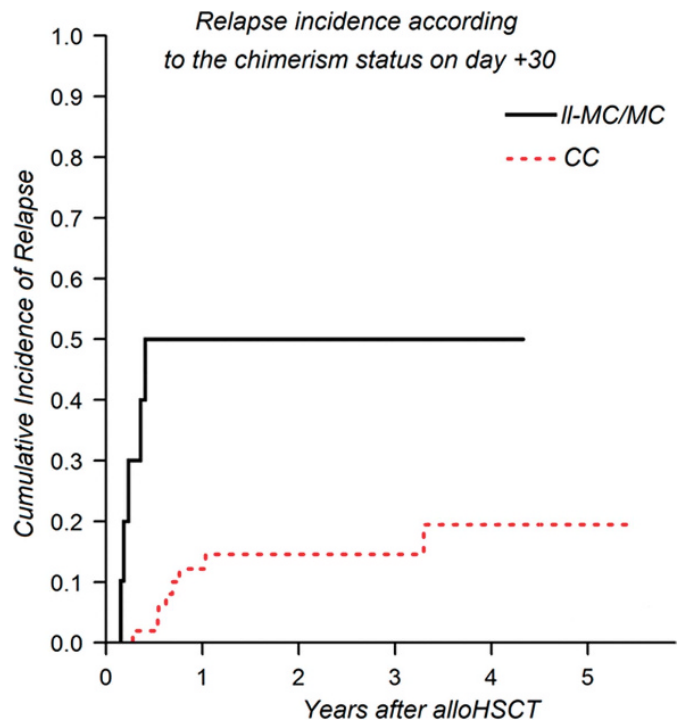


(4 isolated bone marrow and 3 combined relapses), the cumulative incidence of relapse was $2 \%$ at 6 months; $12.2 \%$ at 1 -year, $14.5 \%$ at 2-year, $19.4 \%$ at 4 -year, median time to relapse was 238.5 (100-1203) days. Patients with II-MC/MC on 30 day had earlier relapses than patients with $C C, P=0.0084$. TRM had no significant differences between patients with CC and II-MC/MC: $16.9 \%$ vs $20 \%$ ( $9.8 \%$ vs $10 \%$ at 6 months). Interesting observation that $3 / 5$ of the relapsed patients with MC/II-MC showed decreasing of $\mathrm{MC} / \mathrm{II}-\mathrm{MC}$ or conversion to $\mathrm{CC}$ in $\mathrm{PB} / \mathrm{BM}$ after 30 day with subsequent reappearing/increasing of autologous signal before relapse.

Conclusion: II-MC/MC on +30 in peripheral blood or bone marrow can predict development of early relapses during the first 6 month after alloHSCT.

Disclosure of Interest: None declared.

\section{P634}

Hematopoietic stem cell transplantation in children with hemophagocytic lymfohistiocytosis (HLH) - report of Polish Pediatric Stem Cell Transplantation Group

K. Drabko ${ }^{1, *}, M$. Salomonowicz ${ }^{2}$ A. Pieczonka ${ }^{3}$,J. Gozdzik ${ }^{4}$ I. Malinowska ${ }^{5}$, K. Kalwak ${ }^{2}$, J. Wachowiak ${ }^{3}$, A. Zaucha-Prazmo $^{\prime}$, J. Kowalczyk ${ }^{1}$ on behalf of Polish Pediatric Stem Cell Transplantation Group

${ }^{7}$ Pediatric Hematology Oncology and Transplantology, Medical University, Lublin, ${ }^{2}$ Department of Pediatric BMT, Hematology and Oncology, Medical University, Wrocław, ${ }^{3}$ Pediatric Hematology Oncology and Transplantology, Medical University, Poznan, ${ }^{4}$ Stem Cell Transplant Center, Jagiellonian University Collegium Medicum, Krakow, ${ }^{5}$ Department of Pediatrics, Hematology and Oncology, Medical University, Warsaw, Poland

Introduction: Hemophagocytic lymphohistiocytosis (HLH) become a standard indication for allogeneic stem cell transplantation (alloHSCT) in following clinical situations: identification of characteristic genetic defect (PRF1, UND13D, STX11 or STXBP2), other immune deficiency with symptoms of HLH like XLP or resistant, relapsed malignancy or infection related $\mathrm{HLH}$.

Material (or patients) and methods: Between 2006 and 2014 in Polish Pediatric Hematolology-Oncology centres 37 children were diagnosed as having $\mathrm{HLH}$ and were treated with etoposide, dexamethason and cyclosporine according to HLH2004 protocol (1). Eighteen (49\%) based on above criteria underwent alloHSCT in four Pediatric Transplant Centres (Wrocław-6, Lublin-5, Poznan-5 and Krakow-2). Transplanted group consisted of 12 boys and 6 girls; median age at time of alloHSCT was 3.2 years (range $1-18.5$ years).

$7 / 18$ transplanted patients had familiar HLH (FLH), in 10 children mutation were not found and those patients were considered as HLH secondary to viral infection (HHV6 $n=4$, CMV $n=2$, EBV $n=2$, mycoplasma pneumoniae $n=1$ ) or autoimmune disease $n=1$ and no data about one patient.

Match unrelated donor was used in majority cases $n=15$, match related donor in 2 patients and one child was transplanted from mismatched related donor. Stem cell source were bone marrow $n=9$ and peripheral blood $n=9$. Conditioning regimen was myeloablative in 14 patients (9 treosulfan based and 5 busulfan based) and four children received fludarabin and melphalan. Serotherapy was used in all procedures: ATG in 13 and alemtuzumab in 5. Median number of CD34 cells infused was $5 \times 106$ (range 2.48-17.45). GvHD prophylaxis consisted of cyclosporine in all cases with addition of MMF in 8, MTX in 5 and steroids in one.

Results: Median follow-up of the group was 1.5 years (range 6 days-9 years). All but one patient engrafted and 12 of 17 evaluated patients achieved stable complete chimerism, 2 children have stable mixed chimerism and data about 2 patients are missing.

Probability of overall survival was 0.71 . Thirteen patients are alive and well. Causes of death were infections $n=3$ (CMV and fungal), multiorgan failure in 2 patients (day +6 before engraftment in one boy and day +47$)$. Acute GvHD grade II

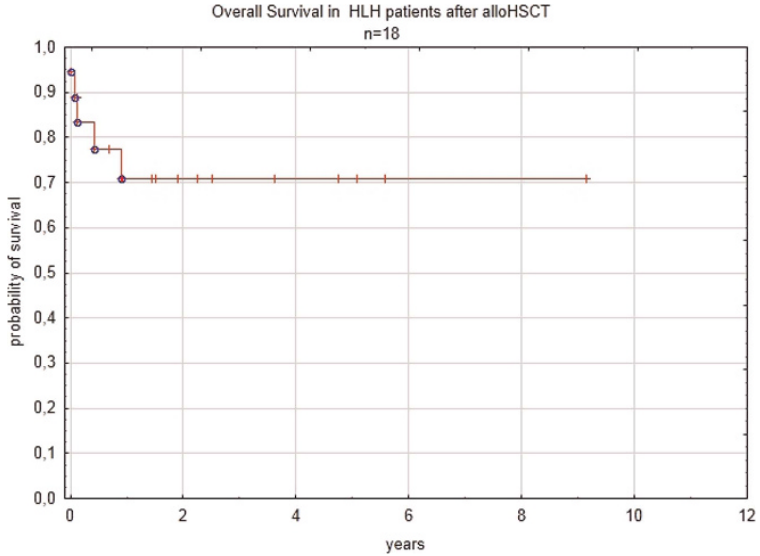

or more occurred in 9 patients (50\%), chronic GvHD contributed to the death of one patient.

Conclusion: This study confirmed that alloHSCT led to long term survival in familiar or resistant to standard therapy hemophagocytic lymphohistiocytosis comparable with other studies (2). Better survival could be achieved with more efficient management of early complications: infections and organ toxicities.

References: 1 . https://clinicaltrials.gov/ct2/show/NCT00426101

2. Hematopoietic Stem Cell Transplantation for Hemophagocytic Lymphohistiocitosis: A National Retrospective Analysis of Data from the Italian Association of Pediatric Hematology Oncology (AIEOP), Blood Abstracts: 57th Annual Meeting Abstracts Vol. 126, Issue 23, 3 Dec 2015.

Sponsored by National Science Centre (Poland) 0989/B/ P01/2011/40.

Disclosure of Interest: None declared.

\section{P635}

Outcome of Reduced-Intensity Allogeneic Stem Cell Transplantation for Pediatric Patients with Acute Myeloid Leukemia in Second Complete Remission in Our Institute K. Higuchi, ${ }^{1, *}$ M. Sato ${ }^{1}$, A. Ioi ${ }^{1}$, A. Mayumi ${ }^{1}$, M. Shimizu', A. Sawada ${ }^{7}$, M. Yasui ${ }^{1}$, M. Inoue

${ }^{7}$ Hematology/Oncology, Osaka Medical Center And Research Institute For Maternal And Child Health, Izumi, Osaka, Japan

Introduction: We have been performing reduced-intensity stem cell transplantation (RIST) to avoid preconditioningrelated complications. However, the effectiveness of RIST in pediatric patients with acute leukemia remains to be clarified. We reported the results of RIST for acute lymphoblastic leukemia in second complete remission (CR2), which suggested that RIST was comparable to MAST, at the 41st annual meeting of EBMT in Istanbul, Turkey. This time we evaluated the effectiveness of RIST in pediatric patients with acute myeloid leukemia (AML) in CR2.

Material (or patients) and methods: We retrospectively reviewed 16 pediatric patients with AML in CR2 who underwent first hematopoietic stem cell transplantation (HSCT) between 1995 and 2015. We compared the outcomes of RIST with those of myeloablative stem cell transplantation (MAST). Results: The median age at HSCT was 8.5 years (range, 1 to 21 years). Nine patients received HLA-matched bone marrow (MAST 8, RIST 1), 2 HLA-mismatched bone marrow (MAST 1, RIST 1), and 5 cord blood (MAST 1, RIST 4). Six patients underwent RIST and 10 patients underwent MAST. The median follow-up durations of RIST and MAST groups were 21.5 months (range, 10 to 75 months) and 176 months (range, 6 to 240 months), respectively. The 2-year overall survival (OS) rates in RIST and MAST groups were $83.3 \%$ and $90.0 \%$ $(P=0.87)$, and the 2-year event free survival (EFS) rates were $66.7 \%$ and $80.0 \%(P=0.59)$, respectively. The 2 -year cumulative 
transplant-related mortality (TRM) rates in RIST and MAST groups were $16.7 \%$ and $10.0 \%(P=0.74)$, and the 2 -year cumulative relapse rates were $20.0 \%$ and $11.1 \%(P=0.66)$, respectively.

Conclusion: In our series, the outcomes of RIST group don't seem to be so different from those of MAST group. Although further studies are needed because of the small size of patients and short follow-up duration, RIST can be considered as the transplantation for AML in CR2.

Disclosure of Interest: None declared.

P636

Fertility preservation in the Nordic Countries prior to HSCT in childhood or adolescence

K. Jahnukainen ${ }^{1,2, *}$, M. Ifversen ${ }^{3}$, H. Hasle ${ }^{4}$, C. Rechnitzer,

G. Greggains ${ }^{6}$, R. Bjarnason ${ }^{7}$, B. Borgström ${ }^{8}$, C. Petersen ${ }^{9}$,

K. A. Rodriguez-Wallberg ${ }^{10}$, J.-B. Stukenborg ${ }^{1}$, K. Tryde Macklon ${ }^{11}$ on behalf of the Nordic Network for Gonadal Preservation and the Nordic Society of Pediatric Hematology and Oncology (NOPHO)

${ }^{7}$ Pediatric Endocrinology Unit, Karolinska Institutet and University Hospital, Stockholm, Sweden, ${ }^{2}$ Children's Hospital, University of Helsinki and Helsinki University Central Hospital, Helsinki, Finland, ${ }^{3}$ Paediatric Stem Cell Transplant and Immune Deficiency Dept for Children and Adolescents, Rigshospitalet, Copenhagen University Hospital, Copenhagen, ${ }^{4}$ Department of Pediatrics, Aarhus University Hospital Skejby, Århus, ${ }^{5}$ Department of Paediatrics and Adolescent Medicine, Rigshospitalet, Copenhagen University Hospital, Copenhagen, Denmark, ${ }^{6}$ Section for Reproductive Medicine, Department of Gynaecology, Oslo University Hospital, Oslo, Norway, ${ }^{7}$ Children's Medical Center, Landspitali and University of Iceland, Reykjavik, Iceland, ${ }^{8}$ Department of Pediatrics, Huddinge, ${ }^{9}$ Pediatric oncology unit, ${ }^{10}$ Obstetrics and Gynecology and Reproductive Medicine, Karolinska Institutet and University Hospital, Stockholm, Sweden, ${ }^{11}$ The Fertility Clinic, Rigshospitalet, Copenhagen University Hospital, Copenhagen, Denmark

Introduction: An increasing number of children and young adults become long time survivors after hematopoietic stem cell transplantation (HSCT) in childhood or adolescence. The issue of fertility preservation is emerging since the treatment with HSCT is known to cause infertility. Cryopreservation of ovarian tissue was first introduced in the Nordic countries in 1999. Cryopreservation of semen in postpubertal boys is a routine procedure in all hematologic-oncologic departments, whereas cryopreservation of ovarian and testicular tissue is centralized. We here review the experiences of fertility preservation from the Nordic countries and present the recent guidelines for optimized fertility preservation in both genders prior to HSCT.

Material (or patients) and methods: Children and adolescents younger than 18 years at HSCT with an indication for autologous or allogeneic HSCT were included. For males sperm cryopreservation or testicular biopsy and for females laparoscopic ovarian biopsy or ovariectomy were performed. Cryopreservation is paid by the national health services in all Nordic countries.

Results: Sperm cryopreservation is state of the art in all pediatric hematology/oncology units throughout the Nordic countries. A total of 172 girls have had ovarian tissue cryopreserved before HSCT since 2000. One case of ovarian tissue transplantation with resulting puberty induction has been performed. 35 of boys have had testicular tissue cryopreserved since 2004.

Conclusion: Present Nordic recommendations for optimized fertility preservation prior to HSCT in childhood and adolescence state that:

- The patient and her/his parents should be informed of possibilities for fertility preservation by a health care professional, specially assigned for this purpose.

- All males who are physically mature enough should be offered cryopreservation of sperm.
- If the boy is unable to produce an ejaculate, alternative methods like vibrator stimulation, electrostimulation or invasive procedures with retrieval of testicular or epididymal sperm could be offered.

- Prepubertal boys are eligible for the procedure of testicular biopsy and testicular tissue cryopreservation.

- All girls are eligible for ovarian biopsy or total ovariectomy and ovarian tissue cryopreservation.

- If the girl has had menarche, is sexually active and able to give informed consent, ovarian hyperstimulation and cryopreservation of oocytes could be considered provided HSCT can be delayed 2 weeks.

- Cancer contamination in the cryopreserved tissue is a contraindication for re-transplantation.

Disclosure of Interest: None declared.

\section{P637}

Unrelated cord blood transplantation in severe congenital neutropenia

L. Alonso 1,*, I. Elorza ${ }^{1}$, T. Olive ${ }^{1}$, J. L. Dapena ${ }^{1}$, J. Sanchez de Toledo ${ }^{1}$, C. 'Diaz de Heredia ${ }^{1}$

${ }^{1}$ Department of Pediatric Oncology and Hematology, Hospital Vall d'Hebron, Barcelona, Spain

Introduction: Severe congenital neutropenia (SCN) is a bone marrow failure syndrome characterised by severe neutropenia and myeloid precursor maturation arrest. Mutations in the serine protease neutrophil elastase transcribed by the ELANE gene are the most common in these patients.

Patients with this condition are at risk of two serious complications: life-threatening infections and the development of MDS/AML. The prognosis of SCN patients has changed dramatically in the last 3 decades with the improvement in antibiotic support and the development of G-CSF. however, there are concerns that the chronic administration of high doses of G-CSF might raise the risk of MDS/ AML. In addition, some patients do not respond to G-CSF. Haematopoietic stem cell transplantation (HSCT) remains as yet the only curative treatment.

The approach to HSCT in SCN has been heterogeneous and data regarding the percentage of patients who actually undergo HSCT are scant.

Material (or patients) and methods: Patients diagnosed with SCN and transplanted between 2006 and 2013 with an unrelated cord blood unit were included. The patients did not have a suitable matched related or unrelated donor.

Indications for HSCT were non-response to G-CSF and severe infections or progression to malignant transformation, considered to be either the development of overt acute leukaemia or the diagnosis of MDS

Results:

Six patients diagnosed of severe congenital neutropenia treated with unrelated cord blood transplant at our institution are reported.

Indication for transplant was poor response to G-CSF and severe infections in 3 patients, MDS monosomy 7 in two and secondary AML in one. Patient age at transplant ranged from 8 months to 4 years. One patient harboured an ELANE mutation; the other five did not have a genetic diagnosis. All patients received myeloablative conditioning based on busulfan. One received a 6/6 HLA-matched graft, 3 a 5/6 and 2 a $4 / 6$. All patients engrafted. No relapses occurred and all patients remain alive. However, the post-transplant course was complicated by HHV6 encephalitis in one patient and autoimmune haemolytic anaemia in two; three patients experienced limited acute skin or gut GVHD and the patient with secondary AML suffered a secondary graft rejection 7 months post-transplant.

Conclusion: Our data indicate that UCBT is a feasible treatment option for children with SCN considered candidates for HSCT. This appears to be so even for patients with evidence of malignant transformation.

Disclosure of Interest: None declared. 


\begin{tabular}{|c|c|c|c|c|c|c|c|c|c|}
\hline Patient & $\begin{array}{l}\text { Age at } \\
\text { diagnosis } \\
\text { (months) }\end{array}$ & $\begin{array}{l}\text { Age at } \\
\text { transplant } \\
\text { (years) }\end{array}$ & $\begin{array}{c}\text { HSCT } \\
\text { indication }\end{array}$ & Match & Conditioning & GVHD proph & $\begin{array}{c}\text { Cell dosel } \\
\mathrm{Kg}\end{array}$ & $\begin{array}{c}\text { PostHSCT } \\
\text { complications }\end{array}$ & $\begin{array}{l}\text { Status/ } \\
\text { Follow up }\end{array}$ \\
\hline 1 & 1 & 3 & $\begin{array}{l}\text { no response } \\
\text { to G-CSF+ser- } \\
\text { ious infection }\end{array}$ & $5 / 6$ & BuCyATG & $\begin{array}{l}\text { ciclosporin } \\
+ \text { methylpred }\end{array}$ & $\begin{array}{l}\text { TNC 7,1x } \\
10^{7}, \text { CD34 } \\
1.2 \times 10^{5}\end{array}$ & $\begin{array}{c}\text { skin grade2 acute } \\
\text { GVHD }\end{array}$ & 3.8 years \\
\hline 2 & 2 & 4 & $\begin{array}{l}\text { no response } \\
\text { to G-CSF+ser- } \\
\text { ious infection }\end{array}$ & $5 / 6$ & BuCyATG & $\begin{array}{l}\text { ciclosporin } \\
+ \text { MMF }\end{array}$ & $\begin{array}{c}\text { TNC } \\
5.6 \times 10^{7} \\
\text { CD34 } \\
2,26 \times 10^{5}\end{array}$ & $\begin{array}{c}\text { AlHA, ITP, HHV6 } \\
\text { encephalopathy, skin } \\
\text { and gut grade } 2 \\
\text { GVHD }\end{array}$ & 5 years \\
\hline 3 & 1 & 0,7 & $\begin{array}{c}\text { No response } \\
\text { to G-CSF }\end{array}$ & $6 / 6$ & BuMelATG & ciclosporin & $\begin{array}{c}\text { TNC } \\
11 \times 10^{7} \\
\text { CD34 } \\
4.2 \times 10^{5}\end{array}$ & & 8 years \\
\hline 4 & 4 & 1 & $\begin{array}{l}\text { serious infec- } \\
\text { tions, monos- } \\
\text { omy } 7\end{array}$ & $5 / 6$ & BuCyATG & $\begin{array}{l}\text { ciclosporin } \\
+ \text { methylpred }\end{array}$ & $\begin{array}{c}\text { TNC } \\
14.5 \times 10^{7}\end{array}$ & refractory AlHA & 8.8 years \\
\hline 5 & 2 & 2 & $\begin{array}{l}\text { MDS/ monos- } \\
\text { omy } 7\end{array}$ & $4 / 6$ & BuCyMelATG & $\begin{array}{l}\text { ciclosporin } \\
+\mathrm{MMF}\end{array}$ & $\begin{array}{c}\text { TNC } \\
9.7 \times 10^{7}\end{array}$ & $\begin{array}{c}\text { skin grade } 1 \text { acute } \\
\text { GVHD }\end{array}$ & $\begin{array}{c}\text { Alive and } \\
\text { well/4,5 } \\
\text { years }\end{array}$ \\
\hline 6 & 1 & 4 & $\begin{array}{c}\text { secondary } \\
\text { AML }\end{array}$ & $4 / 6$ & TTFluBu ATG & $\begin{array}{c}\text { ciclosporin } \\
+ \text { methylpred }\end{array}$ & $\begin{array}{c}\text { TNC } \\
4.9 \times 10^{7} \\
\text { CD34 } \\
2.8 \times 10^{5}\end{array}$ & $\begin{array}{l}\text { secondary graft } \\
\text { failure }\end{array}$ & $\begin{array}{l}\text { alive } 1.6 \\
\text { years } \\
\text { after } \\
\text { transplant }\end{array}$ \\
\hline
\end{tabular}

P638

Hematopoietic stem cell transplantation in children affected by RASopathies who developed hematological disorders

P. Merli ${ }^{1}$, L. Strocchio, ${ }^{1, *}$, B. Lucarelli ${ }^{1}$, G. M. Milano ${ }^{1}$, L. P. Brescia ${ }^{1}$, F. Di Florio ${ }^{1}$, V. Ferruzzi ${ }^{1}$, C. Gurnari ${ }^{1}$, V. Trevisan ${ }^{1}$, D. Pagliara ${ }^{\prime}$, A. Bertaina ${ }^{\prime}$

${ }^{1}$ Hemato-Oncology, IRCCS Bambino Gesù Children's Hospital, Rome, Italy

Introduction: RASopathies are a group of developmental disorders (about $1 / 1000$ births) caused by germline mutations in genes that encode components of the RAS-MAPK pathway. They are characterized by a broad spectrum of abnormalities, including cancer predisposition [Kratz, Am J Med Genet C Semin Med Genet. 2011]. To date, no data about hematopoietic stem cell transplantation (HSCT) in this group of disorders is currently available in the medical literature. Here we report 3 cases of children affected by RASopathies who underwent allogeneic HSCT due to either malignant and non-malignant hematological disorders associated with their condition.

Material (or patients) and methods: Patient (Pt) 1 is affected by Noonan's syndrome (NS) (PTPN11-mutated), displaying characteristic facies and mild pulmonary valve stenosis, developmental delay and failure to thrive. He developed bone marrow failure at the age of 2 months. Also Patient 2, a 15-year old male, is affected by PTPN11-mutated NS, with typical dysmorphic features, short stature and mild intellectual impairment. He developed Acute Myeloid Leukemia at the age of 14, treated with an AIEOP AML-like protocol. Patient 3 is affected by Cardiofaciocutaneous (CFC) syndrome (MEK2-mutated), characterized by peculiar facies, pulmonary valve stenosis, developmental delay, intellectual disability and glaucoma. At 9 year of age he developed a B Cell Precursor Acute Lymphoblastic Leukemia and was treated with an AIEOP ALL-like protocol.

Results: After accurate discussion with the local Ethical Committee, transplantation was approved for all 3 patient. Pt1 underwent a TCRaß/CD19-depleted HSCT from his haploidentical mother, after a Treosulfan-based conditioning regimen

[P638]

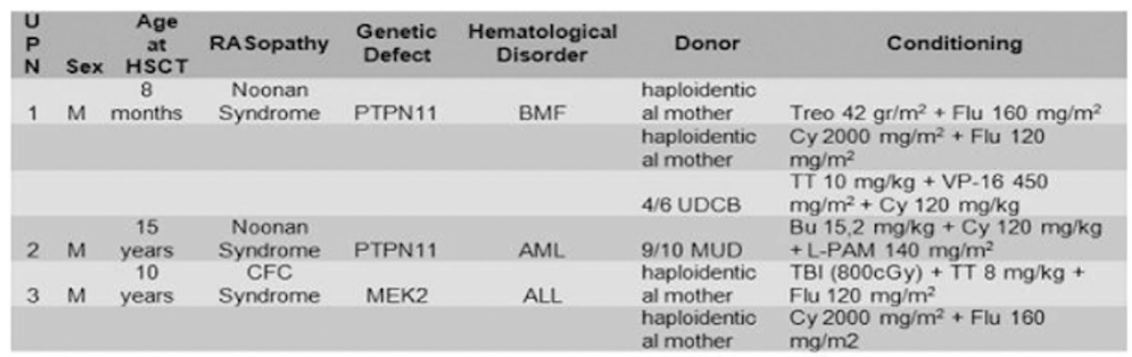

CFC indicates Cardiofaciocutaneous Syndrome; BMF, bone marrow fallure; AML, Acute Myeloid Leukemia: ALL, Acute Lymphoblastic Leukemia, UDCB, Unrelated Donor Cord Blood; MUD, Matched Unrelated Donor. Cy, Cyclophosphamide; Flu, Fludarabine; L-PAM, Melphalan; TT, Thiotepa; Treo, Treosulfan; TBI, Total Body Irradiation. 
(Table I). Due to graft rejection occurring on day +18 after donor stem cell infusion, the patient was given a second TCRaß/CD19-depleted haploidentical HSCT from the same donor (the father was not available), after a Cyclophosphamide/Fludarabine preparative regimen. He rejected the second allograft and was finally rescued by a third successful HSCT using a 4/6 compatible unrelated cord blood unit, after a Thiotepa-based protocol (neutrophil engraftment on day +34). Pt2 underwent HSCT from a 9/10 Matched Unrelated Donor, after a Busulfan-based conditioning. Engraftment occurred on day +20; he subsequently developed Grade III acute intestinal graft-versus-host disease (GVHD) requiring steroids and extracorporeal photopheresis (ECP). Pt3 was given a TCRaß/CD19depleted transplant from his haploidentical mother after a TBIbased conditioning regimen. He developed severe cutaneous toxicity, requiring topical and systemic steroids. He rejected the graft on day +17 and was rescued by a second T cell-depleted transplant from the same donor, following a Cyclophosphamide/Fludarabine preparative regimen (neutrophil engraftment on day +16). With a mean follow-up of 242 days, all 3 patients are alive and well, without any sign of GVHD.

Conclusion: HSCT is a feasible option for selected children affected by NS and CFC syndrome who developed hematological disorders. The intensity of conditioning regimen has to be accurately evaluated according to the genetic condition and the hematological disease.

Disclosure of Interest: None declared.

\section{P639}

Children undergoing hematopoietic stem cell

transplantation are more likely to die in hospital and PICU with shorter time to end of life care than those dying at home

L. M. Ball ${ }^{1, *}$, A. Dalissier ${ }^{2}$, E. Trigoso Arjona ${ }^{3}$ on behalf of Nurse research group, T. A. Neuman-van Eijk', A. M. S. van Walraven", Z. Nademi ${ }^{5}$, M. Sundin ${ }^{6}$, T. Natanzon', M. Renard ${ }^{8}$, N. Andersson', A. I. Benito ${ }^{10}$, J. Stringer ${ }^{11}$ on behalf of Nurses Research Group, S. Mary ${ }^{12}$, P. Bader ${ }^{13}$ on behalf of Pediatric Diseases Working Party

${ }^{7}$ Pediatrics, Willem Alexander Childrens Hospital, LUMC, Leiden, Netherlands, ${ }^{2}$ Data management, EBMT office, Paris, France, ${ }^{3} E B M T$ Paediatric Nurse Committee, Enfermera Hospital U y P LA FE Unidad Pediátrica de Trasplantes, Valencia, Spain, ${ }^{4}$ Pediatrics, Willem Alexander Children's Hospital, LUMC, Leiden, Netherlands, ${ }^{5}$ Pediatrics, Newcastle-Upon-Tyne Royal Victoria, Newcastle-Upon-Tyne, United Kingdom, ${ }^{6}$ Pediatrics, Stockholm University, Stockholm, Sweden, ${ }^{7}$ Pediatrics, Petach-Tikva [Schneider Children], Jersualem, Israel, ${ }^{8}$ Pediatrics, Leuven University Hospital, Leuven, ${ }^{9}$ Pediatrics, Jules Bordet, Brussels, Belgium, ${ }^{10}$ Pediatrics, Salamanca [H Clinico], Salamanca, Spain, ${ }^{11}$ Chair EBMT Nurses Research Group, Christies Hospital, Manchester, ${ }^{12}$ Pediatrics, Newcastle-Upon-Tyne [Royal Victoria], Newcastle-Upon-Tyne, United Kingdom, ${ }^{13}$ Chair of EBMT Paediatric Diseases Working Group, Dept. for Stem Cell Transplantation \& Immunology, University Hospital for Children and Adolescents, Frankfurt/Main, Germany

Introduction: Transplant related mortality (TRM) and malignancy relapse means pediatric HSCT centres need to provide palliative and end of life care (PC/ELC). Treatment exclusively aimed at cure may lead to unrealistic expectations and impede timely provision of appropriate PC/ELC.

Material (or patients) and methods: We undertook a survey of EBMT registered pediatric SCT centres to retrospectively analyse number and place of death of children undergoing HSCT, who died between January 2011 and December 2015. Epidemiological data, clinical details, place and cause of death were requested and documented. Time from medical discussion with parents/child about "no possibility of cure" and time to death was documented from a single centre (WAKZ, LUMC Leiden) in order to explore ELC provision and withdrawal of life saving support.

Results: In a preliminary analysis, 8 participating centres who had completed the registration entered 209 transplanted children who had died, 128 boys and 81 girls median aged $7 y$ $4 \mathrm{~m}$ (range $0,1-21 \mathrm{y}$ ) at the time of death. Underlying reason for HSCT was haematological malignancy $n=79$, bone marrow failure $\mathrm{n}=15$, immune-deficiencies/inherited disease $n=49$, hemoglobinopathies $n=6$, other $n=19$, missing data $n=14$. Death was transplant related in $57 \%(n=119)$, relapse or progression of disease in 74 children (36\%). Graft failure, secondary malignancies, and other causes were separately recorded.

Place of death was available for 168 patients, with 20 children $(12 \%)$ dying at home, 3 hospice (2\%), with the majority dying either in hospital $(20 \% n=33)$, SCT unit $(22 \% n=37)$ or PICU based $(39 \% n=65)$, with 10 unknown. Children undergoing HSCT for non-malignant disease died more often in PICU setting $(n=42 / 86)$ compared to those with malignant disease (23/59.) $P=<0.009$ Chi square.

In a single centre analysis $14 / 55$ children died at home, with ELC discussion initiated at a median of 1 mo before death (range 3dy-3mo). Children were admitted to PICU (26/55) at a median of 14 days before death (range 1-49) with ELC discussion initiated at median 18 hours before death (range $<1-120)$.

Conclusion: In children undergoing HSCT, few children die at home and over a third die in the PICU, especially children transplanted for non-malignant disease. In a single centre, we confirmed children with relapsed malignancies are more likely to die at home, with ELC discussion introduced earlier than those dying in the PICU setting. Late introduction of ELC discussion has been shown in other clinical settings to compromise parental grieving. The intensity and complexity of HSCT warrants further discussions, recommendations and improvements in PC and ELC. The survey continues to enrol patients to confirm these findings but presently inter- centre consistency of results suggest these findings will be maintained at study completion.

Disclosure of Interest: None declared.

\section{P640}

Timing for ovarian cryopreservation (OCP) in acute leukemia (AL) pediatric patients before HSCT: Do we all agree?

M. Andres ${ }^{1, *}$, B. Torres ${ }^{1}$, J. M. Fernández Navarro' ${ }^{1}$ S. García Williams' ${ }^{1}$, S. Herraiz', E. Novella-Maestre ${ }^{3}$

${ }^{1}$ Pediatric Oncology, ${ }^{2}$ Fertility Preservation Unit, ${ }^{3}$ Genetic Department, Hospital Universitario y Politécnico La Fe, Valencia, Spain

Introduction: OCP remains the only fertility preservation option in pediatric patients who need HSCT. Deciding the adequate moment for OCP in AL patients is not easy due to clinical situation at diagnosis, urgent need of starting treatment and no clear indications of HSCT until evaluating response to chemotherapy (ChT). As per our protocol we perform OCP just before HSCT at the same time as central line (double lumen Hickman) is inserted.

The aim of this study is to analyze ovarian cortex after ChT, but prior to HSCT to determine whether the selected timing is appropriate for tissue viability.

Material (or patients) and methods: Retrospective casecontrol study. Ovarian tissue variables analysed: follicular density, growing follicles percentage, apoptotic cells number and apoptosis degree. Prepubertal and pubertal ovarian cortex from AL patients were compared with other pediatric oncology patients' ovarian tissue without prior ChT at the time of OCP. Statistical analysis performed through SPSS, considering statistically significant differences if $P<0.05$.

Results: Study group: twenty-two patients (14 ALL and $8 \mathrm{AML}$ ). Fourteen prepubertal patients, median age of 6.8 years (IQR 4.3 to 8 ) and 8 pubertal girls, median age of 13.1 years (IQR 12.7 to 14 )

Control group: ten patients. Underlying diseases: Myelodisplastic syndrome $(n=4)$, Ewing $(n=2)$, medulloblastoma $(n=1)$, germ cell tumour $(n=1)$, chronic granulomatous disease $(n=1)$ and Hodgkin disease $(n=1)$. Four prepubertal 
patients, median age of 9.9 years (IQR 9.4 to 10.3) and 6 pubertal girls, median age of 13.4 years (IQR 12.2 to 13.4). In the structural study of ovarian tissue, we found no differences in primordial, primary or secondary follicular density. No significant differences were observed in the percentage of growing follicles (primaries follicles / total follicles). Apoptotic cells number in ovarian cortex from all groups was very small with no differences compared to controls or concerning pubertal development. We found no differences in apoptosis degree quantification either.

Conclusion: Ovarian tissue of AL pediatric patients collected according to our protocol showed a normal tissue structure and absence of apoptotic cell death despite previous ChT. On the scope of these results we can consider that the timing chosen by our program is adequate. This schedule allows to perform various procedures at a time (central line and OCP), to offer OCP only to HSCT patients, an stable complete remission and clinical stability at the time of collection.

Disclosure of Interest: None declared.

\section{P641}

Ovarian cryopreservation (OCP) in paediatric population before HSCT: from bench to bedside

M. Andres ${ }^{1, *}$, S. García Williams ${ }^{1}$, S. Carreño ${ }^{1}$, J. Gomez Chacon ${ }^{2}$ F. Carolina ${ }^{\prime}$, J. M. Fernández Navarro ${ }^{1}$

${ }^{1}$ Pediatric Oncology, ${ }^{2}$ Pediatric Surgery, Hospital Universitario y Politécnico La Fe, Valencia, Spain

Introduction: Patients who undergoing HSCT are at high risk of premature ovarian failure (POF) especially if they are conditioned with $\mathrm{TBI}$ or Busulfan based regimes. OCP remains the only fertility preservation option for this population.

Material (or patients) and methods: Descriptive study. Clinical records of children undergoing OCP before HSCT in our centre were retrospectively analyzed. Study Time period: October 2008 - September 2015.

Results: OCP was performed in 51 girls in the study time period. Thirty-two patients (62.7\%) underwent HSCT as part of their treatment. Their characteristics are: mean age at the time of the OCP 10.3 years (IQR 7.4 to 13.4); 56,3\% were prepubertal girls. Forty five percent of patients were referred from other hospitals exclusively for this procedure. Underlying diseases: ALL (16), AML (8), MDS (4), Ewing sarcoma (3), disseminated medulloblastoma (1), Hodgkin disease (1), chronic granulomatous disease (1). HSCT description: 15 autologous and 17 allogenic procedures. HLA identity and source were: MRD/PB $(n=5), \mathrm{MUD} / \mathrm{PB}(n=1), \mathrm{HLA}-\mathrm{MMRD} / \mathrm{PB}(1), \mathrm{HLA}-\mathrm{MMUD} / \mathrm{PB}(1)$, MUD/UCB $(n=1), \mathrm{HLA}-\mathrm{MMUD} / \mathrm{UCB}(n=3), \mathrm{MUD} / \mathrm{BM}(n=1)$, haploidentical $(n=1)$, not available $(n=3)$. TBI and Busulfan based regimens were the most prevalent conditioning schedules (85\%). Median time between diagnosis and OCP was 304 days (IQR 186-522). Median time between OCP and TPH was 13 days (IQR 12 to 25.8). Partial (most cases) or entire laparoscopic ovariectomy was carried out without surgical complications. Oncological planned treatment was started without any delay. The tissue was successfully obtained at the same time as other surgical procedures in $69,7 \%$ of cases $(60 \%$ venous central line insertion). Twenty-three patients (72,5\%) had previously received non gonadotoxic chemotherapy (ChT) before the OCP; despite this fact normal count of primordial follicles was found in most samples ( $>90 \%)$. Current status: sixty-six percent of patients remain alive in complete remission, 3,1\% alive with progressive disease and 30,3\% died from disease progressions or medical complications during HSCT. Regarding gonadal function, although evaluable group is small $(n=12)$, no ovarian activity after HSCT was detected in $90 \%$ of patients.

Conclusion: In our series OCP was safely and efficiently carried out. Prior low gonadotoxic ChT may be considerer if ovarian failure risk is high.

In our series the high rate of premature ovarian failure confirms the need to considerer preserving fertility strategies in these patients.

Disclosure of Interest: None declared.

\section{P642}

Ovarian premature failure (POF) and other endocrine complications after HSCT. Preliminary results of a pediatric fertility preservation programme

M. Andres ${ }^{1, *}$, F. Carolina ${ }^{1}$, B. Torres ${ }^{1}$, S. Carreño ${ }^{1}$, C. De Mingo ${ }^{2}$, J. M. Fernández Navarro

${ }^{1}$ Pediatric Oncology, ${ }^{2}$ Endocrine Pediatric Department, Hospital Universitario y Politécnico La Fe, Valencia, Spain

Introduction: Long-term survivors of pediatric HSCT are at risk of developing endocrine complications, particularly gonadal failure. Regular follow-up is essential for early detection and proper management. Ovarian cryopreservation (OCP) plays a leading role in pediatric fertility preservation (FP).

Material (or patients) and methods: Descriptive study. Clinical records of children undergoing OCP before HSCT in our centre were analyzed. Study time period: October 2008 September 2015. Study variables: POF presence and other endocrine complications during follow-up. Persistently elevated levels of FSH (>25IU/I) were required for POF diagnosis. Results: In the time period OCP was performed in 32 girls before HSCT. Mean age at time of the OCP was 10.3 years (IQR 7.4 to 13.4). TBI and busulfan based regimen were the most

[P642]

\begin{tabular}{|c|c|c|c|c|}
\hline \multirow[t]{2}{*}{ Endocrine Evaluation } & \multicolumn{2}{|c|}{ Prepubertal patients $(\mathrm{n}=18)$} & \multicolumn{2}{|c|}{ Pubertal patients $(\mathrm{n}=14)$} \\
\hline & $\begin{array}{l}\text { Not evaluable } \\
(\mathrm{n}=11) \\
\text { Exitus }(\mathrm{n}=5) \\
\text { Too young or recent } \mathrm{HSCT} \\
(\mathrm{n}=6)\end{array}$ & $\begin{array}{l}\text { Evaluable } \\
(n=7)\end{array}$ & $\begin{array}{l}\text { Not evaluable } \\
(\mathrm{n}=9) \\
\text { Exitus }(\mathrm{n}=4) \\
\text { Too young or recent HSCT } \\
(\mathrm{n}=5)\end{array}$ & $\begin{array}{l}\text { Evaluable } \\
(n=5)\end{array}$ \\
\hline Presence POF & & $6 / 7$ & & $5 / 5$ \\
\hline No Spontaneous puberty & & $4 / 6$ & & \\
\hline Gonadal Hormone replacement & & $3 / 6$ & & $4 / 5$ \\
\hline $\begin{array}{l}\text { Median age at the last visit } \\
\text { (years) }\end{array}$ & $\begin{array}{c}6.7 \text { years } \\
\text { (IQR } 5.4 \text { to } 8.4)\end{array}$ & $\begin{array}{c}13.3 \text { years } \\
\text { (IQR } 12.4 \text { to } \\
13.8)\end{array}$ & $\begin{array}{c}13,5 \text { years } \\
\text { (IQR } 13 \text { to } 15.7)\end{array}$ & $\begin{array}{c}16.7 \text { years } \\
\text { (IQR 16.5 to } \\
17.1)\end{array}$ \\
\hline Median follow-up time (years) & $\begin{array}{l}0.7 \text { years } \\
\text { (IQR } 0.3-1.1)\end{array}$ & $\begin{array}{c}4 \text { years } \\
\text { (IQR } 3.4 \text { to } 4.6)\end{array}$ & $\begin{array}{l}0.2 \text { years } \\
\text { (IQR } 0.2-0.7)\end{array}$ & $\begin{array}{c}3.1 \text { years } \\
\text { (IQR } 3.1 \text { to } 3.8\end{array}$ \\
\hline
\end{tabular}


prevalent conditioning schedules (85\%). Main characteristics of this cohort are summarized in table 1.

In the evaluable prepubertal group additionally we report subclinical hypothyroidism in 2 cases (previous cranial radiotherapy and TBI based conditioning), insufficient growth hormone secretion in 2 patients (Busulfan based conditioning and previous cranial radiotherapy) and adrenal insufficiency by prolonged steroid therapy in one girl. Current status: $6 / 7$ alive in complete remission and 1 died (disease progression).

In the evaluable pubertal group at present we didn't find any other hormonal insufficiency, although short stature was diagnosed in one patient. All of them remain alive in complete remission, starting hormone replacement therapy at median age of 15.1 years (IQR 14.7 to 15.6 ).

Conclusion: Patients conditioned with TBI or Bu-based regimes are at very high risk of POF.

In our series even very young girls show high rates of POF post HCST.

Fertility preservation and its options should be considered in girls undergoing HSCT.

Disclosure of Interest: None declared.

P643

MIBG megatherapy as preconditioning is more effective than stand-alone therapy in neuroblastoma

M. Ussowicz, ${ }^{1,}$, A. Pieczonka ${ }^{2}$, W. Balwierz ${ }^{3}$, J. Goździk ${ }^{4}$, K. Drabko $^{5}$, R. Dębski ${ }^{6}$, E. Wawrzyniak-Dzierżek', D. Handkiewicz-Junak ${ }^{8}$, J. Wachowiak ${ }^{9}$

${ }^{1}$ Department of Pediatric BMT, Hematology and Oncology, Wroclaw Medical University, Wrocław, ${ }^{2}$ Department of Pediatric Oncology, Hematology and Transplantology (EBMT CIC 641, CIBMTR Center 10797), University of Medical Sciences, Poznań, ${ }^{3}$ Department of Paediatric Oncology and Haematology, University Children's Hospital, Jagiellonian University Collegium Medicum, ${ }^{4}$ Stem Cell Transplant Center, University Children's Hospital, Department of Clinical Immunology and Transplantology, Jagiellonian University Collegium Medicum, Kraków, ${ }^{5}$ Department of Paediatric Haematology, Oncology and Stem Cell Transplantation, Medical University, Lublin, ${ }^{6}$ Department of Paediatric Haematology and Oncology, Collegium Medicum,
Nicolaus Copernicus University Torun, Bydgoszcz, ${ }^{7}$ Department of Pediatric Hematology and Oncology, BMT Unit CIC 817, Wroclaw Medical University, Wrocław, ${ }^{8}$ Nuclear Medicine and Endocrine Oncology Department, Maria Sklodowska Curie Memorial Cancer Center and Institute of Oncology Gliwice Branch, Gliwice, ${ }^{9}$ Department of Pediatric Oncology, Hematology and Transplantology (EBMT CIC 641, CIBMTR Center 10797), University of Medical Sciences, Poznan, Poland

Introduction: The MIBG I-131 therapy is a promising targeted treatment in refractory and relapsed neuroblastoma. We performed a retrospective analysis of this approach outcomes in patients (pts) treated in Poland between 2006-2015.

Material (or patients) and methods: 38 pts ( $20 \mathrm{M}: 18 \mathrm{~F})$ with median age of 90 months (range 19-338) with neuroblastoma underwent one (36 pts) or more courses of MIBG I-131 therapy. The prerequirement for therapy was an avid MIBG disease, and unfavourable prognostic factors like relapse (15 patients), primary chemoresistance (12) or progressing disease (4 patients). Patients received $100-400 \mathrm{mCi}$ of MIBG. Two children received standard dose chemotherapy with SCT. Among 12 patients left without chemotherapy, 2 required SCT due to prolonged cytopenia. In 21 patients the megachemotherapy was performed- (busilvex-melfalan in 12 pts, treosulfan-based in 7 pts, and other in 2 pts). Grafting material was autologous in 12 children, haploidentical from parent in 5, allogeneic in 3, and one - syngeneic. Average time from MIBG to SCT was 19 days (ranged 13-80).

Results: The probability of 5 year OS (5 yr pOS) was 32\%, and probability of EFS was $29 \%$. The stand-alone MIBG therapy was never curative (5 $\mathrm{yr}$ pOS $0 \%$ ), and only addition of megachemotherapy with stem cell rescue irrespective of their donor type (autologous, allogeneic or haploidentical) could have led to durable remission $(P=0.07)$. Patients treated with progressing disease had inferior survival $(5 \mathrm{yr}$ pOS $0 \%)$ results than those treated with relapsing ( $5 \mathrm{yr}$ pOS $41 \%)$ or stable/ refractory (5 yr pOS $44 \%$ ) disease. In $37 \%$ of treated children a hypothyroidism was diagnosed within 30 months form MIBG therapy.

[P643]

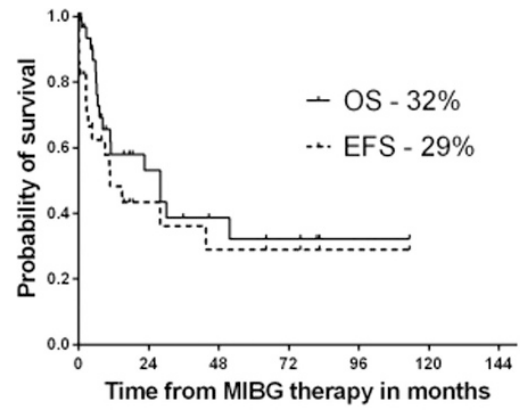

Effect of megachemotherapy

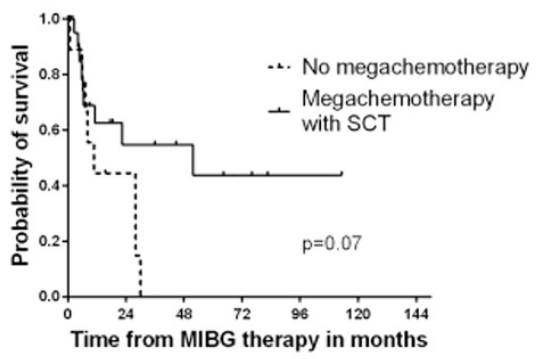

Survival according

to megachemotherapy and SCT

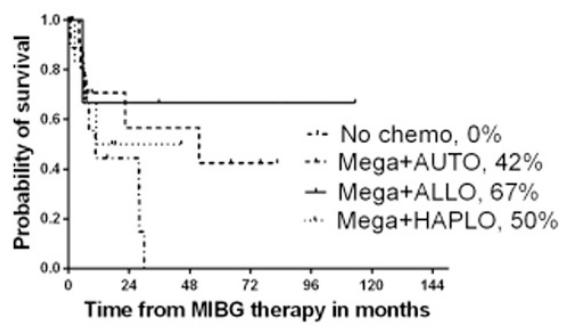

Probability of thyroid failure

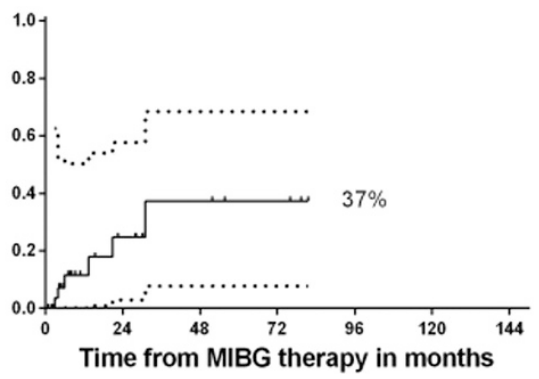


Conclusion: According to different publications the MIBG therapy can be incorporated into therapy of relapsed or resistant neuroblastoma patients, but most advantage is achieved by using the MIBG therapy as pre-conditioning with megachemotherapy. Therapy is eligible in heavily pretreated patients, but requires endocrinological follow-up due to high incidence of thyroid gland failure.

Disclosure of Interest: None declared.

\section{P644}

Immune Reconstitution in pediatric patients after T- Cell replete vs T-Cell deplete Hematopoietic stem cell transplantation for Leukemia

M. S. Villegas ${ }^{1, *}$, F. Yeap ${ }^{1}$, P.-L. Tan ${ }^{1,2}$

${ }^{1}$ Khoo Teck Puat - National University Children's Medical Institute, National University Hospital, ${ }^{2}$ Department of Pediatrics, National University of Singapore, Singapore, Singapore

Introduction: There is limited data on immune reconstitution comparing T-replete (TCR) vs T-Deplete (TCD) hematopoietic stem cell transplantation (HSCT), in children with leukemia.

Material (or patients) and methods: A retrospective review of pediatric leukemia patients post HSCT who had lymphocyte subsets (CD4, CD20, CD56) from March 2005 to April 2012 (TCR) and December 2012 to September 2015 (TCD).

Results: There were 38 TCR HSCTs performed at our institution with preparative regimens with backbone of $A T G+C y / T B I$ or ATG+FluBuMel. Nineteen TCD HSCTs had preparative regimens with backbone of TLI or Treosulfan+Rituximab+/- ATG +FluThioMel. Time points that were followed post HSCT were $\mathrm{D}+30, \mathrm{D}+100, \mathrm{D}+200$ and 1 year. T-Cell depletion methods were CD3 depletion, TCRaß depletion, CD34+/CD45RA depletion, CD3/CD45RA depletion and post cyclophosphamide in vivo depletion. The results are shown in (Table 1).

Conclusion: Immune reconstitution for CD4 is comparable except for D+100 in the TCD group, likely owing to the small population. CD20 reconstitution is likewise comparable, even though we used Rituximab in the TCD, and none in the TCR group. There might be an advantage for TCD group when it comes to CD56. Although the numbers are smaller, there is a clear trend towards fast and sustained immune reconstitution for CD56. NK cells are the important effector cells in TCD HSCT and this may show a benefit towards better leukemia control. These are important observations as we continue to perform TCD HSCT in patients without suitable donors. A follow up study is needed.

References: $C$ de Konig, M Plantinga, P Besseling, Jan Bolens, S Nierkens. Immune Reconstitution after Allogeneic Cell Transplantation in Children. Biol Blood Marrow TransBiol Blood Marrow Transplant. 2015 Sep 1. pii: S1083-8791(15)00576-5. doi: 10.1016/j.bbmt.2015.08.028. [Epub ahead of print]. Disclosure of Interest: None declared.
P645

T-cell replete haploidentical hematopoietic stem cell transplantation (HSCT) as salvage therapy for acute rejection/graft failure in pediatric patients with nonmalignant disorders

M. Sirin ${ }^{1, *}$, D. Moshous ${ }^{2,3}$, M. Hoenig ${ }^{1}$, C. Schuetz ${ }^{1}$, B. Neven ${ }^{2,3}$ M. Cavazzana ${ }^{3,4}$, A. Fischer ${ }^{2,3}$, S. Blanche ${ }^{2,3}$, J. Tischer', A. Schulz ${ }^{\prime}$, M. H. Albert ${ }^{6}$

${ }^{1}$ University Children's Hospital, Ulm, Germany, ${ }^{2}$ Immunologie et Hématologie Pédiatriques, Hôpital Necker-Enfants Malades, ${ }^{3}$ Institut Imagine, Université Paris Descartes-Sorbonne Paris Cité, ${ }^{4}$ Département de Biothérapie, Centre d'Investigation Clinique intégré en Biothérapies, Hôpital Necker-Enfants Malades, Paris, France, ${ }^{5}$ Department III of Internal Medicine, Hematopoietic Stem Cell Transplantation, Ludwig-Maximilians-University Hospital of Munich-Grosshadern, ${ }^{6}$ Department of Pediatric Hematology/ Oncology, Dr. von Hauner University Children's Hospital, Munich, Germany

Introduction: Acute graft rejection and graft failure occur in approximately $10 \%$ of patients with non-malignant disorders after matched unrelated or haploidentical HSCT. Stringent ex vivo T-cell depletion of the graft may contribute to graft rejection/failure especially in patients with inborn errors and residual $\mathrm{T}$ cell function. In case of infection or prolonged aplasia there is the need for urgent salvage HSCT. There is no universally accepted approach for donor choice or conditioning in this situation, and recruitment of a second unrelated donor often is not possible in a timely manner. We hypothesized that $\mathrm{T}$ cell replete haploidentical BMT after a serotherapy-based, radiation-free, reduced intensity conditioning would be able to overcome engraftment barriers in children who rejected their primary graft, possibly due to the tolerizing effect of post-transplant cyclophosphamide and/or the large number of graft-facilitating cells infused including donor CD8 T-cells.

Material (or patients) and methods: Eight consecutive patients (refractory cytopenia $n=3$, primary immunodeficiency $n=4$, osteopetrosis $n=1$ ) from 3 centers received secondary T-replete bone marrow grafts from haploidentical donors for acute rejection/graft failure at a median of 43 days (range 3059) after one or two previous HSCTs (6 mismatched family donors and 2 matched unrelated donors). The secondary donors were different from the previous ones in $6 / 8$ patients. Conditioning comprised alemtuzumab $0.4-0.5 \mathrm{mg} / \mathrm{kg}(n=6)$ or ATG-G $7.5 \mathrm{mg} / \mathrm{kg} \quad(n=2) \pm$ rituximab $375 \mathrm{mg} / \mathrm{m}^{2} \quad(n=6)$ as serotherapy and cyclophosphamide $29 \mathrm{mg} / \mathrm{kg}$, fludarabine $150-160 \mathrm{mg} / \mathrm{m}^{2} \pm$ treosulfan $20-24 \mathrm{~g} / \mathrm{m}^{2}(n=5)$. GVHD prophylaxis consisted of cyclophosphamide $2 \times 50 \mathrm{mg} / \mathrm{kg}$ on days 3 and 4 followed by MMF and tacrolimus $(n=5)$ or CSA $(n=3)$. Results: After a median follow-up of 6.1 months (4.3-38.4) all patients are alive and free of disease. Two patients experienced acute GVHD $\|^{\circ}$, while acute GVHD $\geq \|^{\circ}$ or chronic GVHD were not observed. All patients exhibit complete donor chimerism at last follow-up. Neutrophil and platelet engraftment occurred on day +18 (median; range 15-63) and +63 (30191) respectively. Viral reactivations (CMV $n=4, \operatorname{ADV} n=3$ and HHV6 $n=1$ ) were detected in 6 patients, but no transplantrelated mortality occurred. T-cell immune reconstitution was

Table 1

\begin{tabular}{|l|l|l|l|l|l|l|l|l|l|l|l|l|l|}
\hline \multicolumn{9}{|c|}{ CD4\%(200/ cu mn) } & \multicolumn{3}{c|}{ CD20(200/ cu mm) } & \multicolumn{3}{c|}{ CD56 (100/ cu mm) } \\
\hline Treplate & $34 \%$ & $26 \%$ & $55 \%$ & $88 \%$ & $3 \%$ & $26 \%$ & $51 \%$ & $65 \%$ & $71 \%$ & $68 \%$ & $75 \%$ & $88 \%$ \\
\hline N & 35 & 38 & 33 & 26 & 35 & 38 & 33 & 26 & 31 & 38 & 33 & 26 \\
\hline D+ & 30 & 100 & 200 & 1 ycar & 30 & 100 & 200 & $1 \%$ rar & 30 & 100 & 200 & 1 rear \\
\hline T Deolete & $25 \%$ & $100 \%$ & $50 \%$ & $83 \%$ & $0 \%$ & $21 \%$ & $54 \%$ & $66 \%$ & $94 \%$ & $79 \%$ & $91 \%$ & $100 \%$ \\
\hline N & 16 & 14 & 11 & 6 & 16 & 14 & 11 & 6 & 16 & 11 & 11 & 6 \\
\hline
\end{tabular}


swift with a median of $293 / \mu \mathrm{l} C D 3+$ cells (range 12-2018) on day+100.

Conclusion: In this multicenter preliminary study of pediatric patients requiring an urgent salvage HSCT due to graft failure/ rejection, a serotherapy-containing, radiation-free approach with reduced intensity conditioning, T-cell replete haploidentical graft and post-transplant cyclophosphamide resulted in excellent engraftment in all patients and absence of severe GVHD or transplant related mortality. Interestingly, in two patients this strategy led to robust engraftment even with the same haploidentical donor as for the previous HSCT. This strategy makes a salvage donor available for virtually every patient in this emergency situation.

Disclosure of Interest: None declared.

\section{P646}

Later events in pediatric leukemia survivors post hematopoietic cell transplantation: A landmark analysis

M. Bitan ${ }^{1, *}$, N. N. Shah ${ }^{2}$, A. S. Nelson ${ }^{3}$, K. Woo Ahn ${ }^{4}$, H. R. Millard ${ }^{4}$,

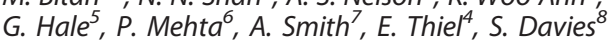

${ }^{1}$ Department of Pediatric Hematology/ Oncology, Tel-Aviv Sourasky Medical Center, Tel-Aviv, Israel, ${ }^{2}$ Pediatric Oncology Branch, Center for Cancer Research (CCR), National Cancer Institute (NIH), Bethesda, ${ }^{3}$ Cincinnati Children's Hospital Medical Center, Cincinnati, ${ }^{4}$ CIBMTR, Medical College of Wisconsin, Milwaukee, ${ }^{5}$ Department of Hematology/Oncology, All Children's Hospital, St. Petersburg, ${ }^{6}$ Department of Pediatrics, Cincinnati Children's Hospital Medical Center, Cincinnati, "University of Minnesota Medical Center, Fairview, Minneapolis, ${ }^{8}$ Cincinnati Children's Medical Center, Cincinnati, United States

Introduction: The majority of the mortality and morbidity in children with leukemia undergoing allogeneic hematopoietic cell transplantation occurs in the first year after transplant. Many parents want to know if their child can be considered cured if they are doing well one year after transplant, and specifically may want to know what transplant related factors may enhance long-term survival beyond first year (e.g., chronic GVHD). To attempt to answer this question we performed a landmark analysis of outcomes, and identified risk factors for later mortality.

Material (or patients) and methods: We analyzed 856 patients with acute myeloid leukemia (AML) and 1159 patients with acute lymphoblastic leukemia (ALL) under the age of 18 years old, who received myeloablative conditioning and related or unrelated stem cell HCTs from 2000 to 2012. AML and ALL disease cohorts were analyzed separately. Patient-, disease-, and transplant-related variables were summarized for all patients alive and disease free at one-year post transplant. The probabilities of death and death or treatment failure were calculated using the Kaplan-Meier estimator, and by cumulative incidence function for relapse and transplant related mortality (TRM). The time to event for all outcomes starts at one year post-transplant. Cox proportional hazard models were used to investigate risk factors for death and death or treatment failure at the landmark times of 1, 2, and 3 years of disease-free survival after transplant.

Results: Leukemia free survival (LFS) for AML patients was $86 \%, 78 \%$ and $75 \%$ at 1 year ( 2 years after transplant), 3 years and 5 years after landmark, respectively. For ALL it was $88 \%$, $81 \%$ and $78 \%$, respectively. In multivariate analysis for AML patients, age at transplant (0-9 vs $10-17)(P=0.004)$, disease status prior to transplant (complete first and second remission vs relapsed/primary induction failure) $(P=0.001), A M L$ type (primary vs treatment related) $(P=0.029)$, donor type (HLA identical siblings vs cord blood vs other relative vs well matched unrelated donor vs partially mismatched/mismatched unrelated donor $))(P=0.045)$ and presence of extensive chronic GVHD prior to landmark $(P=0.002)$ were significant risk factors of death or treatment failure after 1 year of disease-free survival. After living disease-free for 2 years post-transplant, AML type $(P=0.013)$, donor type $(P=0.002)$ and history of extensive chronic GVHD at landmark
$(P=<0.0001)$ remain significant. After 3 years of diseasefree survival, only AML type remains significant $(P=0.007)$. In contrast, for ALL the significant risk factors for death or treatment failure from 1 year after transplant were age at transplant $(P=0.009)$, history of extensive chronic GVHD at landmark $(P=<0.0001)$, and year of transplant $(P=0.017)$. Only history of extensive chronic GVHD at landmark remains a significant risk factor of death or treatment failure after 2 years $(P=<0.0001)$ and 3 years $(P=<0.0001)$ of disease-free survival.

Conclusion: Approximately $25 \%$ of children alive one year after transplant will have a further event in the next 5 years, and $20 \%$ will not be alive 5 years after transplant. Risk factor analysis shows increased risk of adverse events in children with AML with secondary leukemia, and in AML and ALL from extensive chronic GVHD.

Disclosure of Interest: None declared.

\section{P647}

T-cell replete haploidentical bone marrow transplantation with serotherapy-containing conditioning and posttransplant cyclophosphamide for pediatric patients with non-malignant disorders

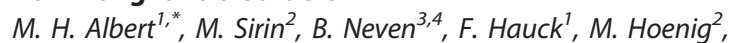
M. Cavazzana', A. Fischer ${ }^{4,5}$, C. Schuetz', S. Blanche ${ }^{4,5}$, K.-M. Debatin', C. Klein', A. Schulz', D. Moshous'

${ }^{1}$ Dr. von Hauner University Children's Hospital, Munich, ${ }^{2}$ University Children's Hospital, Ulm, Germany, ${ }^{3}$ Hôpital NeckerEnfants Malades, ${ }^{4}$ Université Paris Descartes-Sorbonne Paris Cité, ${ }^{5}$ Hôpital Necker-Enfants Malades, Paris, France

Introduction: Allogeneic hematopoietic stem cell transplantation (HSCT) from matched related and unrelated donors results in excellent cure rates of around $90 \%$ in children with most inborn, non-malignant disorders. In patients lacking a matched donor in vivo or in vitro T-cell depletion allows the alternative use of mismatched (haploidentical) family donors [1]. However, concerns about rejection, GVHD and infectious complications remain $[2,3]$. With the aim of reducing GVHD and rejection rates, we combined an upfront serotherapy-based conditioning regimen with T-cell replete haploidentical bone marrow grafts and post-transplant cyclophosphamide in pediatric patients with non-malignant disorders.

Material (or patients) and methods: Eleven patients from 3 centers received T-cell replete haploidentical parental bone marrow grafts at a median age of 4.3 years (range $0.4-10.3$ ). Nine patients suffered from primary immunodeficiencies [familiar lymphohistiocytosis $(n=2)$, IPEX syndrome $(n=2)$, IL-10 R deficiency $(n=1)$, XIAP $(n=1)$, WAS $(n=1)$, CORO1A deficiency $(n=1)$, Hyper-IgM-like syndrome $(n=1)$ ] and two patients from sickle cell disease. The grafts contained a median of $6.1 \times 10 \wedge 8$ total nucleated (5-13.6), $8.3 \times 10 \wedge 6 \mathrm{CD} 34^{+}$ (4-30.5) and $6.5 \times 10 \wedge 7 \mathrm{CD}^{+}$cells (3.9-17.4). Conditioning was adjusted for disease pathology and center preference, but contained a serotherapy backbone of alemtuzumab 0.4$0.7 \mathrm{mg} / \mathrm{kg} \quad(n=10)$ or ATG-G $10 \mathrm{mg} / \mathrm{kg} \quad(n=1) \pm$ rituximab $375 \mathrm{mg} / \mathrm{m}^{2}(n=7)$ administered prior to day -7 . All patients received fludarabine $150-160 \mathrm{mg} / \mathrm{m}^{2} \pm$ cyclophosphamide $29 \mathrm{mg} / \mathrm{kg}(n=9)$ combined with either treosulfan $36-42 \mathrm{~g} / \mathrm{m}^{2}$ $(n=7) \pm$ thiotepa $10 \mathrm{mg} / \mathrm{kg}(n=5)$ or busulfan with myeloablative AUC $(n=3)$ or TBI 2 Gy $(n=1)$. GVHD prophylaxis consisted of cyclophosphamide $2 \times 50 \mathrm{mg} / \mathrm{kg}$ on days +3 and +4 , followed by MMF with tacrolimus $(n=7)$ or CSA $(n=4)$. Results: After a median follow-up of 2.9 months (0.2-34) all patients are alive and 8 of 9 evaluable patients have a $100 \%$ donor chimerism; one patient (IPEX) is $35 \%$ donor. Acute GVHD was observed in one patient (II ${ }^{\circ}$ overall); chronic GVHD was not observed so far. Neutrophil and platelet engraftment occurred on day +17 (median; 12-34) and +24 (14-44) respectively. T-cell immune reconstitution was timely with a median of $946 / \mu \mathrm{l} \mathrm{CD3}{ }^{+}$cells $(45-1782)$ on day +100 . Four patients had at least one detectable virus at time of HSCT (CMV, ADV, EBV, BK). Two CMV reactivations and one CMV 
infection were observed, but no transplant-related mortality occurred.

Conclusion: In this preliminary multicenter study a "Baltimore"-like approach containing upfront serotherapy resulted in excellent engraftment and absence of severe GVHD or relevant transplantation related morbidity and mortality in children with non-malignant diseases. Larger prospective and comparative studies are warranted to evaluate this approach in comparison to technically more demanding and more expensive in vitro T-cell depletion strategies for this particular patient group.

References: 1. Brodsky et al, Bone Marrow Transplant. 2008 Oct;42(8):523-7. 2. Bolanos-Meade et al, Blood. 2012 Nov 22;120(22):4285-91. 3. Balashov et al, Biol Blood Marrow Transplant. 2015 Nov;21(11):1955-62. AS and DM contributed equally.

Disclosure of Interest: None declared.

\section{P648}

Post-transplant cyclophosphamide as graft-versus-host disease prophylaxis in pediatric patients with hematologic malignancies transplanted from haploidentical and matched unrelated donors

A. Abrosimov', L. Shelikhova', Z. Shekhovtsova', J. Skvortsova', I. Muzalevsky ${ }^{2}$, A. Kazachenok ${ }^{3}$, M. Ilushina ${ }^{1}$, I. Shipitsina',

D. Shasheleva', R. Khismatullina', S. Blagov', E. Gutovskaya', N. Myakova ${ }^{4}$ D. Litvinov ${ }^{4}$, G. Novichkova ${ }^{3}$, A. Maschan ${ }^{3}$, M. Maschan,

${ }^{1} \mathrm{HSCT},{ }^{2}$ Laboratory of Transplant Processing and Cell Preparations, "Dmitry Rogachev Federal Research Centre of Pediatric Hematology, Oncology and Immunology", Moscow, Russian Federation, ${ }^{4}$ Oncohematology, "Dmitry Rogachev Federal Research Centre of Pediatric Hematology, Oncology and Immunology", Moscow, Russian Federation

Introduction: Standard GvHD prophylaxis regimens impair the graft-versus-tumor (GVT) effect, delay immune reconstitution and are associated with high rate of infections. High-dose post-transplantation cyclophosphamide (PTCy) targets alloreactive donor $\mathrm{T}$ cells proliferating early after BMT, promotes regulatory $\mathrm{T}$ cell and permits rapid immune reconstitution. In this pilot trial we evaluate the safety and effects of PTCy in unmanipulated haploidentical and matched unrelated transplantation (MUD) in pediatric patients with hematologic malignancies.

Material (or patients) and methods: Twenty eight pediatric patients with high risk hematologic malignancies underwent unmanipulated allogeneic bone marrow (BM) $(n=26)$ or peripheral blood stem cell $(\mathrm{PBSC})(n=2)$ transplantation followed by PTCy between April 2014 and September 2015 with a median follow-up of 9 months (1,5-19). Nineteen patients were transplanted from haploidentical donors and 9 from MUD. The median age was 10,4 years (range 1,9-18); 14 patients with ALL, $4 \mathrm{AML}, 8 \mathrm{JMML}$ and 2 with $\mathrm{HD} ; 16$ patients were in remission (CR) (14-ALL, 1-AML, 1-HD), 12 had active disease (AD) (8-JMML, 3-AML, 1-HD).

In 11 pts this was a second allograft. Twenty-six pts received myeloablative Treosulfan/Melphalan/Fludarabine and 2 pts reduced-intensity Fludarabine/TAO 2 Gy/Thiotepa as conditioning regimen. GvHD prophylaxis consisted of PTCy on day $+3,+4$ and tacrolimus from day +5 in all pts., $12(43 \%)$ pts. also received ATG (rabbit, thymoglobuline) at $5 \mathrm{mg} / \mathrm{kg} /$ course.

Results: Primary engraftment was achieved in $27(96,4 \%)$ of 28 pts., the median time to neutrophil and platelet recovery was 20 days (14-45) and 21 days (10-97). Twenty seven (96,4\%) patients were in full donor chimerism on day +30 . Early mortality was $14,9 \%$ (95\% Cl: 6-36), causes of death included viral infections $(n=3)$; GvHD and viral infection $(n=1)$. Cumulative incidence $(\mathrm{Cl})$ of acute $\mathrm{GvHD}$ grade $\geq$ II was $50 \%$ (95\%Cl:34-74), grade III-IV - 34\% (95\%Cl:19-62) and chronic GvHD - 19\% (95\%Cl:8-45). No correlation between the use of ATG and acute/chronic GvHD was noted. $\mathrm{Cl}$ of relapse was $15,4 \%$ (95\% Cl: 6-38) and was significantly lower in CR vs $A D$ group: $0 \%$ vs $36 \%$ (95\% Cl: $17-77), P=0,015$. One year eventfree survival (EFS) was $69,7 \%(95 \% \mathrm{Cl}$ : $52-87)$ and overall survival (OS) - 76\% (95\%Cl: 59-93). One year pOS was significantly lower in the ATG group $58 \%(95 \% \mathrm{Cl}: 30-86)$ vs non-ATG group 93\% (95\%Cl: 79-100), $P=0,039$. One year $\mathrm{pEFS}$ was $87 \%$ for patients in remission and $48 \%$ for patients with active disease $(P=0,028)$. There was no significant difference in survival and relapse rate according to donor type.

Conclusion: We demonstrate that unmanipulated HSCT and posttransplantation cyclophosphamide allows for high rate of engraftment with acceptable transplant-related mortality in pediatric patients with hematological malignancies. All major outcomes were equivalent between transplantation from unrelated and haploidentical donor. GvHD prophylaxis including PTCy and tacrolimus was revealed a high rate of acute and chronic GvHD rate independently of ATG use. Event-free survival is significantly higher and relapse rate is significantly lower in patients grafted in complete remission.

Disclosure of Interest: None declared.

\section{P649}

Successful allogeneic unrelated bone marrow transplantation in a female patient with a heterozygous splice-site mutation in PIK3R1

M. Sugiyama, ${ }^{1,}$, Y. Terashita ${ }^{1}$, J. Ohshima ${ }^{1}$, Y. Cho ${ }^{1}$, A. Iguchi ${ }^{1}$, M. Ueki ${ }^{1}$,Y. Tozawa ${ }^{1}$, S. Takezaki ${ }^{1}$, M. Yamada ${ }^{1}$, I. Kobayashi ${ }^{\prime}$, T. Ariga ${ }^{\prime}$

${ }^{1}$ pediatrics, Hokkaido University Graduate School of Medicine, Sapporo, Japan

Introduction: Phosphoinositide 3-kinase (PI3K) signal promotes diverse intracellular responses including proliferation, survival, and differentiation. PIK3R1 encodes the regulatory subunits of PI3K, p85a, p55a and p50a. A recently reported heterozygous splice-site mutation in PIK3R1 affecting all of the 3 subunits leads to increased PI3K signals and causes susceptibility to infection and lymphoproliferation. Hematopoietic stem cell transplantation (HSCT) is the only curative treatment but has not been reported in this disease. Here we report the first successful allogeneic HSCT for a patient with this disease who developed EBV-associated lymphoproliferation.

Material (or patients) and methods: In the present report, the patient was a 20-year-old Japanese female. At the age of 2 years, her serum IgG and IgA levels were low and her IgM level was high. A heterozygous splice-site mutation in PIK3RI was confirmed by sequence analysis of the patient's DNA and CDNA. The same abnormality was also confirmed in her mother who died of Epstein-Barr virus (EBV) associated diffuse large B-cell lymphoma. She suffered from recurrent bacterial infections, such as pneumonia and otitis media, and therefore needed antibiotics, trimethoprim/sulfamethoxazole, and immunoglobulin replacement. Computed tomography revealed an enlargement of the bilateral cervical lymph node, and pathological examination confirmed lymphoproliferative disease associated with primary immune disorders driven by EBV. Because her mother had died of lymphoma at the age of 41, HSCT was planned for the patient. As HLA-matched related donors were not available, the patient received bone marrow transplantation at the age of 21 years from an unrelated male donor who was HLA-matched in A, B, C, and DRB1 loci by DNA typing. After conditioning consisting of busulfan $(0.8 \mathrm{mg} / \mathrm{kg})$ four times daily on days -9 to -6 (total dose, $12.8 \mathrm{mg} / \mathrm{kg}$ ) and cyclophosphamide $(50 \mathrm{mg} / \mathrm{kg})$ once daily on days -5 to -2 (total dose, $200 \mathrm{mg} / \mathrm{kg}$ ), she received intravenous infusion of bone marrow cells (a total $3.7 \times 10^{8} / \mathrm{kg}, 1.7 \times 10^{6} / \mathrm{kg}$ as CD34 positive cells).Tacrolimus $(0.02 \mathrm{mg} / \mathrm{kg}$ ) from day -1 and shortterm methotrexate (MTX)( $15 \mathrm{mg}$ on day +1 and $10 \mathrm{mg}$ on days $+3,+6$, and +11) were used for graft-versus-host disease (GVHD) prophylaxis.

Results: The patient achieved engraftment (neutrophils: $>500 \times 10^{8} / \mathrm{L}$ ) on day +15 with full donor-type chimerism. The final transfusion to support red blood cells and platelets 
was given on days +21 and +32 . She had terminal ileitis with cytomegalovirus reactivation on day +41 , and therefore ganciclovir was administered. Although pre-engraftment syndrome and grade II acute GVHD involving the skin developed on day +9 , the symptoms disappeared after administration of immunosuppressive agents, such as methylprednisolone and additional MTX. Chronic GVHD of the skin and oral mucosa was revealed at 6 months, and prednisolone was needed until 21 months after transplantation. The patient has been doing well with full donor-type chimerism for 24 months after SCT without chronic GVHD, EBV reactivation and malignancy.

Conclusion: HSCT was an effective treatment for a patient with a heterozygous splice-site mutation in PIK3R1, and we think that HSCT is necessary before the onset of malignancy. Further studies are necessary to determine the appropriate conditioning, GVHD prophylaxis and donor.

Disclosure of Interest: None declared.

P650

Endocrine and Metabolic Complications After

Hematopoietic Stem Cell Transplantation in Children and Adolescents

D. Gurlek Gokcebay ${ }^{1}$, N. Ozbek ${ }^{1, *}$, F. Azik', A. Yazal Erdem', C. Bayram ', Z. Avci', P. Isik', N. Yarali' ', B. Tavil', F. Demirel', B. Tunc ${ }^{1}$ ${ }^{1}$ Pediatric Hematology, ${ }^{2}$ Pediatric Endocrinology, Ankara Children's Hematology and Oncology Hospital, Ankara, Turkey

Introduction: There has been an increasing trend of hematopoietic stem cell transplantation (HSCT) in children. Endocrine organs are highly susceptible to effects of high dose chemotherapy, as well as immunosuppressive therapy. In this study we aimed to evaluate endocrine and metabolic complications after HSCT.

Material (or patients) and methods: We retrospectively analysed children who underwent HSCT in our center from April 2010 to July 2013 with at least two years follow-up without relapse or progression of primary disease. Data were collected from medical records of the patients.

Results: Seventy-seven children [mean age at HSCT 8.8 (0.519) years, mean follow-up time 3.7 (2.1-5.3) years, male/female 46/31] included in the study. 71 (93\%) of them underwent allogeneic transplantation from matched-related donors, and $6(7 \%)$ of them were autologous. Indications for HSCT were malignancy in $30(39 \%)$ patients [9 received fractionated total body irradiation (TBI)], and non-malignant diseases in 39 (50\%) [26 (34\%) with thalassemia major]. Twenty patients (28\%) developed chronic graft-versus-host disease (cGvHD). Mean body mass index SD score was significantly increased from $-0.67 \pm 1.34$ to $-0.48 \pm 1.24$ at $1^{\text {st }}$ year of HSCT $(P=0.017)$. Three months after HSCT, the number of patients with dyslipidemia increased from $26(33.7 \%)$ to $35(45.5 \%)$, but a significant decrease was noted at $6^{\text {th }}$ and $12^{\text {th }}$ months $(P<0.001$, and $P=0.014$ ) (Table). Twenty patients (14\%) with elevated fasting serum insulin and glucose underwent further endocrinological evaluation. One of the patients developed diabetes mellitus during HSCT and received insulin therapy. Four patients, all with CGvHD who were on immunosuppressive therapy, fulfilled the criteria of metabolic syndrome during follow-up. Two of the patients had hypertension, and one had obesity. Totally 17 of the girls (55\%) showed pubertal development. Hypogonadism was diagnosed in 11 girls (35\%) at median 1 year (range, 0.5-4 years) after HSCT. Two of the patients (one girl and one boy) were diagnosed with precocious puberty and treatment was initiated. The risk of hypogonadism was significantly higher in the girls who underwent HSCT $>10$ years than those who underwent HSCT $\leq 10$ years $(75 \%$ vs $10.5 \%, P=0.003)$. Hypothyroidism developed in 9 of the patients (11\%). Dyslipidemia, insulin resistance, and hypothyroidism showed no relation with sex, age at HSCT, conditioning with $\mathrm{TBI}$, or presence of CGvHD.

Conclusion: Previous studies showed the risk of endocrine complications was associated with age at HSCT, gender, type of transplantation, type of conditioning regimen, use of steroids or other immunosuppressive medications. In our study, there was no relation between these factors and endocrine or metabolic dysfunction, except the risk of hypogonadism that was significantly higher in girls who underwent HSCT >10 years. However, larger, multicenter studies are needed for a definite conclusion. We believe, children should be monitored regularly for endocrine and metabolic functions after HSCT.

References: Dvorak CC, Gracia CR, Sanders JE, et al. Biol Blood Marrow Transplant 2011;17(12):1725-38.

Disclosure of Interest: None declared.

\section{P651}

Long-term outcome and prognostic factors of second and subsequent allogeneic hematopoietic stem cell transplants in pediatric patients with hematologic malignancies

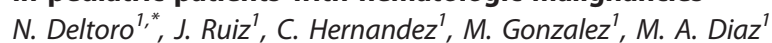
${ }^{1}$ Hospital Infantil Universitario "Niño Jesus", Madrid, Spain

Introduction: Second or subsequent allogeneic hematopoietic stem cell transplantation (HSCT) is a treatment option for graft failure or relapse after a first one. However, data on longterm outcome and risk factors for survival in pediatric patients are limited.

Material (or patients) and methods: We retrospectively analyzed risk factors for survival in a cohort of 65 children that received a 2 HSCT (12 of them received a 3th allogeneic transplant) between 1997 and 2015 due to hematologic malignancies. Patients were aged between 1 to 16 years old (median 7 y). There were 44 boys and 21 girls. They had undergone a first auto-HSCT in $13(20 \%)$ of cases and a first allo-HSCT in 52 (80\%). Diagnoses were as follows: ALL in 32 cases, AML in 23, NHL in 8 and MDS in 2. Time to second transplantation was 6 months ( range, from 1 to 133). At time of transplant, patients were in 1st CR in 8 cases, 2 nd CR in 28 , 3 th $C R$ in 13 and not in remission in 16. The main source of hematopoietic stem cells was mobilized peripheral blood in 52 cases followed by cord blood in 9 and bone marrow in 2. Same donor was used in 30 cases and a different donor in 22. The need of a second-HSCT derived from relapse in 41 cases (63\%), graft failure in $22(33 \%)$ and a secondary event in $2(3 \%)$.

[P650]

Table. Changes in lipid profiles

\begin{tabular}{|c|c|c|c|c|c|c|}
\hline Mean $\pm S D(m g / d L)$ & Before HSCT & 3rd month & 6th month & 9th month & 12th month & 2nd year \\
\hline Cholesterol & $149 \pm 43^{*}$ & $171 \pm 44^{*}$ & $163 \pm 43$ & $179 \pm 153$ & $163 \pm 43$ & $155 \pm 36$ \\
\hline Triglyceride & $125 \pm 69 \dagger$ & $203 \pm 254 \dagger$ & $134 \pm 86$ & $129 \pm 106$ & $116 \pm 66$ & $107 \pm 53$ \\
\hline HDL (high density lipoprotein) & $42 \pm 15^{\S}$ & $41 \pm 10$ & $49 \pm 17^{\S}$ & $49 \pm 15$ & $51 \pm 13$ & $51 \pm 12$ \\
\hline
\end{tabular}

${ }^{*} P<0.001,+P=0.046,{ }^{\S} P=0.001$. 
Results: With a median follow-up of 5 years for survivors, the cumulative incidence of relapse and NRM following transplantation were $43 \pm 8 \%$ and $39 \pm 8 \%$ respectively. Causes of death $(n=39)$ were as follows: primary disease in 18 , infection in 10, microangiopathy in 8, GvHD in 2 and idiopathic pneumonia syndrome in one case. The probability of DFS was $37 \pm 7 \%$ for the whole group. Patients receiving a 2nd transplant for graft failure did better than those transplanted due to relapsing disease ( $53 \pm 10 \%$ vs $28 \pm 8 \%$, respectively). For those patients transplanted due to relapsing disease, we have found no impact of disease status at transplant in DFS (33 $\pm 9 \%$ in 2nd CR vs $32 \pm 18 \%$ in 3 th $C R$ vs $31 \pm 14 \%$ in not remission). No impact in DFS was also seeing for patients transplanted from same or different donor ( $30 \pm 11 \%$ vs $42 \pm 8 \%$, respectively) or for those transplanted after a previous autologous or allogeneic transplantation ( $38 \pm 16 \%$ vs $36 \pm 7 \%$, respectively). Four of 12 patients that received a 3th allo transplantation are alive and doing well.

Conclusion: Despite the high incidence of relapse and NRM, a second or subsequent allogeneic HSCT remains as a reasonable and potentially curative option for patients experiencing graft failure or relapse after a first transplant procedure.

References: Long-term outcome and prognostic factors of second allogeneic hematopoietic stem cell transplant for acute leukemia in patients with a median follow-up of $\geqslant 10$ years. Andreola G et al. Bone Marrow Transplant. 2015 Dec;50 (12):1508-12.

Second reduced intensity conditioning allogeneic transplant as a rescue strategy for acute leukaemia patients who relapse after an initial RIC allogeneic transplantation: analysis of risk factors and treatment outcomes. Vrhovac R et al. Bone Marrow Transplant. 2015 Oct 5.

Disclosure of Interest: None declared.

\section{P652}

Impact of neuroblastoma-specific features and donorspecific characteristics on NK and gd T cell alloreativity P. Schlegel ${ }^{1, *}$, A. Jöchner ${ }^{1}$, M. Wiedenmann ${ }^{1}$, D. Syring ${ }^{1}$, A. Schilling ${ }^{1}$, C. Seitz ${ }^{\top}$, A. Rabsteyn ${ }^{1}$, C. Mainardi ${ }^{1}$, R. Handgretinger ${ }^{1}$, P. Lang ${ }^{1}$ Hematology/Oncology, University Children's Hospital, Tübingen, Germany

Introduction: The ongoing study NCT02258815 has shown favorable survival of relapsed stage IV neuroblastoma by intensification of treatment via haploidentical hematopoietic stem cell transplantation and subsequent anti-GD2 immunotherapy. Donor selection by KIR-genotyping has been shown to dramatically determine event-free survival in AML and pediatric ALL $[1,2]$. The impact on survival in neuroblastoma remains unclear. Nevertheless, the genetic background of the KIR genes alone does not define the NK alloreactive potential of donors. Many other genes are involved in regulating the NK cell activity. Therefore, functional donor assessment needs implementation for donor selection strategies as well as the immune characterization of the primary tumor and metastasis that will profile the tumor and will help to estimate the susceptibility for immune recognition Material (or patients) and methods: Different neuroblastoma cell lines $(n=8)$ have been immune characterized for HLA-I-genotype and quantitative HLA-I expression, NKG2D ligand, DNAM-1 ligand and GD2-expression as to be the most prominent parameters to determine NK cell mediated recognition, cytolysis and ADCC. Additionally, PD-1 ligand expression was assessed. Further, NK profiling of healthy volunteer donors $(n=15)$ by KIR genotyping, KIR phenotyping and assessment of NKG2D receptor, DNAM-1 coreceptor, NCRs and PD-1 R were functionally analyzed by CD107a assay, intracellular IFN-gamma staining and cytotoxicity assays (Calcein release; Luciferase based kill, xCELLigence) in primary PBMCs and preactivated NK and TcRy $\delta$ T cells.

Results: HLA-I upregulation by IFN-gamma led to significant reduction of NK cytolysis and ADCC and a significant correlation of NK cytolysis with inhibitory and activatory ligands was revealed in blocking experiments and quantitative GD2-expression clearly dictated susceptibility for ADCC using the chimeric mAb ch14.18. However, blocking PD-1 R/L interaction with pembrolizumab led to reduced CD107a degranulation but increased intracellular IFN-gamma production of NK cells in neuroblastoma targets whereas in pre-B-ALL cell lines to increased overall activation, cytolysis and ADCC. KIR receptor-ligand mismatch and NK cell education showed an impact on NK activity. Irrespective of targets, superior and inferior donors were identified by in vitro functional testing added to the donor selection algorithm of KIR genotyping.

Conclusion: In conclusion, we demonstrate that defined tumor-specific features determine NK cell recognition, cytolysis and ADCC. Further, donor-specific characteristics allow to graduate donors for their NK and TcR $\gamma \delta$ T cell activity, cytolysis and ADCC implicating to extend conventional donor selection strategies by functional effector cell testing. The role of checkpoint inhibitors in neuroblastoma needs further evaluation.

References: 1. Michaelis, S.U., et al., KIR haplotype B donors but not KIR-ligand mismatch result in a reduced incidence of relapse after haploidentical transplantation using reduced intensity conditioning and CD3/CD19-depleted grafts. Ann Hematol, 2014. 93(9): p. 1579-86.

2. Cooley, S., et al., Donor selection for natural killer cell receptor genes leads to superior survival after unrelated transplantation for acute myelogenous leukemia. Blood, 2010. 116(14): p. 2411-9.

Disclosure of Interest: None declared.

\section{P653}

Impact of activatory and inhibitory NK cell receptors on recognition, cytolysis and ADCC in B-lineage precursor ALL P. Schlegel ${ }^{1, *}$, M. Wiedenmann ${ }^{1}$, A. Jöchner ${ }^{1}$, D. Syring ${ }^{1}$, S. Christian ${ }^{1}$, A. Rabsteyn ${ }^{1}$, C. Mainardi ${ }^{1}$, A. Schilling ${ }^{1}$, R. Handgretinger ${ }^{1}$, P. Lang

${ }^{1}$ Hematology/Oncology, University Children's Hospital, Tübingen, Germany

Introduction: The treatment of refractory and relapsed B-lineage precursor leukemia (ALL) has improved by advancements in allogeneic SCT and CD19-targeting approaches. KIR/ HLA interaction has been shown absolutely relevant for the survival of patients suffering from leukemia [1]. This was shown in AML and childhood ALL in haploidentical SCT [2-4]. Fc modified antibodies can enhance ADCC efficiently irrespective of KIR geno- and phenotype and FCyllla polymorphism [5]. We analyzed the influence of activatory and inhibitory receptors on NK and $\gamma \delta \mathrm{T}$ cells, using an Fc-optimized monoclonal CD19 antibody.

Material (or patients) and methods: NK profiling of healthy donors $(n=15)$ by KIR genotyping, KIR phenotyping and assessment of NKG2D receptor, DNAM-1 coreceptor, NCRs and PD-1 receptor was done by CD107a assay, intracellular IFNy staining and cytotoxicity assays using primary PBMCs and preactivated NK and $\gamma \delta T$ cells with and without the CD19 mAb 4G7SDIE. Different BCP-ALL cell lines $(n=4$, NALM-16, NALM-6, MHH-CALL-4 and SEM) were immune characterized for HLA-I-genotype and quantitative HLA-I expression, NKG2D ligand, DNAM-1 ligand and CD19-expression as to be the most prominent parameters to determine NK cell mediated recognition, cytolysis and ADCC in CD19+ BCP-ALL. Additionally, the influence of PD-1 receptor-ligand interaction was assessed. Further experiments included upregulation and blockade of HLA-I, blockade of NKG2D-R and DNAM-1- R.

Results: All donors showed low antileukemic activity of PBMCs. Preactivation and expansion of donors' PBMCs significantly increased activation of NK cells and cytolysis. In all patients coincubation of CD19 mAb led to enhanced cytolysis and NK cell degranulation as well as IFNy production. RL-mismatch and NK education showed an impact on NK activity. Irrespective of the BCP-ALL cell line, superior and 
inferior donors were identified by in vitro testing partly controversial to KIR genotyping results.

Conclusion: In conclusion, we demonstrate that defined tumor-specific features determine NK cell recognition, cytolysis and ADCC. Further, donor-specific characteristics indentify donors for their NK and $\gamma \delta \mathrm{T}$ cell activity, cytolysis and ADCC implicating to extend donor selection strategies by functional effector cell assessment. The role of checkpoint inhibitors in NK alloreactivity of BCP-ALL needs further evaluation.

References: 1. Velardi, A., L. Ruggeri, and A. Mancusi, Killer-cell immunoglobulin-like receptors reactivity and outcome of stem cell transplant. Curr Opin Hematol, 2012. 19(4): p. 319-23.

2. Oevermann, L., et al., KIR B haplotype donors confer a reduced risk for relapse after haploidentical transplantation in children with ALL. Blood, 2014. 124(17): p. 2744-7.

3. Michaelis, S.U., et al., KIR haplotype B donors but not KIRligand mismatch result in a reduced incidence of relapse after haploidentical transplantation using reduced intensity conditioning and CD3/CD19-depleted grafts. Ann Hematol, 2014. 93(9): p. 1579-86

4. Cooley, S., et al., Donor selection for natural killer cell receptor genes leads to superior survival after unrelated transplantation for acute myelogenous leukemia. Blood, 2010. 116(14): p. 2411-9.

5. Terszowski, G., C. Klein, and M. Stern, KIR/HLA interactions negatively affect rituximab- but not GA101 (obinutuzumab)induced antibody-dependent cellular cytotoxicity. J Immunol, 2014. 192(12): p. 5618-24.

Disclosure of Interest: None declared.

\section{P654}

Low TRM rate and excellent survival after haploidentical stem cell transplantation in children and adolescents with acute leukemia

P. Bader ${ }^{1, *}$, J. Soerensen ${ }^{1}$, A. Willasch ${ }^{1}$, E. Salzmann-Manrique ${ }^{1}$, T. Krenn ${ }^{2}$, M. Dürken ${ }^{3}$, J. Faber ${ }^{4}, H$. Kreyenberg ${ }^{7}$, R. Bager ${ }^{\prime}$, S. Huenecke ${ }^{1}$, M. Bremm ${ }^{1}$, E. Ullrich ${ }^{1}$, S. Bakhtiar ${ }^{1}$, E. Rettinger ${ }^{\prime}$, A. Jarisch ${ }^{7}$, T. Klingebiel ${ }^{1}$

${ }^{7}$ Division for Stem Cell Transplantation and Immunology, Department for Children and Adolescent Medicine, Frankfurt, ${ }^{2}$ Pediatric Hemato-Oncolgy, University Hospital Saarland, Homburg/Saar, ${ }^{3}$ Pediatric Hemato-Oncology, University Hospital Mannheim, Mannheim, ${ }^{4}$ Department for Pediatric Hemato-Oncology and Hemostasiology, University Hospital Mainz, Mainz, Germany

Introduction: In patients with high risk acute leukemia haploSCT may be life saving when an HLA-identical donor is not available or when patients relapsed after a first allo-SCT from a matched donor. Rapid recovery of NK-cells and the use of KIRligand mismatch donors are known factors of increased GvLeffect. Rapid T-cell regeneration is of utmost importance to avoid mortality caused by viral reactivation and infections. Therefore immediate sustained engraftment is desired. Important factors to achieve these goals are number of transplanted hematopoietic stem cells, type of T-cell depletion and type of serotherapy to deplete i) recipient T-cells and ii) to allow cotransfused peripheral T-cells to expand until thymus depended T-cell regeneration is possible. Here we present our results of haplo-transplantation using CD3/CD19 depleted $\mathrm{HCT}$ after reduced intensity conditioning.

Material (or patients) and methods: 50 children and adolescents with ALL $(n=26), \operatorname{AML}(n=24)$ and CML after blast crisis $(n=2)$ received a haplo-SCT in our institution between $1 / 2005$ and $8 / 2015$. Patients (pts) were transplanted in CR1 $(n=7)$, CR2 $(n=17) ;$ CR3 $(n=7)$, CR4 $(n=1)$, and 18 pts while not being in remission. $16 / 50$ pts received a second transplant after having relapsed after a first transplant from a matched donor. Conditioning regimens consisted of Fludarabine (FLU), Thiotepa (THIO), Melphalan (MEL) and OKT-3 $(n=30)$. After OKT-3 was taken from the market, pts received THIO-FLU-MEL and ATG $(n=8)$ preceded by a 3 day pre-phase with Clofarabine (CLO), Cyclophosphamide (CYC) and Etoposide (ETO). As these pts showed a higher rate of primary rejection and TRM, the conditioning regimen was changed to CLO-CYC-ETO followed by THIO-FLU-MEL and Campath in 12 pts.

Results: Median follow-up of the surviving pts was 63 month (0.4-130 months). Time to achieve more than 1000 leukocytes was 12 (1-30 days), time for thrombocyte recovery was 11 days (8-30 days). Immune regeneration was rapid for pts who received OKT-3 as serotherapy, delayed for ATG pts and better for pts who received Campath.

For the whole cohort $(n=50)$, pEFS (at 3 years) was $44 \% \pm 8$, pOS was $50 \% \pm 8$; pCIR was $36 \% \pm 7$ and pTRM was $19 \% \pm 6$.

Patients in remission: For all pts who were transplanted in remission pEFS was $61 \% \pm 9$, pOS was $65 \% \pm 9$, pCIR was $26 \% \pm 08$, and pTRM was $10 \% \pm 6$.

Patients not in remission: Pts not in remission had a pEFS of $14 \% \pm 9$, pOS of $15 \% \pm 9$, pCIR was $55 \% \pm 14$ and pTRM was $30 \% \pm 13$.

ALL vs. AML: The analysis of the subgroup of patients who were transplanted in remission $(n=32 ; \mathrm{ALL}, n=16$; myeloid leukemia $(n=16$; AML, $n=14 ; C M L, n=2)$ showed for ALL pts a pEFS of $50 \% \pm 14$, and for $A M L+C M L$ pts a pEFS of $72 \% \pm 12$.

Patients in CR with $1^{\text {st }}$ transplantation: Pts who received a first allogeneic transplantation $(n=19)$ and were in remission at the time of transplant had an excellent survival probability. Their pEFS was $62 \% \pm 11$, pOS of $67 \% \pm 11$, pCIR was $27 \% \pm 10$ and PTRM was $11 \% \pm 8$.

OKT3 vs. ATG vs. Campath: Pts treated in remission or not, had a pEFS of $65 \% \pm 9$ if they received OKT3, of $25 \% \pm 15$ if they received ATG, and of $71 \% \pm 18$ if they received Campath for conditioning. CIR of relapse was $20 \% \pm 9$ for OKT-3, $50 \% \pm 24$ for ATG and $0 \%$ for Campath pts and the TRM rate was $15 \% \pm 7,25 \pm 16$, and $18 \% \pm 18$ for OKT-3, ATG and Campath, respectively.

Conclusion: Haplo-SCT has a profound anti leukemia effect; serotherapy has considerable influence on CIR and on TRM. Disclosure of Interest: None declared.

\section{P655}

Final results of a multicenter phase I/II study using CD3/ CD19 depleted stem cells for haploidentical transplantation in children

P. Lang1,*, T. Eichholz ${ }^{1}$, T. Feuchtinger ${ }^{2}$, H.-M. Teltschik ${ }^{1}$, I. Mueller ${ }^{3}, M$. Pfeiffer ${ }^{7}$ M. Schumm ${ }^{1}$, M. Ebinger ${ }^{\prime}$, C.-P. Schwarze', B. Gruhn ${ }^{4}$, J. Alten ${ }^{5}$, M. Albert, J. Greil $6^{2}$, C. Urban ${ }^{7}$, R. Handgretinger ${ }^{7}$

${ }^{1}$ Hematology / Oncology, University Children's Hospital, Tuebingen, 'Pediatric Hematology / Oncology, von Haunersches Kinderspital, Muenchen, 'University Children's Hospital, Hamburg, "University Hospital of Jena, Jena, ${ }^{5}$ University Children's Hospital, Kiel, ${ }^{6}$ Universtiy Children's Hospital, Heidelberg, Germany, 'University Children's Hospital, Graz, Austria

Introduction: A total of 163 pediatric patients with ALL, AML/ MDS, CML/JMML $(n=67)$ relapsed/refractory solid tumors (neuroblastoma, soft tissue sarcomas, $n=68$ ) and nonmalignant diseases (immune deficiencies, hemoglobinopathies) $(n=28)$ received T and B cell depleted peripheral stem cells from full haplotype mismatched family donors within this phase I/II trial. $34 / 163$ patients received a $2^{\text {nd }}$ or $3^{\text {rd }}$ HSCT.

Material (or patients) and methods: AntiCD3/antiCD19 coated magnetic microbeads and the CliniMACS ${ }^{\circledR}$ device were used for graft manipulation. A short course single agent prophylaxis with MMF was given. Patients received either myeloablative TBI or Busulfan based conditioning regimens $(n=26)$ or a toxicity reduced regimen (Melphalan $\left(140 \mathrm{mg} / \mathrm{m}^{2}\right)$ fludarabine $\left(160 \mathrm{mg} / \mathrm{m}^{2}\right)$ thiotepa $\left.(10 \mathrm{mg} / \mathrm{kg}), n=131\right)$ and serotherapy with ATG-F or OKT3.

Results: A median number of $15.2 \times 10 \mathrm{E} 6 / \mathrm{kg}$ CD34+ progenitors, $77 \times 10 E 6 / \mathrm{kg} \mathrm{NK}$ cells and 48×10E3/kg residual T cells were infused. Primary engraftment occurred in $90.8 \%$ of patients. In a multivariate analysis, the influence of diagnosis, conditioning regimen, number of infused progenitors/T cells/NK cells and type of serotherapy was investigated. Only diagnosis remained significant. Median time to reach $>500$ neutrophiles/ $\mu$ l was 
10 days. GvHD grade $0-1$ occurred in $78 \% .16 \%$ and $6 \%$ had GvHD grade II and III-IV, respectively. Chronic limited and extensive GvHD was observed in $13 \%$ and $12 \%$. In an univariate analysis, only use of ATG-F significantly reduced the incidence of aGvHD, whereas the above factors did not.

Transplant related mortality was low with $8 \%$ at one year. Regeneration of NK cells was very fast (mean number CD56 $+/ \mu \mathrm{l}$ at day +90/180: 234/233), whereas $\mathrm{T}$ cells recovered slower (mean number CD3+/ $\mu$ lat day $+90 / 180: 205 / 426$ ). Event free survival at 3 years was $41 \%$ for patients with acute leukemias CR 1-3 (1 $\left.1^{\text {st }} \mathrm{HSCT}\right)$ and $14 \%$ for patients with active disease. Patients with nonmalignant diseases had a favorable EFS at 3 years of $60 \%$ whereas patients with solid tumors did worse (25\%).

Conclusion: Transplantation of CD3/CD19 depleted haploidentical stem cells resulted in a robust myeloic engraftment and in a fast recovery of donor NK cells. The method helped to prevent GvHD very effectively and to reduce TRM, despite intensive pretreatment of the patients. Patients with acute leukemias in any CR had a favorable EFS whereas potential anti tumor effects were not sufficient in patients with active disease. Since only short course MMF was used, the method offers a basis for further post-transplant immune therapies.

Disclosure of Interest: None declared.

\section{P656}

Viral reactivations are predicted by CD4+ T-cell recovery, and are associated with morbidity and mortality after pediatric hematopoietic cell transplantation

R. Admiraal ${ }^{1,2}$, C. de Koning ${ }^{2}$, C. Lindemans ${ }^{1}$, M. Bierings ${ }^{1}$ T. Wolfs ${ }^{3}$, A. Wensing ${ }^{4}$, B. Versluijs ${ }^{7}$, S. Nierkens ${ }^{2,5}$, J. J. Boelens ${ }^{1,2, "}$ ${ }^{1}$ Pediatric Blood and Marrow Transplant Program, ${ }^{2} U-D A N C E$, ${ }^{3}$ Pediatric Infectious Diseases, ${ }^{4}$ Virology, ${ }^{5} U-D A I R$, University Medical Center, Utrecht, Netherlands

Introduction: Viral reactivations (VR) following hematopoietic cell transplantation (HCT) in children have been shown to negatively influence outcome, and may be influenced by early immune reconstitution (IR). In the current study, we evaluate IR as a predictor of VR, and determine the effect of VR and IR on outcomes.
Material (or patients) and methods: Patients receiving a first HCT for any indication with any cell source in the pediatric BMT program of the University Medical Center in Utrecht from Jan-2004 to Okt-2014 were included. IR (CD3+, CD4+ and CD8 + T-cells, B-cells and NK-cells) was measured every other week, VR; (cytomegalovirus; CMV, human herpesvirus 6; HHV6, BKvirus; BK, Epstein-Barr virus; EBV and adenovirus; AdV) twice weekly. Continuous cell counts over time were evaluated for associations with VR's (defined as $>1000$ copies $/ \mathrm{mL}$ ). VR's and CD4 IR (defined as CD4+ $>50 \times 10^{6}$ within 100 days), both introduced as time dependent variable, were evaluated as a predictor for clinical outcome (OS, EFS, TRM, acute and chronic GvHD, graft failure and lung injury). Cox proportional hazards models were used.

Results: A total of 273 patients were included, with cord blood $(n=142 ; 52 \%)$ and bone marrow $(n=123 ; 45 \%)$ being the most frequent cell sources. Median follow-up was 5 years (0.2130 months). AdV, EBV and HHV6 reactivations were associated with absence of $\mathrm{CD} 4 \mathrm{IR}(P=0.022, P=0.029$ and $P=0.022$, respectively), while CMV and $B K$ were not correlated to any IRparameter. Duration of AdV reactivation as well as antiviral treatment was significantly shorter in patients with CD4 IR $(P=0.004$; fig 1a). Both AdV (HR 2.2, $P=0.0039)$ and CD4+ IR $(\mathrm{HR}=0.5, P=0.0045)$ were predictors for OS. However, those with AdV and successful IR had comparable OS to those without AdV (Fig 1b). AdV and EBV reactivations were a predictor for TRM (HR 3.0, $P=0.0008$ and $\mathrm{HR}=2.0, P=0.046$, respectively). Early $\mathrm{CD} 4+\mathrm{IR}$ predicted a lower TRM $(\mathrm{HR}=0.3, P=0.0005)$. A higher incidence of acute-GvHD grade 2-4 was associated with preceding HHV6 (HR 3.5, $P<0.0001)$, with a trend seen in preceding EBV $(P=0.08)$. Likewise, aGvHD grade 3-4 was influenced by both HHV6 (HR= 2.7, $P=0.015$ ) and EBV (HR 4.8, $P=0.018$ ). The incidence of extensive chronic-GvHD was higher in those with EBV (HR 3.6, $P=0.03$ ). Lung injury and graft failure were not predicted by any VR. Interestingly, CMV and BK were no predictors for any of the investigated outcomes.

Conclusion: Probability and duration of AdV, EBV and HHV6 reactivation were predicted by time to $C D 4+I R$, while CMV and HHV6 were not predicted by any immune reconstitution markers. Reactivations of AdV, EBV and HHV6 significantly impacted morbidity and mortality. Strategies to better predict

[P656]

Adenovirus load over time

A

\section{Only patients with Adeno reactivation}

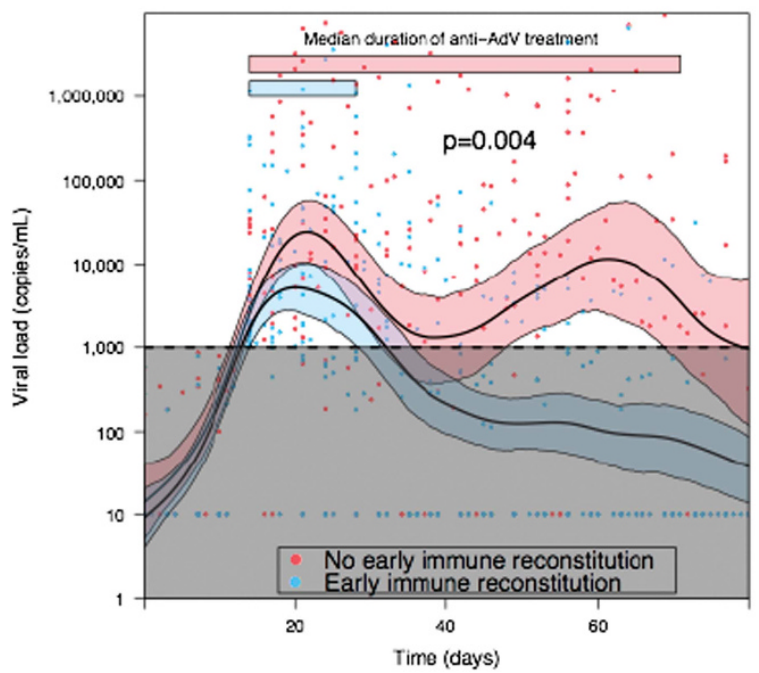

OS according to Adenovirus reactivation

B

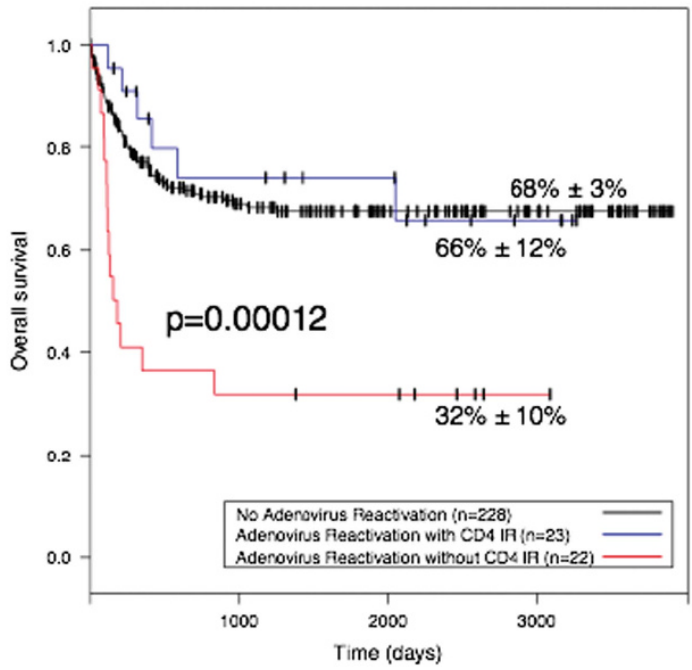

Panel A: Spline regression analysis of Adenovirus (AdV) bad (sclid curved lines with $95 \% \mathrm{Cl}$ as shaded area) over time (normalised to start of reactvation) in pavents with AdV reactivation, with (blue area/cothar) or without (red areidotoar) successtul immune reconstibtion, showing longer AdV-reactivation and higher AdV loads in those without early immune reoansttution. Dots: individual raw AdV lad. Bars: median duration of AOV load > $1000 \mathrm{cs} / \mathrm{mL}$ Grey area: cinically insigrnctint AdV load. Panel B: S.rvival plot showing pasents not having an AdV-reactivation (black, heving AdV-reactivation with suocessful early immune reconstituton (blue) and having AdV-reactivation without immune reconstutution (res). 
and accelerate CD4 T-cell immune reconstitution are imperative to improve the survival chances.

Disclosure of Interest: None declared.

P657

T-cell reconstitution and Event Free Survival are predicted by low ATG exposure after Cord Blood Transplantation in Children: Towards Individualized ATG dosing to improve survival chances

R. Admiraal ${ }^{1,2}$, C. van Kesteren ${ }^{1,2}$, C. Lindemans ${ }^{1}$, M. Bierings $^{1}$, B. Versluijs ${ }^{1}, S^{\prime}$. Nierkens ${ }^{2,3}$, J. J. Boelens , $^{1, *^{*}}$

${ }^{1}$ Pediatric Blood and Marrow Transplant Program, ${ }^{2} U-D A N C E$,

${ }^{3} U$-DAIR, University Medical Center, Utrecht, Netherlands

Introduction: Anti-thymocyte globulin (ATG) is frequently used in unrelated cord blood transplantation (CBT) to prevent graft-versus-host disease (GvHD) and graft failure (GF). However, in vivo exposure of ATG to the infused CB-graft (i.e. post-CBT exposure) is suggested to hamper T-cell immune reconstitution (IR) significantly. This effect is more pronounced in CBT compared to bone marrow or peripheral blood stem cell sources ${ }^{1}$. We studied the influence of various ATG exposures after CBT on the outcomes in a large homogenous group of pediatric patients.

Material (or patients) and methods: All pediatric patients who underwent a first HCT with unrelated cord blood (uCB) after myelo-ablative conditioning in the UMC Utrecht between 1-2004 and 3-2015 were included. The cumulative dose of ATG was given intravenously over 4 days consecutively. From 2013 onwards, patients with AML and MRD+ ALL did not receive ATG. Individual area under the curve (AUC) of active-ATG preand post-CBT was calculated using a validated pharmacokinetic model. The calculated active-ATG exposures were studied in exposure groups as previously described ${ }^{1}$ : noATG, $<20,20-50$ and $>50 \mathrm{AU} * \mathrm{~d} / \mathrm{L}$. Main outcome of interest was IR (defined as CD4+ T-cells $>50 \times 10^{6} / \mathrm{L}$ in two consecutive measurements within 100 days); other outcomes of interest were overall survival (OS), event free survival (EFS), therapy related mortality (TRM), acute- and chronic GvHD and GF. IR was considered as a time-varying predictor. Cox proportional hazard and logistic regression models were used. Results: A total of 127 patients (49 malignant, 78 nonmalignant) were included: 49 patients received a $6 / 6,60$ a 5/6, and 18 a $4 / 6$ matching uCB-unit. The median follow-up was 42 (range 0.3-131) months. Seventeen patients (13\%) did not receive ATG. Indications were equally distributed over the 3 ATG exposure groups. Lower AUC of active ATG after CBT (continuous variable) was the best predictor for successful IR (HR 0.97, $P=0.0001$; In Fig 1a depicted in the 4 groups). In multivariate analyses, successful IR is associated with higher
OS (HR 0.33, $P=0.0020$ ), EFS (HR 0.38, $P=0.0016$; fig 1c) and TRM (HR 0.31, $P=0.008$ ). Low active-ATG exposure after CBT (continuous) predicted higher EFS (HR 1.005, $P=0.015$, figure $1 \mathrm{~b})$, and showed a trend to higher OS $(P=0.08)$. High ATG exposure before CBT was a predictor for a lower incidence of acute GvHD 2-4 (HR 0.40, P=0.031). Acute-GvHD 3-4 (12\% @ 180d), extensive chronic-GvHD (6\% @1 yr) and GF (12\% @ $1 \mathrm{yr}$ ) were not associated with ATG exposure, possibly due to the low incidence.

Conclusion: Low exposure of active-ATG after CBT is a predictor for IR and EFS, while presence of IR is associated with higher OS and EFS chances. Individualized dosing of ATG targeted to optimal exposures before and after CBT may result in better survival chances. A clinical trial investigating the influence of individualized dosing of ATG on IR is recruiting in the Netherlands.

References: ${ }^{1} \mathrm{Admiraal}$ et al, Lancet Haematology 2015.

Disclosure of Interest: None declared.

\section{P658}

Predictable Immune Reconstitution and Prevention of Graft Failure in High-Risk Patients following Cord Blood Transplantation by Individualized Dosing and Therapeutic Drug Monitoring of Anti-Thymocyte Globulin

R. Admiraal ${ }^{1,2,{ }^{*}}, C_{\text {. van Kesteren }}{ }^{1,2}$, A. Lacna ${ }^{3}$, L. Ebskamp-van Raaij $^{3}$, S. Nierkens ${ }^{2,3}$, J. J. Boelens ${ }^{1,2}$

${ }^{1}$ Pediatric stem cell transplantation unit, ${ }^{2} U-D A N C E,{ }^{3} U-D A I R$, University Medical Center, Utrecht, Netherlands

Introduction: Survival chances after unrelated cord blood transplantation (CBT) is associated with presence of immune reconstitution (IR) ${ }^{1}$. IR may be significantly hampered by high exposure of anti-thymocyte globulin (ATG) after CBT resulting in significant in vivo T-cell depletion. ATG exposure prior CBT however is suggested to reduce the probability of graft-versushost-disease (GvHD) and graft failure (GF). By individualizing ATG dosing and thereby optimizing ATG exposure both before and after CBT, the outcomes may improve. Here, we describe the preliminary results of a study using individualized dosing and therapeutic drug monitoring (TDM) of ATG (Thymoglobulin) in high-risk patients receiving a CBT.

Material (or patients) and methods: From 2014, patients (children and young adults) receiving a CBT, after standard myeloablative busulfan (Bu; $90 \mathrm{mg}^{*} \mathrm{l} / \mathrm{h}$ ) and fludarabine (Flu) in the UMC Utrecht at high-risk for GF and/or for whom fast IR was essential because of ongoing infections, were included. High-risk for GF was defined as increased inflammation with an intact or activated T-cell function; e.g. hemoglobulinopathies, Chronic Granulomatous Disease (CGD), Hemophagocytic Lympho-Histiocytosis (HLH) and a

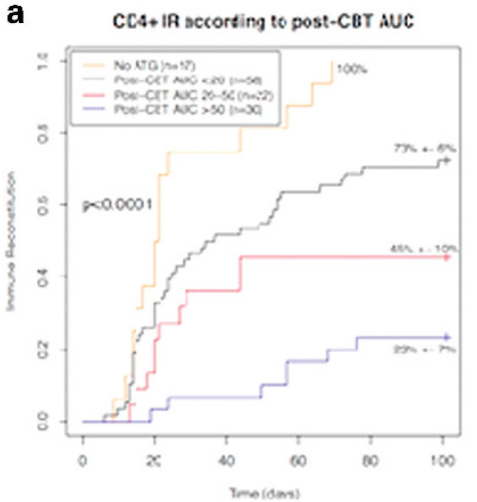

b

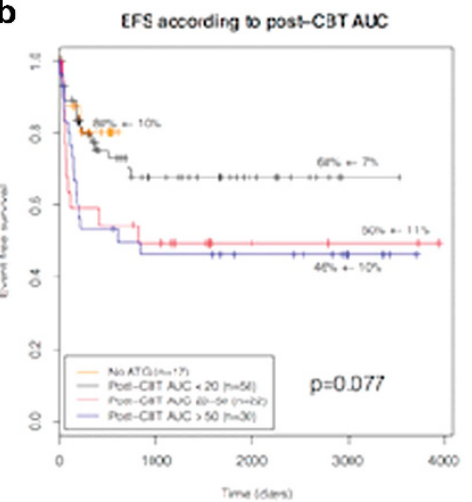

c

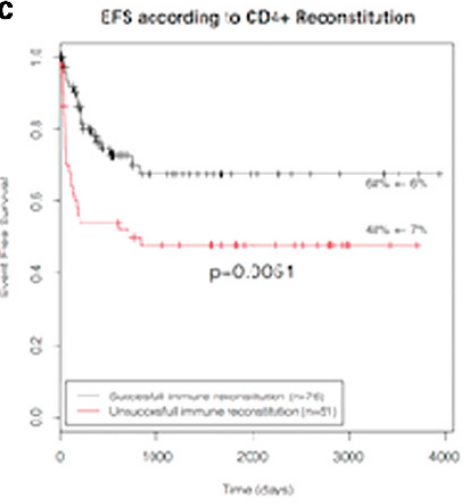

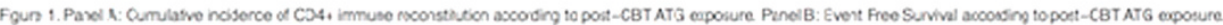


CD4+ T-cell IR in Cordblood transplants

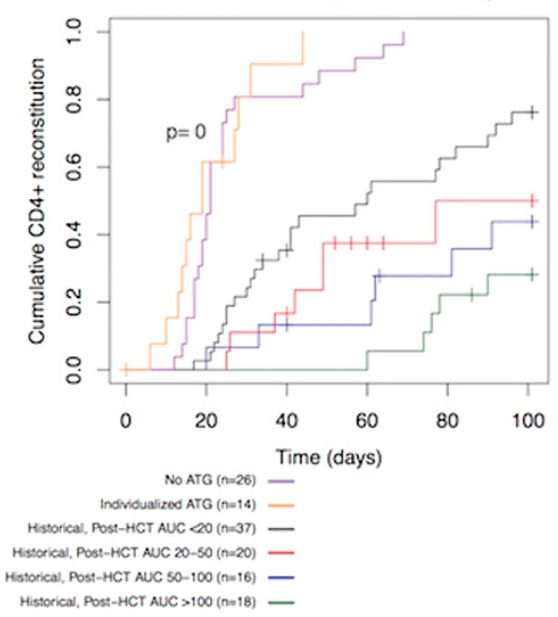

Immune Deficiencies with auto-immune phenomena. Early 2015, we amended the study protocol by adding TDM. Dosage and timing of ATG was calculated with a validated population pharmacokinetic (PK)-model (Admiraal et al, Clin. PK 2014). We targeted to a pre-CBT AUC of $>40 \mathrm{AU}^{*}$ day $/ \mathrm{mL}$ ( $>80 \mathrm{AU}$ day/ $\mathrm{mL}$ for TDM-patients) while target post-CBT exposure was very low ( $<10 \mathrm{AU}^{*}$ day/mL). In TDM-patients, doses were adjusted according to individual PK if necessary. Main endpoints were CD4+ IR (twice >50/uL within 100 days) and GF. Other endpoints: acute-GvHD, viral reactivations (CMV, EBV, Adv, HHV6) and overall survival (OS). CD4+ IR was compared to recent cohort ${ }^{1}$.

Results: Fourteen patients are included (3 CGD, 2 CID, 2 SAA, $1 \beta$-thalassemia, $1 \mathrm{HLH}, 5$ other), of whom 5 received TDM. Median follow up is 199 days. Mean cumulative dose of ATG was $11.8(3-30) \mathrm{mg} / \mathrm{kg}$, starting at a median of 12 (7-17) days before HCT. In all TDM-patients, both actual pre-CBT AUC (mean 104, range 73-140) and post-CBT AUC (3.4, 0.1-6) were within the target. CD4+ IR was significantly improved when compared to historical controls (all had CD4+ IR before day 40), and comparable to patients not receiving ATG (Fig 1a). No GF (all full donor) occurred, 1 patient had aGvHD II. Two patients experienced a transient ( $<2 \mathrm{wk}$ ) viral reactivation of CMV/Adenovirus for which no anti-viral treatment was started. OS at 6 months is $89 \%+-10 \%$ with 1 TRM due to pulmonary failure associated with pre-existent lung disease (Fig $1 b$ ).

Conclusion: Individualized dosing of ATG (on FluBu-platform), with added TDM is feasible, showed fast CD4+ IR and encouraging other outcomes. It may improve the outcomes of CBT in patients with high-risk features.

References: 1 Admiraal $\mathrm{R}$ et al. Association between antithymocyte globulin exposure and CD4+ immune reconstitution in paediatric haemopoietic cell transplantation: a multicentre, retrospective pharmacodynamic cohort analysis. Lancet Haematol 2015; 3026: 8-11.

Disclosure of Interest: None declared.

\section{P659}

Proactive Interventional Feeding in Pediatric BMT

Recipients: Is it needed?

S. Anak ${ }^{1, *}$, O. Ocal Doğan ${ }^{2}$, G. Baysoy ${ }^{3}$, A. Kacar ${ }^{4}$, Y. Yaman ${ }^{1}$, E. Saribeyoglu ${ }^{5}$, K. Ozdilli, ${ }^{5}$ V. Hazar ${ }^{7}$

${ }^{1}$ Pediatric Hematology-Oncology, ${ }^{2}$ Pediatrics, ${ }^{3}$ Pediatric Gastroenterology, ${ }^{4}$ Dietician, ${ }^{5}$ Pediatric Hematology, Medipol Mega University Hospital, Istanbul, Turkey

Introduction: The increased survival of transplant patients evokes quality of life problems such as adequate feeding,

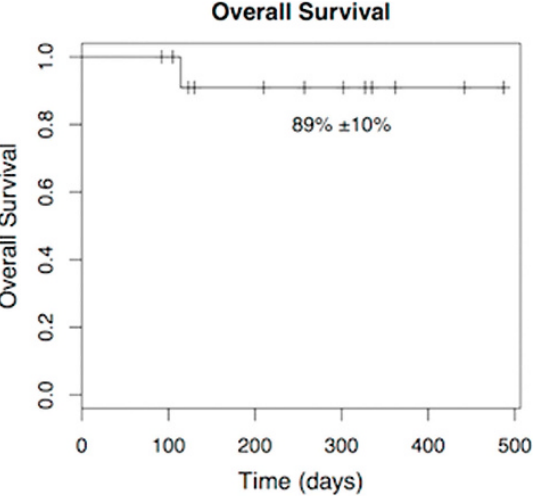

especially in pediatric patients. The morbidity and mortality is increased in patients with inadequate calorie intake, which is very common in transplant recipients. Most patients are referred to transplant centers with already developed undernourishment. This prospective study was conducted to determine the effect of proactive feeding support of transplant recipients in terms of weight and anthropometric measurements.

Material (or patients) and methods: In this ongoing study, as a first time point, 18 patients ( 11 boys and 7 girls, 9 autologous and 9 allogeneic, 11 malign diseases, 7 nonmalignant diseases) were enrolled in this preliminary study. Nine patients ( 5 boys $/ 4$ girls) received proactive treatment with nasogastric tube feeding while the remaining received supported oral feeding. The weight, height, BMI z score and anthropometric measurements such as mid-upper arm circumference, triceps-biceps skin fold thickness were checked before transplantation and at day 30 . Results: The groups were comparable for age and gender. The $\mathrm{BMI} z$ score and anthropometric measurements at day 30 were not significantly different in both groups, the decrease in weight was more prominent and statistically significant in proactive feeding group $(P=0,05)$. This was also true for $\mathrm{BMI} \mathrm{z}$ score change $(P=0,04)$.

Conclusion: Although proactive enteral feeding is supposed to be effective in the adequate feeding of pediatric BMT patients, our preliminary results could not support this hypothesis. Our controversial results may be due to low number of patients included in the study. Underlying disease, complications of BMT, type of BMT might also be effecting this outcome. Prospective studies with more patients are needed. Disclosure of Interest: None declared.

\section{P660}

Successful curative therapy with Rituximab and haemotopoietic stem cell transplant in a child with idiopathic CD4+ lymphocytopenia and EBV-driven MALT lymphoma

S. H. Lum ${ }^{1, *}$, S. Hughes ${ }^{2}$, D. Bonney ${ }^{3}$, E. Cheesman ${ }^{4}$, R. Wynn ${ }^{1}$ ${ }^{1}$ Department of Blood and Marrow Transplant, ${ }^{2}$ Department of Paediatric Allergy and Immunology, Royal Manchester Children's Hospital, Manchester, United Kingdom, ${ }^{3}$ Department of Blood and Marrow Transplant, Royal Manchester Children's Hospital, Manchester, Malaysia, ${ }^{4}$ Diagnostic Paediatric Histopathology Service, Royal Manchester Children's Hospital, Manchester, United Kingdom

Introduction: Idiopathic CD4+ lymphocytopenia (ICL) is a rare, autosomal recessive disorder, which results in recurrent infections, malignancies and autoimmune disorders. Here, 
we report the first case of EBV-associated MALT lymphoma arising in the salivary gland in a child with ICL.

Material (or patients) and methods: The patient was diagnosed with ICL at 4 years old following an immune assessment for recurrent, life threatening and multi-systemic infections since infancy. She first presented with cryptosporidial gastroenteritis at 12 months of age and viral croup needing ventilatory support at 15 months of age. Following these, she had multiple bacterial sinopulmonary infections (Streptococcus pneumoniae and Pseudomonas sp.), severe herpes gingivostomatitis and persistent molluscum contagiosum infection. Because of previous experience of two affected children died of transplant related complications, parents declined stem cell transplant for the patient. Despite having prophylactic immunoglobulin replacement and antibiotics, her quality of life was poor due to frequent hospitalizations for invasive infective episodes. Consequently, she developed bronchiectasis and hearing impairment secondary to chronic pseudomonas and streptococcal otitis. In addition, she had persistent mucocutaneous candidiasis, difficult control eczema and chronic relapsing EBV viraemia in spite of rituximab therapy since August 2009.

Results: At 12 years old, the patient developed a rapidly enlarging left submandibular swelling in July 2013. The diagnosis of extranodal marginal zone B-cell lymphoma of MALT-lymphoma was established on histopathological examination of surgically excised left submandibular salivary gland, which showed diffuse infiltration of the salivary gland by lymphoid cells with loss of normal structure. These lymphoid cells were positive for CD20, CD 19 and CD79a and some demonstrated CD 10 positivity, which suggested the possibility of blast transformation. EBV in-situ hybridization testing revealed the presence of Epstein Barr virus. PET scan showed very high intensity FDG update at left posterior nasal mucosa and cervical lymph nodes (Figure $2 \mathrm{~A}$ and 2B). Marrow studies and $C T$ images at staging did not identify any distant focal lesions. JD was administered four courses of Rituximab and her disease demonstrated good remission. HLA-matched sibling donor marrow transplantation was done in November 2013 following conditioning regimen with fludarabine, treosulfan and alemtuzumab and post-transplant GVHD prophylaxis with ciclosporin alone. Neutrophil engraftment was demonstrated on Day +12 and her early transplant course was complicated by Streptococcus oralis neutropenic bacteraemia, CMV viraemia and asymptomatic BK viruria. The patient continues to be well at 24 months post transplant. Her pre-transplant lymphoproliferative disease has fully resolved. She has normal blood counts and is fully immune reconstituted. She has been free from any unusual infections and flare of eczema.

Conclusion: MALT lymphoma is extremely rare in children and adolescents and there is no standardised treatment protocol. Our patient showed good response to rituximab monotherapy and her underlying disease is cured with haematopoietic stem cell transplant. In addition, this case also illustrated the potential of PET scan in monitoring disease response in patients with MALT-lymphoma.

Disclosure of Interest: None declared.

\section{P661}

Effects of conditioning intensity on growth after hematopoietic stem cell transplantation in children

S. Uemura ${ }^{1, *}$, D. Hasegawa ${ }^{1}$, A. Kozaki ${ }^{1}$, N. Nino ${ }^{1}$, T. Yokoi ${ }^{1}$, S. Takafuji ${ }^{1}$, A. Saito ${ }^{1}$, T. Ishida ${ }^{1}$, K. Kawasaki ${ }^{1}$, Y. Kosaka ${ }^{7}$ 'DEPARTMENT OF HEMATOLOGY \& ONCOLOGY, KOBE CHILDREN'S HOSPITAL, Kobe, Japan

Introduction: Allogeneic hematopoietic stem cell transplantation (allo-HSCT) is an effective therapeutic option for high risk hematologic disease; however, it may cause a wide range of inevitable late sequelaes especially in children who are more vulnerable than adults. For allo-HSCT in children, it is important to prevent late gonadal disorder and a short stature. In this study, we examined the effects of the conditioning intensity on growth in children using an HSCT cohort in our hospital.

Material (or patients) and methods: The subjects were 72 prepubertal children who underwent initial allogeneic HSCT in our hospital between 2003 and 2015, and could be followedup without the necessity of additional transplantation for 1 year or more. We analyzed 70 children, excluding 2 with Fanconi's anemia. The annual heights after transplantation were extracted from medical records, and standardized based on the standard height of Japanese children, prepared by the Japanese Society for Pediatric Endocrinology, to calculate the SD score (Z-score). In addition, we analyzed the association between the SD score every year after transplantation and conditioning intensity using statistical methods. Conditioning consisting of total-body irradiation (TBI) at $£ 8 \mathrm{~Gy}$ and Busulfan administration at $£ 8 \mathrm{mg} / \mathrm{kg}\left(£ 280 \mathrm{mg} / \mathrm{m}^{2}\right)$ was defined as nonmyeloablative conditioning (RIC). That consisting of other conditions was defined as myeloablative conditioning (MAC). Results: Overall, the height SD scores on transplantation and after $1,2,3,4$, and 5 years were $-0.83 \pm 1.02,-1.14 \pm 1.03$, $-1.22 \pm 1.15,-1.44 \pm 1.04,-1.64 \pm 1.19$, and $-1.77 \pm 1.13$, respectively. The height SD scores in the MAC group $(n=36)$ on transplantation and after $1,2,3,4$, and 5 years were $-0.88 \pm 0.91,-1.30 \pm 1.07,-1.46 \pm 1.22,-1.71 \pm 1.01,-1.74 \pm 1.27$, and $-2.05 \pm 1.17$, respectively, whereas the values in the RIC group $(n=34)$ were $-0.79 \pm 1.13,-1.00 \pm 0.98,-0.92 \pm 0.99$, $-1.08 \pm 1.01,-1.45 \pm 1.06$, and $-1.06 \pm 0.57$, respectively. In the former, the height SD scores 2 and 3 years after transplantation were significantly lower than in the latter. Two patients in the MAC group received growth hormone replacement therapy for growth hormone deficiency dwarfism after transplantation.

Conclusion: Allogeneic HSCT with RIC may reduce the risk of a short stature post-transplantation.

Disclosure of Interest: None declared.

\section{P662}

Evaluation of engraftment syndrome in children following full matched related donor hematopoietic stem cell transplantations

T. Ileri ${ }^{1, *}$, E. Ünal Ince ${ }^{7}, H$. Çakmaklı ${ }^{1}$, Z. Uysal ${ }^{1}$, M. Ertem ${ }^{1}$

${ }^{1}$ Pediatric Bone Marrow Transplantation Unit, Ankara University School of Medicine, Ankara, Turkey

Introduction: The term of Engraftment Syndrome (ES) has been widely used for describing the clinical conditions in such as skin rash, noninfectious fever, weight gain which occur during neutrophil recovery period following both the autologous and allogeneic hematopoietic stem cell transplantation (HSCT). In most of the studies reporting the risk factors, study populations are non-homogeneous resulting in consistent risk factors reported among these studies. To determine the risk factors, studies with homogeneous recipient and donor populations are critical.

Material (or patients) and methods: In this study, the incidence, clinical features, risk factors, and outcomes of ES were evaluated in 169 children following allogeneic HSCT from full matched related donor. Patients were evaluated according to the Spitzer criteria.

Results: Seventeen patients (10.1\%) presented with clinical conditions suggesting the ES and noninfectious fever (100\%) and skin rash $(76.5 \%)$ are the most frequent clinical findings of the patients with ES. Using the a univariate analysis, the significant risk factors for the development of ES appear to be the underlying malignant disease, ATG use in conditioning regimen, using only CsA for GVHD prophylaxis, and early release of monocytes to the peripheral blood. Multivariate analysis showed a higher risk of ES in children having underlying malignant diseases and early release of monocytes to the peripheral blood. Patients with ES experienced significantly higher incidence of grade $2-4$ acute graft vs host disease $(29.3 \%$ vs $7.5 \%, P<0.001)$ and propensity towards to a higher rate of transplantation related mortality $(P<0.001)$. 
Overall survival did not differ between the patients with and without ES. Thirteen of 17 patients received steroid therapy and all but one patient responded to therapy.

Conclusion: Monitoring for early detection of ES and subsequent early intervention of steroid therapy is the key for recovery successfully. The most crucial approach for this purpose, mainly is to find out and use the most useful and feasible diagnostic criteria for routine medical practice. One of the most accepted criteria was defined by Spitzer et al in 2001 and they proposed that the clinical conditions should develope within $96 \mathrm{~h}$ of engraftment. From a prospective standpoint, it is not possible to predict the exact engraftment date before it occurs. We believe that meeting at least two positive criteria after the day of first apperance of monocytes in peripheral blood is a clinically sensible diagnostic criteria.

References: 1. SPITZER TR. Engraftment syndrome following hematopoietic stem cell transplantation. Bone Marrow Transplantation 2001; 27: 893-898.

2. SCHMID I, STACHEL D, PAGEL P, ALBERT MH. Incidence, Predisposing Factors, and Outcome of Engraftment Syndrome in Pediatric Allogeneic Stem Cell Transplant Recipients. Biology of Blood and Marrow Transplantation 2008; 14:438-444.

3. NISHIO N, YAGASAKI H, TAKAHASHI $\mathrm{Y}$ et al. Engraftment syndrome following allogeneic hematopoietic stem cell transplantation in children. Pediatr Transplantation 2009; 13: 831-837.

Disclosure of Interest: None declared.

\section{P663}

Fertility preservation in paediatric and adolescent patients undergoing HSCT: Update of the cross-sectional survey of the EBMT Paediatric WP

T. Diesch-Furlanetto 1,*, A. Rovó ${ }^{2}$, N. von der Weid ${ }^{3}$, M. Faraci ${ }^{4}$, M. Pillon ${ }^{5}$, A. Dalissier ${ }^{6}, P$. Bader ${ }^{7}$ on behalf of Paediatric WP

${ }^{7}$ Paediatrc Oncology/Haematology, University Children's Hospital of Basel, Basel, ${ }^{2}$ Haematology, University Hopsital of Bern, Bern,

${ }^{3}$ Paed. Oncology/Haematology, University Children's Hospital of Basel, UKBB, Basel, Switzerland, ${ }^{4}$ Haematology/HSCT Unit, Research Insitute G. Gaslini, Genova, ${ }^{5}$ Paediatric Oncology/ Haematology, Hospital of Padua, Padua, Italy, ${ }^{6}$ EBMT Paediatric Disease Working Party, Paris, France, ${ }^{7}$ Diviosn of Stem Cell Transplanation and Immunology, Hospital for Children and Adolescents of Frankfurt, Frankfurt, Germany

Introduction: With improvement in survival rates for paediatric and young adult cancer patients, considerations regarding long-term effects of therapy have become more important. Many chemotherapeutic agents and irradiation can affect gonads leading to impairment of pubertal development and infertility. Infertility causes a substantial restriction in quality of life of patients. In the last years many fertility preservation guidelines for cancer patients have been published. However, strategies for preserving fertility in this age group are not yet well established. Despite these recommendations options for fertility preservation are discussed only in about $42 \%$ (Terenziani et al. PBC 2014) of all eligible patients. We present here a survey conducted by the Paediatric WP of the EBMT. We aimed to analyse and compare the different fertility preservation practices for children and adolescents across the EBMT centres and to identify hence the unmet needs in the field.

Material (or patients) and methods: The survey was initiated in September 2014. 177 paediatric centres reporting to the EBMT were asked to fill-out a form with 24 questions related to fertility preservation strategies. Questions focused on availability of a fertility preservation program and its options, number of patients included in the program, payers and suggestions for improvement.

Results: Until November $30^{\text {th }} 2015,36(20 \%)$ centres from 15 countries replied (range of HSCT patients 5-60 in 2013).). Data from 781 of 3789 patients with HSCT in 2013 could be obtained. 298/781 (38\%) patients received a fertility preservation counselling and $125(16 \%)$ of them were offered a fertility preservation procedure. Half of the 36 centres reported to have a standard operating procedure to perform counselling about ways to preserve fertility. Timing for counselling was in $50 \%$ of the centres during the first week after diagnosis. Advisory concept involving more than one specialist was largely preferred, in $60 \%$ of the centres, an interdisciplinary team involving haematologists, gynaecologists and /or reproductive medicine specialists was responsible for patients counselling. All responding centres considered the topic of fertility preservation as very relevant. Better education, more resources and financial funding emerged as pivotal factors to support and implement such programs.

Conclusion: This EBMT survey revealed that participating centres have a great interest for fertility preservation. The number of responders (20\%) cannot exclude a representatively bias of these results. The collected data showed that $38 \%$ of the patients received counselling on fertility preservation and fertility preservation procedures were performed in only $16 \%$ of this cohort. This emphasizes the need for enhanced education of the transplant team and active multidisciplinary collaboration in the field. Fertility preservation should become a standard of care; this data represents the basis to further efforts toward fertility preservation standardization in the EBMT community.

Disclosure of Interest: None declared.

\section{P664}

Second Allogeneic Hematopoietic Stem Cell Transplantation in Pediatric Patients

T. Aksu', B. Kuşkonmaz ${ }^{1, *}$, S. Aytaç ${ }^{2}$, B. Tavil ${ }^{2}$, D. Uçkan Cetinkaya

${ }^{1}$ Department of Pediatrics, Division of Bone Marrow Transplantation Unit, ${ }^{2}$ Department of Pediatrics, Division of Hematology, Hacettepe University Faculty of Medicine, Ankara, Turkey

Introduction: Second allogeneic hematopoietic stem cell transplantation (HSCT2) is a treatment option especially for graft failure or relapse of primary disease. However there is insufficient data on long-term outcome and cure for primary disease.

Material (or patients) and methods: We retrospectively analyzed 30 patients with 8 malignant, 22 non-malignant diseases who underwent HSCT2 between January 1996 and November 2015.

Results: The characteristics of patients and HSCT2 were shown on Table 1. Malignant disorders (MD) were acute myeloid leukemia (3), chronic myeloid leukemia (3), myelodysplastic syndrome (1), and juvenile myelomonocytic leukemia (1). Nonmalignant disorders (NMD) were various immune deficiencies (12), beta thalassemia major (5), Fanconi anemia (2), hypereosinophilic syndrome (1), acquired aplastic anemia (1), and osteopetrosis (1). The indications for HSCT2 were graft failure or relapse of primary disease. The patients either received myeloablative or reduced / non-myeloablative conditioning regimens or no conditioning at all (Table 1). Cyclosporine $A \pm$ methotrexate/ methylprednisolone/ mycophenolate mofetil were used for graft versus host disease (GVHD) prophylaxis. Engraftment was achieved in 6 of $8 \mathrm{MD}$ group (75\%) and 13 of 22 NMD group (59\%). Survival rates were $50 \%(4 / 8)$ in patients with MD, 45\% (10/22) in patients with NMD. The causes of death in MD group were; engraftment failure and associated infection (2), relapse (1) and venoocclusive disease (1). Among patients with NMD, causes of death were; engraftment failure and associated infection (7), poor graft function associated infection (2), infection (2) and GVHD associated infection (1). Four patients underwent third HSCT due to engraftment failure and eventually died. The median time between transplants was 18.7 months (range 1.5-52) for $\mathrm{MD}$ and 4.7 months (0.6-24.0) for NMD. Median follow-up was 85 months (1-176) for MD. Median follow-up was 97.5 months (17-222) for NMD. Disease free survival was $50 \%$ for MD and $40 \%$ for NMD. 
Table 1. Characteristics of the patients and second HSCT

\begin{tabular}{|c|c|c|}
\hline & Malignant & $\begin{array}{l}\text { Non- } \\
\text { malignant }\end{array}$ \\
\hline Number of patients & 8 & 22 \\
\hline $\begin{array}{l}\text { Age of patients (median year } \\
\text { / min-max) }\end{array}$ & $13.1(5.3-19)$ & $5.8(0.5-16.5)$ \\
\hline Gender (Male/female) & $6 / 2$ & $13 / 9$ \\
\hline \multicolumn{3}{|l|}{ Conditioning regimen } \\
\hline Myeloablative & 2 & 5 \\
\hline $\begin{array}{l}\text { Reduced/Non- } \\
\text { myeloablative }\end{array}$ & 5 & 13 \\
\hline Without conditioning & 1 & 4 \\
\hline \multicolumn{3}{|l|}{ Stem cell source } \\
\hline BM & 3 & 11 \\
\hline PB & 5 & 11 \\
\hline Acute GvHD ( $\geq$ grade 2 ) & $\begin{array}{l}1 \text { (N/A for } 2 \\
\text { patients) }\end{array}$ & $\begin{array}{l}1 \text { (N/A for } 8 \\
\text { patients) }\end{array}$ \\
\hline Chronic GvHD & $\begin{array}{l}2 \text { (N/A for } 3 \\
\text { patients) }\end{array}$ & $\begin{array}{l}1 \text { (N/A for } 11 \\
\text { patients) }\end{array}$ \\
\hline VOD & 1 & 3 \\
\hline $\begin{array}{l}\text { Median follow-up (median } \\
\text { month / min-max) }\end{array}$ & $85(1-176)$ & $97.5(17-222)$ \\
\hline
\end{tabular}

BM: bone marrow, PB: peripheral blood, CB: cord blood, GvHD: graft versus host disease, VOD: venoocclusive disease, N/A: Not applicable.

Conclusion: In MD, survival rate is reported to be about $32-$ $59 \%$ after HSCT2. In our study, survival rate is found as $50 \%$ in MD, and 45\% in NMD after HSCT2. Graft failure was the leading cause for HSCT2 in our series. Therefore, a great concern should be given to prevent graft failure in the first HSCT. Eventually, HSCT2 offers the chance of cure or long-term remission for MD as well as NMD. But according to our results, HSCT3 fails to cure primary diseases of the patients.

Disclosure of Interest: None declared.
P665

Favorable Response to Rituximab in Refractory Cytopenias Occurring After Pediatric Hematopoietic Stem Cell Transplantation

T. Aksu' ${ }^{1}$, B. Kuşkonmaz ${ }^{1, *}$, F. V. Okur ${ }^{1}$, S. Ünal ${ }^{2}$, D. Uçkan Çetinkaya ${ }^{1}$ ${ }^{1}$ Department of Pediatrics, Division of Bone Marrow Transplantation Unit, ${ }^{2}$ Department of Pediatrics, Division of Hematology, Hacettepe University Faculty of Medicine, Ankara, Turkey

Introduction: Rituximab is a promising agent in the treatment of cytopenias after HSCT. We report 3 pediatric patients, who suffered from refractory cytopenia after allogeneic hematopoietic stem cell transplantation (HSCT), successfully treated with rituximab.

Material (or patients) and methods: Characteristics of patients and transplantation and treatment for cytopenias was recorded.

Results: Case presentation: Patients, transplantation characteristics and therapies for cytopenias are shown on Table 1. All patients underwent HSCT from 10/10 HLA matched sibling donors with myeloablative conditioning. Patient 1 and 2 did not develop acute or chronic graft versus host disease (GVHD), patient 3 showed mild oral symptoms related to chronic GVHD, 15 months after HSCT. All patients developed hemorrhagic cystitis, and patient 3 developed moderate sinus obstruction syndrome. Neutrophil engraftment was achieved at day +15, 16, and 14 after HSCT, respectively. Thrombocyte engraftment was achieved at day +36 and 20 after HSCT for patient 2 and 3, respectively. Patient 1 never achieved thrombocyte independence. Chimerism analysis (day +30 ) showed $98 \%$ donor profile in case $1,97 \%$ in case 2, \%89 in case 3. Patients developed cytopenias $+55,61$, and 84 days after HSCT respectively. The vitamin deficiencies, immune causes, and viral etiologies were excluded. Simultaneous bone marrow findings were all normal, including moderate cellularity. Cytopenias did not respond to trial of corticosteroids (CS), G-CSF, and intravenous immunoglobulin (IVIG). Rituximab therapy $\left(375 \mathrm{mg} / \mathrm{m}^{2}\right.$ weekly $\left.\mathrm{x} 4\right)$ was initiated to patients at day $+134,78$, and 93 . The cytopenia of all lineages of patients recovered after rituximab therapy. Response to rituximab took two months for patients 1 and 2, and 34 days for patient 3. Rituximab therapy was well tolerated without any complications except infusion related symptoms.

Conclusion: In the posttransplant setting, rituximab was demonstrated to be effective both in children and adults with refractory cytopenias. We have shown that rituximab may be effective to treat cytopenias after HSCT, non-associated with GVHD. Also long-term response to rituximab was obtained in the patients in terms of anemia, leukopenia and thrombocytopenia. Disclosure of Interest: None declared.

[P665]

Table 1. Characteristics of transplantation and therapy for cytopenias

\begin{tabular}{|c|c|c|c|}
\hline & Patient 1 & Patient 2 & Patient $3^{*}$ \\
\hline Age/Gender & $10,1 / F$ & $17,3 / \mathrm{M}$ & $9,8 / \mathrm{M}$ \\
\hline Diagnosis & MDS & AML & TM Pesaro Class 3 \\
\hline Conditioning regimen & $\mathrm{Bu} / \mathrm{Cy} / \mathrm{ATG}$ & $\mathrm{Bu} / \mathrm{Cy}$ & Protocol 26 \\
\hline Source of stem cell & BM & BM & PBSC \\
\hline CD34+cell dose $\left(x 10^{6}\right.$ per $\left.\mathrm{kg}\right)$ & 2.5 & 2.1 & 18.2 \\
\hline Hematological parameters, before rituximab & $\begin{array}{l}\text { PLT: transfusion dependent } \\
\text { WBC: } 1.6 \times 10^{9} / \mathrm{I}\end{array}$ & $\begin{array}{c}\mathrm{Hb}: 7.4 \mathrm{~g} / \mathrm{dl}, \mathrm{WBC}: 1.5 \times 10^{9} / \mathrm{I} \\
\text { PLT: } 19 \times 10^{9} / \mathrm{l}\end{array}$ & $\begin{array}{c}\text { Hb: } 7.2 \mathrm{~g} / \mathrm{dl} \\
\text { WBC: } 2 \times 10^{9} / \mathrm{I} \\
\text { PLT: } 20 \times 10^{9} / \mathrm{l}\end{array}$ \\
\hline Prior therapy & CS, G-CSF, IVlg & CS, G-CSF & CS, G-CSF, IVIG \\
\hline Start of rituximab (days) & +134 & +78 & +93 \\
\hline Follow-up (months) & 75 & 27 & 50 \\
\hline
\end{tabular}

*Second $\mathrm{HSCT}$ due to primary engraftment failure $\mathrm{AML}=$ Acute myeloid leukemia, MDS = Myelodysplastic syndrome, $\mathrm{TM}=\mathrm{Thalassemia}$ major, $\mathrm{Bu}=$ Busulfan, $\mathrm{Cy}=$ Cyclophosphamide, $\mathrm{ATG}=$ Anti thymocyte globulin, $\mathrm{BM}=$ Bone marrow, PBSC = Peripheral blood stem cells, PLT: platelets, $\mathrm{CS}=$ Corticosteroid, $\mathrm{G}-\mathrm{CSF}=$ Granulocyte colony-stimulating factor, IVIg = Intravenous immunoglobulin . 


\section{P666}

Leukemia related co-stimulation / co-inhibition predict graft-versus-leukemia effect and T-cell attack of acute lymphoblastic leukemia mediated by CD19/CD3-bispecific T-cell engager

J. Feucht ${ }^{1}$, S. Kayser ${ }^{1}$, D. Gorodezki ${ }^{1}$, M. Döring ${ }^{2}$, F. Blaeschke ${ }^{2}$ P. Schlegel', H. Bösmüller', M. Ebinger', P. Lang',

R. Handgretinger ${ }^{1}, T$. Feuchtinger,

${ }^{1}$ Pediatric Hematology / Oncology, University Children's Hospital, Tübingen, ${ }^{2}$ Pediatric Hematology / Oncology, Hauner University Children's Hospital, Munich, Institute of Pathology, Eberhard Karls University, Tübingen, Germany

Introduction: Refractory B-precursor acute lymphoblastic leukemia (ALL) remains an unsolved therapeutic challenge. Graft-versus-leukemia effect (GVL) has been limited in ALL, but T-cell responses against ALL can be induced by the CD19/CD3bispecific T-cell engaging antibody Blinatumomab. Until now factors for susceptibility to T-cell attack against leukemia have not been characterized. Immune-checkpoint molecules regulate immune escape of malignant cells and antibody blockade of these inhibitory pathways enhances antitumor immune responses.

Material (or patients) and methods: We investigated the role of co-stimulatory and co-inhibitory molecules for effector-target cell interactions and influence on T-cell attack against leukemia. $\mathrm{CD} 19^{+}$lymphoblast lines, primary pediatric B-ALL bone marrow blasts $(n \geq 10)$ and physiologic $C D 19^{+} C D 10^{+}$pre-B precursors from healthy bone marrow were screened for surface expression of 20 different co-signaling molecules. Surface expression of PDL1, PD-1, LAG3, CD40, CD86, CD27, CD70 and HVEM revealed differences in stimulatory and inhibitory profiles of pediatric ALL blasts as compared to physiologic cells.

Results: Pediatric ALL patients refractory to Blinatumomabtreatment $(n=5)$ as well as patients with relapsed leukemia $(n=11)$ showed increased expression of PD-L1 on blasts. Expression of exhaustion markers PD-1 and TIM-3 was significantly higher on patients' T cells as compared to healthy donors and is induced by T-cell attack against blasts. Blinatumomab-mediated T-cell function was examined in healthy donors as compared to pediatric patients with ALL through analysis of proliferation and effector function. Significant differences in Blinatumomab-induced T-cell function were found to be target-cell dependent and correlated to expression of co-signaling molecules on target cells. Blockade of inhibitory PD-1-PD-L and CTLA-4-CD80/CD86 interactions could further enhance effector T-cell function of healthy donors and patients whereas blockade of co-stimulatory CD28-CD80/86 interactions resulted in reduced T-cell effector and proliferation potential $(\geq 15)$. Combined treatment with Blinatumomab and PD-1 blocking antibody Pembrolizumab was feasible and induced an anti-leukemic immune response in a 12-year-old patient with refractory ALL post SCT.

Conclusion: We show that regulation of T-cell activation and inhibition by co-signaling molecules guides GVL and the efficacy of T-cell attack against ALL. Inhibitory interactions between leukemia-induced checkpoint molecules on T cells and their counterparts on ALL regulate in vivo resistance to T-cell immunotherapy and will guide future therapeutic interventions.

Disclosure of Interest: None declared.
P667

Quality of life improvement in terminal state of disease after hematopoietic stem cell transplantation, after the implementation of "Palliative Care" procedure in Transplantation Centre of University Children's Hospital in Cracow from 2012 to 2015

M. Wozniak ${ }^{7}$, W. Czogala ${ }^{2}$, A. Krasowska - Kwieciwenn ${ }^{3, *}$, O. Wiecha ${ }^{4}$, A. Dłużniewska ${ }^{4}$, S. Skoczen ${ }^{4}$, J. Goździk $^{5}$

${ }^{1}$ Transplantation, University Children Hospital, ${ }^{2}$ Transplantation, University Children's Hospital, ${ }^{3}$ Transplantation, University Children Haospital, ${ }^{4}$ Transplantation, University hospital, ${ }^{5}$ Transplantation, Children University Hospital, Krakow, Poland

Introduction: Hematopoietic stem cell transplantation (HSCT) is an aggressive therapy, aimed to resolve severe diseases, not to be cured by other methods. Despite of a continuous progress in this area of medicine there are still 10 to 30 percent of patients dying during the first year after HSCT. Since 2011, there has been a striving for implementation of quality management system JACIE, concerning, apart from others, the procedure of appointing to and running the palliative care. Before the procedure was implemented, many of patients in end-stage condition had been treated inappropriately to their state. Some of patients had been subjected to exaggerated pain and struggle associated with overtreatment due to the lack of relevant guidelines (first congruent guidelines were published by Polish Pediatric Society in 2011). The main purpose is to indicate the quality of life improvement as a result of delimitation to palliative care in those patients, who develop untreatable complications or the primary disease relapse after having been subjected to transplantation procedure in Transplantation Centre of University Children's Hospital of Cracow through the years 2011-2015, during and after having implemented "Palliative Care" standard operating procedure (SOP).

Material (or patients) and methods: Through the years: 2012-2015 76 allogeneic HSCT were performed. In 33 cases the procedure was accomplished due to neoplastic disease and in 43 - in non - neoplastic disease. During following years 12 patients died and in 4 the palliative care SOP was implemented.

Results: Through 2012-2015 4 patients aged 8 to 17 years were met the inclusion criteria to palliative care based on the appropriate procedure. In three cases the reason to delimit the patients were untreatable infectious complications and in one case there was second early - onset relapse of the primary disease. In one case the patient was referred to the home hospice. In subsequent case, the patient was simultaneously taken care by both Intensive Care Unit and Transplantation Ward. In case of two subsequent patients the care was performed merely by Transplantation Ward. Due to the procedure, the decision to discard the non-effective causative therapy and implement palliative therapy was the result of mutual agreement of both patients' parents and medical team. Neither mechanical ventilation, intravenous hydration nor blood products transfusion were performed. The therapy was focused of treating pain, dyspnea, anxiety, depression, constipation and a vast psychological support to the family and the patients.

One boy passed away in his home, surrounded by family, the rest of the patients died in their parents' attendance, with no exacerbated medical treatment carried out, in the most comfortable conditions attainable, after having the families and patients themselves congruently prepared for the death. Conclusion: The implementation the fitting outcome of SOP "Palliative Care" makes the process of delimiting patients to the palliative care efficient and straightens the course of the performing, especially in hospital conditions. This leads to considerable improvement of quality of life of the patients subjected to HSCT, facing the end-stage condition of the disease.

Disclosure of Interest: None declared. 
P668

Umbilical cord blood as suitable alternative donor of hematopoietic stem cell transplantation in childhood acute myeloid leukemia: a multi-center retrospective study of Yeungnam region, South Korea

Y. J. Shim 1,*, H. S. Kim ${ }^{1}$, J. M. Lee ${ }^{2}$, J. O. Hah ${ }^{2}$, K. S. Lee ${ }^{3}$, J. Y. Kim ${ }^{3}$,

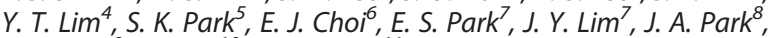
H. Chueh ${ }^{9}, J$. Park $^{10}$, S. G. Kong ${ }^{11}$ on behalf of on the behalf of Youngnam Pediatric Hematology and Oncology Society

${ }^{1}$ Pediatrics, Keimyung University Dongsan Medical Center, ${ }^{2}$ Pediatrics, Yeungnam University Hospital, ${ }^{3}$ Pediatrics, Kyungpook National University Hospital, Daegu, ${ }^{4}$ Pediatrics, Busan National University Hospital, Busan, ${ }^{5}$ Pediatrics, Ulsan University Hospital, Ulsan, ${ }^{6}$ Pediatrics, Daegu Catholic University Hospital, Daegu, 7 Pediatrics, Gyeongsang National University Hospital, Jinju, ${ }^{8}$ Pediatrics, Inje University Haeundae-paik Hospital, ${ }^{9}$ Pediatrics, Dong-A University Hospital, ${ }^{10}$ Pediatrics, Inje University Busan-Paik Hospital, ${ }^{11}$ Pediatrics, Kosin University Gospel Hospital, Busan, Korea, Republic Of

Introduction: An alternative source, umbilical cord blood (UCB) is now prevalently used for hematopoietic stem cell transplantation (HSCT) in children with no available donor. We intended to compare the HSCT outcome between stem cell sources and demonstrate the usefulness of UCB in childhood acute myeloid leukemia (AML) by multi-center retrospective study.

Material (or patients) and methods: The AML children who received HSCT and are followed until now in Yeungnam region, South Korea from 2000 to 2015 were retrospectively reviewed. The 5-year probabilities of overall survival (OS) and event-free survival (EFS) were estimated by Kaplan-Meier methodology. Event was considered as leukemia relapse or death. Statistical analysis was performed by SPSS ver. 22 .

Results: There were a total of 75 AML children (male: female $=50: 25)$ who received HSCT. Age at diagnosis of AML was $9.2 \pm 5.4$ years. Among $75 \mathrm{AML}$ children, 15 patients $(20 \%)$ received bone marrow $(\mathrm{BM}), 48$ patients $(64 \%)$ received peripheral blood $(\mathrm{PB})$, and 12 patients $(16 \%)$ received $\mathrm{UCB}$. In HSCT using BM group, nucleated cells were $3.6 \pm 2.1 \times 10^{8} / \mathrm{kg}$ and CD34+ cells were $3.4 \pm 2.7 \times 10^{6} / \mathrm{kg}$. In HSCT using PB group, nucleated cells were $10.8 \pm 6.1 \times 10^{8} / \mathrm{kg}$ and CD34+ cells were $6.8 \pm 6.1 \times 10^{6} / \mathrm{kg}$. In HSCT using UCB group, nucleated cells were $6.0 \pm 2.7 \times 10^{7} / \mathrm{kg}$ and CD34+ cells were $2.8 \pm 2.4 \times 10^{5} / \mathrm{kg}$. HLA matches were as follows in UCB group: 1 patient in $8 / 8$ match, 2 patients in $6 / 8$ match, 1 patient in $6 / 6$ match, 5 patients in $5 / 6$ match, and 3 patients in $4 / 6$ match. By stem cell source, there was no statistical difference of 5 -year OS (BM 63.0\%, PB 68.5\%, and UCB 71.4\%, $P=0.783$ ). And there was no difference of 5 -year EFS (BM $60.0 \%$, PB $69.3 \%$, and UCB $75.0 \%, P=0.691)$.

Conclusion: An alternative donor source, UCB, didn't affect the transplantation outcome comparing $\mathrm{BM}$ or $\mathrm{PB}$ in this study. Thus, HSCT using UCB may be a suitable method to improve the treatment outcome of AML children lacking an available donor.

Disclosure of Interest: None declared.

\section{P669}

Volume Reduction and RBC Depletion of Apheresis Products for Pediatric Transplantation

Y. Cheng ${ }^{1, *}$, P. Bresnahan ${ }^{1}$, D. Sethi ${ }^{2}$, N. Bruque ${ }^{3}$, S. Wilcox ${ }^{3}$

${ }^{1}$ Marketing, ${ }^{2}$ Clinical Affairs, Cesca Therapeutics, Emeryville,

${ }^{3}$ Engineering, Cesca Therapeutics, Rancho Cordova, United States

Introduction: It is often desirable to volume-reduce apheresis product in order to deplete plasma from mobilized peripheral blood prior to transfusion, particularly in the cases of $A B O$ major mismatch. Current methods involve manual manipulation and concentration by centrifugation in non-closed systems, potentially compromising the safety of the final product. We have addressed this issue by developing a rapid, semi-automated protocol which is composed of a functionally closed system for rapid apheresis product processing with minimal manipulation and without additives.

Material (or patients) and methods: Apheresis product ( $>4.5 \times 10^{9}$ WBCs and $4 \%$ hematocrit) was subjected to density stratification by centrifugation without the use of density gradient media such as HES or Ficoll. We used a semiautomated platform which delivers the red blood cells (RBC), white blood cells (WBC), and plasma into separate sample chambers without user intervention within 60 minutes. The system was programmed to deliver the WBC fraction volume of $21 \mathrm{ml}$ into a freezing bag with $\sim 0.6 \pm 0.2 \mathrm{ml} \mathrm{RBC}$ contamination. The product sample was characterized for WBC count, viability, Neutrophil and Lymphocyte count. Our system offers a rapid ( 1hr protocol), simple, functionally closed system for producing transplant sample without additives.

Results: Processing of $50-200 \mathrm{ml}$ of apheresis product in our system produced a mean cell recovery of $75-85 \%$, with a RBC mean volume of $0.6 \pm 0.2 \mathrm{ml}$ volume in a $21 \mathrm{ml}$ product volume, and cell viabviability on average $>90 \%$.

Conclusion: Our rapid protocol for leukopak volume reduction enables processing and RBC depletion of apheresis samples within hours of harvesting, with excellent WBC recovery and viability.

Disclosure of Interest: Y. Cheng Funding from: Cesca Therapeutics, Employee of: Cesca Therapeutics, P. Bresnahan Employee of: Cesca Therapeutics, D. Sethi Employee of: Cesca Therapeutics, N. Bruque Employee of: Cesca Therapeutics, S. Wilcox Employee of: Cesca Therapeutics.

\section{P670}

HIV-1 Reservoir Dynamics after Allogeneic Stem Cell Transplantation: Clues to eradicate HIV

M. Kwon ${ }^{1, *}$, M. Salgado ${ }^{2}$, P. Balsalobre ${ }^{1}$, M. Nijhuis ${ }^{3}$, J. Blanco ${ }^{2}$, P. Miralles , D. Serrano ${ }^{1}$, J. Gayoso', J. Anguita ${ }^{1}$, I. Buno $^{1}$, A. M. Wensing ${ }^{3}$, J. Martinez Picado ${ }^{2}$, J. L. Diez Martin ${ }^{1}$ on behalf of EPISTEM Consortium

Hematology, Hospital Gral. Univ. Gregorio Marañon, Madrid, ${ }^{2}$ AIDS Research Institute irsiCaixa, Badalona, Spain, ${ }^{3}$ University Medical Center Utrecht, Utrecht, Netherlands, ${ }^{4}$ Infectious Diseases, Hospital Gral. Univ. Gregorio Marañon, Madrid, Spain

Introduction: To date, the Berlin patient's case provides the only evidence of long-term HIV-1 control after allogeneic stem cell transplantation (SCT), potentially due to the CCR5 $\Delta 32 / \Delta 32$ donor genotype. The case of the two Boston patients, without resulting in HIV cure, showed that the SCT procedure itself involves a tremendous reduction of the viral reservoir. The underlying mechanisms are unraveled yet. Herein we analyzed two patients with HIV infection from the European cohort EpiStem receiving different types of allogeneic SCT.

Material (or patients) and methods: Patient 1 (Pt1) and Patient 3 (Pt3) were transplanted in 2012 and 2013 in Hospital Gregorio Marañón due to Burkitt's NHL and NK-NHL, respectively. Analysis of the HIV-1 reservoir was performed 29 (Pt1) and 20 (Pt3) months post-transplant using qVOA, ddPCR, and semiquantitative PCR in CD4 $T$ cells from peripheral blood and ileum, and CD3+ cells from bone marrow (BM). SCA was performed in plasma and CSF. Chimerism was performed by short tandem repeat PCR (STR$P(R)$. Antibody titers were determined by ELISA. Activation markers (CD38 and HLA-DR) were determined in CD4 and CD8 T-cells.

Results: Pt 1 received a myeloablative single cord blood SCT, supported with third party HLA-mismatched CD34+ cells (haplo-cord SCT). Pt 3 underwent reduced intensity conditioning SCT with peripheral blood progenitor cells (PBPC) of an HLA-matched sibling. Both patients were kept on CART. Donors were wild type for CCR5. Pt 3 reached full chimera 1 month after SCT, while Pt 1 still harbors $0.2 \%$ of host cell in BM and $0.1 \%$ in PB. Pt 3 developed chronic GvHD but not Pt 1. CD4 T cells from both patients were susceptible to in vitro R5 

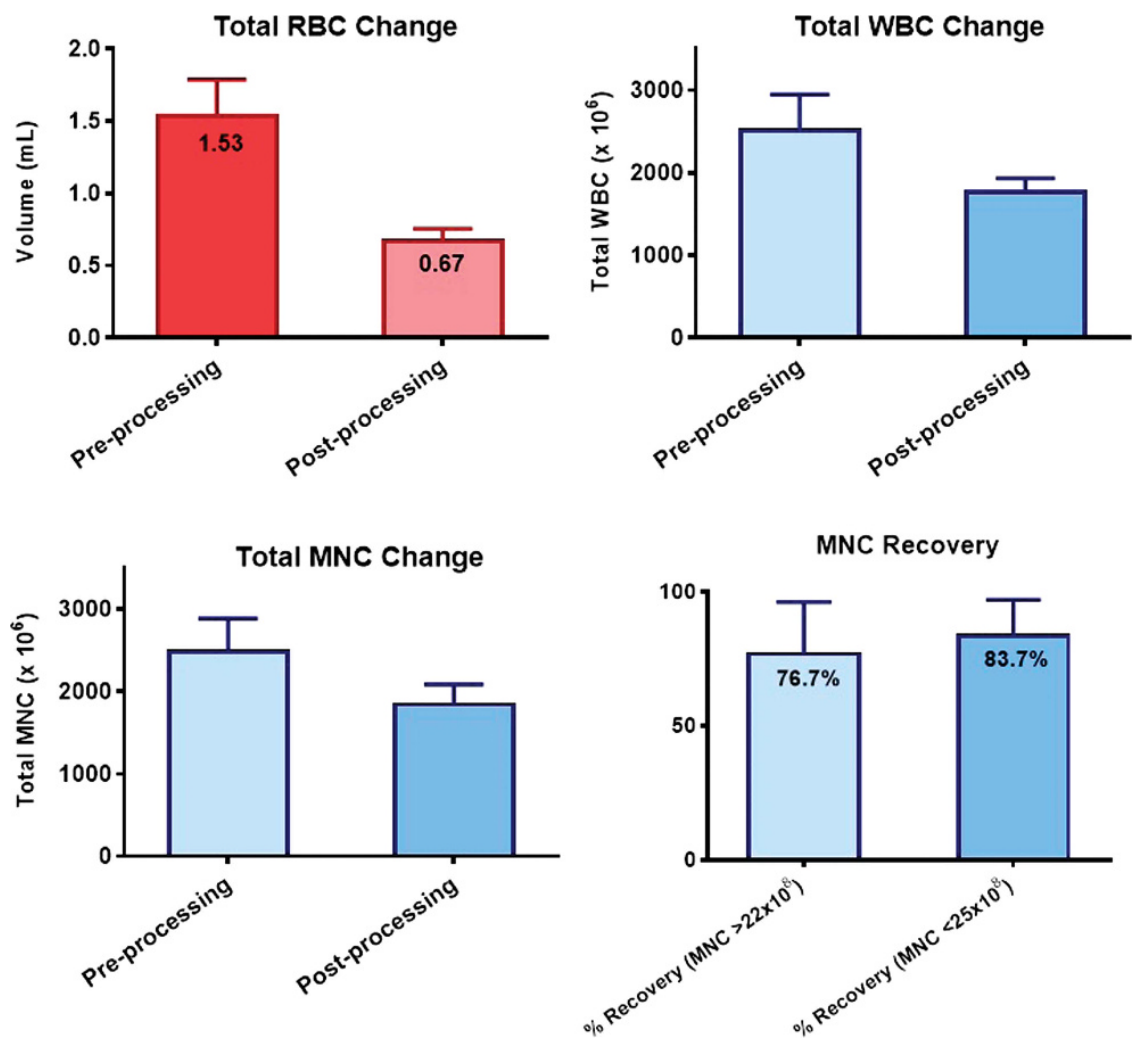

and X4-tropic HIV infection. IUPM in Pt 1 were 0.034 but undetectable (IUPM $<0.006$ ) in Pt3. Total DNA in peripheral CD4 were 25 copies/106cells in Pt1, with 5 HIV RNA copies/ml in plasma. On the contrary, virus is not detectable in peripheral $\mathrm{CD} 4$, ileum CD4, BM, plasma or CSF in Pt3. Although both patients had a reduction in HIV-specific antibody titers, Pt 3 showd lower levels. CD4 and CD8 activation levels were lower in Pt 3 than Pt 1.

Conclusion: Within the EPISTEM cohort, we compared two different SCT procedures, with different outcome regarding viral reservoir. Allogeneic SCT with PBPC of an HLA-matched sibling donor, with a rapid achievement of full donor chimera and the development of chronic GvHD resulted in a more drastic reduction of the latent reservoir down to undetectable levels. We hypothesize that the "graft versus HIV-1 reservoir effect" contributes to facilitate the clearance of the viral reservoir.

References: Hutter et al. N Engl J Med. 2009;360(7):692-8.

Henrich et al. J Infect Dis. 2013;207(11):1694-702.

Disclosure of Interest: None declared.

\section{P671}

Atorvastatin Improves Functions of Bone Marrow Endothelial Progenitor Cells in Poor Graft Function Patients

M.-M. Shi ${ }^{1,2, *}$, Y. Kong ${ }^{1}$, X.-J. Huang ${ }^{1,2}$

${ }^{1}$ Peking University People's Hospital, Peking University Institute of Hematology, Beijing Key Laboratory of Hematopoietic Stem Cell Transplantation, Collaborative Innovation Center of Hematology, ${ }^{2}$ Peking-Tsinghua Center for Life Sciences, Academy for Advanced Interdisciplinary Studies, Peking University, Beijing, China

Introduction: Poor graft function (PGF) is a serious complication after allogeneic hematopoietic stem cell transplantation (allo-HSCT). Murine studies suggested that endothelial progenitor cells (EPCs) are preferential supporting cells for HSCs in

the bone marrow (BM) microenvironment[1]. Our case control study showed that lower frequency of EPCs was the independent risk factor of PGF[2]. Atorvastatin was found to improve the EPCs mobilization and functions in heart disease patients[3]. In this study, we speculated that the occurrence of PGF is most likely associated with the dysfunction of EPCs, and we also investigated whether Atorvastatin can enhance the number and functions of cultured BM EPCs in PGF patients.

Material (or patients) and methods: The 15 patients with PGF, 15 matched patients with good graft function (GGF), and 15 healthy donors(HDs) were enrolled. The BM EPCs in PGF patients were cultured for 7 days and treated with Atorvastatin $(0.5 \mathrm{nM}, 5 \mathrm{nM}, 500 \mathrm{nM})$. The number and functions of CD34(+)/CD133(+)/KDR(+)EPCs were evaluated by flow cytometry, cell counting, Dil-Ac-LDL and FITC-lectin-UEA-1 double staining, migration and tube formation test. Reactive Oxygen Species (ROS) level was evaluated by flow cytometry and DCFH-DA staining. Cell proliferation was determined by cell counting kit-8 test.

Results: The functions of BM EPCs in PGF patients were impaired comparing to GGF patients and HDs, including decreased Dil-Ac-LDL and FITC-lectin-UEA-1 double positive staining cells, lower tube formation capability, less migration capacity and higher the ROS level. Treatment of $500 \mathrm{nM}$ Atorvastatin to the cultured BM EPCs in PGF patients significantly enhanced their number and proliferation rate comparing to PGF control group. The tube formation capability, cell migration and Dil-Ac-LDL and FITC-lectinUEA-1 double positive staining cells were also increased after $500 \mathrm{nM}$ Atorvastatin treatment. The ROS level in BM EPCs of PGF patients was significantly decreased in $500 \mathrm{nM}$ Atorvastatin group on day 7 in culture.

Conclusion: The functions of BM EPCs in PGF patients were impaired compared to GGF patients and healthy donors. Atorvastatin enhanced the number and functions of cultured BM EPCs in PGF patients, indicating Atovastatin may have 
therapeutic effect for PGF patients by improving the EPCs' number and functions in BM microenvironment.

References: 1. Calvi LM, Adams GB, Weibrecht KW, Weber JM, Olson DP, Knight MC, Martin RP, Schipani $E$, Divieti $P$, Bringhurst FR et al: Osteoblastic cells regulate the haematopoietic stem cell niche. Nature 2003, 425(6960):841-846.

2. Kong Y, Chang YJ, Wang YZ, Chen YH, Han W, Wang Y, Sun YQ, Yan CH, Wang FR, Liu YR et al: Association of an impaired bone marrow microenvironment with secondary poor graft function after allogeneic hematopoietic stem cell transplantation. Biology of blood and marrow transplantation: journal of the American Society for Blood and Marrow Transplantation 2013, 19(10):1465-1473.

3. Oikonomou E, Siasos G, Zaromitidou M, Hatzis G, Mourouzis K, Chrysohoou C, Zisimos K, Mazaris S, Tourikis P, Athanasiou D et al: Atorvastatin treatment improves endothelial function through endothelial progenitor cells mobilization in ischemic heart failure patients. Atherosclerosis 2015, 238(2):159-164.

Disclosure of Interest: None declared.

\section{P672}

vascular and perivascular niches but not endosteal niche restoration in patients with aplastic anemia after allogeneic hematopoietic stem cell transplantation by dynamic observation

L. Wu ${ }^{1}$, S. Wang ${ }^{1, *}$ on behalf of Senior Author, W. Mo on behalf of Presenting Author, Y. Zhang ${ }^{1}$ on behalf of Guangzhou Medical University

${ }^{1}$ Department of Hematology, Guangzhou First People's Hospital, Guangzhou, China

Introduction: It remains unclear whether restoration of the bone marrow (BM) niches in patients with $A A$ after allogenic hematopoietic stem cell transplantation (allo-HSCT). To investigate the changes of BM niches, we dynamic observed 24 patients with AA who had undergone allo-HSCT.

Material (or patients) and methods: 24 patients with AA who received allo-HSCT in our center. All patients had trephine BM biopsies available before allo-HSCT and at 1,2,3,6 and 12 months after allo-HSCT. Hematoxylin eosin staining and immunohistochemistry with anti-osteopontin, anti-CD34, and anti-CD146 were performed on each slide, as our previously described ${ }^{1}$. For evaluation, each slide was first scanned at $100 \times$ magnification to determine three "hot spots" that had a high number of positive cells. Each of the hot spots was consequently detected at $400 \times$ and the target cells were recorded. The mean number of cells was counted. The number of endosteal, vascular, and perivascular cells were assessed as reported before ${ }^{1}$. A total of 15 patients in whom BM biopsy was normal on histopathological examination, were enrolled as controls.

Results: Patients with AA showed markedly fewer vascular cells $(7.30 \pm 3.51$ vs $12.67 \pm 3.85$ high-power field (hpf); $P<0.05)$, perivascular cells $(6.68 \pm 3.59$ vs $10.67 \pm 5.34$ per hpf; $P<0.05)$ and endosteal cells $[0.90 \pm 1.85$ vs $3.67 \pm 1.18$ per; $P<0.05]$ compared with controls before allo-HSCT. At +1 month post alloHSCT, vascular cells $(12.75 \pm 3.94$ vs $7.30 \pm 3.51$ per hpf; $P<0.05)$

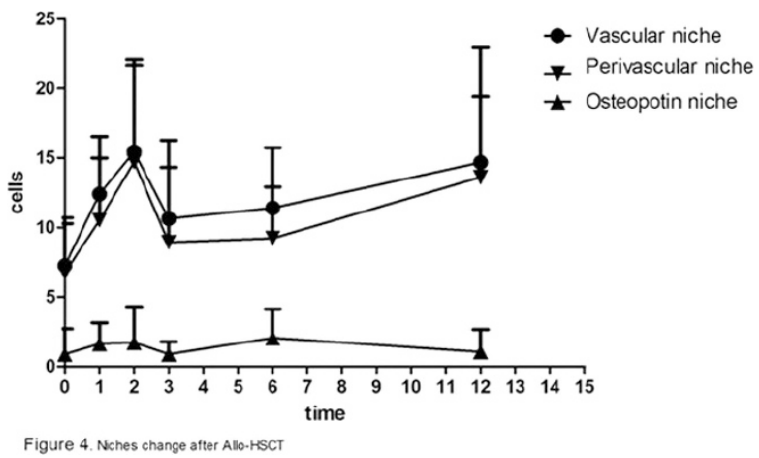

Figure 4. Nches change after Allo-HSCT and perivascular cells $(10.84 \pm 4.65$ vs $6.68 \pm 3.59$ per hpf; $P<0.05)$ exhibited remarkable increase compared with pretransplantation. In addition, no significant difference in the levels of vascular cells $(12.75 \pm 3.94$ vs $12.67 \pm 3.85$ per hpf; $P>0.05)$ and perivascular cells $(10.84 \pm 4.65$ vs $10.67 \pm 5.34$ per hpf; $P>0.05)$ compared with the control group. The count of endosteal cells $(1.69 \pm 1.56$ vs $0.90 \pm 1.85$ per hpf) was elevated after allo-HSCT. However, there was no significant difference $(P>0.05)$ before and after transplantation. At +2 month after allo-HSCT, the number of vascular cells and perivascular cells actually peaked were $15.67 \pm 6.81$ and $14.55 \pm 7.19$, respectively. Also reached peak in the endosteal cells $(1.79 \pm 2.66)$, however, there was still no significant difference $(P>0.05)$ before and after transplantation. At $+3,+6,+12$ month after allo-HSCT, the levels of aforementioned cells increased compared with +2 month. Moreover, except the endosteal cells, there was significant difference in vascular cells and perivascular cells compared with before allo-HSCT.

Conclusion: In conclusion, these data indicate that vascular and perivascular niches been repaired and endosteal niche remains numerical impairment in patients with AA after alloHSCT.

References: 1. Wu L, Mo W, Zhang Y, Deng H, Li Y, Zhou R et al. Impairment of hematopoietic stem cell niches in patients with aplastic anemia. Int J Hematol 2015; 102: 645-653.

Disclosure of Interest: None declared.

\section{P673}

Reactive oxygen species-induced apoptosis contributes to exhaustion of CD34-positive bone marrow cells in poor graft function after allotransplants

Y. Kong ${ }^{1, *}, Y$. Song ${ }^{1,2}$, X.-J. Huang ${ }^{1,2}$

${ }^{1}$ Peking University Peoples Hospital, Peking University Institute of Hematology, Beijing Key Laboratory of HSCT, ${ }^{2}$ Peking-Tsinghua Center for Life Sciences, Academy for Advanced Interdisciplinary Studies, Peking University, Beijing, China

Introduction: Poor graft function (PGF), which was defined as the presence of the following 2-3 cytopenic counts: neutrophils $\leq 0.5 \times 10 \mathrm{E}+9 / \mathrm{L}$, platelets $\leq 20 \times 10 \mathrm{E}+9 / \mathrm{L}$, and/or hemoglobin concentration $\leq 70 \mathrm{~g} / \mathrm{L}$ for $\geq 3$ consecutive days beyond day +28 post-transplant or in accordance with platelet and/or RBC transfusion and/or G-CSF support requirements with complete donor chimerism, is a serious complication after allogeneic hematopoietic cell transplants for hematologic neoplasms. However, the mechanisms underlying PGF are unclear. In mice, effective cross-talk between hematopoietic stem cells (HSCs) and the bone marrow (BM) microenvironment is important for normal hematopoiesis. Normal HSCs reside in a hypoxic BM microenvironment protecting them from oxidative stress, which would otherwise inhibit their selfrenewal resulting in exhaustion and BM failure. We recently reported an impaired $B M$ endosteal and vascular microenvironment in persons with PGF posttransplant. However, it is unknown whether defective donor HSCs or an abnormal BM microenvironment is causal. Thus, the aim of the study is to determine whether quantitative and/or functional abnormalities donor $\mathrm{CD}_{3}{ }^{+}$cells result in PGF posttransplant.

Material (or patients) and methods: The frequency, intracellular reactive oxygen species (ROS) levels, apoptosis, cellcycle state and colony-forming capacity of donor $\mathrm{CD} 34^{+} \mathrm{BM}$ cells were quantified in subjects with PGF and good graft function (GGF) posttransplant. To study the effect of oxidative stress on posttransplant hematopoiesis, $\mathrm{CD} 34^{+}$cells from subjects with GGF were treated of $\mathrm{H}_{2} \mathrm{O}_{2}$ with and without $\mathrm{N}$-acetyl-L-cysteine (NAC). Total RNA was extracted from the $\mathrm{CD}_{4}{ }^{+}$cells using an RNA easy micro kit. The Bcl-2, Bcl-xl, Bax, Puma, Caspase 3 and GAPDH genes were evaluated by quantitative reverse-transcription (qRT)-PCR. The expression of intracellular proteins, including $\mathrm{Y}-\mathrm{H} 2 \mathrm{AX}, \mathrm{p} 53$, phospho-p53, and p21 were analyzed by flow cytometry.

Results: The frequency of $\mathrm{CD} 34^{+} \mathrm{BM}$ cells and colony-forming capacity were decreased in subjects with PGF posttransplant. 
Significantly increased ROS levels, higher percentages of $\mathrm{CD}^{2} 4^{+} \mathrm{\gamma}-\mathrm{H} 2 \mathrm{AX}^{+}$cells with DNA double-strand breaks were observed in $\mathrm{CD}_{3} 4^{+}$cells, especially in the quiescent $\mathrm{CD} 34^{+}$ $\mathrm{CD}^{\text {low }}$ fraction, from subjects with PGF. This resulted in exhaustion of quiescent $\mathrm{CD} 34^{+}$cells, which could be partially reversed by treatment with NAC. Moreover, the mRNA levels of anti-apoptotic $\mathrm{BCl}-2$ and $\mathrm{BCl}-\mathrm{xl}$ were lower in $\mathrm{CD}_{3} 4^{+}$cells of PGF, whereas those of pro-apoptotic Bax, Puma and Caspase 3 were significantly higher than those in GGF. Furthermore, markedly increased levels of p53, phospho-p53 and p21 were observed in $\mathrm{CD}_{34}{ }^{+}$cells of PGF, indicating that p53-dependent apoptosis may contribute to ROS-induced exhaustion of CD34 ${ }^{+}$BM cells in subjects with PGF after allotransplants.

Conclusion: Increased levels of ROS and exhaustion of quiescent $\mathrm{CD}_{34}^{+} \mathrm{BM}$ cells may operate in PGF, implicating an abnormal BM microenvironment rather than defective HSCs as the aetiology of PGF posttransplant.

References: 1. Kong $\mathrm{Y}_{-}^{\#}, \ldots$, Huang $\mathrm{XJ}^{*}$. Biol Blood Marrow Transplant. 2013;19(10):1465-1473.

2. Kong $\mathrm{Y}^{\#}, \ldots$, Huang $\mathrm{XJ}^{*}$. Bone Marrow Transplant. doi:10.1038/bmt.2015.229.

Disclosure of Interest: None declared.

\section{P674}

Immunemodulation of AML-blasts with clinically approved response modifiers improves antileukemic T-cell reactivity ex vivo and leads to blast-reduction in vivo (rat-Modell)

M. Inngjerdingen ${ }^{1}$ on behalf of schmetzer, A. Rabe ${ }^{2}, D$. Deen ${ }^{2}$ Z. Stankova ${ }^{2}$, A. Hirn' ${ }^{2}$, J. Tischer', C. Schmid ${ }^{3}$, H. Schmetzer ${ }^{2, *}$

${ }^{1}$ Dept Immunology, Oslo, Norway, ${ }^{2}$ DEPT OF HEMATOPOETIC TRANSPLANTATIONS, MED DEPT 3, UNIVERSITY HOSPITAL MUNICH, Munich, ${ }^{3}$ Dept of Hematopoetic transplantations, Municipal Hospital Augsburg, Augsburg, Germany

Introduction: Antileukemic T-cell-reactivity has to be improved in AML-pts. Leukemia-derived $D C\left(D_{\text {leu }}\right)$, converted from AML-blasts are most effective antileukemic T-cellstimulators.

Material (or patients) and methods: We tested 11 IMMUNEMODULATORY KITS, combinations of 2 clinically approved compounds (GMCSF+ INTRON/ CALCIMYCIN/ PICIBANIL (HS-I)/ PROSTAGLANDIN E1 (HS-M)/-2 (HS-K)/ TNFa) to convert blasts into a 'DC $\mathrm{leu}_{\text {lev }}$-vaccine', that induces antileukemic T-cell(memory) without induction of blast proliferation in a Whole Blood ('WB') culture system with human as well as rat AML-samples. Moreover we treated leukemically diseased rats with 3 best KITS (HS-I, HS-K, HS-M) and evaluated safety as well as efficacy compared to untreated rats.

Results: 1 . Generation of $D C / D C_{\text {leu }}$ from WB and ranking of KITS: 3 KITS were selected as' best kits'; 3 KITS were not successful to generate $\mathrm{DC} / \mathrm{DC}_{\mathrm{leu}}$ from human as well as ratAML-WB. On average $20(22) \%$ DC and $14(22) \%$ DC leu $_{\text {could be }}$ generated from human (rat)WB with Kits HS-I,K and $M$, although individual variations occured 2. Exceptionally KITS induced ex vivo blast proliferation in few individual AML-pts WB. 3. Antileukemic reactivity of human (rat)T-cells stimulated with KIT-treated WB was regularly 20 (29)-fold improved compared to controls. 4. Blast lysis correlated with proportions of DC-subtypes (DC, DC $C_{\text {leu }}, \mathrm{CD}^{+}$Tcells after mixed lymphocyte culture and concentrations of IL-12, IFNg, IL-6 and -8 in culture supernatants. 5 . Kit-treatment in vivo of rats diseased with AML showed a (highly significant) reduction of blasts with KIT 'HS-M' (0.03/0.0001 in spleen/blood) and tendentially with 'HS-I' in only 9 days. Successful KIT treatment correlated with an increase of CD62L+ probably Tmem Tcells in the spleen $(P=0.07)$ and in addition a reduction of CD4+Treg $(P=0.037)$. Interestingly a reduction of blasts correlated with an increase of CD62L+ Tcells.

Conclusion: KITs can create $\mathrm{DC}_{\text {leu }}$ from blasts in WB cultures without induction of blast proliferation, but regularly result in the generation/activation of antileukemic T-cells after coculture. AML-blasts are reduced in vivo in rats after KITtreatment. A ranking based on $\mathrm{DC}, \mathrm{DC}_{\text {leu }}$ subtypes, antileukemic reactivity and cytokine profiles should be created to allow an individualised selection of ,best $\mathrm{KIT}^{\prime}$ for in vivo applications in man.

Disclosure of Interest: Patent pending, None declared.

P675

The Outcome of AML and High-grade MDS Relapsed after Allogeneic Transplantation, Single Centre Evaluation

M. Marková ${ }^{1, *}$, V. Valkova ${ }^{1}$, J. Vydra ${ }^{1}$, L. Novakova ${ }^{1}$, M. Kolar ${ }^{7}$, P. Cetkovsky ${ }^{1}$, A. Vitek ${ }^{1}$

${ }^{1}$ Institute o Hematology and Blood Transfusion, Prague, Czech Republic, Praha 2, Czech Republic

Introduction: Although allogeneic transplantation is a substantial step in intermediate and higher risk AML treatment and actually the only curative approach in MDS treatment, post-transplant relapse remains a major complication leading to treatment failure.

Material (or patients) and methods: A retrospective survival evaluation of post-transplant relapsed AML $(n=77)$ and high grade MDS $(n=33)$ patients after allogeneic transplantation between the years 1989 and 2015 was done. The relapse treatment approach was decided individually with the respect to patient's physical status and will. For the evaluation purpose it was divided to 3 groups $-A$ ) no treatment except of immunosuppression tapering $(n=25)$, B) "low intensity" treatment including low dose ARA-C, 5-azacytidine with DLI or DLI only $(n=28)$ and C) "high intensity" treatment including regimens $3+7$, or other intensive regimens with high dose ARA-C with DLI or second transplants $(n=58)$.

Results: The overall survival in 1, 2 and 5 years was $31 \%, 16 \%$ and $12 \%$. There was an expected significant difference between not treated group $(10 \%, 1 \%$, and $1 \%)$ and treated group with no significant further difference between low intensity and high intensity groups (34\%, $21 \%$ and $18 \%)$. The median time of relapse in AML and MDS was 5, resp. 12 months from transplant, though not significant. Patients who relapsed earlier from transplant did worse in 6 and 12 months, but the difference disappeared in 24 months of relapse treatment. There was no difference between MDS and AML survival outcome and remission achievement as well. Not surprisingly patients who had achieved complete remission had significant OS advantage ( $70 \%$ vs $6 \%$ in 1 year and $46 \%$ vs $1 \%$ in 2 years). There was no survival advantage in treated patients without remission achievement to untreated patients at all, though among untreated patients there was one $(1 / 25)$ fully responding to immunosuppression tapering. $47 \%$ of patients with remission achievement relapsed again. GVHD was a major contributor to death in $22 \%$ patients in remission. Conclusion: The prognosis of patients relapsing after transplant is very poor despite various treatment approaches. Those who succeeded to achieve a further remission benefited from the treatment, while it seems that the treatment did not prolong OS in non-responders. Further emphasis to prophylactic and preemptive approaches with tight residual disease monitoring could be one way to improvement.

Disclosure of Interest: None declared.

\section{P676}

Hematopoietic Cell Transplantation including Autologous Transplantation leads to superior Free Disease Survival in young Adults with $\mathbf{t}(8 ; 21) \mathrm{AML}$

M. Shaheen ${ }^{1, *}$, S. O. Ahmed ${ }^{1}$, W. Rasheed ${ }^{1}$, S. Y. Mohamed ${ }^{1}$ N. Chaudhri', F. Alsharif', H. Alzahrani ${ }^{1}$, G. AL Dawsari ${ }^{\prime}$ A. Hanbali', F. Alfraih ${ }^{1}$, R. El Fakih ${ }^{1}$, S. Ahmed ', W. Chebbo', G. El Gohary ${ }^{1}$, M. H. Almahayni ${ }^{1}$, O. Ali ${ }^{1}$, A. Mannan ${ }^{\prime}$, M. Alsermani ${ }^{1}$, F. Anjum ${ }^{1}$, M. Hassanein ${ }^{1}$, M. Aljurf ${ }^{1}$, F. Almohareb ${ }^{1}$

${ }^{7}$ Adult Hematology \& Hematopoietic Cell Transplantation, King Faisal Specialist Hospital \& Research Centre, Riyadh, Saudi Arabia 


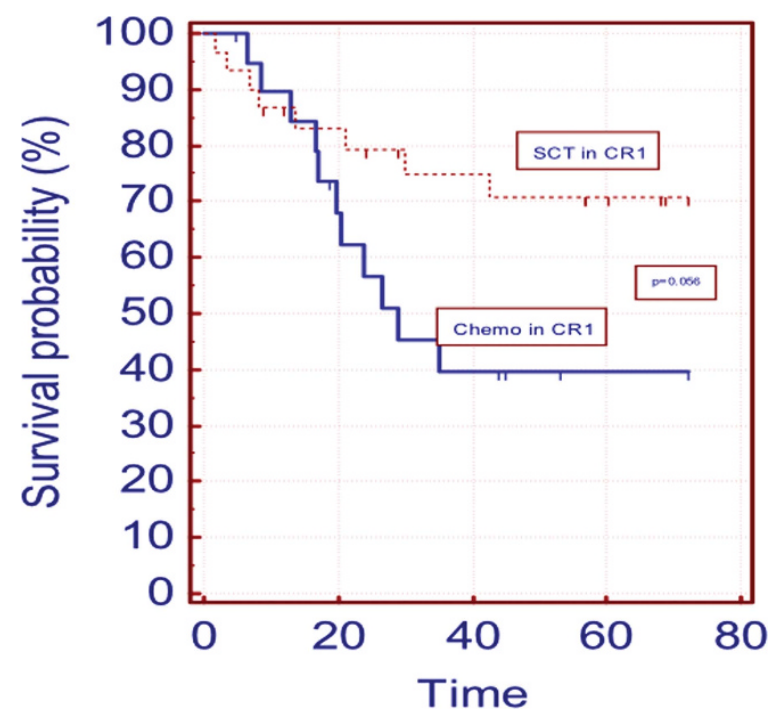

Introduction: AML $\mathrm{t}(8 ; 21)$ is considered good risk category, and the standard recommendation is for patient's to receive 3 -4 cycles of high dose Ara-C consolidation. A previous NCCN recommendation of subjecting these patients at an autologous stem cell transplant (ASCT) has been withdrawn in 2015. Here we report on our outcomes of a population of mainly young patients who were treated in our center.

Material (or patients) and methods: A retrospective analysis was carried out of the institutional database for patient, disease characteristics and outcome was correlated to post remission therapy, either chemotherapy alone, or a form of transplantation in CR1.

Results: We identified 51 patients who were wholly treated at our center. Induction was with $3+7$ with or without etoposide as a $3^{\text {rd }}$ agent; 45 patients $(88 \%)$ achiever a complete remission. Six patients either were refractory or had disease progression before they were able to receive post-remission therapy. Twenty patients received post remission chemotherapy with sequential high dose Ara-C (HiDAC); 31 patients received a form of transplantation in $1^{\text {st }}$ CR: 10 patients received an autograft and 21 received an allogeneic SCT (AlloSCT). The overall survival was superior in the cohort of patients who received a HCT: $70 \% 5 \mathrm{yr}$ OS in the HCT group vs $40 \%$ in the chemotherapy group. Relapse rates were significantly higher in the chemotherapy group.

Conclusion: In our cohort of young adults, HCT was associated with a superior relapse free survival. ASCT had the best outcomes though the numbers were too small for a statistically significant improvement over other therapies. Where the risk of treatment related mortality is low, a transplant, especially ASCT, should still be an option.

Disclosure of Interest: None declared.

\section{P677}

Outcome of allogeneic hematopoietic stem cell transplantation in normal karyotype AML with NPM1 mutation in complete remission is not affected by FLT3/ITD positivity - potentially crucial importance of pre-transplant level of minimal residual disease

M. Karas ${ }^{1, *}$, K. Steinerová ${ }^{1}$, D. Lysák ${ }^{1}$, M. Hrabětová ${ }^{1}$, A. Jungová ${ }^{1}$, M. Pachner ${ }^{1}$, P. Jindra ${ }^{1}$

${ }^{1}$ Hematology and Oncology dpt., CHARLES UNIVERSITY HOSPITAL PILSEN, Pilsen, Czech Republic

Introduction: Background: detection of FLT3/ITD in normal karyotype (NK) AML even in case of NPM1 mutation affects negatively prognosis of $A M L$ patients (pts) treated with intensive chemotherapy alone. But impact of FLT3/ITD positivity in the setting of allogeneic hematopoietic stem cell transplantation (alloHSCT) in pts with NK AML with NPM1 mutation in complete remission (CR) is less clear. Aims: with the aim to evaluate the potential role of FLT3/ITD positivity in NK AML with NPM1 mutation on the outcome of alloSCT we compared alloHSCT results in pts with NK AML with NPM1 mutation in CR with alloHSCT results of pts with NK AML in CR with NPM1 mutation and concurrent FLT3/ITD positivity transplanted in our centre from 2005. We also tried to identify the impact of other known pre-transplant prognostic factors on alloHSCT outcome in this cohort of pts.

Material (or patients) and methods: from 2/2005 to 9/2014 60 consecutive pts with median of age 54 years (range, 30-66 years) with normal normal karyotype AML harboring NPM1 mutations - 53\% FLT3/ITD positivity - in $1^{\text {st }} \mathrm{CR}$ (45 pts) and $2^{\text {nd }}$ CR (15pts) underwent myeloablative (16 pts) or reducedintensity (44 pts) alloHSCT (27\% HLA identical related, 50\% HLA matched unrelated, $23 \%$ HLA mismatched unrelated). Source of stem cells was in $80 \%$ peripheral blood and in $20 \%$ bone marrow. Pre-transplant prognostic factors (age, type of donor, donor recipient sex combination, CMV status, type of conditioning regimen, source of stem cells, number of CR, FLT3/ITD status and MRD level before alloHSCT - determined using quantitative real-time polymerase chain reaction for detection of NPM1 mutations) were included in the univariate and multivariate statistical analysis.

Results: all pts fully engrafted with ongoing CR after alloHSCT. 36 pts (60\%) developed aGVHD and 24 pts (40\%) developed chGVHD. With median follow-up 52 months (range, 4-101 months) 35 pts (58\%) are alive. 16 pts (27\%) relapsed and 13 of them died. 12 pts $(20 \%)$ died due to NRM. The estimated probabilities of 3-years EFS and OS are 54\% and 59\%. Statistical analysis showed that only age over 63 years and MRD level affected alloHSCT outcome in univariate and multivariate analysis. FLT3/ITD positivity did not influence alloSCT results. Pre-transplant MRD level 10 NPM1mut/10000 $\mathrm{ABL}$ copies had the strongest statistical significance. The estimated probabilities of 3-years relapse incidence, EFS and OS were for pts with low MRD level ( $\leq 10$ NPM1mut copies) $6 \%, 72 \%$ and $75 \%$ and for pts with high MRD level $(>10$ NPM1mut copies) $48 \%, 35 \%$ and $40 \%$.

Conclusion: our data show that FLT3/ITD positivity did not influence alloSCT results in pts with NK AML with NPM1 mutation in CR and also show that level of MRD in pts with normal karyotype AML harboring NPM1 mutation in CR provides important prognostic information, which as an independent prognostic factor predicts transplant results.

References: 1 . ayne, Radich JP. Pretransplant MRD: the light is yellow, not red. Blood. 2012;120:244-245.

2. Walter RB, et al. Significance of minimal residual disease before myeloablative allogeneic hematopoietic cell transplantation for AML in first and second complete remission. Blood.2013;122:1813-1821.

Disclosure of Interest: None declared.

\section{P678}

Reduced-intensity transplantation as a part of standard treatment strategy in patients aged 60 to 70 years with acute myeloid leukemia - single centre experience

M. Karas ${ }^{1, *}$, K. Steinerova ${ }^{1}$, D. Lysák ${ }^{1}$, M. Hrabetova ${ }^{1}$, A. Jungova ${ }^{7}$, P. Jindra ${ }^{1}$

${ }^{7}$ Hematology and Oncology dpt., CHARLES UNIVERSITY HOSPITAL PILSEN, Pilsen, Czech Republic

Introduction: outcome of patients (pts) over 60 years of age with acute myeloid leukemia (AML) treated only with intensive chemotherapy is poor. To improve treatment results reducedintensity transplantation (RIT) was establish as a part of standard treatment strategy for pts aged 60 to70 years with AML (except of pts with prognostically favourable $A M L$ ) in our centre from 2003. With the aim to evaluate transplant feasibility and the role of RIT in the treatment of pts aged 60 to 70 years with AML we analysed outcome of such pts in our centre from 2003. 
Material (or patients) and methods: from 1/2003 to 11/2014 AML was diagnosed in 188 pts aged 60 to 70 years. 120 pts were intensively treated and 61 pts with median of age 63 years (range, 60-68 years) with AML in $1^{\text {st }} C R\left(35\right.$ pts), in $2^{\text {nd }} C R$ (5pts), with primarily resistant AML (9 pts) and with $A M L$ beyond CR (12 pts) underwent RIT (30\% HLA identical related, $41 \%$ HLA matched unrelated, $26 \%$ HLA mismatched unrelated, $3 \%$ haploidentical related). Source of stem cells was in $85 \%$ peripheral blood and in $15 \%$ bone marrow with the median of infused CD $34+$ cells $4,8 \times 10^{\wedge} 6 / \mathrm{kg}$ (range, $1,7-14,9 \times 10^{\wedge} 6 / \mathrm{kg}$ ). The conditioning regimen consisted of fludarabine $(30 \mathrm{mg} / \mathrm{m} 2$ for 4 days) and melphalan ( $140 \mathrm{mg} / \mathrm{m} 2$ for 1 day), in 14 pts with ATG (ATG Fresenius $15 \mathrm{mg} / \mathrm{kg}$ ). CsA and methotrexate were used as GVHD prophylaxis except haploidentical RIT where posttransplant CPA and combination of CsA and MMF was used.

Results: the main reasons of impossibility to implement RIT in treatment of older pts with AML were death during remission induction treatment (39\%), resistant or progressive AML (24\%) non-availability of donor (16\%), severe comorbidities (12\%) and refusal of RIT (9\%). However $51 \%$ of intensively treated pts with AML eventually underwent RIT. All pts fully engrafted and achieved complete remission (CR). 37 pts (60\%) developed aGVHD ( 6 pts grade III-IV) and among 50 evaluable pts 22 (44\%) of them developed chGVHD (9 limited, 13 extensive). With median follow-up 46 months (range, 4-123 months) 27 pts (44\%) are alive (26 pts in CR). 14 pts (23\%) relapsed and 13 of them died. 21 pts (34\%) died due to NRM, 2 (3\%) of them till day 100 after RIT and 11 (18\%) of them till day 365 after RIT. The estimated probabilities of 3-years EFS and OS are $44 \%$ and $47 \%$. 3-years OS for all intensively treated elderly pts was 38\%. Conclusion: our data show that half of intensively treated pts aged 60 to 70 years with AML were able to undergo RIT and that RIT even in case of unrelated or HLA mismatched donor is associated with acceptable NRM and encouraging disease control of unfavourable AML (3-years OS 47\%). The relatively high percentage of transplanted pts undoubtedly influenced the overall results of the group of intensively treated elderly patients with AML with a median survival of 15 months and estimated 3-year OS of $38 \%$. RIT should be consider as standard treatment option for consolidation therapy in elderly patients with AML

References: 1) Julliusson $G$ et al. Age and acute myeloid leukemia: real world data on decision to treat and outcomes from Swedish Acute Leukemia Registry.Blood.2009.

2) Estey E et al. Prospective feasibility analysis of reducedintensity conditioning regiment for hemopoietic stem cell transplantation (HSCT) in elderly patiens with acute myeloid leukemia (AML) and high-risk myelodysplastic syndrome (MDS).Blood.2007.

Disclosure of Interest: None declared.

\section{P679}

Improved outcome of children transplanted for high-risk leukemia by using goal-oriented monitoring of cyclosporine without methotrexate or mycophenolate

N. Bleyzac ${ }^{1, *}$, D. Cuzzubbo ${ }^{2}$, C. Rénard ${ }^{2}$, N. Garnier ${ }^{3}$, V. Dubois ${ }^{4}$ C. Domenech, M.P. Goutagny', A. Plesa , N. Grardel ${ }^{2}$ S. Goutelle ${ }^{7}, A$. Janoly-Duménil', Y. Bertrand ${ }^{2}$

${ }^{1}$ Hematology, ${ }^{2}$ Institute of Pediatric Hematology and Oncology, ${ }^{3}$ Institute of Pediatrice Hematology and Oncology, ${ }^{4}$ Etablissement Français du sang, ${ }^{5}$ Lyon-Sud hospital, Lyon, ${ }^{6} \mathrm{CHU}$ Lille, Lille, ${ }^{7}$ Université Lyon I, Lyon, France

Introduction: There is currently a major concern regarding the optimal immunosuppression therapy to be administered after hematopoietic stem cell transplantation (HSCT) to reduce both the toxicity of graft-versus-host disease (GvHD) and the rate of relapses. Cyclosporine is usually associated to methotrexate or mycophenolate in GvHD prophylaxis regimens, despite their inhibitory effect on NK cells compromising graft-versusleukemia (GvL) effect. We report the outcome of high-risk leukemia children transplanted with a new way of managing cyclosporine-based GvHD prophylaxis, without using methotrexate or mycophenolate.

Material (or patients) and methods: A total of $110 \mathrm{HSCT}$ in 109 ALL or AML children in CR1 to 3 (47 sibling and 63 unrelated donors) who received 2 daily 2 -hour infusions of cyclosporine alone (without mycophenolate or methotrexate) in matched related as well as in matched or mismatched unrelated stem cell transplantation were included. Cyclosporine dosage regimens were individualized to obtain specific trough blood concentrations values $(120 \mathrm{ng} / \mathrm{ml})$.

Results: The incidence of grades I-II and III-IV acute GvHD was $69.1 \%$ and $1.8 \%$, and $8.4 \%$ for chronic GVHD. Most of acute GvHD (94\%) involved skin only. GvHD was neither more frequent nor severe in unrelated than in related HSCT. GvHD occurred in $87 \%$ of patients with a mean CsA trough concentration $\leq 120 \mathrm{ng} / \mathrm{ml}$ versus $43 \%$ with concentration $>120 \mathrm{ng} / \mathrm{ml}(P<0.0001)$. Transplantation-related-mortality was low (9.5\%). Five-year disease-free survival (DFS) and overall survival were $78 \%$ and $83.6 \%$ and respectively. DFS was 76.9\% for ALL and $80.4 \%$ for AML patients. There was no difference in DFS between matched siblings, matched unrelated or mismatched unrelated HSCT. DFS in patients with MRD $\geq 10^{-3}$ and in those with MRD $<10^{-3}$ before HSCT were comparable. A majority of patients (71.4\%) who did not relapse had grade I-II GvHD. Most patients (73.7\%) with MRD $\geq 10^{-3}$ before HSCT and available MRD post-transplantation became $\mathrm{MRD}<10^{-3}$ post HSCT. There was a trend to lower mean CsA TBC in patients who reversed MRD: $114 \pm 15$ versus $144 \pm 32 \mathrm{ng} / \mathrm{ml}(P=0.056)$

Conclusion: Our results indicate that a GvHD prophylaxis regimen based on CsA without mycophenolate or methotrexate, but with specific drug monitoring, is safe and effective whatever the donor compatibility is. These results suggest that graft-versus-leukemia effect may be enhanced by this strategy of GvHD prophylaxis.

Disclosure of Interest: None declared.

\section{P680}

Sequential chemotherapy followed by reduced intensity conditioning and allogeneic hematopoietic stem cell transplantation in adult patients with relapse or refractory acute myeloid leukemia (AML): a survey from the Acute Leukemia Working Party of EBMT

O. Ringdén 1,*, M. Labopin ${ }^{2}$, C. Schmid ${ }^{3}$, B. Sadeghi ${ }^{1}$, E. Polge ${ }^{2}$ J. Tischer ${ }^{4}$, A. Ganser ${ }^{5}$, M. Michallet ${ }^{6}$, L. Kanz $^{7}$, R. Schwerdtfeger ${ }^{8}$, A. Nagler ${ }^{\prime, 10,11}$, M. Mohty ${ }^{12}$ on behalf of Acute Leukemia Working Party of the EBMT

'Laboratory Medicine, Therapeutic Immunology, KAROLINSKA INSTITUTET, STOCKHOLM, Sweden, ${ }^{2}$ EBMT-ALWP Study Office, Hopital Saint Antoine, Paris, France, ${ }^{3}$ Medizinische Klinik, Klinikum Augsburg, Augsburg, ${ }^{4}$ Medizinische Klinik III, Klinikum Grosshadern, Munich, ${ }^{5}$ Dept. of Hematology/Oncology, Hannover Medical University, Hannover, Germany, ${ }^{6}$ BMT Unit, Hospital Herriot, Lyon, France, 'Dept. of Medicine, University of Tuebingen, Tuebingen, ${ }^{8}$ KMT Zentrum, Deutsche Klinik fur Diagnostik, Wiesbaden, Germany, ${ }^{9}$ ALWP Office, Hopital Saint-Antoine, $A P-H P,{ }^{10}$ Universite Pierre \& Marie Curie, Paris, France,

${ }^{11}$ Chaim Sheba Medical Center, Tel-Hashomer, Israel, ${ }^{12}$ Service d'Hematologie et Thérapie Cellulaire, Hopital Saint Antoine, Paris, France

Introduction: We analyzed the outcome of 280 patients with refractory acute myeloid leukemia (AML) who received sequential chemotherapy including fludarabine, cytarabine and amsacrine, followed by reduced intensity conditioning (RIC) and allogeneic hematopoietic stem cell transplantation (HSCT).

Material (or patients) and methods: The transplants were from matched sibling (MSD) for 77 patients and matched unrelated donors (MUD) for 191 patients. Almost all patients (95\%) were given anti-T-cell antibodies.

Results: The incidence of grades II-IV acute graft-versus-host disease (GVHD) was 31\% and of chronic GVHD 30\%. The 3-year 
probability of non-relapse mortality (NRM) was $25 \%$, of relapse was $50 \%$, of GVHD and relapse-free survival (GRFS) $18 \%$, and leukemia-free survival (LFS) $25 \%$. In multivariate analysis, MSD recipients had less acute GVHD grades II-IV (hazards ratio (HR) $1.86, P=0.03)$ and more relapse $(\mathrm{HR}=0.67, P=0.03)$, compared to MUD recipients. Cytomegalovirus (CMV) seronegative recipients of grafts from seronegative donors had a decreased risk of relapse $(\mathrm{HR}=0.62, P=0.02)$ and an improved LFS $(\mathrm{HR}=0.72, P=0.04)$, compared to the other patients. Treatment with anti-T-cell antibodies decreased NRM $(H R=0.35$, $P=0.01)$, and improved survival $(\mathrm{HR}=0.50, P=0.01)$ and GRFS $(\mathrm{HR}=0.38, P=0.001)$, and LFS (HR=0.47, $P=0.01)$.

Conclusion: Sequential chemotherapy, followed by RIC HSCT and use of anti-T-cell antibodies can resque $25 \%$ of patients with refractory AML.

Disclosure of Interest: None declared.

P681

Outcome of allogeneic hematopoietic stem cell transplant in therapy related myeloid neoplasms. Comparative study with a de novo myeloid neoplasms group

O. Ferré ${ }^{1, *}$, E. Pérez ${ }^{1}$, M. Alpoim ${ }^{1}$, O. López-Godino ${ }^{1}$, J. M. Bastida ${ }^{1}$, L. López-Corral ${ }^{1}$, F. López-Cadenas ${ }^{1}$, L. García-Martín ${ }^{\prime}$, N. Arratibel', M. Baile ${ }^{i}$, A. Veiga ${ }^{1}$, S. Alonso ${ }^{1}$, M. Diez-Campelo $^{\prime}$, L. Vásquez", C. Del Cañizo ${ }^{1}$, D. Caballero' ${ }^{1}$, M. Cabrero ${ }^{1}$

${ }^{1}$ Hematology, Salamanca University Hospital, Salamanca, Spain

Introduction: Therapy related myeloid neoplasms (t-MN) are caused by the use of cytotoxic chemotherapy and/or radiotherapy for previous neoplasms or non-malignant diseases. According to literature these diseases are associated with worse prognosis, resulting in a more complex therapeutic approach and a lack of an accepted standard therapy.

Material (or patients) and methods: In our center 298 allogeneic hematopoietic stem cell transplants (HSCT) were performed between 1998 and 2014 for de novo myeloid neoplasms $(n-M N)$, from which $10.4 \%(n=31)$ were $t-M N$. We retrospectively analyzed this group of patients in terms of overall survival (OS), event free survival (EFS, being event death or relapse) and transplant related mortality (TRM); we compared it with a control group of 62 n-MN with similar clinical characteristics from the same period of time (No differences in age, cytogenetic risk, status at HSCT, donor or conditioning regimen; $P>0.05$ ) (Table. 1)

Results: t-MN patients median age was 52 years old [34-66], diagnosis was MDS in 19 (61\%) and AML in 12 (40\%). Sixteen (52\%) had an unfavorable risk cytogenetic. Median time from first disease to t-MN was 67.5 months [12-356]. Ten (32\%) had active disease at HSCT. All patients but one engrafted. Acute graft versus host disease (aGVHD) incidence was $61 \%$ (grades III-IV, 23\%), and chronic graft versus host disease (cGVHD) incidence was $65 \%$ (extensive $48 \%$ ).

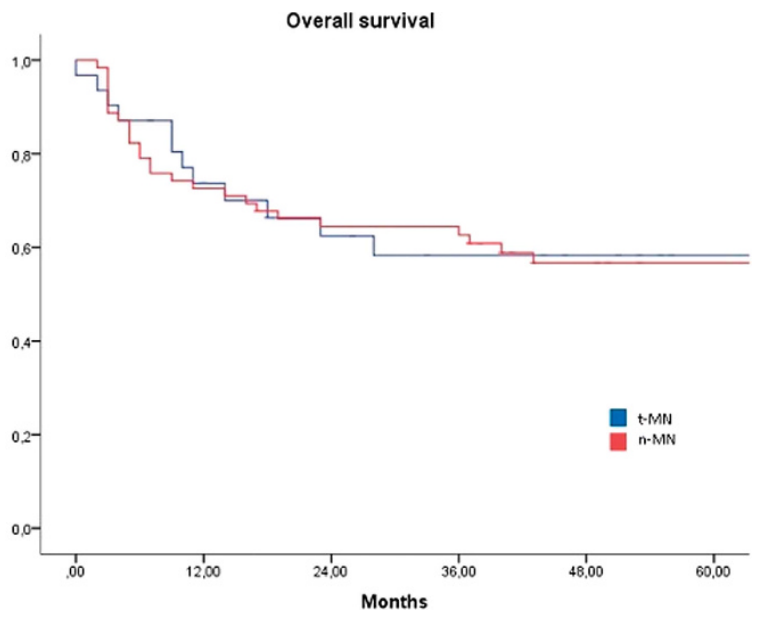

With a median follow up of 26 months [1-163] from the date of HSCT, the estimated OS and EFS at 2 years was $62.1 \%$ and $57.1 \%$ respectively. Overall mortality was $42 \%(n=13 / 31)$ and the main cause was relapse $54 \%(n=7 / 13)$; TRM was $13 \%$ $(n=4 / 31)$ at 2 years.

In the univariate analysis none of the variables tested had significant impact on OS and EFS, including cytogenetics and pre HSCT status (all $P>0.05$ ).

In a second step, we compared this group with the control group (Table 1); we did not observe significant differences in $\mathrm{OS}$ at 2 years between patients with t-MN and $\mathrm{n}-\mathrm{MN}(62.4 \%$ vs. $64.4 \%, P=0.91)$ (Figure 1), in EFS $(57.1 \%$ vs. $61.2 \%, P=0.77)$, and TRM $(15.9 \%$ vs. $15.6 \%, P=0.54)$.

Table. 1

\begin{tabular}{|c|c|c|c|}
\hline & $t-M N$ & $n-M N$ & $\mathrm{P}$ value \\
\hline \multicolumn{4}{|l|}{ Age } \\
\hline$<55$ years & $19(61.3 \%)$ & $35(56.5 \%)$ & 0.82 \\
\hline$\geq 55$ years & $12(38.7 \%)$ & $27(43.5 \%)$ & \\
\hline \multicolumn{4}{|c|}{ Cytogenetic prognostic group } \\
\hline Good & $4(12.9 \%)$ & $3(4.8 \%)$ & 0.16 \\
\hline Intermediate & $10(32.3 \%)$ & $40(64.5 \%)$ & \\
\hline Poor & $16(51.6 \%)$ & $19(30.6 \%)$ & \\
\hline \multicolumn{4}{|l|}{ Pre-HSCT disease status } \\
\hline Complete remission & $12(38.7 \%)$ & $35(56.5 \%)$ & 0.24 \\
\hline Active disease & $10(32.3 \%)$ & $16(25.8 \%)$ & \\
\hline No treatment & $9(29 \%)$ & $11(17.7 \%)$ & \\
\hline \multicolumn{4}{|c|}{ HSCT conditioning regimen } \\
\hline Myeloablative & $8(25.8 \%)$ & $23(37.1 \%)$ & 0.27 \\
\hline Non-myeloablative & $23(74.2 \%)$ & $39(62.9 \%)$ & \\
\hline \multicolumn{4}{|l|}{ Donor type } \\
\hline Related & $17(54.8 \%)$ & $45(72.6 \%)$ & 0.12 \\
\hline Unrelated & $13(41.9 \%)$ & $17(27.4 \%)$ & \\
\hline Haploidentical & $1(3.2 \%)$ & $0(0 \%)$ & \\
\hline aGVHD & 19 (61.3\%) & $37(59.7 \%)$ & 0.73 \\
\hline cGVHD & $20(64.5 \%)$ & $34(54.8 \%)$ & 0.62 \\
\hline Relapse & $10(32.3 \%)$ & $28(45.2 \%)$ & 0.23 \\
\hline TRM & $4(12.9 \%)$ & $15(24.1 \%)$ & 0.2 \\
\hline
\end{tabular}

Conclusion: Despite the worse prognosis associated with t-MN, according to our results, HSCT is a potentially curative treatment in this group of patients, showing similar results than those obtained in $\mathrm{n}-\mathrm{MN}$ and thus overcoming its bad prognosis.

Disclosure of Interest: None declared.

P682

MFC-MRD assessment may drive post induction consolidation in intermediate risk AML. Is it time for MRD-DRIVEN approach?

P. Minetto 1,*, F. Guolo', M. Clavio ${ }^{1}$, A. Kunkl, D. Guardo ${ }^{1}$, F. Ballerini ${ }^{1}$, M. Miglino ${ }^{7}$, C. Di Grazia ${ }^{3}$, A. Bacigalupo ${ }^{3}$, M. Gobbi ${ }^{1}$ ${ }^{1}$ Chair of Hematology, Department of Internal Medicine (DiMI), University of Genoa, ${ }^{2}$ Service of Flow-cytometry, ${ }^{3}$ Second division of Hematology, IRCSS AOU San Martino-IST, Genoa, Italy

Introduction: Since the induction therapy for acute myeloid leukemia (AML) has not changed in the last decades, the optimization of post remissional strategies is fundamental to improve patients outcome. Allogeneic hematopoietic stem cell transplantation (BMT) offers the greatest chance of cure for most patients, however in intermediate cytogenetic risk patients (int-risk), the role of BMT in $1^{\text {st }}$ complete remission 
Table 1.

\begin{tabular}{|c|c|c|c|c|c|c|}
\hline & Num. (\%) & Relapse (\%) & median RFS & 3-year RFS (\%) & $p$ (univ.) & $p$ (univ.) \\
\hline ALL PATIENTS & 55 & $16(29)$ & 69 & 71.4 & - & - \\
\hline \multicolumn{7}{|c|}{ MFC MRD after 1st cycle negative } \\
\hline BMT $1^{\text {st }} \mathrm{CR}$ & 9/23 (39) & $1(11)$ & 62 & 100 & 0.748 & 0.003 \\
\hline No $B M T / 2^{\text {nd }} C R$ & $14 / 23(61)$ & $2(14)$ & NR & 83.6 & & \\
\hline \multicolumn{7}{|c|}{ MFC MRD after 1st cycle positive } \\
\hline BMT $1^{\text {st }} \mathrm{CR}$ & $12 / 32(38)$ & $1(8)$ & NR & 87.5 & 0.048 & \\
\hline No $B M T / 2^{\text {nd }} C R$ & $20 / 32(62)$ & $12(60)$ & 36 & 45.2 & & \\
\hline \multicolumn{7}{|c|}{ MFC MRD after 2nd cycle negative } \\
\hline BMT $1^{\text {st }} \mathrm{CR}$ & $9 / 30(30)$ & $2(22)$ & 62 & 87.5 & 0.790 & 0.098 \\
\hline No $B M T / 2^{\text {nd }} C R$ & $21 / 30(70)$ & $6(29)$ & NR & 77.9 & & \\
\hline \multicolumn{7}{|c|}{ MFC MRD after 2nd cycle positive } \\
\hline BMT $1^{\text {st }} \mathrm{CR}$ & $8 / 19(42)$ & $0(0)$ & NR & 100 & 0.023 & \\
\hline No $B M T / 2^{\text {nd }} C R$ & $11 / 19(58)$ & $7(64)$ & 15 & 33.3 & & \\
\hline
\end{tabular}

$(C R)$ is not well defined. The aim of the present study was to evaluate the prognostic role of minimal residual disease (MRD) assessment through multiparameter flow cytometry (MFCMRD) after $1^{\text {st }}$ and $2^{\text {nd }}$ induction courses in int-risk patients undergoing or not BMT in $1^{\text {st }} \mathrm{CR}$.

Material (or patients) and methods: Fifty-five consecutive int-risk AML patients, according to MRC classification, achieving CR after induction therapy, with available post induction MFC-MRD evaluation, were retrospectively included. All patients had been treated with a fludarabine-containing induction. Median age was 49 years. BMT in $1^{\text {st }}$ CR was scheduled if an HLA identical sibling donor or, for selected patients, if an haploidentical donor was available. The remaining 34 patients proceeded to high dose cytarabine consolidation and underwent BMT in $2^{\text {nd }} C R$ from any donor if available ( 6 patients). A positive MFC-MRD was defined by the presence of no less than 25 clustered leukemic cells $/ 10^{5}$ total events at four-color flow-cytometry. Twenty-three and 30 patients achieved MFC-MRD negativity after one or two induction cycles, respectively. Median follow up was 44 months. Relapse-free survival (RFS) was calculated from the time of diagnosis until last follow-up or documented leukemic relapse.

Results: Relapse occurred in 16 patients (29\%). Three-year RFS was $71.4 \%$ (median not reached). MFC-MRD after induction 1 (MFC -MRD1) significantly affected outcome with a 3 year RFS of $88.4 \%$ for MFC-MRD1 negative patients compared to $57.8 \%$ for MFC-MRD1 positive $(P<0.005)$; MFC-MRD after induction 2 (MFC-MRD2) had only a borderline impact, with a 3 year RFS of $80.1 \%$ and $56.7 \%$ for MFC-MRD2 negative and positive patients, respectively $(P=0.098)$. Performing BMT in $1^{\text {st }} C R$ did not significantly improve RFS of patients who were MFCMRD negative after cycle 1 or after cycle 2 (Tab.1) whereas performing early BMT provided a significant advantage for patients with positive MFC MRD at any of the two timepoints $(P<0.05$ and $P<0.03$ for positive MFC-MRD after cycle 1 or cycle 2 , respectively).

Conclusion: MFC-MRD assessment after induction is a strong predictor of relapse risk and could improve risk stratification for int-risk patients. A deeper evaluation of MRD may overcome morphologic CR assessment becoming a powerful tool to delineate the therapeutic iter.

References: Buccisano F, et al. Prognostic and therapeutic implications of minimal residual disease detection in acute myeloid leukemia.Blood. 2012;119:332-41.
Disclosure of Interest: None declared.

\section{P683}

Efficacy and Safety of Defibrotide (DF) in the Treatment of Hepatic Veno-Occlusive Disease/Sinusoidal Obstruction Syndrome (VOD/SOS) Following Chemotherapy in an Ongoing Study

N. Kernan ${ }^{1}$, P. Richardson 2,*, A. Smith ${ }^{3}$, B. Triplett ${ }^{4}$, S. Grupp ${ }^{5}$ J. Antin ${ }^{2}$, L. Lehmann ${ }^{2}$, W. Liang ${ }^{6}$, R. Hume ${ }^{6}$, A. Hannah ${ }^{6}$, R. Soiffer ${ }^{2}$

${ }^{1}$ Memorial Sloan Kettering Cancer Center, New York, NY, ${ }^{2}$ DanaFarber Cancer Institute, Boston, MA, ${ }^{3}$ University of Minnesota Minneapolis, MN, ${ }^{4}$ St. Jude Children's Research Hospital, Memphis, TN, ${ }^{5}$ The Children's Hospital of Philadelphia, Philadelphia, PA, ${ }^{6} \mathrm{Jazz}$ Pharmaceuticals, Palo Alto, CA, United States

Introduction: VOD/SOS, which is unpredictable and potentially life-threatening, is typically thought of as a complication of hematopoietic stem cell transplantation (HSCT); however, there is also a known risk in patients (pts) following chemotherapy in a non-HSCT setting. Severe hepatic VOD/ SOS (ie, with multi-organ dysfunction [MOD]) may be associated with $>80 \%$ mortality. Endothelial cell (EC) damage is a critical pathophysiologic factor, and preclinical data suggest that DF stabilizes ECs with direct, as well as ECmediated, restoration of the thrombo-fibrinolytic balance. DF is approved for treatment of severe hepatic VOD/SOS in adult and pediatric pts in the EU. In the US, a new drug application was submitted and filed in 2015.

Material (or patients) and methods: DF is available in the US through an ongoing expanded-access protocol for pts with hepatic VOD/SOS (Baltimore/modified Seattle criteria or biopsy) post-HSCT or chemotherapy, with/without MOD defined by renal and/or pulmonary dysfunction. DF $25 \mathrm{mg} /$ $\mathrm{kg} / \mathrm{d}$ in 4 divided doses was given for a recommended $\geq 21$ days. Here, we describe updated efficacy and safety results of DF for the subset of pts with VOD/SOS postchemotherapy.

Results: Out of 857 pts who developed VOD/SOS and received $\geq 1$ dose of DF as of April 18, 2015, 101 (11.8\%) pts received chemotherapy without HSCT; 49 (49\%) had MOD and 52 (51\%) did not. Median age was 7 yrs (range, 0 mos-69.0 yrs), and 81 pts $(80 \%)$ were $\leq 16$ yrs (59 pts were children aged $2-11$ ); $55 \%$ of pts were male. The most common primary diseases were acute lymphocytic leukemia (49\%) and acute myelogenous 
Figure. Kaplan-Meier Estimates of Day +100 Survival Following Chemotherapy

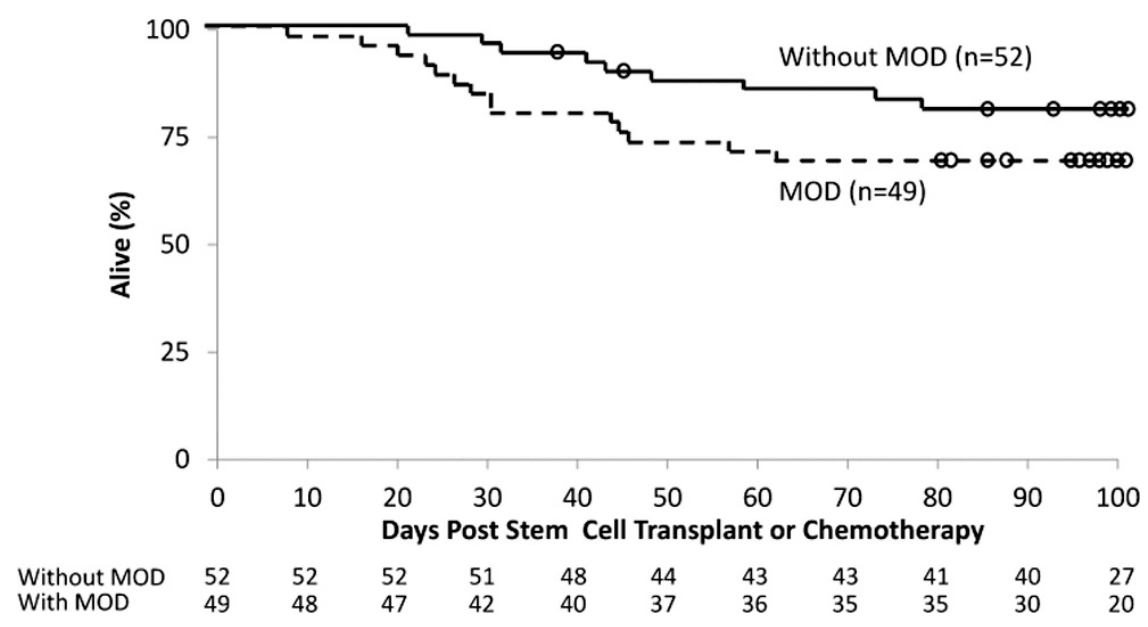

leukemia (13\%). Chemotherapeutic agents received by $>30 \%$ of pts were vincristine, cyclophosphamide, cytarabine, doxorubicin, methotrexate and PEG-L-asparaginase. Antibody-drug conjugates linked to ozogamicin that are associated with development of VOD/SOS (gemtuzumab and inotuzumab) were received by 2 and $0 \mathrm{pt}$, respectively.

The Kaplan-Meier estimated Day+100 survival rates were $77.0 \%$ overall $(95 \% \mathrm{Cl}, 67.5-84.1 \%), 71.4 \%(56.6-82.0 \%)$ for pts with MOD, and $82.3 \%$ (68.7-90.4\%) for pts without MOD.

Overall, $\geq 1$ adverse event (AE) was reported in 62 pts (61.4\%). Of these, $22(21.8 \%)$ had AEs assessed as at least possibly related to $\mathrm{DF}$, most commonly $(\geq 2.0 \%)$ : hypotension $(4.0 \%)$; nausea (3.0\%); vomiting, epistaxis, hematochezia, gastrointestinal (GI) and pulmonary hemorrhages (2.0\% each). Hemorrhagic AEs of any severity occurring in $\geq 2.0 \%$ were: pulmonary (6.9\%); epistaxis (5.0\%); hematochezia, gastric, and GI $(2.0 \%$ each). Serious AEs were reported in 37 pts (36.6\%), most commonly: multi-organ failure (6.9\%); pulmonary hemorrhage (5.9\%); hypoxia (4.0\%). AEs led to discontinuation in 7 pts (gastric, Gl, mouth, intraventricular, and esophagitis hemorrhages; epistaxis; hypotension). Two deaths (pleural hemorrhage and pulmonary hemorrhage plus hypotension) were at least possibly related to DF.

Conclusion: Day +100 survival of $77.0 \%$ in pts $(80 \%$ of which were pediatric) developing VOD/SOS following a variety of chemotherapy regimens without HSCT is a clinically encouraging finding. In this group of 101 pts with VOD/SOS postchemotherapy, DF was generally well tolerated, with 2 possibly treatment-related fatalities and $6.9 \%$ of pts discontinuing due to an $\mathrm{AE}$.

Support: Jazz Pharmaceuticals.

Disclosure of Interest: N. Kernan: None declared, P. Richardson Funding from: Jazz Pharmaceuticals, Personal Interest: Served on advisory committees with Jazz Pharmaceuticals, A. Smith: None declared, B. Triplett: None declared, S. Grupp Personal Interest: Served as a consultant to Jazz Pharmaceuticals, J. Antin Personal Interest: Served on advisory committees with Jazz Pharmaceuticals, L. Lehmann: None declared, W. Liang Employee of: Jazz Pharmaceuticals, and in the course of employment has received stock options exercisable for, and other stock awards of, ordinary shares of Jazz Pharmaceuticals, R. Hume Employee of: Jazz Pharmaceuticals, and in the course of employment has received stock options exercisable for, and other stock awards of, ordinary shares of Jazz Pharmaceuticals, A. Hannah Personal Interest: Consultant to Jazz Pharmaceuticals, R. Soiffer Personal Interest: Served on advisory committees with Jazz Pharmaceuticals.

\section{P684}

Allogeneic haematopoietic cell transplantation in patients with FLT3-ITD mutated AML: transplantation in 1st CR is the decisive factor for good outcome

P. Jindra ${ }^{1, *}$, M. Karas ${ }^{1}$, T. Szotkowski ${ }^{2}$ D. Lysák ${ }^{1}$, L. Raida ${ }^{2}$ A. Jungová ', A. Kuba', M. Hrabětová', C. Martin ${ }^{2}, K$ K. Steinerová ${ }^{1}$ ${ }^{1}$ Haematology\&Oncology, University Hospital, Pilsen, ${ }^{2}$ Haematology\&Oncology, University Hospital, Olomouc, Czech Republic

Introduction: Patients with karyotypic normal (CN) FLT3-ITD mutated AML have dismal prognosis and the only curative option seems to be alloHCT. However, the timing of alloHCT is still matter of debate and some advocates HCT only beyond $1^{\text {st }}$ CR. The consistent policy in 2 Czech centres since 2003 is to refer all FLT3-ITD+ AML pts to upfront alloHCT in CR1. The aim of this study was to analyze the outcomes of this strategy. Material (or patients) and methods: 73 consecutive adults with FLT3-ITD+ CN-AML allografted in 2 centres $(56+17$ pts) from 2003-2014 either with matched sibling (MSD, $n=23$, $32 \%$ ) or unrelated donors (UD, $n=50,68 \%$ ) were studied. Conditioning regimen was either RIC $(n=54)$ or myeloablative (MAC, $n=19)$. The median age of cohort was 53 years (20-68). $33(45 \%)$ were NPM1mutated. The original aim of our policy was to perform HCT in CR1 $(n=56)$, however in some patients due to various reasons (donor availability, disease resistance or patient preference), HCT was performed in more advanced stage ( $n=17,1 \times P R 1,2 \times C R 2 / P R 2,4 \times P I F, 10 x R E L)$. Median interval dg.- HCT was 5 months (2-13) with median of 2 (1-5) cycles of chemotherapy before HCT. For analysis, the status at the time of HCT was classified as either early (CR1) or advanced (CR2, PR1, PR2, REL, PIF).

Results: With median follow up of 44 months (range 5-135) altogether 35 pts $(48 \%)$ has died mainly of relapse $(n=26$, $74 \%)$. The 5 -year probabilities of OS and DFS were $49 \%$ and $47 \%$. Cumulative incidences of relapse (RI) and NRM at 5-y were $37 \%$ and $17 \%$, respectively. Cox regression hazard model identified status at HCT outside the CR1 (= advanced disease) as the strongest factor predicting extremely poor OS/DFS (HR 6.665, $P<0.0001 / \mathrm{HR}$ 5.474, $P<0.0001$ ) and $\mathrm{RI}$ (HR 8.270, $P<0.0001)$. The estimated 5 -y OS for patients transplanted in CR1 was $62 \%$ vs. $0 \%$ for pts transplanted outside CR1 The higher patient age (cut-off 57 years) predicted higher NRM (HR $5.496, P=0.0136$ ) as well as the higher aGVHD grade (HR 2.577, $P=0.0058$ ). The presence of chronic GVHD was associated with superior OS/DFS (HR 0.178, $P=0.0059 / \mathrm{HR} 0.238, P=0.01$ ) probably because of the relapse protection (HR 0.214, 
$P=0.0145$ ). No association of patient age, NPM mutational status, time to HCT, no. of cycles pre-HCT, donor type (MSD vs. UD), donor age and sex, conditioning regimen (MAC vs. RIC), stem cell source, CD34+ cell dose and CMV status on the main outcomes was detected. Multivariable analysis identified HCT outside CR1 as the key factor for poor OS (HR 5.419, $P<0.0001)$, DFS (HR 4.415, $P=0.0002$ ) and high RI (HR 8.080, $P<0.0001$ ). aGVHD grade $\geq$ III predicted higher NRM (HR 3.798, $P=0.059$ ) as well as inferior OS (HR 2.041, $P=0.0079$ ) and DFS (HR 1.693, $P=0.0439$ ).

Conclusion: Despite the limitations of a retrospective study, our data suggests alloHCT is a valuable therapeutic option for FLT3-ITD mutated CN-AML patients with potential to provide durable remission if patient is transplanted in CR1. Until prospective studies are completed, our study strongly supports to prioritize the allografting in CR1 regardless of donor type, i.e. alloHCT should be regarded as the urgency in CR1 in this subset of AML patients.

References: Schmid C et al. Outcome and risk factor analysis of molecular subgroups in cytogenetically normal AML treated by allogeneic transplantation. Blood 2015, 126, 2062-2069.

Disclosure of Interest: None declared.

\section{P685}

Integrin alphavbeta 3 enhances $\beta$-catenin signaling in acute myeloid leukemia harboring Fms-like tyrosine kinase-3 internal tandem duplication mutations: implications for microenvironment influence on sorafenib sensitivity

H. Hai ${ }^{1}$, Z. Dongfeng ${ }^{1}$, Z. Shen ${ }^{1}$, J. Liao ${ }^{1}$, X. Wang ${ }^{1}$, Y. Liu ${ }^{1}$, X. Zhang ${ }^{1}$, P. Kong ${ }^{1,}$

${ }^{1}$ Department of hematology, XINQIAO HOSPITAL, THIRD MILITARY MEDICAL UNIVERSITY, Chongqing, China

Introduction: Communication of cancer cells and the extracellular matrix (ECM) in bone marrow environment might influence drug response and the survival of acute myeloid leukemia (AML). Integrin plays an important role in cell-ECM interaction. However, the expression and prognostic value of integrin and the mechanisms involved in drug sensitivity in AML are largely unknown.

Material (or patients) and methods: We retrieved transcriptome sequencing data and clinical data from The Cancer Genome Atlas database, and analyzed integrin $\beta 3$ (ITGB3) expression and prognostic significance for AML patients. We used human bone marrow stromal cells (BMSCs) to mimic bone marrow environment, to investigate their effects on sorafenib sensitivity of FLT3-ITD AML cells in vitro and the underlined mechanisms especially for integrin alphavbeta3 (av $\beta 3)$. Cell apoptosis was measured by flow cytometry; protein expression was analyzed by western blotting; cell growth was measured by CCK-8 assay.

Results: We demonstrate that ITGB3 expression was positively correlated with risk stratification and prognosis of $\mathrm{AML}$ patients, especially in patients with Fms-like tyrosine kinase3 internal tandem duplication (FLT3-ITD) mutation. Co-culture of FLT3-ITD mutated AML cell line MV4-11 and BMSCs caused leukemia cell insensitivity to sorafenib treatment, which was partly mediated by enhancing integrin av $\beta 3$ signaling pathway. Mechanically, av $\beta 3$ could enhance $\beta$-catenin activation through phosphatidylinositol 3-kinase (PI3K)/Akt/Glycogen synthase kinase-3 beta (GSK3 $\beta$ ) pathway. Moreover, genetic inhibition of $\beta$-catenin by shRNA could increase MV4-11 cell sensitivity to sorafenib.

Conclusion: Our study suggested that integrin $\beta 3$ could be a prognostic factor for AML especially for FLT3-ITD positive AML patients. BMSCs protected MV4-11 against sorafenib through integrin av $\beta 3$, partly through av $\beta 3$ mediated $\beta$-catenin activation. Disruption of integrin av $\beta 3 / \beta$-catenin pathway may represent a potential target therapy to improve survival of AML patients with FLT3-ITD mutation.

Disclosure of Interest: None declared.
P686

Relevance of donor choice for patients with Acute Myeloid Leukemia undergoing allogeneic stem cell transplantation in complete remission: impact of donor age on overall outcome

P. Hemmati, ${ }^{1, *}$, C. Jehn ${ }^{1}$, T. Terwey ${ }^{1}$, L. Vuong ${ }^{1}$, P. le Coutre ${ }^{1}$,

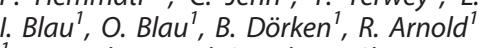

${ }^{1}$ Hematology and Oncology, Charite University Medicine, Berlin, Germany

Introduction: For patients with Acute Myeloid Leukemia (AML) undergoing allogeneic stem cell transplantation (alloSCT) in first complete remission (CR), a 10/10 human leukocyte antigen- (HLA) matched sibling is regarded as donor of first choice. However, recent reports suggest that donor age may have a significant impact on the outcome of patients with AML undergoing alloSCT. Specifically, patients transplanted from a young unrelated donor might have a better outcome as compared to those transplanted from an older matched sibling. This should be of particular relevance, since the majority of patients with AML referred to alloSCT is beyond the age of 50 years. Furthermore, unrelated donor availability has substantially increased within the last decade.

Material (or patients) and methods: A total of 277 patients with AML (median age: 50 , range: $17-70$ years) transplanted at our center between 2000 and 2014 was retrospectively analyzed. All patients were in CR (CR1: N=213 patients, $C R>1$ : 64 patients). Standard myeloablative conditioning (MAC) was given in 115 patients, whereas 162 patients received reduced intensity conditioning $(\mathrm{RIC})$ prior to alloSCT. Cytogenetic risk was categorized according to the Southwest Oncology Group/Eastern Cooperative Oncology Group (SWOG/ECOG) classification, i.e. favorable $(N=24)$, intermediate $(N=155)$, poor $(N=68)$, or undetermined/unknown significance $(N=30)$. Grafts were from either matched related donors (MRD; $N=120$; median age: 49 , range: $17-76$ years) or matched unrelated donors (MUD; $N=157$; median age: 30, range: $19-56$ years).

Results: For the surviving patients the median duration of follow-up was 43 months. At 3 years after alloSCT, overall survival (OS) or disease-free survival (DFS) of the entire cohort was $62 \%$ and $58 \%$. The cumulative incidence of relapse (Cl-R) or non-relapse mortality (CI-NRM) was $27 \%$ or $15 \%$, respectively. According to donor source and age, the patients were grouped into 4 subgroups, i.e. group 1: MUD/age below median, group 2: MUD/age median or higher, group 3: MRD/ age below median, group 4: MRD/age median or higher. DFS at 3 years after alloSCT was almost identical between the 4 subgroups and ranged between $58 \%$ and $60 \%$ (overall p: 0.57 ). Likewise, there were no significant differences in the $\mathrm{Cl}-\mathrm{R}$ or the Cl-NRM between the four 4 subgroups (overall $p$ for relapse: 0.95 , overall $p$ for NRM: 0.47 ). In patients aged 50 years and higher treated with RIC before alloSCT similar results were obtained. Notably, at 5+ years after alloSCT, patients transplanted from an older donor (either MRD or MUD) tended to a have lower DFS due to an increased in NRM.

Conclusion: These data suggest that, in general, donor age may have an impact on overall outcome of patients with AML undergoing alloSCT in CR. However, our results do not delineate that a matched unrelated donor should be preferred over an older matched sibling. Nonetheless, total number of patients is small and, therefore, analysis of larger patient cohorts is warranted.

Disclosure of Interest: None declared.

\section{P687}

Adult patients with ALL not in complete remission: longterm results after allogeneic stem cell transplantation

R. Arnold ${ }^{1, *}$, T. Terwey ${ }^{1}$, N. Waldhüter ${ }^{1}$, I. Blau ${ }^{1}$, O. Penack', C. Jehn ${ }^{1}$, L. Vuong ${ }^{1}$, P. Hemmati ${ }^{1}$

${ }^{1}$ Hematology and Oncology, Charite University Medicine, Berlin, Germany 
Introduction: In the German Multicenter ALL (GMALL) study high-risk and very high-risk ALL patients are candidates for allogeneic stem cell transplantation (allo SCT) in first complete remission (CR1). High-risk factors are B-lineage ALL: at diagnosis $>30 / \mathrm{nl}$, pro-B ALL, CR after 4 weeks, and in T-lineage ALL pre-T ALL and mature T-ALL. Ph-positive ALL is regarded as very highrisk group. All other patients are treated in the standard-risk group and are candidates for allo SCT after relapse. All patients with primary refractory ALL are candidates for allo SCT.

Material (or patients) and methods: Between 7/1995 and 8/2014 63 patients ( 40 males, 24 females) with a median age of 33 years (range: 16-57 years) underwent allo SCT in a single center. Subtypes of ALL were B-lineage in 36 patients, 13 patients had Ph-positve ALL, and 14 patients had T-lineage ALL. Conditioning regimens were mainly myeloablative (12 Gy $\mathrm{TBI}+/-\mathrm{CY}+/$ - VP16) and donors were HLA-identical siblings in 21 patients and 42 patients received a matched unrelated donor transplant.

Results: $14 / 63$ patients are alive in $C R$ with a median follow-up of 86 months (range: 26-180 months). 2/12 patients transplanted with primary refractory ALL are alive in CCR, 11/43 patients transplanted in first relapse are alive in CCR and $1 / 8$ patients transplanted in second relapse is alive and in CCR. 49 patients are dead, due to relapse/progression of leukemia in $35 / 49$ patients or due to transplant-related mortality, i.e. infections +/- GvHD, in 14/49 patients. Probability of survival for the whole group $(N=63)$ is 0.25 at 5 years after allo SCT, probability of disease-free survival is 0.21 at five years, and probability of transplant-related mortality at five years is 0.32 Conclusion: Adult ALL patients with active disease at conditioning can be cured by allo SCT, but despite myeloablative conditioning the rate of relapse/progression of leukemia after allo SCT is high. Regular MRD analysis of the patients will allow earlier transplantation before hematologic relapse occurs and so improve the results.

Disclosure of Interest: None declared.

\section{P688}

Impact of Cytomegalovirus serological status and viral reactivation on outcome of AML patients after allogeneic stem cell transplantation

S. Guenounou ${ }^{1,2, *}$, E. Berard ${ }^{3,4}$, E. Yon ${ }^{3,4}$, S. Bertoli ${ }^{1,2,5}$, C. Recher ${ }^{1,2,5}$, C. Borel ${ }^{1}$, A. Huynh ${ }^{i}$

${ }^{1}$ Hematology, Institut Universitaire du Cancer de Toulouse, ${ }^{2}$ Université Toulouse III Paul Sabatier, ${ }^{3}$ UMR 1027, INSERM Université de Toulouse III, ${ }^{4}$ Epidemiology, Centre Hospitalier Universitaire de Toulouse, ${ }^{5}$ UMR 1037, INSERM Université de Toulouse III, Toulouse, France

Introduction: According to recent data, donor or recipient's seropositivity for cytomegalovirus (CMV) and CMV replication can have a protective effect on relapse in acute myeloid leukemia (AML) patients after allogeneic stem cell transplantation (SCT). The aim of our study is to investigate the impact of
CMV serological status and viral reactivation on outcome of patients who underwent allogeneic STC.

Material (or patients) and methods: We evaluated retrospectively in univariate and multivariate analysis the effect of CMV serological status and viral reactivation (CMV, EBV, BKV, VZV, ...) on relapse and survival in a cohort of $136 \mathrm{AML}$ patients undergoing SCT after first complete remission (CR1) at Toulouse University Hospital between 2000 and 2012. Regimens were myeloablative $(n=71)$ and reduced intensity conditioning $(n=65)$. Graft versus host disease (GVHD) prophylaxis was cyclosporine alone, or in association with either methotrexate or mycophenolate mofetil. Risk of relapse, overall survival (OS), progression free-survival (PFS) and nonrelapse mortality (NRM) were calculated.

Results: The median follow up was 5.6 years. Between 01/01/ 2000 and 12/31/2013, 46 patients with AML in CR1 undergoing SCT had negative CMV serology for donor and recipient (CMV-/-) (cohort 1) and 90 patients had at least one positive CMV serology (CMV+/-, CMV+/+, CMV-/+) for donor or recipient (cohort 2). AML features were comparable in the two cohorts. Patients in cohort 1 were younger (median age at 45.4 years old compared to 50.7 years old in cohort2, $P=0.03$ ), and more likely to have bone marrow as stem cell source $(65.2 \%$ in cohort 1 compared to $52.9 \%$ in cohort $2, P=0.04$ ) and ciclosporine combined to methotrexate as GVHD prophylaxis (62.2\% versus $50.0 \%$ respectively, $P=0.04$ ). Our study shows that occurrence of viral reactivation post SCT significantly decreases the risk of relapse (HR $0.14 ; 95 \% \mathrm{Cl} 0.07-$ $0.30 ; P<0.001$ ), improves OS (HR $0.44 ; 95 \% \mathrm{Cl} 0.25-0.77 ; P=0.005$ ) and PFS (HR 0.29; 95\% $0.16-0.54 ; P<0.001)$. Comparatively with cohort 2, patients allografted with CMV-/- status had, at 10 years, an increase of $20 \%$ for risk of relapse $(P=0.03)$ (Figure1) and a decrease of $18 \%$ for OS $(P=0.02)$. These results are independent of $A M L$ characteristics, conditioning regimen, acute or chronic GVHD incidence. NRM at 10 years is not different regarding CMV status $(18 \%$ for cohort 1 versus $15 \%$ for cohort $2(P=0.68)$. However, NRM at 10 years was significantly higher for patients who had viral reactivation compared to patients who had not, $24 \%$ versus $7 \%$ respectively $(P=0.02)$.

Conclusion: Our retrospective and monocentric study confirms that CMV status other than CMV-/- and viral reactivation post SCT improves outcome of AML patients undergoing allogeneic SCT in CR1. The main hypothesis is that viral antileukemic effect could be mediated by natural killer cells. Prospective and multicentric studies and better understanding of immunological aspects are needed and could lead to new recommendations regarding $\mathrm{CMV}$ serological donor/recipient status and to develop new anti-viral strategies to prevent the risk of relapse.

References: Elmaagacli et al. Blood 2011;118:1402-12.

Green et al. Blood 2013;122:1316-24.

Manjappa et al. Biology of blood and marrow transplantation 2014;20:46-52.

Jang et al. Annals of hematology 2015;94:275-82.

Lewis et al. Abstract EBMT 2015;0013.

Disclosure of Interest: None declared.
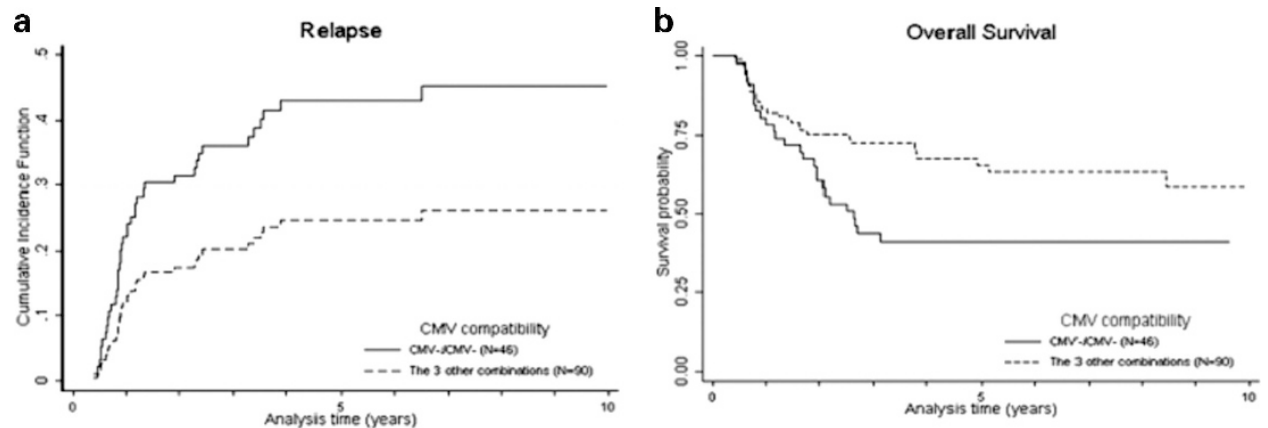

Figure 1.Impact of Cytomegalovirus serological status on cumulative incidence of relapse $(A)(\mathrm{p} 0,03)$ and overall survival (B) $(\mathrm{p0}, 02)$, 


\section{P689}

Improving results of unrelated donor - HCT for adults with acute lymphoblastic leukemia in first complete remission: an analysis from Acute Leukemia Working Party of the EBMT

S. Giebel ${ }^{1, *}$, M. Labopin ${ }^{2,3}$, G. Socié ${ }^{4}$, L. Volin ${ }^{5}$, M. Michallet ${ }^{6}$, R. Arnold ${ }^{7}$, O. Ringden ${ }^{8}$, R. Tabrizi ${ }^{9}$, J. Passweg ${ }^{10}$, I. YakoubAgha $^{11}$, S. Lenhoff ${ }^{12}$, L. Kanz $^{13}$, A. Nagler ${ }^{3,14}$

${ }^{1}$ Bone Marrow Transplantation and Onco-Hematology, Maria Sklodowska-Curie Memorial Cancer Center and Institute of Oncology, Gliwice Branch, Gliwice, Poland, ${ }^{2}$ Hospital St. Antoine, ${ }^{3}$ Acute Leukemia Working Party EBMT, ${ }^{4}$ Hopital St. Louis, Paris, France, ${ }^{5}$ Helsinki University Hospital, Helsinki, Finland, ${ }^{6}$ Centre Hospitalier Lyon Sud - Service Hematologie, Lyon, France, ${ }^{7}$ Charité Universitätsmedizin Berlin - Campus Virchow Klinikum, Berlin Germany, ${ }^{8}$ Karolinska University Hospital, Stockholm, Sweden, ${ }^{9} \mathrm{CHU}$ Bordeaux, Pessac, France, ${ }^{10}$ University Hospital, Basel, Switzerland, ${ }^{11}$ Hôpital Huriez, Lille, France, ${ }^{12}$ Skanes University Hospital, Lund, Sweden, ${ }^{13}$ University Tubingen, Tubingen, Germany, ${ }^{14}$ Chaim Sheba Medical Center, Tel-Hashomer, Israel

Introduction: Allogeneic HCT with myeloablative conditioning is considered a standard of care for adults with high risk acute lymphoblastic leukemia (ALL). In recent years transplantations from unrelated donors (URD-HCT) are more frequent compared to those from HLA-identical siblings. The aim of this study was to analyze if results of myeloablative URD-HCT for patients with ALL in first complete remission (CR1) changed over time and to identify prognostic factors.

Material (or patients) and methods: 2178 patients, aged 1855 years, treated with URD-HCT in CR1 between 1993-2012 were included. The outcome was analyzed for subsequent periods (1993-2002, 2003-2007, 2008-2012) in various age groups.

Results: For the whole study population, the 2-year probability of OS increased from $51 \%$ for URD-HCT performed between 1993-2002 to $61 \%$ between 2003-2007 and 65\% between 2008-2012 $(P=0.003)$. Respective LFS rates were $43 \%, 54 \%$ and $59 \%(P=0.0001)$. Relapse incidence $(\mathrm{Rl})$ was $29 \%, 22 \%$, and $19 \%(0.006)$, while non-relapse mortality was $28 \%, 24 \%$, and $22 \%(P=0.27)$, respectively. Significant improvement of LFS rates could be demonstrated for patients aged $36-45$ years $(24 \%, 50 \%$, and $55 \%$ in subsequent study periods, $P<0.0001)$ and $46-55$ years $(31 \%, 40 \%$, and $58 \%$, respectively, $P<0.0001)$, while for younger adults the differences did not reach statistical significance. The improvement of LFS over time was particularly prominent among patients with Ph-positive ALL: $36 \%, 47 \%$, and $61 \%$ in subsequent periods $(P=0.001)$. In a multivariate analysis adjusted to time periods and restricted to patients with $\mathrm{Ph}$-negative $\mathrm{ALL}$, the risk of relapse was increased for those with high initial $W B C(H R=2.2$, $P=0.01$ ) while reduced in case of TBI-based conditioning $(\mathrm{HR}=0.46, P=0.03)$. The overall mortality was increased with increasing patient age $(\mathrm{HR}=1.22$ for each 10 years, $P=0.03)$, CMV-positive recipient status $(H R=1.51, P=0.04)$ and high initial WBC $(H R=1.89, P=0.002)$. No independent prognostic factors could be identified for Ph-positive ALL.

Conclusion: Results of myeloablative URD-HCT for adults with ALL in CR1 improved significantly over time with the overall survival rate reaching $65 \%$. The improvement is in major part related to reduced risk of relapse and is particularly prominent for 36-55 years old patients and those with Ph-positive disease. Disclosure of Interest: None declared.

\section{P690}

Successfull treatment of a child with natural killer (NK) cell lymphoma/leukemia with allogeneic hematopoeitic stem cell transplantation

S. Aytac ${ }^{1, *}$, B. Kuskonmaz ${ }^{1}$, B. Aydin ${ }^{2}$, M. Cetin ${ }^{1}$, C. Akyuz D. Uckan-Cetinkaya ${ }^{3}$

${ }^{1}$ Pediatric Hematology, ${ }^{2}$ Pediatric oncology, ${ }^{3}$ Bone marrow transplantation Unit, Hacettepe University, Ankara, Turkey

Introduction: NK cell neoplasms derived from precursor NK cells represent two distinct phenotypes, blastic NK cell leukemia/lymphoma and myeloid/NK cell precursor acute leukemia. Both of these two entity share some of those clinical features such as some immunophenotypic features and lymphoblastoid morphology but mainly differed in the involvement sites on which blastic NK cell leukemia/lymphoma exhibited more frequent involvement of lymph nodes and skin, in some way theymay overlapping. There were limited number of case reports for the childhood period in the literature and they generally showed a poor response to chemotherapy. However, it was reported that the prognosis for patients who were treated with hematopoietic stem cell transplantation was better than that for patients who were not. We present here, a case of blastic NK cell leukemia/lymphoma who was successfully treated with allogeneic hematopoeitic stem cell transplantation. Material (or patients) and methods: A 10 year-old boy was referred to Hacettepe University Children's Hospital for bilateral periorbital ecchymosis and swelling, extensive lymphadenopathy and massive splenomegaly. On physical examination, he had bilateral multiple submandibular, axillar and cervical lymph nodes up to $2 \times 2 \mathrm{~cm}$, bilateral ecchymosis and swelling in the periorbital region, hepatomegaly $(10 \mathrm{~cm}$ below the costal margin) and splenomegaly (15 cm below the costal margin). Complete blood count revealed a Hb:6.8 g/dl, WBC: 194x10^9/l

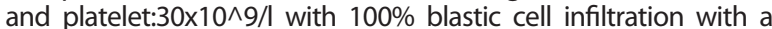
lymphoblastoid morphology, prominent nucleoli and some of them have azurophilic granules on the peripheral smear. Leukemic blasts in the bone marrow were positive for CD2, CD5,CD7,CD33,CD34 and HLA-DR. Abdominal USG did show hepatosplenomegaly with multiple lymph node groupings in the abdomen. Computarized tomography(CT) of the chest demonstrated a huge anterior mediastinal mass. Modified St Jude Total XV therapy was given however at the end of the induction $6 \%$ blasts were persist and neither splenomegaly nor thoracal mass remarkably decrease in size. Lymphoma based therapy with modified LMT-89 was started after showing a pathologic FDG uptake of spleen, lymph nodes by PET-CT which were disappeared after consolidation phase.

Results: The disease considered to refraftory to the standard chemotherapy and allogeneic hemaopoetic stem cell transplantation from a HLA identical sibling donor was performed. The conditioning regimen included TBI(1200 cGy) and Vp-16 (60 mg/ $\mathrm{kg})$. Cyclosporine A and short term methotrexate were given for graft versus host disease (GVHD) prophylaxis. Neutrophil and platelet engraftment were achieved at day +23 and +35 day, respectively. During post-transplant period grade 1 acute GVHD and mild venoocclusive disease were observed and both were resolved without any complication.The patient has been in remission for more than thirty-six months after HSCT and he is doing well with full donor chimerism.

Conclusion: There are only a few cases of HSCT in children with NK leukemia/lymphoma. Here, we report another patient with NK leukemia/lymphoma who underwent HSCT with myeloablative conditioning regimen and successful outcome with limited toxicity was achieved.

Disclosure of Interest: None declared.

\section{P691}

Allogeneic hematopoietic stem cell transplantation for acute myeloid leukemia patients in first complete remission: significant impact of the interval between diagnosis and transplantation on different outcomes

S. Ducreux 1,* , M. Sobh' ${ }^{2}$ S. Morisset ${ }^{2}$, M. Balsat ${ }^{2}$, X. Thomas ${ }^{2}$, C. Lejeune ${ }^{2}$, S. Ducastelle Leprêtre ${ }^{2}, F$. Barraco ${ }^{2}, H$. Labussière Wallet ${ }^{2}$, F. E. Nicolini' ${ }^{2}$, V. Dubois ${ }^{1}, M$. Michallet ${ }^{2}$

${ }^{1}$ Histocompatibility, EFS Rhone Alpes, ${ }^{2}$ Haematology, Lyon Sud Hospital, Lyon, France

Introduction: Allogeneic hematopoietic stem cell transplantation (allo-HSCT) is the main therapeutic option for most patients with high risk acute myeloid leukemia (AML).

Material (or patients) and methods: In order to determine whether time between diagnosis and allo-HSCT could have any impact on transplant outcomes, we screened 700 
consecutive adult AML patients diagnosed with between January 2007 and June 2014 at the Lyon Sud Hospital transplant center. Inclusion criteria were: (1) newly diagnosed $A M L$ in patients with age $\leq 65$ years, (2) AML classified in the intermediate-2 and unfavorable risk groups according to the Acute Leukemia French Association (3) patients fit for receiving chemotherapy (4) patients candidates for allo-HSCT and in first complete remission (CR1) at transplantation.

Results: Two hundred and one patients met the inclusion criteria and were enrolled in the study. Among them, 137 (68\%) received allo-HSCT of whom 83 (41\%) only received HSCT in CR1 after a median time of 143 days (range: 69-265) from diagnosis. We collected within this interval different delays for donor search, including patients HLA typing and time to unrelated donor identification when a sibling donor was not available. Patients were split into 2 groups based on their time to transplant. An "early transplant group" included 28 (33\%) patients transplanted after a median time of 108 days (range: 69-133) after diagnosis. The other $55(67 \%)$ patients transplanted after a median time of 163 days (range: 134-265) were qualified as "late transplant group". After a median follow-up of 16 months (range: 0-60) the 5 -year probability of overall survival (OS) and disease-free survival (DFS) for the whole population were respectively, $63.4 \%$ and $48.3 \%$. The cumulative incidences of non-relapse mortality (NRM) at 1 and 5 years were constant at $17.5 \%$. The multivariate analysis using proportional hazards modeling showed that conditioning regimen and sex mismatching were independent prognostic factors for DFS, with no significant impact on OS. To evaluate the long term impact of transplantation timing on OS and DFS, we performed a landmark analysis for patients surviving at 1 year post-allo-HSCT. This analysis showed that patients in the early transplant group had a higher probability of OS at 3 and 5 years with $100 \%$ survival respectively compared to the late transplantation group with $85.5 \%$ and $79.4 \%$ respectively $(P=0.09)$; accordingly, we found a significant difference in terms of DFS with $100 \%$ probability at 3 and 5 years for the early transplantation group compared to $80 \%$ and $56 \%$ respectively in the late transplantation group $(P=0.02)$.

Conclusion: These results confirm the important impact of allogeneic HSCT timing in high-risk AML patients, early alloHSCT for patients transplanted in CR1 is associated with a better OS and a very significant benefit in terms of DFS. Further analyses are ongoing including disease monitoring from diagnosis to the last follow-up to identify the potential of transplantation timing on the graft versus leukemia effect.

Disclosure of Interest: None declared.

\section{P692}

Impact of additional cytogenetic aberrations on outcome of allogeneic hematopoietic stem cell transplantation in patients with RUNX1-RUNX1T1 acute myeloid leukemia

T. L. Gindina 1,*, N. Mamaev', S. Bondarenko ${ }^{1}$, A. Alyanskiy ${ }^{1}$, A. Borovkova', S. Razumova ${ }^{\prime}$, O. Slesarchuk', O. Pirogova ${ }^{\prime}$, E. Nikolaeva ${ }^{1}$, I. Petrova ${ }^{1}$, L. Zubarovskaya ${ }^{1}$, B. Afanasyev

${ }^{1}$ R.M. Gorbacheva Memorial Research Institute of Pediatric Oncology, Hematology and Transplantation, 1st Pavlov State Medical University, Saint-Petersburg, Russian Federation

Introduction: RUNX1-RUNX1T1-positive acute myeloid leukemia (AML) is considered as a favorable group. Allo-HSCT has been used for relapsed patients (pts), although prognostic factors are still unclear and significance of additional cytogenetic aberrations (ACA) in transplanted pts is contradictory [1-3].

Material (or patients) and methods: In this study, outcomes in 25 RUNX1-RUNX1T1-AML pts, who were transplanted in a single institution between 2008 and 2015, were analyzed. All patients and transplant characteristics are listed in Table I.

Results: The median follow-up was $566(8-2127)$ days. Overall survival (OS) was 33\% (95\% Cl 14-53) and relapse free survival (RFS) was $26 \%(95 \% \mathrm{Cl} 9-45)$ at 4 years estimated with KaplanMeier method. In univariate analysis, prognostic factors associated with increased OS and RFS were age ( $>18$ vs
$<18$ years; $P=0.03, P=0.0006$, respectively), donor type (match related vs match unrelated vs haploidentical; $P=0.0003, \quad P=0.02$, respectively), the disease status at transplant (complete remission vs active disease; $P=0.0002$, $P=0.005$, respectively), the interval from diagnosis to transplant ( $<360$ vs $>360$ days; $P=0.008, P=0.9$, respectively), ACA (ACA- vs ACA+; $P=0.02, P=0.009$, respectively)(Figure 1 ), complex karyotype (CK- vs CK+; $P=0.004, P=0.0003$, respectively). In multivariate analysis, the ACA (HR-13.5; $P=0.04$ ), the donor type (HR-6.86; $P=0.01)$, the interval from diagnosis to HSCT (HR-6.80; $P=0.02$ ) remained statistically significant for OS. Moreover, age (HR-0.11;P=0.004) and the donor type (HR-4.16; $P=0.04$ ) were independent predictors of RFS.

Table 1. Patient and Transplant Characteristics

\begin{tabular}{|c|c|}
\hline Number of patients & $25(100 \%)$ \\
\hline \multicolumn{2}{|l|}{ Patient sex, $n$ (\%) } \\
\hline Male & $15(60 \%)$ \\
\hline Female & $10(40 \%)$ \\
\hline Age at $\mathrm{HSCT}$, median, (range) years & $20.2(2-58)$ \\
\hline \multicolumn{2}{|l|}{ Age group } \\
\hline$\leq 18$ yo & $12(48 \%)$ \\
\hline$\geq 18$ yo & $13(52 \%)$ \\
\hline \multicolumn{2}{|l|}{ Cytogenetics, n (\%) } \\
\hline $\begin{array}{l}\mathrm{t}(8 ; 21) \text { without additional cytoge- } \\
\text { netic aberration }\end{array}$ & $12(48 \%)$ \\
\hline $\begin{array}{l}\mathrm{t}(8 ; 21) \text { with additional cytogenetic } \\
\text { aberration }\end{array}$ & $13(52 \%)$ \\
\hline Complex karyotype - & $16(64 \%)$ \\
\hline Complex karyotype + & $9(36 \%)$ \\
\hline \multicolumn{2}{|l|}{ Time from diagnosis to HSCT, $n$ (\%) } \\
\hline$<360$ days & $14(56 \%)$ \\
\hline$>360$ days & $11(44 \%)$ \\
\hline \multicolumn{2}{|l|}{ Clinical stage at HSCT, $n$ (\%) } \\
\hline$C R$ & $13(52 \%)$ \\
\hline Active disease & $12(48 \%)$ \\
\hline \multicolumn{2}{|l|}{ HSC source, $n$ (\%) } \\
\hline Bone marrow & $13(52 \%)$ \\
\hline Peripheral blood & $11(44 \%)$ \\
\hline Both & $1(4 \%)$ \\
\hline \multicolumn{2}{|l|}{ Conditioning regimen, $n$ (\%) } \\
\hline MA & $12(48 \%)$ \\
\hline Non-MA & $13(52 \%)$ \\
\hline \multicolumn{2}{|l|}{ Donor type, $n$ (\%) } \\
\hline HLA-id sibling & $7(28 \%)$ \\
\hline Matched unrelated & $12(48 \%)$ \\
\hline Haploidentical & $6(24 \%)$ \\
\hline
\end{tabular}

Conclusion: The study showed the ACA to be independent prognostic factor for RUNX1-RUNX1T1-AML in transplanted pts. Since cytotoxic conditioning regimen may be responsible for production of new ACA, substitution of more toxic alkylating agents to less toxic would be available.

\section{References:}

1. Mosna F., Papayannidis C., Martinelli G. Am J Hematol 2015; doi:10.1002/ajh.24000

2. Yoon J.H., Kim H.J., Jeon Y.W. Bone marrow transplant 2014; doi:10.1038/bmt2014.180

3. Shin H.J., Kim H.J., Sohn S.K. Jpn J Clin Oncol 2010; 40(6)556566

Disclosure of Interest: None declared. 


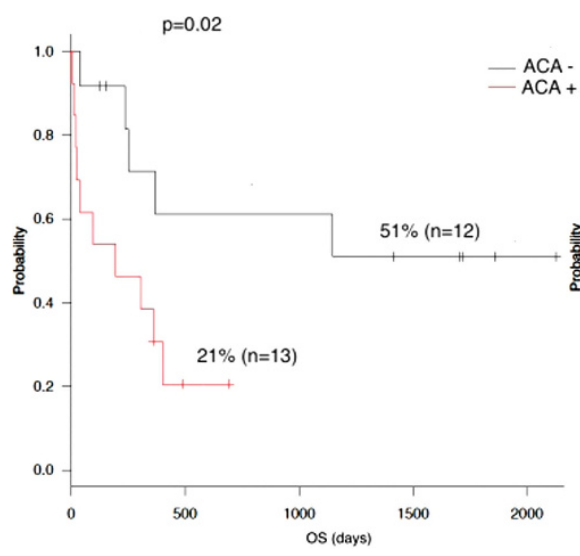

P693

Efficiency of 5-azacytidine administration after allogeneic hematopoietic stem cell transplantation in acute myeloid leukemia and myelodisplastic syndrome

V. Ovechkina ${ }^{1, *}$, S. Bondarenko ${ }^{1}$, E. Morozova ${ }^{1}$, I. Moiseev ${ }^{1}$, O. Slesarchuk ${ }^{1}$, S. Razumova ${ }^{1}$, A. Osipova ${ }^{1}$, L. Zubarovskaya ${ }^{1}$, B. Afanasyev ${ }^{7}$

${ }^{1}$ Raisa Gorbacheva Memorial Research Institute of Children Oncology, Hematology and Transplantation, First Pavlov State Medical University of St.Petersburg, St Petersburg, Russian Federation

Introduction: The aim of this study was to assess the effect of 5-azacitidine (5-aza) administration as prophylaxis and treatment of disease relapse after allogeneic hematopoietic stem cell transplantation (allo-HSCT) in patients (pts) with acute myeloid leukemia (AML) and myelodisplastic syndrome (MDS). Material (or patients) and methods: We analyzed 136 (pts) with AML and MDS, who underwent allo-HSCT. Among them, 68 patients received 5-aza after allo-HSCT as prophylaxis or preemptive therapy (5-aza group). At the beginning of 5-aza treatment all patients were in the morphological remission after allo-HSCT. Patient's demographics and clinical characteristics are shown in the Table 1. There is no significant difference between 5-aza group and the control group. Prophylactic 5-aza was administered in pts with high disease relapse risk $(43,63 \%)$ : active disease at the moment of alloHSCT, unfavorable cytogenetic or minimal residual disease (MRD), registered before allo-HSCT. Preemptive 5-aza was administered in pts, who had the minimal residual disease or dropping chimerism after allo-HSCT $(25,37 \%)$. The median time for the administration of 5-aza in the prophylactic group was day+94 (28-180) after allo-HSCT, in the preemptive group - day+112 (27-721); the main criteria for the 5-aza administration was Leu $>1.0 \times 10^{9} / \mathrm{l}, \operatorname{Tr}>20 \times 10^{9} / \mathrm{l}$, absence of severe infection complications and renal or hepatic failure. 5-aza was injected subcutaneously $35 \mathrm{mg} / \mathrm{m} 2 /$ daily, for 5 or 7 consecutive days of 28- day cycle, 3 (1-8) cycles. For 24 (35\%) patients 5 -aza treatment was combined with donor lymphocyte infusion (DLI). Indications for DLI were MRD or decreasing donor chimerism after allo-HSCT.

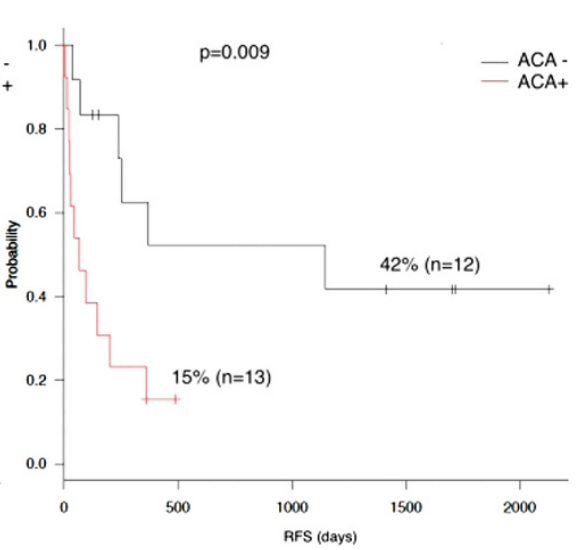

Results:

\begin{tabular}{|c|c|c|c|}
\hline \multirow[t]{2}{*}{ Characteristic } & \multicolumn{2}{|c|}{$\mathrm{N}$} & \multirow[t]{2}{*}{$\mathrm{P}$} \\
\hline & \multicolumn{2}{|c|}{$\begin{array}{c}\text { 5-aza group } \\
\text { Comparison group }\end{array}$} & \\
\hline No. of patients & 68 & 68 & 0.4 \\
\hline \multicolumn{4}{|l|}{ Sex } \\
\hline Male & 40 & 35 & 0.9 \\
\hline Female & 28 & 33 & \\
\hline Age & $28(1-68)$ & $27(2-60)$ & \\
\hline \multicolumn{4}{|l|}{ Diagnosis } \\
\hline AML, de novo & 51 & 48 & 0.7 \\
\hline $\mathrm{AML}$, secondary & 10 & 14 & \\
\hline MDS, de novo & 3 & 4 & \\
\hline MDS, secondary & 4 & 2 & \\
\hline \multicolumn{4}{|l|}{ Karyotype } \\
\hline Favorable & 8 & 5 & 0.3 \\
\hline Intermediate & 37 & 45 & \\
\hline Adverse & 23 & 18 & \\
\hline \multicolumn{4}{|l|}{ Disease status at alloHST } \\
\hline CR1 & 24 & 23 & 0.2 \\
\hline CR2 & 6 & 13 & \\
\hline Relapse & 28 & 18 & \\
\hline Induction failure & 9 & 11 & \\
\hline Untreated & 1 & 3 & \\
\hline \multicolumn{4}{|l|}{ Conditioning } \\
\hline Standard-dose & 23 & 24 & 0.9 \\
\hline Dose-reduced & 45 & 44 & \\
\hline \multicolumn{4}{|l|}{ Donor type } \\
\hline HLA-identical related & 17 & 24 & 0.4 \\
\hline HLA-identical unrelated & 33 & 24 & \\
\hline HLA-mismatched unrelated & 8 & 10 & \\
\hline Haploidentical & 10 & 10 & \\
\hline \multicolumn{4}{|l|}{ Graft source } \\
\hline BM & 36 & 38 & 0.1 \\
\hline PBSV & 32 & 26 & \\
\hline Mixed & 0 & 4 & \\
\hline \multicolumn{4}{|l|}{$D L I$} \\
\hline Yes & 24 & 22 & 0.4 \\
\hline No & 44 & 46 & \\
\hline
\end{tabular}




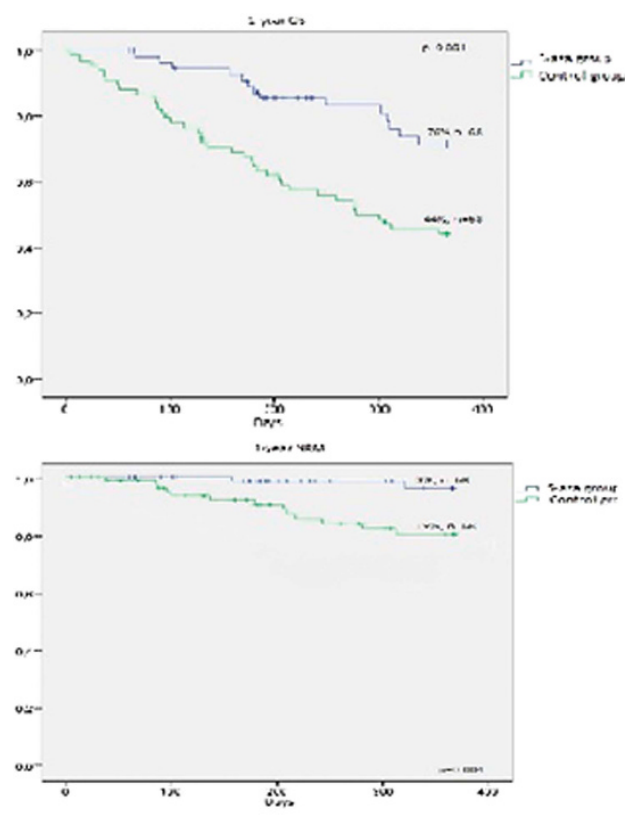

Conclusion: Based on our preliminary analysis 5-aza is effective for prevention of relapse and improvement of overall and disease-free survival in pts with high risk of disease relapse. This drug has low toxicity and can be used in early post-transplant period of allo-HSCT. Further larger prospective, randomized trials are needed for more confirmed conclusions. Disclosure of Interest: None declared.

P694

Donor-Recipient Combinations rather than HSC Sources Significantly Affect Long-term Survival of Patients Transplanted for Acute Myeloid Leukemia

W. Arcese ${ }^{1, *}$ on behalf of Rome Transplant Network, P. Di Bartolomeo ${ }^{2}$, R. Cerretti ${ }^{1}$, S. Santarone ${ }^{2}$, L. Cudillo ${ }^{1}$, G. De Angelis ${ }^{1}$, G. Papalinetti ${ }^{2}$, P. Accorsi ${ }^{2}$, A. Venditti ${ }^{1}$, F. Buccisano ${ }^{1}$, T. Bonfini ${ }^{2}$, P. Olioso ${ }^{2}$, P. De Fabritiis ${ }^{3}$, T. Dentamaro $^{3}$, G. Avvisati $^{4}$, M. C. Tirindelli ${ }^{4}$, A. Mengarelli ${ }^{5}$, F. Marchesi ${ }^{5}$, A. Chierichini ${ }^{6}$, B. Anaclerico ${ }^{6}$, A. Tafuri ${ }^{7}$, A. Ferrari ${ }^{7}$, M. Andreani $^{8}$, S. Miccichè ${ }^{7}$, I. Mangione ${ }^{7}$, F. Di Piazza ${ }^{7}$, F. Lo-Coco ${ }^{1}$, S. Amadori ${ }^{1}$, A. Picardi ${ }^{1}$ ${ }^{1}$ Hematology and Stem Cell Transplant Unit, "Tor Vergata" University, Rome, ${ }^{2}$ Bone Marrow Transplant Center, Department of Hematology, Spirito Santo Hospital, Pescara, ${ }^{3}$ Hematology, Sant' Eugenio Hospital, ${ }^{4}$ Hematology, University Campus BioMedico, ${ }^{5}$ Hematology, Regina Elena National Cancer Institute, ${ }^{6}$ Hematology, San Giovanni-Addolorata Hospital, ${ }^{7}$ Hematology, Sant'Andrea Hospital, "Sapienza" University, ' ${ }^{2}$ aboratory of Immunogenetics and Transplant Biology, IME Foundation, Policlinic of Tor Vergata, Rome, Italy

Introduction: Similar probabilities of survival have been reported for patients transplanted from HLA Id-siblings (Idsib), MUD or Haplo donors. However, all reported studies are retrospective and include patients for whom criteria of eligibility and donor selection were not predefined. We report here the results of an analysis conducted by donor/recipient (D/R) characteristics on 101 adult patients with acute myeloid leukemia (AML) prospectively transplanted in high-risk CR1 or CR2 according to a uniform transplant policy. Patients were enrolled in the JACIE accredited transplant programs of Rome Transplant Network (RTN: $n=69$ ) and Pescara Transplant Center (PTC: $n=32$ ).

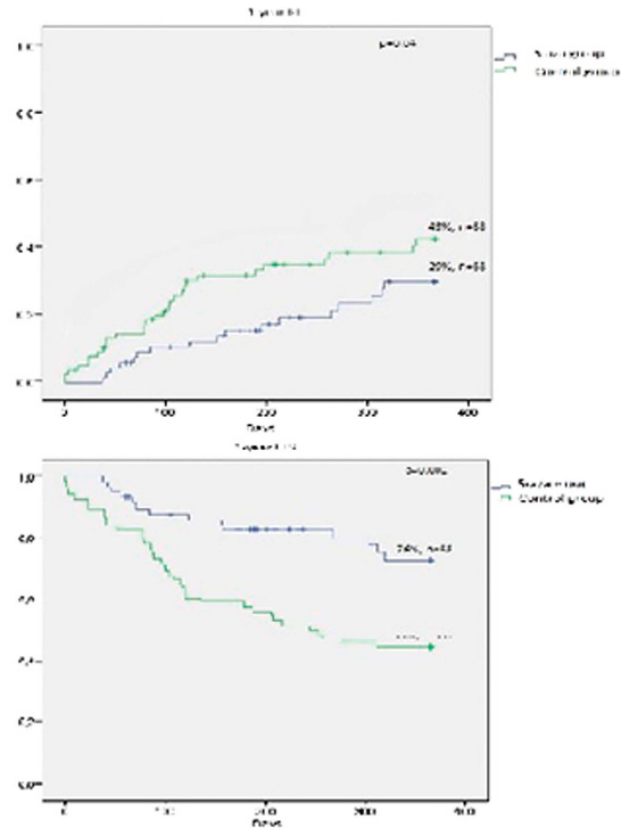

Material (or patients) and methods: According to established criteria, the hierarchy of donor choice was as follows: 1) HLA identical sibling; 2) Matched Unrelated Donor (MUD; 3) Umbilical Cord Blood (UCB); 4) unmanipulated bone marrow haploidentical donor. The combination of Thiotepa, Busulfan and Fludarabine (TBF) as myeloablative conditioning (MAC) was the same for all patients, GVHD prophylaxis adapted to each categories of transplant (Id-sib, MUD, UCB, Haplo) was uniform, anti-infective management, supportive care and transfusion policy were identical for all patients. Because of the lowest patient number, recipients of UCB were excluded from the analysis.

Results: From January 2008 to December 2014, 101 consecutive patients with AML in high-risk CR1 $(n=73)$ or CR2 $(n=28)$ conditioned with an identical MAC TBF regimen were transplanted from Id-sib $(n=27)$, MUD $(n=32)$ or Haplo $(n=42)$ donors. For these 101 patients, the cumulative incidence of III-IV grade acute GVHD was $8 \pm 3 \%$, of TRM $19 \pm 4 \%$ and of relapse $23 \pm 4 \%$ without significant differences between the 3 donor sources. With a median follow-up of 3 (range, 0.3-6.8) years, the 6-yr probability of overall (OS) survival was $65 \pm 5 \%$, without significant difference between patients transplanted in CR1 and CR2 (66 $\pm 6 \%$ vs $62 \pm 10 \%$; $P=\mathrm{ns})$. The $6-\mathrm{yr}$ OS by donor was $75 \pm 9 \%$ for Id-sibs, $67 \pm 9 \%$ for MUD and $58 \pm 8 \%$ for Haplo recipients $(P=n s)$. The results were analyzed by $D / R$ combinations known to influence outcomes such as age, sex and CMV status. The median patient and donor age was 42 (range, 16-59) and 38 years (range, 18-70) respectively. Although not significant, the 6-yr OS of patients $(n=28)$ with younger D/R combination ( $D<38 y-R<42 y)$ was higher $(79 \pm 9 \%)$ than that observed in the 3 other $(n=73) \mathrm{D} / \mathrm{R}$ combinations $(59 \pm 6 \%)$. The $4 \mathrm{D} / \mathrm{R}$ sex combinations were homogeneously distributed among Id-sib, MUD and Haplo recipients: for 52 sex mismatched patients, the $6-y r$ OS was $76 \pm 6 \%$, significantly higher than $53 \pm 8 \%$ observed for 49 sex matched patients $(P=0.05)$. Finally, the 6-yr OS by D/R CMV status was $72 \pm 6 \%$ for 67 D/R CMV matched and $47 \pm 11 \%$ for $\mathrm{D} / \mathrm{R}$ CMV mismatched patients $(P=0.06)$. In multivariate analysis, the factors influencing survival were $\mathrm{D} / \mathrm{R}$ age $(P=0.02)$, sex $(P=0.005)$ and CMV status $(P=0.04)$. The donor source did not affect survival. 

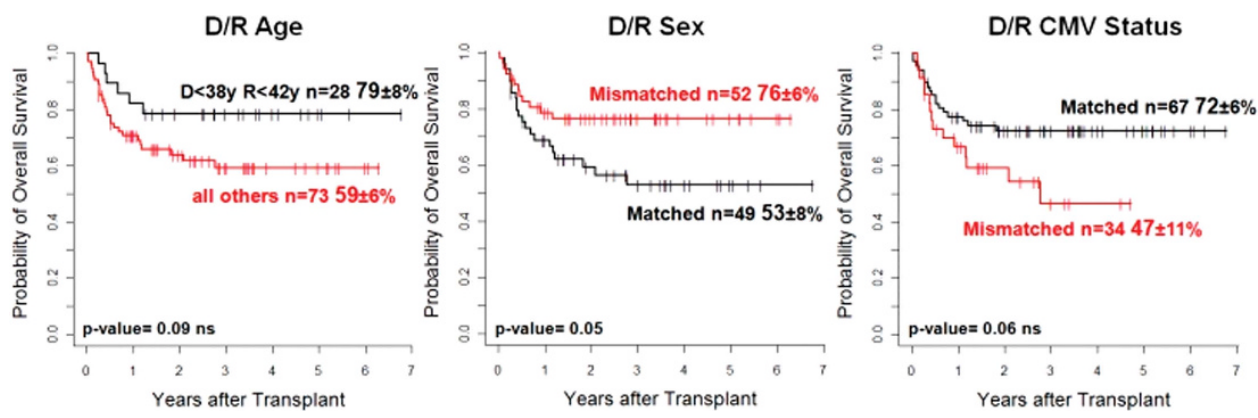

Conclusion: For AML patients transplanted in high-risk CR1 or $C R 2$, the HLA matching is unlikely to remain the first criterion for donor identification. Other factors known to affect the transplant outcomes such as $\mathrm{D} / \mathrm{R}$ age, sex and CMV status combined together should drive the search for the best donor, who might be found in one of the 3 donor sources: Id-sib, MUD or Haplo. This proposal should be tested in a prospective study.

Disclosure of Interest: None declared.

\section{P695}

Allogeneic stem cell transplantation for elderly patients with intermediate-risk cytogenetic acute myeloid leukemia and internal tandem duplication of FLT3; a study from the Acute Leukemia Working Party of the EBMT X. Poiré ${ }^{1, *}$, M. Labopin ${ }^{2}$, J. Passweg ${ }^{3}$, C. Craddock ${ }^{4}$, D. Blaise C $^{5}$ J. J. Cornelissen ${ }^{6}$, L. Volin', N. H. Russel ${ }^{8}$, G. Socié ${ }^{9}$, M. Michallet $^{10}$ N. Fegueux ${ }^{11}$, P. Chevallier ${ }^{12}$, G. Stuhler ${ }^{13}$, N. Ifrah ${ }^{14}$, M. Mohty ${ }^{15^{\prime}}$, J. Esteve ${ }^{16}$, A. Nagler ${ }^{17}$

${ }^{1}$ Hematology, Cliniques Universitaires Saint-Luc, Brussels, Belgium, ${ }^{2}$ Acute Leukemia Working Party of the EBMT, Paris, France, ${ }^{3}$ Hematology, University Hospital, Basel, Switzerland, ${ }^{4}$ Hematology, Queen Elisabeth Hospital, Birmingham, United Kingdom, ${ }^{5}$ Centre de Recherche en Cancérologie de Marseille, Institut Paoli Calmettes, Marseille, France, ${ }^{6}$ Daniel den Hoed Cancer Centre, Erasmus, Rotterdam, Netherlands, "7tem Cell Transplantation Unit, HUCH Comprehensive Cancer Center, Helsinki, Finland, ${ }^{8}$ Hematology, Nottingham City Hospital, Nottingham, United Kingdom, ${ }^{9} \mathrm{Hema}$ tology, Hôpital St-Louis, Paris, ${ }^{10}$ Hematology, Centre Hospitalier Lyon Sud, Lyon, ${ }^{11}$ Hematology, CHU Lapeyronie, Montpellier, ${ }^{12}$ Hematology, CHU Nantes, Nantes, France, ${ }^{13}$ Deutsche Klinik für Diagnostik, KMT Zentrum, Wiesbaden, Germany, ${ }^{14}$ Service des Maladies du Sang, CHRU, Angers, ${ }^{15}$ Hematology, Hôpital StAntoine, Paris, France, ${ }^{16}$ Hematology, Institute of Hematology \& Oncology, Barcelona, Spain, ${ }^{17}$ Hematology, Chaim Sheba Medical Center, Tel-Hashomer, Israel

Introduction: The presence of an internal tandem duplication of FLT3 (FLT3-ITD) confers a higher risk of relapse and is now a current indication of allogeneic stem cell transplantation (SCT) in patients with intermediate-risk cytogenetic acute myeloid leukemia (IRC-AML) in first complete remission (CR1). Most studies encouraging this strategy have been performed in patients below 60 year-old, after a myeloablative conditioning (MAC) and using a sibling donor. Because age remains associated with a worse outcome after SCT, we decided to analyse outcomes of SCT in patients aged 60 year-old or older with IRC-AML and FLT3-ITD.

Material (or patients) and methods: Using the EBMT registry, we selected de novo acute myeloid leukemia (AML) harboring IRC-AML and FLT3-ITD in patients transplanted from a related or matched unrelated donor ( $9 / 10$ or $10 / 10)$ between January 2000 and July 2014.
Results: Two hundred and five patients have been allocated. Median age at the time of SCT was 64 (range, 60-75) year-old and median follow-up was 20 (range, 2-139) months. Ninetyfour percent of the patients had a good performance status (Karnofsky at SCT $\geq 80 \%$ ). Most patients had a normal karyotype at diagnosis $(90 \%$ versus $10 \%$ with other intermediate-risk karyotype) and NPM1 status was reported in 131 patients out of which $100(76 \%)$ were mutated. Thirtyfour patients received a MAC, 142 had a reduced-intensity conditioning (RIC) and 29 a non-myeloablative conditioning (NMA). One hundred forty-six patients received their SCT in CR1, 24 in second remission (CR2) and 35 in more advanced stage of the disease, respectively. The 2-year leukemia-free survival (LFS) was $52 \%$ in patients in CR1, $17 \%$ in those in CR2 and $11 \%$ in patients with advanced disease, respectively $(P<0.005)$. Similarly, the 2 -year overall survival (OS) was $54 \%$ in CR1,24\% in CR2 and $11 \%$ in advanced disease, respectively $(P<0.005)$. The 2 -year non-relapse mortality (NRM) for the all cohort was $20 \%$. The cumulative incidence of grade II to IV acute graft-versus-host disease (GvHD) was 24\% and that of chronic GvHD was 30\%.

In multivariate analysis, disease status at SCT was the most powerful predictor of worse LFS and OS. Age, as a continuous variable, was not significantly associated with outcomes. Donor type (unrelated versus sibling donor) and donor CMV positivity correlated with worse OS and higher NRM. Next, we performed a second analysis focusing on patients transplanted in CR1. A multivariate analysis performed in this subgroup showed that age, as a continuous variable, did not translate into worse LFS, OS or NRM. While interval from diagnosis to CR1 was significantly associated with LFS, being $63 \%$ in patients achieving CR1 in less than 43 days and $45 \%$ in patients achieving CR1 in more than 43 days, respectively $(P<0.005)$. NMA conditioning was associated with a trend to a worse OS.

Conclusion: Allogeneic SCT in elderly ( $\geq 60$ up to 75 year-old) patients with IRC-AML harboring the FLT3-ITD mutation, similar to current strategy in younger patients, appears as a good treatment strategy if performed in CR1, independently of age, with somewhat inferior outcome in transplants from unrelated donors. Nevertheless, indication for transplantation should still be evaluated taking into account donor type, co-morbidities and performance status.

Disclosure of Interest: None declared.

\section{P696}

Induction remission for refractory acute lymphoblastic leukemia: Role of interleukin-2 priming chemotherapy

C. Zhang ${ }^{1}$, X.-H. Chen ${ }^{1}$, S.-J. Yang ${ }^{1}$, X.-Q. Luo ${ }^{1}$, Y. Liu ${ }^{1}$, L. Gao', L.Gao', P. Wang ${ }^{1}$, P.-Y. kong ${ }^{1}$, A.-H. Sun ${ }^{1}, X^{\prime}$ X.-G. Peng ${ }^{\prime}$, X. Zhang ${ }^{i, *}$

${ }^{7}$ Department of hematology, XINQIAO HOSPITAL,THIRD MILITARY MEDICAL UNIVERSITY, Chongqing, China 
Introduction: Outcome of allogeneic hematopoietic stem cell transplantation is very poor when a patient with refractory acute lymphoblastic leukemia (ALL) is not in complete remission (CR). The quiescent and self-renewal characteristics of leukemia stem cells contribute to drug resistance. Interleukin-2 (IL-2) plays a critical role in the progression of lymphocytes from the G0 to S phase of the cell cycle. Whether IL-2 priming can increase the rate of reinduction remission with cell cycle-specific cytotoxic agents in refractory ALL is still unknown. This is study is to explore the role of IL-2 priming in increasing the rate of reinduction remission with cell cyclespecific cytotoxic agents in refractory ALL.

Material (or patients) and methods: The cell lines were cultured with IL-2 in vitro, the ratio of the S phase of cell cycle was detected. The apoptosis of cell lines cultured with IL-2 treated with cell cycle-specific drugs was observed. The eight patients with refractory ALL were treated with cell cyclespecific drugs with IL-2 priming, the CR was observed and the primary mechanism was explored.

Results: IL-2 priming increased the ratio of cells in the S phase of the cell cycle and TNF- $\beta$ in the cell lines of ALL and in the patients with refractory ALL. The percentage of apoptosis and necrosis significantly increased when the cell cycle-specific drugs were added to the cell lines. The rate of complete remission for patients reached $62.5 \%$ after chemotherapy with cell cycle-specific drugs with IL-2 priming. The ratio of $\mathrm{CD}^{+} /$ $\mathrm{CD}^{+}, \mathrm{CD}^{+} \mathrm{T}$ cells and $\mathrm{CD} 56^{+}$NK cells significantly increased in the patients.

Conclusion: It is a new strategy to reinduction remission of refractory ALL with IL-2 priming chemotherapy possibly via its influence on the cell cycle, T-cell subtype and cytokines.

Disclosure of Interest: None declared.

P697

Pre-emptive therapy with IFN- $a-2 b$ for Acute Leukemia patients with high risk of relapsing tendency post alloHSCT

A. Wang ${ }^{1}$, F. Chen ${ }^{1}$, X. Ma ${ }^{1}$, A. Sun ${ }^{1}$, X. Zhu ${ }^{1}$, H. Qiu ${ }^{1}$, Z. Jin ${ }^{1}$, S. Xue ${ }^{1}, D . W u^{1}, X$. Tang $^{1, *}$

${ }^{1}$ The First Affiliated Hospital of Soochow University, Jiangsu Institute of Hematology, Suzhou, China

Introduction: To determine the efficacy and safety of IFN- $a-2 b$ pre-emptive therapy for acute leukemia(AL) patients with relapsing tendencies after allogeneic hematopoietic stem cell transplantation (allo-HSCT).

Material (or patients) and methods: Retrospectively analyzed 986 acute leukemia patients undergoing allo-HSCT from Jan,2006 to Mar,2014 in our hospital. After allo-HSCT, 986 AL patients were periodically monitored the minimal residual disease(MRD) including: bone marrow smear, leukemiaassociated immunophenotype (LAIP), leukemia specific or related fusion genes, and donor chimerism through multiparameter detection to evaluate disease status. Patients were given IFN- a -2b 3 million units / day by subcutaneous injection for preemptive treatment once a relapse tendency was detected, such as: increasing proportion of blast in bone marrow between $3-5 \%$, or MRD $>1.0 \times 10^{-3}$, or leukemia specific fusion gene transfrom negative to positive, or dynamic incressing copy number of WT1 more than 200 copies $/ 10^{4}$ abl, or decreasing of donor chimerism( $\leq 90 \%)$. There were 98 patients who presented increasing tendency of MRD and were enrolled in this study. Among them, 31 patients received IFN$a-2 b$ pre-emptive therapy, and 67 patients received non-IFN-a$2 \mathrm{~b}$ therapy such as: withdraw immunosupressant, traditional DLI or DC-CIK immunotherapy.

Results: There were no significant differences in disease characteristics between two groups. For the 31 patients who received IFN- $a-2 b$ pre-emptive therapy (IFN group), the median time of IFN-atreatment was 60 days (range: 5-720 days), Twenty five patients had responsed to the treatment without progressing to hematological relapse (response rate $80.6 \%$ ). 2 patients developed to hematological relapse again after temporary response; 3 patients had no response and eventually progressed to hematological relapse. Regarding 67patients who received non-IFN- $a-2 b$ therapy (non IFN group), 22 patients responsed to the treatment (RR $32.8 \%$ ), 45 patients failed to the treatment and progressed to hematological relapse at a median time of 35 (range: 6-940) days, There was significant difference of RR between two group $(P=0.000)$. 31 patients of IFN group tolerate well and no patient terminated therapy due to side effects. During the treatment of IFN, 18 patients(58.1\%) developed GVHD: 6 patients (19.4\%) with aGVHD and 14(45.2\%) with limited cGVHD. The median follow-up time was 21(4.5-78.5) months. 22 of 31 cases of IFN group maintained disease-free survival. The 5-year overall survival rate (OS) and the leukemia-free survival rate (LFS) of IFN group were $47.0 \% \pm 13.9 \%$ and $38.7 \% \pm 13.1 \%$ respectively. However, the $5-\mathrm{yr}$ OS and LFS of non IFN group were $14.5 \% \pm 10.7 \%$ and $12.5 \% \pm 9.4 \%$ respectively. The difference were significantly $(P=0.000, P=0.002$ respectively). Patients with GVHD had significantly better response than patients without GVHD (88.9\% vs $53.8 \%$, $P=0.043, P<0.05)$.

Conclusion: IFN- $a-2 b$ pre-emptive therapy can effectively prevent high-risk patients with relapsing tendencies for disease progression post allo-HSCT. Further large-scale investigation is warranted.

Disclosure of Interest: None declared.

\section{P698}

Outcome Predictors for Adult Patients with Acute Myeloid Leukemia Undergoing Allogeneic Hematopoietic Stem Cell Transplantation

J.-L. Tang ${ }^{1,2}$, X.-W. Liao ${ }^{3, *}$ on behalf of Taiwan Blood and Marrow Transplantation Registry Group

${ }^{1}$ Tai Cheng Stem Cell Therapy Center, National Taiwan University, ${ }^{2}$ Department of Internal Medicine, National Taiwan University Hospital, ${ }^{3}$ Tai Cheng Stem Cell Therapy Center, National Taiwan University, Taipei, Taiwan, Province of China

Introduction: We validated the utility of EBMT risk score and cytogenetics to predict the outcome after allo-HSCT for adult $A M L$ in previous study. In the present study, we aim to incorporate EBMT risk score, cytogenetics at diagnosis, the Hematopoietic Cell Transplantation-Specific Comorbidity Index $(\mathrm{HCT}-\mathrm{Cl})$, and other known predictors of outcome into a simple predictive score with improved prediction of overall survival (OS), non-relapse mortality (NRM), and cumulative incidence of relapse (CIR) for adult AML patients who underwent Allo-HSCT in the Taiwan Society of Blood and Marrow Transplantation registry (TBMTR) cohort data.

Material (or patients) and methods: There were 468 patients in the nationwide cohort from TBMT registry during 2009-2013 (development cohort), and were 255 in a cohort of National Taiwan University Hospital during 1992-2008 (validation cohort). The sensitivity and specificity of the predictive scores in discrimination between the good risk and poor risk of clinical outcome was evaluated using the receiver operating characteristic (ROC) curves. The area under ROC curves (AUROC) of the development cohort and the validation cohort were compared. Results: With a median follow-up of 39 months (ranges 1$79 \mathrm{~m}$ ), the estimated 3-year OS and NRM were $44.9 \%$ and $30.8 \%$ for the whole cohort. The independent risk predictors for OS (AUROC $=0.6840,95 \% \mathrm{Cl}: 0.63-0.74$,Fig 1a) were EBMT risk score, unfavorable-risk cytogenetics, WBC count, and delayed remission. The risk predictors for NRM (AUROC = 0.6719, 95\% Cl: 0.61-0.73,Fig 1c) included modified EBMT risk score, unfavorable-risk cytogenetics, HCT-Cl, and donor age. The EBMT risk score, unfavorable-risk cytogenetics, WBC count, delayed remission, as well as reduced-intensity conditioning regimen were predictors for CIR (AUROC $=0.6283,95 \% \mathrm{Cl}$ : $0.57-0.68$,Fig 1e). Moreover, the AUROC of OS, NRM and CIR in the validation cohort was $0.7114(95 \% \mathrm{Cl}$ : $0.64-0.78$,Fig $1 \mathrm{~b})$, 0.5994 (95\% Cl: 0.51-0.69,Fig 1d), and 0.6393 (95\% Cl: 0.55-0.72, Fig $1 \mathrm{f}$ ), respectively. 


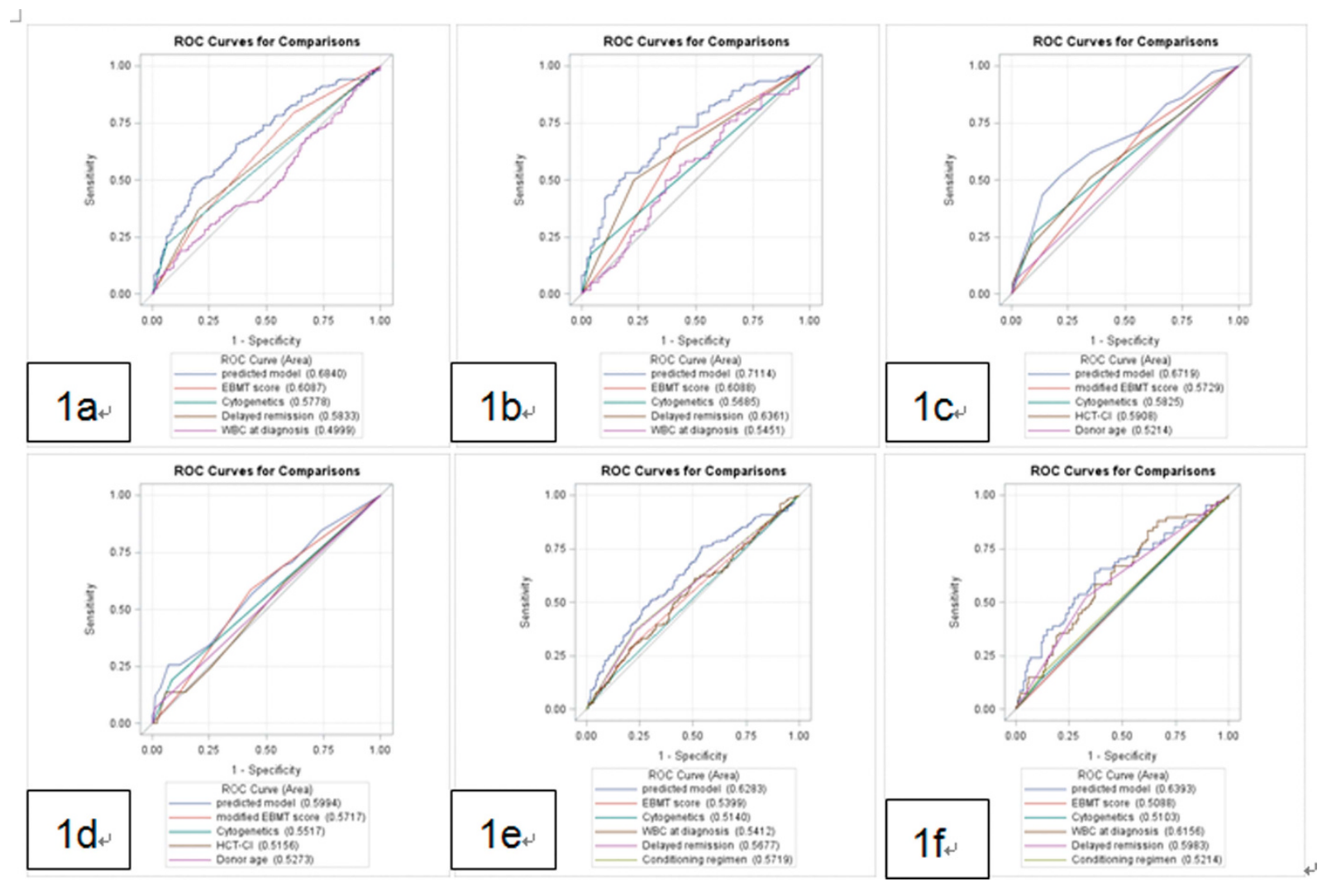

Conclusion: The EBMT risk score and cytogenetics improved the AUROC of OS, NRM, and CIR compared with EBMT risk score as a single predictor. In addition, high WBC count and delayed remission were independent risk factor for both OS and $\mathrm{CIR}$. Modified EBMT risk score and $\mathrm{HCT}-\mathrm{Cl}$ were important predictors for NRM. Modification of the current EBMT risk scoring system with cytogenetic and molecular alteration is warranted in the future study.

References: 1. Matsumoto $\mathrm{K}$, Yamamoto $\mathrm{W}$, Ogusa $\mathrm{E}_{\mathrm{d}}$ Ishigatsubo $\mathrm{Y}$, Kanamori $\mathrm{H}$. Prognostic index for relapsed acute leukemia after allogeneic stem cell transplant. Leukemia \& Lymphoma, 2014; 55(12): 2808-2812.

2. Sorror ML, Storb RF, Sandmaier BM, Maziarz RT, Pulsipher $M A$, Maris MB, et al. Comorbidity-Age index: a clinical measure of biologic age before allogeneic hematopoietic cell transplantation. J Clin Oncol, 2014; 32(29): 3249-3256.

3. Raimondi R, Tosetto A, Oneto R, Cavazzina R, Rodeghiero F, Bacigalupo $A$, et al. Validation of the hematopoietic cell transplantation-specific comorbidity index: a prospective, multicenter GITMO study. Blood, 2012; 120(6): 1327-1333.

4. Macmillan ML, DeFor TE, Weisdorf DJ. What predicts high risk acute graft-versus-host disease (GVHD) at onset?: identification of those at highest risk by a novel acute GVHD risk score. Br J Haematol, 2012; 157(6): 732-741.

5. Gratwohl, A., The EBMT risk score. Bone Marrow Transplant 2012; 47(6):749-756.

Disclosure of Interest: None declared.

Haploidentical Blood and Marrow Transplantation with GIAC/GIAC-3 protocol in Acute Lymphoblastic Leukemia: A Single-Center Study of $\mathbf{2 0 7}$ cases

Y.-L. Zhao ${ }^{1, *}$, T. Wu ${ }^{1}$, Y. Lu ${ }^{1}$, X.-Y. Cao ${ }^{1}$, D.-Y. Liu ${ }^{1}$, M. Xiong ${ }^{1}$ J.-P. Zhang ${ }^{1}$, Z.-J. Wei ', J.-R. Zhou', C.-R. Tong ${ }^{1}$, X. Zhang ${ }^{3}$, H.-X. Liu ${ }^{3}$, H. Wang ${ }^{3}$, T. Wang ${ }^{3}$, S.-Q. Ji ${ }^{1}$, D. $-P$. Lu $^{1}$ on behalf of Hebei Yanda Ludaopei Hospital

${ }^{7}$ Bone Marrow Transplantation, ${ }^{2}$ Hematology and Immunotherapy, ${ }^{3}$ Hematopathology, Hebei Yanda Ludaopei Hospital, Langfang, China

Introduction: Haploidentical blood and marrow transplantation (haplo-BMT) has achieved comparable outcomes with identical sibling transplant with GIAC regimen (Dao-Pei Lu et al., Blood 2006; 107:3065). Our previous study has shown that the third party cell co-infusion in haplo-BMT (GIAC-3 regimen) could significantly reduce aGVHD and transplantrelated mortality (TRM). Objective: To investigate the outcomes of our haplo-BMT with GIAC/GIAC-3 protocol in acute lymphoblastic leukemia (ALL).

Material (or patients) and methods: Between April 2012 and December 2014, consecutive 207 patients with ALL who underwent haplo-BMT in our hospital were analyzed retrospectively. The median age was 16 (3 to 48) years old. Transplants at CR1(high risk or persistent MRD), $\geq C R 2$ or advanced disease were 92 (44.4\%), 69 (33.3\%), 46 (22.2\%), respectively. High-resolution HLA typing for A, B, C, DRB1 and DQB1 were done. Donors were from sibling $45(21.7 \%)$ or parent $155(74.9 \%)$ or child $6(2.9 \%)$ or other relative $1(0.5 \%)$. All patients received unmanipulated bone marrow (BM) and peripheral blood stem cells as graft after myeloablative conditioning plus ATG. Majority patients received TBICybased regimen. For refractory/relapsed diseases, individualized chemotherapy followed by conditioning was administered. 
Cyclosporine/tacrolimus, short-term methotrexate, and mycophenolate mofetil were employed for GVHD prophylaxis. The $3^{\text {rd }}$ party cells with either haplo-BM 124 (59.9\%) or unrelated cord blood 66 (31.9\%) were infused right after haplo-BMT. The patients with persistent MRD were interfered by immunosuppressant withdrew, adoptive immunotherapy with cytokine induced killer or NK cells or donor lymphocyte infusion.

Results: The median MNCs were 8.49 (3.61 to 21.3$) \times 10^{8} / \mathrm{kg}$ and the median CD34 ${ }^{+}$cells were $4.15(1.66$ to 17.2$) \times 10^{6} / \mathrm{kg}$. All patients but 1 achieved durable engraftment. The cumulative incidences of grade II to IV and grade III to IV aGVHD were $32.6 \pm 3.3 \%, 20 \pm 2.6 \%$, respectively. The cumulative incidences of CGVHD and extensive CGVHD were $53.4 \pm 4.0 \%$, 32.9 $\pm 3.4 \%$, respectively. Two-year TRM and relapse rate were $19.4 \pm 3.5 \%, 22.6 \pm 4.1 \%$, respectively. With the median follow up 17.5 (6 to 38 ) months, two-year LFS in CR1, $\geq$ CR2 and advanced disease were $84.5 \pm 4.3 \%$, $69.6 \pm 6.3 \%, 41.6 \pm 9.4 \%$, respectively (CR1 vs. $\geq C R 2, P=0.040$; CR1 vs. NR, $P=0.000 ; \geq C R 2$ vs. NR, $P=0.025$ ). Multivariate analysis indicated that disease status before transplant was the only impact factor on LFS after haplo-BMT in ALL $(P<0.001)$ but not recipient's age, HLA disparity and recipient-donor relationship.

Conclusion: With our protocol, overall outcomes of haplo-BMT in ALL have been improved remarkably and very encouraging. The patients with high-risk ALL or persistent MRD should receive haplo-BMT in $\mathrm{CR} 1$ if no matched sibling or unrelated donor available.

Disclosure of Interest: None declared.

P700

Nilotinib has more effective anti-leukemia propagating cells activity than imatinib in Philadelphia chromosomepositive acute lymphoblastic leukemia

Y. Kong ${ }^{1, *}$, Y.-L. Wu ${ }^{1,2}$, Y. Song ${ }^{1,2}$, M.-M. Shi ${ }^{1,2}$, D.-L. Hong ${ }^{3}$, X.-J. Huang ${ }^{1,2}$

${ }^{1}$ Peking University Peoples Hospital, Peking University Institute of Hematology, Beijing Key Laboratory of HSCT, ${ }^{2}$ Peking-Tsinghua Center for Life Sciences, Academy for Advanced Interdisciplinary Studies, Peking University, Beijing, ${ }^{3}$ Department of Pathophysiology, Key Laboratory of Cell Differentiation and Apoptosis of Ministry of Education, Shanghai Jiao Tong University School of Medicine, Shanghai, China

Introduction: Relapse, the major cause of treatment failure in Philadelphia chromosome-positive acute lymphoblastic leukemia $\left(\mathrm{Ph}^{+} \mathrm{ALL}\right)$, seriously affects the long-term survival of $\mathrm{Ph}^{+} \mathrm{ALL}$ patients. Leukemia propagating cells (LPCS), defined by their ability to initiate human leukemia and self-renew in immunocompromised mice, almost equal to leukemia stem cells. Relapse of $\mathrm{Ph}^{+} \mathrm{ALL}$ may result from the persistence of LPCs. Using an anti-CD122-conditioned NOD/SCID xenograft mouse assay, we recently reported that LPCs are enriched in the $\mathrm{CD} 34^{+} \mathrm{CD} 38^{-} \mathrm{CD} 58^{-}$fraction in human $\mathrm{Ph}^{+} \mathrm{ALL}$. Our cohort study demonstrated that $\mathrm{Ph}^{+} \mathrm{ALL}$ patients with LPCs phenotype at diagnosis exhibited significantly higher cumulative incidence of relapse than the other phenotype group even receiving uniform first-line imatinib-based therapy pre- and post-allotransplant. Recently, in vitro and clinical studies have shown that nilotinib is more potent than imatinib against $\mathrm{Ph}^{+} \mathrm{ALL}$. The aim of our study is to investigate whether nilotinib is able to more effectively eliminate LPCs than imatinib in $\mathrm{Ph}^{+} \mathrm{ALL}$

Material (or patients) and methods: The LPCs $\left(\mathrm{CD} 34^{+}\right.$ CD38 ${ }^{-}$D58 $8^{-}$) were sorted from bone marrow cells of patients with de novo $\mathrm{Ph}^{+} \mathrm{ALL}$ by flow cytometry. In order to assess the effects of nilotinib and imatinib on $\mathrm{Ph}^{+} \mathrm{ALL}$ LPCs, drug-induced apoptosis of LPCs was investigated in vitro. Furthermore, the anti-LPCs activities of nilotinib and imatinib were evaluated in vivo using sublethally irradiated and anti-mouse CD122 monoclonal antibody conditioned NOD/SCID mice by intrabone marrow-injection.
Results: In vitro study, nilotinib induced significantly higher levels apoptosis of LPCs than imatinib. In $\mathrm{Ph}^{+} \mathrm{ALL}$ humanized mouse model, bone marrow and spleen were efficiently engrafted with human leukemia cells with an aberrant phenotype similar to that in the donor $\mathrm{Ph}^{+} \mathrm{ALL}$ patients. The engrafted human cells were derived from the $\mathrm{Ph}^{+} \mathrm{ALL}$ clone indicated by the presence of $B C R / A B L$ transcripts. At 2 months post drugs intervention, similar high levels of human leukemia cells engraftment were demonstrated in imatinib-treated mice and no-treatment control mice. In contrast, nilotinib significantly reduced the engraftment levels of human leukemia cells than imatinib. Consistent with our flow cytometry data, hematoxylin-eosin staining and immune histochemistry with rabbit anti-hCD34 and CD19 demonstrated that the infiltrating levels of the transplanted LPCs cells were significantly lower in brain, liver, kidney and spleen tissues of nilotinib-treated mice than those in imatinib-treated mice.

Conclusion: Nilotinib has more effective anti-LPCs activity than imatinib, which may consequently lead to more effective disease eradication and better prognosis in $\mathrm{Ph}^{+} \mathrm{ALL}$.

References: 1.Kong $Y$, Ying-jun Chang, Yan-rong Liu, et al. CD34+CD38-CD58- cells are leukemia-propagating cells in Philadelphia chromosome-positive acute lymphoblastic leukemia.Leukemia. 2014;28(12):2398-2401.

2.Kong $Y$, Lan-ping $\mathrm{Xu}$, Yan-rong Liu, et al. Presence of CD34 + CD38-CD58- Leukemia-Propagating Cells at Diagnosis Identifies Patients at High-risk for Relapse with Philadelphia Chromosome-Positive Acute Lymphoblastic Leukemia after Allogeneic Hematopoietic Stem Cell Transplantation. Bone Marrow Transplant. 2015;50(3);348-353.

Disclosure of Interest: None declared.

\section{P701}

Comparison of autologous versus allogeneic hematopoietic cell transplantation in patients with intermediate-risk acute myeloid leukemia in first complete remission

Y. Kim ${ }^{1, *}$, H. Chung ${ }^{1}$, H. Cho ${ }^{1}$, J. Y. Lee ${ }^{1}$, H. S. Park', J. E. Jang ${ }^{1}$, S. J. Kim ', J. S. Kim ${ }^{\prime}$, J.-W. Cheong

Internal Medicine, Yonsei University Severance hospital, Seoul, Korea, Republic Of

Introduction: Autologous hematopoietic cell transplantation (auto-HCT) has been investigated as a potential therapeutic option to improve the outcome in acute myeloid leukemia (AML) patients. However, in 2014, auto-HCT was removed from the National Comprehensive Cancer Network guidelines as a recommended treatment for patients with intermediate-risk AML in first complete remission (CR1) although still utilized relatively frequently in Europe and Asia. In the present study, we analyzed the outcomes of HCT to evaluate the safety and efficacy of auto-HCT, RIC-HCT and MA-HCT in these patients who were treated at our institution.

Material (or patients) and methods: We included adult AML patients between the age of 15 and 69 years who had intermediate cytogenetics at the time of diagnosis. Patients in whom CR1 had been achieved on our institutional protocol were recommended auto-HCT or allo-HCT after consolidation chemotherapy according to donor availability.

Results: Between 1998 and 2014, we performed HCT in a total of 74 AML patients with intermediate-risk cytogenetics in CR1; 24 patients received autografts and 50 patients received allografts. There were 40 men and 34 women with a median age of 40 years (range, 15-69 years). Of the 76 total patients, 46 patients (62.2\%) had documented mutational testing for FLT3ITD, and 36 patients (48.6\%) for NPM1. Two of the 46 patients (2.7\%) had a FLT3-ITD mutation (all of 2 patients also had a NPM1 mutation). The median time to CR and HCT from the time of diagnosis was 28 and 178 days, respectively. The pretransplantation characteristics were well balanced among the auto-HCT, RIC-HCT and MA-HCT groups except for time to HCT. Allograft recipient underwent transplantation earlier than autograft recipients $(P<0.001)$. Donors were sibling for 27 
patients and were unrelated for 23 patients. The requirement of pheresis was higher and the neutrophil recovery was longer in the auto-HCT group ( $P=0.005$ and $P<0.001$, respectively). After a median follow-up of 78 months (range, 13-144 months) for surviving transplants, the disease free survival (DFS) at $6 \mathrm{yr}$ was $62.2 \pm 11.9 \%$ for auto-HCT, $62.3 \pm 10.9 \%$ for RIC-HCT, and $79.5 \pm 0.9 \%$ for MA-HCT, which was not significantly different $(P=0.544)$. Overall survival (OS) at $6 \mathrm{yr}$ was $65.9 \pm 10.7 \%$ for auto-HCT, $43.2 \pm 10.2 \%$ for RIC-HCT, and $43.5 \pm 10.5 \%$ for MAHCT $(P=0.064)$, and OS was lower in MA-HCT than auto-HCT $(P=0.025)$, while there was no difference between auto-HCT and RIC-HCT $(P=0.077)$. The cumulative incidence of nonrelapse mortality (NRM) at 6 yr was $46.5 \pm 12.8 \%$ for auto-HCT $43.0 \pm 10.2 \%$ for RIC-HCT, and $44.3 \pm 10.4 \%$ for MA-HCT $(P=0.202)$. MA-HCT had a higher NRM than auto-HCT (RR $1.425 ; 95 \% \mathrm{Cl} 0.954-2.129)$, although it was not stastically difference $(P=0.077)$. RIC-HCT had a similar NRM compared with auto-HCT (RR 1.683; 95\% Cl 0.752-3.768; $P=0.201)$. Age was a factor affecting OS $(P=0.004)$ and DFS $(P=0.028)$ in the auto-HCT group, but not in the RIC-HCT group. In the auto-HCT group, an age of $>50$ years were associated with inferior survival; however, these differences disappeared in the RICHCT group.

Conclusion: These results indicate that auto-HCT was feasible option in AML in CR1 with intermediate cytogenetics, when lacking the available donor. The subgroup with advanced age should be preferentially considered for RIC-SCT, rather than RIC-HCT.

Disclosure of Interest: None declared.

P702

Plasma levels of Soluble HLA-E and HLA-G, but not MICA are increased in Acute Leukemia patients

L. $L u^{1}, Y . X u^{1, *}$

'transplantation immunology, Shenzhen blood center, Shenzhen, China

Introduction: The higher expression of soluble Human Leukocyte Antigens (HLA) appears to be an important mechanism for tumor escape. This study aims to investigate the soluble levels of non-classical HLA class I molecules, HLA-E and HLA-G, and the MHC class I chain-related gene A (MICA) in acute leukemia patients. Plasma soluble levels of $H L A-E,-G$, and MICA of 54 acute leukemia patients (including cases 28 of ALL, 26cases of AML) and 64 controls were quantitatively analyzed in Chinese individuals using double antibody sandwich Elisa method. Based on our results, the average levels of sHLA-E and sHLA-G in controls are $18.34 \mathrm{pg} / \mathrm{ml}$ and $11.08 \mathrm{pg} / \mathrm{ml}$, respectively. In patients, both of the levels of sHLA-E $(P<0.001)$ and sHLA-G $(P<0.001)$ are significantly increased, and the average level attain to $31.32 \mathrm{pg} / \mathrm{ml}$ and $19.05 \mathrm{pg} / \mathrm{ml}$, respectively. The average levels of sMICA in patients and controls are $38.92 \mathrm{pg} / \mathrm{ml}$ and $38.72 \mathrm{pg} / \mathrm{ml}$ respectively, and no correlation was observed between sMICA level in patients and controls.

Material (or patients) and methods: Samples Leukemia Patients were collected from Shenzhen second hospital. Healthy donors are regular non-paid blood donors recruited from the Shenzhen blood center with the information of age and gender. Detection of sHLA-E by Elisa Human HLA-E, $-\mathrm{G}$ and MICA Elisa kit were purchased from Shanghai JiangLai Biotech Co. Ltd. Plasma of biological samples were diluted by 5 times and operated following the kit manual. Statistical analysis The quantitative expression of $\mathrm{SHLA}-\mathrm{E},-\mathrm{G}$ and MICA in plasma of leukemia patients were compared with plasma of healthy donors. Data were presented as means, medians, and ranges of sHLA-E, -G and sMICA expression. Statistical analysis was performed by two-tails T test. A $P$-value $<0.05$ was considered statistically significant. All statistical analyses were performed using Prism 5 Software (GraphPad Software Inc., La Jolla, (A, USA).

[P702]
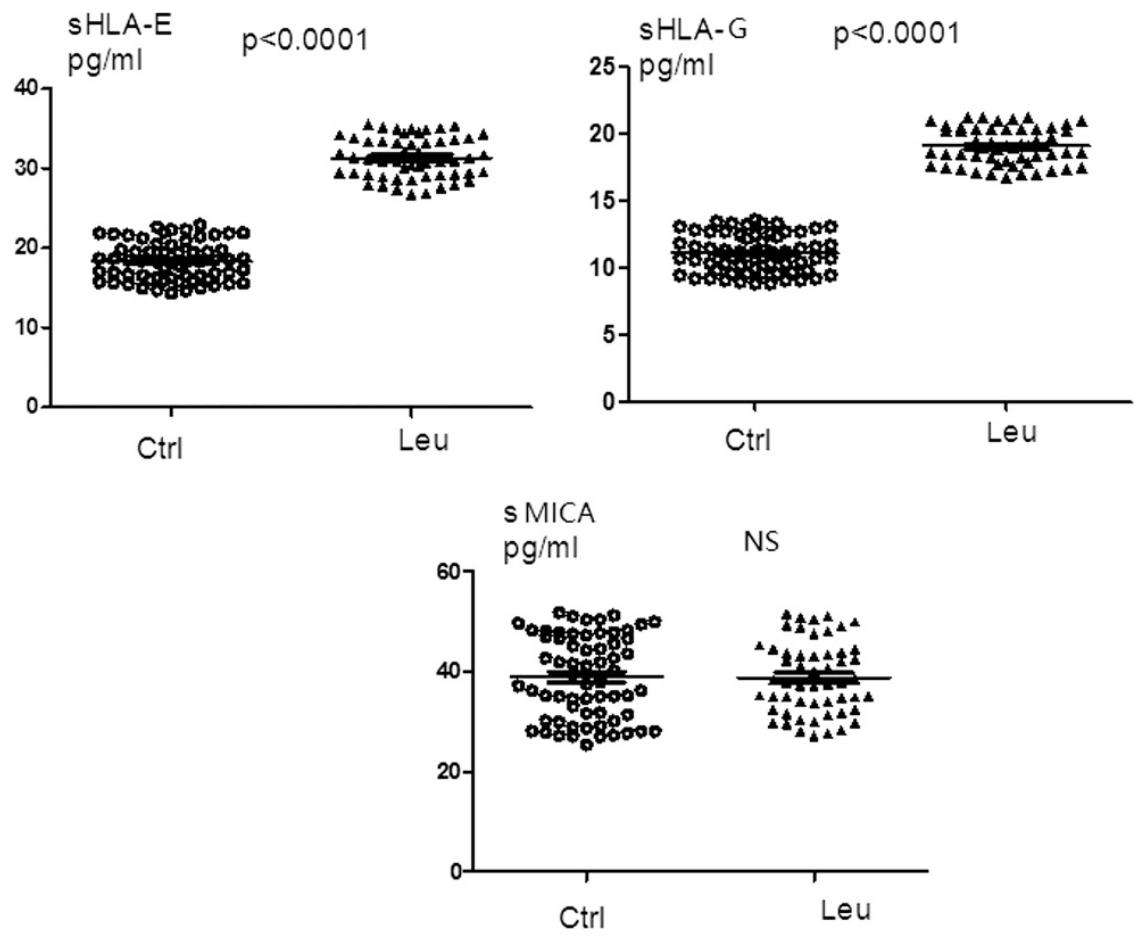
Results:

Table 1 Comparation of sHLA-E, -G and MICA levels in healthy donors and leukemia patients

\begin{tabular}{|c|c|c|c|c|c|c|c|}
\hline & $\mathrm{N}$ & Max & Min & Mean & $\begin{array}{l}\text { Std. } \\
\text { Dev }\end{array}$ & $t$ & $P$ \\
\hline \multicolumn{8}{|l|}{$s H L A-E$} \\
\hline Controls & 64 & 22.94 & 14.4 & 18.34 & 2.43 & - & - \\
\hline Leukemia & 54 & 35.49 & 26.71 & 31.32 & 2.58 & 28.09 & $<0.0001$ \\
\hline \multicolumn{8}{|l|}{$s H L A-G$} \\
\hline Controls & 64 & 13.59 & 8.83 & 11.08 & 1.44 & - & - \\
\hline Leukemia & 54 & 21.26 & 16.72 & 19.05 & 1.38 & 30.52 & $<0.0001$ \\
\hline \multicolumn{8}{|l|}{$s M I C A$} \\
\hline Controls & 64 & 51.81 & 25.42 & 38.92 & 8.28 & - & - \\
\hline Leukemia & 54 & 51.53 & 27.12 & 38.72 & 7.21 & 0.14 & NS \\
\hline
\end{tabular}

Conclusion: Plasma levels of Soluble HLA-E and HLA-G, but not MICA are significantly increased in Acute Leukemia patients.

References: Bassani-Sternberg M, Barnea E, Beer I, Avivi I, Katz T, Admon A. Soluble plasma HLA peptidome as a potential source for cancer biomarkers.

Proc Natl Acad Sci U S A. 2010;107(44):18769-76.

Disclosure of Interest: None declared.

P703

A study on the correlation of HLA-E gene polymorphism with Acute Leukemia

S. Wang ${ }^{1}, Y . X u^{1, *}$

${ }^{1}$ transplantation immunology, Shenzhen blood center, Shenzhen, China

Introduction: We studied the correlation between the gene polymorphism of HLA-E and leukemia, aimed to offer a new molecular markers for the mechanism of acute leukemia occurrence and development.

Material (or patients) and methods: The peripheral blood DNA were extracted from patients with acute leukemia $(n=59)$ collected from shenzhen second hospotal and healthy individuals $(n=132)$ collected from shenzhen blood center. The full-length $H L A-E$ was amplified by long-range high-fidelity $\mathrm{PCR}$ and the exon 3 was sequenced to identify the genotype of HLA-E. The genotype and allele frequency were calculated respectively in the leukemia patients and healthy controls.

Results: In Leukemia group, we detected 35 homozygous cases (the detection rate is 59.3\%, 35/59), of which $29(49.2 \%)$ were homozygous for $E^{*} 01: 03$, and only 6 cases (10.2\%) were homozygous for $E^{*} 01: 01$. In healthy individuals, we detected 62 homozygous cases from healthy donors (the detection rate is $47.0 \%, 62 / 132), 36$ cases $(27.3 \%)$ were homozygous for HLA-E*01:03, 26 cases (19.7\%) were homozygous for HLA$E^{*} 01: 01$. The allele frequency of $H L A-E^{*} 01: 03$ in leukemia patients and healthy individuals were $69.5 \%$ vs $53.7 \%$ $(P=0.004, \mathrm{OR}=2.0)$. The detection rate of $H L A-E^{*} 01: 03$ homozygous in two groups were $49.2 \%$ vs $27.3 \%(P=0.003, \mathrm{OR}=2.6)$.
Table 1 frequency of HLA-E genotype in controls and acute leukemia patiens

\begin{tabular}{lrrrrr}
\hline genotype & $\begin{array}{c}\text { controls } \\
(\mathrm{n}=132)\end{array}$ & $\begin{array}{c}\text { leukemia } \\
(\mathrm{n}=59)\end{array}$ & $X^{2}$ & $\mathrm{P}$ & $\mathrm{OR}$ \\
\hline $\begin{array}{l}\mathrm{HLA}- \\
\mathrm{E}^{*} 01: 03 /\end{array}$ & $70(53.0 \%)$ & $24(40.7 \%)$ & 2.489 & 0.115 & 0.607 \\
${ }^{*} 01: 01$ & & & & & \\
$\begin{array}{l}\mathrm{HLA}- \\
\mathrm{E}^{*} 01: 03 /\end{array}$ & $36(27.3 \%)$ & $29(49.2 \%)$ & 8.695 & 0.003 & 2.578 \\
${ }^{*} 01: 03$ & & & & & \\
$\mathrm{HLA}-$ & $26(19.7 \%)$ & $6(10.2 \%)$ & 2.654 & 0.103 & 0.462 \\
$\mathrm{E}^{*} 01: 01 /$ & & & & & \\
${ }^{*} 01: 01$ & & & & & \\
\hline
\end{tabular}

Conclusion: We disclose that $E^{*} 01: 03$ is the susceptibility gene for leukemia.

Disclosure of Interest: None declared.

\section{P704}

Long term follow up of Fanconi's anemia patients following reduced intensity allogeneic stem cell transplantation

M. Y. Shapira ${ }^{1,2, *}$, S. Vipul', S. Grisariu ${ }^{1}$, B. Avni ${ }^{1}$, P. Stepensky ${ }^{2}$, R. $\mathrm{Or}^{7}$

${ }^{1}$ Department of Bone Marrow Transplantation, ${ }^{2}$ Pediatric hematooncology, HADASSAH UNIVERSITY HOSPITAL, Jerusalem, Israel

Introduction: Reduced intensity conditioning has been suggested as a desirable therapeutic modality for the treatment of patients with malignant and non-malignant disorders, but it seems especially attractive for patients with Fanconi's anemia due to their increased sensitivity to chemoradiotherapy.

Material (or patients) and methods: Between November 1996 and October 2015, 25 patients were conditioned with a fludarabine-low dose cyclophosphamide based protocol for stem cell transplantation without radiation. In vivo T-cell depletion was accomplished with anti-thymocyte globulin (22 patients) or alemtuzumab (3 patients). Nineteen patients (76\%) received transplants from matched related donors and six (24\%) from unrelated donors.

Results: Out of 25 patients, 22 (88\% DFS and OS, median follow-up of survivors 93 months (3.4-230), figure 1) are alive and well, fully reconstituted with donor cells and a performance status of $100 \%$. The 3 patients that expired (2 transplant related mortality, 1 late meduloblastoma) received grafts from matched related donors. Two of them had AML prior to transplant, and in all the conditioning regimen was augmented by the addition of busulfan. None of the patients currently alive suffers from secondary malignancies or renal dysfunction. Two patients with single kidneys had short term peri-transplant acute renal failure during transplant and have completely recovered.

Conclusion: in Fanconi's anemia fludarabine-low dose cyclophosphamide based preparative protocols are well tolerated and facilitate rapid engraftment with minimal toxicity and should be considered an essential choice. Augmentation with busulfan should be avoided.

Disclosure of Interest: None declared. 


\section{P705}

Alemtuzumab is Effective for the Prevention of GvHD in Patients with Fanconi's Anaemia undergoing HLA Identical Stem Cell Transplantation

N. Novitzky ${ }^{1, *}$, D. Pillay ${ }^{2}$, V. Thomas ${ }^{3}$, M. Hendricks ${ }^{4}$, A. Davidson ${ }^{4}$ ${ }^{1}$ Division of Haematology, UNIVERSITY OF CAPE TOWN AND GROOTE SCHUUR HOSPITAL, ${ }^{2}$ Blood Centre Transplant Program, Univeristy of Cape Town Private Academic Hospital, ${ }^{3}$ Division of Haematology, Groote Schuur Hospital, ${ }^{4}$ Department of Paediatric Oncology, Red Cross War Memorial Children's Hospital, Cape Town, South Africa

Introduction: FA is a rare genetic disease characterized by progressive bone marrow failure, malformations, and a high propensity of malignancies. The underlying cause is genomic instability resulting from the deficiency in the replication dependent DNA cross-link repair pathway commonly referred to as the FA/BRCA pathway. FA cells develop increased DNA damage with alkylating agents such as cyclophosphamide. In addition patients with FA tolerate poorly severe forms of GvHD. To ensure maximum protection from GvHD the value of alemtuzumab treatment of the graft ("in the bag") was studied. Material (or patients) and methods: Patients with somatic and cytogenetic features of Fanconi's anaemia who had become symptomatic of transfusion dependent anaemia or thrombocytopenia and had an HLA identical donor were entered into the study. The conditioning was with busulfan $8 \mathrm{mg} / \mathrm{kg}$ (or Busulfex $6.4 \mathrm{mg} / \mathrm{kg}$ ), cyclophosphamide $10 \mathrm{mg} / \mathrm{kg}$ $x 4$ days in 5 and 6 had additionally to busulfan, fludarabine $125 \mathrm{mg} / \mathrm{m}^{2}$, cyclophosphamide $10 \mathrm{mg} / \mathrm{kg} \times 2$ days. ATG (Fresinius $9 \mathrm{mg} / \mathrm{kg}$ ) was prescribed in those receiving unrelated grafts. Filgrastim mobilised PBPC were obtained by apheresis from HLA compatible donors and each $1 \times 10^{10}$ mono nuclear cells were incubated with $1 \mathrm{mg}$ of alemtuzumab ex vivo. Patients were given post-transplant therapeutic doses of cyclosporine until day 90-120.

Results: From September 2006, 11 patients were entered into the study. The median (range) age at transplant was 7 (4-17) years and 6 were female. Two donors were unrelated. The median CD34+ cell number was $8.3 \times 10^{6} / \mathrm{kg}$ (4.3-13.9), the median total mononuclear cell number in the graft was $1.3 \mathrm{x}$ $10^{10}$ and the median alemtuzumab dose "in the bag" was 5.3 (2-10) $\mathrm{mg}$ incubated in median concentration of $1 \times 10^{8}$ mononuclear cells $/ \mathrm{mL}$ and in $100 \mathrm{~mL}$. Following infusion of the graft all patients engrafted and the median time to 0.5 $\mathrm{x} 10^{9} / \mathrm{L}$ granulocytes and platelets of $>20 \times 10^{9} / \mathrm{L}$ was 13 days. Pyrexia that required antibiotic therapy occurred in 9 children but there were no grade 3 or 4 toxicities. One patient developed grade $>2$ GvHD which was steroid responsive but died of infection. Two patients had CMV reactivation and responded to gancyclovir. Another patient developed haemorrhagic cystitis and then post-transplant lymphoproliferative disorder that responded to rituximab. Chimerism studies are available in 6 and were of donor origin $(>90 \%)$ in all. Ten patients survive disease free at a median of 1288 days (330 - 3360).

Conclusion: The combination of purine analogue and low dose alkylators resulted in universal engraftment, while alemtuzumab in the bag provided effective protection from GvHD to this particularly vulnerable patient population.

Disclosure of Interest: None declared.
P706

Cyclophosphamide (CY) Conditioning In Patients with Severe Aplastic Anaemia (SAA) Given Unrelated Marrow Transplantation: Final Results of a Phase 1-2 Dose De-Escalation Study from the Blood and Marrow Transplant Clinical Trials Network (BMT CTN 0301; NCT 00326417)

P. Anderlini, ${ }^{1, *}$, J. Wu ${ }^{2}$, H. J. Deeg ${ }^{3}$, I. Gersten ${ }^{2}$, M. Ewell ${ }^{2}$, J. Tolar ${ }^{4}$, J. Antin ${ }^{5}$, S. Arai, ${ }^{6}$ M. Horwitz', J. McCarty ${ }^{8}$, R. Nakamura9 M. Pulsipher ${ }^{10}$, R. Adams ${ }^{11}$ ' E. Leifer ${ }^{12}, N$. DiFronzo ${ }^{13}$, M. H. Horowitz ${ }^{14}$, D. Confer ${ }^{15}, M$. Eapen ${ }^{14}$ on behalf of Bone Marrow Transplant Clinical Trials Network

${ }^{1}$ Stem Cell Transplantation, MD Anderson Cancer Center, Houston, Texas, ${ }^{2}$ EMMES Corporation, Rockville, Maryland, ${ }^{3}$ FHCRC, Seattle, ${ }^{4}$ University of Minnesota, Minneapolis, ${ }^{5}$ Dana Farber Cancer Center, Boston, ${ }^{6}$ Stanford Hospital and Clinics, Stanford, 'Duke University, Durham, NC, ${ }^{8}$ Virginia Commonwealth University, Richmond, VA, ${ }^{9}$ City of Hope Medical Center, Duarte, CA, ${ }^{10}$ Children's Hospital, Los Angeles, CA, ${ }^{11}$ Phoenix Children's Hospital, Phoenix, AZ, ${ }^{12} \mathrm{NIH} / \mathrm{NHLBI},{ }^{13} \mathrm{NHLBI} / \mathrm{NIH}$, Bethesda, MD, ${ }^{14}$ CIBMTR, Milwaukee, WI, ${ }^{15}$ NMDP, Minneapolis, $M N$, United States

Introduction: There is no consensus on the optimum preparative regimen for unrelated donor transplantation in SAA. We elected to investigate whether adding fludarabine (FLU) to a preparative regimen of low-dose total body irradiation (TBI), anti-thymocyte globulin (ATG) and CY (1) would allow the de-escalation of the CY dose.

Material (or patients) and methods: Between May 2006 and December 2013, the BMT CTN (sponsored by the NHLBI and $\mathrm{NCl}$ ) conducted a Phase I/II trial of unrelated donor BMT in SAA (BMT CTN 0301; NCT 00326417). Patient eligibility criteria were reported previously (2). The trial accrued 96 patients. All patients received TBI (200 cGy x1), ATG (thymoglobulin: $3 \mathrm{mg} /$ $\mathrm{kg} \times 3)$ and FLU (30 mg/m $\left.\mathrm{m}^{2} \times 4\right)(2)$. The Phase I portion of the trial tested four CY dose levels: $150 \mathrm{mg} / \mathrm{kg}, 100 \mathrm{mg} / \mathrm{kg}, 50 \mathrm{mg} /$ $\mathrm{kg}$ and $0 \mathrm{mg} / \mathrm{kg}$. The Phase I design allowed enrollment of up to six patients at each $\mathrm{CY}$ dose level unless toxicity or graft failure boundaries were crossed. In the Phase II portion, enrollment started at the optimal $\mathrm{CY}$ dose level, using adaptive Bayesian criteria as already outlined (2). The primary endpoint of the study was determination of the optimal CY dose based on Day 100 assessments of graft failure, major (grade 3 or higher) regimen-related toxicity (3) and early death. Early stopping guidelines were based on a composite endpoint of graft failure and treatment-related mortality through day 100. Secondary endpoints (which we are now reporting) included 2year survival, chronic graft-vs-host disease (GVHD) and late/ secondary graft failures.

Results: Twenty-one patients accrued to the Phase I portion of the trial. CY dose level $0 \mathrm{mg} / \mathrm{kg}(n=3)$ and the initial Phase II CY dose of $150 \mathrm{mg} / \mathrm{kg}(n=14)$ were closed for excess graft failure and excess toxicity, respectively (2). Patients were subsequently assigned to CY dose level $100 \mathrm{mg} / \mathrm{kg}$ (CY DL 100; $n=41$ ) or $\mathrm{CY}$ dose level $50 \mathrm{mg} / \mathrm{kg}$ (CY DL 50; $n=38$ ), depending on Bayesian assessment of criteria noted above (2). The median age for CY DL 50 and DL 100 was 24.5 (0.5-65) and $17.6(1.9-63)$ years. The number of transplants with a mismatched (i.e. 7/8) donor was 7 (18\%) in the CY DL 50 group and $14(34 \%)$ in the CY DL 100 group. All patients reached their Day 100 endpoint. Day 100 outcomes (already reported) suggested that the most desirable CY dose is $50 \mathrm{mg} / \mathrm{kg}$, followed by CY DL $100 \mathrm{mg} / \mathrm{kg}$ (2). All surviving patients have now reached the planned follow up of 24 months. Overall 2year survival for CY DL 50 and CY DL 100 is $91 \%$ (95\% Cl 76-97) and $76 \%(95 \% \mathrm{Cl} 59-86)$, respectively. For patients receiving transplants from an 8/8 matched donor, overall 2-year survival is $93 \%(95 \% \mathrm{Cl} 76-98)$ and $70 \%(95 \% \mathrm{Cl} 49-84)$. At two years, cumulative incidence of graft failure is $11 \%$ and $15 \%$, respectively. Cumulative incidence of chronic GVHD is $37 \%$ and $34 \%$. 
Conclusion: Two-year follow-up data confirm the desirability of CY DL $50 \mathrm{mg} / \mathrm{kg}$. However, interpretation of the results should take into account the imbalance of age and donorrecipient HLA disparity between the CY 50 and CY $100 \mathrm{mg} / \mathrm{kg}$ dose levels.

References: 1. Deeg J et al, Blood 2006; 108: 1485.

2. Anderlini P et al, Lancet Haematol 2015; 2: e367.

3. Bearman SI et al, JCO 1989; 7: 1288.

4. Tolar J et al, BBMT 2012; 18: 1007.

Disclosure of Interest: None declared.

\section{P707}

Donor Telomere Length and Post-HCT Survival in SAA Patients by Age: Preliminary Results

S. Gadalla 1,*, T. Wang ${ }^{2}$, C. Dagnall 3 , M. Haagenson ${ }^{4}$, S. Spellman ${ }^{4}$, B. Hicks ${ }^{3}$, K. Jones ${ }^{3}$, H. Katki ${ }^{5}$, S. Lee ${ }^{6}$, S. Savage ${ }^{1}$ Clinical Genetics Branch, National Cancer Institute, Rockville, ${ }^{2}$ Center for International Blood and Marrow Transplant Research, Medical college of Wisconsin, Milwaukee, ${ }^{3}$ Cancer Genomics Research Laboratory, Leidos Biomedical Research, Inc., Frederick, ${ }^{4}$ Center for International Blood and Marrow Transplant Research, Center for International Blood and Marrow Transplant Research, Minneapolis, ${ }^{5}$ Biostatistics Branch, National Cancer Institute, Rockville, ${ }^{6}$ Clinical Research Division, Fred Hutchinson Cancer Research Center, Seattle, United States

Introduction: Telomeres are markers of cellular replicative capacity, and aging. ${ }^{1}$ We have recently showed that longer donor relative telomere length (RTL) was associated with improved survival after unrelated allogeneic hematopoietic cell transplant $(\mathrm{HCT})$ in a cohort of severe aplastic anemia (SAA) patients $<40$ years old who received HCT between 1989-2007. ${ }^{2}$ Because of the clinical significance and the translational potential of such a finding, a validation study including all age groups and reflecting recent HCT modalities was needed.

Material (or patients) and methods: We identified a new set of patients who received a first HCT for SAA from an unrelated donor at any age between 1995-2013, and who had an available pre-transplant donor blood in the Center for International Blood and Marrow Transplant Research (CIBMTR) Research Repository. We used QIAamp Maxi Kit procedure to extract DNA and monoplex quantitative real-time PCR (qPCR) to measure RTL. ${ }^{3,4,5}$ Kaplan-Meier estimator and Cox proportional hazard models were used to evaluate the association between donor RTL and patient overall survival (OS) by patient age at HCT $(<40[n=305]$, and $\geq 40$ years $[n=122])$.

Results: The study included 427 SAA patients who received $\mathrm{HCT}$ at a median age of 22 years (range $<1-77$ years). The majority of patients received bone marrow grafts $(74 \%), 38 \%$ received a myeloablative conditioning regimen, and $63 \%$ had an 8/8 HLA matched donor graft. Median post-HCT follow-up for survivors was 3 years (range $=3$ months- 14 years). In SAA patients $<40$ years of age, the OS probabilities for the tertile with the longest RTL compared with those with shorter RTL were: $85 \%$ vs. $76 \%, P=0.07$ at 1 year; and $81 \%$ vs. $71 \%$ at 5 years, $P=0.06$. In patients $\geq 40$ years, the OS was $56 \%$ vs. $61 \%$, $P=0.6$ at 1 year, and $47 \%$ vs. $51 \%, P=0.7$ at 5 years. Multivariable models adjusting for patient race, HLA matching, and stem cell source suggested that the association between donor RTL and OS may be affected by patient age, although not statistically significant (hazard ratio $[\mathrm{HR}]=0.67,95 \%$ $\mathrm{Cl}=0.39-1.14$ for patients $<40$ vs. $\mathrm{HR}=1.39,95 \% \mathrm{Cl}=0.69-2.8$ for patients $\geq 40$ years, test for interaction $P=0.37$ ). In analysis restricted on patients who received bone marrow grafts, and adjusted for donor age, the HR was $0.64(95 \% \mathrm{Cl}=0.34-1.22)$ for patients $<40$ years of age.

Conclusion: Although not statistically significant, this analysis of an independent cohort of SAA patients is consistent with our prior work. It also suggests that recipient age and stem cell source may modify the association between donor RTL and post-HCT survival in patients receiving unrelated allogeneic HCT for SAA. A larger cohort is needed to confirm such finding.
References: 1. Lin J, Kaur P, Countryman P, Opresko PL, Wang $\mathrm{H}$. Unraveling secrets of telomeres: One molecule at a time. DNA Repair (Amst). 2014.

2. Gadalla SM, Wang T, Haagenson M, et al. Association between donor leukocyte telomere length and survival after unrelated allogeneic hematopoietic cell transplantation for severe aplastic anemia. JAMA. 2015;313(6):594-602.

3. Cawthon RM. Telomere measurement by quantitative PCR. Nucleic Acids Res. 2002;30(10):e47.

4. Cawthon RM. Telomere length measurement by a novel monochrome multiplex quantitative PCR method. Nucleic Acids Res. 2009;37(3):e21.

5. Callicot RJ, Womack JE. Real-time PCR assay for measurement of mouse telomeres. Comp. Med. 2006;56:17-22.

Disclosure of Interest: None declared.

\section{P708}

Outcomes of Cord Blood Transplantation from an HLA-Identical Sibling for Patients with Inherited or Acquired Bone Marrow Failure Disorders: A Report from Eurocord, Cord Blood committee-CTIWP and SAAWP of EBMT

S. Pagliuca ${ }^{1, *}$, R. Peffault de Latour ${ }^{2}$, F. Locatelli ${ }^{3}$, J.-H. Dalle ${ }^{4}$ K. Vettenranta ${ }^{5}$, M. A. Diaz ${ }^{6}$, O. Reuven , C. Diaz de Heredia ${ }^{8}$ A. Nagler ${ }^{9}$ A. Ghavamzadeh ${ }^{10}$, S. Sufliarska ${ }^{11}$ S. Lawson ${ }^{12}$ C. Kenzey $^{13}{ }^{\prime}$ V. Rocha ${ }^{14}$, C. Dufour '15, E. Gluckman ${ }^{16}$, J. Passweg ${ }^{17}$, A. Ruggeri ${ }^{18,19}$

${ }^{7}$ University of Naples Federico II, Naples, Italy, ${ }^{2}$ Hematology and Transplantation, Hôpital Saint-Louis - Assistance Publique-Hôpitaux de Paris., Paris, France, ${ }^{3}$ Oncoematologia Pediatrica, Fondazione IRCCS Policlinico S. Matteo, Università di Pavia, Pavia, Italy, ${ }^{4}$ Pediatric hematology immunology, Robert Debre Hospital, Paris, France, ${ }^{5}$ University of Helsinki Children's Hospital, Helsinki, Finland, ${ }^{6}$ Niño Jesus Children's Hospital, Madrid, Spain, ${ }^{7}$ Hadassah University Hospital, Jerusalem, Israel, ${ }^{8}$ Servicio de Hematologia y Oncologia Pediátrica, Hospital Vall d'Hebron, Barcelona, Spain, ${ }^{9}$ Hematology Division, Chaim Sheba Medical Center and Tel Aviv University, Tel-Hashomer Ramat-Gan, Israel, ${ }^{10}$ HematologyOncology and Stem Cell Transplantation Research Center, Tehran University of Medical Sciences, Tehran, Iran, Islamic Republic Of, ${ }^{11}$ Bone Marrow Transplantation Unit, Comenius University Children's Hospital, Bratislava, Slovakia, ${ }^{12}$ Department of Haematology, Birmingham Childrens Hospital, Birmingham, United Kingdom,

${ }^{13}$ Eurocord - Monacord, AP-HP Hôpital Saint Louis, Paris, France; Monacord, Centre Scientifique de Monaco, Monaco, Paris, France, ${ }^{14}$ Churchill Hospital, Oxford University, Oxford, United Kingdom, ${ }^{15}$ stituto Giannina Gaslini, Genoa, Italy, ${ }^{16}$ Hôpital Saint-Louis Assistance Publique-Hôpitaux de Paris, Paris, France, ${ }^{17}$ Hopitaux Universitaires de Geneve, Geneve, Switzerland, ${ }^{18}$ Senvice d'Hématologie et Thérapie Cellulaire, Hôpital Saint-Antoine, Paris, ${ }^{19}$ Eurocord - Monacord, AP-HP Hôpital Saint Louis, Paris, France; Monacord, Centre Scientifique de Monaco, Monaco, Paris, Monaco, France

Introduction: Cord blood transplantation (CBT) from an HLAidentical sibling donor is of interest in non-malignant diseases, like bone marrow failure (BMF) disorders, due to the low risk of graft-versus-host disease (GVHD) and the absence of risk to the donor.

We analyzed outcomes of 121 children with inherited or acquired BMF syndromes, who received a CBT from an HLAidentical unaffected related donor.

Material (or patients) and methods: Patients were transplanted in EBMT centers between 1988 and 2014 and reported to Eurocord. Ninety-five patients had an inherited and 26 an acquired BMF.

Results: Forty-eight patients had Fanconi Anemia, 26 had Diamond Blackfan Anemia, 5 had Amegakariocytic Thrombocytopenia, 4 had Kostamann syndrome, 2 had Dyskeratosis Congenita, 2 had Schwachman-Diamond syndrome and 25 had Idiopathic Severe Aplastic Anemia.

Eighty-eight patients received a single cord blood (CB) unit and 33 received a combination of $\mathrm{CB}$ and bone marrow (BM) from the same donor. 
Median age at CBT was 6.7 (1-16) years (5.6 years for acquired and 6.9 years for inherited group). Median interval between diagnosis and CBT was 49 months ( 21 for acquired and 55 for inherited group).

Sixty-six patients $(60 \%)$ received a reduced intensity (RIC) and $49(40 \%)$ a myeloablative regimen (MAC). The most common protocols were cyclophosphamide (Cy) and fludarabine (54\%) in RIC setting and Cy and busulfan (46\%) for patients receiving a MAC. Total body irradiation was used in 8 patients (4 at dose of 2 Gy). ATG was used in $46 \%$ of acquired and $37 \%$ of inherited BMF, respectively. GVHD prophylaxis consisted mainly of cyclosporine alone (CSA) in 53 patients $(43 \%)$ and CSA+methotrexate in 23 patients (19\%). Median number of infused total nucleated cells was $6.2(1-25) \times 10^{7} / \mathrm{Kg}$ for patients receiving a single $C B$ unit and $\left.27(5.3-41) \times 10^{7} / \mathrm{Kg}\right)$ for those receiving $\mathrm{CB}+\mathrm{BM}$. Median follow-up was 6 (range 0.6-27) years. The cumulative incidence $(\mathrm{Cl})$ of neutrophil recovery at day 60 was $91 \% \pm 9 \%$, with a median time of 22 days. Eleven patients experienced primary graft failure (PFG, 4 of them had acquired and 7 had inherited BMF). Among them 9 died and two are alive after a second BMT.

The 100 - day $\mathrm{Cl}$ of grade II-IV acute GVHD was $11 \pm 3 \%$ and the 1 -year $\mathrm{Cl}$ of chronic GVHD was $12 \pm 4 \%$. The 1 -year $\mathrm{Cl}$ of transplant-related mortality was $13 \pm 3 \%$. Sixteen patients died: 9 due to PGF, 1 for CGVHD, and 6 of infections and other transplant related causes.

Overall survival (OS) was $91 \%$ at 6 years. At last follow-up OS was $73 \pm 9 \%$ for acquired and $84 \pm 5 \%$ for inherited BMF $(P=0.09)$.

Information on long-term outcomes were available for 56 patients, of them 3 patients were reported as experiencing secondary neoplasia.

Conclusion: In children with inherited or acquired BMF syndrome, CBT from an HLA-identical sibling donor is associated with excellent long-term outcomes with particular low incidence of GVHD. In case of inherited BMF collecting cord blood unit at the birth of a new sibling is recommended.

Disclosure of Interest: None declared.

\section{P709}

Long-term follow up of patients with immune-mediated Bone Marrow Failure Syndromes treated with Alemtuzumab-based immunosuppression

S. Pagliuca ${ }^{1, *}$, S. Marotta ${ }^{2}$, F. Grimaldi ${ }^{2}$, P. Ricci ${ }^{3}$, L. Luciano ${ }^{4}$, A. Risitano ${ }^{4}$

${ }^{7}$ Department of Hematology and Transplantation Unit, ${ }^{2} \mathrm{Hema-}$ tology and Transplantation Unit, University of Naples Federico II,

${ }^{3}$ University of Naples Federico II, ${ }^{4}$ University of Naples Federico II, Naples, Italy

Introduction: Acquired immune-mediated bone marrow failure syndromes (aBMF) include a range of diseases which may affect the bone marrow broadly (as in idiopathic acquired aplastic anemia, AA) or in a lineage-specific way (e.g., pure red cell aplasia, PRCA, and poor white cell aplasia, PWCA), which may benefit from intensive immunosuppressive treatment (IST). Here we report the long-term follow up of a pilot phase II prospective trial (NCT00895739) which investigated the use of the anti-CD52 alemtuzumab in combination with low-dose cyclosporine A for the treatment of aBMF (CyA; Risitano, BJH 2010;148:791).

Material (or patients) and methods: The study enrolled twenty-nine patients with either SAA $(n=14)$, PRCA $(n=13)$ or PWCA $(n=2)$; about half of the patients have not received any previous IST regimen (6 SAA, 9 PRCA and 1 PWCA). Three of the patients enrolled in this trial also had a concomitant haemolytic paroxysmal nocturnal hemoglobinuria (PNH) requiring anti-complement therapy with eculizumab.Details of patient population, treatment protocol and injection-related adverse events have been previously reported.

Results: The overall response rates were $77 \%(38.5 \% \mathrm{CR})$ in $\mathrm{AA}$, $84.5 \%(61.5 \%$ CR) in PRCA and $100 \%$ in PWCA patients, as previously reported. The median follow up now is about 60 months. Late infectious events were uncommon, irrespective of late lymphocyte recovery (especially the CD4 compartment remained substantially reduced for at least 12 months, with normalization not expected before 24 months). However, this long-lasting immune-ablation did not translate in protection from disease relapse, since many patients required further alemtuzumab treatment to sustain haematological response. Notably, the 3 patients with concomitant PNH, experienced the same complete lymphocyte ablation seen in noneculizumab treated patients, irrespective of the anticomplement treatment (which was pharmacologically effective, as proven by normal lactate dehydrogenase levels). Current stable remission was achieved in $38.5 \%$ of $\mathrm{AA}$, in $23 \%$ of both PRCA and PWCA. Nevertheless, long-term failures were frequent. Indeed, $15 \%$ of AA and $7.5 \%$ of PRCA experienced refractory relapses; in addition, $15 \%$ of $A A$ and $23 \%$ of PRCA developed a clonal evolution. One of the 2 PWCA developed a secondary tumour (a Hodgkin Lymphoma). All surviving patients were screened for clonal evolution by standard karyotype; none of them showed cytogenetic abnormalities, including abnormalities of the $5 q$. Overall survival was $69 \%$ for AA (all deaths due to refractory disease) and only 31\% for PRCA. This was due to an unexpected rate of disease evolution ( 3 leukemias and $1 \mathrm{AA}$ ) and other complications, both associated to PRCA (1 thymoma complicated with PML, 1 refractory connective tissue disease) or independent (cardiovascular comorbidities).

Conclusion: In conclusion, our study confirms that alemtuzumab can be biologically effective in immune-mediated aBMF; its long-term follow up is influenced by the underlying disease, especially in patients not achieving a stable response. This is also the first report which demonstrated that clearance of lymphocytes by alemtuzumab is largely independent from complement, and likely occurs through antibody-dependent cell-mediated cytolysis $(A D C C)$ rather than complement-dependent cytolysis $(C D C)$.

Disclosure of Interest: None declared.

\section{P710}

The role of Autologous Haematopoietic Stem Cell Transplant in MS - Updated results from the Pan-London MS group

F. Collins ${ }^{1, *}$, R. Nicholas ${ }^{2}$, O. Malik ${ }^{2}$, E. Silber ${ }^{3}$, P. Muraro ${ }^{4}$, A. Pagliuca ${ }^{3}$, M. Kazmi ${ }^{3}$

${ }^{7}$ King's College London Medical School, ${ }^{2}$ Imperial Healthcare, Charing Cross Hospital, ${ }^{3}$ King's College Hospital, ${ }^{4}$ Imperial Healthcare, Hammersmith Hospital, London, United Kingdom

Introduction: Recent years have witnessed an emerging role for Autologous Haematopoietic Stem Cell Transplantation (AHSCT) in the treatment of multiple sclerosis (MS), where existing disease modifying therapies (DMT) fail to stabilise the disease. A Pan-London MS AHSCT group, which selects patients for this procedure, reviews existing results and refines criteria for AHSCT, was set up earlier this year. We report on the updated results from this group.

Material (or patients) and methods: Retrospective analysis of outcome of AHSCT, from 2009-2015. Patients were selected based on persistent clinical relapses, or secondary progressive neurological disability, with MRI lesion activity despite use of at least 1 DMT. Follow-up included clinical evaluation, EDSS assessment and MRI scanning.

Results: As of December 2015, 21 patients (11 female, 9 SPMS, 12 RRMS) had received AHSCT. Mean age at transplant was 40 years (range 22-57). Mean EDSS at time of transplant was 5.5 (range 2.0-8.5). The majority of patients $(n=19)$ underwent Cyclophosphamide/ATG conditioning, with the remaining receiving BEAM/ATG conditioning. Whilst conditioning and stem cell infusion were well tolerated, 20/21 patients experienced early infectious complications. Delayed herpes zoster infection, beyond six months post-transplant, was seen in $(5 / 16)$ patients where data was available. 
Median follow-up was 576 days (range 57-1442). 100 day assessment EDSS scores are available for 10/12 RRMS patients and 6/9 SPMS patients, with the remaining having too short follow-up. Of the RRMS patients, 3 had a stable EDSS score, 4 patients had an improved EDSS of median improvement 1.0 (range $0.5-1.5$ ) and 3 patients had a deterioration in their EDSS score of 0.5. Of the SPMS patients, 3 patients had a stable EDSS score, 1 patient had an improvement in their EDSS score by 1.0 and 2 patients had deterioration in their EDSS score of 0.5 and 1.0 .

Conclusion: This study demonstrated the feasibility of AHSCT as a treatment for both RR and SPMS patients in this cohort. Transplant related mortality was $0 \%$ despite significant baseline disability. Notably, a high rate of early infectious complications was seen. The majority of patients showed improvement or stabilisation of functional status (EDSS) at 100 days post-transplant. The Pan London MS group has used this data to inform its revised criteria for AHSCT.

Disclosure of Interest: None declared.

\section{P711}

intermediate intensity regimen in autologous HSCT for severe Multiple Sclerosis: a a large, single center experience

R. Saccardi ${ }^{1, *}$, A. Barilaro ${ }^{2}$, A. Fani ${ }^{1}$, M. Giannini ${ }^{2}$, A. Gozzini ${ }^{1}$ S. Guidi ${ }^{1}$, C. Innocenti ${ }^{3}$, A. Mariottini ${ }^{2}$, C. Nozzoli ${ }^{1}$, E. Portaccio ${ }^{2}$, A. M. Repice ${ }^{2}$, M. P. Amato ${ }^{2}$, L. Massacesi ${ }^{2}$

${ }^{7}$ Hematology dept, ${ }^{2}$ Neurology Dept, ${ }^{3}$ Hematology, Careggi University Hospital, Florence, Italy

Introduction: High dose chemotherapy with autologous hematopoietic stem cell transplantation (aHSCT) is a promising approach for treatment of aggressive forms of multiple sclerosis (MS). Data reported in the literature are often derived from either small, single center or larger registry analysis, with a wide variability in the transplant techniques and clinical conditions at baseline. We report here a large retrospective, single-center analysis of 62 consecutive MS patients treated with an uniform transplant protocol.

Material (or patients) and methods: Sixty-two patients diagnosed with MS according either Poser or McDonald criteria were included in our transplant program between 1999 and 2015. Indication to the procedure was assessed by a mixed neuro-hematological team. Female/male ratio was 0.76 , median age was 36 (range 20-54). Thirty-seven patients (59.7\%) were diagnosed with Relapsing-Remitting (RR) MS, $23(37.1 \%)$ with Secondary Progressive (SP) MS, 2(3.2\%) had Primary Progressive (PP) MS. RR patients were more frequent after 2010. Median EDSS score at transplant was 6.0 (range 17.5). PBSC were mobilized with cyclophosphamide (CTX) $4 \mathrm{gr} /$ sqm+G-CSF and conditioned with BEAM/ATG, except for 2 patients who could not accomplish the conditioning with

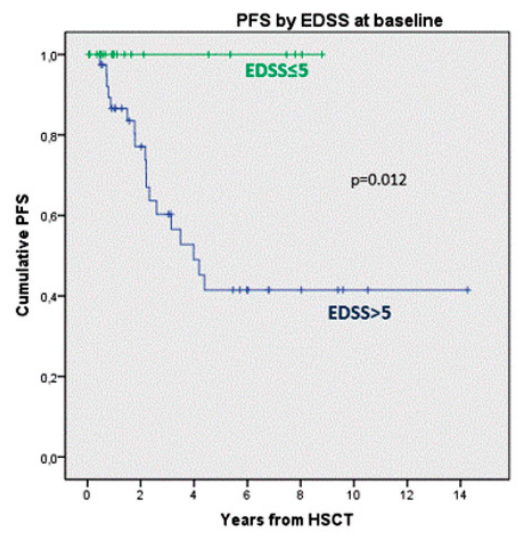

Figure 1: Progression free survival by EDSS at baseline

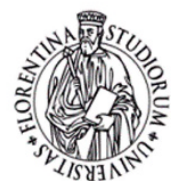

BEAM due to either BCNU reaction or detection of Meropenem- resistant Klebsiella respectively.

Results: All patients mobilized adequately except one, who required 1 dose of Plerixafor to achieve the planned cellular target. Median duration of follow up is 4.2 years (range 0.114.9). Three out of 62 patients relapsed at 3.9, 4.9 and 5 years from transplant, respectively, without disability progression thereafter. Overall progression rate was $27.4 \%$ at a median time of $2.2(0.47-4.39)$ years from HSCT: among progressed patients, 11 out of 23 had SPMS, 4 out of 37 RRMS and 2 out of 2 PPMS (RR vs SP, $P<0.004$ ).

The overall progression-free survival (PFS) at 5 and 10 years is $52.7 \%$. When stratified for MS form at baseline, the PFS at 5 years is $79.8 \%$ in RR and $17.6 \%$ in progressive MS $(P=0,002)$. A major prognostic factor for the neurological outcome was the EDSS score at baseline: patients transplanted with EDSS $\leq 5$ showed a $100 \%$ PFS at 5 years vs $41.5 \%$ for patient with EDSS $>5(P=0.012)$, Figure 1 .

We had no transplant-associated mortality. Two severe adverse event occurred during the procedure: an anaphylactic shock to carmustine and a E. Coli-related sepsis. FUO in 39 patients $(62.9 \%)$ and diarrhea in $25(40.3 \%)$, were the most frequently recorded adverse events in the first 100 days after HSCT. CMV and EBV reactivations occurred in 18(29\%) and 32 (51\%) patients within 3 months from HSCT, respectively; a preemptive treatment was administered in case of high viral titer with prompt negativization. Adverse events occurred beyond 100 days were documented pneumonia (3), HVZ reactivation (4), transient monoclonal gammopathy (9) and autoimmune thyroiditis (3).

Conclusion: Patients with RRMS in an early phase of disease, showing clinical or radiological evidence of high inflammatory activity, with EDSS score $\leq 5$ at baseline, had an excellent outcome. CTX/G-CSF mobilization followed by BEAM/ATG regimen provided a favourable risk/benefit equipoise and might be considered as a valuable clinical option for MS patients not responding to $1 / 2$ lines of approved treatments. Disclosure of Interest: None declared.

\section{P712}

HSCT in patients with Multiple Sclerosis - "cure", therapy goals and accepted risks of treatment

W. Chacińska ${ }^{1, *}$, M. Brzostowska ${ }^{7}$, E. Snarski ${ }^{1}$, M. Nojszewska ${ }^{2}$, W. Wiktor Jędrzejczak

${ }^{1}$ Department of Hematology, Oncology and Internal Diseases, ${ }^{2}$ Department of Neurology, Medical University of Warsaw, Warsaw, Poland

Introduction: Multiple sclerosis (MS) is the most common autoimmune inflammatory disease of the central nervous system. Autologous Hematopoietic Stem Cell Transplantation (AHSCT) brings hope for halt of the disease progression in many patients with this disease. Neurologist often point patients the mortality risk connected with the procedure and dissuade transplantation. However, MS patients' opinion on risk acceptance for this treatment option has never been studied. In our study we aimed at analysis of risk acceptance for HSCT among the patients with MS on various stages of the disease.

Material (or patients) and methods: The study was designed as a prospective online questionnaire. It was distributed to the members of internet forums of MS patients. Responders were asked about: their basic personal data, details of their illness, experienced symptoms, the influence on personal life, process of treatment, definition of the "cure" for MS and crucial goals in the treatment, and the risk of death accepted by them if their therapy could lead to cure or satisfactory target for therapy at the current stage of the disease.

Results: One hundred eighty patients filled the online questionnaire (129 women and 51 men). Average age of the patients was 33 years (range 18 - 64). The average EDDS score was 3 points. The types of the disease reported by patients were: $65 \%$ relapsing-remitting MS, $14 \%$ secondary-progressive, 


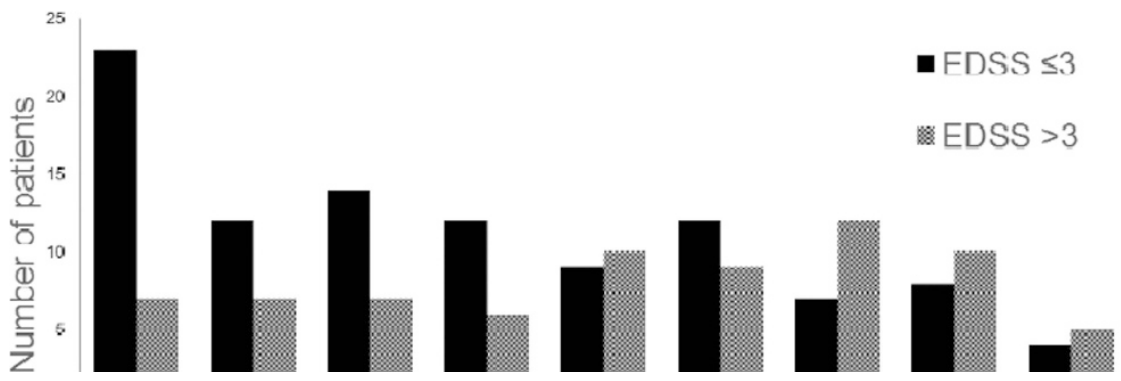

$10 \%$ primary-progressive, $4 \%$ another type, $6 \%$ did not know what type of MS they had. The symptoms which were reported as the biggest problems included: fatigue (72\%), paresis $(66 \%)$, balance disorders $(65 \%)$, urination and defecation disorders $(63 \%)$, depression and mood swings (63\%), erectile and sexual dysfunction (60\%), increased muscle tonus (58\%), eye disorders (55\%).

For $50 \%$ of patients relief of those symptoms was synonymous with "cure". When asked about acceptable therapy risk for achievement of this selfdefined "cure" $78 \%$ of patients with EDSS $\leq 3$ would undergo treatment with mortality rates of $1 \%$ or higher, and $55 \%$ of patients with EDSS $>3$ would undergo treatment with mortality rates over $10 \%$. Just to stop disease progression $73 \%$ of patients would undergo treatment with mortality of $1 \%$ or higher (Image 1).

When considering the best aim of the treatment, $56 \%$ of patients would choose to stop disease progression with low risk treatment and $21 \%$ would rather "cure" the disease even with high mortality rate.

Image 1. Mortality risk of treatment that stops disease progression that is accepted by multiple sclerosis patients.

Conclusion: To our knowledge it is the first study assesing what the "cure" of MS is for the patients. We also show patients' accepted mortality rates for AHSCT in MS patients. The disease is a heavy burden for patients with MS and they would accept risk much higher than connected with AHSCT to achieve alleviation of the symptoms and to stop progression of the disease.

Disclosure of Interest: None declared.

P713

Similar outcome of calreticulin type I and calreticulin type II mutations following RIC allogeneic hematopoietic stem cell transplantation for myelofibrosis

M. Christopeit ${ }^{1, *}$, A. Badbaran ${ }^{1}$, T. Zabelina ${ }^{1}$, G. Zeck ${ }^{1}$, F. Ayuk ${ }^{7}$, C. Wolschke ${ }^{1}$, N. Kröger

${ }^{1}$ Department of Stem Cell Transplantation, University Medical Center Hamburg, Hamburg, Germany

Introduction: Primary Myelofibrosis (PMF) frequently is accompanied by a mutation in JAK2 (V617F, 60\% of all PMF patients), CALR (20-25\%), or MPL (4-8\%). Lack of any of the three "classic" mutations ("triple negative") is prognostically adverse. Mutations in exon 9 of CALR in $80 \%$ of affected patients are either of type I (deletion of 52 basepairs at L367) or type II (insertion of 5 basepairs at K385). The favorable outcome of CALR mutated patients was hypothesized to be confined to patients with a type I CALR mutation as presence of a type II CALR mutation was associated with significant worse survival. In our recent analysis, we showed that CALR mutated patients who received allogeneic stem cell transplantation had an excellent OS (82\% at 4 years, significantly better than for JAK2V617F mutated or triple negative patients). Here, we analyzed whether the type of the CALR mutation impacted outcome after RIC followed by allogeneic stem cell transplantation.

Material (or patients) and methods: CALR type I mutation was found in $26 / / 40$ patients $(65 \%), 7$ patients (18\%) each had the type II mutation or a different mutation. Different mutations were frameshifts at K374 (2x), at K368, at E380, at D384fs and at K386 (2x). Median age of the 13 female and 27 male patients at transplantation was 58 years (, range 28-75 years). Myelofibrosis was of post-ET or post-PV origin in 11 patients $(28 \%)$. Bone marrow fibrosis was scored as $1^{\circ}$ in 2 patients $(5 \%), 2^{\circ}$ in 6 patients $(15 \%)$, and $3^{\circ}$ in 31 patients (78\%). Normal karyotype was observed in 18 patients (45\%). DIPSS was low risk in 1 patient (3\%), intermediate-1 in 8 patients (20\%), intermediate- 2 in 22 patients (55\%), and high in 9 patients $(23 \%)$. Conditioning for most of the patients consisted of intravenous Busulfan (10 doses of $0.8 \mathrm{mg} / \mathrm{kg} \mathrm{BW}$ ) and Fludarabin ( 6 doses of $30 \mathrm{mg} / \mathrm{m}^{2}$ ), combined with AntiLymphocyte Globulin at $30-90 \mathrm{mg} / \mathrm{kg}$. All patients received PBSC with a median of $6.5 \times 10^{6} \mathrm{CD} 34$ positive stem cells $/ \mathrm{kgBW}$ (, range $2.2-13 \times 106) .8$ patients $(20 \%)$ were transplanted from a matched related donor, 20 patients $(50 \%)$ were grafted from a matched unrelated donor, and 12 patients (30\%) received their transplant from a mismatched unrelated donor. Immunosuppression mainly consisted of cyclosporine and mycophenolic acid.

Results: Cumulative incidence $(\mathrm{Cl})$ of relapse at 3 years was $7 \pm 5 \%$ (type I), $14 \pm 14 \%$ (type II), and $29 \pm 19 \%$ (other CALR mutations, $P=0.838)$. Cl of non-relapse mortality (NRM) at 1 year was $12 \pm 7 \%$ (type I), 0 (type II), and 0 (other CALR mutations, $P=0.303$ ). Kaplan-Meier estimated $O S$ at 3 years was $75 \pm 9 \%$ (type I mutation), $86 \pm 13 \%$ (type II mutation), and $71 \pm 17 \%$ (other mutation, $P=0.903$ ). For the entire cohort, $\mathrm{Cl}$ of relapse at 3 years and NRM at 1 year were $13 \pm 6 \%$ and $5 \pm 4 \%$, resulting in OS of $75 \pm 7 \%$ at 3 years.

Conclusion: The negative prognostic impact of type II mutations in CALR that had been observed in the nontransplant setting can be overcome by allogeneic hematopoietic stem cell transplantation. RIC plus allogeneic HSCT for myelofibrosis with type I and type II CALR mutations as well as with other CALR mutations resulted in excellent OS.

Disclosure of Interest: None declared. 
P714

Outcome of patients $\geq 65$ years of age suffering myelofibrosis and treated with RIC allogeneic hematopoietic stem cell transplantation

M. Christopeit ${ }^{1, *}$, H. Alchalby ${ }^{1}$, T. Zabelina ${ }^{1}$, G. Zeck ${ }^{1}$, F. Ayuk ${ }^{1}$, C. Wolschke ${ }^{1}$, N. Kröger ${ }^{1}$

${ }^{1}$ Department of Stem Cell Transplantation, University Medical Center Hamburg, Hamburg, Germany

Introduction: Median age of patients with myelofibrosis is 65 years at diagnosis. Frequently, patients of higher age are considered not fit for allogeneic transplantation. We hypothesized that RIC plus allogeneic transplantation is feasible for this population of patients and assessed outcomes of 53 patients $\geq 65$ years with myelofibrosis.

Material (or patients) and methods: Patients' (22 female, 42\%) median age at transplantation was 68 (65-75) years. Primary Myelofibrosis (PMF) had been diagnosed in 37 patients $(70 \%)$, the disease was of post-ET/-PV origin in 16 patients (30\%). At diagnosis, 24 patients showed normal karyotype $(45 \%)$. Bone marrow fibrosis scored $1^{\circ}$ in 3 patients $(6 \%), 2^{\circ}$ in 7 patients (13\%), and $3^{\circ}$ in 34 patients (64\%). DIPSS was intermediate- 1 in 3 patients (6\%), intermediate-2 in 21 patients (40\%), and high in 22 patients (42\%). CMV IgG pairing between donor/ patient was negative/ negative in 17 patients (32\%), positive/ positive in 26 patients (49\%), positive/ negative in 4 patients (8\%), and negative/ positive in 6 patients (11\%). Intravenous Busulfan (10 doses of $0.8 \mathrm{mg} / \mathrm{kg}$ BW) and Fludarabin ( 6 doses of $30 \mathrm{mg} / \mathrm{m}^{2}$ ), combined with Anti-Lymphocyte Globulin at $30-90 \mathrm{mg} / \mathrm{kg}$, was used for conditioning in 33 patients (62\%), 17 patients (32\%) received an intensified RIC scheme consisting of sequential FLAMSAchemotherapy and Busulfan/ Fludarabine RIC, combined with $30-90 \mathrm{mg} / \mathrm{kg}$ Anti-Lymphocyte Globulin. The median of transplanted PBSC was $6.3 \times 10^{6}\left(1.5-16.1 \times 10^{6}\right) / \mathrm{kgBW}$. A matched related donor was present for 7 patients $(13 \%)$, matched unrelated for 27 patients (51\%), 18 patients $(34 \%)$ received their transplant from a mismatched unrelated donor, 1 patient from a haploidentical donor. Immunosuppression mainly $(85 \%)$ consisted of cyclosporine and mycophenolic acid.

Results: Leucocyte engraftment occurred in 49 patients (92\%), 4 patients (8\%) experienced primary graft failure. Median time to leucocyte engraftment was 13 (7-34) days, median time to platelet engraftment 20.5 (5-293) days. Acute GVHD grades 2-4 was present in 22 patients (42\%), chronic GVHD in 19 patients (36\%). Cumulative incidence $(\mathrm{Cl})$ of relapse was $21 \pm 6 \%$ after 3 years. $\mathrm{Cl}$ of non-relapse mortality (NRM) was $28 \pm 6 \%$ after 1 year. OS at 3 years was $46 \pm 8 \%$.

Conclusion: Allogeneic stem cell transplantation of patients with myelofibrosis is feasible even at and above 65 years of age. Further research will have to focus on further reducing NRM in this elderly population.

Disclosure of Interest: None declared.

\section{P715}

Sequential intensified RIC versus standard RIC conditioning followed by allogeneic SCT for high risk myelofibrosis patients

M. Christopeit ${ }^{1, *}$, T. Zabelina ${ }^{1}$, H. Alchalby ${ }^{1}$, G. Zeck ${ }^{1}$, F. Ayuk ${ }^{1}$, C. Wolschke", N. Kröger ${ }^{1}$

${ }^{1}$ Department of Stem Cell Transplantation, University Medical Center Hamburg, Hamburg, Germany

Introduction: Patients suffering myelofibrosis with high risk according to DIPSS are at significantly increased risk of relapse and death. In order to improve the results of BusulfanFludarabine-based conditioning, we investigated conditioning with sequential FLAMSA-chemotherapy followed by BusulfanFludarabine-based conditioning in 17 patients with DIPSS "high risk". We compared their outcomes to the outcomes of 21 historical patients who received RIC Busulfan-Fludarabine with DIPSS "high risk".
Material (or patients) and methods: At transplantation, the 11 female and 27 male patients had a median age of 64 years (, range 37-75 years). Myelofibrosis was classified as primary (PMF) in 25 patients (66\%), and as post-PV/ -ET myelofibrosis in 13 patients (34\%). Conditioning consisted of intravenous Busulfan (10x0.8 mg/kgBW) and Fludarabine $\left(6 \times 30 \mathrm{mg} / \mathrm{m}^{2}\right)$ for 21 patients. The remaining 17 patients were conditioned with Fludarabine $30 \mathrm{mg} / \mathrm{m}^{2}$, Amsacrine $100 \mathrm{mg} / \mathrm{m}^{2}$, Cytarabine $2000 \mathrm{mg} / \mathrm{m}^{2}$ on 4 consecutive days, briefly "FLAMSA", followed by intravenous Busulfan (10x0.8 mg/kgBW) and Fludarabine (additional $2 \times 30 \mathrm{mg} / \mathrm{m}^{2}$ ). Both regimens were combined with Anti-Lymphocyte Globulin at $30-90 \mathrm{mg} / \mathrm{kg}$. All patients received $\mathrm{PBSC}$ with a median of $6 \times 10^{6} \mathrm{CD} 34$ positive stem

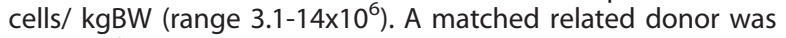
chosen for 5 patients (13\%), 20 patients $(53 \%)$ received a transplant from a matched unrelated donor, and 13 patients (34\%) were transplanted from a mismatched unrelated donor. Immunosuppression mainly consisted of cyclosporine and mycophenolic acid.

Results: Leucocyte engraftment was reached after a median of 12 (range 9-32) days (FLAMSA-Busulfan), and 14 (range 10-30) days (Busulfan-Fludarabine, $P=0.163$ ). Platelet recovery occurred after 20 (range 9-118) days (FLAMSA-Busulfan), and 22 (range 10-293) days (Busulfan-Fludarabine, $P=0.560$ ). Acute GVHD grades 2-4 was experienced by 6 patients (FLAMSABusulfan) and 10 patients, respectively (Busulfan-Fludarabine, $P=0.444$ ). Eleven patients in the FLAMSA-Busulfan group versus 8 patients in the Busulfan-Fludarabine group experienced chronic GVHD to some extent $(P=0.103)$. Cumulative incidence $(\mathrm{Cl})$ of relapse at 3 years was $18 \pm 10 \%$ (FLAMSABusulfan) and $22 \pm 10 \%$ (Busulfan-Fludarabine, $P=0.958)$. $\mathrm{Cl}$ of non-relapse mortality (NRM) at 1 year was $26 \pm 12 \%$ (FLAMSABusulfan) and $27 \pm 11 \%$ (Busulfan-Fludarabine, $P=0.726$ ). Kaplan-Meier estimated OS at 3 years was $63 \pm 11 \%$ (Busulfan-Fludarabine) and $62 \pm 12 \%$ (FLAMSA-Busulfan, $P=0.863$ ).

Conclusion: In this series of patients treated in one institution, $\mathrm{OS}, \mathrm{Cl}$ of relapse and $\mathrm{Cl}$ of NRM of patients with myelofibrosis after FLAMSA-Busulfan-based conditioning was comparable to corresponding outcomes following Busulfan-Fludarabinebased conditioning.

Disclosure of Interest: None declared.

\section{P716}

Pathway inhibitors in the context of allogeneic hematopoietic stem cell transplantation (HSCT) for chronic lymphocytic leukemia (CLL): a single center safety analysis M. Hahn ${ }^{1}$, S. Dietrich ${ }^{1}$, M. Görner ${ }^{2}$, M. Welslau ${ }^{3}$, F. Kleinsorge ${ }^{4}$, M. Rummel ${ }^{5}$, A. Köhler ${ }^{6}$, T. Zenz ${ }^{1}$, S. Stilgenbauer ${ }^{7}$ A. D. Ho ${ }^{1}$, P. Dreger ${ }^{1, *}$

${ }^{1}$ Internal Medicine V, UNIVERSITY HOSPITAL HEIDELBERG, Heidelberg, ${ }^{2}$ Klinik für Hämatologie, Onkologie und Palliativmedizin, Klinikum Bielefeld, Bielefeld, ${ }^{3}$ Praxis für Onkologie, Klinikum Aschaffenburg, Aschaffenburg, "Internistische Gemeinschaftspraxis mit onkologischem Schwerpunkt, Medicum Detmold, Detmold, ${ }^{5}$ Internal Medicine IV, University Hospital Gießen, Gießen, 'Gemeinschaftspraxis für Hämatologie und Onkologie, Fachärztezentrum an der Asklepiosklinik Langen, Langen, 7 Internal Medicine III, University Hospital UIm, Ulm, Germany

Introduction: Pathway inhibitors (PI), such as ibrutinib (IBR) and idelalisib (IDE), have dramatically increased the treatment options for high-risk CLL. There is only limited information on the interference of $\mathrm{PI}$ on a subsequent HSCT, and on the feasibility of PI treatment of CLL relapse after HSCT.

Material (or patients) and methods: Objectives of this single centre retrospective analysis were (I) to investigate the outcome of HSCT after previous PI exposure; and (II) to investigate safety and efficacy of post-transplant relapse treatment with $\mathrm{PI}$ in patients with CLL.

Results: (I) Between Nov 2013 and Nov 2015, 8 patients $(\mathrm{m} / \mathrm{f}=$ 5/3; median age 53y (33-61)) underwent HSCT for relapsed/ refractory high-risk CLL (RR-CLL) after prior exposure to IBR (5), CC-292 (2), IDE (2), and/or venetoclax (1) (multiple exposure 
included). Indications for HSCT were (hierarchical) RR-CLL with poor-risk cytogenetics (5), previous PI failure (1), and Richter transformation (RT)(2). Before start of conditioning, 7 patients were still responding to the most recent $\mathrm{PI}$ with incipient progression under IBR in 2 of them. Patients had been on their most recent PI for 9 (1-15) months. Donors were well-matched unrelated (7) or related (1); conditioning comprised fludarabine/ATG with Treosulfan (6) or TBI 8 Gy (2). PI was generally stopped on the day before conditioning. However, a single patient developed a fulminant CLL relapse under fludarabine conditioning within 3 days (!) after IBR discontinuation and was put back on IBR until day -1 . With 5 (1-18) months of follow-up, low-grade acute and chronic GVHD each occurred in one patient. Whilst unexpected toxicity was not observed, one patient who had been bridged with IBR dynamically progressed at day +72 , and another patient had a relapse of her RT on day +214 resulting in rapid death. The 6 remaining patients live progression-free, 2 of them MRD-negative.

(II) 14 patients $(\mathrm{m} / \mathrm{f}=10 / 4$; age 50y (33-64) started IBR (12), IDE (1) or CC-292 (1) for CLL (14) or RT (2) relapse 33 months (1-88) after HSCT. 13/14 had been allografted from an unrelated donor (10 MUD, 3 MMUD), 4/14 were $17 p$ - at relapse. The median PI therapy duration is currently $5+(1-20)$ months. IBR had to be discontinued in 4 patients because of grade 3-4 skin toxicity (2), stomach cancer (1), or CLL progression (1), and CC-292 because of RT relapse. No other grade 3-4 toxicity has been observed. GVHD was absent in all patients at PI commencement and remained so except for one patient who developed extensive chronic GVHD while receiving DLI along with IBR. At a median observation time of 12 months, 6 patients are in ongoing response ( 5 in PR, and one patient in sustained MRD-negative CR despite IBR discontinuation), 4 have progressed, and 4 are too early. With a single RT-related death, 12-month survival after start of post-HSCT PI is $89 \%$.

Conclusion: This preliminary data does not raise safety concerns about the use of $\mathrm{PI}$ in the context of HSCT. IBR seems to be effective both for bridging patients with high-risk CLL to HSCT and for treating relapse afterwards. Multicenter studies are underway to define the optimal integration of $\mathrm{PI}$ in the HSCT pathway including their peritransplant use and posttransplant maintenance / pre-emptive strategies.

Disclosure of Interest: None declared.

\section{P717}

SETBP1 mutations are associated with early relapse after allogeneic hematopoietic stem cell transplantation

N. Winkelmann ${ }^{1, *}$, V. Schaefer ${ }^{1}$, J. Rinke ${ }^{1}$, A. Markusch ${ }^{1}$ A. Waldau' ${ }^{1}$, J. Reichert ${ }^{1}$, A. Hochhaus ${ }^{1}$, I. Hilgendorf ${ }^{1}$, T. Ernst $^{1}$

${ }^{7}$ Hematology/Oncology, Jena University Hospital, Jena, Germany

Introduction: SETBP1 mutations have recently been discovered and characterized in myelodysplastic syndrome (MDS), secondary acute myeloid leukemia (AML), myelodysplastic/ myeloproliferative neoplasms (MDS/MPN), and aplastic anemia. Even though their rare incidence $(1-5 \%$ in the above mentioned diseases), they are associated with a poor prognosis due to rapid disease progression. We sought to investigate the impact of SETBP1 mutations in a cohort of patients with myeloid disorders eligible for allogeneic hematopoietic stem cell transplantation (HSCT).

Material (or patients) and methods: To address this subject, we have screened an unselected cohort of 74 patients with primary AML $(n=23)$, secondary $\operatorname{AML}(n=24)$, MDS $(n=10)$, classical MPN $(n=4), \operatorname{MDS} / \mathrm{MPN}(n=10)$ and aplastic anemia $(n=3)$ who underwent allogeneic SCT from 2011 to 2015 Mutational screening was performed from bone marrow samples (70 patients) or peripheral blood (4 patients) at diagnosis or disease progression prior to allogenic SCT. We applied bidirectional Sanger sequencing of genomic DNA covering the entire SKI homology domain of SETBP1.

Results: In total, we found SETBP1 mutations in 3/74 (4\%) patients (atypical CML, $n=2, \mathrm{CMML}-2, n=1$ ). All three patients were 60 years of age at diagnosis and underwent allogeneic SCT from matched unrelated donors after reduced-toxicity conditioning due to aggressive disease. Karyotype was normal in two patients and one patient harbored a trisomy 21 and loss of chromosome X. All mutations were located within known somatic SETBP1 hotspots according to the COSMIC database (codons $868,870,871$ ). The somatic origin of all mutations was confirmed in germline material. All patients with SETBP1 mutations relapsed after SCT. In one patient, the SETBP1-clone dissolved for 6 months after allotransplant, but re-appeared at time of relapse (day +183 ), while in another patient the clone harboring SETBP1 mutation persisted through engraftment and relapse $($ day +62$)$. The last patient relapsed recently (day +375 ) and mutational analysis for SETBP1 is currently ongoing. Conclusion: Mutated SETBP1 is a rare event but associated with rapid disease progression and early relapse after allogeneic SCT in this small cohort of patients. Multicenter studies of mutational status before and after allogeneic SCT with determination of cooperating mutations should be conducted. Since SETBP1 mutations are present in disease evolution, they may drive disease progression and may guide future treatment decisions.

Disclosure of Interest: None declared.

\section{P718}

MAC and RIC conditioning regimens have the same effectiveness in case of allogenic hematopoietic stem cell transplantation for patients with Hurler syndrome: longtime follow-up experience of the Russian joint study

K. Kirgizov 1,2,* A. Borovkova ${ }^{3}$, E. Semenova ${ }^{3}$, E. Pristanskova ${ }^{1}$ S. Mikhailova ${ }^{4}$, N. Sidorova ${ }^{7}$, D. Balashov ${ }^{2}$, V. Konstantinova ${ }^{7}$ B. Purbueva , O. Blagonravova', O. Paina ${ }^{3}$, S. Razumova ${ }^{3}$, P. Kozhokar, A. Osipova ${ }^{3}$, K. Ekushov', L. Zubarovskaya ${ }^{3}$, A. Bologov ${ }^{5}$, E. Skorobogatova ${ }^{7}$, A. Maschan ${ }^{2}$, B. Afanasyev ${ }^{3}$, A. Rumyantsev ${ }^{2}$

${ }^{1}$ BMT Department, The Russian Children's Research Hospital, ${ }^{2}$ Dmitry Rogachev Federal Research Center of Pediatric Hematology, Oncology and Immunology, Moscow, ${ }^{3}$ R.M.Gorbacheva Memorial Institute of Children Oncology, Hematology and Transplantation, First Pavlov State Medical University of St.Petesburg, Saint-Petersburg, ${ }^{4}$ Genetics department, ${ }^{5}$ The Russian Children's Research Hospital, Moscow, Russian Federation

Introduction: In-time HSCT for pts. with Hurler syndrome (HS) can significantly improve the results. Long-term follow-up required to find a better conditioning regimen and graft source on a background of very high rejection potential. We aimed to analyze our experience with $\mathrm{HS}$ and estimate a safety and efficacy of RIC and MAC conditioning.

Material (or patients) and methods: $33 \mathrm{HSCT}$ during the 2004-2015 were performed at 31 pts. with HS. Median age at the diagnosis was 15 months (3-24 months), at HSCT 22 months (9-42 months). BM used in $63,6 \%(n=21)$, PBSC $31,2 \%(n=10), \mathrm{CB}-5,2 \%(n=2)$. CD34+ dose median was $8,3 \times 10^{6} / \mathrm{kg}(2,7-13,1)$. MAC conditioning was used for $24 \mathrm{HSCT}$, RIC - for 9. RIC regimen: Flu+Mel+ATG, MAC: Bu/Treo+Flu +Thio/Mel (Bu was used in early 2000) and ATG+Rituximab (in case of MUD HSCT). All pts. with RIC received MUD HSCT, pts. with MAC MUD - 18 pts., MRD - 5 pts. Pts. received CsA/Tacrobased GvHD prophylaxis. MMF/MTX was additionally added in all cases. In 3 RIC HSCT immunomagnetic CD3/CD19+ depletionof PBSC (by CliniMACS) was used.

Results: All pts. engrafted with of full donor chimerism on $D$ +30 . Median of engraftment day - 20 (11-29 days). Median follow-up - 50 months (7-140 months). 27 pts. survived. All pts. with complete donor's chimerism have good response - based on AIDU activity (best response correlated with short interval to transplant after diagnosis establishing). Reasons of death MAC: infection - 3 pts., RIC: TRALI - 1 pt., aGvHD - 1 pt. All infection deaths were registered before 2009. No severe toxicity revealed. GvHD: Grade II - developed 14 pts., Grade IIIIV -2 pts. (after RIC), local cGVHD - 3 pts. (RIC). No pts. had extensive $C G v H D .5$ pts. rejected (MAC and RIC rejection rate 


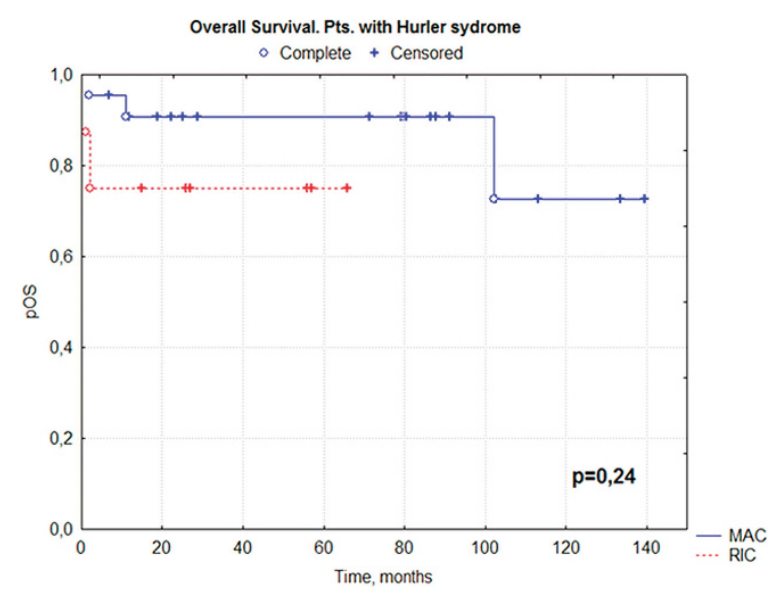

was same). Our data showed statistically same OS for MAC and RIC (see picture).

Conclusion: In-time HSCT is an effective way to stop neurodegenerative process for pts. with HS. OS for MAC and $\mathrm{RIC}$ conditioning regimens is statistically same. MAC conditioning regimen was associated with the higher risk of infections, but infection control improving with years. RIC was associated with higher risk of aGvHD. Rejection rate was close between these regimens (MAC rejections were associated with Thiotepa usage). Our study showed possibility of using of nonmanipulated graft. Both these regimens can be used on clinics choice. Russian Joint study showed effective cooperation for treatment pts. with HS.

Disclosure of Interest: None declared.

P720

Allogeneic hematopoietic stem cell transplantation with major $\boldsymbol{\beta}$-thalassemia in children: Algerian experience M. Benakli ${ }^{1, *}$, R. Ahmed Nacer ${ }^{1}$, F. Mehdid ${ }^{1}$, R. Belhadj ${ }^{1}$, F. Tensaout ${ }^{1}$, N. Rahmoune ${ }^{1}$, D. Ait Ouali ${ }^{1}$, M. Baazizi ${ }^{1}, H$. Bouarab ${ }^{1}$, S. Zerkout ${ }^{1}$, N. Boukhenfouf', R.-M. Hamladji ${ }^{1}$

${ }^{1}$ Hematology-Bone Marrow Transplantation Department. Pierre and Marie Curie Center, Algiers, ${ }^{2}$ Pediatrics Department, Rouiba, Algeria

Introduction: Allogeneic hematopoietic stem cell transplantation (Allo-HSCT) remains the only curative approach for children with major $\beta$-thalassemia. HLA-matched related donor allo-HSCT should be performed as early as possible if donor is available. This retrospective study analyzes the outcome of 47 consecutive pediatric patients (pts) with $\beta$ thalassemia who underwent Allo HSCT with HLA-identical donors in a our center

Material (or patients) and methods: From June 1999 until May 2015, 47 consecutive pts affected by transfusiondependent major $\beta$-thalassemia underwent allo-HSCT from matched related donors in our institution (Sibling HLA identical: 38, pheno-identical: 6 , cord blood: 3$)$. The median age:7,6 years (3-15) within 28 (59,7\%) older than 7 years. Sexratio: 1,47 . The median red blood cell transfusions was 69,5 units/pt (20-177). Irregular iron chelation in 39 pts (82,9\%) and median serum ferritin at transplant was $2238 \mathrm{ng} / \mathrm{ml}$ (2507424). Liver biopsy was performed in all pts and Risk class Pesaro was determined in all pts: $6(13 \%)$ were assigned to risk class $1,19(41,3 \%)$ to class 2 and $22(45,7 \%)$ to class 3. Nineteen pts $(41,3 \%)$ had splenectomy. The median time diagnosis-graft was 80,7 months $(27-174)$. The preparative regimen consist on Misulban $500 \mathrm{mg} / \mathrm{m}^{2}$ per os or Busilvex doses adapted, Ciclophosphamide $200 \mathrm{mg} / \mathrm{kg}$ and Thymoglobuline $10 \mathrm{mg} / \mathrm{kg}$ (Pesaro 1-2); Misulban $14 \mathrm{mg} / \mathrm{kg}$ or Busilvex dose adapted, Ciclophosphamide $120 \mathrm{mg} / \mathrm{kg}$ and Thymoglobuline $10 \mathrm{mg} / \mathrm{kg}$ (Pesaro 3); Busilvex dose adapted, Thiotepa $10 \mathrm{mg} / \mathrm{kg}$ and Fludarabine $160 \mathrm{mg} / \mathrm{m}^{2}$ (Cord blood).
Prevention of GVHD consisted of the association Ciclosporine and Methotrexate short cycle (Seattle) or Ciclosporine alone (cord blood). The grafts used are peripheral blood stem cells in 41 pts (84\%) with an average rate of CD34+ cell: $10,3 \times 10^{6} / \mathrm{kg}$ $(4,47-37,90)$, bone marrow in 4 pts with an average rate of Nuclear Cells (NC): $5.1210^{8} / \mathrm{kg}$ and cord blood in 3 pts with a rate of NC: $4.810^{7} / \mathrm{kg}$. At November 2015, the minimal followup was 7 months and the maximal 198 months.

Results: Median time of aplasia, observed in all pts, was 17 days (6-71). Neutrophil engraftment was observed at day 16 (9-68). Transfusions were required in all pts with an average of red blood cells: 5,4 units/pt (2-15) and Platelets concentrates: 5,3 units/pt (0-34). Seven pts (5 with Pesaro score 3 ) presented an early rejection and received boost without any benefit. Veinous occlusive disease observed in 4 pts $(8,7 \%)$. Acute GVHD grade II-IV was seen in 13 cases (28\%) and extensive chronic GVHD in 4 pts $(8,7 \%)$. Thirty-five pts $(74,4 \%)$ are alive with a median follow up of 71 months (7-185) within 29 pts $(68 \%)$ with total donor chimerism. Twelve pts $(25,5 \%)$ died (graft rejection: 4, earlier infection: 2, VOD: 1, GVH: 4, hydrocephalus: 1). Overall survival (OS) and event-free survival (EFS) at 16 years are $75,7 \%$ and $66,8 \%$ respectively. According to Pesaro score, OS and EFS are $76,7 \%$ and $70,5 \%$ (Pesaro 1-2) and $74,6 \%$ and $62,8 \%$ (Pesaro 3 ).

Conclusion: In our series, most of pts have higher Pesaro score and are older than 7 years. Allo-HSCT should be performed early to have a best results

Disclosure of Interest: None declared.

\section{P721}

Allogeneic stem cell transplantation with a reduced toxicity conditioning regimen for thalassemia major - long term results and follow up

M. Y. Shapira ${ }^{1,2, *}$, S. Vipul', B. Avni ${ }^{1}$, S. Grisariu' ${ }^{1}$, P. Stepensky ${ }^{2}$, R. Or ${ }^{1}$

${ }^{1}$ Department of Bone Marrow Transplantation, ${ }^{2}$ Pediatric hematooncology, HADASSAH UNIVERSITY HOSPITAL, Jerusalem, Israel

Introduction: The only radical cure for patients with thalassemia major is the replacement of the defective hematopoietic system by allogeneic stem cell transplantation (allo-SCT). The major obstacles for the application of allo-SCT have been the transplant-related morbidity and mortality rates and graft failure that is usually associated with the recurrence of the thalassemia hematopoiesis.

Material (or patients) and methods: In this report we address our experience of allo-SCT from matched related and unrelated donors, using a reduced toxicity (yet myeloablative) conditioning with fludarabine-busulphan in a cohort of 51 patients with thalassemia major (41 related and 10 unrelated donors). The primary endpoint was overall survival and secondary endpoint was thalassemia free survival.

Results: The regimen-related toxicity was minimal, and engraftment failure or primary rejection was $7 / 51$, (13\%) out of which $4(60 \%$ of failures) were in unrelated grafts. With a median follow-up period of 96 months (range 2- 186 months) the overall survival was $90 \%$ and the thalassemia-free survival was $68 \%$ (figure 1). When comparing the related and unrelated groups, in the group of 41 patients who received matched related transplants the overall survival was $97 \%$ and thalassemia free survival was $82 \%$. Whereas, in the matched or mismatched unrelated donor group, overall survival was $60 \%$ and thalassemia free survival was a dismal 10\%. Interestingly, $17(37 \%)$ of the surviving patients had prolonged mixed chimerism while thalassemia free, out of whom only one recovered thalassemic hematopoiesis and successfully underwent a second allo-SCT.

Conclusion: We conclude that overall survival and thalassemia free survival are excellent with related donors. Much still needs to be done regarding development of protocols regarding unrelated donors in thalassemia.

Disclosure of Interest: None declared. 


\section{P722}

The efficacy of hematopoietic stem cell transplantation in modifying disease phenotype in Glycogen Storage Disease type $\mathbf{1 b}$

S. O'Brien ${ }^{1, *}$, S. Jones ${ }^{2}$, A. Broomfield ${ }^{2}$, F. White ${ }^{2}$, C. Murray ${ }^{3}$, A. Fagbemi ${ }^{4}$, D. Bonney ${ }^{1}$, R. Wynn ${ }^{1}$

${ }^{1}$ Haematology Department, ${ }^{2}$ Metabolic Department, ${ }^{3}$ Respiratory Department, ${ }^{4}$ Gastroenterology Department, Royal Manchester Children's Hospital, Manchester, United Kingdom

Introduction: Glycogen storage disease (GSD) type $1 \mathrm{~b}$ is a metabolic disease caused by glucose-6-phosphate translocase deficiency; hypoglycaemia during minimal fasting occurs. Severe neutropenia is also a prominent feature and is associated with recurrent bacterial infections, poor wound healing and inflammatory bowel disease (IBD) ${ }^{1}$.

Haematopoietic stem cell transplantation (HSCT) is widely used in the management of children with severe constitutional neutropenia but its role in correcting the neutropenia and other disease manifestations of GSD1b is poorly understood. However a single previous case report of HSCT in GSD1b has suggested that transplantation corrected neutropenia, and ameliorated metabolic control ${ }^{2}$. We report a second case of GSD1b treated with HSCT to further address the value of transplant on symptomatology. (Table 1)

Material (or patients) and methods: Our patient presented with hypoglycemia at 7 months of age. Genetic testing confirmed GSD1b. Management was regular daytime feeds and continuous overnight feeds via nasogastric tube gastrostomy insertion was avoided due to poor wound healing in GSD1b. He developed neutropenia around 9 months old with multiple infections requiring hospitalization. By 2.5 years there was increasing concern about the development of IBD. Granulocyte-colony stimulating factor use was complicated by extra-medullary haemopoiesis and hypersplenism. HSCT was offered when the patient was 4 years old. Transplant aims are outlined in Table 1 . He received a 5/6 matched unrelated cord transplant after conditioning with Fludrabine, Thioepa, Treofulfan and ATG. Graft-versus-host disease (GvHD) prophylaxis was ciclosporin and prednisolone.

Results: The transplant was initially uncomplicated until grade 4, steroid-refractory skin GvHD developed by day 45 . Subsequently, frequent diarrhoea with abdominal cramping occurred, indicating likely gut GvHD. He received mesenchymal stem cells and his skin disease remitted. Neutropenia completely resolved and 8 months post-transplant a gastrostomy was inserted and the site healed well. Diarrhoea persisted and biopsies showed a granulomatous colitis, which was managed as Crohns disease with Mesalazine and Infliximab. This was discontinued as diarrhoea resolved. Fasting interval was not improved by HSCT. Some chronic GVHD of the chest persists but he is rarely in hospital.

Table 1

\begin{tabular}{lcc}
\hline Aims & Intended outcome & Achieved \\
\hline $\begin{array}{l}\text { Correct } \\
\text { Neutropenia }\end{array}$ & $\begin{array}{c}\text { 1) Decrease infections } \\
\text { Decrease G-CSF driven extra- } \\
\text { medullary haemopoiesis } \\
\text { Improve bowel symptoms }\end{array}$ & Yes \\
$\begin{array}{l}\text { Improve } \\
\text { Wound } \\
\text { Healing } \\
\text { Improve meta- } \\
\text { bolic control }\end{array}$ & Allow gastrostomy insertion & Yes \\
\hline
\end{tabular}

Conclusion: We conclude that HSCT has significant utility in GSDIb and has the potential to improve the quality of life in carefully selected patients. Metabolic control will probably not be improved by HSCT and liver transplant may still be necessary in older children.

References: 1) Visser G, Rake JP, Fernandes J, Labrune P, Leonard JV, Moses S, et al. Neutropenia, neutrophil dysfunction, and inflammatory bowel disease in glycogen storage disease type lb: Results of the European Study on Glycogen Storage Disease Type I. J Pediatr. 2000;137:187-91.

2) Pierre G, Chakupurakal G, McKiernan P, Hendriksz C, Lawson S, Chakrapani A. Bone Marrow Transplantation in Glycogen Storage Disease Type 1b. J Pediatr. 2008;152:286-8.

Disclosure of Interest: None declared.

\section{P723}

Efficacy of the second CD34 cell mobilization cycle in poormobilizing patients with lymphoma and myeloma

J. Romejko-Jarosinska ${ }^{1, *}$, L. Poplawska ${ }^{1}$, L. Targonski ${ }^{1}$, M. Szymanski ${ }^{1}$, E. Mroz-Zycinska ${ }^{1}$, Z. Pojda ${ }^{1}$, J. Walewski ${ }^{1}$ MARIA SKLODOWSKA CURIE MEMORIAL CANCER CENTRE, Warsaw, Poland

Introduction: Feasibility of hematopoietic cell transplantation (HCT) depends on a successful CD34 cell harvest. Poormobilizing patients need another mobilization cycle to collect a minimum cell dose of $>2 \times 10^{6} / \mathrm{kg}$. We evaluated efficacy of the second mobilization cycle and searched for potential factors predicting for the successful second harvest.

Material (or patients) and methods: Between January 2010 and October 2015, 451 adult patients were mobilized with chemotherapy and G-CSF at our institution, and 57 (13\%) failed the first cycle. Of 57 patients, 20 had Hodgkin lymphoma, 12 - DLBCL, 14 - plasma cell myeloma, and 11 other lymphoma. 26 patients (46\%) did not produce sufficient CD34 cell peak in peripheral blood for initiating aphereses, and 31 patients $(54 \%)$ had inadequate harvest after the first collection cycle. Median patient age was 48 (range, 21-69) and 15 patients were $60 \mathrm{y} / \mathrm{o}$ or more. Six patients received plerixafor in the first mobilization. Median time from the first to the second procedure was 2 months (range, 1-30). The following mobilization regimens were used: G-CSF+ high dose cyclophosphamide $(n=12), \mathrm{G}-\mathrm{CSF}+$ high or intermediate dose cytarabine $(n=22), \mathrm{G}-\mathrm{CSF}+\mathrm{ICE} / \mathrm{CED}(n=19), \mathrm{G}-\mathrm{CSF}$ alone $(n=5)$. Plerixafor was used in 20 patients. The mobilization was considered effective if CD34 cell dose of $2.0 \times 10^{\wedge} 6 / \mathrm{kg}$ was collected.

Results: Overall, 54\% of patients had a peak CD34 cell count of $>10 /$ uL in peripheral blood, and $21 \%$ had a maximum cell count $>20 /$ uL with a median (range) 9.5/uL (0-99). Sufficient cell dose (median harvest $1.59 \times 10^{\wedge} 6 / \mathrm{kg}$ (range, $0-10.6 \times 10^{\wedge} 6$ / $\mathrm{kg}$ ) was obtained in $42 \%$ patients in the first course. Out of patients who collected suboptimal cell count in the first mobilization, 19\% completed an adequate harvest in the second cycle. Overall, $61 \%$ of patients collected sufficient cell number in order to proceed to HCT. We were unable to identify clinical or treatment related factors that would predict for successful mobilization. None of the number of chemotherapeutic regimens or the use of plerixafor were associated with improved mobilization in univariate analysis $(P=0.17)$. Absolute CD34 cell count $>10 u \mathrm{~L}(P=0.0001)$ and CD34 cell percentage $>0.05 \%$ of WBC count (median $0.06 \%$ [0-2.2\%]) on day 1 of planned harvest $(P=0.0001)$ was predictive for the successful collection. Of 57 poor-mobilizing patients, 32 (54\%) eventually proceeded to HCT.

Conclusion: About $60 \%$ of poor mobilizing patients collected sufficient CD34 cell dose in the second mobilization cycle including $42 \%$ patients with successful second mobilization itself, and $19 \%$ of patients with an adequate cell harvest form the first and the second collection combined. Only the absolute CD34 cell count $>10 / \mathrm{uL}$ in peripheral blood and CD34 cell percentage $>0.05 \%$ of WBC were predictive for successful second mobilization.

Disclosure of Interest: None declared. 
P724

Hodgkin Lymphoma relapsing after Allogeneic Stem Cell Transplantation: The efficacy of Brentuximab Vedotin in combination with Donor Lymphocyte Infusion

P. Tsirigotis ${ }^{1}$, R. Yerushalmi ${ }^{2}$, K. Gkirkas ${ }^{1, *}$, N. Shemtov ${ }^{2}$, I. Danylesko ${ }^{2}$, M. Stamouli ${ }^{1}$, A. Avigdor ${ }^{2}$, A. Spyridonidis ${ }^{3}$,

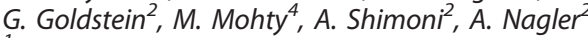

${ }^{1}$ 2nd Dept of Internal Medicine, ATTIKO University Hospital, National and Kapodistrian University of Athens, Athens, Greece, ${ }^{2}$ Hematology Division, Chaim Sheba Medical Center, Tel Hashomer, Tel Aviv, Israel, ${ }^{3}$ Bone Marrow Transplantation Unit, University of Patras, Rio, Patras, Greece, ${ }^{4}$ Department of Hematology, Hospital St. Antoine, Paris, France

Introduction: Brentuximab Vedotin (BV) administered as maintenance after auto-SCT in pts with Hodgkin lymphoma $(\mathrm{HL})$ is safe and effective. In contrast, there is a paucity of data regarding the efficacy and safety of BV administration, combined or not with donor lymphocyte infusion (DLI), after allo-SCT.

Material (or patients) and methods: Fifteen pts with relapsed $\mathrm{HL}$ after allo-SCT were included in this report. There were 8 males and 7 females, with a median age of 28 years (range, 15 - 40). BV was administered before allo-SCT in 11 pts (5 responded to BV). Graft was PBSC from a matched related or an unrelated donor in 8 and 6 pts respectively, while 1 pt received a double umbilical cord blood graft. Seven and 4 pts achieved CR and PR after allo-SCT, while 4 pts had no response. Twelve pts progressed in a median of 5.5 months (range, 2-17) after allo-SCT. Two pts in PR and 1 in CR but with high risk for relapse after allo-SCT received consolidation with $B V$. All pts received BV at a dose of $1.8 \mathrm{mg} / \mathrm{kg}$ every 3 weeks (max 16 doses). A median of 4 (range, 1-16) BV cycles was administered. DLI was co-administered in 9 pts.

Results: Eight out of 12 pts with relapsed $\mathrm{HL}$ after allo-SCT achieved response to treatment with BV (6 CR, 2 PR). All 5 pts who were refractory to $\mathrm{BV}$ responded to re-treatment with $\mathrm{BV}$ after allo-SCT. GVHD developed in 6 out of 9 pts post DLI. Acute grade I, acute grade II, mild chronic, and severe chronic GVHD was observed in 1, 2, 2, and 1 pts, respectively. Of note is the observation that GVHD resolved in all cases after a short course of low dose steroids. Disease progression was observed in 8 pts in a median of 5.5 months, (range, 4-10), while 7 pts remain progression free after a median follow up of 4 (range, 2-43) months. Twelve pts are still alive, while 3 died due to disease progression. No serious adverse effect was observed in any of the pts.

Conclusion: In our study, we observed that administration of $\mathrm{BV}$ in $\mathrm{HL}$ pts after allo-SCT was effective while toxicity was minimal. Of notice is the observation that 5 pts who were refractory to $\mathrm{BV}$ post auto-SCT, responded to re-administration of BV post allo-SCT. Our observations are in accordance with the results of a previous report, showing that BV plus DLI after allo-SCT creates a vaccination like-effect against $\mathrm{HL}$ [1]. Moreover, we observed a lower than expected severity of GVHD post DLI that was of transient duration and easily manageable with low dose steroids, suggesting an immunemodulating effect produced by BV. Indeed previous studies have shown that CD30 is expressed on the surface of activated T-cells present in inflammatory infiltrates of GVHD lesions [2]. Administration of BV plus DLI should be tested in larger group of pts at high risk of relapse after allo-SCT.

References: 1) Theurich S, et al. J Clin Oncol. 2013, 31; 59-63. 2) Chen Y, et al. Blood. 2012; 120(3):691-696.

Disclosure of Interest: None declared.
P725

Allografting as salvage treatment in refractory/relapsed Hodgkin Lymphoma

L. Giaccone ${ }^{1}$ L. Brunello ${ }^{2}, G_{\text {. }}$ lovino $^{2}$, M. Festuccia ${ }^{2}$, C. Dellacasa ${ }^{1}$, R. Passera ${ }^{3}$, S. Aydin ${ }^{2}$, A. Busca ${ }^{7}$, B. Bruno,

SSCVD Trapianto di Cellule Staminali, AOU Città della salute e della Scienza di Torino, ${ }^{2}$ Ematologia, AOU Città della Salute e della Scienza di Torino, ${ }^{3}$ Division of Nuclear Medicine, Statistical Consultant, AOU Città della salute e della Scienza di Torino, Torino, Italy

Introduction: Patients with refractory/relapsed (RR) Hodgkin lymphoma (HL) have very poor prognosis. Allogeneic stem cell transplantation has been used in this setting with controversial results ${ }^{1,2}$.

Material (or patients) and methods: This study reports on 46 consecutive patients (median age 35 years, 17-64) with RR HL who underwent an allografting between 2000 and 2015 at the BMT Unit of the AOU Città della Salute e della Scienza di Torino, Torino, Italy). Twenty-nine/46 (63\%) patients had received $\geq 3$ lines of prior therapy; $43 / 46$ (93\%) had a previous autologous transplant. At the time of allografting, 35 patients had responsive disease (at least partial remission), whereas 11 were in progression or refractoriness. Forty-two/46 patients reduced-intensity conditionings. Donors were matched unrelated $(N=29)$ and siblings $(N=17)$. Stem cells source was bone marrow in 5 patients only.

Results: Overall, treatment related mortality (TRM) was $15.4 \%$ at 5 years. Patients younger than 35 had a lower TRM compare to those older (4.0\% vs $29.7 \%, P=0.023$ ). After a median follow-up of 87 months (range 3-154), the incidence of acute and chronic graftversus-host disease (GVHD) was $42.2 \%$ and $48.7 \%$ respectively. Six additional patients developed chronic GVHD after disease relapse due to abrupt discontinuation of immunosuppression or donor lymphocyte infusion. Overall, the relapse incidence (RI) was 31.5\%, $41.1 \%, 44.2 \%$ at 1,3 and 5 years, however $\mathrm{Rl}$ was $24.1 \%$ in responsive and $54.5 \%$ in non-responsive patients at the time of transplant $(P=0.002)$. The impact of pre-transplant disease status was confirmed by multivariate analysis (SDHR 0.31, $P<0.001$ ). Five-year overall survival and event-free survival (EFS) were $52.9 \%$ and $40.4 \%$, respectively. Median EFS was not reached in responsive patients and 6.4 months in the others $(P=0.032)$. Brentuximab-Venotin (BV) was used in 12 patients. Eight/12 received $\mathrm{BV}$ as a bridge to allografting for a median of 6 cycles (310), all but one achieved at least a partial response. In 4 patients BV was used as rescue for post-allografting relapse (median 11 cycles, 7-16) and all of them had at least a partial response. None of the patients treated with BV had unexpected toxicity or GVHD worsening. Donor lymphocytes were infused in 7 patients for disease relapse, at follow-up 5/7 are alive and 4/5 free of disease and GVHD, 2 patients died for disease progression.

Conclusion: Given the poor outcome of $\mathrm{RR} \mathrm{HL}$, allografting represents an efficacious salvage therapy in $R R H L$, with a key role played by pre-transplant disease status. Most patients were treated in the pre-BV era. However, the introduction of BV will hopefully increase the number of patients in at least a partial response at the time of transplant and will lead to further improved clinical outcomes after allografting.

References: 1. Hertzberg M. Hematol Oncol Clin North Am. 2014;28(1):123-47.

2. Martino M et al. Expert Opin Biol Ther. 2015 Dec 10. [Epub ahead of print].

Disclosure of Interest: None declared. 


\section{P726}

Haploidentical Stem Cell Transplant for Relapsed or Refractory Hodgkin Lymphoma in Brazil

M. P. LACERDA ${ }^{1, *}$, C. ARRAIS-RODRIGUES ${ }^{2}$, A. PEREIRA ${ }^{3}$, Y. NOVIS ${ }^{4}$ M. FONSECA ${ }^{5}, R$. SILVA ${ }^{6}$, M. MACEDO ${ }^{6}$, N. HAMERSCHLAK I. ESTEVES ${ }^{7}$, J. SCHMIDT FILHO ${ }^{8}$, V. ROCHA ${ }^{4}$

${ }^{1}$ Department of Clinical and Experimental Oncology, UNIFESP, ${ }^{2}$ Department of Clinical and Experimental Oncology, UNIFESP/ Hospital Sírio Libanês, ${ }^{3}$ Hospital Sírio Libanes/Hospital São Camilo, ${ }^{4}$ Hospital Sirio Libanes, ${ }^{5}$ Hospital São Camilo, ${ }^{6}$ IBCC, ${ }^{7}$ Hospital Israelita Albert Einstein, ${ }^{8}$ AC Camargo Cancer Center, SAO PAULO, Brazil

Introduction: Patients with relapsed/refractory Hodgkin Lymphoma (HL) after an autologous stem cell transplant have a very poor prognosis. The use of reduced-intensity conditioning allogeneic stem cell transplantation remains a potentially curative approach although limited by a relatively low longterm progression-free survival (PFS) and by the lack of a suitable HLA-matched related or unrelated donor in many cases. In this setting, haploidentical stem cell transplant (haplo-SCT) with posttransplant cyclophosphamide (PT-Cy) has been evaluated with favorable preliminary results, showing a possibly higher PFS probability than observed other transplant modalities.

Material (or patients) and methods: We evaluated 24 patients (median age, 27 years, range 13-43, 56\% male) who underwent a haplo-SCT for relapsed/refractory HL. The median time from disease diagnosis to haplo-SCT was 44 months (range 17-154). Most patients (96\%) had a prior autologous transplant. Thirteen patients $(54 \%)$ were in complete remission, $9(38 \%)$ in partial remission, and $2(8 \%)$ had refractory disease at the time of the transplant. All patients received cyclophosphamide, fludarabine and TBI as conditioning regimen. GVHD prophylaxis consisted of a calcineurin inhibitor (cyclosporine in 50\%, tacrolimus in 50\%), mycophenolate mofetil and high-dose PT-Cy $(50 \mathrm{mg} / \mathrm{kg} / \mathrm{day})$ in all cases. The source of stem cells was bone marrow in thirteen (54\%) patients, and peripheral blood in twelve patients (46\%). Median follow-up was 18 months (range 4-57).

Results: All patients engrafted after a median time of 17 days. Cumulative incidence (Cl) of non-relapse-related mortality (NRM) was $22 \%$, and $\mathrm{Cl}$ of relapse or progression was $25 \%$ at 20 months. $\mathrm{Cl}$ of grade II-IV acute GVHD and chronic GVHD was $17 \%$ and $19 \%$, respectively. The probability of progression-free survival and overall survival at 20 months was $58 \%$ and $78 \%$, respectively. Six patients died during follow-up: four patients from infectious complications, and two from pulmonary toxicity.

Conclusion: Haplo-SCT is a feasible and valuable option for relapsed/refractory $\mathrm{HL}$ patients after a failed autologous stem cell transplant, with favorable survival and relatively low risk of GVHD. Further studies are needed to clarify if a haploidentical donor would be preferable to a matched unrelated donor in these high-risk $\mathrm{HL}$ patients.

Disclosure of Interest: None declared.

P727

Outcomes in B non-Hodgkin lymphoma (B-NHL) patients undergonig autologous stem cell transplant (ASCT) in the Rituximab era: risk of hypogammaglobulinaemia and impact on post-ASCT infections

M. Sola Soto ${ }^{1, *}$, M. J. Serna Muñoz ${ }^{1}$, J. Muñoz Ballester ${ }^{1}$, P. Iniesta López Matencio ${ }^{1}$, J. J. Sanchez Blanco ${ }^{1}$, C. Castilla Llorente ${ }^{1}$, F. De Arriba de La Fuente, M. D. Garcia Malo ${ }^{7}$, E. Pérez Ceballos', O. López Godino ${ }^{1}$, I. Heras ${ }^{1}$, V. Vicente García ${ }^{1}$

${ }^{1}$ Hematology, Hospital Morales Meseguer, Murcia, Spain

Introduction: Although Rituximab (R) improves outcomes in ASCT, the hypogammaglobulinaemia (hypoGG) associated represents a potential side effect in patients with $\mathrm{NHL}$, and to date few data are reported concerning infection risk in this group of patients.
Material (or patients) and methods: We retrospectively analyzed B-NHL patients undergoing ASCT at Morales Meseguer Hospital between 1992-2014, excluding patients undergoing tandem auto-allo, those who relapse before day +100 , and those without available plasma Igs (immunoglobulins) levels. We determined hypoGG as a level of $\operatorname{lgG}<400 \mathrm{mg} / \mathrm{dL}$ and lymphopenia as $<1000$ lymphocytes/ $\mu \mathrm{L}$. Serious infections complications were considered as those episodes requiring intravenous treatment and/or hospitalization. Data was analyzed with SPSS v20.

Results: Patient's characteristic and ASCT outcomes are represented in table 1 . There were statistical differences among patients $R$ and no-R treated in the media of lgG pre-ASCT (630 vs $840 \mathrm{mg} /$ $\mathrm{dL}, P=0,014)$ and $\mathrm{lgG}$ at day +100 (580 vs $780 \mathrm{mg} / \mathrm{dL}, P=0,02)$, without differences in the mean of pre-ASCT lymphocytes number. Among pre-ASCT characteristics, no factors were identified as risk factor for hypoGG, pre-ASCT $\mathrm{R}$ use or not included; however those patients with hypoGG pre-ASCT, maintained hypoGG at $+100(P=0,002$, OR 8,4 , IC 2,2-31,5). The most clinically documented infections was pneumonia (14\%), and the incidence of shock septic was $5 \%$. According to risk factors to develop severe infections, there were not differences between $\mathrm{R}$ vs no-R patients, hypoGG pre-ASCT, and lymphopenia with a trend of statistical significance in patients with hypoGG at day +100 $(P=0,06$, OR 2,9 , IC $0,9-9,4)$. Rituximab was the only factor that had impact in both: disease free survival $(P=0,013 ; 29$ months vs not achieved) and overall survival ( $P=0,045$, not achieved) in KaplanMeier analysis (Figure 1).

\begin{tabular}{|c|c|c|}
\hline Characteristics & $\begin{array}{l}\text { Patients } R \\
\text { treated } \\
\text { Value } n / \%\end{array}$ & $\begin{array}{c}\text { Patients no } R \\
\text { treated } \\
\text { Value } n / \%\end{array}$ \\
\hline $\begin{array}{l}\text { No. of patients } \\
\text { Male } \\
\text { Median age }\end{array}$ & $\begin{array}{c}71 / 78 \% \\
47 / 66 \% \\
52(19-72)\end{array}$ & $\begin{array}{c}20 / 22 \% \\
9 / 45 \% \\
48(14-65)\end{array}$ \\
\hline $\begin{array}{l}\text { NHL subtype } \\
\text { FL } \\
\text { MCL } \\
\text { DLBCL } \\
\text { Others } \\
\text { Median prior lines } \\
\text { Median } R \text { cycles }(375 \mathrm{mg} / \mathrm{m} 2) \\
\text { Time from last } \mathrm{R}<3 \text { months }\end{array}$ & $\begin{array}{l}13 / 18 \% \\
12 / 17 \% \\
37 / 52 \% \\
9 / 13 \% \\
2(1-6) \\
39 / 55 \% \\
7(2-26)\end{array}$ & $\begin{array}{c}6 / 30 \% \\
0 / 0 \% \\
8 / 40 \% \\
6 / 30 \% \\
2(1-4) \\
- \\
-\end{array}$ \\
\hline $\begin{array}{l}\text { Igs } \\
\qquad \text { Median IgG pre-ASCT }(\mathrm{mg} / \mathrm{dL}) \\
\text { Median } \lg G \text { at day }+100(\mathrm{mg} / \mathrm{dL})\end{array}$ & $\begin{array}{l}600 \\
(110-1480) \\
540 \\
(150-1740)\end{array}$ & $\begin{array}{l}630 \\
(396-2500) \\
750 \\
(300-1300)\end{array}$ \\
\hline $\begin{array}{l}\text { Disease status at ASCT } \\
\text { Complete Response } \\
\text { Partial Response } \\
\text { Progression Disease } \\
\text { BEAM conditioning regimen } \\
\text { Median CD34x106/Kg } \\
\text { Serious infections }\end{array}$ & $\begin{array}{c}44 / 62 \% \\
12 / 17 \% \\
14 / 21 \% \\
71 / 100 \% \\
2,8(1,3-15) \\
20(28 \%)\end{array}$ & $\begin{array}{l}13 / 65 \% \\
5 / 25 \% \\
2 / 10 \% \\
16 / 80 \% \\
3(1,3-10,5) \\
2(10 \%)\end{array}$ \\
\hline $\begin{array}{l}\text { Relapse \& Death (time in months) } \\
\text { Relapse } \\
\text { Median time to relapse } \\
\text { Death } \\
\text { Median follow-up }\end{array}$ & $\begin{aligned} 22 & (31 \%) \\
30 & (3-106) \\
5 & (17 \%) \\
76 & (6-156)\end{aligned}$ & $\begin{aligned} 12 & (60 \%) \\
15 & (3-115) \\
4 & (20 \%) \\
50 & (3-223)\end{aligned}$ \\
\hline
\end{tabular}

Conclusion: In contrast to previous studies, $\mathrm{R}$ did not suppose a risk factor to develop hypoGG and had not impact in development of infectious complications post-ASCT, maintaining its advantage in overall survival and disease free survival. Disclosure of Interest: None declared. 
P727

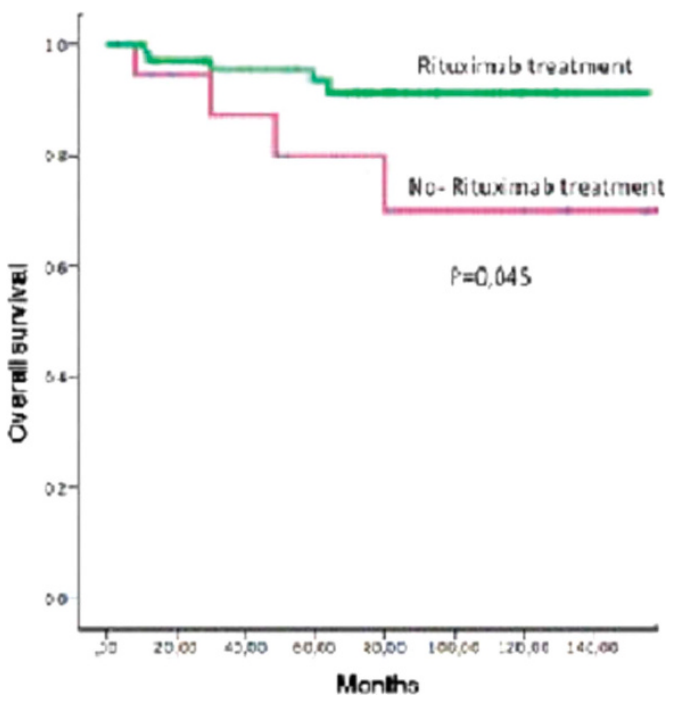

P728

Brentuximab vedotin for relapse or progression therapy after allogeneic hematopoietic stem cell transplantation in relapsed / refractory classic Hodgkin lymphoma

N. Mikhailova ${ }^{1, *}$, M. Popova ${ }^{1}$, E. Kondakova ${ }^{1}$, M. Ivanova', E. Borzenkova', A. Alaynskiy', S. Bondarenko', B. Afanasyev', E. Babenko

${ }^{1}$ First St.-Petersburg Pavlov State Medical University, St. Petersburg, Russian Federation

Introduction: The relapsed/refractory classical Hodgkin Lymphoma $(\mathrm{r} / \mathrm{r}-\mathrm{cHL})$ patients comprise about $30 \%$ of overall $\mathrm{cHL}$ patient's population. Allo-HSCT is considered as a therapeutic option for patients with relapse after auto-HSCT. The main problem in patient with $\mathrm{r} / \mathrm{r}-\mathrm{cHL}$ after allo-HSCT is relapse. Brentuximab-vedotin (BV) is an effective drug, which allowed achieving good response in majority of $\mathrm{r} / \mathrm{r}-\mathrm{cHL}$ patients. The aim of this study was to estimate safety and efficacy of BV for relapse therapy after allo-HSCT.

Material (or patients) and methods: From Jan 2008 to Jul 201547 pts with $\mathrm{r} / \mathrm{r}$-cHL have undergone RIC allo-HSCT from MUD (32), MRD (13), Haplo (2). Conditioning regimen with Fludarabine-Melphalan/Busulfane $\quad(n=28), \quad$ FludarabineBendamustine $(n=17)$ or Fludarabine-2GyTBI $(n=2)$. GVHD prophylaxis included calcineurin inhibitors and short-course methotrexate or MMF (from MRD), and ATG (from MUD) or PTcyclophosphamide, calcineurin inhibitors and MMF (from MUD or MMUD). Study group consist of 25 patients with relapse or progression after allo-HSCT. Conventional chemotherapy was administrated in 15 patient and BV in 10 patients for relapse therapy. BV was administrated at a dose $1.8 \mathrm{mg} / \mathrm{m}^{2}$ every 3 weeks intravenous. ORR (complete and partial remission) was evaluated by PET/CT.

Results: Relapse rate was 53\% $(n=25)$ after allo-HSCT with the median time - 88 days (32-298). BV after allo-HSCT was administrated in 10 patients, BV with DLI - 8 (80\%). Control group included 15 patients with conventional chemotherapy (CT), together with DLI - 5 (50\%). ORR to BV therapy (+/- DLI) was $100 \%$, including $25 \%$ who achieved PET - negative status vs $33.3 \%$ in CT group. PFS at 1 year after post allo-HSCT relapse or progression was $50 \%$ in BV group and $20 \%$ in CT group $(P=0,07)$. OS at 1 year after post allo-HSCT relapse or progression was $64 \%$ : in BV group OS was $100 \%$ vs $40 \%$ in CT group $(P=0,007)$. We did not observe toxicity grade 3-4 after BV administration.TRM at 1 year after post allo-HSCT relapse or progression was $0 \%$ in both groups.
Conclusion: Brentuximab vedotin in heavily pretreated population for relapse or progression treatment after alloHSCT has high clinical efficacy, could be safely administrated and have been associated with significant better PFS and OS then conventional chemotherapy. Prospective studies on BV after allo-HSCT are needed.

Disclosure of Interest: None declared.

\section{P729}

A Prognostic model for survival of patients with Hodgkin lymphoma after failure of HDC auto-SCT

S. Akhtar ${ }^{1, *}$, M. Elshenawy ${ }^{1}$, T. A. Elhassan ${ }^{1}$, A. Rehman ${ }^{1}$, S. M. Rauf $^{7}$, I. Maghfoor ${ }^{7}$

${ }^{1}$ Oncology Center, King Faisal Specialist Hospital and Research Center, Riyadh, Saudi Arabia

Introduction: Hodgkin lymphoma $(\mathrm{HL})$ patients generally have poor outcomes after failure of HDC auto-SCT. However, some patients can have long term disease control or cure with chemotherapy and/or radiation therapy (XRT). We evaluated various prognostic factors and their impact on overall survival (OS).

Material (or patients) and methods: From 1996 - 2014, 125 (40.6\%) of $308 \mathrm{HL}$ patients who underwent HDC auto-SCT and failed (defined as persistent, progressive and relapsed disease post HDC auto-SCT) were identified. Overall survival (OS) was defined as death due to any cause; calculated from last followup or date of death. Probability of survival was calculated using Kaplan-Meir method and compared using log-rank test. Multivariate analysis was utilized using COX proportional hazard Model. Presence or absence of 14 factors at the time of failure were analyzed; gender, histology, B symptom, extranodal disease, HL-International prognostic score, age at post HDC event, albumin $<4 \mathrm{gm} / \mathrm{dl}$, hemoglobin $<10.5 \mathrm{gm} / \mathrm{dl}$ or lower, WBC $>15000 \mathrm{~K} / \mathrm{ul}$, lymphocytes along with various combinations of type of failure, timing of post HDC auto-SCT failure, largest tumor size and stage at failure. Extranodal diseases was not included in multivariate analysis due to overlapping multi-co-linearity with stage IV as almost all stage IV had extranodal disease. Response to treatment at failure was not included in the primary analysis as this is a surrogate marker of survival / death in this setting.

Results: Male: female ratio was $62: 38 \%$, median age at HDC auto-SCT 23.4 years, median follow-up was 57 months (9-131 months). Type of failure was progressive disease (59 patients, 47\%; median OS 15.2 months), relapsed disease (42 patients, 34\%; median OS 18.6 months) and persistent disease (24 patient, 19\%; median OS 36.8 months). Kaplan-Meier estimated median OS for all patients was 18.8 months; only $27 / 125$ patients are alive. Significant factors on univariate analysis and their OS is shown in Table 1. Multivariate analysis identified presence of B symptoms (hazard ratio: $2.69, \mathrm{Cl} 1.25-5.76, P$-value 0.011 ), stages III-IV (hazard ratio:2.77, $\mathrm{Cl}$ 1.34-5.71, $P$-value 0.006 ) and hemoglobin $<10.5 \mathrm{gm} / \mathrm{dl}$ (hazard ratio:1.93, Cl $0.97-3.83, P$-value 0.06 ) were significant and the model had a $P$-value of $<0.001$. Eighty-eight patients had complete values for these three variables. OS for patients with 0 factor (not reached): 1 factor (31 months): 2 factors (12.5 months) or 3 risk factor were 4.4 months respectively $(P=<0.001)$. All patients (except one) with 2 or 3 factors have died.

Conclusion: Patients with HL who fail HDC auto-SCT have varying survivals depending upon the presence of several prognostic factors. Our model identifies important prognostic factors and can predict survival in this very high risk group of patient. Large cohort is needed to validate this model.

Disclosure of Interest: None declared. 


\begin{tabular}{|c|c|c|c|c|}
\hline Prognostic Factors & Total Patients & $\begin{array}{c}\text { KM-Median OS in } \\
\text { months }\end{array}$ & $\begin{array}{l}\text { Univariate } \\
\text { P-value }\end{array}$ & $\begin{array}{c}\text { Multivariate } \\
\text { P-value }\end{array}$ \\
\hline \multicolumn{5}{|l|}{ Overall Survival Analysis } \\
\hline Whole group & 125 & 18.8 & $x$ & $x$ \\
\hline Stage III-IV vs I-II & 77 vs 38 & 14.4 vs 57.6 & $<0.001$ & 0.006 \\
\hline B symptom YES vs NO & 17 vs 72 & 5.2 vs 38 & $<0.001$ & 0.011 \\
\hline Hemoglobin $<10.5$ vs $\geq 10.5$ & 25 vs 80 & 9.6 vs 30.6 & $<0.001$ & 0.06 \\
\hline Extranodal YES vs NO & 64 vs 51 & 13.2 vs 53.6 & $<0.001$ & $\mathrm{x}$ \\
\hline Albumin $<4$ vs $\geq 4$ & 43 vs 51 & 13.2 vs 24 & 0.006 & $x$ \\
\hline Largest node $\geq 5 \mathrm{~cm}$ vs $<5 \mathrm{~cm}$ & 22 vs 79 & 8.3 vs 24.3 & $<0.001$ & $x$ \\
\hline Relapsed+PD vs persistent & 101 vs 24 & 16.5 vs 36.8 & 0.005 & $x$ \\
\hline
\end{tabular}

[P729]

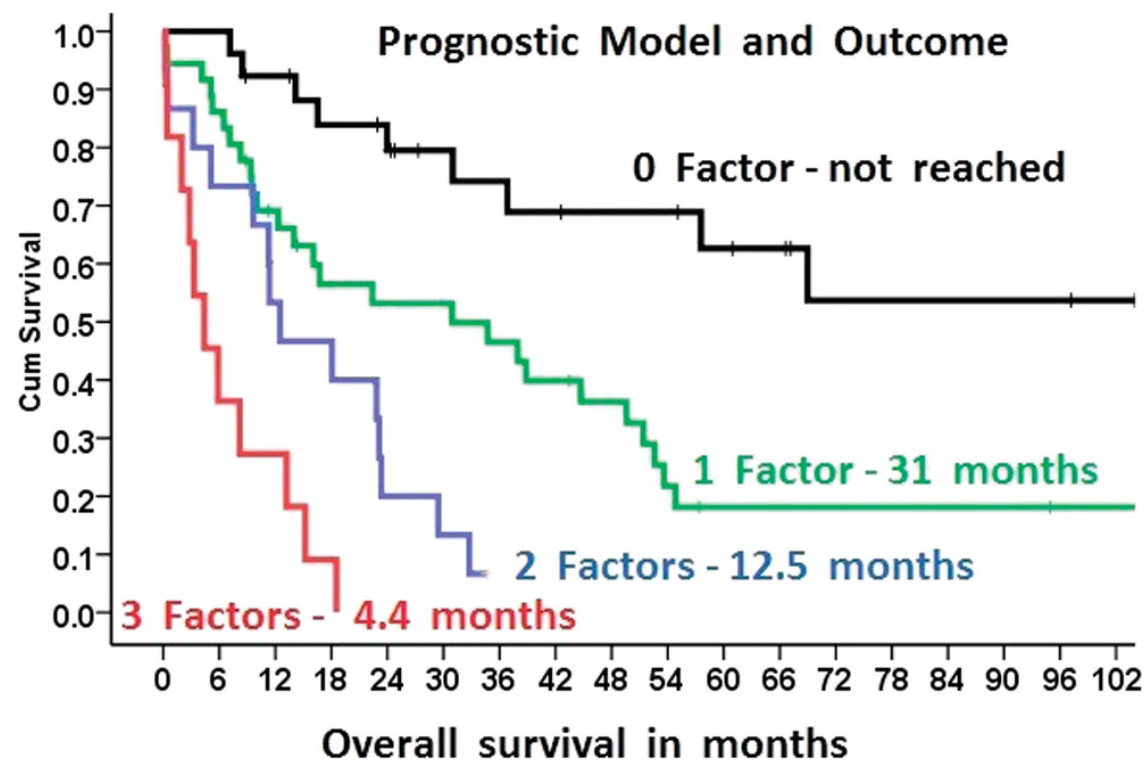

P730

A retrospective analysis of prognostic indices at diagnosis for adult T-cell leukemia/lymphoma patients receiving allogeneic stem cell transplantation

S. Takeuchi, ${ }^{1, *}$, N. Nakano ${ }^{1}$, A. Kubota ${ }^{1}$, T. Miyazono ${ }^{1}$, M. Tokunaga ${ }^{1}$, K. Yonekura ${ }^{1}$, Y. Takatsuka' ${ }^{1}$ A. Utsunomiya ${ }^{1}$

${ }^{1}$ Hematology, IMAMURA BUN-IN HOSPITAL, Kagoshima, Japan

Introduction: Allogeneic hematopoietic stem cell transplantation (allo-HSCT) has been used as a curative option for adult T-cell leukemia/lymphoma (ATLL). However the suitable prognostic index at diagnosis for ATLL patients receiving allo-HSCT is not well known. Here we intend to clarify the relation between prognostic indices at diagnosis and overall survival (OS) after allo-HSCT in our single institute located in an endemic area of ATLL.

Material (or patients) and methods: There were 102 ATLL patients receiving allo-HSCT at Imamura Bun-in Hospital from June 1998 to December 2014. We analyzed the relation between prognostic indices at diagnosis such as Japan Clinical Oncology Group prognosis index (JCOG-PI), the prognostic index for acute- and lymphoma-type adult T-cell leukemia/ lymphoma (ATL-PI), NCCN-IPI, PIT and OS after allo-HSCT retrospectively. OS was analyzed with Kaplan-Meier method. Statistical significance defined $P<0.05$ in log-rank test. All statistical analyses were performed with EZR (R commander).
Results: In 102 patients (60 male, 40 female), median age was 52 (range: 32-69) years. Median OS was 217 (range: 10-6140) days. Performance status (PS) at diagnosis was 0-1 in 93 and 2 in 9 patients. At diagnosis median value of soluble IL-2 receptor was 13356 (range: 540-293454) U/ml, median value of LDH was 388 (range: 145-6338) IU/I, hypercalcemia in 23 patients. JCOG-PI was low in 76, intermediate in 20 and high in 6 patients, respectively. ATL-PI was low in 52, intermediate in 47 and high in 3 patients, respectively. NCCN-IPI was low in 1 , low-intermediate in 22, high-intermediate in 73 and high in 6 patients, respectively. PIT was low in 2, low-intermediate in 19, high-intermediate in 61 and high in 20 patients, respectively. In univariate analysis, JCOG-PI high $(\mathrm{P}=0.30)$, ATL-PI high $(P=0.32)$ and $P I T$ high $(P=0.79)$ did not contribute to inferior OS. However, NCCN-IPI high $(P=0.02)$ contributed to inferior OS.

Conclusion: Our results suggest that among prognostic indices at diagnosis for ATLL patients, NCCN-IPI high could only predict the poor outcome after allo-HSCT. It should be considered not only pre-transplant indices such as HCT-Cl and EBMT score, but also NCCN-PI when planning to undergo alloHSCT for ATLL patients.

Disclosure of Interest: None declared. 


\section{P731}

Significance of Positron Emission Tomography/ Computed Tomography in Lymphoma Patients before Allogeneic Stem Cell Transplantation

V. Valkova ${ }^{1, *}$, A. Vitek ${ }^{1}$, M. Markova ${ }^{1}$, L. Novakova ${ }^{1}$, J. Vydra ${ }^{1}$, K. Benesova ${ }^{2}$, D. Pohlreich ${ }^{2}$, B. Vackova ${ }^{2}$, M. Trneny ${ }^{2}$, P. Cetkovsky ${ }^{3}$ ${ }^{1}$ Bone Marrow Transplantation, Institute of Haematology and Blood Transfusion, 21st Dept of Medicine, Charles University General Hospital, ${ }^{3}$ Institute of Haematology and Blood Transfusion, Prague, Czech Republic

Introduction: The role of fluorine-18-deoxyglucose positrone emission tomography/ computed tomography (PET/CT) as prognostic factor prior to allogeneic stem cell transplantation (allo-SCT) for Hodgkin or non-Hodgkin lymphoma ( $\mathrm{HL}$ or $\mathrm{NHL}$ ) is not yet clearly defined. While it has been suggested that allo-SCT can overcome PET/CT positivity, there are some reports on the contrary that PET/CT positive patients have poor outcome regardless of allo-SCT. We performed a retrospective analysis of 83 patients (pts) with NHL or HL who underwent allo-SCT in our center between March 1997 and December 2014

Material (or patients) and methods: Patient characteristics were as follows: 57/83 were males, 28 pts received myeloablative/55 reduced intensity conditioning, median age was 47 years (range; 21-63). Histological subtypes were as follows: FCL 16 (19\%), DLBCL 12 (15\%), T-NHL 20 (27\%), MCL 15 (18\%) HL $14(17 \%)$ and 4 others. Twenty-five (30\%) pts had identical sibling donor, 58 (70\%) pts were transplanted from an unrelated donor. Forty-five (54\%) pts had previous autologous SCT (ASCT). Median of previous chemotherapy lines was 2 (range; 1-9). At allo-SCT, 52 (63\%) pts were chemosensitive. PET/CT scans before allo-SCT were performed in 46 (55\%) pts, 26 pts were PET/CT positive and 20 were PET/CT negative. The distribution of histological types was similar between the two groups.

Results: Median follow-up of survivors was 79 months (range; 4 -156). Overall $44(53 \%)$ patients died, 16 of disease progression, 28 of non-relapse mortality (NRM). Estimated overall survival (OS) and event-free survival (EFS) at 5 years were $44 \%$ and $34 \%$ resp. The cumulative incidence of NRM and relapse incidence at 5 years were $41 \%$ and $37 \%$ resp. Acute GVHD occurred in $45 \%$ pts, chronic GVHD in $44 \%$ pts. Significantly worse results $(P=0.02)$ were observed in patients with DLBCL (5-years OS 17\%), while in MCL, PTCL, HL and FCL the results were better (5-years OS of $47 \%, 42 \%, 45 \%$ and $78 \%$ respectively).Analysis of other pre-transplant factors showed a significantly better EFS $(P=0.01)$ and borderline OS $(P=0.06)$ in patients without prior ASCT. We found no significant differences in terms of donor type, number of previous chemotherapy lines, or conditioning intensity. Significantly better results were observed in patients with chemosensitive disease (according to standard clinical/haematological criteria) at time of allo-SCT; 5-years OS was $52 \%$ and $29 \%$ for chemosensitive and chemoresistant disease $(P=0.01)$ and 5years EFS was $42 \%$ and $20 \%$ resp $(P=0.008)$. Five-years OS, EFS, relapse incidence and NRM was $46 \%, 27 \%, 44 \%$ and $43 \%$ for PET/CT negative pts and 35\%, 24\%, 54\% and $47 \%$ for PET/ CT positive pts resp. We found no significant difference between PET/CT positive and PET/CT negative pts in terms of OS, EFS, RR or NRM.

Conclusion: The results suggest that allo-SCT can overcome poor prognosis of PET/CT positive patients. The limitation of our study is the small number of patients, heterogenous lymphoma histology, and the fact that majority of failure events were NRM in context of GVHD. Chemosensitivity is prognostically essential, and it is necessary to search for new procedures and treatment modalities to achieve the best possible response before allo-SCT.

Disclosure of Interest: The study was supported by IGA of the Czech Ministry of Health - grant No. NT 11299-6. None declared.

\section{P732}

Retrospective analysis of MEL200 and MEL140 conditioning in patients with Multiple Myeloma J. Santos ${ }^{1, *}$, N. Miranda ${ }^{2}$, S. Esteves ${ }^{3}$, F. Moita ${ }^{2}$, G. Teixeira ${ }^{2}$, I. Ferreira ${ }^{2}$, M. J. Gutierrez, M. Abecasis ${ }^{2}$

${ }^{1}$ Hematology, ${ }^{2}$ Bone Marrow Transplant Unit, ${ }^{3}$ Clinical Research Unit, Instituto Português de Oncologia de Lisboa, Lisboa, Portugal

Introduction: treatment intensification with autologous support increases overall survival in patients with Multiple Myeloma (MM). Patient related factors such as age and comorbilities account on the elegilibility and choice of conditioning. The aim of this study was to characterize toxicity and to evaluate the endpoints progression free survival (PFS), time to next treatment (TNT) and overall survival (OS) in patients receiving MEL200 or MEL140.

Material (or patients) and methods: retrospective unicenter analysis (February 2005 to June 2014) of newly diagnosed patients, older than 60 years submitted to MEL200, and patients who received MEL140 for having renal clearance using EDTA $<60 \mathrm{ml} / \mathrm{min} / 1.73 \mathrm{~m}^{2}$ and/or other comorbilities.

Results: 34 patients received conditioning with MEL200; 38 received MEL140; median age was similar between groups (63). $62 \%$ and 53\% were male, respectively. Staging according to ISS was similar in both groups (Mel200/Mel140 - ISS 1, 44\%/ 26\%; ISS 2, 24\%/29\%; ISS 3 24\%/32\%; ND 8\%/13\%). First line treatment consisted of bortezomib in $85 \%$ and $66 \%$ of patients and with thalidomide in $6 \%$ and $18 \%$, respectively. Other patients were treated with alkylating agentes or anthracyclines. On admission patients receiving MEL200 had RCs/RC (32\%), VGPR/RP (56\%), SD/PD (12\%); comparing to patients treated with MEL140: RCs/RC (15\%), VGPR/RP (77\%), SD/PD $(8 \%)$. Of the patients selected to MEL140, 26\% $(n=10)$ were younger than 60 years old: 8 had EDTA clearance $<60$, 1 had ischemic cardiopathy, and other had lung disease. Toxicity evaluated as: time to hematological recovery (13 days for neutrophils >500; 11 days for platelets $>20 \mathrm{k}$ ), time to discharge (20 days) was similar in both groups, mucositis (97\% vs $n=79 \%$ ) and febril neutropenia ( $n=91 \%$ vs $n=79 \%$ ). Response to ATMO (IWG) defined as RCs/RC (65\% vs $42 \%$, $P=0,53)$ and VGPR/RP $(32 \%$ vs $47 \%, P=0,4)$ was not different. Median follow up was 30,8 months ( 1,7 months -8 years). PFS was 33 months (MEL200) and 20,1 months (MEL140), $P=0,08$. We found a difference in TNT, - 52,2 (MEL200) vs 18,2 months (MEL140), $P=0,0002$, and in OS, - not reached at follow up (MEL200) vs 39,8 months $(P=0,002)$.

Conclusion: patients submitted to MEL200 did not have more toxicity and presented advantage in terms of TNT and OS. Conditioning with MEL200 should be proposed whenever possible.

Disclosure of Interest: None declared.

\section{P733}

Atypical serum immunofixation patterns after autologous stem cell transplantation in patients with multiple myeloma

J. Bastos ${ }^{1, *}$, R. Bergantim ${ }^{1}$, F. Trigo ${ }^{1}$, J. E. Guimarães ${ }^{1}$ ${ }^{1}$ Hematológia Clínica, Hospital São João, Porto, Portugal

Introduction: The emergence of atypical serum immunofixation patterns - oligoclonal bands/different immunoglobulin isotype - has been described in patients with multiple myeloma (MM) after autologous stem cell transplantation (ASCT) and/or treatment with proteasome inhibitors and immunomodulators. The biological significance and prognostic relevance of these patterns are not fully understood.

Material (or patients) and methods: We retrospectively analysed a series of consecutive patients with MM who underwent ASCT in our centre between 2000-2014, regarding serum immunofixation (IF) patterns, clinical data of patient, disease status, induction treatment, overall survival (OS) and progression free survival (PFS). Atypical patterns were defined 
by different serum IF from the original monoclonal protein resulting in a different serum IF pattern (DSIP) or multiple bands resulting in an oligoclonal pattern (OP). PFS, OS and relapse were assessed using the International Myeloma Working Group criteria.

Results: 119 patients were included with a median age of 56 years. $35.3 \%$ presented with extramedullary disease, $19.3 \%$ with high $\mathrm{LDH}$ and $43.7 \%$ in ISS-II stage. $46.2 \%$ had cytogenetic characterization at diagnosis, with del17p in $5.9 \%$; $\mathrm{t}(4 ; 14)$ in $7.6 \% ; \mathrm{t}(14 ; 16)$ in $1.7 \%$ and $\mathrm{t}(11 ; 14)$ in $5 \%$ of patients. ASCT was single $(61.3 \%)$ or double $(37.8 \% ; 30.3 \%$ tandem-ASCT). OP was observed after ASCT in $53.8 \%$ of patients (median period of 9 months) and DSIP in $7.6 \%$ of patients. Median PFS and OS were 43 and 103 months respectively. The characteristics associated with survival (OS and PFS) in univariate analysis were ISS stage, del17p, induction therapy, response after induction, response after ASCT and tandem-ASCT (for PFS), but only del17p remained significantly associated with OS after multivariate analysis. In DSIP group, PFS was significantly higher $(P<0.001$ in univariate and multivariate analysis); with no statistical difference in OS $(P=0.16)$. OP was not significantly associated with PFS $(P=0.654)$ or OS $(P=0.473)$. Within this group, PFS was superior according to extended expression of OP and loss of oligoclonal bands with re-expression of the initial isotype was associated with clinical relapse of MM within a median period of 4.5 months. Conclusion: The emergence of DSIP had a low prevalence in this series of patients, showing a favourable prognostic impact. The OP had a high prevalence, with no prognostic association in terms of survival. Nonetheless, loss of OP was frequently associated with re-expression of the original serum IF pattern being a hallmark of an early clinical relapse, suggesting the importance of an attentive follow-up of these patterns during this transition period.

Disclosure of Interest: None declared.

\section{P734}

Identification of prognostic factors in patients with mature lymphoid malignancies undergoing autologous stem cell transplantation-a single center experience

K. Troppan ${ }^{1, *}$, G. Pregartner ${ }^{2}$, V. Zach ${ }^{1}$, H. Greinix ${ }^{1}$, P. Neumeister ${ }^{1}$ ${ }^{1}$ Hematology, ${ }^{2}$ Institute for Medical Informatics, Statistics and Documentation, Medical University Graz, Graz, Austria

Introduction: Autologous stem cell transplantation (ASCT) represents an established treatment strategy for patients with lymphoma and multiple myeloma either as first line therapy or at relapse depending on the underlying subtype. Patients up to the age of 75 years in good clinical condition without significant comorbidities and undergoing ASCT for various mature $B$ and $T$ cell malignancies at the Division of Hematology of the Medical University of Graz have been retrospectively analysed for the presence of risk factors at diagnosis influencing survival.

Material (or patients) and methods: Outcome of 357 patients with lymphoid malignancies (217 multiple myeloma and 140 relapsed non-Hodgkin's lymphoma), consecutively diagnosed and transplanted between 2004 and 2014 at our institution, was evaluated. The prognostic influence of various patient characteristics at diagnosis, including age, gender, pretransplant disease status, and body-mass index (BMI) as well as laboratory parameters like haemoglobin, absolute neutrophil count (ANC), thrombocytes, lactate dehydrogenase, creatinine, bilirubin, albumin, total protein, C-reactive protein (CRP) levels, lymphocyte to monocyte ratio (LMR), and neutrophil to lymphocyte ratio (NLR) on overall survival (OS) and progression free survival (PFS), was studied by univariate and by stepwise Cox regression.

Results: Of 357 patients, with a median age of 57 (range, 1974) years, 239 (67\%) were alive whereas 118 (33\%) have died. $188(53 \%)$ patients had a relapse after ASCT, at a median follow-up of 2.6 years. Univariate analysis demonstrated a statistical significance for BMI (BMI $20-24$ vs $\geq 25 ; p=0.004$ ), pre-transplant disease status (CR/PR/VGPR vs. SD/PD; $p=0.034)$, LMR (LMR $<1.2$ vs. $\geq 1.2 ; P=0.001)$, haemoglobin $(<12 \mathrm{~g} / \mathrm{dl}$ vs. $\geq 12 \mathrm{~g} / \mathrm{dl} ; p=0.002)$, thrombocytes $(<100 \mathrm{G} / \mathrm{l}$ vs $\geq 100 \mathrm{G} / \mathrm{l} ; p<0.001)$, creatinine $(<1 \mathrm{mg} / \mathrm{dl}$ vs. $\geq 1 \mathrm{mg} / \mathrm{dl}$; $p=0.020)$ total protein $(<6.6 \mathrm{~g} / \mathrm{dl}$ vs. $\geq 6.6 \mathrm{~g} / \mathrm{dl} ; p=0.012)$, albumin $(<3.5 \mathrm{~g} / \mathrm{dl} \mathrm{vs.} \geq 3.5 \mathrm{~g} / \mathrm{dl} ; p=0.005)$ and CRP $(<5 \mathrm{mg} /$ dl vs. $\geq 5 \mathrm{mg} / \mathrm{dl} ; p<0.001)$ as predictive factors regarding $O S$. For PFS, haemoglobin ( $<12 \mathrm{~g} / \mathrm{dl}$ vs. $\geq 12 \mathrm{~g} / \mathrm{dl} ; p=0.005)$, NLR $(<2.8$ vs. $\geq 2.8 ; p=0.032)$, protein, $(<6.6 \mathrm{~g} / \mathrm{dl}$ vs. $\geq 6.6 \mathrm{~g} / \mathrm{dl}$; $p=0.006)$ and albumin ( $<3.5 \mathrm{~g} / \mathrm{dl}$ vs. $\geq 3.5 \mathrm{~g} / \mathrm{dl} ; p=0.001)$ were significant predictive factors. To evaluate these parameters for their independent value, a stepwise regression analysis was performed LMR, BMI and CRP were factors for OS, and pre-transplant disease status and NLR for PFS (Table 1).

\begin{tabular}{lccc}
\hline OS & $H R$ & $\mathrm{Cl}$ & P-value \\
\hline $\mathrm{BMI}=20-25$ & 0.40 & {$[0.19-0.84]$} & 0.007 \\
$\mathrm{BMI} \geq 25$ & 0.28 & {$[0.13-0.59]$} & \\
$\mathrm{LMR} \geq 1.2$ & 0.52 & {$[0.32-0.83]$} & 0.007 \\
$\mathrm{CRP} \geq 5$ & 2.44 & {$[1.62-3.66]$} & 0.000 \\
PFS & HR & $\mathbf{C l}$ & $\boldsymbol{P}$-value \\
Pre-Transplant- & 1.72 & {$[1.20-2.46]$} & 0.003 \\
Status =SD/PD & & & \\
NLR $\geq 2.8$ & 1.30 & {$[0.95-1.79]$} & 0.100 \\
\hline
\end{tabular}

Conclusion: In this large single center cohort of patients with lymphoid malignancies undergoing ASCT, we could identify LMR, BMI and CRP at diagnosis as predictive parameters for survival after autologous stem cell transplantation.

Disclosure of Interest: None declared.

P735

The relevance of minimal residual disease after autologous stem cell transplantation in patients with multiple myeloma (first results of a prospective study)

M. Solovev ${ }^{1}$, L. Mendeleeva ${ }^{1, *}$, I. Galtseva ${ }^{2}$, O. Pokrovskaya ${ }^{1}$, M. Firsova', M. Nareyko', 'L. Kuzmina', E. Gemdzhian ${ }^{\prime}$, V. Savchenko ${ }^{4}$

${ }^{1}$ Bone Marrow Transplantation, ${ }^{2}$ Immunophenotyping of Bllod Cells and Bone Marrow Laboratory, ${ }^{3}$ Biostatistics laboratory, ${ }^{4}$ Research Center for Hematology, Moscow, Russian Federation

Introduction: High-dose therapy plus autologous stem cell transplantation (ASCT) has increased the rate of achievement of complete remission (CR), and has improved progressionfree survival (PFS) and overall survival (OS) for patients (pts) with multiple myeloma (MM) over the last decade and remains the standard of care for patients 65 years of age or younger. The residual clone of plasma cells (minimal residual disease (MRD)), detected by flow cytometry is a subject of study, as a major factor of early relapse. The aim of our study was to determine the frequency of achievement stringent $C R$ by flow cytometry after ASCT, to assess the probability of relapse depending on the presence of MRD in the bone marrow after ASCT in pts with MM.

Material (or patients) and methods: 48 pts with MM (17 men, 31 women) who had achieved CR after ASCT were included in a prospective study from 1 January 2014 to 1 June 2015. The median age was 54 years (from 25 to 66 years). On the day +100 after ASCT bone marrow examination was performed to determine MRD in all pts. A single 6-color antibody combination (CD38/CD138/CD45/CD56/CD117/ CD19) was used to detect phenotypically aberrant clonal plasma cells. MRD-negativity was defined when $<50$ clonal plasma cells were detected among 500000 leukocytes $(<0.01 \%$ and the detection limit of $10-4)$. Median follow-up since MRD determination was 8 months.

Results: Stringent CR (MRD-negativity) after ASCT was detected in $50 \%$ of patients $(n=24)$, other 24 pts were MRDpositive. During follow-up 6 pts relapsed. The cumulative 


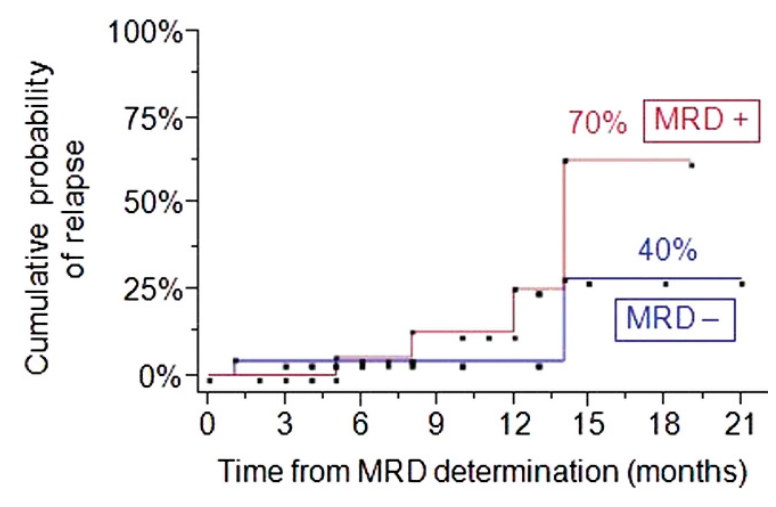

probability of disease relapse in the MRD-positive group was $70 \%$ vs $40 \%$ in the group of MRD-negativity (figure 1).

Figure 1 . The cumulative probability of relapse in the MRDpositive group is higher than in the group of MRD-negativity: $70 \%(4 / 24)$ vs $40 \%(2 / 24)$, respectively.

Conclusion: The study showed that the achievement of stringent CR after ASCT is relevant to prevent relapse, and may be considered as a factor of favorable prognosis for patients with MM.

Disclosure of Interest: None declared.

\section{P736}

Heavy/light chain ratio - early prognostic marker for PFS in multiple myeloma patients undergoing autologous stem cell transplantation

L. Gartcheva ${ }^{1, *}$, G. Arnaudov ${ }^{1}$, P. Ganeva ${ }^{1}$ on behalf of Violeta Petkova, Keranka Dimitrova, Veronika Petkova, Yavor Petrov

${ }^{1}$ National Hospital for Active Treatment of Hematological Diseases, Sofia, Bulgaria

Introduction: Hevylite ${ }^{\mathbb{R}}$ (HLC) allows measurement of both the monoclonal involved protein and polyclonal non-involved HLC pair. Abnormal HLC ratios and suppression of the uninvolved HLC pair correlate with shorter overall survival in MM patients. Both reduction in M-protein and reconstitution of the immune system are associated with increased survival in multiple myeloma (MM) patients receiving an autologous stem cell transplant (ASCT).

Material (or patients) and methods: We prospectively evaluated 28 intact immunoglobulin MM (IIMM) patients (12 Female, 16 Male; $20 \mathrm{lgG}$, and $8 \mathrm{lgA}$; median age: 54 years; range 37-67 years) who received ASCT subsequent to high dose melphalan. Median follow up time from ASCT was 391 days (range: 61-603 days). Patient responses were assigned according to international response criteria guidelines prior to ASCT. Serum samples were analysed with Hevylite, prior to ASCT (median: 2 days; range 0-77 days) and at least one month following ASCT $(n=25)$ (median: 52 days; range 40-119 days), on a SPAPLUS turbidimeter. $\mathrm{HLC}$ concentrations and ratios were compared to normal ranges (lgGk: $3.85-12.07 \mathrm{~g} / \mathrm{L}$; IgGl: $1.91-6.74 \mathrm{~g} / \mathrm{L}$; IgAk: 0.57$2.08 \mathrm{~g} / \mathrm{L} ; \lg \mathrm{A} \lambda$ : $0.44-2.04 \mathrm{~g} / \mathrm{L} ; \mathrm{HLC}$ ratio reference range: IgGk /lgG $\lambda$ : 1.12-3.21; IgAk / $\lg A \lambda$ : 0.78-1.94). Progression free survival (PFS) was determined using Kaplan Meier analysis (Graphpad Prism).

Results: Prior to ASCT, 1 patient achieved CR, 13 obtained VGPR, 9 obtained PR, 2 obtained (MR), 2 PD and 1 patient had no response assigned. An abnormal HLC ratio ( $\mathrm{K}$ median 23.87 range 3.59-474.6; $\lambda$ median: 0.16 , range $0.017-0.32$ ) prior to ASCT was associated with significantly poorer PFS (17.1 months versus median not reached $P=0.036$ ). In comparison, achievement of $\geq$ VGPR $(14 / 28 ; 50 \%)$ prior to ASCT was not associated with increased PFS $(P=0.74)$. Addition of a normal $\mathrm{HLC}$ ratio to the assignment of response (patients achieving a $\geq V G P R$ ) added significant prognostic information and was significantly associated with improved PFS (median not reached versus 11.5 months, $P=0.025$ ). Suppression of the uninvolved HLC pair prior to ASCT had no significant impact on PFS $(P=0.19)$. Post-ASCT, an abnormal HLC ratio was not significantly associated with poorer PFS $(P=0.34)$. However, the presence of HLC paired suppression was significantly associated with poorer PFS (median: (13.9 months versus median not reached $P=0.031)$ ).

Conclusion: Analysis of HLC ratio is an early prognostic marker for PFS in MM patients receiving an ASCT. The achievement of a normalised HLC ratio prior to ASCT and reduced HLC paired suppression post-ASCT may be a significant indicator of improved PFS and may add prognostic information to current response criteria.

Disclosure of Interest: None declared.

\section{P737}

Iron balance in multiple myeloma patients undergoing autologous stem cell transplantation: hepcidin and ferritin assessment

N. Piccirillo ${ }^{1, *}$, G. Ausoni ${ }^{1}$, P. Chiusolo ${ }^{1}$, L. Laurenti ${ }^{1}$, F. Sorà ${ }^{1}$, S. Giammarco ${ }^{1}$, E. Rossi ${ }^{1}$, T. Ża ${ }^{1}$, G. Zini ${ }^{1}$, V. De Stefano ${ }^{1}$, S. Sica ${ }^{1}$ ${ }^{1}$ Haematology Institute, Catholic University of Rome, Rome, Italy

Introduction: In our clinical practice we observed an unexpected high incidence of low ferritin levels in multiple myeloma (MM) patients undergoing autologous bone marrow transplant (aPBSCT).

Material (or patients) and methods: We collected data on iron assessment, including hepcidin, in 23 patients affected by MM undergoing aPBSCT (12 females, 11 males; median age of 57 years); after the verification of close link between hepcidin and ferritin, we decided to use the ferritin as iron balance marker in a larger cohort of MM patients. Thus we report data about 96 patients affected by MM undergoing to aPBSCT (43 females, 53 males; median age of 57 years). We used T-test for continuous variables, chi square for categorical factors and Pearson $r$ for correlation; $P$ value 0,05 was considered significant.

Results: We confirm our clinical observation finding a statistical significant difference between ferritin levels at diagnosis and post induction $(170 \mathrm{ng} / \mathrm{ml}$ vs $51 \mathrm{ng} / \mathrm{ml}$ $P$ 0.0039); the same finding was observed using hepcidin levels $(9.75 \mathrm{nmol} / \mathrm{L}$ vs $4.31 \mathrm{nmol} / \mathrm{L} \mathrm{p} \mathrm{0.0058).} \mathrm{We} \mathrm{found} \mathrm{a} \mathrm{direct}$ correlation between ferritin and hepcidin levels at time of aPBSCT $\left(r^{2}\right.$ 0.63, p 0.002). These observations underline the close link with ferritin allowing us to use the latter molecule as iron balance marker in a larger cohort of MM patients. In this group of patients we found a significant difference between ferritin levels at diagnosis and post induction $(185 \mathrm{ng} / \mathrm{ml}$ vs 58 $\mathrm{ng} / \mathrm{ml} \mathrm{p}<0.0001$ ). In our series $\mathrm{Gl}$ endoscopy was carried out in case of iron depletion and no concurrent disease was detected. No patients received erythropoietin. So we investigated the possible role of new drugs focusing on Bortezomib. Moreover Campanella et al observed how Bortezomib prevents upregulation of ferritin enhancing toxic activity of iron (1). We found a significant difference in incidence of high ferritin levels between patients receiving Bortezomib and patients not receiving it ( $9 \%$ vs $27 \%$ ); interestingly no patient showing high ferritin levels at post induction evaluation achieved complete response compared to $24.3 \%$ of patients showing low or normal ferritin levels (fig1).

Conclusion: Iron balance in MM patients undergoing aPBSCT could be duly investigated as a response marker in order to correct iron deficiency. Bortezomib play a central role in decreasing ferritin levels by two mechanism: interrupting paracrine loop by curing disease and preventing the ferritin upregulation. The low complete response rate in patients with high ferritin levels need to be investigated in further studies in order to clarify the relationship between Bortezomib administration, iron balance and response to therapy. 


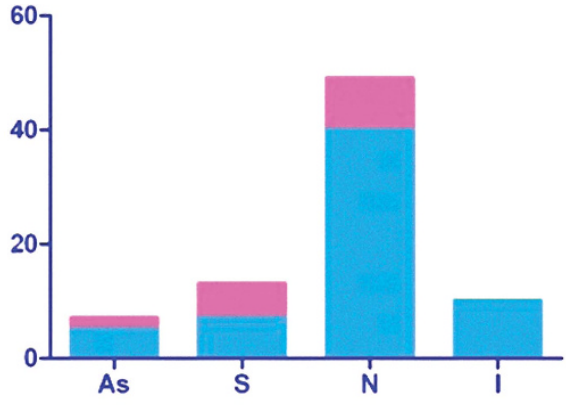

Figure 1: Patsents distnbution according to uron levels astron defiency anemia, s=iron deficiency, $n=$ nomal uron storage, inncreased tron storge, in the upper part of the columns patients achuering complete remussion

References: Campanella A, Santambrogio P, Fontana F et al Iron increases the susceptibility of multiple myeloma cells to bortezomib. Haematologica. 2013 Jun;98(6):971-9

Disclosure of Interest: None declared.

\section{P738}

Autologus stem cell transplantation for multiple myeloma: experience of the tunisian national center of stem cell transplantation

R. El Fatmi ${ }^{1, *}$, N. Ben Abdeljelil ${ }^{1}$, S. Ladeb ${ }^{1}$, L. Torjemane ${ }^{1}$, A. Lakhal ${ }^{1}, T$. Ben Othman ${ }^{1}$

${ }^{1}$ Centre National de Greffe de Moelle Osseuse de Tunis (CNGMO). Faculté de médecine de Tunis. Université Tunis El Manar, Tunis, Tunisia

Introduction: High dose melphalan and autologous stem cell transplantation (ASCT) remains the standard treatment for eligible patients with multiple myeloma even in the era of the novel drugs.

Material (or patients) and methods: We retrospectively analyzed 115 patients with multiple myeloma autografted in the CNGMO between June 2011 and February 2015. As induction therapy before ASCT, 99 patients $(86 \%)$ received a median of 3 cycles of thalidomide-Dexamethasone (TD), 5 patients (4\%) a median of 4 cycles of bortezomib-TD and 11 patients other protocols.

Results: Sixty six patients (57\%) were male. Median age at transplantation was 58 years (25- 65 years). Myeloma subtype was $\lg G, \lg A$ and light chain in respectively $56 \%, 21 \%$ and $17 \%$. ISS was evaluable in 82 patients $(65 \%)$ : Thirty patients $(36 \%)$ were classified ISS (1), 31 patients (38\%) ISS (2) and 21 patients (26\%) ISS (3). Forty one patients (36\%) were transplanted in $\geq$ very good partial response (VGPR), 29 patients (25\%) in PR and 45 patients $(39 \%)$ in failure or progression. Non relapse mortality (NRM) was $1,7 \%$. Among the 110 patients evaluable at 3 months post transplantation: $68(61 \%)$ were in $\geq$ VGPR, $24(22 \%)$ in PR and $18(17 \%)$ in failure or progression. Fifty six patients (49\%) progressed after ASCT with a median time to progression of 18 months. With a median follow up of 21 months after ASCT (6-50 months), progression free survival (PFS) and overall survival (OS) at 3 years were respectively $35 \%$ and $70 \%$. Outcome was statistically better in patients transplanted in $\geq$ VGPR versus others with a median PFS of 32 versus 20 months (p: 0,02) and an OS at 3 years of $80 \%$ versus $60 \%$ (p:0,03). Patients transplanted in failure or progression had a worse outcome compared with patients transplanted in response with an OS of $57 \%$ versus $72 \%$ $(P: 0,008)$ and a trend to a shorter PFS: 18 versus 26 months (P: 0,058$)$.

Conclusion: ASCT for multiple myeloma is associated with low NRM. Pre-transplantation status is a major prognostic factor influencing statistically the post ASCT outcome.

Disclosure of Interest: None declared.
P739

Early relapse after novel agent induction is a an important prognostic marker: An analysis by Asian Myeloma Network S. K. Gopalakrishnan 1, , S. Y. Ong ', S. J. Kim ${ }^{2}$, H. Kim ${ }^{2}$, J. Y. Lee ${ }^{2}$ S. H. Lim ${ }^{2}$, Y. Chen ${ }^{1}$, S. DeMel ${ }^{3}, M . \mathrm{Ooi}^{3}$, S. Surendran ${ }^{7}$, A. $\mathrm{Lin}^{3}$, D. Tan ${ }^{1}$, B. Durie ${ }^{4}$, W. J. Chng ${ }^{5}$, K. Kim ${ }^{2}$

${ }^{1}$ Haematology, Singapore general hospital, Singapore, Singapore, 'Haematology, Samsung medical centre, Seoul, Korea, Republic Of, ${ }^{3}$ Haematology, National university hospital, SIngapore, Singapore, ${ }^{4}$ Haematology, Cedars-Sinai Outpatient Cancer Center, Hollywood, United States, ${ }^{5}$ Natinonal university hospital, SIngapore, Singapore

Introduction: Multiple myeloma is a heterogeneous disease with variable outcomes based on a number of new predictors including cytogenetics, suboptimal response to induction therapy, and early relapse. We performed a retrospective analysis to identify whether these markers are relevant to prognosis in Asian patients who were treated with autologous transplant after novel agents.

Material (or patients) and methods: We retrospectively analysed outcomes of 246 patients who were treated with upfront autologous transplant in two centers in Singapore $(n=173)$ and one center in Korea $(n=73)$ after receiving novel agent based induction (bortezomib, thalidomide, and lenalidomide) between 2006 and 2014. Patients were staged according to International Staging System (ISS), and responses were defined according to International Myeloma Working Group criteria. Early relapse was defined as relapse within 12 months post-transplant. High risk cytogenetics includes the presence of $17 \mathrm{p} 13$ deletion, $\mathrm{t}(14 ; 16)$ or $\mathrm{t}(4 ; 14)$ by FISH.

Results: Early relapse occurred in $30.8 \%$ of patients. Compared to patients who did not relapse early, patients who relapsed within 12 months were more likely to have high risk cytogenetics $(P=0.003)$ and higher ISS stage at diagnosis $(P<0.001)$. The median overall survival $(O S)$ for patients who relapsed early was significantly shorter at 18 months, compared to 67 months $(P<0.001)$ in patients who did not (Fig 1). Multivariate regression analysis revealed that the factors independently associated with a shorter OS were high risk cytogenetics (HR 1.76, $P=0.021$ ), failure to achieve complete or very good partial response to induction chemotherapy (HR $2.29, P=0.001$ ), and early relapse (HR $7.25, P<0.001)$, but not advanced ISS stage or response post-transplant. Median overall survival for patients who relapsed within 12 months was 18 months compared with 67 months $(P<0.001)$.

Conclusion: Early relapse is an important clinical risk factor for poorer OS, independent of baseline characteristics. Our data also supports the use of high risk cytogenetics by FISH and response to novel agent induction, rather than ISS alone to improve risk stratification.

Disclosure of Interest: None declared.

\section{P740}

The influence of additional treatment prior to ASCT in newly diagnosed patients who failed to achieve at least a VGPR to initial therapy

S. Park', , S. H. Kim ${ }^{1}$, J. Yun ${ }^{1}$, H. J. Kim ${ }^{1}$, C. K. Kim ${ }^{1}$, J. H. Won ${ }^{2}$, D. S. Hong ${ }^{1}$, H. J. Yoon ${ }^{3}$

${ }^{1}$ Hematology Oncology, Soonchunhyang University Bucheon Hospital, Bucheon, ${ }^{2}$ Hematology Oncology, Soonchunhyang University Hospital, ${ }^{3}$ Hematology Oncology, Kyung Hee University Hospital, Seoul, Korea, Republic Of

Introduction: A recently published report suggested that transplant-eligible patients who achieve a suboptimal response to initial induction therapy should move on to their planned transplants rather than receiving additional therapy in order to deepen the level of response. Given the extraordinary circumstances that the National insurance benefit for the multi-novel agents combination is not available, the study to evaluate the influence of additional treatment to deepen 
response prior to ASCT may be helpful for our own decision making in patients who failed to achieve at least a very good partial response (VGPR) to initial therapy.

Material (or patients) and methods: A total of 46 patients who received ASCT were recruited. The patients were divided into two groups: those who received additional treatment before the ASCT $(n=19)$, and those who did not received additional treatment and proceeded directly to the ASCT $(n=27)$. The initial therapy consists of two drugs including one novel agent. And all patients were administered second ASCT or novel agents as consolidation therapy and maintenance therapy with thalidomide.

Results: Median follow-up duration was 51 months. The median cycles of additional treatment were 8 (7-12). After the first transplants, the rate of deep response including VGPR was similar between two groups (47.4\% for no additional treatment vs $55.6 \%$ for additional treatment). And the time to next treatment in the patients who received additional treatment was longer than the patients who went straight to transplant (38 months vs 22 months, $P=0.05$ ). Additional treatment might improve PFS rate at 3 years $(56.4 \%$ vs $36.2 \%$ for no additional treatment, $P=0.15$ ), although no difference in overall survival at 3 years was seen between two groups $(87.4 \%$ vs $75.9 \%, P=0.72)$. There also little difference in the clinical outcomes between the patients received second ASCT as consolidation and the patients received consolidation therapy with novel agents.

Conclusion: Considering three drug combinations including bortezomib and dexamethasone are currently the standard of care prior to ASCT, the additional treatment including one novel agent to deepen response prior to ASCT may not improve survival in newly diagnosed multiple myeloma.

Disclosure of Interest: None declared.

P741

Relapse with extramedullary disease after front-line autologous stem cell transplant in patients with multiple myeloma

S.-H. Jung, ${ }^{1, *}$, S.-S. Lee ${ }^{1}$, D.-H. Yang ${ }^{1}$, J.-S. Ahn ${ }^{1}$, Y.-K. Kim ${ }^{1}$, H.-J. Kim ${ }^{\top}$, J.-J. Lee

${ }^{1}$ CHONNAM NATIONAL UNIVERSITY HWASUN HOSPITAL, Hwasun-Eup, Korea, Republic Of

Introduction: Prognostic impact of extramedullary disease (EMD) at the time of diagnosis or relapse has been reported in patients with multiple myeloma (MM). In this study, we evaluated the incidence and prognostic significance of relapse with EMD after front-line high-dose therapy and autologous stem cell transplantation (HDT/ASCT) in young patients with MM.

Material (or patients) and methods: Ninety-seven patients with MM treated with front-line HDT/ASCT were enrolled between July 2003 and June 2015. EMD assessment was performed by imaging (magnetic resonance imaging, computed tomography, or positron emission tomography) or tissue biopsy in patients with palpable mass, localized pain, or neurologic signs.

Results: After completion of induction chemotherapy, HDT/ ASCT was performed at median 5.8 months (range, 3.3-11.8). Over a median follow up of 40.6 months (range, 11.5-145.1 months) in survivors, 64 patients showed disease progression after ASCT and $8(8.2 \%)$ had EMD at the time of relapse. Patients with EMD at the time of relapse had significantly shorter progression free survival (PFS) and overall survival (OS) from ASCT compared to patients without EMD (PFS: 6.5 vs. 24.0 months, $P<0.001$, OS: 33.3 vs. 139.9 months, $P<0.001)$. Although all patients with EMD received the salvage regimen consisting of bortezomib, cyclophosphamide, and dexamethasone, they showed the very poor survival outcome compare to patients without EMD (PFS2: 2.5 vs. 14.2 months, $P=0.045$ ). Presence of EMD at the time of diagnosis was only factor associated with development of EMD at the time of relapse in univariate analysis (hazard ratio $=7.900, P=0.014$ )
Conclusion: This data suggests that EMD at the time of relapse is associated with poor survival outcome in patients treated with upfront ASCT, and effective salvage therapy is needed.

Disclosure of Interest: None declared.

\section{P742}

A meta-analysis of hypomethylating agents as bridging therapy to hematopoietic stem cell transplantation in patients with myelodysplastic syndromes

X. Wang ${ }^{1}$, X. Liang ${ }^{2}$, D. Zeng ${ }^{1}$, C. Zhang ${ }^{1}$, X. Zhang ', J. Liao', Y. Ma ${ }^{1}$, A. Yi ${ }^{1}$, A.-H. Sun ${ }^{1}$, P.-Y. Kong ${ }^{1, *}$

${ }^{1}$ Department of hematology, Xinqiao Hospital, Third Military Medical University, Chongqing, ${ }^{2}$ Department of hematology, The 150th Hospital of PLA, Luoyang Henan, China

Introduction: Myelodysplastic syndrome (MDS) is a malignancy characterized by ineffective hematopoiesis. Allogeneic hematopoietic stem cell transplantation (HSCT) is the only potential cure for myelodysplastic syndrome (MDS). However, relapse remains a reason for treatment failure after hematopoietic stem cell transplantation (HSCT) in patients with intermediate- or high-risk myelodysplastic syndrome (MDS). Pre-transplant cytoreduction has been able to reduce relapse rates, but the significant toxicity and mortality of induction chemotherapy (IC) make it faces great challenge. Several scholars has reported that hypomethylating agents can achieve cytoreduction with limited toxicity and mortality before transplantation and improve the prognosis, especially in patients with intermediate- or high-risk myelodysplastic syndrome(MDS). But in some studies else, the authors also put forward that the patients with MDS have no beneficence from hypomethylating agents(HMA) compared with IC pretransplantion. To expound the question, this study proves a systematic review and meta-analysis of patients with MDS receiving HMA or non-HMA containing IC and best supportive care(BSC)before hematopoietic stem cell transplantation (HSCT).

Material (or patients) and methods: We has searched the databases combined PubMed,Embase, Wanfang,CNKI and OVID.Two researchers screen the trials according to inclusion and exclusion criteria, and assessed the methodology quality independently.Finally, six articles including 502 patients were retained and we used RevMan5.3 software to analysis databases.

Results: We acquired that compared with IC or BSC pretransplant, hypomethylating agents as bridging therapy to hematopoietic stem cell transplantation in patients with MDS has higher overall survival $(\mathrm{OR}=1.54,95 \% \mathrm{Cl}$ [1.04-2.30], $\left.P=0.28,\left[I^{2}=20 \%<25 \%, P=0.03\right]\right)$ and similar relapse-free survival(RFS) or disease-free survival (DFS).And, it hasn't increased cumulative incidence of relapse (CIR) and nonrelapse mortality (NRM). They has no significant variation in II-IV and III-IV aGVHD. Otherwise, pre-transplant hypomethylating agents can reduce the $\operatorname{cGVHD}(P=0.03)$.

Conclusion: Our meta analysis showed that pre-HSCT with HMA therapy increased OS patients with MDS or AML after HSCT compare to non-HMA therapy $(p=0.03)$ and improved the living quality of these patients. Based on these observations that HMA as bridging therapy have a positive impact on the HSCT outcome.Prospective randomized trials are required to explicitly demonstrate this in the future.

Disclosure of Interest: None declared. 


\section{P743}

No benefit of hypomethylating agents compared to supportive care for myelodysplastic syndrome of high or very-high risk by IPSS-R, but without its adverse effect as a pre-transplant use on the outcome of allogeneic transplantation

S. K. Sohn ${ }^{1, *}$, J. H. Moon ${ }^{1}$, Y. J. Lee', S. W. Park', J. S. Ahn ${ }^{1}$ H. J. Kim ${ }^{2}$, J. H. Lee ${ }^{3}$, I. C. Song ${ }^{4}$ D. Y. Jo ${ }^{5}$, H. S. Lee ${ }^{6}$, Y. S. Kim ${ }^{6}$ S. H. Bae

${ }^{1}$ Hematology, KYUNGPOON NATL. UNIV. HOSPITAL, Daegu,

${ }^{2}$ Hematology, Chunnam National University Hwasun Hospital, Kwang Ju, ${ }^{3}$ Hematology, Dong-A University Hospital, Pusan, ${ }^{4}$ Hematology, 4Chungnam National University Hospital, ${ }^{5} \mathrm{Hema}$ tology, Chunnam National University Hwasun Hospital, Daejun, ${ }^{6}$ Hematology, Kosin University Gospel Hospital, Pusan, ${ }^{7}$ Hematology, 6Daegu Catholic University Medical Center, Daegu, Korea, Republic Of

Introduction: The revised International Prognostic Scoring System (IPSS-R) is known to stratify more definitely the prognosis of myelodysplastic syndrome (MDS). The current study evaluated the role of hypomethylating agent (HMA) as a pre-transplant use compared to supportive care for high or very-high risk $(\mathrm{H} / \mathrm{VH}) \mathrm{MDS}$ patients classified by IPSS-R system. Material (or patients) and methods: A total of 279 H/VH MDS patients classified by the IPSS-R were included for this retrospective analysis. Overall survival (OS) rate was analyzed with Kaplan-Meier test and each treatment groups were compared with log-rank test. Cox-proportional hazard model was used to define the prognostic factors affecting OS.

Results: Median age of the patients was 67 years (range 18-86 years) and 136 patients (48.7\%) were under age 65 years. ECOG performance status (PS) was 0 to 1 in 213 patients $(77.6 \%)$ and 2 to 4 in $62(22.2 \%)$. The IPSS-R H/VH risk groups were 160 patients $(57.3 \%)$ and $119(42.7 \%)$, respectively. HMA was used for 173 patients (62\%), allogeneic stem cell transplantation (allo-SCT) with HMA for 31 (11.1\%), allo-SCT without HMA for $5(1.8 \%)$ and best supportive care (BSC) for 70 (25.1\%). The response (CR/PR/HI) rate of HMA before allo-SCT was $19.4 \%$. Median survival duration was $1113 \pm 183$ days in allo-SCT with HMA, $538 \pm 37$ days in HMA group, and $361 \pm 98$ days in BSC group. Median survival duration was not reached in allo-SCT without HMA group. The 3-year OS rate was $53.1 \pm 10.7 \%$ in allo-SCT with HMA, $75 \pm 21.7 \%$ in alloSCT without HMA, $17.3 \pm 3.6 \%$ in HMA group, and $20.8 \pm 6.9 \%$ in BSC $(P<0.001)$, respectively. In the multivariate analysis, allo-SCT were only related with favorable OS $(\mathrm{HR}=0.356,95 \%$ $\mathrm{Cl}$ : 0.186-0.681, $P<0.002$ ) in patients with $\mathrm{H} / \mathrm{VH}$ risk. While $\mathrm{BM}$ blast $\geq 10 \%(\mathrm{HR}=9.502,95 \% \mathrm{Cl}: 1.065-84.758, P=0.044)$ and very poor cytogenetic risk by IPSS-R (HR 5.696, 95\% Cl: 1.068 $30.374, P=0.042$ ) adversely affected on OS.

Conclusion: No benefit of HMA was observed in terms of OS compared to BSC in $\mathrm{H} / \mathrm{VH}$ risk group. Allo-SCT was the only factor predicting favorable long-term outcome in MDS patients with $\mathrm{H} / \mathrm{VH}$ risk based on IPSS-R system. The pretransplant use of HMA for higher risk MDS did not seem to adversely effect on transplantation outcome.

Disclosure of Interest: None declared.
P744

Allogeneic Stem Cell Transplantation for MDS Patients More Than 70 Years of Age: Update of a Retrospective Study of the MDS Subcommittee of the Chronic Malignancies Working Party (CMWP) of the EBMT

S. Heidenreich, ${ }^{*}$, D. Ziagkos, ${ }^{2}$ A. van Biezen ${ }^{3}$, J. Finke U. Platzbecker, ${ }^{5}$ D. Niederwieser ${ }^{6}, H$. Einsele ${ }^{7}$, W. A. Bethge ${ }^{8}$ M. Schleuning ${ }^{\prime}$, D. W. Beelen ${ }^{10}$, J. Tischer ${ }^{11}$, A. Nagler $^{12}$, B. Glass ${ }^{13}$

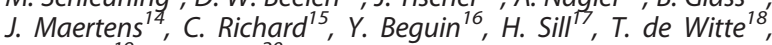
M. Robin ${ }^{19}$, N. Kröger ${ }^{20}$

${ }^{1}$ University Medical Center Hamburg, Hamburg, Germany, ${ }^{2}$ Department of Medical Statistics and Bioinformatics Leiden, Leiden University Medical Center, ${ }^{3}$ EBMT Data Office, University Medical Center, Leiden, Netherlands, ${ }^{4}$ University Medical Center Freiburg, Freiburg, ${ }^{5}$ Universitätsklinikum Carl Gustav Carus an der Technischen Universität Dresden, Dresden, ${ }^{6}$ Hematology and Oncology, University of Leipzig, Leipzig, ${ }^{7}$ Department of Internal Medicine, University Medical Center, Wuerzburg, ${ }^{8}$ University of Tübingen Medical Center, Tübingen, ${ }^{9}$ Centre for hematopoietic cell transplantation, Wiesbaden, ${ }^{10}$ Department of Bone Marrow Transplantation, West German Cancer Center, University Hospital Essen, Essen, ${ }^{11}$ Department of Internal Medicine III, Hematopoietic Cell Transplantation, Ludwig-Maximilians-University Hospital of Munich-Grosshadern, Munich, Germany, ${ }^{12}$ Division of Hematology, Sheba Medical Center, Ramat Gan, Israel, ${ }^{13}$ Hematology, Oncology and Stem Cell Transplantation, Asklepios Hospital St Georg, Hamburg, Germany, ${ }^{14}$ Department of Hematology, University Hospital Gasthuisberg Leuven, Leuven, Belgium, ${ }^{15}$ Hematology Department, University Hospital Marques de Valdecilla, Santander, Spain, "16 Laboratory of Hematology, GIGA-I3, University of Liège CHU Sart-Tilman, Liège, Belgium, "1'Division of Hematology, Medical University of Graz, Graz, Germany, ${ }^{18}$ Nijmegen Center for Molecular Life Sciences, Dep. of Tumor Immunology, Radboud University of Nijmegen Medical Centre, Nijmegen, Netherlands, ${ }^{19}$ Hematology Bone Marrow Transplantation, Saint-Louis Hospital, Paris, France, ${ }^{20}$ Department of Stem Cell Transplantation, University Medical Center Hamburg-Eppendorf, Hamburg, Germany

Introduction: Median age at diagnosis for Myelodysplastic syndromes (MDS) is 70 years of age. Treatment guidelines suggest allogeneic stem cell transplantation (HSCT) for intermediate-ll and high risk constellations up to the age of 65 , and reduced intensity conditioning (RIC) regimens are commonly used up to 70 years of age. We performed a retrospective analysis to investigate results after HSCT and influence of KPS on outcome of patients 70 years or older.

Material (or patients) and methods: We analyzed data of 337 patients in the EMBT database $\geq 70$ years with MDS/sAL: RA/RARS/del5q/RCDM-RS $(n=36)$, RAEB/RAEB-1/RAEB-2 ( $n=$ 91), RAEB-t/transformed to $\mathrm{AL}(n=30)$, secondary $\mathrm{AL}$ from diagnosis onwards $(n=100)$. Donor were: related $(n=86)$ and unrelated $(n=251)$. Median age at transplantation was 72 years (70-79 years) with 243 male and 94 female patients. KPS was defined in 294 cases, being $\geq 90 \%$ in $61 \%$ and $80 \%$ or less in $39 \%$. Stem cell source was mainly peripheral blood (94\%). Conditioning regimen was RIC (78\%) or myeloablative (MAC, 22\%).

Results: The number of HSCT for MDS patients $\geq 70$ years increased over time. While 2000-2004 only 19 patients received allogeneic transplantation, the following 3-year periods included 28, 92 and 197 patients, respectively. Estimated 3-year overall survival (OS) was 33\% (95\% Cl: $27-$ $38 \%)$. A significantly better 3-year OS in the univariate analysis was seen for KPS $\geq 90 \%$ vs. $80 \%$ or less ( 41 vs. $22 \%, P=0.008$ ) and for CMV negative sero-status ( $46 \%$ vs. $27 \%, P=0.002)$. Cumulative incidence of relapse at 3 years was $39 \%(95 \% \mathrm{Cl}$ : $31-47 \%)$ and significantly lower with unrelated than related donors ( $23 \%$ vs $43 \%, p=0.003$ ). Disease status was associated with a significantly higher incidence of relapse for "RAEB-t /transformed to AL" compared to "RAEB/RAEB-1/RAEB-2" (49\% vs $23 \%, P=0.012)$. There was only a trend for a lower incidence of relapse after MAC compared to RIC ( $22 \%$ vs $30 \%, P=0.12)$. Cumulative incidence of non-relapse mortality (NRM) at 1 year 
was 36\% (95\% Cl: 30-42). NRM at three years was 51\% (95\% Cl: 43-58\%) and significantly influenced by CMV sero-negativity of the recipient ( $32 \%$ vs $48 \%, P=0.02$ ), by $\mathrm{KPS} \geq 90 \%$ ( $33 \%$ vs $53 \%, P=0.01$ and at 2 years: $32 \%$ vs $47 \%$ ) and related donors ( $34 \%$ vs $45 \%, P=0.04)$. A trend for lower NRM was seen after RIC ( $40 \%$ vs $47 \%, P=0.08)$. In a multivariate analysis (MVA) significant factor for improved OS was KPS $\geq 90 \%$ (HR 0.62: $95 \% \mathrm{Cl}: 0.46-0.85, P<0.001)$ and for worse survival CMV seropositivity (HR 1.57; 95\% Cl: 1.11-2.21, $P=0.01$ ). For relapse the only significant factor was disease status at transplantation for "RAEB-t/transformed to AL" (HR 2.83; 95\% Cl 1.03-7.75, $P=0.04$ ). Significant factors for NRM were KPS $\geq 90 \%$ (HR $0.57 ; 95 \% \mathrm{Cl}: 0.37-0.87, P=0.01)$ and CMV sero-positivity (HR $1.82 ; 95 \% \mathrm{Cl}: 1.15-2.89, P=0.01)$. Use of unrelated donors showed a trend towards worse NRM (HR 1.65; 95\% Cl: $0.97-$ 2.81, $P=0.06$ )

Conclusion: HSCT from related or unrelated donor after MAC or RIC for advanced MDS patients $\geq 70$ is a potential curative treatment option with a 3-year OS of 33\%. Good performance and sero-negativity for CMV in the patient increase the 3-year estimated OS to 41 and $46 \%$, respectively. After careful patient selection, allogeneic HSCT can be offered also to older patients with MDS.

Disclosure of Interest: S. Heidenreich: None declared, D. Ziagkos: None declared, A. van Biezen: None declared, J. Finke: None declared, U. Platzbecker Funding from: Celgene, Novartis, Boehringer, D. Niederwieser Conflict with: Membership on an entity's Board of Directors or advisory committees and Speakers Bureau, H. Einsele Funding from: Celgene, Janssen, Conflict with: Celgene, Janssen, Novartis, Amgen/ Onyx, W. A. Bethge: None declared, M. Schleuning: None declared, D. W. Beelen: None declared, J. Tischer Conflict with: Sanofi-Aventis, A. Nagler Funding from: Novaratis Pharmaceuticals Corporation, B. Glass Funding from: Roche, MSD, Takeda, Riemser, Ctilifesciences, J. Maertens: None declared, C. Richard: None declared, Y. Beguin: None declared, H. Sill Funding from: Celgene, T. de Witte Funding from: Novartis, M. Robin: None declared, N. Kröger: None declared.

\section{P745}

Haploidentical transplant for myelodysplastic syndrome: registry-based comparison with identical-sibling transplant

Y. Wang ${ }^{1, *}$, H.-X. wang $^{2}$, Y.-R. lai ${ }^{3}$, Z.-M. Sun ${ }^{4}$, D.-P. Wu $u^{5}$, M. Jiang ${ }^{6}$, D.-H. Liu', K.-L. Xu ${ }^{8}$, Q.-F. Liu' ${ }^{7}$, L. Liu ${ }^{10}$, J.-B. Wang ${ }^{11}$, F. Gao ${ }^{12}$, J. Ou-Yang ${ }^{13}$, S.-J. $\mathrm{GaO}^{14}{ }^{14}$, L.-P. Xu', X.-J. Huang ${ }^{1}$ on behalf of XJH

${ }^{1}$ Peking University People's Hospital, Peking University Institute of Hematology, ${ }^{2}$ Air Force General Hospital, PLA, beijing, ${ }^{3}$ the First Affiliated Hospital of Guangxi Medical University, Nanning, ${ }^{4}$ Anhui Provincial Hospital, hefei, ${ }^{5}$ The first affiliated hospital of Soochow University, suzhou, ${ }^{6}$ the First Affiliated Hospital of Xinjiang Medical University, Wulumuqi, ${ }^{7}$ PLA General Hospital, beijing, ${ }^{8}$ the Affiliated Hospital of Xuzhou Medical College, xuzhou, ${ }^{9}$ Nanfang Hospital, Southern Medical Universit, guangzhou, ${ }^{10}$ The first Affiliated Hospital of Chongqing Medical University, chongqing, ${ }^{11}$ Aero Center Space Hospital, beijing, ${ }^{12}$ the Affiliated Hospital of North China University Science and Technology, tangshan, ${ }^{13}$ Nanjing Drum Tower Hospital, The Affiliated Hospital of Nanjing University Medical School, nanjing, ${ }^{14}$ the First Affiliated Hospital of Jilin University, changchun, China

Introduction: Encouraging results from small sample of haploidentical donor (HID) hematopoietic stem cell transplantation (HSCT) for patients with myelodysplastic syndrome (MDS) need to be extended. Furthermore, algorithm deriving from outcome comparison between haploidentcial and identical-sibling donors (ISDs) HSCT need to be established.

Material (or patients) and methods: outcomes of 456 MDS patients who received HSCT from HIDs $(n=228)$ or ISDs $(n=228)$ between 2003 and 2013 and reported to the Chinese Bone Marrow Transplantation Registry were analyzed.

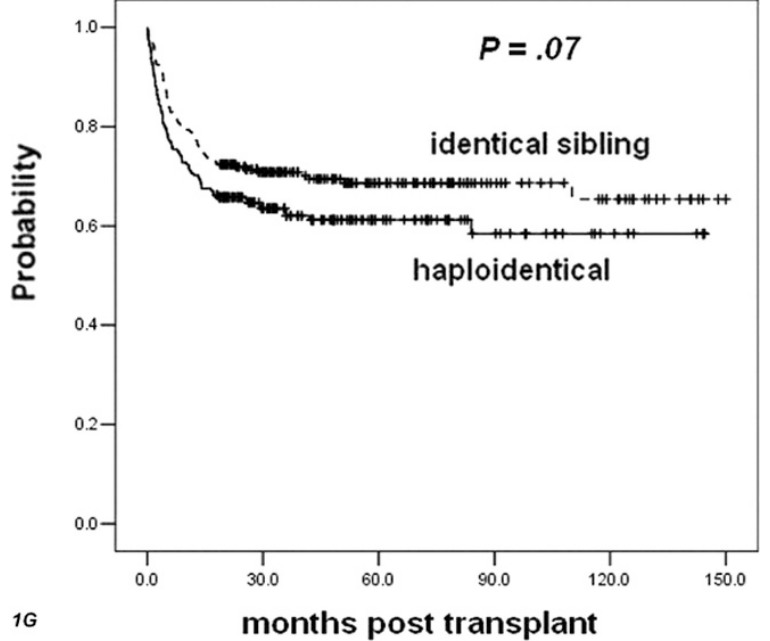

Results: By day 28 post-HSCT, the cumulative incidence of neutrophil recovery was comparable between the HID and ISD group ( $94 \%$ to $98 \% ; P=0.61$ ). By day 100 , the cumulative incidence of grades 2 to 4 acute GVHD was significantly higher in recipients of HID transplantation than ISD transplantation ( $28 \%$ to $15 \% ; P=0.0004$ ); however, the cumulative incidence of grades 3 to 4 aGVHD did not differ between the 2 groups ( $8 \%$ to $8 \% ; P=0.87$ ). In contrast, the 4-year cumulative incidence of chronic GVHD was significantly higher among recipients after ISD than HID transplantation (52\% to $41 \%$, $P=0.04$ )

Among the HID and ISD patients, 4-year cumulative incidences of non-relapse mortality (NRM) were $29 \%$ and $20 \%(P=0.02)$ and of relapse were $9 \%$ and $13 \%$, respectively $(P=0.19)$. Fouryear probabilities of overall-survival were $62 \%$ and $69 \%$ $(P=0.07)$ and of relapse-free-survival were $62 \%$ and $67 \%$ $(P=0.13)$, respectively. However, similar survival was achieved with HID-HSCT in comparison with ISD-HSCT for patients with advanced MDS. In multivariate analysis, a significant differences in NRM $(P=0.03)$ and a trend of difference in survival rates $(P=0.13)$ were found between the 2 cohorts.

Conclusion: The data suggest that in patients with MDS, ISDs remains the best donor source while HIDs could be a valid alternative when an ISD is not available, especially for patients with advanced MDS.

Disclosure of Interest: None declared.

P746

Long-term survival in patients with metastatic breast cancer receiving intensified chemotherapy and stem cell rescue: data from the EBMT registry

M. Martino ${ }^{1, *}$, P. Pedrazzoli ${ }^{2}$, F. Lanza $^{3}$ on behalf of on behalf of Solid Tumor Working Party of EBMT

${ }^{1}$ Oncology and Hematology, Hematology, Stem Cell Collection and Transplantation Unit, Azienda Ospedaliera BMM, Reggio Calabria, ${ }^{2}$ Medical Oncology Unit, IRCCS Policlinico S. Matteo Foundation, Pavia, ${ }^{3}$ Section of Hematology and Bone Marrow Transplant Unit, Cremona, Italy

Introduction: Metastatic breast cancer (MBC) remains a leading cause of cancer death in western countries. Despite advances in diagnosis and treatment, the median survival of women with $\mathrm{MBC}$ that is no longer hormonally responsive or is estrogen receptor (ER)-negative is 18-24 months, and fewer than $5 \%$ are alive and disease free at 5 years. Recently metaanalyses obtained individual patient data from MBC randomized trials that compared HDC to a control therapy without stem-cell support. The settings, HDC achieved a statistically significant improvement in EFS but without a significant improvement in OS. An Italian study suggested that could be a 
role for HDC and AHSCT in delaying disease progression in $\mathrm{MBC}$ and possibly cure a subset of patients harboring chemosensitive tumors.

Material (or patients) and methods: As a contribution to the field of HDC for MBC, we report herethe results of this therapeutic approach in women treated in Europe between 2000 and 2014. Data set for survival analysis has been obtained for 242 patients (median age 47 years, range 2666). Clinical parameters including probability of transplantrelated mortality (TRM), PFS and OS.

Results: The most of transplants were performed using PBSC $(>95 \%)$ and all high-dose conditioning regimens employed included alkylating agents Twenty-nine percent of patients received two courses of HDC with stem cell support (tandem transplant).With a median follow-up of 14 months (range 2172), OS and PFS at 5 and 10 years in the whole population were $46 / 31$ and $21 / 17 \%$, respectively. A total 151 patients are alive with a median OS of 4.13 years (95\% C.I. 2.09-6.17). TRM was $2 \%$. Full data of the study, including subset analysis, will be presented at the meeting.

Conclusion: Our preliminary findings suggest that could be a role for HDC and AHSCT in delaying disease progression and possibly cure a subset of $M B C$ patient.

References: Berry DA, Ueno NT, Johnson MM, Lei X, Caputo J, Rodenhuis $S$ et al.High-dose chemotherapy with autologous stem-cell support as adjuvant therapyin breast cancer: overview of 15 randomized trials. J Clin Oncol 2011; 29:3214-3223. Martino M, Ballestrero A, Zambelli A, Secondino S, Aieta M, Bengala $C$, et al. Long-term survival in patients with metastatic breast cancer receiving intensified chemotherapy and stem cell rescue: data from the Italian registry. Bone Marrow Transplant. 2013 Mar;48(3):414-8

Disclosure of Interest: None declared.

\section{P747}

Preclinical data supporting an adoptive immunotherapy approach in KRAS mutated metastatic colorectal cancer patients with refractory disease

P. Pedrazzoli ${ }^{1, *}$, S. Brugnatelli ${ }^{1}$, I. Turin ${ }^{1}$, F. Ferulli ${ }^{1}$, S. Delfanti ${ }^{1}$, M. Tanzi ${ }^{1}$, D. Lisini ${ }^{2}$, D. Montagna ${ }^{3}$

${ }^{1}$ Fondazione IRCCS Policlinico San Matteo, Pavia, ${ }^{2}$ Fond. IRCCS Istituto Neurologico C. Besta, Milano, ${ }^{3}$ Fondazione IRCCS Pol. San Matteo, Università di Pavia, Pavia, Italy

Introduction: Evidence from randomized studies has established that subjects with KRAS-mutated metastatic colorectal cancer ( $\mathrm{mCRC}$ ) do not derive benefit from the administration of anti-EGFR MAbs, thus causing a poorer outcome in this cohort of patients. With the aim to look for a potential synergistic anti-tumor effect of an anti-EGFR drug and activated autologous NK cells, we investigated whether KRAS mutated mCRC cells were susceptible to anti-EGFR-induced ADCC mediated by ex vivo cytokine-activated autologous NK cells.

Material (or patients) and methods: After obtaining signed informed consent, $10 \mathrm{mCRC}$ patients have been enrolled. $\mathrm{mCRC}$ cells, from metastatic tissue, were expanded in vitro and analyzed to confirm their neoplastic origin. Ligand expression was evaluated by cytofluorimetric analysis and gene expression on mCRC cells. Purified NK cells were activated overnight (ON) with IL-2 or IL-15 and were analyzed for expression of triggering and inhibitory receptors and for their ability to kill autologous $\mathrm{mCRC}$ cells alone or coated with anti-EGFR mAbs in $4-\mathrm{h}$ cytotoxicity assay.

Results: In spite of the mCRC cells express the most important NK ligands, patients resting NK cells were unable to lyse mCRC cells ( $<10 \%$ lysis at E:T ratio of 40:1). The anti-EGFR-induced ADCC was evaluated in 7 patients ( 4 wild type,WT and 3 KRAS mutated). The coating of mCRC cells with control lgG did not influence their susceptibility to NK lysis. Cetuximab-coating of mCRC cells increase NK cell mediated lysis by ADCC mechanism against both wild type and KRAS mutated cells. NK cells incubated ON with medium alone displayed measurable levels of cytotoxic activity against cetuximabcoated mCRC cells (WT, media:22\%, DS 8\%; KRAS, media:21\%, DS 7\%at E:T ratio of 40:1) which were increased after IL-2 (WT, media:35\%, DS 11\%; KRAS, media:39\%, DS 11\%) and IL-15 (WT, media:57\%, DS 19\%; KRAS, media:48\%, DS 8\%at E:T ratio of $40: 1)$.

Conclusion: Results of this study confirm that KRAS mutated mCRC cells can be efficiently lysed by ex vivo activated NK cells which support an ongoing study of immunotherapy with anti-EGFR monoclonal antibody and ex vivo activated autologous NK cells $\mathrm{mCRC}$ patients failing conventional therapies.

References: Normanno N, Tejpar S, Morgillo F, et al. Implication for KRAS status and EGFR targeted therapies in metastatic CRC. Nat Rev Clin Oncol 2009 6(9):519-27.

Ljunggren HG1, Malmberg KJ. Prospects for the use of NK cells in immunotherapy of human cancer. Nat Rev Immunol. 2007 May;7(5):329-39.

Lanier LL. Up on the tightrope: natural killer cell activation and inhibition. Nat. Immunol. 2008 9(10):1091-4.

Roda JM, Joshi T, Butchar JP, McAlees, et al. The activation of natural killer cell effector functions by cetuximab-coated, epidermal growth factor receptor positive tumor cells is enhanced by cytokines. Clin Cancer Res 2007 13(21):6419-28. I Turin, R Schiavo, M Maestri, et al. In Vitro Efficient Expansion of Tumor Cells Deriving from different Types of Human Tumor Samples. Med. Sci. 2014, 2, 70-81.

Montagna Turin I, Schiavo R, Montini E, et al. Feasibility and safety of adoptive immunotherapy with ex vivo-generated autologous, cytotoxic T lymphocytes in patients with solid tumor. Cytotherapy. 2012 Jan;14(1):80-90.

Disclosure of Interest: None declared.

\section{P748}

Salvage high-dose chemotherapy in patients with refractory gestational trophoblastic neoplasia: the EBMT experience

U. De Giorgi i, , E. Kanfer ${ }^{2}$, S. Richard ${ }^{3}$, M. Badoglio ${ }^{4}$, E. Scarpi ${ }^{1}$ J. Apperley ${ }^{2}$, F. Selle, J. H. Bourrhis ${ }^{5}$, E. Nicolas-Virelizier ${ }^{6}$ G. Mangili', M. Renard ${ }^{8}$, A. Bazarbachi ${ }^{9}$, K. Schaefer-Eckart ${ }^{10}$, B. Lioure ${ }^{11}$, C. Bokemeyer ${ }^{12}, F$. Lanza ${ }^{13}$, M. Bregni $^{14}$ on behalf of EBMT Solid Tumours Working Party

${ }^{1}$ Istituto Scientifico Romagnolo per lo Studio e la Cura dei Tumori (IRST) IRCCS, Meldola, Italy, ${ }^{2}$ Imperial College Healthcare NHS Trust, London, United Kingdom, ${ }^{3}$ Hopital Tenon, ${ }^{4} E B M T$ Office, Paris, Institut Gustave-Roussy, Villejuif, ${ }^{6}$ Centre Leon Berard, Lyon, France, ${ }^{7}$ San Raffaele Scientific Institute, Milan, Italy, ${ }^{8}$ University Hospital, Leuven, Belgium, ${ }^{9}$ American University of Beirut Medical Center, Beirut, Lebanon, ${ }^{10}$ Nuernberg klinikum, Nuernberg, Germany, ${ }^{11}$ Hôpital Civil, Strasbourg, France, ${ }^{12}$ Universitätsklinikum Hamburg-Eppendorf, Hamburg, Germany,

${ }^{13}$ Istituti Ospitalieri di Cremona, Cremona, ${ }^{14}$ Ospedale di Busto Arsizio, Varese, Italy

Introduction: A few case reports and small case series reported of salvage high-dose chemotherapy (HDC) and hematopoietic stem cell support (HSCS) in patients with gestational trophoblastic neoplasia (GTN). To date no clear evidence of benefit from HDC has been reported. We conducted a retrospective analysis on patients GTN treated with salvage HDC registered with the European Group for Blood and Marrow Transplantation (EBMT).

Material (or patients) and methods: Between 1985 and 2012, 30 registered patients with GNT, median age 37 years (range 25-60), were treated with salvage HDC. All had received standard-chemotherapeutic treatments before HDC. Seven pts $(23 \%)$ received HDC as second-line therapy, 10 $(33 \%)$ as third-line, and $13(43 \%)$ as fourth- or fifth-line. The conditioning HDC regimens comprised carboplatin in 28 of 30 cases $(93 \%)$. HSCS consisted of peripheral blood progenitor cells in 22 cases $(73 \%)$ and autologous bone marrow in $8(27 \%)$. 
Results: Nine patients (30\%) achieved a complete response (CR), 3 (10\%) a marker-negative partial remission (PRm-), 1 (3\%) a marker-positive partial remission, 3 (10\%) stable disease, 9 (30\%) progressive disease, whereas five toxic deaths were reported $(17 \%)$ including two in the first two cases treated with HDC in 80 's. With a median follow-up of 11 months (range 1-312), 8 of 30 patients (27\%) continue to be free of relapse/progression. Three of the 4 patients who had a disease recurrence after a CR/PRm- from HDC received further treatments and were alive after 12, 44 and 96 months, respectively. In total, 12 of 30 patients (40\%) were progressionfree following HDC at a median follow-up of 44 months (range 6-312 months).

Conclusion: HDC based on carboplatin seems to be active in this heavily pretreated patient population with refractory GTN. Salvage HDC might represent a possible option for patients with refractory GTN.

Disclosure of Interest: None declared.

P749

Results of tandem high-dose chemotherapy and autologous stem cell transplantation using thiotepa in children with high-risk medulloblastoma

V. Daylidite ${ }^{1, *}$ on behalf of Vidmante V. Daylidite, Natalia N. Subbotina, Igor S. Dolgopolov, Stepan S. Babelyan, Andrey S. Levashow, Georgy L. Mentkevich

'bone marrow transplantation, 1Institute of Pediatric oncology and hematology, RCRC Moscow, Russia, Moscow, Russian Federation

Introduction: High-risk (HR) medulloblastoma $(\mathrm{MB})$ includes pts with R1(except desmoplastic MB) M+, C-myc amplification and/or isochromosome 17. This group of pts is characterized by poor long-term survival. Administration of high-dose thiotepa may improve the prognosis in high-risk patients.

Material (or patients) and methods: Our strategy is based on tandem HDCT with thiophosphamide (TT) $300 \mathrm{mg} / \mathrm{m} 2$ (for pts under 36 months $-10 \mathrm{mg} / \mathrm{kg}$ ) on days $-4,-3$ and carboplatin (Carbo) $510 \mathrm{mg} / \mathrm{m} 2$ (for pts under 36 months $-17 \mathrm{mg} / \mathrm{kg}$ ) on days $-4,-3$ on consolidation stage followed by autologous stem cell transplantation (ASCT). From 2012 till 201513 patients with $\mathrm{MB}$ of poor prognosis were eligible for tandem HDCT based on $\Pi$ and Carbo. The median age was 7 (2-25) years. All pts got CT and age adjusted RT at induction. Regimen-related toxicity was mild, there was no toxic mortality. Toxicity didn't increase after course 2 .

Results: The median time for hematologic recovery after the first course: $\mathrm{WBC}>1 \times 10^{9} / \mathrm{I}$ - on day $+10, \mathrm{PLT}>20 \times 10^{9} / \mathrm{I}$ on $\mathrm{d}$ +15 ; after the second course: $W B C>1 \times 10^{9} / /$ - on day +11 , PLT $>20 \times 10^{9} / \mathrm{l}$ - on $\mathrm{d}+15$. Three (23\%) patients relapsed 17,20 and 23 months after end of treatment. Six (46\%) pts are in complete remission with a median follow-up 13.8 (7 - 24) months, 4 pts $(30.7 \%)$ have stable disease without signs of progression with a median follow-up 13.5 (5 - 25) months. Conclusion: This study demonstrates that tandem HDCT using $\Pi$ and Carbo was generally tolerable. $46 \%$ pts achieved complete remission. General survival on protocol $77 \%$ with a median follow-up 13.7 (5 - 25) months in this HR group.

Disclosure of Interest: None declared. 